\title{
EVALUATING GEOMORPHIC CHANGE IN \\ LITTLE CREEK USING REPEATED CROSS-SECTIONAL AND LONGITUDINAL PROFILE SURVEYS
}

A Thesis Presented to the Faculty of California Polytechnic State University, San Luis Obispo

\author{
In Partial Fulfillment of \\ the Requirements for the Degree \\ Master of Science in Forestry Sciences
}

Drew Allen Perkins

March 2012 
(C) 2012

Drew Allen Perkins

ALL RIGHTS RESERVED 
COMMITTEE MEMBERSHIP

TITLE:

EVALUATING GEOMORPHIC CHANGE IN LITTLE CREEK USING REPEATED CROSSSECTIONAL AND LONGITUDINAL PROFILE SURVEYS.

AUTHOR:

Drew Allen Perkins

DATE SUBMITTED: $\quad$ March 2012

COMmitee CHAIR: Brian C. Dietterick, Ph.D., P.H. Professor of Hydrology and Watershed Management, Natural Resources Management and Environmental Sciences Department

COMMITTEE MEMBER: Samantha J. Gill, Ph.D., RPF. Professor of Forest Measurements and Management, Natural Resources Management and Environmental Sciences Department \& BioResource and Agricultural Engineering Department

COMMITTEE MEMBER: Antonio F. Garcia, Ph.D. Associate Professor of Geology, Physics Department 


\section{Abstract}

\section{EVALUATING GEOMORPHIC CHANGE IN LITTLE CREEK USING REPEATED CROSS-SECTIONAL AND LONGITUDINAL PROFILE SURVEYS.}

Six geomorphic study reaches were established in 2002 along a forested mountain stream (gradients range from 0.02 to 0.05) on Cal Poly's Swanton Pacific Ranch in Santa Cruz County, California. These study reaches are a component of paired and nested watershed studies in the approximately 500 hectare Little Creek watershed. The overall goal of this study was to monitor water quality and channel conditions before, during, and after a selective harvest of redwood. A selective harvest occurred in the North Fork of Little Creek in Summer 2008. In August 2009, approximately $90 \%$ of the Little Creek Watershed was burned in the Lockheed Fire. Channel change was evaluated by measuring ground profiles using traditional survey methods. Cross section and longitudinal profiles are surveyed annually every summer in the six study reaches. Change is assessed through evaluation of cross sections and longitudinal profiles, analysis of bed elevation and cross-sectional area change data, and analysis of residual pool characteristics and longitudinal profile variability. Changes in the channel during this time have been relatively small and are typically associated with movement or introduction of coarse woody debris to the stream channel. However, during the study period no large streamflow events occurred (return interval at the closest USGS gaging station does not exceed 5 years). Historically, large debris flow events have occurred in this watershed, with well documented events in 1955 and 1998. The survey data is an important tool for understanding change detection in channel characteristics before and after harvesting, and following fire disturbance. 


\section{Acknowledgments}

This project would not have happened without the work and support of many different people. I would like to thank all of the graduate students that have worked on the Little Creek Project, Brooke Akers, Ryan Hilburn, Mike Gaedeke, Russ White, Drew Loganbill, and Mary Crable, as well as the many interns who helped collect the annual survey data. I would like to thank my advisor Brian Dietterick for all his help, guidance, and for giving me the opportunity to work at Swanton. I would like to thank my committee for the excellent feedback that greatly improved this document. I would like to thank my family and wonderful girlfriend Kerri for all of their love and support. Finally, I would like to thank $\mathrm{Al}$ Smith for sharing Swanton Pacific Ranch with Cal Poly students. Being here has changed my life and I will be forever thankful for his wonderful gift.

This project was funded by the McIntire-Stennis and Agricultural Research Initiative grant programs. 


\section{Contents}

$\begin{array}{ll}\text { List of Tables } & \text { ix }\end{array}$

List of Figures $\quad$ x

1 Introduction 1

1.1 Goals and objectives . . . . . . . . . . . . . . . . 2

2 Background 4

2.1 Little Creek Study . . . . . . . . . . . . . . . . . . . . 4

2.1.1 Paired and Nested Watershed Study . . . . . . . . . . . 5

2.1.2 Sediment Source Survey . . . . . . . . . . . . . . 6

$2.1 .3 \quad$ LiDAR . . . . . . . . . . . . . . . . 6

2.2 Study Location . . . . . . . . . . . . . . . . . . . . . . 7

2.2 .1 Climate . . . . . . . . . . . . . . . . 7

2.2.2 Geology and Geomorphology . . . . . . . . . . . . . 9

2.2.3 Vegetation ...................... . . . . 11

2.2.4 Ownership and Land Use . . . . . . . . . . . . . . . . 12

3 Literature Review $\quad 15$ 
4 Methodology 20

4.1 Study Design . . . . . . . . . . . . . . . . . . . 20

4.2 Reach Descriptions . . . . . . . . . . . . . . . . . . . 22

4.3 Equipment and Procedures . . . . . . . . . . . . . . . . 25

4.3 .1 Cross sections . . . . . . . . . . . . . 25

4.3.2 Longitudinal profiles . . . . . . . . . . . . . . 26

4.4 Measurement Errors . . . . . . . . . . . . . . . . . . . 27

4.4.1 Sources of error . . . . . . . . . . . . . . 27

4.4.2 Estimates of error from literature . . . . . . . . . . . . 32

4.4 .3 Repeated surveys . . . . . . . . . . . . . . 33

5 Analysis and Results $\quad 36$

5.1 Data Management .................. 36

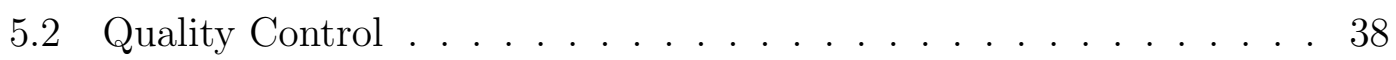

5.2.1 Longitudinal profile adjustment . . . . . . . . . . . . . 39

5.3 Qualitative Analysis . . . . . . . . . . . . . . 41

$5.3 .1 \quad$ Reach A . . . . . . . . . . . . . . . . . . 42

5.3 .2 Reach B ..................... . . . 43

5.3 .3 Reach C . . . . . . . . . . . . . . . . . . 45

5.3 .4 Reach D . . . . . . . . . . . . . . . 48

5.3 .5 Reach E .................... 50

5.3 .6 Reach $\mathrm{F} \ldots \ldots \ldots \ldots$. . . . . . . . . . . . . . . . 51

5.4 Quantitative Analysis . . . . . . . . . . . . . . . . 52

5.4 .1 Cross sections . . . . . . . . . . . . . . . . 52

5.4 .2 Longitudinal profiles . . . . . . . . . . . . . . . . 62 
$\begin{array}{lll}6 & \text { Discussion } & 77\end{array}$

6.1 Limitations of Data . . . . . . . . . . . . . . . . 77

6.1.1 Spatial data limitations . . . . . . . . . . . . . . 78

6.1.2 Data limitations due to sampling interval . . . . . . . . 79

6.1.3 Limitations of data interpretation . . . . . . . . . . . . 81

6.2 Observed Changes and Interpretations _ . . . . . . . . . . 82

6.3 Flow Regime During Study Period . . . . . . . . . . . . . . 83

6.4 Observations in Context of Morphology and Processes in Little Creek 87

6.4.1 Stream classification . . . . . . . . . . . . 87

6.4.2 Streamside landslides and debris flows . . . . . . . . . . 89

6.4.3 Influence of large woody debris . . . . . . . . . . . . . 91

6.4.4 Conceptual model of morphologic changes in Little Creek . . 92

7 Conclusion

7.1 Future Work and Opportunities . . . . . . . . . . . . . . 96

7.2 Data Archiving and Preservation . . . . . . . . . . . . . 97

Bibliography

A Graphs of All Cross Sections 
C.1 Cross section Instructions _ . . . . . . . . . . . . . . . . 133

C.1.1 Equipment . . . . . . . . . . . . . . . . . 133

C.2 Longitudinal Profile Instructions . . . . . . . . . . . . . . . 139

C.2.1 Equipment . . . . . . . . . . . . . . . . . . . . 139

C.2.2 Setup . . . . . . . . . . . . . . . . . . 140

C.2.3 Surveying . . . . . . . . . . . . . . . . . . . . . 140

C.3 Pebble Count . . . . . . . . . . . . . . . . . . . . . . . . . . . 142

D Survey Data for All Cross Sections and Longitudinal Profiles

D.1 Cross Section Data . . . . . . . . . . . . . . . . . . . . 143

D.2 Cross Section Offsets . . . . . . . . . . . . . . . . 297

D.3 Longitudinal Profile Data . . . . . . . . . . . . . . . . . 305

E VBA code 


\section{List of Tables}

4.1 Table showing who collected channel geometry data each year. . . . 21

4.2 Median particle diameters for each study reach . . . . . . . . . . 33

4.3 Thalweg and cross-sectional area measurements for 30 cross sections repeated in $2002 \ldots \ldots \ldots$

5.1 Table showing missing data . . . . . . . . . . . . . . 40

5.2 Table of reach gradients . . . . . . . . . . . . . . 64

5.3 Standard deviation of longitudinal profile residuals calculated from a best fit line. . . . . . . . . . . . . . . . . . 6 65

5.4 Table of residual pool statistics . . . . . . . . . . . . . 71

6.1 Peak flows and return intervals for the San Lorenzo River at Big Trees from 1994-2008. . . . . . . . . . . . . . . 85

6.2 Table of cross section metric standard deviation for all reaches compared to annual peak flows. . . . . . . . . . . . 86

6.3 Pearson's correlation matrix between standard deviation of annual cross section changes and peak flows. . . . . . . . . . . 86

6.4 Table of sensivity of alluvial channel types to increases in sediment supply. From Montgomery \& MacDonald (2002). Used with permission. . . . . . . . . . . . . . . . . . . . . 88

C.1 Table of existing benchmark elevations. . . . . . . . . . . . . 136

D.1 All cross section survey data . . . . . . . . . . . . . . 143

D.2 Cross section offsets _ . . . . . . . . . . . . . . 298

D.3 Permanent point and rebar coordinates. Units are in feet, CA State Plane Coordinates. . . . . . . . . . . . . . . . 305

D.4 Adjusted longitudinal profile data. . . . . . . . . . . . . . . 308 


\section{List of Figures}

2.1 Little Creek Location Map . . . . . . . . . . . . . . . . . . . 8

2.2 Geologic map and topography of the Little Creek Watershed . . . . 10

2.3 Photo of 1955 flood damage in Swanton. . . . . . . . . . . . . . 11

2.4 Aerial photo from 1957 showing mouth of Little Creek and extent of 1955 debris flow deposits. Image from Scotts Creek Watershed Council website. . . . . . . . . . . . . . . . . . 12

2.5 Burn severity map from the 2009 Lockheed Fire. . . . . . . . . . . . 14

4.1 Map of study reach locations in Little Creek. . . . . . . . . . . . . . 21

4.2 Diagram showing how lack of a rod level could cause errors in measurement . . . . . . . . . . . . . . . . . . . 29

4.3 Map of points surveyed for cross sections and longitudinal profiles

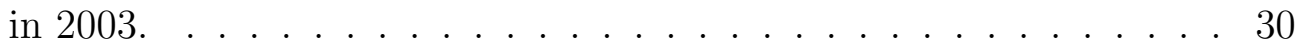

4.4 Figure showing repeated surveys in 2002. Units are in feet. . . . . . 34

5.1 Screen shot of cross section viewer spreadsheet. . . . . . . . . . . 37

5.2 Figure showing before and after rebar correction in the A6 cross section. . . . . . . . . . . . . . . . . . . . 39

5.3 Reach D longitudinal profile before and after adjustment. . . . . . . 41

5.4 Cross section A6 . . . . . . . . . . . . . . . . . . . . . . 42 
5.5 Cross section A10 . . . . . . . . . . . . . . . . 43

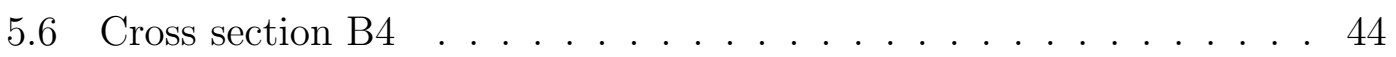

5.7 Cross section B8 f . . . . . . . . . . . . . . . 44

5.8 Cross section B10 . . . . . . . . . . . . . . . . 44

5.10 Cross section $\mathrm{C} 4 \ldots \ldots \ldots \ldots . \ldots . \ldots . \ldots 45$

5.9 Upper Reach C, Fall 2009 after the Lockheed Fire . . . . . . . . . . 46

5.11 Cross section C5 . . . . . . . . . . . . . . 46

5.12 Cross section $\mathrm{C} 7 \ldots \ldots \ldots$. . . . . . . . . . . . . . . 47

5.13 Cross section C 8 . . . . . . . . . . . . . . . . . . . 47

5.14 Reach C upper section longitudinal profile . . . . . . . . . . . . 48

5.15 Cross section D5 . . . . . . . . . . . . . . . . 49

5.16 Cross section D9 . . . . . . . . . . . . . . . . . 49

5.17 Reach D longitudinal profile . . . . . . . . . . . . . . . . 49

5.18 Reach E, Fall 2009 after the Lockheed Fire. . . . . . . . . . . . . . 50

5.19 Cross section $\mathrm{E} 1 \ldots \ldots \ldots \ldots$

5.20 Cross section E4 f . . . . . . . . . . . . . . . 51

5.21 Cross section F4 . . . . . . . . . . . . . . 52

5.22 Cross section F5 . . . . . . . . . . . . . . . . 52

5.23 Thalweg elevation change calculated from cross sections and longitudinal profile from Reach A. . . . . . . . . . . . . . . 53

5.24 Boxplot of Thalweg Elevation Change . . . . . . . . . . . . . . 55

5.25 Boxplot of Bankfull Area Change . . . . . . . . . . . . . . 58

5.26 Boxplot of Mean Bed Elevation Change . . . . . . . . . . . . . . . 60 
5.27 Standard deviations of longitudinal profile residuals . . . . . . . . 66

5.28 Residual pool depths in each reach . . . . . . . . . . . . . . 68

5.29 Graph showing mean residual depths. . . . . . . . . . . . . . . 72

5.30 Graph showing standing deviations of residual depths. . . . . . . . . 73

5.31 Graph showing third quartile of residual depths. . . . . . . . . . . 74

5.32 Cumulative frequency graph of residual depths in all reaches. . . . . 76

6.1 Annual peak flows at USGS San Lorenzo River at Big Trees gage . 84

6.2 Comparison of peak flows in Scotts Creek and the San Lorenzo River 85

6.3 Map of nearstream landslides . . . . . . . . . . . . . . . . . 90

6.4 Log and Boulder Jam in Mainstem of Little Creek. . . . . . . . . . 91

6.5 Conceptual model of channel change in Little Creek. . . . . . . . . 94

A.1 Little Creek Cross Section A1 . . . . . . . . . . . . . . . . . . 105

A.2 Little Creek Cross Section A2 . . . . . . . . . . . . . . 105

A.3 Little Creek Cross Section A3 . . . . . . . . . . . . 106

A.4 Little Creek Cross Section A4 . . . . . . . . . . . . . . . 106

A.5 Little Creek Cross Section A5 . . . . . . . . . . . 106

A.6 Little Creek Cross Section A6 . . . . . . . . . . . . . . . 107

A.7 Little Creek Cross Section A7 . . . . . . . . . . . . . . 107

A.8 Little Creek Cross Section A8 . . . . . . . . . . . . . 107

A.9 Little Creek Cross Section A9 . . . . . . . . . . . . . . . . 108

A.10 Little Creek Cross Section A10 . . . . . . . . . . . . . . 108

A.11 Little Creek Cross Section B1 . . . . . . . . . . . . . . . 108 
A.12 Little Creek Cross Section B2 . . . . . . . . . . . . . . 109

A.13 Little Creek Cross Section B3 . . . . . . . . . . . . . . . . . 109

A.14 Little Creek Cross Section B4 . . . . . . . . . . . . . . . . . 109

A.15 Little Creek Cross Section B5 . . . . . . . . . . . . . . . . . 110

A.16 Little Creek Cross Section B6 . . . . . . . . . . . . . . . . . . 110

A.17 Little Creek Cross Section B7 . . . . . . . . . . . . . . . . . 110

A.18 Little Creek Cross Section B8 . . . . . . . . . . . . . . . . 111

A.19 Little Creek Cross Section B9 . . . . . . . . . . . . . . . . . 111

A.20 Little Creek Cross Section B10 . . . . . . . . . . . . . . 111

A.21 Little Creek Cross Section C1 . . . . . . . . . . . . . . . . . 112

A.22 Little Creek Cross Section C2 . . . . . . . . . . . . . . . . . 112

A.23 Little Creek Cross Section C3 . . . . . . . . . . . . . . . . . 112

A.24 Little Creek Cross Section C4 . . . . . . . . . . . . . . . . 113

A.25 Little Creek Cross Section C5 . . . . . . . . . . . . . . . . 113

A.26 Little Creek Cross Section C6 . . . . . . . . . . . . . . . . . 113

A.27 Little Creek Cross Section C7 . . . . . . . . . . . . . . . 114

A.28 Little Creek Cross Section C8 . . . . . . . . . . . . . . . . 114

A.29 Little Creek Cross Section C9 . . . . . . . . . . . . . . . . . . . . 114

A.30 Little Creek Cross Section C10 . . . . . . . . . . . . . 115

A.31 Little Creek Cross Section D1 . . . . . . . . . . . . . . 115

A.32 Little Creek Cross Section D2 . . . . . . . . . . . . . . 115

A.33 Little Creek Cross Section D3 . . . . . . . . . . . . 116

A.34 Little Creek Cross Section D4 . . . . . . . . . . . . . . . 116 
A.35 Little Creek Cross Section D5 . . . . . . . . . . . . . . . . 116

A.36 Little Creek Cross Section D6 . . . . . . . . . . . . . . . 117

A.37 Little Creek Cross Section D7 . . . . . . . . . . . . . . . . . 117

A.38 Little Creek Cross Section D8 . . . . . . . . . . . . . . . . . . 117

A.39 Little Creek Cross Section D9 . . . . . . . . . . . . . . . . . . . 118

A.40 Little Creek Cross Section D10 . . . . . . . . . . . . . . 118

A.41 Little Creek Cross Section E1 . . . . . . . . . . . . . . . . 118

A.42 Little Creek Cross Section E2 . . . . . . . . . . . . . . . . . . 119

A.43 Little Creek Cross Section E3 . . . . . . . . . . . . . . . . . 119

A.44 Little Creek Cross Section E4 . . . . . . . . . . . . . . . . . 119

A.45 Little Creek Cross Section E5 . . . . . . . . . . . . . . . . 120

A.46 Little Creek Cross Section E6 . . . . . . . . . . . . . . . . 120

A.47 Little Creek Cross Section E7 . . . . . . . . . . . . . . . . 120

A.48 Little Creek Cross Section E8 . . . . . . . . . . . . . . . . . 121

A.49 Little Creek Cross Section E9 . . . . . . . . . . . . . . . 121

A.50 Little Creek Cross Section E10 . . . . . . . . . . . . . . . . 121

A.51 Little Creek Cross Section F1 . . . . . . . . . . . . . . . . . . . 122

A.52 Little Creek Cross Section F2 . . . . . . . . . . . . . . . . . . 122

A.53 Little Creek Cross Section F3 . . . . . . . . . . . . . . . . 122

A.54 Little Creek Cross Section F4 . . . . . . . . . . . . . . . 123

A.55 Little Creek Cross Section F5 . . . . . . . . . . . . . . . . 123

A.56 Little Creek Cross Section F6 . . . . . . . . . . . . . . 123

A.57 Little Creek Cross Section F7 . . . . . . . . . . . . . . . . . . 124 
A.58 Little Creek Cross Section F8 . . . . . . . . . . . . . . . . . . . . . 124

A.59 Little Creek Cross Section F9 . . . . . . . . . . . . . . . . . 124

A.60 Little Creek Cross Section F10 . . . . . . . . . . . . . . . . . 125

B.1 Reach A longitdudinal profile. . . . . . . . . . . . . . 127

B.2 Reach B longitdudinal profile. . . . . . . . . . . . . . . 128

B.3 Reach C longitdudinal profile. . . . . . . . . . . . . . . . . 129

B.4 Reach D longitdudinal profile. . . . . . . . . . . . . . 130

B.5 Reach E longitdudinal profile. . . . . . . . . . . . . . . 131

B.6 Reach F longitdudinal profile. . . . . . . . . . . . . . . . . 132 


\section{Chapter 1}

\section{Introduction}

The shape, size, and gradient of an alluvial stream channel are functions of the amount of sediment supplied to the channel, vegetation on the banks, woody debris in the channel, and the magnitude and frequency of streamflow events (Montgomery \& Buffington, 1998). Channel shape and condition have direct impacts on aquatic habitat quality. An understanding of channel stability and rates of change is also important to planning, managing, and mitigating human activities and development in the riparian area. Land use history (both upslope and in the riparian zone) and variation in climate have the ability to change all of the factors that influence channel shape and form. Observing changes in the channel may provide insights into the impacts on the riparian system from land management and changing climatic conditions that may be difficult or impossible to detect in other ways.

The Little Creek study is a multi-faceted study designed primarily to evaluate the ability of the current California Forest Practice Rules to maintain water quality and channel conditions during and after a selective timber harvest in the Santa 
Cruz Mountains. In 2002, six channel morphology study reaches were established in Little Creek. In each reach there are several permanent benchmarks and ten monumented, permanent cross sections. The cross sections and a longitudinal profile through the reach are surveyed annually. This thesis evaluates the channel morphology data collected from 2002 through 2008 when the North Fork of Little Creek was selectively harvested.

Luna Leopold, an influential pioneer of quantitative geomorphology, stated that "Monumented benchmarks from which a survey may be repeated are a gift to the future" (Leopold et al., 2005). Besides providing a baseline for evaluating the direct impacts that may have occurred from this timber harvest, the data collected in this project provides valuable information that will help future scientists interpret field observations. Geomorphic processes (and the tectonic and climatic factors that drive them) often occur at rates that are highly variable over time. It is almost impossible within one person's lifetime, let alone a graduate study, to make observations over a period long enough to understand the rates and variability of these processes. Observations that are well documented and repeatable allow future scientists to understand the timescales that these natural systems may operate on. An important part of this thesis is providing a long-term repository for these data, as well as ensuring that continued work meets the standards needed for this data set to be valuable in the future.

\subsection{Goals and objectives}

This project and document have several objectives:

1. Evaluate magnitude and characteristics of geomorphic change observed in study reaches by analyzing data collected from 2002-2008. 
2. Determine factors that influence channel change in Little Creek.

Besides analyzing and interpreting data collected, another important part of this project is ensuring this data is useful to future scientists. There are two other goals of this project addressed in this thesis.

1. Critically evaluate methods used in past and modify them as needed to provide a robust long-term dataset of channel geometry.

2. Provide baseline information that can be used in the future to evaluate effects of the fire, future flood events, and human activity in the watershed. Ensure that data and benchmarks can be found in the future. 


\section{Chapter 2}

\section{Background}

\section{$2.1 \quad$ Little Creek Study}

The work described in this thesis is part of the long-term Little Creek Study. The Little Creek Study (study) was designed to evaluate water quality and channel conditions, before, during and after a selective timber harvest. Little Creek is located within Swanton Pacific Ranch which serves as the school forest for the Natural Resources Management and Environmental Sciences Department at Cal Poly San Luis Obispo. The 3280-acre ranch is located in the Santa Cruz Mountains north of Davenport, CA. Little Creek is an approximately $5.5 \mathrm{~km}^{2}$ tributary to Scotts Creek, which drains to the Pacific Ocean. Little Creek contains 1st and 2nd order channels as delineated on the 1:24,000 scale Davenport Quadrangle USGS topographic map. The long-term study began in 1997 with the construction of the Mainstem, North Fork, and South Fork flumes on Little Creek and installation of two rain gages, one near the Red House near the confluence with Scotts Creek, and the other at Landing 23 on the ridge between the North Fork and South Fork 
tributaries. In the winter of 1997-1998 flood flows damaged the flumes. Repairs were made and additional instrumentation was purchased; successful monitoring began during the 2000-2001 winter. An additional natural channel gaging station was added in 2001 at the upper property line (Upper North Fork Flume). Crosssectional and longitudinal profiles were established and first measured in 2002. The selective timber harvest occurred in the North Fork of Little Creek in the summer of 2008 using ground-based and cable yarding methods.

\subsubsection{Paired and Nested Watershed Study}

The primary purpose of the watershed study is to evaluate potential changes from the North Fork harvest on flow, turbidity, and suspended sediment load using both paired (North Fork/South Fork) and nested (North Fork/Upper North Fork) comparisons. The North Fork, South Fork, and Mainstem monitoring stations utilize natural-bottom, rated-section flumes constructed with redwood walls and a concrete sill at the downstream end. The Upper North Fork station utilizes a rated natural channel. Additional monitoring stations were added on two small tributaries in the 2007-2008 winter. Hourly water samples are taken during storm events and analyzed for turbidity and suspended sediment concentration. The methodology is based on the Caspar Creek Study (operated by the USDA Forest Service Pacific Southwest Research Station) on Jackson State Demonstration Forest. The suspended sediment data collected in Little Creek during the pre-harvest calibration period is discussed in a thesis by Gaedeke (2006). Temperature data is also measured at each station during the summer months. 


\subsubsection{Sediment Source Survey}

Another aspect of the paired watershed study is an annual near-stream sediment source survey developed by former graduate student Brooke Akers. The purpose of the near-stream sediment source survey is to identify, quantify and monitor sediment inputs to Little Creek from near-stream landslides and streambank erosion, which provides another way of documenting changes along the watercourse. The location, size, and characteristics of streamside landslides and bank erosion features greater than $0.75 \mathrm{~m}^{3}$ are documented in the survey. The initial survey was performed in 2002, with subsequent surveys in 2006, 2009, 2010, and 2011. The initial survey primarily identified features created by the February 1998 flood events where significant bank erosion and streamside landsliding occurred.

\subsubsection{LiDAR}

LiDAR data covering the Little Creek watershed has been collected in 2002, 2008, 2010 and 2011. Hilburn (2010) compared LiDAR-generated cross-sectional profiles to ground-surveyed profiles using the 2002 data. LiDAR generated cross sections had a vertical error of $0.61 \mathrm{~m}$ RMSE compared to ground-surveyed cross sections. The relatively low horizontal resolution (average of one ground return every $5.2 \mathrm{~m}^{2}$ ) and vertical error made reliable detection of changes in the bankfull channel difficult. Comparisons of the 2008 and $2010 \mathrm{LiDAR}$ data to the ground-surveyed cross sections has also been completed (Dietterick et al., 2012). Improvements in technology allow much higher sampling intensities and closer-spaced ground returns. Bankfull width was more accurately measured than bankfull depth or cross-sectional area. In 2010, two thirds of the LiDAR-derived cross-sections provided bankfull width within 10 percent $(0.46 \mathrm{~m})$ of the field-surveyed width. 
The ground-surveyed longitudinal profiles were also compared in both plan and profile to LiDAR-derived longitudinal profiles (White, 2010). The LiDAR-derived channel planform position had an average 95 percent buffer width of $2.73 \mathrm{~m}$ (meaning 95 percent of the derived channel position was within $2.73 \mathrm{~m}$ of the surveyed channel position), a mean absolute difference in channel gradient of 0.0049 , and a mean absolute difference in channel length of 2 percent.

\subsection{Study Location}

The study location is Cal Poly's Swanton Pacific Ranch in Little Creek, a tributary to Scotts Creek in western Santa Cruz County, California (Figure 2.1).

\subsubsection{Climate}

The Santa Cruz mountains have a Mediterranean climate with mild, wet winters and warm, dry summers. The Scotts Creek watershed drains the western side of Ben Lomond Mountain and local climate is highly variable and is driven primarily by distance from the ocean, elevation and aspect. In summer, temperatures are usually cool near the ocean where summer fog is common, and warmer inland and at higher elevations. In winter, rainfall is primarily driven by orographic processes and varies greatly with elevation. The elevation of Little Creek at its confluence with Scotts Creek is $9 \mathrm{~m}$ and the elevation of the highest ridgeline is $550 \mathrm{~m}$. Mean annual precipitation is $75 \mathrm{~cm}$ at the mouth of the watershed and $125 \mathrm{~cm}$ at the upper ridgelines. Almost all precipitation falls between October and May and snow is very rare. Swanton Pacific Ranch operates 10 rain gages in the Scotts Creek Watershed; 8 are located in the Little Creek subwatershed. 


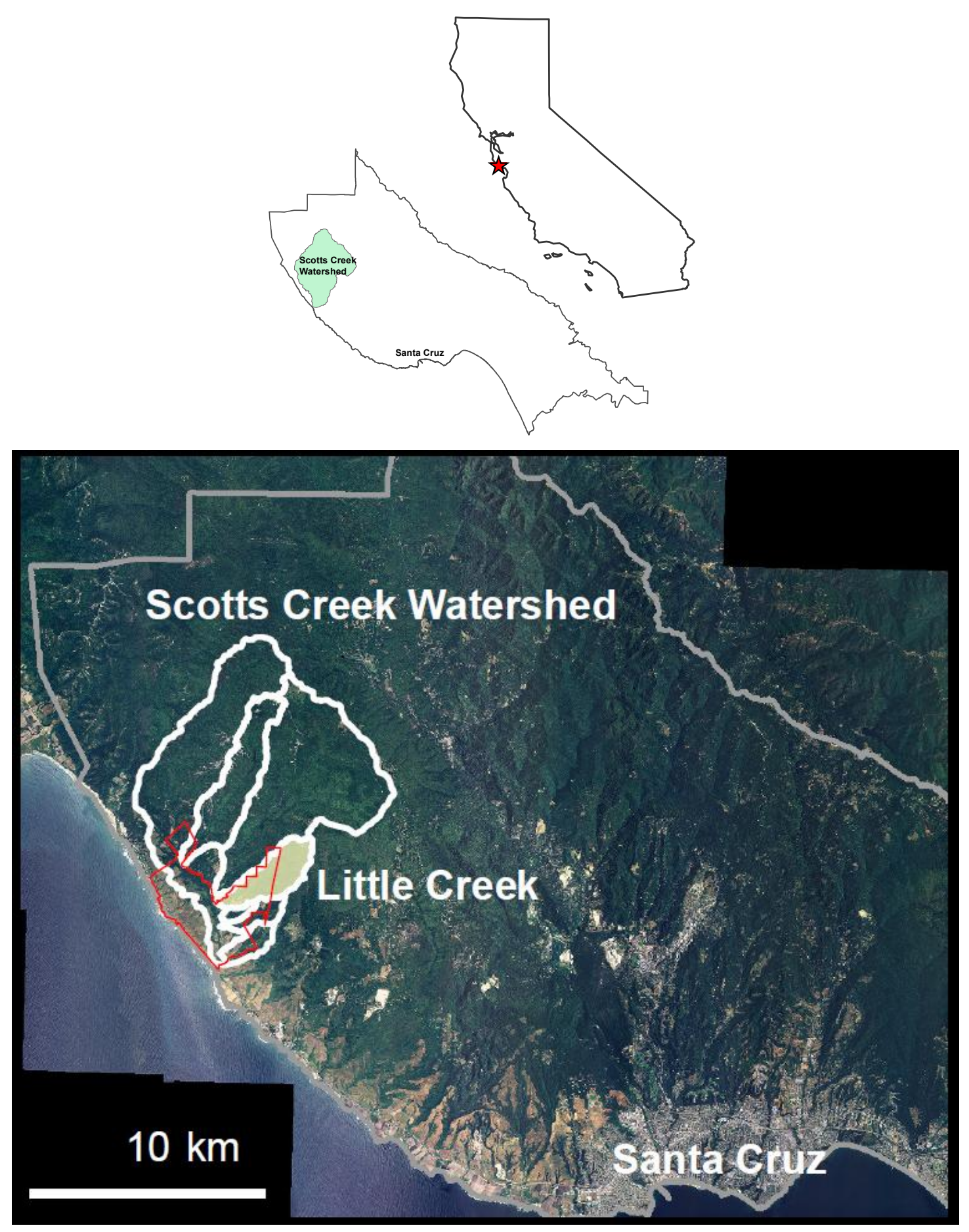

Figure 2.1: Little Creek Location Map. Swanton Pacific Ranch boundary is shown in red. 


\subsubsection{Geology and Geomorphology}

The Little Creek watershed is located in the tectonically active Santa Cruz Mountains. A restraining bend in the San Andreas Fault has resulted in rapid regional uplift and associated stream incision (Anderson, 1994). Much of Little Creek has inner gorge characteristics with steep side slopes leading down to the stream channel (slopes of 70 percent and steeper are common). Streamside landslides and bank failures as well as excavated colluvial hollows are common along most of the channel length. Topographic evidence (scarps and associated midslope benches, hummocky topography) for larger-scale, rotational, deep-seated slides of varying ages covers most of the watershed.

The watershed is underlain by four different rock types. Westward dipping Santa Cruz Mudstone and Santa Margarita Sandstone (Upper Miocene) comprise the western half of the watershed and the upper ridgelines. These sedimentary rocks unconformably overlay the igneous Ben Lomond Mountain quartz diorite (Cretaceous) and metamorphic rocks (schist and small amounts of marble) that are exposed in the lower slopes of the stream valleys and at upper elevations at the eastern edge of the watershed (Figure 2.2). 


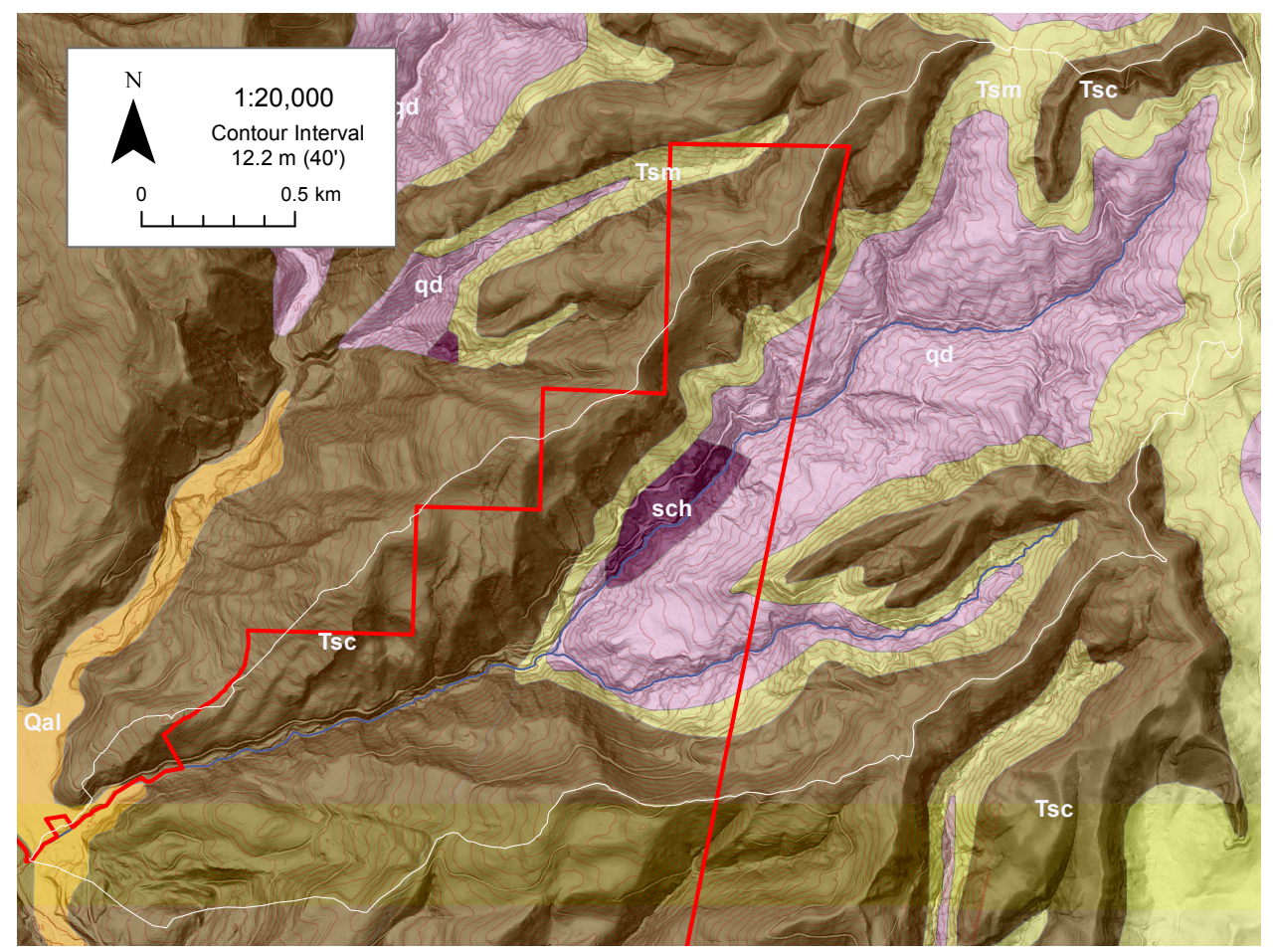

Figure 2.2: Geologic map and topography of the Little Creek Watershed (geology from Brabb (1989)). Topography and hillshade from 1m LiDAR derived DEM from 2010 flight. Contour Interval 40' (12.2m). Swanton Pacific Ranch Boundary shown in red. Key to geologic units: Tsc (brown) - Santa Cruz Mudstone; Tsm (tan) - Santa Margarita Sandstone; qd (pink) - quartz diorite; sch (purple) schist; Qal (orange) - Quaternary alluvial deposits.

Evidence for large-scale landsliding and debris flows is common in the Little Creek watershed. In December of 1955, a debris flow originated in the South Fork of Little Creek and traveled downstream to the confluence with Scotts Creek destroying structures and causing loss of life in Swanton Valley. This debris flow transported logs and large boulders (larger than $1 \mathrm{~m}$ diameter) and deposited up to a meter of debris and mud in the Scotts Creek floodplain (B. McCrary pers. comm.) (see Figures 2.3 and 2.4). This debris flow possibly originated at a large landslide in the South Fork of Little Creek, $0.5 \mathrm{~km}$ upstream from the confluence. Flood events in 1982, 1986, and 1998 also caused significant landsliding and streambank erosion. Major changes in the channel of Little Creek were also 
observed during these events.

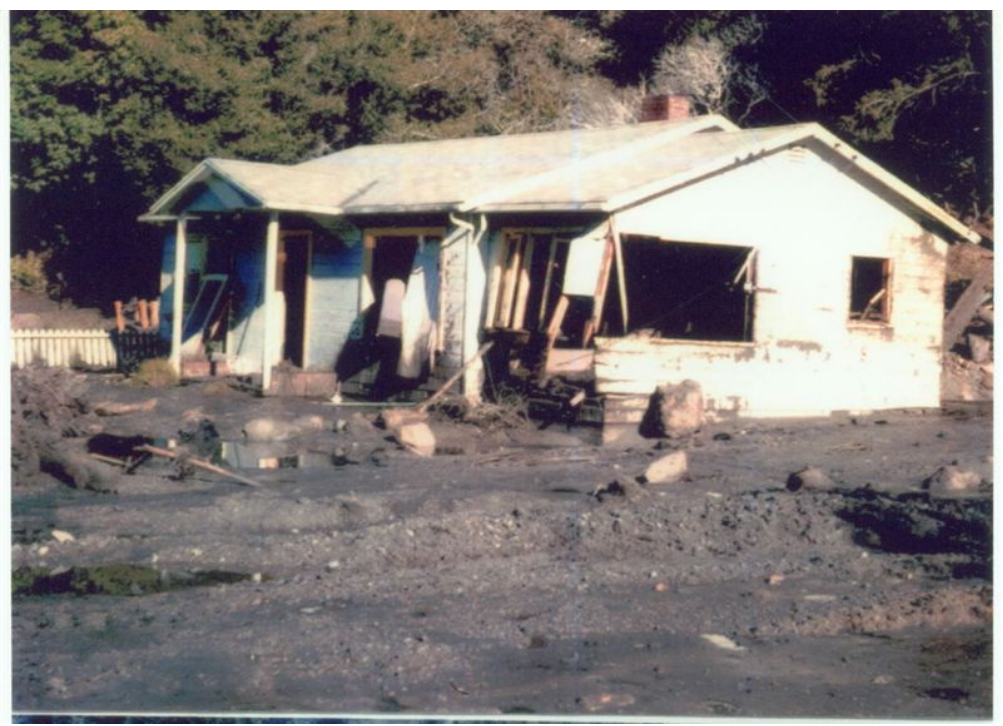

Figure 2.3: Damage to house at mouth of Little Creek from 1955 flood. Photographer unknown.

\subsubsection{Vegetation}

Vegetation in the watershed varies with aspect, elevation and distance from the ocean. The ridgelines are dominated by chaparral vegetation and knobcone pine (Pinus attenuata). A mix of Douglas-fir (Psuedotsuga menziesii var. menziesii) and hardwoods are the predominant vegetation on midslope areas. Near stream channels and on north facing slopes vegetation is dominated by redwood (Sequoia sempervirens) with tanoak (Notholithocarpus densiflorus) and Douglas-fir as associates. The riparian corridor along the Mainstem of Little Creek is dominated by an even-aged stand of red alder (Alnus rubra) along with redwood, bay laurel (Umbellularia californica), Douglas-fir and maple (Acer macrophylum). Much of the alder was recruited after the 1955 debris flow event. Above the confluence of the North and South Forks, red alder becomes less common and the riparian corridor is dominated by redwood and Douglas-fir. 


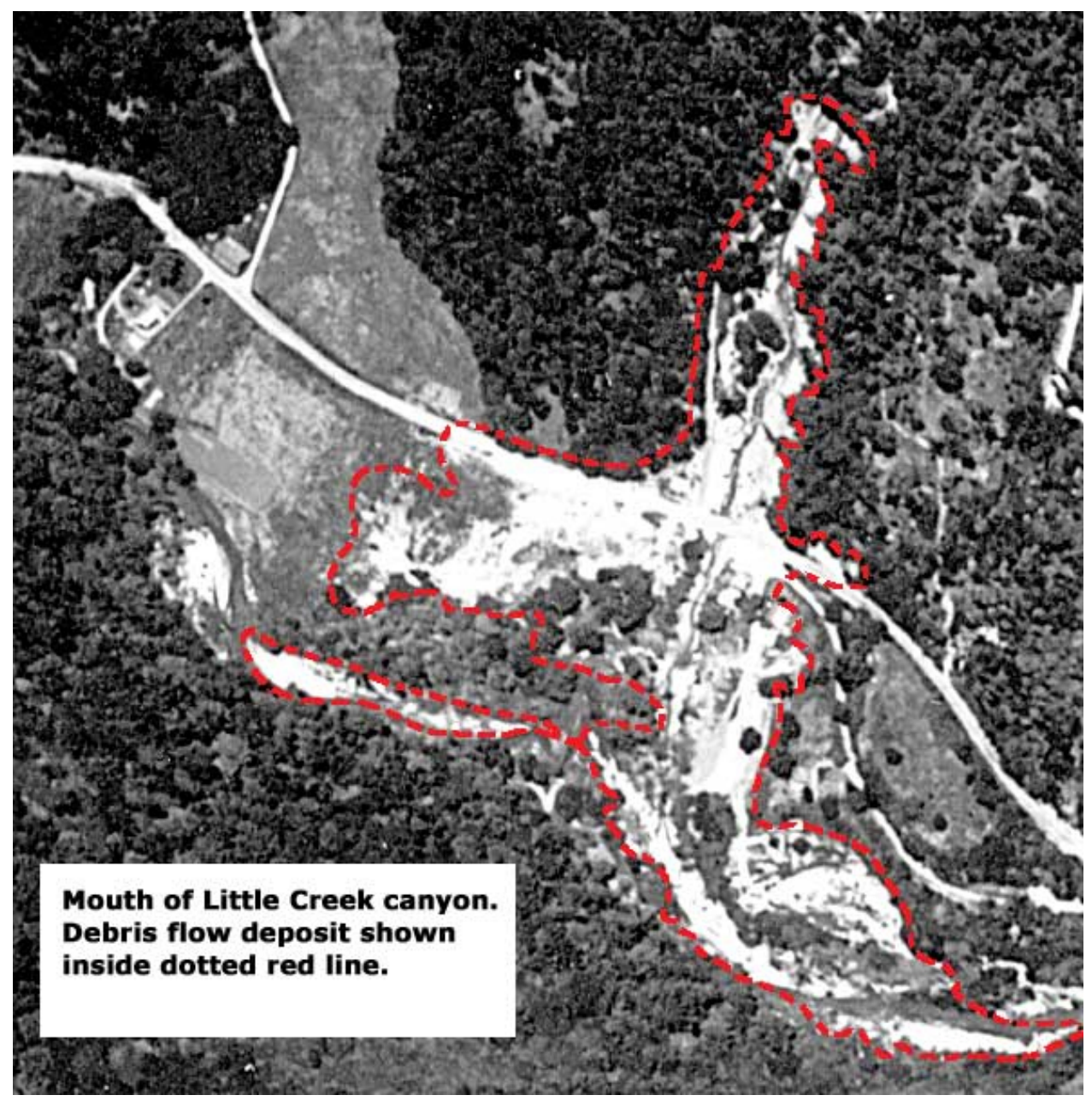

Figure 2.4: Aerial photo from 1957 showing mouth of Little Creek and extent of 1955 debris flow deposits. Image from Scotts Creek Watershed Council website.

\subsubsection{Ownership and Land Use}

The majority of the Little Creek watershed is owned by the Peninsula Open Space Trust (POST) and Sempervirens Fund (42.8\%) and Cal Poly Corporation (49.4\%). POST/Sempervirens owns the eastern (upstream) portion of the watershed, and Cal Poly Corporation owns the western (downstream) portion. Small portions along the northern edge of the watershed are owned by Big Creek Lumber and other private landowners. The entire watershed was clearcut, burned and logged using railroads by the San Vicente Lumber Company around 1915. The primary 
land use is still timber production. Both Cal Poly and POST/Sempervirens perform selective harvesting of second-growth redwood, with harvest entries every 10-18 years. Small amounts of Douglas-fir and hardwoods are also harvested for firewood. A small amount of the watershed on the southern ridgeline above the Staub House has been used for cattle grazing in the past, and Swanton Pacific Ranch is currently working to reintroduce grazing to these areas.

The Lockheed Fire occurred in August 2009 and burned most of the Little Creek Watershed (approximately 90\%). Five of the six study reaches were located in the burned area. Much of the upper slopes of the watershed, especially the southerly facing slopes with chaparral and knobcone pine vegetation, burned at high or extreme intensity resulting in complete loss of canopy and ground cover. Small debris flows occurred in several tributaries draining these high burn severity areas in the 2009-2010 winter. Figure 2.5 is a burn severity map of the Lockheed Fire. 


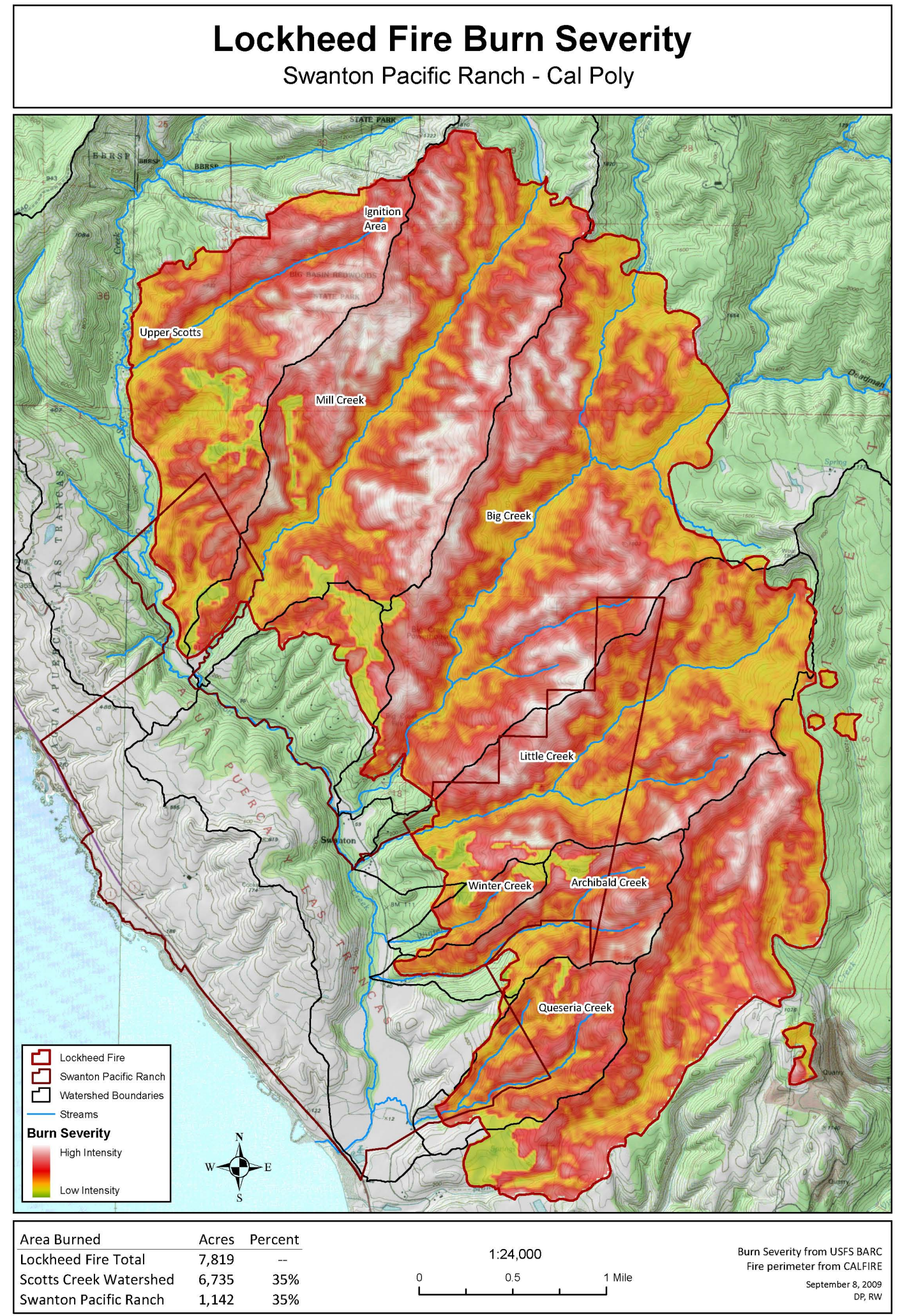

Figure 2.5: Burn severity map from the 2009 Lockheed Fire. 


\section{Chapter 3}

\section{Literature Review}

Variations in sediment delivery, hydraulic discharge, and vegetation cause spatial and temporal variations in channel morphology. Over geologic time channels respond to tectonic uplift, landscape erosion, and climate variability. On historical time scales, channels respond to changes in sediment and water supply from land use history and channel modification (dams, levees, straightening, etc.) as well as those resulting from floods and droughts (Montgomery \& Buffington, 1998). The ability of an alluvial channel to transport sediment is based on the shape and gradient of the channel, the character of the sediment, and quantity of water flowing through the reach. Differences in the amount of sediment supplied to a reach and the amount of sediment transported out of a reach result in a net change of sediment storage within a reach, which results in a change in channel geometry (Anderson, 2008). Understanding changes in the channel geometry (fill and scour) can provide insight into other processes and factors that are more difficult to measure directly. 
Cross-sectional and longitudinal profiles have been used extensively to help understand the processes at work in rivers as well as the impacts and recovery rates from floods, land use changes, and wildfire in a variety of physical settings.

One of the earliest sets of repeated cross sections was on Watts Branch in Maryland (drainage area $9.6 \mathrm{~km}^{2}$ ). Data from this channel was used to help understand some of the bank erosion and point bar formation processes that lead to river meandering (Leopold et al., 1964) and impacts to streams from urbanization (Leopold et al., 2005). A series of repeated surveys were performed between 1953 and 1994, which showed that the stream and floodplain had aggraded over time. In many cross sections, the original channel had been completely filled by point bar deposition and the current channel existed in what was earlier the floodplain.

Madej \& Ozaki (1996) used repeated cross sections on Redwood Creek in northwest California (drainage area $720 \mathrm{~km}^{2}$ ) to document downstream development and propagation of a sediment wave resulting from extensive poor logging practices and floods in 1955, 1964, 1972, and 1975. Surveys were repeated several times between 1973 and 1991 and generally documented scour of stored sediment from upper reaches of the channel and aggradation of lower reaches. Mean bed elevations at cross sections changed up to $1 \mathrm{~m}$ (both scour and fill) during the survey period, and up to $9 \mathrm{~m}$ of aggradation and channel widening of 150 percent to 300 percent associated with the 1964 flood was documented at some locations.

Nolan \& Marron $(1985,1988)$ evaluated impacts of large storms on stream channel morphology in the Santa Cruz Mountains and northwestern California. The storm of January 4, 1982 caused flooding and landsliding throughout most of Santa Cruz County. Channel changes were evaluated at USGS gaging stations using data collected during stream discharge measurements. General patterns were 
observed, but overall the response was highly variable, and depended on local geology and rainfall characteristics. General effects on channel morphology were scour in small first and second-order channels and some filling of larger higherorder channels. In localized reaches, deposition adjacent to large scale streamside landslides or abundant small scale landslides caused significant reductions in crosssectional area. Streambanks in the Santa Cruz Mountains study area were generally bedrock and resistant to erosion, and most sediment appeared to originate from upper slope landslides and debris flows driven by high pore water pressure as well as scour of low order channels. Channels generally had high transport capacity and were only overwhelmed by sediment in localized areas.

This contrasts strongly with changes due to large flows in northwestern California (Nolan \& Marron, 1985). Floods in 1964, 1972, and 1975 caused major changes in the channels (Lisle, 1981; Madej \& Ozaki, 1996). Lisle (1981) observed mean bed elevation changes from $1 \mathrm{~m}-4 \mathrm{~m}$ and channel widening of 7 percent to 105 percent at gaging sites on north coast rivers. The primary source of sediment in these higher-order river systems appears to be streamside landslides caused by streambank erosion undercutting the toe of the hillslope. This process may drive a positive feedback loop, as more material is deposited to the channel, it aggrades and widens or deflects flow, eroding banks and causing more streamside landsliding (Nolan \& Marron, 1985). The difference in response is a function of the geology, channel type, and associated fluvial processes.

Cross sections at a variety of channel scales (rills developing in unchanneled swales to perennial low gradient streams) have been used to understand impacts to channels resulting from wildfires in Colorado's front range (Eccleston, 2008). Cross sections, aerial photographs, and field observations were used to understand responses and recovery of the Eel River and tributaries to floods in 1955, 1964, 
and 1997 (Sloan, 2001). Cross sections and residual pool depths were used to study channel responses to logging in the North Fork of Caspar Creek. Lisle \& Napolitano (1998) identified channel aggradation and changes in pool volumes associated with large woody debris inputs from buffer strips along clearcuts blocks. Cross sections and aerial-photo analysis were used to understand channel dynamics resulting from riparian vegetation changes that occurred after reintroduction of wolves to Yellowstone National Park (Beschta \& Ripple, 2006).

Besides direct measures of channel change (scour, fill, widening, etc), other authors have observed that channel complexity is often impacted by large sediment inputs and have evaluated variability of channel geometry as a surrogate of habitat complexity. Madej $(1999,2001)$ studied recovery of channels from disturbance at a range of sizes and gradients. Autoregression analysis of residual channel depths was used to identify development of regular patterns in longitudinal geometry as streams recovered from large sediment inputs or other disturbance.

Bartley \& Rutherfurd (2005) assessed measures of geomorphic variability in Chreitons Creek (drainage area $141 \mathrm{~km}^{2}$ ) in Victoria, Australia, a low gradient, sand bed stream impacted by a sediment slug. Measured stream characteristics were cross-sectional shape, thalweg profile, and sediment size distribution. Initially, 13 different measures were assessed on synthetic channel geometries developed to have differing types and scales of variability. Of these, six were determined reasonable metrics for identifying complexity changes in the geometry of real channel. Cross section measures were trapezoidal (which fits a trapezoid to the channel cross section and uses standard errors as a measure of variability), vector dispersion (a measure of angular variance), and sum of the squared height deviations (differences of consecutive bed elevations normalized to channel 
width). Measures of thalweg variability used were degree of wiggliness ${ }^{1}$, standard deviations of residuals of a fit to the channel profile, and the fractal dimension of the profile. These methods were able to differentiate between disturbed and undisturbed reaches, but did not rank reaches consistently and may have biases toward certain stream shapes or scales of measurement. The results also showed that sediment size distribution may have been more sensitive to disturbance than larger geomorphic changes.

A study of streams in Southern Alaska was also able to distinguish between disturbed vs. non-disturbed catchments using pools per reach, pool depth as function of bankfull depth, and ratio of critical shear of D50 sediment to bankfull shear stress (Wood-Smith \& Buffington, 1996).

Most studies utilizing cross-sectional and longitudinal profiles study channel change on relatively low-gradient $(<1 \%)$, gravel-bed channels with pool-riffle morphology that is generally more sensitive to disturbance (Montgomery \& MacDonald, 2002). All of the reaches in Little Creek are steeper than 2\%. A majority of studies are associated with investigating response and recovery of a channel from major changes in sediment supply, runoff characteristics, woody debris loading, and riparian vegetation condition. Generally, observed changes reflect those that can be predicted using process-based conceptual models of channel response (Montgomery \& Buffington, 1998).

\footnotetext{
${ }^{1}$ The degree of wiggliness measures the deviation, from the mean elevation, of angles between points along a profile.
} 


\section{Chapter 4}

\section{Methodology}

\subsection{Study Design}

Six study reaches were established in spring of 2002, each with 10 monumented permanent cross sections and a thalweg longitudinal profile. Three reaches are in the Mainstem of Little Creek, two in the North Fork, and one in the South Fork. An additional reach was established in the upper South Fork, but was never resurveyed due to poor access and lack of total station control. Figure 4.1 shows the location of these study reaches. The study reach locations chosen provided easy access, were representative of overall channel conditions in the vicinity of the reach, and were areas that had exhibited some change in the past, especially in the 1997-1998 winter (B. Dietterick, pers. comm.). These study reaches were also used for other projects that compared LiDAR-derived cross sections to ground surveyed cross sections (Hilburn, 2010; Dietterick et al., 2012) and LiDAR-derived longitudinal profiles to ground-surveyed longitudinal profiles (White, 2010). The 


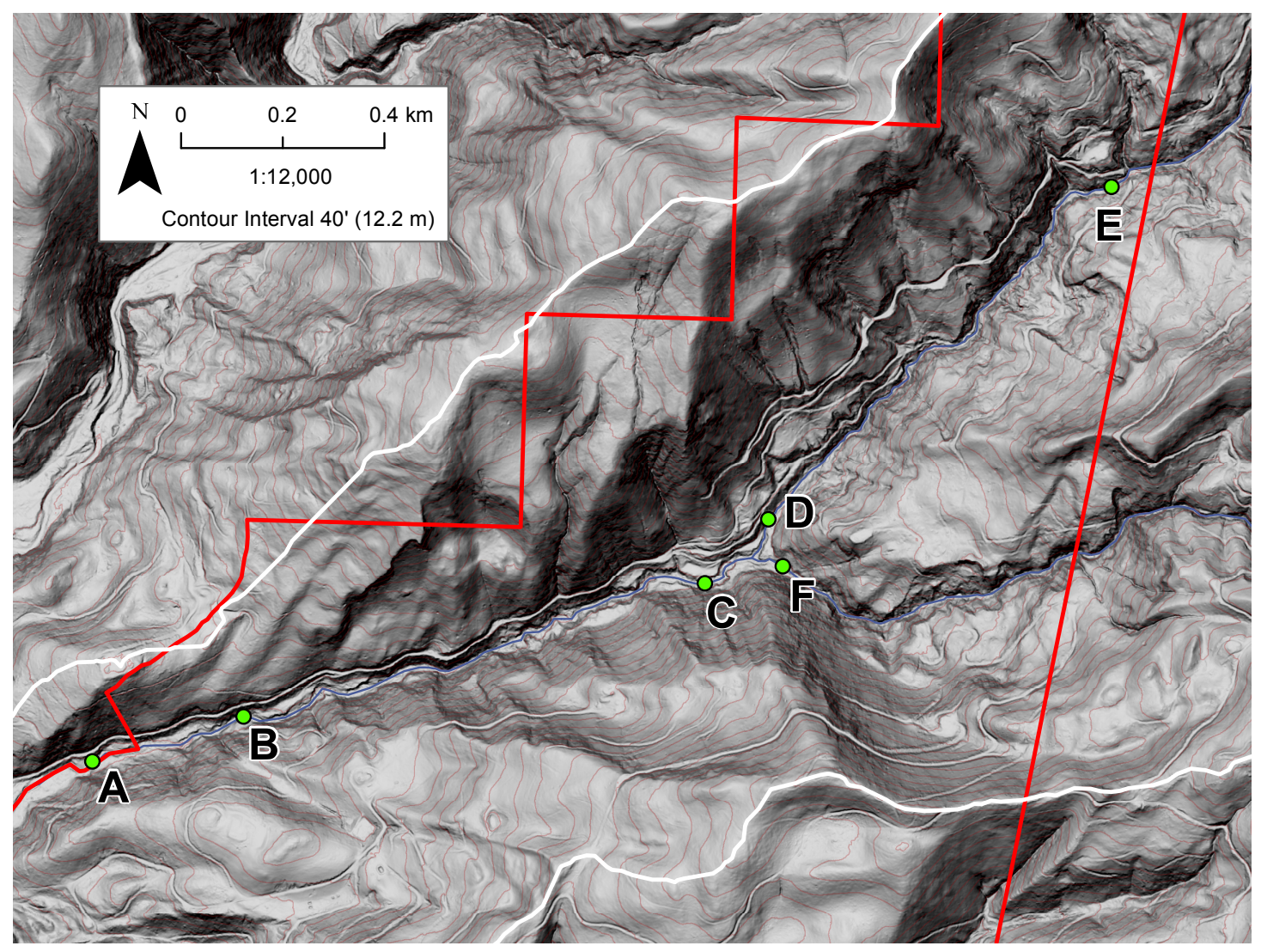

Figure 4.1: Map of study reach locations in Little Creek. Contour interval is 40' $(12.2 \mathrm{~m})$. Hillshade is derived from a $1 \mathrm{~m}$ DEM produced from LiDAR data collected in 2010. Red line is Swanton Pacific Ranch boundary.

Table 4.1: Table showing who collected channel geometry data each year.

\begin{tabular}{cc}
\hline Year & Surveyors \\
\hline 2002 & Brooke Akers, Brian Kong \\
2003 & Michael Gaedeke, Susan Burgess \\
2004 & Tim Dodson, Sasha Cichowski \\
2005 & Sasha Cichowski, Alicia Moss \\
2006 & Alicia Moss, Ben Jansen \\
2007 & Drew Perkins, David Haas \\
2008 & Colin Nicol, Drew Gaffney, Tom Sanford \\
\hline
\end{tabular}


study reaches are surveyed annually (Table 4.1 shows who collected the data each year.)

Originally, evenly-spaced cross-sectional profiles and complete longitudinal profiles were planned for the entire length of Little Creek on the Swanton Pacific Ranch property. After discussion with researchers at the USFS Redwood Sciences Lab and USGS Western Ecological Research Center, the cluster design was adopted. Evenly-spaced cross sections separated by large distances make it difficult to reach definitive conclusions about changes (or lack of changes) in any length of stream because there is only one sampling point within a reach and Little Creek is highly variable on small scales. With ten closely-spaced cross sections, there is much higher confidence in the inferences that can be made about changes occurring in a reach. There was an intentional choice made to have higher-resolution data in a few areas instead of low-resolution data spanning the entire channel length.

\subsection{Reach Descriptions}

Each study reach is approximately 100 meters long and vary in gradient from 0.02 to 0.05 . The bed material in each reach ranges from fine sand to boulders - no bedrock is exposed in any of the reaches. Sand content of the bed is generally high (2 mm ranges from D30 to D50) and most of the coarse bed material is highly embedded. Sediment size distribution data was collected by pebble count in Fall 2009. 


\section{Reach A}

Reach A is located in the lower mainstem of Little Creek approximately 0.4 kilometers upstream of mouth (Figure 4.1). The channel is incised into older floodplain terraces which have two prominent surfaces. The valley bottom has low relief and is and approximately 35 meters wide, with steep side slopes (70 percent+). Gradient through reach is approximately 0.018. The bed substrate is primarily cobbles $(\mathrm{D} 84=78 \mathrm{~mm})$ with high sand content $(\mathrm{D} 45=2 \mathrm{~mm})$ and occasional boulders. This was the only reach that did not burn in the 2009 Lockheed Fire.

\section{Reach B}

Reach B is located in the middle mainstem of Little Creek approximately 0.8 kilometers from the mouth (Figure 4.1). The reach is downstream of an old $4 \mathrm{wd}$ road bridge crossing (bridge removed in 2008). The valley bottom is narrower than in Reach A (18-25 meters). There are terraces 3-4.5 meters above the current channel on both sides. About $1 / 3$ of the reach has a vertical 2-3 meter bank on the south side. Side-slopes are very steep (70 percent to 100 percent+ on south side). Average gradient through reach is 0.024. Substrate is similar to Reach A $(\mathrm{D} 84=78 \mathrm{~mm}$ and $\mathrm{D} 30=2 \mathrm{~mm})$.

\section{Reach C}

Reach $\mathrm{C}$ is located in the upper mainstem of Little Creek 1.8 kilometers from the mouth (Figure 4.1). The reach is located below the former boy scout camp swimming pool (Camp Arroyo, Troop 539). There are two sets of five cross sections above and below a log/boulder jam in the middle of reach. The upper section of 
the reach was identified as a depositional area following the 1998 floods. The channel is in an approximately 12 meter wide valley cut into terraces that are 7-9 meters above the channel. Valley width including the terraces is 40-45 meters. Average reach gradient is 0.041 . Bed substrate is similar to Reaches A and B $(\mathrm{D} 84=88 \mathrm{~mm}$ and D32 $=2 \mathrm{~mm})$, except the $\mathrm{log} /$ boulder jam which has several boulders with diameters greater than 1 meter.

\section{Reach D}

Reach D is located in the lower North Fork of Little Creek above the North Fork Flume (Figure 4.1). A landslide occurred in middle of the reach in 1998 and deposited a large quantity of rock, soil, and a clump of redwood trees into the channel. There are five cross sections above and five cross sections below the landslide deposit. This reach is incised into terraces that appear to be formed from reworked debris flow deposits. The surface is generally 2 meters above the current channel. The valley width is 15 meters. The average reach gradient is 0.049. The substrate is slightly coarser than the mainstem reaches and small boulders are more common $(\mathrm{D} 84=91 \mathrm{~mm}$ and D33 $=2 \mathrm{~mm})$. There are several logs embedded in the bed in the lower section of the reach.

\section{Reach E}

Reach E is located in the Upper North Fork of Little Creek downstream of property line (Figure 4.1). The bridge on the Little Creek haul road crosses the upstream end of the reach. The bed in this reach has many large boulders $(0.5 \mathrm{~m}$ to $1+\mathrm{m}$ diameter) and is primarily a cascade with few developed pools. There is a low 
terrace about 1.5 meters above the current channel which appears to be remnants of a debris fan at the mouth of a tributary which enters Little Creek just upstream. The valley width is 8 meters. Average gradient is 0.049 and the substrate is the coarest of the study reaches $(\mathrm{D} 84=120 \mathrm{~mm})$, however this reach has the highest percentage of bed covered by sand (D56 $=2 \mathrm{~mm})$.

\section{Reach F}

Reach F is located in the lower South Fork of Little Creek located above the South Fork Flume (Figure 4.1). There are several terrace surfaces on the north side of channel (up to 2 meters above the channel) and the south side has a very steep sideslope $(100 \%+)$. The valley width is 12 meters. There is a bedrock waterfall (2 $\mathrm{m}$ high) about 30 meters upstream of the F1 cross section. Average reach gradient is 0.041 . The composition of the substrate is similar to the mainstem reaches $(\mathrm{D} 84=63 \mathrm{~mm}$ and $\mathrm{D} 37=2 \mathrm{~mm})$.

\subsection{Equipment and Procedures}

\subsubsection{Cross sections}

Cross section endpoints were established with $10 \mathrm{~mm}\left(3 / 8^{\prime \prime}\right)$ diameter bent steel stakes (rebar) cemented into place on the banks of the stream; the rebar were typically located 2 to 2.5 meters above the thalweg. Additionally, $19 \mathrm{~mm}(3 / 4 ")$ diameter steel rebar were installed higher up the slopes to mark the cross section alignment if the lower rebar were ever lost due to slope failure. Typically, the cross section lines are spaced 5 meters apart. In Reaches C and D there is a gap 
between the lower 5 cross sections and the upper 5 cross sections. In Reach $\mathrm{C}$ this gap contains a $\log$ /boulder jam and in Reach D this gap is where a debris flow/landslide deposited a mass of sediment and clump of redwood trees into the channel in 1998. One reason for these gaps was to determine if there were differences in changes above and below these features.

Each cross section is surveyed with a cloth tape strung from rebar to rebar and a rod and level. Elevations and distances are measured at the top and bottom of each rebar, bankfull indicators, edge of water, thalweg, and other breaks in slope. The downstream left rebar is set at 0 distance in all cross sections and the top of the left rebar is given an arbitrary elevation of 100 meters. Elevations are measured with a Philadelphia rod and Topcon automatic level. Starting in 2009, a measurement was also made to one of the permanent benchmarks in each reach to establish an elevation reference independent of the rebar endpoints. Detailed surveyor instructions are located in Appendix C.

\subsubsection{Longitudinal profiles}

Thalweg longitudinal profiles were surveyed in each reach using a total station. $5 \mathrm{~cm}(2 ")$ diameter galvanized steel capped pipes were installed in 2002 to establish permanent benchmarks in each study reach. In 2003, these benchmarks were then tied into a baseline survey performed by Dunbar and Craig Inc. along the Little Creek haul road. These surveyed benchmarks allow comparison of the crosssectional and longitudinal profile survey data to LiDAR-derived channel data.

Longitudinal profiles were surveyed in 2002-2008 using a Leica TCR307 total station and TDS Survey Pro on the HP48gx calculator as a data collector. In 
2009 a Leica TCR407 and Carlson Explorer data collector were used. In 2010, a Leica TCR407 and TDS Recon data collector were used.

The total station is set up on one of the permanent benchmark locations and a backsight is set up at another. Measurements (side shots) are taken in the thalweg of the stream at all breaks in slope for as far upstream and downstream as can be seen from the permanent benchmark locations. Start and stop locations were established in 2010 to help provide year-to-year consistency. Points are identified as riffle or head/middle/tail of a pool. Shots are also taken to the top of each cross section endpoint rebar pin. Detailed longitudinal profile surveying instructions are located in Appendix C.

\subsection{Measurement Errors}

\subsubsection{Sources of error}

In order to have a relevant discussion about channel changes seen with the crosssectional profile and longitudinal profile surveys, it is imperative to have an understanding on the amount of error in the measurements. Ideally, with stable benchmarks and good surveying techniques, measurement error would be equal to the precision of the instrument. However, there are several sources of error present in the both the cross-sectional and longitudinal profile surveys. This section describes the sources of error present in the surveys, ways to quantify those errors, and develops an estimate of the amount of change in the channel that could be attributed to surveying error. There are five primary causes of error in these surveys. 
1. Philadelphia rod or prism rod not level.

2. Surveyor judgment in survey point location and associated errors.

3. Instrument errors.

4. Typos and measurement errors (reading rod incorrectly or incorrect total station setup).

5. Rebar end point movement.

Before 2007, a rod level was not used for the cross section surveys. This has the potential to add an unknown, but significant amount of error to the crosssectional profile survey. Figure 4.2 shows how the surveying rod not being level can cause measurement error. This problem is amplified on steep side slopes and when the tape is far above the stream channel. If distance is being measured 3 meters above the channel just a 2 degrees tilt of the survey rod could cause 10 centimeters error in the distance measurement and several milimeters error in elevation measurement.

Errors resulting from surveyor's judgment generally has two forms. The first is based on the surveyor's choice of survey points. The surveyor is tasked with representing a smooth, continuous surface with a series of points connected by straight lines. Generally, the surveyor chooses points to survey that are located at "breaks in slope" or locations where the slope of the ground surface changes significantly and interpolation between points represents the ground's surface accurately. It is clear from reviewing the cross sections that different surveyors interpreted the surface differently, and that some surveyors put more effort into capturing smaller scale features than others. These features were often boulders or logs and difficult and time consuming to portray accurately in the survey. Some surveyors 

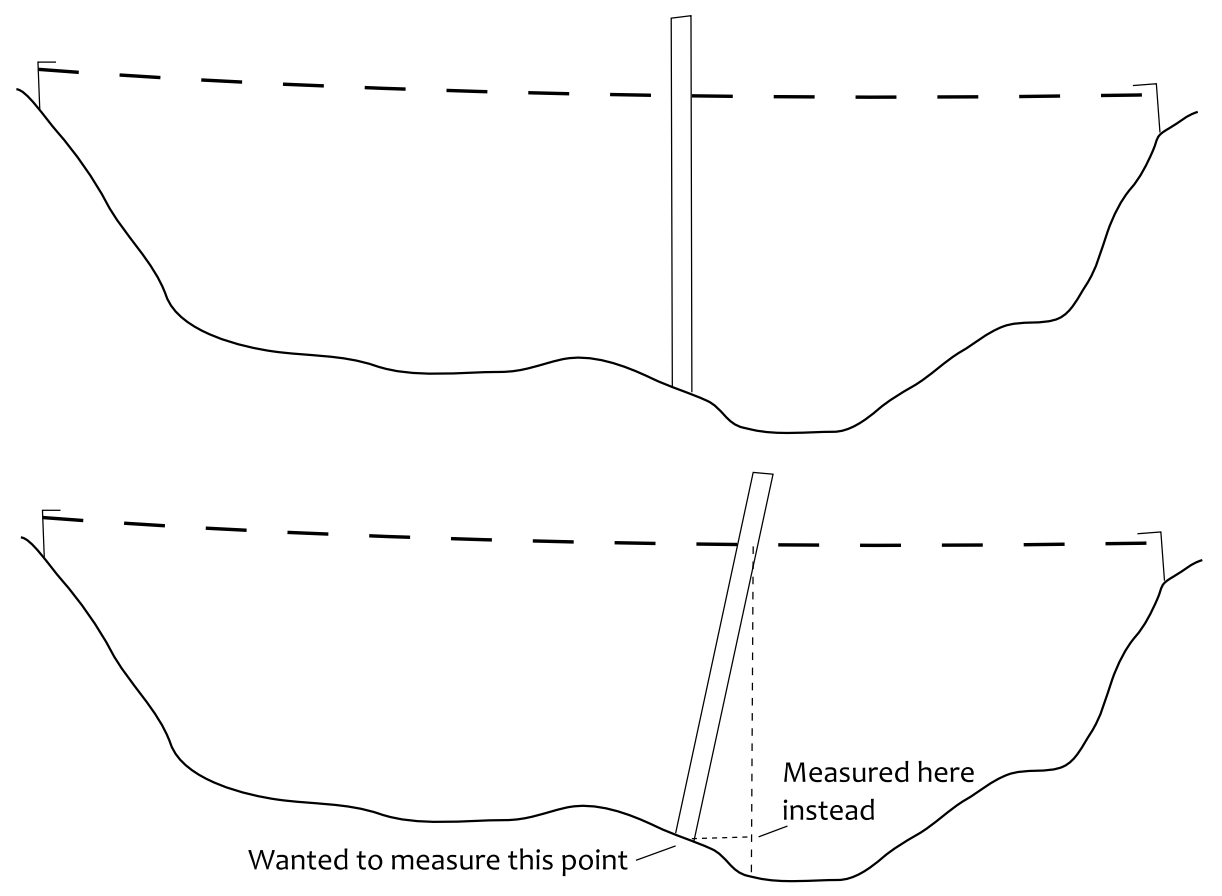

Figure 4.2: Diagram showing how lack of a rod level could cause errors in measurement

skipped these features entirely and the instructions for surveyors (Appendix C) now provide guidance on how to consistently record these features. Discrepancies due to surveyor judgment likely have a larger impact on cross-sectional area measurements than thalweg elevation measurements.

The second surveyor-induced source of error is also associated with the survey rod not being level. This error occurs when measurements are taken upstream or downstream of the actual cross section line. Depending on the shape of the channel and the orientation and location of features like rocks, banks, etc., moving the measurement location upstream or downstream just $0.15 \mathrm{~m}$ of the cross section line can cause differences that can be misinterpreted to represent change in the channel. In 2003, the cross sections were surveyed using the total station which allows the route taken during the survey to be seen visually (see Figure 4.3). In this 


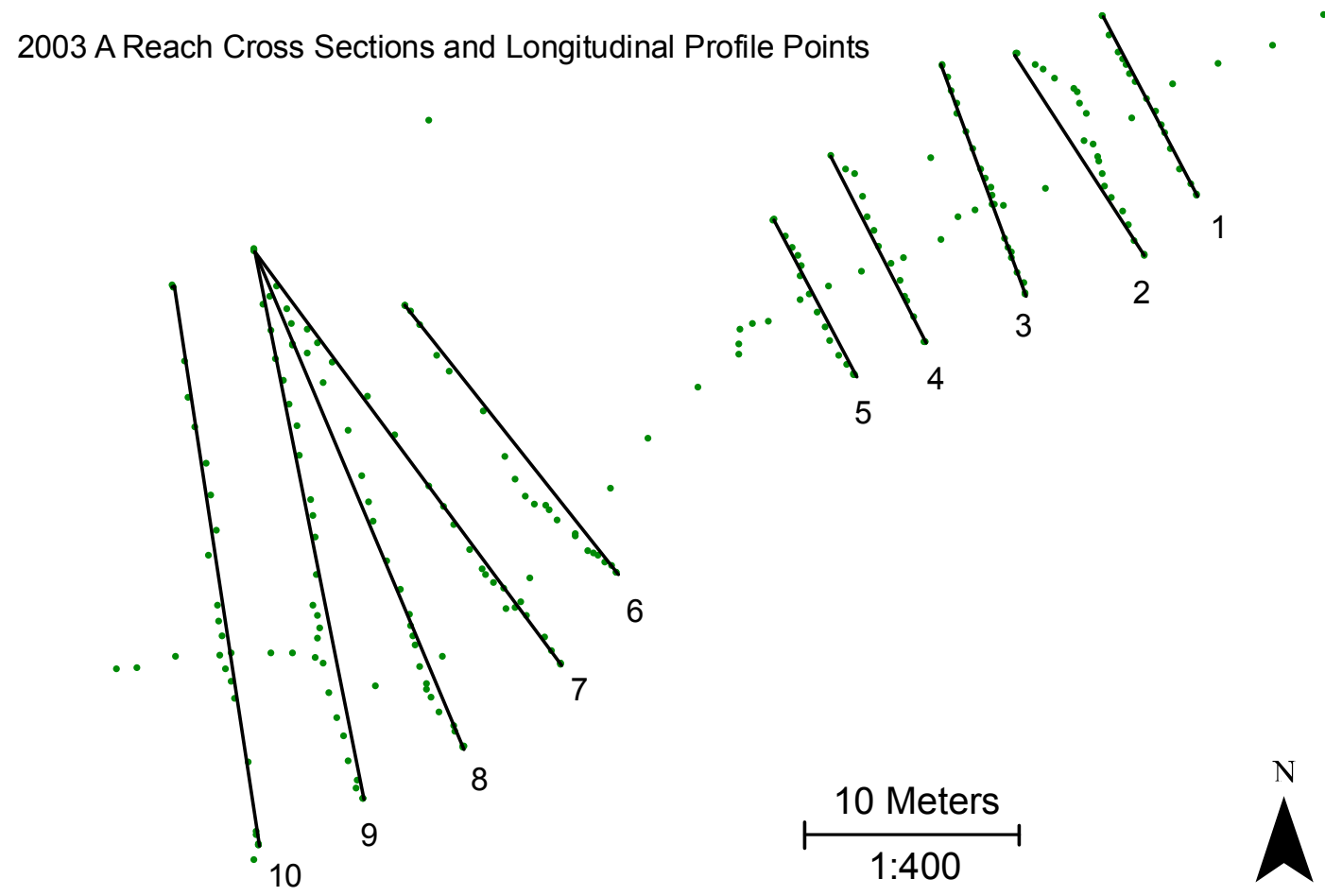

Figure 4.3: Map of points surveyed for cross sections and longitudinal profiles in 2003. Note how for many of the cross sections the points are not taken directly under the cross section line.

survey, some of the measured locations were over 1 meter from the cross section line. Ensuring that measurements are taken directly underneath the cross section line has been emphasized for new surveyors and in the instructions (Appendix C). It is almost impossible to identify rod level errors and differentiate them from what appears to be channel change. Surveyor judgment and rod level errors are likely responsible for much of the "noise" in the cross sections, especially where slopes are steeper. This error is very difficult to quantify.

Instrument errors are likely not a significant source of error relative to other sources. A two-peg test (Harrelson et al., 1994) is performed to check that the automatic level is accurate. Error with the total station is impossible to quantify when a closed traverse is not performed and all of the data collected for the longitudinal profiles is taken with side shots. When setting up the surveying 
equipment, extra care is made to ensure that the backsight prism is as level as possible and the total station stays level during the survey. If the distance error on the backsight was more than $1.5 \mathrm{~cm}$ in either the horizontal or vertical direction, the equipment was repositioned and instrument heights remeasured. Surveyors are also instructed to frequently check that the total station is level and backsight angle is zero during the survey.

Typographical errors and misreading of instruments is another source of error. The surveying has generally been performed by student interns who do not have extensive surveying experience and are more prone to making errors in data collection. Major typographical errors in data collection, data entry mistakes, or incorrect readings of the Philidelphia rod were usually obvious when the data was reviewed for quality control and could usually be corrected. However, there are many smaller errors that are more difficult or impossible to trace down or identify that cause some amount of error that cannot be quantified.

Since the cross sections were established and surveyed in 2002, the rebar endpoints in several of the cross sections have moved. This has happened for a variety of reasons. Soil creep on steep sideslopes has caused some rebar to rotate or slide and move toward the stream. In other instances, bank erosion has undermined the bank supporting the rebar, causing it to move. In one cross section, a tree fell on the rebar end point and severely bent it. In the past, strings were tied between the rebar to identify the cross section alignment. Trees and branches fell on these strings, pulling the rebar inward towards the stream. When the left bank (looking downstream) rebar moved (which was used as both the position and elevation benchmark) this would cause apparent changes in the cross section and position of the right rebar. Usually this could be corrected by matching up the cross sections on the right rebar. If only the right rebar moved, this did not typically cause 
problems in the channel portion of the cross section. In cross sections where both rebar appeared to have moved, the cross sections were aligned so they matched up away from the channel and banks where the assumption of minimal change was reasonable. Correction of the rebar movement is discussed in the quality control section of Chapter 5.

In the summer of 2010, new $16 \mathrm{~mm}\left(5 / 8^{\prime \prime}\right)$, diameter $1.5 \mathrm{~m}$ (5') long rebar endpoints were installed outside of existing cross sections where the previous rebar endpoints had moved or showed potential to move. These new rebar are expected to provide a more stable control on both position and elevation than the previous $10 \mathrm{~mm}(3 / 8$ ") bent rebar. In addition, since 2009, surveyors have also shot a backsight to one of the capped pipe permanent points to use as an elevation control. The most obvious cases of rebar movement were corrected as much as possible, however, rebar movement does add some unknown amount of error to the analysis.

\subsubsection{Estimates of error from literature}

Several authors have reported measurement error of stream channel surveys. Lisle (1987) estimates that error in residual pool depth measurements are approximately twice the median particle diameter. This could be interpreted as the error in surveying the true bed elevation when you take into account the irregularities present in the substrate. The median particle (D50) diameters for each reach in 2009 are shown in Table 4.2. Little Creek does not have a normally distributed particle size distribution and has a large amount of sand sized particles along with cobble and boulder sized material. Therefore, the median diameter may underrepresent the size of particles that define the bed surface and that would cause error in 
Table 4.2: Median particle diameters for each study reach measured in Fall 2009 and twice the diameter in meters. This is an estimate of vertical error in the measurement of the bed based on Lisle (1987) and could be reasonably interpreted as the error associated with surveys across an irregular substrate.

\begin{tabular}{ccccc}
\hline Reach & D50 $(\mathrm{mm})$ & D84 $(\mathrm{mm})$ & 2x D50 Diameter $(\mathrm{m})$ & 2x D84 Diameter $(\mathrm{m})$ \\
\hline A & 11 & 78 & 0.02 & 0.16 \\
B & 13 & 48 & 0.03 & 0.10 \\
C & 11 & 88 & 0.02 & 0.17 \\
D & 10 & 91 & 0.02 & 0.18 \\
E & 3 & 120 & 0.01 & 0.24 \\
F & 9 & 63 & 0.02 & 0.12 \\
\hline
\end{tabular}

bed elevation surveying and the D $84^{1}$ may be a more appropriate metric. Hickey (1969) suggested that an error of $0.15 \mathrm{~m}$ is associated with comparisons of bed elevation from stream gaging measurements. Madej \& Ozaki (1996) reported error in change of measured mean bed elevation of $0.05 \mathrm{~m}$.

\subsubsection{Repeated surveys}

Thirty randomly selected cross-sectional profiles were re-surveyed by different surveyors in 2002 for quality control purposes (Initial surveys in March and resurveyed in May/June). See Table 4.3 for data. No large storms ${ }^{2}$ occurred between surveys, so it is reasonable to expect bed conditions to be very similar. Comparing surveys from these two dates shows the level of repeatability that is possible in measurements of cross-sectional area or thalweg depth. This integrates most of the possible sources of error and estimates an average level of precision in the determination of the cross section metrics. The repeated surveys also help un-

\footnotetext{
${ }^{1}$ D84 is the particle size most often used to estimate channel roughness in flow resistance equations. It is the particle size one standard deviation above the geometric mean.

${ }^{2}$ On April 17th, the nearest USGS gage, San Lorenzo River at Big Trees, had a peak flow of $49 \mathrm{~m}^{3} / \mathrm{s}$ (1720 cfs), a 1.2 year return interval flow using an annual peak series.
} 


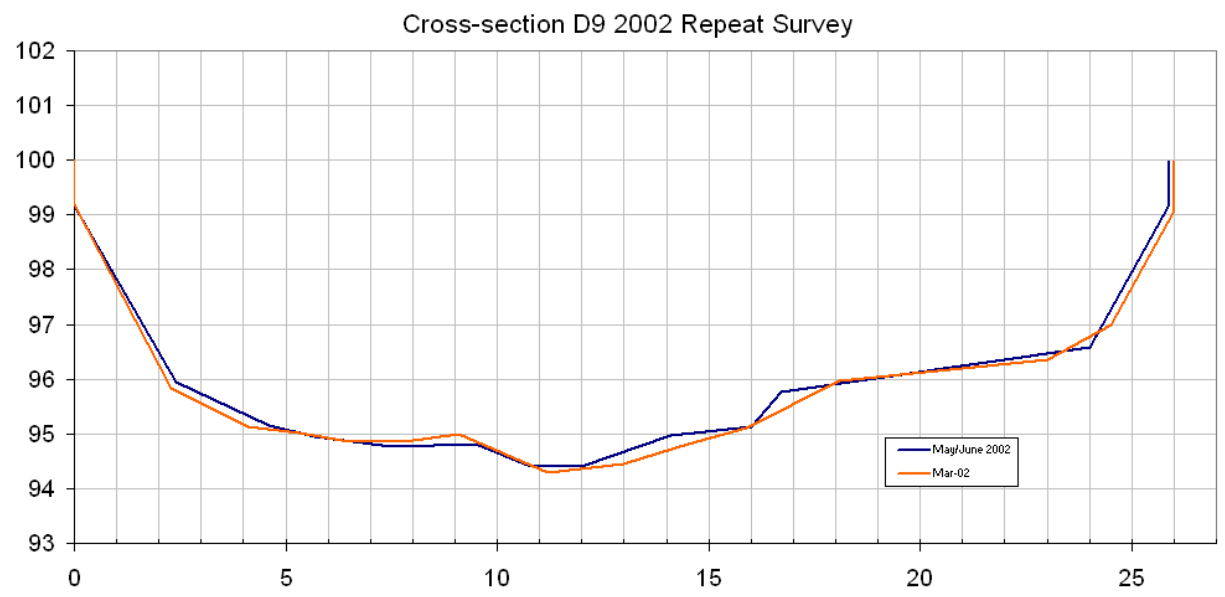

Figure 4.4: Figure showing repeated surveys in 2002. Units are in feet.

derstand visually the impact that variations in the surveyor's judgment may have had (see Figure 4.4). It is assumed that no rod level was used in these surveys.

Mean error in the thalweg elevation was $0.021 \mathrm{~m}$ with a standard deviation of $0.018 \mathrm{~m}$. Mean error in area calculations was $0.18 \mathrm{~m}^{2}$ with a standard deviation of $0.20 \mathrm{~m}^{2}$. Eccleston (2008) used the mean error $+1 \mathrm{SD}$ calculated from repeated surveys as an estimate of error in channel bed elevation measurement. Based on these different sources, the estimated error in bed elevation measurement is $\pm 0.04 \mathrm{~m}$ and area measurement is $\pm 0.4 \mathrm{~m}^{2}$. Change less than this could be be reasonably attributed to error in surveying. 
Table 4.3: Thalweg and cross-sectional area measurements for 30 cross sections repeated in 2002. Cross-sectional areas were calculated under the 100 meter elevation (height of top of left rebar). The means and standard deviations are calculated using the absolute values of the errors.

\begin{tabular}{|c|c|c|c|c|c|c|}
\hline \multirow[b]{2}{*}{ Cross section } & \multicolumn{3}{|c|}{ Area $\left(\mathrm{m}^{2}\right)$} & \multicolumn{3}{|c|}{ Thalweg Depth (m) } \\
\hline & May/June 2002 & March 2002 & Difference & May/June 2002 & March 2002 & Difference \\
\hline A1 & 6.80 & 6.86 & -0.06 & 98.686 & 98.677 & 0.009 \\
\hline A5 & 6.87 & 6.77 & 0.09 & 98.442 & 98.452 & -0.009 \\
\hline A 8 & 24.89 & 24.34 & 0.55 & 97.748 & 97.744 & 0.003 \\
\hline B1 & 18.56 & 18.41 & 0.15 & 97.519 & 97.528 & -0.009 \\
\hline B2 & 20.76 & 21.02 & -0.26 & 97.366 & 97.339 & 0.027 \\
\hline B3 & 12.92 & 13.03 & -0.11 & 97.900 & 97.894 & 0.006 \\
\hline B4 & 7.78 & 7.70 & 0.07 & 98.458 & 98.470 & -0.012 \\
\hline B7 & 13.55 & 13.48 & 0.07 & 97.830 & 97.781 & 0.049 \\
\hline $\mathrm{B} 8$ & 5.18 & 5.17 & 0.02 & 98.668 & 98.656 & 0.012 \\
\hline B10 & 28.67 & 28.41 & 0.26 & 96.928 & 96.925 & 0.003 \\
\hline $\mathrm{C} 1$ & 15.73 & 15.75 & -0.02 & 98.305 & 98.269 & 0.037 \\
\hline C3 & 18.30 & 18.27 & 0.03 & 98.125 & 98.110 & 0.015 \\
\hline $\mathrm{C} 4$ & 16.96 & 17.17 & -0.20 & 98.077 & 98.083 & -0.006 \\
\hline C6 & 18.53 & 18.54 & -0.02 & 97.912 & 97.885 & 0.027 \\
\hline D2 & 9.30 & 8.87 & 0.43 & 98.433 & 98.433 & 0.000 \\
\hline D5 & 14.24 & 14.01 & 0.23 & 97.943 & 97.903 & 0.040 \\
\hline D6 & 16.57 & 16.72 & -0.15 & 97.562 & 97.476 & 0.085 \\
\hline D7 & 11.86 & 12.79 & -0.93 & 97.647 & 97.641 & 0.006 \\
\hline D9 & 10.23 & 10.42 & -0.20 & 98.293 & 98.260 & 0.034 \\
\hline E3 & 10.66 & 10.58 & 0.07 & 97.760 & 97.751 & 0.009 \\
\hline E7 & 6.15 & 6.30 & -0.15 & 98.412 & 98.378 & 0.034 \\
\hline E8 & 5.74 & 5.94 & -0.20 & 98.592 & 98.552 & 0.040 \\
\hline E9 & 14.88 & 14.95 & -0.07 & 97.555 & 97.586 & -0.030 \\
\hline E10 & 8.97 & 8.76 & 0.20 & 98.016 & 97.991 & 0.024 \\
\hline F6 & 9.89 & 9.96 & -0.07 & 98.043 & 98.025 & 0.018 \\
\hline F7 & 8.92 & 8.93 & -0.01 & 98.296 & 98.314 & -0.018 \\
\hline G2 & 9.84 & 9.73 & 0.11 & 97.988 & 97.982 & 0.006 \\
\hline G3 & 10.54 & 10.57 & -0.04 & 97.772 & 97.738 & 0.034 \\
\hline G5 & 12.07 & 11.66 & 0.41 & 97.211 & 97.168 & 0.043 \\
\hline \multirow[t]{3}{*}{ G9 } & 5.47 & 5.47 & 0.00 & 98.101 & 98.138 & -0.037 \\
\hline & & Mean Error & 0.18 & & Mean Error & 0.021 \\
\hline & & SD Error & 0.20 & & SD Error & 0.018 \\
\hline
\end{tabular}




\section{Chapter 5}

\section{Analysis and Results}

This chapter details the analysis methods used to evaluate change and also contains a discussion of what was observed in the surveys. Quality control, qualitative analysis and quantitative analysis are also discussed in this chapter. All surveys were measured using U.S. feet and data was converted to metric units for analysis.

\subsection{Data Management}

The first task undertaken for this project was developing a method to store and view the results from the successive cross sections. The seven years of data analyzed in this thesis encompasses approximately 540 surveyed cross sections (some cross sections were not surveyed every year) and 7,519 distance-elevation pairs. Graphing each cross section in an individual spreadsheet would have been extremely time consuming and cumbersome. A system was developed that utilized a Microsoft Access ${ }^{\mathrm{TM}}$ database to store the data and a single Microsoft 


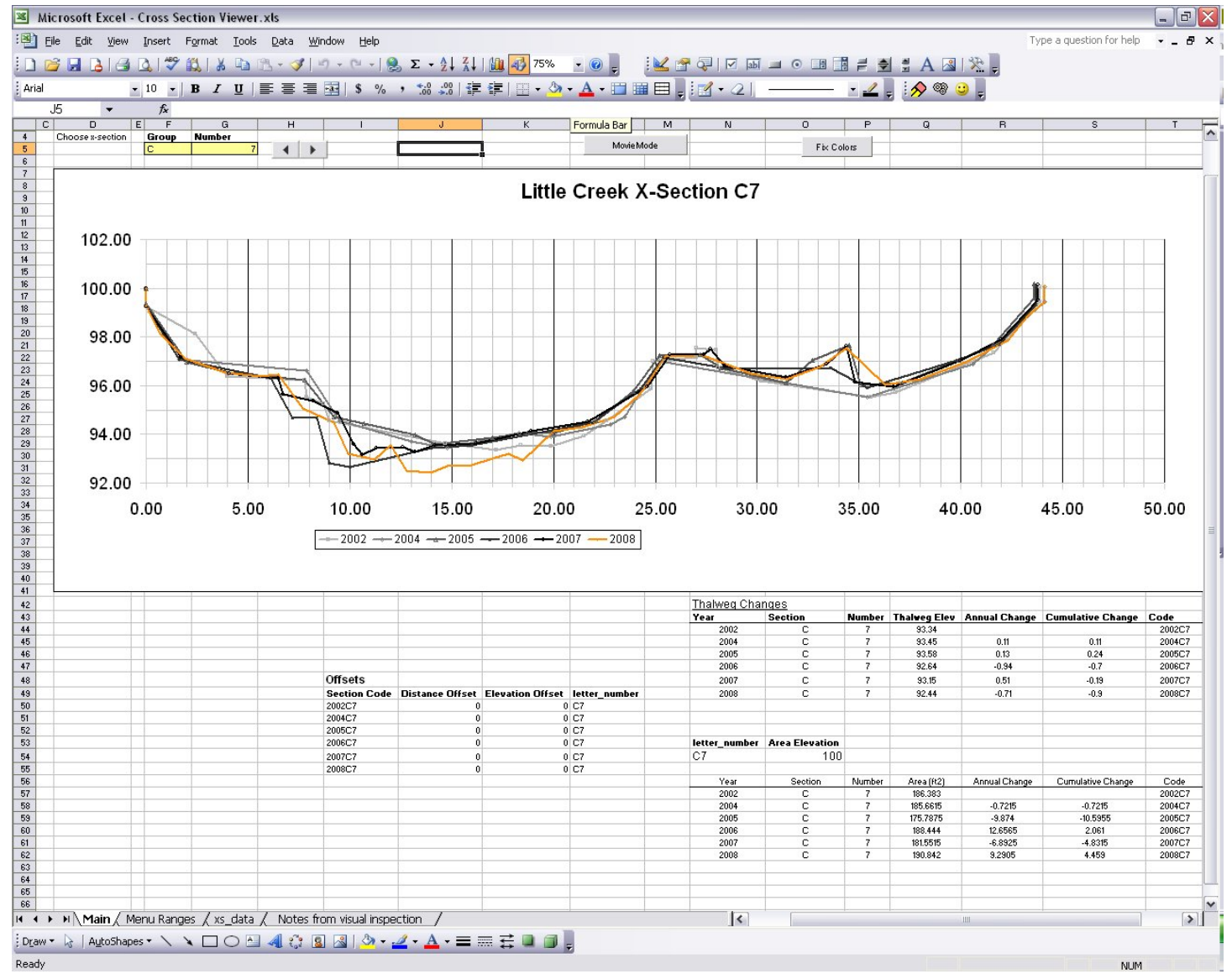

Figure 5.1: Screen shot of cross section viewer spreadsheet.

Excel $^{\mathrm{TM}}$ spreadsheet with custom programing to query the data and graph a chosen cross section with data from each year. The spreadsheet also applies the offsets needed to correct for rebar movement and calculates successive and cumulative changes in thalweg elevation, mean bed elevation, and cross-sectional area under a specified datum (for example, the field-identified bankfull elevation). Figure 5.1 is an example of the cross section viewer Excel ${ }^{\mathrm{TM}}$ spreadsheet. VBA code written for the custom Excel ${ }^{\mathrm{TM}}$ program is located in Appendix E. 


\subsection{Quality Control}

Quality control for the cross section data consisted of two main tasks. The first was looking for and correcting typos and errors in both the field notes and data entered electronically. These were primarily identified by visually reviewing the cross sections and identifying points that did not match previous cross sections or seemed out of place. The most common error was single points that were a foot $(0.30 \mathrm{~m})$ lower than the previous cross sections. This error commonly occurs when reading the Philadelphia rod through the automatic level telescope. When looking at number just below a whole foot marker, the number written down was often a foot higher than the actual measurement (for example, 9.87 instead of 8.87). All corrected measurements were noted in the field notes and in the database. Additionally, logs and small rocks not surveyed every year were removed from the cross sections so only the channel bottom was analyzed.

The second type of quality control involved correcting for movement of the endpoint rebar. The cross section viewer software and database (discussed below) were set up so that a given cross section could be offset vertically and horizontally to correct for movement of the rebar. If the right bank rebar was in a stable location, setting the offset so that the right bank rebar became the horizontal and vertical datum corrected the problem. In some cross sections, both the left and right rebar had moved. These were corrected by trying to identify another feature in the cross section that could be used to provide controls on the horizontal and vertical position. Figure 5.2 shows before and after correcting for rebar movement in a cross section.

There is data missing from several survey years. Table 5.1 shows what data is missing and the reason it is missing. In 2003 only cross sections in Reach A and 

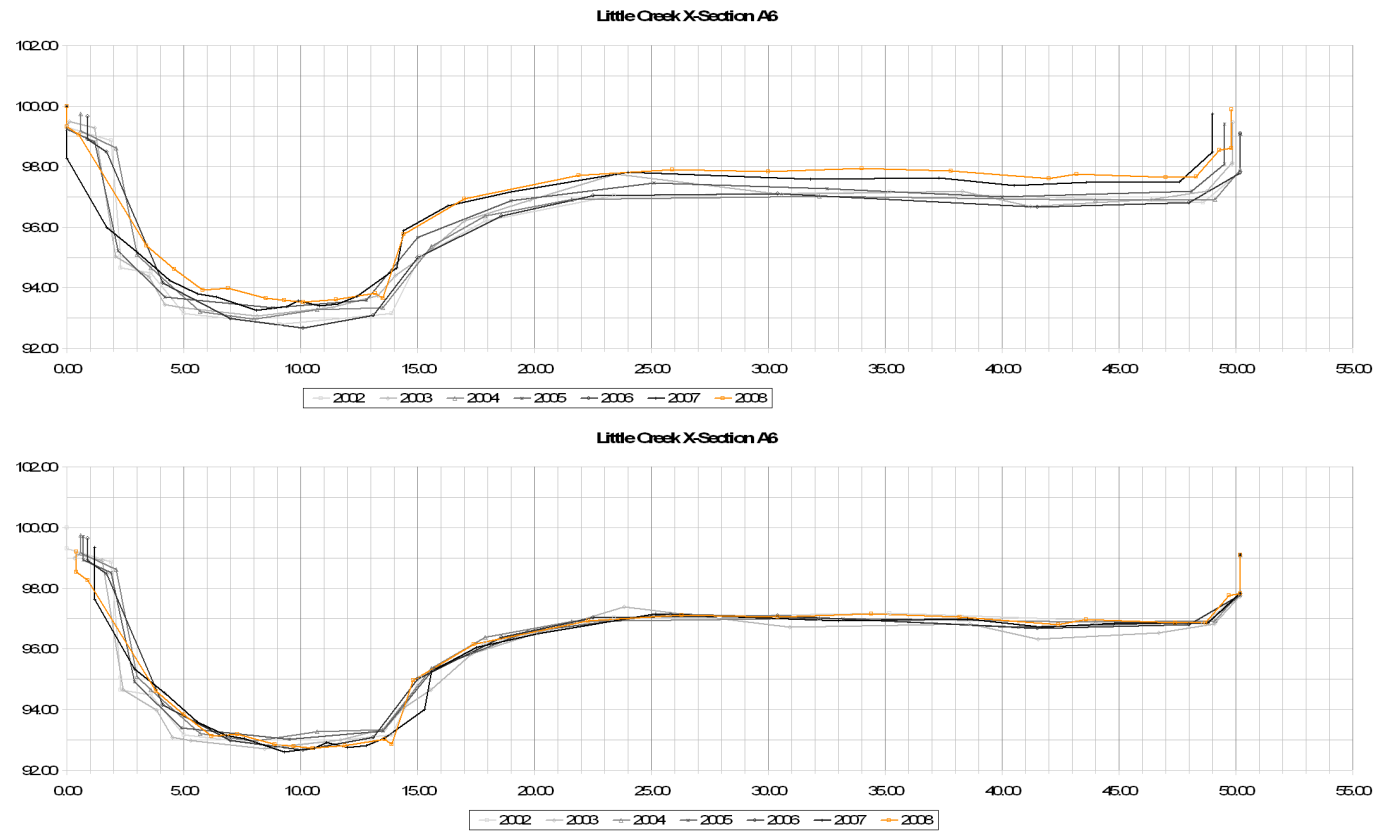

Figure 5.2: Figure showing before and after rebar correction in the A6 cross section. Cross sections were adjusted to match on the right rebar. View in all cross sections is looking downstream.

Reach B were surveyed due to time and staffing constraints. The 2002 data from Reach E was overwritten by 2004 data during data entry, however, original data from five of these surveys was located in the replicate cross section spreadsheet. The original field data is missing from 2002 (except from Reach A).

\subsubsection{Longitudinal profile adjustment}

The longitudinal profiles in each reach did not have specific start locations and also followed different paths up the stream channel. This meant that the profile between two identical plan view locations could have a different length. To help make comparisons between annual surveys, the longitudinal profiles were stretched and translated to match the distances where the profile crossed the cross sections in 2002. This allows much better comparison of the annual profiles because features (pools, steps, etc.) appear in roughly the same location each year. 
Table 5.1: Table showing missing survey data

\begin{tabular}{|c|c|c|}
\hline Profile & Year & Notes \\
\hline Reaches C, D, E, and F & 2003 & $\begin{array}{l}\text { Not surveyed due to staffing } \\
\text { and time constraints (lower half } \\
\text { of longitudinal profile in Reach } \\
\text { C was surveyed) }\end{array}$ \\
\hline $\mathrm{A} 4$ & 2002,2003 & $\begin{array}{l}\text { Right end point and cross sec- } \\
\text { tion alignment changed in } 2004\end{array}$ \\
\hline $\mathrm{C} 8$ & 2005 & Yellow jacket nest near section \\
\hline C9 & 2005 & Yellow jacket nest near section \\
\hline $\mathrm{C} 10$ & 2005,2007 & $\begin{array}{l}\text { Yellow jacket nest near section, } \\
\text { right bank endpoint had been } \\
\text { buried }\end{array}$ \\
\hline D4 & 2002 & Spreadsheet says "no stakes" \\
\hline $\mathrm{E} 1, \mathrm{E} 2, \mathrm{E} 4, \mathrm{E} 5, \mathrm{E} 6$ & 2002 & $\begin{array}{l}\text { Original data overwritten dur- } \\
\text { ing } 2004 \text { data entry, field data } \\
\text { could not be located }\end{array}$ \\
\hline
\end{tabular}

The first step in this procedure was identifying the points in each longitudinal profile where the cross section lines crossed the longitudinal profile. A spreadsheet using custom functions and lookup tables was developed that used the coordinates from the longitudinal profile and the cross section rebar to determine the threedimensional coordinates of the intersection point. This point was then added to the longitudinal profile. The distance where the cross section crossed the 2002 longitudinal profile was set as the standard distance. The longitudinal profile from each subsequent year was then translated, stretched, or compressed so that the distances at the cross section intersections matched the 2002 survey. Sections below Cross Section 10 and above Cross Section 1 were only translated. All other sections between cross sections were linearly re-interpolated so the start and end distances matched the 2002 survey. Figure 5.3 shows the lower section of Reach D before and after adjustment of the longitudinal profiles. The unadjusted data was translated to match at a point in the upper part of the reach which caused 
poor alignment in the lower reach. The adjusted data is much easier to interpret and it is more obvious that this reach has been very stable over the study period.
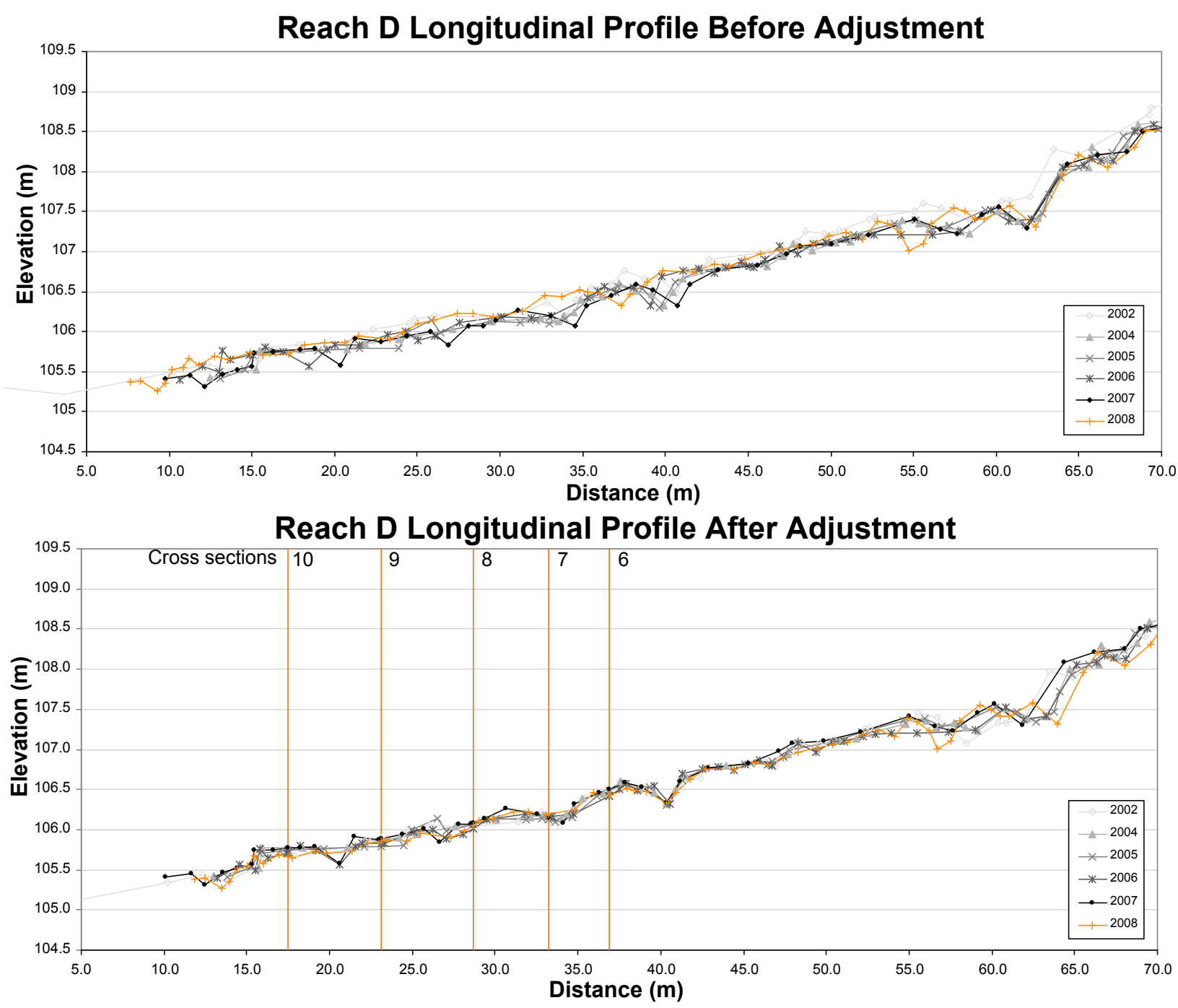

Figure 5.3: Reach D longitudinal profile before and after adjustment.

\subsection{Qualitative Analysis}

When evaluated visually, most of the reaches do not appear show much change. There are a few cross sections, especially in Reach C, which had some substantial changes. However, the observed changes are relatively minor compared to the 
magnitude of changes discussed in much of the literature (Madej \& Ozaki, 1996; Leopold et al., 2005; Nolan \& Marron, 1985). All cross sections are contained in Appendix A and all longitudinal profiles are contained in Appendix B. All cross sections are shown looking downstream.

\subsubsection{Reach A}

Overall, there was little change in Reach A during the study period. Changes in the cross sections can primarily be traced to influence of forced channel features (boulders) or unstable/eroding banks. The A6 left bank has eroded and even undercut the left rebar endpoint. Except in Cross Section A10, there was not much change in the active channel. The A10 cross section is through a pool created by flow around several boulders; the arrangement of this pool appears to have changed several times during the study period due to flow routing around these boulders. Some of this change may also be due to slight deviations in the measurement location upstream or downstream of the cross section line (as discussed in 4.4.1). Overall, bed elevations and channel dimensions have remained about the same in this reach.

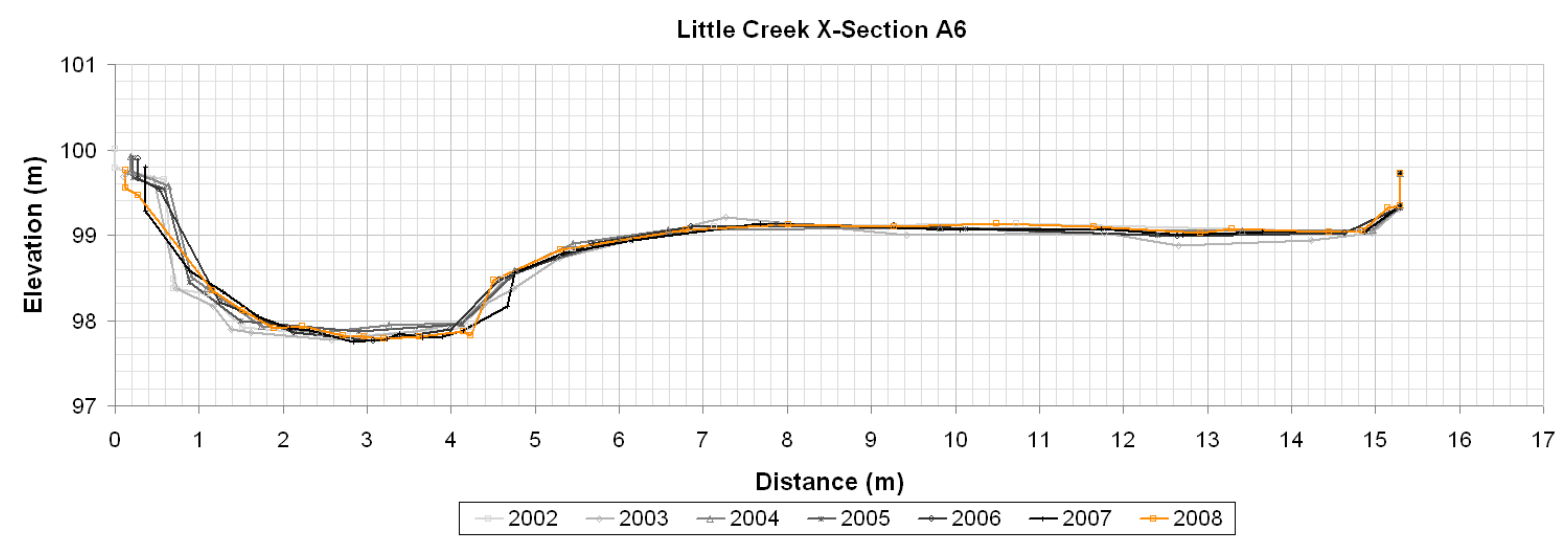

Figure 5.4: Cross section A6 


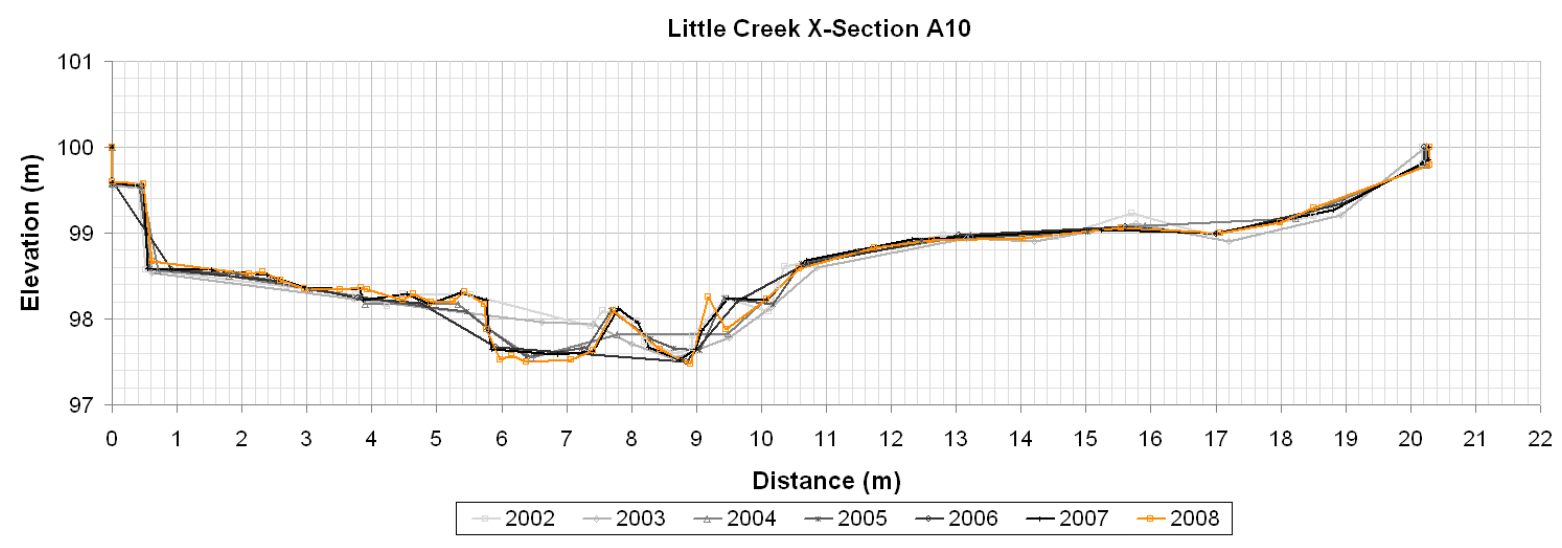

Figure 5.5: Cross section A10

\subsubsection{Reach B}

Similar to Reach A, there were no major changes in the Reach B cross sections. Cross sections B1, B2 and B3 show minimal change occurred in the channel. The B4 cross section had some change during the study period. The top of a bay laurel tree had fallen across the channel downstream and began to collect debris. This created a dynamic grade control that appears to have changed slightly each year as the debris shifted, accumulated, or flushed away. The B8 cross section had a tree fall across it and the tree landed on the right bank rebar. This made surveying this cross section difficult and explains the amount of variability in the right bank. Cross sections B9 and B10 have a very steep left bank, and it appears that the toe of this bank was eroded and scoured in the 2006 and 2007 cross sections, with some filling occurring in 2008. 


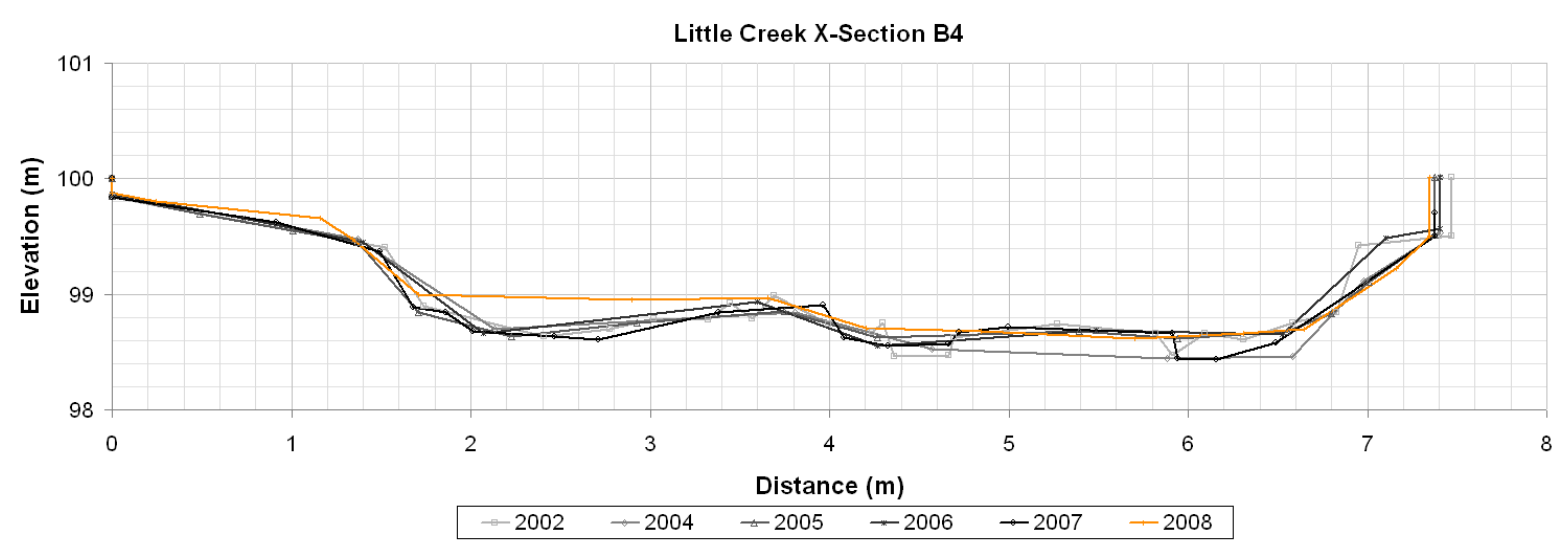

Figure 5.6: Cross section B4. A tree fell just downstream of this cross section.

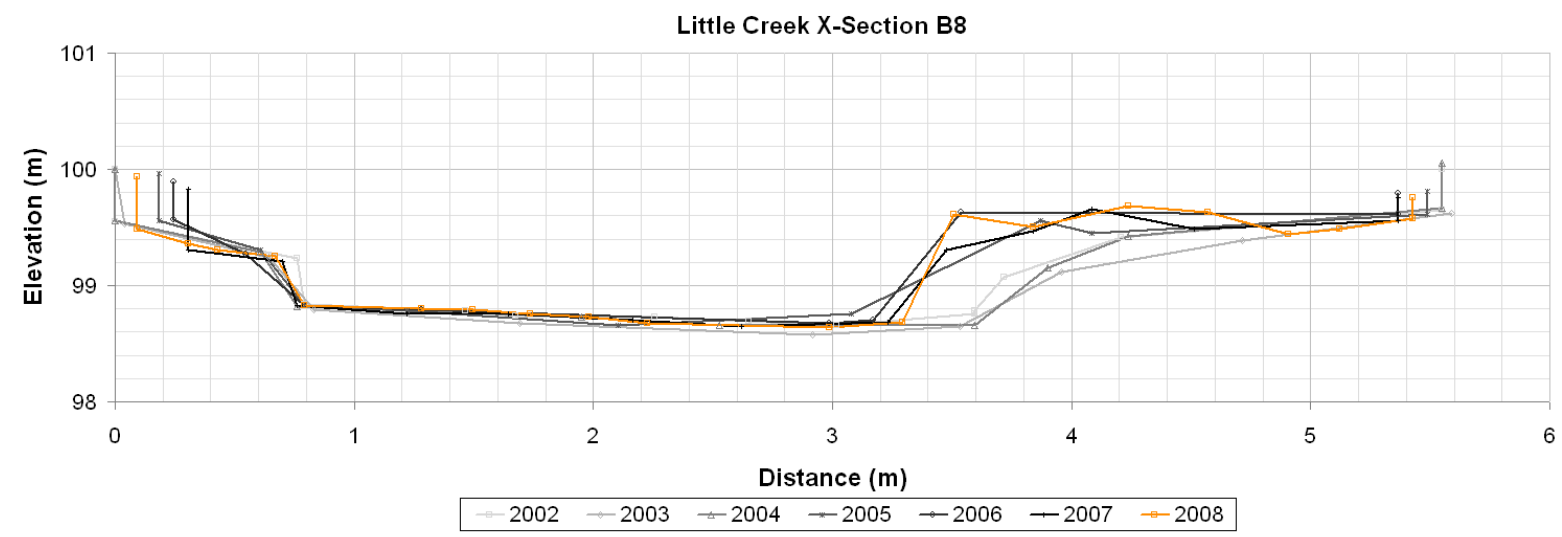

Figure 5.7: Cross section B8. A tree fell on rebar and trunk is perpendicular to cross section. The left bank also has been eroding slightly.

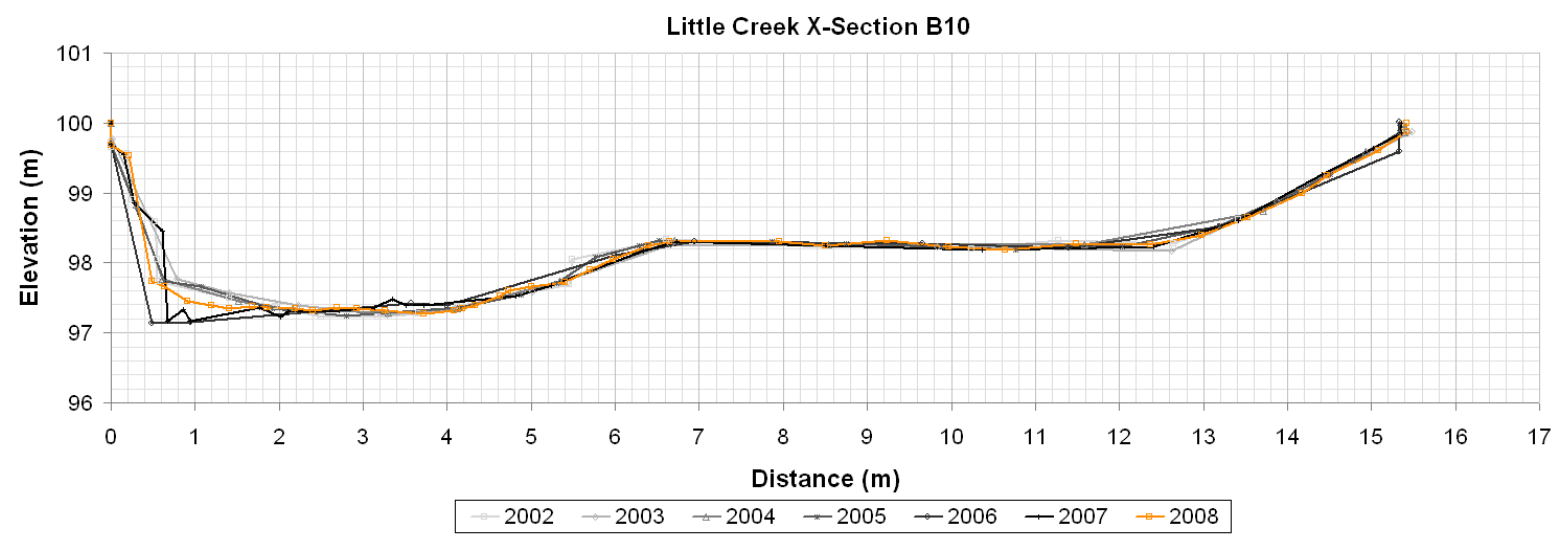

Figure 5.8: Cross section B10 


\subsubsection{Reach C}

Reach $\mathrm{C}$ was the most dynamic reach during the study period. This reach has two groups of five cross sections separated by a log/boulder jam. The upstream portion is an aggradational area where sediment was stored behind the log/boulder jam. As the arrangement of wood in the logjam has been modified by high streamflows and/or decomposition, the stream has been downcutting into this stored sediment. This change was most drastic in between the 2005 and 2006 surveys. Annual precipitation that winter was well above average and there were several high flow events. The change is most evident in the C4 and C5 cross sections and in the longitudinal profile. There has also been change in several of the downstream cross sections as well. The channel thalweg has migrated and/or downcut in cross sections $\mathrm{C} 7, \mathrm{C} 8$ and $\mathrm{C} 9$. Causes for this are not clear, but changes in the flow patterns out of the logjam area may be important. Reach $\mathrm{C}$ was not surveyed in 2003.

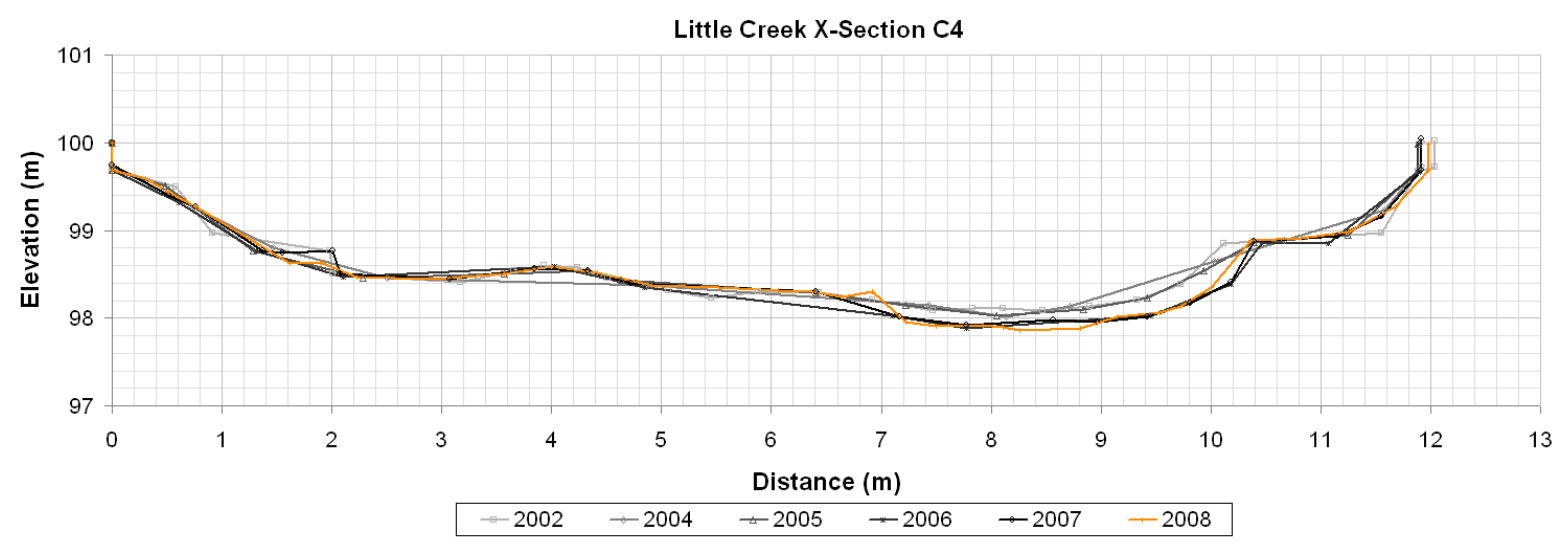

Figure 5.10: Cross section C4 


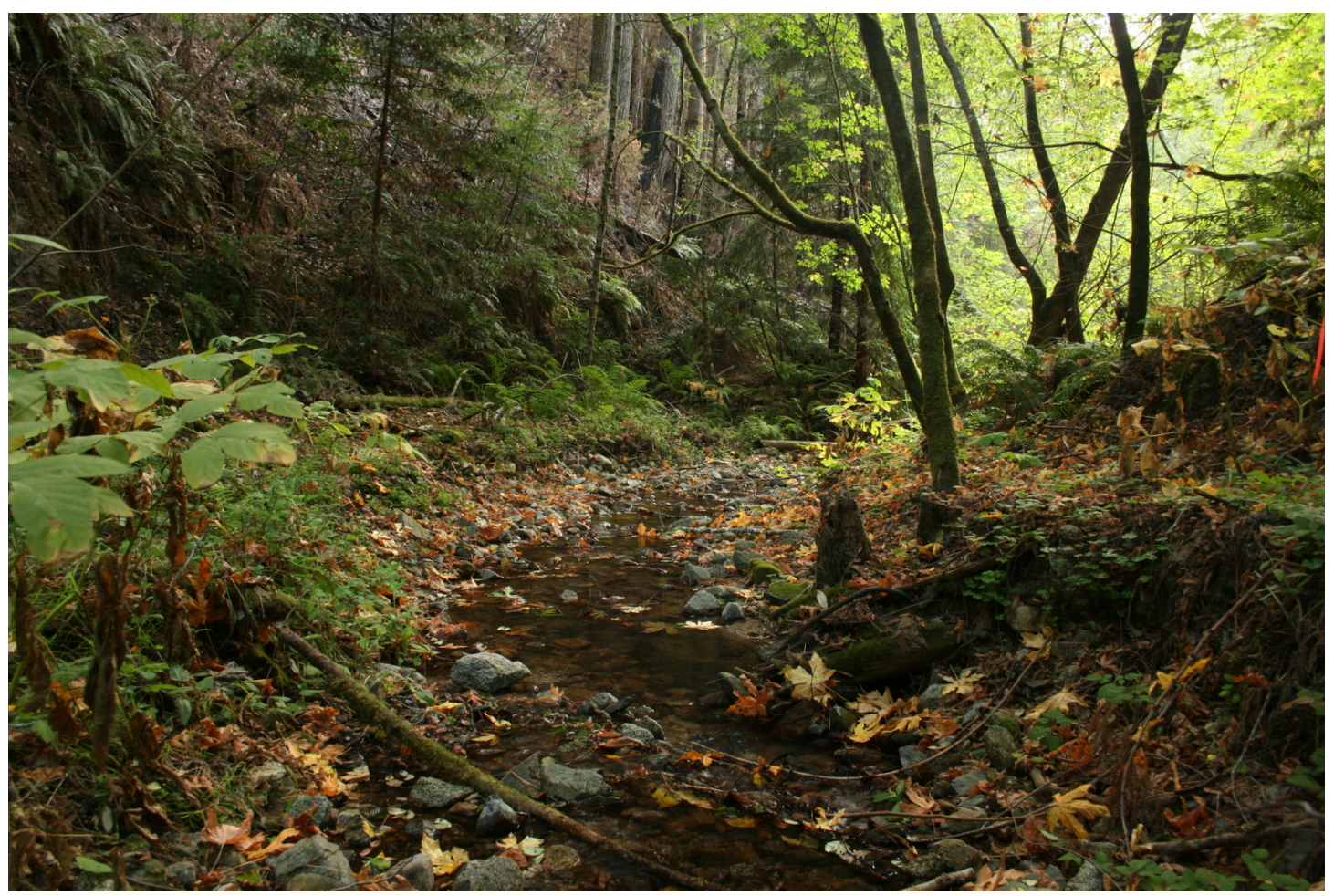

Figure 5.9: Upper Reach C, Fall 2009 after the Lockheed Fire. View is looking downstream near cross section C3.

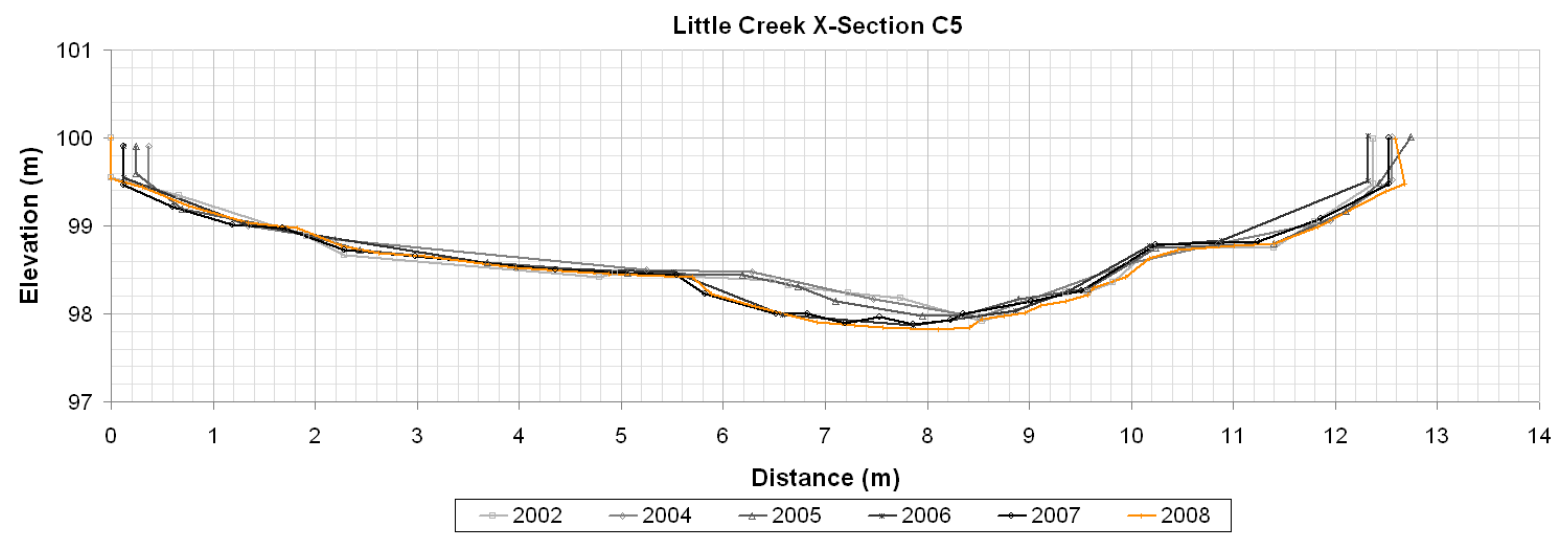

Figure 5.11: Cross section C5 


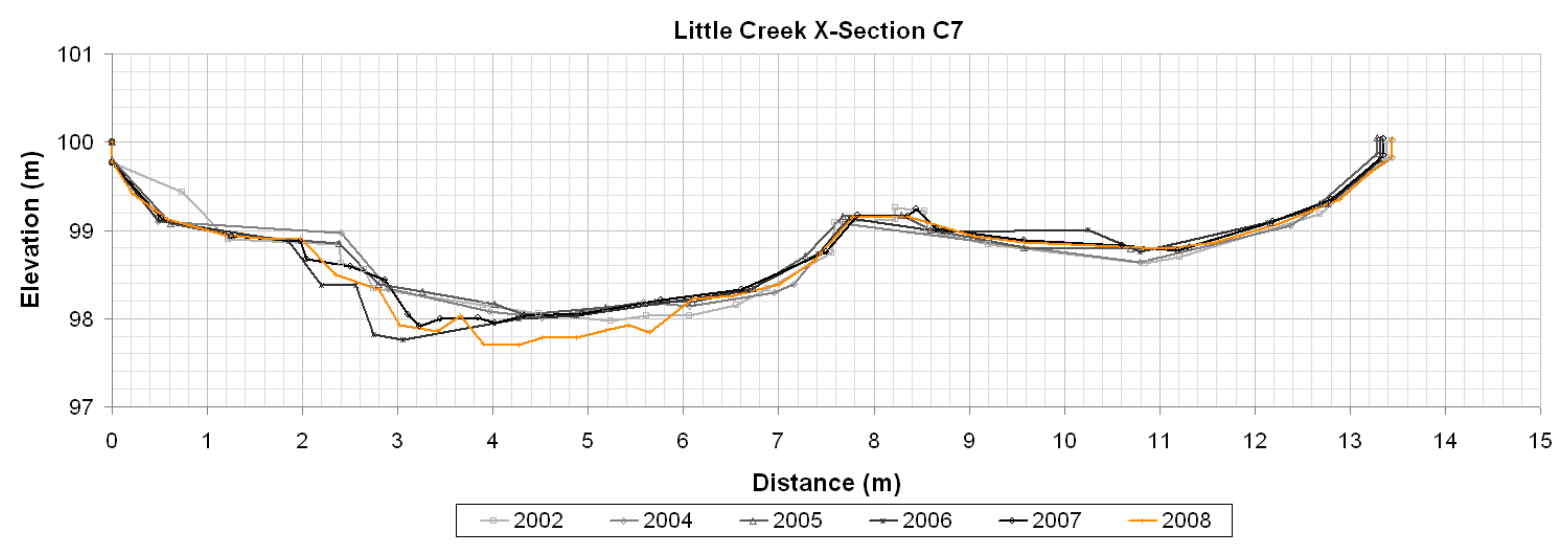

Figure 5.12: Cross section C7

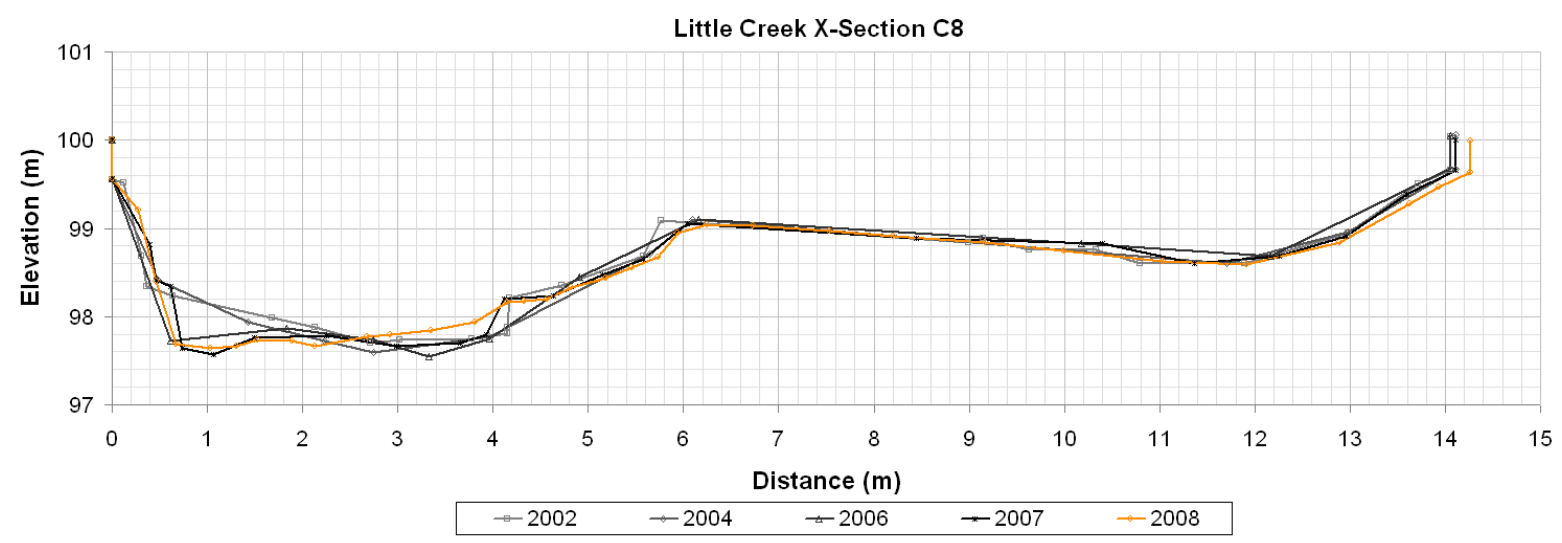

Figure 5.13: Cross section C8 
Reach C Longitudinal Profile (Upper)

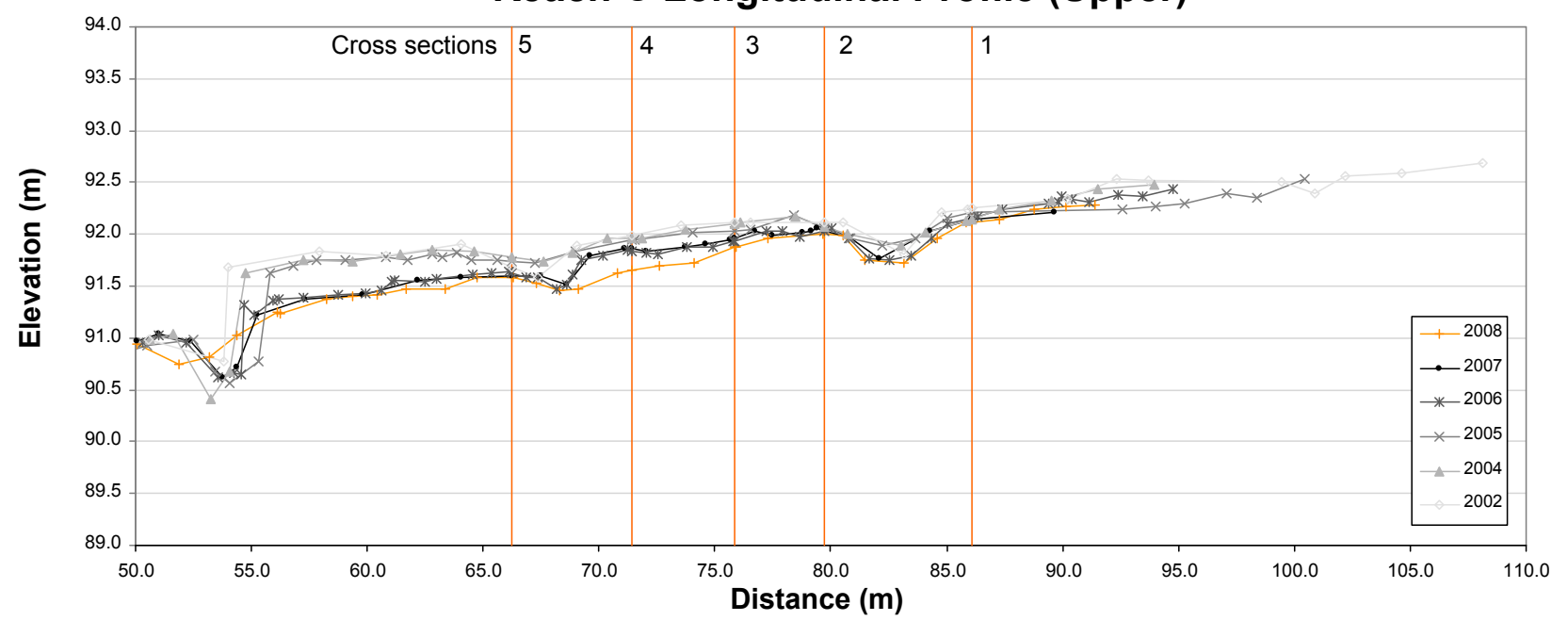

Figure 5.14: Little Creek upper Reach C longitudinal profile. This shows the downcutting behind the log step (at approximately 55 meters) that occurred between the 2005 and 2006 surveys.

\subsubsection{Reach D}

Reach $\mathrm{D}$ was the most stable reach during the study period. This reach also has two groups of cross sections which are separated by a clump of redwood rootwads and other debris deposited by a landslide that occurred in 1998. Unlike Reach C, this deposit appears to be relatively stable, and there has been little change in the cross sections above this feature. Several of the rebar in the lower section were placed on steep, unstable banks and have moved during the study period. Reach D was not surveyed in 2003. 


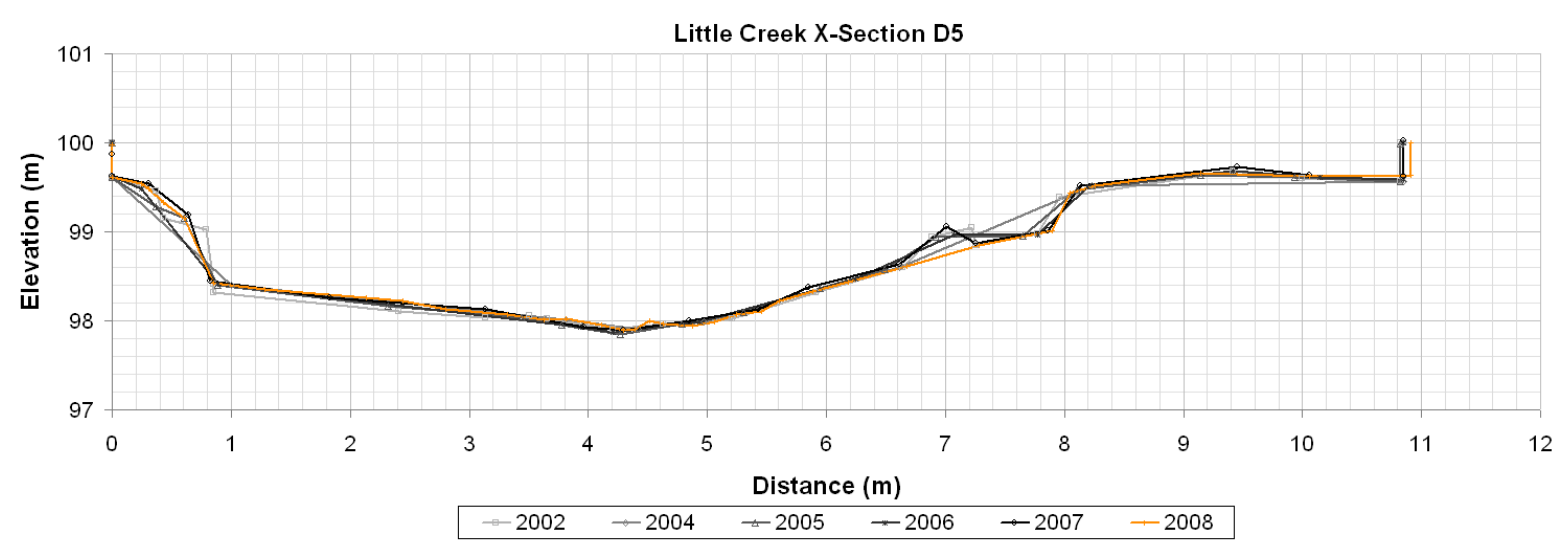

Figure 5.15: Cross section D5.

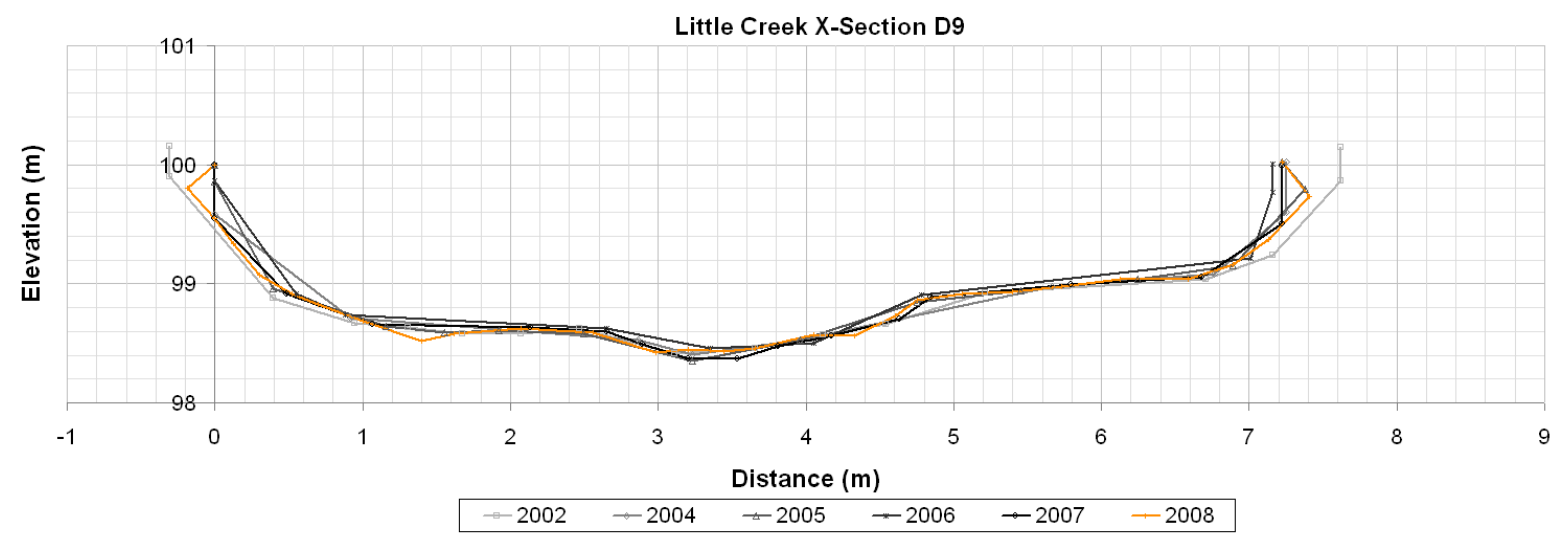

Figure 5.16: Cross section D9. Notice the banks have eroded slightly and the rebar endpoints have moved, but the channel has been stable.

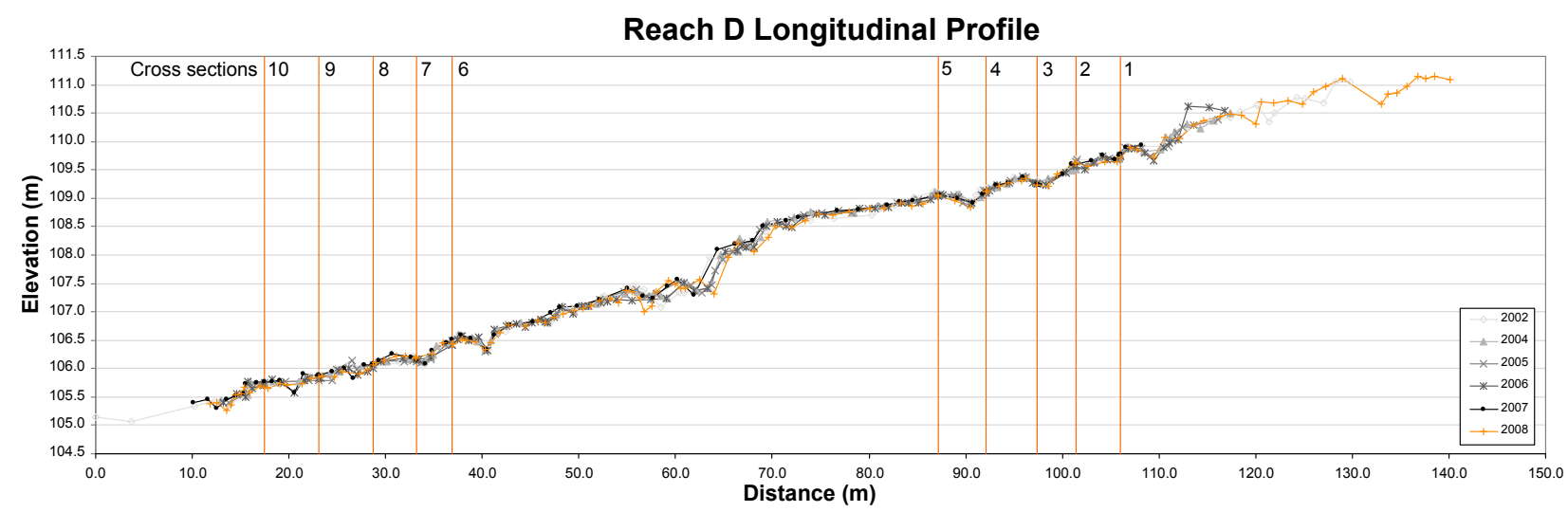

Figure 5.17: Reach D longitudinal profile. 


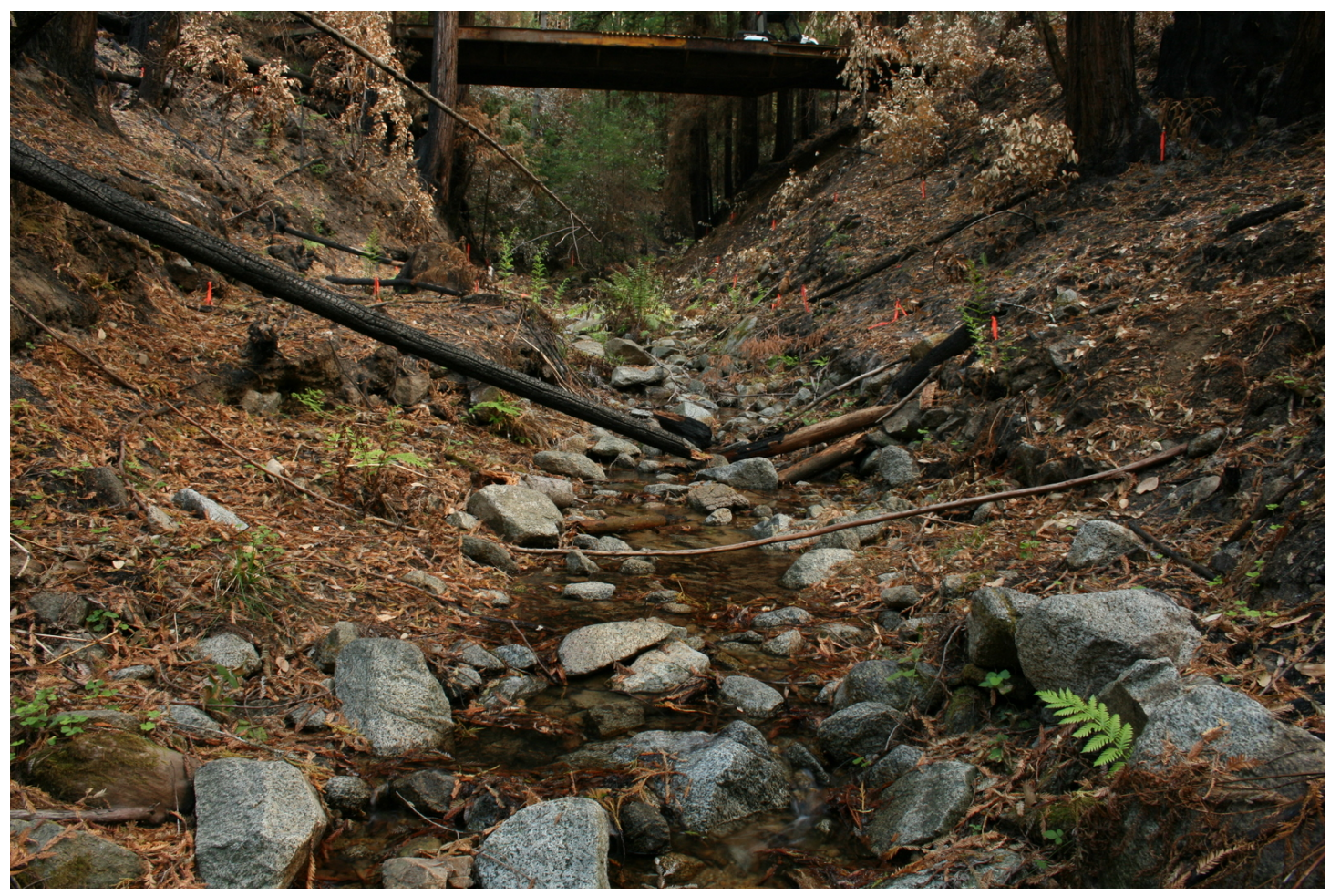

Figure 5.18: Reach E, Fall 2009 after the Lockheed Fire. View is looking upstream near cross section E8

\subsubsection{Reach E}

Reach E has also been relatively stable during the study period. This reach is bouldery and has a relatively steep gradient ( 5 percent). Much of the survey variation in this reach is likely due to the high local variability (measuring slightly upstream/downstream of section can cause large changes in elevation) and inconsistencies in how the surveyors dealt with surveying the large boulders. One section that is hard to interpret is E1, where there is a significant undercut bank. Previous survey methods were not able to characterize the undercut bank. The instructions for surveyors (Appendix C) include procedures for dealing with undercut banks in the cross section utilizing a hand level and additional measuring rod. The E reach was not surveyed in 2003 and is missing data from many of the cross sections in 2002. 


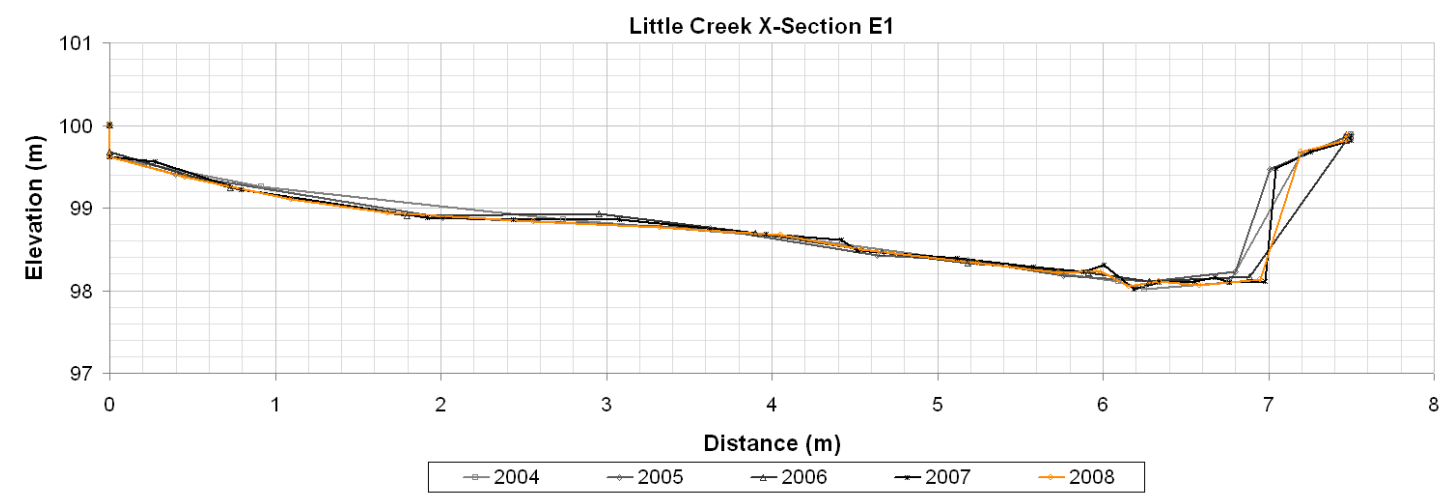

Figure 5.19: Cross section E1. Right bank is undercut. Surveyor instructions (Appendix C) provide instructions on how to survey undercut banks.

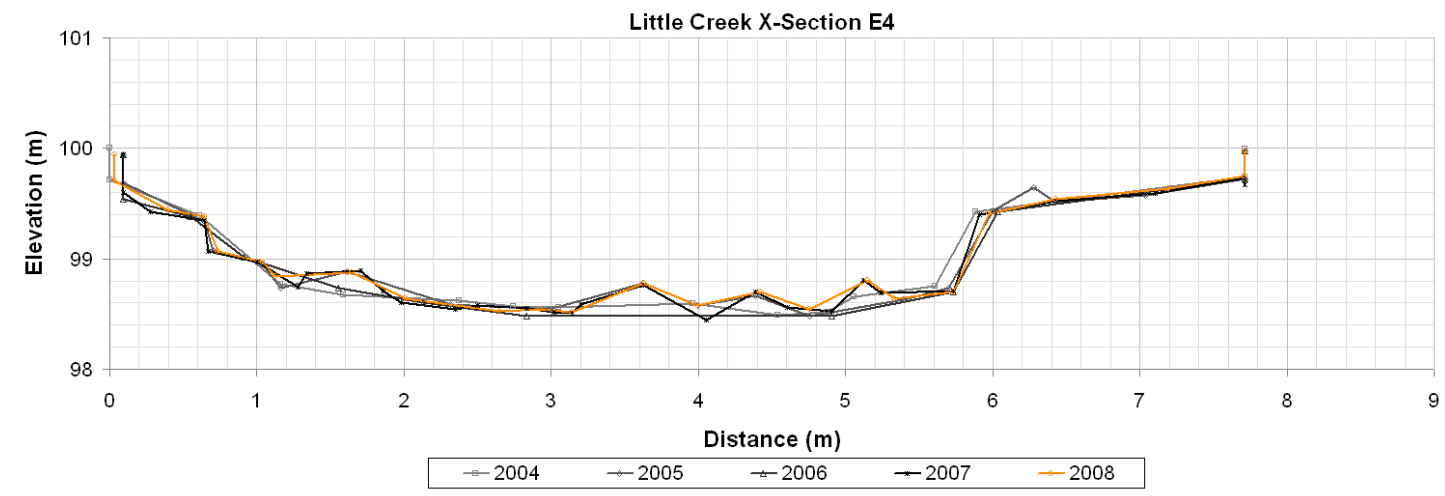

Figure 5.20: Cross section E4. Much of the variability in the cross section is likely due to inconsistencies in how the large rocks were surveyed.

\subsubsection{Reach F}

Reach F aggraded during the study period. Cross sections F2, F3, F4, F5, F6 and F10 all show this aggradational trend. The 2006 and 2008 surveys have the strongest aggradation signal. In contrast to the changes in upper Reach $\mathrm{C}$, there is no obvious cause for the trend in Reach F. A boulder may have been deposited in the F5 cross section in 2007, or it was not surveyed in previous years. Reach F was not surveyed in 2003. 


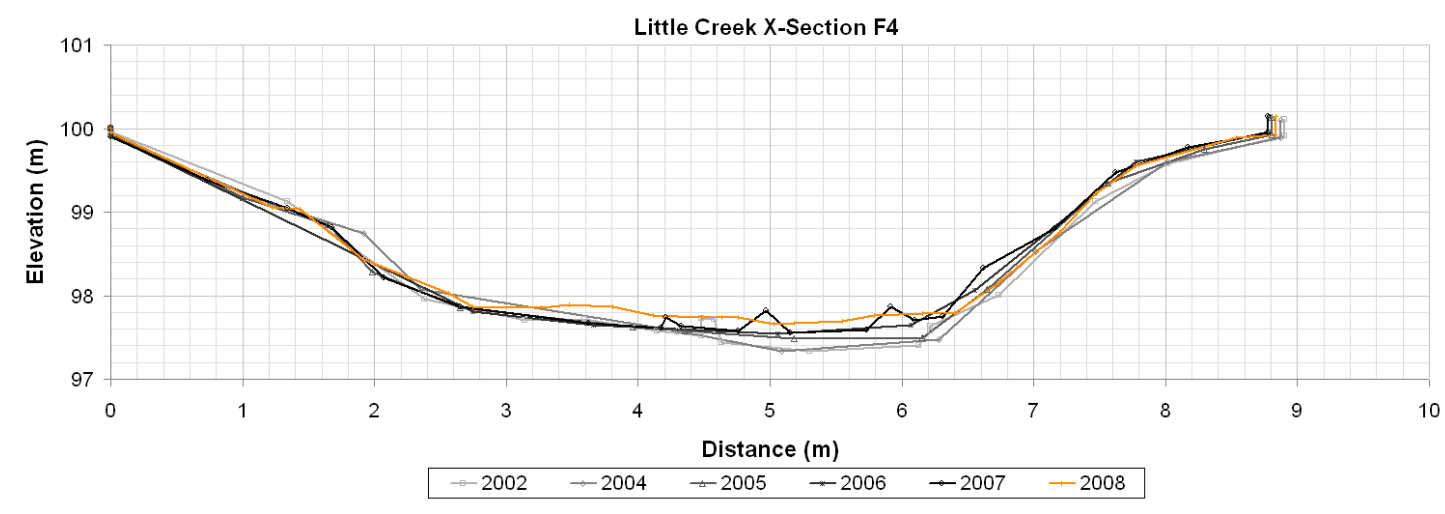

Figure 5.21: Cross section F4.

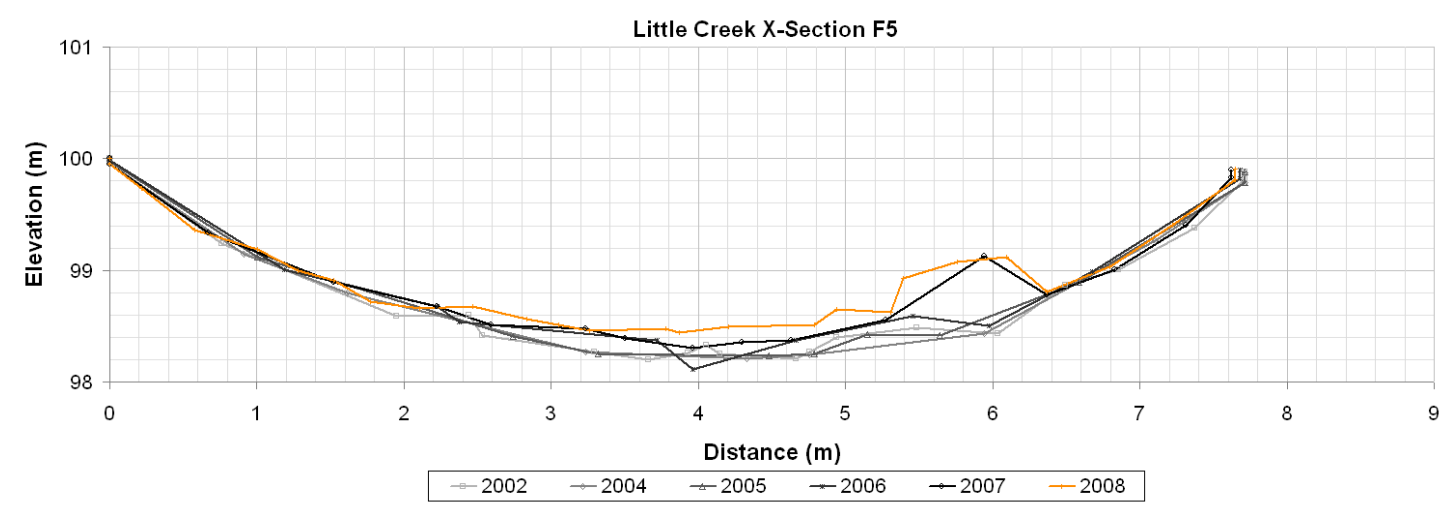

Figure 5.22: Cross section F5. A boulder was deposited on the right bank in 2007 (or the boulder was not surveyed in previous years).

\subsection{Quantitative Analysis}

\subsubsection{Cross sections}

There are three primary numerical properties of the cross sections that were calculated and compared: thalweg elevation, cross-sectional area, and mean bankfull channel elevation. 


\section{Thalweg elevation}

Thalweg elevation is the elevation of the deepest part of the channel along the cross-sectional profile. This is a measure of vertical change in the channel elevation and is an indication of scour or fill of the channel. Thalweg elevation can change with no corresponding change in mean elevation or loss or addition of material from a reach. However, thalweg elevation changes typically correspond with fill or scour of the channel. Thalweg elevations can also be calculated by interpolating the elevation of the longitudinal profile where it crosses through the plane of the cross section. Figure 5.23 compares elevation changes calculated from each method in Reach A. The magnitude of change measured at individual cross sections is not always consistent, but overall trends are similar.

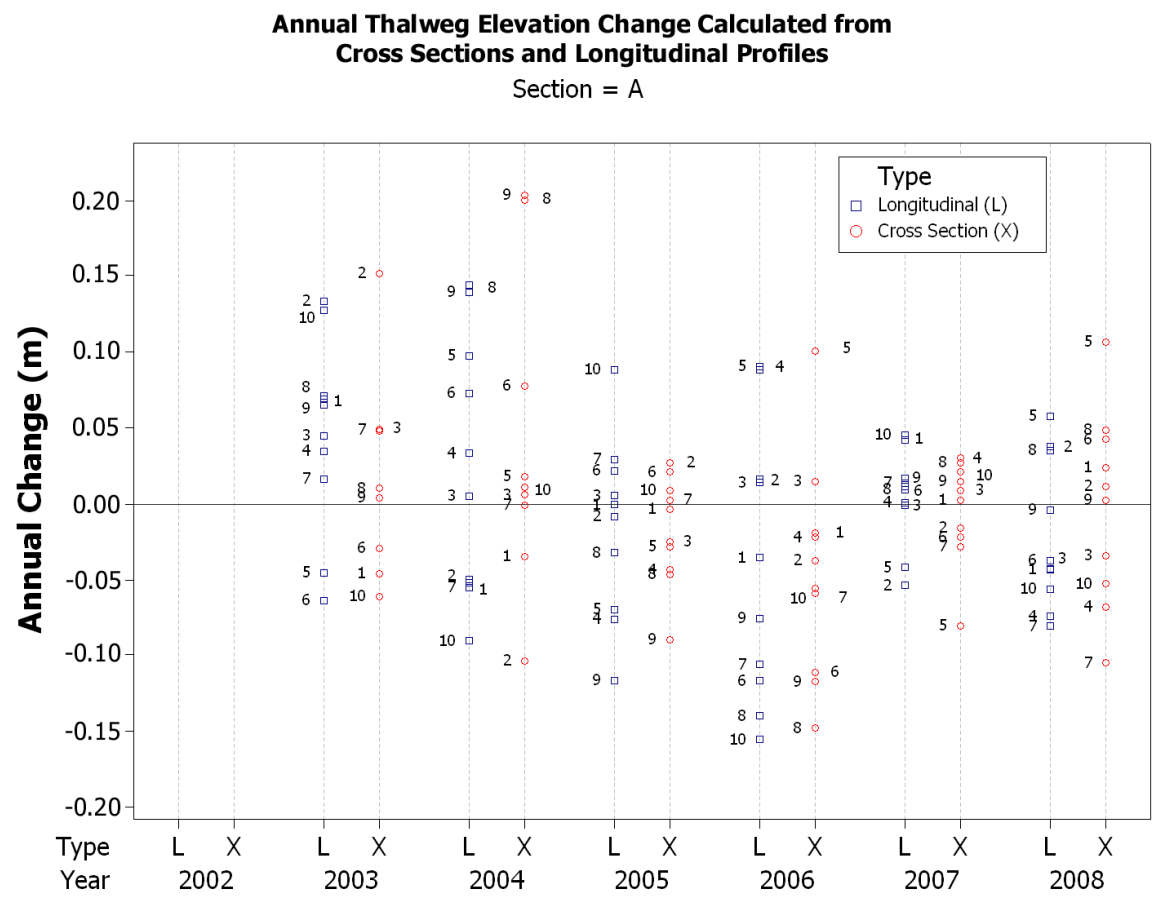

Figure 5.23: Individual value plot comparing annual thalweg elevation changes calculated from cross sections (X) to annual thalweg elevations changes calculated from the longitudinal profile (L). The number indicates the cross section. Negative changes indicate channel scour, and positive changes indicate channel filling. 
The most useful way of viewing the cross section data utilizes boxplots that show change in all cross sections in a reach. Both annual change and cumulative change since the first survey are calculated and shown in the boxplots. These boxplots are a way of integrating all of the observations made in a reach. The boxplot shows the maximum, minimum, interquartile range, median, and mean of the changes observed in each reach. The boxplots confirm some patterns observed in the qualitative analysis. The degradational trend in Reach $\mathrm{C}$, and the aggradational trend in Reach $\mathrm{F}$ are both apparent in the annual and cumulative change boxplots. Another pattern that emerges is the overall variability of change was greater in some years than others.

Thalweg elevation changes are shown in Figure 5.24. Reach A appears to have aggraded slightly during 2002-2004, degraded during 2005-2006, and remained relatively stable during 2007-2008. The changes in Reach A have been relatively minor, with only a few cross sections changing more than 0.15 meters during the study period, and mean changes less than 0.06 meters. Reach B shows a slight aggradational trend during the study period; overall change was minor with few cross sections changing more than 0.05 meters and reach mean changes less than 0.03 meters. Reach $\mathrm{C}$ had the most pronounced changes in thalweg elevation. The degradational trend after 2006 noted in the qualitative analysis is also evident here. By 2008, reach mean thalweg elevation dropped about 0.12 meters, with some cross sections approaching 0.30 meters of lowering. Both Reach D and Reach E were very stable during the study period with no elevation changes greater than 0.15 meters and reach mean changes less than 0.03 meters. Reach F shows an aggradation trend between 2005 and 2008. Mean thalweg elevation increased each year, and by 2008 all Reach F cross sections had aggraded. The mean thalweg elevation gain was almost 0.15 meters and thalweg elevations in three cross sections 

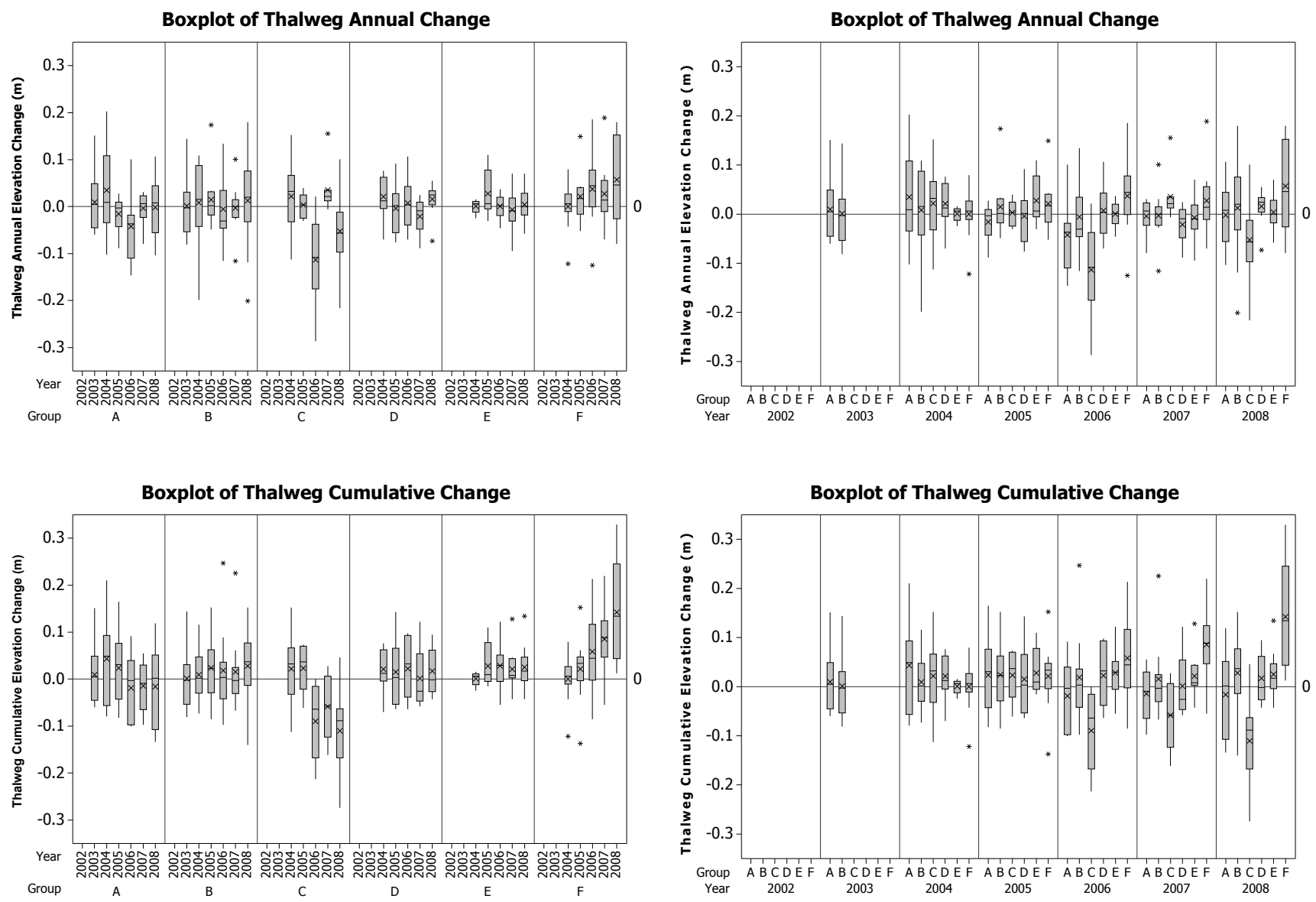

Figure 5.24: Boxplot shows thalweg elevation change grouped by year and by reach. Cumulative change is change since the first survey, annual change is change since the previous survey (typically annually, but not every cross section was surveyed every year). The boxplot shows the median (middle bar), interquartile ranges, and the mean (small $\mathrm{x}$ ) for the group of 10 cross sections in each reach for each year. Scour is a negative change and fill is a positive change.

increased over 0.24 meters.

\section{Cross-sectional area}

Cross-sectional area is another commonly used metric to evalute changes in cross sections. Changes in cross-sectional area can be multiplied by a representative 
length to determine sediment volume changes in a channel reach. One consideration when using area as a metric is how the location of the the top of the channel cross section is defined. Theoretically, the cross section could extend all the way to the ridgeline. The choice of elevation depends on the intended interpretations made from the data. If the only concern is net change of area, any area that encompasses the entire channel where change occurred would be appropriate.

Hilburn (2010) used areas under the 100 meter elevation (the arbitrary elevation given to the top of the left bank rebar) to compare of areas of ground-surveyed and LiDAR-derived cross sections in Little Creek. Using the rebar as the elevation datum worked well for his analysis, however, it also imparts several challenges when using area as a change-detection metric. One issue with using the rebar as the elevation datum for change comparisons is that the surveyed channel is typically much larger than the active channel that experiences water flow, even at flood level. This means that changes in the active channel can be "drowned out" by surveying error, rebar movement, and other noise in the larger cross section. Also, when comparing different cross sections, it is easier to make comparisons of relative change if the cross-sectional areas are relatively similar. Because of the high variability in shape of banks and the constraints of installing and accessing the endpoints on steep slopes, areas under the rebar datum vary greatly, even within adjacent cross sections in the same reach.

For this analysis bankfull areas are used instead. One challenge with using bankfull elevation to define an area measurement is identifying the bankfull elevation, especially in a steep banked, incising channel like Little Creek where bankfull indicators are typically vague (Roper et al., 2008; Rosgen \& Silvey, 1996). Bankfull areas were calculated using the bankfull elevation identified in the 2002 survey. Surveying in that year was performed by a graduate student (Brooke Akers) and 
there is an unjustified assumption that her assessment is the most reliable and consistent. Some elevations had to be adjusted slightly $(<0.1 \mathrm{~m})$ due to the relatively simple area calculation algorithm, which could not handle points between banks that are above the bankfull channel level. The field-identified bankfull elevations were also adjusted when a visual check of the cross section indicated that they were too low or too high. Even if the field identified bankfull stage is inaccurate, it provides an elevation datum that allows better comparisons of relative changes in area, because the range of bankfull areas is much smaller than the range of areas under the elevation of the rebar. For example, the range of areas in Reach D calculated under the rebar elevation varies from approximately $9 \mathrm{~m}^{2}$ to $36 \mathrm{~m}^{2}$, and the calculated areas under the bankfull elevation varies from $0.7 \mathrm{~m}^{2}$ to $2.6 \mathrm{~m}^{2}$. The smaller difference in bankfull areas makes it easier to compare changes between cross sections because the changes will be more proportional. In most cross sections, using the bankfull area removed parts of the cross sections where the banks are very steep and surveying errors are higher, and also helped minimize area changes due to rebar movement. It is also important to note that the bankfull area analysis is more prone to errors resulting from discrepancies in how surveyors interpreted complicated bed features (e.g. boulders) than the thalweg elevation analysis because the surveyor typically can consistently identify the lowest point of the cross section.

The cross-sectional area metric provides a quantification of the amount of material added or removed from the channel. However, there are also limitations in interpreting the change in area of the channel cross section. Area changes could result from a change in width or depth in the channel. Figure 5.25 shows the boxplot of observed changes in bankfull area.

Trends in bankfull area change are similar to those in seen in thalweg elevation. 

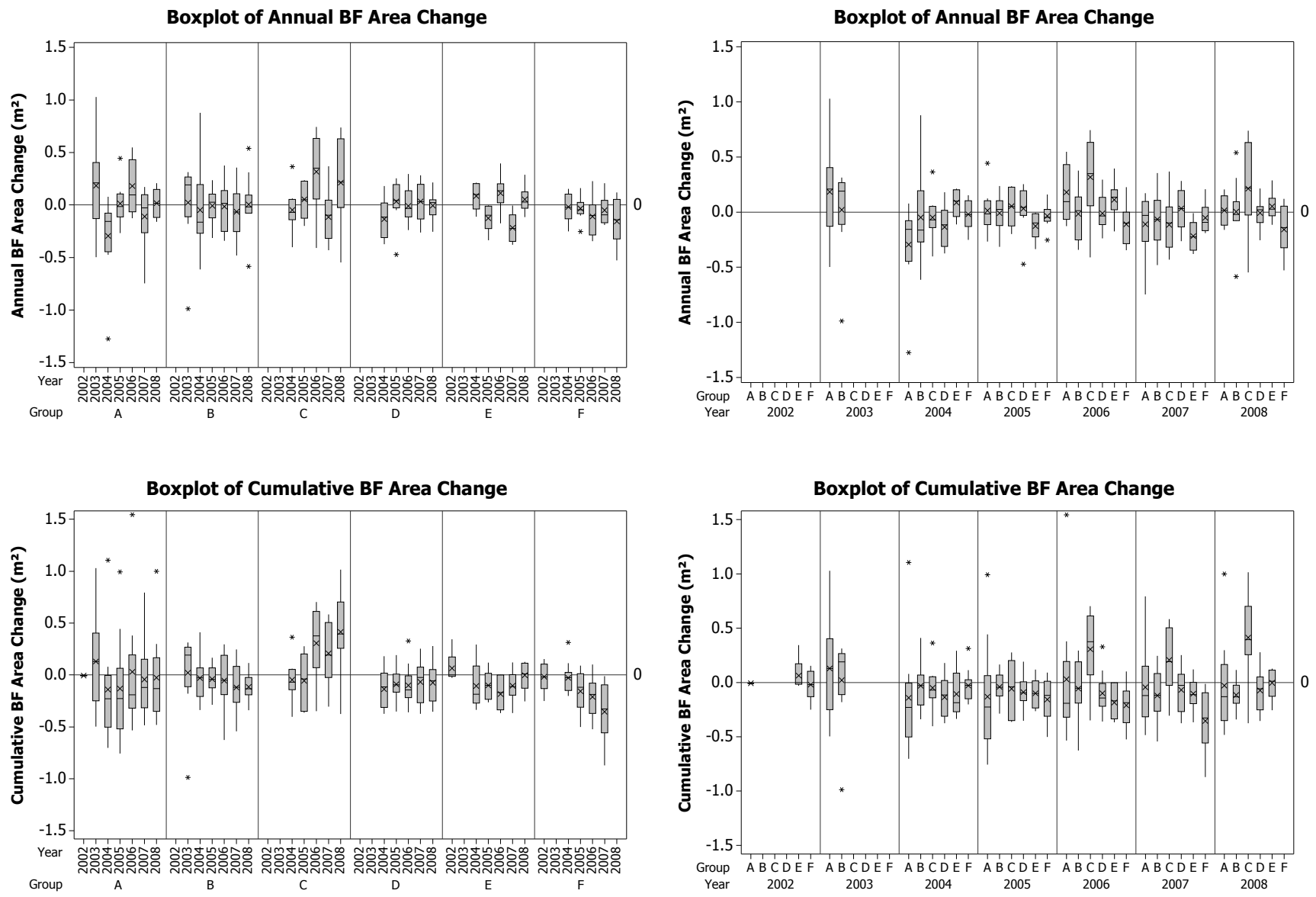

Figure 5.25: Boxplot shows bankfull area change grouped by year and by reach. Cumulative change is change since the first survey, annual change is change since the previous survey (typically annually, but not every cross section was surveyed every year). The boxplot shows the median (middle bar), interquartile ranges, and the mean (small $\mathrm{x}$ ) for the group of 10 cross sections in each reach for each year. Note that scour of the channel is a positive change and fill of the channel is a negative change (opposite direction of mean bed elevation and thalweg elevation change graphs). 
There was a loss of material from Reach C (scour) and a gain of material in Reach F (fill). As noted in the qualitative analysis, there was significant scour in A10, but overall change in Reach A was minimal with mean decrease of cross-sectional area of $0.2-0.3 \mathrm{~m}^{2}$. The range of change in this reach was generally larger than the range of changes observed in other reaches, but this reach has the largest drainage area and cross-sectional area. When considered as a percentage of bankfull area, the magnitude of change in Reach A is similar to the magnitude of change in other reaches. No reach-wide trends are obvious in Reaches B, D or E and mean changes in cross-sectional area are small $\left(<0.2 \mathrm{~m}^{2}\right)$.

\section{Mean bankfull channel bed elevation}

A third metric, mean bankfull channel bed elevation, was also used to compare the annual surveys. This is a commonly used metric in many studies that utilize cross section surveys to study channel changes. Mean elevation was calculated using the following equation:

$$
\text { mean bankfull bed elevation }=\text { bankfull elevation }-\frac{\text { bankfull area }}{\text { bankfull width }}
$$

The mean elevation has similar interpretation limitations as cross-sectional area, but is less sensitive to changes in channel width.

The mean bed elevation trends are similar to thalweg elevation and bankfull area trends. There are slight variations in the relative magnitude of change, but overall patterns are very similar. Only a few cross sections had a mean bankfull elevation change of over $0.15 \mathrm{~m}$ during the study period, and except for Reach $\mathrm{C}$ and Reach F, reachwide mean changes were less than $0.06 \mathrm{~m}$. This is only slightly 

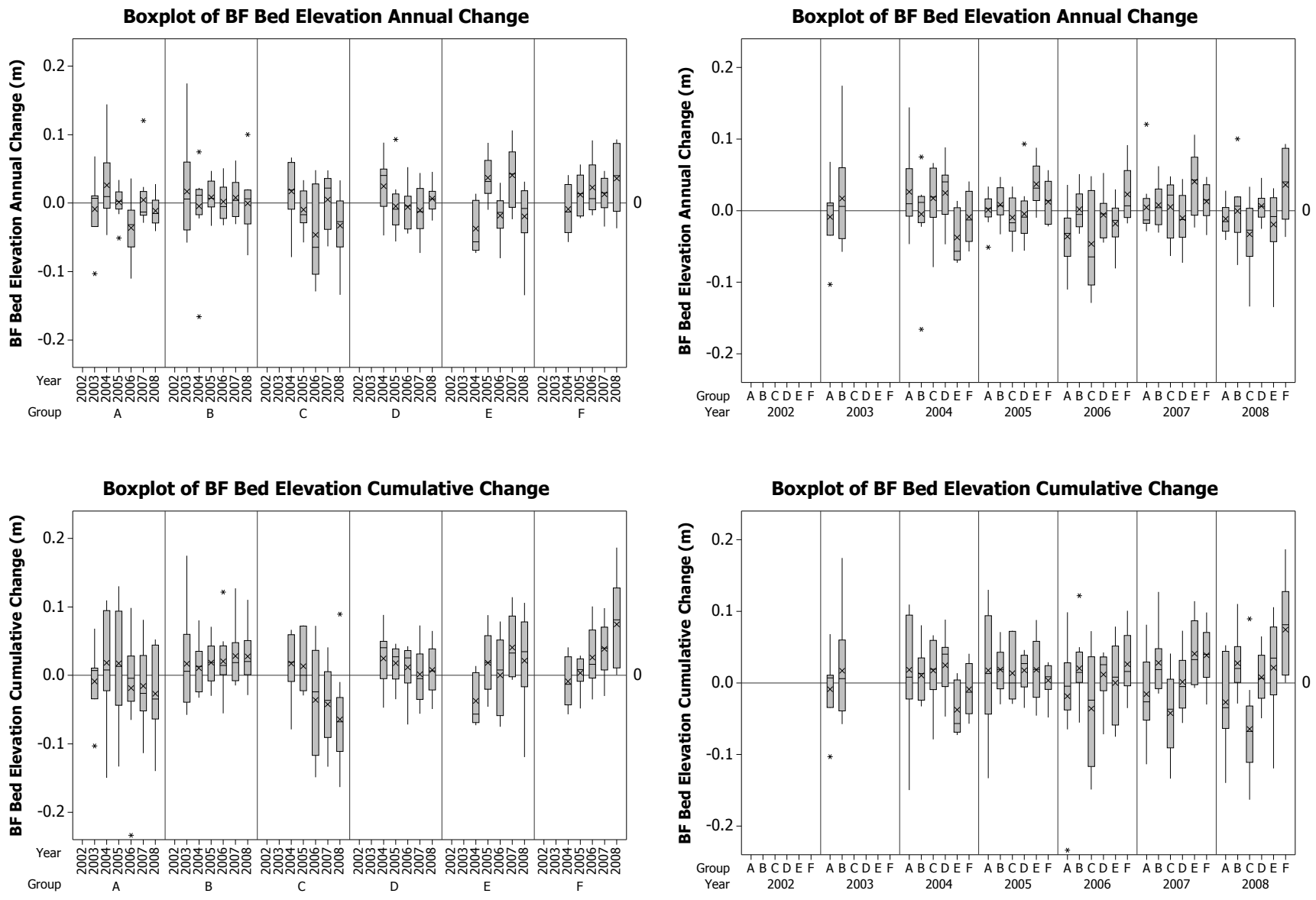

Figure 5.26: Boxplot shows mean bed elevation change grouped by year and by reach. Cumulative change is change since the first survey, annual change is change since the previous survey (typically annually, but not every cross section was surveyed every year). The boxplot shows the median (middle bar), interquartile ranges, and the mean (small $\mathrm{x}$ ) for the group of 10 cross sections in each reach for each year. Negative change is scour of the channel and positive change is filling of the channel. 
larger than error identified in Section 4.4 (Measurement Errors) that is inherent to surveying the bed elevation.

\section{Statistical analysis}

It is difficult to utilize more rigorous statistical tests than basic statistics to compare changes observed in cross sections. The study design is primarily observational and the study was not designed to test factors influencing channel changes or determine if environmental variables and management influences could be used to predict channel characteristics. Chapter 4.4 identifies an average level of precision in the measurement of channel features (cross-sectional area and thalweg elevation). It can be assumed that changes in these metrics greater than the identified error represent real change in the channel, but this cannot be identified with a statistical test.

One potential statistical method to compare two cross sections is sampling the cross section at a fixed interval and then using the measurements from the same location in different years to perform a paired T-test comparing mean changes between the two surveys. Because cross sections sampled at surveyor determined breaks in slope and not at an even interval, the values needed for the analysis would need to be interpolated, introducing some error. There is also some question of what a statistically significant change would mean as detected by this method. A small change in either direction (gain or loss of elevation) by a majority of points would be interpreted as "significant" even though the mean bed elevation may have only changed one or two centimeters, because the standard deviation of the changes is small compared to the mean change. In a cross section where a portion of the channel gains elevation and a portion loses elevation, the change may 
be considered "insignificant" even if the mean elevation change is much greater than the earlier example. The statistically significant changes detected by this method have little relationship to channel changes that may be morphologically or ecologically significant. Because of its very limited utility, this analysis was not performed.

\subsubsection{Longitudinal profiles}

Channel gradient, variability of the thalweg, as well as pool spacing and depth are all metrics that can be calculated from channel longitudinal profile data. Quantity of pool habitat and variability of the bed surface have been linked with habitat quality for a variety of aquatic organisms (Mossop \& Bradford, 2006). Plan view characteristics like sinuosity and meander pattern can also be determined from longitudinal profile survey data collected with a total station. Change in these metrics is unlikely in a mountainous headwater stream like Little Creek, which has a steep gradient and narrow valley. Changes in plan form shape and meander pattern have not been observed in Little Creek and plan view characteristics were not analyzed for this project.

\section{Reach gradient}

Channel gradient is most often calculated by fitting a linear best fit line through the points of the profile. Gradients in Little Creek are locally highly variable, and in the measured profiles the gradient of the fit line often depends on how far above and below the cross sections the reach was surveyed. To compare annual surveys, reach gradients were only calculated for the section of the reach where 
longitudinal profile data existed for all years. Gradients were calculated using the General Linear Model routine in Minitab using reach and year as categorical variables. The calculated gradients are more strongly influenced by the upper and lower ends of the profile, and changes in those parts of the reach would most likely change the gradient. There was little variation in reach gradient during the study period. Table 5.2 shows the reach gradients for each year.

\section{Thalweg profile variability}

Vertical variability in thalweg profile has been identified as a geomorphic characteristic that is reduced by inputs of sediment or other stream disturbance (e.g. large woody debris removal) (Madej, 2001; Lisle, 1995; Bartley \& Rutherfurd, 2005). To calculate profile variability, a least squares best fit line is applied to the profile data and the residuals calculated. Changes in the standard deviation of the residuals are interpreted as a change in variability of the thalweg profile. To weight each section of stream equally, the thalweg profile needs to be reinterpolated at a constant interval. Custom formulas and an Excel ${ }^{\mathrm{TM}}$ spreadsheet were used to reinterpolate each longitudinal profile at $0.305 \mathrm{~m}(1 \mathrm{ft})$ intervals. This interval was chosen because it could accurately represent small-scale features like steps and would also likely sample the deepest portion of pools.

Thalweg profile variability was analyzed using a subset of the longitudinal profile data that included only the portion of the reach that had been surveyed in all years. A function was fit to each profile using the General Linear Model routine in Minitab and residuals were calculated and stored. For some profiles a linear fit did not match the larger scale trend of the profile and the residual plots showed large scale curvature. For these profiles a second and/or third order 
Table 5.2: Table of reach gradients calculated in each reach where longitudinal data from all years exists. Slopes were calculated with a least squares linear regression fit to the profile.

\begin{tabular}{|c|c|c|c|}
\hline Reach & Year & $\begin{array}{l}\text { Intercept } \\
\text { (elevation) }\end{array}$ & $\begin{array}{l}\text { Gradient } \\
\text { (slope) }\end{array}$ \\
\hline \multirow[t]{7}{*}{ A } & 2002 & 84.73 & 0.0171 \\
\hline & 2003 & 84.74 & 0.0179 \\
\hline & 2004 & 85.14 & 0.0168 \\
\hline & 2005 & 85.04 & 0.0168 \\
\hline & 2006 & 84.33 & 0.0190 \\
\hline & 2007 & 84.35 & 0.0196 \\
\hline & 2008 & 84.62 & 0.0184 \\
\hline \multirow[t]{7}{*}{ B } & 2002 & 120.28 & 0.0281 \\
\hline & 2003 & 120.56 & 0.0255 \\
\hline & 2004 & 120.50 & 0.0268 \\
\hline & 2005 & 120.85 & 0.0244 \\
\hline & 2006 & 120.61 & 0.0259 \\
\hline & 2007 & 120.76 & 0.0257 \\
\hline & 2008 & 120.64 & 0.0261 \\
\hline \multirow[t]{6}{*}{$\mathrm{C}$} & 2002 & 290.28 & 0.0467 \\
\hline & 2004 & 290.17 & 0.0475 \\
\hline & 2005 & 290.04 & 0.0484 \\
\hline & 2006 & 289.90 & 0.0470 \\
\hline & 2007 & 290.06 & 0.0466 \\
\hline & 2008 & 289.76 & 0.0464 \\
\hline \multirow[t]{6}{*}{$\mathrm{D}$} & 2002 & 343.28 & 0.0496 \\
\hline & 2004 & 342.30 & 0.0492 \\
\hline & 2005 & 342.31 & 0.0495 \\
\hline & 2006 & 342.33 & 0.0492 \\
\hline & 2007 & 342.50 & 0.0487 \\
\hline & 2008 & 342.30 & 0.0489 \\
\hline \multirow[t]{6}{*}{$\mathrm{E}$} & 2002 & 617.52 & 0.0483 \\
\hline & 2004 & 617.64 & 0.0476 \\
\hline & 2005 & 617.59 & 0.0480 \\
\hline & 2006 & 617.32 & 0.0495 \\
\hline & 2007 & 617.76 & 0.0467 \\
\hline & 2008 & 617.30 & 0.0491 \\
\hline \multirow[t]{6}{*}{$\mathrm{F}$} & 2002 & 344.46 & 0.0414 \\
\hline & 2004 & 344.28 & 0.0422 \\
\hline & 2005 & 344.41 & 0.0415 \\
\hline & 2006 & 344.69 & 0.0402 \\
\hline & 2007 & 344.43 & 0.0424 \\
\hline & 2008 & 344.45 & 0.0424 \\
\hline
\end{tabular}


polynomial term was added to the model so only smaller-scale variability (i.e. filling of pools) would influence standard deviation calculations. Table 5.3 shows standard deviations of the residual calculated for each longitudinal profile. Figure 5.27 shows these values plotted over time.

Table 5.3: Standard deviation of longitudinal profile residuals calculated from a best fit line. Units are in meters. Type of best fit line is indicated. Higher standard deviation of residuals indicates higher thalweg variability. $n$ is the number of observations from each reach and is equivalent to the reach length in feet that was modeled.

\begin{tabular}{ccccccc}
\hline & \multicolumn{6}{c}{ Reach $(n)$} \\
\cline { 2 - 7 } Year & $\mathrm{A}(202)$ & $\mathrm{B}(145)$ & $\mathrm{C}(248)$ & $\mathrm{D}(309)$ & $\mathrm{E}(164)$ & $\mathrm{F}(174)$ \\
\hline 2002 & 0.099 & 0.054 & 0.242 & 0.140 & 0.106 & 0.079 \\
2003 & 0.075 & 0.032 & - & - & - & - \\
2004 & 0.106 & 0.032 & 0.181 & 0.150 & 0.095 & 0.064 \\
2005 & 0.101 & 0.056 & 0.164 & 0.146 & 0.101 & 0.074 \\
2006 & 0.100 & 0.058 & 0.149 & 0.145 & 0.092 & 0.088 \\
2007 & 0.100 & 0.047 & 0.128 & 0.145 & 0.111 & 0.096 \\
2008 & 0.092 & 0.060 & 0.150 & 0.139 & 0.098 & 0.119 \\
\hline Fit order & 1st & 2nd & 3rd & 3rd & 1st & 3rd \\
\hline
\end{tabular}

Reaches $\mathrm{C}$ and $\mathrm{D}$ have the largest overall variability. Both of these reaches contain a boulder/debris jam and this feature is likely responsible for some of the variability because there is a large change in bed elevation over a relatively short length of stream. Reach B has the lowest variability. This reach has few pools and mostly has a plane-bed morphology. Two obvious trends over time are the decrease in variability in Reach $\mathrm{C}$ and the increase in variability in Reach $\mathrm{F}$. These were also the reaches that had the largest amount of fill and scour. The decrease in variability in Reach $\mathrm{C}$ is likely due to the loss of the step and deep pool and subsequent scour upstream between 2005 and 2006. Reach F appears to have developed more pool features over the study period (discussed below). Standard deviations in the other reaches have some annual variations but do not show any trends over the study period. 


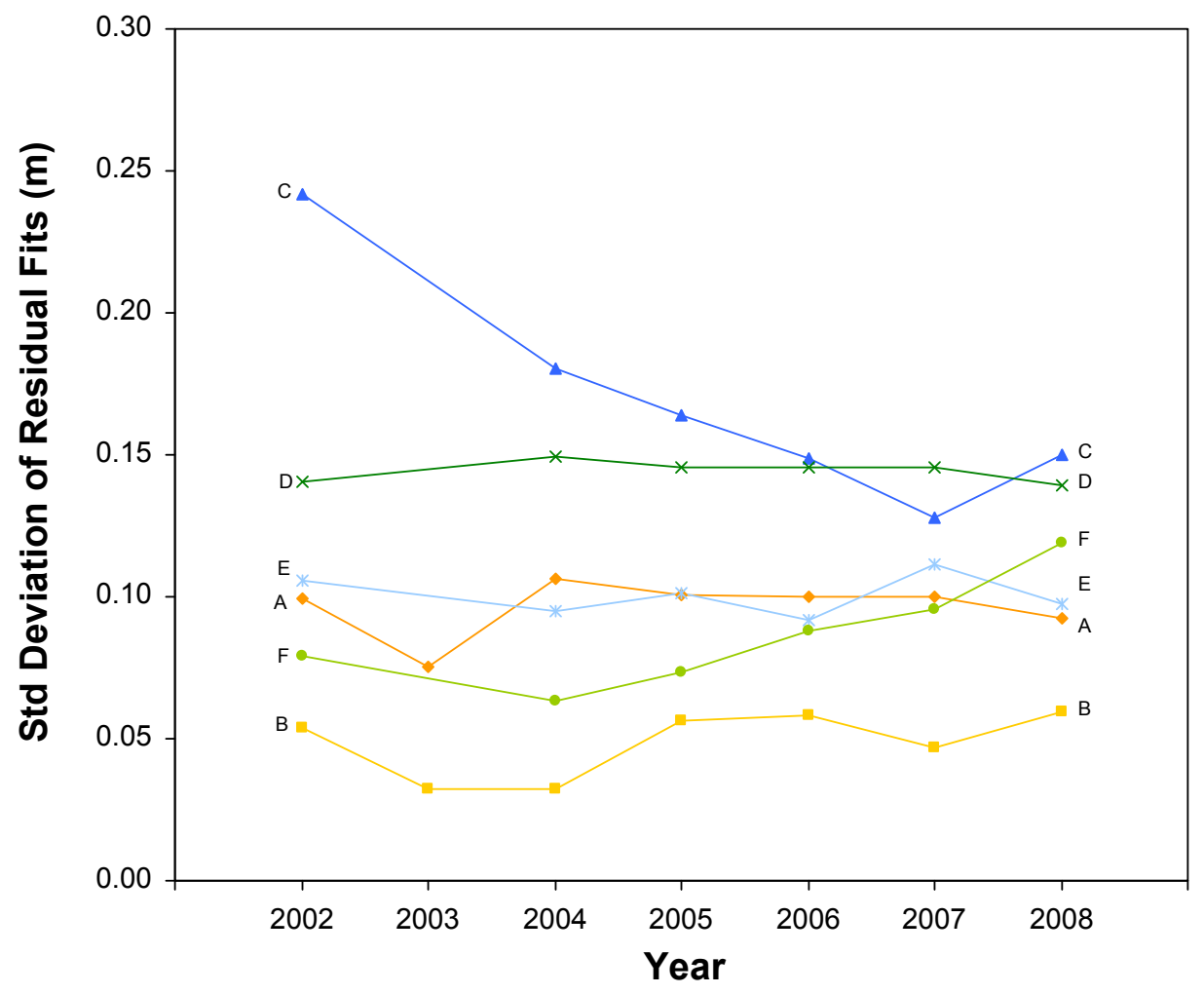

Figure 5.27: Standard deviations of longitudinal profile residuals. Two noticeable changes are the decrease in variability in Reach $\mathrm{C}$ and the increase in Reach $\mathrm{F}$. 


\section{Residual pool depths}

Residual pool depth is the difference in elevation between the tail of a pool and the deepest point, or the depth of the pool at zero flow (Lisle, 1987). It is a discharge-independent method of measuring pool depth and allows meaningful comparisons of pool depth from year to year. Deep pools are an important rearing habitat component for salmonids. Moreover, changes in pool depths could be an indicator of changes sediment inputs to the stream system (Wood-Smith \& Buffington, 1996). Figure 5.28 shows residual pool depths for pools in each reach. It is sometimes challenging to correlate pools in longitudinal profiles measured in different years. Even though they are normalized to the cross sections, survey distances vary at the deepest point of the pool depending on pool shape and how the surveyor interpreted the longitudinal profile. Pools in each reach were assigned a unique identifier manually using the distance of the tail of the pool and a visual analysis of the longitudinal profile for identification. The pools are numbered sequentially upstream. Only pools deeper than $0.12 \mathrm{~m}$ were included in this analysis. If a pool is not included in a particular year the residual depth was less than $0.12 \mathrm{~m}$ in that portion of the profile. Pools that were not included in all longitudinal profiles are noted with an asterisk $\left(^{*}\right)$. Note vertical scale varies between graphs.

Reach A has several pools that persisted the entire study period (A2 and A9). The A2 pool started relatively shallow in 2002 and 2003 and became quite deep by 2008. The A3 pool, approximately $7.5 \mathrm{~m}$ upstream, lost depth during this period. All pools except A2 lost depth in 2004.

Reach B has very few pools deeper than $0.12 \mathrm{~m}$, and none that persisted throughout the study period. This is consistent with the low variability in the thalweg residual analysis and the observed morphology of the reach. 

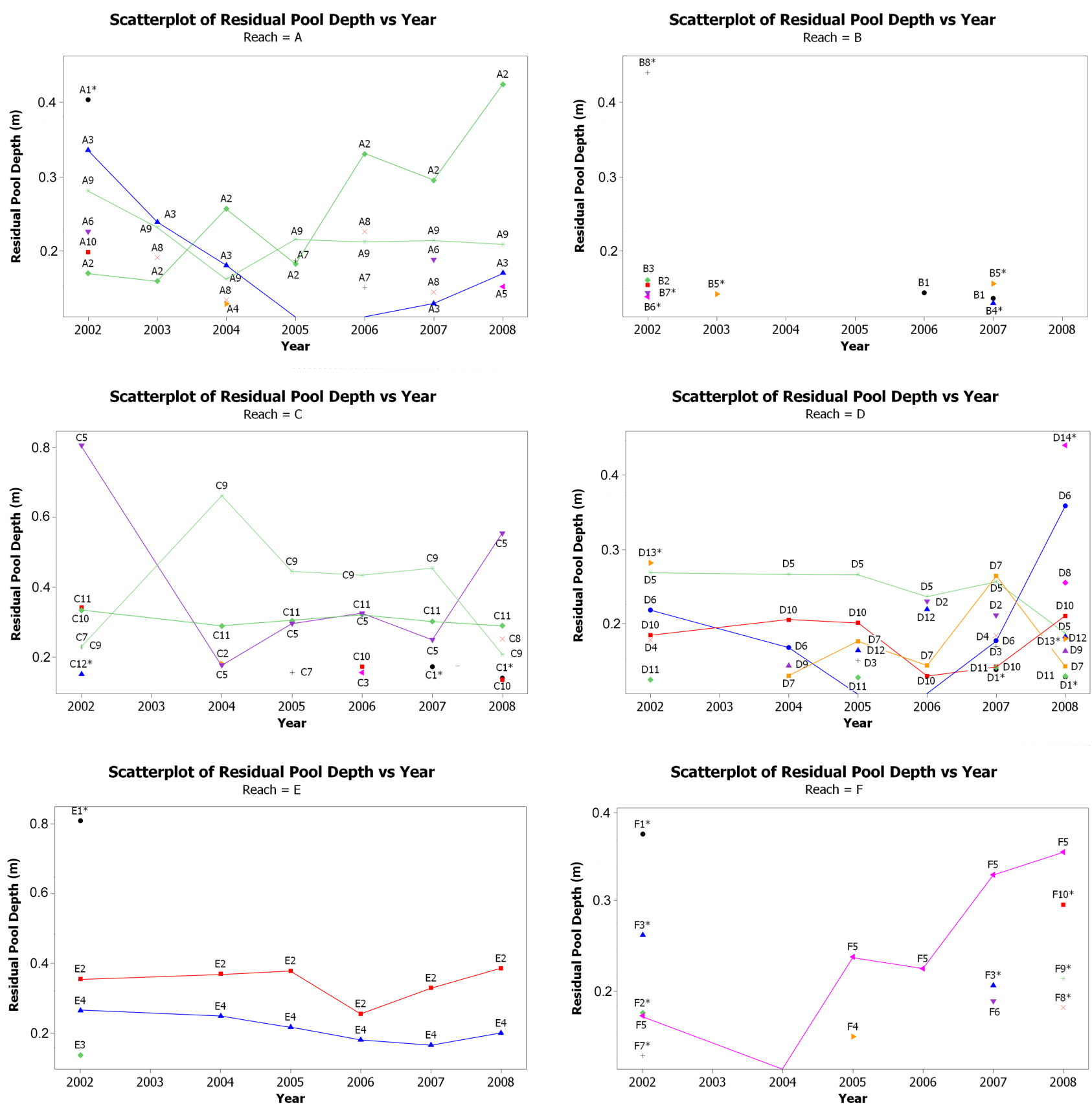

Figure 5.28: Residual pool depths in each study reach. Each pool is given a unique identifier to track year to year change. Lines connect pools that persisted throughout much of the study period. If a pool is absent its residual depth was less than $0.12 \mathrm{~m}$ during that survey. Pools marked with an asterisk are located outside of the region covered by all surveys and that portion of the channel may not have been surveyed every year. 
Reach C has several deep pools. The C5 pool in 2002 was the deepest surveyed during the study period. This pool lost almost $0.60 \mathrm{~m}$ of residual depth between the 2002 and 2004 surveys (approximately a 75\% reduction). The C9 pool had the opposite response during this time, gaining almost $0.45 \mathrm{~m}$ of depth. These two pools are at either end of the log/boulder jam and during the study period the residual depths of these pools seem to be inversely related. Generally, pool volumes were quite variable during the study period, and maximum depths appear to decrease over time. This may explain the decrease in thalweg variability seen in the residual analysis.

Reach D has the highest number of pools among the study reaches, however maximum pool depths are typically lower than Reach C and Reach A. Besides the D6 pool, which had a large increase in residual depth in 2008, pool depths were stable.

Reach E has only two pools with depths greater than $0.15 \mathrm{~m}$. They persisted throughout the study period and their depths follow similar patterns. Both pools depth decreased in 2006 and increased in 2008.

Reach F also has few pools deeper than $0.12 \mathrm{~m}$. The F5 pool persisted for the whole study period (except in 2004 when no pools were deeper than $0.12 \mathrm{~m}$ ) and was deeper than $0.31 \mathrm{~m}$ in 2007 and 2008. Several other pools also developed between 2005 and 2008. This corresponds to the increase in thalweg variability seen in the residual analysis.

Interpreting the results of this analysis is rather difficult. Residual pool depths appear to have a large amount of variability within a reach and from year-to-year, but no clear trends are apparent. However, residual pool depths are sensitive to changes in sediment load (Montgomery \& MacDonald, 2002) and if major changes 
in sediment dynamics were occurring, one could reasonably expect to identify them using this analysis.

\section{Residual depth statistics}

As in the analysis of residuals of a function fit to a longitudinal profile, it is possible to evaluate statistical variation in the residual depths along the profile. The residual depths were calculated from the reinterpolated longitudinal profile. Areas outside pools have a residual depth of zero.

Mean pool depth can be influenced by the depth of pools and the length of the channel composed of pools. If the median pool depth is greater than zero, at least half of the channel is within a pool. One-quarter of the channel has a residual depth deeper than the third quartile. Descriptive statistics for residual depths are shown in in Table 5.4 and graphs of the mean, standard deviation, and third quartile are shown in in Figures 5.29, 5.30, and 5.31.

There are some apparent patterns in the reach-wide residual depth characteristics, but similar to the residual depths of individual pools, there is a high amount of variability. There is a decrease in both residual depth (mean and Q3) and variability (standard deviation) in most reaches between 2002 and 2004. The A reach longitudinal profile has an anomaly (a large gap between two surveyed points that effectively creates a very long pool that may not have actually existed), which would explain the very high mean and Q3 depths in 2002. It is important to note that surveyor interpretation of the channel is a likely contributor to some of the variability in these graphs.

Another way of visualizing the residual depth distribution in a reach is a cumulative frequency graph (Lisle, 1986). This graph shows the percentage of each 
Table 5.4: Table of reach wide residual pool depth statistics showing mean, standard deviation, median, and third quartile. Units are in meters.

\begin{tabular}{|c|c|c|c|c|c|c|}
\hline Reach & Year & $\mathrm{N}$ & Mean & StDev & Median & Q3 \\
\hline \multirow[t]{7}{*}{ A } & 2002 & 202 & 0.067 & 0.082 & 0.035 & 0.127 \\
\hline & 2003 & 202 & 0.038 & 0.058 & 0.003 & 0.061 \\
\hline & 2004 & 202 & 0.034 & 0.049 & 0.009 & 0.057 \\
\hline & 2005 & 202 & 0.037 & 0.054 & 0.009 & 0.055 \\
\hline & 2006 & 202 & 0.053 & 0.080 & 0.013 & 0.080 \\
\hline & 2007 & 202 & 0.048 & 0.070 & 0.012 & 0.066 \\
\hline & 2008 & 202 & 0.053 & 0.093 & 0.020 & 0.051 \\
\hline \multirow[t]{7}{*}{ B } & 2002 & 145 & 0.019 & 0.035 & 0.000 & 0.028 \\
\hline & 2003 & 145 & 0.007 & 0.019 & 0.000 & 0.000 \\
\hline & 2004 & 145 & 0.005 & 0.011 & 0.000 & 0.000 \\
\hline & 2005 & 145 & 0.020 & 0.029 & 0.000 & 0.034 \\
\hline & 2006 & 145 & 0.012 & 0.026 & 0.000 & 0.013 \\
\hline & 2007 & 145 & 0.011 & 0.026 & 0.000 & 0.009 \\
\hline & 2008 & 145 & 0.008 & 0.019 & 0.000 & 0.004 \\
\hline \multirow[t]{7}{*}{$\mathrm{C}$} & 2002 & 248 & 0.055 & 0.127 & 0.000 & 0.040 \\
\hline & 2003 & 148 & 0.034 & 0.081 & 0.000 & 0.018 \\
\hline & 2004 & 248 & 0.042 & 0.091 & 0.000 & 0.042 \\
\hline & 2005 & 248 & 0.040 & 0.082 & 0.000 & 0.043 \\
\hline & 2006 & 248 & 0.041 & 0.081 & 0.000 & 0.045 \\
\hline & 2007 & 248 & 0.028 & 0.067 & 0.000 & 0.018 \\
\hline & 2008 & 248 & 0.038 & 0.086 & 0.000 & 0.025 \\
\hline \multirow[t]{6}{*}{$\mathrm{D}$} & 2002 & 309 & 0.027 & 0.058 & 0.000 & 0.029 \\
\hline & 2004 & 309 & 0.020 & 0.041 & 0.000 & 0.014 \\
\hline & 2005 & 309 & 0.020 & 0.042 & 0.000 & 0.013 \\
\hline & 2006 & 309 & 0.021 & 0.039 & 0.000 & 0.029 \\
\hline & 2007 & 309 & 0.025 & 0.050 & 0.000 & 0.022 \\
\hline & 2008 & 309 & 0.027 & 0.054 & 0.000 & 0.025 \\
\hline \multirow[t]{6}{*}{$\mathrm{E}$} & 2002 & 164 & 0.023 & 0.053 & 0.000 & 0.018 \\
\hline & 2004 & 164 & 0.021 & 0.053 & 0.000 & 0.007 \\
\hline & 2005 & 164 & 0.019 & 0.056 & 0.000 & 0.003 \\
\hline & 2006 & 164 & 0.018 & 0.043 & 0.000 & 0.005 \\
\hline & 2007 & 164 & 0.013 & 0.040 & 0.000 & 0.000 \\
\hline & 2008 & 164 & 0.021 & 0.055 & 0.000 & 0.006 \\
\hline \multirow[t]{6}{*}{$\mathrm{F}$} & 2002 & 174 & 0.017 & 0.039 & 0.000 & 0.010 \\
\hline & 2004 & 174 & 0.006 & 0.019 & 0.000 & 0.000 \\
\hline & 2005 & 174 & 0.012 & 0.029 & 0.000 & 0.007 \\
\hline & 2006 & 174 & 0.018 & 0.037 & 0.000 & 0.016 \\
\hline & 2007 & 174 & 0.021 & 0.053 & 0.000 & 0.003 \\
\hline & 2008 & 174 & 0.019 & 0.050 & 0.000 & 0.020 \\
\hline
\end{tabular}




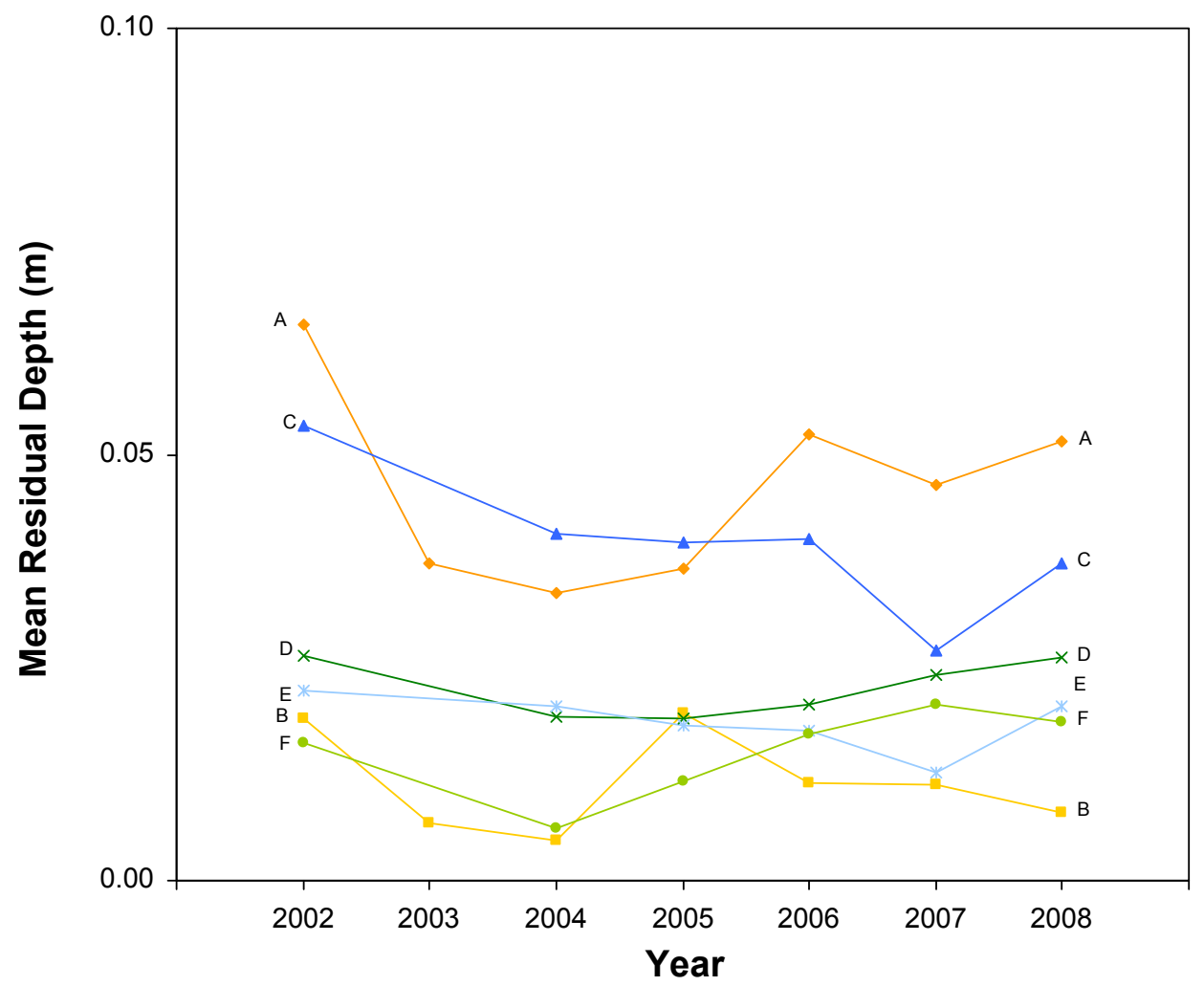

Figure 5.29: Graph showing mean residual depths for each year. 


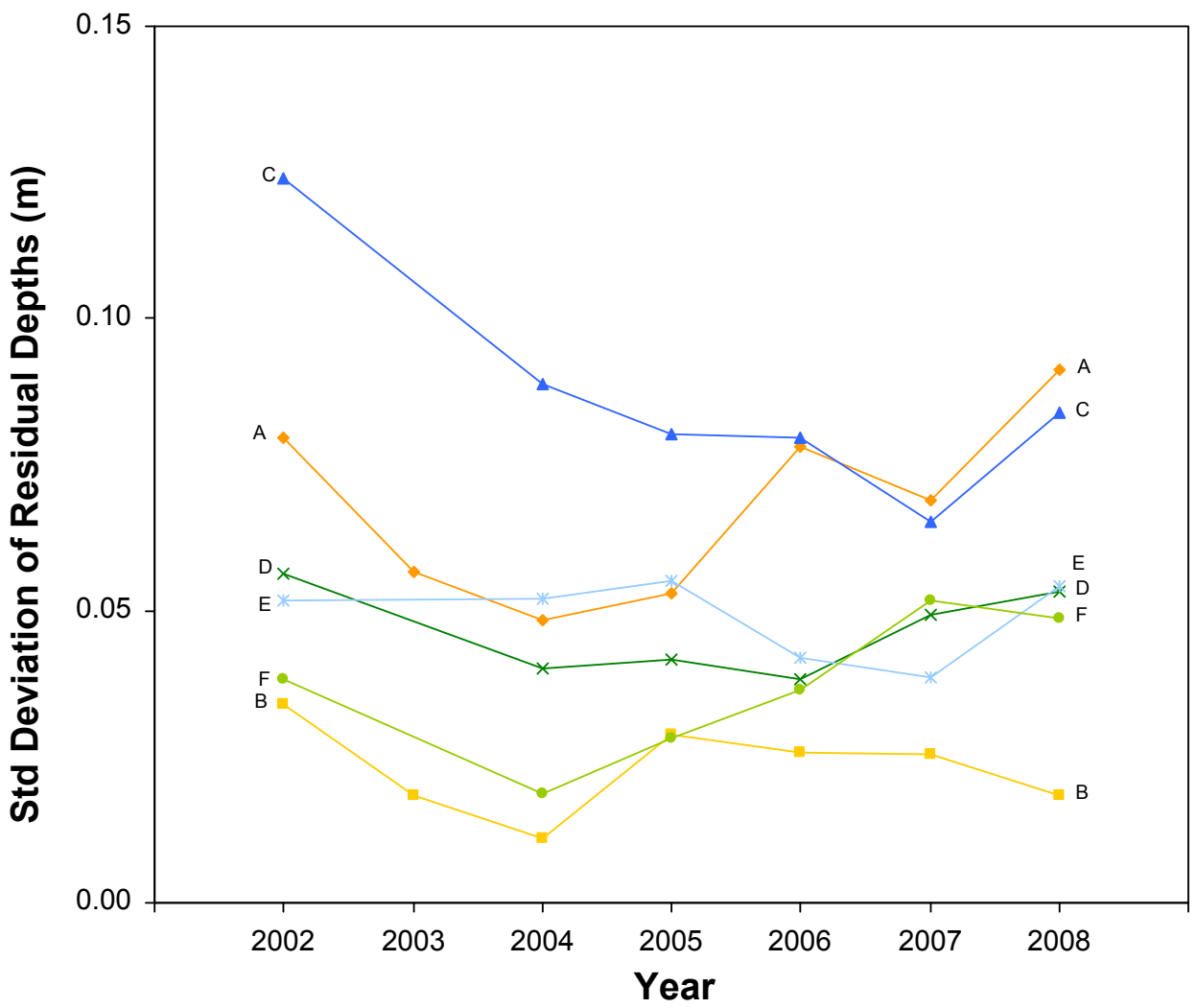

Figure 5.30: Graph showing standing deviations of residual depths from each year. 


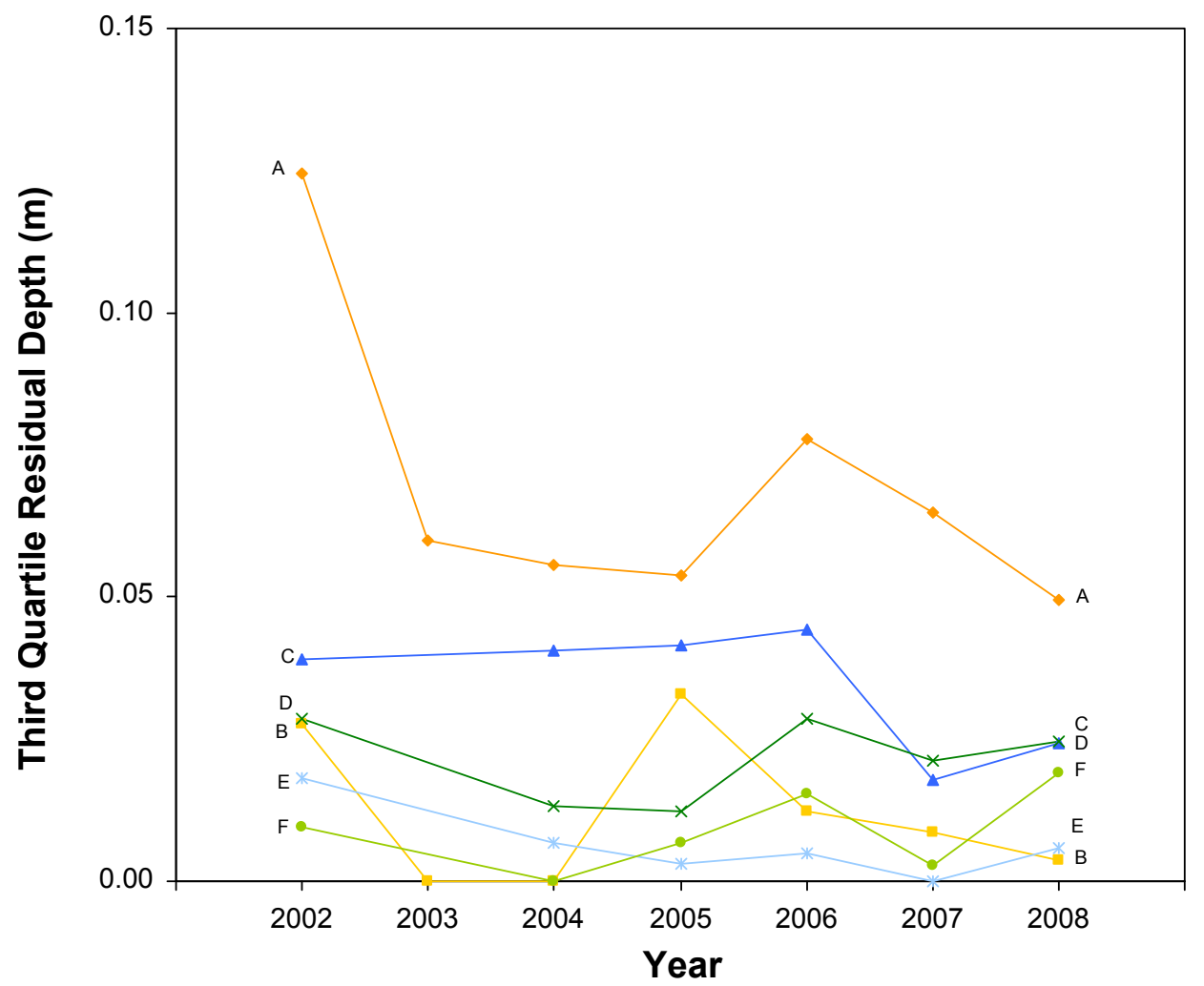

Figure 5.31: Graph showing third quartile of residual depths from each year. 
longitudinal profile with a residual depth lower than the value of the x-axis. It is a way of visualizing the distribution of pools in the reach. The y-axis intercept is the percentage of the reach that is not in a pool (percent with residual depth $=$ 0 ) and the $\mathrm{x}$-axis intercept (at the top) is the depth of the deepest pool. A shift of a line downward and to the right indicates an increase in quantity and depth of pools in the reach. A shift upward and to the left indicates a loss of pool quantity and depth. Figure 5.32 shows the cumulative frequency graphs for all reaches. There are some notable changes evident in these graphs. In 2008, Reach A had an increase in the depth of the deepest 5 percent of the channel. In Reach $\mathrm{C}$ there is a contrast of both quantity and maximum depth of pools between the 2002 and 2007 surveys. Pools are much deeper in 2002 and a larger portion of the reach is in relatively deep pools compared to 2007. 


\section{Cumulative distribution graphs of residual depth}
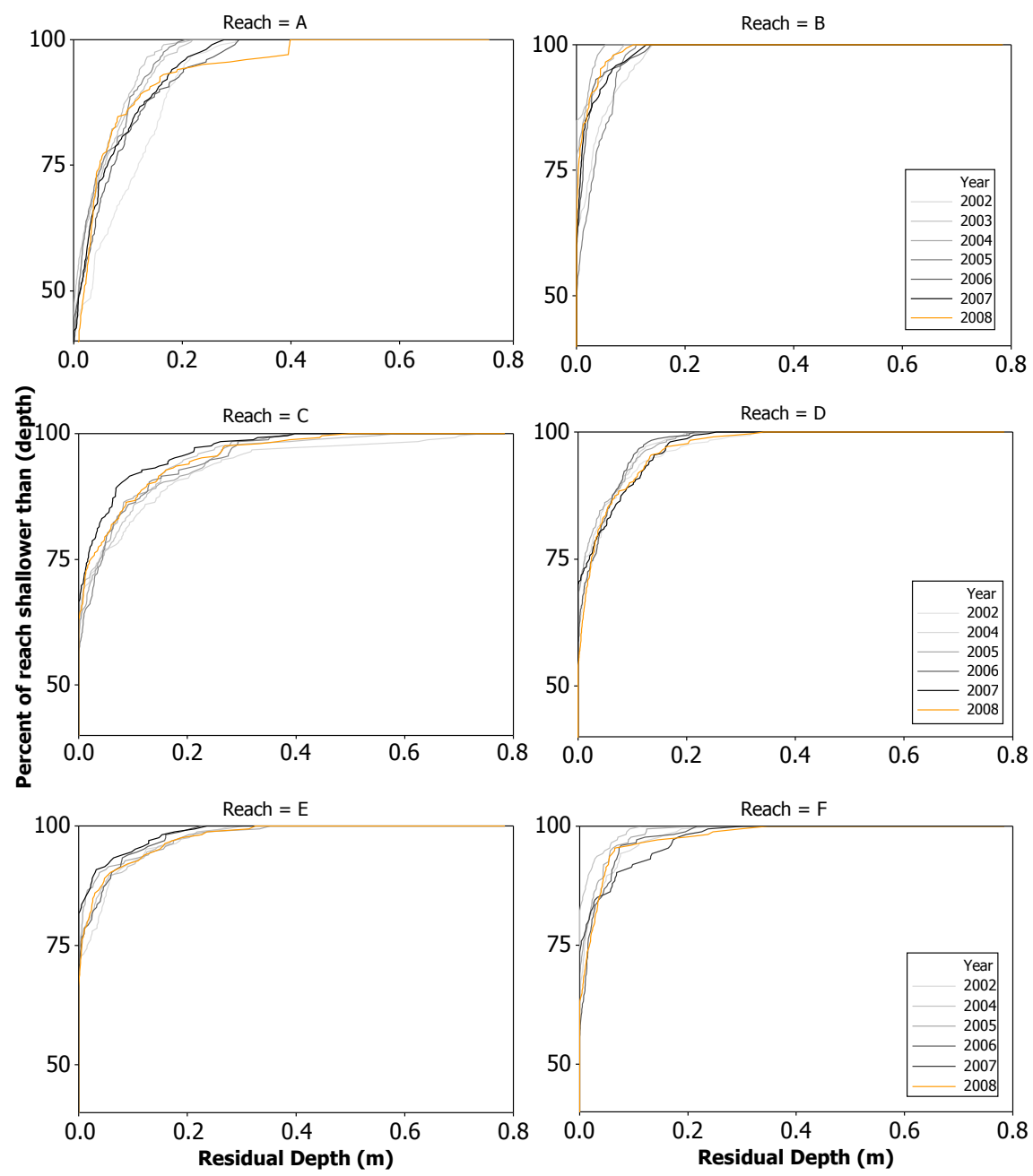

Figure 5.32: Cumulative frequency graph of residual depths in all reaches. The graph shows the percentage of the reach with residual depths less than a certain value. The $\mathrm{y}$-intercept is the percentage of the reach not in pools, and the $\mathrm{x}$ intercept (at the top of the graph) is the depth of the deepest pool. 


\section{Chapter 6}

\section{Discussion}

This chapter interprets the data collected from the study reaches. Many hydrolo-

gists and geomorphologists have used surveys similar to those used in this thesis to understand fluvial processes as well as impacts and recovery of stream morphology after large disturbances. Changes in stream geometry can result from a wide variety of factors including: the intersection between hillslope and fluvial processes; local geology, vegetation, and land use; and stochastic variation in the hydrologic regime and sediment inputs. This chapter assesses the observations from Little Creek during the study period in the context of these factors.

\subsection{Limitations of Data}

There are several aspects of the type and scope of data collected, as well as the study design, that are important to consider when making interpretations of channel changes in Little Creek. 


\subsubsection{Spatial data limitations}

The primary limitation of the survey data is the spatial extent of the surveys. The reaches covered by cross sections represent approximately 10 percent of the Mainstem, 5 percent of the North Fork and 3 percent of the South Fork. Most of the stream is not being surveyed and may be behaving in a completely different way than the study reaches. However, this sacrifice is consciously made to increase the resolution of data within the study reaches. Another problem with the spatial scale is at the reach level. The stream system is a highly dynamic three-dimensional environment and there are limitations to measuring it using two-dimensional methods. Cross sections only describe a very small length of stream, especially in a highly-variable channel like Little Creek. There are several cases where two adjacent cross sections behaved quite differently in a particular year or over the course of the study period. Likewise, the longitudinal profile also has limitiations in interpretation and might not provide a complete picture of morphological changes in a reach.

A three-dimensional digital terrain model (DTM) is a way to collect topographic data about the reach that can eliminate some of the limitations from traditional cross section and longitudinal profile data collection (Keim et al., 1999). DTMs are typically created using a total-station theodolite, however stereo photography and LiDAR (both aerial and ground-based) can be used to create DTMs. DTMs can become the basis for many different types of analysis and cover the entire channel. This eliminates problems that stem from the limited extent that cross sections or longitudinal profiles represent. Creating and using DTMs to study channel morphology also has several drawbacks. Equipment is more expensive and data collection is very time consuming, especially in heavy understory 
vegetation. Keim et al. (1999) reported up to six days of surveying necessary for a $300 \mathrm{~m}$ study reach - typical surveying time for a longitudinal profile and 10 cross sections in Little Creek is 1.5 days. Another challenge is the spatial scale of data collected. Surveys described in Keim et al. (1999) did not attempt to detect change less than $0.15 \mathrm{~m}$. This is greater than the magnitude of almost all of the bed elevation changes observed in Little Creek over the study period. Collecting data at a spatial resolution that could detect these changes is possible, but it would require significantly more work to collect.

One advantage of creating a DTM with a theodolite is that the spatial resolution can be varied so more data is collected where it is more useful. More detailed data can be collected in the active channel and detailed cross sections can be surveyed along with general topographic data. Ground-based LiDAR has been used to create a DTM in the upper portion of Reach C in Little Creek. This equipment can create a DTM with a very high spatial resolution $(<1 \mathrm{~cm}$ grid). However, this method still has several disadvantages: (1) the equipment is extremely expensive (the unit used in Little Creek cost over $\$ 300,000$ ) and difficult to transport; (2) vegetation typically hides the true ground surface outside of the active channel; and (3) several set up locations are needed, especially when vegetation or topography shadows portions of the study reach.

\subsubsection{Data limitations due to sampling interval}

Except in 2003, all of the reaches have been surveyed annually. This temporal scale can detect changes that occur in the stream each winter period, however, nothing is known about changes that happen between storm events in a given year. Especially in a year with several large storms, it is unclear what combination of storm events 
caused the observed changes. Conducting surveys during the winter between storm events would provide this information, but is highly unlikely due to other winter workloads related to the water quality monitoring and the considerable time and effort it takes to survey all of the reaches. There likely are changes occurring storm to storm that on an annual basis appear like little or no change. For example, a pool may aggrade with fine sediment during the earlier storms where the assumed sediment supply/flow ratio is higher, and then degrade towards the end of the season when "clean" water originating from base flow is higher, providing more available energy for transport. Anecdotally, this sequence was observed in the plunge pool where stage is measured at the Upper North Fork flume in the 20072008 winter. After the Jan 25th storm, which had high flows and sediment loads, the pool was filled with fine sediment (fine gravel and finer). A week later on Feb 2nd, a moderate storm (in terms of rainfall) occurred, but streamflows were still high because of high antecedent baseflow and soil moisture condition. This storm had lower sediment loads and transported most of the sediment deposited the earlier storm out of the pool. A similar situation also occurred in the 2010-2011 winter.

On the other hand, with the relatively small changes seen since the start of this project, one could reasonably argue that the surveys could occur less often. This argument has merit, but there are several problems associated with less frequent surveys. It becomes harder to decide when to survey and determine what would trigger a survey year. Surveys might only be performed after a flow of a certain return interval occurred, however, it then becomes unclear when and what events caused the observed changes to occur. Annual surveys performed in summer occur on the same time scale as the major changes in the flow regime of Little Creek (high flow in winter, low flow in summer). There is a general understanding of 
what storm and flow characteristics may have been responsible for the observed changes in a survey performed annually. Another advantage of annual surveys is that it helps to keep the system of benchmarks maintained and provides continuity of procedures, data, and knowledge of stream conditions. These are both essential elements needed for a long term monitoring effort like this to be successful. Annual surveys seem to be an appropriate temporal scale of measurement and should be continued in Little Creek, especially to document changes that might be associated with the Lockheed Fire.

\subsubsection{Limitations of data interpretation}

The third major limitation to the data collected for this project is that crosssectional and longitudinal profiles do not directly identify processes or causes of change. Other data and observations are necessary to make these interpretations. It is difficult to understand why the changes seen so far during this study have occurred without this other information. The study design also results in some limitations in interpretation. Many other geomorphic studies utilizing cross sections and longitudinal profiles compare disturbed and undisturbed reaches of a stream or similar adjacent streams. They are typically established after a major disturbance (flood, fire, etc.) to help understand impacts and/or recovery of channels after disturbance. This study is fundamentally observational and its primary goal is understanding conditions in Little Creek in the context of the larger Little Creek project. The study was not designed as an experiment to determine if harvesting had an impact on channel morphology in Little Creek; instead it provides data regarding the occurrences and magnitude of channel change, albeit spatially limited, during the study. The surveys do not serve as primary means of understanding landuse impacts from timber harvesting or impacts from natural 
disturbances like floods or the Lockheed Fire. However, additional observations, including flow and precipitation records, photographs, field notes, and personal observations over extended time periods, provide a context which allows interpretation and understanding of the changes that have occurred in Little Creek during the study period and the potential changes (or lack of change) that may result from forest management activities or the Lockheed Fire.

\subsection{Observed Changes and Interpretations}

The results discussed in Chapter 5 show that most of the changes observed in Little Creek during the study period occurred in Reach C and Reach F. Other individual cross sections also changed during the study period, but Reach $\mathrm{C}$ and Reach $\mathrm{F}$ were the only ones where similar changes occurred in a majority of the cross sections. A discrete event in the upper section of Reach $\mathrm{C}$, the shifting of a log in 2005-2006 winter, appears to have caused much of the observed change that occurred. The causes of other changes are much harder to interpret, and the data needed to document these causes is very difficult or impossible to obtain. We can develop correlations between phenomenon observed in the field and changes observed in the stream. However, because of complicated relationships between channel morphology and the factors that influence it, without very strong signals, it is impossible to determine why the observed changes in Little Creek occurred.

The largest reach-wide changes observed were in Reach C, notably between C3 and C7. A channel spanning piece of large woody debris shifted, which allowed water to scour under it. This removed a hardpoint and the channel downcut upstream through sediment stored behind the log jam, widening and deepening 
the cross sections. This change was also very obvious in the longitudinal profile. The loss of this step and deep pool is one of the reasons thalweg complexity and residual pool depths decreased in Reach C. To a lesser extent, there have also been woody-debris influenced changes in cross-sectional form in Reaches B, E, and F.

Residual pool depths also had relatively high variability during the study period. Residual pool depths are primarily influenced by fine sediment that is mobilized even during relatively moderate flows. Bedload transport in step-pool channels typically demonstrates complicated relationships between discharge magnitude and duration, nature of sediment inputs, and antecedent events (Montgomery \& Buffington, 1997). There are some general patterns in the residual depths (drops in depth in 2003 and 2004) but most changes are likely a result of flow conditions at individual pools and year to year variability of the size and timing of storms.

Besides changes associated with large woody debris and residual pool depths discussed above, there generally has been minimal change observed during the study period. This is especially obvious when compared to changes that have occurred historically $(1955,1998)$ and channel changes in Scotts Creek during the same time period. The relative lack of change during the study period provides a good baseline to evaluate changes that may result from the Lockheed Fire or future flood events.

\subsection{Flow Regime During Study Period}

There have been no large floods during the study period, however there have been several storms that produced flows at or above the bankfull stage. Figure 6.1 shows the peak flows at the San Lorenzo River at Big Trees stream gage - the closest 


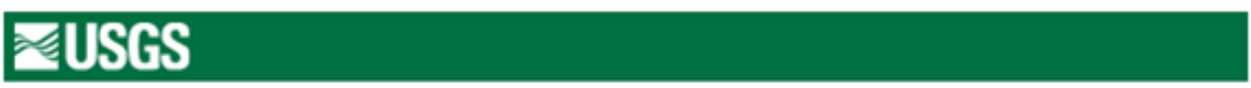

USGS 11160500 SAN LORENZO R A BIG TREES CA

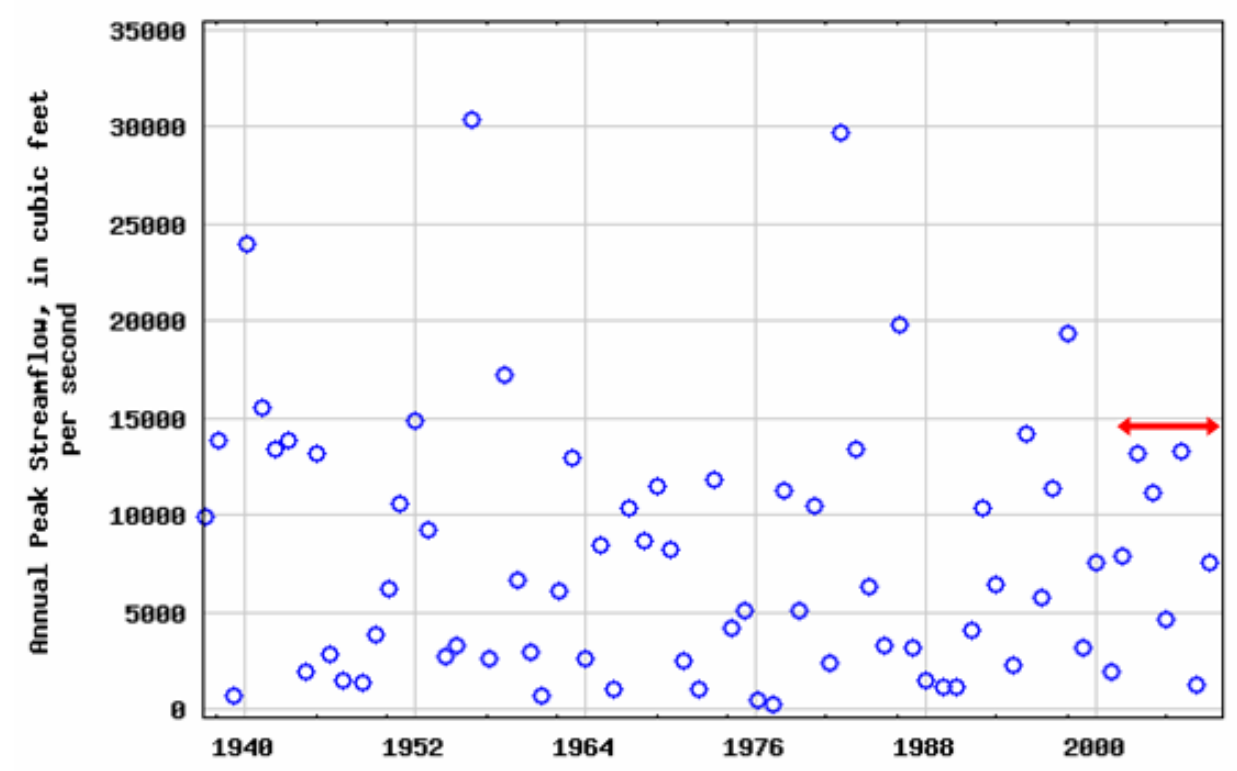

Figure 6.1: Annual peak flows at USGS San Lorenzo River at Big Trees gage. The red arrow shows the flows experienced during the study period.

stream gage to Little Creek with a long term record. Table 6.1 shows peak flows and return intervals from the period 1994-2008. The San Lorenzo River is orders of magnitude larger than Little Creek and rainfall characteristics can definitely differ between the two basins, however large flows in the San Lorenzo typically correspond to large flows in Little Creek. A stream gage on Scotts Creek was operated for several years (the record includes 20 annual peak flow measurements between 1937 and 1982) and a comparison of concurrent peak flows shows that relatively large flows in the San Lorenzo generally correspond with relatively large flows in Scotts Creek, and most of the annual peak flows occurred during the same storm event at each station (Figure 6.2). Even though no very large flows have occurred during the study period, peak flows have not been uncommonly low either.

A comparison of Little Creek annual changes in thalweg elevation, mean bed 
Table 6.1: Peak flows and return intervals for the San Lorenzo River at Big Trees from 1994-2008.

\begin{tabular}{cccc}
\hline & & & $\begin{array}{c}\text { Return } \\
\text { Water Year }\end{array}$ \\
\hline 1994 & Feb. 19, 1994 & 65 & 1.2 \\
1995 & Mar. 10, 1995 & 402 & 8.1 \\
1996 & Feb. 19, 1996 & 164 & 1.8 \\
1997 & Dec. 10, 1996 & 323 & 3.7 \\
1998 & Feb. 03, 1998 & 549 & 14.6 \\
1999 & Feb. 09, 1999 & 91 & 1.5 \\
2000 & Feb. 13, 2000 & 214 & 2.2 \\
2001 & Mar. 04, 2001 & 54 & 1.2 \\
2002 & Dec. 02, 2001 & 223 & 2.3 \\
2003 & Dec. 16, 2002 & 374 & 4.9 \\
2004 & Jan. 01, 2004 & 317 & 3.3 \\
2005 & Jan. 07, 2005 & 131 & 1.7 \\
2006 & Dec. 31, 2005 & 377 & 5.2 \\
2007 & Feb. 10, 2007 & 34 & 1.1 \\
2008 & Jan. 25, 2008 & 214 & 2.2 \\
\hline
\end{tabular}

Comparison of San Lorenzo River and Scotts Creek Peak Flows

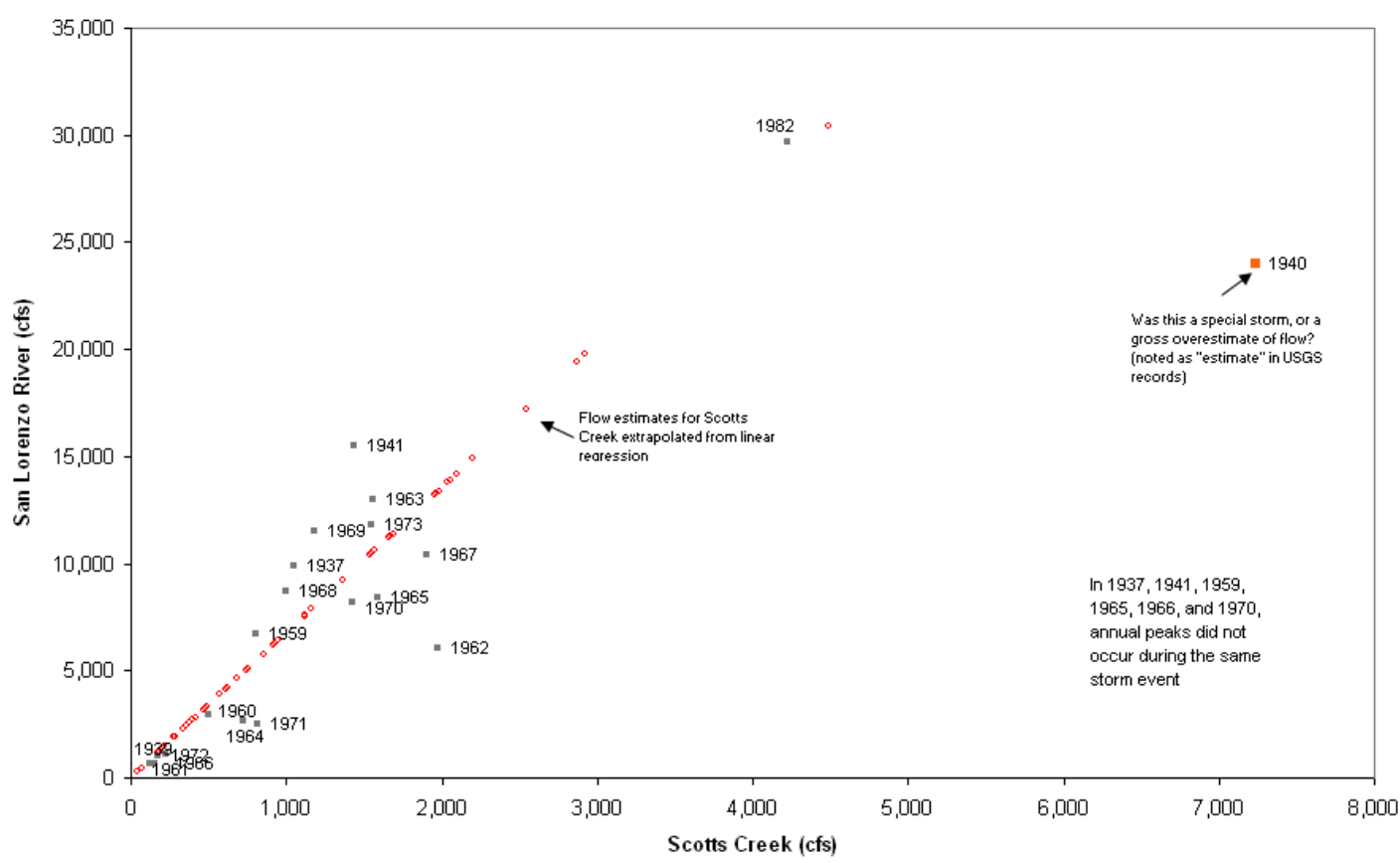

Figure 6.2: Comparison of peak flows in Scotts Creek and the San Lorenzo River 
elevation, and bankfull area across all cross sections shows a correlation between standard deviation (larger variability of changes) and years with larger flow magnitude (especially when the 2003 data is omitted when only 2 reaches were surveyed and changes in one or two cross sections has a much higher influence on the standard deviation). Table 6.3 shows the Pearson's correlation coefficients between annual changes and peakflows. Larger flows have more available stream power to rearrange the substrate and result in higher variability of annual change within the study reaches.

Table 6.2: Table of thalweg elevation annual change means and standard deviations and annual peakflows from the San Lorenzo River at Big Trees (USGS 11160500) and the Little Creek North Fork Flume. Standard deviations are larger in years with larger storms (2003, 2004, 2006 and 2008 contrasted with 2005 and 2007). Only the A and B reaches were surveyed in 2003.

\begin{tabular}{cccccc}
\hline Year & $\begin{array}{c}\text { Mean Bed } \\
\text { Elevation SD }(\mathrm{m})\end{array}$ & $\begin{array}{c}\text { Thalweg } \\
\text { SD }(\mathrm{m})\end{array}$ & $\begin{array}{c}\text { Bankfull Area } \\
\mathrm{SD}\left(\mathrm{m}^{2}\right)\end{array}$ & $\begin{array}{c}\text { San Lorenzo River } \\
\text { Peakflow }\left(\mathrm{m}^{3} / \mathrm{s}\right)\end{array}$ & $\begin{array}{c}\text { Little Creek NF } \\
\text { Peakflow }\left(\mathrm{m}^{3} / \mathrm{s}\right)\end{array}$ \\
\hline 2003 & 0.062 & 0.065 & 0.422 & 374 & 0.50 \\
2004 & 0.050 & 0.072 & 0.291 & 317 & 1.36 \\
2005 & 0.032 & 0.048 & 0.168 & 131 & 0.55 \\
2006 & 0.047 & 0.082 & 0.264 & 377 & 0.90 \\
2007 & 0.039 & 0.051 & 0.218 & 34 & 0.41 \\
2008 & 0.046 & 0.079 & 0.251 & 214 & 1.03 \\
\hline
\end{tabular}

Table 6.3: Pearson's correlation matrix between standard deviation of annual cross section changes and peak flows.

\begin{tabular}{clccc}
\hline & & \multicolumn{3}{c}{ Pearson's $r$} \\
\cline { 3 - 5 } All Years & $\begin{array}{l}\text { Mean bed } \\
\text { elevation }\end{array}$ & $\begin{array}{c}\text { Thalweg } \\
\text { elevation }\end{array}$ & $\begin{array}{c}\text { Bankfull } \\
\text { area }\end{array}$ \\
\hline \multirow{2}{*}{2003 omitted Creek } & 0.27 & 0.74 & 0.14 \\
& $\begin{array}{l}\text { Peakflow } \\
\text { San Lorenzo } \\
\text { River Peakflow }\end{array}$ & 0.76 & 0.73 & 0.76 \\
& $\begin{array}{l}\text { Little Creek } \\
\text { Peakflow } \\
\text { San Lorenzo } \\
\text { River Peakflow }\end{array}$ & 0.85 & 0.76 & 0.84 \\
\hline
\end{tabular}




\subsection{Observations in Context of Morphology and Processes in Little Creek}

\subsubsection{Stream classification}

Morphology in the study reaches in Little Creek typically vary between planebed, forced step-pool and step-pool (Montgomery \& Buffington, 1998) depending on gradient and presence, size, and arrangement of large woody debris and large boulders (forcing agents). The lower gradient reaches (A and B) could be generally classified as confined plane-bed, however Reach A does have a boulder-forced pool near the bottom of the reach. The higher gradient reaches have step-pool and forced step-pool morphologies. These reaches are primarily considered transport reaches and are generally morphologically resistant to changes induced by moderate changes in sediment supply or discharge. Montgomery \& MacDonald (2002) identify likely morphologic responses to increased coarse and fine sediment supply for different channel types (Table 6.4). The channel types in Little Creek are likely to have little response in channel geometry (width and depth) resulting from increases in fine sediment supply (primary potential impact from logging) and moderate responses from increases in coarse sediment supply. In channels like Little Creek, Montgomery \& MacDonald (2002) predict that impacts from increased sediment loads are more likely to be realized as changes in pool characteristics and sediment size distribution rather than channel dimensions or reach morphology. Under the Rosgen stream classification system, the channel types in Little Creek (A3 for the higher gradient reaches, B2 to B4 for lower gradient reaches) are identified as having low to moderate sensitivity to disturbance (Rosgen \& Silvey, 1996) 


\begin{tabular}{|c|c|c|c|c|c|}
\hline Response Variables & $\mathbf{C}$ & SP & PB & PR & DR \\
\hline \multicolumn{6}{|c|}{ Channel Dimensions } \\
\hline Bankfull Width & $\square$ & 口 & $\bullet$ & - & $\square$ \\
\hline Bankfull Depth & 口 & $\square$ & $\bullet$ & $\bullet$ & $\square$ \\
\hline \multicolumn{6}{|c|}{ Bed Material (particle size) } \\
\hline $\mathrm{D}_{84}$ & 0 & $\square$ & $\bullet$ & $\bullet$ & $\bullet$ \\
\hline$D_{50}$ & $\square$ & 口 & $\bullet$ & 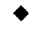 & $\bullet$ \\
\hline $\mathrm{D}_{50}$ in Pools & $\square$ & $\bullet$ & - & $\bullet$ & o \\
\hline Percent Fines $(<2 \mathrm{~mm})$ & 0 & 0 & 0 & 0 & 0 \\
\hline Embeddedness & 0 & 0 & 0 & 0 & 0 \\
\hline \multicolumn{6}{|c|}{ Pool Characteristics } \\
\hline Number & - & 0 & - & $\bullet$ & 口 \\
\hline Area & - & 0 & - & $\bullet$ & ㅁ \\
\hline Volume & $\square$ & • & - & $\bullet$ & $\square$ \\
\hline Residual Depth & $\square$ & $\bullet$ & - & $\bullet$ & $\square$ \\
\hline $\mathrm{V}^{*}$ & - & 0 & - & 0 & $\square$ \\
\hline \multicolumn{6}{|c|}{ Reach Morphology } \\
\hline Thalweg Profiles/Bedforms & 0 & - & o & - & 0 \\
\hline Bank Erosion & 口 & 口 & 口 & $\bullet$ & 口 \\
\hline Habitat Units & 0 & 0 & 0 & $\bullet$ & 0 \\
\hline Channel Scour & $\square$ & 口 & $\bullet$ & $\bullet$ & $\square$ \\
\hline \multicolumn{6}{|c|}{ Sediment Transport } \\
\hline Suspended Load & 0 & 0 & 0 & 0 & o \\
\hline Bedload & $\bullet$ & $\bullet$ & $\bullet$ & $\bullet$ & 0 \\
\hline
\end{tabular}

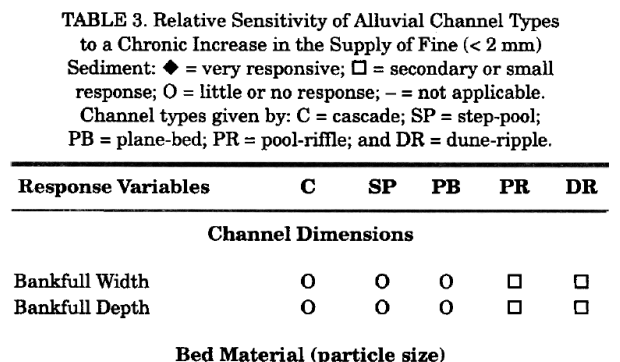

\begin{tabular}{|c|c|c|c|c|}
\hline$D_{84}$ & 0 & o & $\square$ & $\square$ \\
\hline $\mathrm{D}_{50}$ & o & o & $\bullet$ & $\bullet$ \\
\hline $\mathrm{D}_{16}$ & 口 & ㅁ & $\bullet$ & $\bullet$ \\
\hline $\mathrm{D}_{50}$ in Pools & $\square$ & 口 & - & $\bullet$ \\
\hline Percent Fines $(<2 \mathrm{~mm}$ ) & $\square$ & $\square$ & $\bullet$ & $\bullet$ \\
\hline Embeddedness & $\square$ & ㅁ & $\bullet$ & $\bullet$ \\
\hline
\end{tabular}

Pool Characteristics

$\begin{array}{llllll}\text { Number } & - & 0 & - & 0 & 0 \\ \text { Area } & - & \square & - & \square & 0 \\ \text { Volume } & 0 & \square & - & \bullet & \bullet \\ \text { Residual Depth } & \square & \square & - & \bullet & \bullet \\ \mathrm{V}^{*} & - & \square & - & \bullet & -\end{array}$

Reach Morphology

\begin{tabular}{lccccc} 
Thalweg Profiles & 0 & $\square$ & 0 & $\bullet$ & $\square$ \\
Bank Erosion & 0 & 0 & 0 & 0 & $\square$ \\
Habitat Units & 0 & 0 & 0 & $\square$ & 0 \\
Channel Scour & 0 & 0 & 0 & $\square$ & $\square$ \\
& Sediment Transport & & & \\
$\begin{array}{l}\text { Suspended Load } \\
\text { Bedload }\end{array}$ & $\bullet$ & $\bullet$ & $\bullet$ & $\bullet$ & $\bullet$ \\
\hline
\end{tabular}

Table 6.4: Table of sensivity of alluvial channel types to increases in sediment supply. From Montgomery \& MacDonald (2002). Used with permission. 


\subsubsection{Streamside landslides and debris flows}

There is morphological, sedimentary, and historical evidence that landslides and debris flows exert primary controls on channel morphology in Little Creek. All of the study reaches in Little Creek as well as much of channel length (especially in the Mainstem) is incised into fill terraces that appear to be debris flow deposits or sediment deposited behind debris flow or streamside landslide deposits. In the Mainstem of Little Creek these boulder jams and terrace deposits consist of poorly sorted sediment (diameters $<2 \mathrm{~mm}$ to $>2 \mathrm{~m}$ ) that are derived from granitic rock, shist, or sandstone. These rock types are all stratigraphically below the channel bed along almost the entire length of the Mainstem channel ${ }^{1}$ indicating that these terrace materials were transported from upstream and are not primarily hillslope derived. Some boulder $/ \log$ deposits create large steps $(>3-4 \mathrm{~m})$ in the channel profile and have large amounts of sediment stored behind them (see Figure 6.4).

Large streamside landslides are also a major influence on channel morphology. These landslides likely have the greatest influence on the channel directly impacted by the landslide deposits, but also have impacts both up and downstream. Downstream impacts would primarily result from increased sediment loads as the sediment deposited by the landslide is transported downstream. The landslide may also be a triggering mechanism for the initiation of debris flows which could scour the channel downstream. Upstream impacts could result from the change in local baselevel above the deposit; this would lower the gradient upstream and cause aggradation behind the landslide deposit. Most of the hillslopes along Little Creek show evidence for relatively recent landslides (young vegetation, sharply defined scarps, etc.) — both shallow and deep-seated. Figure 6.3 shows relatively

\footnotetext{
${ }^{1}$ The contact between the Santa Cruz mudstone and underlying rocks is in the vicintity of the swimming pool just downstream of the confluence of the North and South forks
} 


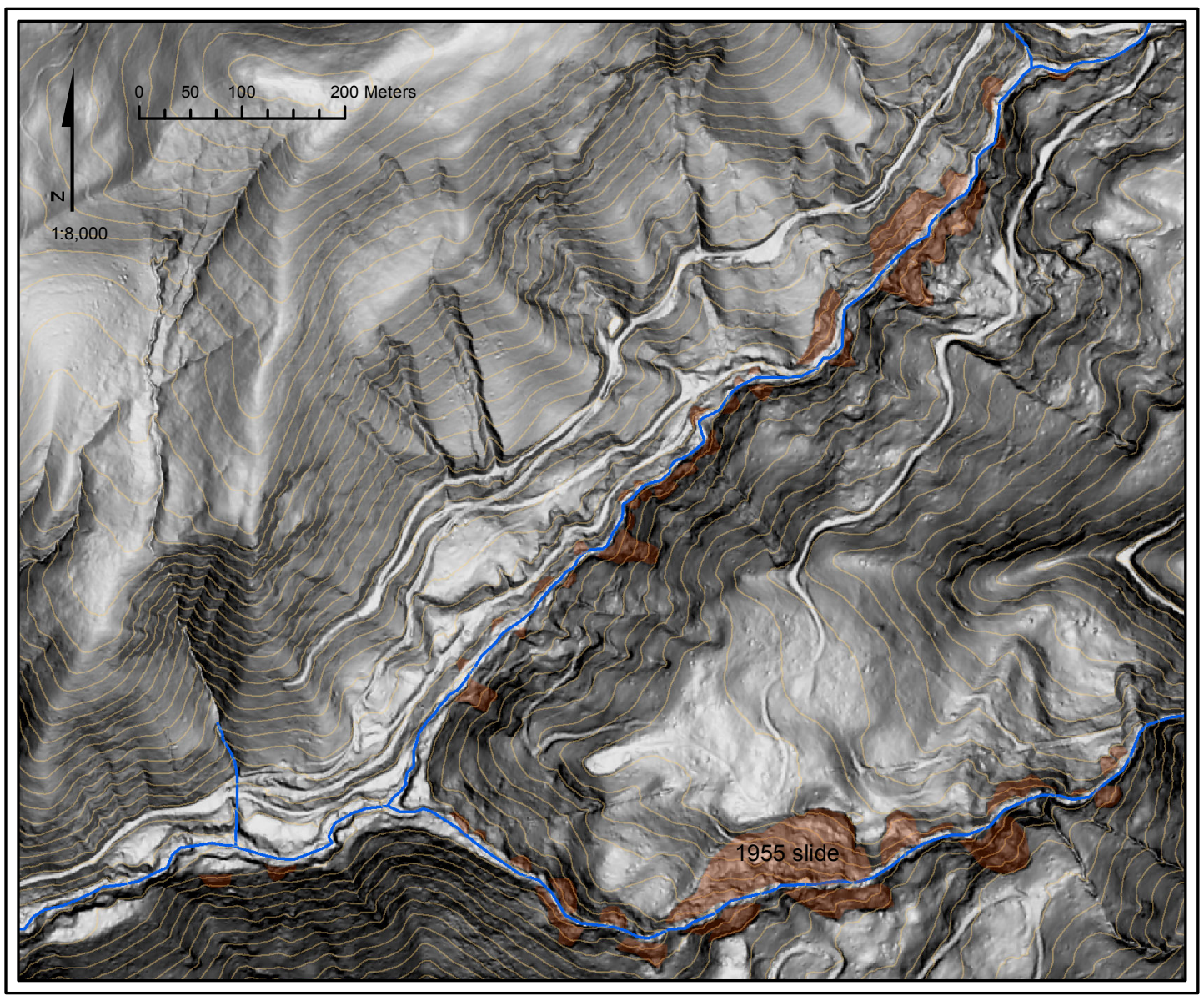

Figure 6.3: Map showing recent near-stream landslides in the area around the confluence of the North and South Forks of Little Creek. Landslide areas are highlighted in orange and were mapped from LiDAR topography data and field observations. Contour interval $=12.2 \mathrm{~m}$ (40 feet).

recent (well defined scarps and voids) streamside landslide features mapped using the 2010 LiDAR-derived 1m DEM and field observations. These landslides are likely also a major input mechanism of large woody debris to the channel.

It appears that most of the terraces in Little Creek result from deposition behind landslide and debris flow deposits, and that flow events that can mobilize and rearrange the large boulders and logs that constitute these deposits (like the 1955 storm) are the primary driver of large-scale morphologic change. 


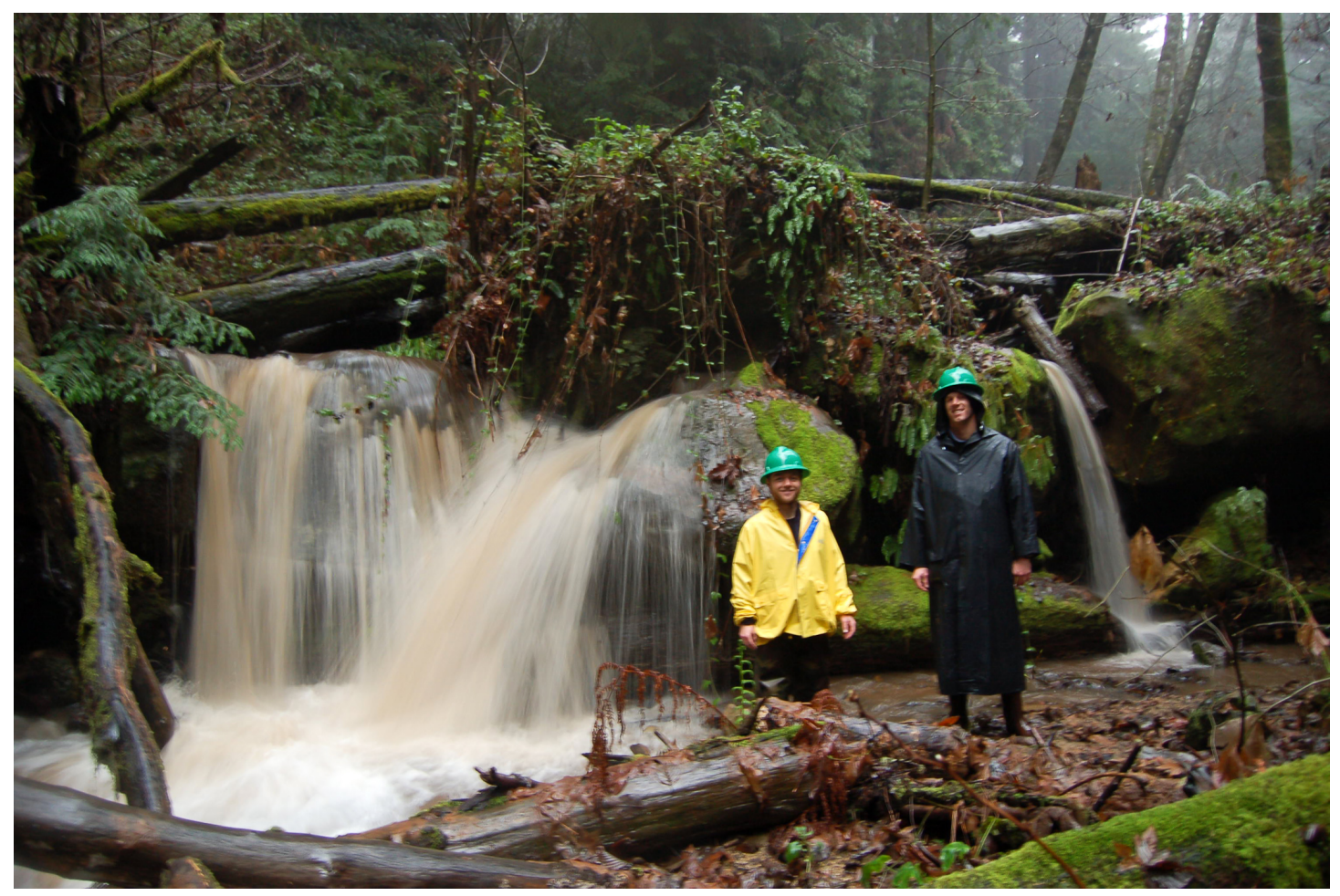

Figure 6.4: Log and Boulder Jam in Mainstem of Little Creek.

\subsubsection{Influence of large woody debris}

The influence of large woody debris on channel morphology has been recognized in numerous studies (Keller \& Swanson, 1979; Faustini \& Jones, 2003; Montgomery \& Buffington, 1998; Montgomery et al., 2003). Large woody debris is an important forcing agent in Little Creek, and a majority of the steps and pools in the channel are influenced by large woody debris. Much of the observed change in Little Creek during the study period can be related to inputs or reorganization of woody debris in the channel. The type of wood introduced to channel appears to have an impact on the longevity of influence. Most of the larger steps in the channel are formed by redwood logs or rootwads that are typically larger, decay resistant and long lived. This makes them more likely to accumulate other wood pieces over time to create wood complexes that have a larger influence on morphology (Montgomery et al., 2003). In contrast, as trees from the even-aged riparian stand (that was 
established after the 1955 flood) reach maturity and succumb to disease and decay, large quantities of red alder have been recruited to the Mainstem channel in recent years. These trees typically have smaller diameters than the conifer species, are decay prone, and typically only survive in the channel for 3-5 years before they decay and are destroyed by streamflows. Depending on orientation and location, red-alder derived woody debris can have a significant influence on the channel, but it is typically only for a short time period. It is likely that several large bedload-mobilizing flows must interact with woody debris in order for significant morphological changes to occur, and the red alder typically decays before this can occur. An estimated 80 percent of the large woody debris influenced pools and steps in Little Creek result from redwood logs or rootwads, and some of these features may influence the channel for hundreds of years.

\subsubsection{Conceptual model of morphologic changes in Little Creek}

This section presents a model of morphologic change in Little Creek based on the observations from the study period, historic geomorphic evidence, and the morphology of Little Creek (Figure 6.5). Most of the channel morphology is dominated by patterns that develop after debris-flow events that can mobilize

and rearrange a majority of the larger particle sizes present in the bed. There is then an adjustment period where normal high flows (2-5 year return intervals) reorganize the bed until a relatively stable configuration develops that is controlled by the arrangement of the larger bed particles and large woody debris. Between these large debris-flow events, the channel form appears to be relatively stable with only small adjustments in shape or bed configuration, even though flow events can 
mobilize a majority of the clasts forming the channel bed. The particle size that can be mobilized by streamflows is highly dependent on local gradient and flow configuration, but particles at least up to $256 \mathrm{~mm}$ have been observed moving through the flumes during regular high flow events. The channel upstream of the 1998 streamside-landslide deposit in Reach D appears to be an example of this model. The channel likely had some initial adjustment after the landslide, especially within the deposit itself, but the reach has been stable since 2002. This model is consistent with what others have observed in steep mountainous channels influenced by debris flows (Montgomery \& Buffington, 1998). It will be interesting to observe how the channel responds to the Lockheed Fire. Initial observations are that channel changes have been minor compared to responses observed in other systems (Eccleston, 2008; Meyer et al., 1992; Jackson \& Roering, 2009).

Between large debris-flow events, it appears that most channel change is associated with input or modification of large woody debris in the channel. These changes are localized and not necessarily influenced by the flow regime. For example, many trees were recruited to the channel after the 2009 Lockheed Fire either directly through fire damage to roots and stems, or indirectly through mortality. There is likely a strong correlation between large woody debris recruitment and debris-flow events; the storms that produce debris flows also likely cause many stream-side landslides and fluvially-related bank failures which appear to be primary large woody debris recruitment mechanisms in Little Creek. There also is some adjustment period after large woody debris modification, but stable conditions likely develop within a relatively short time period. 


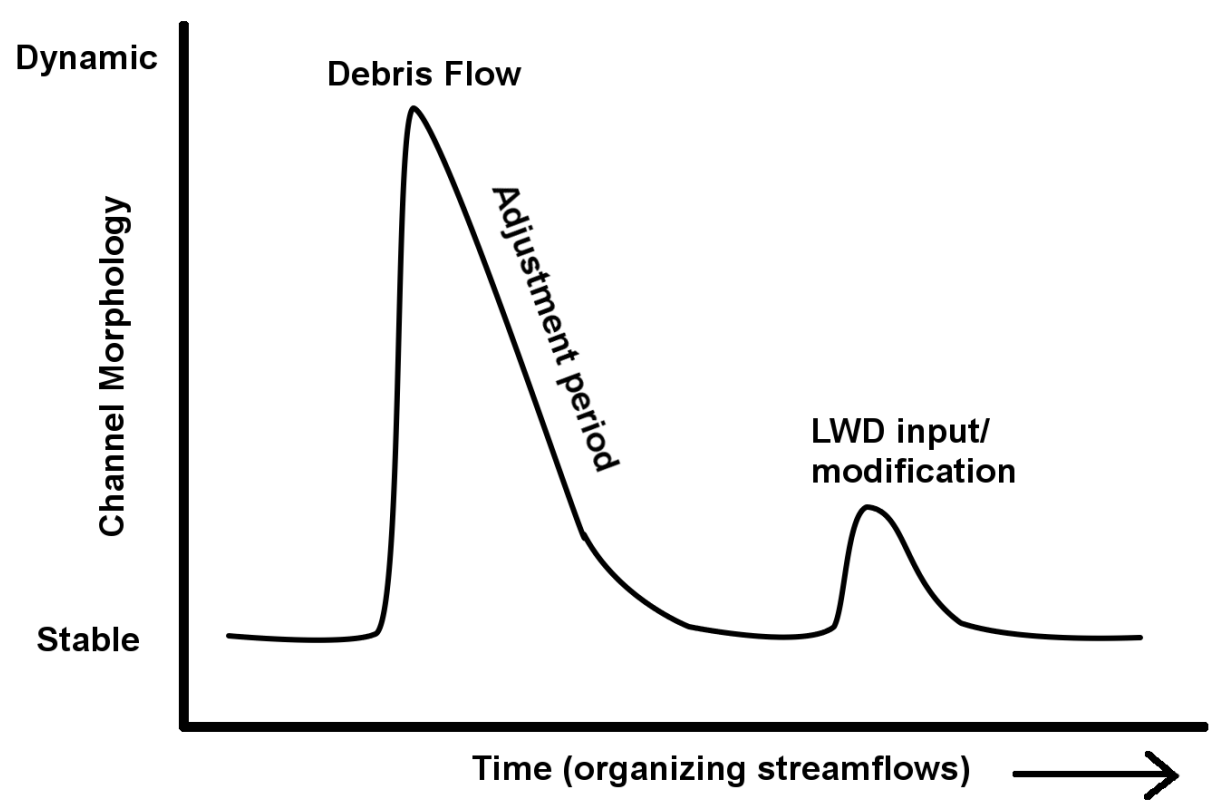

Figure 6.5: Conceptual model of channel change in Little Creek. 


\section{Chapter 7}

\section{Conclusion}

The channel morphology in Little Creek has been relatively stable during the period 2002-2008. Changes were primarily observed in Reach C and Reach F. Reach $\mathrm{C}$ had a degradational trend during the study period, primarily associated erosion of material stored behind a large woody debris deposit. The reach-wide average mean bankfull bed elevation dropped $0.08 \mathrm{~m}$ with drops of up to $0.15 \mathrm{~m}$ in some cross sections. Reach $\mathrm{F}$ had an aggradational trend during the study period. The reach-wide average mean bankfull bed elevation increased $0.08 \mathrm{~m}$ with increases of up to $0.15 \mathrm{~m}$ in some cross sections. Reach-wide average mean bankfull bed elevations in the other study reaches changed less than $0.05 \mathrm{~m}$ during the study period, which is also the approximate error in surveying the bed elevation. Similar patterns were observed in measurements of thalweg elevation and cross-sectional area. Variability of year-to-year changes appears to correlate with magnitude of annual peakflows, and pool residual depths are generally more dynamic than cross section geometry. It appears that in the the absence of debris-flow events or input or modification of large woody debris, even flows that can potentially mobilize 
most of the bed material do not cause major changes in channel morphology in Little Creek.

This is a strong contrast to the lower gradient portions of Scotts Creek, where bankfull flows can completely transform the bed morphology and transport and reorganize large woody debris. Many reaches in Scotts Creek have changed significantly during the last five years, and current work (Goin, in prep.) has reestablished surveys that were conducted in 1997,1998, and 2000 along the length of Scotts Creek on Swanton Pacific Ranch.

An understanding of channel dynamics in Little Creek provides an excellent baseline to help understand changes that may result from the Lockheed fire. The data collected will also be a valuable resource to future researchers trying to understand channel changes and recovery that may result from future events like debris-flows, earthquakes, and timber harvest. The channel metrics identified and calculated in this thesis will facilitate these analyses.

\subsection{Future Work and Opportunities}

To ensure that this dataset has value in the future, modifications and additional data collection are recommended for future annual surveys. First, the importance of reliable, immobile benchmarks must be stressed. The steep side slopes in Little Creek are a challenging location to establish cross section endpoints, and most of the original 3/8" cemented benchmarks were not installed deep enough to provide a stable control of position and elevation. This rebar movement added an unknown source of error to estimates of channel change. In 2010, new rebar endpoints were established at approximately 35 of the cross sections. These endpoints should 
provide a more stable benchmark and new endpoints should be established and documented as needed. Secondly, more consistent guidelines for surveyors need to be established and followed. New instructions for surveyors (Appendix C) were developed and added to the Little Creek Field Manual, which should help with the collection of more consistent, accurate data. Third, additional data should be collected which can help provide other information to help understand changes in the channel and provide context for interpretation. It is recommended that a pebble count in each reach be added to the annual survey based on the methodology in Harrelson et al. (1994). Pebble counts were performed before and after the first winter following the Lockheed Fire (2009-2010), and this data collection effort should continue annually. Additionally, an annual photo set should be taken of each reach. At a minimum, this set should have a photo of each cross section showing at least the bankfull channel looking both upstream and downstream. This photo set should provide visual information that can help future researchers understand conditions in Little Creek at the time of each study and provide information needed to make interpretations from changes seen in cross sections or longitudinal profiles.

\subsection{Data Archiving and Preservation}

The final goal of this thesis is ensuring that these data and benchmarks will be available to future researchers. This data will be archived in several ways. Appendix D contains the quality controlled data for all cross sections and longitudinal profiles. This document will provide a permanent record of this data that will be stored in the Cal Poly library's collection which will be accessible for future use. The true coordinates of the permanent points and cross section endpoints will also 
allow re-establishment of these locations if they are ever lost in the field. Appendix E contains the Visual Basic code used for all of the analysis so it can be referenced or checked by future researchers. A file will also be established at Swanton Pacific Ranch library that will contain all of the field books, a copy of this thesis, and a DVD and flash drive containing all of the spreadsheets, databases, and Minitab worksheets used for the analysis. A text version of the data will also be provided to assist future researchers in case there is difficulty opening the spreadsheets due to software changes. 


\section{Bibliography}

Anderson, R. S. (1994). Evolution of Santa Cruz Mountains, California, through tectonic growth and geomorphic decay. Journal of Geophysical Research, 99 (B10), 20,161-20,179.

Anderson, R. S. (2008). THE LITTLE BOOK OF GEOMORPHOLOGY: Exercising the Principle of Conservation.

Bartley, R., \& Rutherfurd, I. (2005). Measuring the reach-scale geomorphic diversity of streams: application to a stream disturbed by a sediment slug. River Research and Applications, 21(1), 39-59.

Beschta, R. L., \& Ripple, W. J. (2006). River channel dynamics following extirpation of wolves in northwestern Yellowstone National Park, USA. Earth Surface Processes and Landforms, 1539, 1525-1539.

Brabb, E. (1989). Geologic map of Santa Cruz County, California. United States Geological Survey.

Dietterick, B., White, R., \& Hilburn, R. (2012). Comparing LiDAR-Generated to Ground-Surveyed Channel Cross-Sectional Profiles in a Forested Mountain Stream. In R. Standiford, T. Weller, D. Piirto, \& J. Stuart (Eds.) Proceedings of coast redwood forests in a changing California: A symposium for scientists 
and managers. Gen. Tech. Rep. PSW-GTR-238. Pacific Southwest Research Station, Forest Service, U.S. Department of Agriculture.

Eccleston, D. T. (2008). POST-FIRE CHANNEL CHANGE IN TWO SMALL WATERSHEDS IN THE COLORADO FRONT RANGE. Master's thesis, Colorado State University.

Faustini, J. M., \& Jones, J. A. (2003). Influence of large woody debris on channel morphology and dynamics in steep, boulder-rich mountain streams, western Cascades, Oregon. Geomorphology, 51, $187-205$.

Gaedeke, M. C. (2006). Preharvest Calibration of the Little Creek Watershed: A Paired and Nested Watershed Analysis. Master's thesis, California Polytechnic State University San Luis Obispo.

Harrelson, C. C., Rawlins, C. L., \& Potyondy, J. P. (1994). Stream Channel Reference Sites : An Illustrated Guide to Field Technique. USFS RMRS Gen Tech Rpt RM-245, (p. 61 p.).

Hickey, J. (1969). Variations in low-water streambed elevations at selected streamgaging stations in northwestern California. USGS Water Supply Paper 1879-E, (p. 33).

Hilburn, R. M. (2010). A Comparison of LiDAR Generated Channel Features with Ground-Surveyed Channel Features in the Little Creek Watershed. Master's thesis, Cal Poly San Luis Obispo.

Jackson, M., \& Roering, J. J. (2009). Post-fire geomorphic response in steep, forested landscapes: Oregon Coast Range, USA. Quaternary Science Reviews, 28(11-12), 1131-1146. 
Keim, R. F., Skaugset, A. E., \& Bateman, D. S. (1999). Digital terrain modeling of small stream channels with a total-station theodolite. Advances in Water Resources, 23, 41-48.

Keller, E. A., \& Swanson, F. J. (1979). Effects of large organic material on channel form and fluvial processes. Earth Surface Processes, 4 (4), 361-380.

Leopold, L., Wolman, M., \& Miller, J. (1964). Fluvial processes in geomorphology. Dover Pubns.

Leopold, L. B., Huppman, R., \& Miller, A. (2005). GEOMORPHIC EFFECTS OF URBANIZATION IN FORTY-ONE YEARS OF OBSERVATION. Proceedings Of The American Philosophical Society, $149(3)$.

Lisle, T. (1995). Effects of coarse wood debris and its removal on a channel affected by the 1980 eruption of Mount St. Helens, Washington. Water Resources Research, $31(7), 1797-1808$.

Lisle, T. E. (1981). The recovery of aggraded stream channels at gauging stations in northern California and southern Oregon. In Erosion and Sediment Transport in Pacific Rim Steeplands. I.A.H.S Publ. No. 132, vol. 132, (pp. 189-211). Christchurch, New Zealand: IAHS.

Lisle, T. E. (1986). Effects of Woody Debris on Anadromous Salmonid Habitat, Prince of Wales Island, Southeast Alaska. North American Journal of Fisheries Management, 6(4), 538-550.

Lisle, T. E. (1987). Using "Residual Depths" to Monitor Pool Depths Independently of Discharge. Research Note PSW-394. Tech. rep., Pacific Southwest Forest and Range Experiment Station, Forest Service, U.S. Department of Agriculture, Berkeley, CA. 
Lisle, T. E., \& Napolitano, M. B. (1998). Effects of Recent Logging on the Main Channel of North North Fork Caspar Creek. In R. R. Ziemer (Ed.) Proceedings of the Conference on Coastal Watersheds: The Caspar Creek Story USFS General Technical Report PSW-GTR-168, (pp. 81-85). Ukiah, CA: USFS Pacific Southwest Research Station.

Madej, M. A. (1999). Temporal and spatial variability in thalweg profiles of a gravel-bed river. Earth Surface Processes and Landforms, 24(12), 1153-1169.

Madej, M. A. (2001). Development of channel organization and roughness following sediment pulses in single-thread, gravel bed rivers. Water Resources Research, 37(8), 2259.

Madej, M. A., \& Ozaki, V. (1996). Channel Response To Sediment Wave Propagation and Movement, Redwood Creek, California, Usa. Earth Surface Processes and Landforms, $21(10), 911-927$.

Meyer, G. A., Wells, S. G., Balling, R. C., \& Jull, A. T. (1992). Response of alluvial systems to fire and climate change in Yellowstone National Park. Nature, 357.

Montgomery, D. R., \& Buffington, J. M. (1997). Channel-reach morphology in mountain drainage basins. Geological Society Of America Bulletin, 109, 596611.

Montgomery, D. R., \& Buffington, J. M. (1998). Channel Processes, Classification, and Response, chap. 2, (pp. 13-42). New York: Springer-Verlag New York, Inc.

Montgomery, D. R., Collins, B., Buffington, J., \& Abbe, T. (2003). Geomorphic effects of wood in rivers. American Fisheries, 37, 21-47. 
Montgomery, D. R., \& MacDonald, L. H. (2002). Diagnostic Approach To Stream Channel Assessment and Monitoring. Journal of the American Water Resources Association, 38(1), 1-16.

Mossop, B., \& Bradford, M. (2006). Using thalweg profiling to assess and monitor juvenile salmon (oncorhynchus spp.) habitat in small streams. Canadian Journal of Fisheries and Aquatic Sciences, 63(7), 1515-1525.

Nolan, K. M., \& Marron, D. C. (1985). Contrast in stream-channel response to major storms in two mountainous areas of California. Geology, 13, 135-138.

Nolan, K. M., \& Marron, D. C. (1988). Stream-channel response to the storm in the Santa Cruz Mountains, (p. 319). United States Geological Survey.

Roper, B., Buffington, J., Archer, E., Moyer, C., \& Ward, M. (2008). The Role of Observer Variation in Determining Rosgen Stream Types in Northeastern Oregon Mountain Streams1. JAWRA Journal of the American Water Resources Association, $44(2), 417-427$.

Rosgen, D., \& Silvey, H. (1996). Applied river morphology, vol. 1481. Wildland Hydrology Pagosa Springs, Colorado.

Sloan, J. (2001). Response and recovery of the Eel River, California, and its tributaries to floods in 1955, 1964, and 1997. Geomorphology, 36(3-4), 129154 .

White, R. A. (2010). ACCURACY OF FOREST ROAD AND STREAM CHANNEL CHARACTERISTICS DERIVED FROM LIDAR IN FORESTED MOUNTAIN CONDITIONS. Master's thesis, Cal Poly SLO. 
Wood-Smith, R., \& Buffington, J. (1996). Multivariate geomorphic analysis of forest streams: implications for assessment of land use impacts on channel condition. Earth Surface Processes and Landforms, 21(4), 377-393. 


\section{Appendix A}

\section{Graphs of All Cross Sections}

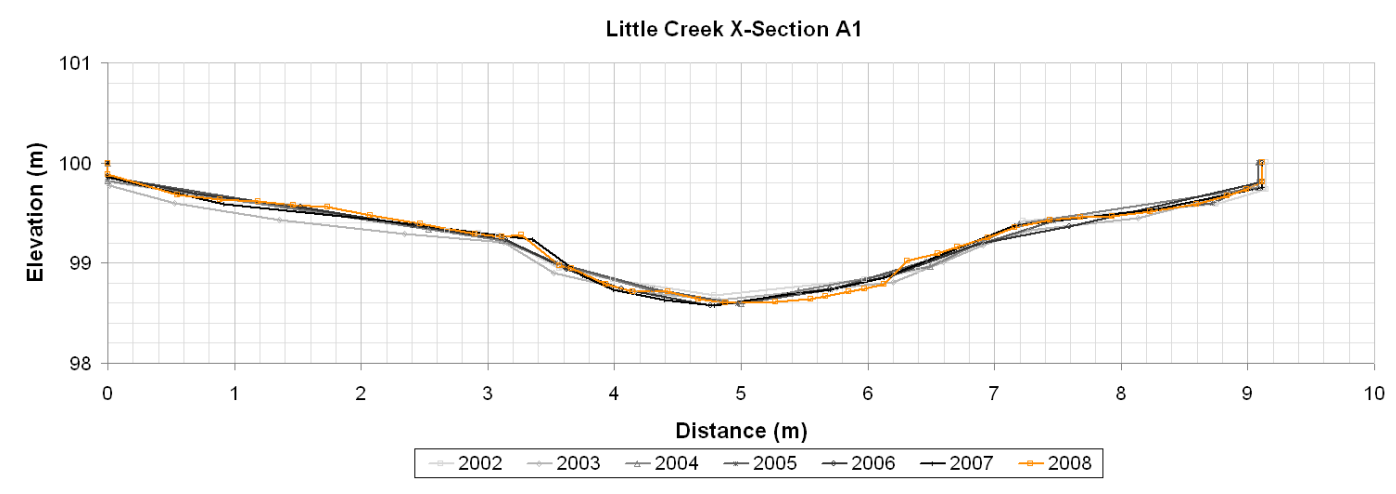

Figure A.1: Little Creek Cross Section A1

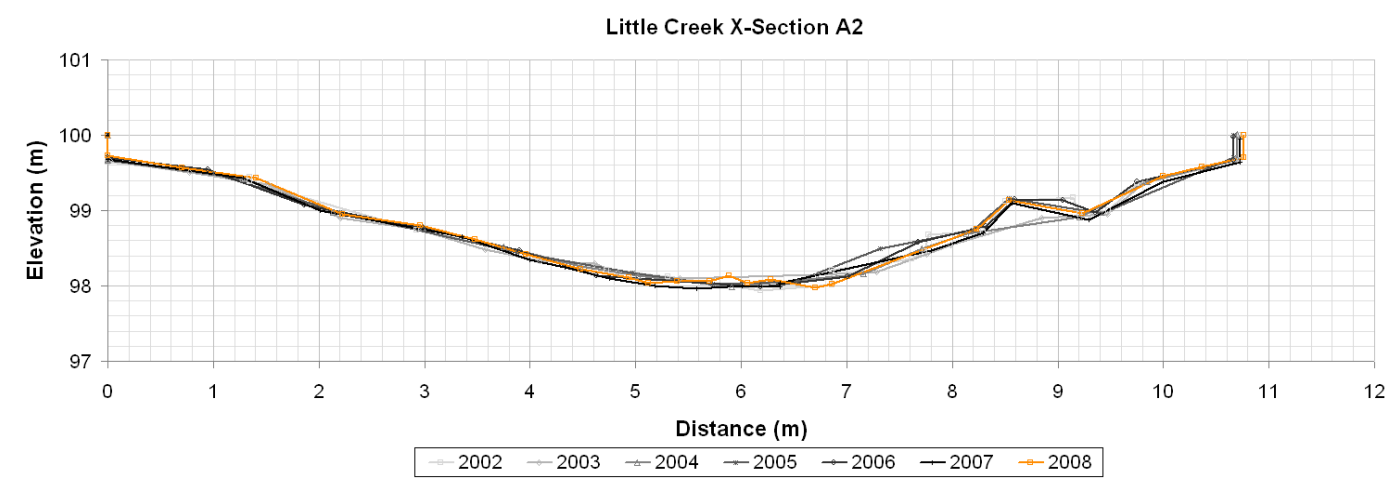

Figure A.2: Little Creek Cross Section A2 


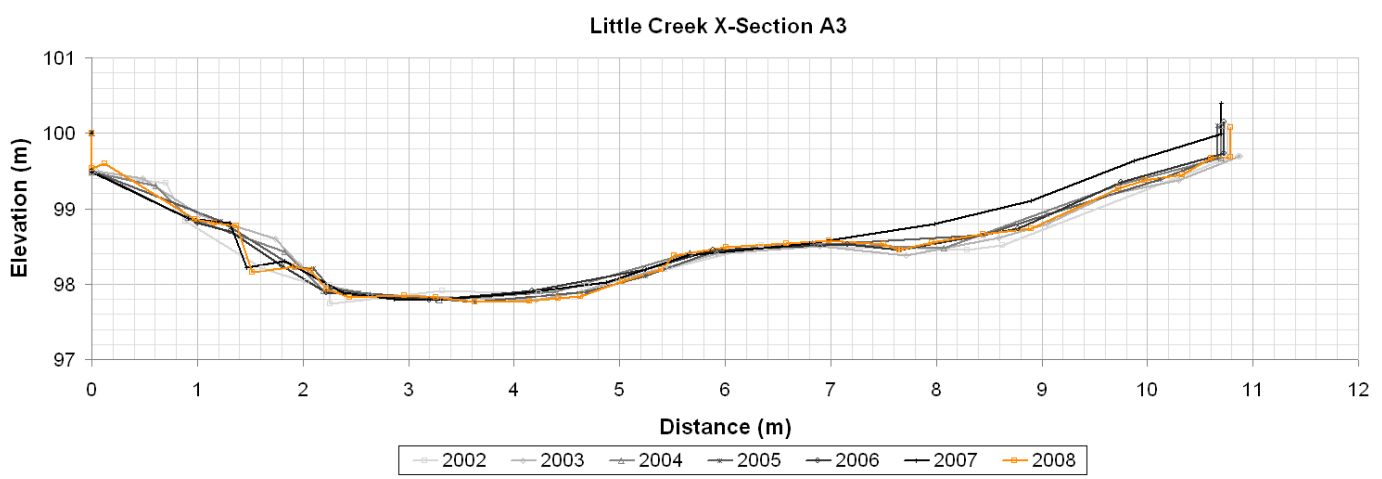

Figure A.3: Little Creek Cross Section A3

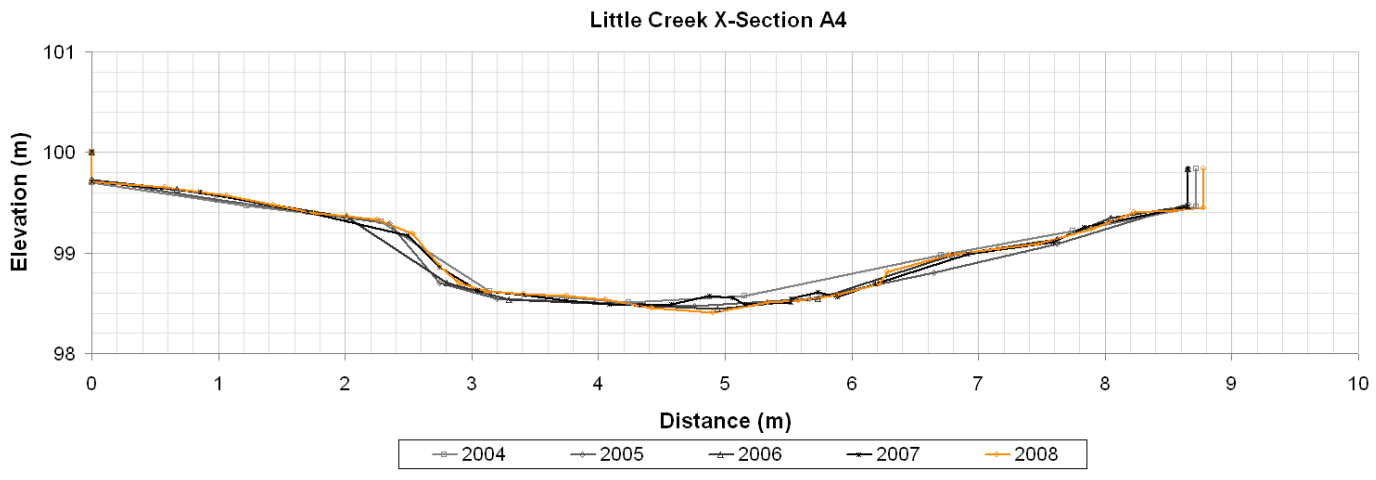

Figure A.4: Little Creek Cross Section A4

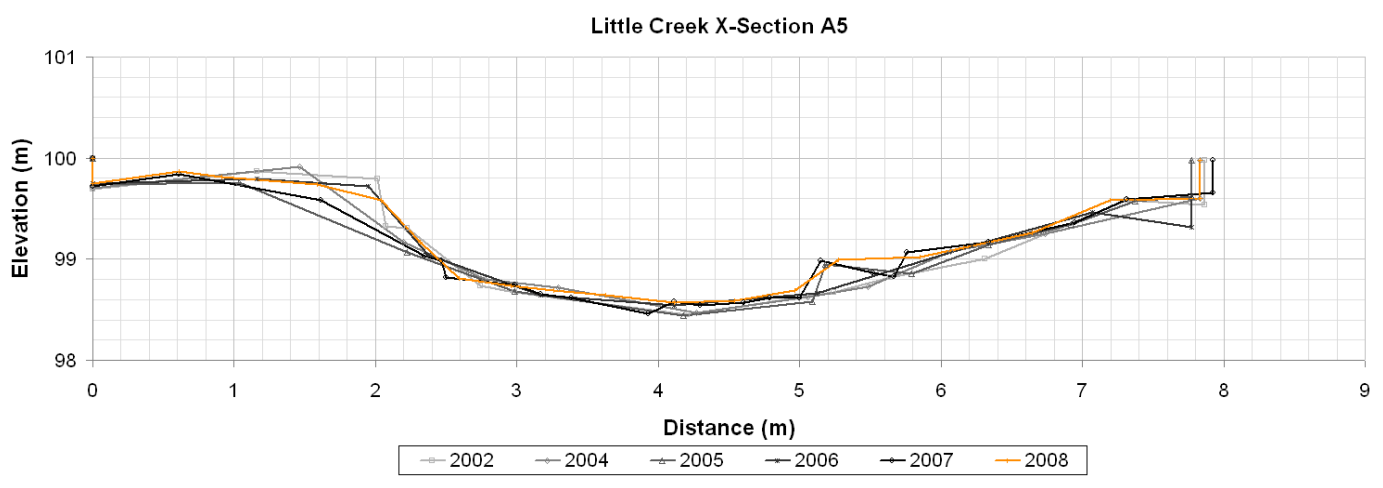

Figure A.5: Little Creek Cross Section A5 


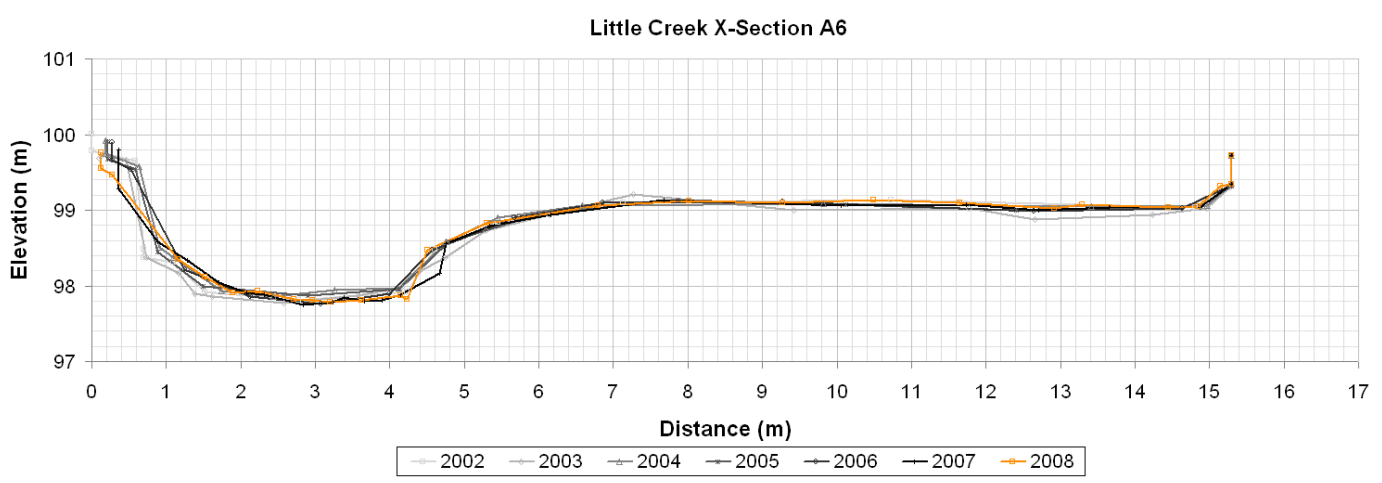

Figure A.6: Little Creek Cross Section A6

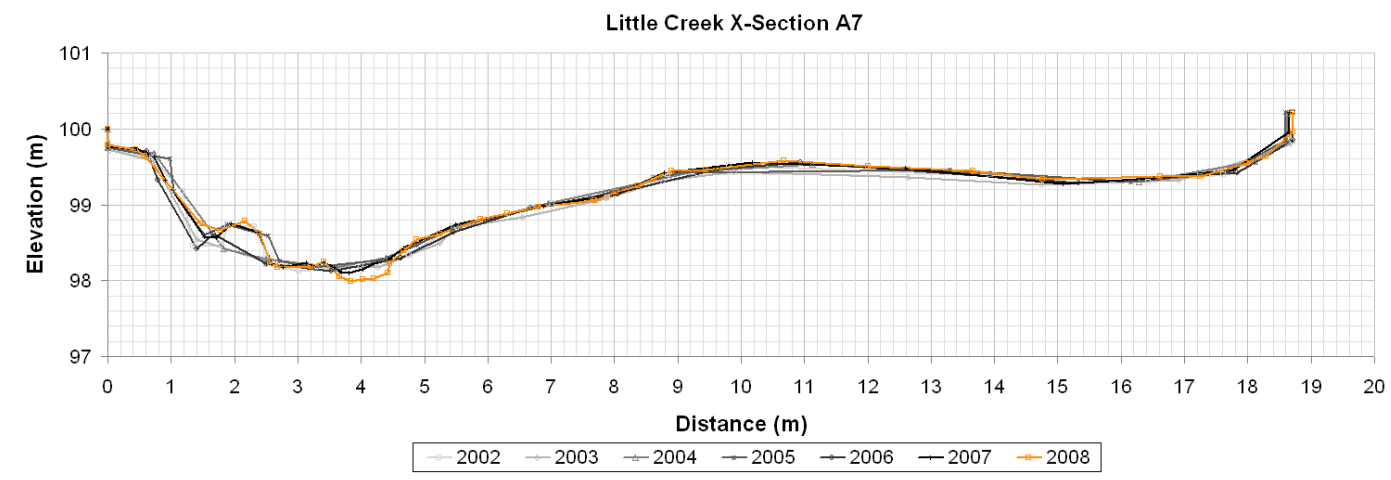

Figure A.7: Little Creek Cross Section A7

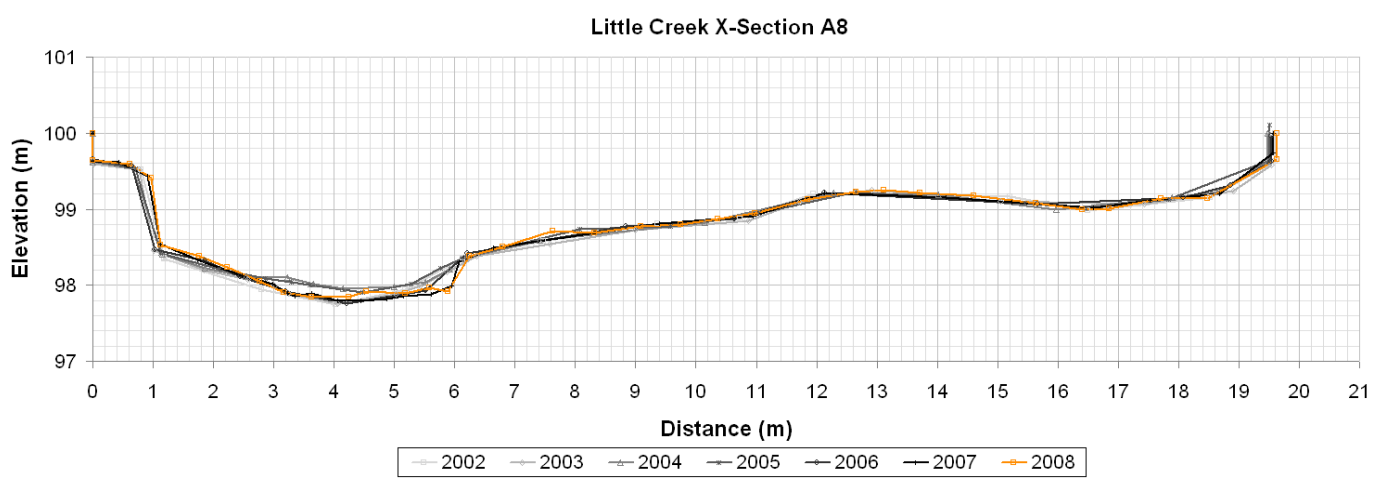

Figure A.8: Little Creek Cross Section A8 


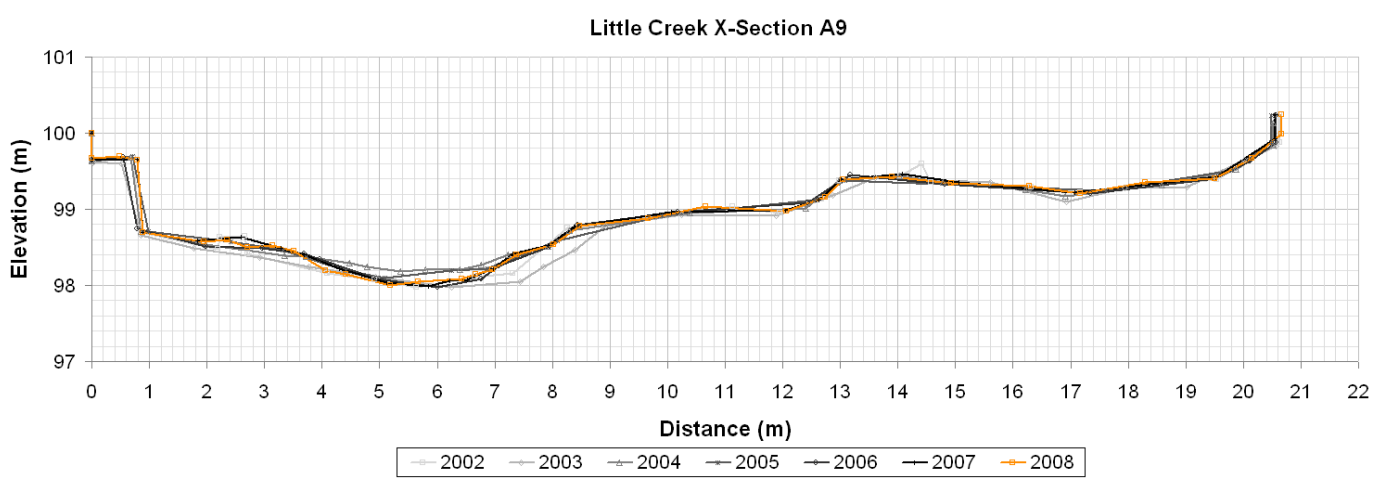

Figure A.9: Little Creek Cross Section A9

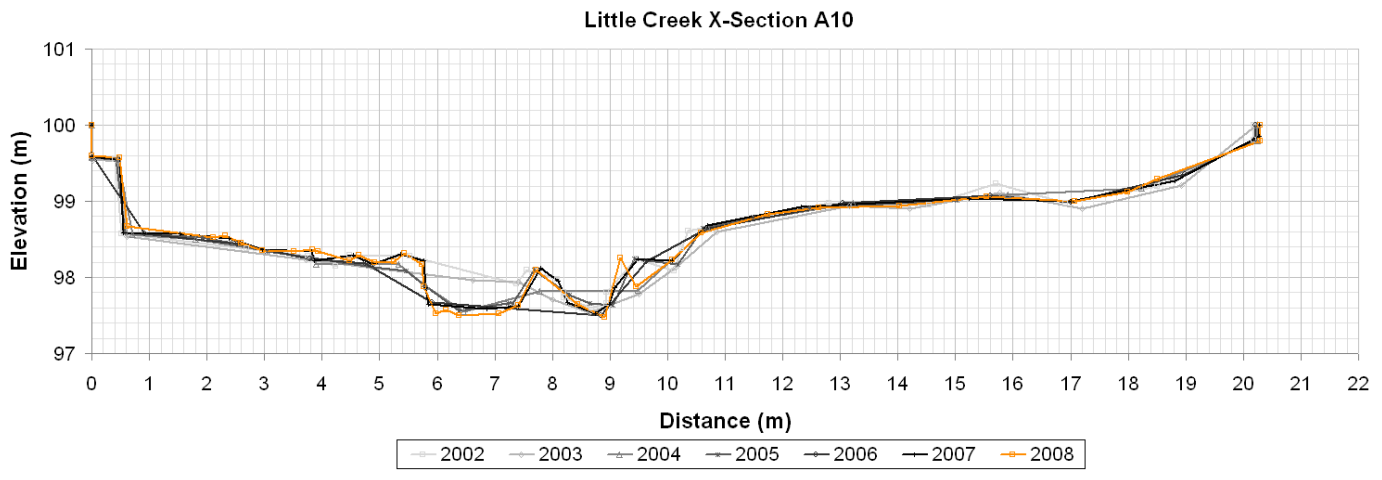

Figure A.10: Little Creek Cross Section A10

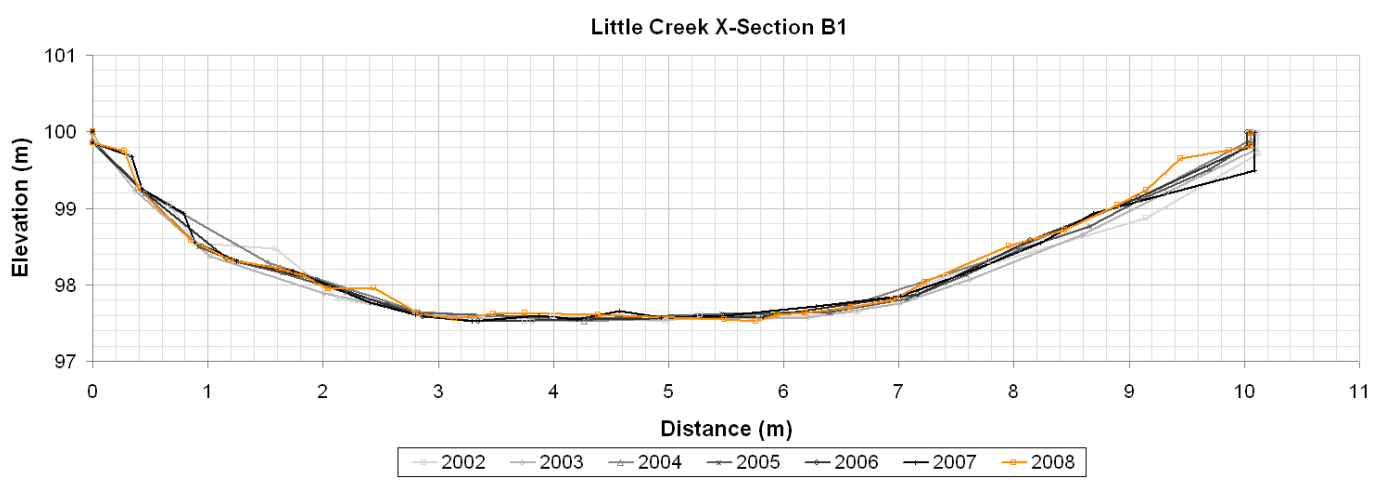

Figure A.11: Little Creek Cross Section B1 


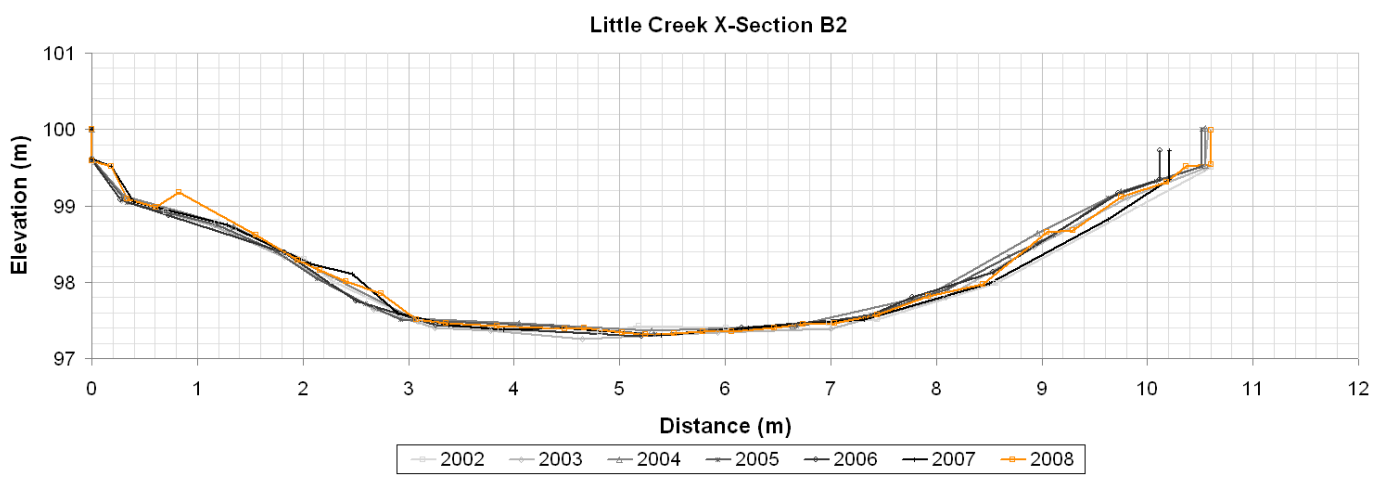

Figure A.12: Little Creek Cross Section B2

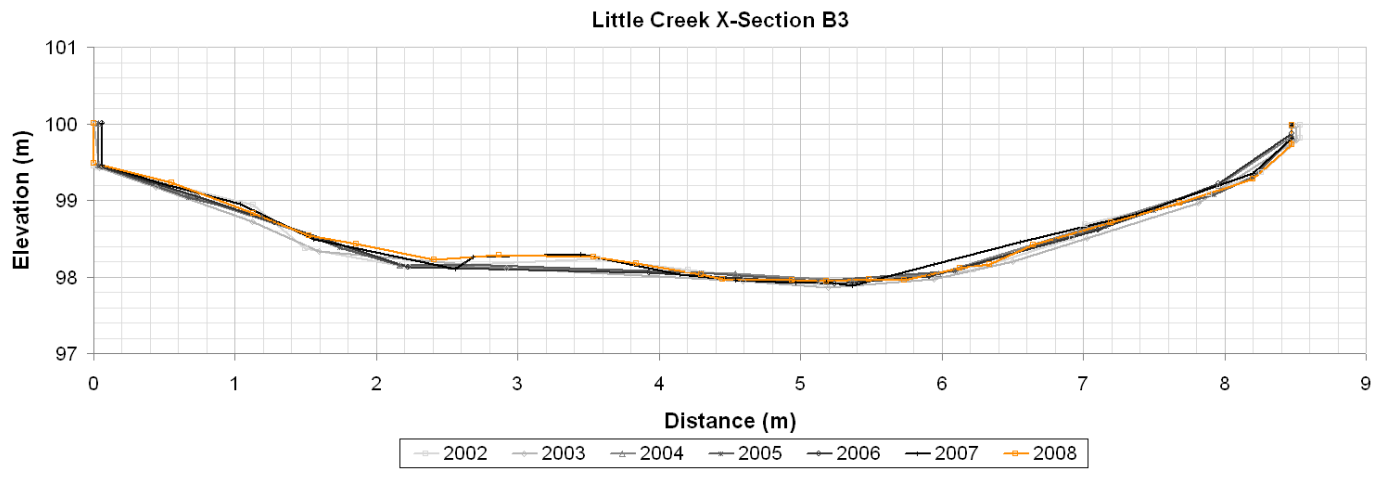

Figure A.13: Little Creek Cross Section B3

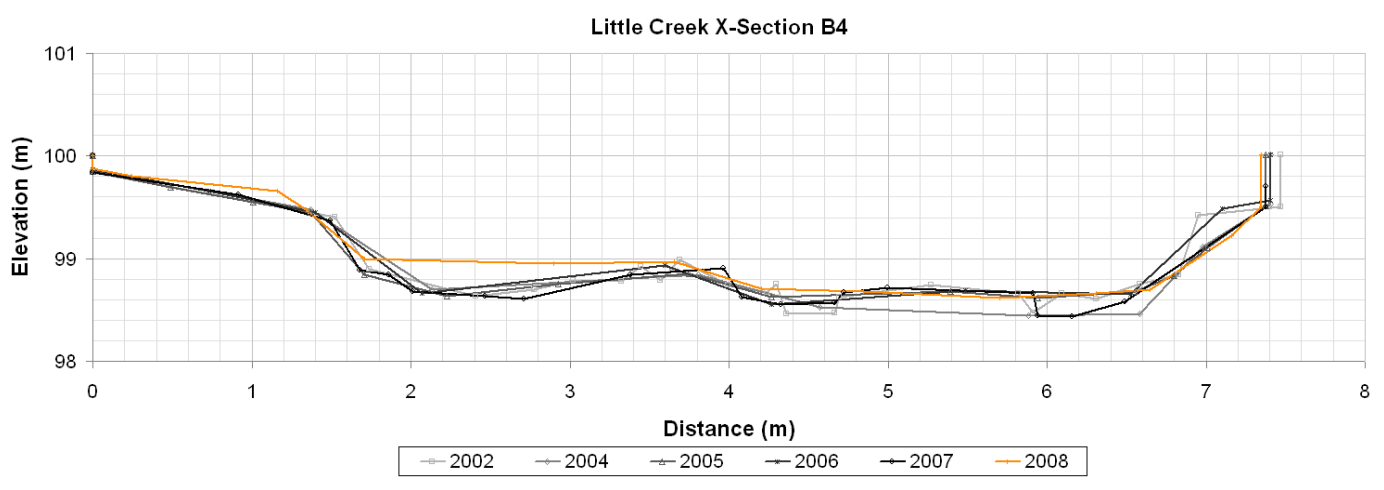

Figure A.14: Little Creek Cross Section B4 


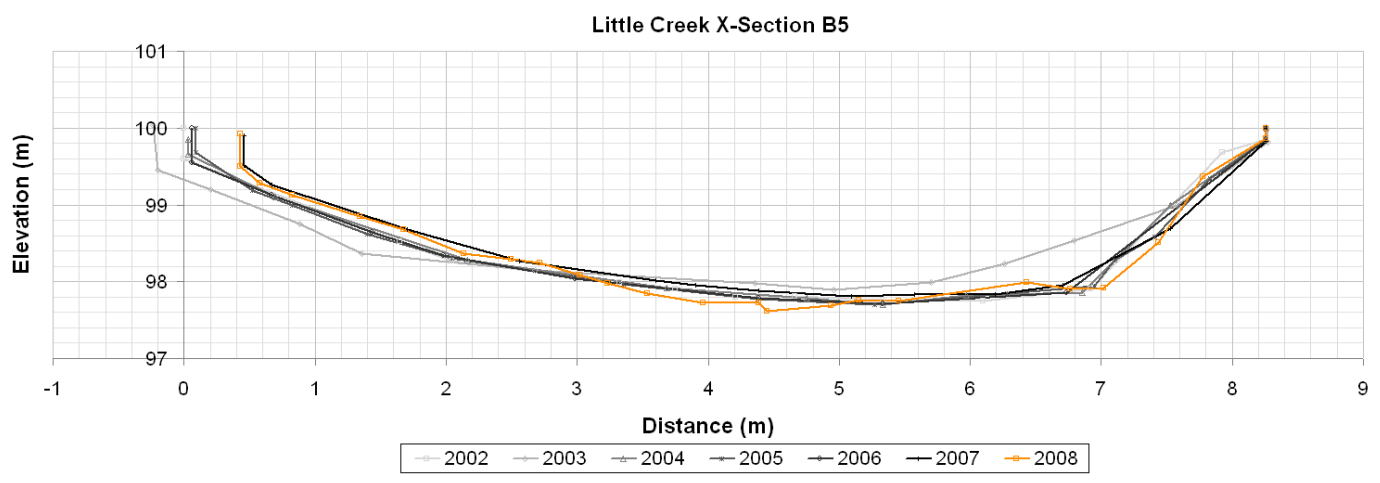

Figure A.15: Little Creek Cross Section B5

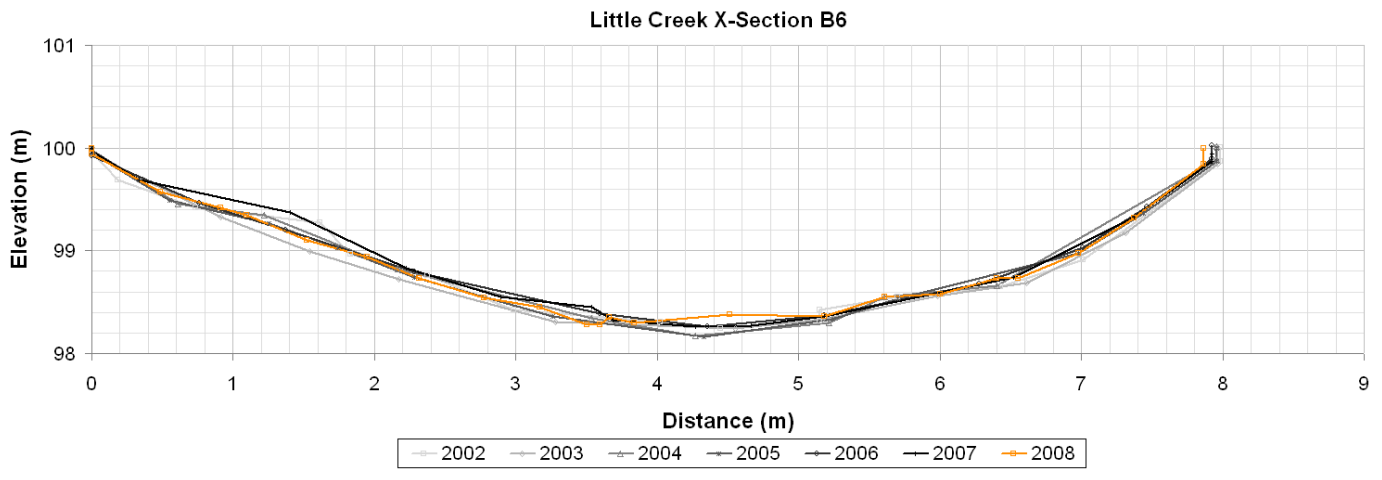

Figure A.16: Little Creek Cross Section B6

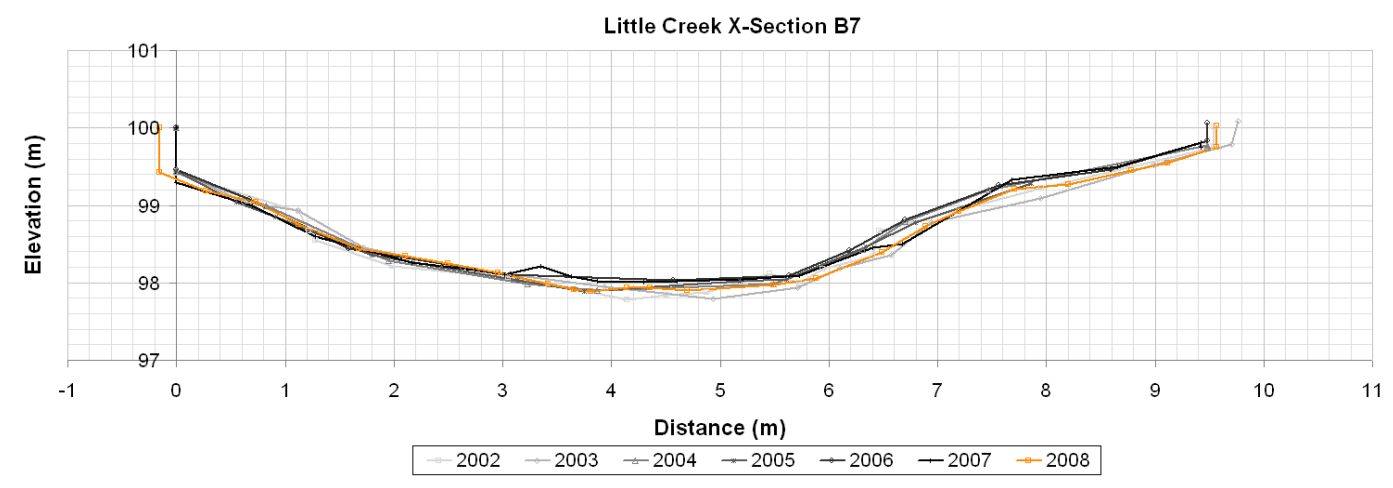

Figure A.17: Little Creek Cross Section B7 


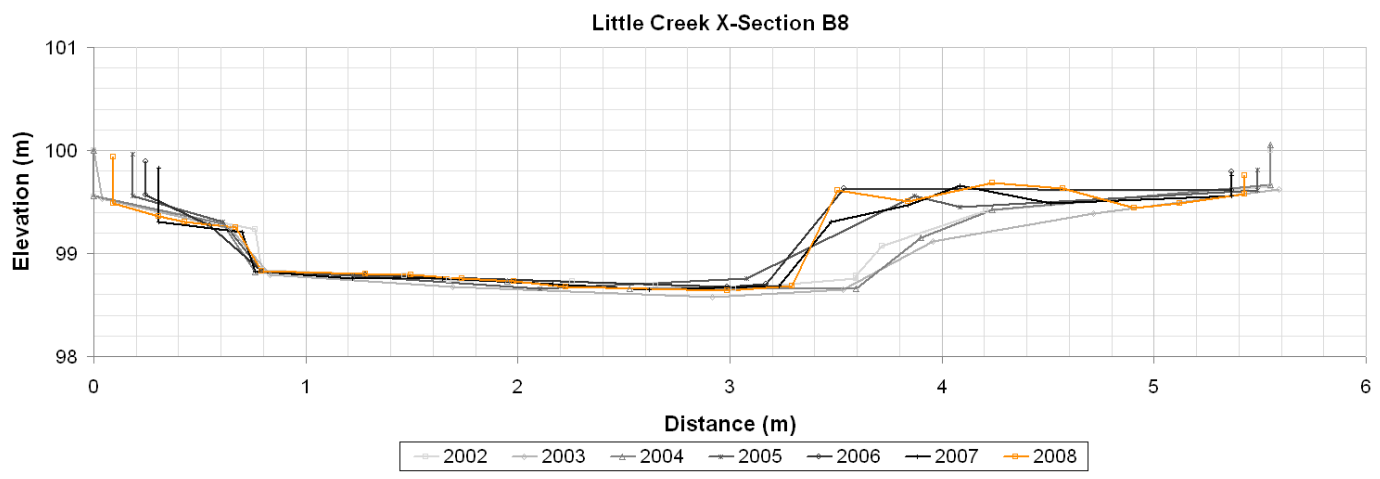

Figure A.18: Little Creek Cross Section B8

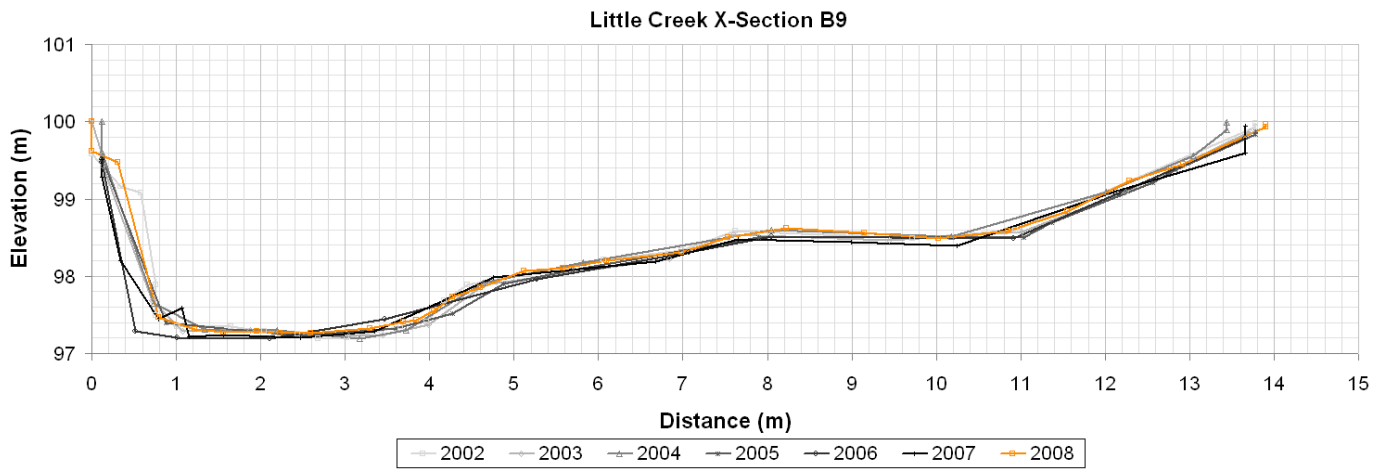

Figure A.19: Little Creek Cross Section B9

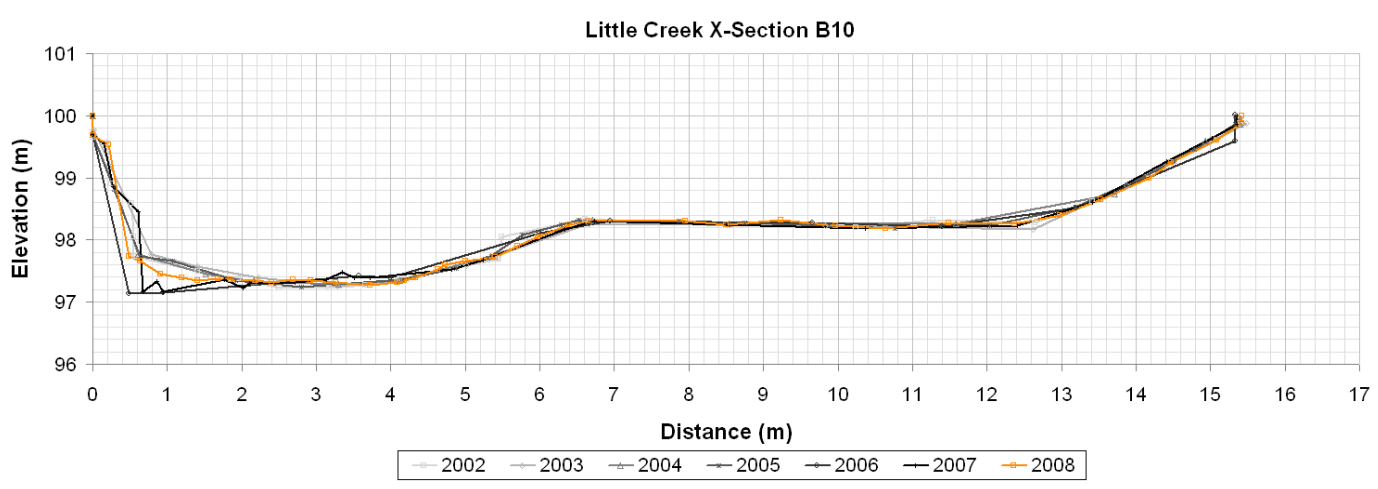

Figure A.20: Little Creek Cross Section B10 


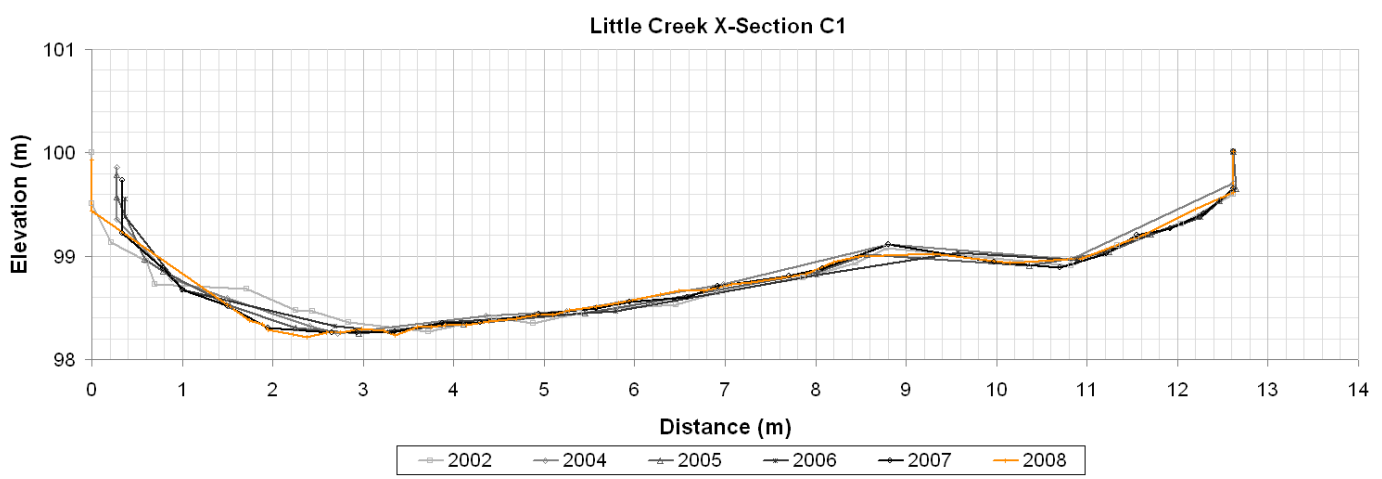

Figure A.21: Little Creek Cross Section C1

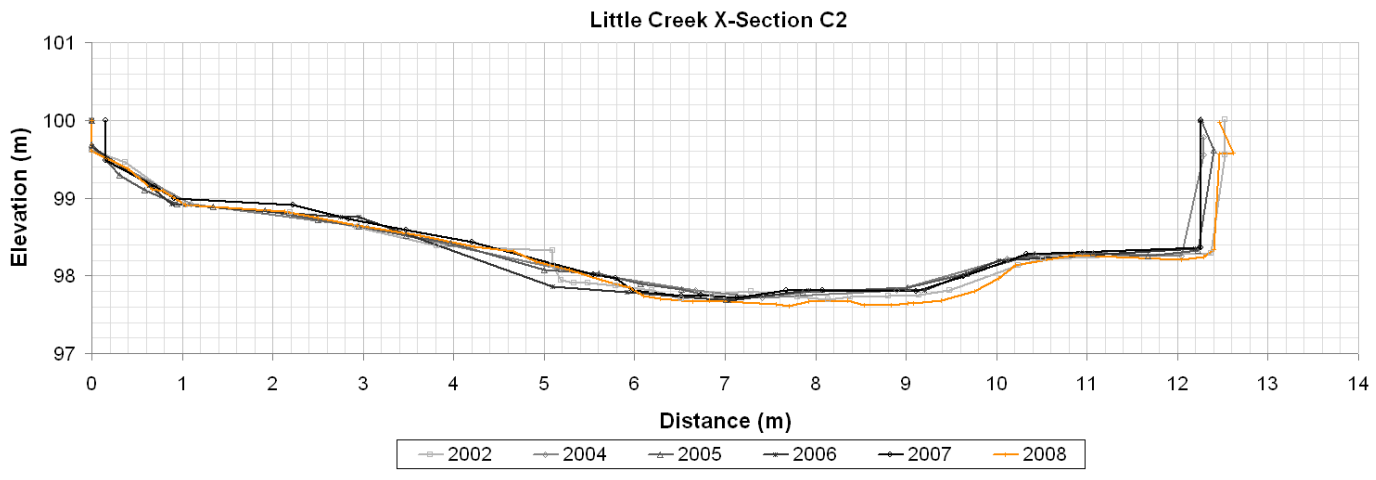

Figure A.22: Little Creek Cross Section C2

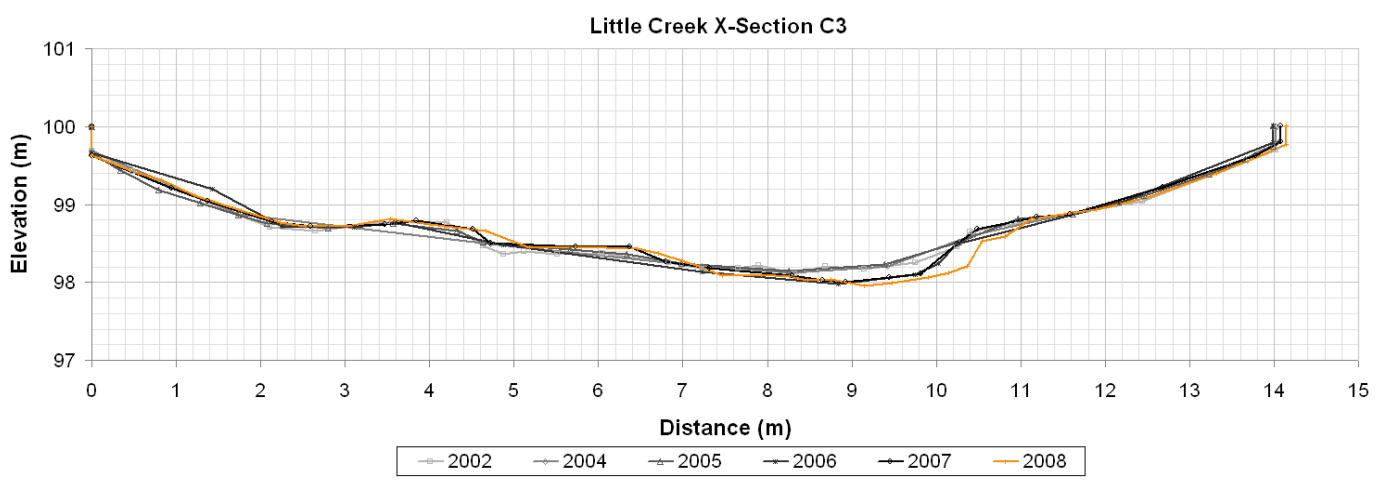

Figure A.23: Little Creek Cross Section C3 


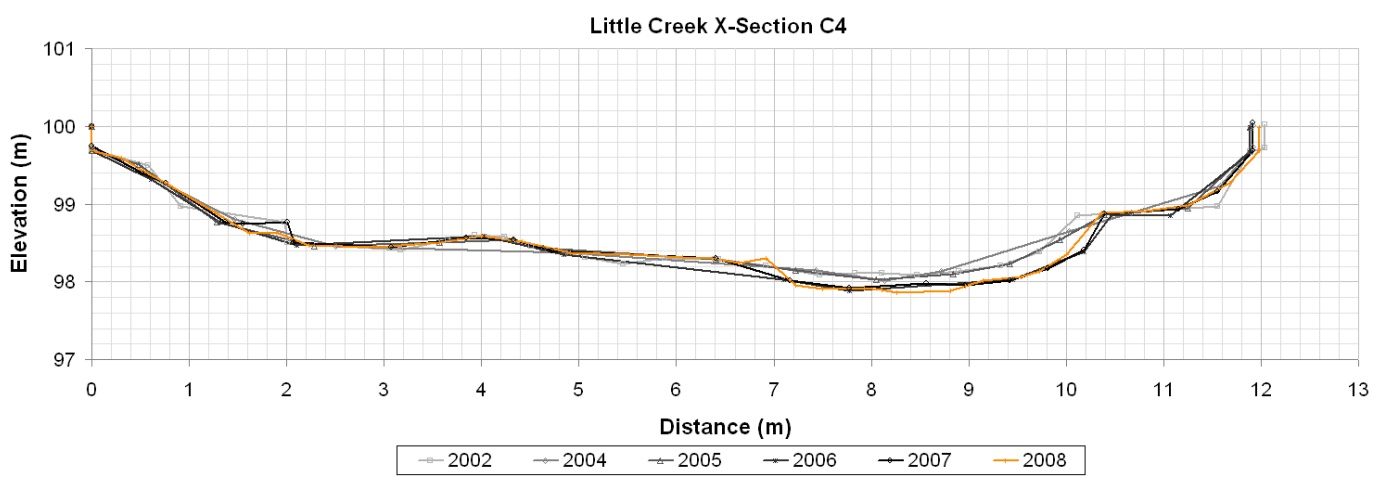

Figure A.24: Little Creek Cross Section C4

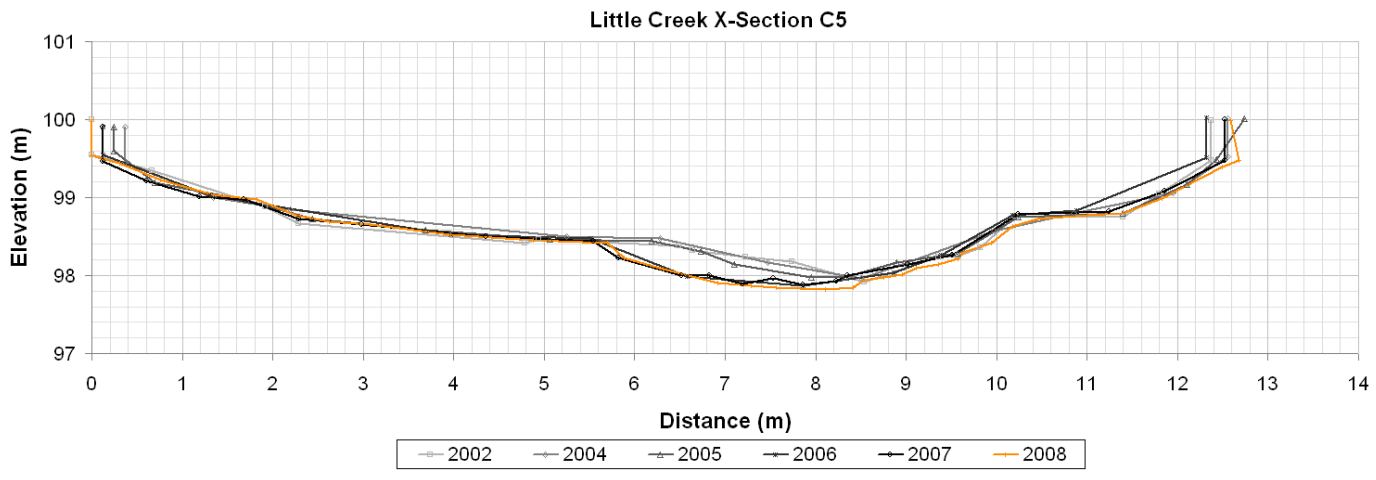

Figure A.25: Little Creek Cross Section C5

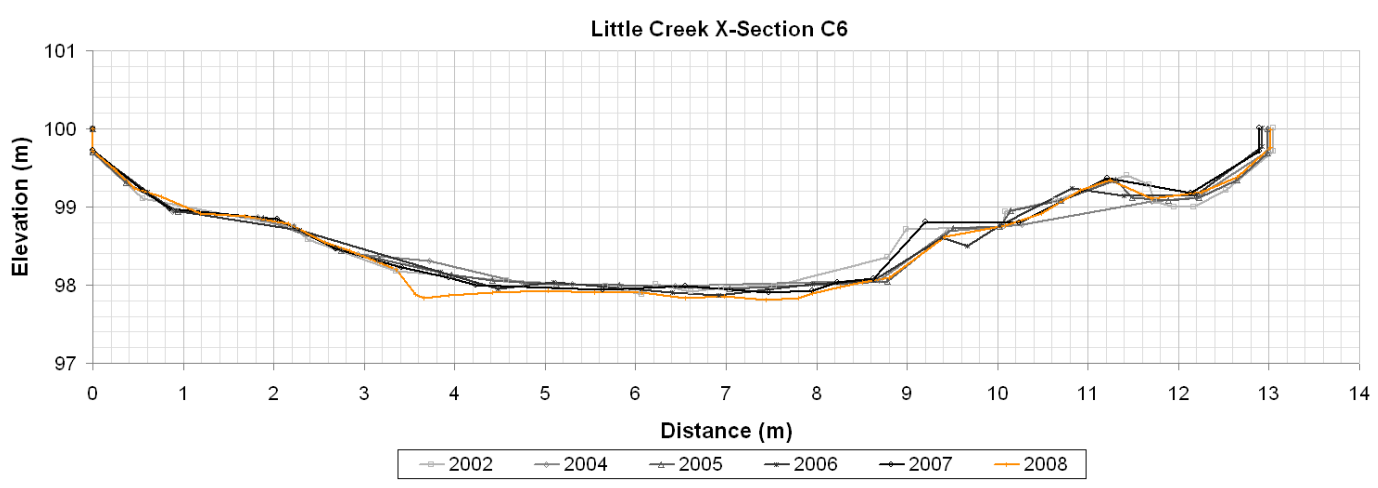

Figure A.26: Little Creek Cross Section C6 


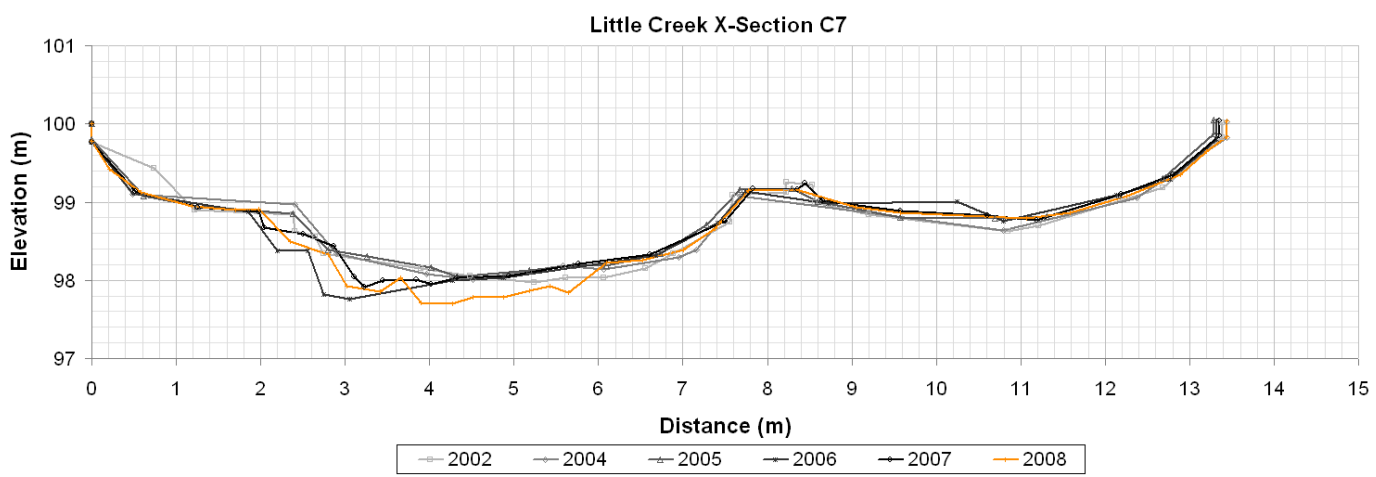

Figure A.27: Little Creek Cross Section C7

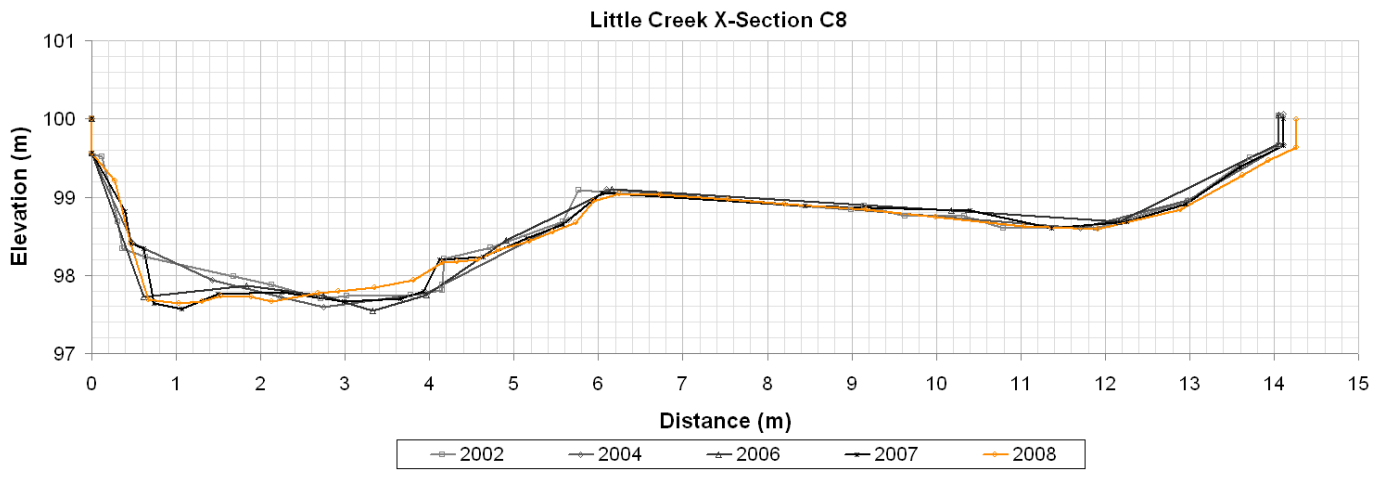

Figure A.28: Little Creek Cross Section C8

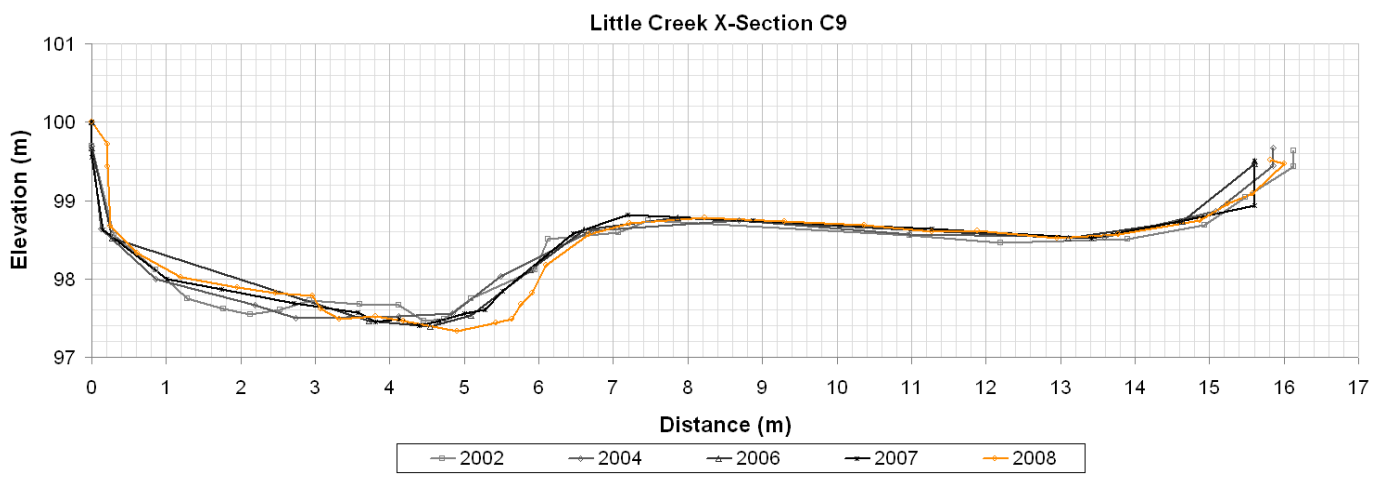

Figure A.29: Little Creek Cross Section C9 


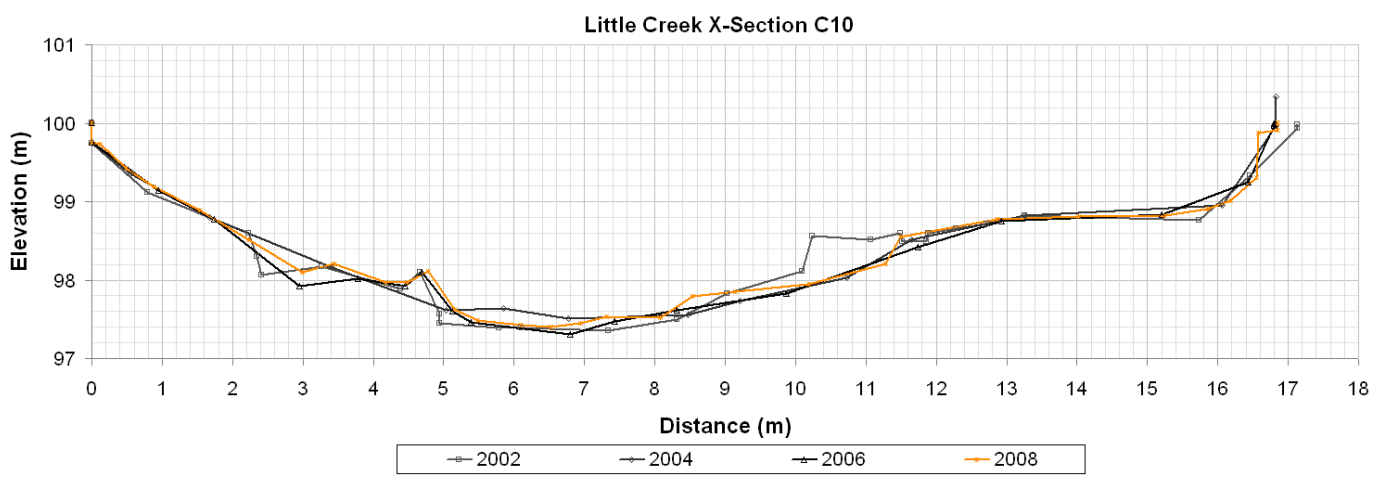

Figure A.30: Little Creek Cross Section C10

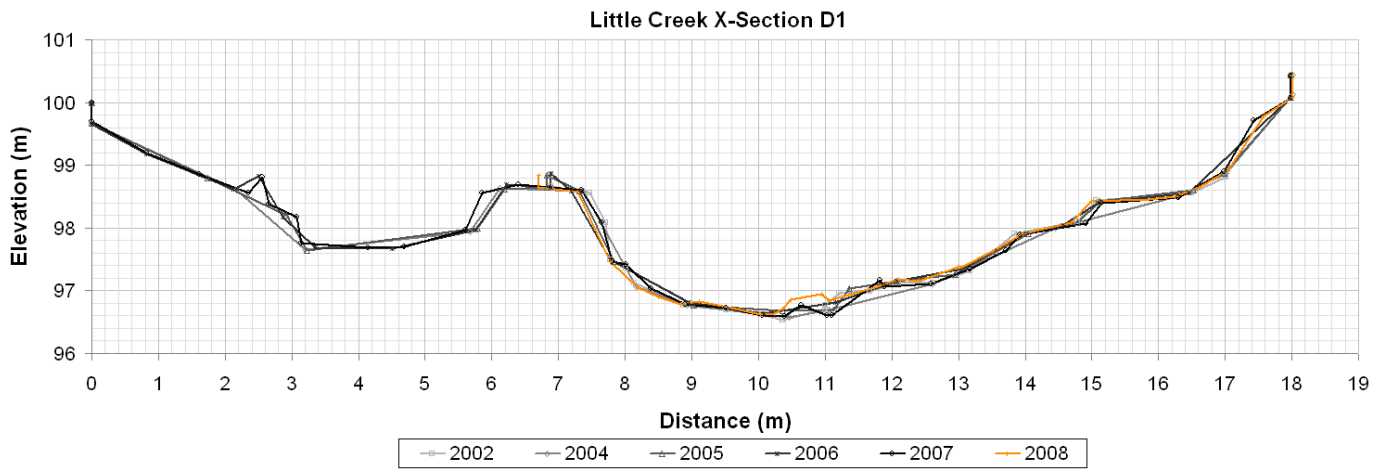

Figure A.31: Little Creek Cross Section D1

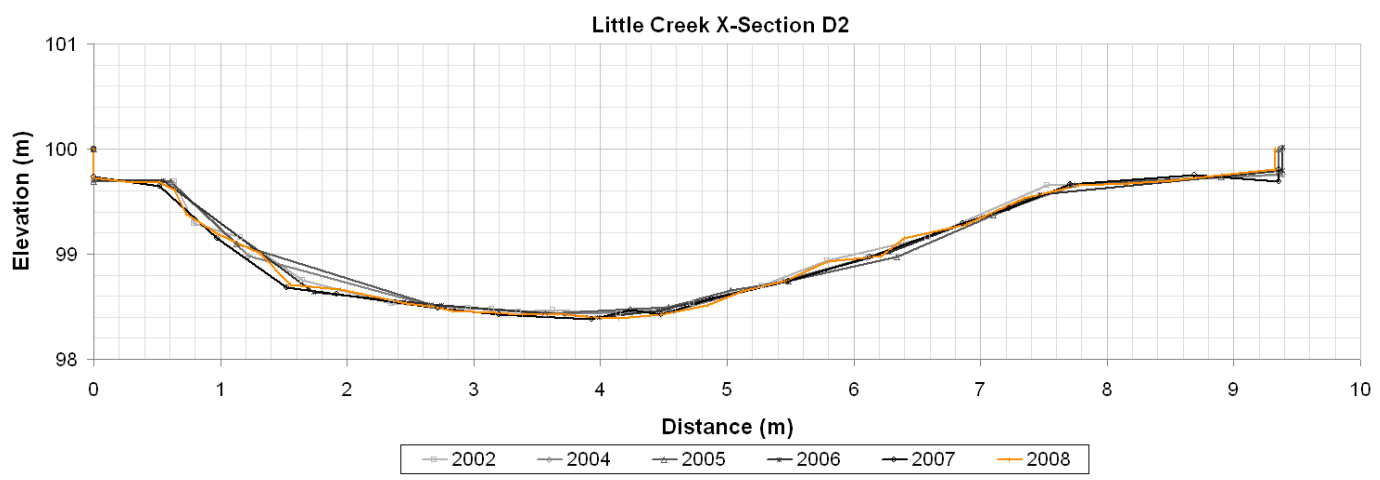

Figure A.32: Little Creek Cross Section D2 


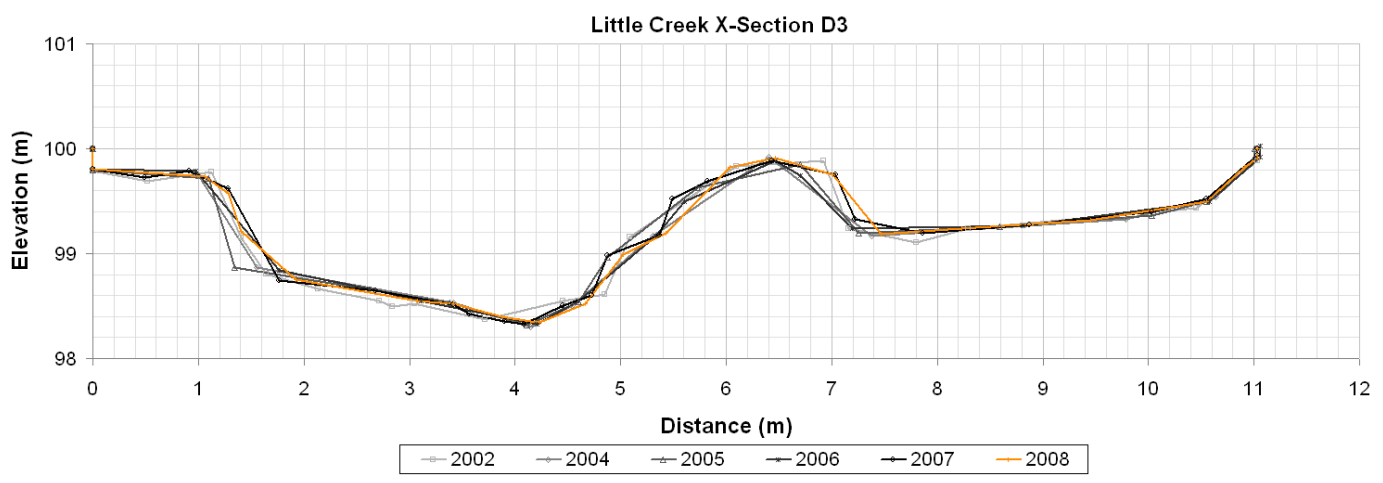

Figure A.33: Little Creek Cross Section D3

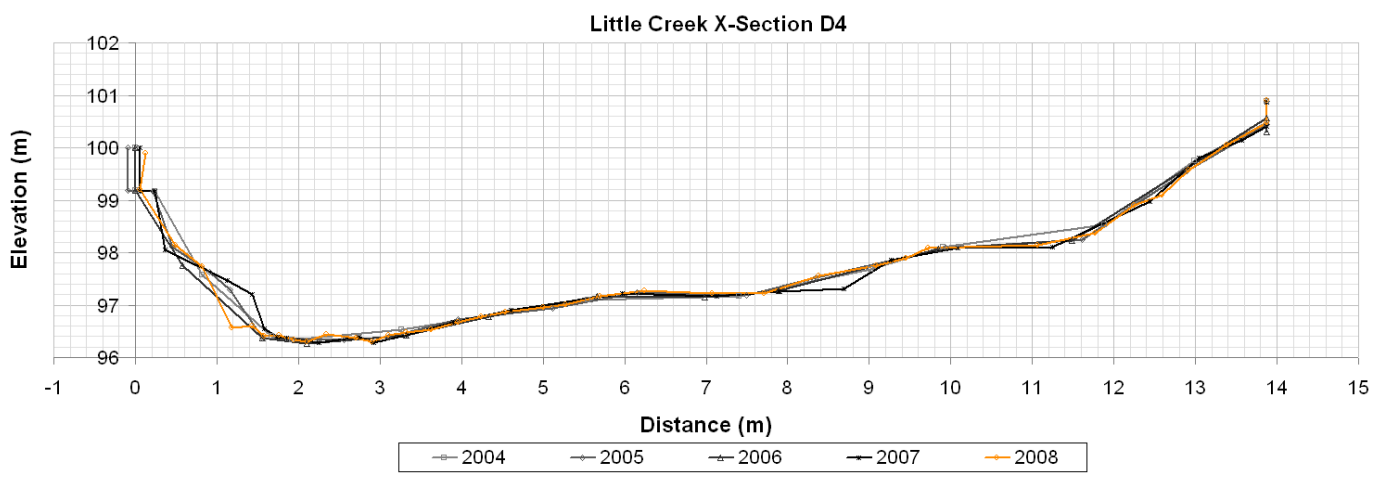

Figure A.34: Little Creek Cross Section D4

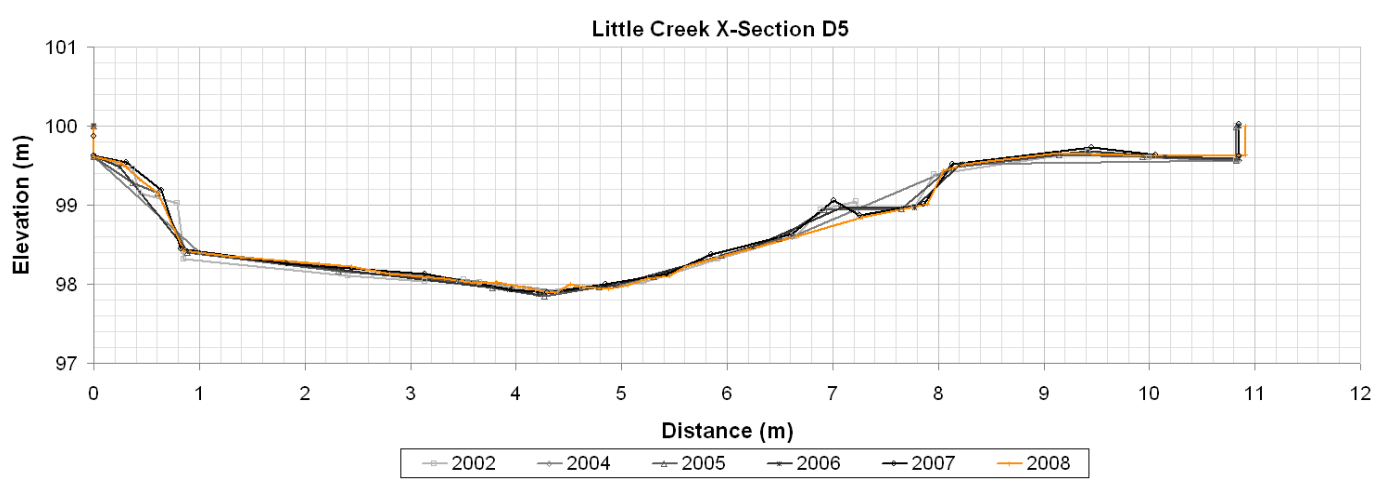

Figure A.35: Little Creek Cross Section D5 


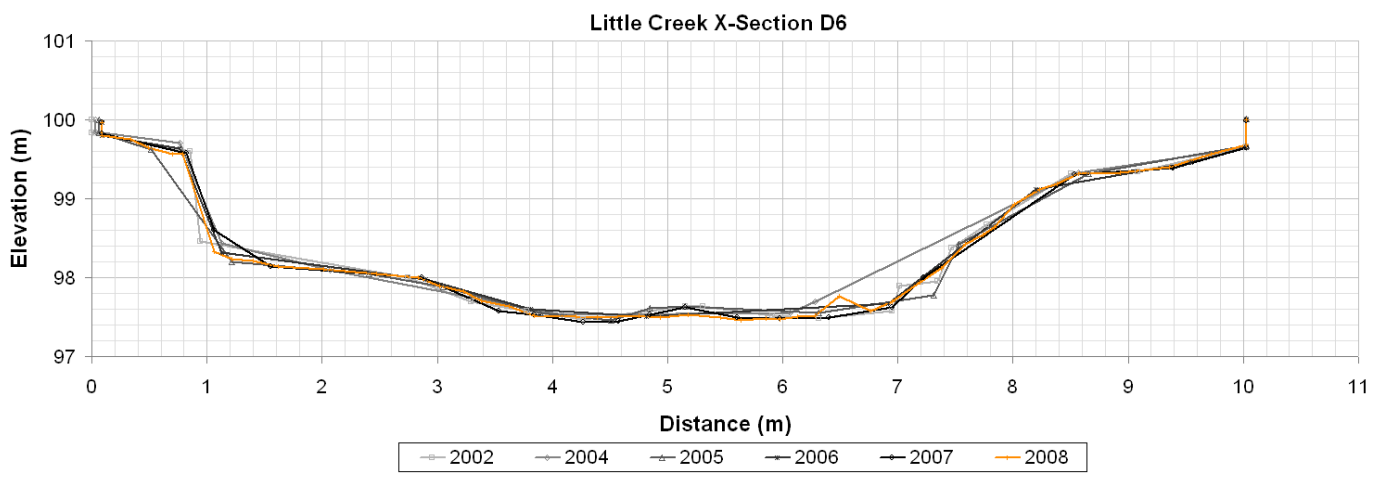

Figure A.36: Little Creek Cross Section D6

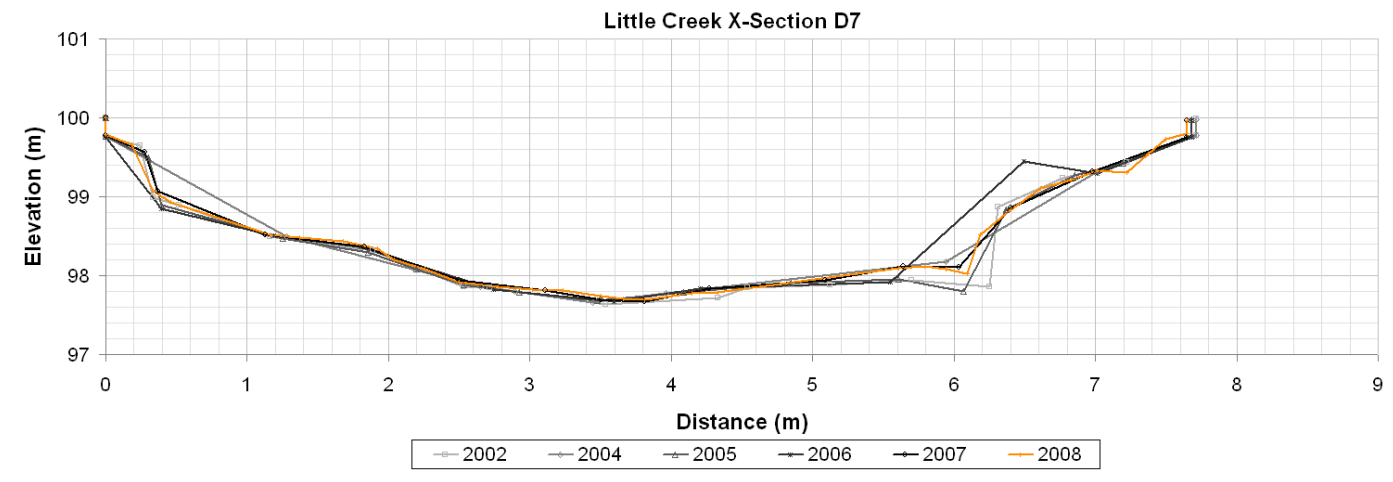

Figure A.37: Little Creek Cross Section D7

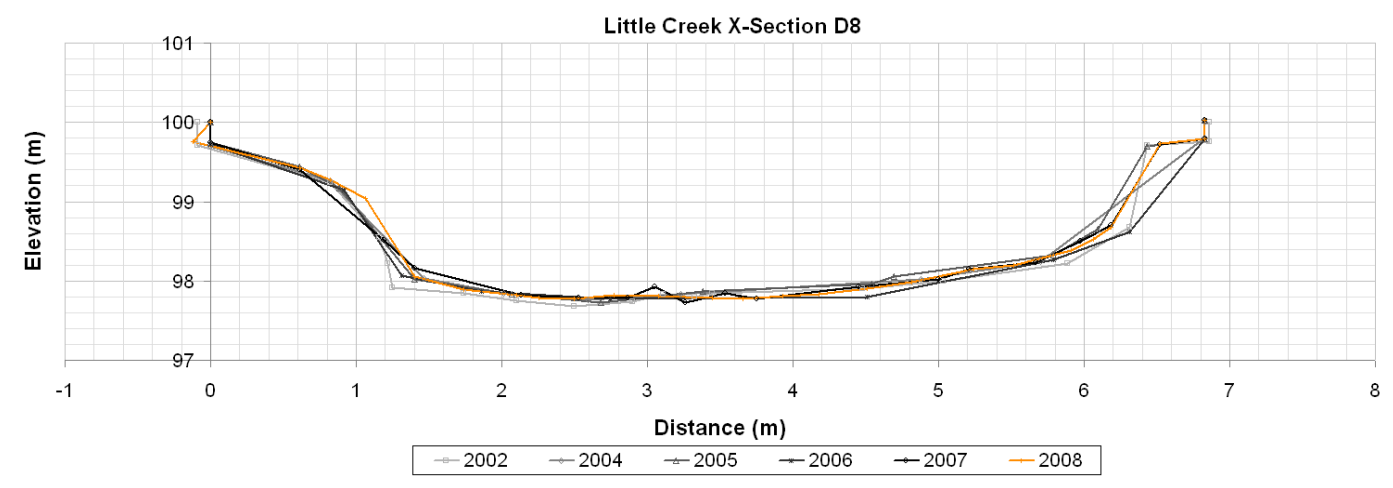

Figure A.38: Little Creek Cross Section D8 


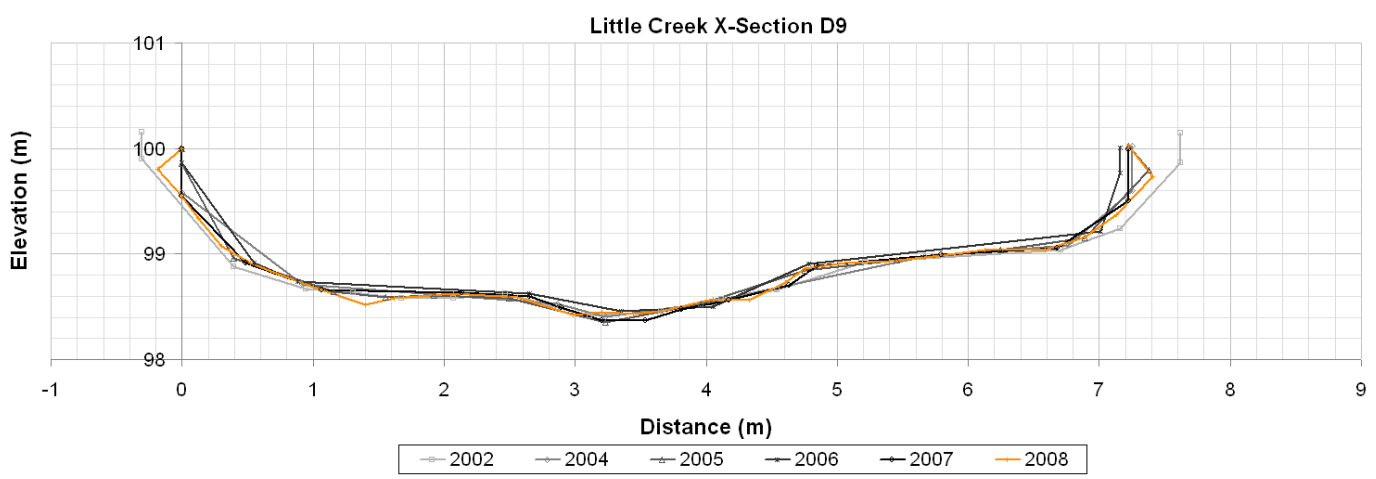

Figure A.39: Little Creek Cross Section D9

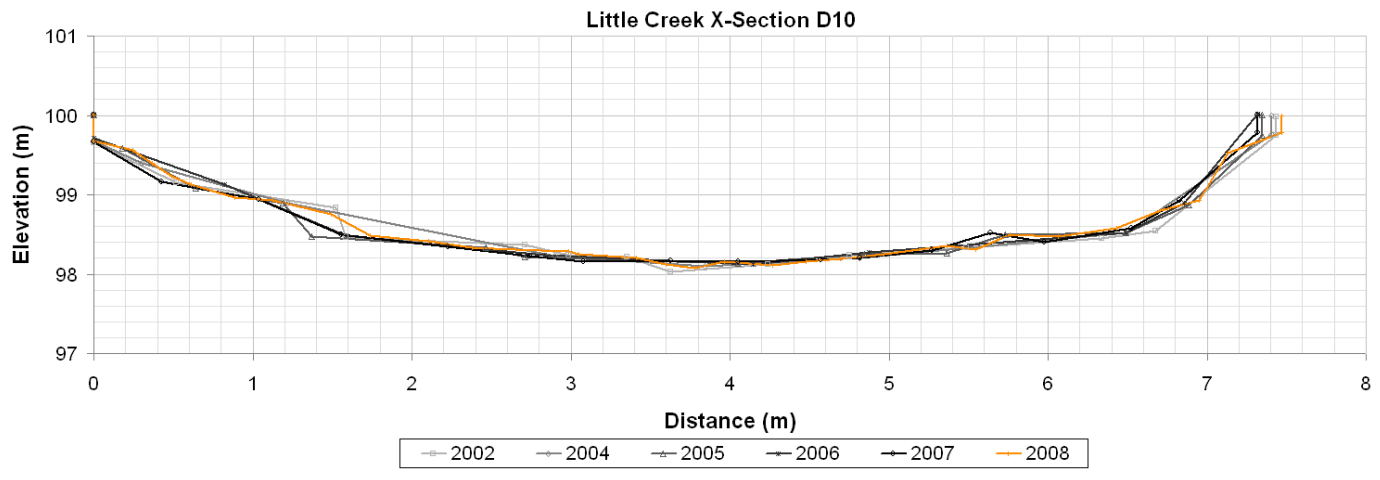

Figure A.40: Little Creek Cross Section D10

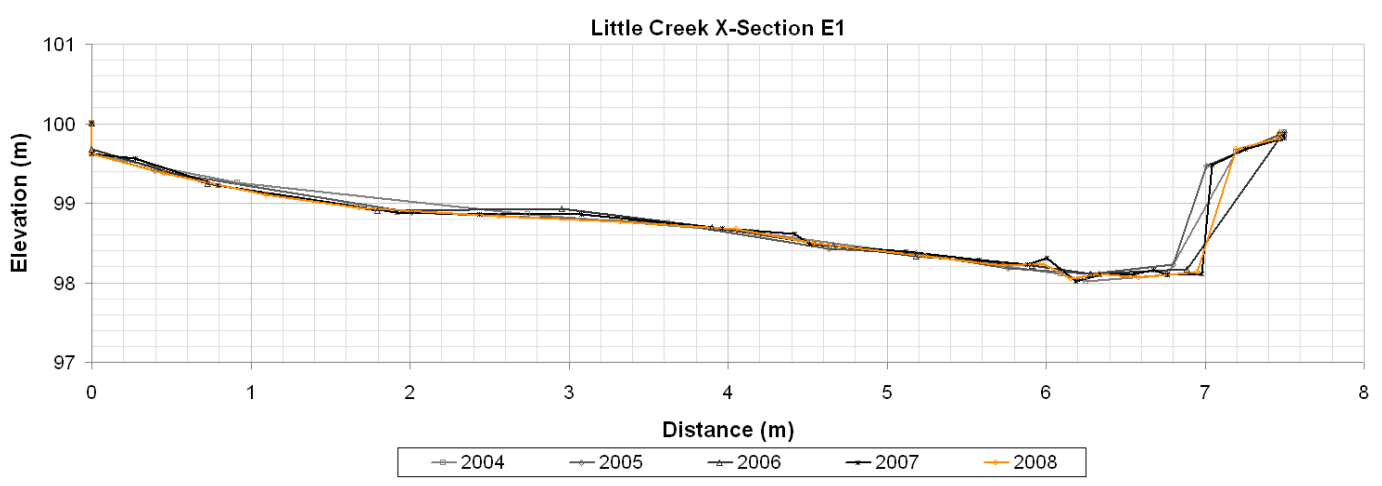

Figure A.41: Little Creek Cross Section E1 


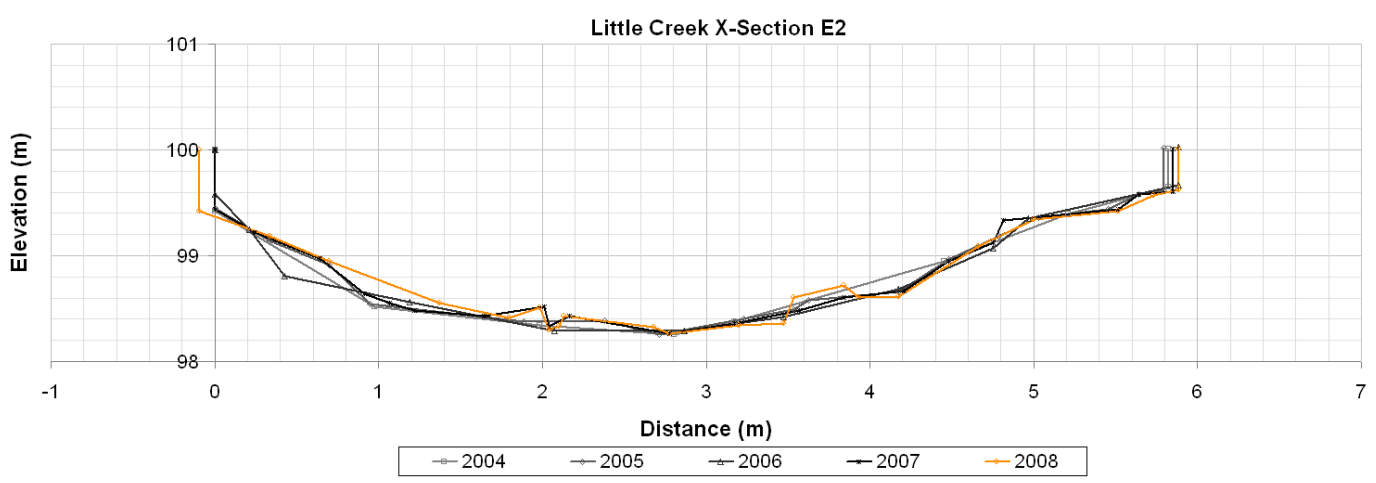

Figure A.42: Little Creek Cross Section E2

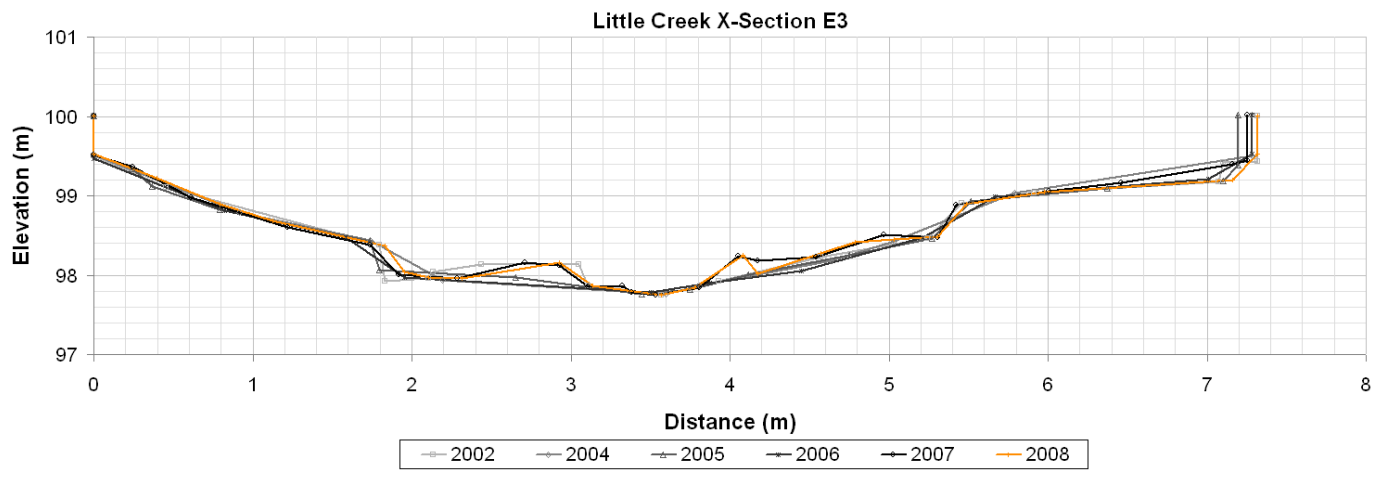

Figure A.43: Little Creek Cross Section E3

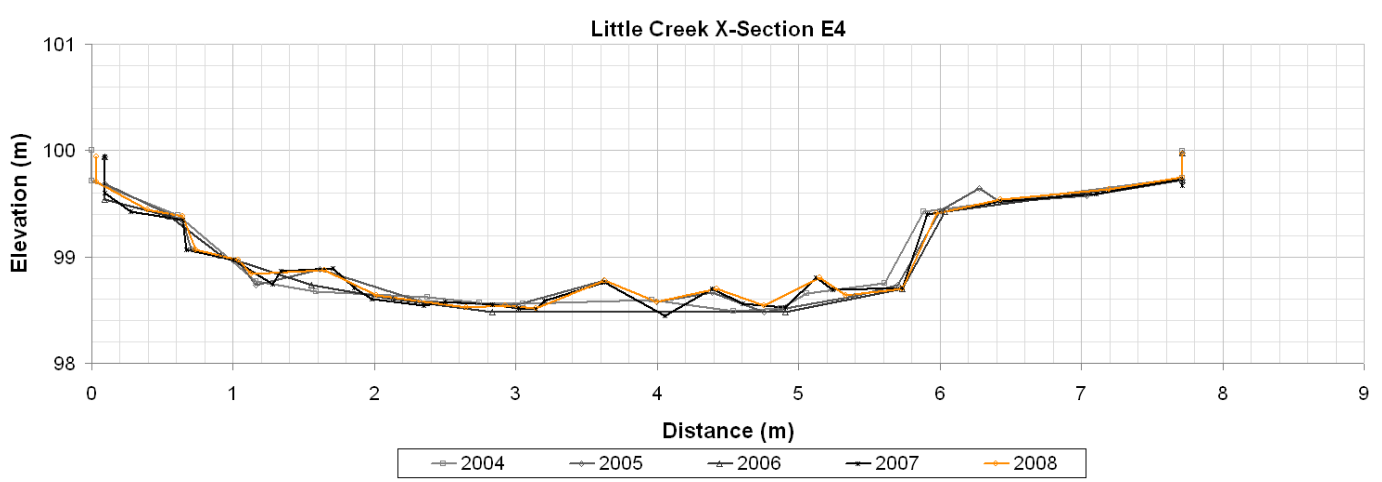

Figure A.44: Little Creek Cross Section E4 


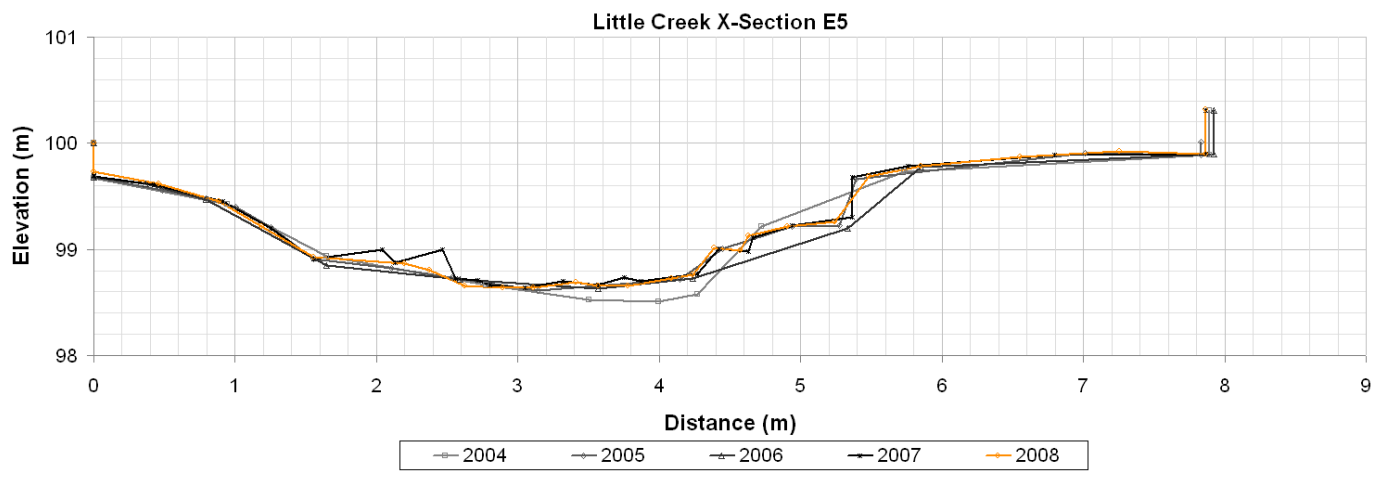

Figure A.45: Little Creek Cross Section E5

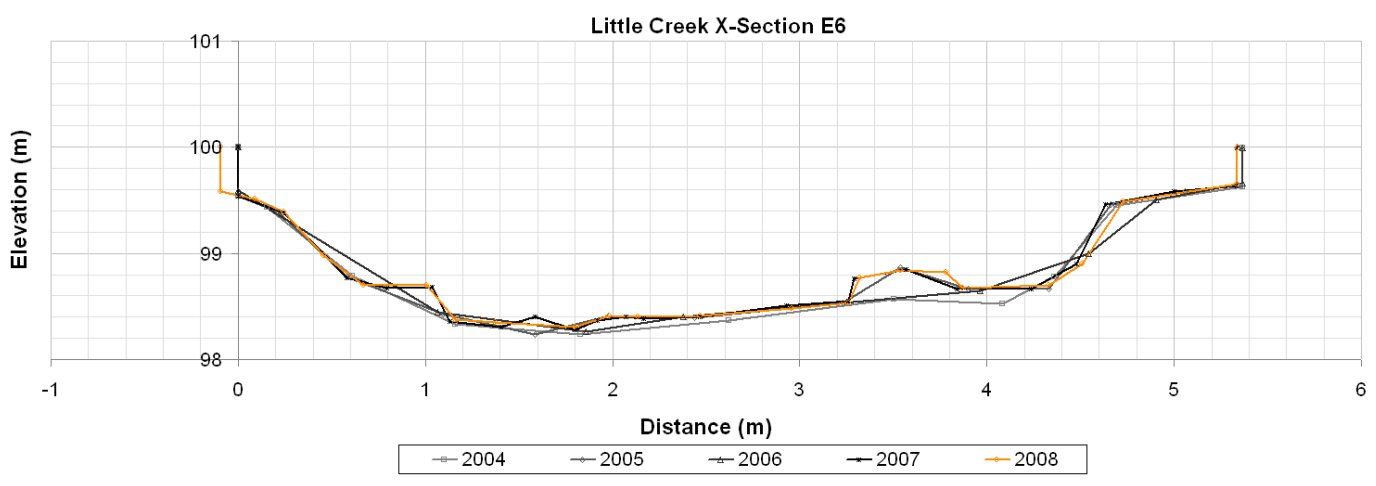

Figure A.46: Little Creek Cross Section E6

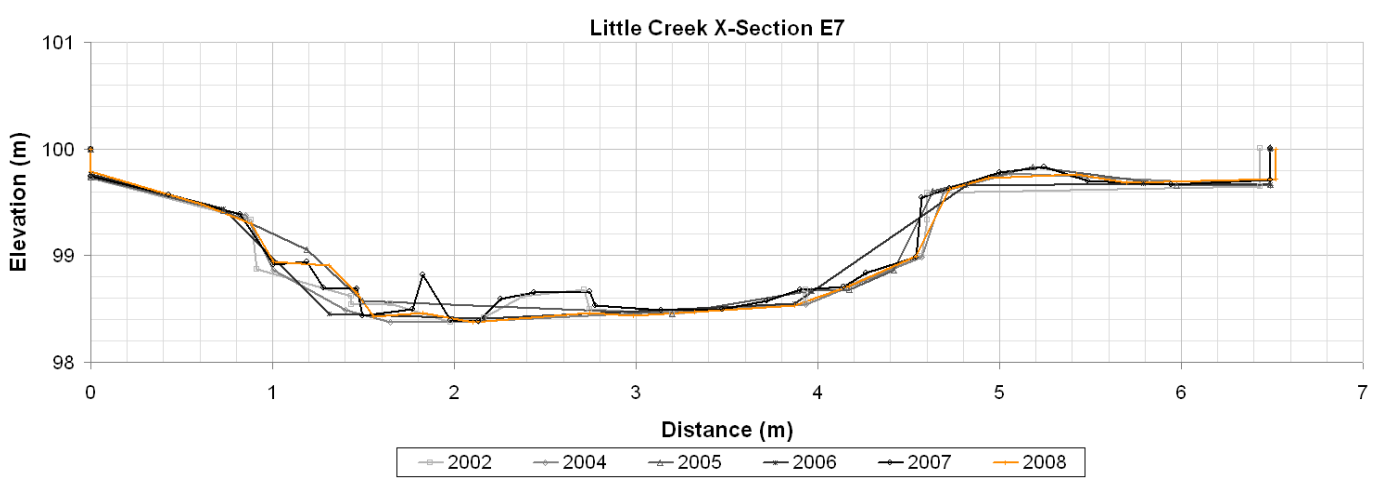

Figure A.47: Little Creek Cross Section E7 


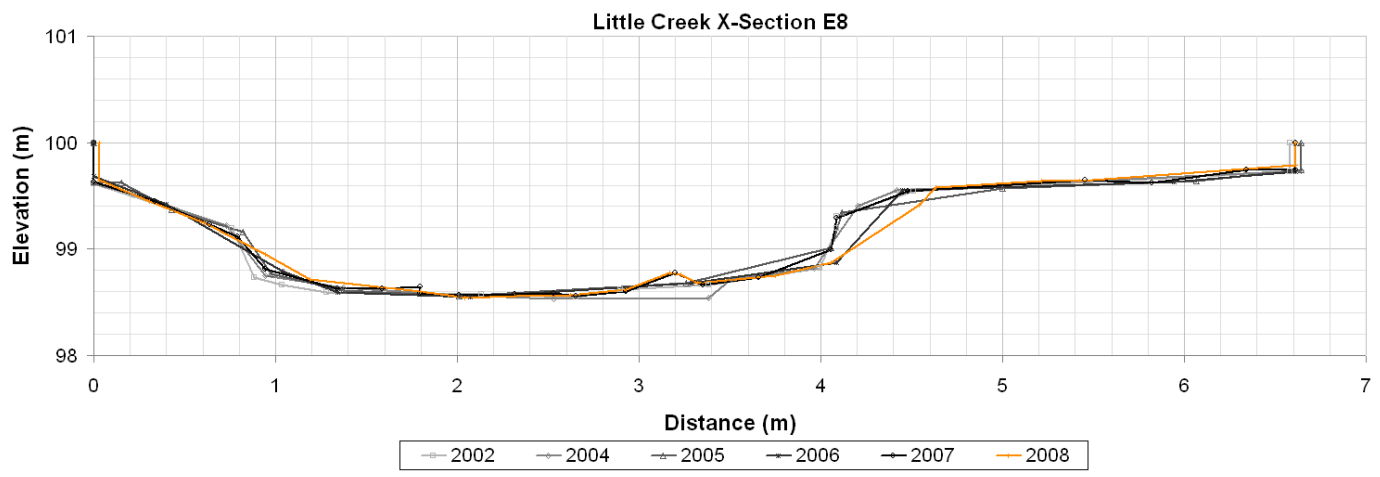

Figure A.48: Little Creek Cross Section E8

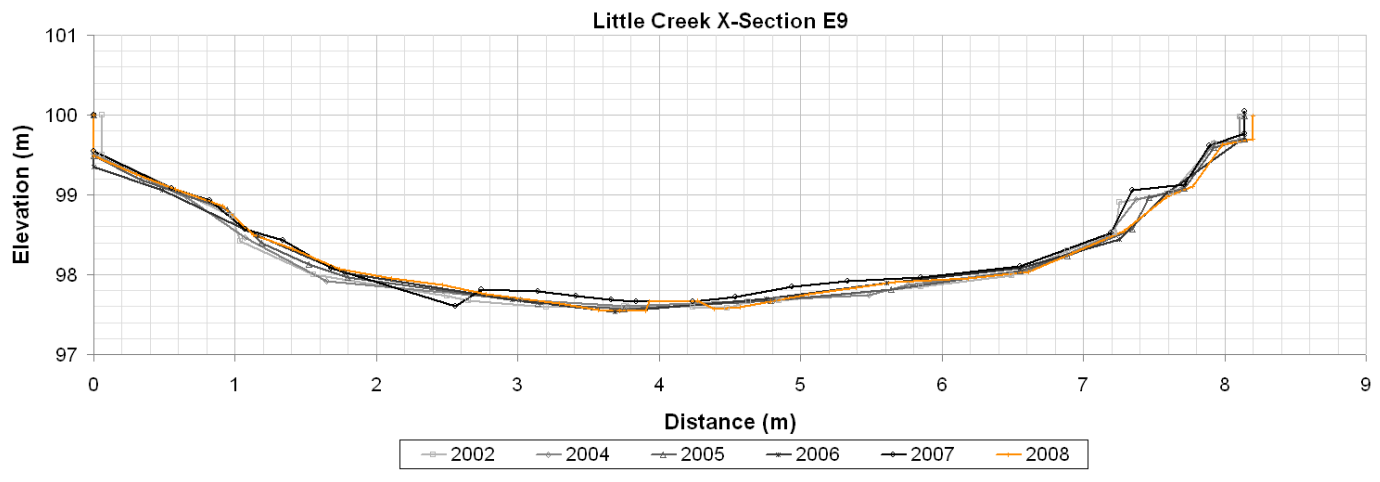

Figure A.49: Little Creek Cross Section E9

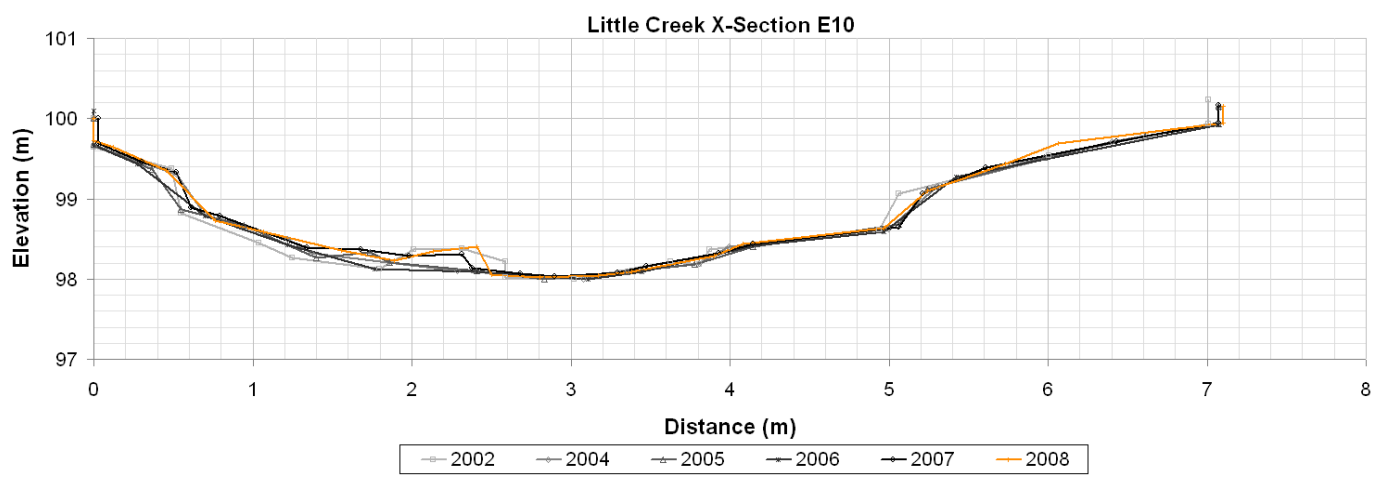

Figure A.50: Little Creek Cross Section E10 


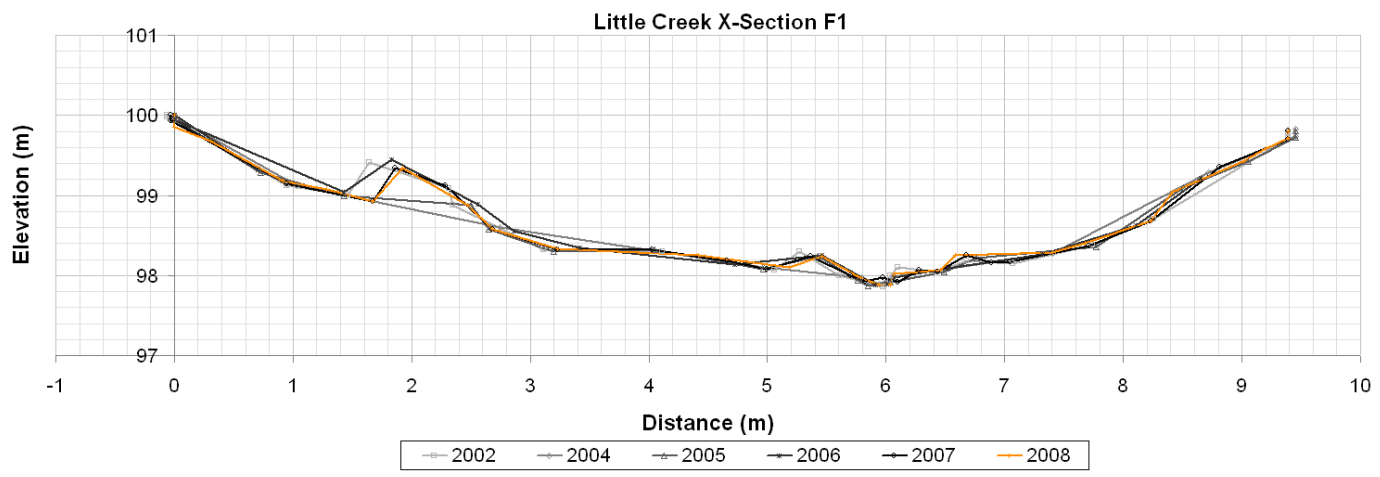

Figure A.51: Little Creek Cross Section F1

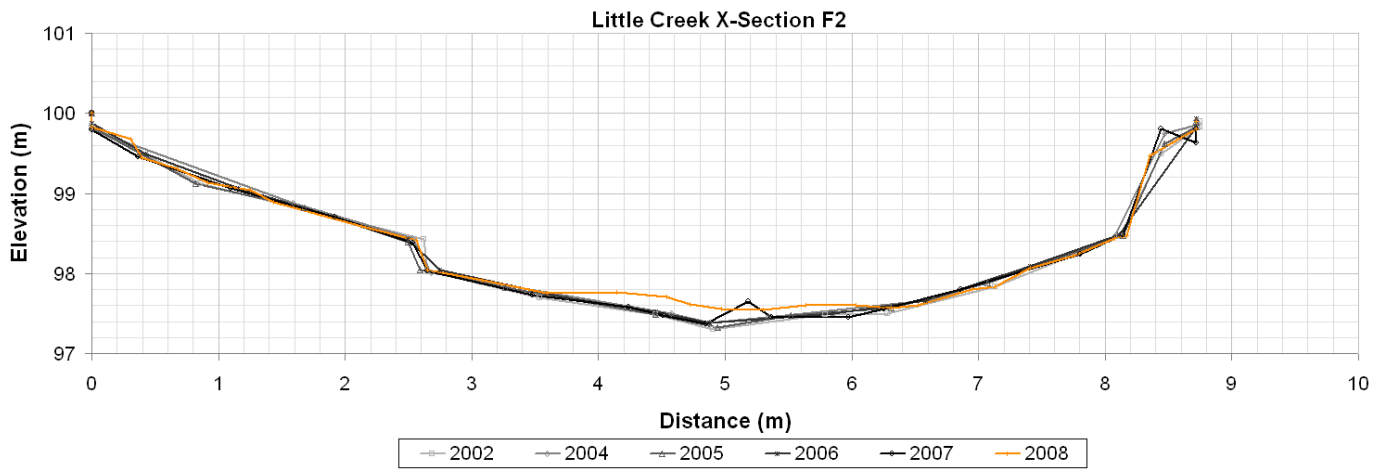

Figure A.52: Little Creek Cross Section F2

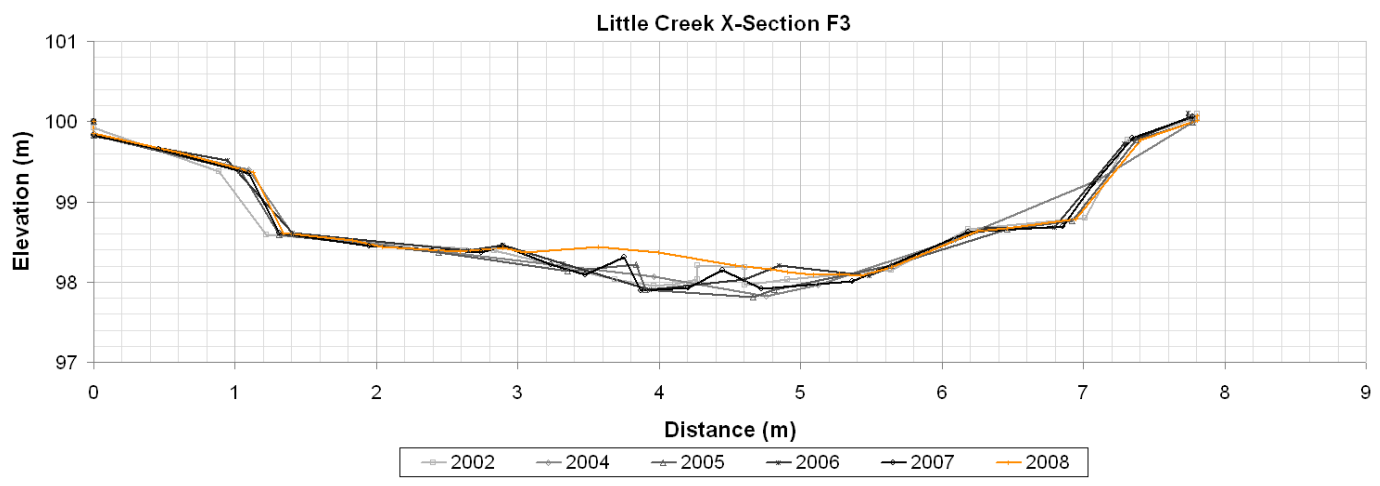

Figure A.53: Little Creek Cross Section F3 


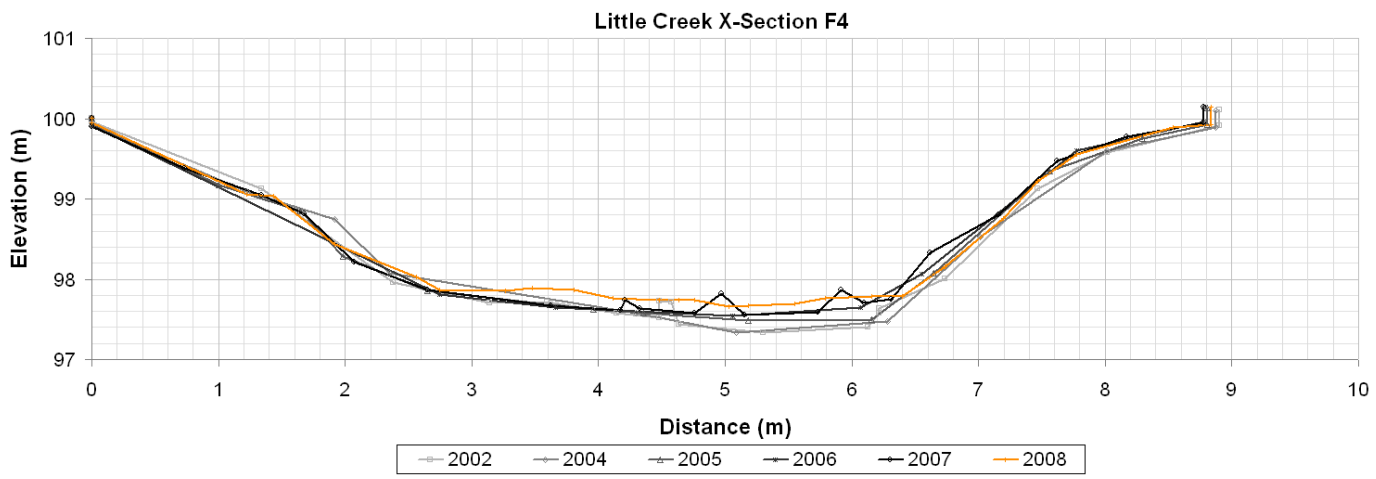

Figure A.54: Little Creek Cross Section F4

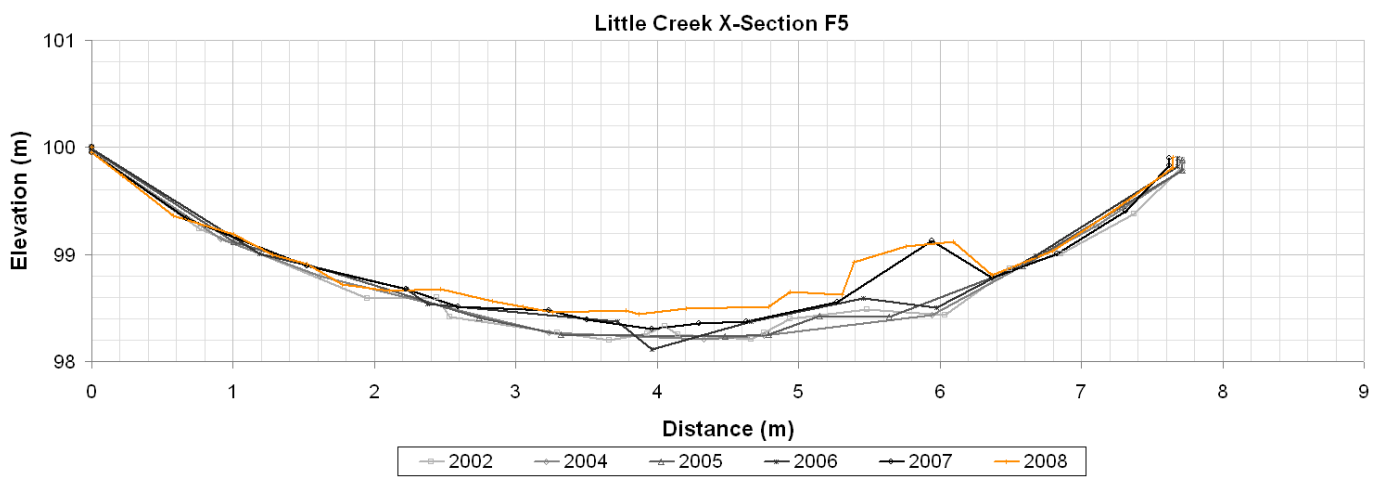

Figure A.55: Little Creek Cross Section F5

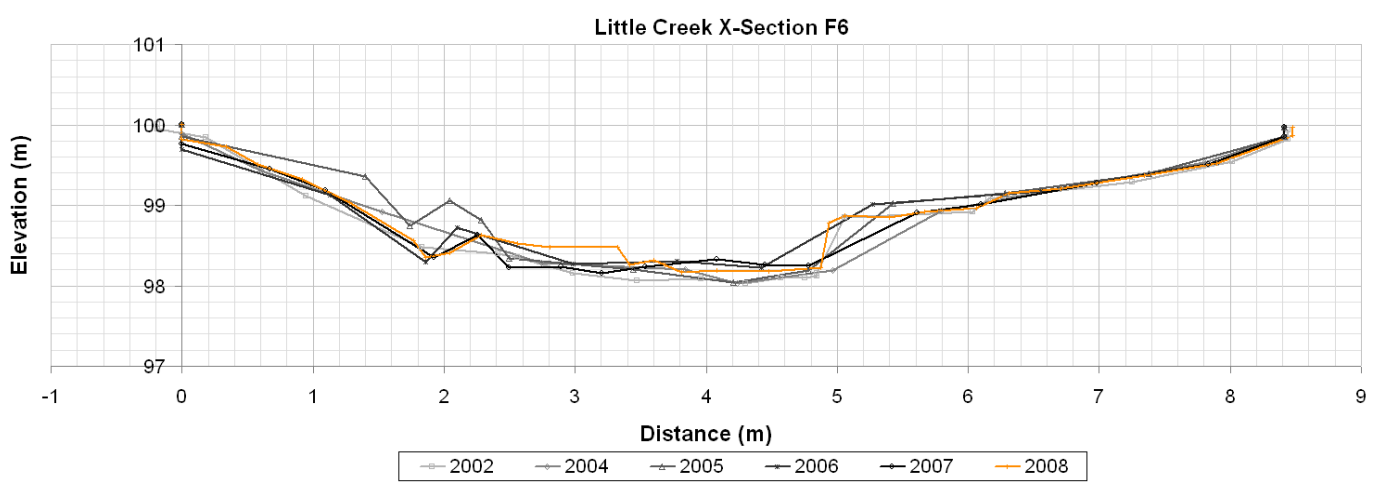

Figure A.56: Little Creek Cross Section F6 


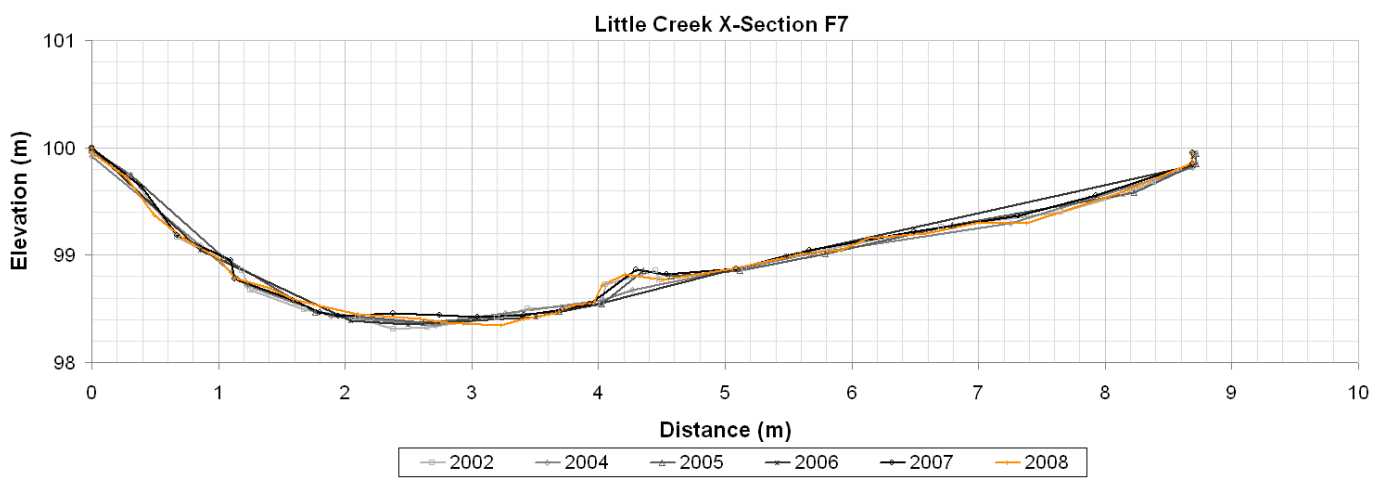

Figure A.57: Little Creek Cross Section F7

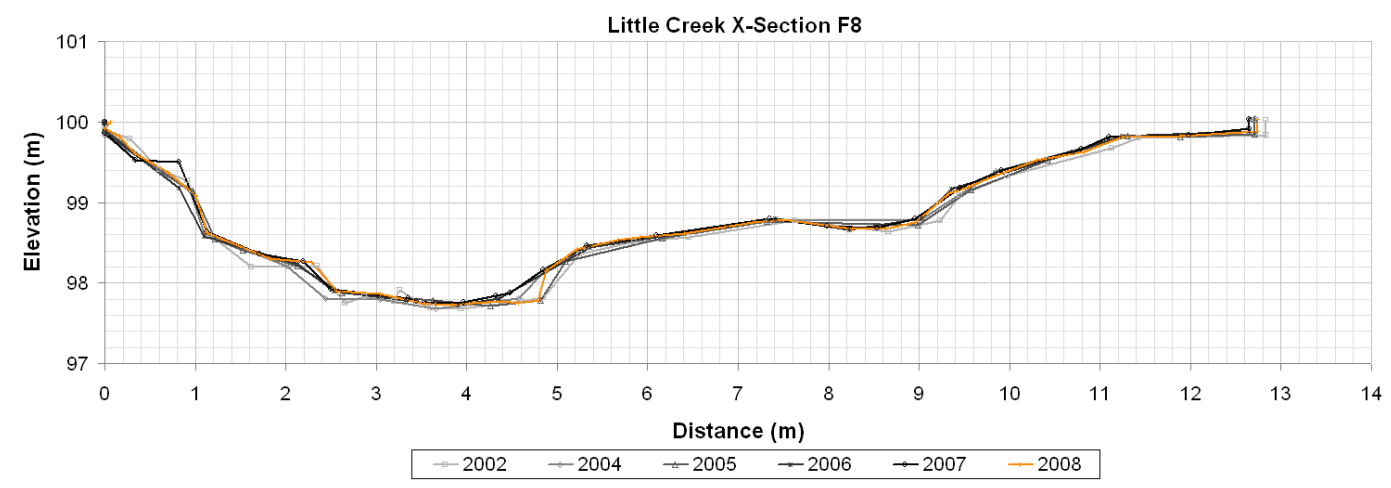

Figure A.58: Little Creek Cross Section F8

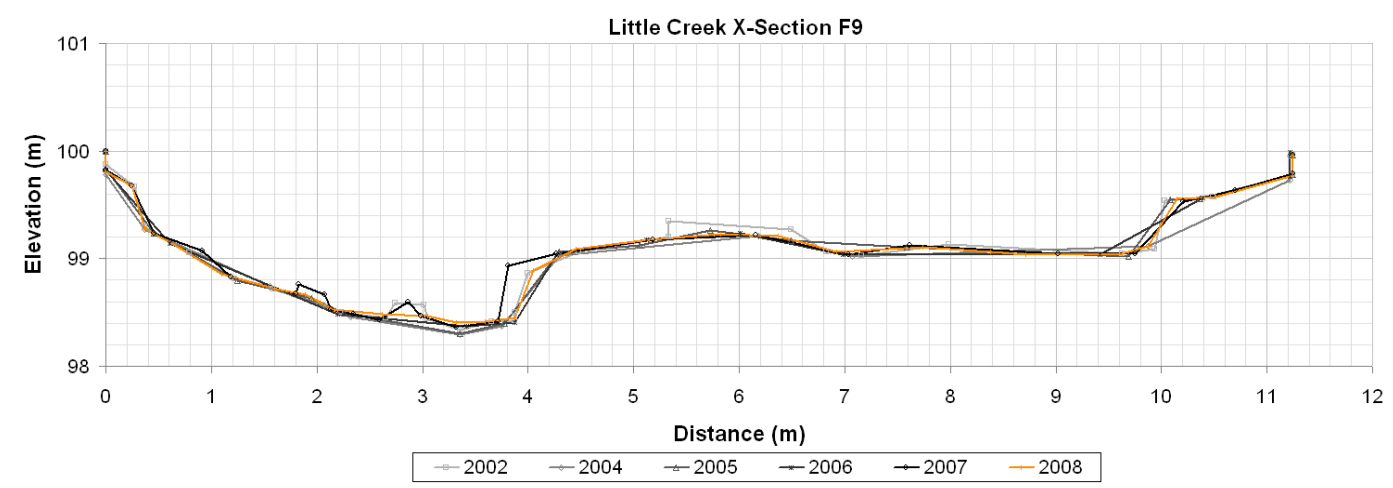

Figure A.59: Little Creek Cross Section F9 


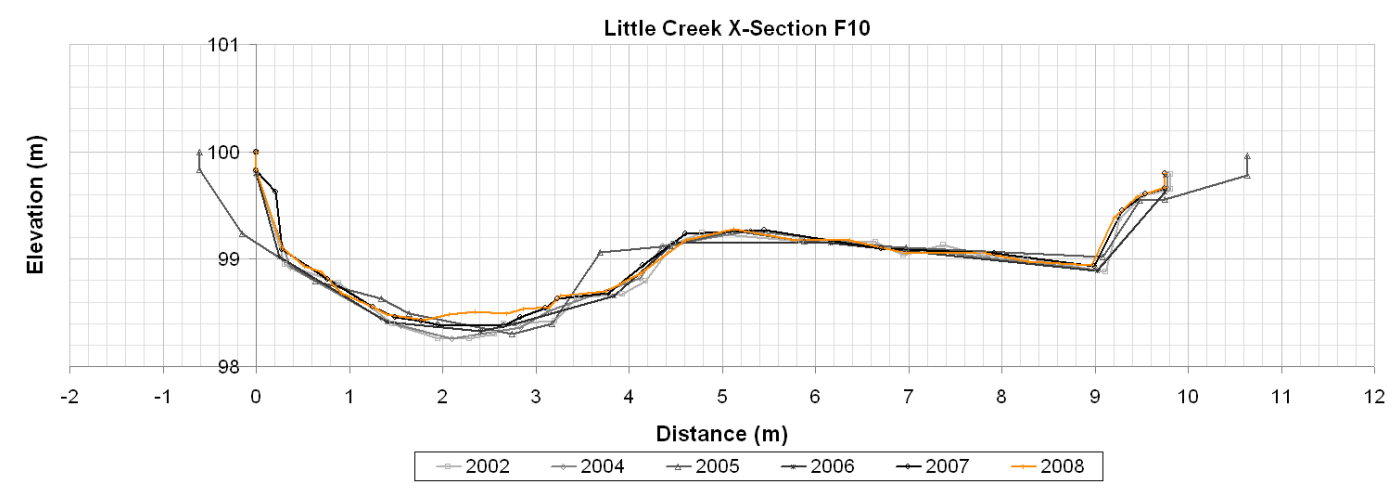

Figure A.60: Little Creek Cross Section F10 
Appendix B

Graphs of All Longitudinal

\section{Profiles}




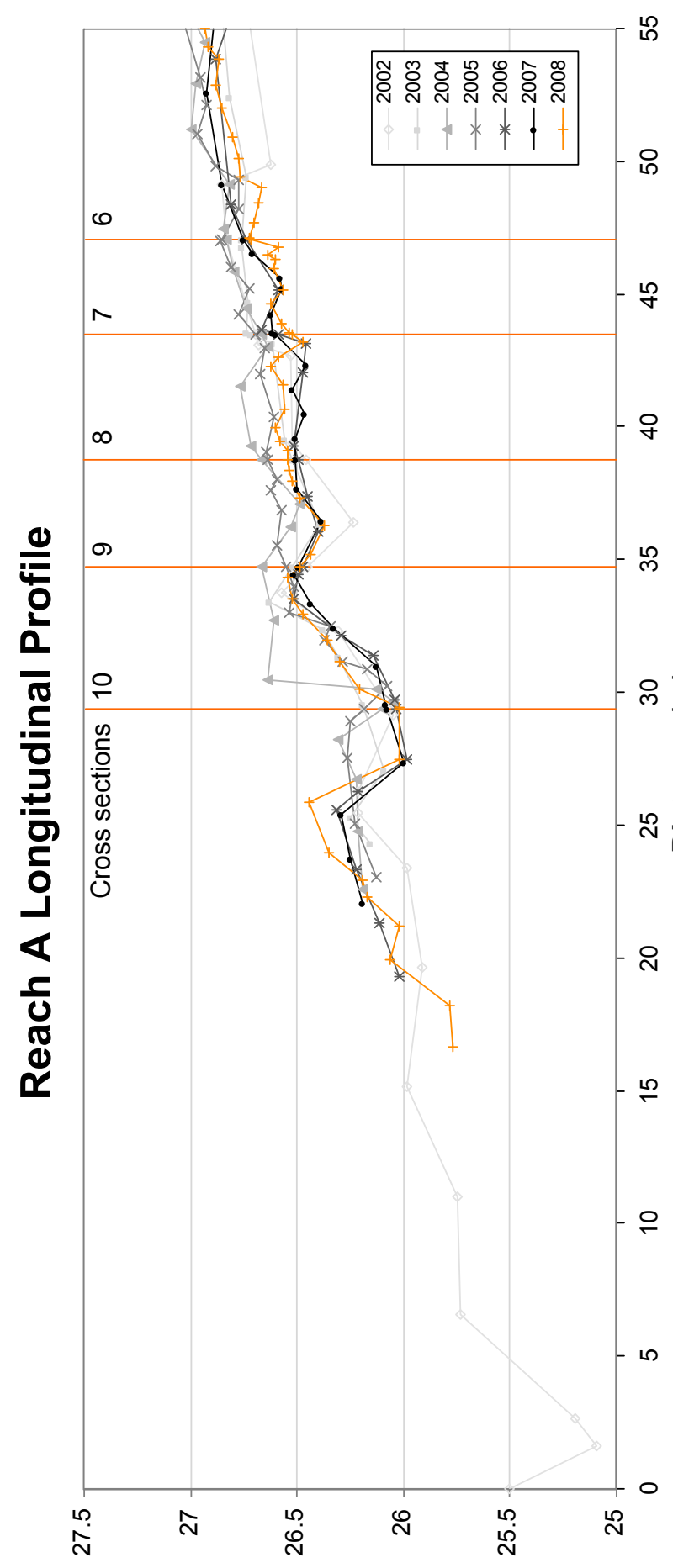

(u) ио!ฺе^әㅋ

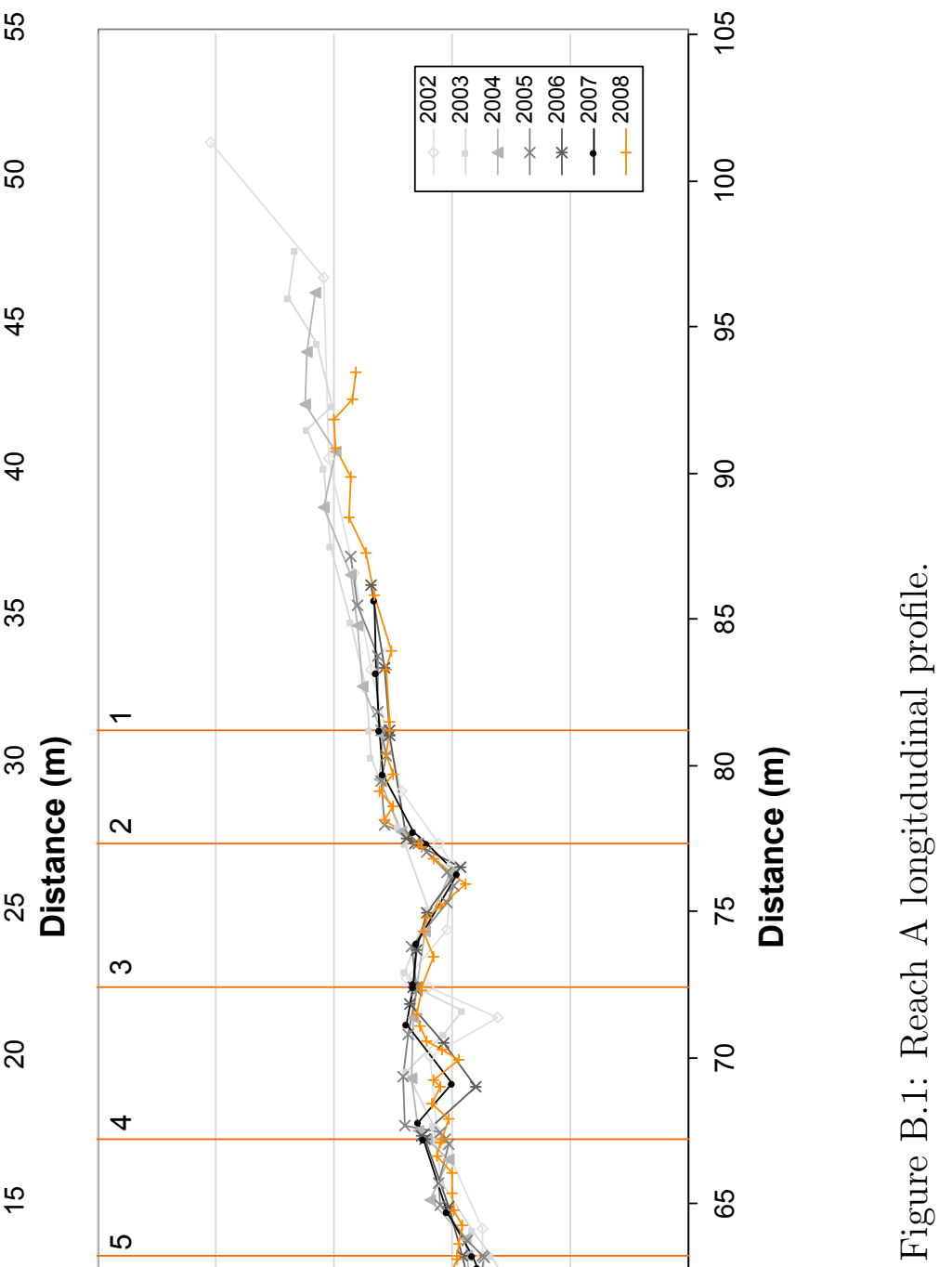




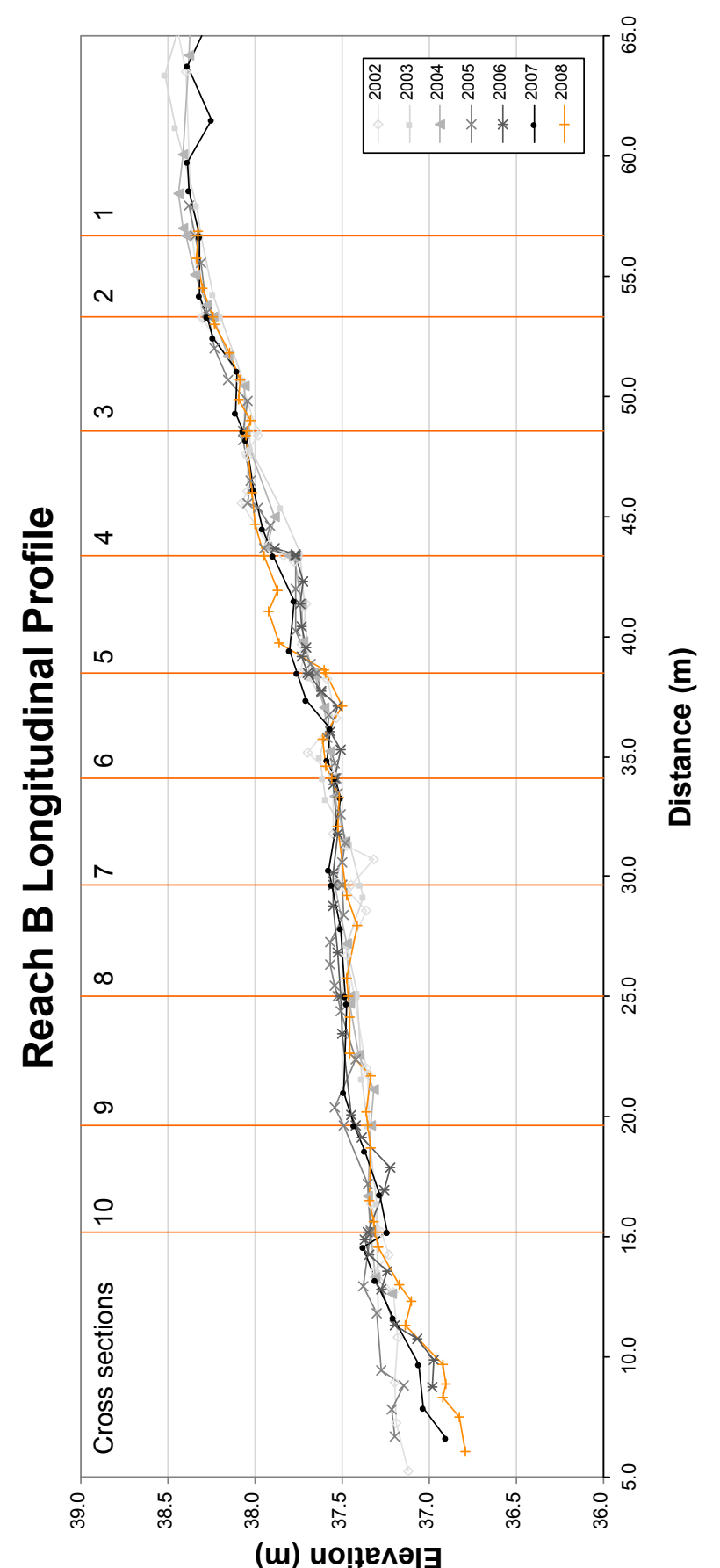

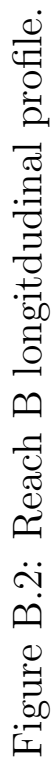




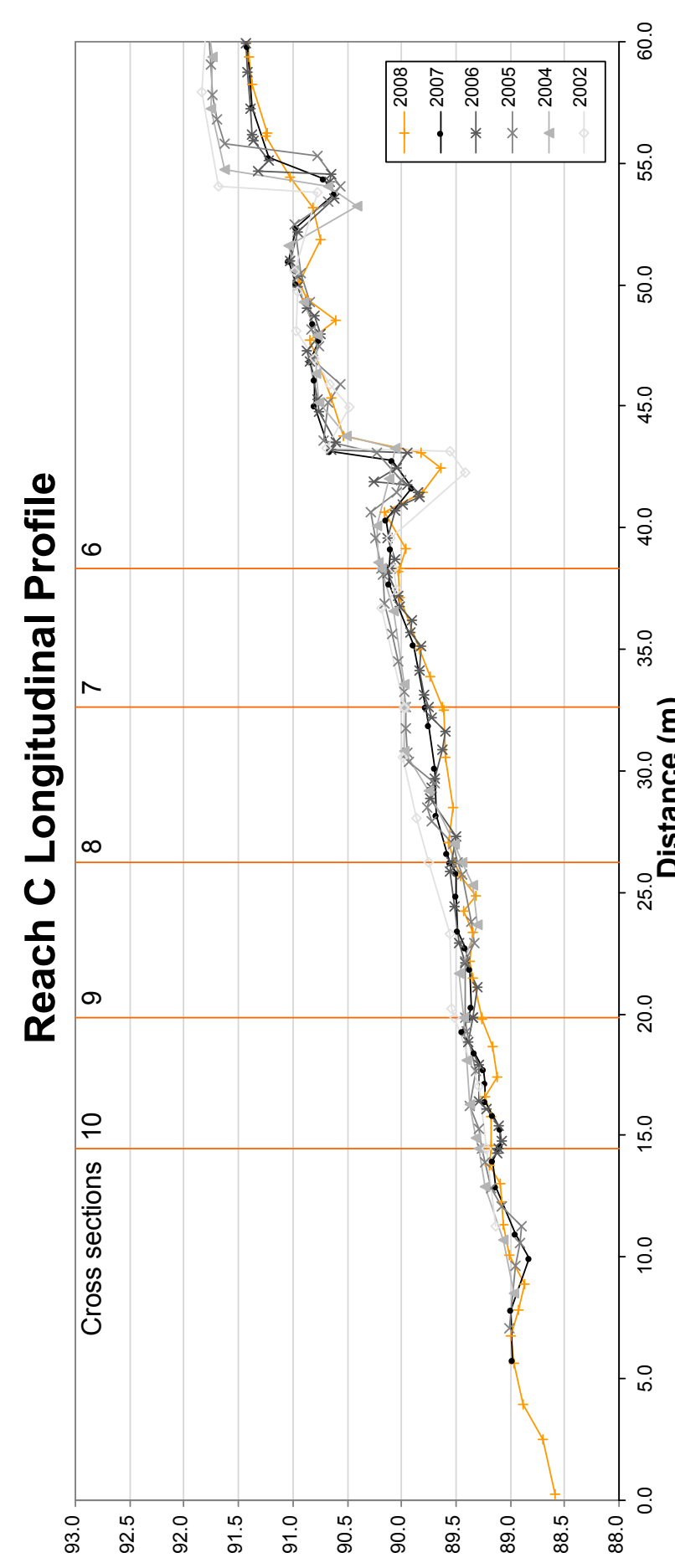

(w) ио!ฺе^әэᄏ

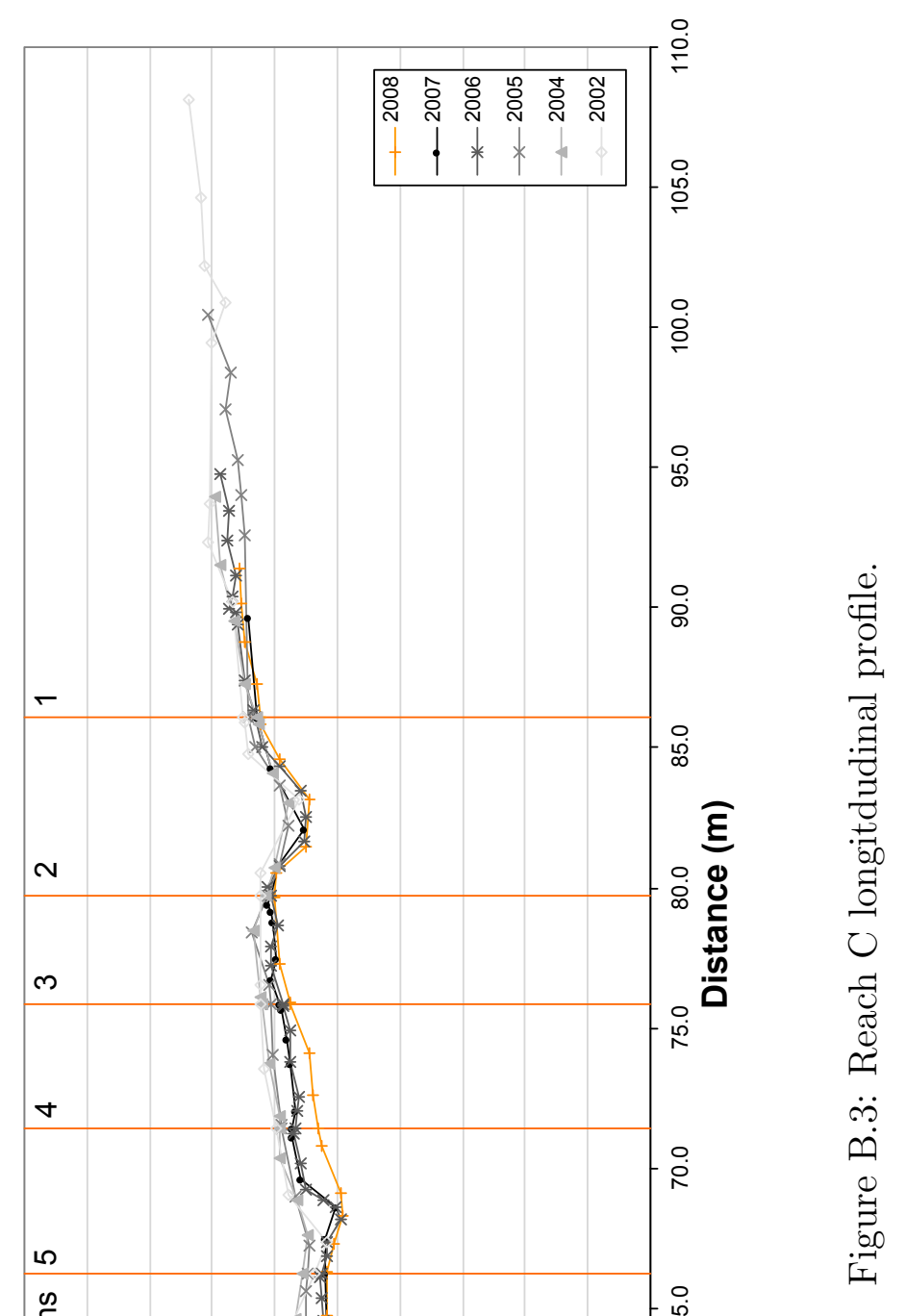




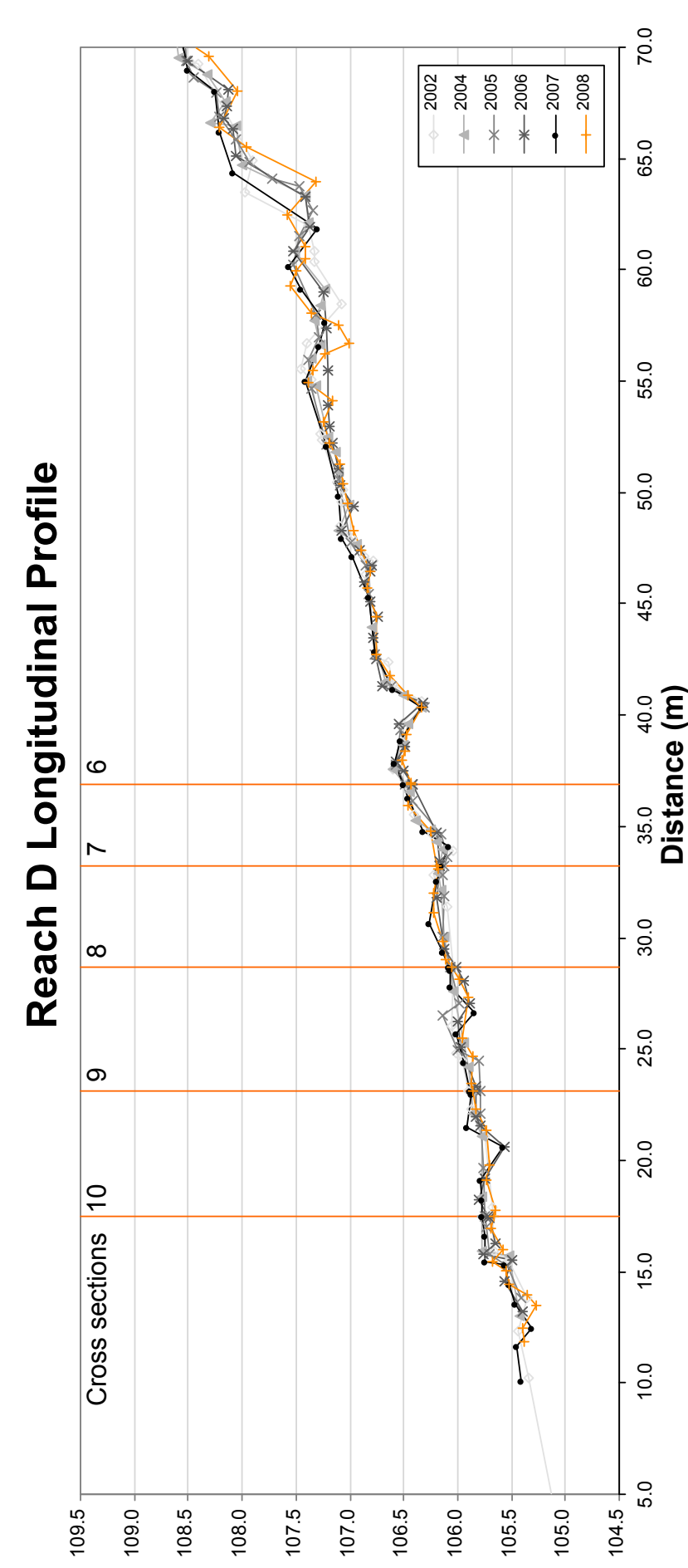

(ui) บо!ฺел키

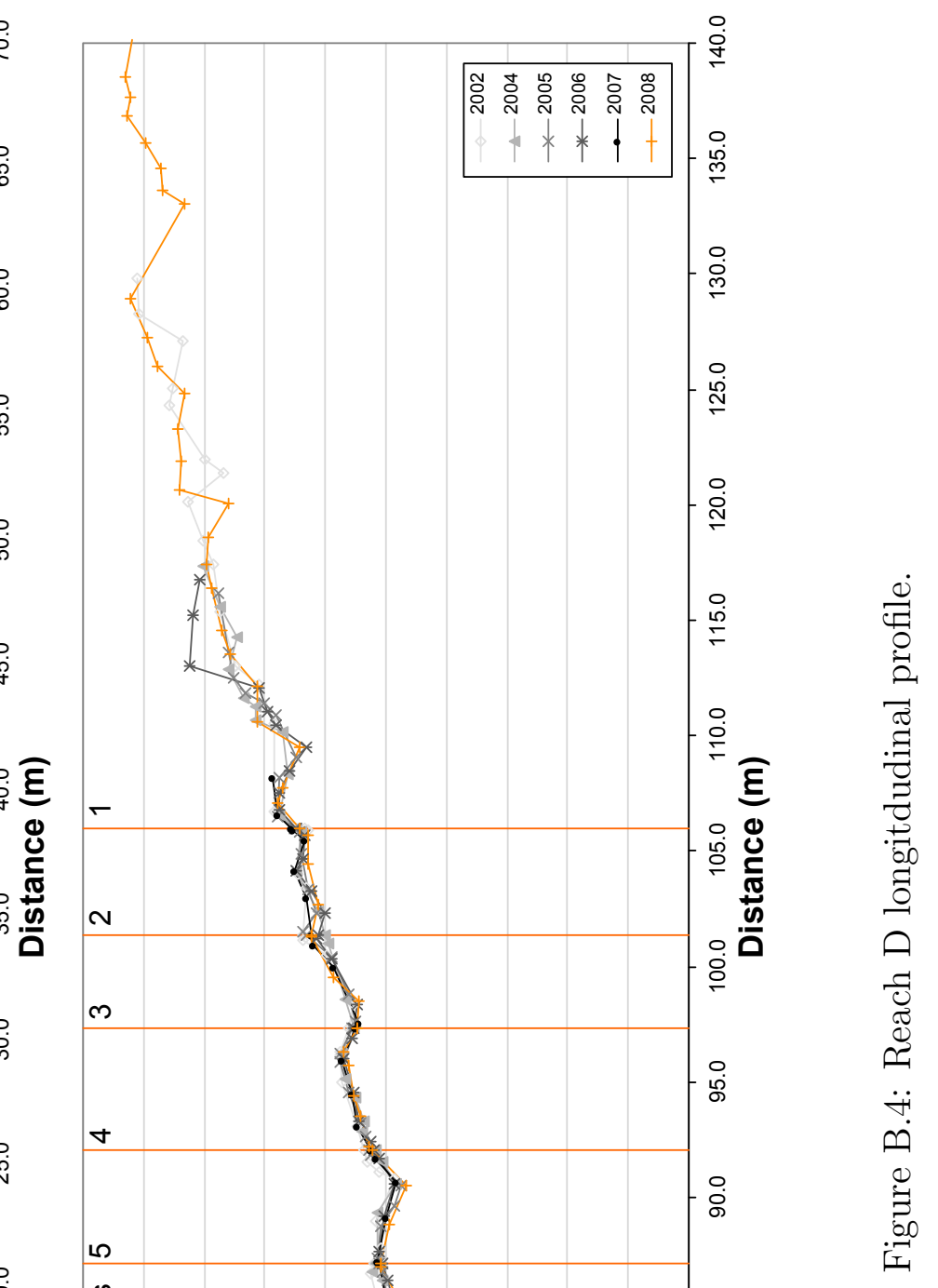




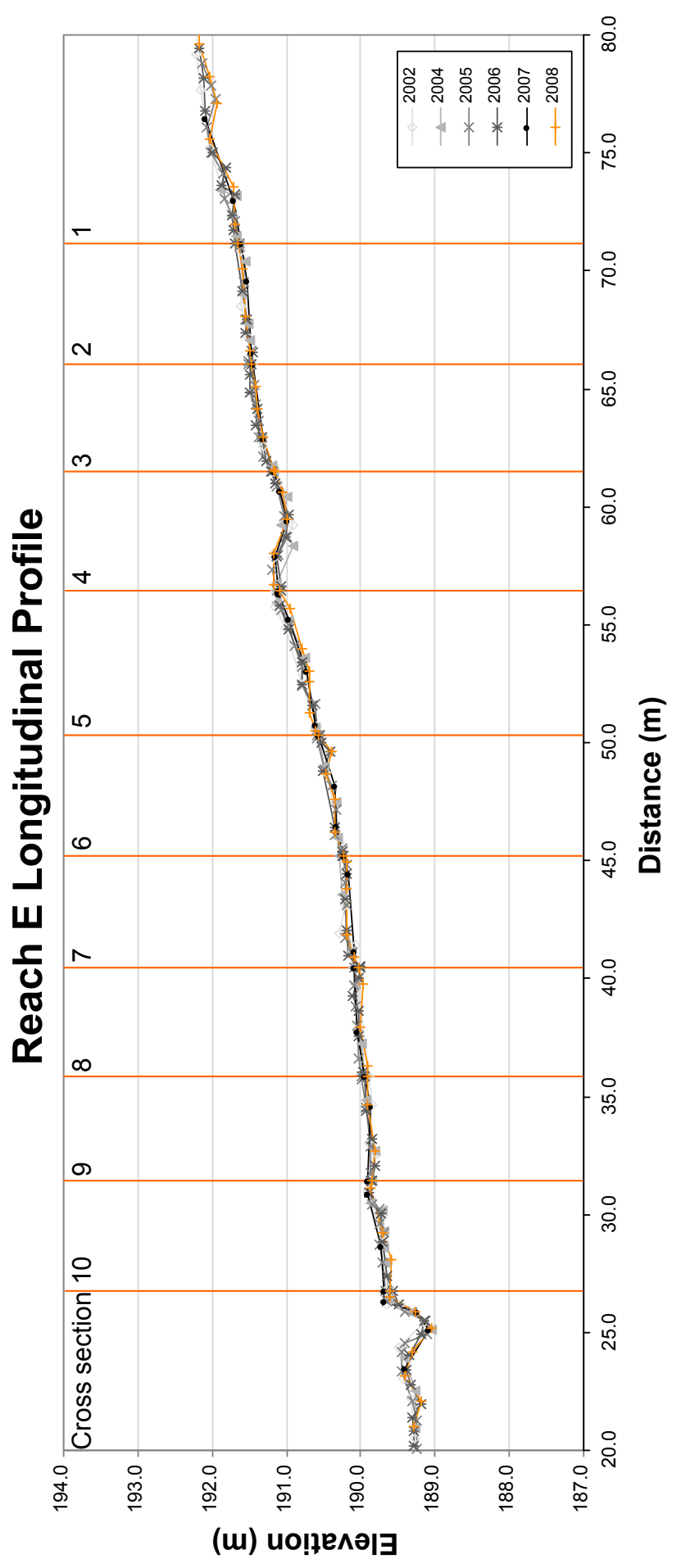




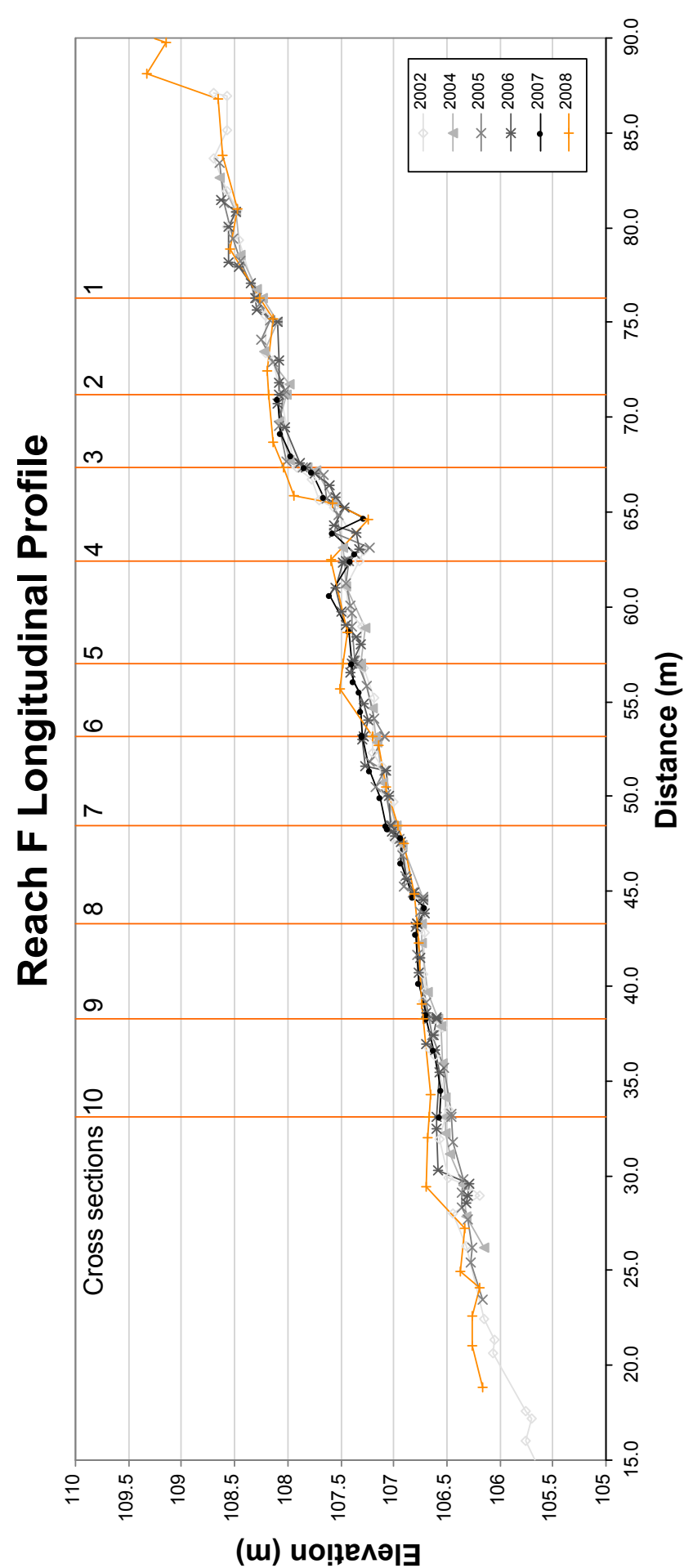




\section{Appendix C}

\section{Instructions for Surveyors}

This appendix contains specific instructions for surveying the permanent cross section and longitudinal profile surveys in Little Creek. Surveyors are encouraged to first review Harrelson et al. (1994) published by the USFS Rocky Mountain Research Station, which covers channel surveying procedures and theory in more depth than provided here. The intent of these instructions is to provide guidelines that will minimize errors in surveying with the overall goal of aiding the interpretation of future changes in Little Creek. Confident data interpretation depends on the confidence in the accuracy of the survey data.

\section{C.1 Cross section Instructions}

\section{C.1.1 Equipment}

Below is a list of the equipment needed to survey in the permanent cross sections. Treat the equipment with care (especially the level) and replace any defective or damaged equipment. 
1. Auto-level

2. Tripod

3. Philadelphia rod

4. Rod level (bullseye)

5. Field Book

6. These instructions

7. Machete

8. Handsaw

9. Flagging (Pink or Orange)

10. (if needed) New rebar (5/8" diameter and minimum 5' long) and hammer

11. 0.1' ruled PVC pipe w/ level (for undercut banks)

\section{Instructions}

Before surveying, make sure that the level has not been damaged or is out of calibration by performing a peg test (see Harrelson et al. (1994)). Extend a cloth tape from the left rebar (steel rod that marks the end of the cross section) to the right rebar (looking downstream) so the zero mark is on the left rebar. Make sure the tape is snug but not so tight that it bends the rebar. Set up and level the tripod and attach the automatic level. Make sure that the level is set up above the top of both rebar endpoints and at least one of the permanent point benchmarks (2" diameter pipes). Inspect the rebar and if flagging is missing or deteriorated reflag and label with the cross section number. Flagging should be 
several feet long to aid in find rebar that becomes buried. Check if the rebar is bent or loose. If it is loose or has moved, measure and note the offset and draw a sketch in the field book that illustrates the orientation of the rebar. Install a new rebar endpoint outside of the existing cross section (in the same plane) if the existing rebar seems unstable (with 5/8" rebar at least 5' long). If possible, choose a location that is relatively close to the same elevation as the opposite rebar. In 2010, new rebar endpoints were installed in approximately half of the reaches. Make sure you include the top and base of the old rebar in the new survey.

\section{Field book setup}

Set up the field book as shown in Harrelson et al. (1994). Use one of the permanent benchmarks as your backsight to set the height of instrument. Elevations of all the benchmarks are in Table C.1.

\section{Making measurements}

Take measurements starting from the top and bottom of the left rebar (looking downstream). If the rebar endpoint is tilted or bent, place the zero mark of the tape at the bottom of the rebar. Take measurements at all breaks in slope. Also take measurements at bankfull indicators, top of bank (if terrace or other feature), edge of water (both sides) and thalweg (deepest part of channel). If no bankfull indicator exists, write NO BANKFULL INDICATOR. Include as much detail as possible. More points are much better than fewer points when trying to interpret the data. Detailed descriptions and many data points are especially important on steep banks or complicated sections of stream. There should be a measurement at least every foot except in areas that are planar or featureless. 


\begin{tabular}{cc}
\hline Benchmark & Elevation $(\mathrm{ft})$ \\
\hline PPA & 93.81 \\
PPA2 & 91.37 \\
PPA3 & 93.96 \\
PPB & 143.43 \\
PPB2 & 139.72 \\
PPB3 & 152.06 \\
PPC & 318.45 \\
PPC2 & 297.64 \\
PPC3 & 321.92 \\
PPD & 362.94 \\
PPD2 & 356.55 \\
PPD3 & 363.37 \\
PPE & 631.69 \\
PPE2 & 627.37 \\
PPF & 362.51 \\
PPF2 & 355.36 \\
PPF3 & 351.78 \\
\hline
\end{tabular}

Table C.1: Table of existing benchmark elevations.

Make sure the surveying rod is level on each measurement and make sure the rod is directly under the tape. When surveying rocks, boulders, logs, etc., measure enough points to represent the true shape (not just a triangle) and note the feature in the fieldbook. Be specific and provide details — what is the diameter of a boulder or species of a log; is the rock or log embedded in substrate or lying on top? Typically, logs are removed from the survey, but the information is still valuable. When surveying in the active channel, ignore features that are small enough that they can be easily moved with your foot and either kick them out of the way or survey between them.

Special care must be taken when taking measurements that are close to a "whole" foot on the Philadelphia rod. For example, 4.95 is often mistakenly read as 5.95 because the large 5 is what is seen when looking through the scope. Be conscious of this and this mistake will be minimized. Also make sure the rod is 
completely extended the necessary distance when making a reading. A reading of $7 \mathrm{ft}$ followed by one of $11 \mathrm{ft}$ should mean the rod moved down four feet, it should not mean that the rod was not fully extended. Think about each measurement as you take it and make sure it makes sense. The largest foresight reading should be the thalweg and measurements should only change drastically when the rod is on a steep slope.

\section{Undercut banks}

To survey an undercut bank, level the Philadelphia rod and position it so it is touching the top of the undercut bank. Use a ruled PVC pipe or pocket rod (with a carpenters level to ensure the ruler is horizontal) to measure the shape of the bank in the same direction of the survey. Measure the top of the undercut bank first on downstream left bank and the bottom of the undercut bank first on the downstream right bank. Measureing the start of the undercut bank will be the same as a normal reading, however, make sure to write "start undercut bank in the notes". The next reading will use the distance measured with the ruler from the Philadelphia rod to the bank as the distance measurement, with a plus or minus to indicate direction towards the undercut bank $(+$ will be used the right bank, - on left banks). The foresight recorded is the height of the rod where the distance to the undercut bank was measured (put a minus sign in front to indicate that is subtracted from the initial foresight). Continue to take measurements in this manner until the shape of the bank is accurately described. The last measurement will be the location where the Philadelphia rod is touching the bank. This measurement will have the same distance as the measurement at the start of the undercut bank, and the foresight will be the height where the 
bank touches the rod. Sketch the shape of the bank and where measurements were taken in the field book. See the example below.

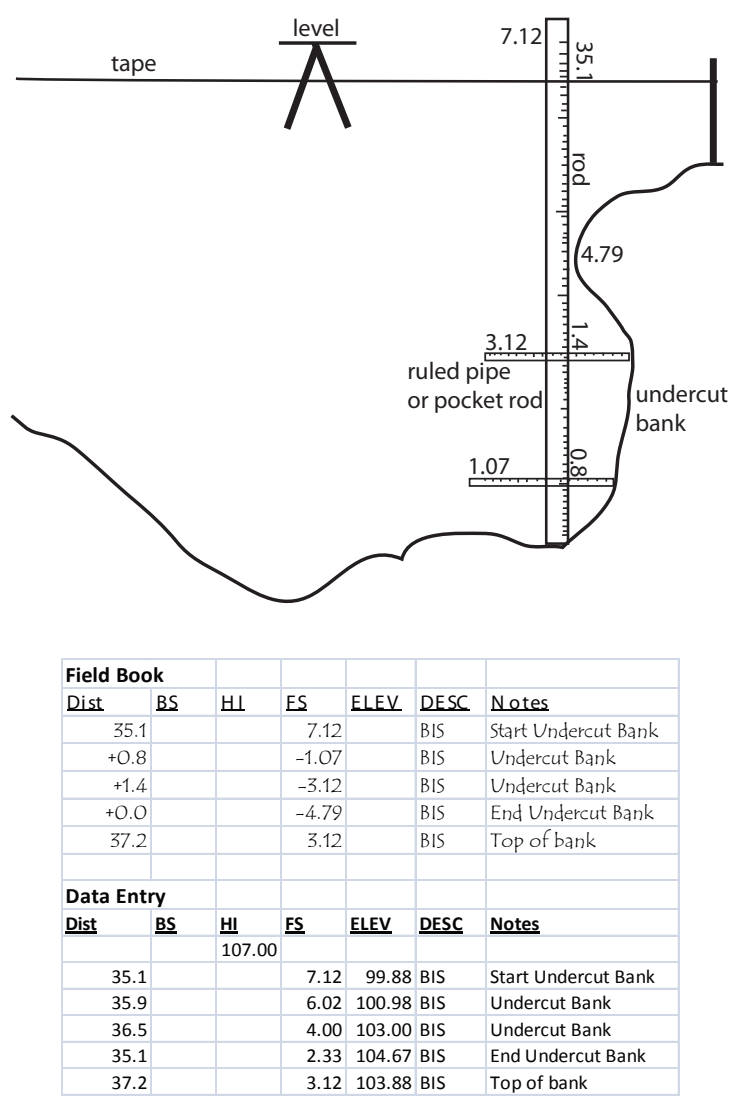

At the end of the survey shoot back to first rebar to check backsight and close the survey the reading should be the same as the initial backsight. This ensures that the level did not move during the survey. 


\section{C.2 Longitudinal Profile Instructions}

\section{C.2.1 Equipment}

Below is a list of the equipment needed to survey in the permanent cross sections. Treat the equipment with care (especially the total station) and replace any defective or damaged equipment.

1. Total station

2. Data collector

3. Cables and extra battery

4. Tripod

5. Survey rods (2) - one with tripod legs

6. Prisms (2)

7. Pocket rod

8. Field Book

9. These instructions

10. Machete

11. Handsaw

12. Flagging (Pink or Orange) 


\section{C.2.2 Setup}

Equipment used varies but the general setup is the same for total station surveying regardless of the equipment used. Verify that the data collector and total station can communicate before going into the field (a good test is setting the backsight angle to zero on the instrument). Familiarize yourself with the surveying software. Set up a job file for each reach and enter the coordinates of the permanent points.

\section{C.2.3 Surveying}

At the study reach identify the permanent benchmark points. They are 2" capped steel pipes painted orange with a drilled indentation marking the exact benchmark location. The benchmarks should be labeled with the point name ("PPA2" for example). If the point name is missing or faded relabel it with a permanent marker. The longitudinal profile should be surveyed from downstream to upstream. Set up the total station on the point which allows you to see farthest downstream, and set up your backsight on another permanent point. Ensure both the backsight and total station are leveled and centered directly above the mark on the pipe. A plumb bob (a string tied to a nut works well) helps to set up the tripod directly over the permanent point. Measure the height from the pipe to the center of the backsight prism (HR-height of rod) and to the axis of the scope (HI-height of instrument). Do not use the measurements on the rod, because they are not accurate with the prisms we use. Set up the total-station with these values and very precisely aim at the center of the backsight scope. Send the backsight circle of zero to the instrument and then take a shot to check the distance. The error should be less than $0.05^{\prime}$ in both the horizontal or vertical direction. If the error is greater, recheck the level and height of the total station and backsight rod. 
Once the total station is set up correctly you can begin making measurements. Set the foresight rod to 5.0' and measure the true height. This height will likely need to be adjusted during the survey and it is easiest if it is adjusted in whole feet $\left(5^{\prime}, 6^{\prime}, 7^{\prime}, 8^{\prime}\right)$ so the total station operator can easily adjust the height of instrument in the data collector. The foresight rod should be kept as short as possible during surveying to minimize errors that occur from the rod not being level. Identify the longitudinal profile start rebar/flagging and replace if faded, damaged, or missing. The start location should be at farthest downstream location visible from the benchmark points. Start taking side shots at breaks in slope of the thalweg longitudinal profile. Points should be stored with a descriptive label (typically riffle, tail of pool, middle of pool, head of pool) but add other useful information that will aid interpretation of the profile to the point labels. More points is better than fewer points. It is especially important to accurately represent pools in the longitudinal profile. Take special care to accurately survey the tail of the pool (downstream end where water surface begins to slope downstream) and deepest points of pools to ensure accurate measurement of the residual depth. When the channel is split, survey the side that has the lowest elevation in cross section.

As you survey, frequently check that the total station is level. If the legs are in direct sunlight they expand as they heat, and tilt the total station slightly. After re-leveling check the backsight circle (it should be 0) and reset as needed. Once you finish surveying the profile visible from a permanent point you will survey the tops of the rebar pins visible from that location before moving to the next benchmark. Store these points in a different number series than the longitudinal points (you want to make sure all the longitudinal points are in sequential order from downstream to upstream when you are done surveying). For example, survey the 
longitudinal profile starting with point 100, and start the cross sections endpoints with point 600 . Survey the top of the rebar and where the rebar intersects the ground. Label points like this: XS1Rtop, XS1Rbot, XS1Ltop, XS1Lbot. After surveying the rebar visible from the permanent point, move to next benchmark upstream and repeat. Make sure you set the point number back to the series containing the longitudinal data before taking longitudinal profile measurements again.

\section{C.3 Pebble Count}

Perform pebble count as described in Harrelson et al. (1994). Collect 100 measurements between the uppermost and lowermost cross sections, stratifying the sampling locations based on channel condition (pools and riffles). 


\section{Appendix D}

\section{Survey Data for All Cross}

\section{Sections and Longitudinal Profiles}

\section{D.1 Cross Section Data}

Table D.1: All cross section survey data

\begin{tabular}{lccccclc}
\hline Year & Reach & Section & Point & Dist. $(\mathrm{m})$ & Elev. $(\mathrm{m})$ & Desc. & Notes \\
\hline 2002 & A & 1 & 1 & 0.00 & 100.00 & TSL & Exclude \\
2002 & A & 1 & 2 & 0.00 & 99.81 & BS & FALSE \\
2002 & A & 1 & 3 & 2.68 & 99.32 & B @ EL & FALSE \\
2002 & A & 1 & 4 & 2.93 & 99.30 & B @ EL & FALSE \\
2002 & A & 1 & 5 & 3.08 & 99.27 & BF & FALSE \\
2002 & A & 1 & 6 & 3.57 & 98.95 & BIS & FALSE \\
2002 & A & 1 & 7 & 3.99 & 98.83 & WE & FALSE \\
2002 & A & 1 & 8 & 4.79 & 98.68 & TW & FALSE \\
2002 & A & 1 & 9 & 5.97 & 98.83 & WE & FALSE \\
2002 & A & 1 & 10 & 6.40 & 98.93 & BIS & FALSE \\
2002 & A & 1 & 11 & 7.22 & 99.42 & BF & FALSE \\
2002 & A & 1 & 12 & 8.75 & 99.59 & BIS & FALSE \\
2002 & A & 1 & 13 & 9.14 & 99.74 & BS & FALSE \\
2002 & A & 1 & 14 & 9.14 & 100.01 & TSR & FALSE \\
2002 & A & 2 & 1 & 0.00 & 100.00 & TSL & FALSE \\
2002 & A & 2 & 2 & 0.00 & 99.64 & BS & FALSE \\
2002 & A & 2 & 3 & 1.34 & 99.45 & TB & FALSE \\
2002 & A & 2 & 4 & 1.34 & 99.38 & BF & FALSE \\
2002 & A & 2 & 5 & 2.93 & 98.74 & BIS & FALSE
\end{tabular}


Table D.1 - Continued

\begin{tabular}{|c|c|c|c|c|c|c|c|c|}
\hline Year & Reach & Section & Point & Dist. $(\mathrm{m})$ & Elev. $(\mathrm{m})$ & Desc. & Notes & Exclude \\
\hline 2002 & A & 2 & 6 & 4.72 & 98.21 & WE & & FALSE \\
\hline 2002 & A & 2 & 7 & 5.30 & 98.13 & BIS & & FALSE \\
\hline 2002 & A & 2 & 8 & 6.19 & 97.94 & TW & & FALSE \\
\hline 2002 & A & 2 & 9 & 6.86 & 98.01 & SB @ Edge & & FALSE \\
\hline & & & & & & Log & & \\
\hline 2002 & $\mathrm{~A}$ & 2 & 10 & 6.86 & 98.21 & $\begin{array}{l}\text { WE - actu- } \\
\text { ally under de- }\end{array}$ & & FALSE \\
\hline & & & & & & bris & & \\
\hline 2002 & $\mathrm{~A}$ & 2 & 11 & 6.92 & 98.92 & $\mathrm{TL}$ & & TRUE \\
\hline 2002 & A & 2 & 12 & 7.25 & 98.92 & $\mathrm{TL}$ & & TRUE \\
\hline 2002 & A & 2 & 13 & 7.35 & 98.50 & EL - Debris & & TRUE \\
\hline 2002 & $\mathrm{~A}$ & 2 & 14 & 7.62 & 98.36 & $\begin{array}{l}\text { TL - WE un- } \\
\text { derneath }\end{array}$ & & FALSE \\
\hline 2002 & A & 2 & 15 & 7.74 & 98.46 & BB & & FALSE \\
\hline 2002 & A & 2 & 16 & 7.77 & 98.68 & BIS - debris & & FALSE \\
\hline 2002 & $\mathrm{~A}$ & 2 & 17 & 8.29 & 98.73 & BIS - debris & & FALSE \\
\hline 2002 & A & 2 & 18 & 8.50 & 99.13 & BIS - debris & & FALSE \\
\hline 2002 & $\mathrm{~A}$ & 2 & 19 & 9.14 & 99.18 & BIS - debris & & FALSE \\
\hline 2002 & A & 2 & 20 & 9.21 & 98.95 & BIS - debris & & FALSE \\
\hline 2002 & A & 2 & 21 & 9.48 & 99.00 & BIS - debris & & FALSE \\
\hline 2002 & $\mathrm{~A}$ & 2 & 22 & 9.75 & 99.36 & TВ & & FALSE \\
\hline 2002 & A & 2 & 23 & 10.73 & 99.67 & BS & & FALSE \\
\hline 2002 & A & 2 & 24 & 10.73 & 100.00 & TSR & & FALSE \\
\hline 2002 & A & 3 & 1 & 0.00 & 100.00 & TSL & & FALSE \\
\hline 2002 & A & 3 & 2 & 0.00 & 99.46 & BS & & FALSE \\
\hline 2002 & A & 3 & 3 & 0.70 & 99.34 & TB & & FALSE \\
\hline 2002 & $\mathrm{~A}$ & 3 & 4 & 0.88 & 98.83 & BIS & & FALSE \\
\hline 2002 & A & 3 & 5 & 1.62 & 98.20 & $\mathrm{BF}$ & & FALSE \\
\hline 2002 & A & 3 & 6 & 2.04 & 98.02 & BIS & & FALSE \\
\hline 2002 & A & 3 & 7 & 2.26 & 97.98 & WE & & FALSE \\
\hline 2002 & $\mathrm{~A}$ & 3 & 8 & 2.26 & 97.74 & $\mathrm{BB} / \mathrm{TW}$ & & FALSE \\
\hline 2002 & A & 3 & 9 & 3.32 & 97.91 & BIS & & FALSE \\
\hline 2002 & A & 3 & 10 & 4.24 & 97.87 & BIS & & FALSE \\
\hline 2002 & $\mathrm{~A}$ & 3 & 11 & 4.69 & 97.98 & WE & & FALSE \\
\hline 2002 & $\mathrm{~A}$ & 3 & 12 & 5.43 & 98.20 & BIS & raised 1 foot & FALSE \\
\hline 2002 & A & 3 & 13 & 6.34 & 98.51 & $\mathrm{BF}$ & raised 1 foot & FALSE \\
\hline 2002 & A & 3 & 14 & 8.29 & 98.45 & BIS & & FALSE \\
\hline 2002 & $\mathrm{~A}$ & 3 & 15 & 8.63 & 98.52 & BIS & & FALSE \\
\hline 2002 & $\mathrm{~A}$ & 3 & 16 & 10.76 & 99.67 & BS & & FALSE \\
\hline 2002 & A & 3 & 17 & 10.76 & 100.09 & TSR & & FALSE \\
\hline 2002 & A & 4 & 1 & 0.00 & 100.00 & TSL & & TRUE \\
\hline 2002 & $\mathrm{~A}$ & 4 & 2 & 0.00 & 99.69 & BS & & TRUE \\
\hline 2002 & $\mathrm{~A}$ & 4 & 3 & 0.91 & 99.58 & BIS & & TRUE \\
\hline 2002 & $\mathrm{~A}$ & 4 & 4 & 1.25 & 99.48 & BIS & & TRUE \\
\hline 2002 & A & 4 & 5 & 2.38 & 99.41 & TB & & TRUE \\
\hline 2002 & $\mathrm{~A}$ & 4 & 6 & 2.47 & 99.18 & $\mathrm{BF}$ & 2004 survey different & TRUE \\
\hline 2002 & A & 4 & 7 & 2.47 & 98.75 & BS & & TRUE \\
\hline 2002 & A & 4 & 8 & 2.71 & 98.69 & $\mathrm{BR}$ & & TRUE \\
\hline 2002 & A & 4 & 9 & 2.77 & 98.79 & $\mathrm{TR}$ & & TRUE \\
\hline 2002 & A & 4 & 10 & 3.05 & 98.77 & $\mathrm{TR}$ & & TRUE \\
\hline 2002 & $\mathrm{~A}$ & 4 & 11 & 3.08 & 98.69 & WE & & TRUE \\
\hline 2002 & A & 4 & 12 & 3.08 & 98.57 & $\mathrm{BR}$ & & TRUE \\
\hline
\end{tabular}

Continued on Next Page... 
Table D.1 - Continued

\begin{tabular}{|c|c|c|c|c|c|c|c|c|}
\hline Year & Reach & Section & Point & Dist. (m) & Elev. $(\mathrm{m})$ & Desc. & Notes & Exclude \\
\hline 2002 & A & 4 & 13 & 4.05 & 98.47 & $\mathrm{TW}$ & & TRUE \\
\hline 2002 & A & 4 & 14 & 5.36 & 98.69 & WE & & TRUE \\
\hline 2002 & A & 4 & 15 & 5.73 & 98.74 & BIS & & TRUE \\
\hline 2002 & A & 4 & 16 & 6.49 & 99.18 & $\mathrm{BF}$ & 2004 survey different & TRUE \\
\hline 2002 & A & 4 & 17 & 7.59 & 99.19 & BIS & & TRUE \\
\hline 2002 & $\mathrm{~A}$ & 4 & 18 & 8.20 & 99.44 & BIS & & TRUE \\
\hline 2002 & $\mathrm{~A}$ & 4 & 19 & 8.32 & 99.87 & BIS & & TRUE \\
\hline 2002 & $\mathrm{~A}$ & 4 & 20 & 9.69 & 99.92 & BS & & TRUE \\
\hline 2002 & $\mathrm{~A}$ & 4 & 21 & 9.69 & 100.01 & TSR & & TRUE \\
\hline 2002 & $\mathrm{~A}$ & 5 & 1 & 0.00 & 100.00 & TSL & & FALSE \\
\hline 2002 & A & 5 & 2 & 0.00 & 99.70 & BS & & FALSE \\
\hline 2002 & $\mathrm{~A}$ & 5 & 3 & 1.16 & 99.87 & BIS/ALDER & & FALSE \\
\hline 2002 & $\mathrm{~A}$ & 5 & 4 & 2.01 & 99.79 & BIS/ALDER & & FALSE \\
\hline 2002 & $\mathrm{~A}$ & 5 & 5 & 2.07 & 99.33 & BIS & & FALSE \\
\hline 2002 & $\mathrm{~A}$ & 5 & 6 & 2.23 & 99.31 & $\mathrm{~TB} / \mathrm{BF}$ & & FALSE \\
\hline 2002 & $\mathrm{~A}$ & 5 & 7 & 2.74 & 98.74 & BIS & & FALSE \\
\hline 2002 & $\mathrm{~A}$ & 5 & 8 & 2.99 & 98.67 & WE & & FALSE \\
\hline 2002 & $\mathrm{~A}$ & 5 & 9 & 4.21 & 98.45 & $\mathrm{TW}$ & & FALSE \\
\hline 2002 & $\mathrm{~A}$ & 5 & 10 & 5.24 & 98.67 & WE & & FALSE \\
\hline 2002 & $\mathrm{~A}$ & 5 & 11 & 5.64 & 98.82 & BIS & & FALSE \\
\hline 2002 & $\mathrm{~A}$ & 5 & 12 & 6.31 & 99.01 & $\mathrm{BF}$ & & FALSE \\
\hline 2002 & $\mathrm{~A}$ & 5 & 13 & 7.32 & 99.58 & BIS & & FALSE \\
\hline 2002 & $\mathrm{~A}$ & 5 & 14 & 7.86 & 99.54 & BS & & FALSE \\
\hline 2002 & $\mathrm{~A}$ & 5 & 15 & 7.86 & 99.98 & TSR & & FALSE \\
\hline 2002 & A & 6 & 1 & 0.00 & 100.00 & TSL & & FALSE \\
\hline 2002 & $\mathrm{~A}$ & 6 & 2 & 0.00 & 99.79 & BS & & FALSE \\
\hline 2002 & $\mathrm{~A}$ & 6 & 3 & 0.58 & 99.66 & TB/ROOT & & FALSE \\
\hline 2002 & $\mathrm{~A}$ & 6 & 4 & 0.70 & 98.49 & $\mathrm{BF}$ & & FALSE \\
\hline 2002 & $\mathrm{~A}$ & 6 & 5 & 0.70 & 98.37 & $\mathrm{BS}$ & & FALSE \\
\hline 2002 & $\mathrm{~A}$ & 6 & 6 & 1.10 & 98.32 & BIS & & FALSE \\
\hline 2002 & $\mathrm{~A}$ & 6 & 7 & 1.52 & 97.92 & WE & & FALSE \\
\hline 2002 & $\mathrm{~A}$ & 6 & 8 & 2.77 & 97.81 & TW & & FALSE \\
\hline 2002 & $\mathrm{~A}$ & 6 & 9 & 4.24 & 97.92 & WE & & FALSE \\
\hline 2002 & $\mathrm{~A}$ & 6 & 10 & 4.60 & 98.49 & $\mathrm{BF}$ & & FALSE \\
\hline 2002 & $\mathrm{~A}$ & 6 & 11 & 5.46 & 98.84 & TB/ROOT & & FALSE \\
\hline 2002 & $\mathrm{~A}$ & 6 & 12 & 7.13 & 99.10 & BIS & & FALSE \\
\hline 2002 & $\mathrm{~A}$ & 6 & 13 & 10.73 & 99.14 & BIS & & FALSE \\
\hline 2002 & $\mathrm{~A}$ & 6 & 14 & 14.81 & 99.03 & BIS & & FALSE \\
\hline 2002 & $\mathrm{~A}$ & 6 & 15 & 15.30 & 99.34 & $\mathrm{BS}$ & & FALSE \\
\hline 2002 & $\mathrm{~A}$ & 6 & 16 & 15.30 & 99.73 & TSR & & FALSE \\
\hline 2002 & $\mathrm{~A}$ & 7 & 1 & 0.00 & 100.00 & TSL & & FALSE \\
\hline 2002 & $\mathrm{~A}$ & 7 & 2 & 0.00 & 99.74 & $\mathrm{BS}$ & & FALSE \\
\hline 2002 & A & 7 & 3 & 0.70 & 99.62 & TB & & FALSE \\
\hline 2002 & A & 7 & 4 & 1.19 & 99.18 & BIS & & FALSE \\
\hline 2002 & $\mathrm{~A}$ & 7 & 5 & 1.28 & 98.71 & $\mathrm{BF}$ & & FALSE \\
\hline 2002 & A & 7 & 6 & 1.34 & 98.46 & $\mathrm{BB}$ & & FALSE \\
\hline 2002 & A & 7 & 7 & 1.80 & 98.47 & $\mathrm{BR}$ & & FALSE \\
\hline 2002 & A & 7 & 8 & 1.89 & 98.73 & $\mathrm{TR}$ & & FALSE \\
\hline 2002 & $\mathrm{~A}$ & 7 & 9 & 2.32 & 98.72 & $\mathrm{TR}$ & & FALSE \\
\hline 2002 & $\mathrm{~A}$ & 7 & 10 & 2.50 & 98.34 & WE & & FALSE \\
\hline 2002 & A & 7 & 11 & 2.50 & 98.21 & $\mathrm{BR}$ & & FALSE \\
\hline 2002 & A & 7 & 12 & 3.02 & 98.13 & TW & & FALSE \\
\hline
\end{tabular}

Continued on Next Page... 
Table D.1 - Continued

\begin{tabular}{|c|c|c|c|c|c|c|c|c|}
\hline Year & Reach & Section & Point & Dist. (m) & Elev. $(\mathrm{m})$ & Desc. & Notes & Exclude \\
\hline 2002 & A & 7 & 13 & 3.99 & 98.19 & BIS & & FALSE \\
\hline 2002 & A & 7 & 14 & 4.75 & 98.34 & WE & & FALSE \\
\hline 2002 & A & 7 & 15 & 5.46 & 98.71 & $\mathrm{BF} / \mathrm{TB}$ & & FALSE \\
\hline 2002 & A & 7 & 16 & 7.01 & 99.02 & BIS & & FALSE \\
\hline 2002 & $\mathrm{~A}$ & 7 & 17 & 7.89 & 99.09 & BIS & & FALSE \\
\hline 2002 & A & 7 & 18 & 8.84 & 99.40 & BIS & & FALSE \\
\hline 2002 & $\mathrm{~A}$ & 7 & 19 & 9.81 & 99.52 & BIS & & FALSE \\
\hline 2002 & $\mathrm{~A}$ & 7 & 20 & 16.43 & 99.30 & BIS & & FALSE \\
\hline 2002 & $\mathrm{~A}$ & 7 & 21 & 17.62 & 99.40 & BIS & & FALSE \\
\hline 2002 & $\mathrm{~A}$ & 7 & 22 & 18.71 & 99.83 & BS & & FALSE \\
\hline 2002 & A & 7 & 23 & 18.71 & 100.20 & TSR & & FALSE \\
\hline 2002 & A & 8 & 1 & 0.00 & 100.00 & TSL & & FALSE \\
\hline 2002 & $\mathrm{~A}$ & 8 & 2 & 0.00 & 99.62 & BS & & FALSE \\
\hline 2002 & $\mathrm{~A}$ & 8 & 3 & 0.79 & 99.52 & ТВ & & FALSE \\
\hline 2002 & $\mathrm{~A}$ & 8 & 4 & 1.19 & 98.36 & $\mathrm{BF}$ & & FALSE \\
\hline 2002 & $\mathrm{~A}$ & 8 & 5 & 1.19 & 98.35 & $\mathrm{BB}$ & & FALSE \\
\hline 2002 & $\mathrm{~A}$ & 8 & 6 & 2.80 & 97.94 & WE & & FALSE \\
\hline 2002 & $\mathrm{~A}$ & 8 & 7 & 4.02 & 97.74 & $\mathrm{TW}$ & & FALSE \\
\hline 2002 & A & 8 & 8 & 5.18 & 97.94 & WE & & FALSE \\
\hline 2002 & $\mathrm{~A}$ & 8 & 9 & 6.13 & 98.36 & $\mathrm{BF} / \mathrm{TB}$ & & FALSE \\
\hline 2002 & $\mathrm{~A}$ & 8 & 10 & 7.47 & 98.58 & BIS & & FALSE \\
\hline 2002 & $\mathrm{~A}$ & 8 & 11 & 9.36 & 98.81 & BIS & & FALSE \\
\hline 2002 & $\mathrm{~A}$ & 8 & 12 & 10.73 & 98.87 & BIS & & FALSE \\
\hline 2002 & $\mathrm{~A}$ & 8 & 13 & 11.95 & 99.20 & BIS & & FALSE \\
\hline 2002 & A & 8 & 14 & 15.21 & 99.16 & BIS & & FALSE \\
\hline 2002 & $\mathrm{~A}$ & 8 & 15 & 16.49 & 98.98 & BIS & & FALSE \\
\hline 2002 & $\mathrm{~A}$ & 8 & 16 & 18.53 & 99.15 & BIS & & FALSE \\
\hline 2002 & $\mathrm{~A}$ & 8 & 17 & 19.60 & 99.63 & $\mathrm{BS}$ & & FALSE \\
\hline 2002 & $\mathrm{~A}$ & 8 & 18 & 19.60 & 100.00 & TSR & & FALSE \\
\hline 2002 & $\mathrm{~A}$ & 9 & 1 & 0.00 & 100.00 & TSL & & FALSE \\
\hline 2002 & $\mathrm{~A}$ & 9 & 2 & 0.00 & 99.63 & $\mathrm{BS}$ & & FALSE \\
\hline 2002 & $\mathrm{~A}$ & 9 & 3 & 0.73 & 99.67 & TB & & FALSE \\
\hline 2002 & A & 9 & 4 & 0.82 & 98.76 & $\mathrm{BF}$ & & FALSE \\
\hline 2002 & $\mathrm{~A}$ & 9 & 5 & 0.88 & 98.70 & $\mathrm{BB}$ & & FALSE \\
\hline 2002 & $\mathrm{~A}$ & 9 & 6 & 2.19 & 98.47 & $\mathrm{BR}$ & & FALSE \\
\hline 2002 & $\mathrm{~A}$ & 9 & 7 & 2.23 & 98.63 & $\mathrm{TR}$ & & FALSE \\
\hline 2002 & $\mathrm{~A}$ & 9 & 8 & 2.65 & 98.64 & $\mathrm{TR}$ & & FALSE \\
\hline 2002 & A & 9 & 9 & 2.71 & 98.42 & $\mathrm{BR}$ & & FALSE \\
\hline 2002 & A & 9 & 10 & 4.08 & 98.16 & WE & & FALSE \\
\hline 2002 & $\mathrm{~A}$ & 9 & 11 & 4.91 & 98.07 & BIS & & FALSE \\
\hline 2002 & $\mathrm{~A}$ & 9 & 12 & 5.64 & 97.97 & TW & & FALSE \\
\hline 2002 & $\mathrm{~A}$ & 9 & 13 & 6.64 & 98.11 & BIS & & FALSE \\
\hline 2002 & A & 9 & 14 & 7.32 & 98.16 & WE & & FALSE \\
\hline 2002 & A & 9 & 15 & 8.26 & 98.76 & $\mathrm{BF} / \mathrm{TB}$ & & FALSE \\
\hline 2002 & $\mathrm{~A}$ & 9 & 16 & 11.13 & 99.04 & BIS & & FALSE \\
\hline 2002 & A & 9 & 17 & 11.95 & 98.95 & BIS & & FALSE \\
\hline 2002 & A & 9 & 18 & 12.68 & 99.14 & BIS & & FALSE \\
\hline 2002 & A & 9 & 19 & 12.98 & 99.38 & BIS & & FALSE \\
\hline 2002 & $\mathrm{~A}$ & 9 & 20 & 14.05 & 99.44 & $\mathrm{BR}$ & & FALSE \\
\hline 2002 & $\mathrm{~A}$ & 9 & 21 & 14.42 & 99.59 & $\mathrm{TR}$ & & FALSE \\
\hline 2002 & A & 9 & 22 & 14.57 & 99.35 & $\mathrm{BR}$ & & FALSE \\
\hline 2002 & A & 9 & 23 & 16.31 & 99.29 & BIS & & FALSE \\
\hline
\end{tabular}

Continued on Next Page... 
Table D.1 - Continued

\begin{tabular}{|c|c|c|c|c|c|c|c|c|}
\hline Year & Reach & Section & Point & Dist. $(\mathrm{m})$ & Elev. $(\mathrm{m})$ & Desc. & Notes & Exclude \\
\hline 2002 & A & 9 & 24 & 17.16 & 99.21 & BIS & & FALSE \\
\hline 2002 & A & 9 & 25 & 19.54 & 99.39 & BIS & & FALSE \\
\hline 2002 & A & 9 & 26 & 20.64 & 99.88 & BS & & FALSE \\
\hline 2002 & A & 9 & 27 & 20.64 & 100.24 & TSR & & FALSE \\
\hline 2002 & A & 10 & 1 & 0.00 & 100.00 & TSL & & FALSE \\
\hline 2002 & A & 10 & 2 & 0.00 & 99.58 & $\mathrm{BS}$ & & FALSE \\
\hline 2002 & A & 10 & 3 & 0.40 & 99.54 & ТВ & & FALSE \\
\hline 2002 & A & 10 & 4 & 0.52 & 98.57 & $\mathrm{BB}$ & & FALSE \\
\hline 2002 & A & 10 & 5 & 2.68 & 98.38 & $\mathrm{BF}$ & & FALSE \\
\hline 2002 & A & 10 & 6 & 3.75 & 98.28 & BIS & & FALSE \\
\hline 2002 & A & 10 & 7 & 4.24 & 98.15 & BIS & & FALSE \\
\hline 2002 & A & 10 & 8 & 4.48 & 98.29 & BIS & & FALSE \\
\hline 2002 & A & 10 & 9 & 5.52 & 98.28 & BIS & & FALSE \\
\hline 2002 & A & 10 & 10 & 7.38 & 97.91 & $\mathrm{BR}$ & & FALSE \\
\hline 2002 & A & 10 & 11 & 7.56 & 98.09 & $\mathrm{TR}$ & & FALSE \\
\hline 2002 & A & 10 & 12 & 8.08 & 97.97 & $\mathrm{TR}$ & & FALSE \\
\hline 2002 & A & 10 & 13 & 8.20 & 97.81 & WE & & FALSE \\
\hline 2002 & A & 10 & 14 & 8.20 & 97.73 & $\mathrm{BR}$ & & FALSE \\
\hline 2002 & A & 10 & 15 & 8.69 & 97.60 & $\mathrm{TW}$ & & FALSE \\
\hline 2002 & A & 10 & 16 & 8.93 & 97.66 & $\mathrm{BR}$ & & FALSE \\
\hline 2002 & A & 10 & 17 & 8.93 & 97.81 & WE & & FALSE \\
\hline 2002 & A & 10 & 18 & 9.45 & 98.24 & $\mathrm{TR}$ & & FALSE \\
\hline 2002 & A & 10 & 19 & 10.12 & 98.08 & BR-BB & & FALSE \\
\hline 2002 & A & 10 & 20 & 10.24 & 98.38 & $\mathrm{BF}$ & & FALSE \\
\hline 2002 & A & 10 & 21 & 10.36 & 98.61 & TB & & FALSE \\
\hline 2002 & A & 10 & 22 & 12.80 & 98.97 & BIS & & FALSE \\
\hline 2002 & A & 10 & 23 & 14.54 & 98.95 & BIS & & FALSE \\
\hline 2002 & A & 10 & 24 & 15.70 & 99.23 & BIS & & FALSE \\
\hline 2002 & A & 10 & 25 & 16.92 & 98.98 & BIS & & FALSE \\
\hline 2002 & A & 10 & 26 & 18.50 & 99.20 & BIS & & FALSE \\
\hline 2002 & A & 10 & 27 & 20.18 & 99.80 & $\mathrm{BS}$ & & FALSE \\
\hline 2002 & A & 10 & 28 & 20.18 & 100.00 & TSR & & FALSE \\
\hline 2002 & B & 1 & 1 & 0.00 & 100.00 & TSL & & FALSE \\
\hline 2002 & B & 1 & 2 & 0.00 & 99.85 & BS & & FALSE \\
\hline 2002 & B & 1 & 3 & 0.30 & 99.71 & BIS & & FALSE \\
\hline 2002 & B & 1 & 4 & 0.43 & 99.25 & $\mathrm{TR}$ & & FALSE \\
\hline 2002 & B & 1 & 5 & 0.67 & 99.05 & $\mathrm{TR}$ & & FALSE \\
\hline 2002 & B & 1 & 6 & 0.88 & 98.55 & $\mathrm{BR}$ & & FALSE \\
\hline 2002 & B & 1 & 7 & 1.58 & 98.47 & $\mathrm{BF}$ & & FALSE \\
\hline 2002 & B & 1 & 8 & 2.13 & 97.82 & BIS & & FALSE \\
\hline 2002 & B & 1 & 9 & 2.80 & 97.64 & WE & & FALSE \\
\hline 2002 & B & 1 & 10 & 3.75 & 97.53 & TW1 & & FALSE \\
\hline 2002 & B & 1 & 11 & 3.99 & 97.60 & BIS & & FALSE \\
\hline 2002 & B & 1 & 12 & 4.98 & 97.53 & TW2 & & FALSE \\
\hline 2002 & B & 1 & 13 & 5.27 & 97.61 & BIS & & FALSE \\
\hline 2002 & B & 1 & 14 & 5.79 & 97.54 & BIS & & FALSE \\
\hline 2002 & B & 1 & 15 & 6.64 & 97.64 & WE & & FALSE \\
\hline 2002 & B & 1 & 16 & 7.41 & 98.11 & $\mathrm{BF}$ & & FALSE \\
\hline 2002 & B & 1 & 17 & 8.17 & 98.43 & BIS & & FALSE \\
\hline 2002 & B & 1 & 18 & 9.14 & 98.88 & BIS & & FALSE \\
\hline 2002 & B & 1 & 19 & 10.12 & 99.72 & BS & & FALSE \\
\hline 2002 & B & 1 & 20 & 10.12 & 99.98 & TSR & & FALSE \\
\hline
\end{tabular}

Continued on Next Page... 
Table D.1 - Continued

\begin{tabular}{|c|c|c|c|c|c|c|c|c|}
\hline Year & Reach & Section & Point & Dist. (m) & Elev. $(\mathrm{m})$ & Desc. & Notes & Exclude \\
\hline 2002 & B & 2 & 1 & 0.00 & 100.00 & TSL & & FALSE \\
\hline 2002 & B & 2 & 2 & 0.00 & 99.59 & BS & & FALSE \\
\hline 2002 & B & 2 & 3 & 0.34 & 99.09 & BIS & & FALSE \\
\hline 2002 & B & 2 & 4 & 0.70 & 98.91 & BIS & & FALSE \\
\hline 2002 & B & 2 & 5 & 1.19 & 98.79 & BIS & & FALSE \\
\hline 2002 & B & 2 & 6 & 2.01 & 98.30 & BIS & & FALSE \\
\hline 2002 & B & 2 & 7 & 2.29 & 98.01 & $\mathrm{BF}$ & & FALSE \\
\hline 2002 & B & 2 & 8 & 3.11 & 97.47 & WE & & FALSE \\
\hline 2002 & B & 2 & 9 & 3.87 & 97.37 & BIS & & FALSE \\
\hline 2002 & B & 2 & 10 & 4.82 & 97.34 & $\mathrm{TW}$ & & FALSE \\
\hline 2002 & B & 2 & 11 & 5.18 & 97.42 & BIS & & FALSE \\
\hline 2002 & B & 2 & 12 & 6.00 & 97.40 & BIS & & FALSE \\
\hline 2002 & B & 2 & 13 & 6.98 & 97.46 & WE & & FALSE \\
\hline 2002 & B & 2 & 14 & 7.44 & 97.51 & BIS & & FALSE \\
\hline 2002 & B & 2 & 15 & 8.56 & 97.98 & $\mathrm{BF}$ & & FALSE \\
\hline 2002 & B & 2 & 16 & 10.61 & 99.51 & $\mathrm{BS}$ & & FALSE \\
\hline 2002 & B & 2 & 17 & 10.61 & 99.99 & TSR & & FALSE \\
\hline 2002 & B & 3 & 1 & 0.00 & 100.00 & TSL & & FALSE \\
\hline 2002 & B & 3 & 2 & 0.00 & 99.45 & $\mathrm{BS}$ & & FALSE \\
\hline 2002 & B & 3 & 3 & 1.13 & 98.95 & BIS & & FALSE \\
\hline 2002 & B & 3 & 4 & 1.49 & 98.37 & $\mathrm{BF}$ & & FALSE \\
\hline 2002 & B & 3 & 5 & 2.23 & 98.13 & BIS & & FALSE \\
\hline 2002 & B & 3 & 6 & 3.57 & 98.24 & BIS & & FALSE \\
\hline 2002 & B & 3 & 7 & 4.21 & 98.09 & WE & & FALSE \\
\hline 2002 & B & 3 & 8 & 5.15 & 97.89 & $\mathrm{TW}$ & & FALSE \\
\hline 2002 & B & 3 & 9 & 6.22 & 98.09 & WE & & FALSE \\
\hline 2002 & B & 3 & 10 & 6.92 & 98.50 & $\mathrm{BF}$ & & FALSE \\
\hline 2002 & B & 3 & 11 & 7.01 & 98.68 & BIS & & FALSE \\
\hline 2002 & B & 3 & 12 & 7.89 & 99.08 & BIS & & FALSE \\
\hline 2002 & B & 3 & 13 & 8.26 & 99.36 & BIS & & FALSE \\
\hline 2002 & B & 3 & 14 & 8.53 & 99.81 & BS & & FALSE \\
\hline 2002 & B & 3 & 15 & 8.53 & 99.98 & TSR & & FALSE \\
\hline 2002 & B & 4 & 1 & 0.00 & 100.00 & TSL & & FALSE \\
\hline 2002 & B & 4 & 2 & 0.00 & 99.84 & $\mathrm{BS}$ & & FALSE \\
\hline 2002 & B & 4 & 3 & 1.52 & 99.41 & $\mathrm{BF}$ & & FALSE \\
\hline 2002 & B & 4 & 4 & 1.74 & 98.90 & $\mathrm{BB}$ & & FALSE \\
\hline 2002 & B & 4 & 5 & 2.41 & 98.64 & BIS & & FALSE \\
\hline 2002 & B & 4 & 6 & 2.77 & 98.70 & BIS & & FALSE \\
\hline 2002 & B & 4 & 7 & 3.02 & 98.79 & BIS & & FALSE \\
\hline 2002 & B & 4 & 8 & 3.32 & 98.78 & $\mathrm{BR}$ & & FALSE \\
\hline 2002 & B & 4 & 9 & 3.44 & 98.93 & $\mathrm{TR}$ & & FALSE \\
\hline 2002 & B & 4 & 10 & 3.57 & 98.79 & $\mathrm{BR}$ & & FALSE \\
\hline 2002 & B & 4 & 11 & 3.69 & 98.99 & $\mathrm{TR}$ & & FALSE \\
\hline 2002 & B & 4 & 12 & 3.99 & 98.74 & $\mathrm{TR}$ & & FALSE \\
\hline 2002 & B & 4 & 13 & 4.24 & 98.67 & $\mathrm{TR}$ & & FALSE \\
\hline 2002 & B & 4 & 14 & 4.30 & 98.75 & WE & & FALSE \\
\hline 2002 & B & 4 & 15 & 4.36 & 98.46 & $\mathrm{BR}$ & & FALSE \\
\hline 2002 & B & 4 & 16 & 4.66 & 98.47 & TW1 & & FALSE \\
\hline 2002 & B & 4 & 17 & 4.69 & 98.63 & $\mathrm{TR}$ & & FALSE \\
\hline 2002 & B & 4 & 18 & 5.27 & 98.74 & $\mathrm{TR}$ & & FALSE \\
\hline 2002 & B & 4 & 19 & 5.82 & 98.66 & $\mathrm{TR}$ & & FALSE \\
\hline 2002 & B & 4 & 20 & 5.91 & 98.47 & TW2 & & FALSE \\
\hline
\end{tabular}

Continued on Next Page... 
Table D.1 - Continued

\begin{tabular}{|c|c|c|c|c|c|c|c|c|}
\hline Year & Reach & Section & Point & Dist. (m) & Elev. $(\mathrm{m})$ & Desc. & Notes & Exclude \\
\hline 2002 & B & 4 & 21 & 6.10 & 98.66 & $\mathrm{TR}$ & & FALSE \\
\hline 2002 & B & 4 & 22 & 6.31 & 98.60 & $\mathrm{TR}$ & & FALSE \\
\hline 2002 & B & 4 & 23 & 6.58 & 98.75 & WE & & FALSE \\
\hline 2002 & B & 4 & 24 & 6.83 & 98.84 & BIS & & FALSE \\
\hline 2002 & B & 4 & 25 & 6.95 & 99.42 & $\mathrm{BF}$ & & FALSE \\
\hline 2002 & B & 4 & 26 & 7.47 & 99.51 & BS & & FALSE \\
\hline 2002 & B & 4 & 27 & 7.47 & 100.01 & TSR & & FALSE \\
\hline 2002 & B & 5 & 1 & 0.00 & 100.00 & TSL & & FALSE \\
\hline 2002 & B & 5 & 2 & 0.00 & 99.60 & BS & & FALSE \\
\hline 2002 & B & 5 & 3 & 1.65 & 98.52 & $\mathrm{BF}$ & & FALSE \\
\hline 2002 & B & 5 & 4 & 2.04 & 98.27 & BIS & & FALSE \\
\hline 2002 & B & 5 & 5 & 3.32 & 97.99 & BIS & & FALSE \\
\hline 2002 & B & 5 & 6 & 3.75 & 97.90 & WE & & FALSE \\
\hline 2002 & B & 5 & 7 & 4.21 & 97.80 & BIS & & FALSE \\
\hline 2002 & B & 5 & 8 & 4.66 & 97.81 & BIS & & FALSE \\
\hline 2002 & B & 5 & 9 & 5.33 & 97.76 & BIS & & FALSE \\
\hline 2002 & B & 5 & 10 & 6.10 & 97.75 & $\mathrm{TW}$ & & FALSE \\
\hline 2002 & B & 5 & 11 & 6.92 & 97.90 & WE & & FALSE \\
\hline 2002 & B & 5 & 12 & 7.92 & 99.69 & BIS & & FALSE \\
\hline 2002 & B & 5 & 13 & 8.26 & 99.85 & BS & & FALSE \\
\hline 2002 & B & 5 & 14 & 8.26 & 100.00 & TSR & & FALSE \\
\hline 2002 & B & 6 & 1 & 0.00 & 100.00 & TSL & & FALSE \\
\hline 2002 & B & 6 & 2 & 0.00 & 99.96 & BS & & FALSE \\
\hline 2002 & B & 6 & 3 & 0.18 & 99.69 & BIS & & FALSE \\
\hline 2002 & B & 6 & 4 & 0.73 & 99.42 & BIS & & FALSE \\
\hline 2002 & B & 6 & 5 & 1.62 & 99.27 & BIS & & FALSE \\
\hline 2002 & B & 6 & 6 & 1.83 & 98.96 & $\mathrm{BF}$ & & FALSE \\
\hline 2002 & B & 6 & 7 & 2.38 & 98.76 & BIS & & FALSE \\
\hline 2002 & B & 6 & 8 & 2.99 & 98.42 & WE & & FALSE \\
\hline 2002 & B & 6 & 9 & 4.02 & 98.31 & BIS & & FALSE \\
\hline 2002 & B & 6 & 10 & 4.45 & 98.25 & $\mathrm{TW}$ & & FALSE \\
\hline 2002 & B & 6 & 11 & 5.15 & 98.32 & $\mathrm{BR}$ & & FALSE \\
\hline 2002 & B & 6 & 12 & 5.15 & 98.42 & WE & & FALSE \\
\hline 2002 & B & 6 & 13 & 5.43 & 98.65 & $\mathrm{TR}$ & rock & TRUE \\
\hline 2002 & B & 6 & 14 & 5.70 & 98.55 & $\mathrm{BR}$ & & FALSE \\
\hline 2002 & B & 6 & 15 & 6.43 & 98.65 & BIS & & FALSE \\
\hline 2002 & B & 6 & 16 & 7.01 & 98.91 & $\mathrm{BF}$ & & FALSE \\
\hline 2002 & B & 6 & 17 & 7.99 & 99.87 & BS & & FALSE \\
\hline 2002 & B & 6 & 18 & 7.99 & 100.00 & TSR & & FALSE \\
\hline 2002 & B & 7 & 1 & 0.00 & 100.00 & TSL & & FALSE \\
\hline 2002 & B & 7 & 2 & 0.00 & 99.41 & BS & & FALSE \\
\hline 2002 & B & 7 & 3 & 1.13 & 98.93 & TB & & FALSE \\
\hline 2002 & B & 7 & 4 & 1.22 & 98.67 & $\mathrm{BF}$ & & FALSE \\
\hline 2002 & B & 7 & 5 & 1.28 & 98.55 & $\mathrm{BB}$ & & FALSE \\
\hline 2002 & B & 7 & 6 & 1.98 & 98.21 & BIS & & FALSE \\
\hline 2002 & B & 7 & 7 & 3.05 & 98.07 & BIS & & FALSE \\
\hline 2002 & B & 7 & 8 & 3.41 & 97.99 & WE & & FALSE \\
\hline 2002 & B & 7 & 9 & 4.15 & 97.78 & $\mathrm{TW}$ & & FALSE \\
\hline 2002 & B & 7 & 10 & 4.51 & 97.85 & BIS & & FALSE \\
\hline 2002 & B & 7 & 11 & 4.88 & 97.87 & BIS & & FALSE \\
\hline 2002 & B & 7 & 12 & 5.46 & 98.12 & BIS & & FALSE \\
\hline 2002 & B & 7 & 13 & 5.73 & 97.99 & WE & & FALSE \\
\hline
\end{tabular}

Continued on Next Page... 
Table D.1 - Continued

\begin{tabular}{|c|c|c|c|c|c|c|c|}
\hline Year & Reach & Section & Point & Dist. (m) & Elev. $(\mathrm{m})$ & Notes & Exclude \\
\hline 2002 & B & 7 & 14 & 5.94 & 98.17 & BIS & FALSE \\
\hline 2002 & B & 7 & 15 & 6.40 & 98.34 & BIS & FALSE \\
\hline 2002 & B & 7 & 16 & 6.46 & 98.67 & $\mathrm{BF}$ & FALSE \\
\hline 2002 & B & 7 & 17 & 7.96 & 99.22 & BIS & FALSE \\
\hline 2002 & B & 7 & 18 & 9.54 & 99.75 & $\mathrm{BS}$ & FALSE \\
\hline 2002 & B & 7 & 19 & 9.54 & 100.02 & TSR & FALSE \\
\hline 2002 & B & 8 & 1 & 0.00 & 100.00 & TSL & FALSE \\
\hline 2002 & B & 8 & 2 & 0.00 & 99.55 & BS & FALSE \\
\hline 2002 & B & 8 & 3 & 0.76 & 99.24 & $\mathrm{BF}$ & FALSE \\
\hline 2002 & B & 8 & 4 & 0.79 & 98.84 & BIS & FALSE \\
\hline 2002 & B & 8 & 5 & 1.46 & 98.79 & WE & FALSE \\
\hline 2002 & B & 8 & 6 & 1.68 & 98.74 & BIS & FALSE \\
\hline 2002 & B & 8 & 7 & 2.26 & 98.73 & BIS & FALSE \\
\hline 2002 & B & 8 & 8 & 2.65 & 98.69 & BIS & FALSE \\
\hline 2002 & B & 8 & 9 & 3.02 & 98.66 & $\mathrm{TW}$ & FALSE \\
\hline 2002 & B & 8 & 10 & 3.60 & 98.76 & $\mathrm{BB}$ & FALSE \\
\hline 2002 & B & 8 & 11 & 3.60 & 98.79 & WE & FALSE \\
\hline \multirow[t]{2}{*}{2002} & B & 8 & 12 & 3.72 & 99.07 & BIS - Alder & FALSE \\
\hline & & & & & & Roots & \\
\hline 2002 & B & 8 & 13 & 4.21 & 99.41 & $\mathrm{BF}$ & FALSE \\
\hline 2002 & B & 8 & 14 & 5.55 & 99.65 & BS & FALSE \\
\hline 2002 & B & 8 & 15 & 5.55 & 100.00 & TSR & FALSE \\
\hline 2002 & B & 9 & 1 & 0.00 & 100.00 & TSL & FALSE \\
\hline 2002 & B & 9 & 2 & 0.00 & 99.59 & $\mathrm{BS}$ & FALSE \\
\hline 2002 & B & 9 & 3 & 0.34 & 99.16 & BIS & FALSE \\
\hline 2002 & B & 9 & 4 & 0.58 & 99.07 & BIS & FALSE \\
\hline 2002 & B & 9 & 5 & 0.76 & 97.89 & $\mathrm{BF}$ & FALSE \\
\hline 2002 & B & 9 & 6 & 0.76 & 97.49 & BIS & FALSE \\
\hline 2002 & B & 9 & 7 & 1.01 & 97.35 & $\mathrm{BB} / \mathrm{WE}$ & FALSE \\
\hline 2002 & B & 9 & 8 & 1.65 & 97.35 & BIS & FALSE \\
\hline 2002 & B & 9 & 9 & 2.04 & 97.29 & BIS & FALSE \\
\hline 2002 & B & 9 & 10 & 2.44 & 97.26 & BIS & FALSE \\
\hline 2002 & B & 9 & 11 & 2.68 & 97.20 & $\mathrm{TW}$ & FALSE \\
\hline 2002 & B & 9 & 12 & 3.02 & 97.21 & BIS & FALSE \\
\hline 2002 & B & 9 & 13 & 3.66 & 97.35 & WE & FALSE \\
\hline 2002 & B & 9 & 14 & 3.96 & 97.41 & BIS & FALSE \\
\hline 2002 & B & 9 & 15 & 4.18 & 97.69 & BIS & FALSE \\
\hline 2002 & B & 9 & 16 & 4.45 & 97.89 & $\mathrm{BF}$ & FALSE \\
\hline 2002 & B & 9 & 17 & 6.07 & 98.21 & BIS & FALSE \\
\hline 2002 & B & 9 & 18 & 6.98 & 98.33 & BIS & FALSE \\
\hline 2002 & B & 9 & 19 & 7.62 & 98.59 & BIS & FALSE \\
\hline 2002 & B & 9 & 20 & 9.72 & 98.49 & BIS & FALSE \\
\hline 2002 & B & 9 & 21 & 10.94 & 98.59 & BIS & FALSE \\
\hline 2002 & B & 9 & 22 & 13.78 & 99.93 & BS & FALSE \\
\hline 2002 & B & 9 & 23 & 13.78 & 99.98 & TSR & FALSE \\
\hline 2002 & B & 10 & 1 & 0.00 & 100.00 & TSL & FALSE \\
\hline 2002 & B & 10 & 2 & 0.00 & 99.65 & BS & FALSE \\
\hline 2002 & B & 10 & 3 & 0.18 & 99.57 & TB & FALSE \\
\hline 2002 & B & 10 & 4 & 0.24 & 98.90 & BIS & FALSE \\
\hline 2002 & B & 10 & 5 & 0.52 & 98.59 & BIS & FALSE \\
\hline 2002 & B & 10 & 6 & 0.55 & 98.06 & $\mathrm{BF}$ & FALSE \\
\hline 2002 & B & 10 & 7 & 0.55 & 97.74 & $\mathrm{BB}$ & FALSE \\
\hline
\end{tabular}

Continued on Next Page... 
Table D.1 - Continued

\begin{tabular}{|c|c|c|c|c|c|c|c|c|}
\hline Year & Reach & Section & Point & Dist. (m) & Elev. $(\mathrm{m})$ & Desc. & Notes & Exclude \\
\hline 2002 & B & 10 & 8 & 1.40 & 97.50 & BIS & & FALSE \\
\hline 2002 & B & 10 & 9 & 1.95 & 97.38 & WE & & FALSE \\
\hline 2002 & B & 10 & 10 & 2.50 & 97.23 & BIS & & FALSE \\
\hline 2002 & B & 10 & 11 & 3.17 & 97.23 & TW & & FALSE \\
\hline 2002 & B & 10 & 12 & 3.66 & 97.27 & BIS & & FALSE \\
\hline 2002 & B & 10 & 13 & 4.11 & 97.38 & WE & & FALSE \\
\hline 2002 & B & 10 & 14 & 5.46 & 97.70 & BIS & & FALSE \\
\hline 2002 & B & 10 & 15 & 5.49 & 98.06 & $\mathrm{BF}$ & & FALSE \\
\hline 2002 & B & 10 & 16 & 6.61 & 98.34 & BIS & & FALSE \\
\hline 2002 & B & 10 & 17 & 10.24 & 98.20 & BIS & & FALSE \\
\hline 2002 & B & 10 & 18 & 11.28 & 98.31 & BIS & & FALSE \\
\hline 2002 & B & 10 & 19 & 12.65 & 98.28 & BIS & & FALSE \\
\hline 2002 & B & 10 & 20 & 13.41 & 98.62 & BIS & & FALSE \\
\hline 2002 & B & 10 & 21 & 15.42 & 99.84 & BS & & FALSE \\
\hline 2002 & B & 10 & 22 & 15.42 & 100.00 & TSR & & FALSE \\
\hline 2002 & $\mathrm{C}$ & 1 & 1 & 0.00 & 100.00 & TSL & & FALSE \\
\hline 2002 & $\mathrm{C}$ & 1 & 2 & 0.00 & 99.51 & BS & & FALSE \\
\hline 2002 & $\mathrm{C}$ & 1 & 3 & 0.21 & 99.13 & BIS & & FALSE \\
\hline 2002 & $\mathrm{C}$ & 1 & 4 & 0.61 & 98.95 & BIS & & FALSE \\
\hline 2002 & $\mathrm{C}$ & 1 & 5 & 0.70 & 98.73 & $\mathrm{BF}$ & & FALSE \\
\hline 2002 & $\mathrm{C}$ & 1 & 6 & 1.71 & 98.68 & BIS & & FALSE \\
\hline 2002 & $\mathrm{C}$ & 1 & 7 & 2.26 & 98.48 & BIS & & FALSE \\
\hline 2002 & $\mathrm{C}$ & 1 & 8 & 2.44 & 98.46 & WE & & FALSE \\
\hline 2002 & $\mathrm{C}$ & 1 & 9 & 2.83 & 98.36 & BIS & & FALSE \\
\hline 2002 & C & 1 & 10 & 3.72 & 98.27 & $\mathrm{TW}$ & & FALSE \\
\hline 2002 & $\mathrm{C}$ & 1 & 11 & 4.45 & 98.40 & BIS & & FALSE \\
\hline 2002 & $\mathrm{C}$ & 1 & 12 & 4.88 & 98.35 & BIS & & FALSE \\
\hline 2002 & $\mathrm{C}$ & 1 & 13 & 5.40 & 98.45 & BIS & & FALSE \\
\hline 2002 & $\mathrm{C}$ & 1 & 14 & 5.79 & 98.49 & WE & & FALSE \\
\hline 2002 & $\mathrm{C}$ & 1 & 15 & 6.46 & 98.53 & BIS & & FALSE \\
\hline 2002 & $\mathrm{C}$ & 1 & 16 & 6.92 & 98.64 & $\mathrm{BF}$ & & FALSE \\
\hline 2002 & $\mathrm{C}$ & 1 & 17 & 7.86 & 98.79 & BIS & & FALSE \\
\hline 2002 & $\mathrm{C}$ & 1 & 18 & 8.44 & 98.94 & BIS & & FALSE \\
\hline 2002 & $\mathrm{C}$ & 1 & 19 & 8.78 & 99.07 & BIS & & FALSE \\
\hline 2002 & $\mathrm{C}$ & 1 & 20 & 10.82 & 98.91 & BIS & & FALSE \\
\hline 2002 & $\mathrm{C}$ & 1 & 21 & 11.34 & 99.10 & BIS & & FALSE \\
\hline 2002 & $\mathrm{C}$ & 1 & 22 & 11.55 & 99.18 & BIS & & FALSE \\
\hline 2002 & $\mathrm{C}$ & 1 & 23 & 12.04 & 99.31 & BIS & & FALSE \\
\hline 2002 & $\mathrm{C}$ & 1 & 24 & 12.62 & 99.60 & BS & & FALSE \\
\hline 2002 & $\mathrm{C}$ & 1 & 25 & 12.62 & 100.01 & TSR & & FALSE \\
\hline 2002 & $\mathrm{C}$ & 2 & 1 & 0.00 & 100.00 & TSL & & FALSE \\
\hline 2002 & $\mathrm{C}$ & 2 & 2 & 0.00 & 99.62 & BS & & FALSE \\
\hline 2002 & $\mathrm{C}$ & 2 & 3 & 0.37 & 99.45 & BS & & FALSE \\
\hline 2002 & $\mathrm{C}$ & 2 & 4 & 1.04 & 98.91 & BIS & & FALSE \\
\hline 2002 & $\mathrm{C}$ & 2 & 5 & 2.19 & 98.81 & BIS & & FALSE \\
\hline 2002 & $\mathrm{C}$ & 2 & 6 & 2.93 & 98.62 & BIS & & FALSE \\
\hline 2002 & $\mathrm{C}$ & 2 & 7 & 3.81 & 98.39 & BIS & & FALSE \\
\hline 2002 & $\mathrm{C}$ & 2 & 8 & 5.09 & 98.33 & T Stump & & FALSE \\
\hline 2002 & $\mathrm{C}$ & 2 & 9 & 5.09 & 98.13 & $\mathrm{BF}$ & & FALSE \\
\hline 2002 & $\mathrm{C}$ & 2 & 10 & 5.18 & 97.94 & B Stump & & FALSE \\
\hline 2002 & $\mathrm{C}$ & 2 & 11 & 5.33 & 97.91 & BIS & & FALSE \\
\hline 2002 & C & 2 & 12 & 5.49 & 97.91 & BIS & & FALSE \\
\hline
\end{tabular}

Continued on Next Page... 
APPENDIX D. SURVEY DATA

Table D.1 - Continued

\begin{tabular}{|c|c|c|c|c|c|c|c|c|}
\hline Year & Reach & Section & Point & Dist. (m) & Elev. $(\mathrm{m})$ & Desc. & Notes & Exclude \\
\hline 2002 & $\mathrm{C}$ & 2 & 13 & 5.79 & 97.87 & BIS & & FALSE \\
\hline 2002 & $\mathrm{C}$ & 2 & 14 & 6.19 & 97.81 & WE & & FALSE \\
\hline 2002 & $\mathrm{C}$ & 2 & 15 & 6.52 & 97.70 & BIS & & FALSE \\
\hline 2002 & $\mathrm{C}$ & 2 & 16 & 6.86 & 97.77 & BIS & & FALSE \\
\hline 2002 & $\mathrm{C}$ & 2 & 17 & 7.28 & 97.80 & BIS & & FALSE \\
\hline 2002 & $\mathrm{C}$ & 2 & 18 & 7.80 & 97.73 & BIS & & FALSE \\
\hline 2002 & $\mathrm{C}$ & 2 & 19 & 8.14 & 97.69 & TW & & FALSE \\
\hline 2002 & $\mathrm{C}$ & 2 & 20 & 8.38 & 97.73 & BIS & & FALSE \\
\hline 2002 & $\mathrm{C}$ & 2 & 21 & 8.81 & 97.74 & BIS & & FALSE \\
\hline 2002 & $\mathrm{C}$ & 2 & 22 & 9.14 & 97.75 & BIS & & FALSE \\
\hline 2002 & $\mathrm{C}$ & 2 & 23 & 9.48 & 97.81 & WE & & FALSE \\
\hline 2002 & $\mathrm{C}$ & 2 & 24 & 10.24 & 98.13 & $\mathrm{BF}$ & & FALSE \\
\hline 2002 & $\mathrm{C}$ & 2 & 25 & 10.52 & 98.22 & BIS & & FALSE \\
\hline 2002 & $\mathrm{C}$ & 2 & 26 & 12.38 & 98.29 & B Stump & & FALSE \\
\hline 2002 & $\mathrm{C}$ & 2 & 27 & 12.53 & 99.56 & $\mathrm{BS}$ & & FALSE \\
\hline 2002 & $\mathrm{C}$ & 2 & 28 & 12.53 & 100.00 & TSR & & FALSE \\
\hline 2002 & $\mathrm{C}$ & 3 & 1 & 0.00 & 100.00 & TSL & & FALSE \\
\hline 2002 & $\mathrm{C}$ & 3 & 2 & 0.00 & 99.69 & BS & & FALSE \\
\hline 2002 & $\mathrm{C}$ & 3 & 3 & 0.49 & 99.43 & BIS & & FALSE \\
\hline 2002 & $\mathrm{C}$ & 3 & 4 & 2.10 & 98.70 & BIS & & FALSE \\
\hline 2002 & $\mathrm{C}$ & 3 & 5 & 2.65 & 98.66 & BIS & & FALSE \\
\hline 2002 & $\mathrm{C}$ & 3 & 6 & 3.51 & 98.78 & BIS & & FALSE \\
\hline 2002 & $\mathrm{C}$ & 3 & 7 & 4.21 & 98.77 & BIS & & FALSE \\
\hline 2002 & $\mathrm{C}$ & 3 & 8 & 4.63 & 98.47 & $\mathrm{BF}$ & & FALSE \\
\hline 2002 & $\mathrm{C}$ & 3 & 9 & 4.88 & 98.36 & BIS & & FALSE \\
\hline 2002 & $\mathrm{C}$ & 3 & 10 & 5.12 & 98.40 & BIS & & FALSE \\
\hline 2002 & $\mathrm{C}$ & 3 & 11 & 5.52 & 98.36 & BIS & & FALSE \\
\hline 2002 & $\mathrm{C}$ & 3 & 12 & 6.04 & 98.38 & BIS & & FALSE \\
\hline 2002 & $\mathrm{C}$ & 3 & 13 & 6.83 & 98.25 & WE & & FALSE \\
\hline 2002 & $\mathrm{C}$ & 3 & 14 & 7.25 & 98.17 & BIS & & FALSE \\
\hline 2002 & $\mathrm{C}$ & 3 & 15 & 7.65 & 98.18 & BIS & & FALSE \\
\hline 2002 & $\mathrm{C}$ & 3 & 16 & 7.89 & 98.22 & BIS & & FALSE \\
\hline 2002 & $\mathrm{C}$ & 3 & 17 & 8.32 & 98.11 & TW & & FALSE \\
\hline 2002 & $\mathrm{C}$ & 3 & 18 & 8.69 & 98.20 & BIS & & FALSE \\
\hline 2002 & $\mathrm{C}$ & 3 & 19 & 9.14 & 98.17 & BIS & & FALSE \\
\hline 2002 & $\mathrm{C}$ & 3 & 20 & 9.75 & 98.25 & WE & & FALSE \\
\hline 2002 & $\mathrm{C}$ & 3 & 21 & 10.24 & 98.46 & $\mathrm{BF}$ & & FALSE \\
\hline 2002 & $\mathrm{C}$ & 3 & 22 & 10.39 & 98.65 & BIS & & FALSE \\
\hline 2002 & $\mathrm{C}$ & 3 & 23 & 12.44 & 99.04 & BIS & & FALSE \\
\hline 2002 & $\mathrm{C}$ & 3 & 24 & 14.02 & 99.70 & BS & & FALSE \\
\hline 2002 & $\mathrm{C}$ & 3 & 25 & 14.02 & 99.99 & TSR & & FALSE \\
\hline 2002 & $\mathrm{C}$ & 4 & 1 & 0.00 & 100.00 & TSL & & FALSE \\
\hline 2002 & $\mathrm{C}$ & 4 & 2 & 0.00 & 99.70 & BS & & FALSE \\
\hline 2002 & $\mathrm{C}$ & 4 & 3 & 0.58 & 99.50 & BIS & & FALSE \\
\hline 2002 & $\mathrm{C}$ & 4 & 4 & 0.91 & 98.97 & $\mathrm{~T}$ roots & & FALSE \\
\hline 2002 & $\mathrm{C}$ & 4 & 5 & 1.98 & 98.77 & $\mathrm{~T}$ roots & & FALSE \\
\hline 2002 & $\mathrm{C}$ & 4 & 6 & 2.01 & 98.50 & B roots & & FALSE \\
\hline 2002 & $\mathrm{C}$ & 4 & 7 & 3.17 & 98.41 & BIS & & FALSE \\
\hline 2002 & $\mathrm{C}$ & 4 & 8 & 3.66 & 98.54 & BIS & & FALSE \\
\hline 2002 & $\mathrm{C}$ & 4 & 9 & 3.93 & 98.60 & BIS & & FALSE \\
\hline 2002 & $\mathrm{C}$ & 4 & 10 & 4.24 & 98.57 & BIS & & FALSE \\
\hline 2002 & C & 4 & 11 & 4.51 & 98.45 & $\mathrm{BF}$ & & FALSE \\
\hline
\end{tabular}

Continued on Next Page... 
APPENDIX D. SURVEY DATA

Table D.1 - Continued

\begin{tabular}{|c|c|c|c|c|c|c|c|c|}
\hline Year & Reach & Section & Point & Dist. (m) & Elev. $(\mathrm{m})$ & Desc. & Notes & Exclude \\
\hline 2002 & $\mathrm{C}$ & 4 & 12 & 5.06 & 98.31 & BIS & & FALSE \\
\hline 2002 & $\mathrm{C}$ & 4 & 13 & 5.46 & 98.23 & BIS & & FALSE \\
\hline 2002 & $\mathrm{C}$ & 4 & 14 & 5.88 & 98.30 & BIS & & FALSE \\
\hline 2002 & $\mathrm{C}$ & 4 & 15 & 6.43 & 98.29 & BIS & & FALSE \\
\hline 2002 & $\mathrm{C}$ & 4 & 16 & 6.92 & 98.20 & WE & & FALSE \\
\hline 2002 & $\mathrm{C}$ & 4 & 17 & 7.47 & 98.08 & BIS & & FALSE \\
\hline 2002 & $\mathrm{C}$ & 4 & 18 & 7.83 & 98.11 & BIS & & FALSE \\
\hline 2002 & $\mathrm{C}$ & 4 & 19 & 8.11 & 98.12 & BIS & & FALSE \\
\hline 2002 & $\mathrm{C}$ & 4 & 20 & 8.47 & 98.09 & BIS & & FALSE \\
\hline 2002 & $\mathrm{C}$ & 4 & 21 & 8.90 & 98.13 & BIS & & FALSE \\
\hline 2002 & $\mathrm{C}$ & 4 & 22 & 9.33 & 98.20 & WE & & FALSE \\
\hline 2002 & $\mathrm{C}$ & 4 & 23 & 9.72 & 98.39 & $\mathrm{BF}$ & & FALSE \\
\hline 2002 & $\mathrm{C}$ & 4 & 24 & 10.12 & 98.85 & BIS & & FALSE \\
\hline 2002 & $\mathrm{C}$ & 4 & 25 & 11.55 & 98.97 & BIS & & FALSE \\
\hline 2002 & $\mathrm{C}$ & 4 & 26 & 11.89 & 99.67 & BIS & & FALSE \\
\hline 2002 & $\mathrm{C}$ & 4 & 27 & 12.04 & 99.73 & BS & & FALSE \\
\hline 2002 & $\mathrm{C}$ & 4 & 28 & 12.04 & 100.02 & TSR & & FALSE \\
\hline 2002 & $\mathrm{C}$ & 5 & 1 & 0.00 & 100.00 & TSL & & FALSE \\
\hline 2002 & $\mathrm{C}$ & 5 & 2 & 0.00 & 99.55 & BS & & FALSE \\
\hline 2002 & $\mathrm{C}$ & 5 & 3 & 0.67 & 99.35 & BIS & $\begin{array}{l}\text { elevation lowered } 1.2 \text { feet, likely } \\
\text { on } \log d p\end{array}$ & FALSE \\
\hline 2002 & $\mathrm{C}$ & 5 & 4 & 1.92 & 98.88 & BIS & & FALSE \\
\hline 2002 & $\mathrm{C}$ & 5 & 5 & 2.29 & 98.66 & BIS & & FALSE \\
\hline 2002 & $\mathrm{C}$ & 5 & 6 & 4.79 & 98.42 & $\mathrm{BF}$ & & FALSE \\
\hline 2002 & $\mathrm{C}$ & 5 & 7 & 4.94 & 98.47 & BIS & & FALSE \\
\hline 2002 & $\mathrm{C}$ & 5 & 8 & 6.49 & 98.38 & BIS & & FALSE \\
\hline 2002 & $\mathrm{C}$ & 5 & 9 & 6.64 & 98.32 & BIS & & FALSE \\
\hline 2002 & $\mathrm{C}$ & 5 & 10 & 7.22 & 98.24 & WE & & FALSE \\
\hline 2002 & $\mathrm{C}$ & 5 & 11 & 7.74 & 98.18 & BIS & & FALSE \\
\hline 2002 & $\mathrm{C}$ & 5 & 12 & 8.53 & 97.92 & TW & & FALSE \\
\hline 2002 & $\mathrm{C}$ & 5 & 13 & 9.36 & 98.24 & WE & & FALSE \\
\hline 2002 & $\mathrm{C}$ & 5 & 14 & 9.57 & 98.25 & BIS & & FALSE \\
\hline 2002 & $\mathrm{C}$ & 5 & 15 & 9.81 & 98.35 & $\mathrm{BF}$ & & FALSE \\
\hline 2002 & $\mathrm{C}$ & 5 & 16 & 10.21 & 98.76 & BIS & & FALSE \\
\hline 2002 & $\mathrm{C}$ & 5 & 17 & 11.40 & 98.75 & BIS & & FALSE \\
\hline 2002 & $\mathrm{C}$ & 5 & 18 & 11.80 & 99.05 & BIS & & FALSE \\
\hline 2002 & $\mathrm{C}$ & 5 & 19 & 12.38 & 99.48 & BS & & FALSE \\
\hline 2002 & C & 5 & 20 & 12.38 & 99.99 & TSR & & FALSE \\
\hline 2002 & $\mathrm{C}$ & 6 & 1 & 0.00 & 100.00 & TSL & & FALSE \\
\hline 2002 & $\mathrm{C}$ & 6 & 2 & 0.00 & 99.70 & BS & & FALSE \\
\hline 2002 & $\mathrm{C}$ & 6 & 3 & 0.55 & 99.10 & BIS & & FALSE \\
\hline 2002 & $\mathrm{C}$ & 6 & 4 & 2.23 & 98.74 & $\mathrm{BF}$ & & FALSE \\
\hline 2002 & $\mathrm{C}$ & 6 & 5 & 2.38 & 98.58 & BIS & & FALSE \\
\hline 2002 & $\mathrm{C}$ & 6 & 6 & 3.35 & 98.18 & BIS & & FALSE \\
\hline 2002 & $\mathrm{C}$ & 6 & 7 & 4.57 & 98.06 & BIS & & FALSE \\
\hline 2002 & $\mathrm{C}$ & 6 & 8 & 5.67 & 97.99 & WE & & FALSE \\
\hline 2002 & $\mathrm{C}$ & 6 & 9 & 6.07 & 97.88 & TW & & FALSE \\
\hline 2002 & $\mathrm{C}$ & 6 & 10 & 6.22 & 98.01 & BIS & & FALSE \\
\hline 2002 & $\mathrm{C}$ & 6 & 11 & 6.61 & 97.92 & BIS & & FALSE \\
\hline 2002 & $\mathrm{C}$ & 6 & 12 & 6.95 & 97.96 & BIS & & FALSE \\
\hline 2002 & $\mathrm{C}$ & 6 & 13 & 7.53 & 97.99 & WE & & FALSE \\
\hline 2002 & $\mathrm{C}$ & 6 & 14 & 8.78 & 98.35 & BIS & & FALSE \\
\hline
\end{tabular}

Continued on Next Page... 
APPENDIX D. SURVEY DATA

Table D.1 - Continued

\begin{tabular}{|c|c|c|c|c|c|c|c|c|}
\hline Year & Reach & Section & Point & Dist. (m) & Elev. $(\mathrm{m})$ & Desc. & Notes & Exclude \\
\hline 2002 & $\mathrm{C}$ & 6 & 15 & 8.99 & 98.72 & $\mathrm{BF}$ & Tree Trunk Adjacent & FALSE \\
\hline 2002 & C & 6 & 16 & 10.06 & 98.75 & $\mathrm{BR}$ & & FALSE \\
\hline 2002 & $\mathrm{C}$ & 6 & 17 & 10.09 & 98.94 & $\mathrm{TR}$ & & FALSE \\
\hline 2002 & $\mathrm{C}$ & 6 & 18 & 10.64 & 99.08 & $\mathrm{TR}$ & & FALSE \\
\hline 2002 & $\mathrm{C}$ & 6 & 19 & 11.43 & 99.40 & TR & & FALSE \\
\hline 2002 & $\mathrm{C}$ & 6 & 20 & 11.67 & 99.29 & $\mathrm{TR}$ & & FALSE \\
\hline 2002 & $\mathrm{C}$ & 6 & 21 & 11.73 & 99.07 & $\mathrm{BR}$ & & FALSE \\
\hline 2002 & $\mathrm{C}$ & 6 & 22 & 11.95 & 99.00 & BIS & & FALSE \\
\hline 2002 & $\mathrm{C}$ & 6 & 23 & 12.16 & 99.00 & BIS & & FALSE \\
\hline 2002 & $\mathrm{C}$ & 6 & 24 & 12.53 & 99.21 & BIS & & FALSE \\
\hline 2002 & $\mathrm{C}$ & 6 & 25 & 13.05 & 99.71 & BS & & FALSE \\
\hline 2002 & $\mathrm{C}$ & 6 & 26 & 13.05 & 100.01 & TSR & & FALSE \\
\hline 2002 & $\mathrm{C}$ & 7 & 1 & 0.00 & 100.00 & TSL & & FALSE \\
\hline 2002 & $\mathrm{C}$ & 7 & 2 & 0.00 & 99.77 & BS & & FALSE \\
\hline 2002 & $\mathrm{C}$ & 7 & 3 & 0.73 & 99.43 & BIS & & FALSE \\
\hline 2002 & $\mathrm{C}$ & 7 & 4 & 1.22 & 98.90 & BIS & & FALSE \\
\hline 2002 & $\mathrm{C}$ & 7 & 5 & 2.38 & 98.84 & BIS & & FALSE \\
\hline 2002 & $\mathrm{C}$ & 7 & 6 & 2.41 & 98.63 & $\mathrm{BF}$ & & FALSE \\
\hline 2002 & $\mathrm{C}$ & 7 & 7 & 2.65 & 98.56 & BIS & & FALSE \\
\hline 2002 & $\mathrm{C}$ & 7 & 8 & 2.74 & 98.34 & BIS & & FALSE \\
\hline 2002 & $\mathrm{C}$ & 7 & 9 & 3.90 & 98.15 & WE & & FALSE \\
\hline 2002 & $\mathrm{C}$ & 7 & 10 & 4.48 & 98.06 & BIS & & FALSE \\
\hline 2002 & $\mathrm{C}$ & 7 & 11 & 5.24 & 97.97 & TW & & FALSE \\
\hline 2002 & $\mathrm{C}$ & 7 & 12 & 5.61 & 98.03 & BIS & & FALSE \\
\hline 2002 & $\mathrm{C}$ & 7 & 13 & 6.07 & 98.03 & BIS & & FALSE \\
\hline 2002 & $\mathrm{C}$ & 7 & 14 & 6.55 & 98.15 & WE & & FALSE \\
\hline 2002 & $\mathrm{C}$ & 7 & 15 & 6.89 & 98.34 & BIS & & FALSE \\
\hline 2002 & $\mathrm{C}$ & 7 & 16 & 7.56 & 98.74 & $\mathrm{BF}$ & & FALSE \\
\hline 2002 & $\mathrm{C}$ & 7 & 17 & 7.59 & 99.09 & BIS & & FALSE \\
\hline 2002 & $\mathrm{C}$ & 7 & 18 & 8.23 & 99.13 & $\mathrm{BL}$ & & FALSE \\
\hline 2002 & $\mathrm{C}$ & 7 & 19 & 8.23 & 99.25 & $\mathrm{TL}$ & & FALSE \\
\hline 2002 & $\mathrm{C}$ & 7 & 20 & 8.53 & 99.22 & TL & & FALSE \\
\hline 2002 & $\mathrm{C}$ & 7 & 21 & 8.56 & 99.00 & $\mathrm{BL}$ & & FALSE \\
\hline 2002 & $\mathrm{C}$ & 7 & 22 & 9.21 & 98.84 & BIS & & FALSE \\
\hline 2002 & $\mathrm{C}$ & 7 & 23 & 10.85 & 98.63 & BIS & & FALSE \\
\hline 2002 & $\mathrm{C}$ & 7 & 24 & 11.22 & 98.70 & BIS & & FALSE \\
\hline 2002 & $\mathrm{C}$ & 7 & 25 & 12.68 & 99.19 & BIS & & FALSE \\
\hline 2002 & $\mathrm{C}$ & 7 & 26 & 13.38 & 99.83 & BS & & FALSE \\
\hline 2002 & $\mathrm{C}$ & 7 & 27 & 13.38 & 100.02 & TSR & & FALSE \\
\hline 2002 & $\mathrm{C}$ & 8 & 1 & 0.00 & 100.00 & TSL & & FALSE \\
\hline 2002 & $\mathrm{C}$ & 8 & 2 & 0.00 & 99.55 & BS & & FALSE \\
\hline 2002 & $\mathrm{C}$ & 8 & 3 & 0.12 & 99.52 & BIS & & FALSE \\
\hline 2002 & $\mathrm{C}$ & 8 & 4 & 0.30 & 98.68 & $\mathrm{BF}$ & & FALSE \\
\hline 2002 & $\mathrm{C}$ & 8 & 5 & 0.37 & 98.34 & BIS & & FALSE \\
\hline 2002 & $\mathrm{C}$ & 8 & 6 & 0.64 & 98.23 & BIS & & FALSE \\
\hline 2002 & $\mathrm{C}$ & 8 & 7 & 1.68 & 97.99 & BIS & & FALSE \\
\hline 2002 & $\mathrm{C}$ & 8 & 8 & 2.13 & 97.88 & WE & & FALSE \\
\hline 2002 & $\mathrm{C}$ & 8 & 9 & 2.71 & 97.70 & $\mathrm{TW}$ & & FALSE \\
\hline 2002 & $\mathrm{C}$ & 8 & 10 & 3.02 & 97.73 & BIS & & FALSE \\
\hline 2002 & $\mathrm{C}$ & 8 & 11 & 3.78 & 97.75 & BIS & & FALSE \\
\hline 2002 & $\mathrm{C}$ & 8 & 12 & 4.15 & 97.81 & $\mathrm{BL}$ & & FALSE \\
\hline 2002 & C & 8 & 13 & 4.15 & 97.88 & WE & & FALSE \\
\hline
\end{tabular}

Continued on Next Page... 
APPENDIX D. SURVEY DATA

Table D.1 - Continued

\begin{tabular}{|c|c|c|c|c|c|c|c|}
\hline Year & Reach & Section & Point & Dist. (m) & Elev. $(\mathrm{m})$ & Notes & Exclude \\
\hline 2002 & $\mathrm{C}$ & 8 & 14 & 4.18 & 98.21 & TL & FALSE \\
\hline 2002 & C & 8 & 15 & 4.72 & 98.35 & BIS & FALSE \\
\hline 2002 & $\mathrm{C}$ & 8 & 16 & 5.58 & 98.68 & $\mathrm{BF}$ & FALSE \\
\hline 2002 & $\mathrm{C}$ & 8 & 17 & 5.76 & 99.09 & BIS & FALSE \\
\hline 2002 & $\mathrm{C}$ & 8 & 18 & 8.99 & 98.84 & BIS & FALSE \\
\hline 2002 & $\mathrm{C}$ & 8 & 19 & 9.14 & 98.89 & BIS & FALSE \\
\hline 2002 & $\mathrm{C}$ & 8 & 20 & 9.63 & 98.76 & BIS & FALSE \\
\hline 2002 & $\mathrm{C}$ & 8 & 21 & 10.33 & 98.76 & BIS & FALSE \\
\hline 2002 & $\mathrm{C}$ & 8 & 22 & 10.79 & 98.60 & BIS & FALSE \\
\hline 2002 & $\mathrm{C}$ & 8 & 23 & 11.89 & 98.60 & BIS & FALSE \\
\hline 2002 & $\mathrm{C}$ & 8 & 24 & 12.98 & 98.95 & BIS & FALSE \\
\hline 2002 & $\mathrm{C}$ & 8 & 25 & 13.72 & 99.51 & BIS & FALSE \\
\hline 2002 & $\mathrm{C}$ & 8 & 26 & 14.05 & 99.66 & BS & FALSE \\
\hline 2002 & $\mathrm{C}$ & 8 & 27 & 14.05 & 100.03 & TSR & FALSE \\
\hline 2002 & $\mathrm{C}$ & 9 & 1 & 0.00 & 100.00 & $\begin{array}{l}\text { BS (temp) - } \\
\text { must replace }\end{array}$ & FALSE \\
\hline 2002 & $\mathrm{C}$ & 9 & 2 & 0.30 & 98.86 & BIS & FALSE \\
\hline 2002 & $\mathrm{C}$ & 9 & 3 & 0.85 & 98.42 & $\mathrm{BF}$ & FALSE \\
\hline 2002 & $\mathrm{C}$ & 9 & 4 & 1.28 & 98.05 & WE & FALSE \\
\hline 2002 & $\mathrm{C}$ & 9 & 5 & 1.77 & 97.92 & BIS & FALSE \\
\hline 2002 & $\mathrm{C}$ & 9 & 6 & 2.13 & 97.85 & BIS & FALSE \\
\hline 2002 & $\mathrm{C}$ & 9 & 7 & 2.53 & 97.91 & BIS & FALSE \\
\hline 2002 & $\mathrm{C}$ & 9 & 8 & 2.93 & 98.03 & BIS & FALSE \\
\hline 2002 & $\mathrm{C}$ & 9 & 9 & 3.60 & 97.98 & BIS & FALSE \\
\hline 2002 & $\mathrm{C}$ & 9 & 10 & 4.11 & 97.97 & BIS & FALSE \\
\hline 2002 & $\mathrm{C}$ & 9 & 11 & 4.45 & 97.77 & TW & FALSE \\
\hline 2002 & $\mathrm{C}$ & 9 & 12 & 4.72 & 97.80 & BIS & FALSE \\
\hline 2002 & $\mathrm{C}$ & 9 & 13 & 5.09 & 98.05 & WE & FALSE \\
\hline 2002 & $\mathrm{C}$ & 9 & 14 & 5.94 & 98.42 & $\mathrm{BF}$ & FALSE \\
\hline 2002 & $\mathrm{C}$ & 9 & 15 & 6.13 & 98.81 & BIS & FALSE \\
\hline 2002 & $\mathrm{C}$ & 9 & 16 & 7.07 & 98.90 & BIS & FALSE \\
\hline 2002 & $\mathrm{C}$ & 9 & 17 & 7.47 & 99.05 & BIS & FALSE \\
\hline 2002 & $\mathrm{C}$ & 9 & 18 & 10.97 & 98.86 & BIS & FALSE \\
\hline 2002 & $\mathrm{C}$ & 9 & 19 & 12.19 & 98.77 & BIS & FALSE \\
\hline 2002 & $\mathrm{C}$ & 9 & 20 & 13.90 & 98.81 & BIS & FALSE \\
\hline 2002 & $\mathrm{C}$ & 9 & 21 & 14.94 & 98.99 & BIS & FALSE \\
\hline 2002 & $\mathrm{C}$ & 9 & 22 & 15.48 & 99.34 & BIS & FALSE \\
\hline 2002 & $\mathrm{C}$ & 9 & 23 & 16.12 & 99.73 & BS & FALSE \\
\hline 2002 & C & 9 & 24 & 16.12 & 99.94 & TSR & FALSE \\
\hline 2002 & $\mathrm{C}$ & 10 & 1 & 0.00 & 100.00 & TSL & FALSE \\
\hline 2002 & $\mathrm{C}$ & 10 & 2 & 0.00 & 99.75 & BS & FALSE \\
\hline 2002 & $\mathrm{C}$ & 10 & 3 & 0.79 & 99.11 & BIS & FALSE \\
\hline 2002 & $\mathrm{C}$ & 10 & 4 & 2.23 & 98.60 & BIS & FALSE \\
\hline 2002 & $\mathrm{C}$ & 10 & 5 & 2.35 & 98.30 & $\mathrm{BF}$ & FALSE \\
\hline 2002 & $\mathrm{C}$ & 10 & 6 & 2.41 & 98.06 & BIS & FALSE \\
\hline 2002 & $\mathrm{C}$ & 10 & 7 & 3.26 & 98.17 & BIS & FALSE \\
\hline 2002 & $\mathrm{C}$ & 10 & 8 & 4.39 & 97.89 & $\mathrm{BR}$ & FALSE \\
\hline 2002 & $\mathrm{C}$ & 10 & 9 & 4.66 & 98.10 & $\mathrm{TR}$ & FALSE \\
\hline 2002 & $\mathrm{C}$ & 10 & 10 & 4.94 & 97.57 & WE & FALSE \\
\hline 2002 & $\mathrm{C}$ & 10 & 11 & 4.94 & 97.45 & $\mathrm{BR}$ & FALSE \\
\hline 2002 & $\mathrm{C}$ & 10 & 12 & 5.79 & 97.39 & BIS & FALSE \\
\hline 2002 & C & 10 & 13 & 6.10 & 97.39 & BIS & FALSE \\
\hline
\end{tabular}

Continued on Next Page... 
Table D.1 - Continued

\begin{tabular}{|c|c|c|c|c|c|c|c|c|}
\hline Year & Reach & Section & Point & Dist. (m) & Elev. $(\mathrm{m})$ & Desc. & Notes & Exclude \\
\hline 2002 & $\mathrm{C}$ & 10 & 14 & 7.35 & 97.36 & TW & & FALSE \\
\hline 2002 & $\mathrm{C}$ & 10 & 15 & 8.32 & 97.49 & WE & & FALSE \\
\hline 2002 & $\mathrm{C}$ & 10 & 16 & 9.02 & 97.82 & BIS & & FALSE \\
\hline 2002 & $\mathrm{C}$ & 10 & 17 & 10.09 & 98.11 & $\mathrm{BF}$ & & FALSE \\
\hline 2002 & $\mathrm{C}$ & 10 & 18 & 10.24 & 98.56 & BIS & & FALSE \\
\hline 2002 & $\mathrm{C}$ & 10 & 19 & 11.06 & 98.52 & BIS & & FALSE \\
\hline 2002 & $\mathrm{C}$ & 10 & 20 & 11.49 & 98.60 & BIS & & FALSE \\
\hline 2002 & $\mathrm{C}$ & 10 & 21 & 11.52 & 98.49 & BIS & & FALSE \\
\hline 2002 & $\mathrm{C}$ & 10 & 22 & 11.86 & 98.49 & BIS & & FALSE \\
\hline 2002 & $\mathrm{C}$ & 10 & 23 & 11.89 & 98.59 & BIS & & FALSE \\
\hline 2002 & $\mathrm{C}$ & 10 & 24 & 12.28 & 98.66 & BIS & & FALSE \\
\hline 2002 & $\mathrm{C}$ & 10 & 25 & 12.71 & 98.72 & BIS & & FALSE \\
\hline 2002 & $\mathrm{C}$ & 10 & 26 & 13.26 & 98.82 & BIS & & FALSE \\
\hline 2002 & $\mathrm{C}$ & 10 & 27 & 15.73 & 98.77 & BIS & & FALSE \\
\hline 2002 & $\mathrm{C}$ & 10 & 28 & 16.46 & 99.33 & BIS & & FALSE \\
\hline 2002 & $\mathrm{C}$ & 10 & 29 & 17.13 & 99.94 & $\mathrm{BS}$ & & FALSE \\
\hline 2002 & $\mathrm{C}$ & 10 & 30 & 17.13 & 99.98 & TSR & & FALSE \\
\hline 2002 & $\mathrm{D}$ & 1 & 1 & 0.00 & 100.00 & TSL & & FALSE \\
\hline 2002 & $\mathrm{D}$ & 1 & 2 & 0.00 & 99.82 & BS & & FALSE \\
\hline 2002 & $\mathrm{D}$ & 1 & 3 & 0.58 & 99.72 & TB & & FALSE \\
\hline 2002 & $\mathrm{D}$ & 1 & 4 & 0.82 & 99.25 & BIS & & FALSE \\
\hline 2002 & $\mathrm{D}$ & 1 & 5 & 0.88 & 98.66 & BIS & & FALSE \\
\hline 2002 & $\mathrm{D}$ & 1 & 6 & 1.13 & 98.52 & $\mathrm{BF}$ & & FALSE \\
\hline 2002 & $\mathrm{D}$ & 1 & 7 & 1.25 & 98.28 & BIS & & FALSE \\
\hline 2002 & $\mathrm{D}$ & 1 & 8 & 1.98 & 97.92 & WE & & FALSE \\
\hline 2002 & $\mathrm{D}$ & 1 & 9 & 2.62 & 97.86 & BIS & & FALSE \\
\hline 2002 & $\mathrm{D}$ & 1 & 10 & 3.17 & 97.78 & BIS & & FALSE \\
\hline 2002 & $\mathrm{D}$ & 1 & 11 & 3.47 & 97.69 & $\mathrm{TW}$ & & FALSE \\
\hline 2002 & $\mathrm{D}$ & 1 & 12 & 4.11 & 97.87 & $\mathrm{BR}$ & & FALSE \\
\hline 2002 & $\mathrm{D}$ & 1 & 13 & 4.11 & 97.92 & WE & & FALSE \\
\hline 2002 & $\mathrm{D}$ & 1 & 14 & 4.33 & 98.13 & $\mathrm{TR}$ & & FALSE \\
\hline 2002 & $\mathrm{D}$ & 1 & 15 & 4.79 & 98.15 & $\mathrm{BR}$ & & FALSE \\
\hline 2002 & $\mathrm{D}$ & 1 & 16 & 5.21 & 98.28 & $\mathrm{TR}$ & & FALSE \\
\hline 2002 & $\mathrm{D}$ & 1 & 17 & 5.73 & 98.28 & BIS & & FALSE \\
\hline 2002 & $\mathrm{D}$ & 1 & 18 & 6.28 & 98.48 & $\mathrm{BF}$ & & FALSE \\
\hline 2002 & $\mathrm{D}$ & 1 & 19 & 6.95 & 99.07 & BIS & & FALSE \\
\hline 2002 & $\mathrm{D}$ & 1 & 20 & 7.89 & 99.26 & BIS & & FALSE \\
\hline 2002 & $\mathrm{D}$ & 1 & 21 & 8.17 & 99.60 & TB & & FALSE \\
\hline 2002 & $\mathrm{D}$ & 1 & 22 & 9.60 & 99.71 & BIS & & FALSE \\
\hline 2002 & $\mathrm{D}$ & 1 & 23 & 10.12 & 99.96 & BS & & FALSE \\
\hline 2002 & $\mathrm{D}$ & 1 & 24 & 10.12 & 99.99 & $\mathrm{TS}$ & & FALSE \\
\hline 2002 & $\mathrm{D}$ & 2 & 1 & 0.00 & 100.00 & TSL & & FALSE \\
\hline 2002 & $\mathrm{D}$ & 2 & 2 & 0.00 & 99.70 & BS & & FALSE \\
\hline 2002 & $\mathrm{D}$ & 2 & 3 & 0.64 & 99.69 & TB & & FALSE \\
\hline 2002 & $\mathrm{D}$ & 2 & 4 & 0.79 & 99.29 & BIS & & FALSE \\
\hline 2002 & $\mathrm{D}$ & 2 & 5 & 1.16 & 99.16 & $\mathrm{BF}$ & & FALSE \\
\hline 2002 & $\mathrm{D}$ & 2 & 6 & 1.65 & 98.75 & BIS & & FALSE \\
\hline 2002 & $\mathrm{D}$ & 2 & 7 & 2.35 & 98.53 & WE & & FALSE \\
\hline 2002 & $\mathrm{D}$ & 2 & 8 & 2.96 & 98.49 & BIS & & FALSE \\
\hline 2002 & $\mathrm{D}$ & 2 & 9 & 3.14 & 98.48 & BIS & & FALSE \\
\hline 2002 & $\mathrm{D}$ & 2 & 10 & 3.35 & 98.45 & BIS & & FALSE \\
\hline 2002 & $\mathrm{D}$ & 2 & 11 & 3.63 & 98.47 & BIS & & FALSE \\
\hline
\end{tabular}

Continued on Next Page... 
Table D.1 - Continued

\begin{tabular}{|c|c|c|c|c|c|c|c|c|}
\hline Year & Reach & Section & Point & Dist. $(\mathrm{m})$ & Elev. $(\mathrm{m})$ & Desc. & Notes & Exclude \\
\hline 2002 & $\mathrm{D}$ & 2 & 12 & 4.15 & 98.43 & TW & & FALSE \\
\hline 2002 & $\mathrm{D}$ & 2 & 13 & 4.72 & 98.53 & WE & & FALSE \\
\hline 2002 & $\mathrm{D}$ & 2 & 14 & 5.27 & 98.70 & BIS & & FALSE \\
\hline 2002 & $\mathrm{D}$ & 2 & 15 & 5.79 & 98.94 & BIS & & FALSE \\
\hline 2002 & $\mathrm{D}$ & 2 & 16 & 6.58 & 99.16 & $\mathrm{BF}$ & & FALSE \\
\hline 2002 & $\mathrm{D}$ & 2 & 17 & 7.53 & 99.66 & TB & & FALSE \\
\hline 2002 & $\mathrm{D}$ & 2 & 18 & 9.39 & 99.76 & BS & & FALSE \\
\hline 2002 & $\mathrm{D}$ & 2 & 19 & 9.39 & 100.00 & TSR & & FALSE \\
\hline 2002 & $\mathrm{D}$ & 3 & 1 & 0.00 & 100.00 & TSL & & FALSE \\
\hline 2002 & $\mathrm{D}$ & 3 & 2 & 0.00 & 99.79 & BS & & FALSE \\
\hline 2002 & $\mathrm{D}$ & 3 & 3 & 0.52 & 99.69 & BIS & & FALSE \\
\hline 2002 & $\mathrm{D}$ & 3 & 4 & 1.13 & 99.78 & TB & & FALSE \\
\hline 2002 & $\mathrm{D}$ & 3 & 5 & 1.40 & 99.15 & $\mathrm{BF}$ & & FALSE \\
\hline 2002 & $\mathrm{D}$ & 3 & 6 & 1.65 & 98.81 & $\mathrm{BB}$ & & FALSE \\
\hline 2002 & $\mathrm{D}$ & 3 & 7 & 2.13 & 98.66 & BIS & & FALSE \\
\hline 2002 & $\mathrm{D}$ & 3 & 8 & 2.71 & 98.55 & WE & & FALSE \\
\hline 2002 & $\mathrm{D}$ & 3 & 9 & 2.83 & 98.50 & BIS & & FALSE \\
\hline 2002 & $\mathrm{D}$ & 3 & 10 & 3.05 & 98.52 & BIS & & FALSE \\
\hline 2002 & $\mathrm{D}$ & 3 & 11 & 3.72 & 98.37 & TW & & FALSE \\
\hline 2002 & $\mathrm{D}$ & 3 & 12 & 4.45 & 98.55 & WE & & FALSE \\
\hline 2002 & $\mathrm{D}$ & 3 & 13 & 4.85 & 98.61 & BIS & & FALSE \\
\hline 2002 & $\mathrm{D}$ & 3 & 14 & 5.09 & 99.16 & $\mathrm{BF}$ & & FALSE \\
\hline 2002 & $\mathrm{D}$ & 3 & 15 & 6.10 & 99.83 & TB & & FALSE \\
\hline 2002 & $\mathrm{D}$ & 3 & 16 & 6.92 & 99.88 & BIS & & FALSE \\
\hline 2002 & $\mathrm{D}$ & 3 & 17 & 7.16 & 99.24 & BIS & & FALSE \\
\hline 2002 & $\mathrm{D}$ & 3 & 18 & 7.80 & 99.10 & BIS & & FALSE \\
\hline 2002 & $\mathrm{D}$ & 3 & 19 & 8.29 & 99.23 & BIS & & FALSE \\
\hline 2002 & $\mathrm{D}$ & 3 & 20 & 10.45 & 99.43 & BIS & & FALSE \\
\hline 2002 & $\mathrm{D}$ & 3 & 21 & 11.03 & 99.88 & $\mathrm{BS}$ & & FALSE \\
\hline 2002 & $\mathrm{D}$ & 3 & 22 & 11.03 & 100.00 & TSR & & FALSE \\
\hline 2002 & $\mathrm{D}$ & 5 & 1 & 0.00 & 100.00 & TSL & & FALSE \\
\hline 2002 & $\mathrm{D}$ & 5 & 2 & 0.00 & 99.62 & BS & & FALSE \\
\hline 2002 & $\mathrm{D}$ & 5 & 3 & 0.37 & 99.46 & TB & & FALSE \\
\hline 2002 & $\mathrm{D}$ & 5 & 4 & 0.43 & 99.16 & BIS & & FALSE \\
\hline 2002 & $\mathrm{D}$ & 5 & 5 & 0.79 & 99.02 & BIS & & FALSE \\
\hline 2002 & $\mathrm{D}$ & 5 & 6 & 0.85 & 98.41 & $\mathrm{BF}$ & & FALSE \\
\hline 2002 & $\mathrm{D}$ & 5 & 7 & 0.85 & 98.32 & $\mathrm{BB}$ & & FALSE \\
\hline 2002 & $\mathrm{D}$ & 5 & 8 & 2.41 & 98.10 & BIS & & FALSE \\
\hline 2002 & $\mathrm{D}$ & 5 & 9 & 3.14 & 98.03 & BIS & & FALSE \\
\hline 2002 & $\mathrm{D}$ & 5 & 10 & 3.51 & 98.06 & BIS & & FALSE \\
\hline 2002 & $\mathrm{D}$ & 5 & 11 & 3.66 & 98.03 & WE & & FALSE \\
\hline 2002 & $\mathrm{D}$ & 5 & 12 & 4.21 & 97.90 & BIS & & FALSE \\
\hline 2002 & $\mathrm{D}$ & 5 & 13 & 4.63 & 97.96 & BIS & & FALSE \\
\hline 2002 & $\mathrm{D}$ & 5 & 14 & 5.21 & 98.03 & WE & & FALSE \\
\hline 2002 & $\mathrm{D}$ & 5 & 15 & 5.91 & 98.32 & BIS & & FALSE \\
\hline 2002 & $\mathrm{D}$ & 5 & 16 & 6.64 & 98.60 & $B R$ & & FALSE \\
\hline 2002 & $\mathrm{D}$ & 5 & 17 & 6.89 & 98.94 & $\mathrm{TR}$ & & FALSE \\
\hline 2002 & $\mathrm{D}$ & 5 & 18 & 7.22 & 99.05 & $\mathrm{TR}$ & & FALSE \\
\hline 2002 & $\mathrm{D}$ & 5 & 19 & 7.25 & 98.84 & $\mathrm{BR}$ & & FALSE \\
\hline 2002 & $\mathrm{D}$ & 5 & 20 & 7.77 & 98.98 & BIS & & FALSE \\
\hline 2002 & $\mathrm{D}$ & 5 & 21 & 7.96 & 99.39 & TB & & FALSE \\
\hline 2002 & $\mathrm{D}$ & 5 & 22 & 9.42 & 99.69 & BIS & & FALSE \\
\hline
\end{tabular}

Continued on Next Page... 
Table D.1 - Continued

\begin{tabular}{|c|c|c|c|c|c|c|c|}
\hline Year & Reach & Section & Point & Dist. (m) & Elev. $(\mathrm{m})$ & Notes & Exclude \\
\hline 2002 & $\mathrm{D}$ & 5 & 23 & 10.00 & 99.59 & BIS & FALSE \\
\hline 2002 & D & 5 & 24 & 10.82 & 99.56 & BS & FALSE \\
\hline 2002 & $\mathrm{D}$ & 5 & 25 & 10.82 & 100.00 & TSR & FALSE \\
\hline 2002 & $\mathrm{D}$ & 6 & 1 & 0.00 & 100.00 & TSL & FALSE \\
\hline 2002 & $\mathrm{D}$ & 6 & 2 & 0.00 & 99.84 & BS & FALSE \\
\hline 2002 & $\mathrm{D}$ & 6 & 3 & 0.85 & 99.60 & TB & FALSE \\
\hline 2002 & $\mathrm{D}$ & 6 & 4 & 0.94 & 98.46 & $\mathrm{BB}$ & FALSE \\
\hline 2002 & $\mathrm{D}$ & 6 & 5 & 2.74 & 98.00 & $\mathrm{BF}$ & FALSE \\
\hline 2002 & $\mathrm{D}$ & 6 & 6 & 3.29 & 97.69 & BIS & FALSE \\
\hline 2002 & $\mathrm{D}$ & 6 & 7 & 3.78 & 97.58 & WE & FALSE \\
\hline 2002 & $\mathrm{D}$ & 6 & 8 & 4.27 & 97.48 & TW & FALSE \\
\hline 2002 & $\mathrm{D}$ & 6 & 9 & 5.15 & 97.63 & BIS & FALSE \\
\hline 2002 & $\mathrm{D}$ & 6 & 10 & 5.30 & 97.63 & BIS & FALSE \\
\hline 2002 & $\mathrm{D}$ & 6 & 11 & 5.91 & 97.52 & BIS & FALSE \\
\hline 2002 & $\mathrm{D}$ & 6 & 12 & 6.31 & 97.48 & B Rootwad & FALSE \\
\hline 2002 & $\mathrm{D}$ & 6 & 13 & 6.95 & 97.58 & WE & FALSE \\
\hline 2002 & $\mathrm{D}$ & 6 & 14 & 7.01 & 97.89 & B Rootwad & FALSE \\
\hline 2002 & $\mathrm{D}$ & 6 & 15 & 7.35 & 97.95 & $\mathrm{BB} / \mathrm{BF}$ & FALSE \\
\hline 2002 & $\mathrm{D}$ & 6 & 16 & 7.47 & 98.38 & BIS & FALSE \\
\hline 2002 & $\mathrm{D}$ & 6 & 17 & 7.77 & 98.66 & BIS & FALSE \\
\hline 2002 & $\mathrm{D}$ & 6 & 18 & 8.50 & 99.32 & TВ & FALSE \\
\hline 2002 & $\mathrm{D}$ & 6 & 19 & 9.08 & 99.34 & BIS & FALSE \\
\hline 2002 & $\mathrm{D}$ & 6 & 20 & 10.03 & 99.66 & BS & FALSE \\
\hline 2002 & $\mathrm{D}$ & 6 & 21 & 10.03 & 100.00 & TSR & FALSE \\
\hline 2002 & $\mathrm{D}$ & 7 & 1 & 0.00 & 100.00 & TSL & FALSE \\
\hline 2002 & $\mathrm{D}$ & 7 & 2 & 0.00 & 99.75 & BS & FALSE \\
\hline 2002 & $\mathrm{D}$ & 7 & 3 & 0.24 & 99.65 & TВ & FALSE \\
\hline 2002 & $\mathrm{D}$ & 7 & 4 & 0.34 & 98.99 & BIS & FALSE \\
\hline 2002 & $\mathrm{D}$ & 7 & 5 & 0.52 & 98.90 & BIS & FALSE \\
\hline 2002 & $\mathrm{D}$ & 7 & 6 & 1.16 & 98.50 & BIS & FALSE \\
\hline 2002 & $\mathrm{D}$ & 7 & 7 & 1.89 & 98.32 & $\mathrm{BF}$ & FALSE \\
\hline 2002 & $\mathrm{D}$ & 7 & 8 & 2.53 & 97.86 & BIS & FALSE \\
\hline 2002 & $\mathrm{D}$ & 7 & 9 & 2.71 & 97.85 & WE & FALSE \\
\hline 2002 & $\mathrm{D}$ & 7 & 10 & 3.54 & 97.64 & TW & FALSE \\
\hline 2002 & $\mathrm{D}$ & 7 & 11 & 4.33 & 97.72 & BIS & FALSE \\
\hline 2002 & $\mathrm{D}$ & 7 & 12 & 4.54 & 97.85 & WE & FALSE \\
\hline 2002 & $\mathrm{D}$ & 7 & 13 & 5.12 & 97.89 & BIS & FALSE \\
\hline 2002 & $\mathrm{D}$ & 7 & 14 & 5.70 & 97.95 & BIS & FALSE \\
\hline 2002 & $\mathrm{D}$ & 7 & 15 & 6.25 & 97.85 & $\mathrm{BB}$ & FALSE \\
\hline 2002 & $\mathrm{D}$ & 7 & 16 & 6.31 & 98.87 & BIS & FALSE \\
\hline 2002 & $\mathrm{D}$ & 7 & 17 & 6.77 & 99.23 & BIS & FALSE \\
\hline 2002 & $\mathrm{D}$ & 7 & 18 & 7.22 & 99.43 & BIS & FALSE \\
\hline 2002 & $\mathrm{D}$ & 7 & 19 & 7.71 & 99.77 & BS & FALSE \\
\hline 2002 & $\mathrm{D}$ & 7 & 20 & 7.71 & 99.99 & TSR & FALSE \\
\hline 2002 & $\mathrm{D}$ & 8 & 1 & 0.00 & 100.00 & TSL & FALSE \\
\hline 2002 & $\mathrm{D}$ & 8 & 2 & 0.00 & 99.71 & BS & FALSE \\
\hline 2002 & $\mathrm{D}$ & 8 & 3 & 0.91 & 99.23 & $\mathrm{~TB}$ & FALSE \\
\hline 2002 & $\mathrm{D}$ & 8 & 4 & 1.25 & 98.55 & BIS & FALSE \\
\hline 2002 & $\mathrm{D}$ & 8 & 5 & 1.31 & 98.22 & $\mathrm{BF}$ & FALSE \\
\hline 2002 & $\mathrm{D}$ & 8 & 6 & 1.34 & 97.91 & $\mathrm{BB}$ & FALSE \\
\hline 2002 & $\mathrm{D}$ & 8 & 7 & 1.83 & 97.84 & WE & FALSE \\
\hline 2002 & $\mathrm{D}$ & 8 & 8 & 2.19 & 97.75 & BIS & FALSE \\
\hline
\end{tabular}

Continued on Next Page... 
Table D.1 - Continued

\begin{tabular}{|c|c|c|c|c|c|c|c|c|}
\hline Year & Reach & Section & Point & Dist. (m) & Elev. $(\mathrm{m})$ & Desc. & Notes & Exclude \\
\hline 2002 & $\mathrm{D}$ & 8 & 9 & 2.59 & 97.68 & TW & & FALSE \\
\hline 2002 & $\mathrm{D}$ & 8 & 10 & 2.99 & 97.74 & BIS & & FALSE \\
\hline 2002 & $\mathrm{D}$ & 8 & 11 & 3.20 & 97.84 & BIS & & FALSE \\
\hline 2002 & $\mathrm{D}$ & 8 & 12 & 3.41 & 97.80 & BIS & & FALSE \\
\hline 2002 & $\mathrm{D}$ & 8 & 13 & 3.54 & 97.84 & WE & & FALSE \\
\hline 2002 & $\mathrm{D}$ & 8 & 14 & 4.57 & 97.91 & BIS & & FALSE \\
\hline 2002 & $\mathrm{D}$ & 8 & 15 & 5.12 & 97.99 & BIS & & FALSE \\
\hline 2002 & $\mathrm{D}$ & 8 & 16 & 5.97 & 98.22 & $\mathrm{BF}$ & & FALSE \\
\hline 2002 & $\mathrm{D}$ & 8 & 17 & 6.40 & 98.68 & $\mathrm{BB}$ & & FALSE \\
\hline 2002 & $\mathrm{D}$ & 8 & 18 & 6.52 & 99.71 & TB & & FALSE \\
\hline 2002 & $\mathrm{D}$ & 8 & 19 & 6.95 & 99.76 & BS & & FALSE \\
\hline 2002 & $\mathrm{D}$ & 8 & 20 & 6.95 & 100.00 & TSR & & FALSE \\
\hline 2002 & $\mathrm{D}$ & 9 & 1 & 0.00 & 100.00 & TSL & & FALSE \\
\hline 2002 & $\mathrm{D}$ & 9 & 2 & 0.00 & 99.75 & BS & & FALSE \\
\hline 2002 & $\mathrm{D}$ & 9 & 3 & 0.70 & 98.73 & $\mathrm{BF}$ & & FALSE \\
\hline 2002 & $\mathrm{D}$ & 9 & 4 & 1.25 & 98.51 & BIS & & FALSE \\
\hline 2002 & $\mathrm{D}$ & 9 & 5 & 1.62 & 98.48 & WE & & FALSE \\
\hline 2002 & $\mathrm{D}$ & 9 & 6 & 1.98 & 98.43 & BIS & & FALSE \\
\hline 2002 & $\mathrm{D}$ & 9 & 7 & 2.38 & 98.43 & BIS & & FALSE \\
\hline 2002 & $\mathrm{D}$ & 9 & 8 & 2.77 & 98.48 & BIS & & FALSE \\
\hline 2002 & $\mathrm{D}$ & 9 & 9 & 3.41 & 98.26 & $\mathrm{TW}$ & & FALSE \\
\hline 2002 & $\mathrm{D}$ & 9 & 10 & 3.96 & 98.31 & BIS & & FALSE \\
\hline 2002 & $\mathrm{D}$ & 9 & 11 & 4.42 & 98.42 & WE & & FALSE \\
\hline 2002 & $\mathrm{D}$ & 9 & 12 & 4.85 & 98.51 & BIS & & FALSE \\
\hline 2002 & $\mathrm{D}$ & 9 & 13 & 5.52 & 98.77 & $\mathrm{BF}$ & & FALSE \\
\hline 2002 & $\mathrm{D}$ & 9 & 14 & 7.01 & 98.89 & $\mathrm{BB}$ & & FALSE \\
\hline 2002 & $\mathrm{D}$ & 9 & 15 & 7.47 & 99.09 & BIS & & FALSE \\
\hline 2002 & $\mathrm{D}$ & 9 & 16 & 7.92 & 99.72 & BS & & FALSE \\
\hline 2002 & $\mathrm{D}$ & 9 & 17 & 7.92 & 100.00 & TSR & & FALSE \\
\hline 2002 & $\mathrm{D}$ & 10 & 1 & 0.00 & 100.00 & TSL & & FALSE \\
\hline 2002 & $\mathrm{D}$ & 10 & 2 & 0.00 & 99.66 & BS & & FALSE \\
\hline 2002 & $\mathrm{D}$ & 10 & 3 & 0.52 & 99.15 & BIS & & FALSE \\
\hline 2002 & $\mathrm{D}$ & 10 & 4 & 1.52 & 98.84 & BIS & & FALSE \\
\hline 2002 & $\mathrm{D}$ & 10 & 5 & 1.58 & 98.47 & $\mathrm{BF}$ & & FALSE \\
\hline 2002 & $\mathrm{D}$ & 10 & 6 & 2.71 & 98.37 & BIS & & FALSE \\
\hline 2002 & $\mathrm{D}$ & 10 & 7 & 2.99 & 98.24 & WE & & FALSE \\
\hline 2002 & $\mathrm{D}$ & 10 & 8 & 3.35 & 98.22 & BIS & & FALSE \\
\hline 2002 & $\mathrm{D}$ & 10 & 9 & 3.63 & 98.03 & TW & & FALSE \\
\hline 2002 & $\mathrm{D}$ & 10 & 10 & 4.24 & 98.12 & BIS & & FALSE \\
\hline 2002 & $\mathrm{D}$ & 10 & 11 & 4.75 & 98.21 & BIS & & FALSE \\
\hline 2002 & $\mathrm{D}$ & 10 & 12 & 4.75 & 98.24 & WE & & FALSE \\
\hline 2002 & $\mathrm{D}$ & 10 & 13 & 5.49 & 98.33 & BIS & & FALSE \\
\hline 2002 & $\mathrm{D}$ & 10 & 14 & 6.34 & 98.45 & $\mathrm{BF}$ & & FALSE \\
\hline 2002 & $\mathrm{D}$ & 10 & 15 & 6.68 & 98.55 & BIS & & FALSE \\
\hline 2002 & $\mathrm{D}$ & 10 & 16 & 7.44 & 99.75 & BS & & FALSE \\
\hline 2002 & $\mathrm{D}$ & 10 & 17 & 7.44 & 99.99 & TSR & & FALSE \\
\hline 2002 & $\mathrm{E}$ & 1 & 1 & 0.00 & 100.00 & $\begin{array}{l}\text { Top X } 1 \text { L } \\
\text { Reb/low }\end{array}$ & incorrect-same as 2004 & TRUE \\
\hline 2002 & $\mathrm{E}$ & 1 & 2 & 0.00 & 99.62 & $\begin{array}{l}\text { Bot X } 1 \text { L Re- } \\
\text { b/low }\end{array}$ & incorrect-same as 2004 & TRUE \\
\hline 2002 & $\mathrm{E}$ & 1 & 3 & 0.91 & 99.25 & ТОВ & incorrect-same as 2004 & TRUE \\
\hline 2002 & $\mathrm{E}$ & 1 & 4 & 2.74 & 98.86 & BIS & incorrect-same as 2004 & TRUE \\
\hline
\end{tabular}

Continued on Next Page... 
Table D.1 - Continued

\begin{tabular}{|c|c|c|c|c|c|c|c|c|}
\hline Year & Reach & Section & Point & Dist. $(\mathrm{m})$ & Elev. $(\mathrm{m})$ & Desc. & Notes & Exclude \\
\hline 2002 & $\mathrm{E}$ & 1 & 5 & 4.15 & 98.64 & $\mathrm{BF}$ & incorrect-same as 2004 & TRUE \\
\hline 2002 & $\mathrm{E}$ & 1 & 6 & 6.10 & 98.12 & EW & incorrect-same as 2004 & TRUE \\
\hline 2002 & $\mathrm{E}$ & 1 & 7 & 6.25 & 98.02 & TW & incorrect-same as 2004 & TRUE \\
\hline 2002 & $\mathrm{E}$ & 1 & 8 & 6.77 & 98.10 & EW & incorrect-same as 2004 & TRUE \\
\hline 2002 & $\mathrm{E}$ & 1 & 9 & 7.19 & 99.65 & BIS & incorrect-same as 2004 & TRUE \\
\hline 2002 & $\mathrm{E}$ & 1 & 10 & 7.50 & 99.84 & $\begin{array}{lll}\text { Top X } & 1 & R \\
\text { Reb/low } & \end{array}$ & incorrect-same as 2004 & TRUE \\
\hline 2002 & $\mathrm{E}$ & 1 & 11 & 7.50 & 99.89 & 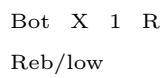 & incorrect-same as 2004 & TRUE \\
\hline 2002 & $\mathrm{E}$ & 2 & 1 & 0.00 & 100.00 & $\begin{array}{l}\text { Top X } 2 \text { L } \\
\text { Reb/low }\end{array}$ & incorrect-same as 2004 & TRUE \\
\hline 2002 & $\mathrm{E}$ & 2 & 2 & 0.00 & 99.42 & $\begin{array}{l}\text { Bot X } 2 \text { L Re- } \\
\text { b/low }\end{array}$ & incorrect-same as 2004 & TRUE \\
\hline 2002 & $\mathrm{E}$ & 2 & 3 & 0.98 & 98.52 & BIS & incorrect-same as 2004 & TRUE \\
\hline 2002 & $\mathrm{E}$ & 2 & 4 & 1.98 & 98.34 & EW & incorrect-same as 2004 & TRUE \\
\hline 2002 & $\mathrm{E}$ & 2 & 5 & 2.80 & 98.25 & TW & incorrect-same as 2004 & TRUE \\
\hline 2002 & $\mathrm{E}$ & 2 & 6 & 3.17 & 98.38 & EW & incorrect-same as 2004 & TRUE \\
\hline 2002 & $\mathrm{E}$ & 2 & 7 & 4.45 & 98.95 & $\mathrm{BF}$ & incorrect-same as 2004 & TRUE \\
\hline 2002 & $\mathrm{E}$ & 2 & 8 & 5.21 & 99.39 & ТОВ & incorrect-same as 2004 & TRUE \\
\hline 2002 & $\mathrm{E}$ & 2 & 9 & 5.82 & 99.65 & $\begin{array}{l}\text { Top X } 2 \text { R } \\
\text { Reb/low }\end{array}$ & incorrect-same as 2004 & TRUE \\
\hline 2002 & $\mathrm{E}$ & 2 & 10 & 5.82 & 100.01 & $\begin{array}{l}\text { Bot } X \quad 2 \quad R \\
\text { Reb/low }\end{array}$ & incorrect-same as 2004 & TRUE \\
\hline 2002 & $\mathrm{E}$ & 3 & 1 & 0.00 & 100.00 & & TSL & FALSE \\
\hline 2002 & $\mathrm{E}$ & 3 & 2 & 0.00 & 99.51 & & $\mathrm{BS}$ & FALSE \\
\hline 2002 & $\mathrm{E}$ & 3 & 3 & 0.46 & 99.11 & & BIS & FALSE \\
\hline 2002 & $\mathrm{E}$ & 3 & 4 & 1.80 & 98.38 & & $\mathrm{BF}$ & FALSE \\
\hline 2002 & $\mathrm{E}$ & 3 & 5 & 1.83 & 97.92 & & WE & FALSE \\
\hline 2002 & $\mathrm{E}$ & 3 & 6 & 2.10 & 97.95 & & $\mathrm{BB}$ & FALSE \\
\hline 2002 & $\mathrm{E}$ & 3 & 7 & 2.13 & 98.04 & & BR & FALSE \\
\hline 2002 & $\mathrm{E}$ & 3 & 8 & 2.44 & 98.13 & & $\mathrm{TR}$ & FALSE \\
\hline 2002 & $\mathrm{E}$ & 3 & 9 & 3.05 & 98.13 & & $\mathrm{TR}$ & FALSE \\
\hline 2002 & $\mathrm{E}$ & 3 & 10 & 3.11 & 97.87 & & $\mathrm{BR}$ & FALSE \\
\hline 2002 & $\mathrm{E}$ & 3 & 11 & 3.57 & 97.75 & & TW & FALSE \\
\hline 2002 & $\mathrm{E}$ & 3 & 12 & 3.93 & 97.92 & & WE & FALSE \\
\hline 2002 & $\mathrm{E}$ & 3 & 13 & 4.57 & 98.23 & & BIS & FALSE \\
\hline 2002 & $\mathrm{E}$ & 3 & 14 & 5.24 & 98.46 & & $\mathrm{BF}$ & FALSE \\
\hline 2002 & $\mathrm{E}$ & 3 & 15 & 5.46 & 98.91 & & BIS & FALSE \\
\hline 2002 & $\mathrm{E}$ & 3 & 16 & 5.91 & 99.04 & & BIS & FALSE \\
\hline 2002 & $\mathrm{E}$ & 3 & 17 & 7.07 & 99.18 & & BIS & FALSE \\
\hline 2002 & $\mathrm{E}$ & 3 & 18 & 7.10 & 99.42 & & BIS & FALSE \\
\hline 2002 & $\mathrm{E}$ & 3 & 19 & 7.32 & 99.43 & & BS & FALSE \\
\hline 2002 & $\mathrm{E}$ & 3 & 20 & 7.32 & 100.01 & & TSR & FALSE \\
\hline 2002 & $\mathrm{E}$ & 4 & 1 & 0.00 & 100.00 & $\begin{array}{l}\text { Top X } 4 \text { L } \\
\text { Reb/low }\end{array}$ & Same as 2004 & TRUE \\
\hline 2002 & $\mathrm{E}$ & 4 & 2 & 0.00 & 99.00 & $\begin{array}{l}\text { Bot X } 4 \text { L Re- } \\
\text { b/low }\end{array}$ & & TRUE \\
\hline 2002 & $\mathrm{E}$ & 4 & 3 & 0.61 & 98.68 & BIS & & TRUE \\
\hline 2002 & $\mathrm{E}$ & 4 & 4 & 1.16 & 98.06 & BIS & & TRUE \\
\hline 2002 & $\mathrm{E}$ & 4 & 5 & 1.58 & 97.97 & BIS & & TRUE \\
\hline 2002 & $\mathrm{E}$ & 4 & 6 & 2.38 & 97.92 & EW & & TRUE \\
\hline 2002 & $\mathrm{E}$ & 4 & 7 & 2.74 & 97.86 & TW & & TRUE \\
\hline
\end{tabular}

Continued on Next Page... 
Table D.1 - Continued

\begin{tabular}{|c|c|c|c|c|c|c|c|}
\hline Year & Reach & Section & Point & Dist. $(\mathrm{m})$ & Elev. $(\mathrm{m})$ & Notes & Exclude \\
\hline 2002 & $\mathrm{E}$ & 4 & 8 & 3.05 & 97.85 & $\begin{array}{l}\text { BIS *left side } \\
\text { of rock in } \\
\text { creek }\end{array}$ & TRUE \\
\hline 2002 & $\mathrm{E}$ & 4 & 9 & 3.96 & 97.88 & $\begin{array}{l}\text { BIS *osland } \\
\text { sand bar on } \\
\text { center }\end{array}$ & TRUE \\
\hline 2002 & $\mathrm{E}$ & 4 & 10 & 4.54 & 97.78 & $\begin{array}{l}\text { BIS *to right } \\
\text { of rock, edge } \\
\text { of sand bar }\end{array}$ & TRUE \\
\hline 2002 & $\mathrm{E}$ & 4 & 11 & 4.88 & 97.80 & EW & TRUE \\
\hline 2002 & $\mathrm{E}$ & 4 & 12 & 5.06 & 97.95 & BIS & TRUE \\
\hline 2002 & $\mathrm{E}$ & 4 & 13 & 5.61 & 98.04 & $\mathrm{BF}$ & TRUE \\
\hline 2002 & $\mathrm{E}$ & 4 & 14 & 5.88 & 98.72 & ТОВ & TRUE \\
\hline 2002 & $\mathrm{E}$ & 4 & 15 & 7.71 & 99.03 & $\begin{array}{l}\text { Top X } \quad 4 \quad \mathrm{R} \\
\text { Reb/low }\end{array}$ & TRUE \\
\hline 2002 & $\mathrm{E}$ & 4 & 16 & 7.71 & 99.29 & $\begin{array}{l}\text { Bot } \mathrm{X} \quad 4 \quad \mathrm{R} \\
\text { Reb/low }\end{array}$ & TRUE \\
\hline 2002 & $\mathrm{E}$ & 5 & 1 & 0.00 & 100.00 & $\begin{array}{llll}\text { Top } & \mathrm{X} & 5 & \mathrm{~L} \\
\text { Reb/low } & \end{array}$ & TRUE \\
\hline 2002 & $\mathrm{E}$ & 5 & 2 & 0.00 & 99.67 & $\begin{array}{l}\text { Bot X } 5 \text { L Re- } \\
\text { b/low }\end{array}$ & TRUE \\
\hline 2002 & $\mathrm{E}$ & 5 & 3 & 0.94 & 99.43 & BIS & TRUE \\
\hline 2002 & $\mathrm{E}$ & 5 & 4 & 1.65 & 98.93 & BIS & TRUE \\
\hline 2002 & $\mathrm{E}$ & 5 & 5 & 2.77 & 98.66 & EW & TRUE \\
\hline 2002 & $\mathrm{E}$ & 5 & 6 & 3.51 & 98.53 & $\mathrm{TW}$ & TRUE \\
\hline 2002 & $\mathrm{E}$ & 5 & 7 & 3.99 & 98.51 & $\begin{array}{l}\text { BIS * pool in } \\
\text { creek }\end{array}$ & TRUE \\
\hline 2002 & $\mathrm{E}$ & 5 & 8 & 4.27 & 98.57 & $\begin{array}{l}\text { EW } * \text { left side } \\
\text { of rock }\end{array}$ & TRUE \\
\hline 2002 & $\mathrm{E}$ & 5 & 9 & 4.72 & 99.22 & $\mathrm{BF}$ & TRUE \\
\hline 2002 & $\mathrm{E}$ & 5 & 10 & 5.73 & 99.73 & TOB & TRUE \\
\hline 2002 & $\mathrm{E}$ & 5 & 11 & 7.89 & 99.89 & $\begin{array}{llll}\text { Bot } & \mathrm{X} & 5 & \mathrm{R} \\
\text { Reb/low } & & \end{array}$ & TRUE \\
\hline 2002 & $\mathrm{E}$ & 5 & 12 & 7.89 & 100.30 & 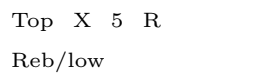 & TRUE \\
\hline 2002 & $\mathrm{E}$ & 6 & 1 & 0.00 & 100.00 & $\begin{array}{llll}\text { Top } & \mathrm{X} & 6 & \mathrm{~L} \\
\text { Reb/low } & \end{array}$ & TRUE \\
\hline 2002 & $\mathrm{E}$ & 6 & 2 & 0.00 & 99.54 & $\begin{array}{l}\text { Bot X } 6 \text { L Re- } \\
\text { b/low }\end{array}$ & TRUE \\
\hline 2002 & $\mathrm{E}$ & 6 & 3 & 0.15 & 99.44 & BIS & TRUE \\
\hline 2002 & $\mathrm{E}$ & 6 & 4 & 0.61 & 98.78 & BIS & TRUE \\
\hline 2002 & $\mathrm{E}$ & 6 & 5 & 1.16 & 98.34 & $\begin{array}{l}\text { EW } *^{*} \text { next to } \\
\text { rock }\end{array}$ & TRUE \\
\hline 2002 & $\mathrm{E}$ & 6 & 6 & 1.83 & 98.24 & $\mathrm{TW}$ & TRUE \\
\hline 2002 & $\mathrm{E}$ & 6 & 7 & 2.62 & 98.37 & $\begin{array}{l}\mathrm{EW} * \text { next to } \\
\text { rock }\end{array}$ & TRUE \\
\hline 2002 & $\mathrm{E}$ & 6 & 8 & 3.51 & 98.57 & BIS & TRUE \\
\hline 2002 & $\mathrm{E}$ & 6 & 9 & 4.08 & 98.52 & BIS & TRUE \\
\hline 2002 & $\mathrm{E}$ & 6 & 10 & 4.36 & 98.77 & $\mathrm{BF}$ & TRUE \\
\hline 2002 & $\mathrm{E}$ & 6 & 11 & 4.69 & 99.45 & ТОВ & TRUE \\
\hline 2002 & $\mathrm{E}$ & 6 & 12 & 5.36 & 99.63 & $\begin{array}{llll}\text { Bot } & X & 6 & R \\
\text { Reb/low } & & \end{array}$ & TRUE \\
\hline
\end{tabular}

Continued on Next Page... 
Table D.1 - Continued

\begin{tabular}{|c|c|c|c|c|c|c|c|c|}
\hline Year & Reach & Section & Point & Dist. (m) & Elev. $(\mathrm{m})$ & Desc. & Notes & Exclude \\
\hline 2002 & $\mathrm{E}$ & 6 & 13 & 5.36 & 100.00 & Top $\mathrm{X} \quad 6 \quad \mathrm{R}$ & & TRUE \\
\hline & & & & & & Reb/low & & \\
\hline 2002 & $\mathrm{E}$ & 7 & 1 & 0.00 & 100.00 & & TSL & FALSE \\
\hline 2002 & $\mathrm{E}$ & 7 & 2 & 0.00 & 99.73 & & BS & FALSE \\
\hline 2002 & $\mathrm{E}$ & 7 & 3 & 0.88 & 99.34 & & $\mathrm{BF}$ & FALSE \\
\hline 2002 & $\mathrm{E}$ & 7 & 4 & 0.91 & 98.87 & & $\mathrm{BB}$ & FALSE \\
\hline 2002 & $\mathrm{E}$ & 7 & 5 & 1.43 & 98.62 & & WE & FALSE \\
\hline 2002 & $\mathrm{E}$ & 7 & 6 & 1.43 & 98.54 & & BIS & FALSE \\
\hline 2002 & $\mathrm{E}$ & 7 & 7 & 1.65 & 98.55 & & BIS & FALSE \\
\hline 2002 & $\mathrm{E}$ & 7 & 8 & 1.98 & 98.38 & & TW & FALSE \\
\hline 2002 & $\mathrm{E}$ & 7 & 9 & 2.16 & 98.42 & & $\mathrm{BR}$ & FALSE \\
\hline 2002 & $\mathrm{E}$ & 7 & 10 & 2.38 & 98.62 & & $\mathrm{TR}$ & FALSE \\
\hline 2002 & $\mathrm{E}$ & 7 & 11 & 2.71 & 98.68 & & $\mathrm{TR}$ & FALSE \\
\hline 2002 & $\mathrm{E}$ & 7 & 12 & 2.74 & 98.50 & & $\mathrm{BR}$ & FALSE \\
\hline 2002 & $\mathrm{E}$ & 7 & 13 & 3.20 & 98.46 & & BIS & FALSE \\
\hline 2002 & $\mathrm{E}$ & 7 & 14 & 3.93 & 98.55 & & $\mathrm{BR}$ & FALSE \\
\hline 2002 & $\mathrm{E}$ & 7 & 15 & 3.93 & 98.62 & & WE & FALSE \\
\hline 2002 & $\mathrm{E}$ & 7 & 16 & 3.93 & 98.68 & & $\mathrm{TR}$ & FALSE \\
\hline 2002 & $\mathrm{E}$ & 7 & 17 & 4.15 & 98.71 & & TR & FALSE \\
\hline 2002 & $\mathrm{E}$ & 7 & 18 & 4.57 & 98.99 & & $\mathrm{BB}$ & FALSE \\
\hline 2002 & $\mathrm{E}$ & 7 & 19 & 4.60 & 99.34 & & $\mathrm{BF}$ & FALSE \\
\hline 2002 & $\mathrm{E}$ & 7 & 20 & 4.60 & 99.59 & & TB & FALSE \\
\hline 2002 & $\mathrm{E}$ & 7 & 21 & 6.43 & 99.65 & & $\mathrm{BS}$ & FALSE \\
\hline 2002 & $\mathrm{E}$ & 7 & 22 & 6.43 & 100.00 & & TSR & FALSE \\
\hline 2002 & $\mathrm{E}$ & 8 & 1 & 0.00 & 100.00 & & TSL & FALSE \\
\hline 2002 & $\mathrm{E}$ & 8 & 2 & 0.00 & 99.61 & & BS & FALSE \\
\hline 2002 & $\mathrm{E}$ & 8 & 3 & 0.76 & 99.19 & & $\mathrm{BF}$ & FALSE \\
\hline 2002 & $\mathrm{E}$ & 8 & 4 & 0.88 & 98.73 & & BIS & FALSE \\
\hline 2002 & $\mathrm{E}$ & 8 & 5 & 1.04 & 98.66 & & WE & FALSE \\
\hline 2002 & $\mathrm{E}$ & 8 & 6 & 1.28 & 98.59 & & BIS & FALSE \\
\hline 2002 & $\mathrm{E}$ & 8 & 7 & 2.13 & 98.58 & & BIS & FALSE \\
\hline 2002 & $\mathrm{E}$ & 8 & 8 & 2.65 & 98.55 & & TW & FALSE \\
\hline 2002 & $\mathrm{E}$ & 8 & 9 & 2.90 & 98.62 & & BIS & FALSE \\
\hline 2002 & $\mathrm{E}$ & 8 & 10 & 3.38 & 98.66 & & WE & FALSE \\
\hline 2002 & $\mathrm{E}$ & 8 & 11 & 3.99 & 98.82 & & $\mathrm{BB}$ & FALSE \\
\hline 2002 & $\mathrm{E}$ & 8 & 12 & 4.08 & 99.19 & & $\mathrm{BF}$ & FALSE \\
\hline 2002 & $\mathrm{E}$ & 8 & 13 & 4.08 & 99.31 & & TВ & FALSE \\
\hline 2002 & $\mathrm{E}$ & 8 & 14 & 4.51 & 99.54 & & BIS & FALSE \\
\hline 2002 & $\mathrm{E}$ & 8 & 15 & 6.58 & 99.72 & & $\mathrm{BS}$ & FALSE \\
\hline 2002 & $\mathrm{E}$ & 8 & 16 & 6.58 & 99.99 & & TSR & FALSE \\
\hline 2002 & $\mathrm{E}$ & 9 & 1 & 0.00 & 100.00 & & TSL & FALSE \\
\hline 2002 & $\mathrm{E}$ & 9 & 2 & 0.00 & 99.50 & & BS & FALSE \\
\hline 2002 & $\mathrm{E}$ & 9 & 3 & 0.91 & 98.73 & & BIS & FALSE \\
\hline 2002 & $\mathrm{E}$ & 9 & 4 & 0.98 & 98.42 & & BIS & FALSE \\
\hline 2002 & $\mathrm{E}$ & 9 & 5 & 1.49 & 98.00 & & $\mathrm{BF}$ & FALSE \\
\hline 2002 & $\mathrm{E}$ & 9 & 6 & 2.44 & 97.74 & & BIS & FALSE \\
\hline 2002 & $\mathrm{E}$ & 9 & 7 & 2.59 & 97.68 & & WE & FALSE \\
\hline 2002 & $\mathrm{E}$ & 9 & 8 & 3.14 & 97.60 & & BIS & FALSE \\
\hline 2002 & $\mathrm{E}$ & 9 & 9 & 3.69 & 97.60 & & BIS & FALSE \\
\hline 2002 & $\mathrm{E}$ & 9 & 10 & 4.18 & 97.59 & & BIS & FALSE \\
\hline 2002 & $\mathrm{E}$ & 9 & 11 & 4.42 & 97.59 & & TW & FALSE \\
\hline 2002 & $\mathrm{E}$ & 9 & 12 & 4.57 & 97.67 & & BIS & FALSE \\
\hline
\end{tabular}

Continued on Next Page... 
Table D.1 - Continued

\begin{tabular}{|c|c|c|c|c|c|c|c|c|}
\hline Year & Reach & Section & Point & Dist. $(\mathrm{m})$ & Elev. $(\mathrm{m})$ & Desc. & Notes & Exclude \\
\hline 2002 & $\mathrm{E}$ & 9 & 13 & 4.79 & 97.68 & & WE & FALSE \\
\hline 2002 & $\mathrm{E}$ & 9 & 14 & 5.79 & 97.85 & & BIS & FALSE \\
\hline 2002 & $\mathrm{E}$ & 9 & 15 & 6.43 & 97.99 & & $\mathrm{BF}$ & FALSE \\
\hline 2002 & $\mathrm{E}$ & 9 & 16 & 6.83 & 98.30 & & BIS & FALSE \\
\hline 2002 & $\mathrm{E}$ & 9 & 17 & 7.16 & 98.52 & & BIS & FALSE \\
\hline 2002 & $\mathrm{E}$ & 9 & 18 & 7.19 & 98.90 & & BIS & FALSE \\
\hline 2002 & $\mathrm{E}$ & 9 & 19 & 7.56 & 99.02 & & BIS & FALSE \\
\hline 2002 & $\mathrm{E}$ & 9 & 20 & 7.86 & 99.64 & & BIS & FALSE \\
\hline 2002 & $\mathrm{E}$ & 9 & 21 & 8.05 & 99.68 & & BS & FALSE \\
\hline 2002 & $\mathrm{E}$ & 9 & 22 & 8.05 & 99.98 & & TSR & FALSE \\
\hline 2002 & $\mathrm{E}$ & 10 & 1 & 0.00 & 100.00 & & TSL & FALSE \\
\hline 2002 & $\mathrm{E}$ & 10 & 2 & 0.00 & 99.65 & & BS & FALSE \\
\hline 2002 & $\mathrm{E}$ & 10 & 3 & 0.49 & 99.37 & & BIS & FALSE \\
\hline 2002 & $\mathrm{E}$ & 10 & 4 & 0.55 & 98.82 & & BIS & FALSE \\
\hline 2002 & $\mathrm{E}$ & 10 & 5 & 1.04 & 98.45 & & $\mathrm{BF}$ & FALSE \\
\hline 2002 & $\mathrm{E}$ & 10 & 6 & 1.25 & 98.26 & & BIS & FALSE \\
\hline 2002 & $\mathrm{E}$ & 10 & 7 & 1.80 & 98.13 & & $\mathrm{BR}$ & FALSE \\
\hline 2002 & $\mathrm{E}$ & 10 & 8 & 2.01 & 98.37 & & $\mathrm{TR}$ & FALSE \\
\hline 2002 & $\mathrm{E}$ & 10 & 9 & 2.32 & 98.38 & & $\mathrm{TR}$ & FALSE \\
\hline 2002 & $\mathrm{E}$ & 10 & 10 & 2.59 & 98.22 & & WE & FALSE \\
\hline 2002 & $\mathrm{E}$ & 10 & 11 & 2.59 & 98.02 & & $\mathrm{BR}$ & FALSE \\
\hline 2002 & $\mathrm{E}$ & 10 & 12 & 3.02 & 97.99 & & TW & FALSE \\
\hline 2002 & $\mathrm{E}$ & 10 & 13 & 3.63 & 98.22 & & WE & FALSE \\
\hline 2002 & $\mathrm{E}$ & 10 & 14 & 3.81 & 98.18 & & $\mathrm{BB}$ & FALSE \\
\hline 2002 & $\mathrm{E}$ & 10 & 15 & 3.87 & 98.36 & & BIS & FALSE \\
\hline 2002 & $\mathrm{E}$ & 10 & 16 & 4.94 & 98.61 & & $\mathrm{BF}$ & FALSE \\
\hline 2002 & $\mathrm{E}$ & 10 & 17 & 5.06 & 99.07 & & BIS & FALSE \\
\hline 2002 & $\mathrm{E}$ & 10 & 18 & 6.00 & 99.54 & & BIS & FALSE \\
\hline 2002 & $\mathrm{E}$ & 10 & 19 & 7.01 & 99.93 & & $\mathrm{BS}$ & FALSE \\
\hline 2002 & $\mathrm{E}$ & 10 & 20 & 7.01 & 100.24 & & TSR & FALSE \\
\hline 2002 & $\mathrm{~F}$ & 1 & 1 & 0.00 & 100.00 & TSL & & FALSE \\
\hline 2002 & $\mathrm{~F}$ & 1 & 2 & 0.00 & 99.98 & BS & & FALSE \\
\hline 2002 & $\mathrm{~F}$ & 1 & 3 & 1.01 & 99.12 & BIS & & FALSE \\
\hline 2002 & $\mathrm{~F}$ & 1 & 4 & 1.52 & 99.00 & B Rootwad & & FALSE \\
\hline 2002 & $\mathrm{~F}$ & 1 & 5 & 1.71 & 99.41 & T Rootwad & & FALSE \\
\hline 2002 & $\mathrm{~F}$ & 1 & 6 & 2.38 & 99.08 & T Rootwad & & FALSE \\
\hline 2002 & $\mathrm{~F}$ & 1 & 7 & 2.41 & 98.88 & B Rootwad & & FALSE \\
\hline 2002 & $\mathrm{~F}$ & 1 & 8 & 3.17 & 98.32 & $\mathrm{BF}$ & & FALSE \\
\hline 2002 & $\mathrm{~F}$ & 1 & 9 & 4.18 & 98.30 & BIS & & FALSE \\
\hline 2002 & $\mathrm{~F}$ & 1 & 10 & 5.12 & 98.07 & $\mathrm{BR}$ & & FALSE \\
\hline 2002 & $\mathrm{~F}$ & 1 & 11 & 5.33 & 98.30 & $\mathrm{TR}$ & & FALSE \\
\hline 2002 & $\mathrm{~F}$ & 1 & 12 & 5.67 & 98.00 & WE & & FALSE \\
\hline 2002 & $\mathrm{~F}$ & 1 & 13 & 6.04 & 97.86 & BR/TW & & FALSE \\
\hline 2002 & $\mathrm{~F}$ & 1 & 14 & 6.10 & 97.93 & $\mathrm{BR}$ & & FALSE \\
\hline 2002 & $\mathrm{~F}$ & 1 & 15 & 6.10 & 98.00 & WE & & FALSE \\
\hline 2002 & $\mathrm{~F}$ & 1 & 16 & 6.16 & 98.10 & $\mathrm{TR}$ & & FALSE \\
\hline 2002 & $\mathrm{~F}$ & 1 & 17 & 6.49 & 98.05 & BIS & & FALSE \\
\hline 2002 & $\mathrm{~F}$ & 1 & 18 & 6.74 & 98.18 & BIS & & FALSE \\
\hline 2002 & $\mathrm{~F}$ & 1 & 19 & 7.13 & 98.15 & BIS & & FALSE \\
\hline 2002 & $\mathrm{~F}$ & 1 & 20 & 7.80 & 98.38 & $\mathrm{BF}$ & & FALSE \\
\hline 2002 & $\mathrm{~F}$ & 1 & 21 & 8.32 & 98.70 & BIS & & FALSE \\
\hline 2002 & $\mathrm{~F}$ & 1 & 22 & 9.45 & 99.71 & BS & & FALSE \\
\hline
\end{tabular}

Continued on Next Page... 
APPENDIX D. SURVEY DATA

Table D.1 - Continued

\begin{tabular}{|c|c|c|c|c|c|c|c|c|}
\hline Year & Reach & Section & Point & Dist. (m) & Elev. $(\mathrm{m})$ & Desc. & Notes & Exclude \\
\hline 2002 & $\mathrm{~F}$ & 1 & 23 & 9.45 & 99.79 & TSR & & FALSE \\
\hline 2002 & $\mathrm{~F}$ & 2 & 1 & 0.00 & 100.00 & TSL & & FALSE \\
\hline 2002 & $\mathrm{~F}$ & 2 & 2 & 0.00 & 99.84 & BS & & FALSE \\
\hline 2002 & $\mathrm{~F}$ & 2 & 3 & 0.85 & 99.12 & BIS & & FALSE \\
\hline 2002 & $\mathrm{~F}$ & 2 & 4 & 2.62 & 98.42 & BIS & & FALSE \\
\hline 2002 & $\mathrm{~F}$ & 2 & 5 & 2.65 & 98.03 & $\mathrm{BF}$ & & FALSE \\
\hline 2002 & $\mathrm{~F}$ & 2 & 6 & 3.54 & 97.70 & BIS & & FALSE \\
\hline 2002 & $\mathrm{~F}$ & 2 & 7 & 4.45 & 97.49 & WE & & FALSE \\
\hline 2002 & $\mathrm{~F}$ & 2 & 8 & 4.91 & 97.30 & TW & & FALSE \\
\hline 2002 & $\mathrm{~F}$ & 2 & 9 & 5.79 & 97.49 & WE & & FALSE \\
\hline 2002 & $\mathrm{~F}$ & 2 & 10 & 6.28 & 97.50 & BIS & & FALSE \\
\hline 2002 & $\mathrm{~F}$ & 2 & 11 & 7.13 & 97.83 & $\mathrm{BF}$ & & FALSE \\
\hline 2002 & $\mathrm{~F}$ & 2 & 12 & 8.11 & 98.46 & BB & & FALSE \\
\hline 2002 & $\mathrm{~F}$ & 2 & 13 & 8.44 & 99.50 & BIS & & FALSE \\
\hline 2002 & $\mathrm{~F}$ & 2 & 14 & 8.75 & 99.82 & BS & & FALSE \\
\hline 2002 & $\mathrm{~F}$ & 2 & 15 & 8.75 & 99.89 & TSR & & FALSE \\
\hline 2002 & $\mathrm{~F}$ & 3 & 1 & 0.00 & 100.00 & TSL & & FALSE \\
\hline 2002 & $\mathrm{~F}$ & 3 & 2 & 0.00 & 99.92 & BS & & FALSE \\
\hline 2002 & $\mathrm{~F}$ & 3 & 3 & 0.88 & 99.38 & TВ & & FALSE \\
\hline 2002 & $\mathrm{~F}$ & 3 & 4 & 1.22 & 98.59 & $\mathrm{BB} / \mathrm{BF}$ & & FALSE \\
\hline 2002 & $\mathrm{~F}$ & 3 & 5 & 2.83 & 98.39 & BIS & & FALSE \\
\hline 2002 & $\mathrm{~F}$ & 3 & 6 & 3.69 & 98.03 & WE & & FALSE \\
\hline 2002 & $\mathrm{~F}$ & 3 & 7 & 3.96 & 97.95 & BIS & & FALSE \\
\hline 2002 & $\mathrm{~F}$ & 3 & 8 & 4.11 & 97.97 & BIS & & FALSE \\
\hline 2002 & $\mathrm{~F}$ & 3 & 9 & 4.27 & 98.03 & $\mathrm{BR}$ & & FALSE \\
\hline 2002 & $\mathrm{~F}$ & 3 & 10 & 4.27 & 98.21 & $\mathrm{TR}$ & & FALSE \\
\hline 2002 & $\mathrm{~F}$ & 3 & 11 & 4.60 & 98.19 & $\mathrm{TR}$ & & FALSE \\
\hline 2002 & $\mathrm{~F}$ & 3 & 12 & 4.60 & 97.97 & $\mathrm{BR}$ & & FALSE \\
\hline 2002 & $\mathrm{~F}$ & 3 & 13 & 4.91 & 98.03 & WE & & FALSE \\
\hline 2002 & $\mathrm{~F}$ & 3 & 14 & 5.64 & 98.15 & BIS & & FALSE \\
\hline 2002 & $\mathrm{~F}$ & 3 & 15 & 6.19 & 98.65 & $\mathrm{BF}$ & & FALSE \\
\hline 2002 & $\mathrm{~F}$ & 3 & 16 & 7.01 & 98.79 & BIS & & FALSE \\
\hline 2002 & $\mathrm{~F}$ & 3 & 17 & 7.32 & 99.77 & BIS & & FALSE \\
\hline 2002 & $\mathrm{~F}$ & 3 & 18 & 7.80 & 100.05 & BS & & FALSE \\
\hline 2002 & $\mathrm{~F}$ & 3 & 19 & 7.80 & 100.09 & TSR & & FALSE \\
\hline 2002 & $\mathrm{~F}$ & 4 & 1 & 0.00 & 100.00 & TSL & & FALSE \\
\hline 2002 & $\mathrm{~F}$ & 4 & 2 & 0.00 & 99.96 & BS & & FALSE \\
\hline 2002 & $\mathrm{~F}$ & 4 & 3 & 1.34 & 99.13 & BIS & & FALSE \\
\hline 2002 & $\mathrm{~F}$ & 4 & 4 & 2.38 & 97.96 & $\mathrm{BF}$ & & FALSE \\
\hline 2002 & $\mathrm{~F}$ & 4 & 5 & 3.14 & 97.71 & BIS & & FALSE \\
\hline 2002 & $\mathrm{~F}$ & 4 & 6 & 3.60 & 97.71 & BIS & & FALSE \\
\hline 2002 & $\mathrm{~F}$ & 4 & 7 & 4.15 & 97.57 & WE & & FALSE \\
\hline 2002 & $\mathrm{~F}$ & 4 & 8 & 4.48 & 97.52 & $\mathrm{BR}$ & & FALSE \\
\hline 2002 & $\mathrm{~F}$ & 4 & 9 & 4.48 & 97.73 & $\mathrm{TR}$ & & FALSE \\
\hline 2002 & $\mathrm{~F}$ & 4 & 10 & 4.57 & 97.71 & $\mathrm{TR}$ & & FALSE \\
\hline 2002 & $\mathrm{~F}$ & 4 & 11 & 4.63 & 97.44 & $\mathrm{BR}$ & & FALSE \\
\hline 2002 & $\mathrm{~F}$ & 4 & 12 & 5.30 & 97.33 & BIS & & FALSE \\
\hline 2002 & $\mathrm{~F}$ & 4 & 13 & 6.13 & 97.41 & $\mathrm{BB}$ & & FALSE \\
\hline 2002 & $\mathrm{~F}$ & 4 & 14 & 6.22 & 97.57 & WE & & FALSE \\
\hline 2002 & $\mathrm{~F}$ & 4 & 15 & 6.22 & 97.64 & BIS & & FALSE \\
\hline 2002 & $\mathrm{~F}$ & 4 & 16 & 6.74 & 98.00 & $\mathrm{BF}$ & & FALSE \\
\hline 2002 & $\mathrm{~F}$ & 4 & 17 & 7.47 & 99.13 & BIS & & FALSE \\
\hline
\end{tabular}

Continued on Next Page... 
APPENDIX D. SURVEY DATA

Table D.1 - Continued

\begin{tabular}{|c|c|c|c|c|c|c|c|c|}
\hline Year & Reach & Section & Point & Dist. (m) & Elev. $(\mathrm{m})$ & Desc. & Notes & Exclude \\
\hline 2002 & $\mathrm{~F}$ & 4 & 18 & 8.02 & 99.57 & BIS & & FALSE \\
\hline 2002 & $\mathrm{~F}$ & 4 & 19 & 8.90 & 99.91 & BS & & FALSE \\
\hline 2002 & $\mathrm{~F}$ & 4 & 20 & 8.90 & 100.11 & TSR & & FALSE \\
\hline 2002 & F & 5 & 1 & 0.00 & 100.00 & TSL & & FALSE \\
\hline 2002 & $\mathrm{~F}$ & 5 & 2 & 0.00 & 99.96 & $\mathrm{BS}$ & & FALSE \\
\hline 2002 & $\mathrm{~F}$ & 5 & 3 & 0.76 & 99.24 & BIS & & FALSE \\
\hline 2002 & $\mathrm{~F}$ & 5 & 4 & 1.95 & 98.59 & $\mathrm{BF}$ & & FALSE \\
\hline 2002 & F & 5 & 5 & 2.44 & 98.60 & BIS & & FALSE \\
\hline 2002 & $\mathrm{~F}$ & 5 & 6 & 2.53 & 98.42 & BIS & & FALSE \\
\hline 2002 & $\mathrm{~F}$ & 5 & 7 & 3.29 & 98.27 & WE & & FALSE \\
\hline 2002 & $\mathrm{~F}$ & 5 & 8 & 3.66 & 98.20 & TW & & FALSE \\
\hline 2002 & F & 5 & 9 & 3.93 & 98.26 & BIS & & FALSE \\
\hline 2002 & $\mathrm{~F}$ & 5 & 10 & 4.05 & 98.33 & $\mathrm{TR}$ & & FALSE \\
\hline 2002 & $\mathrm{~F}$ & 5 & 11 & 4.15 & 98.25 & BIS & & FALSE \\
\hline 2002 & $\mathrm{~F}$ & 5 & 12 & 4.66 & 98.20 & BIS & & FALSE \\
\hline 2002 & $\mathrm{~F}$ & 5 & 13 & 4.75 & 98.27 & WE/BR & & FALSE \\
\hline 2002 & $\mathrm{~F}$ & 5 & 14 & 4.94 & 98.40 & $\mathrm{TR}$ & & FALSE \\
\hline 2002 & $\mathrm{~F}$ & 5 & 15 & 5.49 & 98.49 & $\mathrm{TR}$ & & FALSE \\
\hline 2002 & $\mathrm{~F}$ & 5 & 16 & 6.04 & 98.43 & BIS & & FALSE \\
\hline 2002 & $\mathrm{~F}$ & 5 & 17 & 6.49 & 98.87 & BIS & & FALSE \\
\hline 2002 & F & 5 & 18 & 6.86 & 99.01 & BIS & & FALSE \\
\hline 2002 & F & 5 & 19 & 7.38 & 99.38 & BIS & & FALSE \\
\hline 2002 & $\mathrm{~F}$ & 5 & 20 & 7.71 & 99.82 & BS & & FALSE \\
\hline 2002 & $\mathrm{~F}$ & 5 & 21 & 7.71 & 99.88 & TSR & & FALSE \\
\hline 2002 & $\mathrm{~F}$ & 6 & 1 & 0.00 & 100.00 & TSL & & FALSE \\
\hline 2002 & F & 6 & 2 & 0.00 & 99.95 & BS & & FALSE \\
\hline 2002 & F & 6 & 3 & 0.37 & 99.85 & BIS & & FALSE \\
\hline 2002 & $\mathrm{~F}$ & 6 & 4 & 1.13 & 99.12 & BIS & & FALSE \\
\hline 2002 & $\mathrm{~F}$ & 6 & 5 & 2.01 & 98.49 & $\mathrm{BF}$ & & FALSE \\
\hline 2002 & $\mathrm{~F}$ & 6 & 6 & 2.59 & 98.40 & BIS & & FALSE \\
\hline 2002 & $\mathrm{~F}$ & 6 & 7 & 2.74 & 98.35 & BIS & & FALSE \\
\hline 2002 & $\mathrm{~F}$ & 6 & 8 & 3.17 & 98.16 & WE & & FALSE \\
\hline 2002 & $\mathrm{~F}$ & 6 & 9 & 3.66 & 98.06 & BIS & & FALSE \\
\hline 2002 & $\mathrm{~F}$ & 6 & 10 & 4.15 & 98.09 & BIS & & FALSE \\
\hline 2002 & $\mathrm{~F}$ & 6 & 11 & 4.48 & 98.02 & TW & & FALSE \\
\hline 2002 & $\mathrm{~F}$ & 6 & 12 & 4.75 & 98.10 & BIS & & FALSE \\
\hline 2002 & $\mathrm{~F}$ & 6 & 13 & 4.94 & 98.09 & BIS & & FALSE \\
\hline 2002 & $\mathrm{~F}$ & 6 & 14 & 5.03 & 98.12 & $\mathrm{BR}$ & & FALSE \\
\hline 2002 & $\mathrm{~F}$ & 6 & 15 & 5.03 & 98.16 & WE & & FALSE \\
\hline 2002 & F & 6 & 16 & 5.24 & 98.84 & $\mathrm{TR}$ & & FALSE \\
\hline 2002 & F & 6 & 17 & 6.22 & 98.92 & $\mathrm{TR}$ & & FALSE \\
\hline 2002 & $\mathrm{~F}$ & 6 & 18 & 6.34 & 99.08 & BIS & & FALSE \\
\hline 2002 & $\mathrm{~F}$ & 6 & 19 & 7.44 & 99.29 & BIS & & FALSE \\
\hline 2002 & $\mathrm{~F}$ & 6 & 20 & 8.20 & 99.55 & BIS & & FALSE \\
\hline 2002 & F & 6 & 21 & 8.63 & 99.82 & BS & & FALSE \\
\hline 2002 & $\mathrm{~F}$ & 6 & 22 & 8.63 & 99.93 & TSR & & FALSE \\
\hline 2002 & $\mathrm{~F}$ & 7 & 1 & 0.00 & 100.00 & TSL & & FALSE \\
\hline 2002 & F & 7 & 2 & 0.00 & 99.97 & BS & & FALSE \\
\hline 2002 & $\mathrm{~F}$ & 7 & 3 & 0.30 & 99.73 & BIS & & FALSE \\
\hline 2002 & F & 7 & 4 & 0.67 & 99.16 & BIS & & FALSE \\
\hline 2002 & $\mathrm{~F}$ & 7 & 5 & 1.19 & 98.86 & BIS & & FALSE \\
\hline 2002 & $\mathrm{~F}$ & 7 & 6 & 1.25 & 98.67 & $\mathrm{BF}$ & & FALSE \\
\hline
\end{tabular}

Continued on Next Page... 
APPENDIX D. SURVEY DATA

Table D.1 - Continued

\begin{tabular}{|c|c|c|c|c|c|c|c|c|}
\hline Year & Reach & Section & Point & Dist. (m) & Elev. $(\mathrm{m})$ & Desc. & Notes & Exclude \\
\hline 2002 & $\mathrm{~F}$ & 7 & 7 & 1.68 & 98.49 & BIS & & FALSE \\
\hline 2002 & $\mathrm{~F}$ & 7 & 8 & 2.10 & 98.42 & WE & & FALSE \\
\hline 2002 & F & 7 & 9 & 2.38 & 98.31 & TW & & FALSE \\
\hline 2002 & F & 7 & 10 & 2.65 & 98.33 & BIS & & FALSE \\
\hline 2002 & $\mathrm{~F}$ & 7 & 11 & 3.20 & 98.42 & WE & & FALSE \\
\hline 2002 & $\mathrm{~F}$ & 7 & 12 & 3.44 & 98.50 & BIS & & FALSE \\
\hline 2002 & F & 7 & 13 & 4.02 & 98.54 & $\mathrm{BR}$ & & FALSE \\
\hline 2002 & F & 7 & 14 & 4.05 & 98.73 & $\mathrm{TR}$ & & FALSE \\
\hline 2002 & $\mathrm{~F}$ & 7 & 15 & 4.45 & 98.86 & $\mathrm{TR}$ & & FALSE \\
\hline 2002 & $\mathrm{~F}$ & 7 & 16 & 4.48 & 98.77 & $\mathrm{BR}$ & & FALSE \\
\hline 2002 & F & 7 & 17 & 5.15 & 98.87 & $\mathrm{BR}$ & & FALSE \\
\hline 2002 & $\mathrm{~F}$ & 7 & 18 & 5.52 & 99.18 & $\mathrm{TR}$ & $\begin{array}{l}\text { root not surveyed elsewhere, ex- } \\
\text { cluded }\end{array}$ & TRUE \\
\hline 2002 & $\mathrm{~F}$ & 7 & 19 & 5.58 & 99.00 & $\mathrm{BR}$ & & FALSE \\
\hline 2002 & F & 7 & 20 & 6.71 & 99.26 & BIS & & FALSE \\
\hline 2002 & F & 7 & 21 & 7.65 & 99.40 & BIS & & FALSE \\
\hline 2002 & $\mathrm{~F}$ & 7 & 22 & 8.14 & 99.56 & BIS & & FALSE \\
\hline 2002 & F & 7 & 23 & 8.38 & 99.68 & BIS & & FALSE \\
\hline 2002 & $\mathrm{~F}$ & 7 & 24 & 8.72 & 99.85 & $\mathrm{BS}$ & & FALSE \\
\hline 2002 & $\mathrm{~F}$ & 7 & 25 & 8.72 & 99.94 & TSR & & FALSE \\
\hline 2002 & F & 8 & 1 & 0.00 & 100.00 & TSL & & FALSE \\
\hline 2002 & F & 8 & 2 & 0.00 & 99.90 & BS & & FALSE \\
\hline 2002 & $\mathrm{~F}$ & 8 & 3 & 0.27 & 99.79 & BIS & & FALSE \\
\hline 2002 & $\mathrm{~F}$ & 8 & 4 & 0.61 & 99.41 & BIS & & FALSE \\
\hline 2002 & $\mathrm{~F}$ & 8 & 5 & 0.91 & 99.27 & BIS & & FALSE \\
\hline 2002 & F & 8 & 6 & 1.16 & 98.60 & BIS & & FALSE \\
\hline 2002 & $\mathrm{~F}$ & 8 & 7 & 1.62 & 98.20 & $\mathrm{BF} / \mathrm{TR}$ & & FALSE \\
\hline 2002 & $\mathrm{~F}$ & 8 & 8 & 2.35 & 98.21 & $\mathrm{TR}$ & & FALSE \\
\hline 2002 & $\mathrm{~F}$ & 8 & 9 & 2.65 & 97.74 & $\mathrm{BR}$ & & FALSE \\
\hline 2002 & $\mathrm{~F}$ & 8 & 10 & 2.87 & 97.83 & BIS & & FALSE \\
\hline 2002 & $\mathrm{~F}$ & 8 & 11 & 3.20 & 97.78 & WE & & FALSE \\
\hline 2002 & $\mathrm{~F}$ & 8 & 12 & 3.26 & 97.91 & $\mathrm{TR}$ & & FALSE \\
\hline 2002 & $\mathrm{~F}$ & 8 & 13 & 3.51 & 97.73 & $\mathrm{BR}$ & & FALSE \\
\hline 2002 & $\mathrm{~F}$ & 8 & 14 & 3.60 & 97.69 & BIS & & FALSE \\
\hline 2002 & F & 8 & 15 & 3.93 & 97.68 & BIS & & FALSE \\
\hline 2002 & $\mathrm{~F}$ & 8 & 16 & 4.60 & 97.77 & WE & & FALSE \\
\hline 2002 & $\mathrm{~F}$ & 8 & 17 & 4.85 & 97.81 & BIS & & FALSE \\
\hline 2002 & $\mathrm{~F}$ & 8 & 18 & 5.24 & 98.36 & $\mathrm{BF}$ & & FALSE \\
\hline 2002 & $\mathrm{~F}$ & 8 & 19 & 5.85 & 98.52 & BIS & & FALSE \\
\hline 2002 & F & 8 & 20 & 6.46 & 98.56 & BIS & & FALSE \\
\hline 2002 & F & 8 & 21 & 7.62 & 98.77 & BIS & & FALSE \\
\hline 2002 & $\mathrm{~F}$ & 8 & 22 & 8.66 & 98.63 & BIS & & FALSE \\
\hline 2002 & $\mathrm{~F}$ & 8 & 23 & 9.24 & 98.78 & BIS & & FALSE \\
\hline 2002 & $\mathrm{~F}$ & 8 & 24 & 9.45 & 99.09 & BIS & & FALSE \\
\hline 2002 & $\mathrm{~F}$ & 8 & 25 & 9.97 & 99.33 & BIS & & FALSE \\
\hline 2002 & $\mathrm{~F}$ & 8 & 26 & 11.13 & 99.67 & BIS & & FALSE \\
\hline 2002 & $\mathrm{~F}$ & 8 & 27 & 11.43 & 99.80 & BIS & & FALSE \\
\hline 2002 & F & 8 & 28 & 12.83 & 99.84 & BS & & FALSE \\
\hline 2002 & $\mathrm{~F}$ & 8 & 29 & 12.83 & 100.02 & TSR & & FALSE \\
\hline 2002 & $\mathrm{~F}$ & 9 & 1 & 0.00 & 100.00 & TSL & & FALSE \\
\hline 2002 & $\mathrm{~F}$ & 9 & 2 & 0.00 & 99.87 & BS & & FALSE \\
\hline 2002 & $\mathrm{~F}$ & 9 & 3 & 0.27 & 99.67 & BIS & & FALSE \\
\hline
\end{tabular}

Continued on Next Page... 
Table D.1 - Continued

\begin{tabular}{|c|c|c|c|c|c|c|c|c|}
\hline Year & Reach & Section & Point & Dist. (m) & Elev. $(\mathrm{m})$ & Desc. & Notes & Exclude \\
\hline 2002 & $\mathrm{~F}$ & 9 & 4 & 0.37 & 99.29 & BIS & & FALSE \\
\hline 2002 & $\mathrm{~F}$ & 9 & 5 & 0.79 & 99.06 & BIS & & FALSE \\
\hline 2002 & $\mathrm{~F}$ & 9 & 6 & 1.22 & 98.81 & $\mathrm{BF}$ & & FALSE \\
\hline 2002 & F & 9 & 7 & 1.58 & 98.72 & BIS & & FALSE \\
\hline 2002 & $\mathrm{~F}$ & 9 & 8 & 2.23 & 98.50 & WE & & FALSE \\
\hline 2002 & $\mathrm{~F}$ & 9 & 9 & 2.65 & 98.45 & $\mathrm{BR}$ & & FALSE \\
\hline 2002 & $\mathrm{~F}$ & 9 & 10 & 2.74 & 98.59 & $\mathrm{TR}$ & & FALSE \\
\hline 2002 & F & 9 & 11 & 3.02 & 98.57 & $\mathrm{TR}$ & & FALSE \\
\hline 2002 & $\mathrm{~F}$ & 9 & 12 & 3.05 & 98.44 & $\mathrm{BR}$ & & FALSE \\
\hline 2002 & $\mathrm{~F}$ & 9 & 13 & 3.35 & 98.34 & $\mathrm{TW}$ & & FALSE \\
\hline 2002 & $\mathrm{~F}$ & 9 & 14 & 3.66 & 98.42 & BIS & & FALSE \\
\hline 2002 & F & 9 & 15 & 3.87 & 98.43 & BB & & FALSE \\
\hline 2002 & F & 9 & 16 & 3.87 & 98.50 & WE & & FALSE \\
\hline 2002 & $\mathrm{~F}$ & 9 & 17 & 3.99 & 98.87 & $\mathrm{BF}$ & & FALSE \\
\hline 2002 & $\mathrm{~F}$ & 9 & 18 & 4.48 & 99.06 & ТВ & & FALSE \\
\hline 2002 & $\mathrm{~F}$ & 9 & 19 & 5.33 & 99.20 & BIS & & FALSE \\
\hline 2002 & $\mathrm{~F}$ & 9 & 20 & 5.33 & 99.35 & BIS & & FALSE \\
\hline 2002 & $\mathrm{~F}$ & 9 & 21 & 6.49 & 99.27 & BIS & & FALSE \\
\hline 2002 & $\mathrm{~F}$ & 9 & 22 & 6.83 & 99.06 & BIS & & FALSE \\
\hline 2002 & $\mathrm{~F}$ & 9 & 23 & 7.16 & 99.03 & BIS & & FALSE \\
\hline 2002 & $\mathrm{~F}$ & 9 & 24 & 7.99 & 99.13 & BIS & & FALSE \\
\hline 2002 & F & 9 & 25 & 9.63 & 99.04 & BIS & & FALSE \\
\hline 2002 & $\mathrm{~F}$ & 9 & 26 & 9.94 & 99.09 & BIS & & FALSE \\
\hline 2002 & $\mathrm{~F}$ & 9 & 27 & 10.03 & 99.54 & BIS & & FALSE \\
\hline 2002 & $\mathrm{~F}$ & 9 & 28 & 10.49 & 99.57 & BIS & & FALSE \\
\hline 2002 & F & 9 & 29 & 11.25 & 99.78 & $\mathrm{BS}$ & & FALSE \\
\hline 2002 & $\mathrm{~F}$ & 9 & 30 & 11.25 & 99.97 & TSR & & FALSE \\
\hline 2002 & $\mathrm{~F}$ & 10 & 1 & 0.00 & 100.00 & TSL & & FALSE \\
\hline 2002 & $\mathrm{~F}$ & 10 & 2 & 0.00 & 99.83 & BS & & FALSE \\
\hline 2002 & $\mathrm{~F}$ & 10 & 3 & 0.30 & 98.95 & $\mathrm{BF}$ & & FALSE \\
\hline 2002 & $\mathrm{~F}$ & 10 & 4 & 0.88 & 98.78 & BIS & & FALSE \\
\hline 2002 & $\mathrm{~F}$ & 10 & 5 & 1.46 & 98.40 & WE & & FALSE \\
\hline 2002 & $\mathrm{~F}$ & 10 & 6 & 1.95 & 98.26 & BIS & & FALSE \\
\hline 2002 & $\mathrm{~F}$ & 10 & 7 & 2.29 & 98.26 & BIS & & FALSE \\
\hline 2002 & $\mathrm{~F}$ & 10 & 8 & 2.56 & 98.31 & BIS & & FALSE \\
\hline 2002 & $\mathrm{~F}$ & 10 & 9 & 2.65 & 98.40 & WE & & FALSE \\
\hline 2002 & $\mathrm{~F}$ & 10 & 10 & 3.20 & 98.43 & BIS & & FALSE \\
\hline 2002 & $\mathrm{~F}$ & 10 & 11 & 3.44 & 98.64 & BIS & & FALSE \\
\hline 2002 & $\mathrm{~F}$ & 10 & 12 & 3.93 & 98.68 & BIS & & FALSE \\
\hline 2002 & $\mathrm{~F}$ & 10 & 13 & 4.18 & 98.80 & BIS & & FALSE \\
\hline 2002 & $\mathrm{~F}$ & 10 & 14 & 4.48 & 99.14 & $\mathrm{BF}$ & & FALSE \\
\hline 2002 & $\mathrm{~F}$ & 10 & 15 & 4.79 & 99.25 & BIS & & FALSE \\
\hline 2002 & $\mathrm{~F}$ & 10 & 16 & 5.85 & 99.16 & BIS & & FALSE \\
\hline 2002 & $\mathrm{~F}$ & 10 & 17 & 6.64 & 99.16 & BIS & & FALSE \\
\hline 2002 & $\mathrm{~F}$ & 10 & 18 & 6.95 & 99.04 & BIS & & FALSE \\
\hline 2002 & $\mathrm{~F}$ & 10 & 19 & 7.38 & 99.13 & BIS & & FALSE \\
\hline 2002 & $\mathrm{~F}$ & 10 & 20 & 7.77 & 99.04 & BIS & & FALSE \\
\hline 2002 & $\mathrm{~F}$ & 10 & 21 & 8.26 & 99.01 & BIS & & FALSE \\
\hline 2002 & $\mathrm{~F}$ & 10 & 22 & 9.11 & 98.88 & BIS & & FALSE \\
\hline 2002 & $\mathrm{~F}$ & 10 & 23 & 9.27 & 99.38 & BIS & & FALSE \\
\hline 2002 & $\mathrm{~F}$ & 10 & 24 & 9.51 & 99.59 & BIS & & FALSE \\
\hline 2002 & $\mathrm{~F}$ & 10 & 25 & 9.81 & 99.65 & BS & & FALSE \\
\hline
\end{tabular}

Continued on Next Page... 
Table D.1 - Continued

\begin{tabular}{|c|c|c|c|c|c|c|c|}
\hline Year & Reach & Section & Point & Dist. $(\mathrm{m})$ & Elev. $(\mathrm{m})$ & Notes & Exclude \\
\hline 2002 & $\mathrm{~F}$ & 10 & 26 & 9.81 & 99.79 & TSR & FALSE \\
\hline \multirow[t]{2}{*}{2003} & A & 1 & 1 & 0.00 & 99.82 & $\mathrm{BOT}$ & FALSE \\
\hline & & & & & & REB/LOW & \\
\hline \multirow[t]{2}{*}{2003} & A & 1 & 2 & 0.01 & 99.77 & GRND $\quad \mathrm{XS} 1$ & FALSE \\
\hline & & & & & & REBAR & \\
\hline 2003 & A & 1 & 3 & 0.52 & 99.60 & GB & FALSE \\
\hline 2003 & A & 1 & 4 & 1.35 & 99.43 & TOP BANK & FALSE \\
\hline 2003 & A & 1 & 5 & 2.34 & 99.29 & GB & FALSE \\
\hline 2003 & A & 1 & 6 & 3.13 & 99.20 & GB & FALSE \\
\hline 2003 & A & 1 & 7 & 3.52 & 98.90 & GB & FALSE \\
\hline 2003 & A & 1 & 8 & 4.16 & 98.72 & EW & FALSE \\
\hline 2003 & A & 1 & 9 & 4.82 & 98.63 & THALWEG & FALSE \\
\hline 2003 & A & 1 & 10 & 5.76 & 98.75 & EW & FALSE \\
\hline 2003 & A & 1 & 11 & 6.20 & 98.81 & GB & FALSE \\
\hline 2003 & A & 1 & 12 & 6.60 & 99.01 & $\mathrm{BF}$ & FALSE \\
\hline 2003 & A & 1 & 13 & 6.91 & 99.18 & TOP BANK & FALSE \\
\hline 2003 & A & 1 & 14 & 7.27 & 99.33 & GB & FALSE \\
\hline 2003 & A & 1 & 15 & 8.13 & 99.45 & GB & FALSE \\
\hline \multirow[t]{2}{*}{2003} & A & 1 & 16 & 9.03 & 99.74 & GRND $\quad \mathrm{XS} 1$ & FALSE \\
\hline & & & & & & REBAR & \\
\hline \multirow[t]{2}{*}{2003} & A & 1 & 17 & 9.06 & 99.75 & BOT $\quad \mathrm{X} 1 \mathrm{R}$ & FALSE \\
\hline & & & & & & REB/LOW & \\
\hline \multirow[t]{2}{*}{2003} & A & 2 & 1 & 0.00 & 99.66 & BOT $\mathrm{X} 2 \mathrm{~L}$ & FALSE \\
\hline & & & & & & REB/LOW & \\
\hline \multirow[t]{2}{*}{2003} & A & 2 & 2 & 0.01 & 99.66 & GRND $\quad \mathrm{XS} 2$ & FALSE \\
\hline & & & & & & REBAR & \\
\hline 2003 & A & 2 & 3 & 0.77 & 99.51 & & FALSE \\
\hline 2003 & A & 2 & 4 & 1.52 & 99.34 & & FALSE \\
\hline 2003 & A & 2 & 5 & 2.20 & 98.90 & & FALSE \\
\hline 2003 & A & 2 & 6 & 2.93 & 98.76 & & FALSE \\
\hline 2003 & A & 2 & 7 & 3.57 & 98.49 & & FALSE \\
\hline 2003 & A & 2 & 8 & 4.10 & 98.34 & & FALSE \\
\hline 2003 & A & 2 & 9 & 4.61 & 98.29 & GB & FALSE \\
\hline 2003 & A & 2 & 10 & 4.83 & 98.17 & EW & FALSE \\
\hline 2003 & A & 2 & 11 & 5.42 & 98.09 & THALWEG & FALSE \\
\hline 2003 & A & 2 & 12 & 6.72 & 98.14 & EW & FALSE \\
\hline 2003 & A & 2 & 13 & 7.28 & 98.18 & GB & FALSE \\
\hline 2003 & A & 2 & 14 & 7.76 & 98.42 & $\mathrm{BF}$ & FALSE \\
\hline 2003 & A & 2 & 15 & 7.98 & 98.54 & GB & FALSE \\
\hline 2003 & A & 2 & 16 & 8.85 & 98.91 & GB & FALSE \\
\hline 2003 & A & 2 & 17 & 9.47 & 98.95 & GB & FALSE \\
\hline 2003 & A & 2 & 18 & 9.80 & 99.35 & TOP BANK & FALSE \\
\hline \multirow[t]{2}{*}{2003} & A & 2 & 19 & 10.68 & 99.68 & GRND $\quad \mathrm{XS} 2$ & FALSE \\
\hline & & & & & & REBAR & \\
\hline \multirow[t]{2}{*}{2003} & A & 2 & 20 & 10.69 & 99.69 & BOT $\quad \mathrm{X} 2 \mathrm{R}$ & FALSE \\
\hline & & & & & & REB/LOW & \\
\hline \multirow[t]{2}{*}{2003} & A & 3 & 1 & 0.00 & 99.48 & BOT X3L & FALSE \\
\hline & & & & & & REB/LOW & \\
\hline \multirow[t]{2}{*}{2003} & A & 3 & 2 & 0.04 & 99.50 & GRND $\quad \mathrm{XS} 3$ & FALSE \\
\hline & & & & & & REBAR & \\
\hline 2003 & A & 3 & 3 & 0.49 & 99.40 & TOP BANK & FALSE \\
\hline 2003 & A & 3 & 4 & 1.01 & 98.92 & GB & FALSE \\
\hline
\end{tabular}

Continued on Next Page... 
Table D.1 - Continued

\begin{tabular}{|c|c|c|c|c|c|c|c|}
\hline Year & Reach & Section & Point & Dist. (m) & Elev. $(\mathrm{m})$ & Notes & Exclude \\
\hline 2003 & A & 3 & 5 & 1.74 & 98.60 & GB & FALSE \\
\hline 2003 & A & 3 & 6 & 1.96 & 98.27 & GB & FALSE \\
\hline 2003 & A & 3 & 7 & 2.21 & 97.98 & THALWEG & FALSE \\
\hline 2003 & A & 3 & 8 & 2.63 & 97.86 & EW & FALSE \\
\hline 2003 & $\mathrm{~A}$ & 3 & 9 & 4.21 & 97.79 & THALWEG & FALSE \\
\hline 2003 & A & 3 & 10 & 4.64 & 97.90 & EW & FALSE \\
\hline 2003 & A & 3 & 11 & 4.99 & 98.06 & GB & FALSE \\
\hline 2003 & A & 3 & 12 & 5.47 & 98.23 & $\mathrm{BF}$ & FALSE \\
\hline 2003 & $\mathrm{~A}$ & 3 & 13 & 5.92 & 98.40 & GB & FALSE \\
\hline 2003 & A & 3 & 14 & 6.90 & 98.51 & GB & FALSE \\
\hline 2003 & $\mathrm{~A}$ & 3 & 15 & 7.71 & 98.38 & GB & FALSE \\
\hline 2003 & $\mathrm{~A}$ & 3 & 16 & 8.59 & 98.61 & GB & FALSE \\
\hline 2003 & A & 3 & 17 & 9.04 & 98.79 & GB & FALSE \\
\hline 2003 & $\mathrm{~A}$ & 3 & 18 & 9.64 & 99.19 & TOP BANK & FALSE \\
\hline 2003 & $\mathrm{~A}$ & 3 & 19 & 10.30 & 99.37 & GB & FALSE \\
\hline \multirow[t]{2}{*}{2003} & $\mathrm{~A}$ & 3 & 20 & 10.87 & 99.70 & GRND $\quad \mathrm{XS} 3$ & FALSE \\
\hline & & & & & & REBAR & \\
\hline \multirow[t]{2}{*}{2003} & A & 3 & 21 & 10.86 & 99.69 & BOT $\quad$ X3R & FALSE \\
\hline & & & & & & REB/LOW & \\
\hline \multirow[t]{2}{*}{2003} & $\mathrm{~A}$ & 4 & 1 & 0.00 & 100.00 & TOP $\quad \mathrm{X} 4 \mathrm{~L}$ & TRUE \\
\hline & & & & & & REB/LOW & \\
\hline \multirow[t]{2}{*}{2003} & $\mathrm{~A}$ & 4 & 2 & 0.00 & 99.67 & BOT $\quad \mathrm{X} 4 \mathrm{~L}$ & TRUE \\
\hline & & & & & & REB/LOW & \\
\hline \multirow[t]{2}{*}{2003} & A & 4 & 3 & 0.03 & 99.68 & GRND $\quad \mathrm{XS} 2$ & TRUE \\
\hline & & & & & & REBAR & \\
\hline 2003 & $\mathrm{~A}$ & 4 & 4 & 1.21 & 99.41 & GB & TRUE \\
\hline 2003 & $\mathrm{~A}$ & 4 & 5 & 1.98 & 99.36 & $\mathrm{BF}$ & TRUE \\
\hline 2003 & $\mathrm{~A}$ & 4 & 6 & 2.22 & 98.81 & GB & TRUE \\
\hline 2003 & $\mathrm{~A}$ & 4 & 7 & 2.97 & 98.57 & EW & TRUE \\
\hline 2003 & A & 4 & 8 & 3.80 & 98.48 & THALWEG & TRUE \\
\hline 2003 & $\mathrm{~A}$ & 4 & 9 & 4.73 & 98.54 & EW & TRUE \\
\hline 2003 & $\mathrm{~A}$ & 4 & 10 & 5.47 & 98.60 & GB & TRUE \\
\hline 2003 & $\mathrm{~A}$ & 4 & 11 & 6.16 & 98.94 & $\mathrm{BF}$ & TRUE \\
\hline 2003 & $\mathrm{~A}$ & 4 & 12 & 7.07 & 99.14 & GB & TRUE \\
\hline 2003 & $\mathrm{~A}$ & 4 & 13 & 8.14 & 99.34 & GB & TRUE \\
\hline 2003 & $\mathrm{~A}$ & 4 & 14 & 8.48 & 99.89 & TOP BANK & TRUE \\
\hline \multirow[t]{2}{*}{2003} & $\mathrm{~A}$ & 4 & 15 & 9.32 & 99.92 & GRND $\quad \mathrm{XS} 2$ & TRUE \\
\hline & & & & & & NOREBAR & \\
\hline \multirow[t]{2}{*}{2003} & $\mathrm{~A}$ & 5 & 1 & 0.00 & 100.00 & TOP $\quad \mathrm{X} 5 \mathrm{~L}$ & TRUE \\
\hline & & & & & & REB/LOW & \\
\hline \multirow[t]{2}{*}{2003} & $\mathrm{~A}$ & 5 & 2 & 0.04 & 99.67 & BOT $\quad \mathrm{X} 5 \mathrm{~L}$ & TRUE \\
\hline & & & & & & REB/LOW & \\
\hline 2003 & $\mathrm{~A}$ & 5 & 3 & 0.05 & 99.69 & & TRUE \\
\hline 2003 & A & 5 & 4 & 0.51 & 99.77 & & TRUE \\
\hline 2003 & A & 5 & 5 & 1.01 & 99.74 & & TRUE \\
\hline 2003 & A & 5 & 6 & 1.63 & 99.35 & & TRUE \\
\hline 2003 & A & 5 & 7 & 2.11 & 98.78 & & TRUE \\
\hline 2003 & A & 5 & 8 & 2.68 & 98.60 & & TRUE \\
\hline 2003 & A & 5 & 9 & 3.36 & 98.36 & & TRUE \\
\hline 2003 & A & 5 & 10 & 4.08 & 98.56 & & TRUE \\
\hline 2003 & A & 5 & 11 & 4.23 & 98.69 & GB & TRUE \\
\hline 2003 & A & 5 & 12 & 4.55 & 98.88 & $\mathrm{BF}$ & TRUE \\
\hline
\end{tabular}

Continued on Next Page... 
Table D.1 - Continued

\begin{tabular}{|c|c|c|c|c|c|c|c|}
\hline Year & Reach & Section & Point & Dist. $(\mathrm{m})$ & Elev. $(\mathrm{m})$ & Notes & Exclude \\
\hline 2003 & A & 5 & 13 & 4.96 & 99.09 & GB & TRUE \\
\hline 2003 & A & 5 & 14 & 5.45 & 99.27 & GB & TRUE \\
\hline \multirow[t]{2}{*}{2003} & A & 5 & 15 & 6.23 & 99.51 & GRND $\quad \mathrm{XS} 1$ & TRUE \\
\hline & & & & & & REBAR & \\
\hline \multirow[t]{2}{*}{2003} & A & 5 & 16 & 6.24 & 99.52 & BOT $\quad \mathrm{X} 45 \mathrm{R}$ & TRUE \\
\hline & & & & & & REB/LOW & \\
\hline \multirow[t]{2}{*}{2003} & A & 6 & 1 & 0.00 & 99.80 & BOT $\quad \mathrm{X} 6 \mathrm{~L}$ & FALSE \\
\hline & & & & & & REB/LOW & \\
\hline \multirow[t]{2}{*}{2003} & A & 6 & 2 & 0.04 & 99.84 & GRND $\quad \mathrm{XS} 6$ & FALSE \\
\hline & & & & & & REBAR & \\
\hline 2003 & A & 6 & 3 & 0.36 & 99.78 & TOP BANK & FALSE \\
\hline 2003 & A & 6 & 4 & 0.64 & 98.48 & GB & FALSE \\
\hline 2003 & A & 6 & 5 & 1.07 & 98.28 & GB & FALSE \\
\hline 2003 & A & 6 & 6 & 1.28 & 98.00 & GB & FALSE \\
\hline 2003 & A & 6 & 7 & 1.52 & 97.97 & EW & FALSE \\
\hline 2003 & A & 6 & 8 & 2.48 & 97.89 & THALWEG & FALSE \\
\hline 2003 & A & 6 & 9 & 3.47 & 97.98 & EW & FALSE \\
\hline 2003 & A & 6 & 10 & 4.05 & 98.09 & GB & FALSE \\
\hline 2003 & A & 6 & 11 & 4.28 & 98.29 & $\mathrm{BF}$ & FALSE \\
\hline 2003 & A & 6 & 12 & 4.64 & 98.48 & GB & FALSE \\
\hline 2003 & A & 6 & 13 & 5.18 & 98.84 & GB & FALSE \\
\hline 2003 & A & 6 & 14 & 6.03 & 99.06 & GB & FALSE \\
\hline 2003 & A & 6 & 15 & 7.16 & 99.32 & TOP BANK & FALSE \\
\hline 2003 & A & 6 & 16 & 9.32 & 99.11 & GB & FALSE \\
\hline 2003 & A & 6 & 17 & 11.67 & 99.14 & GB & FALSE \\
\hline 2003 & A & 6 & 18 & 12.56 & 98.99 & GB & FALSE \\
\hline 2003 & A & 6 & 19 & 14.14 & 99.06 & GB & FALSE \\
\hline 2003 & A & 6 & 20 & 14.86 & 99.14 & GB & FALSE \\
\hline \multirow[t]{2}{*}{2003} & A & 6 & 21 & 15.19 & 99.43 & GRND $\quad \mathrm{XS} 6$ & FALSE \\
\hline & & & & & & REBAR & \\
\hline \multirow[t]{2}{*}{2003} & A & 6 & 22 & 15.19 & 99.42 & BOT $\quad \mathrm{x} 6 \mathrm{R}$ & FALSE \\
\hline & & & & & & REB/LOW & \\
\hline \multirow[t]{2}{*}{2003} & A & 6 & 23 & 15.20 & 99.84 & TOP $\quad \mathrm{X} 6 \mathrm{R}$ & FALSE \\
\hline & & & & & & REB/LOW & \\
\hline \multirow[t]{2}{*}{2003} & A & 7 & 1 & 0.00 & 100.00 & TOP $\quad \mathrm{X} 7 \mathrm{~L}$ & FALSE \\
\hline & & & & & & REB/LOW & \\
\hline \multirow[t]{2}{*}{2003} & A & 7 & 2 & 0.03 & 99.71 & BOT $\quad \mathrm{X} 7 \mathrm{~L}$ & FALSE \\
\hline & & & & & & REB/LOW & \\
\hline \multirow[t]{2}{*}{2003} & A & 7 & 3 & 0.01 & 99.73 & GRND $\quad \mathrm{XS} 7$ & FALSE \\
\hline & & & & & & REBAR & \\
\hline 2003 & A & 7 & 4 & 0.70 & 99.58 & TOP BANK & FALSE \\
\hline 2003 & A & 7 & 5 & 1.40 & 98.55 & GB & FALSE \\
\hline 2003 & A & 7 & 6 & 2.64 & 98.22 & EW & FALSE \\
\hline 2003 & A & 7 & 7 & 3.29 & 98.18 & THALWEG & FALSE \\
\hline 2003 & A & 7 & 8 & 4.26 & 98.18 & EW & FALSE \\
\hline 2003 & A & 7 & 9 & 4.75 & 98.33 & GB & FALSE \\
\hline 2003 & A & 7 & 10 & 5.24 & 98.50 & GB & FALSE \\
\hline 2003 & A & 7 & 11 & 5.50 & 98.71 & $\mathrm{BF}$ & FALSE \\
\hline 2003 & A & 7 & 12 & 6.55 & 98.84 & GB & FALSE \\
\hline 2003 & A & 7 & 13 & 7.86 & 99.08 & GB & FALSE \\
\hline 2003 & A & 7 & 14 & 8.76 & 99.31 & GB & FALSE \\
\hline 2003 & A & 7 & 15 & 9.88 & 99.43 & TOP BANK & FALSE \\
\hline
\end{tabular}

Continued on Next Page... 
Table D.1 - Continued

\begin{tabular}{|c|c|c|c|c|c|c|c|}
\hline Year & Reach & Section & Point & Dist. (m) & Elev. $(\mathrm{m})$ & Notes & Exclude \\
\hline 2003 & $\mathrm{~A}$ & 7 & 16 & 12.63 & 99.36 & GB & FALSE \\
\hline 2003 & A & 7 & 17 & 14.75 & 99.26 & GB & FALSE \\
\hline 2003 & A & 7 & 18 & 16.90 & 99.33 & GB & FALSE \\
\hline 2003 & A & 7 & 19 & 18.01 & 99.60 & GB & FALSE \\
\hline \multirow[t]{2}{*}{2003} & $\mathrm{~A}$ & 7 & 20 & 18.67 & 99.80 & BOT X789R & FALSE \\
\hline & & & & & & REB/LW & \\
\hline \multirow[t]{2}{*}{2003} & $\mathrm{~A}$ & 7 & 21 & 18.64 & 100.20 & TOP $\quad \mathrm{X} 789 \mathrm{R}$ & FALSE \\
\hline & & & & & & REB/LW & \\
\hline \multirow[t]{2}{*}{2003} & $\mathrm{~A}$ & 8 & 1 & 0.00 & 100.00 & TOP $\quad \mathrm{X} 8 \mathrm{~L}$ & FALSE \\
\hline & & & & & & REB/LOW & \\
\hline \multirow[t]{2}{*}{2003} & A & 8 & 2 & 0.01 & 99.59 & BOT $\quad \mathrm{X} 8 \mathrm{~L}$ & FALSE \\
\hline & & & & & & REB/LOW & \\
\hline \multirow[t]{2}{*}{2003} & $\mathrm{~A}$ & 8 & 3 & 0.02 & 99.60 & GRND XS8 & FALSE \\
\hline & & & & & & REBAR & \\
\hline 2003 & $\mathrm{~A}$ & 8 & 4 & 0.77 & 99.52 & & FALSE \\
\hline 2003 & A & 8 & 5 & 1.02 & 98.45 & & FALSE \\
\hline 2003 & $\mathrm{~A}$ & 8 & 6 & 1.84 & 98.19 & & FALSE \\
\hline 2003 & A & 8 & 7 & 2.56 & 98.08 & & FALSE \\
\hline 2003 & $\mathrm{~A}$ & 8 & 8 & 3.01 & 97.99 & & FALSE \\
\hline 2003 & $\mathrm{~A}$ & 8 & 9 & 3.25 & 97.89 & & FALSE \\
\hline 2003 & $\mathrm{~A}$ & 8 & 10 & 4.06 & 97.76 & THALWEG & FALSE \\
\hline 2003 & $\mathrm{~A}$ & 8 & 11 & 5.03 & 97.88 & EW & FALSE \\
\hline 2003 & $\mathrm{~A}$ & 8 & 12 & 5.43 & 97.99 & GB & FALSE \\
\hline 2003 & $\mathrm{~A}$ & 8 & 13 & 5.88 & 98.17 & $\mathrm{BF}$ & FALSE \\
\hline 2003 & $\mathrm{~A}$ & 8 & 14 & 6.37 & 98.38 & GB & FALSE \\
\hline 2003 & A & 8 & 15 & 7.57 & 98.55 & GB & FALSE \\
\hline 2003 & $\mathrm{~A}$ & 8 & 16 & 8.99 & 98.73 & GB & FALSE \\
\hline 2003 & $\mathrm{~A}$ & 8 & 17 & 10.87 & 98.85 & GB & FALSE \\
\hline 2003 & $\mathrm{~A}$ & 8 & 18 & 11.72 & 99.10 & TOP BANK & FALSE \\
\hline 2003 & $\mathrm{~A}$ & 8 & 19 & 12.92 & 99.25 & GB & FALSE \\
\hline 2003 & $\mathrm{~A}$ & 8 & 20 & 15.05 & 99.10 & GB & FALSE \\
\hline 2003 & A & 8 & 21 & 17.43 & 99.06 & GB & FALSE \\
\hline 2003 & A & 8 & 22 & 18.91 & 99.23 & GB & FALSE \\
\hline \multirow[t]{2}{*}{2003} & $\mathrm{~A}$ & 8 & 23 & 19.54 & 99.58 & BOT X789R & FALSE \\
\hline & & & & & & REB/LW & \\
\hline \multirow[t]{2}{*}{2003} & $\mathrm{~A}$ & 8 & 24 & 19.51 & 99.99 & TOP $\quad$ X789R & FALSE \\
\hline & & & & & & REB/LW & \\
\hline \multirow[t]{2}{*}{2003} & $\mathrm{~A}$ & 9 & 1 & 0.00 & 100.00 & TOP $\quad$ X9L & FALSE \\
\hline & & & & & & REB/LOW & \\
\hline \multirow[t]{2}{*}{2003} & $\mathrm{~A}$ & 9 & 2 & 0.02 & 99.61 & BOT $\quad \mathrm{X} 9 \mathrm{~L}$ & FALSE \\
\hline & & & & & & REB/LOW & \\
\hline \multirow[t]{2}{*}{2003} & $\mathrm{~A}$ & 9 & 3 & 0.03 & 99.62 & GRND XS9 & FALSE \\
\hline & & & & & & REBAR & \\
\hline 2003 & $\mathrm{~A}$ & 9 & 4 & 0.51 & 99.60 & TOP BANK & FALSE \\
\hline 2003 & $\mathrm{~A}$ & 9 & 5 & 0.85 & 98.65 & GB & FALSE \\
\hline 2003 & A & 9 & 6 & 1.77 & 98.48 & GB & FALSE \\
\hline 2003 & A & 9 & 7 & 2.92 & 98.37 & GB & FALSE \\
\hline 2003 & $\mathrm{~A}$ & 9 & 8 & 3.78 & 98.25 & GB & FALSE \\
\hline 2003 & $\mathrm{~A}$ & 9 & 9 & 4.94 & 98.11 & EW & FALSE \\
\hline 2003 & $\mathrm{~A}$ & 9 & 10 & 6.25 & 97.98 & THALWEG & FALSE \\
\hline 2003 & A & 9 & 11 & 7.44 & 98.04 & EW & FALSE \\
\hline 2003 & A & 9 & 12 & 7.85 & 98.24 & GB & FALSE \\
\hline
\end{tabular}

Continued on Next Page... 
Table D.1 - Continued

\begin{tabular}{|c|c|c|c|c|c|c|c|}
\hline Year & Reach & Section & Point & Dist. (m) & Elev. $(\mathrm{m})$ & Notes & Exclude \\
\hline 2003 & A & 9 & 13 & 8.40 & 98.46 & $\mathrm{BF}$ & FALSE \\
\hline 2003 & A & 9 & 14 & 8.92 & 98.78 & GB & FALSE \\
\hline 2003 & A & 9 & 15 & 10.24 & 98.93 & GB & FALSE \\
\hline 2003 & A & 9 & 16 & 11.89 & 98.91 & GB & FALSE \\
\hline 2003 & $\mathrm{~A}$ & 9 & 17 & 12.88 & 99.19 & GB & FALSE \\
\hline 2003 & A & 9 & 18 & 13.61 & 99.42 & TOP BANK & FALSE \\
\hline 2003 & A & 9 & 19 & 15.60 & 99.36 & GB & FALSE \\
\hline 2003 & A & 9 & 20 & 16.93 & 99.10 & GB & FALSE \\
\hline 2003 & A & 9 & 21 & 17.94 & 99.28 & GB & FALSE \\
\hline 2003 & A & 9 & 22 & 19.02 & 99.30 & GB & FALSE \\
\hline 2003 & $\mathrm{~A}$ & 9 & 23 & 20.03 & 99.65 & GB & FALSE \\
\hline \multirow[t]{2}{*}{2003} & A & 9 & 24 & 20.57 & 99.82 & BOT X789R & FALSE \\
\hline & & & & & & REB/LW & \\
\hline \multirow[t]{2}{*}{2003} & $\mathrm{~A}$ & 9 & 25 & 20.54 & 100.23 & TOP $\quad$ X789R & FALSE \\
\hline & & & & & & REB/LW & \\
\hline \multirow[t]{2}{*}{2003} & $\mathrm{~A}$ & 10 & 1 & 0.00 & 100.00 & TOP $\quad \mathrm{X} 10 \mathrm{~L}$ & FALSE \\
\hline & & & & & & REB/LOW & \\
\hline \multirow[t]{2}{*}{2003} & $\mathrm{~A}$ & 10 & 2 & 0.02 & 99.54 & BOT $\quad \mathrm{X} 10 \mathrm{~L}$ & FALSE \\
\hline & & & & & & REB/LOW & \\
\hline \multirow[t]{2}{*}{2003} & $\mathrm{~A}$ & 10 & 3 & 0.03 & 99.55 & XS $10 \quad$ AT & FALSE \\
\hline & & & & & & REBAR & \\
\hline 2003 & $\mathrm{~A}$ & 10 & 4 & 0.45 & 99.53 & THALWEG & FALSE \\
\hline 2003 & $\mathrm{~A}$ & 10 & 5 & 0.62 & 98.53 & EW & FALSE \\
\hline 2003 & $\mathrm{~A}$ & 10 & 6 & 3.73 & 98.24 & GB & FALSE \\
\hline 2003 & $\mathrm{~A}$ & 10 & 7 & 6.62 & 97.96 & GB & FALSE \\
\hline 2003 & A & 10 & 8 & 7.41 & 97.94 & GB & FALSE \\
\hline 2003 & $\mathrm{~A}$ & 10 & 9 & 8.00 & 97.71 & EW & FALSE \\
\hline 2003 & $\mathrm{~A}$ & 10 & 10 & 8.65 & 97.54 & THALWEG & FALSE \\
\hline 2003 & $\mathrm{~A}$ & 10 & 11 & 9.51 & 97.77 & EW & FALSE \\
\hline 2003 & $\mathrm{~A}$ & 10 & 12 & 10.14 & 98.13 & $\mathrm{BF}$ & FALSE \\
\hline 2003 & $\mathrm{~A}$ & 10 & 13 & 10.86 & 98.60 & GB & FALSE \\
\hline 2003 & $\mathrm{~A}$ & 10 & 14 & 13.16 & 98.94 & TOP BANK & FALSE \\
\hline 2003 & A & 10 & 15 & 14.21 & 98.90 & GB & FALSE \\
\hline 2003 & $\mathrm{~A}$ & 10 & 16 & 15.76 & 99.12 & GB & FALSE \\
\hline 2003 & $\mathrm{~A}$ & 10 & 17 & 17.21 & 98.91 & GB & FALSE \\
\hline 2003 & A & 10 & 18 & 18.92 & 99.21 & GB & FALSE \\
\hline \multirow[t]{2}{*}{2003} & $\mathrm{~A}$ & 10 & 19 & 20.25 & 100.01 & TOP X10R & FALSE \\
\hline & & & & & & REB/LOW & \\
\hline \multirow[t]{2}{*}{2003} & B & 1 & 1 & 0.00 & 100.00 & TOP $\quad \mathrm{X} 1 \mathrm{~L}$ & FALSE \\
\hline & & & & & & REB/LOW & \\
\hline \multirow[t]{2}{*}{2003} & $\mathrm{~B}$ & 1 & 2 & 0.05 & 99.82 & $\mathrm{XS}$ B1 RE- & FALSE \\
\hline & & & & & & BAR & \\
\hline 2003 & B & 1 & 3 & 0.37 & 99.23 & GB & FALSE \\
\hline 2003 & $\mathrm{~B}$ & 1 & 4 & 1.01 & 98.37 & GB & FALSE \\
\hline 2003 & B & 1 & 5 & 2.01 & 97.88 & $\mathrm{BF}$ & FALSE \\
\hline 2003 & B & 1 & 6 & 2.97 & 97.60 & EW & FALSE \\
\hline 2003 & B & 1 & 7 & 3.81 & 97.52 & THALWEG & FALSE \\
\hline 2003 & B & 1 & 8 & 6.21 & 97.57 & GB & FALSE \\
\hline 2003 & $\mathrm{~B}$ & 1 & 9 & 7.00 & 97.75 & GB & FALSE \\
\hline 2003 & B & 1 & 10 & 7.61 & 98.07 & GB & FALSE \\
\hline 2003 & B & 1 & 11 & 8.60 & 98.66 & GB & FALSE \\
\hline 2003 & B & 1 & 12 & 9.32 & 99.20 & GB & FALSE \\
\hline
\end{tabular}

Continued on Next Page... 
Table D.1 - Continued

\begin{tabular}{|c|c|c|c|c|c|c|c|}
\hline Year & Reach & Section & Point & Dist. (m) & Elev. $(\mathrm{m})$ & Notes & Exclude \\
\hline \multirow[t]{2}{*}{2003} & B & 1 & 13 & 10.09 & 99.76 & XS B1 RE- & FALSE \\
\hline & & & & & & BAR & \\
\hline \multirow[t]{2}{*}{2003} & B & 1 & 14 & 10.10 & 99.99 & TOP $\quad \mathrm{X} 1 \mathrm{R}$ & FALSE \\
\hline & & & & & & REB/LOW & \\
\hline \multirow[t]{2}{*}{2003} & B & 2 & 1 & 0.00 & 100.00 & TOP $\quad \mathrm{X} 2 \mathrm{~L}$ & FALSE \\
\hline & & & & & & REB/LOW & \\
\hline \multirow[t]{2}{*}{2003} & B & 2 & 2 & 0.01 & 99.57 & XS B2 RE- & FALSE \\
\hline & & & & & & BAR & \\
\hline \multirow[t]{2}{*}{2003} & B & 2 & 3 & 0.28 & 99.07 & GB FOOT- & FALSE \\
\hline & & & & & & PATH & \\
\hline \multirow[t]{2}{*}{2003} & B & 2 & 4 & 1.15 & 98.73 & GB FOOT- & FALSE \\
\hline & & & & & & РATH & \\
\hline 2003 & B & 2 & 5 & 1.82 & 98.32 & GB & FALSE \\
\hline 2003 & B & 2 & 6 & 2.67 & 97.64 & $\mathrm{BF}$ & FALSE \\
\hline 2003 & B & 2 & 7 & 3.25 & 97.40 & GB & FALSE \\
\hline 2003 & B & 2 & 8 & 3.78 & 97.37 & EW & FALSE \\
\hline 2003 & B & 2 & 9 & 4.64 & 97.26 & THALWEG & FALSE \\
\hline 2003 & B & 2 & 10 & 5.93 & 97.34 & EW & FALSE \\
\hline 2003 & B & 2 & 11 & 7.00 & 97.39 & EW & FALSE \\
\hline 2003 & B & 2 & 12 & 8.09 & 97.80 & GB & FALSE \\
\hline 2003 & B & 2 & 13 & 8.93 & 98.46 & GB & FALSE \\
\hline 2003 & B & 2 & 14 & 9.81 & 99.10 & GB & FALSE \\
\hline \multirow[t]{2}{*}{2003} & B & 2 & 15 & 10.54 & 99.49 & XS B2 RE- & FALSE \\
\hline & & & & & & BAR & \\
\hline \multirow[t]{2}{*}{2003} & B & 2 & 16 & 10.58 & 99.99 & TOP $\quad \mathrm{X} 2 \mathrm{R}$ & FALSE \\
\hline & & & & & & REB/LOW & \\
\hline \multirow[t]{2}{*}{2003} & B & 3 & 1 & 0.00 & 100.00 & TOP $\quad$ X3L & FALSE \\
\hline & & & & & & REB/LOW & \\
\hline \multirow[t]{2}{*}{2003} & B & 3 & 2 & 0.04 & 99.43 & XS B3 RE- & FALSE \\
\hline & & & & & & BAR & \\
\hline 2003 & B & 3 & 3 & 0.44 & 99.17 & GB & FALSE \\
\hline 2003 & B & 3 & 4 & 1.12 & 98.72 & TOP BANK & FALSE \\
\hline 2003 & B & 3 & 5 & 1.59 & 98.34 & $\mathrm{BF}$ & FALSE \\
\hline 2003 & B & 3 & 6 & 2.92 & 98.12 & GB & FALSE \\
\hline 2003 & B & 3 & 7 & 4.59 & 97.95 & EW & FALSE \\
\hline 2003 & B & 3 & 8 & 5.20 & 97.86 & THALWEG & FALSE \\
\hline 2003 & B & 3 & 9 & 5.94 & 97.96 & EW & FALSE \\
\hline 2003 & B & 3 & 10 & 6.50 & 98.21 & GB & FALSE \\
\hline 2003 & B & 3 & 11 & 7.03 & 98.50 & GB & FALSE \\
\hline 2003 & B & 3 & 12 & 7.81 & 98.96 & GB & FALSE \\
\hline \multirow[t]{2}{*}{2003} & B & 3 & 13 & 8.51 & 99.78 & XS B3 RE- & FALSE \\
\hline & & & & & & BAR & \\
\hline \multirow[t]{2}{*}{2003} & B & 3 & 14 & 8.50 & 99.97 & TOP $\quad \mathrm{X} 3 \mathrm{R}$ & FALSE \\
\hline & & & & & & REB/LOW & \\
\hline \multirow[t]{2}{*}{2003} & B & 4 & 1 & 0.00 & 100.00 & TOP $\quad \mathrm{X} 2 \mathrm{~L}$ & TRUE \\
\hline & & & & & & REB/LOW & \\
\hline \multirow[t]{2}{*}{2003} & B & 4 & 2 & 0.01 & 99.57 & XS B2 RE- & TRUE \\
\hline & & & & & & $\mathrm{BAR}$ & \\
\hline \multirow[t]{2}{*}{2003} & B & 4 & 3 & 0.28 & 99.07 & GB FOOT- & TRUE \\
\hline & & & & & & РАTH & \\
\hline 2003 & $\mathrm{~B}$ & 4 & 4 & 1.15 & 98.73 & GB FOOT- & TRUE \\
\hline
\end{tabular}

Continued on Next Page... 
Table D.1 - Continued

\begin{tabular}{|c|c|c|c|c|c|c|c|}
\hline Year & Reach & Section & Point & Dist. (m) & Elev. (m) & Notes & Exclude \\
\hline 2003 & B & 4 & 5 & 1.82 & 98.32 & GB & TRUE \\
\hline 2003 & B & 4 & 6 & 2.67 & 97.64 & BF & TRUE \\
\hline 2003 & $\mathrm{~B}$ & 4 & 7 & 3.25 & 97.40 & GB & TRUE \\
\hline 2003 & B & 4 & 8 & 3.78 & 97.37 & EW & TRUE \\
\hline 2003 & $\mathrm{~B}$ & 4 & 9 & 4.64 & 97.26 & THALWEG & TRUE \\
\hline 2003 & B & 4 & 10 & 5.93 & 97.34 & EW & TRUE \\
\hline 2003 & $\mathrm{~B}$ & 4 & 11 & 7.00 & 97.39 & EW & TRUE \\
\hline 2003 & B & 4 & 12 & 8.09 & 97.80 & GB & TRUE \\
\hline 2003 & $\mathrm{~B}$ & 4 & 13 & 8.93 & 98.46 & GB & TRUE \\
\hline 2003 & B & 4 & 14 & 9.81 & 99.10 & GB & TRUE \\
\hline \multirow[t]{2}{*}{2003} & B & 4 & 15 & 10.54 & 99.49 & $\mathrm{XS}$ B2 RE- & TRUE \\
\hline & & & & & & BAR & \\
\hline \multirow[t]{2}{*}{2003} & B & 4 & 16 & 10.58 & 99.99 & TOP $\quad \mathrm{X} 2 \mathrm{R}$ & TRUE \\
\hline & & & & & & REB/LOW & \\
\hline \multirow[t]{2}{*}{2003} & B & 5 & 1 & 0.00 & 100.00 & TOP $\quad \mathrm{X} 3 \mathrm{~L}$ & FALSE \\
\hline & & & & & & REB/LOW & \\
\hline \multirow[t]{2}{*}{2003} & B & 5 & 2 & 0.04 & 99.43 & XS B3 RE- & FALSE \\
\hline & & & & & & BAR & \\
\hline 2003 & B & 5 & 3 & 0.44 & 99.17 & GB & FALSE \\
\hline 2003 & B & 5 & 4 & 1.12 & 98.72 & TOP BANK & FALSE \\
\hline 2003 & B & 5 & 5 & 1.59 & 98.34 & $\mathrm{BF}$ & FALSE \\
\hline 2003 & B & 5 & 6 & 2.92 & 98.12 & GB & FALSE \\
\hline 2003 & $\mathrm{~B}$ & 5 & 7 & 4.59 & 97.95 & EW & FALSE \\
\hline 2003 & B & 5 & 8 & 5.20 & 97.86 & THALWEG & FALSE \\
\hline 2003 & B & 5 & 9 & 5.94 & 97.96 & EW & FALSE \\
\hline 2003 & B & 5 & 10 & 6.50 & 98.21 & GB & FALSE \\
\hline 2003 & B & 5 & 11 & 7.03 & 98.50 & GB & FALSE \\
\hline 2003 & $\mathrm{~B}$ & 5 & 12 & 7.81 & 98.96 & GB & FALSE \\
\hline \multirow[t]{2}{*}{2003} & B & 5 & 13 & 8.51 & 99.78 & XS B3 RE- & FALSE \\
\hline & & & & & & BAR & \\
\hline \multirow[t]{2}{*}{2003} & $\mathrm{~B}$ & 5 & 14 & 8.50 & 99.97 & TOP $\quad$ X3R & FALSE \\
\hline & & & & & & REB/LOW & \\
\hline \multirow[t]{2}{*}{2003} & B & 6 & 1 & 0.00 & 100.00 & TOP $\quad \mathrm{X} 6 \mathrm{~L}$ & FALSE \\
\hline & & & & & & REB/LOW & \\
\hline \multirow[t]{2}{*}{2003} & $\mathrm{~B}$ & 6 & 2 & 0.01 & 99.94 & $\mathrm{XS}$ B6 RE- & FALSE \\
\hline & & & & & & BAR & \\
\hline 2003 & B & 6 & 3 & 0.91 & 99.33 & GB & FALSE \\
\hline 2003 & B & 6 & 4 & 1.54 & 98.99 & GB & FALSE \\
\hline 2003 & $\mathrm{~B}$ & 6 & 5 & 2.17 & 98.72 & $\mathrm{BF}$ & FALSE \\
\hline 2003 & $\mathrm{~B}$ & 6 & 6 & 3.28 & 98.31 & EW & FALSE \\
\hline 2003 & B & 6 & 7 & 4.55 & 98.24 & THALWEG & FALSE \\
\hline 2003 & B & 6 & 8 & 5.17 & 98.33 & EW & FALSE \\
\hline 2003 & B & 6 & 9 & 5.98 & 98.56 & GB & FALSE \\
\hline 2003 & $\mathrm{~B}$ & 6 & 10 & 6.61 & 98.68 & GB & FALSE \\
\hline 2003 & B & 6 & 11 & 7.31 & 99.17 & GB & FALSE \\
\hline \multirow[t]{2}{*}{2003} & B & 6 & 12 & 7.97 & 99.84 & XS B6 RE- & FALSE \\
\hline & & & & & & $\mathrm{BAR}$ & \\
\hline \multirow[t]{2}{*}{2003} & B & 6 & 13 & 7.96 & 100.01 & TOP $\quad \mathrm{X} 6 \mathrm{R}$ & FALSE \\
\hline & & & & & & REB/LOW & \\
\hline \multirow[t]{2}{*}{2003} & B & 7 & 1 & 0.00 & 100.00 & XS B7 RE- & FALSE \\
\hline & & & & & & BAR & \\
\hline 2003 & $\mathrm{~B}$ & 7 & 2 & 0.62 & 99.59 & GB & FALSE \\
\hline
\end{tabular}

Continued on Next Page... 
Table D.1 - Continued

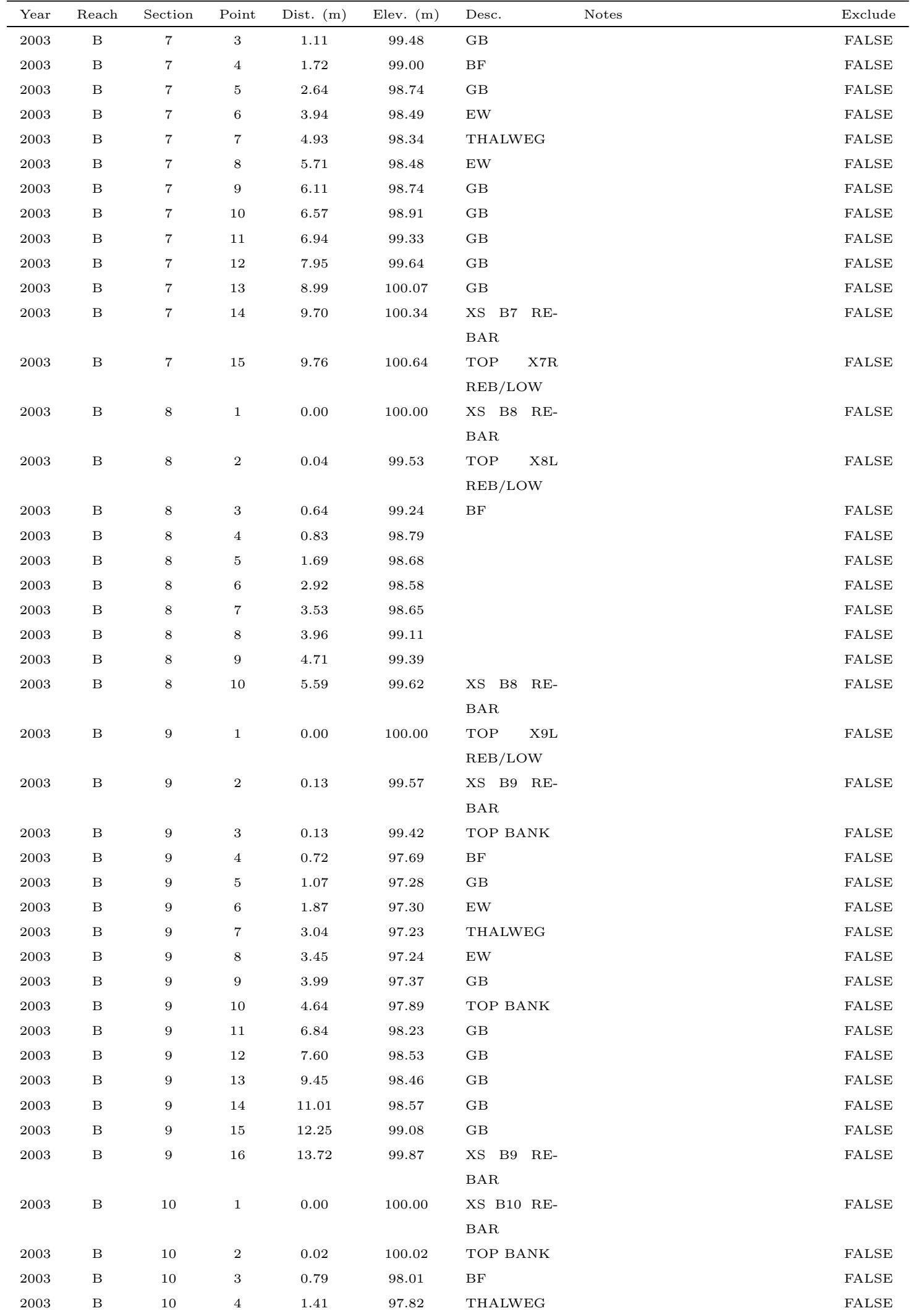

Continued on Next Page... 
Table D.1 - Continued

\begin{tabular}{|c|c|c|c|c|c|c|c|c|}
\hline Year & Reach & Section & Point & Dist. (m) & Elev. $(\mathrm{m})$ & Desc. & Notes & Exclude \\
\hline 2003 & B & 10 & 5 & 2.23 & 97.63 & EW & & FALSE \\
\hline 2003 & $\mathrm{~B}$ & 10 & 6 & 3.28 & 97.50 & GB & & FALSE \\
\hline 2003 & B & 10 & 7 & 4.09 & 97.59 & EW & & FALSE \\
\hline 2003 & $\mathrm{~B}$ & 10 & 8 & 5.02 & 97.83 & GB & & FALSE \\
\hline 2003 & B & 10 & 9 & 6.66 & 98.51 & TOP BANK & & FALSE \\
\hline 2003 & B & 10 & 10 & 9.84 & 98.48 & GB & & FALSE \\
\hline 2003 & B & 10 & 11 & 12.62 & 98.42 & GB & & FALSE \\
\hline 2003 & B & 10 & 12 & 13.88 & 99.11 & GB & & FALSE \\
\hline 2003 & B & 10 & 13 & 15.48 & 100.12 & $\begin{array}{l}\text { XS B10 RE- } \\
\text { BAR }\end{array}$ & & FALSE \\
\hline 2004 & $\mathrm{~A}$ & 1 & 1 & 0.00 & 100.00 & $\begin{array}{llll}\text { Top X } & 1 & \text { L } \\
\text { Reb/low } & \end{array}$ & & FALSE \\
\hline 2004 & $\mathrm{~A}$ & 1 & 2 & 0.00 & 99.82 & $\begin{array}{l}\text { Bot X } 1 \text { L Re- } \\
\text { b/low }\end{array}$ & & FALSE \\
\hline 2004 & $\mathrm{~A}$ & 1 & 3 & 2.53 & 99.34 & BIS & & FALSE \\
\hline 2004 & $\mathrm{~A}$ & 1 & 4 & 3.11 & 99.22 & $\mathrm{BF}$ & & FALSE \\
\hline 2004 & $\mathrm{~A}$ & 1 & 5 & 3.57 & 98.99 & BIS & & FALSE \\
\hline 2004 & A & 1 & 6 & 4.30 & 98.72 & EW & & FALSE \\
\hline 2004 & $\mathrm{~A}$ & 1 & 7 & 5.00 & 98.60 & $\mathrm{TW}$ & & FALSE \\
\hline 2004 & A & 1 & 8 & 5.46 & 98.73 & EW & & FALSE \\
\hline 2004 & $\mathrm{~A}$ & 1 & 9 & 6.49 & 98.97 & BIS & & FALSE \\
\hline 2004 & A & 1 & 10 & 7.19 & 99.39 & ТОВ & & FALSE \\
\hline 2004 & A & 1 & 11 & 9.08 & 99.75 & 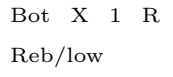 & & FALSE \\
\hline 2004 & $\mathrm{~A}$ & 1 & 12 & 9.08 & 100.01 & $\begin{array}{lll}\text { Top X } & 1 & \mathrm{R} \\
\text { Reb/low } & \end{array}$ & & FALSE \\
\hline 2004 & A & 2 & 1 & 0.00 & 100.00 & $\begin{array}{l}\text { Top X } \quad 2 \quad \text { L } \\
\text { Reb/low }\end{array}$ & & FALSE \\
\hline 2004 & $\mathrm{~A}$ & 2 & 2 & 0.00 & 99.67 & $\begin{array}{l}\text { Bot X } 2 \text { L Re- } \\
\text { b/low }\end{array}$ & & FALSE \\
\hline 2004 & A & 2 & 3 & 1.28 & 99.42 & BIS & & FALSE \\
\hline 2004 & A & 2 & 4 & 2.13 & 98.98 & BIS & & FALSE \\
\hline 2004 & A & 2 & 5 & 2.99 & 98.78 & BIS & & FALSE \\
\hline 2004 & A & 2 & 6 & 3.75 & 98.52 & $\mathrm{BF}$ & & FALSE \\
\hline 2004 & A & 2 & 7 & 4.48 & 98.25 & BIS & & FALSE \\
\hline 2004 & A & 2 & 8 & 5.03 & 98.14 & EW & & FALSE \\
\hline 2004 & A & 2 & 9 & 5.91 & 97.99 & $\mathrm{TW}$ & & FALSE \\
\hline 2004 & A & 2 & 10 & 7.16 & 98.16 & EW & & FALSE \\
\hline 2004 & A & 2 & 11 & 7.71 & 98.49 & BIS & & FALSE \\
\hline 2004 & A & 2 & 12 & 8.14 & 98.70 & BIS & & FALSE \\
\hline 2004 & A & 2 & 13 & 9.21 & 98.92 & ТОВ & & FALSE \\
\hline 2004 & A & 2 & 14 & 9.85 & 99.38 & BIS & & FALSE \\
\hline 2004 & A & 2 & 15 & 10.70 & 99.68 & $\begin{array}{l}\text { Bot } \mathrm{X} \quad 2 \quad \mathrm{R} \\
\text { Reb/low }\end{array}$ & & FALSE \\
\hline 2004 & A & 2 & 16 & 10.70 & 100.02 & $\begin{array}{l}\text { Top X } 2 \quad \mathrm{R} \\
\text { Reb/low }\end{array}$ & & FALSE \\
\hline 2004 & A & 3 & 1 & 0.00 & 100.00 & $\begin{array}{l}\text { Top X3L Re- } \\
\text { b/low }\end{array}$ & & FALSE \\
\hline 2004 & A & 3 & 2 & 0.00 & 99.49 & $\begin{array}{l}\text { Bot X3L Re- } \\
\text { b/low }\end{array}$ & & FALSE \\
\hline 2004 & A & 3 & 3 & 0.61 & 99.30 & BIS & between stump and alder & FALSE \\
\hline 2004 & $\mathrm{~A}$ & 3 & 4 & 0.91 & 98.88 & BIS & & FALSE \\
\hline
\end{tabular}

Continued on Next Page... 
Table D.1 - Continued

\begin{tabular}{|c|c|c|c|c|c|c|c|c|}
\hline Year & Reach & Section & Point & Dist. $(\mathrm{m})$ & Elev. $(\mathrm{m})$ & Desc. & Notes & Exclude \\
\hline 2004 & A & 3 & 5 & 1.83 & 98.43 & ТОВ & & FALSE \\
\hline 2004 & A & 3 & 6 & 2.19 & 97.92 & EW & & FALSE \\
\hline 2004 & A & 3 & 7 & 3.29 & 97.79 & $\mathrm{TW}$ & & FALSE \\
\hline 2004 & A & 3 & 8 & 4.39 & 97.90 & EW & & FALSE \\
\hline 2004 & A & 3 & 9 & 5.67 & 98.42 & $\mathrm{BF}$ & & FALSE \\
\hline 2004 & A & 3 & 10 & 6.83 & 98.53 & ТОВ & & FALSE \\
\hline 2004 & A & 3 & 11 & 8.08 & 98.48 & BIS & one foot low, corrected & FALSE \\
\hline 2004 & A & 3 & 12 & 9.72 & 99.32 & BIS & & FALSE \\
\hline 2004 & A & 3 & 13 & 10.70 & 99.67 & $\begin{array}{l}\text { Bot } \mathrm{X} \quad 3 \quad \mathrm{R} \\
\text { Reb/low }\end{array}$ & & FALSE \\
\hline 2004 & A & 3 & 14 & 10.70 & 100.10 & $\begin{array}{l}\text { Top X } 3 \text { R } \\
\text { Reb/low }\end{array}$ & & FALSE \\
\hline 2004 & A & 4 & 1 & 0.00 & 100.00 & $\begin{array}{l}\text { Top X } 4 \text { L } \\
\text { Reb/low }\end{array}$ & & FALSE \\
\hline 2004 & A & 4 & 2 & 0.00 & 99.70 & $\begin{array}{l}\text { Bot X } 4 \text { L Re- } \\
\text { b/low }\end{array}$ & & FALSE \\
\hline 2004 & A & 4 & 3 & 1.22 & 99.47 & BIS & & FALSE \\
\hline 2004 & A & 4 & 4 & 2.29 & 99.31 & ТОВ & & FALSE \\
\hline 2004 & A & 4 & 5 & 3.14 & 98.62 & EW & & FALSE \\
\hline 2004 & A & 4 & 6 & 4.24 & 98.51 & $\mathrm{TW}$ & & FALSE \\
\hline 2004 & A & 4 & 7 & 5.15 & 98.57 & EW & & FALSE \\
\hline 2004 & A & 4 & 8 & 6.71 & 98.98 & $\mathrm{BF}$ & & FALSE \\
\hline 2004 & A & 4 & 9 & 7.74 & 99.22 & ТОВ & & FALSE \\
\hline 2004 & A & 4 & 10 & 8.72 & 99.46 & $\begin{array}{l}\text { Bot } \mathrm{X} \quad 4 \quad \mathrm{R} \\
\text { Reb/low }\end{array}$ & & FALSE \\
\hline 2004 & A & 4 & 11 & 8.72 & 99.84 & $\begin{array}{l}\text { Top X } 4 \quad \mathrm{R} \\
\text { Reb/low }\end{array}$ & & FALSE \\
\hline 2004 & A & 5 & 1 & 0.00 & 100.00 & $\begin{array}{l}\text { Top X } 5 \text { L } \\
\text { Reb/low }\end{array}$ & & FALSE \\
\hline 2004 & A & 5 & 2 & 0.00 & 99.70 & $\begin{array}{l}\text { Bot X } 5 \text { L Re- } \\
\text { b/low }\end{array}$ & & FALSE \\
\hline 2004 & A & 5 & 3 & 1.46 & 99.92 & $\begin{array}{l}\text { ТОВ *in a } \\
\text { root ball of } \\
\text { Alder }\end{array}$ & & FALSE \\
\hline 2004 & A & 5 & 4 & 2.19 & 99.17 & BIS & & FALSE \\
\hline 2004 & A & 5 & 5 & 2.77 & 98.80 & BIS & & FALSE \\
\hline 2004 & A & 5 & 6 & 3.29 & 98.72 & EW & & FALSE \\
\hline 2004 & A & 5 & 7 & 4.27 & 98.47 & $\mathrm{TW}$ & & FALSE \\
\hline 2004 & A & 5 & 8 & 5.06 & 98.63 & $\begin{array}{ll}\text { BIS } & * \text { in } \\
\text { stream next } \\
\text { to rock }\end{array}$ & & FALSE \\
\hline 2004 & A & 5 & 9 & 5.49 & 98.73 & $\begin{array}{l}\text { EW *other } \\
\text { side of rock }\end{array}$ & & FALSE \\
\hline 2004 & A & 5 & 10 & 6.07 & 99.08 & $\mathrm{BF}$ & & FALSE \\
\hline 2004 & A & 5 & 11 & 6.74 & 99.25 & TB & & FALSE \\
\hline 2004 & A & 5 & 12 & 7.83 & 99.60 & $\begin{array}{lll}\text { Bot } X \quad 5 & R \\
\text { Reb/low } & \end{array}$ & & FALSE \\
\hline 2004 & A & 5 & 13 & 7.83 & 99.98 & $\begin{array}{llll}\text { Top } & X & 5 & R \\
\text { Reb/low } & \end{array}$ & & FALSE \\
\hline 2004 & A & 6 & 1 & 0.00 & 100.00 & $\begin{array}{l}\text { Top X } 6 \text { L } \\
\text { Reb/low }\end{array}$ & & FALSE \\
\hline
\end{tabular}

Continued on Next Page... 
Table D.1 - Continued

\begin{tabular}{|c|c|c|c|c|c|c|c|}
\hline Year & Reach & Section & Point & Dist. (m) & Elev. $(\mathrm{m})$ & Notes & Exclude \\
\hline 2004 & A & 6 & 2 & 0.00 & 99.82 & $\begin{array}{l}\text { Bot X } 6 \text { L Re- } \\
\text { b/low }\end{array}$ & FALSE \\
\hline 2004 & A & 6 & 3 & 0.46 & 99.66 & ТОВ & FALSE \\
\hline 2004 & $\mathrm{~A}$ & 6 & 4 & 0.73 & 98.58 & BIS & FALSE \\
\hline 2004 & A & 6 & 5 & 0.91 & 98.45 & $\mathrm{BF}$ & FALSE \\
\hline 2004 & A & 6 & 6 & 1.55 & 98.01 & EW & FALSE \\
\hline 2004 & A & 6 & 7 & 2.26 & 97.93 & TW & FALSE \\
\hline 2004 & A & 6 & 8 & 3.08 & 98.02 & $\begin{array}{l}\text { BIS } \quad *_{\text {in }} \\
\text { stream }\end{array}$ & FALSE \\
\hline 2004 & A & 6 & 9 & 3.93 & 98.04 & EW & FALSE \\
\hline 2004 & A & 6 & 10 & 4.57 & 98.66 & BIS & FALSE \\
\hline 2004 & A & 6 & 11 & 5.27 & 98.97 & BIS & FALSE \\
\hline 2004 & A & 6 & 12 & 6.40 & 99.13 & ТОВ & FALSE \\
\hline 2004 & A & 6 & 13 & 9.63 & 99.17 & BIS & FALSE \\
\hline 2004 & A & 6 & 14 & 13.23 & 99.13 & BIS & FALSE \\
\hline 2004 & $\mathrm{~A}$ & 6 & 15 & 14.78 & 99.14 & BIS & FALSE \\
\hline 2004 & $\mathrm{~A}$ & 6 & 16 & 15.12 & 99.43 & $\begin{array}{llll}\text { Bot } & \mathrm{X} & 6 & \mathrm{R} \\
\text { Reb/low } & & \end{array}$ & FALSE \\
\hline 2004 & $\mathrm{~A}$ & 6 & 17 & 15.12 & 99.80 & $\begin{array}{llll}\text { Top } & \mathrm{X} & 6 & \mathrm{R} \\
\text { Reb/low } & \end{array}$ & FALSE \\
\hline 2004 & $\mathrm{~A}$ & 7 & 1 & 0.00 & 100.00 & $\begin{array}{llll}\text { Top } & \mathrm{X} & 7 & \mathrm{~L} \\
\text { Reb/low } & \end{array}$ & FALSE \\
\hline 2004 & A & 7 & 2 & 0.00 & 99.76 & $\begin{array}{l}\text { Bot X } 7 \text { L Re- } \\
\text { b/low }\end{array}$ & FALSE \\
\hline 2004 & A & 7 & 3 & 0.73 & 99.68 & ТОВ & FALSE \\
\hline 2004 & A & 7 & 4 & 1.83 & 98.42 & BIS & FALSE \\
\hline 2004 & A & 7 & 5 & 2.50 & 98.28 & EW & FALSE \\
\hline 2004 & A & 7 & 6 & 3.57 & 98.18 & TW & FALSE \\
\hline 2004 & $\mathrm{~A}$ & 7 & 7 & 4.39 & 98.30 & EW & FALSE \\
\hline 2004 & A & 7 & 8 & 5.55 & 98.73 & $\mathrm{BF}$ & FALSE \\
\hline 2004 & A & 7 & 9 & 6.95 & 99.01 & BIS & FALSE \\
\hline 2004 & $\mathrm{~A}$ & 7 & 10 & 9.24 & 99.43 & ТОВ & FALSE \\
\hline 2004 & A & 7 & 11 & 11.13 & 99.53 & BIS & FALSE \\
\hline 2004 & $\mathrm{~A}$ & 7 & 12 & 13.93 & 99.41 & BIS & FALSE \\
\hline 2004 & $\mathrm{~A}$ & 7 & 13 & 16.28 & 99.30 & BIS & FALSE \\
\hline 2004 & A & 7 & 14 & 18.11 & 99.57 & BIS & FALSE \\
\hline 2004 & $\mathrm{~A}$ & 7 & 15 & 18.62 & 99.83 & $\begin{array}{llll}\text { Bot } & X & 7 & R \\
\text { Reb/low } & & \end{array}$ & FALSE \\
\hline 2004 & $\mathrm{~A}$ & 7 & 16 & 18.62 & 100.20 & $\begin{array}{llll}\text { Top } & \mathrm{X} & 7 & \mathrm{R} \\
\text { Reb/low } & & \end{array}$ & FALSE \\
\hline 2004 & A & 8 & 1 & 0.00 & 100.00 & $\begin{array}{l}\text { Top X } 8 \text { L } \\
\text { Reb/low }\end{array}$ & FALSE \\
\hline 2004 & $\mathrm{~A}$ & 8 & 2 & 0.00 & 99.62 & $\begin{array}{l}\text { Bot X } 8 \text { L Re- } \\
\text { b/low }\end{array}$ & FALSE \\
\hline 2004 & A & 8 & 3 & 0.64 & 99.57 & ТОВ & FALSE \\
\hline 2004 & A & 8 & 4 & 1.16 & 98.41 & BIS & FALSE \\
\hline 2004 & A & 8 & 5 & 1.86 & 98.22 & BIS & FALSE \\
\hline 2004 & A & 8 & 6 & 2.53 & 98.11 & $\mathrm{BF}$ & FALSE \\
\hline 2004 & A & 8 & 7 & 3.23 & 98.10 & BIS & FALSE \\
\hline 2004 & A & 8 & 8 & 3.66 & 98.01 & EW & FALSE \\
\hline 2004 & A & 8 & 9 & 4.15 & 97.95 & $\mathrm{TW}$ & FALSE \\
\hline 2004 & A & 8 & 10 & 5.00 & 97.98 & BIS & FALSE \\
\hline
\end{tabular}

Continued on Next Page... 
Table D.1 - Continued

\begin{tabular}{|c|c|c|c|c|c|c|c|}
\hline Year & Reach & Section & Point & Dist. (m) & Elev. $(\mathrm{m})$ & Notes & Exclude \\
\hline 2004 & $\mathrm{~A}$ & 8 & 11 & 5.52 & 98.04 & EW & FALSE \\
\hline 2004 & A & 8 & 12 & 5.91 & 98.21 & BIS & FALSE \\
\hline 2004 & A & 8 & 13 & 6.10 & 98.34 & BIS & FALSE \\
\hline 2004 & A & 8 & 14 & 7.41 & 98.59 & BIS & FALSE \\
\hline 2004 & A & 8 & 15 & 10.15 & 98.82 & BIS & FALSE \\
\hline 2004 & A & 8 & 16 & 12.28 & 99.22 & BIS & FALSE \\
\hline 2004 & A & 8 & 17 & 14.02 & 99.19 & BIS & FALSE \\
\hline 2004 & A & 8 & 18 & 15.97 & 98.99 & BIS & FALSE \\
\hline 2004 & A & 8 & 19 & 17.89 & 99.15 & BIS & FALSE \\
\hline 2004 & A & 8 & 20 & 18.65 & 99.23 & BIS & FALSE \\
\hline 2004 & A & 8 & 21 & 19.48 & 99.63 & $\begin{array}{llll}\text { Bot } & X & 8 & R \\
\text { Reb/low } & & \end{array}$ & FALSE \\
\hline 2004 & A & 8 & 22 & 19.48 & 99.99 & $\begin{array}{llll}\text { Top } & X & 8 & R \\
\text { Reb/low } & \end{array}$ & FALSE \\
\hline 2004 & A & 9 & 1 & 0.00 & 100.00 & $\begin{array}{llll}\text { Top } & \text { X } & 9 & \text { L } \\
\text { Reb/low } & \end{array}$ & FALSE \\
\hline 2004 & A & 9 & 2 & 0.00 & 99.63 & $\begin{array}{l}\text { Bot X } 9 \text { L Re- } \\
\text { b/low }\end{array}$ & FALSE \\
\hline 2004 & A & 9 & 3 & 0.67 & 99.69 & ТОВ & FALSE \\
\hline 2004 & A & 9 & 4 & 0.85 & 98.71 & BIS & FALSE \\
\hline 2004 & A & 9 & 5 & 1.98 & 98.53 & BIS & FALSE \\
\hline 2004 & A & 9 & 6 & 3.35 & 98.39 & BIS & FALSE \\
\hline 2004 & A & 9 & 7 & 3.72 & 98.38 & $\mathrm{BF}$ & FALSE \\
\hline 2004 & A & 9 & 8 & 4.48 & 98.29 & BIS & FALSE \\
\hline 2004 & A & 9 & 9 & 4.79 & 98.24 & EW & FALSE \\
\hline 2004 & A & 9 & 10 & 5.36 & 98.18 & $\begin{array}{l}\text { BIS } \quad *_{\text {in }} \\
\text { stream }\end{array}$ & FALSE \\
\hline 2004 & A & 9 & 11 & 5.79 & 98.20 & $\begin{array}{l}\text { BIS } \quad *_{\text {in }} \\
\text { stream }\end{array}$ & FALSE \\
\hline 2004 & A & 9 & 12 & 6.40 & 98.21 & $\mathrm{TW}$ & FALSE \\
\hline 2004 & A & 9 & 13 & 6.77 & 98.27 & EW & FALSE \\
\hline 2004 & A & 9 & 14 & 7.22 & 98.40 & BIS & FALSE \\
\hline 2004 & A & 9 & 15 & 7.92 & 98.51 & BIS & FALSE \\
\hline 2004 & A & 9 & 16 & 8.29 & 98.72 & ТОВ & FALSE \\
\hline 2004 & A & 9 & 17 & 9.78 & 98.26 & BIS & TRUE \\
\hline 2004 & A & 9 & 18 & 10.39 & 98.95 & BIS & FALSE \\
\hline 2004 & A & 9 & 19 & 12.41 & 99.01 & BIS & FALSE \\
\hline 2004 & A & 9 & 20 & 12.98 & 99.38 & BIS & FALSE \\
\hline 2004 & A & 9 & 21 & 14.02 & 99.44 & BIS & FALSE \\
\hline 2004 & A & 9 & 22 & 14.78 & 99.35 & BIS & FALSE \\
\hline 2004 & A & 9 & 23 & 16.22 & 99.25 & BIS & FALSE \\
\hline 2004 & A & 9 & 24 & 16.92 & 99.17 & BIS & FALSE \\
\hline 2004 & A & 9 & 25 & 18.59 & 99.31 & BIS & FALSE \\
\hline 2004 & A & 9 & 26 & 19.87 & 99.52 & BIS & FALSE \\
\hline 2004 & A & 9 & 27 & 20.51 & 99.84 & 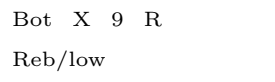 & FALSE \\
\hline 2004 & A & 9 & 28 & 20.51 & 100.13 & $\begin{array}{l}\text { Top } \quad \mathrm{X} \quad 9 \mathrm{R} \\
\text { Reb/low }\end{array}$ & FALSE \\
\hline 2004 & A & 10 & 1 & 0.00 & 100.00 & $\begin{array}{l}\text { Top X } 10 \mathrm{~L} \\
\text { Reb/low }\end{array}$ & FALSE \\
\hline 2004 & A & 10 & 2 & 0.00 & 99.57 & $\begin{array}{l}\text { Bot } X 10 \mathrm{~L} \\
\text { Reb/low }\end{array}$ & FALSE \\
\hline
\end{tabular}

Continued on Next Page... 
Table D.1 - Continued

\begin{tabular}{|c|c|c|c|c|c|c|c|}
\hline Year & Reach & Section & Point & Dist. (m) & Elev. $(\mathrm{m})$ & Notes & Exclude \\
\hline 2004 & $\mathrm{~A}$ & 10 & 3 & 0.46 & 99.56 & ТОВ & FALSE \\
\hline 2004 & A & 10 & 4 & 0.70 & 98.56 & BIS & FALSE \\
\hline 2004 & A & 10 & 5 & 1.80 & 98.49 & BIS & FALSE \\
\hline 2004 & A & 10 & 6 & 3.81 & 98.25 & $\mathrm{BF}$ & FALSE \\
\hline 2004 & A & 10 & 7 & 3.90 & 98.17 & BIS & FALSE \\
\hline 2004 & A & 10 & 8 & 5.33 & 98.17 & BIS & FALSE \\
\hline 2004 & $\mathrm{~A}$ & 10 & 9 & 5.82 & 97.87 & EW & FALSE \\
\hline 2004 & $\mathrm{~A}$ & 10 & 10 & 6.46 & 97.55 & $\begin{array}{l}\mathrm{TW} * \text { thalwag } \\
\text { in a pool }\end{array}$ & FALSE \\
\hline 2004 & A & 10 & 11 & 7.77 & 97.81 & $\begin{array}{l}\text { BIS } \\
\text { stream }\end{array}$ & FALSE \\
\hline 2004 & $\mathrm{~A}$ & 10 & 12 & 9.48 & 97.82 & EW & FALSE \\
\hline 2004 & $\mathrm{~A}$ & 10 & 13 & 10.70 & 98.66 & ТОВ & FALSE \\
\hline 2004 & $\mathrm{~A}$ & 10 & 14 & 13.23 & 98.98 & BIS & FALSE \\
\hline 2004 & $\mathrm{~A}$ & 10 & 15 & 15.91 & 99.08 & BIS & FALSE \\
\hline 2004 & $\mathrm{~A}$ & 10 & 16 & 18.23 & 99.17 & BIS & FALSE \\
\hline 2004 & $\mathrm{~A}$ & 10 & 17 & 20.24 & 99.80 & $\begin{array}{l}\text { Bot X } 10 \mathrm{R} \\
\text { Reb/low }\end{array}$ & FALSE \\
\hline 2004 & $\mathrm{~A}$ & 10 & 18 & 20.24 & 100.00 & $\begin{array}{l}\text { Top X } 10 \mathrm{R} \\
\text { Reb/low }\end{array}$ & FALSE \\
\hline 2004 & B & 1 & 1 & 0.00 & 100.00 & $\begin{array}{llll}\text { Top } & X & 1 & \text { L } \\
\text { Reb/low } & \end{array}$ & FALSE \\
\hline 2004 & B & 1 & 2 & 0.00 & 99.88 & $\begin{array}{l}\text { Bot X } 1 \text { L Re- } \\
\text { b/low }\end{array}$ & FALSE \\
\hline 2004 & B & 1 & 3 & 0.46 & 99.20 & BIS & FALSE \\
\hline 2004 & B & 1 & 4 & 1.52 & 98.29 & BIS & FALSE \\
\hline 2004 & B & 1 & 5 & 2.83 & 97.65 & EW & FALSE \\
\hline 2004 & B & 1 & 6 & 4.27 & 97.52 & TW & FALSE \\
\hline 2004 & B & 1 & 7 & 6.40 & 97.65 & EW & FALSE \\
\hline 2004 & B & 1 & 8 & 7.77 & 98.31 & $\mathrm{BF}$ & FALSE \\
\hline 2004 & B & 1 & 9 & 8.05 & 98.50 & ТОВ & FALSE \\
\hline 2004 & B & 1 & 10 & 9.14 & 99.18 & BIS & FALSE \\
\hline 2004 & B & 1 & 11 & 10.06 & 99.89 & $\begin{array}{llll}\text { Bot } & \mathrm{X} & 1 & \mathrm{R} \\
\text { Reb/low } & & \end{array}$ & FALSE \\
\hline 2004 & B & 1 & 12 & 10.06 & 99.99 & 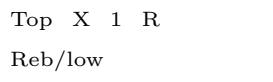 & FALSE \\
\hline 2004 & B & 2 & 1 & 0.00 & 100.00 & $\begin{array}{l}\text { Top X } 2 \text { L } \\
\text { Reb/low }\end{array}$ & FALSE \\
\hline 2004 & B & 2 & 2 & 0.00 & 99.61 & $\begin{array}{l}\text { Bot X } 2 \text { L Re- } \\
\text { b/low }\end{array}$ & FALSE \\
\hline 2004 & B & 2 & 3 & 0.30 & 99.13 & $\begin{array}{l}\text { BIS } *_{\text {left }} \\
\text { edge of trail }\end{array}$ & FALSE \\
\hline 2004 & B & 2 & 4 & 1.34 & 98.73 & $\begin{array}{l}\text { BIS *right } \\
\text { edge of trail }\end{array}$ & FALSE \\
\hline 2004 & B & 2 & 5 & 3.05 & 97.52 & BIS & FALSE \\
\hline 2004 & B & 2 & 6 & 4.05 & 97.46 & EW & FALSE \\
\hline 2004 & B & 2 & 7 & 5.30 & 97.37 & TW & FALSE \\
\hline 2004 & B & 2 & 8 & 6.64 & 97.43 & EW & FALSE \\
\hline 2004 & B & 2 & 9 & 7.92 & 97.83 & $\mathrm{BF}$ & FALSE \\
\hline 2004 & B & 2 & 10 & 8.96 & 98.64 & ТОВ & FALSE \\
\hline 2004 & $\mathrm{~B}$ & 2 & 11 & 9.75 & 99.19 & BIS & FALSE \\
\hline
\end{tabular}


Table D.1 - Continued

\begin{tabular}{|c|c|c|c|c|c|c|c|}
\hline Year & Reach & Section & Point & Dist. $(\mathrm{m})$ & Elev. $(\mathrm{m})$ & Desc. Notes & Exclude \\
\hline 2004 & $\mathrm{~B}$ & 2 & 12 & 10.55 & 99.53 & $\begin{array}{llll}\text { Bot } & X & 2 & R \\
\text { Reb/low } & & \end{array}$ & FALSE \\
\hline 2004 & $\mathrm{~B}$ & 2 & 13 & 10.55 & 100.01 & $\begin{array}{llll}\text { Top } & X & 2 & R \\
\text { Reb/low } & & \end{array}$ & FALSE \\
\hline 2004 & $\mathrm{~B}$ & 3 & 1 & 0.00 & 100.00 & $\begin{array}{l}\text { Top X } 3 \quad \text { L } \\
\text { Reb/low }\end{array}$ & FALSE \\
\hline 2004 & $\mathrm{~B}$ & 3 & 2 & 0.00 & 99.47 & $\begin{array}{l}\text { Bot X } 3 \text { L Re- } \\
\text { b/low }\end{array}$ & FALSE \\
\hline 2004 & B & 3 & 3 & 2.13 & 98.15 & BIS & FALSE \\
\hline 2004 & B & 3 & 4 & 4.51 & 98.04 & EW & FALSE \\
\hline 2004 & $\mathrm{~B}$ & 3 & 5 & 5.33 & 97.94 & TW & FALSE \\
\hline 2004 & B & 3 & 6 & 5.94 & 98.05 & EW & FALSE \\
\hline 2004 & B & 3 & 7 & 6.95 & 98.61 & $\mathrm{BF}$ & FALSE \\
\hline 2004 & $\mathrm{~B}$ & 3 & 8 & 7.92 & 99.20 & ТОВ & FALSE \\
\hline 2004 & B & 3 & 9 & 8.44 & 99.84 & $\begin{array}{llll}\text { Bot } & X & 3 & R \\
\text { Reb/low } & & \end{array}$ & FALSE \\
\hline 2004 & B & 3 & 10 & 8.44 & 99.98 & $\begin{array}{llll}\text { Top } & X & 3 & R \\
\text { Reb/low } & & \end{array}$ & FALSE \\
\hline 2004 & B & 4 & 1 & 0.00 & 100.00 & $\begin{array}{l}\text { Top X } 4 \quad \text { L } \\
\text { Reb/low }\end{array}$ & FALSE \\
\hline 2004 & B & 4 & 2 & 0.00 & 99.87 & $\begin{array}{l}\text { Bot X } 4 \text { L Re- } \\
\text { b/low }\end{array}$ & FALSE \\
\hline 2004 & B & 4 & 3 & 1.37 & 99.48 & BIS & FALSE \\
\hline 2004 & B & 4 & 4 & 2.13 & 98.70 & BIS & FALSE \\
\hline 2004 & B & 4 & 5 & 3.81 & 98.84 & $\begin{array}{l}\text { BIS *in dry } \\
\text { stream chan- } \\
\text { nel }\end{array}$ & FALSE \\
\hline 2004 & B & 4 & 6 & 4.57 & 98.52 & EW & FALSE \\
\hline 2004 & B & 4 & 7 & 5.88 & 98.44 & TW & FALSE \\
\hline 2004 & B & 4 & 8 & 6.58 & 98.46 & EW & FALSE \\
\hline 2004 & B & 4 & 9 & 6.98 & 99.12 & $\mathrm{BF}$ & FALSE \\
\hline 2004 & B & 4 & 10 & 7.41 & 99.53 & $\begin{array}{lcr}\text { Bot } & \text { X } 4 \\
\text { R } & \text { Reb/low } \\
\text { *also } & \text { TOB }\end{array}$ & FALSE \\
\hline 2004 & B & 4 & 11 & 7.41 & 100.01 & $\begin{array}{l}\text { Top X } 4 \text { R } \\
\text { Reb/low }\end{array}$ & FALSE \\
\hline 2004 & B & 5 & 1 & 0.00 & 100.00 & $\begin{array}{l}\text { Top X } 5 \text { L } \\
\text { Reb/low }\end{array}$ & FALSE \\
\hline 2004 & B & 5 & 2 & 0.00 & 99.81 & $\begin{array}{l}\text { Bot X } 5 \text { L Re- } \\
\text { b/low }\end{array}$ & FALSE \\
\hline 2004 & B & 5 & 3 & 0.67 & 99.26 & тов & FALSE \\
\hline 2004 & B & 5 & 4 & 2.13 & 98.43 & $\mathrm{BF}$ & FALSE \\
\hline 2004 & B & 5 & 5 & 3.66 & 98.07 & BIS & FALSE \\
\hline 2004 & B & 5 & 6 & 4.72 & 97.94 & EW & FALSE \\
\hline 2004 & B & 5 & 7 & 5.30 & 97.85 & $\mathrm{TW}$ & FALSE \\
\hline 2004 & B & 5 & 8 & 5.94 & 97.98 & EW & FALSE \\
\hline 2004 & B & 5 & 9 & 6.83 & 98.02 & BIS & FALSE \\
\hline 2004 & B & 5 & 10 & 7.50 & 99.14 & BIS & FALSE \\
\hline 2004 & B & 5 & 11 & 8.23 & 100.02 & $\begin{array}{l}\text { Bot } X \quad 5 \quad \text { R } \\
\text { Reb/low }\end{array}$ & FALSE \\
\hline 2004 & B & 5 & 12 & 8.23 & 100.16 & $\begin{array}{l}\text { Top X } 5 \text { R } \\
\text { Reb/low }\end{array}$ & FALSE \\
\hline
\end{tabular}

Continued on Next Page... 
Table D.1 - Continued

\begin{tabular}{|c|c|c|c|c|c|c|c|}
\hline Year & Reach & Section & Point & Dist. $(\mathrm{m})$ & Elev. $(\mathrm{m})$ & Desc. Notes & Exclude \\
\hline 2004 & B & 6 & 1 & 0.00 & 100.00 & $\begin{array}{l}\text { Top X } 6 \text { L } \\
\text { Reb/low }\end{array}$ & FALSE \\
\hline 2004 & $\mathrm{~B}$ & 6 & 2 & 0.00 & 99.98 & $\begin{array}{l}\text { Bot X } 6 \text { L Re- } \\
\text { b/low }\end{array}$ & FALSE \\
\hline 2004 & B & 6 & 3 & 0.61 & 99.45 & ТОВ & FALSE \\
\hline 2004 & $\mathrm{~B}$ & 6 & 4 & 1.22 & 99.35 & BIS & FALSE \\
\hline 2004 & $\mathrm{~B}$ & 6 & 5 & 2.16 & 98.83 & $\mathrm{BF}$ & FALSE \\
\hline 2004 & B & 6 & 6 & 3.54 & 98.34 & EW & FALSE \\
\hline 2004 & B & 6 & 7 & 4.27 & 98.17 & $\mathrm{TW}$ & FALSE \\
\hline 2004 & B & 6 & 8 & 5.21 & 98.30 & $\begin{array}{l}\text { EW * left side } \\
\text { of granite } \\
\text { rock }\end{array}$ & FALSE \\
\hline 2004 & B & 6 & 9 & 5.61 & 98.56 & $\begin{array}{l}\text { BIS *right } \\
\text { side of gran- } \\
\text { ite rock }\end{array}$ & FALSE \\
\hline 2004 & B & 6 & 10 & 6.40 & 98.66 & BIS & FALSE \\
\hline 2004 & B & 6 & 11 & 7.96 & 99.88 & $\begin{array}{llll}\text { Bot } & X & 6 & R \\
\text { Reb/low } & \end{array}$ & FALSE \\
\hline 2004 & B & 6 & 12 & 7.96 & 100.02 & $\begin{array}{llll}\text { Top } & \mathrm{X} & 6 & \mathrm{R} \\
\text { Reb/low } & \end{array}$ & FALSE \\
\hline 2004 & B & 7 & 1 & 0.00 & 100.00 & $\begin{array}{l}\text { Top X } \quad 7 \quad \text { L } \\
\text { Reb/low }\end{array}$ & FALSE \\
\hline 2004 & B & 7 & 2 & 0.00 & 99.44 & $\begin{array}{l}\text { Bot X } 7 \text { L Re- } \\
\text { b/low }\end{array}$ & FALSE \\
\hline 2004 & B & 7 & 3 & 0.82 & 98.99 & ТОВ & FALSE \\
\hline 2004 & B & 7 & 4 & 1.95 & 98.29 & $\mathrm{BF}$ & FALSE \\
\hline 2004 & B & 7 & 5 & 3.23 & 97.98 & EW & FALSE \\
\hline 2004 & B & 7 & 6 & 3.87 & 97.90 & $\mathrm{TW}$ & FALSE \\
\hline 2004 & B & 7 & 7 & 5.49 & 97.98 & EW & FALSE \\
\hline 2004 & B & 7 & 8 & 6.31 & 98.43 & BIS & FALSE \\
\hline 2004 & B & 7 & 9 & 6.71 & 98.79 & BIS & FALSE \\
\hline 2004 & B & 7 & 10 & 7.47 & 99.19 & BIS & FALSE \\
\hline 2004 & B & 7 & 11 & 9.48 & 99.78 & $\begin{array}{llll}\text { Bot } & \mathrm{X} & 7 & \mathrm{R} \\
\text { Reb/low } & & \end{array}$ & FALSE \\
\hline 2004 & B & 7 & 12 & 9.48 & 99.75 & $\begin{array}{llll}\text { Top } & \mathrm{X} & 7 & \mathrm{R} \\
\text { Reb/low } & & \end{array}$ & FALSE \\
\hline 2004 & B & 8 & 1 & 0.00 & 100.00 & $\begin{array}{llll}\text { Top } & \mathrm{X} & 8 & \mathrm{~L} \\
\text { Reb/low } & \end{array}$ & FALSE \\
\hline 2004 & B & 8 & 2 & 0.00 & 99.56 & $\begin{array}{l}\text { Bot X } 8 \text { L Re- } \\
\text { b/low }\end{array}$ & FALSE \\
\hline 2004 & B & 8 & 3 & 0.61 & 99.29 & $\begin{array}{l}\text { BIS *on top } \\
\text { of alder log } \\
\text { parallel } \\
\text { creek }\end{array}$ & FALSE \\
\hline 2004 & B & 8 & 4 & 0.76 & 98.82 & $\begin{array}{l}\text { BIS *right } \\
\text { side of the } \\
\text { alder log }\end{array}$ & FALSE \\
\hline 2004 & B & 8 & 5 & 1.95 & 98.73 & EW & FALSE \\
\hline 2004 & B & 8 & 6 & 2.53 & 98.66 & TW & FALSE \\
\hline 2004 & B & 8 & 7 & 3.60 & 98.66 & EW & FALSE \\
\hline 2004 & B & 8 & 8 & 3.90 & 99.15 & $\mathrm{BF}$ & FALSE \\
\hline 2004 & B & 8 & 9 & 4.24 & 99.43 & ТОВ & FALSE \\
\hline
\end{tabular}

Continued on Next Page... 
Table D.1 - Continued

\begin{tabular}{|c|c|c|c|c|c|c|c|}
\hline Year & Reach & Section & Point & Dist. (m) & Elev. $(\mathrm{m})$ & Notes & Exclude \\
\hline 2004 & B & 8 & 10 & 5.55 & 99.67 & $\begin{array}{l}\text { Bot } X \quad 8 \quad \mathrm{R} \\
\text { Reb/low }\end{array}$ & FALSE \\
\hline 2004 & B & 8 & 11 & 5.55 & 100.06 & $\begin{array}{l}\text { Top X } 8 \text { R } \\
\text { Reb/low }\end{array}$ & FALSE \\
\hline 2004 & B & 9 & 1 & 0.00 & 100.00 & $\begin{array}{l}\text { Top X } \quad 9 \quad \text { L } \\
\text { Reb/low }\end{array}$ & FALSE \\
\hline 2004 & B & 9 & 2 & 0.00 & 99.60 & $\begin{array}{l}\text { Bot X } 9 \text { L Re- } \\
\text { b/low }\end{array}$ & FALSE \\
\hline 2004 & B & 9 & 3 & 0.61 & 97.65 & BIS & FALSE \\
\hline 2004 & B & 9 & 4 & 1.22 & 97.31 & BIS & FALSE \\
\hline 2004 & B & 9 & 5 & 2.07 & 97.30 & EW & FALSE \\
\hline 2004 & B & 9 & 6 & 3.05 & 97.20 & TW & FALSE \\
\hline 2004 & B & 9 & 7 & 3.60 & 97.30 & EW & FALSE \\
\hline 2004 & B & 9 & 8 & 4.51 & 97.92 & $\mathrm{BF}$ & FALSE \\
\hline 2004 & B & 9 & 9 & 5.70 & 98.17 & ТОВ & FALSE \\
\hline 2004 & B & 9 & 10 & 7.92 & 98.60 & BIS & FALSE \\
\hline 2004 & B & 9 & 11 & 10.06 & 98.51 & BIS & FALSE \\
\hline 2004 & B & 9 & 12 & 11.89 & 99.09 & BIS & FALSE \\
\hline 2004 & B & 9 & 13 & 12.92 & 99.56 & BIS & FALSE \\
\hline 2004 & B & 9 & 14 & 13.32 & 99.90 & $\begin{array}{l}\text { Bot } \quad \mathrm{X} \quad 9 \quad \mathrm{R} \\
\text { Reb/low }\end{array}$ & FALSE \\
\hline 2004 & B & 9 & 15 & 13.32 & 99.99 & 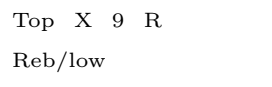 & FALSE \\
\hline 2004 & B & 10 & 1 & 0.00 & 100.00 & $\begin{array}{l}\text { Top X } 10 \mathrm{~L} \\
\text { Reb/low }\end{array}$ & FALSE \\
\hline 2004 & B & 10 & 2 & 0.00 & 99.69 & $\begin{array}{l}\text { Bot X } 10 \mathrm{~L} \\
\text { Reb/low }\end{array}$ & FALSE \\
\hline 2004 & B & 10 & 3 & 0.30 & 98.82 & BIS & FALSE \\
\hline 2004 & B & 10 & 4 & 0.61 & 97.77 & BIS & FALSE \\
\hline 2004 & B & 10 & 5 & 1.52 & 97.45 & BIS & FALSE \\
\hline 2004 & B & 10 & 6 & 2.16 & 97.34 & EW & FALSE \\
\hline 2004 & B & 10 & 7 & 3.29 & 97.28 & TW & FALSE \\
\hline 2004 & B & 10 & 8 & 4.11 & 97.34 & EW & FALSE \\
\hline 2004 & B & 10 & 9 & 5.33 & 97.74 & $\mathrm{BF}$ & FALSE \\
\hline 2004 & B & 10 & 10 & 6.71 & 98.32 & ТОВ & FALSE \\
\hline 2004 & B & 10 & 11 & 8.53 & 98.26 & BIS & FALSE \\
\hline 2004 & B & 10 & 12 & 11.58 & 98.26 & BIS & FALSE \\
\hline 2004 & B & 10 & 13 & 13.72 & 98.74 & BIS & FALSE \\
\hline 2004 & B & 10 & 14 & 14.94 & 99.59 & BIS & FALSE \\
\hline 2004 & B & 10 & 15 & 15.39 & 99.86 & $\begin{array}{l}\text { Bot X } 10 \mathrm{R} \\
\text { Reb/low }\end{array}$ & FALSE \\
\hline 2004 & B & 10 & 16 & 15.39 & 99.95 & $\begin{array}{l}\text { Top } \mathrm{X} 10 \mathrm{R} \\
\text { Reb/low }\end{array}$ & FALSE \\
\hline 2004 & $\mathrm{C}$ & 1 & 1 & 0.00 & 100.00 & $\begin{array}{l}\text { Top X } \quad 1 \quad L \\
\text { Reb/low }\end{array}$ & FALSE \\
\hline 2004 & $\mathrm{C}$ & 1 & 2 & 0.00 & 99.50 & $\begin{array}{l}\text { Bot X } 1 \text { L Re- } \\
\text { b/low }\end{array}$ & FALSE \\
\hline 2004 & $\mathrm{C}$ & 1 & 3 & 0.61 & 98.92 & BIS & FALSE \\
\hline 2004 & $\mathrm{C}$ & 1 & 4 & 1.22 & 98.73 & BIS & FALSE \\
\hline 2004 & $\mathrm{C}$ & 1 & 5 & 2.13 & 98.46 & EW & FALSE \\
\hline 2004 & $\mathrm{C}$ & 1 & 6 & 2.44 & 98.39 & TW & FALSE \\
\hline 2004 & $\mathrm{C}$ & 1 & 7 & 4.08 & 98.56 & $\mathrm{RI}$ & FALSE \\
\hline
\end{tabular}

Continued on Next Page... 
Table D.1 - Continued

\begin{tabular}{|c|c|c|c|c|c|c|c|}
\hline Year & Reach & Section & Point & Dist. $(\mathrm{m})$ & Elev. $(\mathrm{m})$ & Notes & Exclude \\
\hline 2004 & $\mathrm{C}$ & 1 & 8 & 4.97 & 98.61 & EW & FALSE \\
\hline 2004 & $\mathrm{C}$ & 1 & 9 & 6.71 & 98.87 & $\mathrm{BF}$ & FALSE \\
\hline 2004 & $\mathrm{C}$ & 1 & 10 & 8.53 & 99.26 & ТОВ & FALSE \\
\hline 2004 & $\mathrm{C}$ & 1 & 11 & 10.55 & 99.11 & BIS & FALSE \\
\hline 2004 & $\mathrm{C}$ & 1 & 12 & 12.34 & 99.84 & $\begin{array}{lll}\text { Bot } \quad X \quad 1 \quad R \\
\text { Reb/low }\end{array}$ & FALSE \\
\hline 2004 & $\mathrm{C}$ & 1 & 13 & 12.34 & 100.15 & 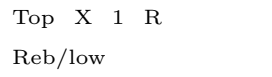 & FALSE \\
\hline 2004 & $\mathrm{C}$ & 2 & 1 & 0.00 & 100.00 & $\begin{array}{l}\text { Top X } \quad 2 \quad \text { L } \\
\text { Reb/low }\end{array}$ & FALSE \\
\hline 2004 & $\mathrm{C}$ & 2 & 2 & 0.00 & 99.61 & $\begin{array}{l}\text { Bot X } 2 \text { L Re- } \\
\text { b/low }\end{array}$ & FALSE \\
\hline 2004 & $\mathrm{C}$ & 2 & 3 & 1.10 & 98.93 & BIS & FALSE \\
\hline 2004 & $\mathrm{C}$ & 2 & 4 & 3.05 & 98.62 & BIS & FALSE \\
\hline 2004 & $\mathrm{C}$ & 2 & 5 & 5.18 & 98.09 & BIS & FALSE \\
\hline 2004 & $\mathrm{C}$ & 2 & 6 & 6.68 & 97.81 & EW & FALSE \\
\hline 2004 & $\mathrm{C}$ & 2 & 7 & 7.41 & 97.72 & TW & FALSE \\
\hline 2004 & $\mathrm{C}$ & 2 & 8 & 8.90 & 97.81 & EW & FALSE \\
\hline 2004 & $\mathrm{C}$ & 2 & 9 & 10.12 & 98.22 & $\mathrm{BF}$ & FALSE \\
\hline 2004 & $\mathrm{C}$ & 2 & 10 & 10.94 & 98.30 & ТОВ & FALSE \\
\hline 2004 & $\mathrm{C}$ & 2 & 11 & 12.04 & 98.25 & BIS & FALSE \\
\hline 2004 & $\mathrm{C}$ & 2 & 12 & 12.28 & 99.56 & $\begin{array}{lrr}\text { Bot } & \text { X } & 2 \\
\mathrm{R} & \text { Reb/low } \\
\text { *in } & \text { redwood } \\
\text { stump }\end{array}$ & FALSE \\
\hline 2004 & $\mathrm{C}$ & 2 & 13 & 12.28 & 99.79 & \begin{tabular}{lcr} 
Top & X & 2 \\
$\mathrm{R}$ & $\mathrm{Reb} /$ low \\
$*_{\text {in }}$ & redwood \\
\multicolumn{2}{l}{ stump }
\end{tabular} & FALSE \\
\hline 2004 & $\mathrm{C}$ & 3 & 1 & 0.00 & 100.00 & $\begin{array}{l}\text { Top X } 3 \quad \text { L } \\
\text { Reb/low }\end{array}$ & FALSE \\
\hline 2004 & $\mathrm{C}$ & 3 & 2 & 0.00 & 99.69 & $\begin{array}{l}\text { Bot X } 3 \text { L Re- } \\
\text { b/low }\end{array}$ & FALSE \\
\hline 2004 & $\mathrm{C}$ & 3 & 3 & 1.74 & 98.88 & BIS & FALSE \\
\hline 2004 & $\mathrm{C}$ & 3 & 4 & 4.72 & 98.49 & BIS & FALSE \\
\hline 2004 & $\mathrm{C}$ & 3 & 5 & 7.22 & 98.21 & EW & FALSE \\
\hline 2004 & $\mathrm{C}$ & 3 & 6 & 8.29 & 98.12 & TW & FALSE \\
\hline 2004 & $\mathrm{C}$ & 3 & 7 & 9.42 & 98.21 & EW & FALSE \\
\hline 2004 & $\mathrm{C}$ & 3 & 8 & 10.45 & 98.62 & $\mathrm{BF}$ & FALSE \\
\hline 2004 & $\mathrm{C}$ & 3 & 9 & 11.80 & 98.93 & ТОВ & FALSE \\
\hline 2004 & $\mathrm{C}$ & 3 & 10 & 13.47 & 99.49 & BIS & FALSE \\
\hline 2004 & $\mathrm{C}$ & 3 & 11 & 14.02 & 99.78 & $\begin{array}{l}\text { Bot } \mathrm{X} \quad 3 \quad \mathrm{R} \\
\text { Reb/low }\end{array}$ & FALSE \\
\hline 2004 & $\mathrm{C}$ & 3 & 12 & 14.02 & 99.99 & $\begin{array}{l}\text { Top } \mathrm{X} \quad 3 \quad \mathrm{R} \\
\text { Reb/low }\end{array}$ & FALSE \\
\hline 2004 & $\mathrm{C}$ & 4 & 1 & 0.00 & 100.00 & $\begin{array}{l}\text { Top X } \quad 4 \quad \text { L } \\
\text { Reb/low }\end{array}$ & FALSE \\
\hline 2004 & $\mathrm{C}$ & 4 & 2 & 0.00 & 99.73 & $\begin{array}{l}\text { Bot X } 4 \text { L Re- } \\
\text { b/low }\end{array}$ & FALSE \\
\hline 2004 & $\mathrm{C}$ & 4 & 3 & 1.46 & 98.81 & BIS & FALSE \\
\hline 2004 & $\mathrm{C}$ & 4 & 4 & 2.50 & 98.46 & BIS & FALSE \\
\hline 2004 & $\mathrm{C}$ & 4 & 5 & 4.88 & 98.38 & BIS & FALSE \\
\hline
\end{tabular}

Continued on Next Page... 
Table D.1 - Continued

\begin{tabular}{|c|c|c|c|c|c|c|c|}
\hline Year & Reach & Section & Point & Dist. $(\mathrm{m})$ & Elev. $(\mathrm{m})$ & Notes & Exclude \\
\hline 2004 & $\mathrm{C}$ & 4 & 6 & 7.44 & 98.14 & EW & FALSE \\
\hline 2004 & $\mathrm{C}$ & 4 & 7 & 8.14 & 98.01 & TW & FALSE \\
\hline 2004 & $\mathrm{C}$ & 4 & 8 & 8.72 & 98.14 & EW & FALSE \\
\hline 2004 & $\mathrm{C}$ & 4 & 9 & 10.03 & 98.65 & $\mathrm{BF}$ & FALSE \\
\hline 2004 & $\mathrm{C}$ & 4 & 10 & 10.67 & 98.89 & ТОВ & FALSE \\
\hline 2004 & $\mathrm{C}$ & 4 & 11 & 11.58 & 99.23 & BIS & FALSE \\
\hline 2004 & $\mathrm{C}$ & 4 & 12 & 11.92 & 99.73 & $\begin{array}{l}\text { Top X } 4 \quad \mathrm{R} \\
\text { Reb/low }\end{array}$ & FALSE \\
\hline 2004 & $\mathrm{C}$ & 4 & 13 & 11.92 & 100.01 & $\begin{array}{l}\text { Bot } \mathrm{X} \quad 4 \quad \mathrm{R} \\
\text { Reb/low }\end{array}$ & FALSE \\
\hline 2004 & $\mathrm{C}$ & 5 & 1 & 0.00 & 100.00 & $\begin{array}{lll}\text { Top X } & 5 & \text { L } \\
\text { Reb/low } & \end{array}$ & FALSE \\
\hline 2004 & $\mathrm{C}$ & 5 & 2 & 0.00 & 99.54 & $\begin{array}{l}\text { Bot X } 5 \text { L Re- } \\
\text { b/low }\end{array}$ & FALSE \\
\hline 2004 & $\mathrm{C}$ & 5 & 3 & 0.98 & 99.09 & BIS & FALSE \\
\hline 2004 & $\mathrm{C}$ & 5 & 4 & 1.83 & 98.94 & BIS & FALSE \\
\hline 2004 & $\mathrm{C}$ & 5 & 5 & 4.88 & 98.59 & BIS & FALSE \\
\hline 2004 & $\mathrm{C}$ & 5 & 6 & 5.91 & 98.57 & $\begin{array}{l}\text { SB *sand } \\
\text { bank on right } \\
\text { side of log }\end{array}$ & FALSE \\
\hline 2004 & $\mathrm{C}$ & 5 & 7 & 7.10 & 98.25 & EW & FALSE \\
\hline 2004 & $\mathrm{C}$ & 5 & 8 & 8.14 & 98.06 & TW & FALSE \\
\hline 2004 & $\mathrm{C}$ & 5 & 9 & 8.63 & 98.25 & EW & FALSE \\
\hline 2004 & $\mathrm{C}$ & 5 & 10 & 9.60 & 98.66 & $\mathrm{BF}$ & FALSE \\
\hline 2004 & $\mathrm{C}$ & 5 & 11 & 10.49 & 98.89 & ТОВ & FALSE \\
\hline 2004 & $\mathrm{C}$ & 5 & 12 & 11.58 & 99.15 & BIS & FALSE \\
\hline 2004 & $\mathrm{C}$ & 5 & 13 & 12.19 & 99.61 & $\begin{array}{lll}\text { Bot } X \quad 5 & R \\
\text { Reb/low } & \end{array}$ & FALSE \\
\hline 2004 & $\mathrm{C}$ & 5 & 14 & 12.19 & 100.11 & 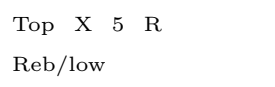 & FALSE \\
\hline 2004 & $\mathrm{C}$ & 6 & 1 & 0.00 & 100.00 & $\begin{array}{l}\text { Top X } \quad 6 \quad \text { L } \\
\text { Reb/low }\end{array}$ & FALSE \\
\hline 2004 & $\mathrm{C}$ & 6 & 2 & 0.00 & 99.70 & $\begin{array}{l}\text { Bot X } 6 \text { L Re- } \\
\text { b/low }\end{array}$ & FALSE \\
\hline 2004 & $\mathrm{C}$ & 6 & 3 & 0.88 & 98.94 & BIS & FALSE \\
\hline 2004 & $\mathrm{C}$ & 6 & 4 & 1.83 & 98.87 & ТОВ & FALSE \\
\hline 2004 & $\mathrm{C}$ & 6 & 5 & 2.93 & 98.39 & $\mathrm{BF}$ & FALSE \\
\hline 2004 & $\mathrm{C}$ & 6 & 6 & 3.72 & 98.31 & BIS & FALSE \\
\hline 2004 & $\mathrm{C}$ & 6 & 7 & 4.75 & 98.03 & EW & FALSE \\
\hline 2004 & $\mathrm{C}$ & 6 & 8 & 5.30 & 98.01 & $\mathrm{SB}$ & FALSE \\
\hline 2004 & $\mathrm{C}$ & 6 & 9 & 6.43 & 97.99 & TW & FALSE \\
\hline 2004 & $\mathrm{C}$ & 6 & 10 & 8.69 & 98.06 & EW & FALSE \\
\hline 2004 & $\mathrm{C}$ & 6 & 11 & 9.45 & 98.69 & ТОВ & FALSE \\
\hline 2004 & $\mathrm{C}$ & 6 & 12 & 10.27 & 98.77 & BIS & FALSE \\
\hline 2004 & $\mathrm{C}$ & 6 & 13 & 11.70 & 99.07 & $\begin{array}{l}\text { BIS *next to } \\
\text { granite rock }\end{array}$ & FALSE \\
\hline 2004 & $\mathrm{C}$ & 6 & 14 & 12.19 & 99.12 & BIS & FALSE \\
\hline 2004 & $\mathrm{C}$ & 6 & 15 & 12.98 & 99.72 & $\begin{array}{llll}\text { Bot } & X & 6 & R \\
\text { Reb/low } & \end{array}$ & FALSE \\
\hline 2004 & $\mathrm{C}$ & 6 & 16 & 12.98 & 100.00 & $\begin{array}{lll}\text { Top } \mathrm{X} & 6 & \mathrm{R} \\
\text { Reb/low } & \end{array}$ & FALSE \\
\hline
\end{tabular}

Continued on Next Page... 
Table D.1 - Continued

\begin{tabular}{|c|c|c|c|c|c|c|c|c|}
\hline Year & Reach & Section & Point & Dist. (m) & Elev. $(\mathrm{m})$ & Desc. & Notes & Exclude \\
\hline 2004 & $\mathrm{C}$ & 7 & 1 & 0.00 & 100.00 & $\begin{array}{l}\text { Top X } \quad 7 \quad \text { L } \\
\text { Reb/low }\end{array}$ & & FALSE \\
\hline 2004 & $\mathrm{C}$ & 7 & 2 & 0.00 & 99.78 & $\begin{array}{l}\text { Bot X } 7 \text { L Re- } \\
\text { b/low }\end{array}$ & & FALSE \\
\hline 2004 & $\mathrm{C}$ & 7 & 3 & 0.49 & 99.10 & BIS & & FALSE \\
\hline 2004 & $\mathrm{C}$ & 7 & 4 & 2.41 & 98.97 & ТОВ & & FALSE \\
\hline 2004 & $\mathrm{C}$ & 7 & 5 & 2.90 & 98.34 & $\begin{array}{l}\text { BIS *on top } \\
\text { of down } \log \end{array}$ & & FALSE \\
\hline 2004 & $\mathrm{C}$ & 7 & 6 & 3.96 & 98.08 & EW & & FALSE \\
\hline 2004 & $\mathrm{C}$ & 7 & 7 & 4.51 & 98.00 & $\mathrm{TW}$ & & FALSE \\
\hline 2004 & $\mathrm{C}$ & 7 & 8 & 5.58 & 98.19 & $\begin{array}{l}\mathrm{SB} * \text { top of is- } \\
\text { land sand bar }\end{array}$ & & FALSE \\
\hline 2004 & $\mathrm{C}$ & 7 & 9 & 6.07 & 98.13 & EW & & FALSE \\
\hline 2004 & $\mathrm{C}$ & 7 & 10 & 6.95 & 98.29 & BIS & & FALSE \\
\hline 2004 & $\mathrm{C}$ & 7 & 11 & 7.16 & 98.39 & $\mathrm{BF}$ & & FALSE \\
\hline 2004 & $\mathrm{C}$ & 7 & 12 & 7.68 & 99.08 & ТОВ & & FALSE \\
\hline 2004 & $\mathrm{C}$ & 7 & 13 & 10.79 & 98.63 & BIS & & FALSE \\
\hline 2004 & $\mathrm{C}$ & 7 & 14 & 12.38 & 99.05 & BIS & & FALSE \\
\hline 2004 & $\mathrm{C}$ & 7 & 15 & 13.44 & 99.83 & $\begin{array}{l}\text { Top } \mathrm{X} \quad 7 \quad \mathrm{R} \\
\text { Reb/low }\end{array}$ & & FALSE \\
\hline 2004 & $\mathrm{C}$ & 7 & 16 & 13.44 & 100.02 & $\begin{array}{l}\text { Bot } \mathrm{X} \quad 7 \quad \mathrm{R} \\
\text { Reb/low }\end{array}$ & & FALSE \\
\hline 2004 & $\mathrm{C}$ & 8 & 1 & 0.00 & 100.00 & $\begin{array}{l}\text { Top X } 8 \text { L } \\
\text { Reb/low }\end{array}$ & & FALSE \\
\hline 2004 & $\mathrm{C}$ & 8 & 2 & 0.00 & 99.55 & $\begin{array}{l}\text { Bot X } 8 \text { L Re- } \\
\text { b/low }\end{array}$ & $\begin{array}{l}\text { Elevation corrected, higher than } \\
\text { top of rebar, seems } 2 \text { feet high, un- } \\
\text { known reasondp }\end{array}$ & FALSE \\
\hline 2004 & $\mathrm{C}$ & 8 & 3 & 0.49 & 98.41 & BIS & & FALSE \\
\hline 2004 & $\mathrm{C}$ & 8 & 4 & 1.43 & 97.94 & $\mathrm{BF}$ & $\begin{array}{l}\text { Elevation corrected, looks like rod } \\
\text { not extended }\end{array}$ & FALSE \\
\hline 2004 & $\mathrm{C}$ & 8 & 5 & 2.23 & 97.73 & EW & & FALSE \\
\hline 2004 & $\mathrm{C}$ & 8 & 6 & 2.74 & 97.59 & TW & & FALSE \\
\hline 2004 & $\mathrm{C}$ & 8 & 7 & 3.96 & 97.77 & EW & & FALSE \\
\hline 2004 & $\mathrm{C}$ & 8 & 8 & 4.57 & 99.15 & BIS & $\log$ & TRUE \\
\hline 2004 & $\mathrm{C}$ & 8 & 9 & 5.64 & 98.71 & BIS & & FALSE \\
\hline 2004 & $\mathrm{C}$ & 8 & 10 & 6.10 & 99.10 & ТОВ & & FALSE \\
\hline 2004 & $\mathrm{C}$ & 8 & 11 & 11.70 & 98.60 & BIS & & FALSE \\
\hline 2004 & $\mathrm{C}$ & 8 & 12 & 12.98 & 98.96 & BIS & & FALSE \\
\hline 2004 & $\mathrm{C}$ & 8 & 13 & 14.11 & 99.67 & $\begin{array}{l}\text { Bot } X \quad 8 \quad R \\
\text { Reb/low }\end{array}$ & & FALSE \\
\hline 2004 & $\mathrm{C}$ & 8 & 14 & 14.11 & 100.06 & $\begin{array}{l}\text { Top } \mathrm{X} \quad 8 \mathrm{R} \\
\text { Reb/low }\end{array}$ & & FALSE \\
\hline 2004 & $\mathrm{C}$ & 9 & 1 & 0.00 & 100.00 & $\begin{array}{l}\text { Top } \quad X \quad 9 \quad L \\
\text { Reb/low }\end{array}$ & & FALSE \\
\hline 2004 & $\mathrm{C}$ & 9 & 2 & 0.00 & 99.70 & $\begin{array}{l}\text { Bot X } 9 \text { L Re- } \\
\text { b/low }\end{array}$ & & FALSE \\
\hline 2004 & $\mathrm{C}$ & 9 & 3 & 0.12 & 98.64 & BIS & & FALSE \\
\hline 2004 & $\mathrm{C}$ & 9 & 4 & 0.85 & 98.00 & BIS & & FALSE \\
\hline 2004 & $\mathrm{C}$ & 9 & 5 & 2.19 & 97.67 & BIS & & FALSE \\
\hline 2004 & C & 9 & 6 & 2.74 & 97.50 & EW & & FALSE \\
\hline 2004 & $\mathrm{C}$ & 9 & 7 & 4.11 & 97.53 & $\mathrm{TW}$ & & FALSE \\
\hline 2004 & $\mathrm{C}$ & 9 & 8 & 4.85 & 97.56 & $\mathrm{EW}$ & & FALSE \\
\hline
\end{tabular}

Continued on Next Page... 
Table D.1 - Continued

\begin{tabular}{|c|c|c|c|c|c|c|c|c|}
\hline Year & Reach & Section & Point & Dist. (m) & Elev. $(\mathrm{m})$ & Desc. & Notes & Exclude \\
\hline 2004 & $\mathrm{C}$ & 9 & 9 & 5.49 & 98.03 & $\mathrm{BF}$ & & FALSE \\
\hline 2004 & $\mathrm{C}$ & 9 & 10 & 6.71 & 98.61 & ТОВ & & FALSE \\
\hline 2004 & $\mathrm{C}$ & 9 & 11 & 8.69 & 98.74 & BIS & & FALSE \\
\hline 2004 & $\mathrm{C}$ & 9 & 12 & 10.97 & 98.57 & BIS & & FALSE \\
\hline 2004 & $\mathrm{C}$ & 9 & 13 & 13.59 & 98.53 & BIS & & FALSE \\
\hline 2004 & $\mathrm{C}$ & 9 & 14 & 15.09 & 98.87 & BIS & & FALSE \\
\hline 2004 & $\mathrm{C}$ & 9 & 15 & 15.85 & 99.45 & 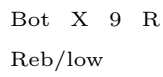 & & FALSE \\
\hline 2004 & $\mathrm{C}$ & 9 & 16 & 15.85 & 99.66 & $\begin{array}{l}\text { Top X } 9 \mathrm{R} \\
\text { Reb/low }\end{array}$ & & FALSE \\
\hline 2004 & $\mathrm{C}$ & 10 & 1 & 0.00 & 100.00 & $\begin{array}{l}\text { Top X } 10 \mathrm{~L} \\
\text { Reb/low }\end{array}$ & originally $R_{\llcorner} L$, fixed & FALSE \\
\hline 2004 & $\mathrm{C}$ & 10 & 2 & 0.00 & 99.75 & $\begin{array}{l}\text { Bot X } 10 \mathrm{~L} \\
\text { Reb/low }\end{array}$ & & FALSE \\
\hline 2004 & $\mathrm{C}$ & 10 & 3 & 0.55 & 99.35 & BIS & & FALSE \\
\hline 2004 & $\mathrm{C}$ & 10 & 4 & 1.68 & 98.80 & ТОВ & & FALSE \\
\hline 2004 & $\mathrm{C}$ & 10 & 5 & 3.41 & 98.16 & $\mathrm{BF}$ & & FALSE \\
\hline 2004 & $\mathrm{C}$ & 10 & 6 & 5.03 & 97.61 & EW & & FALSE \\
\hline 2004 & $\mathrm{C}$ & 10 & 7 & 5.85 & 97.63 & $\mathrm{SB}$ & & FALSE \\
\hline 2004 & $\mathrm{C}$ & 10 & 8 & 6.77 & 97.51 & $\mathrm{TW}$ & & FALSE \\
\hline 2004 & $\mathrm{C}$ & 10 & 9 & 8.47 & 97.56 & EW & & FALSE \\
\hline 2004 & $\mathrm{C}$ & 10 & 10 & 9.21 & 97.73 & BIS & & FALSE \\
\hline 2004 & $\mathrm{C}$ & 10 & 11 & 10.73 & 98.03 & BIS & & FALSE \\
\hline 2004 & $\mathrm{C}$ & 10 & 12 & 11.64 & 98.52 & BIS & & FALSE \\
\hline 2004 & $\mathrm{C}$ & 10 & 13 & 13.26 & 98.83 & BIS & & FALSE \\
\hline 2004 & $\mathrm{C}$ & 10 & 14 & 16.06 & 98.95 & BIS & & FALSE \\
\hline 2004 & $\mathrm{C}$ & 10 & 15 & 16.83 & 99.97 & $\begin{array}{l}\text { Bot } X 10 \mathrm{R} \\
\text { Reb/low }\end{array}$ & & FALSE \\
\hline 2004 & $\mathrm{C}$ & 10 & 16 & 16.83 & 100.33 & $\begin{array}{l}\text { Top X } 10 \mathrm{R} \\
\text { Reb/low }\end{array}$ & & FALSE \\
\hline 2004 & $\mathrm{D}$ & 1 & 1 & 0.00 & 100.00 & $\begin{array}{l}\text { Top X } 1 \text { L } \\
\text { Reb/HIGH }\end{array}$ & & FALSE \\
\hline 2004 & $\mathrm{D}$ & 1 & 2 & 0.00 & 99.67 & $\begin{array}{l}\text { Bot X } 1 \text { L Re- } \\
\text { b/HIGH }\end{array}$ & & FALSE \\
\hline 2004 & $\mathrm{D}$ & 1 & 3 & 2.16 & 98.61 & BIS & & FALSE \\
\hline 2004 & $\mathrm{D}$ & 1 & 4 & 3.20 & 97.65 & BIS & & FALSE \\
\hline 2004 & $\mathrm{D}$ & 1 & 5 & 5.67 & 97.94 & BIS & & FALSE \\
\hline 2004 & $\mathrm{D}$ & 1 & 6 & 6.13 & 98.64 & BIS & & FALSE \\
\hline 2004 & $\mathrm{D}$ & 1 & 7 & 7.38 & 98.58 & ТОВ & & FALSE \\
\hline 2004 & $\mathrm{D}$ & 1 & 8 & 8.17 & 97.07 & BIS & & FALSE \\
\hline 2004 & $\mathrm{D}$ & 1 & 9 & 8.93 & 96.77 & EW & & FALSE \\
\hline 2004 & $\mathrm{D}$ & 1 & 10 & 10.45 & 96.58 & TW & & FALSE \\
\hline 2004 & $\mathrm{D}$ & 1 & 11 & 11.06 & 96.70 & EW & & FALSE \\
\hline 2004 & $\mathrm{D}$ & 1 & 12 & 12.62 & 97.12 & $\mathrm{BF}$ & & FALSE \\
\hline 2004 & $\mathrm{D}$ & 1 & 13 & 14.45 & 97.99 & BIS & & FALSE \\
\hline 2004 & $\mathrm{D}$ & 1 & 14 & 16.28 & 98.49 & BIS & & FALSE \\
\hline 2004 & $\mathrm{D}$ & 1 & 15 & 17.01 & 98.86 & BIS & & FALSE \\
\hline 2004 & $\mathrm{D}$ & 1 & 16 & 18.01 & 100.12 & $\begin{array}{l}\text { Bot X } 1 \text { R } \\
\text { Reb/HIGH }\end{array}$ & & FALSE \\
\hline 2004 & $\mathrm{D}$ & 1 & 17 & 18.01 & 100.44 & $\begin{array}{l}\text { Top X } 1 \text { R } \\
\text { Reb/HIGH }\end{array}$ & & FALSE \\
\hline
\end{tabular}

Continued on Next Page... 
Table D.1 - Continued

\begin{tabular}{|c|c|c|c|c|c|c|c|c|}
\hline Year & Reach & Section & Point & Dist. (m) & Elev. $(\mathrm{m})$ & Desc. & Notes & Exclude \\
\hline 2004 & $\mathrm{D}$ & 2 & 1 & 0.00 & 100.00 & $\begin{array}{l}\text { Top X } 2 \text { L } \\
\text { Reb/low }\end{array}$ & & FALSE \\
\hline 2004 & $\mathrm{D}$ & 2 & 2 & 0.00 & 99.71 & $\begin{array}{l}\text { Bot X } 2 \text { L Re- } \\
\text { b/low }\end{array}$ & & FALSE \\
\hline 2004 & $\mathrm{D}$ & 2 & 3 & 0.61 & 99.68 & ТОВ & & FALSE \\
\hline 2004 & $\mathrm{D}$ & 2 & 4 & 1.22 & 98.98 & $\mathrm{BF}$ & & FALSE \\
\hline 2004 & $\mathrm{D}$ & 2 & 5 & 2.65 & 98.51 & EW & & FALSE \\
\hline 2004 & $\mathrm{D}$ & 2 & 6 & 3.72 & 98.44 & $\mathrm{TW}$ & & FALSE \\
\hline 2004 & $\mathrm{D}$ & 2 & 7 & 4.57 & 98.46 & EW & & FALSE \\
\hline 2004 & D & 2 & 8 & 6.10 & 98.96 & BIS & & FALSE \\
\hline 2004 & $\mathrm{D}$ & 2 & 9 & 7.74 & 99.66 & BIS & & FALSE \\
\hline 2004 & $\mathrm{D}$ & 2 & 10 & 9.39 & 99.76 & $\begin{array}{l}\text { Top X } 2 \text { R } \\
\text { Reb/low }\end{array}$ & & FALSE \\
\hline 2004 & $\mathrm{D}$ & 2 & 11 & 9.39 & 100.00 & $\begin{array}{l}\text { Bot } \mathrm{X} \quad 2 \quad \mathrm{R} \\
\text { Reb/low }\end{array}$ & & FALSE \\
\hline 2004 & $\mathrm{D}$ & 3 & 1 & 0.00 & 100.00 & $\begin{array}{l}\text { Top X } 3 \quad \text { L } \\
\text { Reb/low }\end{array}$ & & FALSE \\
\hline 2004 & $\mathrm{D}$ & 3 & 2 & 0.00 & 99.79 & $\begin{array}{l}\text { Bot X } 3 \text { L Re- } \\
\text { b/low }\end{array}$ & corrected elevation, 1 foot low & FALSE \\
\hline 2004 & $\mathrm{D}$ & 3 & 3 & 1.01 & 99.75 & ТОВ & & FALSE \\
\hline 2004 & $\mathrm{D}$ & 3 & 4 & 1.55 & 98.86 & $\mathrm{BF}$ & & FALSE \\
\hline 2004 & $\mathrm{D}$ & 3 & 5 & 3.41 & 98.53 & EW & & FALSE \\
\hline 2004 & $\mathrm{D}$ & 3 & 6 & 4.15 & 98.30 & TW & & FALSE \\
\hline 2004 & $\mathrm{D}$ & 3 & 7 & 4.54 & 98.50 & EW & & FALSE \\
\hline 2004 & $\mathrm{D}$ & 3 & 8 & 5.30 & 99.17 & BIS & & FALSE \\
\hline 2004 & $\mathrm{D}$ & 3 & 9 & 6.40 & 99.92 & BIS & & FALSE \\
\hline 2004 & $\mathrm{D}$ & 3 & 10 & 7.38 & 99.17 & BIS & & FALSE \\
\hline 2004 & $\mathrm{D}$ & 3 & 11 & 9.78 & 99.32 & BIS & & FALSE \\
\hline 2004 & $\mathrm{D}$ & 3 & 12 & 10.64 & 99.54 & BIS & & FALSE \\
\hline 2004 & $\mathrm{D}$ & 3 & 13 & 11.03 & 99.91 & $\begin{array}{l}\text { Bot } X \quad 3 \quad R \\
\text { Reb/low }\end{array}$ & & FALSE \\
\hline 2004 & $\mathrm{D}$ & 3 & 14 & 11.03 & 100.00 & $\begin{array}{l}\text { Top X } 3 \quad \text { R } \\
\text { Reb/low }\end{array}$ & & FALSE \\
\hline 2004 & $\mathrm{D}$ & 4 & 1 & 0.00 & 100.00 & $\begin{array}{l}\text { Top X } 4 \text { L } \\
\text { Reb/HIGH }\end{array}$ & & FALSE \\
\hline 2004 & $\mathrm{D}$ & 4 & 2 & 0.00 & 99.19 & $\begin{array}{l}\text { Bot X } 4 \text { L Re- } \\
\text { b/HIGH }\end{array}$ & & FALSE \\
\hline 2004 & $\mathrm{D}$ & 4 & 3 & 0.24 & 99.16 & $\begin{array}{l}\text { BIS *edge } \\
\text { of redwood } \\
\text { stump }\end{array}$ & & FALSE \\
\hline 2004 & $\mathrm{D}$ & 4 & 4 & 0.82 & 97.58 & $\begin{array}{l}\text { BIS *on red- } \\
\text { wood stump } \\
\text { rootwad }\end{array}$ & & FALSE \\
\hline 2004 & $\mathrm{D}$ & 4 & 5 & 1.68 & 96.41 & EW & & FALSE \\
\hline 2004 & $\mathrm{D}$ & 4 & 6 & 1.92 & 96.33 & $\mathrm{TW}$ & & FALSE \\
\hline 2004 & $\mathrm{D}$ & 4 & 7 & 3.26 & 96.53 & EW & & FALSE \\
\hline 2004 & $\mathrm{D}$ & 4 & 8 & 5.55 & 97.10 & $\mathrm{BF}$ & & FALSE \\
\hline 2004 & $\mathrm{D}$ & 4 & 9 & 7.41 & 97.16 & BIS & & FALSE \\
\hline 2004 & $\mathrm{D}$ & 4 & 10 & 9.02 & 97.69 & BIS & & FALSE \\
\hline 2004 & $\mathrm{D}$ & 4 & 11 & 9.91 & 98.10 & ТОВ & & FALSE \\
\hline 2004 & D & 4 & 12 & 11.89 & 98.53 & BIS & & FALSE \\
\hline 2004 & $\mathrm{D}$ & 4 & 13 & 12.98 & 99.76 & BIS & & FALSE \\
\hline
\end{tabular}

Continued on Next Page... 
Table D.1 - Continued

\begin{tabular}{|c|c|c|c|c|c|c|c|c|}
\hline Year & Reach & Section & Point & Dist. (m) & Elev. $(\mathrm{m})$ & Desc. & Notes & Exclude \\
\hline 2004 & $\mathrm{D}$ & 4 & 14 & 13.87 & 100.48 & $\begin{array}{l}\text { Bot } \mathrm{X} 4 \mathrm{R} \\
\text { Reb/HIGH }\end{array}$ & & FALSE \\
\hline 2004 & $\mathrm{D}$ & 4 & 15 & 13.87 & 100.88 & $\begin{array}{l}\text { Top X } 4 \text { R } \\
\text { Reb/HIGH }\end{array}$ & & FALSE \\
\hline 2004 & $\mathrm{D}$ & 5 & 1 & 0.00 & 100.00 & $\begin{array}{l}\text { Top X } 5 \text { L } \\
\text { Reb/low }\end{array}$ & & FALSE \\
\hline 2004 & $\mathrm{D}$ & 5 & 2 & 0.00 & 99.62 & $\begin{array}{l}\text { Bot X } 5 \text { L Re- } \\
\text { b/low }\end{array}$ & & FALSE \\
\hline 2004 & $\mathrm{D}$ & 5 & 3 & 1.01 & 98.40 & BIS & & FALSE \\
\hline 2004 & $\mathrm{D}$ & 5 & 4 & 3.81 & 98.01 & EW & & FALSE \\
\hline 2004 & $\mathrm{D}$ & 5 & 5 & 4.42 & 97.90 & TW & & FALSE \\
\hline 2004 & $\mathrm{D}$ & 5 & 6 & 4.97 & 97.99 & EW & & FALSE \\
\hline 2004 & $\mathrm{D}$ & 5 & 7 & 6.61 & 98.60 & $\mathrm{BF}$ & & FALSE \\
\hline 2004 & $\mathrm{D}$ & 5 & 8 & 8.23 & 99.51 & ТОВ & $\begin{array}{l}\text { distance changed from } 47 \text { - obvi- } \\
\text { ous typo and } 27 \text { fits well. }\end{array}$ & FALSE \\
\hline 2004 & $\mathrm{D}$ & 5 & 9 & 10.85 & 99.57 & $\begin{array}{l}\text { Bot } \mathrm{X} \quad 5 \quad \mathrm{R} \\
\text { Reb/low }\end{array}$ & & FALSE \\
\hline 2004 & $\mathrm{D}$ & 5 & 10 & 10.85 & 100.00 & $\begin{array}{l}\text { Top X } 5 \text { R } \\
\text { Reb/low }\end{array}$ & & FALSE \\
\hline 2004 & $\mathrm{D}$ & 6 & 1 & 0.00 & 100.00 & $\begin{array}{l}\text { Top } \quad X \quad 6 \quad L \\
\text { Reb/low }\end{array}$ & & FALSE \\
\hline 2004 & $\mathrm{D}$ & 6 & 2 & 0.00 & 99.85 & $\begin{array}{l}\text { Bot X } 6 \text { L Re- } \\
\text { b/low }\end{array}$ & & FALSE \\
\hline 2004 & $\mathrm{D}$ & 6 & 3 & 0.73 & 99.70 & BIS & & FALSE \\
\hline 2004 & D & 6 & 4 & 1.10 & 98.44 & BIS & & FALSE \\
\hline 2004 & $\mathrm{D}$ & 6 & 5 & 2.04 & 98.09 & $\mathrm{BF}$ & & FALSE \\
\hline 2004 & $\mathrm{D}$ & 6 & 6 & 4.05 & 97.53 & EW & & FALSE \\
\hline 2004 & $\mathrm{D}$ & 6 & 7 & 6.04 & 97.55 & TW & & FALSE \\
\hline 2004 & D & 6 & 8 & 6.25 & 97.69 & EW & & FALSE \\
\hline 2004 & D & 6 & 9 & 8.53 & 99.33 & тОВ & & FALSE \\
\hline 2004 & $\mathrm{D}$ & 6 & 10 & 10.00 & 99.65 & $\begin{array}{llll}\text { Bot } & X & 6 & R \\
\text { Reb/low } & \end{array}$ & & FALSE \\
\hline 2004 & $\mathrm{D}$ & 6 & 11 & 10.00 & 100.00 & $\begin{array}{l}\text { Top } \mathrm{X} \quad 6 \mathrm{R} \\
\text { Reb/low }\end{array}$ & & FALSE \\
\hline 2004 & $\mathrm{D}$ & 7 & 1 & 0.00 & 100.00 & $\begin{array}{l}\text { Top } \quad \mathrm{X} \quad 7 \quad \mathrm{~L} \\
\text { Reb/low }\end{array}$ & & FALSE \\
\hline 2004 & $\mathrm{D}$ & 7 & 2 & 0.00 & 99.77 & $\begin{array}{l}\text { Bot X } 7 \text { L Re- } \\
\text { b/low }\end{array}$ & & FALSE \\
\hline 2004 & $\mathrm{D}$ & 7 & 3 & 0.76 & 99.75 & BIS & & TRUE \\
\hline 2004 & $\mathrm{D}$ & 7 & 4 & 1.28 & 98.50 & $\mathrm{BF}$ & & FALSE \\
\hline 2004 & D & 7 & 5 & 2.65 & 97.86 & BIS & & FALSE \\
\hline 2004 & $\mathrm{D}$ & 7 & 6 & 2.90 & 97.80 & EW & & FALSE \\
\hline 2004 & $\mathrm{D}$ & 7 & 7 & 3.44 & 97.65 & TW & & FALSE \\
\hline 2004 & $\mathrm{D}$ & 7 & 8 & 3.96 & 97.77 & EW & & FALSE \\
\hline 2004 & D & 7 & 9 & 5.94 & 98.17 & BIS & & FALSE \\
\hline 2004 & $\mathrm{D}$ & 7 & 10 & 7.01 & 99.32 & BIS & & FALSE \\
\hline 2004 & $\mathrm{D}$ & 7 & 11 & 7.71 & 99.77 & $\begin{array}{l}\text { Top } \mathrm{X} \quad 7 \quad \mathrm{R} \\
\text { Reb/low }\end{array}$ & & FALSE \\
\hline 2004 & $\mathrm{D}$ & 7 & 12 & 7.71 & 99.98 & $\begin{array}{l}\text { Bot } \mathrm{X} \quad 7 \quad \mathrm{R} \\
\text { Reb/low }\end{array}$ & & FALSE \\
\hline 2004 & $\mathrm{D}$ & 8 & 1 & 0.00 & 100.00 & Top $\quad X \quad 8 \quad L$ & & FALSE \\
\hline
\end{tabular}

Continued on Next Page... 
Table D.1 - Continued

\begin{tabular}{|c|c|c|c|c|c|c|c|c|}
\hline Year & Reach & Section & Point & Dist. (m) & Elev. (m) & Desc. & Notes & Exclude \\
\hline 2004 & $\mathrm{D}$ & 8 & 2 & 0.00 & 99.70 & $\begin{array}{l}\text { Bot X } 8 \text { L Re- } \\
\text { b/low }\end{array}$ & & FALSE \\
\hline 2004 & $\mathrm{D}$ & 8 & 3 & 0.82 & 99.27 & ТОВ & & FALSE \\
\hline 2004 & $\mathrm{D}$ & 8 & 4 & 1.46 & 98.03 & BIS & & FALSE \\
\hline 2004 & $\mathrm{D}$ & 8 & 5 & 2.01 & 97.85 & EW & & FALSE \\
\hline 2004 & $\mathrm{D}$ & 8 & 6 & 2.59 & 97.76 & $\mathrm{TW}$ & & FALSE \\
\hline 2004 & $\mathrm{D}$ & 8 & 7 & 3.23 & 97.84 & EW & & FALSE \\
\hline 2004 & $\mathrm{D}$ & 8 & 8 & 4.88 & 98.02 & BIS & & FALSE \\
\hline 2004 & $\mathrm{D}$ & 8 & 9 & 5.70 & 98.24 & $\mathrm{BF}$ & & FALSE \\
\hline 2004 & $\mathrm{D}$ & 8 & 10 & 6.83 & 99.80 & $\begin{array}{llll}\text { Bot } & X & 8 & R \\
\text { Reb/low } & & \end{array}$ & & FALSE \\
\hline 2004 & $\mathrm{D}$ & 8 & 11 & 6.83 & 100.01 & $\begin{array}{llll}\text { Top } & \mathrm{X} & 8 & \mathrm{R} \\
\text { Reb/low } & & \end{array}$ & & FALSE \\
\hline 2004 & $\mathrm{D}$ & 9 & 1 & 0.00 & 100.00 & $\begin{array}{llll}\text { Top } & X & 9 & \text { L } \\
\text { Reb/low } & \end{array}$ & & FALSE \\
\hline 2004 & $\mathrm{D}$ & 9 & 2 & 0.00 & 99.59 & $\begin{array}{l}\text { Bot X } 9 \text { L Re- } \\
\text { b/low }\end{array}$ & & FALSE \\
\hline 2004 & $\mathrm{D}$ & 9 & 3 & 0.91 & 98.71 & BIS & & FALSE \\
\hline 2004 & $\mathrm{D}$ & 9 & 4 & 2.87 & 98.53 & $\begin{array}{l}\text { EW *on red- } \\
\text { wood log }\end{array}$ & & FALSE \\
\hline 2004 & $\mathrm{D}$ & 9 & 5 & 3.23 & 98.40 & TW & & FALSE \\
\hline 2004 & $\mathrm{D}$ & 9 & 6 & 3.96 & 98.53 & EW & & FALSE \\
\hline 2004 & $\mathrm{D}$ & 9 & 7 & 5.67 & 98.98 & $\mathrm{BF}$ & & FALSE \\
\hline 2004 & $\mathrm{D}$ & 9 & 8 & 6.77 & 99.08 & BIS & corrected, 1 foot low & FALSE \\
\hline 2004 & $\mathrm{D}$ & 9 & 9 & 7.25 & 99.60 & $\begin{array}{llll}\text { Bot } & X & 9 & R \\
\text { Reb/low } & & \end{array}$ & & FALSE \\
\hline 2004 & $\mathrm{D}$ & 9 & 10 & 7.25 & 100.02 & $\begin{array}{llll}\text { Top } & X & 9 & R \\
\text { Reb/low } & \end{array}$ & & FALSE \\
\hline 2004 & $\mathrm{D}$ & 10 & 1 & 0.00 & 100.00 & $\begin{array}{l}\text { Top X } 10 \text { L } \\
\text { Reb/low }\end{array}$ & & FALSE \\
\hline 2004 & $\mathrm{D}$ & 10 & 2 & 0.00 & 99.67 & $\begin{array}{l}\text { Bot } X 10 \quad \text { L } \\
\text { Reb/low }\end{array}$ & & FALSE \\
\hline 2004 & $\mathrm{D}$ & 10 & 3 & 0.30 & 99.40 & тов & & FALSE \\
\hline 2004 & $\mathrm{D}$ & 10 & 4 & 1.22 & 98.89 & $\mathrm{BF}$ & & FALSE \\
\hline 2004 & $\mathrm{D}$ & 10 & 5 & 2.90 & 98.23 & EW & & FALSE \\
\hline 2004 & $\mathrm{D}$ & 10 & 6 & 3.84 & 98.10 & $\mathrm{TW}$ & & FALSE \\
\hline 2004 & $\mathrm{D}$ & 10 & 7 & 4.82 & 98.20 & EW & & FALSE \\
\hline 2004 & $\mathrm{D}$ & 10 & 8 & 6.49 & 98.52 & BIS & & FALSE \\
\hline 2004 & $\mathrm{D}$ & 10 & 9 & 7.41 & 99.76 & $\begin{array}{l}\text { Bot } X 10 \mathrm{R} \\
\text { Reb/low }\end{array}$ & & FALSE \\
\hline 2004 & $\mathrm{D}$ & 10 & 10 & 7.41 & 100.00 & $\begin{array}{l}\text { Top X } 10 \mathrm{R} \\
\text { Reb/low }\end{array}$ & & FALSE \\
\hline 2004 & $\mathrm{E}$ & 1 & 1 & 0.00 & 100.00 & $\begin{array}{l}\text { Top X } \quad 1 \quad \text { L } \\
\text { Reb/low }\end{array}$ & & FALSE \\
\hline 2004 & $\mathrm{E}$ & 1 & 2 & 0.00 & 99.62 & $\begin{array}{l}\text { Bot X } 1 \text { L Re- } \\
\text { b/low }\end{array}$ & & FALSE \\
\hline 2004 & $\mathrm{E}$ & 1 & 3 & 0.91 & 99.25 & тов & & FALSE \\
\hline 2004 & $\mathrm{E}$ & 1 & 4 & 2.74 & 98.86 & BIS & & FALSE \\
\hline 2004 & $\mathrm{E}$ & 1 & 5 & 4.15 & 98.64 & $\mathrm{BF}$ & & FALSE \\
\hline 2004 & $\mathrm{E}$ & 1 & 6 & 6.10 & 98.12 & EW & & FALSE \\
\hline 2004 & $\mathrm{E}$ & 1 & 7 & 6.25 & 98.02 & $\mathrm{TW}$ & & FALSE \\
\hline 2004 & $\mathrm{E}$ & 1 & 8 & 6.77 & 98.10 & EW & & FALSE \\
\hline
\end{tabular}


Table D.1 - Continued

\begin{tabular}{|c|c|c|c|c|c|c|c|}
\hline Year & Reach & Section & Point & Dist. (m) & Elev. $(\mathrm{m})$ & Desc. & Exclude \\
\hline 2004 & $\mathrm{E}$ & 1 & 9 & 7.19 & 99.65 & BIS & FALSE \\
\hline 2004 & $\mathrm{E}$ & 1 & 10 & 7.50 & 99.84 & $\begin{array}{lll}\text { Top X } & 1 & \mathrm{R} \\
\text { Reb/low } & \end{array}$ & FALSE \\
\hline 2004 & $\mathrm{E}$ & 1 & 11 & 7.50 & 99.89 & $\begin{array}{lll}\text { Bot } \quad X \quad 1 \quad R \\
\text { Reb/low }\end{array}$ & FALSE \\
\hline 2004 & $\mathrm{E}$ & 2 & 1 & 0.00 & 100.00 & $\begin{array}{l}\text { Top X } 2 \quad \text { L } \\
\text { Reb/low }\end{array}$ & FALSE \\
\hline 2004 & $\mathrm{E}$ & 2 & 2 & 0.00 & 99.42 & $\begin{array}{l}\text { Bot X } 2 \text { L Re- } \\
\text { b/low }\end{array}$ & FALSE \\
\hline 2004 & $\mathrm{E}$ & 2 & 3 & 0.98 & 98.52 & BIS & FALSE \\
\hline 2004 & $\mathrm{E}$ & 2 & 4 & 1.98 & 98.34 & EW & FALSE \\
\hline 2004 & $\mathrm{E}$ & 2 & 5 & 2.80 & 98.25 & $\mathrm{TW}$ & FALSE \\
\hline 2004 & $\mathrm{E}$ & 2 & 6 & 3.17 & 98.38 & EW & FALSE \\
\hline 2004 & $\mathrm{E}$ & 2 & 7 & 4.45 & 98.95 & $\mathrm{BF}$ & FALSE \\
\hline 2004 & $\mathrm{E}$ & 2 & 8 & 5.21 & 99.39 & ТОВ & FALSE \\
\hline 2004 & $\mathrm{E}$ & 2 & 9 & 5.82 & 99.65 & 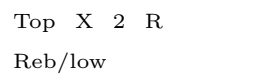 & FALSE \\
\hline 2004 & $\mathrm{E}$ & 2 & 10 & 5.82 & 100.01 & $\begin{array}{l}\text { Bot } \mathrm{X} \quad 2 \quad \mathrm{R} \\
\text { Reb/low }\end{array}$ & FALSE \\
\hline 2004 & $\mathrm{E}$ & 3 & 1 & 0.00 & 100.00 & $\begin{array}{l}\text { Top X } 3 \quad \text { L } \\
\text { Reb/low }\end{array}$ & FALSE \\
\hline 2004 & $\mathrm{E}$ & 3 & 2 & 0.00 & 99.52 & $\begin{array}{l}\text { Bot X } 3 \text { L Re- } \\
\text { b/low }\end{array}$ & FALSE \\
\hline 2004 & $\mathrm{E}$ & 3 & 3 & 0.91 & 98.79 & BIS & FALSE \\
\hline 2004 & $\mathrm{E}$ & 3 & 4 & 1.74 & 98.43 & тОВ & FALSE \\
\hline 2004 & $\mathrm{E}$ & 3 & 5 & 2.19 & 97.94 & EW & FALSE \\
\hline 2004 & $\mathrm{E}$ & 3 & 6 & 3.60 & 97.76 & TW & FALSE \\
\hline 2004 & $\mathrm{E}$ & 3 & 7 & 3.81 & 97.86 & EW & FALSE \\
\hline 2004 & $\mathrm{E}$ & 3 & 8 & 4.63 & 98.18 & BIS & FALSE \\
\hline 2004 & $\mathrm{E}$ & 3 & 9 & 5.03 & 98.42 & $\mathrm{BF}$ & FALSE \\
\hline 2004 & $\mathrm{E}$ & 3 & 10 & 5.79 & 99.03 & BIS & FALSE \\
\hline 2004 & $\mathrm{E}$ & 3 & 11 & 7.28 & 99.50 & $\begin{array}{llll}\text { Bot } & X & 3 & R \\
\text { Reb/low } & \end{array}$ & FALSE \\
\hline 2004 & $\mathrm{E}$ & 3 & 12 & 7.28 & 100.02 & $\begin{array}{llll}\text { Top } & \mathrm{X} & 3 & \mathrm{R} \\
\text { Reb/low } & \end{array}$ & FALSE \\
\hline 2004 & $\mathrm{E}$ & 4 & 1 & 0.00 & 100.00 & $\begin{array}{l}\text { Top X } \quad 4 \quad \text { L } \\
\text { Reb/low }\end{array}$ & FALSE \\
\hline 2004 & $\mathrm{E}$ & 4 & 2 & 0.00 & 99.71 & $\begin{array}{l}\text { Bot X } 4 \text { L Re- } \\
\text { b/low }\end{array}$ & FALSE \\
\hline 2004 & $\mathrm{E}$ & 4 & 3 & 0.61 & 99.39 & BIS & FALSE \\
\hline 2004 & $\mathrm{E}$ & 4 & 4 & 1.16 & 98.77 & BIS & FALSE \\
\hline 2004 & $\mathrm{E}$ & 4 & 5 & 1.58 & 98.68 & BIS & FALSE \\
\hline 2004 & $\mathrm{E}$ & 4 & 6 & 2.38 & 98.63 & EW & FALSE \\
\hline 2004 & $\mathrm{E}$ & 4 & 7 & 2.74 & 98.57 & TW & FALSE \\
\hline 2004 & $\mathrm{E}$ & 4 & 8 & 3.05 & 98.56 & $\begin{array}{l}\text { BIS *left side } \\
\text { of rock in } \\
\text { creek }\end{array}$ & FALSE \\
\hline 2004 & $\mathrm{E}$ & 4 & 9 & 3.96 & 98.59 & $\begin{array}{l}\text { BIS *island } \\
\text { sand bar on } \\
\text { center }\end{array}$ & FALSE \\
\hline
\end{tabular}

Continued on Next Page... 
Table D.1 - Continued

\begin{tabular}{|c|c|c|c|c|c|c|c|c|}
\hline Year & Reach & Section & Point & Dist. (m) & Elev. $(\mathrm{m})$ & Desc. & Notes & Exclude \\
\hline 2004 & $\mathrm{E}$ & 4 & 10 & 4.54 & 98.49 & $\begin{array}{l}\text { BIS *to right } \\
\text { of rock, edge } \\
\text { of sand bar }\end{array}$ & & FALSE \\
\hline 2004 & $\mathrm{E}$ & 4 & 11 & 4.88 & 98.51 & EW & & FALSE \\
\hline 2004 & $\mathrm{E}$ & 4 & 12 & 5.06 & 98.66 & BIS & & FALSE \\
\hline 2004 & $\mathrm{E}$ & 4 & 13 & 5.61 & 98.75 & $\mathrm{BF}$ & & FALSE \\
\hline 2004 & $\mathrm{E}$ & 4 & 14 & 5.88 & 99.43 & ТОВ & & FALSE \\
\hline 2004 & $\mathrm{E}$ & 4 & 15 & 7.71 & 99.74 & $\begin{array}{l}\text { Top X } 4 \text { R } \\
\text { Reb/low }\end{array}$ & & FALSE \\
\hline 2004 & $\mathrm{E}$ & 4 & 16 & 7.71 & 100.00 & $\begin{array}{l}\text { Bot } \mathrm{X} \quad 4 \quad \mathrm{R} \\
\text { Reb/low }\end{array}$ & & FALSE \\
\hline 2004 & $\mathrm{E}$ & 5 & 1 & 0.00 & 100.00 & Top X4L & & FALSE \\
\hline 2004 & $\mathrm{E}$ & 5 & 2 & 0.00 & 99.67 & Bot X4L & & FALSE \\
\hline 2004 & $\mathrm{E}$ & 5 & 3 & 0.94 & 99.43 & BIS & & FALSE \\
\hline 2004 & $\mathrm{E}$ & 5 & 4 & 1.65 & 98.93 & BIS & & FALSE \\
\hline 2004 & $\mathrm{E}$ & 5 & 5 & 2.77 & 98.66 & EW & & FALSE \\
\hline 2004 & $\mathrm{E}$ & 5 & 6 & 3.51 & 98.53 & TW & & FALSE \\
\hline 2004 & $\mathrm{E}$ & 5 & 7 & 3.99 & 98.51 & Pool in Creek & & FALSE \\
\hline 2004 & $\mathrm{E}$ & 5 & 8 & 4.27 & 98.57 & EW & on top of small rocks & FALSE \\
\hline 2004 & $\mathrm{E}$ & 5 & 9 & 4.72 & 99.22 & $\mathrm{BF}$ & & FALSE \\
\hline 2004 & $\mathrm{E}$ & 5 & 10 & 5.73 & 99.73 & ТОВ & & FALSE \\
\hline 2004 & $\mathrm{E}$ & 5 & 11 & 7.89 & 99.89 & BOT X5R & & FALSE \\
\hline 2004 & $\mathrm{E}$ & 5 & 12 & 7.89 & 100.30 & TOP X5R & & FALSE \\
\hline 2004 & $\mathrm{E}$ & 6 & 1 & 0.00 & 100.00 & $\begin{array}{lll}\text { Top X } & 6 & \text { L } \\
\text { Reb/low } & \end{array}$ & & FALSE \\
\hline 2004 & $\mathrm{E}$ & 6 & 2 & 0.00 & 99.54 & $\begin{array}{l}\text { Bot X } 6 \text { L Re- } \\
\text { b/low }\end{array}$ & & FALSE \\
\hline 2004 & $\mathrm{E}$ & 6 & 3 & 0.15 & 99.44 & BIS & & FALSE \\
\hline 2004 & $\mathrm{E}$ & 6 & 4 & 0.61 & 98.78 & BIS & & FALSE \\
\hline 2004 & $\mathrm{E}$ & 6 & 5 & 1.16 & 98.34 & $\begin{array}{l}\text { EW *next to } \\
\text { rock }\end{array}$ & & FALSE \\
\hline 2004 & $\mathrm{E}$ & 6 & 6 & 1.83 & 98.24 & $\mathrm{TW}$ & & FALSE \\
\hline 2004 & $\mathrm{E}$ & 6 & 7 & 2.62 & 98.37 & $\begin{array}{l}\mathrm{EW} * \text { next to } \\
\text { rock }\end{array}$ & & FALSE \\
\hline 2004 & $\mathrm{E}$ & 6 & 8 & 3.51 & 98.57 & BIS & & FALSE \\
\hline 2004 & $\mathrm{E}$ & 6 & 9 & 4.08 & 98.52 & BIS & & FALSE \\
\hline 2004 & $\mathrm{E}$ & 6 & 10 & 4.36 & 98.77 & $\mathrm{BF}$ & & FALSE \\
\hline 2004 & $\mathrm{E}$ & 6 & 11 & 4.69 & 99.45 & ТОВ & & FALSE \\
\hline 2004 & $\mathrm{E}$ & 6 & 12 & 5.36 & 99.63 & $\begin{array}{llll}\text { Bot } & X & 6 & R \\
\text { Reb/low } & \end{array}$ & & FALSE \\
\hline 2004 & $\mathrm{E}$ & 6 & 13 & 5.36 & 100.00 & 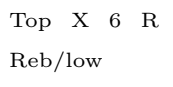 & & FALSE \\
\hline 2004 & $\mathrm{E}$ & 7 & 1 & 0.00 & 100.00 & 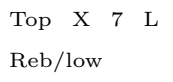 & & FALSE \\
\hline 2004 & $\mathrm{E}$ & 7 & 2 & 0.00 & 99.72 & $\begin{array}{l}\text { Bot X } 7 \text { L Re- } \\
\text { b/low }\end{array}$ & & FALSE \\
\hline 2004 & $\mathrm{E}$ & 7 & 3 & 0.85 & 99.38 & BIS & & FALSE \\
\hline 2004 & $\mathrm{E}$ & 7 & 4 & 1.01 & 98.86 & BIS & & FALSE \\
\hline 2004 & $\mathrm{E}$ & 7 & 5 & 1.40 & 98.49 & EW & & FALSE \\
\hline 2004 & $\mathrm{E}$ & 7 & 6 & 1.65 & 98.38 & $\begin{array}{l}\text { BIS * pool in } \\
\text { creek }\end{array}$ & & FALSE \\
\hline 2004 & $\mathrm{E}$ & 7 & 7 & 2.13 & 98.38 & $\mathrm{TW}$ & & FALSE \\
\hline
\end{tabular}

Continued on Next Page... 
Table D.1 - Continued

\begin{tabular}{|c|c|c|c|c|c|c|c|c|}
\hline Year & Reach & Section & Point & Dist. $(\mathrm{m})$ & Elev. $(\mathrm{m})$ & Desc. & Notes & Exclude \\
\hline 2004 & $\mathrm{E}$ & 7 & 8 & 3.93 & 98.54 & EW & & FALSE \\
\hline 2004 & $\mathrm{E}$ & 7 & 9 & 4.57 & 98.99 & $\mathrm{BF}$ & *on granite rock & FALSE \\
\hline 2004 & $\mathrm{E}$ & 7 & 10 & 4.69 & 99.60 & ТОВ & & FALSE \\
\hline 2004 & $\mathrm{E}$ & 7 & 11 & 5.06 & 99.77 & $\begin{array}{l}\text { BIS * next to } \\
\text { rock }\end{array}$ & & FALSE \\
\hline 2004 & $\mathrm{E}$ & 7 & 12 & 6.49 & 99.66 & $\begin{array}{lll}\text { Top } \mathrm{X} & 7 & \mathrm{R} \\
\text { Reb/low } & & \end{array}$ & & FALSE \\
\hline 2004 & $\mathrm{E}$ & 7 & 13 & 6.49 & 100.00 & $\begin{array}{lll}\text { Bot } \mathrm{X} & 7 & \mathrm{R} \\
\text { Reb/low } & \end{array}$ & & FALSE \\
\hline 2004 & $\mathrm{E}$ & 8 & 1 & 0.00 & 100.00 & $\begin{array}{lll}\text { Top } X \quad 8 & L \\
\text { Reb/low } & \end{array}$ & & FALSE \\
\hline 2004 & $\mathrm{E}$ & 8 & 2 & 0.00 & 99.61 & $\begin{array}{l}\text { Bot X } 8 \text { L Re- } \\
\text { b/low }\end{array}$ & & FALSE \\
\hline 2004 & $\mathrm{E}$ & 8 & 3 & 0.21 & 99.52 & BIS & & FALSE \\
\hline 2004 & $\mathrm{E}$ & 8 & 4 & 0.73 & 99.22 & $\mathrm{BF}$ & & FALSE \\
\hline 2004 & $\mathrm{E}$ & 8 & 5 & 0.94 & 98.75 & BIS & & FALSE \\
\hline 2004 & $\mathrm{E}$ & 8 & 6 & 1.37 & 98.64 & EW & & FALSE \\
\hline 2004 & $\mathrm{E}$ & 8 & 7 & 2.01 & 98.57 & TW & & FALSE \\
\hline 2004 & $\mathrm{E}$ & 8 & 8 & 2.53 & 98.53 & EW & & FALSE \\
\hline 2004 & $\mathrm{E}$ & 8 & 9 & 3.38 & 98.54 & BIS & & FALSE \\
\hline 2004 & $\mathrm{E}$ & 8 & 10 & 3.51 & 98.72 & BIS & & FALSE \\
\hline 2004 & $\mathrm{E}$ & 8 & 11 & 3.96 & 98.82 & BIS & & FALSE \\
\hline 2004 & $\mathrm{E}$ & 8 & 12 & 4.21 & 99.41 & ТОВ & & FALSE \\
\hline 2004 & $\mathrm{E}$ & 8 & 13 & 4.42 & 99.55 & BIS & & FALSE \\
\hline 2004 & $\mathrm{E}$ & 8 & 14 & 6.64 & 99.73 & $\begin{array}{lll}\text { Bot } X \quad 8 & R \\
\text { Reb/low } & \end{array}$ & & FALSE \\
\hline 2004 & $\mathrm{E}$ & 8 & 15 & 6.64 & 99.99 & $\begin{array}{lll}\text { Top } \mathrm{X} & 8 & \mathrm{R} \\
\text { Reb/low } & \end{array}$ & & FALSE \\
\hline 2004 & $\mathrm{E}$ & 9 & 1 & 0.00 & 100.00 & $\begin{array}{l}\text { Top X } 9 \text { L } \\
\text { Reb/low }\end{array}$ & & FALSE \\
\hline 2004 & $\mathrm{E}$ & 9 & 2 & 0.00 & 99.51 & $\begin{array}{l}\text { Bot X } 9 \text { L Re- } \\
\text { b/low }\end{array}$ & & FALSE \\
\hline 2004 & $\mathrm{E}$ & 9 & 3 & 0.67 & 98.96 & BIS & & FALSE \\
\hline 2004 & $\mathrm{E}$ & 9 & 4 & 1.07 & 98.46 & BIS & & FALSE \\
\hline 2004 & $\mathrm{E}$ & 9 & 5 & 1.65 & 97.92 & BIS & & FALSE \\
\hline 2004 & $\mathrm{E}$ & 9 & 6 & 3.02 & 97.68 & EW & & FALSE \\
\hline 2004 & $\mathrm{E}$ & 9 & 7 & 3.78 & 97.60 & $\mathrm{TW}$ & & FALSE \\
\hline 2004 & $\mathrm{E}$ & 9 & 8 & 4.82 & 97.68 & EW & & FALSE \\
\hline 2004 & $\mathrm{E}$ & 9 & 9 & 5.49 & 97.75 & BIS & & FALSE \\
\hline 2004 & $\mathrm{E}$ & 9 & 10 & 5.76 & 97.87 & $\mathrm{BF}$ & & FALSE \\
\hline 2004 & $\mathrm{E}$ & 9 & 11 & 6.61 & 98.06 & BIS & was 3' off, corrected & FALSE \\
\hline 2004 & $\mathrm{E}$ & 9 & 12 & 7.16 & 98.46 & BIS & & FALSE \\
\hline 2004 & $\mathrm{E}$ & 9 & 13 & 7.38 & 98.95 & BIS & & FALSE \\
\hline 2004 & $\mathrm{E}$ & 9 & 14 & 7.68 & 99.06 & BIS & & FALSE \\
\hline 2004 & $\mathrm{E}$ & 9 & 15 & 7.92 & 99.65 & тов & & FALSE \\
\hline 2004 & $\mathrm{E}$ & 9 & 16 & 8.11 & 99.69 & $\begin{array}{l}\text { Bot } \mathrm{X} \quad 9 \mathrm{R} \\
\text { Reb/low }\end{array}$ & & FALSE \\
\hline 2004 & $\mathrm{E}$ & 9 & 17 & 8.11 & 99.98 & 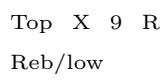 & & FALSE \\
\hline 2004 & $\mathrm{E}$ & 10 & 1 & 0.00 & 100.00 & $\begin{array}{l}\text { Top X } 10 \mathrm{~L} \\
\text { Reb/low }\end{array}$ & & FALSE \\
\hline
\end{tabular}


Table D.1 - Continued

\begin{tabular}{|c|c|c|c|c|c|c|c|}
\hline Year & Reach & Section & Point & Dist. (m) & Elev. (m) & Notes & Exclude \\
\hline 2004 & $\mathrm{E}$ & 10 & 2 & 0.00 & 99.65 & $\begin{array}{l}\text { Bot X } 10 \mathrm{~L} \\
\text { Reb/low }\end{array}$ & FALSE \\
\hline 2004 & $\mathrm{E}$ & 10 & 3 & 0.52 & 99.31 & BIS & FALSE \\
\hline 2004 & $\mathrm{E}$ & 10 & 4 & 0.70 & 98.79 & BIS & FALSE \\
\hline 2004 & $\mathrm{E}$ & 10 & 5 & 1.22 & 98.50 & BIS & FALSE \\
\hline 2004 & $\mathrm{E}$ & 10 & 6 & 1.37 & 98.32 & BIS & FALSE \\
\hline 2004 & $\mathrm{E}$ & 10 & 7 & 2.29 & 98.10 & EW & FALSE \\
\hline 2004 & $\mathrm{E}$ & 10 & 8 & 3.08 & 98.00 & TW & FALSE \\
\hline 2004 & $\mathrm{E}$ & 10 & 9 & 3.35 & 98.09 & EW & FALSE \\
\hline 2004 & $\mathrm{E}$ & 10 & 10 & 3.81 & 98.19 & BIS & FALSE \\
\hline 2004 & $\mathrm{E}$ & 10 & 11 & 3.99 & 98.40 & $\mathrm{BF}$ & FALSE \\
\hline 2004 & $\mathrm{E}$ & 10 & 12 & 5.00 & 98.62 & BIS & FALSE \\
\hline 2004 & $\mathrm{E}$ & 10 & 13 & 5.30 & 99.16 & ТОВ & FALSE \\
\hline 2004 & $\mathrm{E}$ & 10 & 14 & 6.40 & 99.71 & BIS & FALSE \\
\hline 2004 & $\mathrm{E}$ & 10 & 15 & 7.07 & 99.93 & $\begin{array}{l}\text { Bot } X 10 \mathrm{R} \\
\text { Reb/low }\end{array}$ & FALSE \\
\hline 2004 & $\mathrm{E}$ & 10 & 16 & 7.07 & 100.15 & $\begin{array}{l}\text { Top X } 10 \mathrm{R} \\
\text { Reb/low }\end{array}$ & FALSE \\
\hline 2004 & $\mathrm{~F}$ & 1 & 1 & 0.00 & 100.00 & $\begin{array}{l}\text { Top X } 1 \text { L } \\
\text { Reb/low }\end{array}$ & FALSE \\
\hline 2004 & $\mathrm{~F}$ & 1 & 2 & 0.00 & 99.98 & $\begin{array}{l}\text { Bot X } 1 \text { L Re- } \\
\text { b/low }\end{array}$ & FALSE \\
\hline 2004 & $\mathrm{~F}$ & 1 & 3 & 1.04 & 99.12 & ТОВ & FALSE \\
\hline 2004 & $\mathrm{~F}$ & 1 & 4 & 2.74 & 98.59 & $\mathrm{BF}$ & FALSE \\
\hline 2004 & $\mathrm{~F}$ & 1 & 5 & 4.97 & 98.10 & BIS & FALSE \\
\hline 2004 & $\mathrm{~F}$ & 1 & 6 & 5.79 & 97.97 & EW & FALSE \\
\hline 2004 & $\mathrm{~F}$ & 1 & 7 & 5.94 & 97.89 & TW & FALSE \\
\hline 2004 & $\mathrm{~F}$ & 1 & 8 & 6.07 & 97.99 & EW & FALSE \\
\hline 2004 & $\mathrm{~F}$ & 1 & 9 & 7.41 & 98.27 & BIS & FALSE \\
\hline 2004 & $\mathrm{~F}$ & 1 & 10 & 8.72 & 99.28 & BIS & FALSE \\
\hline 2004 & $\mathrm{~F}$ & 1 & 11 & 9.45 & 99.73 & 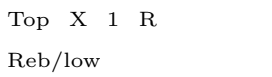 & FALSE \\
\hline 2004 & $\mathrm{~F}$ & 1 & 12 & 9.45 & 99.82 & $\begin{array}{l}\text { Bot } \quad \mathrm{X} \quad 1 \quad \mathrm{R} \\
\text { Reb/low }\end{array}$ & FALSE \\
\hline 2004 & $\mathrm{~F}$ & 2 & 1 & 0.00 & 100.00 & $\begin{array}{l}\text { Top X } \quad 2 \quad \text { L } \\
\text { Reb/low }\end{array}$ & FALSE \\
\hline 2004 & $\mathrm{~F}$ & 2 & 2 & 0.00 & 99.80 & $\begin{array}{l}\text { Bot X } 2 \text { L Re- } \\
\text { b/low }\end{array}$ & FALSE \\
\hline 2004 & $\mathrm{~F}$ & 2 & 3 & 1.58 & 98.88 & ТОВ & FALSE \\
\hline 2004 & $\mathrm{~F}$ & 2 & 4 & 2.53 & 98.44 & BID & FALSE \\
\hline 2004 & $\mathrm{~F}$ & 2 & 5 & 2.68 & 98.01 & $\mathrm{BF}$ & FALSE \\
\hline 2004 & $\mathrm{~F}$ & 2 & 6 & 4.57 & 97.50 & EW & FALSE \\
\hline 2004 & $\mathrm{~F}$ & 2 & 7 & 4.88 & 97.38 & TW & FALSE \\
\hline 2004 & $\mathrm{~F}$ & 2 & 8 & 5.33 & 97.45 & EW & FALSE \\
\hline 2004 & $\mathrm{~F}$ & 2 & 9 & 6.64 & 97.68 & BIS & FALSE \\
\hline 2004 & $\mathrm{~F}$ & 2 & 10 & 8.08 & 98.46 & BIS & FALSE \\
\hline 2004 & $\mathrm{~F}$ & 2 & 11 & 8.47 & 99.75 & BIS & FALSE \\
\hline 2004 & $\mathrm{~F}$ & 2 & 12 & 8.72 & 99.85 & $\begin{array}{l}\text { Top X } 2 \text { R } \\
\text { Reb/low }\end{array}$ & FALSE \\
\hline 2004 & $\mathrm{~F}$ & 2 & 13 & 8.72 & 99.91 & $\begin{array}{l}\text { Bot } \mathrm{X} \quad 2 \quad \mathrm{R} \\
\text { Reb/low }\end{array}$ & FALSE \\
\hline
\end{tabular}

Continued on Next Page... 
Table D.1 - Continued

\begin{tabular}{|c|c|c|c|c|c|c|c|}
\hline Year & Reach & Section & Point & Dist. (m) & Elev. (m) & Notes & Exclude \\
\hline 2004 & $\mathrm{~F}$ & 3 & 1 & 0.00 & 100.00 & $\begin{array}{l}\text { Top X } 3 \text { L } \\
\text { Reb/low }\end{array}$ & FALSE \\
\hline 2004 & $\mathrm{~F}$ & 3 & 2 & 0.00 & 99.85 & $\begin{array}{l}\text { Bot X } 3 \text { L Re- } \\
\text { b/low }\end{array}$ & FALSE \\
\hline 2004 & $\mathrm{~F}$ & 3 & 3 & 1.10 & 99.40 & тов & FALSE \\
\hline 2004 & $\mathrm{~F}$ & 3 & 4 & 1.40 & 98.59 & $\mathrm{BF}$ & FALSE \\
\hline 2004 & $\mathrm{~F}$ & 3 & 5 & 3.96 & 98.07 & EW & FALSE \\
\hline 2004 & $\mathrm{~F}$ & 3 & 6 & 4.75 & 97.83 & TW & FALSE \\
\hline 2004 & $\mathrm{~F}$ & 3 & 7 & 5.12 & 97.96 & EW & FALSE \\
\hline 2004 & $\mathrm{~F}$ & 3 & 8 & 6.10 & 98.52 & BIS & FALSE \\
\hline 2004 & $\mathrm{~F}$ & 3 & 9 & 7.10 & 99.27 & BIS & FALSE \\
\hline 2004 & $\mathrm{~F}$ & 3 & 10 & 7.77 & 99.99 & $\begin{array}{l}\text { Bot } \mathrm{X} \quad 3 \quad \mathrm{R} \\
\text { Reb/low }\end{array}$ & FALSE \\
\hline 2004 & $\mathrm{~F}$ & 3 & 11 & 7.77 & 100.06 & $\begin{array}{l}\text { Top X } 3 \text { R } \\
\text { Reb/low }\end{array}$ & FALSE \\
\hline 2004 & $\mathrm{~F}$ & 4 & 1 & 0.00 & 100.00 & $\begin{array}{l}\text { Top X } 4 \text { L } \\
\text { Reb/low }\end{array}$ & FALSE \\
\hline 2004 & $\mathrm{~F}$ & 4 & 2 & 0.00 & 99.91 & $\begin{array}{l}\text { Bot X } 4 \text { L Re- } \\
\text { b/low }\end{array}$ & FALSE \\
\hline 2004 & $\mathrm{~F}$ & 4 & 3 & 1.22 & 99.09 & BIS & FALSE \\
\hline 2004 & $\mathrm{~F}$ & 4 & 4 & 1.92 & 98.75 & ТОв & FALSE \\
\hline 2004 & $\mathrm{~F}$ & 4 & 5 & 2.35 & 98.07 & $\mathrm{BF}$ & FALSE \\
\hline 2004 & $\mathrm{~F}$ & 4 & 6 & 4.30 & 97.58 & EW & FALSE \\
\hline 2004 & $\mathrm{~F}$ & 4 & 7 & 5.09 & 97.33 & $\mathrm{TW}$ & FALSE \\
\hline 2004 & $\mathrm{~F}$ & 4 & 8 & 6.28 & 97.47 & EW & FALSE \\
\hline 2004 & $\mathrm{~F}$ & 4 & 9 & 7.01 & 98.53 & BIS & FALSE \\
\hline 2004 & $\mathrm{~F}$ & 4 & 10 & 8.02 & 99.60 & BIS & FALSE \\
\hline 2004 & $\mathrm{~F}$ & 4 & 11 & 8.87 & 99.89 & $\begin{array}{l}\text { Top X } 4 \text { R } \\
\text { Reb/low }\end{array}$ & FALSE \\
\hline 2004 & $\mathrm{~F}$ & 4 & 12 & 8.87 & 100.10 & $\begin{array}{l}\text { Bot } \mathrm{X} \quad 4 \quad \mathrm{R} \\
\text { Reb/low }\end{array}$ & FALSE \\
\hline 2004 & $\mathrm{~F}$ & 5 & 1 & 0.00 & 100.00 & $\begin{array}{l}\text { Top X } 5 \text { L } \\
\text { Reb/low }\end{array}$ & FALSE \\
\hline 2004 & $\mathrm{~F}$ & 5 & 2 & 0.00 & 99.95 & $\begin{array}{l}\text { Bot X } 5 \text { L Re- } \\
\text { b/low }\end{array}$ & FALSE \\
\hline 2004 & $\mathrm{~F}$ & 5 & 3 & 0.91 & 99.14 & ТOB & FALSE \\
\hline 2004 & $\mathrm{~F}$ & 5 & 4 & 1.62 & 98.80 & $\mathrm{BF}$ & FALSE \\
\hline 2004 & $\mathrm{~F}$ & 5 & 5 & 3.23 & 98.27 & EW & FALSE \\
\hline 2004 & $\mathrm{~F}$ & 5 & 6 & 4.33 & 98.21 & TW & FALSE \\
\hline 2004 & $\mathrm{~F}$ & 5 & 7 & 4.75 & 98.24 & EW & FALSE \\
\hline 2004 & $\mathrm{~F}$ & 5 & 8 & 5.94 & 98.44 & BIS & FALSE \\
\hline 2004 & $\mathrm{~F}$ & 5 & 9 & 7.10 & 99.26 & BIS & FALSE \\
\hline 2004 & $\mathrm{~F}$ & 5 & 10 & 7.71 & 99.79 & $\begin{array}{l}\text { Bot } \mathrm{X} \quad 5 \quad \mathrm{R} \\
\text { Reb/low }\end{array}$ & FALSE \\
\hline 2004 & $\mathrm{~F}$ & 5 & 11 & 7.71 & 99.87 & $\begin{array}{l}\text { Top X } 5 \mathrm{R} \\
\text { Reb/low }\end{array}$ & FALSE \\
\hline 2004 & $\mathrm{~F}$ & 6 & 1 & 0.00 & 100.00 & $\begin{array}{l}\text { Top X } \quad 6 \quad \text { L } \\
\text { Reb/low }\end{array}$ & FALSE \\
\hline 2004 & $\mathrm{~F}$ & 6 & 2 & 0.00 & 99.89 & $\begin{array}{l}\text { Bot X } 6 \text { L Re- } \\
\text { b/low }\end{array}$ & FALSE \\
\hline 2004 & $\mathrm{~F}$ & 6 & 3 & 0.61 & 99.43 & BIS & FALSE \\
\hline 2004 & $\mathrm{~F}$ & 6 & 4 & 1.52 & 98.92 & $\mathrm{BF}$ & FALSE \\
\hline
\end{tabular}


Table D.1 - Continued

\begin{tabular}{|c|c|c|c|c|c|c|c|}
\hline Year & Reach & Section & Point & Dist. $(\mathrm{m})$ & Elev. $(\mathrm{m})$ & Notes & Exclude \\
\hline 2004 & $\mathrm{~F}$ & 6 & 5 & 2.74 & 98.29 & BIS & FALSE \\
\hline 2004 & $\mathrm{~F}$ & 6 & 6 & 3.84 & 98.21 & EW & FALSE \\
\hline 2004 & $\mathrm{~F}$ & 6 & 7 & 4.24 & 98.03 & $\mathrm{TW}$ & FALSE \\
\hline 2004 & $\mathrm{~F}$ & 6 & 8 & 4.97 & 98.19 & EW & FALSE \\
\hline 2004 & $\mathrm{~F}$ & 6 & 9 & 5.79 & 98.93 & $\begin{array}{l}\text { BIS *on boul- } \\
\text { der }\end{array}$ & FALSE \\
\hline 2004 & $\mathrm{~F}$ & 6 & 10 & 6.71 & 99.21 & ТОВ & FALSE \\
\hline 2004 & $\mathrm{~F}$ & 6 & 11 & 7.80 & 99.54 & BIS & FALSE \\
\hline 2004 & $\mathrm{~F}$ & 6 & 12 & 8.41 & 99.85 & $\begin{array}{l}\text { Bot } \mathrm{X} \quad 6 \quad \mathrm{R} \\
\text { Reb/low }\end{array}$ & FALSE \\
\hline 2004 & $\mathrm{~F}$ & 6 & 13 & 8.41 & 99.97 & $\begin{array}{l}\text { Top X } 6 \text { R } \\
\text { Reb/low }\end{array}$ & FALSE \\
\hline 2004 & $\mathrm{~F}$ & 7 & 1 & 0.00 & 100.00 & $\begin{array}{llll}\text { Top } & X & 7 & \text { L } \\
\text { Reb/low } & \end{array}$ & FALSE \\
\hline 2004 & $\mathrm{~F}$ & 7 & 2 & 0.00 & 99.92 & $\begin{array}{l}\text { Bot X } 7 \text { L Re- } \\
\text { b/low }\end{array}$ & FALSE \\
\hline 2004 & $\mathrm{~F}$ & 7 & 3 & 0.85 & 99.09 & BIS & FALSE \\
\hline 2004 & $\mathrm{~F}$ & 7 & 4 & 1.22 & 98.71 & BIS & FALSE \\
\hline 2004 & $\mathrm{~F}$ & 7 & 5 & 1.89 & 98.43 & EW & FALSE \\
\hline 2004 & $\mathrm{~F}$ & 7 & 6 & 2.71 & 98.35 & TW & FALSE \\
\hline 2004 & $\mathrm{~F}$ & 7 & 7 & 3.05 & 98.42 & EW & FALSE \\
\hline 2004 & $\mathrm{~F}$ & 7 & 8 & 3.26 & 98.45 & BIS & FALSE \\
\hline 2004 & $\mathrm{~F}$ & 7 & 9 & 4.02 & 98.58 & $\mathrm{BF}$ & FALSE \\
\hline 2004 & $\mathrm{~F}$ & 7 & 10 & 4.27 & 98.67 & ТОВ & FALSE \\
\hline 2004 & $\mathrm{~F}$ & 7 & 11 & 5.67 & 99.01 & BIS & FALSE \\
\hline 2004 & $\mathrm{~F}$ & 7 & 12 & 7.25 & 99.30 & BIS & FALSE \\
\hline 2004 & $\mathrm{~F}$ & 7 & 13 & 8.69 & 99.81 & $\begin{array}{llll}\text { Top } & \mathrm{X} & 7 & \mathrm{R} \\
\text { Reb/low } & & \end{array}$ & FALSE \\
\hline 2004 & $\mathrm{~F}$ & 7 & 14 & 8.69 & 99.95 & $\begin{array}{llll}\text { Bot } & X & 7 & \mathrm{R} \\
\text { Reb/low } & & \end{array}$ & FALSE \\
\hline 2004 & $\mathrm{~F}$ & 8 & 1 & 0.00 & 100.00 & $\begin{array}{llll}\text { Top } & X & 8 & \text { L } \\
\text { Reb/low } & \end{array}$ & FALSE \\
\hline 2004 & $\mathrm{~F}$ & 8 & 2 & 0.00 & 99.84 & $\begin{array}{l}\text { Bot X } 8 \text { L Re- } \\
\text { b/low }\end{array}$ & FALSE \\
\hline 2004 & $\mathrm{~F}$ & 8 & 3 & 0.94 & 99.15 & BIS & FALSE \\
\hline 2004 & $\mathrm{~F}$ & 8 & 4 & 1.10 & 98.62 & BIS & FALSE \\
\hline 2004 & $\mathrm{~F}$ & 8 & 5 & 2.01 & 98.20 & BIS *on rock & FALSE \\
\hline 2004 & $\mathrm{~F}$ & 8 & 6 & 2.44 & 97.80 & $\begin{array}{l}\text { BIS *left of } \\
\text { sandy bar }\end{array}$ & FALSE \\
\hline 2004 & $\mathrm{~F}$ & 8 & 7 & 3.05 & 97.80 & EW & FALSE \\
\hline 2004 & $\mathrm{~F}$ & 8 & 8 & 3.66 & 97.68 & TW & FALSE \\
\hline 2004 & $\mathrm{~F}$ & 8 & 9 & 4.57 & 97.81 & $\mathrm{EW}$ & FALSE \\
\hline 2004 & $\mathrm{~F}$ & 8 & 10 & 4.88 & 98.16 & $\mathrm{BF}$ & FALSE \\
\hline 2004 & $\mathrm{~F}$ & 8 & 11 & 5.33 & 98.45 & тОВ & FALSE \\
\hline 2004 & $\mathrm{~F}$ & 8 & 12 & 7.32 & 98.78 & BIS & FALSE \\
\hline 2004 & $\mathrm{~F}$ & 8 & 13 & 9.02 & 98.78 & BIS & FALSE \\
\hline 2004 & $\mathrm{~F}$ & 8 & 14 & 9.85 & 99.39 & BIS & FALSE \\
\hline 2004 & $\mathrm{~F}$ & 8 & 15 & 11.31 & 99.83 & BIS & FALSE \\
\hline 2004 & $\mathrm{~F}$ & 8 & 16 & 12.71 & 99.84 & $\begin{array}{llll}\text { Bot } & X & 8 & R \\
\text { Reb/low } & & \end{array}$ & FALSE \\
\hline 2004 & $\mathrm{~F}$ & 8 & 17 & 12.71 & 100.04 & $\begin{array}{l}\text { Top X } 8 \text { R } \\
\text { Reb/low }\end{array}$ & FALSE \\
\hline
\end{tabular}

Continued on Next Page... 
Table D.1 - Continued

\begin{tabular}{|c|c|c|c|c|c|c|c|c|}
\hline Year & Reach & Section & Point & Dist. (m) & Elev. $(\mathrm{m})$ & Desc. & Notes & Exclude \\
\hline 2004 & $\mathrm{~F}$ & 9 & 1 & 0.00 & 100.00 & $\begin{array}{l}\text { Top X } \quad 9 \quad \text { L } \\
\text { Reb/low }\end{array}$ & & FALSE \\
\hline 2004 & $\mathrm{~F}$ & 9 & 2 & 0.00 & 99.78 & $\begin{array}{l}\text { Bot X } 9 \text { L Re- } \\
\text { b/low }\end{array}$ & & FALSE \\
\hline 2004 & $\mathrm{~F}$ & 9 & 3 & 0.37 & 99.27 & BIS & & FALSE \\
\hline 2004 & $\mathrm{~F}$ & 9 & 4 & 1.55 & 98.74 & $\mathrm{BF}$ & & FALSE \\
\hline 2004 & $\mathrm{~F}$ & 9 & 5 & 2.07 & 98.56 & BIS & & FALSE \\
\hline 2004 & $\mathrm{~F}$ & 9 & 6 & 2.32 & 98.45 & EW & & FALSE \\
\hline 2004 & $\mathrm{~F}$ & 9 & 7 & 3.35 & 98.29 & $\mathrm{TW}$ & & FALSE \\
\hline 2004 & $\mathrm{~F}$ & 9 & 8 & 3.75 & 98.37 & $\begin{array}{l}\mathrm{EW}{ }^{*} \text { next to } \\
\text { rock }\end{array}$ & & FALSE \\
\hline 2004 & $\mathrm{~F}$ & 9 & 9 & 4.30 & 99.03 & тов & & FALSE \\
\hline 2004 & $\mathrm{~F}$ & 9 & 10 & 6.19 & 99.21 & BIS & & FALSE \\
\hline 2004 & $\mathrm{~F}$ & 9 & 11 & 7.07 & 99.02 & BIS & & FALSE \\
\hline 2004 & $\mathrm{~F}$ & 9 & 12 & 9.88 & 99.11 & BIS & & FALSE \\
\hline 2004 & $\mathrm{~F}$ & 9 & 13 & 11.22 & 99.73 & 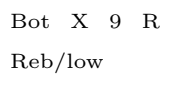 & & FALSE \\
\hline 2004 & $\mathrm{~F}$ & 9 & 14 & 11.22 & 99.95 & $\begin{array}{l}\text { Top } \mathrm{X} \quad 9 \quad \mathrm{R} \\
\text { Reb/low }\end{array}$ & & FALSE \\
\hline 2004 & $\mathrm{~F}$ & 10 & 1 & 0.00 & 100.00 & $\begin{array}{l}\text { Top X } 10 \mathrm{~L} \\
\text { Reb/low }\end{array}$ & & FALSE \\
\hline 2004 & $\mathrm{~F}$ & 10 & 2 & 0.00 & 99.78 & $\begin{array}{l}\text { Bot X } 10 \mathrm{~L} \\
\text { Reb/low }\end{array}$ & & FALSE \\
\hline 2004 & $\mathrm{~F}$ & 10 & 3 & 0.37 & 98.92 & BIS & & FALSE \\
\hline 2004 & $\mathrm{~F}$ & 10 & 4 & 1.34 & 98.45 & BIS & & FALSE \\
\hline 2004 & $\mathrm{~F}$ & 10 & 5 & 1.55 & 98.38 & EW & & FALSE \\
\hline 2004 & $\mathrm{~F}$ & 10 & 6 & 2.10 & 98.26 & TW & & FALSE \\
\hline 2004 & $\mathrm{~F}$ & 10 & 7 & 2.83 & 98.36 & $\begin{array}{l}\text { EW } * \text { left side } \\
\text { of rock (creek } \\
\text { side) }\end{array}$ & & FALSE \\
\hline 2004 & $\mathrm{~F}$ & 10 & 8 & 3.14 & 98.52 & $\begin{array}{l}\text { BIS *right } \\
\text { side of rock }\end{array}$ & & FALSE \\
\hline 2004 & $\mathrm{~F}$ & 10 & 9 & 4.11 & 98.82 & $\mathrm{BF}$ & & FALSE \\
\hline 2004 & $\mathrm{~F}$ & 10 & 10 & 4.36 & 99.13 & TOB *rocky & & FALSE \\
\hline 2004 & $\mathrm{~F}$ & 10 & 11 & 5.30 & 99.27 & BIS *rocky & & FALSE \\
\hline 2004 & $\mathrm{~F}$ & 10 & 12 & 9.02 & 98.90 & BIS & & FALSE \\
\hline 2004 & $\mathrm{~F}$ & 10 & 13 & 9.78 & 99.65 & $\begin{array}{l}\text { Bot } X 10 \mathrm{R} \\
\text { Reb/low }\end{array}$ & & FALSE \\
\hline 2004 & $\mathrm{~F}$ & 10 & 14 & 9.78 & 99.79 & $\begin{array}{l}\text { Top X } 10 \mathrm{R} \\
\text { Reb/low }\end{array}$ & & FALSE \\
\hline 2005 & A & 1 & 1 & 0.00 & 100.00 & TOP X1R & Top X1L reb/low & FALSE \\
\hline 2005 & A & 1 & 2 & 0.00 & 99.86 & BOT X1R & Bot X1L reb/low & FALSE \\
\hline 2005 & A & 1 & 3 & 1.40 & 99.57 & BIS & & FALSE \\
\hline 2005 & A & 1 & 4 & 2.44 & 99.38 & ТОВ & & FALSE \\
\hline 2005 & $\mathrm{~A}$ & 1 & 5 & 3.11 & 99.24 & BIS & & FALSE \\
\hline 2005 & A & 1 & 6 & 3.54 & 99.00 & $\mathrm{BF}$ & & FALSE \\
\hline 2005 & A & 1 & 7 & 4.30 & 98.75 & EW & & FALSE \\
\hline 2005 & A & 1 & 8 & 4.97 & 98.59 & $\mathrm{TW}$ & & FALSE \\
\hline 2005 & A & 1 & 9 & 5.70 & 98.73 & EW & & FALSE \\
\hline 2005 & A & 1 & 10 & 6.46 & 99.02 & BIS & & FALSE \\
\hline 2005 & A & 1 & 11 & 7.47 & 99.42 & ТОВ & & FALSE \\
\hline 2005 & $\mathrm{~A}$ & 1 & 12 & 8.72 & 99.60 & BIS & & FALSE \\
\hline
\end{tabular}

Continued on Next Page... 
Table D.1 - Continued

\begin{tabular}{|c|c|c|c|c|c|c|c|c|}
\hline Year & Reach & Section & Point & Dist. (m) & Elev. $(\mathrm{m})$ & Desc. & Notes & Exclude \\
\hline 2005 & A & 1 & 13 & 9.08 & 99.81 & BOT X1R & Bot $\mathrm{X} 1 \mathrm{R}$ reb/low & FALSE \\
\hline 2005 & A & 1 & 14 & 9.08 & 100.00 & TOP X1R & Top X1R reb/low & FALSE \\
\hline 2005 & A & 2 & 1 & 0.00 & 100.00 & TOP X2L & Top X2L reb/low & FALSE \\
\hline 2005 & A & 2 & 2 & 0.00 & 99.66 & BOT X2L & Bot $\mathrm{X} 2 \mathrm{~L} \mathrm{reb} /$ low & FALSE \\
\hline 2005 & $\mathrm{~A}$ & 2 & 3 & 1.01 & 99.49 & ТОВ & & FALSE \\
\hline 2005 & $\mathrm{~A}$ & 2 & 4 & 2.19 & 98.97 & BIS & & FALSE \\
\hline 2005 & $\mathrm{~A}$ & 2 & 5 & 3.87 & 98.44 & BIS & & FALSE \\
\hline 2005 & $\mathrm{~A}$ & 2 & 6 & 4.97 & 98.17 & EW & & FALSE \\
\hline 2005 & $\mathrm{~A}$ & 2 & 7 & 5.73 & 98.02 & TW & & FALSE \\
\hline 2005 & $\mathrm{~A}$ & 2 & 8 & 6.52 & 98.06 & EW & & FALSE \\
\hline 2005 & $\mathrm{~A}$ & 2 & 9 & 6.68 & 98.70 & BIS & Top of Boulder & TRUE \\
\hline 2005 & $\mathrm{~A}$ & 2 & 10 & 7.04 & 98.91 & BIS & Top of Log (fell across) & TRUE \\
\hline 2005 & $\mathrm{~A}$ & 2 & 11 & 7.32 & 98.50 & $\mathrm{BF}$ & & FALSE \\
\hline 2005 & $\mathrm{~A}$ & 2 & 12 & 8.20 & 98.74 & BIS & & FALSE \\
\hline 2005 & $\mathrm{~A}$ & 2 & 13 & 8.53 & 99.16 & BIS & & FALSE \\
\hline 2005 & $\mathrm{~A}$ & 2 & 14 & 9.42 & 98.96 & BIS & & FALSE \\
\hline 2005 & $\mathrm{~A}$ & 2 & 15 & 10.67 & 99.70 & Bot $\mathrm{X} 2 \mathrm{R}$ & Bot $\mathrm{X} 2 \mathrm{R}$ reb/low & FALSE \\
\hline 2005 & $\mathrm{~A}$ & 2 & 16 & 10.67 & 100.00 & Top X2R & Top X2R reb/low & FALSE \\
\hline 2005 & $\mathrm{~A}$ & 3 & 1 & 0.00 & 100.00 & TOP X3L & Top X3L reb/low & FALSE \\
\hline 2005 & $\mathrm{~A}$ & 3 & 2 & 0.00 & 99.50 & BOT X3L & Bot $\mathrm{X} 3 \mathrm{~L} \mathrm{reb} / \mathrm{low}$ & FALSE \\
\hline 2005 & $\mathrm{~A}$ & 3 & 3 & 1.22 & 98.83 & ТОВ & & FALSE \\
\hline 2005 & $\mathrm{~A}$ & 3 & 4 & 1.92 & 98.22 & BIS & & FALSE \\
\hline 2005 & $\mathrm{~A}$ & 3 & 5 & 2.10 & 98.21 & $\mathrm{BF}$ & & FALSE \\
\hline 2005 & $\mathrm{~A}$ & 3 & 6 & 2.26 & 97.95 & BIS & & FALSE \\
\hline 2005 & $\mathrm{~A}$ & 3 & 7 & 2.53 & 97.89 & EW & & FALSE \\
\hline 2005 & $\mathrm{~A}$ & 3 & 8 & 3.63 & 97.77 & TW & & FALSE \\
\hline 2005 & $\mathrm{~A}$ & 3 & 9 & 4.69 & 97.90 & EW & & FALSE \\
\hline 2005 & $\mathrm{~A}$ & 3 & 10 & 5.24 & 98.11 & BIS & & FALSE \\
\hline 2005 & $\mathrm{~A}$ & 3 & 11 & 5.91 & 98.44 & BIS & & FALSE \\
\hline 2005 & A & 3 & 12 & 8.44 & 98.64 & BIS & & FALSE \\
\hline 2005 & $\mathrm{~A}$ & 3 & 13 & 9.05 & 98.92 & BIS & & FALSE \\
\hline 2005 & $\mathrm{~A}$ & 3 & 14 & 10.12 & 99.39 & ТОВ & & FALSE \\
\hline 2005 & A & 3 & 15 & 10.67 & 99.68 & BOT X3R & Bot $\mathrm{X} 3 \mathrm{R}$ reb/low & FALSE \\
\hline 2005 & $\mathrm{~A}$ & 3 & 16 & 10.67 & 100.10 & TOP X3R & Top X3R reb/low & FALSE \\
\hline 2005 & $\mathrm{~A}$ & 4 & 1 & 0.00 & 100.00 & TOP X4L & Top X4L reb/low & FALSE \\
\hline 2005 & A & 4 & 2 & 0.00 & 99.72 & BOT X4L & Bot $\mathrm{X} 4 \mathrm{~L}$ reb/low & FALSE \\
\hline 2005 & $\mathrm{~A}$ & 4 & 3 & 0.98 & 99.53 & ТОВ & & FALSE \\
\hline 2005 & $\mathrm{~A}$ & 4 & 4 & 2.35 & 99.29 & BIS & & FALSE \\
\hline 2005 & $\mathrm{~A}$ & 4 & 5 & 2.74 & 98.70 & BIS & & FALSE \\
\hline 2005 & $\mathrm{~A}$ & 4 & 6 & 3.20 & 98.55 & EW & & FALSE \\
\hline 2005 & $\mathrm{~A}$ & 4 & 7 & 4.75 & 98.47 & TW & & FALSE \\
\hline 2005 & $\mathrm{~A}$ & 4 & 8 & 5.67 & 98.54 & EW & & FALSE \\
\hline 2005 & $\mathrm{~A}$ & 4 & 9 & 6.64 & 98.81 & $\mathrm{BF}$ & & FALSE \\
\hline 2005 & $\mathrm{~A}$ & 4 & 10 & 7.62 & 99.09 & BIS & & FALSE \\
\hline 2005 & A & 4 & 11 & 8.66 & 99.49 & BOT X4R & Bot $\mathrm{X} 4 \mathrm{R}$ reb/low & FALSE \\
\hline 2005 & A & 4 & 12 & 8.66 & 99.83 & TOP X4R & Top X4R reb/low & FALSE \\
\hline 2005 & A & 5 & 1 & 0.00 & 100.00 & TOP X5L & Top X5L reb/low & FALSE \\
\hline 2005 & $\mathrm{~A}$ & 5 & 2 & 0.00 & 99.74 & BOT X5L & Bot $\mathrm{X} 5 \mathrm{~L} \mathrm{reb} /$ low & FALSE \\
\hline 2005 & $\mathrm{~A}$ & 5 & 3 & 1.04 & 99.76 & ТОВ & & FALSE \\
\hline 2005 & $\mathrm{~A}$ & 5 & 4 & 2.23 & 99.07 & BIS & & FALSE \\
\hline 2005 & A & 5 & 5 & 2.99 & 98.68 & EW & & FALSE \\
\hline 2005 & A & 5 & 6 & 4.18 & 98.44 & TW & & FALSE \\
\hline
\end{tabular}

Continued on Next Page... 
Table D.1 - Continued

\begin{tabular}{|c|c|c|c|c|c|c|c|c|}
\hline Year & Reach & Section & Point & Dist. (m) & Elev. $(\mathrm{m})$ & Desc. & Notes & Exclude \\
\hline 2005 & $\mathrm{~A}$ & 5 & 7 & 5.09 & 98.58 & EW & & FALSE \\
\hline 2005 & A & 5 & 8 & 5.18 & 98.95 & BIS & Top of Boulder & FALSE \\
\hline 2005 & A & 5 & 9 & 5.79 & 98.86 & $\mathrm{BF}$ & & FALSE \\
\hline 2005 & A & 5 & 10 & 6.34 & 99.14 & BIS & & FALSE \\
\hline 2005 & $\mathrm{~A}$ & 5 & 11 & 6.92 & 99.34 & BIS & & FALSE \\
\hline 2005 & $\mathrm{~A}$ & 5 & 12 & 7.38 & 99.57 & ТОВ & & FALSE \\
\hline 2005 & $\mathrm{~A}$ & 5 & 13 & 7.77 & 99.62 & BOT X5R & Bot $\mathrm{X} 5 \mathrm{R}$ reb/low & FALSE \\
\hline 2005 & $\mathrm{~A}$ & 5 & 14 & 7.77 & 99.98 & TOP X5R & Top X5R reb/low & FALSE \\
\hline 2005 & $\mathrm{~A}$ & 6 & 1 & 0.00 & 100.00 & TOP X6L & Top X6L reb/low & FALSE \\
\hline 2005 & A & 6 & 2 & 0.00 & 99.77 & BOT X6L & Bot $\mathrm{X} 6 \mathrm{~L}$ reb/low & FALSE \\
\hline 2005 & $\mathrm{~A}$ & 6 & 3 & 0.37 & 99.64 & ТОВ & & FALSE \\
\hline 2005 & $\mathrm{~A}$ & 6 & 4 & 0.67 & 98.54 & BIS & & FALSE \\
\hline 2005 & $\mathrm{~A}$ & 6 & 5 & 1.28 & 98.08 & EW & & FALSE \\
\hline 2005 & A & 6 & 6 & 2.68 & 97.97 & TW & & FALSE \\
\hline 2005 & $\mathrm{~A}$ & 6 & 7 & 3.90 & 98.05 & EW & & FALSE \\
\hline 2005 & $\mathrm{~A}$ & 6 & 8 & 4.57 & 98.68 & $\mathrm{BF}$ & & FALSE \\
\hline 2005 & $\mathrm{~A}$ & 6 & 9 & 5.79 & 99.05 & BIS & & FALSE \\
\hline 2005 & $\mathrm{~A}$ & 6 & 10 & 7.65 & 99.23 & ТОВ & & FALSE \\
\hline 2005 & $\mathrm{~A}$ & 6 & 11 & 9.91 & 99.17 & BIS & & FALSE \\
\hline 2005 & $\mathrm{~A}$ & 6 & 12 & 12.19 & 99.09 & BIS & & FALSE \\
\hline 2005 & $\mathrm{~A}$ & 6 & 13 & 14.66 & 99.14 & BIS & & FALSE \\
\hline 2005 & $\mathrm{~A}$ & 6 & 14 & 15.09 & 99.41 & BOT X6R & Bot $\mathrm{X} 6 \mathrm{R}$ reb/low & FALSE \\
\hline 2005 & $\mathrm{~A}$ & 6 & 15 & 15.09 & 99.82 & TOP X6R & Top X6R reb/low & FALSE \\
\hline 2005 & $\mathrm{~A}$ & 7 & 1 & 0.00 & 100.00 & TOP X7L & Top X7L reb/low & FALSE \\
\hline 2005 & $\mathrm{~A}$ & 7 & 2 & 0.00 & 99.75 & BOT X7L & Bot $\mathrm{X} 7 \mathrm{~L} \mathrm{reb} /$ low & FALSE \\
\hline 2005 & $\mathrm{~A}$ & 7 & 3 & 0.98 & 99.61 & ТОВ & & FALSE \\
\hline 2005 & $\mathrm{~A}$ & 7 & 4 & 1.04 & 99.18 & BIS & & FALSE \\
\hline 2005 & $\mathrm{~A}$ & 7 & 5 & 1.52 & 98.60 & BIS & & FALSE \\
\hline 2005 & $\mathrm{~A}$ & 7 & 6 & 1.89 & 98.74 & BIS & On top of rock & FALSE \\
\hline 2005 & $\mathrm{~A}$ & 7 & 7 & 2.53 & 98.59 & BIS & On top of rock & FALSE \\
\hline 2005 & $\mathrm{~A}$ & 7 & 8 & 2.71 & 98.26 & EW & & FALSE \\
\hline 2005 & $\mathrm{~A}$ & 7 & 9 & 3.23 & 98.18 & $\mathrm{TW}$ & & FALSE \\
\hline 2005 & $\mathrm{~A}$ & 7 & 10 & 4.30 & 98.27 & EW & & FALSE \\
\hline 2005 & $\mathrm{~A}$ & 7 & 11 & 4.88 & 98.46 & BIS & & FALSE \\
\hline 2005 & $\mathrm{~A}$ & 7 & 12 & 5.43 & 98.70 & $\mathrm{BF}$ & & FALSE \\
\hline 2005 & $\mathrm{~A}$ & 7 & 13 & 7.01 & 99.00 & BIS & & FALSE \\
\hline 2005 & $\mathrm{~A}$ & 7 & 14 & 7.62 & 99.09 & BIS & & FALSE \\
\hline 2005 & $\mathrm{~A}$ & 7 & 15 & 9.02 & 99.43 & ТОВ & & FALSE \\
\hline 2005 & A & 7 & 16 & 13.29 & 99.46 & BIS & & FALSE \\
\hline 2005 & $\mathrm{~A}$ & 7 & 17 & 16.15 & 99.30 & BIS & & FALSE \\
\hline 2005 & A & 7 & 18 & 17.68 & 99.42 & BIS & & FALSE \\
\hline 2005 & $\mathrm{~A}$ & 7 & 19 & 18.59 & 99.84 & BOT X7R & Bot $\mathrm{X} 7 \mathrm{R}$ reb/low & FALSE \\
\hline 2005 & $\mathrm{~A}$ & 7 & 20 & 18.59 & 100.21 & TOP X7R & Top X7R reb/low & FALSE \\
\hline 2005 & $\mathrm{~A}$ & 8 & 1 & 0.00 & 100.00 & TOP X8L & Top X8L reb/low & FALSE \\
\hline 2005 & $\mathrm{~A}$ & 8 & 2 & 0.00 & 99.63 & BOT X8L & Bot $\mathrm{X} 8 \mathrm{~L} \mathrm{reb} /$ low & FALSE \\
\hline 2005 & A & 8 & 3 & 0.70 & 99.53 & тов & & FALSE \\
\hline 2005 & A & 8 & 4 & 1.13 & 98.42 & BIS & & FALSE \\
\hline 2005 & $\mathrm{~A}$ & 8 & 5 & 2.10 & 98.21 & BIS & & FALSE \\
\hline 2005 & $\mathrm{~A}$ & 8 & 6 & 2.87 & 98.09 & BIS & & FALSE \\
\hline 2005 & $\mathrm{~A}$ & 8 & 7 & 3.29 & 98.04 & BIS & sandbar & FALSE \\
\hline 2005 & A & 8 & 8 & 3.60 & 98.01 & EW & & FALSE \\
\hline 2005 & A & 8 & 9 & 4.48 & 97.91 & TW & & FALSE \\
\hline
\end{tabular}

Continued on Next Page... 
Table D.1 - Continued

\begin{tabular}{|c|c|c|c|c|c|c|c|c|}
\hline Year & Reach & Section & Point & Dist. (m) & Elev. $(\mathrm{m})$ & Desc. & Notes & Exclude \\
\hline 2005 & $\mathrm{~A}$ & 8 & 10 & 5.27 & 98.02 & EW & & FALSE \\
\hline 2005 & A & 8 & 11 & 5.76 & 98.23 & BIS & & FALSE \\
\hline 2005 & A & 8 & 12 & 6.19 & 98.37 & $\mathrm{BF}$ & & FALSE \\
\hline 2005 & A & 8 & 13 & 8.08 & 98.74 & BIS & & FALSE \\
\hline 2005 & $\mathrm{~A}$ & 8 & 14 & 9.57 & 98.77 & BIS & & FALSE \\
\hline 2005 & $\mathrm{~A}$ & 8 & 15 & 11.03 & 98.94 & BIS & & FALSE \\
\hline 2005 & $\mathrm{~A}$ & 8 & 16 & 12.65 & 99.22 & ТОВ & & FALSE \\
\hline 2005 & $\mathrm{~A}$ & 8 & 17 & 15.45 & 99.08 & BIS & & FALSE \\
\hline 2005 & A & 8 & 18 & 16.46 & 99.00 & BIS & & FALSE \\
\hline 2005 & $\mathrm{~A}$ & 8 & 19 & 17.83 & 99.13 & BIS & & FALSE \\
\hline 2005 & $\mathrm{~A}$ & 8 & 20 & 19.51 & 99.64 & BOT X8R & Bot $\mathrm{X} 8 \mathrm{R}$ reb/low & FALSE \\
\hline 2005 & $\mathrm{~A}$ & 8 & 21 & 19.51 & 100.11 & TOP X8R & Top X8R reb/low & FALSE \\
\hline 2005 & $\mathrm{~A}$ & 9 & 1 & 0.00 & 100.00 & TOP X9L & Top X9L reb/low & FALSE \\
\hline 2005 & $\mathrm{~A}$ & 9 & 2 & 0.00 & 99.63 & BOT X9L & Bot X9L reb/low & FALSE \\
\hline 2005 & $\mathrm{~A}$ & 9 & 3 & 0.70 & 99.69 & ТОВ & & FALSE \\
\hline 2005 & $\mathrm{~A}$ & 9 & 4 & 0.98 & 98.71 & BIS & & FALSE \\
\hline 2005 & $\mathrm{~A}$ & 9 & 5 & 2.41 & 98.57 & BIS & & FALSE \\
\hline 2005 & $\mathrm{~A}$ & 9 & 6 & 3.32 & 98.46 & $\mathrm{BF}$ & & FALSE \\
\hline 2005 & $\mathrm{~A}$ & 9 & 7 & 4.42 & 98.19 & EW & & FALSE \\
\hline 2005 & $\mathrm{~A}$ & 9 & 8 & 5.09 & 98.09 & TW & & FALSE \\
\hline 2005 & $\mathrm{~A}$ & 9 & 9 & 6.25 & 98.20 & EW & & FALSE \\
\hline 2005 & $\mathrm{~A}$ & 9 & 10 & 6.86 & 98.22 & BIS & edge of sandbar & FALSE \\
\hline 2005 & $\mathrm{~A}$ & 9 & 11 & 8.08 & 98.59 & $\mathrm{BF}$ & & FALSE \\
\hline 2005 & $\mathrm{~A}$ & 9 & 12 & 9.91 & 98.91 & BIS & & FALSE \\
\hline 2005 & $\mathrm{~A}$ & 9 & 13 & 12.56 & 99.13 & BIS & & FALSE \\
\hline 2005 & $\mathrm{~A}$ & 9 & 14 & 13.08 & 99.38 & ТОВ & & FALSE \\
\hline 2005 & $\mathrm{~A}$ & 9 & 15 & 17.62 & 99.24 & BIS & & FALSE \\
\hline 2005 & $\mathrm{~A}$ & 9 & 16 & 19.81 & 99.51 & BIS & & FALSE \\
\hline 2005 & $\mathrm{~A}$ & 9 & 17 & 20.48 & 99.88 & BOT X9R & Bot $\mathrm{X} 9 \mathrm{R}$ reb/low & FALSE \\
\hline 2005 & $\mathrm{~A}$ & 9 & 18 & 20.48 & 100.24 & TOP X9R & Top X9R reb/low & FALSE \\
\hline 2005 & $\mathrm{~A}$ & 10 & 1 & 0.00 & 100.00 & TOP X10L & Top X10L reb/low & FALSE \\
\hline 2005 & $\mathrm{~A}$ & 10 & 2 & 0.00 & 99.57 & BOT X10L & Bot X10L reb/low & FALSE \\
\hline 2005 & $\mathrm{~A}$ & 10 & 3 & 0.43 & 99.55 & ТОВ & & FALSE \\
\hline 2005 & $\mathrm{~A}$ & 10 & 4 & 0.58 & 98.58 & BIS & & FALSE \\
\hline 2005 & $\mathrm{~A}$ & 10 & 5 & 1.92 & 98.52 & BIS & & FALSE \\
\hline 2005 & $\mathrm{~A}$ & 10 & 6 & 2.44 & 98.45 & $\mathrm{BF}$ & & FALSE \\
\hline 2005 & $\mathrm{~A}$ & 10 & 7 & 3.05 & 98.34 & BIS & & FALSE \\
\hline 2005 & A & 10 & 8 & 3.84 & 98.25 & BIS & & FALSE \\
\hline 2005 & A & 10 & 9 & 4.88 & 98.13 & BIS & & FALSE \\
\hline 2005 & $\mathrm{~A}$ & 10 & 10 & 5.46 & 98.08 & BIS & & FALSE \\
\hline 2005 & $\mathrm{~A}$ & 10 & 11 & 5.76 & 97.91 & EW & & FALSE \\
\hline 2005 & $\mathrm{~A}$ & 10 & 12 & 6.37 & 97.56 & BIS & In a pool & FALSE \\
\hline 2005 & A & 10 & 13 & 7.22 & 97.66 & BIS & & FALSE \\
\hline 2005 & A & 10 & 14 & 7.32 & 97.66 & BIS & & FALSE \\
\hline 2005 & $\mathrm{~A}$ & 10 & 15 & 7.68 & 98.09 & $\mathrm{RI}$ & on top of lg. boulder & FALSE \\
\hline 2005 & A & 10 & 16 & 8.29 & 97.77 & BIS & & FALSE \\
\hline 2005 & A & 10 & 17 & 8.66 & 97.65 & $\mathrm{TW}$ & & FALSE \\
\hline 2005 & A & 10 & 18 & 9.05 & 97.63 & EW & & FALSE \\
\hline 2005 & $\mathrm{~A}$ & 10 & 19 & 9.42 & 98.24 & BIS & on top of lg. boulder & FALSE \\
\hline 2005 & $\mathrm{~A}$ & 10 & 20 & 10.18 & 98.17 & $\mathrm{BF}$ & & FALSE \\
\hline 2005 & A & 10 & 21 & 10.61 & 98.65 & VIS & & FALSE \\
\hline 2005 & A & 10 & 22 & 11.49 & 98.79 & BIS & & FALSE \\
\hline
\end{tabular}

Continued on Next Page... 
Table D.1 - Continued

\begin{tabular}{|c|c|c|c|c|c|c|c|c|}
\hline Year & Reach & Section & Point & Dist. (m) & Elev. $(\mathrm{m})$ & Desc. & Notes & Exclude \\
\hline 2005 & A & 10 & 23 & 12.47 & 98.91 & ТОВ & Bot X10R reb/low & FALSE \\
\hline 2005 & A & 10 & 24 & 15.03 & 99.01 & BIS & Top X10R reb/low & FALSE \\
\hline 2005 & $\mathrm{~B}$ & 1 & 1 & 0.00 & 100.00 & & Top X1L reb/low & FALSE \\
\hline 2005 & $\mathrm{~B}$ & 1 & 2 & 0.00 & 99.86 & & Bot $\mathrm{X} 1 \mathrm{~L}$ reb/low & FALSE \\
\hline 2005 & $\mathrm{~B}$ & 1 & 3 & 0.43 & 99.22 & BIS & on boulder & FALSE \\
\hline 2005 & B & 1 & 4 & 0.91 & 98.50 & BIS & & FALSE \\
\hline 2005 & B & 1 & 5 & 1.25 & 98.30 & BIS & & FALSE \\
\hline 2005 & $\mathrm{~B}$ & 1 & 6 & 1.65 & 98.16 & BIS & & FALSE \\
\hline 2005 & $\mathrm{~B}$ & 1 & 7 & 2.19 & 97.90 & $\mathrm{BF}$ & & FALSE \\
\hline 2005 & B & 1 & 8 & 2.83 & 97.64 & EW & & FALSE \\
\hline 2005 & B & 1 & 9 & 4.30 & 97.56 & TW & & FALSE \\
\hline 2005 & $\mathrm{~B}$ & 1 & 10 & 5.46 & 97.62 & BIS & in stream & FALSE \\
\hline 2005 & $\mathrm{~B}$ & 1 & 11 & 6.40 & 97.64 & EW & & FALSE \\
\hline 2005 & B & 1 & 12 & 7.07 & 97.81 & BIS & & FALSE \\
\hline 2005 & B & 1 & 13 & 7.59 & 98.13 & BIS & on boulder & FALSE \\
\hline 2005 & B & 1 & 14 & 8.05 & 98.46 & BIS & & FALSE \\
\hline 2005 & B & 1 & 15 & 8.66 & 98.77 & BIS & & FALSE \\
\hline 2005 & $\mathrm{~B}$ & 1 & 16 & 9.08 & 99.10 & BIS & & FALSE \\
\hline 2005 & $\mathrm{~B}$ & 1 & 17 & 9.69 & 99.50 & BIS & & FALSE \\
\hline 2005 & $\mathrm{~B}$ & 1 & 18 & 10.06 & 99.84 & BOT X1R & Bot $\mathrm{X} 1 \mathrm{R}$ reb/low & FALSE \\
\hline 2005 & B & 1 & 19 & 10.06 & 99.99 & TOP X1R & Top X1R reb/low & FALSE \\
\hline 2005 & B & 2 & 1 & 0.00 & 100.00 & & Top X2L reb/low & FALSE \\
\hline 2005 & B & 2 & 2 & 0.00 & 99.61 & & Bot $\mathrm{X} 2 \mathrm{~L} \mathrm{reb} /$ low & FALSE \\
\hline 2005 & B & 2 & 3 & 0.34 & 99.04 & BIS & & FALSE \\
\hline 2005 & B & 2 & 4 & 1.19 & 98.75 & BIS & & FALSE \\
\hline 2005 & $\mathrm{~B}$ & 2 & 5 & 1.74 & 98.42 & BIS & & FALSE \\
\hline 2005 & B & 2 & 6 & 2.13 & 98.04 & BIS & & FALSE \\
\hline 2005 & B & 2 & 7 & 2.59 & 97.72 & BIS & & FALSE \\
\hline 2005 & B & 2 & 8 & 2.93 & 97.51 & $\mathrm{BF}$ & & FALSE \\
\hline 2005 & B & 2 & 9 & 4.36 & 97.43 & EW & & FALSE \\
\hline 2005 & $\mathrm{~B}$ & 2 & 10 & 5.33 & 97.32 & TW & & FALSE \\
\hline 2005 & B & 2 & 11 & 6.68 & 97.43 & EW & & FALSE \\
\hline 2005 & B & 2 & 12 & 7.38 & 97.57 & BIS & & FALSE \\
\hline 2005 & B & 2 & 13 & 8.11 & 97.91 & BIS & & FALSE \\
\hline 2005 & B & 2 & 14 & 8.69 & 98.33 & BIS & Log Debris & FALSE \\
\hline 2005 & B & 2 & 15 & 9.11 & 98.62 & BIS & & FALSE \\
\hline 2005 & B & 2 & 16 & 9.63 & 99.10 & BIS & & FALSE \\
\hline 2005 & B & 2 & 17 & 10.09 & 99.32 & BIS & & FALSE \\
\hline 2005 & B & 2 & 18 & 10.52 & 99.52 & BOT X2R & Bot $\mathrm{X} 2 \mathrm{R}$ reb/low & FALSE \\
\hline 2005 & B & 2 & 19 & 10.52 & 100.00 & TOP X2R & Top X2R reb/low & FALSE \\
\hline 2005 & B & 3 & 1 & 0.00 & 100.00 & & Top X3L reb/low & FALSE \\
\hline 2005 & B & 3 & 2 & 0.00 & 99.45 & & Bot $\mathrm{X} 3 \mathrm{~L}$ reb/low & FALSE \\
\hline 2005 & B & 3 & 3 & 0.64 & 99.03 & BIS & & FALSE \\
\hline 2005 & B & 3 & 4 & 1.16 & 98.79 & BIS & & FALSE \\
\hline 2005 & B & 3 & 5 & 1.71 & 98.39 & BIS & on boulder & FALSE \\
\hline 2005 & B & 3 & 6 & 2.13 & 98.16 & $\mathrm{BF}$ & & FALSE \\
\hline 2005 & B & 3 & 7 & 2.59 & 98.16 & BIS & sand & FALSE \\
\hline 2005 & B & 3 & 8 & 4.27 & 98.05 & EW & & FALSE \\
\hline 2005 & B & 3 & 9 & 5.09 & 97.94 & $\mathrm{TW}$ & & FALSE \\
\hline 2005 & $\mathrm{~B}$ & 3 & 10 & 6.07 & 98.07 & EW & & FALSE \\
\hline 2005 & B & 3 & 11 & 6.77 & 98.48 & BIS & & FALSE \\
\hline 2005 & B & 3 & 12 & 7.13 & 98.66 & BIS & & FALSE \\
\hline
\end{tabular}

Continued on Next Page... 
Table D.1 - Continued

\begin{tabular}{|c|c|c|c|c|c|c|c|c|}
\hline Year & Reach & Section & Point & Dist. (m) & Elev. $(\mathrm{m})$ & Desc. & Notes & Exclude \\
\hline 2005 & $\mathrm{~B}$ & 3 & 13 & 7.47 & 98.86 & BIS & & FALSE \\
\hline 2005 & B & 3 & 14 & 7.89 & 99.07 & BIS & & FALSE \\
\hline 2005 & $\mathrm{~B}$ & 3 & 15 & 8.17 & 99.30 & BIS & & FALSE \\
\hline 2005 & $\mathrm{~B}$ & 3 & 16 & 8.44 & 99.82 & BOT X3R & Bot $\mathrm{X} 3 \mathrm{R}$ reb/low & FALSE \\
\hline 2005 & B & 3 & 17 & 8.44 & 99.98 & TOP X3R & Top X3R reb/low & FALSE \\
\hline 2005 & B & 4 & 1 & 0.00 & 100.00 & & Top X4L reb/low & FALSE \\
\hline 2005 & B & 4 & 2 & 0.00 & 99.85 & & Bot $\mathrm{X} 4 \mathrm{~L}$ reb/low & FALSE \\
\hline 2005 & $\mathrm{~B}$ & 4 & 3 & 0.49 & 99.70 & BIS & & FALSE \\
\hline 2005 & $\mathrm{~B}$ & 4 & 4 & 1.01 & 99.55 & BIS & & FALSE \\
\hline 2005 & B & 4 & 5 & 1.37 & 99.45 & ТОВ & & FALSE \\
\hline 2005 & B & 4 & 6 & 1.71 & 98.84 & $\mathrm{BF}$ & & FALSE \\
\hline 2005 & B & 4 & 7 & 2.23 & 98.64 & BIS & small side pool & FALSE \\
\hline 2005 & $\mathrm{~B}$ & 4 & 8 & 2.93 & 98.76 & BIS & & FALSE \\
\hline 2005 & $\mathrm{~B}$ & 4 & 9 & 3.75 & 98.85 & BIS & on top of boulders and *(below) & FALSE \\
\hline 2005 & $\mathrm{~B}$ & 4 & 10 & 4.27 & 98.63 & EW & & FALSE \\
\hline 2005 & $\mathrm{~B}$ & 4 & 11 & 5.40 & 98.68 & BIS & rock step & FALSE \\
\hline 2005 & $\mathrm{~B}$ & 4 & 12 & 5.94 & 98.62 & $\mathrm{TW}$ & rock step & FALSE \\
\hline 2005 & $\mathrm{~B}$ & 4 & 13 & 6.55 & 98.68 & EW & & FALSE \\
\hline 2005 & B & 4 & 14 & 6.80 & 98.84 & BIS & & FALSE \\
\hline 2005 & B & 4 & 15 & 7.01 & 99.10 & BIS & on boulder & FALSE \\
\hline 2005 & B & 4 & 16 & 7.38 & 99.51 & BOT X4R & Bot $\mathrm{X} 4 \mathrm{R}$ reb/low & FALSE \\
\hline 2005 & $\mathrm{~B}$ & 4 & 17 & 7.38 & 100.02 & TOP X4R & Top X4R reb/low & FALSE \\
\hline 2005 & $\mathrm{~B}$ & 5 & 1 & 0.00 & 100.00 & & Top X5L reb/low & FALSE \\
\hline 2005 & B & 5 & 2 & 0.00 & 99.68 & & Bot $\mathrm{X} 5 \mathrm{~L} \mathrm{reb} /$ low & FALSE \\
\hline 2005 & B & 5 & 3 & 0.43 & 99.19 & BIS & & FALSE \\
\hline 2005 & B & 5 & 4 & 0.73 & 98.99 & BIS & & FALSE \\
\hline 2005 & B & 5 & 5 & 1.31 & 98.63 & BIS & & FALSE \\
\hline 2005 & B & 5 & 6 & 1.95 & 98.31 & BIS & & FALSE \\
\hline 2005 & B & 5 & 7 & 2.87 & 98.06 & BIS & & FALSE \\
\hline 2005 & B & 5 & 8 & 3.26 & 97.96 & BIS & & FALSE \\
\hline 2005 & B & 5 & 9 & 4.30 & 97.77 & EW & & FALSE \\
\hline 2005 & B & 5 & 10 & 5.18 & 97.70 & TW & & FALSE \\
\hline 2005 & B & 5 & 11 & 6.19 & 97.85 & EW & on rock & FALSE \\
\hline 2005 & B & 5 & 12 & 6.86 & 97.94 & $\mathrm{BF}$ & & FALSE \\
\hline 2005 & B & 5 & 13 & 7.01 & 98.28 & BIS & & FALSE \\
\hline 2005 & B & 5 & 14 & 7.32 & 98.58 & BIS & & FALSE \\
\hline 2005 & B & 5 & 15 & 7.74 & 99.34 & BIS & & FALSE \\
\hline 2005 & B & 5 & 16 & 8.17 & 99.84 & BOT X5R & Bot $\mathrm{X} 5 \mathrm{R}$ reb/low & FALSE \\
\hline 2005 & B & 5 & 17 & 8.17 & 99.99 & TOP X5R & Top X5R reb/low & FALSE \\
\hline 2005 & B & 6 & 1 & 0.00 & 100.00 & & Top X6L reb/low & FALSE \\
\hline 2005 & B & 6 & 2 & 0.00 & 99.95 & & Bot X6L reb/low & FALSE \\
\hline 2005 & B & 6 & 3 & 0.55 & 99.49 & BIS & & FALSE \\
\hline 2005 & B & 6 & 4 & 1.25 & 99.26 & BIS & & FALSE \\
\hline 2005 & B & 6 & 5 & 1.74 & 99.03 & BIS & & FALSE \\
\hline 2005 & B & 6 & 6 & 2.29 & 98.74 & BIS & & FALSE \\
\hline 2005 & B & 6 & 7 & 3.26 & 98.36 & EW & & FALSE \\
\hline 2005 & B & 6 & 8 & 4.33 & 98.16 & TW & & FALSE \\
\hline 2005 & B & 6 & 9 & 5.24 & 98.34 & EW & & FALSE \\
\hline 2005 & B & 6 & 10 & 5.70 & 98.54 & BIS & & FALSE \\
\hline 2005 & B & 6 & 11 & 6.98 & 98.98 & BIS & & FALSE \\
\hline 2005 & B & 6 & 12 & 7.44 & 99.37 & BIS & & FALSE \\
\hline 2005 & B & 6 & 13 & 7.96 & 99.87 & BOT X6R & Bot $\mathrm{X} 6 \mathrm{R}$ reb/low & FALSE \\
\hline
\end{tabular}

Continued on Next Page... 
Table D.1 - Continued

\begin{tabular}{|c|c|c|c|c|c|c|c|c|}
\hline Year & Reach & Section & Point & Dist. (m) & Elev. $(\mathrm{m})$ & Desc. & Notes & Exclude \\
\hline 2005 & B & 6 & 14 & 7.96 & 100.00 & TOP X6R & Top X6R reb/low & FALSE \\
\hline 2005 & B & 7 & 1 & 0.00 & 100.00 & & Top X7L reb/low & FALSE \\
\hline 2005 & $\mathrm{~B}$ & 7 & 2 & 0.00 & 99.44 & & Bot $X 7 \mathrm{~L} \mathrm{reb} /$ low & FALSE \\
\hline 2005 & $\mathrm{~B}$ & 7 & 3 & 0.55 & 99.04 & BIS & & FALSE \\
\hline 2005 & B & 7 & 4 & 0.91 & 98.84 & BIS & & FALSE \\
\hline 2005 & B & 7 & 5 & 1.28 & 98.65 & BIS & & FALSE \\
\hline 2005 & $\mathrm{~B}$ & 7 & 6 & 1.80 & 98.36 & BIS & & FALSE \\
\hline 2005 & $\mathrm{~B}$ & 7 & 7 & 2.44 & 98.20 & BIS & & FALSE \\
\hline 2005 & $\mathrm{~B}$ & 7 & 8 & 3.14 & 98.03 & EW & & FALSE \\
\hline 2005 & B & 7 & 9 & 3.75 & 97.89 & $\mathrm{TW}$ & & FALSE \\
\hline 2005 & B & 7 & 10 & 5.18 & 98.02 & BIS & little sandbar instream & FALSE \\
\hline 2005 & B & 7 & 11 & 5.61 & 98.04 & EW & & FALSE \\
\hline 2005 & $\mathrm{~B}$ & 7 & 12 & 6.34 & 98.47 & $\mathrm{BF}$ & & FALSE \\
\hline 2005 & B & 7 & 13 & 6.80 & 98.77 & BIS & Bot $\mathrm{X} 7 \mathrm{R}$ reb/low & FALSE \\
\hline 2005 & B & 7 & 14 & 7.86 & 99.28 & ТОВ & Top X7R reb/low & FALSE \\
\hline 2005 & B & 8 & 1 & 0.00 & 100.00 & & Top X8L reb/low & FALSE \\
\hline 2005 & B & 8 & 2 & 0.00 & 99.60 & & Bot $\mathrm{X} 8 \mathrm{~L}$ reb/low & FALSE \\
\hline 2005 & $\mathrm{~B}$ & 8 & 3 & 0.43 & 99.34 & BIS & & FALSE \\
\hline 2005 & B & 8 & 4 & 0.61 & 98.87 & $\mathrm{BF}$ & & FALSE \\
\hline 2005 & B & 8 & 5 & 1.04 & 98.82 & EW & & FALSE \\
\hline 2005 & B & 8 & 6 & 1.92 & 98.70 & $\mathrm{TW}$ & & FALSE \\
\hline 2005 & B & 8 & 7 & 2.90 & 98.79 & BIS & & FALSE \\
\hline 2005 & $\mathrm{~B}$ & 8 & 8 & 3.69 & 99.59 & EW & on top of tree & FALSE \\
\hline 2005 & B & 8 & 9 & 3.90 & 99.49 & ТОВ & & FALSE \\
\hline 2005 & B & 8 & 10 & 5.30 & 99.65 & BOT X8R & Bot $\mathrm{X} 8 \mathrm{R}$ reb/low & FALSE \\
\hline 2005 & B & 8 & 11 & 5.30 & 99.85 & TOP X8R & Top X8R reb/low & FALSE \\
\hline 2005 & B & 9 & 1 & 0.00 & 100.00 & & Top X9L reb/low & FALSE \\
\hline 2005 & B & 9 & 2 & 0.00 & 100.02 & & Bot X9L reb/low & FALSE \\
\hline 2005 & B & 9 & 3 & 0.76 & 97.88 & EW & & FALSE \\
\hline 2005 & B & 9 & 4 & 2.35 & 97.72 & $\mathrm{TW}$ & & FALSE \\
\hline 2005 & $\mathrm{~B}$ & 9 & 5 & 3.47 & 97.82 & EW & & FALSE \\
\hline 2005 & B & 9 & 6 & 4.15 & 98.00 & $\mathrm{BF}$ & & FALSE \\
\hline 2005 & B & 9 & 7 & 4.75 & 98.39 & BIS & & FALSE \\
\hline 2005 & B & 9 & 8 & 5.58 & 98.57 & ТОВ & & FALSE \\
\hline 2005 & B & 9 & 9 & 7.77 & 98.99 & BIS & & FALSE \\
\hline 2005 & $\mathrm{~B}$ & 9 & 10 & 10.91 & 98.99 & BIS & & FALSE \\
\hline 2005 & B & 9 & 11 & 11.25 & 99.19 & BIS & & FALSE \\
\hline 2005 & B & 9 & 12 & 12.44 & 99.70 & BIS & & FALSE \\
\hline 2005 & B & 9 & 13 & 12.71 & 99.87 & BIS & & FALSE \\
\hline 2005 & B & 9 & 14 & 13.66 & 100.32 & BOT X9R & Bot $X 9 \mathrm{R}$ reb/low & FALSE \\
\hline 2005 & B & 9 & 15 & 13.66 & 100.36 & TOP X9R & Top X9R reb/low & FALSE \\
\hline 2005 & B & 10 & 1 & 0.00 & 100.00 & & Top X10L reb/low & FALSE \\
\hline 2005 & $\mathrm{~B}$ & 10 & 2 & 0.00 & 99.68 & & Bot X10L reb/low & FALSE \\
\hline 2005 & B & 10 & 3 & 0.64 & 97.74 & BIS & & FALSE \\
\hline 2005 & B & 10 & 4 & 1.07 & 97.66 & BIS & on top of rock & FALSE \\
\hline 2005 & B & 10 & 5 & 1.92 & 97.35 & EW & & FALSE \\
\hline 2005 & B & 10 & 6 & 2.80 & 97.25 & $\mathrm{TW}$ & & FALSE \\
\hline 2005 & B & 10 & 7 & 4.15 & 97.36 & EW & & FALSE \\
\hline 2005 & B & 10 & 8 & 4.88 & 97.55 & $\mathrm{BF}$ & & FALSE \\
\hline 2005 & $\mathrm{~B}$ & 10 & 9 & 5.30 & 97.71 & BIS & & FALSE \\
\hline 2005 & B & 10 & 10 & 5.76 & 98.08 & BIS & & FALSE \\
\hline 2005 & B & 10 & 11 & 6.28 & 98.24 & BIS & & FALSE \\
\hline
\end{tabular}

Continued on Next Page... 
Table D.1 - Continued

\begin{tabular}{|c|c|c|c|c|c|c|c|c|}
\hline Year & Reach & Section & Point & Dist. (m) & Elev. $(\mathrm{m})$ & Desc. & Notes & Exclude \\
\hline 2005 & B & 10 & 12 & 6.52 & 98.32 & ТОВ & & FALSE \\
\hline 2005 & B & 10 & 13 & 7.86 & 98.31 & BIS & & FALSE \\
\hline 2005 & $\mathrm{~B}$ & 10 & 14 & 8.75 & 98.28 & BIS & & FALSE \\
\hline 2005 & B & 10 & 15 & 9.88 & 98.24 & BIS & & FALSE \\
\hline 2005 & B & 10 & 16 & 10.76 & 98.19 & BIS & & FALSE \\
\hline 2005 & B & 10 & 17 & 11.37 & 98.24 & BIS & & FALSE \\
\hline 2005 & $\mathrm{~B}$ & 10 & 18 & 12.04 & 98.23 & BIS & & FALSE \\
\hline 2005 & B & 10 & 19 & 13.20 & 98.53 & BIS & & FALSE \\
\hline 2005 & $\mathrm{~B}$ & 10 & 20 & 14.11 & 99.02 & BIS & & FALSE \\
\hline 2005 & B & 10 & 21 & 14.51 & 99.27 & BIS & & FALSE \\
\hline 2005 & B & 10 & 22 & 14.78 & 99.46 & $?$ & & FALSE \\
\hline 2005 & B & 10 & 23 & 15.33 & 99.87 & BOT X10R & Bot $\mathrm{X} 10 \mathrm{R} \mathrm{reb} / \mathrm{low}$ & FALSE \\
\hline 2005 & B & 10 & 24 & 15.33 & 100.00 & TOP X10R & Top X10R reb/low & FALSE \\
\hline 2005 & $\mathrm{C}$ & 1 & 1 & 0.00 & 100.00 & & Top X1L reb/low & FALSE \\
\hline 2005 & $\mathrm{C}$ & 1 & 2 & 0.00 & 99.79 & & Bot X1L reb/low & FALSE \\
\hline 2005 & $\mathrm{C}$ & 1 & 3 & 0.30 & 99.19 & BIS & & FALSE \\
\hline 2005 & $\mathrm{C}$ & 1 & 4 & 0.52 & 99.07 & BIS & & FALSE \\
\hline 2005 & $\mathrm{C}$ & 1 & 5 & 1.25 & 98.74 & BIS & & FALSE \\
\hline 2005 & $\mathrm{C}$ & 1 & 6 & 1.98 & 98.52 & EW & & FALSE \\
\hline 2005 & $\mathrm{C}$ & 1 & 7 & 2.68 & 98.46 & TW & & FALSE \\
\hline 2005 & $\mathrm{C}$ & 1 & 8 & 3.84 & 98.56 & BIS & In stream (RI) & FALSE \\
\hline 2005 & $\mathrm{C}$ & 1 & 9 & 5.18 & 98.67 & EW & & FALSE \\
\hline 2005 & $\mathrm{C}$ & 1 & 10 & 5.91 & 98.77 & $\mathrm{BF}$ & & FALSE \\
\hline 2005 & $\mathrm{C}$ & 1 & 11 & 6.64 & 98.89 & BIS & & FALSE \\
\hline 2005 & $\mathrm{C}$ & 1 & 12 & 7.74 & 99.06 & BIS & & FALSE \\
\hline 2005 & $\mathrm{C}$ & 1 & 13 & 8.29 & 99.23 & BIS & & FALSE \\
\hline 2005 & $\mathrm{C}$ & 1 & 14 & 10.09 & 99.12 & BIS & & FALSE \\
\hline 2005 & $\mathrm{C}$ & 1 & 15 & 10.97 & 99.26 & BIS & & FALSE \\
\hline 2005 & $\mathrm{C}$ & 1 & 16 & 11.43 & 99.43 & BIS & & FALSE \\
\hline 2005 & $\mathrm{C}$ & 1 & 17 & 11.95 & 99.60 & BIS & & FALSE \\
\hline 2005 & $\mathrm{C}$ & 1 & 18 & 12.19 & 99.75 & BIS & & FALSE \\
\hline 2005 & $\mathrm{C}$ & 1 & 19 & 12.38 & 99.87 & BOT X1R & Bot $\mathrm{X} 1 \mathrm{R}$ reb/low & FALSE \\
\hline 2005 & $\mathrm{C}$ & 1 & 20 & 12.34 & 100.23 & TOP X1R & Top X1R reb/low & FALSE \\
\hline 2005 & $\mathrm{C}$ & 2 & 1 & 0.00 & 100.00 & & Top X2L reb/low & FALSE \\
\hline 2005 & $\mathrm{C}$ & 2 & 2 & 0.00 & 99.69 & & Bot $\mathrm{X} 2 \mathrm{~L} \mathrm{reb} /$ low & FALSE \\
\hline 2005 & $\mathrm{C}$ & 2 & 3 & 0.30 & 99.29 & BIS & & FALSE \\
\hline 2005 & $\mathrm{C}$ & 2 & 4 & 0.58 & 99.11 & BIS & & FALSE \\
\hline 2005 & $\mathrm{C}$ & 2 & 5 & 0.94 & 98.92 & BIS & & FALSE \\
\hline 2005 & $\mathrm{C}$ & 2 & 6 & 1.34 & 98.88 & BIS & & FALSE \\
\hline 2005 & $\mathrm{C}$ & 2 & 7 & 1.92 & 98.84 & BIS & & FALSE \\
\hline 2005 & $\mathrm{C}$ & 2 & 8 & 2.50 & 98.72 & BIS & & FALSE \\
\hline 2005 & $\mathrm{C}$ & 2 & 9 & 2.96 & 98.63 & BIS & & FALSE \\
\hline 2005 & $\mathrm{C}$ & 2 & 10 & 3.47 & 98.52 & BIS & & FALSE \\
\hline 2005 & $\mathrm{C}$ & 2 & 11 & 3.96 & 98.42 & BIS & & FALSE \\
\hline 2005 & $\mathrm{C}$ & 2 & 12 & 5.00 & 98.07 & BIS & & FALSE \\
\hline 2005 & $\mathrm{C}$ & 2 & 13 & 5.61 & 98.02 & BIS & & FALSE \\
\hline 2005 & $\mathrm{C}$ & 2 & 14 & 6.07 & 97.89 & BIS & & FALSE \\
\hline 2005 & $\mathrm{C}$ & 2 & 15 & 6.52 & 97.83 & EW & & FALSE \\
\hline 2005 & $\mathrm{C}$ & 2 & 16 & 7.01 & 97.70 & $\mathrm{TW}$ & & FALSE \\
\hline 2005 & $\mathrm{C}$ & 2 & 17 & 7.86 & 97.79 & BIS & in stream & FALSE \\
\hline 2005 & $\mathrm{C}$ & 2 & 18 & 9.02 & 97.85 & EW & & FALSE \\
\hline 2005 & C & 2 & 19 & 9.66 & 98.02 & $\mathrm{BF}$ & & FALSE \\
\hline
\end{tabular}

Continued on Next Page... 
Table D.1 - Continued

\begin{tabular}{|c|c|c|c|c|c|c|c|c|}
\hline Year & Reach & Section & Point & Dist. $(\mathrm{m})$ & Elev. $(\mathrm{m})$ & Desc. & Notes & Exclude \\
\hline 2005 & $\mathrm{C}$ & 2 & 20 & 10.42 & 98.27 & BIS & & FALSE \\
\hline 2005 & C & 2 & 21 & 11.06 & 98.28 & BIS & & FALSE \\
\hline 2005 & $\mathrm{C}$ & 2 & 22 & 11.67 & 98.26 & BIS & & FALSE \\
\hline 2005 & $\mathrm{C}$ & 2 & 23 & 12.22 & 98.32 & BIS & & FALSE \\
\hline 2005 & $\mathrm{C}$ & 2 & 24 & 12.41 & 99.61 & BOT X2R & Bot $\mathrm{X} 2 \mathrm{R}$ reb/low & FALSE \\
\hline 2005 & $\mathrm{C}$ & 2 & 25 & 12.25 & 100.01 & TOP X2R & Top X2R reb/low & FALSE \\
\hline 2005 & $\mathrm{C}$ & 3 & 1 & 0.00 & 100.00 & & Top X3L reb/low & FALSE \\
\hline 2005 & $\mathrm{C}$ & 3 & 2 & 0.00 & 99.66 & & Bot $\mathrm{X} 3 \mathrm{~L} \mathrm{reb} /$ low & FALSE \\
\hline 2005 & $\mathrm{C}$ & 3 & 3 & 0.34 & 99.43 & BIS & & FALSE \\
\hline 2005 & $\mathrm{C}$ & 3 & 4 & 0.79 & 99.19 & BIS & & FALSE \\
\hline 2005 & $\mathrm{C}$ & 3 & 5 & 1.28 & 99.02 & BIS & & FALSE \\
\hline 2005 & $\mathrm{C}$ & 3 & 6 & 1.74 & 98.86 & BIS & & FALSE \\
\hline 2005 & $\mathrm{C}$ & 3 & 7 & 2.07 & 98.76 & BIS & & FALSE \\
\hline 2005 & C & 3 & 8 & 2.80 & 98.69 & BIS & & FALSE \\
\hline 2005 & $\mathrm{C}$ & 3 & 9 & 3.57 & 98.75 & BIS & & FALSE \\
\hline 2005 & $\mathrm{C}$ & 3 & 10 & 4.33 & 98.66 & BIS & & FALSE \\
\hline 2005 & $\mathrm{C}$ & 3 & 11 & 4.63 & 98.52 & BIS & & FALSE \\
\hline 2005 & $\mathrm{C}$ & 3 & 12 & 5.67 & 98.42 & BIS & & FALSE \\
\hline 2005 & $\mathrm{C}$ & 3 & 13 & 6.34 & 98.36 & BIS & & FALSE \\
\hline 2005 & $\mathrm{C}$ & 3 & 14 & 6.98 & 98.25 & EW & & FALSE \\
\hline 2005 & $\mathrm{C}$ & 3 & 15 & 8.26 & 98.15 & TW & & FALSE \\
\hline 2005 & $\mathrm{C}$ & 3 & 16 & 9.39 & 98.23 & EW & & FALSE \\
\hline 2005 & $\mathrm{C}$ & 3 & 17 & 10.27 & 98.52 & $\mathrm{BF}$ & & FALSE \\
\hline 2005 & $\mathrm{C}$ & 3 & 18 & 10.97 & 98.81 & BIS & & FALSE \\
\hline 2005 & $\mathrm{C}$ & 3 & 19 & 11.61 & 98.88 & BIS & & FALSE \\
\hline 2005 & $\mathrm{C}$ & 3 & 20 & 12.47 & 99.11 & BIS & & FALSE \\
\hline 2005 & $\mathrm{C}$ & 3 & 21 & 13.23 & 99.39 & BIS & & FALSE \\
\hline 2005 & $\mathrm{C}$ & 3 & 22 & 13.66 & 99.58 & BIS & & FALSE \\
\hline 2005 & $\mathrm{C}$ & 3 & 23 & 13.99 & 99.75 & TOP X3R & Top X3R reb/low & FALSE \\
\hline 2005 & $\mathrm{C}$ & 3 & 24 & 13.99 & 100.00 & BOT X3R & Bot X3R reb/low & FALSE \\
\hline 2005 & $\mathrm{C}$ & 4 & 1 & 0.00 & 100.00 & & Top X4L reb/low & FALSE \\
\hline 2005 & $\mathrm{C}$ & 4 & 2 & 0.00 & 99.69 & & Bot $\mathrm{X} 4 \mathrm{~L} \mathrm{reb} /$ low & FALSE \\
\hline 2005 & $\mathrm{C}$ & 4 & 3 & 0.49 & 99.50 & BIS & & FALSE \\
\hline 2005 & $\mathrm{C}$ & 4 & 4 & 1.28 & 98.77 & BIS & & FALSE \\
\hline 2005 & $\mathrm{C}$ & 4 & 5 & 2.29 & 98.45 & BIS & & FALSE \\
\hline 2005 & $\mathrm{C}$ & 4 & 6 & 3.57 & 98.51 & BIS & & FALSE \\
\hline 2005 & $\mathrm{C}$ & 4 & 7 & 4.33 & 98.54 & $\mathrm{BF}$ & & FALSE \\
\hline 2005 & $\mathrm{C}$ & 4 & 8 & 4.63 & 98.43 & BIS & & FALSE \\
\hline 2005 & $\mathrm{C}$ & 4 & 9 & 6.40 & 98.29 & BIS & & FALSE \\
\hline 2005 & $\mathrm{C}$ & 4 & 10 & 7.22 & 98.14 & EW & & FALSE \\
\hline 2005 & $\mathrm{C}$ & 4 & 11 & 8.05 & 98.02 & TW & & FALSE \\
\hline 2005 & $\mathrm{C}$ & 4 & 12 & 8.84 & 98.10 & EW & & FALSE \\
\hline 2005 & $\mathrm{C}$ & 4 & 13 & 9.42 & 98.24 & BIS & & FALSE \\
\hline 2005 & $\mathrm{C}$ & 4 & 14 & 9.94 & 98.55 & BIS & & FALSE \\
\hline 2005 & $\mathrm{C}$ & 4 & 15 & 10.39 & 98.87 & ТОВ & & FALSE \\
\hline 2005 & $\mathrm{C}$ & 4 & 16 & 11.25 & 98.94 & BIS & & FALSE \\
\hline 2005 & $\mathrm{C}$ & 4 & 17 & 11.89 & 99.69 & BOT X4R & Bot $\mathrm{X} 4 \mathrm{R}$ reb/low & FALSE \\
\hline 2005 & $\mathrm{C}$ & 4 & 18 & 11.89 & 99.99 & TOP X4R & Top X4R reb/low & FALSE \\
\hline 2005 & $\mathrm{C}$ & 5 & 1 & 0.00 & 100.00 & & Top X5L reb/low & FALSE \\
\hline 2005 & $\mathrm{C}$ & 5 & 2 & 0.00 & 99.69 & & Bot $\mathrm{X} 5 \mathrm{~L} \mathrm{reb} /$ low & FALSE \\
\hline 2005 & $\mathrm{C}$ & 5 & 3 & 0.46 & 99.29 & BIS & & FALSE \\
\hline 2005 & $\mathrm{C}$ & 5 & 4 & 1.46 & 99.06 & BIS & & FALSE \\
\hline
\end{tabular}

Continued on Next Page... 
Table D.1 - Continued

\begin{tabular}{|c|c|c|c|c|c|c|c|c|}
\hline Year & Reach & Section & Point & Dist. (m) & Elev. $(\mathrm{m})$ & Desc. & Notes & Exclude \\
\hline 2005 & $\mathrm{C}$ & 5 & 5 & 2.19 & 98.82 & BIS & & FALSE \\
\hline 2005 & $\mathrm{C}$ & 5 & 6 & 3.44 & 98.68 & BIS & & FALSE \\
\hline 2005 & $\mathrm{C}$ & 5 & 7 & 4.82 & 98.56 & BIS & & FALSE \\
\hline 2005 & $\mathrm{C}$ & 5 & 8 & 5.94 & 98.53 & $\mathrm{BF}$ & & FALSE \\
\hline 2005 & $\mathrm{C}$ & 5 & 9 & 6.49 & 98.40 & BIS & & FALSE \\
\hline 2005 & $\mathrm{C}$ & 5 & 10 & 6.86 & 98.24 & EW & & FALSE \\
\hline 2005 & $\mathrm{C}$ & 5 & 11 & 7.71 & 98.06 & TW & & FALSE \\
\hline 2005 & $\mathrm{C}$ & 5 & 12 & 8.08 & 98.06 & BIS & in stream & FALSE \\
\hline 2005 & $\mathrm{C}$ & 5 & 13 & 8.66 & 98.26 & EW & & FALSE \\
\hline 2005 & $\mathrm{C}$ & 5 & 14 & 9.33 & 98.38 & BIS & & FALSE \\
\hline 2005 & $\mathrm{C}$ & 5 & 15 & 10.00 & 98.84 & ТОВ & & FALSE \\
\hline 2005 & $\mathrm{C}$ & 5 & 16 & 11.16 & 98.89 & BIS & & FALSE \\
\hline 2005 & $\mathrm{C}$ & 5 & 17 & 11.86 & 99.25 & BIS & & FALSE \\
\hline 2005 & $\mathrm{C}$ & 5 & 18 & 12.19 & 99.58 & BOT X5R & Bot $\mathrm{X} 5 \mathrm{R}$ reb/low & FALSE \\
\hline 2005 & $\mathrm{C}$ & 5 & 19 & 12.50 & 100.10 & TOP X5R & Top X5R reb/low & FALSE \\
\hline 2005 & $\mathrm{C}$ & 6 & 1 & 0.00 & 100.00 & & Top X6L reb/low & FALSE \\
\hline 2005 & $\mathrm{C}$ & 6 & 2 & 0.00 & 99.70 & & Bot $\mathrm{X} 6 \mathrm{~L}$ reb/low & FALSE \\
\hline 2005 & $\mathrm{C}$ & 6 & 3 & 0.37 & 99.31 & BIS & & FALSE \\
\hline 2005 & $\mathrm{C}$ & 6 & 4 & 0.94 & 98.94 & BIS & & FALSE \\
\hline 2005 & $\mathrm{C}$ & 6 & 5 & 1.89 & 98.86 & BIS & & FALSE \\
\hline 2005 & $\mathrm{C}$ & 6 & 6 & 2.23 & 98.75 & BIS & & FALSE \\
\hline 2005 & $\mathrm{C}$ & 6 & 7 & 2.74 & 98.44 & BIS & & FALSE \\
\hline 2005 & $\mathrm{C}$ & 6 & 8 & 3.17 & 98.33 & $\mathrm{BF}$ & & FALSE \\
\hline 2005 & $\mathrm{C}$ & 6 & 9 & 3.96 & 98.13 & BIS & & FALSE \\
\hline 2005 & $\mathrm{C}$ & 6 & 10 & 4.42 & 98.06 & EW & & FALSE \\
\hline 2005 & $\mathrm{C}$ & 6 & 11 & 5.12 & 98.02 & SB & & FALSE \\
\hline 2005 & $\mathrm{C}$ & 6 & 12 & 5.58 & 98.24 & $\mathrm{SB}$ & on top of boulder & TRUE \\
\hline 2005 & $\mathrm{C}$ & 6 & 13 & 5.82 & 98.00 & BIS & in stream & FALSE \\
\hline 2005 & $\mathrm{C}$ & 6 & 14 & 7.04 & 97.95 & $\mathrm{TW}$ & & FALSE \\
\hline 2005 & $\mathrm{C}$ & 6 & 15 & 8.78 & 98.05 & EW & & FALSE \\
\hline 2005 & $\mathrm{C}$ & 6 & 16 & 9.51 & 98.72 & ТОВ & & FALSE \\
\hline 2005 & $\mathrm{C}$ & 6 & 17 & 10.03 & 98.75 & BIS & & FALSE \\
\hline 2005 & $\mathrm{C}$ & 6 & 18 & 10.15 & 98.95 & BIS & on top of boulder & FALSE \\
\hline 2005 & $\mathrm{C}$ & 6 & 19 & 10.70 & 99.08 & BIS & on top of boulder & FALSE \\
\hline 2005 & $\mathrm{C}$ & 6 & 20 & 11.31 & 99.34 & BIS & on top of boulder & FALSE \\
\hline 2005 & $\mathrm{C}$ & 6 & 21 & 11.49 & 99.12 & BIS & on top of boulder & FALSE \\
\hline 2005 & $\mathrm{C}$ & 6 & 22 & 11.89 & 99.09 & BIS & on top of boulder & FALSE \\
\hline 2005 & $\mathrm{C}$ & 6 & 23 & 12.22 & 99.12 & BIS & & FALSE \\
\hline 2005 & $\mathrm{C}$ & 6 & 24 & 12.65 & 99.35 & BIS & & FALSE \\
\hline 2005 & $\mathrm{C}$ & 6 & 25 & 12.98 & 99.69 & BOT X6R & Bot $\mathrm{X} 6 \mathrm{R} \mathrm{reb} /$ low & FALSE \\
\hline 2005 & $\mathrm{C}$ & 6 & 26 & 12.98 & 100.00 & TOP X6R & Top X6R reb/low & FALSE \\
\hline 2005 & $\mathrm{C}$ & 7 & 1 & 0.00 & 100.00 & & Top X7L reb/low & FALSE \\
\hline 2005 & $\mathrm{C}$ & 7 & 2 & 0.00 & 99.80 & & Bot $\mathrm{X} 7 \mathrm{~L} \mathrm{reb} /$ low & FALSE \\
\hline 2005 & $\mathrm{C}$ & 7 & 3 & 0.61 & 99.08 & BIS & & FALSE \\
\hline 2005 & $\mathrm{C}$ & 7 & 4 & 1.25 & 98.94 & BIS & & FALSE \\
\hline 2005 & $\mathrm{C}$ & 7 & 5 & 2.38 & 98.85 & ТОВ & & FALSE \\
\hline 2005 & $\mathrm{C}$ & 7 & 6 & 2.80 & 98.39 & BIS & & FALSE \\
\hline 2005 & $\mathrm{C}$ & 7 & 7 & 3.26 & 98.30 & $\mathrm{BF}$ & & FALSE \\
\hline 2005 & $\mathrm{C}$ & 7 & 8 & 4.02 & 98.16 & EW & & FALSE \\
\hline 2005 & $\mathrm{C}$ & 7 & 9 & 4.33 & 98.04 & $\mathrm{TW}$ & & FALSE \\
\hline 2005 & $\mathrm{C}$ & 7 & 10 & 5.18 & 98.13 & SB & & FALSE \\
\hline 2005 & $\mathrm{C}$ & 7 & 11 & 5.70 & 98.19 & SB & side pool/away from main channel & FALSE \\
\hline
\end{tabular}

Continued on Next Page... 
Table D.1 - Continued

\begin{tabular}{|c|c|c|c|c|c|c|c|c|}
\hline Year & Reach & Section & Point & Dist. $(\mathrm{m})$ & Elev. $(\mathrm{m})$ & Desc. & Notes & Exclude \\
\hline 2005 & $\mathrm{C}$ & 7 & 12 & 6.10 & 98.19 & EW & & FALSE \\
\hline 2005 & C & 7 & 13 & 6.74 & 98.34 & BIS & & FALSE \\
\hline 2005 & $\mathrm{C}$ & 7 & 14 & 7.28 & 98.70 & BIS & & FALSE \\
\hline 2005 & $\mathrm{C}$ & 7 & 15 & 7.68 & 99.16 & ТОВ & & FALSE \\
\hline 2005 & $\mathrm{C}$ & 7 & 16 & 8.29 & 99.17 & BIS & & FALSE \\
\hline 2005 & $\mathrm{C}$ & 7 & 17 & 8.60 & 99.00 & BIS & & FALSE \\
\hline 2005 & $\mathrm{C}$ & 7 & 18 & 9.57 & 98.81 & BIS & & FALSE \\
\hline 2005 & $\mathrm{C}$ & 7 & 19 & 9.97 & 99.09 & BIS & on top of $\log$ & TRUE \\
\hline 2005 & $\mathrm{C}$ & 7 & 20 & 10.52 & 99.29 & BIS & on top of $\log$ & TRUE \\
\hline 2005 & $\mathrm{C}$ & 7 & 21 & 10.70 & 98.79 & BIS & & FALSE \\
\hline 2005 & $\mathrm{C}$ & 7 & 22 & 11.31 & 98.81 & BIS & & FALSE \\
\hline 2005 & $\mathrm{C}$ & 7 & 23 & 12.16 & 99.09 & BIS & & FALSE \\
\hline 2005 & $\mathrm{C}$ & 7 & 24 & 12.68 & 99.30 & BIS & & FALSE \\
\hline 2005 & $\mathrm{C}$ & 7 & 25 & 13.29 & 99.88 & BOT X7R & Bot $\mathrm{X} 7 \mathrm{R}$ reb/low & FALSE \\
\hline 2005 & $\mathrm{C}$ & 7 & 26 & 13.29 & 100.05 & TOP X7R & Top X7R reb/low & FALSE \\
\hline 2005 & $\mathrm{D}$ & 1 & 1 & 0.00 & 100.00 & & Top X1L reb/upper & FALSE \\
\hline 2005 & $\mathrm{D}$ & 1 & 2 & 0.00 & 99.67 & & Bot X1L reb/upper & FALSE \\
\hline 2005 & $\mathrm{D}$ & 1 & 3 & 1.74 & 98.79 & BIS & & FALSE \\
\hline 2005 & $\mathrm{D}$ & 1 & 4 & 2.93 & 98.20 & BIS & & FALSE \\
\hline 2005 & $\mathrm{D}$ & 1 & 5 & 3.23 & 97.64 & BIS & & FALSE \\
\hline 2005 & $\mathrm{D}$ & 1 & 6 & 5.79 & 98.00 & BIS & & FALSE \\
\hline 2005 & $\mathrm{D}$ & 1 & 7 & 6.19 & 98.62 & BIS & & FALSE \\
\hline 2005 & $\mathrm{D}$ & 1 & 8 & 6.83 & 98.65 & BOT X1L & Bot $\mathrm{X} 1 \mathrm{~L}$ reb/low & FALSE \\
\hline 2005 & $\mathrm{D}$ & 1 & 9 & 6.83 & 98.84 & TOP X1L & Top X1L reb/low & FALSE \\
\hline 2005 & $\mathrm{D}$ & 1 & 10 & 7.32 & 98.59 & ТОВ & & FALSE \\
\hline 2005 & $\mathrm{D}$ & 1 & 11 & 7.83 & 97.46 & BIS & & FALSE \\
\hline 2005 & $\mathrm{D}$ & 1 & 12 & 9.02 & 96.77 & EW & & FALSE \\
\hline 2005 & $\mathrm{D}$ & 1 & 13 & 10.36 & 96.67 & TW & & FALSE \\
\hline 2005 & $\mathrm{D}$ & 1 & 14 & 11.13 & 96.69 & EW & & FALSE \\
\hline 2005 & $\mathrm{D}$ & 1 & 15 & 11.37 & 97.03 & BIS & on top of boulder & FALSE \\
\hline 2005 & $\mathrm{D}$ & 1 & 16 & 12.07 & 97.15 & $\mathrm{BF}$ & & FALSE \\
\hline 2005 & $\mathrm{D}$ & 1 & 17 & 12.95 & 97.25 & BIS & & FALSE \\
\hline 2005 & $\mathrm{D}$ & 1 & 18 & 13.56 & 97.63 & BIS & & FALSE \\
\hline 2005 & $\mathrm{D}$ & 1 & 19 & 14.05 & 97.91 & BIS & & FALSE \\
\hline 2005 & $\mathrm{D}$ & 1 & 20 & 14.78 & 98.09 & BIS & & FALSE \\
\hline 2005 & $\mathrm{D}$ & 1 & 21 & 15.12 & 98.43 & BIS & & FALSE \\
\hline 2005 & $\mathrm{D}$ & 1 & 22 & 16.52 & 98.61 & BIS & & FALSE \\
\hline 2005 & $\mathrm{D}$ & 1 & 23 & 16.98 & 98.87 & BIS & & FALSE \\
\hline 2005 & $\mathrm{D}$ & 1 & 24 & 17.98 & 100.08 & BOT X1R & Bot X1R reb/upper & FALSE \\
\hline 2005 & $\mathrm{D}$ & 1 & 25 & 17.98 & 100.44 & TOP X1R & Top X1R reb/upper & FALSE \\
\hline 2005 & $\mathrm{D}$ & 2 & 1 & 0.00 & 100.00 & & Top X2L reb/low & FALSE \\
\hline 2005 & $\mathrm{D}$ & 2 & 2 & 0.00 & 99.69 & & Bot $\mathrm{X} 2 \mathrm{~L} \mathrm{reb} /$ low & FALSE \\
\hline 2005 & $\mathrm{D}$ & 2 & 3 & 0.61 & 99.69 & BIS & & FALSE \\
\hline 2005 & $\mathrm{D}$ & 2 & 4 & 1.13 & 99.10 & BIS & & FALSE \\
\hline 2005 & $\mathrm{D}$ & 2 & 5 & 2.68 & 98.51 & EW & & FALSE \\
\hline 2005 & $\mathrm{D}$ & 2 & 6 & 3.57 & 98.43 & $\mathrm{TW}$ & & FALSE \\
\hline 2005 & $\mathrm{D}$ & 2 & 7 & 4.54 & 98.49 & EW & & FALSE \\
\hline 2005 & $\mathrm{D}$ & 2 & 8 & 5.03 & 98.66 & BIS & & FALSE \\
\hline 2005 & $\mathrm{D}$ & 2 & 9 & 5.49 & 98.74 & $\mathrm{BF}$ & & FALSE \\
\hline 2005 & $\mathrm{D}$ & 2 & 10 & 6.34 & 98.97 & BIS & & FALSE \\
\hline 2005 & $\mathrm{D}$ & 2 & 11 & 7.10 & 99.37 & BIS & & FALSE \\
\hline 2005 & $\mathrm{D}$ & 2 & 12 & 7.71 & 99.66 & ТOB & & FALSE \\
\hline
\end{tabular}

Continued on Next Page... 
Table D.1 - Continued

\begin{tabular}{|c|c|c|c|c|c|c|c|c|}
\hline Year & Reach & Section & Point & Dist. (m) & Elev. $(\mathrm{m})$ & Desc. & Notes & Exclude \\
\hline 2005 & $\mathrm{D}$ & 2 & 13 & 8.90 & 99.73 & BIS & & FALSE \\
\hline 2005 & $\mathrm{D}$ & 2 & 14 & 9.36 & 99.80 & BOT X2R & Bot $\mathrm{X} 2 \mathrm{R}$ reb/low & FALSE \\
\hline 2005 & $\mathrm{D}$ & 2 & 15 & 9.36 & 100.00 & TOP X2R & Top X2R reb/low & FALSE \\
\hline 2005 & $\mathrm{D}$ & 3 & 1 & 0.00 & 100.00 & & Top X3L reb/low & FALSE \\
\hline 2005 & $\mathrm{D}$ & 3 & 2 & 0.00 & 99.80 & & Bot $\mathrm{X} 3 \mathrm{~L}$ reb/low & FALSE \\
\hline 2005 & $\mathrm{D}$ & 3 & 3 & 1.10 & 99.72 & BIS & & FALSE \\
\hline 2005 & $\mathrm{D}$ & 3 & 4 & 1.34 & 98.86 & BIS & & FALSE \\
\hline 2005 & $\mathrm{D}$ & 3 & 5 & 3.38 & 98.53 & EW & & FALSE \\
\hline 2005 & $\mathrm{D}$ & 3 & 6 & 4.11 & 98.32 & $\mathrm{TW}$ & & FALSE \\
\hline 2005 & $\mathrm{D}$ & 3 & 7 & 4.60 & 98.54 & EW & & FALSE \\
\hline 2005 & $\mathrm{D}$ & 3 & 8 & 4.88 & 98.96 & $\mathrm{BF}$ & & FALSE \\
\hline 2005 & $\mathrm{D}$ & 3 & 9 & 5.73 & 99.63 & BIS & & FALSE \\
\hline 2005 & $\mathrm{D}$ & 3 & 10 & 6.71 & 99.85 & ТОВ & & FALSE \\
\hline 2005 & $\mathrm{D}$ & 3 & 11 & 7.25 & 99.20 & BIS & & FALSE \\
\hline 2005 & $\mathrm{D}$ & 3 & 12 & 8.60 & 99.26 & BIS & & FALSE \\
\hline 2005 & $\mathrm{D}$ & 3 & 13 & 10.03 & 99.36 & BIS & & FALSE \\
\hline 2005 & $\mathrm{D}$ & 3 & 14 & 10.55 & 99.49 & BIS & & FALSE \\
\hline 2005 & $\mathrm{D}$ & 3 & 15 & 11.00 & 99.92 & BOT X3R & Bot $\mathrm{X} 3 \mathrm{R}$ reb/low & FALSE \\
\hline 2005 & $\mathrm{D}$ & 3 & 16 & 11.00 & 100.00 & TOP X3R & Top X3R reb/low & FALSE \\
\hline 2005 & $\mathrm{D}$ & 4 & 1 & 0.00 & 100.00 & & Top X4L reb/upper (on stump) & FALSE \\
\hline 2005 & $\mathrm{D}$ & 4 & 2 & 0.00 & 99.18 & & Bot $\mathrm{X} 4 \mathrm{~L}$ reb/upper (on stump) & FALSE \\
\hline 2005 & $\mathrm{D}$ & 4 & 3 & 0.30 & 99.16 & BIS & on top of stump & FALSE \\
\hline 2005 & $\mathrm{D}$ & 4 & 4 & 0.55 & 98.10 & BIS & & FALSE \\
\hline 2005 & $\mathrm{D}$ & 4 & 5 & 1.01 & 97.62 & BIS & & FALSE \\
\hline 2005 & $\mathrm{D}$ & 4 & 6 & 1.25 & 97.28 & BIS & & FALSE \\
\hline 2005 & $\mathrm{D}$ & 4 & 7 & 1.65 & 96.38 & EW & & FALSE \\
\hline 2005 & $\mathrm{D}$ & 4 & 8 & 2.65 & 96.34 & $\mathrm{TW}$ & & FALSE \\
\hline 2005 & $\mathrm{D}$ & 4 & 9 & 3.20 & 96.40 & EW & & FALSE \\
\hline 2005 & $\mathrm{D}$ & 4 & 10 & 4.05 & 96.73 & $\mathrm{BF}$ & & FALSE \\
\hline 2005 & $\mathrm{D}$ & 4 & 11 & 5.21 & 96.93 & BIS & & FALSE \\
\hline 2005 & $\mathrm{D}$ & 4 & 12 & 6.25 & 97.24 & BIS & & FALSE \\
\hline 2005 & $\mathrm{D}$ & 4 & 13 & 7.59 & 97.19 & BIS & & FALSE \\
\hline 2005 & $\mathrm{D}$ & 4 & 14 & 8.44 & 97.52 & BIS & & FALSE \\
\hline 2005 & $\mathrm{D}$ & 4 & 15 & 10.03 & 98.08 & ТОВ & & FALSE \\
\hline 2005 & $\mathrm{D}$ & 4 & 16 & 11.70 & 98.24 & BIS & & FALSE \\
\hline 2005 & $\mathrm{D}$ & 4 & 17 & 12.98 & 99.56 & BIS & & FALSE \\
\hline 2005 & $\mathrm{D}$ & 4 & 18 & 13.96 & 100.44 & BOT X4R & Bot $\mathrm{X} 4 \mathrm{R}$ reb/upper & FALSE \\
\hline 2005 & $\mathrm{D}$ & 4 & 19 & 13.96 & 100.88 & TOP X4R & Top X4R reb/upper & FALSE \\
\hline 2005 & $\mathrm{D}$ & 5 & 1 & 0.00 & 100.00 & & Top X5L reb/low & FALSE \\
\hline 2005 & $\mathrm{D}$ & 5 & 2 & 0.00 & 99.61 & & Bot $\mathrm{X} 5 \mathrm{~L} \mathrm{reb} /$ low & FALSE \\
\hline 2005 & $\mathrm{D}$ & 5 & 3 & 0.37 & 99.28 & BIS & & FALSE \\
\hline 2005 & $\mathrm{D}$ & 5 & 4 & 0.61 & 99.15 & BIS & on top of $\log$ & FALSE \\
\hline 2005 & $\mathrm{D}$ & 5 & 5 & 0.88 & 98.41 & BIS & & FALSE \\
\hline 2005 & $\mathrm{D}$ & 5 & 6 & 2.32 & 98.17 & BIS & & FALSE \\
\hline 2005 & $\mathrm{D}$ & 5 & 7 & 3.44 & 98.03 & BIS & & FALSE \\
\hline 2005 & $\mathrm{D}$ & 5 & 8 & 3.78 & 97.96 & EW & & FALSE \\
\hline 2005 & $\mathrm{D}$ & 5 & 9 & 4.27 & 97.85 & $\mathrm{TW}$ & & FALSE \\
\hline 2005 & $\mathrm{D}$ & 5 & 10 & 4.79 & 97.96 & EW & & FALSE \\
\hline 2005 & $\mathrm{D}$ & 5 & 11 & 5.30 & 98.10 & BIS & & FALSE \\
\hline 2005 & $\mathrm{D}$ & 5 & 12 & 5.94 & 98.37 & BIS & & FALSE \\
\hline 2005 & $\mathrm{D}$ & 5 & 13 & 6.49 & 98.58 & BIS & & FALSE \\
\hline 2005 & $\mathrm{D}$ & 5 & 14 & 6.92 & 98.94 & BIS & on top of boulder & FALSE \\
\hline
\end{tabular}

Continued on Next Page... 
Table D.1 - Continued

\begin{tabular}{|c|c|c|c|c|c|c|c|c|}
\hline Year & Reach & Section & Point & Dist. (m) & Elev. $(\mathrm{m})$ & Desc. & Notes & Exclude \\
\hline 2005 & $\mathrm{D}$ & 5 & 15 & 7.65 & 98.96 & BIS & & FALSE \\
\hline 2005 & D & 5 & 16 & 8.11 & 99.47 & ТОВ & & FALSE \\
\hline 2005 & $\mathrm{D}$ & 5 & 17 & 9.14 & 99.64 & BIS & & FALSE \\
\hline 2005 & $\mathrm{D}$ & 5 & 18 & 9.94 & 99.61 & BIS & & FALSE \\
\hline 2005 & $\mathrm{D}$ & 5 & 19 & 10.82 & 99.56 & BOT X5R & Bot $\mathrm{X} 5 \mathrm{R}$ reb/low & FALSE \\
\hline 2005 & $\mathrm{D}$ & 5 & 20 & 10.82 & 99.99 & TOP X5R & Top X5R reb/low & FALSE \\
\hline 2005 & $\mathrm{D}$ & 6 & 1 & 0.00 & 100.00 & & Top X6L reb/low & FALSE \\
\hline 2005 & $\mathrm{D}$ & 6 & 2 & 0.00 & 99.83 & & Bot $\mathrm{X} 6 \mathrm{~L} \mathrm{reb} /$ low & FALSE \\
\hline 2005 & $\mathrm{D}$ & 6 & 3 & 0.46 & 99.62 & BIS & & FALSE \\
\hline 2005 & $\mathrm{D}$ & 6 & 4 & 1.16 & 98.20 & BIS & & FALSE \\
\hline 2005 & $\mathrm{D}$ & 6 & 5 & 2.35 & 98.05 & BIS & & FALSE \\
\hline 2005 & $\mathrm{D}$ & 6 & 6 & 2.99 & 97.89 & $\mathrm{BF}$ & & FALSE \\
\hline 2005 & $\mathrm{D}$ & 6 & 7 & 3.84 & 97.55 & EW & & FALSE \\
\hline 2005 & $\mathrm{D}$ & 6 & 8 & 4.45 & 97.45 & TW & & FALSE \\
\hline 2005 & $\mathrm{D}$ & 6 & 9 & 4.79 & 97.62 & BIS & in stream; on large rock & FALSE \\
\hline 2005 & $\mathrm{D}$ & 6 & 10 & 5.09 & 97.64 & BIS & rockstep & FALSE \\
\hline 2005 & $\mathrm{D}$ & 6 & 11 & 6.25 & 97.56 & EW & & FALSE \\
\hline 2005 & $\mathrm{D}$ & 6 & 12 & 7.25 & 97.78 & BIS & & FALSE \\
\hline 2005 & $\mathrm{D}$ & 6 & 13 & 7.47 & 98.42 & BIS & & FALSE \\
\hline 2005 & $\mathrm{D}$ & 6 & 14 & 8.60 & 99.32 & ТОВ & & FALSE \\
\hline 2005 & $\mathrm{D}$ & 6 & 15 & 9.97 & 99.67 & BOT X6R & Bot $\mathrm{X} 6 \mathrm{R}$ reb/low & FALSE \\
\hline 2005 & $\mathrm{D}$ & 6 & 16 & 9.97 & 100.01 & TOP X6R & Top X6R reb/low & FALSE \\
\hline 2005 & $\mathrm{D}$ & 7 & 1 & 0.00 & 100.00 & & Top X7L reb/low & FALSE \\
\hline 2005 & $\mathrm{D}$ & 7 & 2 & 0.00 & 99.77 & & Bot $\mathrm{X} 7 \mathrm{~L}$ reb/low & FALSE \\
\hline 2005 & $\mathrm{D}$ & 7 & 3 & 0.30 & 99.50 & BIS & & FALSE \\
\hline 2005 & $\mathrm{D}$ & 7 & 4 & 0.40 & 98.89 & BIS & & FALSE \\
\hline 2005 & $\mathrm{D}$ & 7 & 5 & 0.76 & 99.70 & BIS & on top of $\log$ & TRUE \\
\hline 2005 & $\mathrm{D}$ & 7 & 6 & 1.25 & 98.47 & BIS & & FALSE \\
\hline 2005 & $\mathrm{D}$ & 7 & 7 & 1.86 & 98.30 & BIS & & FALSE \\
\hline 2005 & $\mathrm{D}$ & 7 & 8 & 2.19 & 98.09 & $\mathrm{BF}$ & & FALSE \\
\hline 2005 & $\mathrm{D}$ & 7 & 9 & 2.53 & 97.89 & BIS & & FALSE \\
\hline 2005 & $\mathrm{D}$ & 7 & 10 & 2.93 & 97.78 & EW & & FALSE \\
\hline 2005 & $\mathrm{D}$ & 7 & 11 & 3.47 & 97.68 & $\mathrm{TW}$ & & FALSE \\
\hline 2005 & $\mathrm{D}$ & 7 & 12 & 4.08 & 97.79 & EW & & FALSE \\
\hline 2005 & $\mathrm{D}$ & 7 & 13 & 4.85 & 97.90 & BIS & & FALSE \\
\hline 2005 & $\mathrm{D}$ & 7 & 14 & 5.61 & 97.95 & BIS & & FALSE \\
\hline 2005 & $\mathrm{D}$ & 7 & 15 & 6.07 & 97.80 & BIS & side pool; away from main channel & FALSE \\
\hline 2005 & $\mathrm{D}$ & 7 & 16 & 6.37 & 98.84 & BIS & & FALSE \\
\hline 2005 & $\mathrm{D}$ & 7 & 17 & 6.86 & 99.27 & BIS & & FALSE \\
\hline 2005 & $\mathrm{D}$ & 7 & 18 & 7.19 & 99.43 & ТОВ & & FALSE \\
\hline 2005 & $\mathrm{D}$ & 7 & 19 & 7.68 & 99.77 & BOT X7R & Bot $\mathrm{X} 7 \mathrm{~L} \mathrm{reb} /$ low & FALSE \\
\hline 2005 & $\mathrm{D}$ & 7 & 20 & 7.68 & 99.97 & TOP X7R & Top X7L reb/low & FALSE \\
\hline 2005 & $\mathrm{D}$ & 8 & 1 & 0.00 & 100.00 & & Top X8L reb/low (bent rebar) & FALSE \\
\hline 2005 & $\mathrm{D}$ & 8 & 2 & 0.00 & 99.75 & & Bot $\mathrm{X} 8 \mathrm{~L}$ reb/low (bent rebar) & FALSE \\
\hline 2005 & $\mathrm{D}$ & 8 & 3 & 0.61 & 99.44 & BIS & & FALSE \\
\hline 2005 & $\mathrm{D}$ & 8 & 4 & 0.88 & 99.17 & BIS & & FALSE \\
\hline 2005 & $\mathrm{D}$ & 8 & 5 & 1.40 & 98.02 & BIS & & FALSE \\
\hline 2005 & $\mathrm{D}$ & 8 & 6 & 2.07 & 97.83 & EW & & FALSE \\
\hline 2005 & $\mathrm{D}$ & 8 & 7 & 2.68 & 97.73 & TW & & FALSE \\
\hline 2005 & $\mathrm{D}$ & 8 & 8 & 3.38 & 97.87 & EW & & FALSE \\
\hline 2005 & $\mathrm{D}$ & 8 & 9 & 4.54 & 97.96 & BIS & & FALSE \\
\hline 2005 & $\mathrm{D}$ & 8 & 10 & 4.69 & 98.06 & $\mathrm{BF}$ & & FALSE \\
\hline
\end{tabular}

Continued on Next Page... 
Table D.1 - Continued

\begin{tabular}{|c|c|c|c|c|c|c|c|c|}
\hline Year & Reach & Section & Point & Dist. (m) & Elev. $(\mathrm{m})$ & Desc. & Notes & Exclude \\
\hline 2005 & $\mathrm{D}$ & 8 & 11 & 5.79 & 98.32 & BIS & & FALSE \\
\hline 2005 & $\mathrm{D}$ & 8 & 12 & 6.10 & 98.65 & BIS & & FALSE \\
\hline 2005 & $\mathrm{D}$ & 8 & 13 & 6.43 & 99.69 & ТОВ & & FALSE \\
\hline 2005 & $\mathrm{D}$ & 8 & 14 & 6.83 & 99.79 & BOT X8R & Bot $\mathrm{X} 8 \mathrm{R}$ reb/low & FALSE \\
\hline 2005 & $\mathrm{D}$ & 8 & 15 & 6.83 & 100.02 & TOP X8R & Top X8R reb/low & FALSE \\
\hline 2005 & $\mathrm{D}$ & 9 & 1 & 0.00 & 100.00 & & Top X9L reb/low & FALSE \\
\hline 2005 & $\mathrm{D}$ & 9 & 2 & 0.00 & 99.86 & & Bot $\mathrm{X} 9 \mathrm{~L}$ reb/low & FALSE \\
\hline 2005 & $\mathrm{D}$ & 9 & 3 & 0.40 & 98.96 & BIS & & FALSE \\
\hline 2005 & $\mathrm{D}$ & 9 & 4 & 1.16 & 98.64 & BIS & & FALSE \\
\hline 2005 & $\mathrm{D}$ & 9 & 5 & 1.55 & 98.59 & BIS & & FALSE \\
\hline 2005 & $\mathrm{D}$ & 9 & 6 & 1.92 & 98.61 & BIS & sidepool; apart from main channel & FALSE \\
\hline 2005 & $\mathrm{D}$ & 9 & 7 & 2.50 & 98.58 & EW & sidepool; apart from main channel & FALSE \\
\hline 2005 & $\mathrm{D}$ & 9 & 8 & 3.23 & 98.35 & TW & & FALSE \\
\hline 2005 & $\mathrm{D}$ & 9 & 9 & 3.96 & 98.53 & EW & & FALSE \\
\hline 2005 & $\mathrm{D}$ & 9 & 10 & 4.75 & 98.84 & $\mathrm{BF}$ & & FALSE \\
\hline 2005 & $\mathrm{D}$ & 9 & 11 & 5.46 & 98.95 & BIS & & FALSE \\
\hline 2005 & $\mathrm{D}$ & 9 & 12 & 6.25 & 99.04 & BIS & & FALSE \\
\hline 2005 & $\mathrm{D}$ & 9 & 13 & 6.89 & 99.15 & BIS & & FALSE \\
\hline 2005 & $\mathrm{D}$ & 9 & 14 & 7.38 & 99.80 & BOT X9R & Bot $\mathrm{X} 9 \mathrm{R}$ reb/low & FALSE \\
\hline 2005 & $\mathrm{D}$ & 9 & 15 & 7.22 & 100.03 & TOP X9R & Top X9R reb/low & FALSE \\
\hline 2005 & $\mathrm{D}$ & 10 & 1 & 0.00 & 100.00 & & Top X10L reb/low & FALSE \\
\hline 2005 & $\mathrm{D}$ & 10 & 2 & 0.00 & 99.70 & & Bot $\mathrm{X} 10 \mathrm{~L} \mathrm{reb} / \mathrm{low}$ & FALSE \\
\hline 2005 & $\mathrm{D}$ & 10 & 3 & 0.18 & 99.58 & BIS & $\begin{array}{l}2^{\prime}+\text { lower than all other years. } \\
\text { looks like error }\end{array}$ & FALSE \\
\hline 2005 & $\mathrm{D}$ & 10 & 4 & 0.64 & 99.08 & BIS & & FALSE \\
\hline 2005 & $\mathrm{D}$ & 10 & 5 & 1.19 & 98.89 & BIS & & FALSE \\
\hline 2005 & $\mathrm{D}$ & 10 & 6 & 1.37 & 98.48 & $\mathrm{BF}$ & & FALSE \\
\hline 2005 & $\mathrm{D}$ & 10 & 7 & 2.47 & 98.33 & BIS & & FALSE \\
\hline 2005 & $\mathrm{D}$ & 10 & 8 & 2.71 & 98.22 & EW & & FALSE \\
\hline 2005 & $\mathrm{D}$ & 10 & 9 & 4.05 & 98.14 & $\mathrm{TW}$ & & FALSE \\
\hline 2005 & $\mathrm{D}$ & 10 & 10 & 4.85 & 98.25 & EW & & FALSE \\
\hline 2005 & $\mathrm{D}$ & 10 & 11 & 4.94 & 98.42 & BIS & on top of boulder & TRUE \\
\hline 2005 & $\mathrm{D}$ & 10 & 12 & 5.36 & 98.26 & BIS & & FALSE \\
\hline 2005 & $\mathrm{D}$ & 10 & 13 & 5.73 & 98.50 & BIS & on top of boulder & FALSE \\
\hline 2005 & $\mathrm{D}$ & 10 & 14 & 6.49 & 98.52 & BIS & & FALSE \\
\hline 2005 & $\mathrm{D}$ & 10 & 15 & 6.89 & 98.87 & BIS & & FALSE \\
\hline 2005 & $\mathrm{D}$ & 10 & 16 & 7.35 & 99.75 & BOT X10R & Bot $\mathrm{X} 10 \mathrm{R} \mathrm{reb} / \mathrm{low}$ & FALSE \\
\hline 2005 & $\mathrm{D}$ & 10 & 17 & 7.35 & 100.00 & TOP X10R & Top X10R reb/low & FALSE \\
\hline 2005 & $\mathrm{E}$ & 1 & 1 & 0.00 & 100.00 & & Top X1L reb/low & FALSE \\
\hline 2005 & $\mathrm{E}$ & 1 & 2 & 0.00 & 99.63 & & Bot X1L reb/low & FALSE \\
\hline 2005 & $\mathrm{E}$ & 1 & 3 & 0.40 & 99.41 & BIS & & FALSE \\
\hline 2005 & $\mathrm{E}$ & 1 & 4 & 2.01 & 98.88 & BIS & & FALSE \\
\hline 2005 & $\mathrm{E}$ & 1 & 5 & 3.63 & 98.76 & BIS & & FALSE \\
\hline 2005 & $\mathrm{E}$ & 1 & 6 & 4.63 & 98.42 & BIS & & FALSE \\
\hline 2005 & $\mathrm{E}$ & 1 & 7 & 5.18 & 98.37 & $\mathrm{BF}$ & & FALSE \\
\hline 2005 & $\mathrm{E}$ & 1 & 8 & 5.76 & 98.18 & EW & & FALSE \\
\hline 2005 & $\mathrm{E}$ & 1 & 9 & 6.28 & 98.11 & $\mathrm{TW}$ & & FALSE \\
\hline 2005 & $\mathrm{E}$ & 1 & 10 & 6.80 & 98.23 & BIS & Big, leafy, twig pile in stream & FALSE \\
\hline 2005 & $\mathrm{E}$ & 1 & 11 & 7.01 & 99.47 & BIS & Due to cut bank can't reach EW & FALSE \\
\hline 2005 & $\mathrm{E}$ & 1 & 12 & 7.50 & 99.89 & TOP X1R & Top X1R reb/low & FALSE \\
\hline 2005 & $\mathrm{E}$ & 2 & 1 & 0.00 & 100.00 & & Top X2L reb/low & FALSE \\
\hline 2005 & $\mathrm{E}$ & 2 & 2 & 0.00 & 99.45 & & Bot $\mathrm{X} 2 \mathrm{~L} \mathrm{reb} /$ low & FALSE \\
\hline
\end{tabular}

Continued on Next Page... 
Table D.1 - Continued

\begin{tabular}{|c|c|c|c|c|c|c|c|c|}
\hline Year & Reach & Section & Point & Dist. (m) & Elev. $(\mathrm{m})$ & Desc. & Notes & Exclude \\
\hline 2005 & $\mathrm{E}$ & 2 & 3 & 0.34 & 99.14 & BIS & & FALSE \\
\hline 2005 & $\mathrm{E}$ & 2 & 4 & 0.70 & 98.91 & BIS & & FALSE \\
\hline 2005 & $\mathrm{E}$ & 2 & 5 & 0.94 & 98.55 & BIS & & FALSE \\
\hline 2005 & $\mathrm{E}$ & 2 & 6 & 1.89 & 98.38 & EW & on top of rocks & FALSE \\
\hline 2005 & $\mathrm{E}$ & 2 & 7 & 2.38 & 98.39 & BIS & in stream; on top of rocks & FALSE \\
\hline 2005 & $\mathrm{E}$ & 2 & 8 & 2.71 & 98.25 & TW & & FALSE \\
\hline 2005 & $\mathrm{E}$ & 2 & 9 & 3.23 & 98.40 & EW & & FALSE \\
\hline 2005 & $\mathrm{E}$ & 2 & 10 & 3.54 & 98.49 & $\mathrm{BF}$ & & FALSE \\
\hline 2005 & $\mathrm{E}$ & 2 & 11 & 3.63 & 98.58 & BIS & on top of rocks & FALSE \\
\hline 2005 & $\mathrm{E}$ & 2 & 12 & 4.18 & 98.66 & BIS & & FALSE \\
\hline 2005 & $\mathrm{E}$ & 2 & 13 & 4.48 & 98.97 & BIS & & FALSE \\
\hline 2005 & $\mathrm{E}$ & 2 & 14 & 4.79 & 99.18 & BIS & & FALSE \\
\hline 2005 & $\mathrm{E}$ & 2 & 15 & 5.03 & 99.36 & ТОВ & & FALSE \\
\hline 2005 & $\mathrm{E}$ & 2 & 16 & 5.46 & 99.44 & BIS & & FALSE \\
\hline 2005 & $\mathrm{E}$ & 2 & 17 & 5.64 & 99.58 & BIS & on top of boulder & FALSE \\
\hline 2005 & $\mathrm{E}$ & 2 & 18 & 5.79 & 99.61 & BOT X2R & Bot $\mathrm{X} 2 \mathrm{R}$ reb/low & FALSE \\
\hline 2005 & $\mathrm{E}$ & 2 & 19 & 5.79 & 100.02 & TOP X2R & Top X2R reb/low & FALSE \\
\hline 2005 & $\mathrm{E}$ & 3 & 1 & 0.00 & 100.00 & & Top X3L reb/low & FALSE \\
\hline 2005 & $\mathrm{E}$ & 3 & 2 & 0.00 & 99.52 & & Bot X3L reb/low & FALSE \\
\hline 2005 & $\mathrm{E}$ & 3 & 3 & 0.24 & 99.34 & BIS & & FALSE \\
\hline 2005 & $\mathrm{E}$ & 3 & 4 & 0.37 & 99.12 & BIS & & FALSE \\
\hline 2005 & $\mathrm{E}$ & 3 & 5 & 0.79 & 98.82 & BIS & & FALSE \\
\hline 2005 & $\mathrm{E}$ & 3 & 6 & 1.74 & 98.42 & BIS & & FALSE \\
\hline 2005 & $\mathrm{E}$ & 3 & 7 & 1.80 & 98.06 & EW & on top of rocks & FALSE \\
\hline 2005 & $\mathrm{E}$ & 3 & 8 & 2.65 & 97.98 & BIS & cluster of boulders in stream (RI) & FALSE \\
\hline 2005 & $\mathrm{E}$ & 3 & 9 & 3.44 & 97.77 & TW & & FALSE \\
\hline 2005 & $\mathrm{E}$ & 3 & 10 & 3.75 & 97.82 & EW & & FALSE \\
\hline 2005 & $\mathrm{E}$ & 3 & 11 & 4.11 & 98.00 & $\mathrm{BF}$ & & FALSE \\
\hline 2005 & $\mathrm{E}$ & 3 & 12 & 4.75 & 98.26 & BIS & & FALSE \\
\hline 2005 & $\mathrm{E}$ & 3 & 13 & 5.27 & 98.47 & BIS & & FALSE \\
\hline 2005 & $\mathrm{E}$ & 3 & 14 & 5.52 & 98.93 & ТОВ & & FALSE \\
\hline 2005 & $\mathrm{E}$ & 3 & 15 & 6.37 & 99.09 & BIS & & FALSE \\
\hline 2005 & $\mathrm{E}$ & 3 & 16 & 7.10 & 99.19 & BIS & & FALSE \\
\hline 2005 & $\mathrm{E}$ & 3 & 17 & 7.19 & 99.39 & BOT X3R & Bot X3R reb/low (on log) & FALSE \\
\hline 2005 & $\mathrm{E}$ & 3 & 18 & 7.19 & 100.01 & TOP X3R & Top X3R reb/low & FALSE \\
\hline 2005 & $\mathrm{E}$ & 4 & 1 & 0.00 & 100.00 & & Top X4L reb/low & FALSE \\
\hline 2005 & $\mathrm{E}$ & 4 & 2 & 0.00 & 99.75 & & Bot $\mathrm{X} 4 \mathrm{~L}$ reb/low & FALSE \\
\hline 2005 & $\mathrm{E}$ & 4 & 3 & 0.55 & 99.40 & BIS & & FALSE \\
\hline 2005 & $\mathrm{E}$ & 4 & 4 & 0.61 & 99.13 & BIS & & FALSE \\
\hline 2005 & $\mathrm{E}$ & 4 & 5 & 0.94 & 99.02 & BIS & & FALSE \\
\hline 2005 & $\mathrm{E}$ & 4 & 6 & 1.07 & 98.80 & BIS & & FALSE \\
\hline 2005 & $\mathrm{E}$ & 4 & 7 & 1.52 & 98.95 & BIS & & FALSE \\
\hline 2005 & $\mathrm{E}$ & 4 & 8 & 2.23 & 98.65 & EW & & FALSE \\
\hline 2005 & $\mathrm{E}$ & 4 & 9 & 2.90 & 98.60 & TW & & FALSE \\
\hline 2005 & $\mathrm{E}$ & 4 & 10 & 3.54 & 98.84 & BIS & in stream rock step & FALSE \\
\hline 2005 & $\mathrm{E}$ & 4 & 11 & 3.90 & 98.63 & BIS & in stream rock step & FALSE \\
\hline 2005 & $\mathrm{E}$ & 4 & 12 & 4.30 & 98.72 & BIS & in stream rock step & FALSE \\
\hline 2005 & $\mathrm{E}$ & 4 & 13 & 4.66 & 98.54 & EW & & FALSE \\
\hline 2005 & $\mathrm{E}$ & 4 & 14 & 5.30 & 98.69 & $\mathrm{BF}$ & & FALSE \\
\hline 2005 & $\mathrm{E}$ & 4 & 15 & 5.61 & 98.80 & BIS & adjusted up 1' & FALSE \\
\hline 2005 & $\mathrm{E}$ & 4 & 16 & 5.91 & 99.49 & ТОВ & & FALSE \\
\hline 2005 & $\mathrm{E}$ & 4 & 17 & 6.19 & 99.70 & BIS & on top of $\log$ & FALSE \\
\hline
\end{tabular}

Continued on Next Page... 
Table D.1 - Continued

\begin{tabular}{|c|c|c|c|c|c|c|c|c|}
\hline Year & Reach & Section & Point & Dist. (m) & Elev. $(\mathrm{m})$ & Desc. & Notes & Exclude \\
\hline 2005 & $\mathrm{E}$ & 4 & 18 & 6.34 & 99.58 & BIS & & FALSE \\
\hline 2005 & $\mathrm{E}$ & 4 & 19 & 6.95 & 99.63 & BIS & & FALSE \\
\hline 2005 & $\mathrm{E}$ & 4 & 20 & 7.62 & 99.79 & BOT X4R & Bot $\mathrm{X} 4 \mathrm{R}$ reb/low & FALSE \\
\hline 2005 & $\mathrm{E}$ & 4 & 21 & 7.62 & 100.04 & TOP X4R & Top X4R reb/low & FALSE \\
\hline 2005 & $\mathrm{E}$ & 5 & 1 & 0.00 & 100.00 & & Top X5L reb/low & FALSE \\
\hline 2005 & $\mathrm{E}$ & 5 & 2 & 0.00 & 99.68 & & Bot $\mathrm{X} 5 \mathrm{~L} \mathrm{reb} /$ low & FALSE \\
\hline 2005 & $\mathrm{E}$ & 5 & 3 & 0.49 & 99.56 & BIS & & FALSE \\
\hline 2005 & $\mathrm{E}$ & 5 & 4 & 0.79 & 99.48 & BIS & & FALSE \\
\hline 2005 & $\mathrm{E}$ & 5 & 5 & 1.01 & 99.40 & BIS & & FALSE \\
\hline 2005 & $\mathrm{E}$ & 5 & 6 & 1.25 & 99.20 & BIS & & FALSE \\
\hline 2005 & $\mathrm{E}$ & 5 & 7 & 1.55 & 98.91 & BIS & & FALSE \\
\hline 2005 & $\mathrm{E}$ & 5 & 8 & 2.10 & 98.82 & $\mathrm{BF}$ & on top of small rocks & FALSE \\
\hline 2005 & $\mathrm{E}$ & 5 & 9 & 2.53 & 98.73 & EW & & FALSE \\
\hline 2005 & $\mathrm{E}$ & 5 & 10 & 3.14 & 98.62 & $\mathrm{TW}$ & & FALSE \\
\hline 2005 & $\mathrm{E}$ & 5 & 11 & 4.15 & 98.72 & EW & & FALSE \\
\hline 2005 & $\mathrm{E}$ & 5 & 12 & 4.45 & 99.01 & BIS & on top of large boulder & FALSE \\
\hline 2005 & $\mathrm{E}$ & 5 & 13 & 4.94 & 99.21 & BIS & on top of large boulder & FALSE \\
\hline 2005 & $\mathrm{E}$ & 5 & 14 & 5.27 & 99.23 & BIS & & FALSE \\
\hline 2005 & $\mathrm{E}$ & 5 & 15 & 5.40 & 99.66 & ТОВ & on rock & FALSE \\
\hline 2005 & $\mathrm{E}$ & 5 & 16 & 7.01 & 99.90 & BIS & & FALSE \\
\hline 2005 & $\mathrm{E}$ & 5 & 17 & 7.83 & 99.89 & BOT X5R & Bot $\mathrm{X} 5 \mathrm{R}$ reb/low & FALSE \\
\hline 2005 & $\mathrm{E}$ & 5 & 18 & 7.83 & 100.01 & TOP X5R & Top X5R reb/low & FALSE \\
\hline 2005 & $\mathrm{E}$ & 6 & 1 & 0.00 & 100.00 & & Top X6L reb/low & FALSE \\
\hline 2005 & $\mathrm{E}$ & 6 & 2 & 0.00 & 99.54 & & Bot $\mathrm{X} 6 \mathrm{~L} \mathrm{reb} /$ low & FALSE \\
\hline 2005 & $\mathrm{E}$ & 6 & 3 & 0.21 & 99.39 & BIS & & FALSE \\
\hline 2005 & $\mathrm{E}$ & 6 & 4 & 0.58 & 98.77 & BIS & & FALSE \\
\hline 2005 & $\mathrm{E}$ & 6 & 5 & 1.10 & 98.43 & EW & on top of rock & FALSE \\
\hline 2005 & $\mathrm{E}$ & 6 & 6 & 1.58 & 98.23 & $\mathrm{TW}$ & & FALSE \\
\hline 2005 & $\mathrm{E}$ & 6 & 7 & 1.98 & 98.41 & BIS & in stream; on top of rock & FALSE \\
\hline 2005 & $\mathrm{E}$ & 6 & 8 & 2.44 & 98.39 & EW & & FALSE \\
\hline 2005 & $\mathrm{E}$ & 6 & 9 & 3.23 & 98.54 & BIS & & FALSE \\
\hline 2005 & $\mathrm{E}$ & 6 & 10 & 3.54 & 98.87 & BIS & on top of boulder & FALSE \\
\hline 2005 & $\mathrm{E}$ & 6 & 11 & 3.90 & 98.67 & BIS & & FALSE \\
\hline 2005 & $\mathrm{E}$ & 6 & 12 & 4.33 & 98.66 & BIS & & FALSE \\
\hline 2005 & $\mathrm{E}$ & 6 & 13 & 4.66 & 99.46 & ТОВ & & FALSE \\
\hline 2005 & $\mathrm{E}$ & 6 & 14 & 5.06 & 99.58 & BIS & & FALSE \\
\hline 2005 & $\mathrm{E}$ & 6 & 15 & 5.33 & 99.64 & BOT X6R & Bot $\mathrm{X} 6 \mathrm{R}$ reb/low & FALSE \\
\hline 2005 & $\mathrm{E}$ & 6 & 16 & 5.33 & 100.00 & TOP X6R & Top X6R reb/low & FALSE \\
\hline 2005 & $\mathrm{E}$ & 7 & 1 & 0.00 & 100.00 & & Top X7L reb/low & FALSE \\
\hline 2005 & $\mathrm{E}$ & 7 & 2 & 0.00 & 99.73 & & Bot $\mathrm{X} 7 \mathrm{~L} \mathrm{reb} /$ low & FALSE \\
\hline 2005 & $\mathrm{E}$ & 7 & 3 & 0.73 & 99.42 & ТОВ & & FALSE \\
\hline 2005 & $\mathrm{E}$ & 7 & 4 & 1.19 & 99.06 & BIS & on top of boulder & FALSE \\
\hline 2005 & $\mathrm{E}$ & 7 & 5 & 1.49 & 98.57 & EW & $\begin{array}{l}\text { rock pile; difficult to see EW; } \\
\text { (shelf) on rocks }\end{array}$ & FALSE \\
\hline 2005 & $\mathrm{E}$ & 7 & 6 & 3.20 & 98.45 & TW & & FALSE \\
\hline 2005 & $\mathrm{E}$ & 7 & 7 & 3.96 & 98.67 & EW & on top of rock & FALSE \\
\hline 2005 & $\mathrm{E}$ & 7 & 8 & 4.18 & 98.68 & $\mathrm{BF}$ & & FALSE \\
\hline 2005 & $\mathrm{E}$ & 7 & 9 & 4.42 & 98.86 & BIS & & FALSE \\
\hline 2005 & $\mathrm{E}$ & 7 & 10 & 4.63 & 99.60 & BIS & & FALSE \\
\hline 2005 & $\mathrm{E}$ & 7 & 11 & 5.18 & 99.84 & BIS & on top of rock & FALSE \\
\hline 2005 & $\mathrm{E}$ & 7 & 12 & 5.97 & 99.66 & BIS & & FALSE \\
\hline 2005 & $\mathrm{E}$ & 7 & 13 & 6.49 & 99.67 & BOT X7R & Bot $\mathrm{X} 7 \mathrm{R}$ reb/low & FALSE \\
\hline
\end{tabular}

Continued on Next Page... 
Table D.1 - Continued

\begin{tabular}{|c|c|c|c|c|c|c|c|c|}
\hline Year & Reach & Section & Point & Dist. (m) & Elev. $(\mathrm{m})$ & Desc. & Notes & Exclude \\
\hline 2005 & $\mathrm{E}$ & 7 & 14 & 6.49 & 100.02 & TOP X7R & Top X7R reb/low & FALSE \\
\hline 2005 & $\mathrm{E}$ & 8 & 1 & 0.00 & 100.00 & & Top X8L reb/low & FALSE \\
\hline 2005 & $\mathrm{E}$ & 8 & 2 & 0.00 & 99.63 & & Bot $\mathrm{X} 8 \mathrm{~L}$ reb/low & FALSE \\
\hline 2005 & $\mathrm{E}$ & 8 & 3 & 0.15 & 99.62 & ТОВ & & FALSE \\
\hline 2005 & $\mathrm{E}$ & 8 & 4 & 0.43 & 99.37 & BIS & & FALSE \\
\hline 2005 & $\mathrm{E}$ & 8 & 5 & 0.82 & 99.16 & BIS & & FALSE \\
\hline 2005 & $\mathrm{E}$ & 8 & 6 & 0.98 & 98.77 & $\mathrm{BF}$ & & FALSE \\
\hline 2005 & $\mathrm{E}$ & 8 & 7 & 1.37 & 98.61 & EW & & FALSE \\
\hline 2005 & $\mathrm{E}$ & 8 & 8 & 2.01 & 98.56 & $\mathrm{TW}$ & & FALSE \\
\hline 2005 & $\mathrm{E}$ & 8 & 9 & 3.26 & 98.68 & EW & & FALSE \\
\hline 2005 & $\mathrm{E}$ & 8 & 10 & 4.05 & 99.02 & BIS & & FALSE \\
\hline 2005 & $\mathrm{E}$ & 8 & 11 & 4.11 & 99.34 & BIS & & FALSE \\
\hline 2005 & $\mathrm{E}$ & 8 & 12 & 5.00 & 99.57 & BIS & & FALSE \\
\hline 2005 & $\mathrm{E}$ & 8 & 13 & 6.07 & 99.64 & BIS & & FALSE \\
\hline 2005 & $\mathrm{E}$ & 8 & 14 & 6.64 & 99.74 & BOT X8R & Bot $\mathrm{X} 8 \mathrm{R}$ reb/low & FALSE \\
\hline 2005 & $\mathrm{E}$ & 8 & 15 & 6.64 & 100.00 & TOP X8R & Top X8R reb/low & FALSE \\
\hline 2005 & $\mathrm{E}$ & 9 & 1 & 0.00 & 100.00 & & Top X9L reb/low & FALSE \\
\hline 2005 & $\mathrm{E}$ & 9 & 2 & 0.00 & 99.49 & & Bot X9L reb/low & FALSE \\
\hline 2005 & $\mathrm{E}$ & 9 & 3 & 0.34 & 99.18 & BIS & & FALSE \\
\hline 2005 & $\mathrm{E}$ & 9 & 4 & 0.94 & 98.81 & BIS & & FALSE \\
\hline 2005 & $\mathrm{E}$ & 9 & 5 & 1.19 & 98.39 & BIS & & FALSE \\
\hline 2005 & $\mathrm{E}$ & 9 & 6 & 1.52 & 98.12 & BIS & & FALSE \\
\hline 2005 & $\mathrm{E}$ & 9 & 7 & 1.80 & 97.97 & $\mathrm{BF}$ & & FALSE \\
\hline 2005 & $\mathrm{E}$ & 9 & 8 & 3.14 & 97.64 & EW & & FALSE \\
\hline 2005 & $\mathrm{E}$ & 9 & 9 & 3.75 & 97.57 & $\mathrm{TW}$ & & FALSE \\
\hline 2005 & $\mathrm{E}$ & 9 & 10 & 4.79 & 97.68 & EW & & FALSE \\
\hline 2005 & $\mathrm{E}$ & 9 & 11 & 5.64 & 97.81 & BIS & & FALSE \\
\hline 2005 & $\mathrm{E}$ & 9 & 12 & 6.55 & 98.05 & BIS & & FALSE \\
\hline 2005 & $\mathrm{E}$ & 9 & 13 & 6.89 & 98.24 & BIS & & FALSE \\
\hline 2005 & $\mathrm{E}$ & 9 & 14 & 7.35 & 98.57 & BIS & & FALSE \\
\hline 2005 & $\mathrm{E}$ & 9 & 15 & 7.47 & 98.96 & BIS & & FALSE \\
\hline 2005 & $\mathrm{E}$ & 9 & 16 & 7.71 & 99.08 & BIS & & FALSE \\
\hline 2005 & $\mathrm{E}$ & 9 & 17 & 7.92 & 99.59 & ТОВ & & FALSE \\
\hline 2005 & $\mathrm{E}$ & 9 & 18 & 8.14 & 99.70 & BOT X9R & Bot X9R reb/low & FALSE \\
\hline 2005 & $\mathrm{E}$ & 9 & 19 & 8.14 & 99.98 & TOP X9R & Top X9R reb/low & FALSE \\
\hline 2005 & $\mathrm{E}$ & 10 & 1 & 0.00 & 100.00 & & Top X10L reb/low & FALSE \\
\hline 2005 & $\mathrm{E}$ & 10 & 2 & 0.00 & 99.68 & & Bot $\mathrm{X} 10 \mathrm{~L} \mathrm{reb} /$ low & FALSE \\
\hline 2005 & $\mathrm{E}$ & 10 & 3 & 0.37 & 99.36 & BIS & & FALSE \\
\hline 2005 & $\mathrm{E}$ & 10 & 4 & 0.55 & 98.86 & BIS & & FALSE \\
\hline 2005 & $\mathrm{E}$ & 10 & 5 & 0.94 & 98.67 & BIS & & FALSE \\
\hline 2005 & $\mathrm{E}$ & 10 & 6 & 1.40 & 98.26 & BIS & & FALSE \\
\hline 2005 & $\mathrm{E}$ & 10 & 7 & 1.74 & 98.33 & BIS & on boulder & FALSE \\
\hline 2005 & $\mathrm{E}$ & 10 & 8 & 1.86 & 98.20 & $\mathrm{BF}$ & & FALSE \\
\hline 2005 & $\mathrm{E}$ & 10 & 9 & 2.41 & 98.11 & EW & & FALSE \\
\hline 2005 & $\mathrm{E}$ & 10 & 10 & 2.83 & 98.00 & $\mathrm{TW}$ & & FALSE \\
\hline 2005 & $\mathrm{E}$ & 10 & 11 & 3.35 & 98.09 & EW & & FALSE \\
\hline 2005 & $\mathrm{E}$ & 10 & 12 & 3.78 & 98.18 & BIS & & FALSE \\
\hline 2005 & $\mathrm{E}$ & 10 & 13 & 4.15 & 98.41 & BIS & & FALSE \\
\hline 2005 & $\mathrm{E}$ & 10 & 14 & 5.06 & 98.68 & BIS & & FALSE \\
\hline 2005 & $\mathrm{E}$ & 10 & 15 & 5.24 & 99.12 & ТОВ & & FALSE \\
\hline 2005 & $\mathrm{E}$ & 10 & 16 & 5.97 & 99.52 & BIS & & FALSE \\
\hline 2005 & $\mathrm{E}$ & 10 & 17 & 6.61 & 99.78 & BIS & & FALSE \\
\hline
\end{tabular}

Continued on Next Page... 
Table D.1 - Continued

\begin{tabular}{|c|c|c|c|c|c|c|c|c|}
\hline Year & Reach & Section & Point & Dist. (m) & Elev. $(\mathrm{m})$ & Desc. & Notes & Exclude \\
\hline 2005 & $\mathrm{E}$ & 10 & 18 & 7.07 & 99.94 & BOT X10R & Bot X10R reb/low & FALSE \\
\hline 2005 & $\mathrm{E}$ & 10 & 19 & 7.07 & 100.15 & TOP X10R & Top X10R reb/low & FALSE \\
\hline 2005 & $\mathrm{~F}$ & 1 & 1 & 0.00 & 100.00 & & Top X1L reb/low & FALSE \\
\hline 2005 & $\mathrm{~F}$ & 1 & 2 & 0.00 & 99.95 & & Bot $\mathrm{X} 1 \mathrm{~L} \mathrm{reb} /$ low & FALSE \\
\hline 2005 & $\mathrm{~F}$ & 1 & 3 & 0.73 & 99.28 & BIS & & FALSE \\
\hline 2005 & $\mathrm{~F}$ & 1 & 4 & 1.43 & 98.99 & BIS & & FALSE \\
\hline 2005 & $\mathrm{~F}$ & 1 & 5 & 2.50 & 98.88 & BIS & & FALSE \\
\hline 2005 & $\mathrm{~F}$ & 1 & 6 & 2.65 & 98.58 & BIS & & FALSE \\
\hline 2005 & $\mathrm{~F}$ & 1 & 7 & 3.20 & 98.30 & BIS & & FALSE \\
\hline 2005 & $\mathrm{~F}$ & 1 & 8 & 4.05 & 98.32 & BIS & & FALSE \\
\hline 2005 & $\mathrm{~F}$ & 1 & 9 & 4.97 & 98.08 & BIS & & FALSE \\
\hline 2005 & $\mathrm{~F}$ & 1 & 10 & 5.43 & 98.24 & BIS & on top of boulder & FALSE \\
\hline 2005 & $\mathrm{~F}$ & 1 & 11 & 5.76 & 97.94 & EW & & FALSE \\
\hline 2005 & $\mathrm{~F}$ & 1 & 12 & 5.85 & 97.87 & $\mathrm{TW}$ & & FALSE \\
\hline 2005 & $\mathrm{~F}$ & 1 & 13 & 6.04 & 97.92 & EW & & FALSE \\
\hline 2005 & $\mathrm{~F}$ & 1 & 14 & 6.49 & 98.04 & BIS & & FALSE \\
\hline 2005 & $\mathrm{~F}$ & 1 & 15 & 6.77 & 98.22 & $\mathrm{BF}$ & & FALSE \\
\hline 2005 & $\mathrm{~F}$ & 1 & 16 & 7.77 & 98.35 & BIS & & FALSE \\
\hline 2005 & $\mathrm{~F}$ & 1 & 17 & 8.50 & 99.09 & BIS & & FALSE \\
\hline 2005 & $\mathrm{~F}$ & 1 & 18 & 9.05 & 99.42 & BIS & & FALSE \\
\hline 2005 & $\mathrm{~F}$ & 1 & 19 & 9.45 & 99.72 & BOT X1R & Bot X1R reb/low & FALSE \\
\hline 2005 & $\mathrm{~F}$ & 1 & 20 & 9.45 & 99.80 & TOP X1R & Top X1R reb/low & FALSE \\
\hline 2005 & $\mathrm{~F}$ & 2 & 1 & 0.00 & 100.00 & & Top X2L reb/low & FALSE \\
\hline 2005 & $\mathrm{~F}$ & 2 & 2 & 0.00 & 99.83 & & Bot $\mathrm{X} 2 \mathrm{~L}$ reb/low & FALSE \\
\hline 2005 & $\mathrm{~F}$ & 2 & 3 & 0.82 & 99.13 & BIS & & FALSE \\
\hline 2005 & $\mathrm{~F}$ & 2 & 4 & 1.68 & 98.82 & BIS & & FALSE \\
\hline 2005 & $\mathrm{~F}$ & 2 & 5 & 2.50 & 98.39 & BIS & & FALSE \\
\hline 2005 & $\mathrm{~F}$ & 2 & 6 & 2.59 & 98.05 & BIS & & FALSE \\
\hline 2005 & $\mathrm{~F}$ & 2 & 7 & 3.26 & 97.83 & $\mathrm{BF}$ & & FALSE \\
\hline 2005 & $\mathrm{~F}$ & 2 & 8 & 4.45 & 97.49 & EW & & FALSE \\
\hline 2005 & $\mathrm{~F}$ & 2 & 9 & 4.94 & 97.33 & TW & & FALSE \\
\hline 2005 & $\mathrm{~F}$ & 2 & 10 & 5.52 & 97.47 & EW & & FALSE \\
\hline 2005 & $\mathrm{~F}$ & 2 & 11 & 6.31 & 97.57 & BIS & & FALSE \\
\hline 2005 & $\mathrm{~F}$ & 2 & 12 & 7.07 & 97.88 & BIS & & FALSE \\
\hline 2005 & $\mathrm{~F}$ & 2 & 13 & 8.14 & 98.47 & BIS & & FALSE \\
\hline 2005 & $\mathrm{~F}$ & 2 & 14 & 8.47 & 99.63 & BIS & & FALSE \\
\hline 2005 & $\mathrm{~F}$ & 2 & 15 & 8.72 & 99.83 & BOT X2R & Bot $\mathrm{X} 2 \mathrm{R} \mathrm{reb} /$ low & FALSE \\
\hline 2005 & $\mathrm{~F}$ & 2 & 16 & 8.72 & 99.90 & TOP X2R & Top X2R reb/low & FALSE \\
\hline 2005 & $\mathrm{~F}$ & 3 & 1 & 0.00 & 100.00 & & Top X3L reb/low & FALSE \\
\hline 2005 & $\mathrm{~F}$ & 3 & 2 & 0.00 & 99.83 & & Bot X3L reb/low & FALSE \\
\hline 2005 & $\mathrm{~F}$ & 3 & 3 & 1.04 & 99.38 & BIS & & FALSE \\
\hline 2005 & $\mathrm{~F}$ & 3 & 4 & 1.31 & 98.58 & BIS & & FALSE \\
\hline 2005 & $\mathrm{~F}$ & 3 & 5 & 2.44 & 98.37 & BIS & & FALSE \\
\hline 2005 & $\mathrm{~F}$ & 3 & 6 & 3.35 & 98.13 & BIS & & FALSE \\
\hline 2005 & $\mathrm{~F}$ & 3 & 7 & 3.84 & 98.21 & BIS & on top of boulder & FALSE \\
\hline 2005 & $\mathrm{~F}$ & 3 & 8 & 3.90 & 97.91 & EW & & FALSE \\
\hline 2005 & $\mathrm{~F}$ & 3 & 9 & 4.66 & 97.81 & $\mathrm{TW}$ & & FALSE \\
\hline 2005 & $\mathrm{~F}$ & 3 & 10 & 4.82 & 97.90 & EW & on top of boulder & FALSE \\
\hline 2005 & $\mathrm{~F}$ & 3 & 11 & 5.64 & 98.21 & $\mathrm{BF}$ & & FALSE \\
\hline 2005 & $\mathrm{~F}$ & 3 & 12 & 6.46 & 98.66 & BIS & & FALSE \\
\hline 2005 & $\mathrm{~F}$ & 3 & 13 & 6.92 & 98.76 & BIS & & FALSE \\
\hline 2005 & $\mathrm{~F}$ & 3 & 14 & 7.38 & 99.77 & BIS & & FALSE \\
\hline
\end{tabular}

Continued on Next Page... 
Table D.1 - Continued

\begin{tabular}{|c|c|c|c|c|c|c|c|c|}
\hline Year & Reach & Section & Point & Dist. (m) & Elev. $(\mathrm{m})$ & Desc. & Notes & Exclude \\
\hline 2005 & $\mathrm{~F}$ & 3 & 15 & 7.77 & 100.00 & BOT X3R & Bot $\mathrm{X} 3 \mathrm{R}$ reb/low & FALSE \\
\hline 2005 & $\mathrm{~F}$ & 3 & 16 & 7.77 & 100.05 & TOP X3R & Top X3R reb/low & FALSE \\
\hline 2005 & $\mathrm{~F}$ & 4 & 1 & 0.00 & 100.00 & & Top X4L reb/low & FALSE \\
\hline 2005 & $\mathrm{~F}$ & 4 & 2 & 0.00 & 99.94 & & Bot $\mathrm{X} 4 \mathrm{~L}$ reb/low & FALSE \\
\hline 2005 & $\mathrm{~F}$ & 4 & 3 & 1.01 & 99.18 & BIS & & FALSE \\
\hline 2005 & F & 4 & 4 & 1.65 & 98.84 & BIS & & FALSE \\
\hline 2005 & $\mathrm{~F}$ & 4 & 5 & 1.98 & 98.28 & BIS & & FALSE \\
\hline 2005 & $\mathrm{~F}$ & 4 & 6 & 2.65 & 97.85 & $\mathrm{BF}$ & & FALSE \\
\hline 2005 & $\mathrm{~F}$ & 4 & 7 & 3.96 & 97.62 & BIS & side pool & FALSE \\
\hline 2005 & $\mathrm{~F}$ & 4 & 8 & 4.36 & 97.57 & EW & & FALSE \\
\hline 2005 & F & 4 & 9 & 5.18 & 97.48 & TW & & FALSE \\
\hline 2005 & $\mathrm{~F}$ & 4 & 10 & 6.16 & 97.49 & EW & & FALSE \\
\hline 2005 & $\mathrm{~F}$ & 4 & 11 & 6.64 & 98.07 & BIS & & FALSE \\
\hline 2005 & $\mathrm{~F}$ & 4 & 12 & 7.56 & 99.34 & BIS & & FALSE \\
\hline 2005 & $\mathrm{~F}$ & 4 & 13 & 8.29 & 99.76 & ТОВ & & FALSE \\
\hline 2005 & $\mathrm{~F}$ & 4 & 14 & 8.81 & 99.93 & BOT $\mathrm{X} 4 \mathrm{R}$ & Bot $\mathrm{X} 4 \mathrm{R}$ reb/low & FALSE \\
\hline 2005 & $\mathrm{~F}$ & 4 & 15 & 8.81 & 100.13 & TOP X4R & Top X4R reb/low & FALSE \\
\hline 2005 & $\mathrm{~F}$ & 5 & 1 & 0.00 & 100.00 & & Top X5L reb/low & FALSE \\
\hline 2005 & $\mathrm{~F}$ & 5 & 2 & 0.00 & 99.98 & & Bot $\mathrm{X} 5 \mathrm{~L}$ reb/low & FALSE \\
\hline 2005 & $\mathrm{~F}$ & 5 & 3 & 1.01 & 99.12 & BIS & & FALSE \\
\hline 2005 & $\mathrm{~F}$ & 5 & 4 & 2.74 & 98.41 & BIS & & FALSE \\
\hline 2005 & $\mathrm{~F}$ & 5 & 5 & 3.32 & 98.25 & EW & & FALSE \\
\hline 2005 & $\mathrm{~F}$ & 5 & 6 & 4.48 & 98.24 & $\mathrm{TW}$ & & FALSE \\
\hline 2005 & $\mathrm{~F}$ & 5 & 7 & 4.79 & 98.25 & EW & & FALSE \\
\hline 2005 & $\mathrm{~F}$ & 5 & 8 & 5.15 & 98.42 & BIS & on top of boulder & FALSE \\
\hline 2005 & $\mathrm{~F}$ & 5 & 9 & 5.64 & 98.42 & $\mathrm{BF}$ & & FALSE \\
\hline 2005 & $\mathrm{~F}$ & 5 & 10 & 6.58 & 98.90 & BIS & & FALSE \\
\hline 2005 & $\mathrm{~F}$ & 5 & 11 & 7.71 & 99.79 & BOT X5R & Bot $\mathrm{X} 5 \mathrm{R}$ reb/low & FALSE \\
\hline 2005 & $\mathrm{~F}$ & 5 & 12 & 7.71 & 99.88 & TOP X5R & Top X5R reb/low & FALSE \\
\hline 2005 & $\mathrm{~F}$ & 6 & 1 & 0.00 & 100.00 & & Top X6L reb/low & FALSE \\
\hline 2005 & $\mathrm{~F}$ & 6 & 2 & 0.00 & 99.85 & & Bot $\mathrm{X} 6 \mathrm{~L} \mathrm{reb} /$ low & FALSE \\
\hline 2005 & $\mathrm{~F}$ & 6 & 3 & 1.40 & 99.36 & BIS & & FALSE \\
\hline 2005 & $\mathrm{~F}$ & 6 & 4 & 1.74 & 98.75 & BIS & & FALSE \\
\hline 2005 & $\mathrm{~F}$ & 6 & 5 & 2.04 & 99.05 & BIS & on top of $\log$ & FALSE \\
\hline 2005 & $\mathrm{~F}$ & 6 & 6 & 2.29 & 98.82 & BIS & on top of boulder & FALSE \\
\hline 2005 & $\mathrm{~F}$ & 6 & 7 & 2.50 & 98.34 & BIS & $\begin{array}{l}\text { side poolish; away from main } \\
\text { channel }\end{array}$ & FALSE \\
\hline 2005 & $\mathrm{~F}$ & 6 & 8 & 3.44 & 98.21 & EW & & FALSE \\
\hline 2005 & $\mathrm{~F}$ & 6 & 9 & 4.21 & 98.04 & $\mathrm{TW}$ & & FALSE \\
\hline 2005 & $\mathrm{~F}$ & 6 & 10 & 4.79 & 98.19 & EW & & FALSE \\
\hline 2005 & $\mathrm{~F}$ & 6 & 11 & 5.43 & 99.02 & BIS & on top of boulder & FALSE \\
\hline 2005 & $\mathrm{~F}$ & 6 & 12 & 6.28 & 99.15 & BIS & & FALSE \\
\hline 2005 & $\mathrm{~F}$ & 6 & 13 & 7.38 & 99.39 & BIS & & FALSE \\
\hline 2005 & $\mathrm{~F}$ & 6 & 14 & 8.41 & 99.86 & BOT X1R & Bot $\mathrm{X} 6 \mathrm{R}$ reb/low & FALSE \\
\hline 2005 & $\mathrm{~F}$ & 6 & 15 & 8.41 & 99.97 & TOP X1R & Top X6R reb/low & FALSE \\
\hline 2005 & $\mathrm{~F}$ & 7 & 1 & 0.00 & 100.00 & & Top X7L reb/low & FALSE \\
\hline 2005 & $\mathrm{~F}$ & 7 & 2 & 0.00 & 99.98 & & Bot $\mathrm{X} 7 \mathrm{~L}$ reb/low & FALSE \\
\hline 2005 & $\mathrm{~F}$ & 7 & 3 & 0.30 & 99.75 & BIS & & FALSE \\
\hline 2005 & $\mathrm{~F}$ & 7 & 4 & 1.04 & 98.98 & BIS & & FALSE \\
\hline 2005 & $\mathrm{~F}$ & 7 & 5 & 1.77 & 98.47 & EW & & FALSE \\
\hline 2005 & $\mathrm{~F}$ & 7 & 6 & 2.62 & 98.38 & TW & & FALSE \\
\hline 2005 & $\mathrm{~F}$ & 7 & 7 & 3.69 & 98.48 & EW & & FALSE \\
\hline
\end{tabular}

Continued on Next Page... 
Table D.1 - Continued

\begin{tabular}{|c|c|c|c|c|c|c|c|c|}
\hline Year & Reach & Section & Point & Dist. (m) & Elev. $(\mathrm{m})$ & Desc. & Notes & Exclude \\
\hline 2005 & $\mathrm{~F}$ & 7 & 8 & 4.02 & 98.55 & BIS & & FALSE \\
\hline 2005 & $\mathrm{~F}$ & 7 & 9 & 4.36 & 98.86 & BIS & on top of boulder & FALSE \\
\hline 2005 & $\mathrm{~F}$ & 7 & 10 & 4.57 & 98.80 & $\mathrm{BF}$ & & FALSE \\
\hline 2005 & $\mathrm{~F}$ & 7 & 11 & 5.12 & 98.86 & BIS & & FALSE \\
\hline 2005 & $\mathrm{~F}$ & 7 & 12 & 5.79 & 99.01 & BIS & & FALSE \\
\hline 2005 & $\mathrm{~F}$ & 7 & 13 & 6.80 & 99.28 & BIS & & FALSE \\
\hline 2005 & $\mathrm{~F}$ & 7 & 14 & 8.23 & 99.58 & BIS & & FALSE \\
\hline 2005 & $\mathrm{~F}$ & 7 & 15 & 8.72 & 99.85 & BOT X7R & Bot $\mathrm{X} 7 \mathrm{R}$ reb/low & FALSE \\
\hline 2005 & $\mathrm{~F}$ & 7 & 16 & 8.72 & 99.95 & TOP X7R & Top X7R reb/low & FALSE \\
\hline 2005 & $\mathrm{~F}$ & 8 & 1 & 0.00 & 100.00 & & Top X8L reb/low & FALSE \\
\hline 2005 & $\mathrm{~F}$ & 8 & 2 & 0.00 & 99.90 & & Bot $\mathrm{X} 8 \mathrm{~L}$ reb/low & FALSE \\
\hline 2005 & $\mathrm{~F}$ & 8 & 3 & 0.64 & 99.38 & BIS & & FALSE \\
\hline 2005 & F & 8 & 4 & 0.98 & 99.14 & BIS & & FALSE \\
\hline 2005 & $\mathrm{~F}$ & 8 & 5 & 1.22 & 98.54 & BIS & & FALSE \\
\hline 2005 & $\mathrm{~F}$ & 8 & 6 & 1.52 & 98.41 & BIS & & FALSE \\
\hline 2005 & $\mathrm{~F}$ & 8 & 7 & 2.13 & 98.20 & $\mathrm{BF}$ & & FALSE \\
\hline 2005 & F & 8 & 8 & 2.62 & 97.88 & BIS & & FALSE \\
\hline 2005 & F & 8 & 9 & 3.63 & 97.78 & EW & & FALSE \\
\hline 2005 & $\mathrm{~F}$ & 8 & 10 & 4.27 & 97.72 & $\mathrm{TW}$ & & FALSE \\
\hline 2005 & $\mathrm{~F}$ & 8 & 11 & 4.82 & 97.79 & EW & & FALSE \\
\hline 2005 & F & 8 & 12 & 5.09 & 98.27 & BIS & & FALSE \\
\hline 2005 & F & 8 & 13 & 6.16 & 98.56 & BIS & & FALSE \\
\hline 2005 & $\mathrm{~F}$ & 8 & 14 & 7.32 & 98.77 & BIS & & FALSE \\
\hline 2005 & $\mathrm{~F}$ & 8 & 15 & 8.99 & 98.72 & BIS & & FALSE \\
\hline 2005 & $\mathrm{~F}$ & 8 & 16 & 9.57 & 99.16 & BIS & & FALSE \\
\hline 2005 & F & 8 & 17 & 10.42 & 99.52 & BIS & & FALSE \\
\hline 2005 & F & 8 & 18 & 11.31 & 99.82 & ТОВ & & FALSE \\
\hline 2005 & $\mathrm{~F}$ & 8 & 19 & 11.89 & 99.82 & BIS & & FALSE \\
\hline 2005 & $\mathrm{~F}$ & 8 & 20 & 12.71 & 99.85 & BOT X8R & Bot $\mathrm{X} 8 \mathrm{R}$ reb/low & FALSE \\
\hline 2005 & $\mathrm{~F}$ & 8 & 21 & 12.71 & 100.03 & TOP X8R & Top X8R reb/low & FALSE \\
\hline 2005 & $\mathrm{~F}$ & 9 & 1 & 0.00 & 100.00 & & Top X9L reb/low & FALSE \\
\hline 2005 & $\mathrm{~F}$ & 9 & 2 & 0.00 & 99.84 & & Bot $\mathrm{X} 9 \mathrm{~L} \mathrm{reb} /$ low & FALSE \\
\hline 2005 & $\mathrm{~F}$ & 9 & 3 & 0.46 & 99.23 & BIS & & FALSE \\
\hline 2005 & $\mathrm{~F}$ & 9 & 4 & 1.25 & 98.80 & $\mathrm{BF}$ & & FALSE \\
\hline 2005 & $\mathrm{~F}$ & 9 & 5 & 1.95 & 98.63 & BIS & & FALSE \\
\hline 2005 & $\mathrm{~F}$ & 9 & 6 & 2.26 & 98.49 & EW & & FALSE \\
\hline 2005 & $\mathrm{~F}$ & 9 & 7 & 3.35 & 98.30 & $\mathrm{TW}$ & & FALSE \\
\hline 2005 & $\mathrm{~F}$ & 9 & 8 & 3.78 & 98.40 & EW & & FALSE \\
\hline 2005 & $\mathrm{~F}$ & 9 & 9 & 4.30 & 99.06 & ТОВ & & FALSE \\
\hline 2005 & $\mathrm{~F}$ & 9 & 10 & 5.06 & 99.12 & BIS & & FALSE \\
\hline 2005 & $\mathrm{~F}$ & 9 & 11 & 5.73 & 99.26 & BIS & on top of $\log$ & FALSE \\
\hline 2005 & $\mathrm{~F}$ & 9 & 12 & 6.49 & 99.17 & BIS & & FALSE \\
\hline 2005 & $\mathrm{~F}$ & 9 & 13 & 7.59 & 99.10 & BIS & & FALSE \\
\hline 2005 & $\mathrm{~F}$ & 9 & 14 & 9.69 & 99.02 & BIS & & FALSE \\
\hline 2005 & $\mathrm{~F}$ & 9 & 15 & 10.09 & 99.55 & BIS & on top of boulder & FALSE \\
\hline 2005 & $\mathrm{~F}$ & 9 & 16 & 10.36 & 99.55 & BIS & & FALSE \\
\hline 2005 & $\mathrm{~F}$ & 9 & 17 & 11.25 & 99.78 & BOT X9R & Bot X9R reb/low & FALSE \\
\hline 2005 & $\mathrm{~F}$ & 9 & 18 & 11.25 & 99.96 & TOP X9R & Top X9R reb/low & FALSE \\
\hline 2005 & $\mathrm{~F}$ & 10 & 1 & 0.00 & 100.00 & & Top X9L reb/low & FALSE \\
\hline 2005 & $\mathrm{~F}$ & 10 & 2 & 0.00 & 99.84 & & Bot $\mathrm{X} 9 \mathrm{~L} \mathrm{reb} / \mathrm{low}$ & FALSE \\
\hline 2005 & $\mathrm{~F}$ & 10 & 3 & 0.46 & 99.23 & BIS & & FALSE \\
\hline 2005 & $\mathrm{~F}$ & 10 & 4 & 1.25 & 98.80 & $\mathrm{BF}$ & & FALSE \\
\hline
\end{tabular}

Continued on Next Page... 
Table D.1 - Continued

\begin{tabular}{|c|c|c|c|c|c|c|c|c|}
\hline Year & Reach & Section & Point & Dist. (m) & Elev. $(\mathrm{m})$ & Desc. & Notes & Exclude \\
\hline 2005 & $\mathrm{~F}$ & 10 & 5 & 1.95 & 98.63 & BIS & & FALSE \\
\hline 2005 & $\mathrm{~F}$ & 10 & 6 & 2.26 & 98.49 & EW & & FALSE \\
\hline 2005 & $\mathrm{~F}$ & 10 & 7 & 3.35 & 98.30 & TW & & FALSE \\
\hline 2005 & $\mathrm{~F}$ & 10 & 8 & 3.78 & 98.40 & EW & & FALSE \\
\hline 2005 & $\mathrm{~F}$ & 10 & 9 & 4.30 & 99.06 & ТОВ & & FALSE \\
\hline 2005 & F & 10 & 10 & 5.06 & 99.12 & BIS & & FALSE \\
\hline 2005 & $\mathrm{~F}$ & 10 & 11 & 5.73 & 99.26 & BIS & on top of $\log$ & FALSE \\
\hline 2005 & $\mathrm{~F}$ & 10 & 12 & 6.49 & 99.17 & BIS & & FALSE \\
\hline 2005 & $\mathrm{~F}$ & 10 & 13 & 7.59 & 99.10 & BIS & & FALSE \\
\hline 2005 & $\mathrm{~F}$ & 10 & 14 & 9.69 & 99.02 & BIS & & FALSE \\
\hline 2005 & $\mathrm{~F}$ & 10 & 15 & 10.09 & 99.55 & BIS & on top of boulder & FALSE \\
\hline 2005 & $\mathrm{~F}$ & 10 & 16 & 10.36 & 99.55 & BIS & & FALSE \\
\hline 2005 & $\mathrm{~F}$ & 10 & 17 & 11.25 & 99.78 & BOT X9R & Bot $\mathrm{X} 9 \mathrm{R}$ reb/low & FALSE \\
\hline 2005 & $\mathrm{~F}$ & 10 & 18 & 11.25 & 99.96 & TOP X9R & Top X9R reb/low & FALSE \\
\hline 2006 & $\mathrm{~A}$ & 1 & 1 & 0.00 & 100.00 & TOP X1L & Top X1L reb/low & FALSE \\
\hline 2006 & $\mathrm{~A}$ & 1 & 2 & 0.00 & 99.87 & BOT X1L & Bot $\mathrm{X} 1 \mathrm{~L}$ reb/low & FALSE \\
\hline 2006 & A & 1 & 3 & 0.70 & 99.69 & ТОВ & & FALSE \\
\hline 2006 & A & 1 & 4 & 1.52 & 99.56 & BIS & & FALSE \\
\hline 2006 & $\mathrm{~A}$ & 1 & 5 & 2.41 & 99.38 & BIS & & FALSE \\
\hline 2006 & $\mathrm{~A}$ & 1 & 6 & 3.14 & 99.23 & BIS & & FALSE \\
\hline 2006 & $\mathrm{~A}$ & 1 & 7 & 3.63 & 98.94 & BIS & & FALSE \\
\hline 2006 & $\mathrm{~A}$ & 1 & 8 & 4.05 & 98.74 & EW & & FALSE \\
\hline 2006 & $\mathrm{~A}$ & 1 & 9 & 4.75 & 98.58 & $\mathrm{TW}$ & & FALSE \\
\hline 2006 & $\mathrm{~A}$ & 1 & 10 & 5.70 & 98.74 & EW & & FALSE \\
\hline 2006 & $\mathrm{~A}$ & 1 & 11 & 6.19 & 98.87 & BIS & & FALSE \\
\hline 2006 & $\mathrm{~A}$ & 1 & 12 & 6.86 & 99.19 & $\mathrm{BF}$ & & FALSE \\
\hline 2006 & $\mathrm{~A}$ & 1 & 13 & 7.59 & 99.37 & ТОВ & & FALSE \\
\hline 2006 & $\mathrm{~A}$ & 1 & 14 & 9.11 & 99.81 & BOT X1R & Bot $\mathrm{X} 1 \mathrm{R}$ reb/low & FALSE \\
\hline 2006 & $\mathrm{~A}$ & 1 & 15 & 9.11 & 99.99 & TOP X1R & Top X1R reb/low & FALSE \\
\hline 2006 & $\mathrm{~A}$ & 2 & 1 & 0.00 & 100.00 & TOP X2L & Top X2L reb/low & FALSE \\
\hline 2006 & $\mathrm{~A}$ & 2 & 2 & 0.00 & 99.71 & BOT X2L & Bot $\mathrm{X} 2 \mathrm{~L} \mathrm{reb} /$ low & FALSE \\
\hline 2006 & $\mathrm{~A}$ & 2 & 3 & 0.94 & 99.54 & ТОВ & & FALSE \\
\hline 2006 & A & 2 & 4 & 1.86 & 99.07 & BIS & & FALSE \\
\hline 2006 & $\mathrm{~A}$ & 2 & 5 & 2.99 & 98.77 & $\mathrm{BF}$ & & FALSE \\
\hline 2006 & $\mathrm{~A}$ & 2 & 6 & 3.90 & 98.46 & BIS & & FALSE \\
\hline 2006 & A & 2 & 7 & 4.63 & 98.13 & EW & & FALSE \\
\hline 2006 & $\mathrm{~A}$ & 2 & 8 & 6.19 & 97.98 & TW & & FALSE \\
\hline 2006 & A & 2 & 9 & 7.01 & 98.13 & EW & & FALSE \\
\hline 2006 & A & 2 & 10 & 7.68 & 98.58 & BIS & & FALSE \\
\hline 2006 & $\mathrm{~A}$ & 2 & 11 & 8.32 & 98.80 & BIS & on top of boulder & FALSE \\
\hline 2006 & A & 2 & 12 & 8.60 & 99.15 & BIS & on top of boulder & FALSE \\
\hline 2006 & $\mathrm{~A}$ & 2 & 13 & 9.05 & 99.13 & BIS & on top of boulder & FALSE \\
\hline 2006 & $\mathrm{~A}$ & 2 & 14 & 9.36 & 98.98 & BIS & & FALSE \\
\hline 2006 & A & 2 & 15 & 9.75 & 99.38 & BIS & & FALSE \\
\hline 2006 & A & 2 & 16 & 10.67 & 99.66 & BOT X2R & Bot $\mathrm{X} 2 \mathrm{R}$ reb/low & FALSE \\
\hline 2006 & A & 2 & 17 & 10.67 & 99.97 & TOP X2R & Top X2R reb/low & FALSE \\
\hline 2006 & A & 3 & 1 & 0.00 & 100.00 & TOP X3L & Top X3L reb/low & FALSE \\
\hline 2006 & A & 3 & 2 & 0.00 & 99.50 & BOT X3L & Bot X3L reb/low & FALSE \\
\hline 2006 & A & 3 & 3 & 0.79 & 99.87 & BIS & $\begin{array}{l}\text { On top of alder extending from } \\
\text { bank to bank }\end{array}$ & TRUE \\
\hline 2006 & A & 3 & 4 & 1.01 & 98.81 & BIS & & FALSE \\
\hline 2006 & A & 3 & 5 & 1.31 & 98.70 & BIS & & FALSE \\
\hline
\end{tabular}

Continued on Next Page... 
Table D.1 - Continued

\begin{tabular}{|c|c|c|c|c|c|c|c|c|}
\hline Year & Reach & Section & Point & Dist. (m) & Elev. $(\mathrm{m})$ & Desc. & Notes & Exclude \\
\hline 2006 & A & 3 & 6 & 1.80 & 98.24 & BIS & & FALSE \\
\hline 2006 & A & 3 & 7 & 2.23 & 97.89 & EW & & FALSE \\
\hline 2006 & $\mathrm{~A}$ & 3 & 8 & 3.20 & 97.78 & TW & & FALSE \\
\hline 2006 & A & 3 & 9 & 4.18 & 97.91 & EW & & FALSE \\
\hline 2006 & A & 3 & 10 & 5.24 & 98.20 & BIS & & FALSE \\
\hline 2006 & A & 3 & 11 & 5.88 & 98.46 & BIS & & FALSE \\
\hline 2006 & $\mathrm{~A}$ & 3 & 12 & 7.16 & 98.53 & BIS & & FALSE \\
\hline 2006 & $\mathrm{~A}$ & 3 & 13 & 7.65 & 98.45 & $\mathrm{BF}$ & & FALSE \\
\hline 2006 & A & 3 & 14 & 8.78 & 98.74 & BIS & & FALSE \\
\hline 2006 & $\mathrm{~A}$ & 3 & 15 & 9.75 & 99.35 & BIS & & FALSE \\
\hline 2006 & $\mathrm{~A}$ & 3 & 16 & 10.73 & 99.73 & BOT X3R & Bot X3R reb/low & FALSE \\
\hline 2006 & $\mathrm{~A}$ & 3 & 17 & 10.73 & 100.15 & TOP X3R & Top X3R reb/low & FALSE \\
\hline 2006 & $\mathrm{~A}$ & 4 & 1 & 0.00 & 100.00 & TOP X4L & Top X4L reb/low & FALSE \\
\hline 2006 & $\mathrm{~A}$ & 4 & 2 & 0.00 & 99.72 & BOT X4L & Bot $\mathrm{X} 4 \mathrm{~L} \mathrm{reb} /$ low & FALSE \\
\hline 2006 & $\mathrm{~A}$ & 4 & 3 & 0.67 & 99.64 & BIS & & FALSE \\
\hline 2006 & $\mathrm{~A}$ & 4 & 4 & 2.01 & 99.36 & ТОВ & & FALSE \\
\hline 2006 & $\mathrm{~A}$ & 4 & 5 & 2.80 & 98.70 & $\mathrm{BF}$ & & FALSE \\
\hline 2006 & $\mathrm{~A}$ & 4 & 6 & 3.29 & 98.54 & EW & & FALSE \\
\hline 2006 & $\mathrm{~A}$ & 4 & 7 & 4.94 & 98.45 & TW & & FALSE \\
\hline 2006 & $\mathrm{~A}$ & 4 & 8 & 5.73 & 98.54 & EW & & FALSE \\
\hline 2006 & $\mathrm{~A}$ & 4 & 9 & 6.80 & 98.98 & BIS & & FALSE \\
\hline 2006 & $\mathrm{~A}$ & 4 & 10 & 7.62 & 99.13 & BIS & & FALSE \\
\hline 2006 & $\mathrm{~A}$ & 4 & 11 & 8.05 & 99.34 & BIS & & FALSE \\
\hline 2006 & $\mathrm{~A}$ & 4 & 12 & 8.66 & 99.47 & BOT X4R & Bot $\mathrm{X} 4 \mathrm{R}$ reb/low & FALSE \\
\hline 2006 & $\mathrm{~A}$ & 4 & 13 & 8.66 & 99.83 & TOP X4R & Top X4R reb/low & FALSE \\
\hline 2006 & A & 5 & 1 & 0.00 & 100.00 & TOP X5L & Top X5L reb/low & FALSE \\
\hline 2006 & $\mathrm{~A}$ & 5 & 2 & 0.00 & 99.74 & BOT X5L & Bot $\mathrm{X} 5 \mathrm{~L} \mathrm{reb} /$ low & FALSE \\
\hline 2006 & $\mathrm{~A}$ & 5 & 3 & 1.16 & 99.79 & ТОВ & & FALSE \\
\hline 2006 & $\mathrm{~A}$ & 5 & 4 & 1.95 & 99.72 & BIS & log extending from ТОВ to water & FALSE \\
\hline 2006 & $\mathrm{~A}$ & 5 & 5 & 2.38 & 99.04 & $\mathrm{BF}$ & & FALSE \\
\hline 2006 & A & 5 & 6 & 3.17 & 98.64 & EW & & FALSE \\
\hline 2006 & A & 5 & 7 & 4.11 & 98.54 & TW & & FALSE \\
\hline 2006 & A & 5 & 8 & 5.12 & 98.66 & EW & & FALSE \\
\hline 2006 & A & 5 & 9 & 6.22 & 99.13 & BIS & & FALSE \\
\hline 2006 & $\mathrm{~A}$ & 5 & 10 & 7.07 & 99.46 & BIS & & FALSE \\
\hline 2006 & A & 5 & 11 & 7.77 & 99.31 & BOT X5R & Bot $\mathrm{X} 5 \mathrm{R}$ reb/low & FALSE \\
\hline 2006 & $\mathrm{~A}$ & 5 & 12 & 7.77 & 99.63 & TOP X5R & Top X5R reb/low & FALSE \\
\hline 2006 & $\mathrm{~A}$ & 6 & 1 & 0.00 & 100.00 & TOP X5L & Top X6L reb/low & FALSE \\
\hline 2006 & $\mathrm{~A}$ & 6 & 2 & 0.00 & 99.77 & BOT X5L & Bot $\mathrm{X} 6 \mathrm{~L} \mathrm{reb} /$ low & FALSE \\
\hline 2006 & $\mathrm{~A}$ & 6 & 3 & 0.24 & 99.64 & ТОВ & & FALSE \\
\hline 2006 & A & 6 & 4 & 0.98 & 98.32 & $\mathrm{BF}$ & & FALSE \\
\hline 2006 & $\mathrm{~A}$ & 6 & 5 & 1.86 & 97.97 & EW & & FALSE \\
\hline 2006 & $\mathrm{~A}$ & 6 & 6 & 2.80 & 97.87 & TW & & FALSE \\
\hline 2006 & $\mathrm{~A}$ & 6 & 7 & 3.72 & 98.00 & EW & & FALSE \\
\hline 2006 & A & 6 & 8 & 4.30 & 98.58 & BIS & & FALSE \\
\hline 2006 & $\mathrm{~A}$ & 6 & 9 & 5.40 & 99.00 & ТОВ & & FALSE \\
\hline 2006 & $\mathrm{~A}$ & 6 & 10 & 6.58 & 99.20 & BIS & & FALSE \\
\hline 2006 & A & 6 & 11 & 8.99 & 99.22 & BIS & & FALSE \\
\hline 2006 & $\mathrm{~A}$ & 6 & 12 & 12.38 & 99.09 & BIS & & FALSE \\
\hline 2006 & $\mathrm{~A}$ & 6 & 13 & 14.36 & 99.13 & BIS & & FALSE \\
\hline 2006 & $\mathrm{~A}$ & 6 & 14 & 15.03 & 99.43 & BOT X6R & Bot $\mathrm{X} 6 \mathrm{R}$ reb/low & FALSE \\
\hline 2006 & $\mathrm{~A}$ & 6 & 15 & 15.03 & 99.83 & TOP X6R & Top X6R reb/low & FALSE \\
\hline
\end{tabular}

Continued on Next Page... 
Table D.1 - Continued

\begin{tabular}{|c|c|c|c|c|c|c|c|c|}
\hline Year & Reach & Section & Point & Dist. (m) & Elev. $(\mathrm{m})$ & Desc. & Notes & Exclude \\
\hline 2006 & A & 7 & 1 & 0.00 & 100.00 & TOP X6L & Top X7L reb/low & FALSE \\
\hline 2006 & A & 7 & 2 & 0.00 & 99.78 & BOT X6L & Bot $\mathrm{X} 7 \mathrm{~L}$ reb/low & FALSE \\
\hline 2006 & A & 7 & 3 & 0.61 & 99.70 & TOB & & FALSE \\
\hline 2006 & A & 7 & 4 & 0.79 & 99.33 & BIS & & FALSE \\
\hline 2006 & A & 7 & 5 & 1.40 & 98.42 & BIS & & FALSE \\
\hline 2006 & A & 7 & 6 & 1.68 & 98.62 & $\mathrm{BF}$ & & FALSE \\
\hline 2006 & A & 7 & 7 & 2.50 & 98.22 & EW & & FALSE \\
\hline 2006 & A & 7 & 8 & 3.54 & 98.13 & TW & & FALSE \\
\hline 2006 & $\mathrm{~A}$ & 7 & 9 & 4.63 & 98.29 & EW & & FALSE \\
\hline 2006 & A & 7 & 10 & 5.46 & 98.63 & BIS & & FALSE \\
\hline 2006 & A & 7 & 11 & 6.68 & 98.95 & ТОВ & & FALSE \\
\hline 2006 & A & 7 & 12 & 8.08 & 99.17 & BIS & & FALSE \\
\hline 2006 & A & 7 & 13 & 9.63 & 99.47 & BIS & & FALSE \\
\hline 2006 & $\mathrm{~A}$ & 7 & 14 & 10.94 & 99.56 & BIS & & FALSE \\
\hline 2006 & $\mathrm{~A}$ & 7 & 15 & 15.33 & 99.29 & BIS & & FALSE \\
\hline 2006 & A & 7 & 16 & 17.83 & 99.42 & BIS & & FALSE \\
\hline 2006 & A & 7 & 17 & 18.71 & 99.85 & BOT X7R & Bot $\mathrm{X} 7 \mathrm{R}$ reb/low & FALSE \\
\hline 2006 & A & 7 & 18 & 18.71 & 100.22 & TOP X7R & Top X7R reb/low & FALSE \\
\hline 2006 & A & 8 & 1 & 0.00 & 100.00 & TOP X8L & Top X8L reb/low & FALSE \\
\hline 2006 & A & 8 & 2 & 0.00 & 99.65 & BOT X8L & Bot $\mathrm{X} 8 \mathrm{~L} \mathrm{reb} /$ low & FALSE \\
\hline 2006 & $\mathrm{~A}$ & 8 & 3 & 0.64 & 99.57 & ТОВ & & FALSE \\
\hline 2006 & $\mathrm{~A}$ & 8 & 4 & 1.04 & 98.47 & BIS & & FALSE \\
\hline 2006 & $\mathrm{~A}$ & 8 & 5 & 1.86 & 98.32 & $\mathrm{BF}$ & & FALSE \\
\hline 2006 & $\mathrm{~A}$ & 8 & 6 & 2.44 & 98.11 & BIS & & FALSE \\
\hline 2006 & A & 8 & 7 & 3.20 & 97.92 & EW & & FALSE \\
\hline 2006 & A & 8 & 8 & 4.21 & 97.76 & TW & & FALSE \\
\hline 2006 & $\mathrm{~A}$ & 8 & 9 & 5.52 & 97.93 & EW & & FALSE \\
\hline 2006 & $\mathrm{~A}$ & 8 & 10 & 6.22 & 98.42 & ТОВ & & FALSE \\
\hline 2006 & $\mathrm{~A}$ & 8 & 11 & 8.84 & 98.77 & BIS & & FALSE \\
\hline 2006 & $\mathrm{~A}$ & 8 & 12 & 10.64 & 98.87 & BIS & & FALSE \\
\hline 2006 & A & 8 & 13 & 12.13 & 99.22 & BIS & & FALSE \\
\hline 2006 & A & 8 & 14 & 15.67 & 99.07 & BIS & & FALSE \\
\hline 2006 & $\mathrm{~A}$ & 8 & 15 & 18.07 & 99.15 & BIS & & FALSE \\
\hline 2006 & A & 8 & 16 & 18.87 & 99.31 & BIS & & FALSE \\
\hline 2006 & $\mathrm{~A}$ & 8 & 17 & 19.54 & 99.63 & BOT X8R & Bot $\mathrm{X} 8 \mathrm{R}$ reb/low & FALSE \\
\hline 2006 & $\mathrm{~A}$ & 8 & 18 & 19.54 & 99.96 & TOP X8R & Top X8R reb/low & FALSE \\
\hline 2006 & A & 9 & 1 & 0.00 & 100.00 & TOP X9L & Top X9L reb/low & FALSE \\
\hline 2006 & $\mathrm{~A}$ & 9 & 2 & 0.00 & 99.64 & BOT X9L & Bot X9L reb/low & FALSE \\
\hline 2006 & A & 9 & 3 & 0.55 & 99.68 & ТОВ & & FALSE \\
\hline 2006 & A & 9 & 4 & 0.79 & 98.74 & BIS & & FALSE \\
\hline 2006 & A & 9 & 5 & 1.98 & 98.51 & BIS & & FALSE \\
\hline 2006 & A & 9 & 6 & 2.96 & 98.49 & BIS & & FALSE \\
\hline 2006 & A & 9 & 7 & 3.69 & 98.41 & BIS & & FALSE \\
\hline 2006 & A & 9 & 8 & 3.96 & 98.34 & BIS & & FALSE \\
\hline 2006 & A & 9 & 9 & 4.94 & 98.09 & EW & & FALSE \\
\hline 2006 & A & 9 & 10 & 6.00 & 97.98 & TW & & FALSE \\
\hline 2006 & A & 9 & 11 & 6.77 & 98.08 & EW & & FALSE \\
\hline 2006 & A & 9 & 12 & 7.07 & 98.26 & BIS & & FALSE \\
\hline 2006 & A & 9 & 13 & 7.96 & 98.52 & $\mathrm{BF}$ & & FALSE \\
\hline 2006 & A & 9 & 14 & 8.44 & 98.79 & ТОВ & & FALSE \\
\hline 2006 & A & 9 & 15 & 10.18 & 98.96 & BIS & & FALSE \\
\hline 2006 & A & 9 & 16 & 12.44 & 99.09 & BIS & & FALSE \\
\hline
\end{tabular}

Continued on Next Page... 
Table D.1 - Continued

\begin{tabular}{|c|c|c|c|c|c|c|c|c|}
\hline Year & Reach & Section & Point & Dist. (m) & Elev. $(\mathrm{m})$ & Desc. & Notes & Exclude \\
\hline 2006 & $\mathrm{~A}$ & 9 & 17 & 13.17 & 99.45 & BIS & & FALSE \\
\hline 2006 & A & 9 & 18 & 14.81 & 99.33 & BIS & & FALSE \\
\hline 2006 & A & 9 & 19 & 17.34 & 99.21 & BIS & & FALSE \\
\hline 2006 & A & 9 & 20 & 19.51 & 99.41 & BIS & & FALSE \\
\hline 2006 & A & 9 & 21 & 20.12 & 99.63 & BIS & & FALSE \\
\hline 2006 & A & 9 & 22 & 20.57 & 99.88 & BOT X9R & Bot $\mathrm{X} 9 \mathrm{R}$ reb/low & FALSE \\
\hline 2006 & A & 9 & 23 & 20.57 & 100.23 & TOP X9R & Top X9R reb/low & FALSE \\
\hline 2006 & $\mathrm{~A}$ & 10 & 1 & 0.00 & 100.00 & TOP X10L & Top X10L reb/low & FALSE \\
\hline 2006 & A & 10 & 2 & 0.00 & 99.61 & BOT X10L & Bot X10L reb/low & FALSE \\
\hline 2006 & A & 10 & 3 & 0.91 & 98.57 & ТОВ & & FALSE \\
\hline 2006 & A & 10 & 4 & 2.53 & 98.44 & BIS & & FALSE \\
\hline 2006 & A & 10 & 5 & 3.78 & 98.24 & $\mathrm{BF}$ & & FALSE \\
\hline 2006 & $\mathrm{~A}$ & 10 & 6 & 4.75 & 98.18 & BIS & & FALSE \\
\hline 2006 & $\mathrm{~A}$ & 10 & 7 & 5.91 & 97.67 & BIS & & FALSE \\
\hline 2006 & A & 10 & 8 & 8.84 & 97.50 & EW & & FALSE \\
\hline 2006 & A & 10 & 9 & 9.63 & 98.21 & TW & top of boulder & FALSE \\
\hline 2006 & A & 10 & 10 & 10.67 & 98.64 & EW & & FALSE \\
\hline 2006 & $\mathrm{~A}$ & 10 & 11 & 13.05 & 98.97 & BIS & & FALSE \\
\hline 2006 & A & 10 & 12 & 15.61 & 99.07 & BIS & & FALSE \\
\hline 2006 & A & 10 & 13 & 17.01 & 98.99 & BIS & & FALSE \\
\hline 2006 & $\mathrm{~A}$ & 10 & 14 & 18.99 & 99.35 & BIS & & FALSE \\
\hline 2006 & $\mathrm{~A}$ & 10 & 15 & 20.21 & 99.81 & BOT X10R & Bot $\mathrm{X} 10 \mathrm{R} \mathrm{reb} /$ low & FALSE \\
\hline 2006 & A & 10 & 16 & 20.21 & 100.01 & TOP X10R & Top X10R reb/low & FALSE \\
\hline 2006 & $\mathrm{~B}$ & 1 & 1 & 0.00 & 100.00 & TOP X1L & Top X1L reb/low & FALSE \\
\hline 2006 & $\mathrm{~B}$ & 1 & 2 & 0.00 & 99.87 & BOT X1L & Bot $\mathrm{X} 1 \mathrm{~L}$ reb/low & FALSE \\
\hline 2006 & $\mathrm{~B}$ & 1 & 3 & 0.43 & 99.23 & BIS & & FALSE \\
\hline 2006 & $\mathrm{~B}$ & 1 & 4 & 1.16 & 98.35 & BIS & & FALSE \\
\hline 2006 & B & 1 & 5 & 1.95 & 98.05 & BIS & & FALSE \\
\hline 2006 & $\mathrm{~B}$ & 1 & 6 & 2.56 & 97.73 & BIS & & FALSE \\
\hline 2006 & $\mathrm{~B}$ & 1 & 7 & 2.87 & 97.59 & EW & & FALSE \\
\hline 2006 & $\mathrm{~B}$ & 1 & 8 & 3.35 & 97.52 & TW & & FALSE \\
\hline 2006 & $\mathrm{~B}$ & 1 & 9 & 5.82 & 97.58 & EW & & FALSE \\
\hline 2006 & $\mathrm{~B}$ & 1 & 10 & 7.16 & 97.88 & $\mathrm{BF}$ & & FALSE \\
\hline 2006 & $\mathrm{~B}$ & 1 & 11 & 8.14 & 98.58 & BIS & & FALSE \\
\hline 2006 & B & 1 & 12 & 9.08 & 99.14 & BIS & & FALSE \\
\hline 2006 & $\mathrm{~B}$ & 1 & 13 & 10.03 & 99.80 & BOT X1R & Bot $\mathrm{X} 1 \mathrm{R}$ reb/low & FALSE \\
\hline 2006 & $\mathrm{~B}$ & 1 & 14 & 10.03 & 99.99 & TOP X1R & Top X1R reb/low & FALSE \\
\hline 2006 & $\mathrm{~B}$ & 2 & 1 & 0.00 & 100.00 & TOP X2L & Top X2L reb/low & FALSE \\
\hline 2006 & $\mathrm{~B}$ & 2 & 2 & 0.00 & 99.60 & BOT X2L & Bot $\mathrm{X} 2 \mathrm{~L} \mathrm{reb} /$ low & FALSE \\
\hline 2006 & $\mathrm{~B}$ & 2 & 3 & 0.27 & 99.08 & BIS & & FALSE \\
\hline 2006 & B & 2 & 4 & 0.73 & 98.87 & BIS & & FALSE \\
\hline 2006 & $\mathrm{~B}$ & 2 & 5 & 1.83 & 98.38 & BIS & & FALSE \\
\hline 2006 & $\mathrm{~B}$ & 2 & 6 & 2.50 & 97.76 & BIS & & FALSE \\
\hline 2006 & $\mathrm{~B}$ & 2 & 7 & 3.26 & 97.44 & EW & & FALSE \\
\hline 2006 & $\mathrm{~B}$ & 2 & 8 & 5.21 & 97.29 & TW & & FALSE \\
\hline 2006 & B & 2 & 9 & 6.16 & 97.40 & EW & & FALSE \\
\hline 2006 & B & 2 & 10 & 7.32 & 97.54 & BIS & & FALSE \\
\hline 2006 & B & 2 & 11 & 7.77 & 97.81 & BIS & & FALSE \\
\hline 2006 & B & 2 & 12 & 8.53 & 98.13 & $\mathrm{BF}$ & & FALSE \\
\hline 2006 & B & 2 & 13 & 9.72 & 99.16 & BIS & & FALSE \\
\hline
\end{tabular}

Continued on Next Page... 
Table D.1 - Continued

\begin{tabular}{|c|c|c|c|c|c|c|c|c|}
\hline Year & Reach & Section & Point & Dist. (m) & Elev. $(\mathrm{m})$ & Desc. & Notes & Exclude \\
\hline 2006 & B & 2 & 14 & 10.12 & 99.35 & BOT X2R & $\begin{array}{l}\text { Bot } \mathrm{X} 2 \mathrm{R} \text { reb/low Right bank re- } \\
\text { bar very bent (almost } 90 \text { degrees } \\
\text { from original position), so reading } \\
\text { was taken from top of rebar down }\end{array}$ & FALSE \\
\hline 2006 & B & 2 & 15 & 10.12 & 99.73 & TOP X2R & $\begin{array}{l}\text { Top } \mathrm{X} 2 \mathrm{R} \text { reb/low Note: The dis- } \\
\text { tance between the original top } \\
\text { and bottom right rebar is } 1.2 \text { feet }\end{array}$ & FALSE \\
\hline 2006 & B & 3 & 1 & 0.00 & 100.00 & TOP X3L & Top X3L reb/low & FALSE \\
\hline 2006 & B & 3 & 2 & 0.00 & 99.44 & BOT X3L & Bot X3L reb/low & FALSE \\
\hline 2006 & $\mathrm{~B}$ & 3 & 3 & 0.67 & 99.04 & & & FALSE \\
\hline 2006 & $\mathrm{~B}$ & 3 & 4 & 1.22 & 98.71 & & & FALSE \\
\hline 2006 & B & 3 & 5 & 2.16 & 98.13 & & & FALSE \\
\hline 2006 & B & 3 & 6 & 3.87 & 98.05 & & & FALSE \\
\hline 2006 & B & 3 & 7 & 5.18 & 97.91 & & & FALSE \\
\hline 2006 & $\mathrm{~B}$ & 3 & 8 & 5.85 & 98.00 & & & FALSE \\
\hline 2006 & $\mathrm{~B}$ & 3 & 9 & 7.04 & 98.62 & & & FALSE \\
\hline 2006 & B & 3 & 10 & 7.89 & 99.22 & & & FALSE \\
\hline 2006 & B & 3 & 11 & 8.41 & 99.87 & BOT X3R & Bot $\mathrm{X} 3 \mathrm{R}$ reb/low & FALSE \\
\hline 2006 & B & 3 & 12 & 8.41 & 99.98 & TOP X3R & Top X3R reb/low & FALSE \\
\hline 2006 & B & 4 & 1 & 0.00 & 100.00 & TOP X4L & Top X4L reb/low & FALSE \\
\hline 2006 & B & 4 & 2 & 0.00 & 99.87 & BOT X4L & Bot $\mathrm{X} 4 \mathrm{~L}$ reb/low & FALSE \\
\hline 2006 & B & 4 & 3 & 0.73 & 99.66 & BIS & & FALSE \\
\hline 2006 & $\mathrm{~B}$ & 4 & 4 & 1.40 & 99.45 & BIS & & FALSE \\
\hline 2006 & B & 4 & 5 & 2.07 & 98.66 & BIS & & FALSE \\
\hline 2006 & B & 4 & 6 & 3.60 & 98.94 & BIS & & FALSE \\
\hline 2006 & B & 4 & 7 & 4.27 & 98.55 & EW & & FALSE \\
\hline 2006 & B & 4 & 8 & 5.49 & 98.69 & TW & Top of Rock Step (large boulder) & FALSE \\
\hline 2006 & $\mathrm{~B}$ & 4 & 9 & 6.52 & 98.66 & EW & & FALSE \\
\hline 2006 & $\mathrm{~B}$ & 4 & 10 & 7.10 & 99.49 & BIS & Top of large boulder & FALSE \\
\hline 2006 & B & 4 & 11 & 7.41 & 99.57 & BOT X4R & Bot $\mathrm{X} 4 \mathrm{R}$ reb/low & FALSE \\
\hline 2006 & B & 4 & 12 & 7.41 & 100.02 & TOP X4R & Top X4R reb/low & FALSE \\
\hline 2006 & B & 5 & 1 & 0.00 & 100.00 & TOP X5L & Top X5L reb/low & FALSE \\
\hline 2006 & $\mathrm{~B}$ & 5 & 2 & 0.00 & 99.55 & BOT X5L & Bot $\mathrm{X} 5 \mathrm{~L}$ reb/low & FALSE \\
\hline 2006 & B & 5 & 3 & 0.61 & 99.13 & BIS & & FALSE \\
\hline 2006 & B & 5 & 4 & 1.37 & 98.65 & BIS & & FALSE \\
\hline 2006 & B & 5 & 5 & 1.92 & 98.34 & $\mathrm{BF}$ & & FALSE \\
\hline 2006 & B & 5 & 6 & 2.93 & 98.04 & BIS & & FALSE \\
\hline 2006 & B & 5 & 7 & 4.33 & 97.79 & EW & & FALSE \\
\hline 2006 & B & 5 & 8 & 5.27 & 97.71 & TW & & FALSE \\
\hline 2006 & B & 5 & 9 & 6.68 & 97.86 & EW & & FALSE \\
\hline 2006 & B & 5 & 10 & 8.20 & 99.85 & BOT X5R & Bot X5R reb/low & FALSE \\
\hline 2006 & B & 5 & 11 & 8.20 & 100.00 & TOP X5R & Top X5R reb/low & FALSE \\
\hline 2006 & B & 6 & 1 & 0.00 & 100.00 & TOP X6L & Top X6L reb/low & FALSE \\
\hline 2006 & B & 6 & 2 & 0.00 & 99.92 & BOT X6L & Bot $\mathrm{X} 6 \mathrm{~L} \mathrm{reb} /$ low & FALSE \\
\hline 2006 & B & 6 & 3 & 0.76 & 99.46 & BIS & & FALSE \\
\hline 2006 & B & 6 & 4 & 1.37 & 99.20 & $\mathrm{BF}$ & & FALSE \\
\hline 2006 & B & 6 & 5 & 2.29 & 98.79 & BIS & & FALSE \\
\hline 2006 & B & 6 & 6 & 3.60 & 98.39 & EW & & FALSE \\
\hline 2006 & B & 6 & 7 & 4.36 & 98.26 & TW & & FALSE \\
\hline 2006 & B & 6 & 8 & 5.18 & 98.37 & EW & & FALSE \\
\hline 2006 & $\mathrm{~B}$ & 6 & 9 & 6.28 & 98.67 & BIS & & FALSE \\
\hline 2006 & B & 6 & 10 & 7.01 & 99.03 & BIS & & FALSE \\
\hline
\end{tabular}

Continued on Next Page... 
Table D.1 - Continued

\begin{tabular}{cccccclll}
\hline Year & Reach & Section & Point & Dist. $(\mathrm{m})$ & Elev. $(\mathrm{m})$ & Desc. & Notes & Exclude \\
\hline 2006 & B & 6 & 11 & 7.47 & 99.43 & BIS & & FALSE \\
2006 & B & 6 & 12 & 7.92 & 99.90 & BOT X6R & Bot X6R reb/low & FALSE \\
2006 & B & 6 & 13 & 7.92 & 100.02 & TOP X6R & Top X6R reb/low & FALSE \\
2006 & B & 7 & 1 & 0.00 & 100.00 & TOP X7L & Top X7L reb/low & FALSE \\
2006 & B & 7 & 2 & 0.00 & 99.46 & BOT X7L & Bot X7L reb/low Left bank rebar & FALSE \\
& & & & & & & significantly tilted 6 inches hori-
\end{tabular}
zontal difference between bottom and top

\begin{tabular}{|c|c|c|c|c|c|c|}
\hline 2006 & B & 7 & 3 & 0.67 & 99.07 & $\mathrm{BF}$ \\
\hline 2006 & B & 7 & 4 & 1.58 & 98.45 & BIS \\
\hline 2006 & B & 7 & 5 & 3.08 & 98.11 & EW \\
\hline 2006 & B & 7 & 6 & 4.57 & 98.03 & TW \\
\hline 2006 & B & 7 & 7 & 5.64 & 98.09 & EW \\
\hline 2006 & B & 7 & 8 & 6.19 & 98.42 & BIS \\
\hline 2006 & B & 7 & 9 & 6.71 & 98.81 & BIS \\
\hline 2006 & B & 7 & 10 & 7.56 & 99.26 & BIS \\
\hline 2006 & B & 7 & 11 & 8.60 & 99.46 & BIS \\
\hline 2006 & B & 7 & 12 & 9.48 & 99.83 & BOT X7R \\
\hline 2006 & B & 7 & 13 & 9.48 & 100.07 & TOP X7R \\
\hline 2006 & B & 8 & 1 & 0.00 & 100.00 & TOP X8L \\
\hline 2006 & B & 8 & 2 & 0.00 & 99.67 & BOT X8L \\
\hline 2006 & B & 8 & 3 & 0.30 & 99.38 & BIS \\
\hline 2006 & B & 8 & 4 & 0.55 & 98.93 & BIS \\
\hline 2006 & B & 8 & 5 & 1.04 & 98.90 & EW \\
\hline 2006 & B & 8 & 6 & 2.74 & 98.78 & TW \\
\hline 2006 & B & 8 & 7 & 2.93 & 98.81 & EW \\
\hline 2006 & B & 8 & 8 & 3.29 & 99.74 & BIS \\
\hline 2006 & B & 8 & 9 & 5.12 & 99.72 & BOT X8R \\
\hline 2006 & B & 8 & 10 & 5.12 & 99.90 & TOP X8R \\
\hline 2006 & B & 9 & 1 & 0.00 & 100.00 & TOP X9L \\
\hline 2006 & B & 9 & 2 & 0.00 & 99.97 & BOT X9L \\
\hline
\end{tabular}

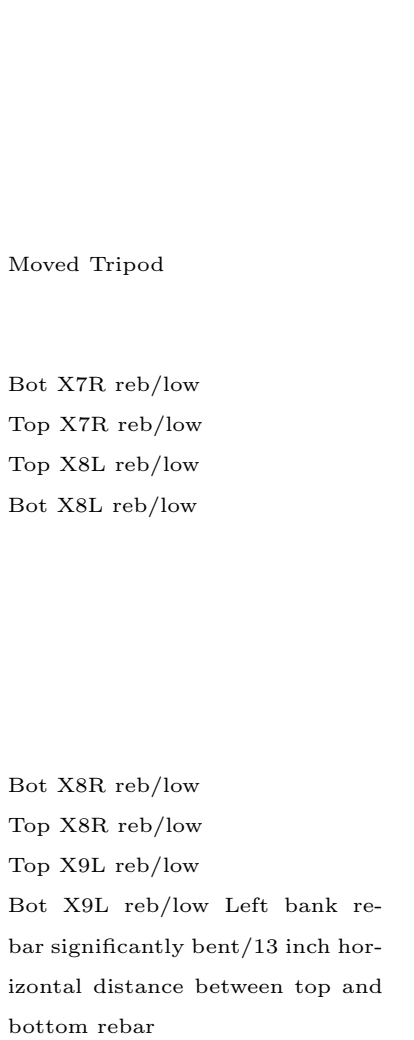

FALSE

FALSE

FALSE

FALSE

FALSE

FALSE

FALSE

FALSE

FALSE

FALSE

FALSE

FALSE

FALSE

FALSE

FALSE

FALSE

FALSE

FALSE

FALSE

FALSE

FALSE

FALSE

FALSE bottom rebar

\begin{tabular}{|c|c|c|c|c|c|c|c|c|}
\hline 2006 & B & 9 & 3 & 0.40 & 97.77 & BIS & & FALSE \\
\hline 2006 & B & 9 & 4 & 0.88 & 97.69 & EW & & FALSE \\
\hline 2006 & B & 9 & 5 & 1.98 & 97.68 & $\mathrm{TW}$ & & FALSE \\
\hline 2006 & B & 9 & 6 & 3.35 & 97.94 & EW & & FALSE \\
\hline 2006 & B & 9 & 7 & 5.15 & 98.45 & BIS & & FALSE \\
\hline 2006 & B & 9 & 8 & 6.95 & 98.80 & $\mathrm{BF}$ & & FALSE \\
\hline 2006 & B & 9 & 9 & 7.92 & 99.00 & BIS & & FALSE \\
\hline 2006 & B & 9 & 10 & 10.79 & 98.98 & BIS & & FALSE \\
\hline 2006 & B & 9 & 11 & 12.04 & 99.57 & BIS & & FALSE \\
\hline 2006 & B & 9 & 12 & 13.53 & 100.27 & BOT X9R & Bot $\mathrm{X} 9 \mathrm{R}$ reb/low & FALSE \\
\hline 2006 & B & 9 & 13 & 13.53 & 100.27 & TOP X9R & Top X9R reb/low & FALSE \\
\hline 2006 & B & 10 & 1 & 0.00 & 100.00 & TOP X10L & Top X10L reb/low & FALSE \\
\hline 2006 & B & 10 & 2 & 0.00 & 99.70 & TOP X10R & Bot $\mathrm{X} 10 \mathrm{~L} \mathrm{reb} / \mathrm{low}$ & FALSE \\
\hline 2006 & B & 10 & 3 & 0.49 & 97.13 & BIS & & FALSE \\
\hline 2006 & B & 10 & 4 & 0.94 & 97.16 & TW & & FALSE \\
\hline 2006 & B & 10 & 5 & 3.57 & 97.42 & SB & & FALSE \\
\hline 2006 & B & 10 & 6 & 3.99 & 97.40 & EW & & FALSE \\
\hline 2006 & B & 10 & 7 & 6.00 & 98.11 & ТОВ & & FALSE \\
\hline
\end{tabular}

Continued on Next Page... 
Table D.1 - Continued

\begin{tabular}{|c|c|c|c|c|c|c|c|c|}
\hline Year & Reach & Section & Point & Dist. (m) & Elev. $(\mathrm{m})$ & Desc. & Notes & Exclude \\
\hline 2006 & B & 10 & 8 & 6.95 & 98.31 & BIS & & FALSE \\
\hline 2006 & B & 10 & 9 & 9.66 & 98.27 & BIS & & FALSE \\
\hline 2006 & $\mathrm{~B}$ & 10 & 10 & 11.40 & 98.22 & BIS & & FALSE \\
\hline 2006 & B & 10 & 11 & 13.20 & 98.52 & BIS & & FALSE \\
\hline 2006 & B & 10 & 12 & 15.33 & 99.59 & BOT X10R & $\begin{array}{l}\text { Bot X10R reb/low Bottom Right } \\
\text { Rebar not representative because } \\
\text { of erosion }\end{array}$ & FALSE \\
\hline 2006 & B & 10 & 13 & 15.33 & 100.01 & TOP X10R & Top X10R reb/low & FALSE \\
\hline 2006 & $\mathrm{C}$ & 1 & 1 & 0.00 & 100.00 & & Top X1L reb/low & FALSE \\
\hline 2006 & $\mathrm{C}$ & 1 & 2 & 0.00 & 99.83 & & Bot X1L reb/low & FALSE \\
\hline 2006 & $\mathrm{C}$ & 1 & 3 & 0.64 & 99.12 & BIS & & FALSE \\
\hline 2006 & $\mathrm{C}$ & 1 & 4 & 0.91 & 99.95 & BIS & Top of Log & TRUE \\
\hline 2006 & $\mathrm{C}$ & 1 & 5 & 1.19 & 99.64 & BIS & & TRUE \\
\hline 2006 & $\mathrm{C}$ & 1 & 6 & 2.32 & 98.77 & EW & & FALSE \\
\hline 2006 & $\mathrm{C}$ & 1 & 7 & 2.93 & 98.71 & TW & & FALSE \\
\hline 2006 & $\mathrm{C}$ & 1 & 8 & 3.54 & 98.80 & BIS & & FALSE \\
\hline 2006 & $\mathrm{C}$ & 1 & 9 & 5.43 & 98.91 & EW & & FALSE \\
\hline 2006 & $\mathrm{C}$ & 1 & 10 & 7.65 & 99.26 & BIS & & FALSE \\
\hline 2006 & $\mathrm{C}$ & 1 & 11 & 9.21 & 99.48 & $\mathrm{BF}$ & & FALSE \\
\hline 2006 & $\mathrm{C}$ & 1 & 12 & 10.52 & 99.40 & BIS & & FALSE \\
\hline 2006 & $\mathrm{C}$ & 1 & 13 & 11.89 & 99.82 & BIS & & FALSE \\
\hline 2006 & $\mathrm{C}$ & 1 & 14 & 12.25 & 100.08 & BOT X1R & Bot X1R reb/low & FALSE \\
\hline 2006 & $\mathrm{C}$ & 1 & 15 & 12.25 & 100.45 & TOP X1R & Top X1R reb/low & FALSE \\
\hline 2006 & $\mathrm{C}$ & 2 & & & & BOT X2R & Bot $\mathrm{X} 2 \mathrm{R}$ reb/low & FALSE \\
\hline 2006 & $\mathrm{C}$ & 2 & & & & TOP X2R & Top X2R reb/low & FALSE \\
\hline 2006 & $\mathrm{C}$ & 2 & 1 & 0.00 & 100.00 & & Top X2L reb/low & FALSE \\
\hline 2006 & $\mathrm{C}$ & 2 & 2 & 0.00 & 99.66 & & Bot $\mathrm{X} 2 \mathrm{~L} \mathrm{reb} /$ low & FALSE \\
\hline 2006 & $\mathrm{C}$ & 2 & 3 & 0.64 & 99.16 & BIS & & FALSE \\
\hline 2006 & $\mathrm{C}$ & 2 & 4 & 0.76 & 99.31 & BIS & Top of Log & TRUE \\
\hline 2006 & $\mathrm{C}$ & 2 & 5 & 0.88 & 98.93 & BIS & & FALSE \\
\hline 2006 & $\mathrm{C}$ & 2 & 6 & 2.74 & 98.76 & BIS & & FALSE \\
\hline 2006 & $\mathrm{C}$ & 2 & 7 & 2.83 & 99.47 & BIS & Top of $\log$ & TRUE \\
\hline 2006 & $\mathrm{C}$ & 2 & 8 & 2.96 & 98.76 & BIS & & FALSE \\
\hline 2006 & $\mathrm{C}$ & 2 & 9 & 5.09 & 97.86 & BIS & & FALSE \\
\hline 2006 & $\mathrm{C}$ & 2 & 10 & 5.91 & 97.79 & EW & & FALSE \\
\hline 2006 & $\mathrm{C}$ & 2 & 11 & 7.04 & 97.68 & $\mathrm{TW}$ & & FALSE \\
\hline 2006 & $\mathrm{C}$ & 2 & 12 & 7.92 & 97.81 & BIS & Rocky Deposition & FALSE \\
\hline 2006 & $\mathrm{C}$ & 2 & 13 & 9.21 & 97.82 & EW & & FALSE \\
\hline 2006 & $\mathrm{C}$ & 2 & 14 & 10.03 & 98.19 & $\mathrm{BF}$ & & FALSE \\
\hline 2006 & $\mathrm{C}$ & 2 & 15 & 12.19 & 98.34 & BIS & & FALSE \\
\hline 2006 & $\mathrm{C}$ & 3 & 1 & 0.00 & 100.00 & & Top X3L reb/low & FALSE \\
\hline 2006 & $\mathrm{C}$ & 3 & 2 & 0.00 & 99.66 & & Bot $\mathrm{X} 3 \mathrm{~L} \mathrm{reb} /$ low & FALSE \\
\hline 2006 & $\mathrm{C}$ & 3 & 3 & 1.43 & 99.19 & BIS & & FALSE \\
\hline 2006 & $\mathrm{C}$ & 3 & 4 & 2.26 & 98.72 & BIS & & FALSE \\
\hline 2006 & $\mathrm{C}$ & 3 & 5 & 3.11 & 98.71 & BIS & & FALSE \\
\hline 2006 & $\mathrm{C}$ & 3 & 6 & 3.60 & 98.76 & BIS & & FALSE \\
\hline 2006 & $\mathrm{C}$ & 3 & 7 & 4.88 & 98.48 & BIS & & FALSE \\
\hline 2006 & $\mathrm{C}$ & 3 & 8 & 7.22 & 98.13 & EW & & FALSE \\
\hline 2006 & $\mathrm{C}$ & 3 & 9 & 8.84 & 97.98 & TW & & FALSE \\
\hline 2006 & $\mathrm{C}$ & 3 & 10 & 9.75 & 98.10 & EW & & FALSE \\
\hline 2006 & $\mathrm{C}$ & 3 & 11 & 10.03 & 98.24 & BIS & & FALSE \\
\hline 2006 & $\mathrm{C}$ & 3 & 12 & 10.24 & 98.49 & BIS & & FALSE \\
\hline
\end{tabular}

Continued on Next Page... 
Table D.1 - Continued

\begin{tabular}{|c|c|c|c|c|c|c|c|c|}
\hline Year & Reach & Section & Point & Dist. (m) & Elev. $(\mathrm{m})$ & Desc. & Notes & Exclude \\
\hline 2006 & $\mathrm{C}$ & 3 & 13 & 12.16 & 99.01 & $\mathrm{BF}$ & & FALSE \\
\hline 2006 & C & 3 & 14 & 13.99 & 99.79 & BOT X3R & Bot $\mathrm{X} 3 \mathrm{R}$ reb/low & FALSE \\
\hline 2006 & $\mathrm{C}$ & 3 & 15 & 13.99 & 100.00 & TOP X3R & Top X3R reb/low & FALSE \\
\hline 2006 & $\mathrm{C}$ & 4 & 1 & 0.00 & 100.00 & & Top X4L reb/low & FALSE \\
\hline 2006 & $\mathrm{C}$ & 4 & 2 & 0.00 & 99.69 & & Bot $\mathrm{X} 4 \mathrm{~L}$ reb/low & FALSE \\
\hline 2006 & $\mathrm{C}$ & 4 & 3 & 0.61 & 99.32 & BIS & & FALSE \\
\hline 2006 & $\mathrm{C}$ & 4 & 4 & 1.31 & 98.77 & BIS & & FALSE \\
\hline 2006 & $\mathrm{C}$ & 4 & 5 & 2.10 & 98.47 & BIS & & FALSE \\
\hline 2006 & $\mathrm{C}$ & 4 & 6 & 4.02 & 98.59 & BIS & & FALSE \\
\hline 2006 & $\mathrm{C}$ & 4 & 7 & 4.85 & 98.35 & BIS & & FALSE \\
\hline 2006 & $\mathrm{C}$ & 4 & 8 & 7.10 & 98.03 & EW & & FALSE \\
\hline 2006 & $\mathrm{C}$ & 4 & 9 & 7.77 & 97.89 & TW & & FALSE \\
\hline 2006 & $\mathrm{C}$ & 4 & 10 & 9.45 & 98.02 & EW & & FALSE \\
\hline 2006 & $\mathrm{C}$ & 4 & 11 & 10.18 & 98.39 & BIS & & FALSE \\
\hline 2006 & $\mathrm{C}$ & 4 & 12 & 10.45 & 98.85 & $\mathrm{BF}$ & & FALSE \\
\hline 2006 & $\mathrm{C}$ & 4 & 13 & 11.06 & 98.84 & BIS & & FALSE \\
\hline 2006 & $\mathrm{C}$ & 4 & 14 & 11.89 & 99.66 & BOT X4R & Bot $\mathrm{X} 4 \mathrm{R}$ reb/low & FALSE \\
\hline 2006 & $\mathrm{C}$ & 4 & 15 & 11.89 & 99.98 & TOP X4R & Top X4R reb/low & FALSE \\
\hline 2006 & $\mathrm{C}$ & 5 & 1 & 0.00 & 100.00 & & Top X5L reb/low & FALSE \\
\hline 2006 & $\mathrm{C}$ & 5 & 2 & 0.00 & 99.64 & & Bot $\mathrm{X} 5 \mathrm{~L} \mathrm{reb} /$ low & FALSE \\
\hline 2006 & $\mathrm{C}$ & 5 & 3 & 1.19 & 99.12 & BIS & & FALSE \\
\hline 2006 & $\mathrm{C}$ & 5 & 4 & 3.84 & 98.61 & BIS & & FALSE \\
\hline 2006 & $\mathrm{C}$ & 5 & 5 & 5.40 & 98.56 & $\mathrm{BF}$ & & FALSE \\
\hline 2006 & $\mathrm{C}$ & 5 & 6 & 6.46 & 98.07 & EW & & FALSE \\
\hline 2006 & $\mathrm{C}$ & 5 & 7 & 7.74 & 97.96 & TW & & FALSE \\
\hline 2006 & $\mathrm{C}$ & 5 & 8 & 8.75 & 98.12 & EW & & FALSE \\
\hline 2006 & $\mathrm{C}$ & 5 & 9 & 9.27 & 98.35 & BIS & & FALSE \\
\hline 2006 & $\mathrm{C}$ & 5 & 10 & 10.06 & 98.87 & BIS & & FALSE \\
\hline 2006 & $\mathrm{C}$ & 5 & 11 & 10.76 & 98.92 & BIS & & FALSE \\
\hline 2006 & $\mathrm{C}$ & 5 & 12 & 12.19 & 99.60 & BOT X5R & Bot $\mathrm{X} 5 \mathrm{R}$ reb/low & FALSE \\
\hline 2006 & $\mathrm{C}$ & 5 & 13 & 12.19 & 100.12 & TOP X5R & Top X5R reb/low & FALSE \\
\hline 2006 & $\mathrm{C}$ & 6 & 1 & 0.00 & 100.00 & & Top X6L reb/low & FALSE \\
\hline 2006 & $\mathrm{C}$ & 6 & 2 & 0.00 & 99.72 & & Bot $\mathrm{X} 6 \mathrm{~L} \mathrm{reb} /$ low & FALSE \\
\hline 2006 & $\mathrm{C}$ & 6 & 3 & 0.61 & 99.20 & BIS & & FALSE \\
\hline 2006 & $\mathrm{C}$ & 6 & 4 & 0.91 & 98.95 & BIS & & FALSE \\
\hline 2006 & $\mathrm{C}$ & 6 & 5 & 2.29 & 98.70 & $\mathrm{BF}$ & & FALSE \\
\hline 2006 & $\mathrm{C}$ & 6 & 6 & 3.84 & 98.17 & BIS & & FALSE \\
\hline 2006 & $\mathrm{C}$ & 6 & 7 & 4.48 & 97.96 & EW & & FALSE \\
\hline 2006 & $\mathrm{C}$ & 6 & 8 & 5.09 & 98.03 & BIS & Sandbar/deposition instream & FALSE \\
\hline 2006 & $\mathrm{C}$ & 6 & 9 & 6.40 & 97.90 & BIS & Sandbar/deposition instream & FALSE \\
\hline 2006 & $\mathrm{C}$ & 6 & 10 & 6.92 & 97.87 & $\mathrm{TW}$ & & FALSE \\
\hline 2006 & $\mathrm{C}$ & 6 & 11 & 7.96 & 98.01 & EW & & FALSE \\
\hline 2006 & $\mathrm{C}$ & 6 & 12 & 8.63 & 98.06 & BIS & & FALSE \\
\hline 2006 & $\mathrm{C}$ & 6 & 13 & 9.39 & 98.61 & BIS & & FALSE \\
\hline 2006 & $\mathrm{C}$ & 6 & 14 & 9.66 & 98.50 & ТОВ & & FALSE \\
\hline 2006 & $\mathrm{C}$ & 6 & 15 & 10.03 & 98.76 & BIS & & FALSE \\
\hline 2006 & $\mathrm{C}$ & 6 & 16 & 10.82 & 99.23 & BIS & On top of rock & FALSE \\
\hline 2006 & $\mathrm{C}$ & 6 & 17 & 11.40 & 99.14 & BIS & & FALSE \\
\hline 2006 & $\mathrm{C}$ & 6 & 18 & 12.19 & 99.16 & BIS & & FALSE \\
\hline 2006 & $\mathrm{C}$ & 6 & 19 & 12.92 & 99.77 & BOT X6R & Bot $\mathrm{X} 6 \mathrm{R}$ reb/low & FALSE \\
\hline 2006 & $\mathrm{C}$ & 6 & 20 & 12.92 & 100.01 & TOP X6R & Top X6R reb/low & FALSE \\
\hline 2006 & $\mathrm{C}$ & 7 & 1 & 0.00 & 100.00 & & Top X7L reb/low & FALSE \\
\hline
\end{tabular}

Continued on Next Page... 
Table D.1 - Continued

\begin{tabular}{|c|c|c|c|c|c|c|c|c|}
\hline Year & Reach & Section & Point & Dist. (m) & Elev. $(\mathrm{m})$ & Desc. & Notes & Exclude \\
\hline 2006 & $\mathrm{C}$ & 7 & 2 & 0.00 & 99.77 & & Bot $X 7 \mathrm{~L}$ reb/low & FALSE \\
\hline 2006 & C & 7 & 3 & 0.49 & 99.11 & BIS & & FALSE \\
\hline 2006 & $\mathrm{C}$ & 7 & 4 & 1.86 & 98.88 & ТОВ & & FALSE \\
\hline 2006 & $\mathrm{C}$ & 7 & 5 & 2.19 & 98.38 & BIS & & FALSE \\
\hline 2006 & $\mathrm{C}$ & 7 & 6 & 2.56 & 98.37 & BIS & & FALSE \\
\hline 2006 & $\mathrm{C}$ & 7 & 7 & 2.74 & 97.81 & EW & & FALSE \\
\hline 2006 & $\mathrm{C}$ & 7 & 8 & 3.05 & 97.76 & $\mathrm{TW}$ & & FALSE \\
\hline 2006 & $\mathrm{C}$ & 7 & 9 & 4.27 & 98.00 & BIS & Deposition in middle of stream & FALSE \\
\hline 2006 & $\mathrm{C}$ & 7 & 10 & 4.88 & 98.04 & EW & & FALSE \\
\hline 2006 & $\mathrm{C}$ & 7 & 11 & 6.68 & 98.32 & BIS & & FALSE \\
\hline 2006 & $\mathrm{C}$ & 7 & 12 & 7.41 & 98.74 & BIS & & FALSE \\
\hline 2006 & $\mathrm{C}$ & 7 & 13 & 7.74 & 99.13 & ТОВ & & FALSE \\
\hline 2006 & $\mathrm{C}$ & 7 & 14 & 8.72 & 98.99 & BIS & & FALSE \\
\hline 2006 & $\mathrm{C}$ & 7 & 15 & 10.24 & 99.00 & BIS & & FALSE \\
\hline 2006 & $\mathrm{C}$ & 7 & 16 & 10.79 & 98.76 & BIS & & FALSE \\
\hline 2006 & $\mathrm{C}$ & 7 & 17 & 12.13 & 99.09 & BIS & & FALSE \\
\hline 2006 & $\mathrm{C}$ & 7 & 18 & 12.77 & 99.30 & BIS & & FALSE \\
\hline 2006 & $\mathrm{C}$ & 7 & 19 & 13.32 & 99.80 & BOT X7R & Bot $\mathrm{X} 7 \mathrm{R}$ reb/low & FALSE \\
\hline 2006 & $\mathrm{C}$ & 7 & 20 & 13.32 & 100.03 & TOP X7R & Top X7R reb/low & FALSE \\
\hline 2006 & $\mathrm{C}$ & 8 & 1 & 0.00 & 100.00 & & Top X8L reb/low & FALSE \\
\hline 2006 & $\mathrm{C}$ & 8 & 2 & 0.00 & 99.57 & & Bot $\mathrm{X} 8 \mathrm{~L} \mathrm{reb} /$ low & FALSE \\
\hline 2006 & $\mathrm{C}$ & 8 & 3 & 0.61 & 97.72 & EW & & FALSE \\
\hline 2006 & $\mathrm{C}$ & 8 & 4 & 1.83 & 97.87 & BIS & in stream & FALSE \\
\hline 2006 & $\mathrm{C}$ & 8 & 5 & 2.74 & 97.73 & BIS & in stream & FALSE \\
\hline 2006 & $\mathrm{C}$ & 8 & 6 & 3.32 & 97.55 & TW & & FALSE \\
\hline 2006 & $\mathrm{C}$ & 8 & 7 & 3.96 & 97.75 & EW & & FALSE \\
\hline 2006 & $\mathrm{C}$ & 8 & 8 & 4.91 & 98.45 & BIS & & FALSE \\
\hline 2006 & $\mathrm{C}$ & 8 & 9 & 6.16 & 99.10 & ТОВ & & FALSE \\
\hline 2006 & $\mathrm{C}$ & 8 & 10 & 10.18 & 98.83 & BIS & & FALSE \\
\hline 2006 & $\mathrm{C}$ & 8 & 11 & 12.16 & 98.69 & BIS & & FALSE \\
\hline 2006 & $\mathrm{C}$ & 8 & 12 & 14.05 & 99.68 & BOT X8R & Bot $\mathrm{X} 8 \mathrm{R}$ reb/low & FALSE \\
\hline 2006 & $\mathrm{C}$ & 8 & 13 & 14.05 & 100.05 & TOP X8R & Top X8R reb/low & FALSE \\
\hline 2006 & $\mathrm{C}$ & 9 & 1 & 0.00 & 100.00 & & Top X9L reb/low & FALSE \\
\hline 2006 & $\mathrm{C}$ & 9 & 2 & 0.00 & 99.67 & & Bot $\mathrm{X} 9 \mathrm{~L} \mathrm{reb} /$ low & FALSE \\
\hline 2006 & $\mathrm{C}$ & 9 & 3 & 0.27 & 98.52 & BIS & & FALSE \\
\hline 2006 & $\mathrm{C}$ & 9 & 4 & 3.72 & 97.46 & EW & & FALSE \\
\hline 2006 & $\mathrm{C}$ & 9 & 5 & 4.54 & 97.39 & $\mathrm{TW}$ & & FALSE \\
\hline 2006 & $\mathrm{C}$ & 9 & 6 & 5.09 & 97.53 & EW & & FALSE \\
\hline 2006 & $\mathrm{C}$ & 9 & 7 & 5.88 & 98.11 & BIS & & FALSE \\
\hline 2006 & $\mathrm{C}$ & 9 & 8 & 6.61 & 98.62 & ТОВ & & FALSE \\
\hline 2006 & $\mathrm{C}$ & 9 & 9 & 7.86 & 98.78 & BIS & & FALSE \\
\hline 2006 & $\mathrm{C}$ & 9 & 10 & 13.11 & 98.52 & BIS & & FALSE \\
\hline 2006 & $\mathrm{C}$ & 9 & 11 & 14.63 & 98.73 & BIS & & FALSE \\
\hline 2006 & $\mathrm{C}$ & 9 & 12 & 15.61 & 99.47 & BOT X9R & Bot X9R reb/low & FALSE \\
\hline 2006 & $\mathrm{C}$ & 9 & 13 & 15.61 & 99.50 & TOP X9R & Top X9R reb/low & FALSE \\
\hline 2006 & $\mathrm{C}$ & 10 & 1 & 0.00 & 100.00 & & Top X10L reb/low & FALSE \\
\hline 2006 & $\mathrm{C}$ & 10 & 2 & 0.00 & 99.76 & & Bot $\mathrm{X} 10 \mathrm{~L} \mathrm{reb} /$ low & FALSE \\
\hline 2006 & $\mathrm{C}$ & 10 & 3 & 0.94 & 99.15 & BIS & & FALSE \\
\hline 2006 & $\mathrm{C}$ & 10 & 4 & 1.74 & 98.77 & BIS & & FALSE \\
\hline 2006 & $\mathrm{C}$ & 10 & 5 & 2.96 & 97.93 & BIS & & FALSE \\
\hline 2006 & $\mathrm{C}$ & 10 & 6 & 3.78 & 98.02 & BIS & & FALSE \\
\hline 2006 & C & 10 & 7 & 4.45 & 97.92 & BIS & & FALSE \\
\hline
\end{tabular}

Continued on Next Page... 
Table D.1 - Continued

\begin{tabular}{|c|c|c|c|c|c|c|c|c|}
\hline Year & Reach & Section & Point & Dist. (m) & Elev. $(\mathrm{m})$ & Desc. & Notes & Exclude \\
\hline 2006 & $\mathrm{C}$ & 10 & 8 & 4.69 & 98.10 & BIS & on top of boulder & FALSE \\
\hline 2006 & $\mathrm{C}$ & 10 & 9 & 5.12 & 97.60 & BIS & & FALSE \\
\hline 2006 & $\mathrm{C}$ & 10 & 10 & 5.40 & 97.47 & EW & & FALSE \\
\hline 2006 & $\mathrm{C}$ & 10 & 11 & 6.80 & 97.31 & TW & & FALSE \\
\hline 2006 & $\mathrm{C}$ & 10 & 12 & 7.44 & 97.47 & EW & & FALSE \\
\hline 2006 & $\mathrm{C}$ & 10 & 13 & 8.32 & 97.62 & BIS & & FALSE \\
\hline 2006 & $\mathrm{C}$ & 10 & 14 & 9.88 & 97.83 & $\mathrm{BF}$ & & FALSE \\
\hline 2006 & $\mathrm{C}$ & 10 & 15 & 11.73 & 98.42 & ТОВ & & FALSE \\
\hline 2006 & $\mathrm{C}$ & 10 & 16 & 12.92 & 98.76 & BIS & & FALSE \\
\hline 2006 & $\mathrm{C}$ & 10 & 17 & 15.21 & 98.84 & BIS & & FALSE \\
\hline 2006 & $\mathrm{C}$ & 10 & 18 & 16.43 & 99.24 & BIS & & FALSE \\
\hline 2006 & $\mathrm{C}$ & 10 & 19 & 16.79 & 99.97 & BOT X10R & Bot $\mathrm{X} 10 \mathrm{R} \mathrm{reb} / \mathrm{low}$ & FALSE \\
\hline 2006 & $\mathrm{C}$ & 10 & 20 & 16.79 & 99.99 & TOP X10R & Top X10R reb/low & FALSE \\
\hline 2006 & $\mathrm{D}$ & 1 & 1 & 0.00 & 100.00 & & Top X1L reb/upper & FALSE \\
\hline 2006 & $\mathrm{D}$ & 1 & 2 & 0.00 & 99.66 & & Bot X1L reb/upper & FALSE \\
\hline 2006 & $\mathrm{D}$ & 1 & 3 & 0.82 & 99.18 & & & FALSE \\
\hline 2006 & $\mathrm{D}$ & 1 & 4 & 2.13 & 98.62 & & & FALSE \\
\hline 2006 & $\mathrm{D}$ & 1 & 5 & 2.50 & 98.83 & & & FALSE \\
\hline 2006 & $\mathrm{D}$ & 1 & 6 & 2.87 & 98.17 & & & FALSE \\
\hline 2006 & $\mathrm{D}$ & 1 & 7 & 3.35 & 97.68 & & & FALSE \\
\hline 2006 & $\mathrm{D}$ & 1 & 8 & 4.51 & 97.67 & & & FALSE \\
\hline 2006 & $\mathrm{D}$ & 1 & 9 & 5.76 & 97.96 & & & FALSE \\
\hline 2006 & $\mathrm{D}$ & 1 & 10 & 6.22 & 98.69 & & & FALSE \\
\hline 2006 & $\mathrm{D}$ & 1 & 11 & 6.89 & 98.65 & BOT X1L & Bot $\mathrm{X} 1 \mathrm{~L}$ reb/low & FALSE \\
\hline 2006 & $\mathrm{D}$ & 1 & 12 & 6.89 & 98.86 & TOP X1L & Top X1L reb/low & FALSE \\
\hline 2006 & $\mathrm{D}$ & 1 & 13 & 7.19 & 98.59 & & & FALSE \\
\hline 2006 & $\mathrm{D}$ & 1 & 14 & 7.77 & 97.50 & & & FALSE \\
\hline 2006 & $\mathrm{D}$ & 1 & 15 & 8.96 & 96.81 & & & FALSE \\
\hline 2006 & $\mathrm{D}$ & 1 & 16 & 10.09 & 96.63 & & & FALSE \\
\hline 2006 & $\mathrm{D}$ & 1 & 17 & 11.16 & 96.82 & & & FALSE \\
\hline 2006 & $\mathrm{D}$ & 1 & 18 & 12.07 & 97.16 & & & FALSE \\
\hline 2006 & $\mathrm{D}$ & 1 & 19 & 13.05 & 97.34 & & & FALSE \\
\hline 2006 & $\mathrm{D}$ & 1 & 20 & 13.99 & 97.91 & & & FALSE \\
\hline 2006 & $\mathrm{D}$ & 1 & 21 & 14.57 & 98.05 & & & FALSE \\
\hline 2006 & $\mathrm{D}$ & 1 & 22 & 15.09 & 98.40 & & & FALSE \\
\hline 2006 & $\mathrm{D}$ & 1 & 23 & 16.46 & 98.56 & & & FALSE \\
\hline 2006 & $\mathrm{D}$ & 1 & 24 & 17.98 & 100.08 & BOT X1R & Bot $\mathrm{X} 1 \mathrm{R}$ reb/upper & FALSE \\
\hline 2006 & $\mathrm{D}$ & 1 & 25 & 17.98 & 100.44 & TOP X1R & Top X1R reb/upper & FALSE \\
\hline 2006 & $\mathrm{D}$ & 2 & 1 & 0.00 & 100.00 & & Top X2L reb/low & FALSE \\
\hline 2006 & $\mathrm{D}$ & 2 & 2 & 0.00 & 99.70 & & Bot $\mathrm{X} 2 \mathrm{~L} \mathrm{reb} /$ low & FALSE \\
\hline 2006 & $\mathrm{D}$ & 2 & 3 & 0.55 & 99.70 & ТОВ & & FALSE \\
\hline 2006 & $\mathrm{D}$ & 2 & 4 & 1.74 & 98.64 & BIS & & FALSE \\
\hline 2006 & $\mathrm{D}$ & 2 & 5 & 2.74 & 98.52 & EW & & FALSE \\
\hline 2006 & $\mathrm{D}$ & 2 & 6 & 3.99 & 98.40 & TW & & FALSE \\
\hline 2006 & $\mathrm{D}$ & 2 & 7 & 4.45 & 98.46 & EW & & FALSE \\
\hline 2006 & $\mathrm{D}$ & 2 & 8 & 5.46 & 98.75 & BIS & & FALSE \\
\hline 2006 & $\mathrm{D}$ & 2 & 9 & 6.28 & 99.02 & $\mathrm{BF}$ & & FALSE \\
\hline 2006 & $\mathrm{D}$ & 2 & 10 & 7.47 & 99.57 & ТОВ & & FALSE \\
\hline 2006 & $\mathrm{D}$ & 2 & 11 & 9.39 & 99.79 & BOT X2R & Bot $\mathrm{X} 2 \mathrm{R}$ reb/low & FALSE \\
\hline 2006 & $\mathrm{D}$ & 2 & 12 & 9.39 & 100.02 & TOP X2R & Top X2R reb/low & FALSE \\
\hline 2006 & $\mathrm{D}$ & 3 & 1 & 0.00 & 100.00 & & Top X3L reb/low & FALSE \\
\hline 2006 & $\mathrm{D}$ & 3 & 2 & 0.00 & 99.80 & & Bot $\mathrm{X} 3 \mathrm{~L} \mathrm{reb} /$ low & FALSE \\
\hline
\end{tabular}

Continued on Next Page... 
Table D.1 - Continued

\begin{tabular}{|c|c|c|c|c|c|c|c|c|}
\hline Year & Reach & Section & Point & Dist. (m) & Elev. $(\mathrm{m})$ & Desc. & Notes & Exclude \\
\hline 2006 & $\mathrm{D}$ & 3 & 3 & 0.98 & 99.79 & ТОВ & & FALSE \\
\hline 2006 & $\mathrm{D}$ & 3 & 4 & 1.77 & 98.84 & BIS & & FALSE \\
\hline 2006 & $\mathrm{D}$ & 3 & 5 & 2.62 & 98.66 & BIS & & FALSE \\
\hline 2006 & $\mathrm{D}$ & 3 & 6 & 3.11 & 98.55 & EW & & FALSE \\
\hline 2006 & $\mathrm{D}$ & 3 & 7 & 4.21 & 98.33 & TW & & FALSE \\
\hline 2006 & $\mathrm{D}$ & 3 & 8 & 4.60 & 98.55 & EW & & FALSE \\
\hline 2006 & $\mathrm{D}$ & 3 & 9 & 5.33 & 99.17 & $\mathrm{BF}$ & & FALSE \\
\hline 2006 & $\mathrm{D}$ & 3 & 10 & 5.61 & 99.50 & BIS & Rootwad & FALSE \\
\hline 2006 & $\mathrm{D}$ & 3 & 11 & 6.46 & 99.88 & BIS & Rootwad & FALSE \\
\hline 2006 & $\mathrm{D}$ & 3 & 12 & 6.71 & 99.74 & BIS & & FALSE \\
\hline 2006 & $\mathrm{D}$ & 3 & 13 & 7.19 & 99.24 & BIS & & FALSE \\
\hline 2006 & $\mathrm{D}$ & 3 & 14 & 8.81 & 99.27 & BIS & & FALSE \\
\hline 2006 & $\mathrm{D}$ & 3 & 15 & 10.58 & 99.49 & BIS & & FALSE \\
\hline 2006 & $\mathrm{D}$ & 3 & 16 & 11.06 & 99.92 & BOT X3R & Bot $\mathrm{X} 3 \mathrm{R}$ reb/low & FALSE \\
\hline 2006 & $\mathrm{D}$ & 3 & 17 & 11.06 & 100.03 & TOP X3R & Top X3R reb/low & FALSE \\
\hline 2006 & $\mathrm{D}$ & 4 & 1 & 0.00 & 100.00 & & $\begin{array}{l}\text { Top X4L reb/upper (on top of } \\
\text { stump) }\end{array}$ & FALSE \\
\hline 2006 & $\mathrm{D}$ & 4 & 2 & 0.00 & 99.18 & & $\begin{array}{l}\text { Bot } \mathrm{X} 4 \mathrm{~L} \text { reb/upper (on top of } \\
\text { stump) }\end{array}$ & FALSE \\
\hline 2006 & $\mathrm{D}$ & 4 & 3 & 0.58 & 97.74 & BIS & $\begin{array}{l}\text { Bottom of stump; bank eroded } \\
\text { under and }\end{array}$ & FALSE \\
\hline 2006 & $\mathrm{D}$ & 4 & 4 & 1.55 & 96.37 & EW & $\begin{array}{l}\text { moved tripod/very high rebar (@ } \\
\text { dist. 1.9) }\end{array}$ & FALSE \\
\hline 2006 & $\mathrm{D}$ & 4 & 5 & 2.10 & 96.27 & TW & & FALSE \\
\hline 2006 & $\mathrm{D}$ & 4 & 6 & 3.32 & 96.42 & EW & & FALSE \\
\hline 2006 & $\mathrm{D}$ & 4 & 7 & 4.33 & 96.78 & BIS & & FALSE \\
\hline 2006 & $\mathrm{D}$ & 4 & 8 & 5.67 & 97.17 & $\mathrm{BF}$ & & FALSE \\
\hline 2006 & $\mathrm{D}$ & 4 & 9 & 6.98 & 97.15 & BIS & & FALSE \\
\hline 2006 & $\mathrm{D}$ & 4 & 10 & 7.89 & 97.27 & BIS & & FALSE \\
\hline 2006 & $\mathrm{D}$ & 4 & 11 & 9.85 & 98.07 & BIS & & FALSE \\
\hline 2006 & $\mathrm{D}$ & 4 & 12 & 11.49 & 98.24 & BIS & moved tripod/very high rebar & FALSE \\
\hline 2006 & $\mathrm{D}$ & 4 & 13 & 13.87 & 100.57 & BOT X4R & Bot $\mathrm{X} 4 \mathrm{R}$ reb/upper & FALSE \\
\hline 2006 & $\mathrm{D}$ & 4 & 14 & 13.87 & 100.29 & TOP X4R & Top X4R reb/upper & FALSE \\
\hline 2006 & $\mathrm{D}$ & 5 & 1 & 0.00 & 100.00 & & Top X5L reb/low & FALSE \\
\hline 2006 & $\mathrm{D}$ & 5 & 2 & 0.00 & 99.63 & & Bot $\mathrm{X} 5 \mathrm{~L} \mathrm{reb} /$ low & FALSE \\
\hline 2006 & $\mathrm{D}$ & 5 & 3 & 0.24 & 99.48 & ТОВ & & FALSE \\
\hline 2006 & $\mathrm{D}$ & 5 & 4 & 0.88 & 98.41 & BIS & & FALSE \\
\hline 2006 & $\mathrm{D}$ & 5 & 5 & 3.93 & 97.93 & EW & & FALSE \\
\hline 2006 & $\mathrm{D}$ & 5 & 6 & 4.21 & 97.88 & $\mathrm{TW}$ & & FALSE \\
\hline 2006 & $\mathrm{D}$ & 5 & 7 & 4.94 & 97.99 & BIS & & FALSE \\
\hline 2006 & $\mathrm{D}$ & 5 & 8 & 6.25 & 98.47 & BIS & & FALSE \\
\hline 2006 & $\mathrm{D}$ & 5 & 9 & 7.07 & 98.96 & $\mathrm{BF}$ & on top of boulder & FALSE \\
\hline 2006 & $\mathrm{D}$ & 5 & 10 & 7.77 & 98.96 & BIS & & FALSE \\
\hline 2006 & $\mathrm{D}$ & 5 & 11 & 8.20 & 99.50 & ТОВ & & FALSE \\
\hline 2006 & $\mathrm{D}$ & 5 & 12 & 9.42 & 99.69 & BIS & & FALSE \\
\hline 2006 & $\mathrm{D}$ & 5 & 13 & 10.15 & 99.62 & BIS & & FALSE \\
\hline 2006 & $\mathrm{D}$ & 5 & 14 & 10.85 & 99.59 & BOT X5R & Bot $\mathrm{X} 5 \mathrm{R}$ reb/low & FALSE \\
\hline 2006 & $\mathrm{D}$ & 5 & 15 & 10.85 & 100.00 & TOP X5R & Top X5R reb/low & FALSE \\
\hline 2006 & $\mathrm{D}$ & 6 & 1 & 0.00 & 100.00 & & Top X6L reb/low & FALSE \\
\hline 2006 & $\mathrm{D}$ & 6 & 2 & 0.00 & 99.83 & & Bot $\mathrm{X} 6 \mathrm{~L} \mathrm{reb} /$ low & FALSE \\
\hline 2006 & $\mathrm{D}$ & 6 & 3 & 0.67 & 99.66 & BIS & & FALSE \\
\hline 2006 & $\mathrm{D}$ & 6 & 4 & 1.04 & 98.34 & BF & & FALSE \\
\hline
\end{tabular}

Continued on Next Page... 
Table D.1 - Continued

\begin{tabular}{|c|c|c|c|c|c|c|c|c|}
\hline Year & Reach & Section & Point & Dist. (m) & Elev. $(\mathrm{m})$ & Desc. & Notes & Exclude \\
\hline 2006 & $\mathrm{D}$ & 6 & 5 & 2.80 & 98.00 & BIS & & FALSE \\
\hline 2006 & $\mathrm{D}$ & 6 & 6 & 3.72 & 97.63 & EW & & FALSE \\
\hline 2006 & $\mathrm{D}$ & 6 & 7 & 4.72 & 97.54 & TW & & FALSE \\
\hline 2006 & $\mathrm{D}$ & 6 & 8 & 6.83 & 97.70 & EW & & FALSE \\
\hline 2006 & $\mathrm{D}$ & 6 & 9 & 8.11 & 99.15 & BIS & & FALSE \\
\hline 2006 & $\mathrm{D}$ & 6 & 10 & 9.45 & 99.49 & BIS & & FALSE \\
\hline 2006 & $\mathrm{D}$ & 6 & 11 & 9.94 & 99.68 & BOT X6R & Bot $\mathrm{X} 6 \mathrm{R}$ reb/low & FALSE \\
\hline 2006 & $\mathrm{D}$ & 6 & 12 & 9.94 & 100.03 & TOP X6R & Top X6R reb/low & FALSE \\
\hline 2006 & $\mathrm{D}$ & 7 & 1 & 0.00 & 100.00 & & Top X7L reb/low & FALSE \\
\hline 2006 & $\mathrm{D}$ & 7 & 2 & 0.00 & 99.75 & & Bot $\mathrm{X} 7 \mathrm{~L}$ reb/low & FALSE \\
\hline 2006 & $\mathrm{D}$ & 7 & 3 & 0.40 & 98.84 & $\mathrm{BF}$ & & FALSE \\
\hline 2006 & $\mathrm{D}$ & 7 & 4 & 0.79 & 99.68 & BIS & on top of log & TRUE \\
\hline 2006 & $\mathrm{D}$ & 7 & 5 & 1.19 & 98.51 & BIS & & FALSE \\
\hline 2006 & $\mathrm{D}$ & 7 & 6 & 1.80 & 98.37 & BIS & & FALSE \\
\hline 2006 & $\mathrm{D}$ & 7 & 7 & 2.74 & 97.82 & EW & & FALSE \\
\hline 2006 & $\mathrm{D}$ & 7 & 8 & 3.60 & 97.67 & $\mathrm{TW}$ & & FALSE \\
\hline 2006 & $\mathrm{D}$ & 7 & 9 & 4.21 & 97.83 & EW & & FALSE \\
\hline 2006 & $\mathrm{D}$ & 7 & 10 & 5.55 & 97.92 & BIS & & FALSE \\
\hline 2006 & $\mathrm{D}$ & 7 & 11 & 6.49 & 99.44 & BIS & & FALSE \\
\hline 2006 & $\mathrm{D}$ & 7 & 12 & 7.01 & 99.29 & BIS & & FALSE \\
\hline 2006 & $\mathrm{D}$ & 7 & 13 & 7.68 & 99.76 & BOT X7R & Bot $\mathrm{X} 7 \mathrm{R}$ reb/low & FALSE \\
\hline 2006 & $\mathrm{D}$ & 7 & 14 & 7.68 & 99.97 & TOP X7R & Top X7R reb/low & FALSE \\
\hline 2006 & $\mathrm{D}$ & 8 & 1 & 0.00 & 100.00 & & Top X8L reb/low & FALSE \\
\hline 2006 & $\mathrm{D}$ & 8 & 2 & 0.00 & 99.73 & & Bot $\mathrm{X} 8 \mathrm{~L} \mathrm{reb} /$ low & FALSE \\
\hline 2006 & $\mathrm{D}$ & 8 & 3 & 0.91 & 99.15 & ТОВ & & FALSE \\
\hline 2006 & $\mathrm{D}$ & 8 & 4 & 1.31 & 98.07 & BIS & & FALSE \\
\hline 2006 & $\mathrm{D}$ & 8 & 5 & 1.86 & 97.86 & EW & & FALSE \\
\hline 2006 & $\mathrm{D}$ & 8 & 6 & 2.74 & 97.77 & TW & & FALSE \\
\hline 2006 & $\mathrm{D}$ & 8 & 7 & 4.51 & 97.80 & EW & & FALSE \\
\hline 2006 & $\mathrm{D}$ & 8 & 8 & 5.79 & 98.27 & BIS & & FALSE \\
\hline 2006 & $\mathrm{D}$ & 8 & 9 & 6.31 & 98.61 & BIS & & FALSE \\
\hline 2006 & $\mathrm{D}$ & 8 & 10 & 6.83 & 99.79 & ВОт X8R & Bot $\mathrm{X} 8 \mathrm{R}$ reb/low & FALSE \\
\hline 2006 & $\mathrm{D}$ & 8 & 11 & 6.83 & 100.03 & TOP X8R & Top X8R reb/low & FALSE \\
\hline 2006 & $\mathrm{D}$ & 9 & 1 & 0.00 & 100.00 & & Top X9L reb/low & FALSE \\
\hline 2006 & $\mathrm{D}$ & 9 & 2 & 0.00 & 99.87 & & Bot X9L reb/low & FALSE \\
\hline 2006 & $\mathrm{D}$ & 9 & 3 & 0.55 & 98.91 & $\mathrm{BF}$ & & FALSE \\
\hline 2006 & $\mathrm{D}$ & 9 & 4 & 0.88 & 98.74 & BIS & & FALSE \\
\hline 2006 & $\mathrm{D}$ & 9 & 5 & 2.65 & 98.63 & EW & top of $\log$ & FALSE \\
\hline 2006 & $\mathrm{D}$ & 9 & 6 & 3.35 & 98.45 & $\mathrm{TW}$ & & FALSE \\
\hline 2006 & $\mathrm{D}$ & 9 & 7 & 4.05 & 98.50 & BIS & & FALSE \\
\hline 2006 & $\mathrm{D}$ & 9 & 8 & 4.79 & 98.90 & BIS & & FALSE \\
\hline 2006 & $\mathrm{D}$ & 9 & 9 & 7.01 & 99.21 & BIS & & FALSE \\
\hline 2006 & $\mathrm{D}$ & 9 & 10 & 7.16 & 99.77 & BOT X9R & Bot $\mathrm{X} 9 \mathrm{R}$ reb/low & FALSE \\
\hline 2006 & $\mathrm{D}$ & 9 & 11 & 7.16 & 100.00 & TOP X9R & Top X9R reb/low & FALSE \\
\hline 2006 & $\mathrm{D}$ & 10 & 1 & 0.00 & 100.00 & & Top X10L reb/low & FALSE \\
\hline 2006 & $\mathrm{D}$ & 10 & 2 & 0.00 & 99.71 & & Bot X10L reb/low & FALSE \\
\hline 2006 & $\mathrm{D}$ & 10 & 3 & 0.82 & 99.13 & $\mathrm{BF}$ & & FALSE \\
\hline 2006 & $\mathrm{D}$ & 10 & 4 & 1.58 & 98.48 & BIS & & FALSE \\
\hline 2006 & $\mathrm{D}$ & 10 & 5 & 2.83 & 98.24 & EW & & FALSE \\
\hline 2006 & $\mathrm{D}$ & 10 & 6 & 4.15 & 98.13 & $\mathrm{TW}$ & & FALSE \\
\hline 2006 & $\mathrm{D}$ & 10 & 7 & 4.88 & 98.27 & EW & & FALSE \\
\hline 2006 & $\mathrm{D}$ & 10 & 8 & 6.49 & 98.52 & BIS & & FALSE \\
\hline
\end{tabular}

Continued on Next Page... 
Table D.1 - Continued

\begin{tabular}{|c|c|c|c|c|c|c|c|c|}
\hline Year & Reach & Section & Point & Dist. (m) & Elev. $(\mathrm{m})$ & Desc. & Notes & Exclude \\
\hline 2006 & $\mathrm{D}$ & 10 & 9 & 6.86 & 98.89 & BIS & & FALSE \\
\hline 2006 & D & 10 & 10 & 7.32 & 100.02 & BOT X10R & Bot $\mathrm{X} 10 \mathrm{R} \mathrm{reb} /$ low & FALSE \\
\hline 2006 & $\mathrm{D}$ & 10 & 11 & 7.32 & 100.00 & TOP X10R & Top X10R reb/low & FALSE \\
\hline 2006 & $\mathrm{E}$ & 1 & 1 & 0.00 & 100.00 & & Top X1L reb/low & FALSE \\
\hline 2006 & $\mathrm{E}$ & 1 & 2 & 0.00 & 99.68 & & Bot $\mathrm{X} 1 \mathrm{~L}$ reb/low & FALSE \\
\hline 2006 & $\mathrm{E}$ & 1 & 3 & 0.73 & 99.25 & BIS & & FALSE \\
\hline 2006 & $\mathrm{E}$ & 1 & 4 & 1.80 & 98.91 & BIS & & FALSE \\
\hline 2006 & $\mathrm{E}$ & 1 & 5 & 2.96 & 98.93 & BIS & & FALSE \\
\hline 2006 & $\mathrm{E}$ & 1 & 6 & 3.90 & 98.70 & BIS & & FALSE \\
\hline 2006 & $\mathrm{E}$ & 1 & 7 & 4.66 & 98.47 & $\mathrm{BF}$ & & FALSE \\
\hline 2006 & $\mathrm{E}$ & 1 & 8 & 5.18 & 98.34 & BIS & & FALSE \\
\hline 2006 & $\mathrm{E}$ & 1 & 9 & 5.91 & 98.21 & EW & & FALSE \\
\hline 2006 & $\mathrm{E}$ & 1 & 10 & 6.28 & 98.11 & $\mathrm{TW}$ & & FALSE \\
\hline 2006 & $\mathrm{E}$ & 1 & 11 & 6.89 & 98.17 & EW & & FALSE \\
\hline 2006 & $\mathrm{E}$ & 1 & 12 & 7.47 & 99.81 & BOT X1R & Bot $\mathrm{X} 1 \mathrm{R}$ reb/low & FALSE \\
\hline 2006 & $\mathrm{E}$ & 1 & 13 & 7.47 & 99.88 & TOP X1R & Top X1R reb/low & FALSE \\
\hline 2006 & $\mathrm{E}$ & 2 & 1 & 0.00 & 100.00 & & Top X2L reb/low & FALSE \\
\hline 2006 & $\mathrm{E}$ & 2 & 2 & 0.00 & 99.58 & & Bot $\mathrm{X} 2 \mathrm{~L} \mathrm{reb} /$ low & FALSE \\
\hline 2006 & $\mathrm{E}$ & 2 & 3 & 0.21 & 99.24 & BIS & & FALSE \\
\hline 2006 & $\mathrm{E}$ & 2 & 4 & 0.43 & 98.81 & BIS & & FALSE \\
\hline 2006 & $\mathrm{E}$ & 2 & 5 & 1.19 & 98.56 & BIS & & FALSE \\
\hline 2006 & $\mathrm{E}$ & 2 & 6 & 2.07 & 98.29 & EW & & FALSE \\
\hline 2006 & $\mathrm{E}$ & 2 & 7 & 2.87 & 98.29 & $\mathrm{TW}$ & & FALSE \\
\hline 2006 & $\mathrm{E}$ & 2 & 8 & 3.47 & 98.42 & EW & & FALSE \\
\hline 2006 & $\mathrm{E}$ & 2 & 9 & 4.18 & 98.68 & BIS & & FALSE \\
\hline 2006 & $\mathrm{E}$ & 2 & 10 & 4.75 & 99.07 & $\mathrm{BF}$ & & FALSE \\
\hline 2006 & $\mathrm{E}$ & 2 & 11 & 4.97 & 99.35 & ТОВ & & FALSE \\
\hline 2006 & $\mathrm{E}$ & 2 & 12 & 5.88 & 99.67 & BOT X2R & Bot $\mathrm{X} 2 \mathrm{R}$ reb/low & FALSE \\
\hline 2006 & $\mathrm{E}$ & 2 & 13 & 5.88 & 100.03 & TOP X2R & Top X2R reb/low & FALSE \\
\hline 2006 & $\mathrm{E}$ & 3 & 1 & 0.00 & 100.00 & & Top X3L reb/low & FALSE \\
\hline 2006 & $\mathrm{E}$ & 3 & 2 & 0.00 & 99.46 & & Bot X3L reb/low & FALSE \\
\hline 2006 & $\mathrm{E}$ & 3 & 3 & 0.82 & 98.81 & BIS & & FALSE \\
\hline 2006 & $\mathrm{E}$ & 3 & 4 & 1.58 & 98.48 & BIS & & FALSE \\
\hline 2006 & $\mathrm{E}$ & 3 & 5 & 1.95 & 97.97 & EW & & FALSE \\
\hline 2006 & $\mathrm{E}$ & 3 & 6 & 3.51 & 97.78 & TW & & FALSE \\
\hline 2006 & $\mathrm{E}$ & 3 & 7 & 3.87 & 97.90 & EW & & FALSE \\
\hline 2006 & $\mathrm{E}$ & 3 & 8 & 4.45 & 98.05 & BIS & & FALSE \\
\hline 2006 & $\mathrm{E}$ & 3 & 9 & 5.21 & 98.48 & $\mathrm{BF}$ & & FALSE \\
\hline 2006 & $\mathrm{E}$ & 3 & 10 & 5.67 & 98.99 & ТОВ & & FALSE \\
\hline 2006 & $\mathrm{E}$ & 3 & 11 & 7.01 & 99.21 & BIS & & FALSE \\
\hline 2006 & $\mathrm{E}$ & 3 & 12 & 7.28 & 99.52 & BOT X3R & Bot X3R reb/low & FALSE \\
\hline 2006 & $\mathrm{E}$ & 3 & 13 & 7.28 & 100.02 & TOP X3R & Top X3R reb/low & FALSE \\
\hline 2006 & $\mathrm{E}$ & 4 & 1 & 0.00 & 100.00 & & Top X4L reb/low & FALSE \\
\hline 2006 & $\mathrm{E}$ & 4 & 2 & 0.00 & 99.60 & & Bot $\mathrm{X} 4 \mathrm{~L} \mathrm{reb} /$ low & FALSE \\
\hline 2006 & $\mathrm{E}$ & 4 & 3 & 0.46 & 99.45 & BIS & & FALSE \\
\hline 2006 & $\mathrm{E}$ & 4 & 4 & 0.82 & 99.08 & $\mathrm{BF}$ & & FALSE \\
\hline 2006 & $\mathrm{E}$ & 4 & 5 & 1.46 & 98.80 & BIS & & FALSE \\
\hline 2006 & $\mathrm{E}$ & 4 & 6 & 2.04 & 98.66 & EW & & FALSE \\
\hline 2006 & $\mathrm{E}$ & 4 & 7 & 2.74 & 98.55 & $\mathrm{TW}$ & & FALSE \\
\hline 2006 & $\mathrm{E}$ & 4 & 8 & 4.82 & 98.54 & EW & & FALSE \\
\hline 2006 & $\mathrm{E}$ & 4 & 9 & 5.64 & 98.76 & BIS & & FALSE \\
\hline 2006 & $\mathrm{E}$ & 4 & 10 & 5.94 & 99.49 & ТOB & & FALSE \\
\hline
\end{tabular}

Continued on Next Page... 
Table D.1 - Continued

\begin{tabular}{|c|c|c|c|c|c|c|c|c|}
\hline Year & Reach & Section & Point & Dist. (m) & Elev. $(\mathrm{m})$ & Desc. & Notes & Exclude \\
\hline 2006 & $\mathrm{E}$ & 4 & 11 & 7.62 & 99.78 & BOT X4R & Bot $\mathrm{X} 4 \mathrm{R}$ reb/low & FALSE \\
\hline 2006 & $\mathrm{E}$ & 4 & 12 & 7.62 & 100.03 & TOP X4R & Top X4R reb/low & FALSE \\
\hline 2006 & $\mathrm{E}$ & 5 & 1 & 0.00 & 100.00 & & Top X5L reb/low & FALSE \\
\hline 2006 & $\mathrm{E}$ & 5 & 2 & 0.00 & 99.68 & & Bot $\mathrm{X} 5 \mathrm{~L}$ reb/low & FALSE \\
\hline 2006 & $\mathrm{E}$ & 5 & 3 & 0.37 & 99.62 & ТОВ & & FALSE \\
\hline 2006 & $\mathrm{E}$ & 5 & 4 & 0.79 & 99.47 & BIS & & FALSE \\
\hline 2006 & $\mathrm{E}$ & 5 & 5 & 1.65 & 98.85 & EW & $\begin{array}{l}\text { side channel; not part of main } \\
\text { stream }\end{array}$ & FALSE \\
\hline 2006 & $\mathrm{E}$ & 5 & 6 & 2.59 & 98.72 & EW & & FALSE \\
\hline 2006 & $\mathrm{E}$ & 5 & 7 & 3.57 & 98.63 & $\mathrm{TW}$ & & FALSE \\
\hline 2006 & $\mathrm{E}$ & 5 & 8 & 4.24 & 98.72 & EW & & FALSE \\
\hline 2006 & $\mathrm{E}$ & 5 & 9 & 5.33 & 99.20 & $\mathrm{BF}$ & & FALSE \\
\hline 2006 & $\mathrm{E}$ & 5 & 10 & 5.85 & 99.78 & ТОВ & & FALSE \\
\hline 2006 & $\mathrm{E}$ & 5 & 11 & 7.92 & 99.90 & BOT X5R & Bot $\mathrm{X} 5 \mathrm{R}$ reb/low & FALSE \\
\hline 2006 & $\mathrm{E}$ & 5 & 12 & 7.92 & 100.31 & TOP X5R & Top X5R reb/low & FALSE \\
\hline 2006 & $\mathrm{E}$ & 6 & 1 & 0.00 & 100.00 & & Top X6L reb/low & FALSE \\
\hline 2006 & $\mathrm{E}$ & 6 & 2 & 0.00 & 99.59 & & Bot $\mathrm{X} 6 \mathrm{~L} \mathrm{reb} /$ low & FALSE \\
\hline 2006 & $\mathrm{E}$ & 6 & 3 & 1.07 & 98.45 & EW & on top of large boulder & FALSE \\
\hline 2006 & $\mathrm{E}$ & 6 & 4 & 1.86 & 98.27 & $\mathrm{TW}$ & & FALSE \\
\hline 2006 & $\mathrm{E}$ & 6 & 5 & 2.38 & 98.40 & EW & & FALSE \\
\hline 2006 & $\mathrm{E}$ & 6 & 6 & 3.96 & 98.65 & BIS & & FALSE \\
\hline 2006 & $\mathrm{E}$ & 6 & 7 & 4.54 & 99.00 & $\mathrm{BF}$ & & FALSE \\
\hline 2006 & $\mathrm{E}$ & 6 & 8 & 4.91 & 99.51 & ТОВ & & FALSE \\
\hline 2006 & $\mathrm{E}$ & 6 & 9 & 5.36 & 99.66 & BOT X6R & Bot $\mathrm{X} 6 \mathrm{R}$ reb/low & FALSE \\
\hline 2006 & $\mathrm{E}$ & 6 & 10 & 5.36 & 100.00 & TOP X6R & Top X6R reb/low & FALSE \\
\hline 2006 & $\mathrm{E}$ & 7 & 1 & 0.00 & 100.00 & & Top X7L reb/low & FALSE \\
\hline 2006 & $\mathrm{E}$ & 7 & 2 & 0.00 & 99.76 & & Bot $\mathrm{X} 7 \mathrm{~L} \mathrm{reb} /$ low & FALSE \\
\hline 2006 & $\mathrm{E}$ & 7 & 3 & 0.73 & 99.44 & BIS & & FALSE \\
\hline 2006 & $\mathrm{E}$ & 7 & 4 & 1.31 & 98.45 & EW & & FALSE \\
\hline 2006 & $\mathrm{E}$ & 7 & 5 & 2.16 & 98.41 & $\mathrm{TW}$ & & FALSE \\
\hline 2006 & $\mathrm{E}$ & 7 & 6 & 3.87 & 98.55 & EW & & FALSE \\
\hline 2006 & $\mathrm{E}$ & 7 & 7 & 4.82 & 99.66 & ТОВ & & FALSE \\
\hline 2006 & $\mathrm{E}$ & 7 & 8 & 5.79 & 99.67 & BIS & & FALSE \\
\hline 2006 & $\mathrm{E}$ & 7 & 9 & 6.49 & 99.67 & BOT X7R & Bot $\mathrm{X} 7 \mathrm{R}$ reb/low & FALSE \\
\hline 2006 & $\mathrm{E}$ & 7 & 10 & 6.49 & 100.00 & TOP X7R & Top X7R reb/low & FALSE \\
\hline 2006 & $\mathrm{E}$ & 8 & 1 & 0.00 & 100.00 & & Top X8L reb/low & FALSE \\
\hline 2006 & $\mathrm{E}$ & 8 & 2 & 0.00 & 99.68 & & Bot $\mathrm{X} 8 \mathrm{~L} \mathrm{reb} /$ low & FALSE \\
\hline 2006 & $\mathrm{E}$ & 8 & 3 & 0.40 & 99.42 & $\mathrm{BF}$ & & FALSE \\
\hline 2006 & $\mathrm{E}$ & 8 & 4 & 1.04 & 98.80 & BIS & & FALSE \\
\hline 2006 & $\mathrm{E}$ & 8 & 5 & 1.34 & 98.59 & EW & & FALSE \\
\hline 2006 & $\mathrm{E}$ & 8 & 6 & 2.07 & 98.55 & TW & & FALSE \\
\hline 2006 & $\mathrm{E}$ & 8 & 7 & 3.29 & 98.68 & EW & & FALSE \\
\hline 2006 & $\mathrm{E}$ & 8 & 8 & 4.08 & 98.87 & BIS & & FALSE \\
\hline 2006 & $\mathrm{E}$ & 8 & 9 & 4.45 & 99.55 & ТОВ & & FALSE \\
\hline 2006 & $\mathrm{E}$ & 8 & 10 & 5.94 & 99.63 & BIS & & FALSE \\
\hline 2006 & $\mathrm{E}$ & 8 & 11 & 6.61 & 99.73 & BOT X8R & Bot $\mathrm{X} 8 \mathrm{R}$ reb/low & FALSE \\
\hline 2006 & $\mathrm{E}$ & 8 & 12 & 6.61 & 99.99 & TOP X8R & Top X8R reb/low & FALSE \\
\hline 2006 & $\mathrm{E}$ & 9 & 1 & 0.00 & 100.00 & & Top X9L reb/low & FALSE \\
\hline 2006 & $\mathrm{E}$ & 9 & 2 & 0.00 & 99.35 & & Bot $\mathrm{X} 9 \mathrm{~L} \mathrm{reb} /$ low & FALSE \\
\hline 2006 & $\mathrm{E}$ & 9 & 3 & 0.49 & 99.06 & BIS & & FALSE \\
\hline 2006 & $\mathrm{E}$ & 9 & 4 & 1.16 & 98.49 & BIS & & FALSE \\
\hline 2006 & $\mathrm{E}$ & 9 & 5 & 1.77 & 98.02 & $\mathrm{BF}$ & & FALSE \\
\hline
\end{tabular}

Continued on Next Page... 
Table D.1 - Continued

\begin{tabular}{|c|c|c|c|c|c|c|c|c|}
\hline Year & Reach & Section & Point & Dist. (m) & Elev. $(\mathrm{m})$ & Desc. & Notes & Exclude \\
\hline 2006 & $\mathrm{E}$ & 9 & 6 & 2.96 & 97.69 & EW & & FALSE \\
\hline 2006 & $\mathrm{E}$ & 9 & 7 & 3.69 & 97.53 & $\mathrm{TW}$ & & FALSE \\
\hline 2006 & $\mathrm{E}$ & 9 & 8 & 4.75 & 97.70 & EW & & FALSE \\
\hline 2006 & $\mathrm{E}$ & 9 & 9 & 5.61 & 97.89 & BIS & & FALSE \\
\hline 2006 & $\mathrm{E}$ & 9 & 10 & 6.55 & 98.08 & BIS & & FALSE \\
\hline 2006 & $\mathrm{E}$ & 9 & 11 & 7.25 & 98.45 & BIS & & FALSE \\
\hline 2006 & $\mathrm{E}$ & 9 & 12 & 7.59 & 99.02 & BIS & & FALSE \\
\hline 2006 & $\mathrm{E}$ & 9 & 13 & 8.14 & 99.73 & BOT X9R & Bot $\mathrm{X} 9 \mathrm{R}$ reb/low & FALSE \\
\hline 2006 & $\mathrm{E}$ & 9 & 14 & 8.14 & 100.00 & TOP X9R & Top X9R reb/low & FALSE \\
\hline 2006 & $\mathrm{E}$ & 10 & 1 & 0.00 & 100.00 & & Top X10L reb/low & FALSE \\
\hline 2006 & $\mathrm{E}$ & 10 & 2 & 0.00 & 99.57 & & Bot X10L reb/low & FALSE \\
\hline 2006 & $\mathrm{E}$ & 10 & 3 & 0.27 & 99.35 & BIS & & FALSE \\
\hline 2006 & $\mathrm{E}$ & 10 & 4 & 0.70 & 98.69 & BIS & & FALSE \\
\hline 2006 & $\mathrm{E}$ & 10 & 5 & 1.22 & 98.34 & BIS & & FALSE \\
\hline 2006 & $\mathrm{E}$ & 10 & 6 & 1.77 & 98.03 & BIS & & FALSE \\
\hline 2006 & $\mathrm{E}$ & 10 & 7 & 2.41 & 98.00 & EW & & FALSE \\
\hline 2006 & $\mathrm{E}$ & 10 & 8 & 3.11 & 97.91 & $\mathrm{TW}$ & & FALSE \\
\hline 2006 & $\mathrm{E}$ & 10 & 9 & 3.44 & 98.00 & EW & & FALSE \\
\hline 2006 & $\mathrm{E}$ & 10 & 10 & 4.15 & 98.33 & BIS & & FALSE \\
\hline 2006 & $\mathrm{E}$ & 10 & 11 & 4.97 & 98.50 & $\mathrm{BF}$ & & FALSE \\
\hline 2006 & $\mathrm{E}$ & 10 & 12 & 5.43 & 99.18 & ТОВ & & FALSE \\
\hline 2006 & $\mathrm{E}$ & 10 & 13 & 7.07 & 99.84 & BOT X10R & Bot $\mathrm{X} 10 \mathrm{R} \mathrm{reb} / \mathrm{low}$ & FALSE \\
\hline 2006 & $\mathrm{E}$ & 10 & 14 & 7.07 & 100.04 & TOP X10R & Top X10R reb/low & FALSE \\
\hline 2006 & $\mathrm{~F}$ & 1 & 1 & 0.00 & 100.00 & & Top X1L reb/low & FALSE \\
\hline 2006 & $\mathrm{~F}$ & 1 & 1 & 0.70 & 99.32 & BIS & & FALSE \\
\hline 2006 & $\mathrm{~F}$ & 1 & 2 & 0.00 & 99.95 & & Bot $\mathrm{X} 1 \mathrm{~L}$ reb/low & FALSE \\
\hline 2006 & $\mathrm{~F}$ & 1 & 2 & 1.43 & 99.05 & BIS & & FALSE \\
\hline 2006 & $\mathrm{~F}$ & 1 & 3 & 1.83 & 99.44 & BIS & $\begin{array}{l}\text { on top of large cobbles and fallen } \\
\text { tree }\end{array}$ & FALSE \\
\hline 2006 & $\mathrm{~F}$ & 1 & 4 & 2.56 & 98.89 & BIS & on top of large cobbles & FALSE \\
\hline 2006 & $\mathrm{~F}$ & 1 & 5 & 2.87 & 98.55 & $\mathrm{BF}$ & & FALSE \\
\hline 2006 & $\mathrm{~F}$ & 1 & 6 & 3.41 & 98.34 & BIS & & FALSE \\
\hline 2006 & $\mathrm{~F}$ & 1 & 7 & 4.72 & 98.14 & BIS & & FALSE \\
\hline 2006 & $\mathrm{~F}$ & 1 & 8 & 5.46 & 98.26 & BIS & on top of large cobbles & FALSE \\
\hline 2006 & $\mathrm{~F}$ & 1 & 9 & 5.91 & 97.88 & EW & & FALSE \\
\hline 2006 & $\mathrm{~F}$ & 1 & 10 & 6.00 & 97.89 & $\mathrm{TW}$ & & FALSE \\
\hline 2006 & $\mathrm{~F}$ & 1 & 11 & 6.07 & 97.97 & EW & & FALSE \\
\hline 2006 & $\mathrm{~F}$ & 1 & 12 & 6.52 & 98.09 & BIS & & FALSE \\
\hline 2006 & $\mathrm{~F}$ & 1 & 13 & 7.32 & 98.27 & BIS & & FALSE \\
\hline 2006 & $\mathrm{~F}$ & 1 & 14 & 8.11 & 98.62 & BIS & & FALSE \\
\hline 2006 & $\mathrm{~F}$ & 1 & 15 & 8.66 & 99.22 & BIS & & FALSE \\
\hline 2006 & $\mathrm{~F}$ & 2 & 3 & 0.00 & 100.00 & & Top X2L reb/low & FALSE \\
\hline 2006 & $\mathrm{~F}$ & 2 & 4 & 0.00 & 99.88 & & Bot $\mathrm{X} 2 \mathrm{~L} \mathrm{reb} /$ low & FALSE \\
\hline 2006 & $\mathrm{~F}$ & 2 & 5 & 0.43 & 99.50 & BIS & & FALSE \\
\hline 2006 & $\mathrm{~F}$ & 2 & 6 & 1.16 & 99.06 & BIS & & FALSE \\
\hline 2006 & $\mathrm{~F}$ & 2 & 7 & 2.50 & 98.43 & BIS & & FALSE \\
\hline 2006 & $\mathrm{~F}$ & 2 & 8 & 2.74 & 98.05 & BIS & & FALSE \\
\hline 2006 & $\mathrm{~F}$ & 2 & 9 & 3.75 & 97.69 & BIS & & FALSE \\
\hline 2006 & $\mathrm{~F}$ & 2 & 10 & 4.45 & 97.52 & EW & & FALSE \\
\hline 2006 & F & 2 & 11 & 4.88 & 97.39 & $\mathrm{TW}$ & & FALSE \\
\hline 2006 & $\mathrm{~F}$ & 2 & 12 & 5.85 & 97.50 & EW & & FALSE \\
\hline 2006 & $\mathrm{~F}$ & 2 & 13 & 6.58 & 97.67 & BIS & & FALSE \\
\hline
\end{tabular}

Continued on Next Page... 
Table D.1 - Continued

\begin{tabular}{|c|c|c|c|c|c|c|c|c|}
\hline Year & Reach & Section & Point & Dist. $(\mathrm{m})$ & Elev. $(\mathrm{m})$ & Desc. & Notes & Exclude \\
\hline 2006 & $\mathrm{~F}$ & 2 & 14 & 7.41 & 98.09 & $\mathrm{BF}$ & & FALSE \\
\hline 2006 & $\mathrm{~F}$ & 2 & 15 & 8.11 & 98.49 & BIS & & FALSE \\
\hline 2006 & $\mathrm{~F}$ & 2 & 16 & 8.72 & 99.83 & $\mathrm{BOT} \times 2 \mathrm{R}$ & Bot $\mathrm{X} 2 \mathrm{R}$ reb/low & FALSE \\
\hline 2006 & $\mathrm{~F}$ & 2 & 17 & 8.72 & 99.93 & TOP X2R & Top X2R reb/low & FALSE \\
\hline 2006 & $\mathrm{~F}$ & 3 & 1 & 0.00 & 100.00 & & Top X3L reb/low & FALSE \\
\hline 2006 & $\mathrm{~F}$ & 3 & 2 & 0.00 & 99.82 & & Bot $\mathrm{X} 3 \mathrm{~L}$ reb/low & FALSE \\
\hline 2006 & $\mathrm{~F}$ & 3 & 3 & 0.94 & 99.51 & BIS & moved tripod; line of sight & FALSE \\
\hline 2006 & $\mathrm{~F}$ & 3 & 4 & 1.40 & 98.61 & $\mathrm{BF}$ & & FALSE \\
\hline 2006 & $\mathrm{~F}$ & 3 & 5 & 2.65 & 98.39 & BIS & & FALSE \\
\hline 2006 & $\mathrm{~F}$ & 3 & 6 & 2.90 & 98.46 & BIS & on top of large boulder & FALSE \\
\hline 2006 & $\mathrm{~F}$ & 3 & 7 & 3.32 & 98.23 & BIS & & FALSE \\
\hline 2006 & $\mathrm{~F}$ & 3 & 8 & 3.93 & 97.90 & $\mathrm{TW}$ & & FALSE \\
\hline 2006 & $\mathrm{~F}$ & 3 & 9 & 4.60 & 98.03 & EW & on top of boulder & FALSE \\
\hline 2006 & $\mathrm{~F}$ & 3 & 10 & 4.85 & 98.20 & BIS & on top of large boulder & FALSE \\
\hline 2006 & $\mathrm{~F}$ & 3 & 11 & 5.49 & 98.08 & BIS & & FALSE \\
\hline 2006 & $\mathrm{~F}$ & 3 & 12 & 6.31 & 98.67 & BIS & & FALSE \\
\hline 2006 & $\mathrm{~F}$ & 3 & 13 & 6.80 & 98.68 & BIS & & FALSE \\
\hline 2006 & $\mathrm{~F}$ & 3 & 14 & 7.28 & 99.73 & BIS & & FALSE \\
\hline 2006 & $\mathrm{~F}$ & 3 & 15 & 7.74 & 100.05 & BOT X3R & Bot X3R reb/low & FALSE \\
\hline 2006 & $\mathrm{~F}$ & 3 & 16 & 7.74 & 100.10 & TOP X3R & Top X3R reb/low & FALSE \\
\hline 2006 & $\mathrm{~F}$ & 4 & 1 & 0.00 & 100.00 & & Top X4L reb/low & FALSE \\
\hline 2006 & $\mathrm{~F}$ & 4 & 2 & 0.00 & 99.91 & & Bot $\mathrm{X} 4 \mathrm{~L} \mathrm{reb} / \mathrm{low}$ & FALSE \\
\hline 2006 & $\mathrm{~F}$ & 4 & 3 & 2.74 & 97.81 & BIS & & FALSE \\
\hline 2006 & $\mathrm{~F}$ & 4 & 4 & 3.66 & 97.65 & EW & & FALSE \\
\hline 2006 & $\mathrm{~F}$ & 4 & 5 & 5.06 & 97.54 & $\mathrm{TW}$ & & FALSE \\
\hline 2006 & $\mathrm{~F}$ & 4 & 6 & 6.07 & 97.65 & EW & & FALSE \\
\hline 2006 & $\mathrm{~F}$ & 4 & 7 & 6.55 & 98.06 & $\mathrm{BF}$ & & FALSE \\
\hline 2006 & $\mathrm{~F}$ & 4 & 8 & 7.77 & 99.60 & BIS & & FALSE \\
\hline 2006 & $\mathrm{~F}$ & 4 & 9 & 8.78 & 99.95 & BOT X4R & Bot $\mathrm{X} 4 \mathrm{R}$ reb/low & FALSE \\
\hline 2006 & $\mathrm{~F}$ & 4 & 10 & 8.78 & 100.15 & TOP X4R & Top X4R reb/low & FALSE \\
\hline 2006 & $\mathrm{~F}$ & 5 & 1 & 0.00 & 100.00 & & Top X5L reb/low & FALSE \\
\hline 2006 & $\mathrm{~F}$ & 5 & 2 & 0.00 & 99.98 & & Bot X5L reb/low & FALSE \\
\hline 2006 & $\mathrm{~F}$ & 5 & 3 & 1.19 & 99.00 & BIS & & FALSE \\
\hline 2006 & $\mathrm{~F}$ & 5 & 4 & 2.23 & 98.68 & $\mathrm{BF}$ & on top of boulder & FALSE \\
\hline 2006 & $\mathrm{~F}$ & 5 & 5 & 2.38 & 98.53 & BIS & & FALSE \\
\hline 2006 & $\mathrm{~F}$ & 5 & 6 & 3.72 & 98.37 & EW & & FALSE \\
\hline 2006 & $\mathrm{~F}$ & 5 & 7 & 3.96 & 98.11 & TW & & FALSE \\
\hline 2006 & $\mathrm{~F}$ & 5 & 8 & 4.66 & 98.37 & EW & & FALSE \\
\hline 2006 & $\mathrm{~F}$ & 5 & 9 & 5.46 & 98.59 & BIS & & FALSE \\
\hline 2006 & $\mathrm{~F}$ & 5 & 10 & 5.97 & 98.50 & BIS & & FALSE \\
\hline 2006 & $\mathrm{~F}$ & 5 & 11 & 6.68 & 98.99 & $\mathrm{BF}$ & & FALSE \\
\hline 2006 & $\mathrm{~F}$ & 5 & 12 & 7.68 & 99.82 & BOT X5R & Bot $\mathrm{X} 5 \mathrm{R}$ reb/low & FALSE \\
\hline 2006 & $\mathrm{~F}$ & 5 & 13 & 7.68 & 99.90 & TOP X5R & Top X5R reb/low & FALSE \\
\hline 2006 & $\mathrm{~F}$ & 6 & 1 & 0.00 & 100.00 & & Top X6L reb/low & FALSE \\
\hline 2006 & $\mathrm{~F}$ & 6 & 2 & 0.00 & 99.69 & & Bot $\mathrm{X} 6 \mathrm{~L} \mathrm{reb} /$ low & FALSE \\
\hline 2006 & $\mathrm{~F}$ & 6 & 3 & 1.13 & 99.13 & BIS & & FALSE \\
\hline 2006 & $\mathrm{~F}$ & 6 & 4 & 1.86 & 98.30 & EW & & FALSE \\
\hline 2006 & $\mathrm{~F}$ & 6 & 5 & 2.10 & 98.72 & BIS & on top of $\log$ & FALSE \\
\hline 2006 & $\mathrm{~F}$ & 6 & 6 & 2.99 & 98.27 & BIS & sandbar/instream & FALSE \\
\hline 2006 & $\mathrm{~F}$ & 6 & 7 & 3.78 & 98.30 & BIS & sandbar/instream & FALSE \\
\hline 2006 & $\mathrm{~F}$ & 6 & 8 & 4.42 & 98.23 & $\mathrm{TW}$ & & FALSE \\
\hline 2006 & $\mathrm{~F}$ & 6 & 9 & 5.27 & 99.01 & EW & on top of large boulder & FALSE \\
\hline
\end{tabular}

Continued on Next Page... 
Table D.1 - Continued

\begin{tabular}{|c|c|c|c|c|c|c|c|c|}
\hline Year & Reach & Section & Point & Dist. $(\mathrm{m})$ & Elev. $(\mathrm{m})$ & Desc. & Notes & Exclude \\
\hline 2006 & $\mathrm{~F}$ & 6 & 10 & 6.55 & 99.18 & BIS & & FALSE \\
\hline 2006 & $\mathrm{~F}$ & 6 & 11 & 7.89 & 99.53 & BIS & & FALSE \\
\hline 2006 & $\mathrm{~F}$ & 6 & 12 & 8.41 & 99.86 & BOT X6R & Bot $\mathrm{X} 6 \mathrm{R}$ reb/low & FALSE \\
\hline 2006 & $\mathrm{~F}$ & 6 & 13 & 8.41 & 99.97 & TOP X6R & Top X6R reb/low & FALSE \\
\hline 2006 & $\mathrm{~F}$ & 7 & 1 & 0.00 & 100.00 & & Bot $X 7 \mathrm{~L}$ reb/low; rebar burried & FALSE \\
\hline 2006 & $\mathrm{~F}$ & 7 & 2 & 0.00 & 100.00 & & Top X7L reb/low; rebar burried & FALSE \\
\hline 2006 & $\mathrm{~F}$ & 7 & 3 & 0.85 & 99.04 & BIS & & FALSE \\
\hline 2006 & $\mathrm{~F}$ & 7 & 4 & 2.04 & 98.39 & EW & & FALSE \\
\hline 2006 & $\mathrm{~F}$ & 7 & 5 & 2.50 & 98.35 & $\mathrm{TW}$ & & FALSE \\
\hline 2006 & $\mathrm{~F}$ & 7 & 6 & 3.51 & 98.42 & EW & & FALSE \\
\hline 2006 & $\mathrm{~F}$ & 7 & 7 & 4.02 & 98.55 & BIS & & FALSE \\
\hline 2006 & $\mathrm{~F}$ & 7 & 8 & 5.49 & 98.99 & $\mathrm{BF}$ & & FALSE \\
\hline 2006 & $\mathrm{~F}$ & 7 & 9 & 8.69 & 99.83 & BOT X7R & Bot $X 7 R$ reb/low & FALSE \\
\hline 2006 & $\mathrm{~F}$ & 7 & 10 & 8.69 & 99.92 & TOP X7R & Top X7R reb/low & FALSE \\
\hline 2006 & $\mathrm{~F}$ & 8 & 1 & 0.00 & 100.00 & & Top X8L reb/low & FALSE \\
\hline 2006 & $\mathrm{~F}$ & 8 & 2 & 0.00 & 99.88 & & Bot $\mathrm{X} 8 \mathrm{~L} \mathrm{reb} / \mathrm{low}$ & FALSE \\
\hline 2006 & $\mathrm{~F}$ & 8 & 3 & 0.82 & 99.18 & BIS & & FALSE \\
\hline 2006 & $\mathrm{~F}$ & 8 & 4 & 1.10 & 98.58 & $\mathrm{BF}$ & & FALSE \\
\hline 2006 & $\mathrm{~F}$ & 8 & 5 & 2.13 & 98.24 & BIS & on top of boulder & FALSE \\
\hline 2006 & $\mathrm{~F}$ & 8 & 6 & 2.53 & 97.90 & BIS & & FALSE \\
\hline 2006 & $\mathrm{~F}$ & 8 & 7 & 3.32 & 97.79 & EW & & FALSE \\
\hline 2006 & $\mathrm{~F}$ & 8 & 8 & 3.90 & 97.73 & TW & & FALSE \\
\hline 2006 & $\mathrm{~F}$ & 8 & 9 & 4.36 & 97.80 & EW & & FALSE \\
\hline 2006 & $\mathrm{~F}$ & 8 & 10 & 5.36 & 98.44 & ТОВ & & FALSE \\
\hline 2006 & $\mathrm{~F}$ & 8 & 11 & 6.89 & 98.70 & BIS & & FALSE \\
\hline 2006 & $\mathrm{~F}$ & 8 & 12 & 7.41 & 98.80 & BIS & & FALSE \\
\hline 2006 & $\mathrm{~F}$ & 8 & 13 & 8.23 & 98.66 & BIS & & FALSE \\
\hline 2006 & $\mathrm{~F}$ & 8 & 14 & 9.02 & 98.79 & BIS & & FALSE \\
\hline 2006 & $\mathrm{~F}$ & 8 & 15 & 9.36 & 99.17 & BIS & on top of large boulder & FALSE \\
\hline 2006 & $\mathrm{~F}$ & 8 & 16 & 10.70 & 99.63 & BIS & & FALSE \\
\hline 2006 & $\mathrm{~F}$ & 8 & 17 & 11.25 & 99.82 & BIS & & FALSE \\
\hline 2006 & $\mathrm{~F}$ & 8 & 18 & 12.65 & 99.88 & Bot X8R & Bot $\mathrm{X} 8 \mathrm{R}$ reb/low & FALSE \\
\hline 2006 & $\mathrm{~F}$ & 8 & 19 & 12.65 & 100.04 & TOP X8R & Top X8R reb/low & FALSE \\
\hline 2006 & $\mathrm{~F}$ & 9 & 1 & 0.00 & 100.00 & & Top X9L reb/low & FALSE \\
\hline 2006 & $\mathrm{~F}$ & 9 & 2 & 0.00 & 99.82 & & Bot X9L reb/low & FALSE \\
\hline 2006 & $\mathrm{~F}$ & 9 & 3 & 0.61 & 99.14 & $\mathrm{BF}$ & & FALSE \\
\hline 2006 & $\mathrm{~F}$ & 9 & 4 & 2.19 & 98.49 & EW & & FALSE \\
\hline 2006 & $\mathrm{~F}$ & 9 & 5 & 3.41 & 98.38 & TW & & FALSE \\
\hline 2006 & $\mathrm{~F}$ & 9 & 6 & 3.87 & 98.41 & EW & Root wad in way; not true EW; & FALSE \\
\hline
\end{tabular}

EW really $+/-1 \mathrm{ft}$. further on right bank

\begin{tabular}{|c|c|c|c|c|c|c|c|c|}
\hline 2006 & $\mathrm{~F}$ & 9 & 7 & 4.27 & 99.04 & ТОВ & & FALSE \\
\hline 2006 & $\mathrm{~F}$ & 9 & 8 & 5.12 & 99.17 & BIS & & FALSE \\
\hline 2006 & $\mathrm{~F}$ & 9 & 9 & 6.00 & 99.24 & BIS & & FALSE \\
\hline 2006 & $\mathrm{~F}$ & 9 & 10 & 6.98 & 99.04 & BIS & & FALSE \\
\hline 2006 & $\mathrm{~F}$ & 9 & 11 & 9.42 & 99.04 & BIS & & FALSE \\
\hline 2006 & $\mathrm{~F}$ & 9 & 12 & 10.39 & 99.56 & BIS & & FALSE \\
\hline 2006 & $\mathrm{~F}$ & 9 & 13 & 11.22 & 99.76 & BOT X9R & Bot $\mathrm{X} 9 \mathrm{R}$ reb/low & FALSE \\
\hline 2006 & $\mathrm{~F}$ & 9 & 14 & 11.22 & 99.98 & TOP X9R & Top X9R reb/low & FALSE \\
\hline 2006 & $\mathrm{~F}$ & 10 & 1 & 0.00 & 100.00 & & Top X10L reb/low & FALSE \\
\hline 2006 & $\mathrm{~F}$ & 10 & 2 & 0.00 & 99.80 & & Bot X10L reb/low & FALSE \\
\hline 2006 & $\mathrm{~F}$ & 10 & 3 & 0.24 & 99.02 & $\mathrm{BF}$ & & FALSE \\
\hline
\end{tabular}

Continued on Next Page... 
Table D.1 - Continued

\begin{tabular}{|c|c|c|c|c|c|c|c|c|}
\hline Year & Reach & Section & Point & Dist. (m) & Elev. $(\mathrm{m})$ & Desc. & Notes & Exclude \\
\hline 2006 & $\mathrm{~F}$ & 10 & 4 & 1.40 & 98.41 & EW & & FALSE \\
\hline 2006 & $\mathrm{~F}$ & 10 & 5 & 2.41 & 98.33 & TW & & FALSE \\
\hline 2006 & $\mathrm{~F}$ & 10 & 6 & 2.77 & 98.40 & EW & & FALSE \\
\hline 2006 & $\mathrm{~F}$ & 10 & 7 & 3.84 & 98.65 & BIS & & FALSE \\
\hline 2006 & $\mathrm{~F}$ & 10 & 8 & 4.48 & 99.15 & тОВ & & FALSE \\
\hline 2006 & $\mathrm{~F}$ & 10 & 9 & 6.16 & 99.15 & BIS & & FALSE \\
\hline 2006 & $\mathrm{~F}$ & 10 & 10 & 9.02 & 98.89 & BIS & & FALSE \\
\hline 2006 & $\mathrm{~F}$ & 10 & 11 & 9.75 & 99.63 & BOT X1R & Bot $\mathrm{X} 10 \mathrm{R} \mathrm{reb} / \mathrm{low}$ & FALSE \\
\hline 2006 & $\mathrm{~F}$ & 10 & 12 & 9.75 & 99.78 & TOP X1R & Top X10R reb/low & FALSE \\
\hline 2007 & A & 1 & 1 & 0.00 & 100.00 & TOP X1L & Top X1L reb/low & FALSE \\
\hline 2007 & A & 1 & 2 & 0.00 & 99.85 & BOT X1L & Bot $\mathrm{X} 1 \mathrm{~L}$ reb/low & FALSE \\
\hline 2007 & A & 1 & 3 & 0.91 & 99.59 & BIS & & FALSE \\
\hline 2007 & A & 1 & 4 & 2.13 & 99.43 & BIS & & FALSE \\
\hline 2007 & A & 1 & 5 & 3.35 & 99.23 & тов & & FALSE \\
\hline 2007 & A & 1 & 6 & 3.75 & 98.88 & $\mathrm{BF}$ & & FALSE \\
\hline 2007 & A & 1 & 7 & 3.99 & 98.74 & EW & & FALSE \\
\hline 2007 & A & 1 & 8 & 4.39 & 98.63 & BIS & & FALSE \\
\hline 2007 & A & 1 & 9 & 4.79 & 98.58 & $\mathrm{TW}$ & & FALSE \\
\hline 2007 & A & 1 & 10 & 5.36 & 98.68 & BIS & & FALSE \\
\hline 2007 & A & 1 & 11 & 5.70 & 98.74 & EW & & FALSE \\
\hline 2007 & A & 1 & 12 & 6.13 & 98.85 & $\mathrm{BF}$ & & FALSE \\
\hline 2007 & $\mathrm{~A}$ & 1 & 13 & 7.16 & 99.38 & тОВ & & FALSE \\
\hline 2007 & A & 1 & 14 & 8.29 & 99.54 & BIS & & FALSE \\
\hline 2007 & A & 1 & 15 & 9.11 & 99.76 & BOT X1R & Bot $X 1 \mathrm{R}$ reb/low & FALSE \\
\hline 2007 & $\mathrm{~A}$ & 1 & 16 & 9.11 & 99.99 & TOP X1R & Top X1R reb/low & FALSE \\
\hline 2007 & A & 2 & 1 & 0.00 & 100.00 & TOP X2L & Top X2L reb/low & FALSE \\
\hline 2007 & $\mathrm{~A}$ & 2 & 2 & 0.00 & 99.68 & BOT X2L & Bot $\mathrm{X} 2 \mathrm{~L} \mathrm{reb} /$ low & FALSE \\
\hline 2007 & A & 2 & 3 & 1.28 & 99.43 & BIS & & FALSE \\
\hline 2007 & A & 2 & 4 & 2.01 & 99.00 & BIS & $\begin{array}{l}30 " \text { dbh redwood fell perpendic- } \\
\text { ular to stream and is suspended } \\
15 \text { ' above channel }\end{array}$ & FALSE \\
\hline 2007 & A & 2 & 5 & 3.35 & 98.66 & BIS & & FALSE \\
\hline 2007 & A & 2 & 6 & 3.99 & 98.34 & BIS & & FALSE \\
\hline 2007 & A & 2 & 7 & 4.33 & 98.24 & $\mathrm{BF}$ & & FALSE \\
\hline 2007 & A & 2 & 8 & 4.75 & 98.09 & EW & & FALSE \\
\hline 2007 & A & 2 & 9 & 5.18 & 98.00 & $\mathrm{TW}$ & & FALSE \\
\hline 2007 & A & 2 & 10 & 5.58 & 97.97 & BIS & & FALSE \\
\hline 2007 & A & 2 & 11 & 6.00 & 98.00 & BIS & & FALSE \\
\hline 2007 & $\mathrm{~A}$ & 2 & 12 & 6.37 & 97.99 & BIS & $\begin{array}{l}\text { Edge of log in stream; water flow- } \\
\text { ing under log }\end{array}$ & FALSE \\
\hline 2007 & $\mathrm{~A}$ & 2 & 13 & 6.37 & 98.03 & BIS & Top of $\log$ & FALSE \\
\hline 2007 & A & 2 & 14 & 7.80 & 98.47 & тов & & FALSE \\
\hline 2007 & A & 2 & 15 & 8.29 & 98.70 & BIS & & FALSE \\
\hline 2007 & $\mathrm{~A}$ & 2 & 16 & 8.56 & 99.10 & BIS & & FALSE \\
\hline 2007 & A & 2 & 17 & 9.30 & 98.87 & BIS & & FALSE \\
\hline 2007 & $\mathrm{~A}$ & 2 & 18 & 10.00 & 99.38 & BIS & & FALSE \\
\hline 2007 & A & 2 & 19 & 10.73 & 99.65 & BOT X2R & Bot $\mathrm{X} 2 \mathrm{R} \mathrm{reb} / \mathrm{low}$ & FALSE \\
\hline 2007 & $\mathrm{~A}$ & 2 & 20 & 10.73 & 99.95 & TOP X2R & Top X2R reb/low & FALSE \\
\hline 2007 & A & 3 & 1 & 0.00 & 100.00 & TOP X3L & Top X3L reb/low & FALSE \\
\hline 2007 & A & 3 & 2 & 0.00 & 99.49 & BOT X3L & Bot $\mathrm{X} 3 \mathrm{~L} \mathrm{reb} /$ low & FALSE \\
\hline 2007 & A & 3 & 3 & 0.30 & 99.89 & BIS & On top of alder extending from & TRUE \\
\hline
\end{tabular}

Continued on Next Page... 
Table D.1 - Continued

\begin{tabular}{|c|c|c|c|c|c|c|c|c|}
\hline Year & Reach & Section & Point & Dist. (m) & Elev. $(\mathrm{m})$ & Desc. & Notes & Exclude \\
\hline 2007 & A & 3 & 4 & 0.82 & 99.80 & BIS & $\begin{array}{l}\text { On top of alder extending from } \\
\text { bank to bank }\end{array}$ & TRUE \\
\hline 2007 & $\mathrm{~A}$ & 3 & 5 & 0.91 & 98.87 & BIS & & FALSE \\
\hline 2007 & A & 3 & 6 & 1.31 & 98.81 & ТОВ & & FALSE \\
\hline 2007 & $\mathrm{~A}$ & 3 & 7 & 1.46 & 98.22 & BIS & & FALSE \\
\hline 2007 & A & 3 & 8 & 1.83 & 98.30 & BIS & & FALSE \\
\hline 2007 & A & 3 & 9 & 2.38 & 97.88 & EW & & FALSE \\
\hline 2007 & $\mathrm{~A}$ & 3 & 10 & 2.87 & 97.80 & BIS & & FALSE \\
\hline 2007 & $\mathrm{~A}$ & 3 & 11 & 3.26 & 97.79 & $\mathrm{TX}$ & & FALSE \\
\hline 2007 & $\mathrm{~A}$ & 3 & 12 & 4.11 & 97.88 & EW & & FALSE \\
\hline 2007 & A & 3 & 13 & 4.88 & 98.02 & $\mathrm{BF}$ & & FALSE \\
\hline 2007 & A & 3 & 14 & 5.64 & 98.38 & BIS & & FALSE \\
\hline 2007 & $\mathrm{~A}$ & 3 & 15 & 6.80 & 98.54 & BIS & & FALSE \\
\hline 2007 & A & 3 & 16 & 7.99 & 98.80 & BIS & & FALSE \\
\hline 2007 & $\mathrm{~A}$ & 3 & 17 & 8.90 & 99.11 & BIS & & FALSE \\
\hline 2007 & A & 3 & 18 & 9.88 & 99.63 & BIS & & FALSE \\
\hline 2007 & $\mathrm{~A}$ & 3 & 19 & 10.70 & 99.99 & BOT X3R & Bot $\mathrm{X} 3 \mathrm{R}$ reb/low & FALSE \\
\hline 2007 & $\mathrm{~A}$ & 3 & 20 & 10.70 & 100.40 & TOP X3R & Top X3R reb/low & FALSE \\
\hline 2007 & $\mathrm{~A}$ & 4 & 1 & 0.00 & 100.00 & TOP X4L & Top X4L reb/low & FALSE \\
\hline 2007 & $\mathrm{~A}$ & 4 & 2 & 0.00 & 99.71 & BOT X4L & Bot $\mathrm{X} 4 \mathrm{~L} \mathrm{reb} /$ low & FALSE \\
\hline 2007 & $\mathrm{~A}$ & 4 & 3 & 0.85 & 99.60 & BIS & & FALSE \\
\hline 2007 & $\mathrm{~A}$ & 4 & 4 & 1.71 & 99.40 & BIS & & FALSE \\
\hline 2007 & $\mathrm{~A}$ & 4 & 5 & 2.50 & 99.17 & ТОВ & & FALSE \\
\hline 2007 & $\mathrm{~A}$ & 4 & 6 & 2.74 & 98.86 & BIS & & FALSE \\
\hline 2007 & $\mathrm{~A}$ & 4 & 7 & 3.05 & 98.63 & BIS & & FALSE \\
\hline 2007 & A & 4 & 8 & 3.26 & 98.76 & BIS & & TRUE \\
\hline 2007 & $\mathrm{~A}$ & 4 & 9 & 3.41 & 98.58 & EW & & FALSE \\
\hline 2007 & $\mathrm{~A}$ & 4 & 10 & 3.75 & 98.52 & BIS & & FALSE \\
\hline 2007 & $\mathrm{~A}$ & 4 & 11 & 4.08 & 98.49 & BIS & & FALSE \\
\hline 2007 & $\mathrm{~A}$ & 4 & 12 & 4.33 & 98.48 & BIS & & FALSE \\
\hline 2007 & A & 4 & 13 & 4.57 & 98.49 & TW & & FALSE \\
\hline 2007 & $\mathrm{~A}$ & 4 & 14 & 4.88 & 98.57 & BIS & & FALSE \\
\hline 2007 & A & 4 & 15 & 5.06 & 98.56 & BIS & & FALSE \\
\hline 2007 & $\mathrm{~A}$ & 4 & 16 & 5.15 & 98.49 & BIS & & FALSE \\
\hline 2007 & A & 4 & 17 & 5.52 & 98.51 & BIS & & FALSE \\
\hline 2007 & $\mathrm{~A}$ & 4 & 18 & 5.52 & 98.54 & EW & & FALSE \\
\hline 2007 & A & 4 & 19 & 5.73 & 98.61 & BIS & & FALSE \\
\hline 2007 & A & 4 & 20 & 5.88 & 98.57 & BIS & & FALSE \\
\hline 2007 & $\mathrm{~A}$ & 4 & 21 & 6.19 & 98.69 & $\mathrm{BF}$ & & FALSE \\
\hline 2007 & $\mathrm{~A}$ & 4 & 22 & 6.92 & 98.99 & BIS & & FALSE \\
\hline 2007 & A & 4 & 23 & 7.59 & 99.11 & BIS & & FALSE \\
\hline 2007 & $\mathrm{~A}$ & 4 & 24 & 7.83 & 99.26 & BIS & & FALSE \\
\hline 2007 & A & 4 & 25 & 8.66 & 99.45 & BOT X4R & Bot $\mathrm{X} 4 \mathrm{R}$ reb/low & FALSE \\
\hline 2007 & A & 4 & 26 & 8.66 & 99.84 & TOPX4R & Top X4R reb/low & FALSE \\
\hline 2007 & A & 5 & 1 & 0.00 & 100.00 & TOP X5L & Top X5L reb/low & FALSE \\
\hline 2007 & A & 5 & 2 & 0.00 & 99.72 & BOT X5L & Bot $\mathrm{X} 5 \mathrm{~L} \mathrm{reb} /$ low & FALSE \\
\hline 2007 & A & 5 & 3 & 0.61 & 99.84 & BIS & & FALSE \\
\hline 2007 & A & 5 & 4 & 1.22 & 99.77 & ТОВ & log extending from ТОВ to water & TRUE \\
\hline 2007 & A & 5 & 5 & 1.62 & 99.59 & BIS & & FALSE \\
\hline 2007 & $\mathrm{~A}$ & 5 & 6 & 2.35 & 99.03 & BIS & & FALSE \\
\hline 2007 & A & 5 & 7 & 2.47 & 98.99 & BIS & & FALSE \\
\hline 2007 & A & 5 & 8 & 2.50 & 98.82 & BIS & & FALSE \\
\hline
\end{tabular}

Continued on Next Page... 
Table D.1 - Continued

\begin{tabular}{|c|c|c|c|c|c|c|c|c|}
\hline Year & Reach & Section & Point & Dist. (m) & Elev. $(\mathrm{m})$ & Desc. & Notes & Exclude \\
\hline 2007 & $\mathrm{~A}$ & 5 & 9 & 2.99 & 98.75 & $\mathrm{BF}$ & & FALSE \\
\hline 2007 & A & 5 & 10 & 3.17 & 98.65 & BIS & & FALSE \\
\hline 2007 & A & 5 & 11 & 3.38 & 98.61 & EW & & FALSE \\
\hline 2007 & A & 5 & 12 & 3.93 & 98.46 & TW & & FALSE \\
\hline 2007 & $\mathrm{~A}$ & 5 & 13 & 4.11 & 98.58 & BIS & & FALSE \\
\hline 2007 & A & 5 & 14 & 4.30 & 98.54 & BIS & & FALSE \\
\hline 2007 & A & 5 & 15 & 4.60 & 98.57 & BIS & & FALSE \\
\hline 2007 & $\mathrm{~A}$ & 5 & 16 & 4.79 & 98.61 & BIS & & FALSE \\
\hline 2007 & $\mathrm{~A}$ & 5 & 17 & 5.00 & 98.62 & EW & & FALSE \\
\hline 2007 & $\mathrm{~A}$ & 5 & 18 & 5.15 & 98.99 & BIS & & FALSE \\
\hline 2007 & A & 5 & 19 & 5.52 & 98.86 & BIS & & FALSE \\
\hline 2007 & A & 5 & 20 & 5.67 & 98.83 & BIS & & FALSE \\
\hline 2007 & $\mathrm{~A}$ & 5 & 21 & 5.76 & 99.06 & ТОВ & & FALSE \\
\hline 2007 & A & 5 & 22 & 6.34 & 99.17 & BIS & & FALSE \\
\hline 2007 & $\mathrm{~A}$ & 5 & 23 & 6.95 & 99.36 & BIS & & FALSE \\
\hline 2007 & A & 5 & 24 & 7.32 & 99.59 & BIS & & FALSE \\
\hline 2007 & $\mathrm{~A}$ & 5 & 25 & 7.92 & 99.65 & BOT X5R & Bot $\mathrm{X} 5 \mathrm{R}$ reb/low & FALSE \\
\hline 2007 & $\mathrm{~A}$ & 5 & 26 & 7.92 & 99.98 & TOP X5R & Top X5R reb/low & FALSE \\
\hline 2007 & $\mathrm{~A}$ & 6 & 1 & 0.00 & 100.00 & TOP X6L & Top X6L reb/low & FALSE \\
\hline 2007 & $\mathrm{~A}$ & 6 & 2 & 0.00 & 99.48 & BOT X6L & Bot X6L reb/low - ТОВ & FALSE \\
\hline 2007 & $\mathrm{~A}$ & 6 & 3 & 0.52 & 98.78 & BIS & & FALSE \\
\hline 2007 & $\mathrm{~A}$ & 6 & 4 & 0.91 & 98.53 & BIS & & FALSE \\
\hline 2007 & $\mathrm{~A}$ & 6 & 5 & 1.34 & 98.24 & $\mathrm{BF}$ & & FALSE \\
\hline 2007 & $\mathrm{~A}$ & 6 & 6 & 1.71 & 98.11 & BIS & & FALSE \\
\hline 2007 & $\mathrm{~A}$ & 6 & 7 & 1.95 & 98.08 & EW & & FALSE \\
\hline 2007 & $\mathrm{~A}$ & 6 & 8 & 2.47 & 97.94 & BIS & & FALSE \\
\hline 2007 & $\mathrm{~A}$ & 6 & 9 & 2.87 & 97.98 & BIS & & FALSE \\
\hline 2007 & $\mathrm{~A}$ & 6 & 10 & 3.02 & 98.04 & BIS & & FALSE \\
\hline 2007 & $\mathrm{~A}$ & 6 & 11 & 3.29 & 97.99 & BIS & & FALSE \\
\hline 2007 & $\mathrm{~A}$ & 6 & 12 & 3.54 & 98.01 & TW & & FALSE \\
\hline 2007 & A & 6 & 13 & 3.78 & 98.08 & EW & & FALSE \\
\hline 2007 & $\mathrm{~A}$ & 6 & 14 & 4.30 & 98.37 & BIS & & FALSE \\
\hline 2007 & A & 6 & 15 & 4.39 & 98.75 & ТОВ & & FALSE \\
\hline 2007 & $\mathrm{~A}$ & 6 & 16 & 4.97 & 98.99 & BIS & & FALSE \\
\hline 2007 & A & 6 & 17 & 5.79 & 99.13 & BIS & & FALSE \\
\hline 2007 & A & 6 & 18 & 7.32 & 99.33 & BIS & & FALSE \\
\hline 2007 & A & 6 & 19 & 9.69 & 99.26 & BIS & & FALSE \\
\hline 2007 & A & 6 & 20 & 11.37 & 99.28 & BIS & & FALSE \\
\hline 2007 & $\mathrm{~A}$ & 6 & 21 & 12.34 & 99.20 & BIS & & FALSE \\
\hline 2007 & $\mathrm{~A}$ & 6 & 22 & 13.29 & 99.23 & BIS & & FALSE \\
\hline 2007 & A & 6 & 23 & 14.51 & 99.24 & BIS & & FALSE \\
\hline 2007 & $\mathrm{~A}$ & 6 & 24 & 14.94 & 99.54 & BOT X6R & Bot $\mathrm{X} 6 \mathrm{R}$ reb/low - 1' low adjusted & FALSE \\
\hline 2007 & $\mathrm{~A}$ & 6 & 25 & 14.94 & 99.92 & TOP X6R & Top X6R reb/low & FALSE \\
\hline 2007 & A & 7 & 1 & 0.00 & 100.00 & TOP X7L & Top X7L reb/low & FALSE \\
\hline 2007 & A & 7 & 2 & 0.00 & 99.77 & BOT X7L & Bot $\mathrm{X} 7 \mathrm{~L} \mathrm{reb} /$ low & FALSE \\
\hline 2007 & $\mathrm{~A}$ & 7 & 3 & 0.46 & 99.74 & BIS & & FALSE \\
\hline 2007 & A & 7 & 4 & 0.73 & 99.63 & ТОВ & & FALSE \\
\hline 2007 & A & 7 & 5 & 0.91 & 99.32 & BIS & & FALSE \\
\hline 2007 & $\mathrm{~A}$ & 7 & 6 & 1.52 & 98.58 & BIS & & FALSE \\
\hline 2007 & A & 7 & 7 & 1.71 & 98.57 & BIS & & FALSE \\
\hline 2007 & A & 7 & 8 & 1.95 & 98.75 & BIS & Top of boulder & FALSE \\
\hline 2007 & A & 7 & 9 & 2.38 & 98.64 & BIS & & FALSE \\
\hline
\end{tabular}

Continued on Next Page... 
Table D.1 - Continued

\begin{tabular}{|c|c|c|c|c|c|c|c|c|}
\hline Year & Reach & Section & Point & Dist. $(\mathrm{m})$ & Elev. $(\mathrm{m})$ & Desc. & Notes & Exclude \\
\hline 2007 & A & 7 & 10 & 2.59 & 98.23 & BIS & & FALSE \\
\hline 2007 & A & 7 & 11 & 2.77 & 98.18 & BIS & & FALSE \\
\hline 2007 & A & 7 & 12 & 3.14 & 98.24 & BIS & Rock & FALSE \\
\hline 2007 & A & 7 & 13 & 3.23 & 98.20 & BIS & Rock & FALSE \\
\hline 2007 & A & 7 & 14 & 3.41 & 98.24 & BIS & Rock & FALSE \\
\hline 2007 & A & 7 & 15 & 3.57 & 98.17 & EW & & FALSE \\
\hline 2007 & A & 7 & 16 & 3.69 & 98.10 & BIS & & FALSE \\
\hline 2007 & A & 7 & 17 & 3.81 & 98.10 & $\mathrm{TW}$ & & FALSE \\
\hline 2007 & A & 7 & 18 & 4.02 & 98.14 & EW & & FALSE \\
\hline 2007 & A & 7 & 19 & 4.21 & 98.23 & BIS & & FALSE \\
\hline 2007 & A & 7 & 20 & 4.42 & 98.27 & BIS & & FALSE \\
\hline 2007 & A & 7 & 21 & 4.66 & 98.44 & BIS & & FALSE \\
\hline 2007 & A & 7 & 22 & 5.49 & 98.73 & BIS & & FALSE \\
\hline 2007 & A & 7 & 23 & 6.89 & 98.98 & BIS & & FALSE \\
\hline 2007 & A & 7 & 24 & 8.05 & 99.15 & BIS & & FALSE \\
\hline 2007 & A & 7 & 25 & 8.78 & 99.43 & BIS & & FALSE \\
\hline 2007 & A & 7 & 26 & 10.18 & 99.56 & BIS & & FALSE \\
\hline 2007 & A & 7 & 27 & 12.59 & 99.48 & BIS & & FALSE \\
\hline 2007 & A & 7 & 28 & 15.09 & 99.28 & BIS & & FALSE \\
\hline 2007 & A & 7 & 29 & 16.52 & 99.34 & BIS & & FALSE \\
\hline 2007 & A & 7 & 30 & 17.77 & 99.46 & BIS & & FALSE \\
\hline 2007 & A & 7 & 31 & 18.65 & 99.96 & BOT X7R & Bot $\mathrm{X} 7 \mathrm{R}$ reb/low & FALSE \\
\hline 2007 & A & 7 & 32 & 18.65 & 100.22 & TOP X7R & Top X7R reb/low & FALSE \\
\hline 2007 & A & 8 & 1 & 0.00 & 100.00 & TOP X8L & Top X8L reb/low & FALSE \\
\hline 2007 & A & 8 & 2 & 0.00 & 99.63 & BOT X8L & Bot $\mathrm{X} 8 \mathrm{~L} \mathrm{reb} /$ low & FALSE \\
\hline 2007 & A & 8 & 3 & 0.43 & 99.62 & BIS & & FALSE \\
\hline 2007 & A & 8 & 4 & 0.91 & 99.43 & ТОВ & & FALSE \\
\hline 2007 & A & 8 & 5 & 1.13 & 98.54 & BIS & & FALSE \\
\hline 2007 & A & 8 & 6 & 1.74 & 98.34 & BIS & & FALSE \\
\hline 2007 & A & 8 & 7 & 2.47 & 98.12 & $\mathrm{BF}$ & & FALSE \\
\hline 2007 & A & 8 & 8 & 2.99 & 98.01 & BIS & & FALSE \\
\hline 2007 & A & 8 & 9 & 3.23 & 97.89 & EW & & FALSE \\
\hline 2007 & A & 8 & 10 & 3.35 & 97.86 & BIS & & FALSE \\
\hline 2007 & A & 8 & 11 & 3.63 & 97.90 & BIS & & FALSE \\
\hline 2007 & A & 8 & 12 & 4.05 & 97.79 & BIS & & FALSE \\
\hline 2007 & A & 8 & 13 & 4.45 & 97.79 & TW & & FALSE \\
\hline 2007 & A & 8 & 14 & 4.88 & 97.82 & BIS & & FALSE \\
\hline 2007 & A & 8 & 15 & 5.15 & 97.85 & BIS & & FALSE \\
\hline 2007 & A & 8 & 16 & 5.61 & 97.88 & EW & & FALSE \\
\hline 2007 & A & 8 & 17 & 5.94 & 97.99 & BIS & & FALSE \\
\hline 2007 & A & 8 & 18 & 6.07 & 98.31 & BIS & & FALSE \\
\hline 2007 & A & 8 & 19 & 6.64 & 98.50 & BIS & & FALSE \\
\hline 2007 & A & 8 & 20 & 9.39 & 98.81 & BIS & & FALSE \\
\hline 2007 & A & 8 & 21 & 10.94 & 98.90 & BIS & & FALSE \\
\hline 2007 & A & 8 & 22 & 12.13 & 99.21 & BIS & & FALSE \\
\hline 2007 & A & 8 & 23 & 14.14 & 99.16 & BIS & & FALSE \\
\hline 2007 & A & 8 & 24 & 15.24 & 99.09 & BIS & & FALSE \\
\hline 2007 & A & 8 & 25 & 16.58 & 99.01 & BIS & & FALSE \\
\hline 2007 & A & 8 & 26 & 17.53 & 99.12 & BIS & & FALSE \\
\hline 2007 & A & 8 & 27 & 18.68 & 99.20 & BIS & & FALSE \\
\hline 2007 & A & 8 & 28 & 19.57 & 99.74 & BOT X8R & Bot $\mathrm{X} 8 \mathrm{R}$ reb/low & FALSE \\
\hline 2007 & A & 8 & 29 & 19.57 & 99.99 & TOP X8R & Top X8R reb/low & FALSE \\
\hline
\end{tabular}

Continued on Next Page... 
Table D.1 - Continued

\begin{tabular}{|c|c|c|c|c|c|c|c|c|}
\hline Year & Reach & Section & Point & Dist. (m) & Elev. $(\mathrm{m})$ & Desc. & Notes & Exclude \\
\hline 2007 & $\mathrm{~A}$ & 9 & 1 & 0.00 & 100.00 & TOP X9L & Top X9L reb/low & FALSE \\
\hline 2007 & A & 9 & 2 & 0.00 & 99.65 & BOT X9L & Bot $\mathrm{X} 9 \mathrm{~L} \mathrm{reb} /$ low & FALSE \\
\hline 2007 & A & 9 & 3 & 0.79 & 99.66 & ТОВ & & FALSE \\
\hline 2007 & A & 9 & 4 & 0.88 & 98.70 & BIS & & FALSE \\
\hline 2007 & $\mathrm{~A}$ & 9 & 5 & 1.83 & 98.59 & BIS & & FALSE \\
\hline 2007 & $\mathrm{~A}$ & 9 & 6 & 2.59 & 98.64 & BIS & & FALSE \\
\hline 2007 & $\mathrm{~A}$ & 9 & 7 & 3.29 & 98.49 & BIS & & FALSE \\
\hline 2007 & $\mathrm{~A}$ & 9 & 8 & 3.69 & 98.40 & $\mathrm{BF}$ & & FALSE \\
\hline 2007 & $\mathrm{~A}$ & 9 & 9 & 4.30 & 98.21 & BIS & & FALSE \\
\hline 2007 & $\mathrm{~A}$ & 9 & 10 & 4.69 & 98.13 & EW & & FALSE \\
\hline 2007 & $\mathrm{~A}$ & 9 & 11 & 5.12 & 98.04 & BIS & & FALSE \\
\hline 2007 & $\mathrm{~A}$ & 9 & 12 & 5.85 & 97.99 & TW & & FALSE \\
\hline 2007 & $\mathrm{~A}$ & 9 & 13 & 6.22 & 98.06 & BIS & & FALSE \\
\hline 2007 & $\mathrm{~A}$ & 9 & 14 & 6.52 & 98.07 & BIS & & FALSE \\
\hline 2007 & $\mathrm{~A}$ & 9 & 15 & 6.64 & 98.13 & EW & & FALSE \\
\hline 2007 & $\mathrm{~A}$ & 9 & 16 & 6.98 & 98.22 & BIS & & FALSE \\
\hline 2007 & $\mathrm{~A}$ & 9 & 17 & 7.32 & 98.42 & $\mathrm{BF}$ & & FALSE \\
\hline 2007 & $\mathrm{~A}$ & 9 & 18 & 7.92 & 98.53 & BIS & & FALSE \\
\hline 2007 & $\mathrm{~A}$ & 9 & 19 & 8.38 & 98.77 & ТОВ & & FALSE \\
\hline 2007 & $\mathrm{~A}$ & 9 & 20 & 10.06 & 98.96 & BIS & & FALSE \\
\hline 2007 & $\mathrm{~A}$ & 9 & 21 & 12.04 & 98.98 & BIS & & FALSE \\
\hline 2007 & $\mathrm{~A}$ & 9 & 22 & 12.62 & 99.13 & BIS & & FALSE \\
\hline 2007 & $\mathrm{~A}$ & 9 & 23 & 13.02 & 99.39 & BIS & & FALSE \\
\hline 2007 & $\mathrm{~A}$ & 9 & 24 & 14.08 & 99.46 & BIS & & FALSE \\
\hline 2007 & A & 9 & 25 & 15.06 & 99.35 & BIS & & FALSE \\
\hline 2007 & $\mathrm{~A}$ & 9 & 26 & 17.07 & 99.22 & BIS & & FALSE \\
\hline 2007 & $\mathrm{~A}$ & 9 & 27 & 19.54 & 99.43 & BIS & & FALSE \\
\hline 2007 & $\mathrm{~A}$ & 9 & 28 & 20.54 & 99.92 & BOT X9R & Bot X9R reb/low & FALSE \\
\hline 2007 & $\mathrm{~A}$ & 9 & 29 & 20.54 & 100.25 & TOP X9R & Top X9R reb/low & FALSE \\
\hline 2007 & $\mathrm{~A}$ & 10 & 1 & 0.00 & 100.00 & TOP X10L & Top X10L reb/low & FALSE \\
\hline 2007 & $\mathrm{~A}$ & 10 & 2 & 0.00 & 99.58 & BOT X10L & Bot $\mathrm{X} 10 \mathrm{~L} \mathrm{reb} / \mathrm{low}$ & FALSE \\
\hline 2007 & $\mathrm{~A}$ & 10 & 3 & 0.49 & 99.55 & ТОВ & & FALSE \\
\hline 2007 & $\mathrm{~A}$ & 10 & 4 & 0.55 & 98.58 & BIS & & FALSE \\
\hline 2007 & $\mathrm{~A}$ & 10 & 5 & 1.52 & 98.58 & BIS & & FALSE \\
\hline 2007 & $\mathrm{~A}$ & 10 & 6 & 2.38 & 98.52 & BIS & & FALSE \\
\hline 2007 & $\mathrm{~A}$ & 10 & 7 & 2.96 & 98.36 & BIS & & FALSE \\
\hline 2007 & $\mathrm{~A}$ & 10 & 8 & 3.81 & 98.34 & BIS & & FALSE \\
\hline 2007 & $\mathrm{~A}$ & 10 & 9 & 3.87 & 98.22 & BIS & & FALSE \\
\hline 2007 & $\mathrm{~A}$ & 10 & 10 & 4.54 & 98.29 & BIS & & FALSE \\
\hline 2007 & $\mathrm{~A}$ & 10 & 11 & 4.88 & 98.17 & BIS & & FALSE \\
\hline 2007 & $\mathrm{~A}$ & 10 & 12 & 5.36 & 98.31 & BIS & Top of rock & FALSE \\
\hline 2007 & $\mathrm{~A}$ & 10 & 13 & 5.76 & 98.22 & BIS & & FALSE \\
\hline 2007 & $\mathrm{~A}$ & 10 & 14 & 5.79 & 97.86 & EW & & FALSE \\
\hline 2007 & A & 10 & 15 & 5.85 & 97.65 & BIS & Top of rock & FALSE \\
\hline 2007 & $\mathrm{~A}$ & 10 & 16 & 6.86 & 97.59 & BIS & & FALSE \\
\hline 2007 & A & 10 & 17 & 7.41 & 97.62 & BIS & Top of rock & FALSE \\
\hline 2007 & A & 10 & 18 & 7.80 & 98.12 & BIS & & FALSE \\
\hline 2007 & A & 10 & 19 & 8.11 & 97.95 & BIS & & FALSE \\
\hline 2007 & $\mathrm{~A}$ & 10 & 20 & 8.26 & 97.67 & BIS & & FALSE \\
\hline 2007 & $\mathrm{~A}$ & 10 & 21 & 8.75 & 97.52 & $\mathrm{TW}$ & & FALSE \\
\hline 2007 & A & 10 & 22 & 8.99 & 97.65 & BIS & & FALSE \\
\hline 2007 & A & 10 & 23 & 9.08 & 97.86 & EW & Top of boulder & FALSE \\
\hline
\end{tabular}

Continued on Next Page... 
Table D.1 - Continued

\begin{tabular}{|c|c|c|c|c|c|c|c|c|}
\hline Year & Reach & Section & Point & Dist. (m) & Elev. $(\mathrm{m})$ & Desc. & Notes & Exclude \\
\hline 2007 & A & 10 & 24 & 9.48 & 98.25 & BIS & & FALSE \\
\hline 2007 & A & 10 & 25 & 10.09 & 98.22 & BIS & & FALSE \\
\hline 2007 & A & 10 & 26 & 10.70 & 98.68 & ТОВ & & FALSE \\
\hline 2007 & A & 10 & 27 & 12.31 & 98.93 & BIS & & FALSE \\
\hline 2007 & $\mathrm{~A}$ & 10 & 28 & 15.24 & 99.03 & BIS & & FALSE \\
\hline 2007 & A & 10 & 29 & 17.01 & 99.00 & BIS & & FALSE \\
\hline 2007 & $\mathrm{~A}$ & 10 & 30 & 18.81 & 99.26 & BIS & & FALSE \\
\hline 2007 & A & 10 & 31 & 20.27 & 99.85 & BOT X9R & Bot $\mathrm{X} 10 \mathrm{~L} \mathrm{reb} / \mathrm{low}$ & FALSE \\
\hline 2007 & A & 10 & 32 & 20.27 & 100.01 & TOP X9R & Top X10L reb/low & FALSE \\
\hline 2007 & B & 1 & 1 & 0.00 & 100.00 & TOP X1L & Top X1L reb/low & FALSE \\
\hline 2007 & B & 1 & 2 & 0.00 & 99.86 & BOT X1L & Bot $\mathrm{X} 1 \mathrm{~L}$ reb/low & FALSE \\
\hline 2007 & $\mathrm{~B}$ & 1 & 3 & 0.34 & 99.67 & BIS & & FALSE \\
\hline 2007 & $\mathrm{~B}$ & 1 & 4 & 0.43 & 99.25 & BIS & & FALSE \\
\hline 2007 & $\mathrm{~B}$ & 1 & 5 & 0.79 & 98.94 & BIS & Rock & FALSE \\
\hline 2007 & B & 1 & 6 & 0.88 & 98.55 & BIS & & FALSE \\
\hline 2007 & B & 1 & 7 & 1.25 & 98.32 & BIS & Between 4.1 and $5.7=$ Trail & FALSE \\
\hline 2007 & B & 1 & 8 & 1.74 & 98.17 & BIS & & FALSE \\
\hline 2007 & $\mathrm{~B}$ & 1 & 9 & 2.41 & 97.77 & BIS & & FALSE \\
\hline 2007 & B & 1 & 10 & 2.80 & 97.61 & EW & & FALSE \\
\hline 2007 & B & 1 & 11 & 3.29 & 97.52 & $\mathrm{TW}$ & & FALSE \\
\hline 2007 & B & 1 & 12 & 3.87 & 97.59 & BIS & & FALSE \\
\hline 2007 & B & 1 & 13 & 4.21 & 97.55 & BIS & & FALSE \\
\hline 2007 & B & 1 & 14 & 4.57 & 97.66 & BIS & & FALSE \\
\hline 2007 & B & 1 & 15 & 4.94 & 97.58 & BIS & & FALSE \\
\hline 2007 & B & 1 & 16 & 5.49 & 97.60 & EW & & FALSE \\
\hline 2007 & B & 1 & 17 & 6.28 & 97.72 & $\mathrm{BF}$ & & FALSE \\
\hline 2007 & B & 1 & 18 & 7.01 & 97.85 & BIS & & FALSE \\
\hline 2007 & B & 1 & 19 & 7.56 & 98.14 & BIS & & FALSE \\
\hline 2007 & B & 1 & 20 & 8.23 & 98.55 & BIS & & FALSE \\
\hline 2007 & B & 1 & 21 & 8.69 & 98.93 & BIS & & FALSE \\
\hline 2007 & B & 1 & 22 & 10.09 & 99.50 & BOT X1R & Bot $\mathrm{X} 1 \mathrm{R}$ reb/low & FALSE \\
\hline 2007 & B & 1 & 23 & 10.09 & 99.99 & TOP X1R & Top X1R reb/low & FALSE \\
\hline 2007 & B & 2 & 1 & 0.00 & 100.00 & TOP X2L & Top X2L reb/low & FALSE \\
\hline 2007 & B & 2 & 2 & 0.00 & 99.61 & BOT X2L & Bot $\mathrm{X} 2 \mathrm{~L} \mathrm{reb} /$ low & FALSE \\
\hline 2007 & B & 2 & 3 & 0.18 & 99.51 & BIS & & FALSE \\
\hline 2007 & B & 2 & 4 & 0.40 & 99.06 & BIS & Bwn 1.3 and $4.2=$ Trail & FALSE \\
\hline 2007 & B & 2 & 5 & 1.28 & 98.75 & BIS & & FALSE \\
\hline 2007 & B & 2 & 6 & 2.07 & 98.24 & BIS & & FALSE \\
\hline 2007 & B & 2 & 7 & 2.47 & 98.11 & ТОВ & & FALSE \\
\hline 2007 & B & 2 & 8 & 2.90 & 97.60 & BIS & & FALSE \\
\hline 2007 & B & 2 & 9 & 3.38 & 97.44 & EW & & FALSE \\
\hline 2007 & B & 2 & 10 & 3.90 & 97.38 & BIS & & FALSE \\
\hline 2007 & B & 2 & 11 & 4.45 & 97.40 & BIS & & FALSE \\
\hline 2007 & B & 2 & 12 & 5.40 & 97.30 & TW & & FALSE \\
\hline 2007 & B & 2 & 13 & 6.22 & 97.40 & EW & & FALSE \\
\hline 2007 & B & 2 & 14 & 7.32 & 97.51 & $\mathrm{BF}$ & $\begin{array}{l}\text { Rock (original BS } 13.88 \text {, graphed } \\
\text { obviously wrong, likely mis- } \\
\text { read philly rod, adjusted } 1 \text { foot } \\
\text { higher.) }\end{array}$ & FALSE \\
\hline 2007 & $\mathrm{~B}$ & 2 & 15 & 7.53 & 97.86 & BIS & & TRUE \\
\hline 2007 & $\mathrm{~B}$ & 2 & 16 & 8.50 & 97.99 & BIS & & FALSE \\
\hline 2007 & B & 2 & 17 & 9.63 & 98.82 & BIS & & FALSE \\
\hline
\end{tabular}

Continued on Next Page... 
Table D.1 - Continued

\begin{tabular}{|c|c|c|c|c|c|c|c|c|}
\hline Year & Reach & Section & Point & Dist. (m) & Elev. $(\mathrm{m})$ & Desc. & Notes & Exclude \\
\hline 2007 & B & 2 & 18 & 10.21 & 99.34 & BOT X2R & Bot $\mathrm{X} 2 \mathrm{R}$ reb/low & FALSE \\
\hline 2007 & B & 2 & 19 & 10.21 & 99.73 & TOP X2R & Top X2R reb/low & FALSE \\
\hline 2007 & B & 3 & 1 & 0.00 & 100.00 & TOP X3L & Top X3L reb/low & FALSE \\
\hline 2007 & B & 3 & 2 & 0.00 & 99.46 & BOT X3L & Bot $\mathrm{X} 3 \mathrm{~L}$ reb/low & FALSE \\
\hline 2007 & B & 3 & 3 & 0.40 & 99.23 & BIS & & FALSE \\
\hline 2007 & B & 3 & 4 & 0.98 & 98.95 & BIS & & FALSE \\
\hline 2007 & B & 3 & 5 & 1.49 & 98.49 & BIS & & FALSE \\
\hline 2007 & B & 3 & 6 & 2.50 & 98.10 & BIS & & FALSE \\
\hline 2007 & B & 3 & 7 & 2.62 & 98.27 & BIS & & FALSE \\
\hline 2007 & B & 3 & 8 & 3.38 & 98.29 & BIS & & FALSE \\
\hline 2007 & B & 3 & 9 & 4.08 & 98.03 & EW & & FALSE \\
\hline 2007 & B & 3 & 10 & 4.48 & 97.96 & BIS & & FALSE \\
\hline 2007 & B & 3 & 11 & 4.91 & 97.92 & BIS & & FALSE \\
\hline 2007 & B & 3 & 12 & 5.12 & 97.94 & BIS & & FALSE \\
\hline 2007 & B & 3 & 13 & 5.30 & 97.89 & $\mathrm{TW}$ & & FALSE \\
\hline 2007 & B & 3 & 14 & 5.88 & 98.16 & $\mathrm{BF}$ & & FALSE \\
\hline 2007 & B & 3 & 15 & 6.49 & 98.45 & ТОВ & & FALSE \\
\hline 2007 & B & 3 & 16 & 7.32 & 98.82 & BIS & & FALSE \\
\hline 2007 & B & 3 & 17 & 8.14 & 99.35 & BIS & & FALSE \\
\hline 2007 & B & 3 & 18 & 8.41 & 99.82 & BOT X3R & Bot $\mathrm{X} 3 \mathrm{R}$ reb/low & FALSE \\
\hline 2007 & B & 3 & 19 & 8.41 & 99.98 & TOP X3R & Top X3R reb/low & FALSE \\
\hline 2007 & $\mathrm{~B}$ & 4 & 1 & 0.00 & 100.00 & TOP X4L & Top X4L reb/low & FALSE \\
\hline 2007 & B & 4 & 2 & 0.00 & 99.84 & BOT X4L & Bot $\mathrm{X} 4 \mathrm{~L}$ reb/low & FALSE \\
\hline 2007 & B & 4 & 3 & 0.91 & 99.62 & BIS & & FALSE \\
\hline 2007 & B & 4 & 4 & 1.49 & 99.37 & ТОВ & & FALSE \\
\hline 2007 & B & 4 & 5 & 1.68 & 98.89 & BIS & & FALSE \\
\hline 2007 & B & 4 & 6 & 1.86 & 98.84 & BIS & & FALSE \\
\hline 2007 & B & 4 & 7 & 2.01 & 98.68 & BIS & Puddle & FALSE \\
\hline 2007 & B & 4 & 8 & 2.47 & 98.63 & BIS & Puddle & FALSE \\
\hline 2007 & B & 4 & 9 & 2.71 & 98.60 & BIS & Puddle & FALSE \\
\hline 2007 & B & 4 & 10 & 3.38 & 98.84 & BIS & & FALSE \\
\hline 2007 & $\mathrm{~B}$ & 4 & 11 & 3.96 & 98.91 & BIS & & FALSE \\
\hline 2007 & B & 4 & 12 & 4.08 & 98.63 & BIS & & FALSE \\
\hline 2007 & B & 4 & 13 & 4.33 & 98.55 & BIS & & FALSE \\
\hline 2007 & B & 4 & 14 & 4.66 & 98.57 & EW & & FALSE \\
\hline 2007 & B & 4 & 15 & 4.72 & 98.67 & BIS & & FALSE \\
\hline 2007 & $\mathrm{~B}$ & 4 & 16 & 5.00 & 98.71 & BIS & & FALSE \\
\hline 2007 & B & 4 & 17 & 5.91 & 98.66 & BIS & In water $0.4^{\prime}$ deep & FALSE \\
\hline 2007 & B & 4 & 18 & 5.94 & 98.45 & BIS & & FALSE \\
\hline 2007 & B & 4 & 19 & 6.16 & 98.44 & TW & & FALSE \\
\hline 2007 & $\mathrm{~B}$ & 4 & 20 & 6.49 & 98.58 & EW & & FALSE \\
\hline 2007 & B & 4 & 21 & 7.38 & 99.50 & BOT X4R & Bot $\mathrm{X} 4 \mathrm{R} \mathrm{reb} /$ low & FALSE \\
\hline 2007 & B & 4 & 22 & 7.38 & 99.71 & TOP X4R & Top X4R reb/low & FALSE \\
\hline 2007 & $\mathrm{~B}$ & 5 & 1 & 0.00 & 100.00 & TOP X5L & Top X5L reb/low & FALSE \\
\hline 2007 & $\mathrm{~B}$ & 5 & 2 & 0.00 & 99.61 & BOT X5L & Bot $\mathrm{X} 5 \mathrm{~L} \mathrm{reb} /$ low & FALSE \\
\hline 2007 & B & 5 & 3 & 0.21 & 99.35 & BIS & & FALSE \\
\hline 2007 & B & 5 & 4 & 1.25 & 98.78 & BIS & & FALSE \\
\hline 2007 & B & 5 & 5 & 2.10 & 98.37 & BIS & & FALSE \\
\hline 2007 & B & 5 & 6 & 3.14 & 98.11 & BIS & & FALSE \\
\hline 2007 & B & 5 & 7 & 3.44 & 98.05 & $\mathrm{BF}$ & & FALSE \\
\hline 2007 & B & 5 & 8 & 3.93 & 97.98 & EW & & FALSE \\
\hline 2007 & B & 5 & 9 & 4.63 & 97.91 & $\mathrm{TW}$ & & FALSE \\
\hline
\end{tabular}

Continued on Next Page... 
Table D.1 - Continued

\begin{tabular}{|c|c|c|c|c|c|c|c|c|}
\hline Year & Reach & Section & Point & Dist. (m) & Elev. $(\mathrm{m})$ & Desc. & Notes & Exclude \\
\hline 2007 & $\mathrm{~B}$ & 5 & 10 & 5.12 & 97.93 & BIS & & FALSE \\
\hline 2007 & B & 5 & 11 & 5.73 & 97.93 & BIS & Woody debris accumulation & FALSE \\
\hline 2007 & $\mathrm{~B}$ & 5 & 12 & 6.25 & 98.05 & EW & & FALSE \\
\hline 2007 & $\mathrm{~B}$ & 5 & 13 & 7.07 & 98.79 & BIS & & FALSE \\
\hline 2007 & B & 5 & 14 & 7.80 & 99.93 & BOT X5R & Bot $\mathrm{X} 5 \mathrm{R}$ reb/low & FALSE \\
\hline 2007 & $\mathrm{~B}$ & 5 & 15 & 7.80 & 100.09 & TOP X5R & Top X5R reb/low & FALSE \\
\hline 2007 & $\mathrm{~B}$ & 6 & 1 & 0.00 & 100.00 & TOP X6L & Top X6L reb/low & FALSE \\
\hline 2007 & $\mathrm{~B}$ & 6 & 2 & 0.00 & 99.97 & BOT X6L & Bot $\mathrm{X} 6 \mathrm{~L}$ reb/low & FALSE \\
\hline 2007 & B & 6 & 3 & 0.34 & 99.69 & BIS & & FALSE \\
\hline 2007 & B & 6 & 4 & 1.40 & 99.37 & BIS & & FALSE \\
\hline 2007 & B & 6 & 5 & 2.23 & 98.84 & BIS & & FALSE \\
\hline 2007 & $\mathrm{~B}$ & 6 & 6 & 2.90 & 98.55 & BIS & & FALSE \\
\hline 2007 & B & 6 & 7 & 3.54 & 98.45 & $\mathrm{BF}$ & & FALSE \\
\hline 2007 & B & 6 & 8 & 3.69 & 98.33 & EW & & FALSE \\
\hline 2007 & $\mathrm{~B}$ & 6 & 9 & 4.27 & 98.26 & TW & & FALSE \\
\hline 2007 & B & 6 & 10 & 4.66 & 98.27 & BIS & & FALSE \\
\hline 2007 & B & 6 & 11 & 5.18 & 98.36 & EW & & FALSE \\
\hline 2007 & B & 6 & 12 & 5.43 & 98.63 & BIS & Top of rock & TRUE \\
\hline 2007 & $\mathrm{~B}$ & 6 & 13 & 6.52 & 98.74 & BIS & & FALSE \\
\hline 2007 & B & 6 & 14 & 7.38 & 99.33 & BIS & & FALSE \\
\hline 2007 & B & 6 & 15 & 7.92 & 99.87 & BOT X6R & Bot $\mathrm{X} 6 \mathrm{R}$ reb/low & FALSE \\
\hline 2007 & B & 6 & 16 & 7.92 & 99.95 & TOP X6R & Top X6R reb/low & FALSE \\
\hline 2007 & $\mathrm{~B}$ & 7 & 1 & 0.00 & 100.00 & TOP X6L & Top X7L reb/low & FALSE \\
\hline 2007 & B & 7 & 2 & 0.00 & 99.29 & BOT X6L & Bot $X 7 \mathrm{~L} \mathrm{reb} /$ low & FALSE \\
\hline 2007 & B & 7 & 3 & 0.70 & 98.99 & BIS & & FALSE \\
\hline 2007 & B & 7 & 4 & 1.28 & 98.60 & BIS & & FALSE \\
\hline 2007 & B & 7 & 5 & 2.16 & 98.26 & BIS & & FALSE \\
\hline 2007 & B & 7 & 6 & 2.74 & 98.16 & $\mathrm{BF}$ & & FALSE \\
\hline 2007 & B & 7 & 7 & 3.02 & 98.11 & BIS & & FALSE \\
\hline 2007 & B & 7 & 8 & 3.35 & 98.21 & BIS & Top of rock & FALSE \\
\hline 2007 & B & 7 & 9 & 3.63 & 98.08 & EW & & FALSE \\
\hline 2007 & $\mathrm{~B}$ & 7 & 10 & 3.87 & 98.02 & BIS & & FALSE \\
\hline 2007 & B & 7 & 11 & 4.30 & 98.01 & $\mathrm{TX}$ & & FALSE \\
\hline 2007 & B & 7 & 12 & 4.60 & 98.02 & BIS & & FALSE \\
\hline 2007 & $\mathrm{~B}$ & 7 & 13 & 5.21 & 98.04 & BIS & & FALSE \\
\hline 2007 & B & 7 & 14 & 5.73 & 98.09 & EW & & FALSE \\
\hline 2007 & $\mathrm{~B}$ & 7 & 15 & 6.40 & 98.45 & BIS & & FALSE \\
\hline 2007 & $\mathrm{~B}$ & 7 & 16 & 6.68 & 98.50 & BIS & & FALSE \\
\hline 2007 & $\mathrm{~B}$ & 7 & 17 & 7.68 & 99.33 & BIS & & FALSE \\
\hline 2007 & $\mathrm{~B}$ & 7 & 18 & 8.66 & 99.50 & BIS & & FALSE \\
\hline 2007 & B & 7 & 19 & 9.42 & 99.81 & BOT X7R & Bot $\mathrm{X} 7 \mathrm{R}$ reb/low & FALSE \\
\hline 2007 & B & 7 & 20 & 9.42 & 99.75 & TOP X7R & Top X7R reb/low & FALSE \\
\hline 2007 & $\mathrm{~B}$ & 8 & 1 & 0.00 & 100.00 & TOP X8L & Top X8L reb/low & FALSE \\
\hline 2007 & $\mathrm{~B}$ & 8 & 2 & 0.00 & 99.48 & BOT X8L & Bot $\mathrm{X} 8 \mathrm{~L} \mathrm{reb} /$ low & FALSE \\
\hline 2007 & $\mathrm{~B}$ & 8 & 3 & 0.40 & 99.37 & ТОВ & Alder log & FALSE \\
\hline 2007 & B & 8 & 4 & 0.46 & 98.99 & $\mathrm{BF}$ & & FALSE \\
\hline 2007 & B & 8 & 5 & 0.91 & 98.93 & BIS & & FALSE \\
\hline 2007 & B & 8 & 6 & 1.34 & 98.93 & EW & & FALSE \\
\hline 2007 & B & 8 & 7 & 1.86 & 98.87 & BIS & & FALSE \\
\hline 2007 & $\mathrm{~B}$ & 8 & 8 & 2.32 & 98.81 & BIS & & FALSE \\
\hline 2007 & B & 8 & 9 & 2.93 & 98.85 & EW & Real EW underneath log, this & FALSE \\
\hline
\end{tabular}

Continued on Next Page... 
Table D.1 - Continued

\begin{tabular}{|c|c|c|c|c|c|c|c|c|}
\hline Year & Reach & Section & Point & Dist. (m) & Elev. $(\mathrm{m})$ & Desc. & Notes & Exclude \\
\hline 2007 & $\mathrm{~B}$ & 8 & 10 & 3.17 & 99.47 & BIS & On $\log$ & FALSE \\
\hline 2007 & $\mathrm{~B}$ & 8 & 11 & 3.54 & 99.63 & BIS & On $\log$ & FALSE \\
\hline 2007 & $\mathrm{~B}$ & 8 & 12 & 3.78 & 99.82 & BIS & On $\log$ & FALSE \\
\hline 2007 & $\mathrm{~B}$ & 8 & 13 & 4.21 & 99.66 & BIS & On $\log m$ but different log & FALSE \\
\hline 2007 & $\mathrm{~B}$ & 8 & 14 & 5.06 & 99.72 & BOT X8R & Bot $\mathrm{X} 8 \mathrm{R}$ reb/low & FALSE \\
\hline 2007 & B & 8 & 15 & 5.06 & 99.92 & TOP X8R & Top X8R reb/low & FALSE \\
\hline 2007 & B & 9 & 1 & 0.00 & 100.00 & TOP X9L & Top X9L reb/low & FALSE \\
\hline 2007 & B & 9 & 2 & 0.00 & 99.77 & BOT X9L & Bot X9L reb/low & FALSE \\
\hline 2007 & $\mathrm{~B}$ & 9 & 3 & 0.21 & 98.69 & BIS & & FALSE \\
\hline 2007 & B & 9 & 4 & 0.67 & 97.93 & BIS & & FALSE \\
\hline 2007 & B & 9 & 5 & 0.94 & 98.07 & BIS & & FALSE \\
\hline 2007 & B & 9 & 6 & 1.04 & 97.71 & EW & & FALSE \\
\hline 2007 & B & 9 & 7 & 1.43 & 97.73 & BIS & & FALSE \\
\hline 2007 & B & 9 & 8 & 2.35 & 97.69 & $\mathrm{TW}$ & & FALSE \\
\hline 2007 & B & 9 & 9 & 2.87 & 97.74 & BIS & & FALSE \\
\hline 2007 & B & 9 & 10 & 3.23 & 97.77 & EW & & FALSE \\
\hline 2007 & B & 9 & 11 & 3.87 & 98.09 & ТОВ & & FALSE \\
\hline 2007 & B & 9 & 12 & 4.63 & 98.47 & BIS & & FALSE \\
\hline 2007 & B & 9 & 13 & 6.55 & 98.68 & BIS & & FALSE \\
\hline 2007 & B & 9 & 14 & 7.53 & 98.97 & BIS & & FALSE \\
\hline 2007 & B & 9 & 15 & 8.87 & 98.93 & BIS & & FALSE \\
\hline 2007 & $\mathrm{~B}$ & 9 & 16 & 10.12 & 98.88 & BIS & & FALSE \\
\hline 2007 & $\mathrm{~B}$ & 9 & 17 & 11.98 & 99.57 & BIS & & FALSE \\
\hline 2007 & B & 9 & 18 & 13.53 & 100.08 & BOT X9R & Bot $\mathrm{X} 9 \mathrm{R}$ reb/low & FALSE \\
\hline 2007 & B & 9 & 19 & 13.53 & 100.43 & TOP X9R & Top X9R reb/low & FALSE \\
\hline 2007 & B & 10 & 1 & 0.00 & 100.00 & TOP X10L & Top X10L reb/low & FALSE \\
\hline 2007 & B & 10 & 2 & 0.00 & 99.69 & BOT X10L & Bot X10L reb/low & FALSE \\
\hline 2007 & B & 10 & 3 & 0.15 & 99.54 & BIS & & FALSE \\
\hline 2007 & B & 10 & 4 & 0.27 & 98.86 & BIS & & FALSE \\
\hline 2007 & B & 10 & 5 & 0.61 & 98.46 & BIS & & FALSE \\
\hline 2007 & B & 10 & 6 & 0.67 & 97.16 & $\mathrm{TW}$ & Undercut bank $8 "$ & FALSE \\
\hline 2007 & B & 10 & 7 & 0.85 & 97.34 & BIS & & FALSE \\
\hline 2007 & B & 10 & 8 & 0.94 & 97.17 & BIS & & FALSE \\
\hline 2007 & B & 10 & 9 & 1.77 & 97.36 & BIS & & FALSE \\
\hline 2007 & B & 10 & 10 & 2.01 & 97.23 & BIS & & FALSE \\
\hline 2007 & B & 10 & 11 & 2.10 & 97.31 & BIS & & FALSE \\
\hline 2007 & B & 10 & 12 & 2.71 & 97.31 & BIS & & FALSE \\
\hline 2007 & B & 10 & 13 & 3.14 & 97.36 & EW & & FALSE \\
\hline 2007 & B & 10 & 14 & 3.35 & 97.48 & BIS & & FALSE \\
\hline 2007 & B & 10 & 15 & 3.51 & 97.39 & BIS & & FALSE \\
\hline 2007 & B & 10 & 16 & 3.72 & 97.39 & $\mathrm{BF}$ & & FALSE \\
\hline 2007 & B & 10 & 17 & 4.82 & 97.53 & BIS & & FALSE \\
\hline 2007 & B & 10 & 18 & 5.24 & 97.68 & BIS & & FALSE \\
\hline 2007 & B & 10 & 19 & 6.34 & 98.17 & тОВ & & FALSE \\
\hline 2007 & B & 10 & 20 & 6.74 & 98.30 & BIS & & FALSE \\
\hline 2007 & B & 10 & 21 & 8.50 & 98.25 & BIS & & FALSE \\
\hline 2007 & B & 10 & 22 & 10.36 & 98.18 & BIS & & FALSE \\
\hline 2007 & B & 10 & 23 & 12.41 & 98.23 & BIS & & FALSE \\
\hline 2007 & B & 10 & 24 & 13.41 & 98.60 & BIS & & FALSE \\
\hline 2007 & $\mathrm{~B}$ & 10 & 25 & 14.42 & 99.26 & BIS & & FALSE \\
\hline 2007 & B & 10 & 26 & 15.03 & 99.64 & BIS & & FALSE \\
\hline 2007 & $\mathrm{~B}$ & 10 & 27 & 15.36 & 99.86 & BOT X10R & Bot X10R reb/low & FALSE \\
\hline
\end{tabular}

Continued on Next Page... 
Table D.1 - Continued

\begin{tabular}{|c|c|c|c|c|c|c|c|c|}
\hline Year & Reach & Section & Point & Dist. $(\mathrm{m})$ & Elev. $(\mathrm{m})$ & Desc. & Notes & Exclude \\
\hline 2007 & B & 10 & 28 & 15.36 & 100.00 & TOP X10R & Top X10R reb/low & FALSE \\
\hline 2007 & $\mathrm{C}$ & 1 & 1 & 0.00 & 100.00 & & Top X1L reb/low & FALSE \\
\hline 2007 & $\mathrm{C}$ & 1 & 2 & 0.00 & 99.48 & & Bot $\mathrm{X} 1 \mathrm{~L}$ reb/low & FALSE \\
\hline 2007 & $\mathrm{C}$ & 1 & 3 & 0.46 & 99.15 & BIS & & FALSE \\
\hline 2007 & $\mathrm{C}$ & 1 & 4 & 0.67 & 98.94 & BIS & & FALSE \\
\hline 2007 & $\mathrm{C}$ & 1 & 5 & 0.94 & 99.77 & & $\begin{array}{l}\text { Suspended Alder log across chan- } \\
\text { nel }\end{array}$ & TRUE \\
\hline 2007 & $\mathrm{C}$ & 1 & 6 & 1.16 & 98.79 & BIS & & FALSE \\
\hline 2007 & $\mathrm{C}$ & 1 & 7 & 1.62 & 98.57 & EW & & FALSE \\
\hline 2007 & $\mathrm{C}$ & 1 & 8 & 2.32 & 98.53 & $\mathrm{TW}$ & & FALSE \\
\hline 2007 & $\mathrm{C}$ & 1 & 9 & 2.59 & 98.53 & BIS & & FALSE \\
\hline 2007 & $\mathrm{C}$ & 1 & 10 & 3.02 & 98.54 & BIS & & FALSE \\
\hline 2007 & $\mathrm{C}$ & 1 & 11 & 3.54 & 98.62 & BIS & & FALSE \\
\hline 2007 & $\mathrm{C}$ & 1 & 12 & 3.96 & 98.63 & BIS & & FALSE \\
\hline 2007 & $\mathrm{C}$ & 1 & 13 & 4.60 & 98.71 & EW & & FALSE \\
\hline 2007 & $\mathrm{C}$ & 1 & 14 & 5.24 & 98.76 & BIS & & FALSE \\
\hline 2007 & $\mathrm{C}$ & 1 & 15 & 5.61 & 98.83 & BIS & & FALSE \\
\hline 2007 & $\mathrm{C}$ & 1 & 16 & 5.97 & 98.97 & BIS & Decaying log & TRUE \\
\hline 2007 & $\mathrm{C}$ & 1 & 17 & 6.25 & 98.87 & BIS & & FALSE \\
\hline 2007 & $\mathrm{C}$ & 1 & 18 & 6.58 & 98.98 & BIS & & FALSE \\
\hline 2007 & $\mathrm{C}$ & 1 & 19 & 7.38 & 99.07 & BIS & & FALSE \\
\hline 2007 & $\mathrm{C}$ & 1 & 20 & 7.74 & 99.15 & BIS & & FALSE \\
\hline 2007 & $\mathrm{C}$ & 1 & 21 & 8.47 & 99.38 & BIS & & FALSE \\
\hline 2007 & $\mathrm{C}$ & 1 & 22 & 9.63 & 99.21 & BIS & & FALSE \\
\hline 2007 & $\mathrm{C}$ & 1 & 23 & 10.36 & 99.16 & BIS & & FALSE \\
\hline 2007 & $\mathrm{C}$ & 1 & 24 & 10.88 & 99.29 & BIS & & FALSE \\
\hline 2007 & $\mathrm{C}$ & 1 & 25 & 11.22 & 99.47 & BIS & & FALSE \\
\hline 2007 & $\mathrm{C}$ & 1 & 26 & 11.58 & 99.53 & BIS & & FALSE \\
\hline 2007 & $\mathrm{C}$ & 1 & 27 & 11.77 & 99.77 & BIS & Top of rock & TRUE \\
\hline 2007 & $\mathrm{C}$ & 1 & 28 & 11.95 & 99.68 & BIS & & FALSE \\
\hline 2007 & $\mathrm{C}$ & 1 & 29 & 12.28 & 99.92 & BOT X1R & Bot $\mathrm{X} 1 \mathrm{R}$ reb/low & FALSE \\
\hline 2007 & $\mathrm{C}$ & 1 & 30 & 12.28 & 100.27 & TOP X1R & Top X1R reb/low & FALSE \\
\hline 2007 & $\mathrm{C}$ & 2 & 1 & 0.00 & 100.00 & Top X2L & Top X2L reb/low & FALSE \\
\hline 2007 & $\mathrm{C}$ & 2 & 2 & 0.00 & 99.48 & Bot X2L & Bot $\mathrm{X} 2 \mathrm{~L} \mathrm{reb} /$ low & FALSE \\
\hline 2007 & $\mathrm{C}$ & 2 & 3 & 0.55 & 99.14 & BIS & bot $\log$ & FALSE \\
\hline 2007 & $\mathrm{C}$ & 2 & 4 & 0.67 & 99.33 & BIS & Top of $\log$ & TRUE \\
\hline 2007 & $\mathrm{C}$ & 2 & 5 & 0.76 & 98.99 & BIS & Bot $\log$ & FALSE \\
\hline 2007 & $\mathrm{C}$ & 2 & 6 & 2.07 & 98.91 & BIS & & FALSE \\
\hline 2007 & $\mathrm{C}$ & 2 & 7 & 2.68 & 98.73 & ТОВ & & FALSE \\
\hline 2007 & $\mathrm{C}$ & 2 & 8 & 3.32 & 98.58 & BIS & & FALSE \\
\hline 2007 & $\mathrm{C}$ & 2 & 9 & 4.05 & 98.44 & EW & & FALSE \\
\hline 2007 & $\mathrm{C}$ & 2 & 10 & 4.39 & 98.43 & BIS & base of $\log$ & TRUE \\
\hline 2007 & $\mathrm{C}$ & 2 & 11 & 4.57 & 98.68 & BIS & Top of $\log$ & TRUE \\
\hline 2007 & $\mathrm{C}$ & 2 & 12 & 5.00 & 98.45 & BIS & & TRUE \\
\hline 2007 & $\mathrm{C}$ & 2 & 13 & 5.40 & 98.01 & BIS & & FALSE \\
\hline 2007 & $\mathrm{C}$ & 2 & 14 & 5.64 & 97.96 & $\mathrm{BF}$ & & FALSE \\
\hline 2007 & $\mathrm{C}$ & 2 & 15 & 5.82 & 97.82 & WEL & & FALSE \\
\hline 2007 & $\mathrm{C}$ & 2 & 16 & 6.37 & 97.74 & BIS & & FALSE \\
\hline 2007 & $\mathrm{C}$ & 2 & 17 & 6.58 & 97.76 & BIS & & FALSE \\
\hline 2007 & $\mathrm{C}$ & 2 & 18 & 6.98 & 97.72 & $\mathrm{TH}$ & & FALSE \\
\hline 2007 & $\mathrm{C}$ & 2 & 19 & 7.53 & 97.81 & BIS & Bar & FALSE \\
\hline 2007 & $\mathrm{C}$ & 2 & 20 & 7.92 & 97.82 & BIS & Bar & FALSE \\
\hline
\end{tabular}

Continued on Next Page... 
Table D.1 - Continued

\begin{tabular}{|c|c|c|c|c|c|c|c|c|}
\hline Year & Reach & Section & Point & Dist. (m) & Elev. $(\mathrm{m})$ & Desc. & Notes & Exclude \\
\hline 2007 & C & 2 & 21 & 8.96 & 97.80 & WER & & FALSE \\
\hline 2007 & C & 2 & 22 & 9.48 & 97.99 & BIS & $\mathrm{BF} ?$ & FALSE \\
\hline 2007 & $\mathrm{C}$ & 2 & 23 & 10.18 & 98.28 & BIS & & FALSE \\
\hline 2007 & $\mathrm{C}$ & 2 & 24 & 12.10 & 98.37 & BOT X2R & Bot $\mathrm{X} 2 \mathrm{R}$ reb/low & FALSE \\
\hline 2007 & $\mathrm{C}$ & 2 & 25 & 12.10 & 99.99 & TOP X2R & Top X2R reb/low & FALSE \\
\hline 2007 & $\mathrm{C}$ & 3 & 1 & 0.00 & 100.00 & & Bot $\mathrm{X} 3 \mathrm{~L}$ reb/low & FALSE \\
\hline 2007 & $\mathrm{C}$ & 3 & 2 & 0.00 & 99.63 & & Top X3L reb/low & FALSE \\
\hline 2007 & $\mathrm{C}$ & 3 & 3 & 0.94 & 99.20 & BIS & & FALSE \\
\hline 2007 & $\mathrm{C}$ & 3 & 4 & 1.37 & 99.05 & BIS & & FALSE \\
\hline 2007 & $\mathrm{C}$ & 3 & 5 & 2.13 & 98.78 & BIS & & FALSE \\
\hline 2007 & $\mathrm{C}$ & 3 & 6 & 2.59 & 98.71 & BIS & & FALSE \\
\hline 2007 & $\mathrm{C}$ & 3 & 7 & 3.47 & 98.75 & BIS & & FALSE \\
\hline 2007 & $\mathrm{C}$ & 3 & 8 & 3.84 & 98.79 & BIS & & FALSE \\
\hline 2007 & $\mathrm{C}$ & 3 & 9 & 4.51 & 98.68 & BIS & & FALSE \\
\hline 2007 & $\mathrm{C}$ & 3 & 10 & 4.72 & 98.50 & BIS & & FALSE \\
\hline 2007 & $\mathrm{C}$ & 3 & 11 & 5.73 & 98.45 & BIS & & FALSE \\
\hline 2007 & $\mathrm{C}$ & 3 & 12 & 6.37 & 98.45 & BIS & & FALSE \\
\hline 2007 & $\mathrm{C}$ & 3 & 13 & 6.80 & 98.27 & BIS & & FALSE \\
\hline 2007 & $\mathrm{C}$ & 3 & 14 & 7.32 & 98.19 & EW & & FALSE \\
\hline 2007 & $\mathrm{C}$ & 3 & 15 & 8.29 & 98.09 & BIS & & FALSE \\
\hline 2007 & $\mathrm{C}$ & 3 & 16 & 8.38 & 98.27 & BIS & top of rock & TRUE \\
\hline 2007 & $\mathrm{C}$ & 3 & 17 & 8.66 & 98.03 & BIS & & FALSE \\
\hline 2007 & $\mathrm{C}$ & 3 & 18 & 8.93 & 98.00 & TW & & FALSE \\
\hline 2007 & $\mathrm{C}$ & 3 & 19 & 9.45 & 98.06 & BIS & & FALSE \\
\hline 2007 & $\mathrm{C}$ & 3 & 20 & 9.81 & 98.11 & EW & & FALSE \\
\hline 2007 & $\mathrm{C}$ & 3 & 21 & 10.24 & 98.49 & BIS & & FALSE \\
\hline 2007 & $\mathrm{C}$ & 3 & 22 & 10.49 & 98.68 & BIS & & FALSE \\
\hline 2007 & $\mathrm{C}$ & 3 & 23 & 11.19 & 98.84 & BIS & & FALSE \\
\hline 2007 & $\mathrm{C}$ & 3 & 24 & 11.58 & 98.88 & BIS & & FALSE \\
\hline 2007 & $\mathrm{C}$ & 3 & 25 & 12.68 & 99.22 & BIS & & FALSE \\
\hline 2007 & $\mathrm{C}$ & 3 & 26 & 13.78 & 99.62 & BIS & & FALSE \\
\hline 2007 & $\mathrm{C}$ & 3 & 27 & 14.08 & 99.80 & BOT X3R & Bot $\mathrm{X} 3 \mathrm{R}$ reb/low & FALSE \\
\hline 2007 & $\mathrm{C}$ & 3 & 28 & 14.08 & 100.01 & TOP X3R & Top X3R reb/low & FALSE \\
\hline 2007 & $\mathrm{C}$ & 4 & 1 & 0.00 & 100.00 & & Top X4L reb/low & FALSE \\
\hline 2007 & $\mathrm{C}$ & 4 & 2 & 0.00 & 99.74 & & Bot $\mathrm{X} 4 \mathrm{~L} \mathrm{reb} /$ low & FALSE \\
\hline 2007 & $\mathrm{C}$ & 4 & 3 & 0.52 & 99.40 & BIS & & FALSE \\
\hline 2007 & $\mathrm{C}$ & 4 & 4 & 0.76 & 99.27 & BIS & On roots & FALSE \\
\hline 2007 & $\mathrm{C}$ & 4 & 5 & 0.82 & 99.57 & BIS & On rootwad & TRUE \\
\hline 2007 & C & 4 & 6 & 1.19 & 99.17 & BIS & On rootwad & TRUE \\
\hline 2007 & $\mathrm{C}$ & 4 & 7 & 1.37 & 98.77 & BIS & & FALSE \\
\hline 2007 & $\mathrm{C}$ & 4 & 8 & 1.55 & 98.75 & BIS & On roots & FALSE \\
\hline 2007 & $\mathrm{C}$ & 4 & 9 & 2.01 & 98.76 & BIS & On roots & FALSE \\
\hline 2007 & $\mathrm{C}$ & 4 & 10 & 2.07 & 98.50 & BIS & & FALSE \\
\hline 2007 & $\mathrm{C}$ & 4 & 11 & 3.08 & 98.44 & BIS & & FALSE \\
\hline 2007 & $\mathrm{C}$ & 4 & 12 & 3.84 & 98.57 & BIS & & FALSE \\
\hline 2007 & $\mathrm{C}$ & 4 & 13 & 4.33 & 98.54 & BIS & & FALSE \\
\hline 2007 & $\mathrm{C}$ & 4 & 14 & 4.72 & 98.39 & BIS & & FALSE \\
\hline 2007 & $\mathrm{C}$ & 4 & 15 & 6.40 & 98.30 & ТОВ & & FALSE \\
\hline 2007 & $\mathrm{C}$ & 4 & 16 & 7.16 & 98.02 & EW & & FALSE \\
\hline 2007 & $\mathrm{C}$ & 4 & 17 & 7.77 & 97.92 & $\mathrm{TW}$ & & FALSE \\
\hline 2007 & $\mathrm{C}$ & 4 & 18 & 8.56 & 97.98 & BIS & & FALSE \\
\hline 2007 & $\mathrm{C}$ & 4 & 19 & 8.96 & 97.96 & BIS & & FALSE \\
\hline
\end{tabular}

Continued on Next Page... 
Table D.1 - Continued

\begin{tabular}{|c|c|c|c|c|c|c|c|c|}
\hline Year & Reach & Section & Point & Dist. $(\mathrm{m})$ & Elev. $(\mathrm{m})$ & Desc. & Notes & Exclude \\
\hline 2007 & $\mathrm{C}$ & 4 & 20 & 9.42 & 98.02 & EW & & FALSE \\
\hline 2007 & C & 4 & 21 & 9.81 & 98.17 & $\mathrm{BF}$ & & FALSE \\
\hline 2007 & $\mathrm{C}$ & 4 & 22 & 10.18 & 98.41 & BIS & & FALSE \\
\hline 2007 & $\mathrm{C}$ & 4 & 23 & 10.39 & 98.88 & ТОВ & & FALSE \\
\hline 2007 & $\mathrm{C}$ & 4 & 24 & 11.16 & 98.94 & BIS & & FALSE \\
\hline 2007 & $\mathrm{C}$ & 4 & 25 & 11.55 & 99.16 & BIS & & FALSE \\
\hline 2007 & $\mathrm{C}$ & 4 & 26 & 11.92 & 99.69 & BOT X4R & Bot $\mathrm{X} 4 \mathrm{R}$ reb/low & FALSE \\
\hline 2007 & $\mathrm{C}$ & 4 & 27 & 11.92 & 100.05 & TOP X4R & Top X4R reb/low & FALSE \\
\hline 2007 & $\mathrm{C}$ & 5 & 1 & 0.00 & 100.00 & & Top X5L reb/low & FALSE \\
\hline 2007 & $\mathrm{C}$ & 5 & 2 & 0.00 & 99.56 & & Bot X5L reb/low & FALSE \\
\hline 2007 & $\mathrm{C}$ & 5 & 3 & 0.49 & 99.30 & BIS & & FALSE \\
\hline 2007 & $\mathrm{C}$ & 5 & 4 & 1.07 & 99.11 & BIS & & FALSE \\
\hline 2007 & $\mathrm{C}$ & 5 & 5 & 1.55 & 99.07 & BIS & & FALSE \\
\hline 2007 & C & 5 & 6 & 2.16 & 98.82 & BIS & & FALSE \\
\hline 2007 & $\mathrm{C}$ & 5 & 7 & 2.87 & 98.75 & BIS & & FALSE \\
\hline 2007 & $\mathrm{C}$ & 5 & 8 & 4.24 & 98.59 & BIS & & FALSE \\
\hline 2007 & $\mathrm{C}$ & 5 & 9 & 5.43 & 98.53 & ТОВ & & FALSE \\
\hline 2007 & $\mathrm{C}$ & 5 & 10 & 5.70 & 98.32 & $\mathrm{BF}$ & & FALSE \\
\hline 2007 & $\mathrm{C}$ & 5 & 11 & 6.40 & 98.09 & BIS & & FALSE \\
\hline 2007 & $\mathrm{C}$ & 5 & 12 & 6.71 & 98.09 & EW & & FALSE \\
\hline 2007 & $\mathrm{C}$ & 5 & 13 & 7.07 & 97.98 & BIS & & FALSE \\
\hline 2007 & $\mathrm{C}$ & 5 & 14 & 7.41 & 98.05 & BIS & On rock & FALSE \\
\hline 2007 & $\mathrm{C}$ & 5 & 15 & 7.74 & 97.97 & TW & & FALSE \\
\hline 2007 & $\mathrm{C}$ & 5 & 16 & 8.11 & 98.02 & BIS & & FALSE \\
\hline 2007 & $\mathrm{C}$ & 5 & 17 & 8.23 & 98.09 & EW & On rock & FALSE \\
\hline 2007 & $\mathrm{C}$ & 5 & 18 & 8.90 & 98.24 & BIS & & FALSE \\
\hline 2007 & $\mathrm{C}$ & 5 & 19 & 9.39 & 98.36 & BIS & & FALSE \\
\hline 2007 & $\mathrm{C}$ & 5 & 20 & 10.12 & 98.87 & ТОВ & & FALSE \\
\hline 2007 & $\mathrm{C}$ & 5 & 21 & 11.13 & 98.92 & BIS & & FALSE \\
\hline 2007 & $\mathrm{C}$ & 5 & 22 & 11.73 & 99.18 & BIS & & FALSE \\
\hline 2007 & $\mathrm{C}$ & 5 & 23 & 12.41 & 99.57 & BOT X5R & Bot $\mathrm{X} 5 \mathrm{R}$ reb/low & FALSE \\
\hline 2007 & $\mathrm{C}$ & 5 & 24 & 12.41 & 100.09 & TOP X5R & Top X5R reb/low & FALSE \\
\hline 2007 & $\mathrm{C}$ & 6 & 1 & 0.00 & 100.00 & & Top X6L reb/low & FALSE \\
\hline 2007 & $\mathrm{C}$ & 6 & 2 & 0.00 & 99.73 & & Bot $\mathrm{X} 6 \mathrm{~L}$ reb/low & FALSE \\
\hline 2007 & $\mathrm{C}$ & 6 & 3 & 0.55 & 99.22 & BIS & & FALSE \\
\hline 2007 & $\mathrm{C}$ & 6 & 4 & 0.85 & 98.98 & BIS & & FALSE \\
\hline 2007 & $\mathrm{C}$ & 6 & 5 & 2.04 & 98.84 & ТОВ & & FALSE \\
\hline 2007 & $\mathrm{C}$ & 6 & 6 & 2.68 & 98.47 & BIS & & FALSE \\
\hline 2007 & $\mathrm{C}$ & 6 & 7 & 3.41 & 98.23 & BIS & & FALSE \\
\hline 2007 & $\mathrm{C}$ & 6 & 8 & 3.93 & 98.10 & $\mathrm{BF}$ & & FALSE \\
\hline 2007 & $\mathrm{C}$ & 6 & 9 & 4.24 & 97.99 & BIS & & FALSE \\
\hline 2007 & $\mathrm{C}$ & 6 & 10 & 5.64 & 97.94 & EW & & FALSE \\
\hline 2007 & $\mathrm{C}$ & 6 & 11 & 6.55 & 97.98 & BIS & & FALSE \\
\hline 2007 & $\mathrm{C}$ & 6 & 12 & 7.47 & 97.90 & $\mathrm{TW}$ & Top of rock & FALSE \\
\hline 2007 & $\mathrm{C}$ & 6 & 13 & 7.96 & 97.93 & BIS & & FALSE \\
\hline 2007 & $\mathrm{C}$ & 6 & 14 & 8.23 & 98.04 & EW & Tapr goes over alder trunk & FALSE \\
\hline 2007 & $\mathrm{C}$ & 6 & 15 & 8.63 & 98.09 & $\mathrm{BF}$ & & FALSE \\
\hline 2007 & $\mathrm{C}$ & 6 & 16 & 9.21 & 98.81 & BIS & & FALSE \\
\hline 2007 & $\mathrm{C}$ & 6 & 17 & 10.24 & 98.79 & BIS & Pile of rocks & FALSE \\
\hline 2007 & $\mathrm{C}$ & 6 & 18 & 11.22 & 99.37 & BIS & Pile of rocks & FALSE \\
\hline 2007 & $\mathrm{C}$ & 6 & 19 & 12.13 & 99.18 & BIS & Pile of rocks & FALSE \\
\hline 2007 & $\mathrm{C}$ & 6 & 20 & 12.89 & 99.72 & BOT X6R & Bot $\mathrm{X} 6 \mathrm{R}$ reb/low & FALSE \\
\hline
\end{tabular}

Continued on Next Page... 
Table D.1 - Continued

\begin{tabular}{|c|c|c|c|c|c|c|c|c|}
\hline Year & Reach & Section & Point & Dist. (m) & Elev. $(\mathrm{m})$ & Desc. & Notes & Exclude \\
\hline 2007 & $\mathrm{C}$ & 6 & 21 & 12.89 & 100.01 & TOP X6R & Top X6R reb/low & FALSE \\
\hline 2007 & $\mathrm{C}$ & 7 & 1 & 0.00 & 100.00 & & Top X7L reb/low & FALSE \\
\hline 2007 & $\mathrm{C}$ & 7 & 2 & 0.00 & 99.77 & & Bot $X 7 \mathrm{~L}$ reb/low & FALSE \\
\hline 2007 & $\mathrm{C}$ & 7 & 3 & 0.52 & 99.13 & BIS & & FALSE \\
\hline 2007 & $\mathrm{C}$ & 7 & 4 & 1.25 & 98.93 & BIS & & FALSE \\
\hline 2007 & $\mathrm{C}$ & 7 & 5 & 1.98 & 98.88 & ТОВ & & FALSE \\
\hline 2007 & $\mathrm{C}$ & 7 & 6 & 2.04 & 98.67 & BIS & & FALSE \\
\hline 2007 & $\mathrm{C}$ & 7 & 7 & 2.50 & 98.59 & BIS & & FALSE \\
\hline 2007 & $\mathrm{C}$ & 7 & 8 & 2.87 & 98.43 & BIS & & FALSE \\
\hline 2007 & $\mathrm{C}$ & 7 & 9 & 3.11 & 98.05 & EW & & FALSE \\
\hline 2007 & $\mathrm{C}$ & 7 & 10 & 3.23 & 97.91 & TW & & FALSE \\
\hline 2007 & $\mathrm{C}$ & 7 & 11 & 3.44 & 98.00 & BIS & & FALSE \\
\hline 2007 & $\mathrm{C}$ & 7 & 12 & 3.84 & 98.01 & BIS & & FALSE \\
\hline 2007 & $\mathrm{C}$ & 7 & 13 & 4.02 & 97.95 & BIS & & FALSE \\
\hline 2007 & $\mathrm{C}$ & 7 & 14 & 4.33 & 98.03 & EW & Undercut bank; EW under bank & FALSE \\
\hline 2007 & $\mathrm{C}$ & 7 & 15 & 4.94 & 98.06 & BIS & & FALSE \\
\hline 2007 & $\mathrm{C}$ & 7 & 16 & 5.76 & 98.21 & BIS & & FALSE \\
\hline 2007 & $\mathrm{C}$ & 7 & 17 & 6.61 & 98.33 & BIS & & FALSE \\
\hline 2007 & $\mathrm{C}$ & 7 & 18 & 7.50 & 98.76 & BIS & & FALSE \\
\hline 2007 & $\mathrm{C}$ & 7 & 19 & 7.83 & 99.17 & BIS & & FALSE \\
\hline 2007 & $\mathrm{C}$ & 7 & 20 & 8.35 & 99.16 & BIS & & FALSE \\
\hline 2007 & $\mathrm{C}$ & 7 & 21 & 8.44 & 99.24 & BIS & $\log$ & FALSE \\
\hline 2007 & $\mathrm{C}$ & 7 & 22 & 8.66 & 99.02 & BIS & & FALSE \\
\hline 2007 & $\mathrm{C}$ & 7 & 23 & 9.57 & 98.88 & BIS & & FALSE \\
\hline 2007 & $\mathrm{C}$ & 7 & 24 & 10.18 & 99.05 & BIS & $\log$ & TRUE \\
\hline 2007 & $\mathrm{C}$ & 7 & 25 & 10.49 & 99.27 & BIS & $\log$ & TRUE \\
\hline 2007 & $\mathrm{C}$ & 7 & 26 & 10.61 & 98.83 & BIS & & FALSE \\
\hline 2007 & $\mathrm{C}$ & 7 & 27 & 11.19 & 98.77 & BIS & & FALSE \\
\hline 2007 & $\mathrm{C}$ & 7 & 28 & 12.19 & 99.10 & BIS & & FALSE \\
\hline 2007 & $\mathrm{C}$ & 7 & 29 & 12.80 & 99.35 & BIS & & FALSE \\
\hline 2007 & $\mathrm{C}$ & 7 & 30 & 13.35 & 99.85 & BOT X7R & Bot $\mathrm{X} 7 \mathrm{R}$ reb/low & FALSE \\
\hline 2007 & $\mathrm{C}$ & 7 & 31 & 13.35 & 100.04 & TOP X7R & Top X7R reb/low & FALSE \\
\hline 2007 & $\mathrm{C}$ & 8 & 1 & 0.00 & 100.00 & & Top X8L reb/low & FALSE \\
\hline 2007 & $\mathrm{C}$ & 8 & 2 & 0.00 & 99.57 & & Bot $\mathrm{X} 8 \mathrm{~L} \mathrm{reb} /$ low & FALSE \\
\hline 2007 & $\mathrm{C}$ & 8 & 3 & 0.40 & 98.82 & BIS & & FALSE \\
\hline 2007 & $\mathrm{C}$ & 8 & 4 & 0.46 & 98.41 & BIS & & FALSE \\
\hline 2007 & $\mathrm{C}$ & 8 & 5 & 0.61 & 98.34 & ТОВ & Undercut bank & FALSE \\
\hline 2007 & $\mathrm{C}$ & 8 & 6 & 0.73 & 97.64 & BIS & & FALSE \\
\hline 2007 & $\mathrm{C}$ & 8 & 7 & 1.07 & 97.57 & BIS & & FALSE \\
\hline 2007 & $\mathrm{C}$ & 8 & 8 & 1.49 & 97.76 & BIS & & FALSE \\
\hline 2007 & $\mathrm{C}$ & 8 & 9 & 2.26 & 97.78 & EW & & FALSE \\
\hline 2007 & $\mathrm{C}$ & 8 & 10 & 2.99 & 97.67 & EW & & FALSE \\
\hline 2007 & $\mathrm{C}$ & 8 & 11 & 3.66 & 97.70 & EW & & FALSE \\
\hline 2007 & $\mathrm{C}$ & 8 & 12 & 3.93 & 97.79 & EW & & FALSE \\
\hline 2007 & $\mathrm{C}$ & 8 & 13 & 4.11 & 98.20 & BIS & & FALSE \\
\hline 2007 & $\mathrm{C}$ & 8 & 14 & 4.63 & 98.23 & BIS & & FALSE \\
\hline 2007 & $\mathrm{C}$ & 8 & 15 & 5.15 & 98.47 & BIS & & FALSE \\
\hline 2007 & $\mathrm{C}$ & 8 & 16 & 5.58 & 98.65 & BIS & & FALSE \\
\hline 2007 & $\mathrm{C}$ & 8 & 17 & 6.04 & 99.06 & ТОВ & & FALSE \\
\hline 2007 & $\mathrm{C}$ & 8 & 18 & 8.44 & 98.89 & BIS & & FALSE \\
\hline 2007 & $\mathrm{C}$ & 8 & 19 & 10.39 & 98.83 & BIS & & FALSE \\
\hline 2007 & $\mathrm{C}$ & 8 & 20 & 11.37 & 98.60 & BIS & & FALSE \\
\hline
\end{tabular}

Continued on Next Page... 
Table D.1 - Continued

\begin{tabular}{|c|c|c|c|c|c|c|c|c|}
\hline Year & Reach & Section & Point & Dist. (m) & Elev. $(\mathrm{m})$ & Desc. & Notes & Exclude \\
\hline 2007 & $\mathrm{C}$ & 8 & 21 & 12.25 & 98.68 & BIS & & FALSE \\
\hline 2007 & C & 8 & 22 & 12.95 & 98.91 & BIS & & FALSE \\
\hline 2007 & $\mathrm{C}$ & 8 & 23 & 13.59 & 99.39 & BIS & & FALSE \\
\hline 2007 & $\mathrm{C}$ & 8 & 24 & 14.11 & 99.66 & BOT X8R & Bot $\mathrm{X} 8 \mathrm{R}$ reb/low & FALSE \\
\hline 2007 & $\mathrm{C}$ & 8 & 25 & 14.11 & 100.01 & TOP X8R & Top X8R reb/low & FALSE \\
\hline 2007 & $\mathrm{C}$ & 9 & 1 & 0.00 & 100.00 & & Top X9L reb/low & FALSE \\
\hline 2007 & $\mathrm{C}$ & 9 & 2 & 0.00 & 99.55 & & Bot X9L reb/low & FALSE \\
\hline 2007 & $\mathrm{C}$ & 9 & 3 & 0.15 & 98.63 & BIS & & FALSE \\
\hline 2007 & $\mathrm{C}$ & 9 & 4 & 1.01 & 97.99 & BIS & & FALSE \\
\hline 2007 & $\mathrm{C}$ & 9 & 5 & 1.74 & 97.87 & BIS & & FALSE \\
\hline 2007 & $\mathrm{C}$ & 9 & 6 & 2.71 & 97.69 & BIS & & FALSE \\
\hline 2007 & $\mathrm{C}$ & 9 & 7 & 3.57 & 97.57 & EW & & FALSE \\
\hline 2007 & $\mathrm{C}$ & 9 & 8 & 3.81 & 97.45 & BIS & & FALSE \\
\hline 2007 & $\mathrm{C}$ & 9 & 9 & 4.11 & 97.49 & BIS & On top of rock & FALSE \\
\hline 2007 & $\mathrm{C}$ & 9 & 10 & 4.39 & 97.41 & TW & & FALSE \\
\hline 2007 & $\mathrm{C}$ & 9 & 11 & 4.66 & 97.46 & BIS & & FALSE \\
\hline 2007 & $\mathrm{C}$ & 9 & 12 & 5.00 & 97.56 & EW & & FALSE \\
\hline 2007 & $\mathrm{C}$ & 9 & 13 & 5.27 & 97.60 & BIS & & FALSE \\
\hline 2007 & $\mathrm{C}$ & 9 & 14 & 5.52 & 97.84 & BIS & & FALSE \\
\hline 2007 & $\mathrm{C}$ & 9 & 15 & 6.46 & 98.58 & ТОВ & & FALSE \\
\hline 2007 & $\mathrm{C}$ & 9 & 16 & 7.19 & 98.81 & BIS & & FALSE \\
\hline 2007 & $\mathrm{C}$ & 9 & 17 & 8.87 & 98.74 & BIS & & FALSE \\
\hline 2007 & $\mathrm{C}$ & 9 & 18 & 11.28 & 98.63 & BIS & & FALSE \\
\hline 2007 & $\mathrm{C}$ & 9 & 19 & 13.41 & 98.52 & BIS & & FALSE \\
\hline 2007 & $\mathrm{C}$ & 9 & 20 & 14.60 & 98.74 & BIS & & FALSE \\
\hline 2007 & $\mathrm{C}$ & 9 & 21 & 15.61 & 98.94 & BOT X9R & Bot $\mathrm{X} 9 \mathrm{R}$ reb/low & FALSE \\
\hline 2007 & $\mathrm{C}$ & 9 & 22 & 15.61 & 99.51 & TOP X9R & Top X9R reb/low & FALSE \\
\hline 2007 & $\mathrm{D}$ & 1 & 1 & 0.00 & 100.00 & TOP REB & Top X1L reb/up & FALSE \\
\hline 2007 & $\mathrm{D}$ & 1 & 2 & 0.00 & 99.70 & BOT REB & Bot X1L reb/up & FALSE \\
\hline 2007 & $\mathrm{D}$ & 1 & 3 & 0.82 & 99.19 & BIS & & FALSE \\
\hline 2007 & $\mathrm{D}$ & 1 & 4 & 1.62 & 98.85 & BIS & & FALSE \\
\hline 2007 & $\mathrm{D}$ & 1 & 5 & 2.35 & 98.55 & BIS & & FALSE \\
\hline 2007 & $\mathrm{D}$ & 1 & 6 & 2.56 & 98.82 & BIS & on $\log$ & FALSE \\
\hline 2007 & $\mathrm{D}$ & 1 & 7 & 2.65 & 98.38 & BIS & & FALSE \\
\hline 2007 & $\mathrm{D}$ & 1 & 8 & 3.08 & 98.17 & BIS & & FALSE \\
\hline 2007 & $\mathrm{D}$ & 1 & 9 & 3.14 & 97.75 & BIS & headcut on overflow channel & FALSE \\
\hline 2007 & $\mathrm{D}$ & 1 & 10 & 4.15 & 97.69 & BIS & & FALSE \\
\hline 2007 & $\mathrm{D}$ & 1 & 11 & 4.69 & 97.70 & BIS & & FALSE \\
\hline 2007 & $\mathrm{D}$ & 1 & 12 & 5.61 & 97.96 & BIS & & FALSE \\
\hline 2007 & $\mathrm{D}$ & 1 & 13 & 5.85 & 98.56 & BIS & & FALSE \\
\hline 2007 & $\mathrm{D}$ & 1 & 14 & 6.40 & 98.69 & BIS & & FALSE \\
\hline 2007 & $\mathrm{D}$ & 1 & 15 & 7.35 & 98.61 & TBL & & FALSE \\
\hline 2007 & $\mathrm{D}$ & 1 & 16 & 7.65 & 98.09 & BIS & & FALSE \\
\hline 2007 & $\mathrm{D}$ & 1 & 17 & 7.80 & 97.46 & BIS & & FALSE \\
\hline 2007 & $\mathrm{D}$ & 1 & 18 & 8.02 & 97.43 & BIS & & FALSE \\
\hline 2007 & $\mathrm{D}$ & 1 & 19 & 8.38 & 97.03 & BIS & & FALSE \\
\hline 2007 & $\mathrm{D}$ & 1 & 20 & 8.90 & 96.79 & WEL & & FALSE \\
\hline 2007 & $\mathrm{D}$ & 1 & 21 & 9.51 & 96.72 & BIS & & FALSE \\
\hline 2007 & $\mathrm{D}$ & 1 & 22 & 10.06 & 96.61 & BIS & & FALSE \\
\hline 2007 & $\mathrm{D}$ & 1 & 23 & 10.39 & 96.59 & $\mathrm{TW}$ & & FALSE \\
\hline 2007 & $\mathrm{D}$ & 1 & 24 & 10.64 & 96.77 & BIS & & FALSE \\
\hline 2007 & $\mathrm{D}$ & 1 & 25 & 11.03 & 96.60 & BIS & & FALSE \\
\hline
\end{tabular}

Continued on Next Page... 
Table D.1 - Continued

\begin{tabular}{|c|c|c|c|c|c|c|c|c|}
\hline Year & Reach & Section & Point & Dist. (m) & Elev. $(\mathrm{m})$ & Desc. & Notes & Exclude \\
\hline 2007 & $\mathrm{D}$ & 1 & 26 & 11.09 & 96.61 & BIS & & FALSE \\
\hline 2007 & $\mathrm{D}$ & 1 & 27 & 11.83 & 97.17 & BIS & $\begin{array}{l}\text { WER under rock - point is top of } \\
\text { rock }\end{array}$ & FALSE \\
\hline 2007 & $\mathrm{D}$ & 1 & 28 & 11.89 & 97.06 & BIS & & FALSE \\
\hline 2007 & $\mathrm{D}$ & 1 & 29 & 12.59 & 97.11 & BIS & & FALSE \\
\hline 2007 & $\mathrm{D}$ & 1 & 30 & 13.17 & 97.35 & BIS & & FALSE \\
\hline 2007 & $\mathrm{D}$ & 1 & 31 & 13.72 & 97.64 & BIS & & FALSE \\
\hline 2007 & $\mathrm{D}$ & 1 & 32 & 13.93 & 97.89 & BIS & & FALSE \\
\hline 2007 & $\mathrm{D}$ & 1 & 33 & 14.90 & 98.06 & BIS & & FALSE \\
\hline 2007 & D & 1 & 34 & 15.15 & 98.40 & BIS & & FALSE \\
\hline 2007 & $\mathrm{D}$ & 1 & 35 & 16.31 & 98.49 & BIS & & FALSE \\
\hline 2007 & $\mathrm{D}$ & 1 & 36 & 16.98 & 98.90 & BIS & & FALSE \\
\hline 2007 & $\mathrm{D}$ & 1 & 37 & 17.43 & 99.72 & BIS & & FALSE \\
\hline 2007 & $\mathrm{D}$ & 1 & 38 & 17.98 & 100.07 & BOT REB & Bot $\mathrm{X} 1 \mathrm{R}$ reb/up & FALSE \\
\hline 2007 & $\mathrm{D}$ & 1 & 39 & 17.98 & 100.42 & TOP REB & Top X1R reb/up & FALSE \\
\hline 2007 & $\mathrm{D}$ & 2 & 1 & 0.00 & 100.00 & TOP REB & Top X2L reb/low & FALSE \\
\hline 2007 & $\mathrm{D}$ & 2 & 2 & 0.00 & 99.73 & BOT REB & Bot $\mathrm{X} 2 \mathrm{~L} \mathrm{reb} /$ low & FALSE \\
\hline 2007 & $\mathrm{D}$ & 2 & 3 & 0.52 & 99.65 & BIS & & FALSE \\
\hline 2007 & $\mathrm{D}$ & 2 & 4 & 0.98 & 99.15 & BIS & & FALSE \\
\hline 2007 & $\mathrm{D}$ & 2 & 5 & 1.52 & 98.68 & BIS & & FALSE \\
\hline 2007 & $\mathrm{D}$ & 2 & 6 & 1.92 & 98.62 & BIS & & FALSE \\
\hline 2007 & $\mathrm{D}$ & 2 & 7 & 2.71 & 98.49 & WEL & & FALSE \\
\hline 2007 & $\mathrm{D}$ & 2 & 8 & 3.20 & 98.43 & BIS & & FALSE \\
\hline 2007 & $\mathrm{D}$ & 2 & 9 & 3.93 & 98.38 & $\mathrm{TW}$ & & FALSE \\
\hline 2007 & $\mathrm{D}$ & 2 & 10 & 4.24 & 98.47 & BIS & & FALSE \\
\hline 2007 & $\mathrm{D}$ & 2 & 11 & 4.48 & 98.43 & WER & & FALSE \\
\hline 2007 & $\mathrm{D}$ & 2 & 12 & 4.88 & 98.58 & BIS & & FALSE \\
\hline 2007 & $\mathrm{D}$ & 2 & 13 & 5.49 & 98.74 & BIS & & FALSE \\
\hline 2007 & $\mathrm{D}$ & 2 & 14 & 6.13 & 98.98 & BIS & & FALSE \\
\hline 2007 & $\mathrm{D}$ & 2 & 15 & 6.86 & 99.29 & BIS & & FALSE \\
\hline 2007 & $\mathrm{D}$ & 2 & 16 & 7.22 & 99.43 & BIS & & FALSE \\
\hline 2007 & $\mathrm{D}$ & 2 & 17 & 7.71 & 99.67 & BIS & & FALSE \\
\hline 2007 & $\mathrm{D}$ & 2 & 18 & 8.69 & 99.75 & BIS & & FALSE \\
\hline 2007 & $\mathrm{D}$ & 2 & 19 & 9.36 & 99.69 & BOT REB & Bot $\mathrm{X} 2 \mathrm{R}$ reb/low & FALSE \\
\hline 2007 & $\mathrm{D}$ & 2 & 20 & 9.36 & 99.80 & TOP REB & Top X2R reb/low & FALSE \\
\hline 2007 & $\mathrm{D}$ & 3 & 1 & 0.00 & 100.00 & TOP REB & Top X3L reb/low & FALSE \\
\hline 2007 & $\mathrm{D}$ & 3 & 2 & 0.00 & 99.81 & BOT REB & Bot $\mathrm{X} 3 \mathrm{~L} \mathrm{reb} /$ low & FALSE \\
\hline 2007 & $\mathrm{D}$ & 3 & 3 & 0.49 & 99.73 & BIS & & FALSE \\
\hline 2007 & $\mathrm{D}$ & 3 & 4 & 0.91 & 99.79 & TBL & & FALSE \\
\hline 2007 & $\mathrm{D}$ & 3 & 5 & 1.28 & 99.62 & BIS & & FALSE \\
\hline 2007 & $\mathrm{D}$ & 3 & 6 & 1.77 & 98.75 & BIS & & FALSE \\
\hline 2007 & D & 3 & 7 & 2.68 & 98.65 & BIS & & FALSE \\
\hline 2007 & $\mathrm{D}$ & 3 & 8 & 3.41 & 98.52 & WEL & & FALSE \\
\hline 2007 & $\mathrm{D}$ & 3 & 9 & 3.57 & 98.42 & BIS & & FALSE \\
\hline 2007 & $\mathrm{D}$ & 3 & 10 & 3.90 & 98.35 & BIS & & FALSE \\
\hline 2007 & $\mathrm{D}$ & 3 & 11 & 4.08 & 98.33 & TW & & FALSE \\
\hline 2007 & $\mathrm{D}$ & 3 & 12 & 4.45 & 98.51 & WER & & FALSE \\
\hline 2007 & $\mathrm{D}$ & 3 & 13 & 4.72 & 98.60 & BIS & & FALSE \\
\hline 2007 & $\mathrm{D}$ & 3 & 14 & 4.88 & 98.98 & BIS & & FALSE \\
\hline 2007 & $\mathrm{D}$ & 3 & 15 & 5.36 & 99.18 & BIS & & FALSE \\
\hline 2007 & $\mathrm{D}$ & 3 & 16 & 5.49 & 99.52 & BIS & & FALSE \\
\hline
\end{tabular}


Table D.1 - Continued

\begin{tabular}{|c|c|c|c|c|c|c|c|c|}
\hline Year & Reach & Section & Point & Dist. (m) & Elev. $(\mathrm{m})$ & Desc. & Notes & Exclude \\
\hline 2007 & $\mathrm{D}$ & 3 & 17 & 5.82 & 99.69 & BIS & $\begin{array}{l}\text { mid channel boulder/debris pile }+ \\
\text { alder }\end{array}$ & FALSE \\
\hline 2007 & $\mathrm{D}$ & 3 & 18 & 6.43 & 99.89 & TBR & & FALSE \\
\hline 2007 & $\mathrm{D}$ & 3 & 19 & 7.04 & 99.76 & BIS & & FALSE \\
\hline 2007 & $\mathrm{D}$ & 3 & 20 & 7.22 & 99.33 & BIS & & FALSE \\
\hline 2007 & $\mathrm{D}$ & 3 & 21 & 7.86 & 99.20 & BIS & & FALSE \\
\hline 2007 & $\mathrm{D}$ & 3 & 22 & 8.87 & 99.28 & BIS & & FALSE \\
\hline 2007 & $\mathrm{D}$ & 3 & 23 & 10.03 & 99.40 & BIS & & FALSE \\
\hline 2007 & $\mathrm{D}$ & 3 & 24 & 10.55 & 99.52 & BIS & & FALSE \\
\hline 2007 & $\mathrm{D}$ & 3 & 25 & 11.03 & 99.94 & BOT REB & Bot X3R reb/low & FALSE \\
\hline 2007 & $\mathrm{D}$ & 3 & 26 & 11.03 & 100.00 & TOP REB & Top X3R reb/low & FALSE \\
\hline 2007 & $\mathrm{D}$ & 4 & 1 & 0.00 & 100.00 & TOP REB & Top X4L reb/up & FALSE \\
\hline 2007 & $\mathrm{D}$ & 4 & 2 & 0.00 & 99.17 & BOT REB & Bot X4L reb/up & FALSE \\
\hline 2007 & $\mathrm{D}$ & 4 & 3 & 0.18 & 99.16 & BIS & edge of stump & FALSE \\
\hline 2007 & $\mathrm{D}$ & 4 & 4 & 0.30 & 98.05 & BIS & & FALSE \\
\hline 2007 & $\mathrm{D}$ & 4 & 5 & 1.07 & 97.46 & BIS & & FALSE \\
\hline 2007 & $\mathrm{D}$ & 4 & 6 & 1.37 & 97.20 & BIS & edge of rootwad & FALSE \\
\hline 2007 & $\mathrm{D}$ & 4 & 7 & 1.52 & 96.55 & BIS & & FALSE \\
\hline 2007 & $\mathrm{D}$ & 4 & 8 & 1.68 & 96.39 & WEL & in water & FALSE \\
\hline 2007 & $\mathrm{D}$ & 4 & 9 & 1.80 & 96.38 & BIS & & FALSE \\
\hline 2007 & $\mathrm{D}$ & 4 & 10 & 2.19 & 96.28 & $\mathrm{TW}$ & & FALSE \\
\hline 2007 & $\mathrm{D}$ & 4 & 11 & 2.68 & 96.39 & BIS & & FALSE \\
\hline 2007 & $\mathrm{D}$ & 4 & 12 & 2.87 & 96.28 & BIS & & FALSE \\
\hline 2007 & $\mathrm{D}$ & 4 & 13 & 3.26 & 96.42 & WER & & FALSE \\
\hline 2007 & $\mathrm{D}$ & 4 & 14 & 3.84 & 96.68 & BIS & & FALSE \\
\hline 2007 & $\mathrm{D}$ & 4 & 15 & 4.54 & 96.91 & BIS & & FALSE \\
\hline 2007 & $\mathrm{D}$ & 4 & 16 & 5.91 & 97.22 & BIS & & FALSE \\
\hline 2007 & $\mathrm{D}$ & 4 & 17 & 7.07 & 97.16 & BIS & & FALSE \\
\hline 2007 & $\mathrm{D}$ & 4 & 18 & 7.83 & 97.26 & BIS & & FALSE \\
\hline 2007 & $\mathrm{D}$ & 4 & 19 & 8.63 & 97.31 & BIS & & FALSE \\
\hline 2007 & $\mathrm{D}$ & 4 & 20 & 9.21 & 97.85 & BIS & & FALSE \\
\hline 2007 & $\mathrm{D}$ & 4 & 21 & 10.03 & 98.09 & BIS & & FALSE \\
\hline 2007 & $\mathrm{D}$ & 4 & 22 & 11.19 & 98.11 & BIS & & FALSE \\
\hline 2007 & $\mathrm{D}$ & 4 & 23 & 12.38 & 98.97 & BIS & & FALSE \\
\hline 2007 & $\mathrm{D}$ & 4 & 24 & 12.98 & 99.80 & BIS & & FALSE \\
\hline 2007 & $\mathrm{D}$ & 4 & 25 & 13.50 & 100.15 & BIS & & FALSE \\
\hline 2007 & $\mathrm{D}$ & 4 & 26 & 13.81 & 100.41 & BOT REB & Bot $\mathrm{X} 4 \mathrm{R} \mathrm{reb} / \mathrm{up}$ & FALSE \\
\hline 2007 & $\mathrm{D}$ & 4 & 27 & 13.81 & 100.86 & TOP REB & Top X4R reb/up & FALSE \\
\hline 2007 & $\mathrm{D}$ & 5 & 1 & 0.00 & 100.00 & TOP REB & Top X5L reb/low & FALSE \\
\hline 2007 & $\mathrm{D}$ & 5 & 2 & 0.00 & 99.75 & BOT REB & Bot $\mathrm{X} 5 \mathrm{~L} \mathrm{reb} /$ low & FALSE \\
\hline 2007 & $\mathrm{D}$ & 5 & 3 & 0.30 & 99.67 & BIS & & FALSE \\
\hline 2007 & $\mathrm{D}$ & 5 & 4 & 0.64 & 99.32 & BIS & root in bank & FALSE \\
\hline 2007 & $\mathrm{D}$ & 5 & 5 & 0.82 & 98.57 & BIS & below root & FALSE \\
\hline 2007 & $\mathrm{D}$ & 5 & 6 & 1.83 & 98.39 & BIS & & FALSE \\
\hline 2007 & $\mathrm{D}$ & 5 & 7 & 3.14 & 98.25 & $\mathrm{BF}$ & & FALSE \\
\hline 2007 & $\mathrm{D}$ & 5 & 8 & 3.96 & 98.06 & WEL & & FALSE \\
\hline 2007 & $\mathrm{D}$ & 5 & 9 & 4.30 & 98.02 & $\mathrm{TW}$ & & FALSE \\
\hline 2007 & $\mathrm{D}$ & 5 & 10 & 4.85 & 98.12 & WER & & FALSE \\
\hline 2007 & $\mathrm{D}$ & 5 & 11 & 5.43 & 98.25 & BIS & & FALSE \\
\hline 2007 & $\mathrm{D}$ & 5 & 12 & 5.85 & 98.51 & BIS & & FALSE \\
\hline 2007 & $\mathrm{D}$ & 5 & 13 & 6.61 & 98.77 & BIS & & FALSE \\
\hline 2007 & $\mathrm{D}$ & 5 & 14 & 7.01 & 99.19 & BIS & top of rock & FALSE \\
\hline
\end{tabular}

Continued on Next Page... 
Table D.1 - Continued

\begin{tabular}{|c|c|c|c|c|c|c|c|c|}
\hline Year & Reach & Section & Point & Dist. (m) & Elev. $(\mathrm{m})$ & Desc. & Notes & Exclude \\
\hline 2007 & $\mathrm{D}$ & 5 & 15 & 7.25 & 99.00 & BIS & & FALSE \\
\hline 2007 & D & 5 & 16 & 7.86 & 99.13 & BIS & & FALSE \\
\hline 2007 & $\mathrm{D}$ & 5 & 17 & 8.14 & 99.64 & BIS & & FALSE \\
\hline 2007 & $\mathrm{D}$ & 5 & 18 & 9.45 & 99.86 & BIS & & FALSE \\
\hline 2007 & $\mathrm{D}$ & 5 & 19 & 10.06 & 99.77 & BIS & & FALSE \\
\hline 2007 & $\mathrm{D}$ & 5 & 20 & 10.85 & 99.76 & BOT REB & Bot $\mathrm{X} 5 \mathrm{R}$ reb/low & FALSE \\
\hline 2007 & $\mathrm{D}$ & 5 & 21 & 10.85 & 100.15 & TOP REB & Top X5R reb/low & FALSE \\
\hline 2007 & $\mathrm{D}$ & 6 & 1 & 0.00 & 100.00 & TOP REB & Top X6L reb/low & FALSE \\
\hline 2007 & $\mathrm{D}$ & 6 & 2 & 0.00 & 99.85 & BOT REB & Bot $\mathrm{X} 6 \mathrm{~L} \mathrm{reb} /$ low & FALSE \\
\hline 2007 & $\mathrm{D}$ & 6 & 3 & 0.73 & 99.60 & ТОВ & & FALSE \\
\hline 2007 & $\mathrm{D}$ & 6 & 4 & 0.98 & 98.63 & BIS & & FALSE \\
\hline 2007 & $\mathrm{D}$ & 6 & 5 & 1.46 & 98.18 & BIS & & FALSE \\
\hline 2007 & $\mathrm{D}$ & 6 & 6 & 2.77 & 98.03 & BIS & & FALSE \\
\hline 2007 & $\mathrm{D}$ & 6 & 7 & 3.44 & 97.60 & BIS & & FALSE \\
\hline 2007 & $\mathrm{D}$ & 6 & 8 & 3.75 & 97.56 & WEL & & FALSE \\
\hline 2007 & $\mathrm{D}$ & 6 & 9 & 4.18 & 97.46 & $\mathrm{TW}$ & & FALSE \\
\hline 2007 & $\mathrm{D}$ & 6 & 10 & 4.48 & 97.48 & BIS & & FALSE \\
\hline 2007 & $\mathrm{D}$ & 6 & 11 & 4.75 & 97.56 & BIS & & FALSE \\
\hline 2007 & $\mathrm{D}$ & 6 & 12 & 5.06 & 97.65 & BIS & & FALSE \\
\hline 2007 & $\mathrm{D}$ & 6 & 13 & 5.52 & 97.52 & BIS & & FALSE \\
\hline 2007 & $\mathrm{D}$ & 6 & 14 & 5.88 & 97.51 & BIS & & FALSE \\
\hline 2007 & $\mathrm{D}$ & 6 & 15 & 6.31 & 97.52 & BIS & & FALSE \\
\hline 2007 & $\mathrm{D}$ & 6 & 16 & 6.86 & 97.66 & WER & & FALSE \\
\hline 2007 & $\mathrm{D}$ & 6 & 17 & 7.13 & 98.03 & BIS & & FALSE \\
\hline 2007 & $\mathrm{D}$ & 6 & 18 & 8.44 & 99.34 & ТОВ & & FALSE \\
\hline 2007 & $\mathrm{D}$ & 6 & 19 & 9.30 & 99.42 & BIS & & FALSE \\
\hline 2007 & $\mathrm{D}$ & 6 & 20 & 9.94 & 99.67 & BOT REB & Bot $\mathrm{X} 6 \mathrm{R}$ reb/low & FALSE \\
\hline 2007 & $\mathrm{D}$ & 6 & 21 & 9.94 & 100.02 & TOP REB & Top X6R reb/low & FALSE \\
\hline 2007 & $\mathrm{D}$ & 7 & 1 & 0.00 & 100.00 & & Top X7L reb/low & FALSE \\
\hline 2007 & $\mathrm{D}$ & 7 & 2 & 0.00 & 99.78 & & Bot $\mathrm{X} 7 \mathrm{~L}$ reb/low & FALSE \\
\hline 2007 & $\mathrm{D}$ & 7 & 3 & 0.27 & 99.57 & BIS & & FALSE \\
\hline 2007 & $\mathrm{D}$ & 7 & 4 & 0.37 & 99.07 & BIS & & FALSE \\
\hline 2007 & $\mathrm{D}$ & 7 & 5 & 0.82 & 99.72 & BIS & Top of log parallel to stream & TRUE \\
\hline 2007 & $\mathrm{D}$ & 7 & 6 & 1.13 & 98.52 & BIS & embedded in right bank & FALSE \\
\hline 2007 & $\mathrm{D}$ & 7 & 7 & 1.83 & 98.36 & BIS & & FALSE \\
\hline 2007 & $\mathrm{D}$ & 7 & 8 & 2.50 & 97.94 & BIS & & FALSE \\
\hline 2007 & $\mathrm{D}$ & 7 & 9 & 3.11 & 97.81 & WEL & & FALSE \\
\hline 2007 & $\mathrm{D}$ & 7 & 10 & 3.51 & 97.69 & BIS & & FALSE \\
\hline 2007 & $\mathrm{D}$ & 7 & 11 & 3.81 & 97.67 & $\mathrm{TW}$ & & FALSE \\
\hline 2007 & $\mathrm{D}$ & 7 & 12 & 4.27 & 97.83 & WER & & FALSE \\
\hline 2007 & $\mathrm{D}$ & 7 & 13 & 5.09 & 97.94 & BIS & & FALSE \\
\hline 2007 & $\mathrm{D}$ & 7 & 14 & 5.64 & 98.11 & BIS & & FALSE \\
\hline 2007 & $\mathrm{D}$ & 7 & 15 & 6.04 & 98.10 & BIS & & FALSE \\
\hline 2007 & $\mathrm{D}$ & 7 & 16 & 6.40 & 98.85 & BIS & & FALSE \\
\hline 2007 & $\mathrm{D}$ & 7 & 17 & 6.98 & 99.32 & BIS & & FALSE \\
\hline 2007 & $\mathrm{D}$ & 7 & 18 & 7.65 & 99.76 & BOT X7R & Bot $\mathrm{X} 7 \mathrm{R}$ reb/low & FALSE \\
\hline 2007 & $\mathrm{D}$ & 7 & 19 & 7.65 & 99.97 & TOP X7R & Top X7R reb/low & FALSE \\
\hline 2007 & $\mathrm{D}$ & 8 & 1 & 0.00 & 100.00 & & Top X8L reb/low & FALSE \\
\hline 2007 & $\mathrm{D}$ & 8 & 2 & 0.00 & 99.74 & & Bot $\mathrm{X} 8 \mathrm{~L} \mathrm{reb} /$ low & FALSE \\
\hline 2007 & $\mathrm{D}$ & 8 & 3 & 0.61 & 99.41 & BIS & & FALSE \\
\hline 2007 & $\mathrm{D}$ & 8 & 4 & 1.19 & 98.52 & BIS & & FALSE \\
\hline 2007 & $\mathrm{D}$ & 8 & 5 & 1.40 & 98.16 & BIS & & FALSE \\
\hline
\end{tabular}

Continued on Next Page... 
Table D.1 - Continued

\begin{tabular}{|c|c|c|c|c|c|c|c|c|}
\hline Year & Reach & Section & Point & Dist. (m) & Elev. $(\mathrm{m})$ & Desc. & Notes & Exclude \\
\hline 2007 & $\mathrm{D}$ & 8 & 6 & 2.13 & 97.82 & WEL & & FALSE \\
\hline 2007 & $\mathrm{D}$ & 8 & 7 & 2.53 & 97.78 & BIS & & FALSE \\
\hline 2007 & $\mathrm{D}$ & 8 & 8 & 2.87 & 97.79 & BIS & & FALSE \\
\hline 2007 & $\mathrm{D}$ & 8 & 9 & 3.05 & 97.92 & BIS & & FALSE \\
\hline 2007 & $\mathrm{D}$ & 8 & 10 & 3.26 & 97.73 & TW & & FALSE \\
\hline 2007 & $\mathrm{D}$ & 8 & 11 & 3.54 & 97.85 & BIS & & FALSE \\
\hline 2007 & $\mathrm{D}$ & 8 & 12 & 3.75 & 97.78 & WER & & FALSE \\
\hline 2007 & $\mathrm{D}$ & 8 & 13 & 4.45 & 97.93 & $\mathrm{BF} ?$ & & FALSE \\
\hline 2007 & $\mathrm{D}$ & 8 & 14 & 5.00 & 98.02 & BIS & & FALSE \\
\hline 2007 & $\mathrm{D}$ & 8 & 15 & 5.21 & 98.15 & BIS & & FALSE \\
\hline 2007 & $\mathrm{D}$ & 8 & 16 & 5.67 & 98.23 & BIS & & FALSE \\
\hline 2007 & $\mathrm{D}$ & 8 & 17 & 5.97 & 98.50 & BIS & & FALSE \\
\hline 2007 & $\mathrm{D}$ & 8 & 18 & 6.19 & 98.70 & BIS & & FALSE \\
\hline 2007 & $\mathrm{D}$ & 8 & 19 & 6.52 & 99.72 & ТОВ & & FALSE \\
\hline 2007 & $\mathrm{D}$ & 8 & 20 & 6.83 & 99.79 & BOT X8R & Bot $\mathrm{X} 8 \mathrm{R}$ reb/low & FALSE \\
\hline 2007 & $\mathrm{D}$ & 8 & 21 & 6.83 & 100.02 & TOP X8R & Top X8R reb/low & FALSE \\
\hline 2007 & $\mathrm{D}$ & 9 & 1 & 0.00 & 100.00 & & Top X9L reb/low & FALSE \\
\hline 2007 & $\mathrm{D}$ & 9 & 2 & 0.00 & 99.55 & & Bot X9L reb/low & FALSE \\
\hline 2007 & $\mathrm{D}$ & 9 & 3 & 0.49 & 98.91 & BIS & & FALSE \\
\hline 2007 & $\mathrm{D}$ & 9 & 4 & 1.07 & 98.66 & BIS & & FALSE \\
\hline 2007 & $\mathrm{D}$ & 9 & 5 & 2.13 & 98.63 & $\mathrm{BF}$ & & FALSE \\
\hline 2007 & $\mathrm{D}$ & 9 & 6 & 2.65 & 98.60 & BIS & & FALSE \\
\hline 2007 & $\mathrm{D}$ & 9 & 7 & 2.90 & 98.49 & WEL & & FALSE \\
\hline 2007 & $\mathrm{D}$ & 9 & 8 & 3.20 & 98.37 & $\mathrm{TW}$ & & FALSE \\
\hline 2007 & $\mathrm{D}$ & 9 & 9 & 3.54 & 98.37 & BIS & & FALSE \\
\hline 2007 & $\mathrm{D}$ & 9 & 10 & 3.81 & 98.48 & WER & & FALSE \\
\hline 2007 & $\mathrm{D}$ & 9 & 11 & 4.18 & 98.57 & BIS & & FALSE \\
\hline 2007 & $\mathrm{D}$ & 9 & 12 & 4.63 & 98.71 & BIS & & FALSE \\
\hline 2007 & $\mathrm{D}$ & 9 & 13 & 4.85 & 98.88 & BIS & & FALSE \\
\hline 2007 & $\mathrm{D}$ & 9 & 14 & 5.79 & 98.99 & BIS & & FALSE \\
\hline 2007 & $\mathrm{D}$ & 9 & 15 & 6.68 & 99.06 & BIS & & FALSE \\
\hline 2007 & $\mathrm{D}$ & 9 & 16 & 7.22 & 99.50 & & Bot $\mathrm{X} 9 \mathrm{R}$ reb/low & FALSE \\
\hline 2007 & $\mathrm{D}$ & 9 & 17 & 7.22 & 99.98 & & Top X9R reb/low & FALSE \\
\hline 2007 & $\mathrm{D}$ & 10 & 1 & 0.00 & 100.00 & & Top X10L reb/low & FALSE \\
\hline 2007 & $\mathrm{D}$ & 10 & 2 & 0.00 & 99.67 & & Bot $\mathrm{X} 10 \mathrm{~L} \mathrm{reb} / \mathrm{low}$ & FALSE \\
\hline 2007 & $\mathrm{D}$ & 10 & 3 & 0.43 & 99.16 & BIS & & FALSE \\
\hline 2007 & $\mathrm{D}$ & 10 & 4 & 1.04 & 98.95 & BIS & & FALSE \\
\hline 2007 & $\mathrm{D}$ & 10 & 5 & 1.55 & 98.50 & BIS & & FALSE \\
\hline 2007 & $\mathrm{D}$ & 10 & 6 & 2.23 & 98.35 & $\mathrm{BF}$ & & FALSE \\
\hline 2007 & $\mathrm{D}$ & 10 & 7 & 2.74 & 98.23 & WEL & & FALSE \\
\hline 2007 & $\mathrm{D}$ & 10 & 8 & 3.08 & 98.15 & BIS & & FALSE \\
\hline 2007 & $\mathrm{D}$ & 10 & 9 & 3.63 & 98.17 & BIS & & FALSE \\
\hline 2007 & $\mathrm{D}$ & 10 & 10 & 4.05 & 98.16 & TW & & FALSE \\
\hline 2007 & $\mathrm{D}$ & 10 & 11 & 4.57 & 98.19 & BIS & & FALSE \\
\hline 2007 & $\mathrm{D}$ & 10 & 12 & 4.82 & 98.21 & WER & & FALSE \\
\hline 2007 & $\mathrm{D}$ & 10 & 13 & 5.09 & 98.49 & BIS & Top of rock & TRUE \\
\hline 2007 & $\mathrm{D}$ & 10 & 14 & 5.27 & 98.29 & BIS & & FALSE \\
\hline 2007 & $\mathrm{D}$ & 10 & 15 & 5.64 & 98.52 & BIS & Top of rock & FALSE \\
\hline 2007 & $\mathrm{D}$ & 10 & 16 & 5.97 & 98.40 & BIS & & FALSE \\
\hline 2007 & $\mathrm{D}$ & 10 & 17 & 6.52 & 98.58 & BIS & & FALSE \\
\hline 2007 & $\mathrm{D}$ & 10 & 18 & 6.83 & 98.93 & BIS & & FALSE \\
\hline 2007 & $\mathrm{D}$ & 10 & 19 & 7.32 & 99.78 & BOT X10R & Bot X10R reb/low & FALSE \\
\hline
\end{tabular}

Continued on Next Page... 
Table D.1 - Continued

\begin{tabular}{|c|c|c|c|c|c|c|c|c|}
\hline Year & Reach & Section & Point & Dist. $(\mathrm{m})$ & Elev. $(\mathrm{m})$ & Desc. & Notes & Exclude \\
\hline 2007 & $\mathrm{D}$ & 10 & 20 & 7.32 & 100.00 & TOP X10R & Top X10R reb/low & FALSE \\
\hline 2007 & $\mathrm{E}$ & 1 & 1 & 0.00 & 100.00 & TOP REB & Top X1L reb/low & FALSE \\
\hline 2007 & $\mathrm{E}$ & 1 & 2 & 0.00 & 99.62 & BOT REB & Bot X1L reb/low & FALSE \\
\hline 2007 & $\mathrm{E}$ & 1 & 3 & 0.27 & 99.56 & BIS & & FALSE \\
\hline 2007 & $\mathrm{E}$ & 1 & 4 & 0.79 & 99.23 & BIS & & FALSE \\
\hline 2007 & $\mathrm{E}$ & 1 & 5 & 1.92 & 98.88 & BIS & & FALSE \\
\hline 2007 & $\mathrm{E}$ & 1 & 6 & 2.44 & 98.85 & BIS & & FALSE \\
\hline 2007 & $\mathrm{E}$ & 1 & 7 & 3.08 & 98.85 & BIS & & FALSE \\
\hline 2007 & $\mathrm{E}$ & 1 & 8 & 3.96 & 98.68 & BIS & & FALSE \\
\hline 2007 & $\mathrm{E}$ & 1 & 9 & 4.42 & 98.62 & BIS & & FALSE \\
\hline 2007 & $\mathrm{E}$ & 1 & 10 & 4.51 & 98.49 & BIS & & FALSE \\
\hline 2007 & $\mathrm{E}$ & 1 & 11 & 5.12 & 98.39 & BIS & & FALSE \\
\hline 2007 & $\mathrm{E}$ & 1 & 12 & 5.58 & 98.29 & $\mathrm{BF}$ & & FALSE \\
\hline 2007 & $\mathrm{E}$ & 1 & 13 & 5.88 & 98.23 & WEL & & FALSE \\
\hline 2007 & $\mathrm{E}$ & 1 & 14 & 6.00 & 98.31 & BIS & On rock & FALSE \\
\hline 2007 & $\mathrm{E}$ & 1 & 15 & 6.19 & 98.02 & BIS & In water, EW underneath rock & FALSE \\
\hline 2007 & $\mathrm{E}$ & 1 & 16 & 6.34 & 98.10 & TW & & FALSE \\
\hline 2007 & $\mathrm{E}$ & 1 & 17 & 6.55 & 98.11 & BIS & & FALSE \\
\hline 2007 & $\mathrm{E}$ & 1 & 18 & 6.68 & 98.16 & WER & & FALSE \\
\hline 2007 & $\mathrm{E}$ & 1 & 19 & 6.77 & 98.10 & BIS & & FALSE \\
\hline 2007 & $\mathrm{E}$ & 1 & 20 & 6.98 & 98.12 & BIS & Bank undercut; EW & FALSE \\
\hline 2007 & $\mathrm{E}$ & 1 & 21 & 7.04 & 99.48 & TOB & & FALSE \\
\hline 2007 & $\mathrm{E}$ & 1 & 22 & 7.25 & 99.68 & BIS & & FALSE \\
\hline 2007 & $\mathrm{E}$ & 1 & 23 & 7.50 & 99.82 & BOT REB & Bot $\mathrm{X} 1 \mathrm{R}$ reb/low & FALSE \\
\hline 2007 & $\mathrm{E}$ & 1 & 24 & 7.50 & 99.88 & TOP REB & Top X1R reb/low & FALSE \\
\hline 2007 & $\mathrm{E}$ & 2 & 1 & 0.00 & 100.00 & TOP REB & Top X2L reb/low & FALSE \\
\hline 2007 & $\mathrm{E}$ & 2 & 2 & 0.00 & 99.44 & BOT REB & Bot $\mathrm{X} 2 \mathrm{~L} \mathrm{reb} /$ low & FALSE \\
\hline 2007 & $\mathrm{E}$ & 2 & 3 & 0.24 & 99.22 & BIS & & FALSE \\
\hline 2007 & $\mathrm{E}$ & 2 & 4 & 0.64 & 98.98 & BIS & & FALSE \\
\hline 2007 & $\mathrm{E}$ & 2 & 5 & 0.91 & 98.64 & BIS & & FALSE \\
\hline 2007 & $\mathrm{E}$ & 2 & 6 & 1.07 & 98.55 & BIS & & FALSE \\
\hline 2007 & $\mathrm{E}$ & 2 & 7 & 1.22 & 98.48 & BIS & & FALSE \\
\hline 2007 & $\mathrm{E}$ & 2 & 8 & 1.65 & 98.43 & BIS & & FALSE \\
\hline 2007 & $\mathrm{E}$ & 2 & 9 & 2.01 & 98.52 & BIS & On rock & FALSE \\
\hline 2007 & $\mathrm{E}$ & 2 & 10 & 2.04 & 98.33 & EW & & FALSE \\
\hline 2007 & $\mathrm{E}$ & 2 & 11 & 2.16 & 98.43 & BIS & & FALSE \\
\hline 2007 & $\mathrm{E}$ & 2 & 12 & 2.77 & 98.26 & TW & & FALSE \\
\hline 2007 & $\mathrm{E}$ & 2 & 13 & 3.17 & 98.36 & WER & & FALSE \\
\hline 2007 & $\mathrm{E}$ & 2 & 14 & 3.57 & 98.48 & BIS & & FALSE \\
\hline 2007 & $\mathrm{E}$ & 2 & 15 & 3.84 & 98.60 & BIS & & FALSE \\
\hline 2007 & $\mathrm{E}$ & 2 & 16 & 4.21 & 98.67 & BIS & & FALSE \\
\hline 2007 & $\mathrm{E}$ & 2 & 17 & 4.48 & 98.95 & BIS & & FALSE \\
\hline 2007 & $\mathrm{E}$ & 2 & 18 & 4.75 & 99.12 & BIS & & FALSE \\
\hline 2007 & $\mathrm{E}$ & 2 & 19 & 4.82 & 99.33 & BIS & & FALSE \\
\hline 2007 & $\mathrm{E}$ & 2 & 20 & 5.52 & 99.44 & BIS & & FALSE \\
\hline 2007 & $\mathrm{E}$ & 2 & 21 & 5.64 & 99.58 & BIS & & FALSE \\
\hline 2007 & $\mathrm{E}$ & 2 & 22 & 5.85 & 99.60 & BOT REB & Bot $\mathrm{X} 2 \mathrm{R}$ reb/low & FALSE \\
\hline 2007 & $\mathrm{E}$ & 2 & 23 & 5.85 & 100.01 & TOP REB & Top X2R reb/low & FALSE \\
\hline 2007 & $\mathrm{E}$ & 3 & 1 & 0.00 & 100.00 & TOP REB & Top X3L reb/low & FALSE \\
\hline 2007 & $\mathrm{E}$ & 3 & 2 & 0.00 & 99.52 & BOT REB & Bot $\mathrm{X} 3 \mathrm{~L} \mathrm{reb} /$ low & FALSE \\
\hline 2007 & $\mathrm{E}$ & 3 & 3 & 0.24 & 99.37 & BIS & & FALSE \\
\hline 2007 & $\mathrm{E}$ & 3 & 4 & 0.61 & 98.98 & BIS & & FALSE \\
\hline
\end{tabular}

Continued on Next Page... 
Table D.1 - Continued

\begin{tabular}{|c|c|c|c|c|c|c|c|c|}
\hline Year & Reach & Section & Point & Dist. (m) & Elev. $(\mathrm{m})$ & Desc. & Notes & Exclude \\
\hline 2007 & $\mathrm{E}$ & 3 & 5 & 1.22 & 98.60 & BIS & & FALSE \\
\hline 2007 & $\mathrm{E}$ & 3 & 6 & 1.74 & 98.38 & ТОВ & & FALSE \\
\hline 2007 & $\mathrm{E}$ & 3 & 7 & 1.92 & 98.00 & BIS & & FALSE \\
\hline 2007 & $\mathrm{E}$ & 3 & 8 & 2.29 & 97.96 & BIS & & FALSE \\
\hline 2007 & $\mathrm{E}$ & 3 & 9 & 2.71 & 98.15 & BIS & On rock & FALSE \\
\hline 2007 & $\mathrm{E}$ & 3 & 10 & 2.93 & 98.13 & BIS & On rock & FALSE \\
\hline 2007 & $\mathrm{E}$ & 3 & 11 & 3.11 & 97.85 & BIS & & FALSE \\
\hline 2007 & $\mathrm{E}$ & 3 & 12 & 3.32 & 97.86 & BIS & & FALSE \\
\hline 2007 & $\mathrm{E}$ & 3 & 13 & 3.38 & 97.78 & In water & & FALSE \\
\hline 2007 & $\mathrm{E}$ & 3 & 14 & 3.54 & 97.75 & $\mathrm{TW}$ & & FALSE \\
\hline 2007 & $\mathrm{E}$ & 3 & 15 & 3.81 & 97.84 & WER & & FALSE \\
\hline 2007 & $\mathrm{E}$ & 3 & 16 & 4.05 & 98.24 & BIS & On rock & FALSE \\
\hline 2007 & $\mathrm{E}$ & 3 & 17 & 4.18 & 98.19 & BIS & On rock & FALSE \\
\hline 2007 & $\mathrm{E}$ & 3 & 18 & 4.54 & 98.23 & BIS & & FALSE \\
\hline 2007 & $\mathrm{E}$ & 3 & 19 & 4.97 & 98.51 & BIS & On rock & FALSE \\
\hline 2007 & $\mathrm{E}$ & 3 & 20 & 5.30 & 98.47 & BIS & & FALSE \\
\hline 2007 & $\mathrm{E}$ & 3 & 21 & 5.43 & 98.88 & ТОВ & & FALSE \\
\hline 2007 & $\mathrm{E}$ & 3 & 22 & 6.00 & 99.06 & BIS & & FALSE \\
\hline 2007 & $\mathrm{E}$ & 3 & 23 & 6.46 & 99.16 & BIS & & FALSE \\
\hline 2007 & $\mathrm{E}$ & 3 & 24 & 7.16 & 99.40 & BIS & & FALSE \\
\hline 2007 & $\mathrm{E}$ & 3 & 25 & 7.25 & 99.44 & BOT REB & Bot X3R reb/low & FALSE \\
\hline 2007 & $\mathrm{E}$ & 3 & 26 & 7.25 & 100.01 & TOP REB & Top X3R reb/low & FALSE \\
\hline 2007 & $\mathrm{E}$ & 4 & 1 & 0.00 & 100.00 & TOP REB & Top X4L reb/low & FALSE \\
\hline 2007 & $\mathrm{E}$ & 4 & 2 & 0.00 & 99.66 & BOT REB & Bot $\mathrm{X} 4 \mathrm{~L}$ reb/low & FALSE \\
\hline 2007 & $\mathrm{E}$ & 4 & 3 & 0.18 & 99.49 & BIS & & FALSE \\
\hline 2007 & $\mathrm{E}$ & 4 & 4 & 0.55 & 99.41 & ТОВ & & FALSE \\
\hline 2007 & $\mathrm{E}$ & 4 & 5 & 0.58 & 99.13 & BIS & & FALSE \\
\hline 2007 & $\mathrm{E}$ & 4 & 6 & 0.91 & 99.03 & BIS & & FALSE \\
\hline 2007 & $\mathrm{E}$ & 4 & 7 & 1.19 & 98.80 & BIS & & FALSE \\
\hline 2007 & $\mathrm{E}$ & 4 & 8 & 1.25 & 98.92 & WEL & On rock & FALSE \\
\hline 2007 & $\mathrm{E}$ & 4 & 9 & 1.62 & 98.95 & BIS & & FALSE \\
\hline 2007 & $\mathrm{E}$ & 4 & 10 & 1.77 & 98.77 & BIS & & FALSE \\
\hline 2007 & $\mathrm{E}$ & 4 & 11 & 1.89 & 98.66 & WEL & & FALSE \\
\hline 2007 & $\mathrm{E}$ & 4 & 12 & 2.26 & 98.60 & BIS & & FALSE \\
\hline 2007 & $\mathrm{E}$ & 4 & 13 & 2.41 & 98.64 & BIS & & FALSE \\
\hline 2007 & $\mathrm{E}$ & 4 & 14 & 2.74 & 98.62 & BIS & & FALSE \\
\hline 2007 & $\mathrm{E}$ & 4 & 15 & 2.93 & 98.58 & TW & & FALSE \\
\hline 2007 & $\mathrm{E}$ & 4 & 16 & 3.05 & 98.56 & BIS & & FALSE \\
\hline 2007 & $\mathrm{E}$ & 4 & 17 & 3.11 & 98.64 & WER & & FALSE \\
\hline 2007 & $\mathrm{E}$ & 4 & 18 & 3.54 & 98.82 & BIS & Top of rock & FALSE \\
\hline 2007 & $\mathrm{E}$ & 4 & 19 & 3.96 & 98.51 & BIS & & FALSE \\
\hline 2007 & $\mathrm{E}$ & 4 & 20 & 4.30 & 98.76 & BIS & Top of rock & FALSE \\
\hline 2007 & $\mathrm{E}$ & 4 & 21 & 4.51 & 98.62 & BIS & & FALSE \\
\hline 2007 & $\mathrm{E}$ & 4 & 22 & 4.82 & 98.59 & BIS & & FALSE \\
\hline 2007 & $\mathrm{E}$ & 4 & 23 & 5.03 & 98.87 & BIS & & FALSE \\
\hline 2007 & $\mathrm{E}$ & 4 & 24 & 5.15 & 98.75 & BIS & & FALSE \\
\hline 2007 & $\mathrm{E}$ & 4 & 25 & 5.64 & 98.77 & BIS & Undercut bank - edge & FALSE \\
\hline 2007 & $\mathrm{E}$ & 4 & 26 & 5.82 & 99.46 & ТОВ & & FALSE \\
\hline 2007 & $\mathrm{E}$ & 4 & 27 & 6.34 & 99.58 & BIS & & FALSE \\
\hline 2007 & $\mathrm{E}$ & 4 & 28 & 7.01 & 99.65 & BIS & & FALSE \\
\hline 2007 & $\mathrm{E}$ & 4 & 29 & 7.62 & 99.80 & BOT REB & Bot $\mathrm{X} 4 \mathrm{R}$ reb/low & FALSE \\
\hline 2007 & $\mathrm{E}$ & 4 & 30 & 7.62 & 99.73 & TOP REB & Top X4R reb/low & FALSE \\
\hline
\end{tabular}

Continued on Next Page... 
Table D.1 - Continued

\begin{tabular}{|c|c|c|c|c|c|c|c|c|}
\hline Year & Reach & Section & Point & Dist. (m) & Elev. $(\mathrm{m})$ & Desc. & Notes & Exclude \\
\hline 2007 & $\mathrm{E}$ & 5 & 1 & 0.00 & 100.00 & TOP REB & Top X5L reb/low & FALSE \\
\hline 2007 & $\mathrm{E}$ & 5 & 2 & 0.00 & 99.99 & BOT REB & Bot $\mathrm{X} 5 \mathrm{~L}$ reb/low & FALSE \\
\hline 2007 & $\mathrm{E}$ & 5 & 3 & 0.43 & 99.92 & BIS & & FALSE \\
\hline 2007 & $\mathrm{E}$ & 5 & 4 & 0.91 & 99.76 & TOB & & FALSE \\
\hline 2007 & $\mathrm{E}$ & 5 & 5 & 1.25 & 99.51 & BIS & & FALSE \\
\hline 2007 & $\mathrm{E}$ & 5 & 6 & 1.55 & 99.21 & BIS & & FALSE \\
\hline 2007 & $\mathrm{E}$ & 5 & 7 & 2.04 & 99.30 & BIS & & FALSE \\
\hline 2007 & $\mathrm{E}$ & 5 & 8 & 2.13 & 99.18 & BIS & & FALSE \\
\hline 2007 & $\mathrm{E}$ & 5 & 9 & 2.47 & 99.30 & BIS & & FALSE \\
\hline 2007 & $\mathrm{E}$ & 5 & 10 & 2.56 & 99.03 & BIS & & FALSE \\
\hline 2007 & $\mathrm{E}$ & 5 & 11 & 2.71 & 99.01 & WEL & & FALSE \\
\hline 2007 & $\mathrm{E}$ & 5 & 12 & 2.80 & 98.97 & BIS & & FALSE \\
\hline 2007 & $\mathrm{E}$ & 5 & 13 & 3.05 & 98.94 & TW & & FALSE \\
\hline 2007 & $\mathrm{E}$ & 5 & 14 & 3.32 & 99.01 & BIS & & FALSE \\
\hline 2007 & $\mathrm{E}$ & 5 & 15 & 3.57 & 98.97 & BIS & & FALSE \\
\hline 2007 & $\mathrm{E}$ & 5 & 16 & 3.75 & 99.04 & BIS & & FALSE \\
\hline 2007 & $\mathrm{E}$ & 5 & 17 & 3.87 & 99.01 & WER & & FALSE \\
\hline 2007 & $\mathrm{E}$ & 5 & 18 & 4.27 & 99.07 & BIS & & FALSE \\
\hline 2007 & $\mathrm{E}$ & 5 & 19 & 4.42 & 99.31 & BIS & & FALSE \\
\hline 2007 & $\mathrm{E}$ & 5 & 20 & 4.63 & 99.29 & BIS & & FALSE \\
\hline 2007 & $\mathrm{E}$ & 5 & 21 & 4.66 & 99.42 & BIS & & FALSE \\
\hline 2007 & $\mathrm{E}$ & 5 & 22 & 4.94 & 99.53 & BIS & & FALSE \\
\hline 2007 & $\mathrm{E}$ & 5 & 23 & 5.36 & 99.61 & BIS & & FALSE \\
\hline 2007 & $\mathrm{E}$ & 5 & 24 & 5.36 & 99.99 & BIS & & FALSE \\
\hline 2007 & $\mathrm{E}$ & 5 & 25 & 5.76 & 100.09 & BIS & & FALSE \\
\hline 2007 & $\mathrm{E}$ & 5 & 26 & 6.80 & 100.20 & BIS & & FALSE \\
\hline 2007 & $\mathrm{E}$ & 5 & 27 & 7.86 & 100.20 & BOT REB & Bot $\mathrm{X} 5 \mathrm{R}$ reb/low & FALSE \\
\hline 2007 & $\mathrm{E}$ & 5 & 28 & 7.86 & 100.61 & TOP REB & Top X5R reb/low & FALSE \\
\hline 2007 & $\mathrm{E}$ & 6 & 1 & 0.00 & 100.00 & TOP REB & $\begin{array}{l}\text { Top X6L reb/low - rebar diago- } \\
\text { nal } 75 \text { degrees from horiz towards } \\
\text { stream }\end{array}$ & FALSE \\
\hline 2007 & $\mathrm{E}$ & 6 & 2 & 0.00 & 99.54 & BOT REB & Bot $\mathrm{X} 6 \mathrm{~L}$ reb/low & FALSE \\
\hline 2007 & $\mathrm{E}$ & 6 & 3 & 0.24 & 99.38 & ТОВ & & FALSE \\
\hline 2007 & $\mathrm{E}$ & 6 & 4 & 0.58 & 98.77 & BIS & & FALSE \\
\hline 2007 & $\mathrm{E}$ & 6 & 5 & 0.79 & 98.67 & BIS & & FALSE \\
\hline 2007 & $\mathrm{E}$ & 6 & 6 & 1.04 & 98.68 & BIS & & FALSE \\
\hline 2007 & $\mathrm{E}$ & 6 & 7 & 1.13 & 98.36 & WEL & & FALSE \\
\hline 2007 & $\mathrm{E}$ & 6 & 8 & 1.40 & 98.31 & BIS & & FALSE \\
\hline 2007 & $\mathrm{E}$ & 6 & 9 & 1.58 & 98.40 & BIS & ON ROCK & FALSE \\
\hline 2007 & $\mathrm{E}$ & 6 & 10 & 1.80 & 98.28 & TW & & FALSE \\
\hline 2007 & $\mathrm{E}$ & 6 & 11 & 1.92 & 98.37 & WER & & FALSE \\
\hline 2007 & $\mathrm{E}$ & 6 & 12 & 2.07 & 98.40 & BIS & & FALSE \\
\hline 2007 & $\mathrm{E}$ & 6 & 13 & 2.16 & 98.38 & BIS & SAND & FALSE \\
\hline 2007 & $\mathrm{E}$ & 6 & 14 & 2.47 & 98.41 & BIS & SAND & FALSE \\
\hline 2007 & $\mathrm{E}$ & 6 & 15 & 2.93 & 98.51 & BIS & SAND & FALSE \\
\hline 2007 & $\mathrm{E}$ & 6 & 16 & 3.26 & 98.55 & BIS & & FALSE \\
\hline 2007 & $\mathrm{E}$ & 6 & 17 & 3.29 & 98.76 & BIS & ON ROCK & FALSE \\
\hline 2007 & $\mathrm{E}$ & 6 & 18 & 3.57 & 98.85 & BIS & ON ROCK & FALSE \\
\hline 2007 & $\mathrm{E}$ & 6 & 19 & 3.84 & 98.66 & BIS & & FALSE \\
\hline 2007 & $\mathrm{E}$ & 6 & 20 & 4.24 & 98.66 & BIS & & FALSE \\
\hline 2007 & $\mathrm{E}$ & 6 & 21 & 4.48 & 98.90 & BIS & & FALSE \\
\hline 2007 & $\mathrm{E}$ & 6 & 22 & 4.63 & 99.46 & TOB & & FALSE \\
\hline
\end{tabular}

Continued on Next Page... 
Table D.1 - Continued

\begin{tabular}{|c|c|c|c|c|c|c|c|c|}
\hline Year & Reach & Section & Point & Dist. (m) & Elev. $(\mathrm{m})$ & Desc. & Notes & Exclude \\
\hline 2007 & $\mathrm{E}$ & 6 & 23 & 5.00 & 99.58 & BIS & & FALSE \\
\hline 2007 & $\mathrm{E}$ & 6 & 24 & 5.33 & 99.64 & BOT REB & Bot $\mathrm{X} 6 \mathrm{R}$ reb/low & FALSE \\
\hline 2007 & $\mathrm{E}$ & 6 & 25 & 5.33 & 100.00 & TOP REB & Top X6R reb/low & FALSE \\
\hline 2007 & $\mathrm{E}$ & 7 & 1 & 0.00 & 100.00 & TOP REB & Bot $X 7 \mathrm{~L} \mathrm{reb} /$ low & FALSE \\
\hline 2007 & $\mathrm{E}$ & 7 & 2 & 0.00 & 99.75 & BOT REB & Top X7L reb/low & FALSE \\
\hline 2007 & $\mathrm{E}$ & 7 & 3 & 0.43 & 99.57 & BIS & & FALSE \\
\hline 2007 & $\mathrm{E}$ & 7 & 4 & 0.82 & 99.38 & ТОВ & & FALSE \\
\hline 2007 & $\mathrm{E}$ & 7 & 5 & 1.01 & 98.91 & BIS & & FALSE \\
\hline 2007 & $\mathrm{E}$ & 7 & 6 & 1.19 & 98.94 & BIS & & FALSE \\
\hline 2007 & $\mathrm{E}$ & 7 & 7 & 1.28 & 98.70 & BIS & & FALSE \\
\hline 2007 & $\mathrm{E}$ & 7 & 8 & 1.46 & 98.69 & BIS & & FALSE \\
\hline 2007 & $\mathrm{E}$ & 7 & 9 & 1.49 & 98.43 & IN WATER & & FALSE \\
\hline 2007 & $\mathrm{E}$ & 7 & 10 & 1.77 & 98.49 & BIS & & FALSE \\
\hline 2007 & $\mathrm{E}$ & 7 & 11 & 1.83 & 98.82 & BIS & & FALSE \\
\hline 2007 & $\mathrm{E}$ & 7 & 12 & 1.98 & 98.39 & BIS & POOL & FALSE \\
\hline 2007 & $\mathrm{E}$ & 7 & 13 & 2.13 & 98.38 & BIS & & FALSE \\
\hline 2007 & $\mathrm{E}$ & 7 & 14 & 2.26 & 98.59 & WATER & ON ROCK & FALSE \\
\hline & & & & & & LEVEL & & \\
\hline 2007 & $\mathrm{E}$ & 7 & 15 & 2.44 & 98.66 & BIS & ON ROCK & FALSE \\
\hline 2007 & $\mathrm{E}$ & 7 & 16 & 2.74 & 98.66 & BIS & ON ROCK & FALSE \\
\hline 2007 & $\mathrm{E}$ & 7 & 17 & 2.77 & 98.53 & BIS & & FALSE \\
\hline 2007 & $\mathrm{E}$ & 7 & 18 & 3.14 & 98.49 & TW & & FALSE \\
\hline 2007 & $\mathrm{E}$ & 7 & 19 & 3.47 & 98.50 & BIS & & FALSE \\
\hline 2007 & $\mathrm{E}$ & 7 & 20 & 3.72 & 98.58 & WER & & FALSE \\
\hline 2007 & $\mathrm{E}$ & 7 & 21 & 3.90 & 98.68 & BIS & & FALSE \\
\hline 2007 & $\mathrm{E}$ & 7 & 22 & 4.15 & 98.71 & $\mathrm{BF}$ & & FALSE \\
\hline 2007 & $\mathrm{E}$ & 7 & 23 & 4.27 & 98.84 & BIS & & FALSE \\
\hline 2007 & $\mathrm{E}$ & 7 & 24 & 4.54 & 98.99 & BIS & $\begin{array}{l}\text { BOTTOM OF MINOR UNDER- } \\
\text { CUT BANK }\end{array}$ & FALSE \\
\hline 2007 & $\mathrm{E}$ & 7 & 25 & 4.57 & 99.55 & BIS & & FALSE \\
\hline 2007 & $\mathrm{E}$ & 7 & 26 & 4.72 & 99.63 & BIS & & FALSE \\
\hline 2007 & $\mathrm{E}$ & 7 & 27 & 5.00 & 99.78 & BIS & & FALSE \\
\hline 2007 & $\mathrm{E}$ & 7 & 28 & 5.24 & 99.84 & BIS & & FALSE \\
\hline 2007 & $\mathrm{E}$ & 7 & 29 & 5.49 & 99.70 & BIS & & FALSE \\
\hline 2007 & $\mathrm{E}$ & 7 & 30 & 5.94 & 99.67 & BIS & & FALSE \\
\hline 2007 & $\mathrm{E}$ & 7 & 31 & 6.49 & 99.71 & TOP REB & Bot $\mathrm{X} 7 \mathrm{R}$ reb/low & FALSE \\
\hline 2007 & $\mathrm{E}$ & 7 & 32 & 6.49 & 100.00 & BOT REB & Top X7R reb/low & FALSE \\
\hline 2007 & $\mathrm{E}$ & 8 & 1 & 0.00 & 100.00 & TOP REB & Bot $\mathrm{X} 8 \mathrm{~L} \mathrm{reb} /$ low & FALSE \\
\hline 2007 & $\mathrm{E}$ & 8 & 2 & 0.00 & 99.63 & BOT REB & Top X8L reb/low & FALSE \\
\hline 2007 & $\mathrm{E}$ & 8 & 3 & 0.34 & 99.45 & BIS & 1' high, corrected & FALSE \\
\hline 2007 & $\mathrm{E}$ & 8 & 4 & 0.64 & 99.23 & BIS & & FALSE \\
\hline 2007 & $\mathrm{E}$ & 8 & 5 & 0.79 & 99.11 & BIS & & FALSE \\
\hline 2007 & $\mathrm{E}$ & 8 & 6 & 0.94 & 98.81 & $\mathrm{BF} ?$ & & FALSE \\
\hline 2007 & $\mathrm{E}$ & 8 & 7 & 1.34 & 98.63 & WEL & & FALSE \\
\hline 2007 & $\mathrm{E}$ & 8 & 8 & 1.58 & 98.63 & BIS & & FALSE \\
\hline 2007 & $\mathrm{E}$ & 8 & 9 & 1.80 & 98.65 & BIS & ROCK & FALSE \\
\hline 2007 & $\mathrm{E}$ & 8 & 10 & 1.80 & 98.59 & BIS & ROCK & FALSE \\
\hline 2007 & $\mathrm{E}$ & 8 & 11 & 2.01 & 98.57 & $\mathrm{TW}$ & & FALSE \\
\hline 2007 & $\mathrm{E}$ & 8 & 12 & 2.32 & 98.58 & BIS & & FALSE \\
\hline 2007 & $\mathrm{E}$ & 8 & 13 & 2.53 & 98.59 & BIS & & FALSE \\
\hline 2007 & $\mathrm{E}$ & 8 & 14 & 2.65 & 98.56 & BIS & & FALSE \\
\hline 2007 & $\mathrm{E}$ & 8 & 15 & 2.93 & 98.60 & WER & & FALSE \\
\hline
\end{tabular}

Continued on Next Page... 
Table D.1 - Continued

\begin{tabular}{|c|c|c|c|c|c|c|c|c|}
\hline Year & Reach & Section & Point & Dist. (m) & Elev. $(\mathrm{m})$ & Desc. & Notes & Exclude \\
\hline 2007 & $\mathrm{E}$ & 8 & 16 & 3.20 & 98.77 & BIS & ROCK & FALSE \\
\hline 2007 & $\mathrm{E}$ & 8 & 17 & 3.35 & 98.66 & BIS & & FALSE \\
\hline 2007 & $\mathrm{E}$ & 8 & 18 & 3.66 & 98.74 & BIS & & FALSE \\
\hline 2007 & $\mathrm{E}$ & 8 & 19 & 4.05 & 98.99 & BIS & & FALSE \\
\hline 2007 & $\mathrm{E}$ & 8 & 20 & 4.08 & 99.29 & BIS & & FALSE \\
\hline 2007 & $\mathrm{E}$ & 8 & 21 & 4.48 & 99.55 & BIS & & FALSE \\
\hline 2007 & $\mathrm{E}$ & 8 & 22 & 5.46 & 99.65 & BIS & & FALSE \\
\hline 2007 & $\mathrm{E}$ & 8 & 23 & 5.82 & 99.63 & BIS & & FALSE \\
\hline 2007 & $\mathrm{E}$ & 8 & 24 & 6.34 & 99.75 & BIS & & FALSE \\
\hline 2007 & $\mathrm{E}$ & 8 & 25 & 6.61 & 99.74 & BOT REB & Bot $\mathrm{X} 8 \mathrm{R}$ reb/low & FALSE \\
\hline 2007 & $\mathrm{E}$ & 8 & 26 & 6.61 & 99.99 & TOP REB & Top X8R reb/low & FALSE \\
\hline 2007 & $\mathrm{E}$ & 9 & 1 & 0.00 & 100.00 & TOP REB & Top X9L reb/low & FALSE \\
\hline 2007 & $\mathrm{E}$ & 9 & 2 & 0.00 & 99.55 & BOT REB & Bot X9L reb/low & FALSE \\
\hline 2007 & $\mathrm{E}$ & 9 & 3 & 0.55 & 99.09 & & & FALSE \\
\hline 2007 & $\mathrm{E}$ & 9 & 4 & 0.82 & 98.93 & & & FALSE \\
\hline 2007 & $\mathrm{E}$ & 9 & 5 & 1.07 & 98.57 & & & FALSE \\
\hline 2007 & $\mathrm{E}$ & 9 & 6 & 1.34 & 98.43 & & & FALSE \\
\hline 2007 & $\mathrm{E}$ & 9 & 7 & 1.68 & 98.08 & & & FALSE \\
\hline 2007 & $\mathrm{E}$ & 9 & 8 & 2.56 & 97.60 & & & FALSE \\
\hline 2007 & $\mathrm{E}$ & 9 & 9 & 2.74 & 97.81 & & & FALSE \\
\hline 2007 & $\mathrm{E}$ & 9 & 10 & 3.14 & 97.79 & & & FALSE \\
\hline 2007 & $\mathrm{E}$ & 9 & 11 & 3.41 & 97.73 & & & FALSE \\
\hline 2007 & $\mathrm{E}$ & 9 & 12 & 3.66 & 97.69 & & & FALSE \\
\hline 2007 & $\mathrm{E}$ & 9 & 13 & 3.84 & 97.66 & & & FALSE \\
\hline 2007 & $\mathrm{E}$ & 9 & 14 & 4.24 & 97.67 & & & FALSE \\
\hline 2007 & $\mathrm{E}$ & 9 & 15 & 4.54 & 97.72 & & & FALSE \\
\hline 2007 & $\mathrm{E}$ & 9 & 16 & 4.94 & 97.85 & & & FALSE \\
\hline 2007 & $\mathrm{E}$ & 9 & 17 & 5.33 & 97.92 & & & FALSE \\
\hline 2007 & $\mathrm{E}$ & 9 & 18 & 5.85 & 97.97 & & & FALSE \\
\hline 2007 & $\mathrm{E}$ & 9 & 19 & 6.55 & 98.11 & & & FALSE \\
\hline 2007 & $\mathrm{E}$ & 9 & 20 & 7.19 & 98.52 & & & FALSE \\
\hline 2007 & $\mathrm{E}$ & 9 & 21 & 7.35 & 99.06 & & & FALSE \\
\hline 2007 & $\mathrm{E}$ & 9 & 22 & 7.71 & 99.13 & & & FALSE \\
\hline 2007 & $\mathrm{E}$ & 9 & 23 & 7.89 & 99.61 & & & FALSE \\
\hline 2007 & $\mathrm{E}$ & 9 & 24 & 8.14 & 99.77 & BOT REB & Bot X9R reb/low & FALSE \\
\hline 2007 & $\mathrm{E}$ & 9 & 25 & 8.14 & 100.05 & TOP REB & Top X9R reb/low & FALSE \\
\hline 2007 & $\mathrm{E}$ & 10 & 1 & 0.00 & 100.00 & TOP REB & Bot $\mathrm{X} 10 \mathrm{~L} \mathrm{reb} / \mathrm{low}$ & FALSE \\
\hline 2007 & $\mathrm{E}$ & 10 & 2 & 0.00 & 99.69 & BOT REB & Top X10L reb/low & FALSE \\
\hline 2007 & $\mathrm{E}$ & 10 & 3 & 0.27 & 99.46 & BIS & & FALSE \\
\hline 2007 & $\mathrm{E}$ & 10 & 4 & 0.49 & 99.33 & BIS & & FALSE \\
\hline 2007 & $\mathrm{E}$ & 10 & 5 & 0.58 & 98.89 & BIS & & FALSE \\
\hline 2007 & $\mathrm{E}$ & 10 & 6 & 0.76 & 98.78 & BIS & & FALSE \\
\hline 2007 & $\mathrm{E}$ & 10 & 7 & 1.31 & 98.39 & BIS & & FALSE \\
\hline 2007 & $\mathrm{E}$ & 10 & 8 & 1.65 & 98.36 & BIS & & FALSE \\
\hline 2007 & $\mathrm{E}$ & 10 & 9 & 1.95 & 98.29 & BIS & & FALSE \\
\hline 2007 & $\mathrm{E}$ & 10 & 10 & 2.29 & 98.31 & BIS & & FALSE \\
\hline 2007 & $\mathrm{E}$ & 10 & 11 & 2.35 & 98.13 & IN WATER & & FALSE \\
\hline 2007 & $\mathrm{E}$ & 10 & 12 & 2.65 & 98.06 & BIS & & FALSE \\
\hline 2007 & $\mathrm{E}$ & 10 & 13 & 2.87 & 98.04 & $\mathrm{TW}$ & & FALSE \\
\hline 2007 & $\mathrm{E}$ & 10 & 14 & 3.26 & 98.08 & BIS & & FALSE \\
\hline 2007 & $\mathrm{E}$ & 10 & 15 & 3.44 & 98.16 & WER & & FALSE \\
\hline 2007 & $\mathrm{E}$ & 10 & 16 & 3.90 & 98.33 & $\mathrm{BF}$ & & FALSE \\
\hline
\end{tabular}

Continued on Next Page... 
Table D.1 - Continued

\begin{tabular}{|c|c|c|c|c|c|c|c|c|c|}
\hline Year & Reach & Section & Point & Dist. (m) & Elev. $(\mathrm{m})$ & Desc. & Notes & & Exclude \\
\hline 2007 & $\mathrm{E}$ & 10 & 17 & 4.11 & 98.44 & BIS & & & FALSE \\
\hline 2007 & $\mathrm{E}$ & 10 & 18 & 5.03 & 98.64 & BIS & & & FALSE \\
\hline 2007 & $\mathrm{E}$ & 10 & 19 & 5.18 & 99.06 & ТОВ & & & FALSE \\
\hline 2007 & $\mathrm{E}$ & 10 & 20 & 5.58 & 99.39 & BIS & & & FALSE \\
\hline 2007 & $\mathrm{E}$ & 10 & 21 & 6.40 & 99.72 & BIS & & & FALSE \\
\hline 2007 & $\mathrm{E}$ & 10 & 22 & 7.04 & 99.95 & BOT REB & Bot $\mathrm{X} 10 \mathrm{R} \mathrm{reb} / \mathrm{low}$ & & FALSE \\
\hline 2007 & $\mathrm{E}$ & 10 & 23 & 7.04 & 100.16 & TOP REB & Top X10R reb/low & & FALSE \\
\hline 2007 & $\mathrm{~F}$ & 1 & 1 & 0.00 & 100.00 & TOP REB & Top X1L reb/low & & FALSE \\
\hline 2007 & $\mathrm{~F}$ & 1 & 2 & 0.00 & 99.93 & BOT REB & Bot $\mathrm{X} 1 \mathrm{~L}$ reb/low & & FALSE \\
\hline 2007 & $\mathrm{~F}$ & 1 & 3 & 0.98 & 99.14 & BIS & & & FALSE \\
\hline 2007 & $\mathrm{~F}$ & 1 & 4 & 1.71 & 98.93 & BIS & & & FALSE \\
\hline \multirow[t]{2}{*}{2007} & $\mathrm{~F}$ & 1 & 5 & 1.89 & 99.34 & BIS & TOP OF FALLEN & ALDER & FALSE \\
\hline & & & & & & & ROOT WAD & & \\
\hline 2007 & $\mathrm{~F}$ & 1 & 6 & 2.32 & 99.12 & BIS & LOG OF ROOT WAD & & FALSE \\
\hline 2007 & $\mathrm{~F}$ & 1 & 7 & 2.71 & 98.58 & BIS & & & FALSE \\
\hline 2007 & $\mathrm{~F}$ & 1 & 8 & 3.26 & 98.33 & BIS & & & FALSE \\
\hline 2007 & $\mathrm{~F}$ & 1 & 9 & 4.05 & 98.33 & BIS & & & FALSE \\
\hline 2007 & $\mathrm{~F}$ & 1 & 10 & 4.69 & 98.18 & BIS & & & FALSE \\
\hline 2007 & $\mathrm{~F}$ & 1 & 11 & 5.03 & 98.08 & BIS & & & FALSE \\
\hline 2007 & $\mathrm{~F}$ & 1 & 12 & 5.40 & 98.24 & BIS & ON A ROCK & & FALSE \\
\hline 2007 & $\mathrm{~F}$ & 1 & 13 & 5.73 & 97.99 & WEL & & & FALSE \\
\hline 2007 & $\mathrm{~F}$ & 1 & 14 & 5.85 & 97.92 & BIS & & & FALSE \\
\hline 2007 & $\mathrm{~F}$ & 1 & 15 & 6.00 & 97.98 & BIS & & & FALSE \\
\hline 2007 & $\mathrm{~F}$ & 1 & 16 & 6.13 & 97.92 & $\mathrm{TW}$ & & & FALSE \\
\hline 2007 & $\mathrm{~F}$ & 1 & 17 & 6.31 & 98.07 & BIS & & & FALSE \\
\hline 2007 & $\mathrm{~F}$ & 1 & 18 & 6.46 & 98.05 & WER & & & FALSE \\
\hline 2007 & $\mathrm{~F}$ & 1 & 19 & 6.71 & 98.25 & BIS & & & FALSE \\
\hline 2007 & $\mathrm{~F}$ & 1 & 20 & 6.92 & 98.16 & BIS & & & FALSE \\
\hline 2007 & $\mathrm{~F}$ & 1 & 21 & 7.07 & 98.17 & BIS & & & FALSE \\
\hline 2007 & $\mathrm{~F}$ & 1 & 22 & 7.74 & 98.37 & BIS & & & FALSE \\
\hline 2007 & $\mathrm{~F}$ & 1 & 23 & 8.26 & 98.68 & BIS & & & FALSE \\
\hline 2007 & $\mathrm{~F}$ & 1 & 24 & 8.84 & 99.35 & BIS & & & FALSE \\
\hline 2007 & $\mathrm{~F}$ & 1 & 25 & 9.42 & 99.70 & BOT REB & Bot $\mathrm{X} 1 \mathrm{R}$ reb/low & & FALSE \\
\hline 2007 & $\mathrm{~F}$ & 1 & 26 & 9.42 & 99.81 & TOP REB & Top X1R reb/low & & FALSE \\
\hline 2007 & $\mathrm{~F}$ & 2 & 1 & 0.00 & 100.00 & TOP REB & Top X2L reb/low & & FALSE \\
\hline 2007 & $\mathrm{~F}$ & 2 & 2 & 0.00 & 99.79 & BOT REB & Bot $\mathrm{X} 2 \mathrm{~L} \mathrm{reb} /$ low & & FALSE \\
\hline 2007 & $\mathrm{~F}$ & 2 & 3 & 0.37 & 99.46 & BIS & & & FALSE \\
\hline 2007 & $\mathrm{~F}$ & 2 & 4 & 1.10 & 99.06 & BIS & & & FALSE \\
\hline 2007 & $\mathrm{~F}$ & 2 & 5 & 1.92 & 98.70 & BIS & & & FALSE \\
\hline 2007 & $\mathrm{~F}$ & 2 & 6 & 2.53 & 98.38 & BIS & & & FALSE \\
\hline 2007 & $\mathrm{~F}$ & 2 & 7 & 2.65 & 98.04 & BIS & & & FALSE \\
\hline 2007 & $\mathrm{~F}$ & 2 & 8 & 3.47 & 97.73 & $\mathrm{BF}$ & & & FALSE \\
\hline 2007 & $\mathrm{~F}$ & 2 & 9 & 4.24 & 97.58 & BIS & & & FALSE \\
\hline 2007 & $\mathrm{~F}$ & 2 & 10 & 4.51 & 97.47 & WEL & & & FALSE \\
\hline 2007 & $\mathrm{~F}$ & 2 & 11 & 4.85 & 97.37 & $\mathrm{TW}$ & & & FALSE \\
\hline 2007 & $\mathrm{~F}$ & 2 & 12 & 5.18 & 97.65 & BIS & ON A ROCK & & FALSE \\
\hline 2007 & $\mathrm{~F}$ & 2 & 13 & 5.36 & 97.46 & BIS & & & FALSE \\
\hline 2007 & $\mathrm{~F}$ & 2 & 14 & 5.97 & 97.45 & WER & & & FALSE \\
\hline 2007 & $\mathrm{~F}$ & 2 & 15 & 6.25 & 97.55 & BIS & & & FALSE \\
\hline 2007 & $\mathrm{~F}$ & 2 & 16 & 6.86 & 97.80 & BIS & & & FALSE \\
\hline 2007 & $\mathrm{~F}$ & 2 & 17 & 7.80 & 98.24 & BIS & & & FALSE \\
\hline 2007 & $\mathrm{~F}$ & 2 & 18 & 8.14 & 98.48 & BIS & & & FALSE \\
\hline
\end{tabular}

Continued on Next Page... 
Table D.1 - Continued

\begin{tabular}{|c|c|c|c|c|c|c|c|c|}
\hline Year & Reach & Section & Point & Dist. (m) & Elev. $(\mathrm{m})$ & Desc. & Notes & Exclude \\
\hline 2007 & $\mathrm{~F}$ & 2 & 19 & 8.44 & 99.80 & BIS & & FALSE \\
\hline 2007 & $\mathrm{~F}$ & 2 & 20 & 8.72 & 99.64 & BOT REB & Bot $\mathrm{X} 2 \mathrm{R}$ reb/low & FALSE \\
\hline 2007 & $\mathrm{~F}$ & 2 & 21 & 8.72 & 99.80 & TOP REB & Top X2R reb/low & FALSE \\
\hline 2007 & $\mathrm{~F}$ & 3 & 1 & 0.00 & 100.00 & TOP REB & Top X3L reb/low & FALSE \\
\hline 2007 & $\mathrm{~F}$ & 3 & 2 & 0.00 & 99.83 & BOT REB & Bot $\mathrm{X} 3 \mathrm{~L}$ reb/low & FALSE \\
\hline 2007 & F & 3 & 3 & 0.46 & 99.65 & BIS & & FALSE \\
\hline 2007 & $\mathrm{~F}$ & 3 & 4 & 1.10 & 99.35 & BIS & & FALSE \\
\hline 2007 & $\mathrm{~F}$ & 3 & 5 & 1.31 & 98.61 & BIS & & FALSE \\
\hline 2007 & $\mathrm{~F}$ & 3 & 6 & 1.95 & 98.45 & BIS & & FALSE \\
\hline 2007 & $\mathrm{~F}$ & 3 & 7 & 2.74 & 98.37 & BIS & & FALSE \\
\hline 2007 & $\mathrm{~F}$ & 3 & 8 & 2.90 & 98.45 & BIS & & FALSE \\
\hline 2007 & $\mathrm{~F}$ & 3 & 9 & 3.23 & 98.22 & BIS & & FALSE \\
\hline 2007 & F & 3 & 10 & 3.47 & 98.09 & BIS & & FALSE \\
\hline 2007 & $\mathrm{~F}$ & 3 & 11 & 3.75 & 98.31 & BIS & & FALSE \\
\hline 2007 & F & 3 & 12 & 3.87 & 97.89 & WEL & $\begin{array}{l}\text { REAL WATERS EDGE UNDER } \\
\text { OVERHANGING ROCKS }\end{array}$ & FALSE \\
\hline 2007 & $\mathrm{~F}$ & 3 & 13 & 4.21 & 97.93 & WER & & FALSE \\
\hline 2007 & F & 3 & 14 & 4.45 & 98.14 & BIS & & FALSE \\
\hline 2007 & $\mathrm{~F}$ & 3 & 15 & 4.72 & 97.92 & BIS & & FALSE \\
\hline 2007 & F & 3 & 16 & 5.36 & 98.01 & BIS & & FALSE \\
\hline 2007 & F & 3 & 17 & 6.19 & 98.62 & BIS & & FALSE \\
\hline 2007 & F & 3 & 18 & 6.86 & 98.69 & BIS & & FALSE \\
\hline 2007 & F & 3 & 19 & 7.07 & 99.23 & BIS & & FALSE \\
\hline 2007 & $\mathrm{~F}$ & 3 & 20 & 7.35 & 99.79 & BIS & & FALSE \\
\hline 2007 & $\mathrm{~F}$ & 3 & 21 & 7.77 & 100.06 & BOT REB & Bot $\mathrm{X} 3 \mathrm{R}$ reb/low & FALSE \\
\hline 2007 & $\mathrm{~F}$ & 3 & 22 & 7.77 & 100.06 & TOP REB & Top X3R reb/low & FALSE \\
\hline 2007 & $\mathrm{~F}$ & 4 & 1 & 0.00 & 100.00 & TOP REB & Top X4L reb/low & FALSE \\
\hline 2007 & $\mathrm{~F}$ & 4 & 2 & 0.00 & 99.90 & BOT REB & Bot $\mathrm{X} 4 \mathrm{~L} \mathrm{reb} /$ low & FALSE \\
\hline 2007 & $\mathrm{~F}$ & 4 & 3 & 0.73 & 99.38 & BIS & & FALSE \\
\hline 2007 & $\mathrm{~F}$ & 4 & 4 & 1.34 & 99.05 & BIS & & FALSE \\
\hline 2007 & $\mathrm{~F}$ & 4 & 5 & 1.68 & 98.82 & BIS & & FALSE \\
\hline 2007 & $\mathrm{~F}$ & 4 & 6 & 2.07 & 98.21 & BIS & & FALSE \\
\hline 2007 & $\mathrm{~F}$ & 4 & 7 & 2.65 & 97.87 & $\mathrm{BF}$ & & FALSE \\
\hline 2007 & $\mathrm{~F}$ & 4 & 8 & 3.63 & 97.67 & WEL & & FALSE \\
\hline 2007 & $\mathrm{~F}$ & 4 & 9 & 4.18 & 97.61 & BIS & & FALSE \\
\hline 2007 & $\mathrm{~F}$ & 4 & 10 & 4.21 & 97.74 & BIS & ROCK & FALSE \\
\hline 2007 & $\mathrm{~F}$ & 4 & 11 & 4.33 & 97.64 & BIS & & FALSE \\
\hline 2007 & $\mathrm{~F}$ & 4 & 12 & 4.75 & 97.57 & BIS & & FALSE \\
\hline 2007 & $\mathrm{~F}$ & 4 & 13 & 4.97 & 97.82 & BIS & & FALSE \\
\hline 2007 & F & 4 & 14 & 5.15 & 97.55 & TW & & FALSE \\
\hline 2007 & F & 4 & 15 & 5.73 & 97.59 & WER & $\begin{array}{l}\text { TRUE WATERS EDGE UNDER } \\
\text { LOG }\end{array}$ & FALSE \\
\hline 2007 & $\mathrm{~F}$ & 4 & 16 & 5.91 & 97.87 & BIS & ON LOG & FALSE \\
\hline 2007 & $\mathrm{~F}$ & 4 & 17 & 6.10 & 97.70 & BIS & & FALSE \\
\hline 2007 & $\mathrm{~F}$ & 4 & 18 & 6.31 & 97.75 & BIS & & FALSE \\
\hline 2007 & $\mathrm{~F}$ & 4 & 19 & 6.61 & 98.33 & BIS & & FALSE \\
\hline 2007 & $\mathrm{~F}$ & 4 & 20 & 7.16 & 98.80 & BIS & & FALSE \\
\hline 2007 & $\mathrm{~F}$ & 4 & 21 & 7.62 & 99.48 & BIS & & FALSE \\
\hline 2007 & $\mathrm{~F}$ & 4 & 22 & 8.17 & 99.77 & BIS & & FALSE \\
\hline 2007 & $\mathrm{~F}$ & 4 & 23 & 8.78 & 99.95 & BOT REB & Bot $\mathrm{X} 4 \mathrm{R}$ reb/low & FALSE \\
\hline 2007 & $\mathrm{~F}$ & 4 & 24 & 8.78 & 100.14 & TOP REB & Top X4R reb/low & FALSE \\
\hline 2007 & $\mathrm{~F}$ & 5 & 1 & 0.00 & 100.00 & TOP REB & Top X5L reb/low & FALSE \\
\hline
\end{tabular}

Continued on Next Page... 
Table D.1 - Continued

\begin{tabular}{|c|c|c|c|c|c|c|c|c|}
\hline Year & Reach & Section & Point & Dist. (m) & Elev. $(\mathrm{m})$ & Desc. & Notes & Exclude \\
\hline 2007 & $\mathrm{~F}$ & 5 & 2 & 0.00 & 99.95 & BOT REB & Bot $\mathrm{X} 5 \mathrm{~L}$ reb/low & FALSE \\
\hline 2007 & $\mathrm{~F}$ & 5 & 3 & 0.67 & 99.33 & BIS & & FALSE \\
\hline 2007 & $\mathrm{~F}$ & 5 & 4 & 1.52 & 98.89 & BIS & & FALSE \\
\hline 2007 & $\mathrm{~F}$ & 5 & 5 & 2.23 & 98.68 & BIS & & FALSE \\
\hline 2007 & $\mathrm{~F}$ & 5 & 6 & 2.59 & 98.51 & $\mathrm{BF}$ & & FALSE \\
\hline 2007 & F & 5 & 7 & 3.23 & 98.48 & BIS & & FALSE \\
\hline 2007 & F & 5 & 8 & 3.51 & 98.39 & WEL & & FALSE \\
\hline 2007 & $\mathrm{~F}$ & 5 & 9 & 3.96 & 98.30 & TW & & FALSE \\
\hline 2007 & $\mathrm{~F}$ & 5 & 10 & 4.30 & 98.35 & BIS & & FALSE \\
\hline 2007 & $\mathrm{~F}$ & 5 & 11 & 4.63 & 98.37 & WER & & FALSE \\
\hline 2007 & F & 5 & 12 & 5.27 & 98.56 & BIS & & FALSE \\
\hline 2007 & $\mathrm{~F}$ & 5 & 13 & 5.94 & 99.13 & BIS & ON A ROCK & FALSE \\
\hline 2007 & $\mathrm{~F}$ & 5 & 14 & 6.37 & 98.78 & BIS & & FALSE \\
\hline 2007 & $\mathrm{~F}$ & 5 & 15 & 6.83 & 99.01 & BIS & & FALSE \\
\hline 2007 & F & 5 & 16 & 7.32 & 99.41 & BIS & & FALSE \\
\hline 2007 & $\mathrm{~F}$ & 5 & 17 & 7.62 & 99.83 & BOT REB & Bot $\mathrm{X} 5 \mathrm{R}$ reb/low & FALSE \\
\hline 2007 & F & 5 & 18 & 7.62 & 99.90 & TOP REB & Top X5R reb/low & FALSE \\
\hline 2007 & $\mathrm{~F}$ & 6 & 1 & 0.00 & 100.00 & TOP REB & Top X6L reb/low & FALSE \\
\hline 2007 & $\mathrm{~F}$ & 6 & 2 & 0.00 & 99.77 & BOT REB & Bot $\mathrm{X} 6 \mathrm{~L}$ reb/low & FALSE \\
\hline 2007 & F & 6 & 3 & 0.67 & 99.45 & BIS & & FALSE \\
\hline 2007 & $\mathrm{~F}$ & 6 & 4 & 1.10 & 99.19 & BIS & & FALSE \\
\hline 2007 & $\mathrm{~F}$ & 6 & 5 & 1.92 & 98.35 & BIS & $\begin{array}{l}\text { small bank failure during survey, } \\
\text { log shifted, dave ate shit }\end{array}$ & FALSE \\
\hline 2007 & $\mathrm{~F}$ & 6 & 6 & 2.26 & 98.63 & BIS & top of $\log$ that fell, WE under $\log$ & FALSE \\
\hline 2007 & F & 6 & 7 & 2.50 & 98.22 & BIS & & FALSE \\
\hline 2007 & $\mathrm{~F}$ & 6 & 8 & 2.90 & 98.24 & BIS & & FALSE \\
\hline 2007 & $\mathrm{~F}$ & 6 & 9 & 3.20 & 98.16 & $\mathrm{TW}$ & & FALSE \\
\hline 2007 & F & 6 & 10 & 3.54 & 98.24 & WE (BAR) & & FALSE \\
\hline 2007 & $\mathrm{~F}$ & 6 & 11 & 4.08 & 98.33 & BIS & & FALSE \\
\hline 2007 & $\mathrm{~F}$ & 6 & 12 & 4.45 & 98.26 & WE (BAR) & & FALSE \\
\hline 2007 & $\mathrm{~F}$ & 6 & 13 & 4.79 & 98.25 & WE UNDER & & FALSE \\
\hline & & & & & & ROCK & & \\
\hline 2007 & $\mathrm{~F}$ & 6 & 14 & 5.61 & 98.91 & BIS & ON ROCK ABOVE WE & FALSE \\
\hline 2007 & $\mathrm{~F}$ & 6 & 15 & 6.10 & 99.01 & BIS & & FALSE \\
\hline 2007 & $\mathrm{~F}$ & 6 & 16 & 6.98 & 99.28 & BIS & & FALSE \\
\hline 2007 & $\mathrm{~F}$ & 6 & 17 & 7.83 & 99.51 & BIS & & FALSE \\
\hline 2007 & $\mathrm{~F}$ & 6 & 18 & 8.41 & 99.86 & BOT REB & Bot $\mathrm{X} 6 \mathrm{R} \mathrm{reb} /$ low & FALSE \\
\hline 2007 & $\mathrm{~F}$ & 6 & 19 & 8.41 & 99.97 & TOP REB & Top X6R reb/low & FALSE \\
\hline 2007 & $\mathrm{~F}$ & 7 & 1 & 0.00 & 100.00 & TOP REB & Bot $\mathrm{X} 7 \mathrm{~L}$ reb/low & FALSE \\
\hline 2007 & $\mathrm{~F}$ & 7 & 2 & 0.00 & 100.00 & BOT REB & Top X7L reb/low - GROUND AT & FALSE \\
\hline & & & & & & & REBAR & \\
\hline 2007 & $\mathrm{~F}$ & 7 & 3 & 0.40 & 99.63 & BIS & & FALSE \\
\hline 2007 & $\mathrm{~F}$ & 7 & 4 & 0.67 & 99.18 & BIS & & FALSE \\
\hline 2007 & $\mathrm{~F}$ & 7 & 5 & 1.10 & 98.95 & BIS & & FALSE \\
\hline 2007 & $\mathrm{~F}$ & 7 & 6 & 1.13 & 98.78 & BIS & & FALSE \\
\hline 2007 & $\mathrm{~F}$ & 7 & 7 & 1.58 & 98.58 & $\mathrm{BF} ?$ & & FALSE \\
\hline 2007 & $\mathrm{~F}$ & 7 & 8 & 1.80 & 98.47 & WEL & & FALSE \\
\hline 2007 & $\mathrm{~F}$ & 7 & 9 & 1.95 & 98.44 & BIS & & FALSE \\
\hline 2007 & $\mathrm{~F}$ & 7 & 10 & 2.38 & 98.45 & BIS & MID CHANNEL BAR & FALSE \\
\hline 2007 & $\mathrm{~F}$ & 7 & 11 & 2.74 & 98.44 & BIS & MID CHANNEL BAR & FALSE \\
\hline 2007 & $\mathrm{~F}$ & 7 & 12 & 3.05 & 98.42 & TW & & FALSE \\
\hline 2007 & $\mathrm{~F}$ & 7 & 13 & 3.41 & 98.44 & BIS & & FALSE \\
\hline
\end{tabular}

Continued on Next Page... 
Table D.1 - Continued

\begin{tabular}{|c|c|c|c|c|c|c|c|c|}
\hline Year & Reach & Section & Point & Dist. (m) & Elev. $(\mathrm{m})$ & Desc. & Notes & Exclude \\
\hline 2007 & $\mathrm{~F}$ & 7 & 14 & 3.72 & 98.49 & WER & & FALSE \\
\hline 2007 & $\mathrm{~F}$ & 7 & 15 & 3.93 & 98.55 & $\mathrm{BF}$ & & FALSE \\
\hline 2007 & $\mathrm{~F}$ & 7 & 16 & 4.30 & 98.87 & BIS & TOP OF ROCK & FALSE \\
\hline 2007 & $\mathrm{~F}$ & 7 & 17 & 4.54 & 98.82 & BIS & & FALSE \\
\hline 2007 & $\mathrm{~F}$ & 7 & 18 & 5.09 & 98.88 & BIS & & FALSE \\
\hline 2007 & $\mathrm{~F}$ & 7 & 19 & 5.67 & 99.04 & BIS & & FALSE \\
\hline 2007 & $\mathrm{~F}$ & 7 & 20 & 6.49 & 99.22 & BIS & & FALSE \\
\hline 2007 & $\mathrm{~F}$ & 7 & 21 & 7.32 & 99.37 & BIS & & FALSE \\
\hline 2007 & $\mathrm{~F}$ & 7 & 22 & 7.92 & 99.55 & BIS & & FALSE \\
\hline 2007 & $\mathrm{~F}$ & 7 & 23 & 8.69 & 99.85 & BOT X7R & Bot $\mathrm{X} 7 \mathrm{R}$ reb/low & FALSE \\
\hline 2007 & $\mathrm{~F}$ & 7 & 24 & 8.69 & 99.95 & TOP X7R & Top X7R reb/low & FALSE \\
\hline 2007 & F & 8 & 1 & 0.00 & 100.00 & TOP REB & Bot $\mathrm{X} 8 \mathrm{~L}$ reb/low & FALSE \\
\hline 2007 & $\mathrm{~F}$ & 8 & 2 & 0.00 & 99.86 & BOT REB & Top X8L reb/low & FALSE \\
\hline 2007 & $\mathrm{~F}$ & 8 & 3 & 0.34 & 99.53 & BIS & & FALSE \\
\hline 2007 & $\mathrm{~F}$ & 8 & 4 & 0.82 & 99.50 & BIS & & FALSE \\
\hline 2007 & $\mathrm{~F}$ & 8 & 5 & 1.13 & 98.63 & BIS & & FALSE \\
\hline 2007 & $\mathrm{~F}$ & 8 & 6 & 1.71 & 98.36 & BIS & & FALSE \\
\hline 2007 & $\mathrm{~F}$ & 8 & 7 & 2.19 & 98.27 & BIS & & FALSE \\
\hline 2007 & $\mathrm{~F}$ & 8 & 8 & 2.50 & 97.93 & $\mathrm{BF}$ & & FALSE \\
\hline 2007 & $\mathrm{~F}$ & 8 & 9 & 3.35 & 97.81 & WEL & & FALSE \\
\hline 2007 & $\mathrm{~F}$ & 8 & 10 & 3.44 & 97.78 & BIS & & FALSE \\
\hline 2007 & $\mathrm{~F}$ & 8 & 11 & 3.66 & 97.75 & $\mathrm{TW}$ & & FALSE \\
\hline 2007 & $\mathrm{~F}$ & 8 & 12 & 3.96 & 97.76 & BIS & & FALSE \\
\hline 2007 & $\mathrm{~F}$ & 8 & 13 & 4.33 & 97.84 & BIS & & FALSE \\
\hline 2007 & $\mathrm{~F}$ & 8 & 14 & 4.48 & 97.87 & BIS & & FALSE \\
\hline 2007 & F & 8 & 15 & 4.85 & 98.17 & BIS & & FALSE \\
\hline 2007 & F & 8 & 16 & 5.33 & 98.46 & TBR & & FALSE \\
\hline 2007 & $\mathrm{~F}$ & 8 & 17 & 6.10 & 98.59 & BIS & & FALSE \\
\hline 2007 & $\mathrm{~F}$ & 8 & 18 & 7.35 & 98.80 & BIS & & FALSE \\
\hline 2007 & $\mathrm{~F}$ & 8 & 19 & 7.99 & 98.71 & BIS & & FALSE \\
\hline 2007 & $\mathrm{~F}$ & 8 & 20 & 8.53 & 98.68 & BIS & & FALSE \\
\hline 2007 & $\mathrm{~F}$ & 8 & 21 & 8.96 & 98.80 & BIS & & FALSE \\
\hline 2007 & $\mathrm{~F}$ & 8 & 22 & 9.45 & 99.18 & BIS & & FALSE \\
\hline 2007 & $\mathrm{~F}$ & 8 & 23 & 9.91 & 99.40 & BIS & & FALSE \\
\hline 2007 & F & 8 & 24 & 10.79 & 99.66 & BIS & & FALSE \\
\hline 2007 & $\mathrm{~F}$ & 8 & 25 & 11.09 & 99.82 & BIS & & FALSE \\
\hline 2007 & $\mathrm{~F}$ & 8 & 26 & 11.98 & 99.83 & BIS & & FALSE \\
\hline 2007 & $\mathrm{~F}$ & 8 & 27 & 12.65 & 99.91 & BOT REB & Bot $\mathrm{X} 8 \mathrm{R}$ reb/low & FALSE \\
\hline 2007 & $\mathrm{~F}$ & 8 & 28 & 12.65 & 100.03 & TOP REB & Top X8R reb/low & FALSE \\
\hline 2007 & $\mathrm{~F}$ & 9 & 1 & 0.00 & 100.00 & TOP REB & Top X9L reb/low & FALSE \\
\hline 2007 & $\mathrm{~F}$ & 9 & 2 & 0.00 & 99.83 & BOT REB & Bot X9L reb/low & FALSE \\
\hline 2007 & $\mathrm{~F}$ & 9 & 3 & 0.24 & 99.67 & BIS & & FALSE \\
\hline 2007 & $\mathrm{~F}$ & 9 & 4 & 0.46 & 99.24 & BIS & & FALSE \\
\hline 2007 & $\mathrm{~F}$ & 9 & 5 & 0.91 & 99.07 & BIS & & FALSE \\
\hline 2007 & $\mathrm{~F}$ & 9 & 6 & 1.19 & 98.83 & BIS & fixed, 1 foot low & FALSE \\
\hline 2007 & $\mathrm{~F}$ & 9 & 7 & 1.80 & 98.67 & BIS & & FALSE \\
\hline 2007 & $\mathrm{~F}$ & 9 & 8 & 1.83 & 98.76 & BIS & on rock & FALSE \\
\hline 2007 & $\mathrm{~F}$ & 9 & 9 & 2.07 & 98.67 & BIS & on rock & FALSE \\
\hline 2007 & $\mathrm{~F}$ & 9 & 10 & 2.13 & 98.53 & $\mathrm{BF} ?$ & & FALSE \\
\hline 2007 & F & 9 & 11 & 2.35 & 98.48 & WE & & FALSE \\
\hline 2007 & $\mathrm{~F}$ & 9 & 12 & 2.59 & 98.43 & BIS & & FALSE \\
\hline 2007 & $\mathrm{~F}$ & 9 & 13 & 2.87 & 98.59 & BIS & on rock & FALSE \\
\hline
\end{tabular}

Continued on Next Page... 
Table D.1 - Continued

\begin{tabular}{|c|c|c|c|c|c|c|c|c|}
\hline Year & Reach & Section & Point & Dist. (m) & Elev. $(\mathrm{m})$ & Desc. & Notes & Exclude \\
\hline 2007 & $\mathrm{~F}$ & 9 & 14 & 2.99 & 98.47 & BIS & & FALSE \\
\hline 2007 & $\mathrm{~F}$ & 9 & 15 & 3.32 & 98.37 & TW & & FALSE \\
\hline 2007 & $\mathrm{~F}$ & 9 & 16 & 3.72 & 98.41 & WER & & FALSE \\
\hline 2007 & $\mathrm{~F}$ & 9 & 17 & 3.81 & 98.93 & BIS & root wad of fern & FALSE \\
\hline 2007 & $\mathrm{~F}$ & 9 & 18 & 4.27 & 99.05 & BIS & & FALSE \\
\hline 2007 & $\mathrm{~F}$ & 9 & 19 & 5.18 & 99.18 & BIS & & FALSE \\
\hline 2007 & $\mathrm{~F}$ & 9 & 20 & 6.16 & 99.22 & BIS & & FALSE \\
\hline 2007 & $\mathrm{~F}$ & 9 & 21 & 7.04 & 99.03 & BIS & & FALSE \\
\hline 2007 & $\mathrm{~F}$ & 9 & 22 & 7.62 & 99.12 & BIS & fixed, 1 foot low & FALSE \\
\hline 2007 & $\mathrm{~F}$ & 9 & 23 & 9.02 & 99.05 & BIS & & FALSE \\
\hline 2007 & $\mathrm{~F}$ & 9 & 24 & 9.75 & 99.05 & BIS & & FALSE \\
\hline 2007 & $\mathrm{~F}$ & 9 & 25 & 10.21 & 99.53 & BIS & & FALSE \\
\hline 2007 & $\mathrm{~F}$ & 9 & 26 & 10.70 & 99.64 & BIS & & FALSE \\
\hline 2007 & $\mathrm{~F}$ & 9 & 27 & 11.25 & 99.79 & BOT REB & Bot $\mathrm{X} 9 \mathrm{R}$ reb/low & FALSE \\
\hline 2007 & $\mathrm{~F}$ & 9 & 28 & 11.25 & 99.96 & TOP REB & Top X9R reb/low & FALSE \\
\hline 2007 & $\mathrm{~F}$ & 10 & 1 & 0.00 & 100.00 & TOP REB & Bot $\mathrm{X} 10 \mathrm{~L} \mathrm{reb} / \mathrm{low}$ & FALSE \\
\hline 2007 & $\mathrm{~F}$ & 10 & 2 & 0.00 & 99.83 & BOT REB & Top X10L reb/low & FALSE \\
\hline 2007 & $\mathrm{~F}$ & 10 & 3 & 0.21 & 99.63 & BIS & & FALSE \\
\hline 2007 & $\mathrm{~F}$ & 10 & 4 & 0.27 & 99.09 & BIS & & FALSE \\
\hline 2007 & $\mathrm{~F}$ & 10 & 5 & 0.76 & 98.81 & BIS & & FALSE \\
\hline 2007 & $\mathrm{~F}$ & 10 & 6 & 1.25 & 98.56 & BIS & & FALSE \\
\hline 2007 & $\mathrm{~F}$ & 10 & 7 & 1.49 & 98.45 & WEL & & FALSE \\
\hline 2007 & $\mathrm{~F}$ & 10 & 8 & 1.77 & 98.43 & BIS & & FALSE \\
\hline 2007 & $\mathrm{~F}$ & 10 & 9 & 1.95 & 98.39 & $\mathrm{TW}$ & & FALSE \\
\hline 2007 & $\mathrm{~F}$ & 10 & 10 & 2.65 & 98.38 & BIS & & FALSE \\
\hline 2007 & F & 10 & 11 & 2.83 & 98.46 & WER & & FALSE \\
\hline 2007 & F & 10 & 12 & 3.11 & 98.55 & $\mathrm{BF}$ & & FALSE \\
\hline 2007 & F & 10 & 13 & 3.23 & 98.63 & BIS & & FALSE \\
\hline 2007 & $\mathrm{~F}$ & 10 & 14 & 3.78 & 98.68 & BIS & & FALSE \\
\hline 2007 & $\mathrm{~F}$ & 10 & 15 & 4.15 & 98.94 & BIS & & FALSE \\
\hline 2007 & F & 10 & 16 & 4.60 & 99.24 & BIS & & FALSE \\
\hline 2007 & F & 10 & 17 & 5.46 & 99.27 & BIS & & FALSE \\
\hline 2007 & $\mathrm{~F}$ & 10 & 18 & 6.71 & 99.09 & BIS & & FALSE \\
\hline 2007 & F & 10 & 19 & 7.92 & 99.05 & BIS & & FALSE \\
\hline 2007 & F & 10 & 20 & 8.99 & 98.94 & BIS & & FALSE \\
\hline 2007 & $\mathrm{~F}$ & 10 & 21 & 9.30 & 99.45 & BIS & & FALSE \\
\hline 2007 & $\mathrm{~F}$ & 10 & 22 & 9.54 & 99.61 & BIS & & FALSE \\
\hline 2007 & $\mathrm{~F}$ & 10 & 23 & 9.75 & 99.66 & BOT REB & Bot $\mathrm{X} 10 \mathrm{R} \mathrm{reb} / \mathrm{low}$ & FALSE \\
\hline 2007 & $\mathrm{~F}$ & 10 & 24 & 9.75 & 99.80 & TOP REB & Top X10R reb/low & FALSE \\
\hline 2008 & $\mathrm{~A}$ & 1 & 1 & 0.00 & 100.00 & TOP X1L & & FALSE \\
\hline 2008 & A & 1 & 2 & 0.00 & 99.88 & BOT X1L & & FALSE \\
\hline 2008 & A & 1 & 3 & 0.55 & 99.68 & BIS & & FALSE \\
\hline 2008 & A & 1 & 4 & 0.88 & 99.64 & BIS & & FALSE \\
\hline 2008 & A & 1 & 5 & 1.19 & 99.61 & BIS & & FALSE \\
\hline 2008 & $\mathrm{~A}$ & 1 & 6 & 1.46 & 99.58 & BIS & & FALSE \\
\hline 2008 & A & 1 & 7 & 1.74 & 99.56 & BIS & & FALSE \\
\hline 2008 & A & 1 & 8 & 2.07 & 99.48 & BIS & & FALSE \\
\hline 2008 & $\mathrm{~A}$ & 1 & 9 & 2.47 & 99.40 & BIS & & FALSE \\
\hline 2008 & $\mathrm{~A}$ & 1 & 10 & 2.68 & 99.63 & BIS & On $\log$ & TRUE \\
\hline 2008 & $\mathrm{~A}$ & 1 & 11 & 2.90 & 99.29 & BIS & & FALSE \\
\hline 2008 & A & 1 & 12 & 3.11 & 99.27 & BIS & & FALSE \\
\hline 2008 & A & 1 & 13 & 3.26 & 99.28 & ТOB & & FALSE \\
\hline
\end{tabular}

Continued on Next Page... 
Table D.1 - Continued

\begin{tabular}{|c|c|c|c|c|c|c|c|c|}
\hline Year & Reach & Section & Point & Dist. (m) & Elev. $(\mathrm{m})$ & Desc. & Notes & Exclude \\
\hline 2008 & A & 1 & 14 & 3.57 & 98.98 & BIS & & FALSE \\
\hline 2008 & A & 1 & 15 & 3.66 & 98.95 & $\mathrm{BF}$ & & FALSE \\
\hline 2008 & A & 1 & 16 & 3.93 & 98.79 & BIS & & FALSE \\
\hline 2008 & A & 1 & 17 & 4.15 & 98.72 & BIS & & FALSE \\
\hline 2008 & $\mathrm{~A}$ & 1 & 18 & 4.42 & 98.71 & EW & & FALSE \\
\hline 2008 & A & 1 & 19 & 4.66 & 98.64 & BIS & & FALSE \\
\hline 2008 & $\mathrm{~A}$ & 1 & 20 & 4.88 & 98.60 & TW & & FALSE \\
\hline 2008 & $\mathrm{~A}$ & 1 & 21 & 5.27 & 98.61 & BIS & & FALSE \\
\hline 2008 & $\mathrm{~A}$ & 1 & 22 & 5.55 & 98.64 & BIS & & FALSE \\
\hline 2008 & $\mathrm{~A}$ & 1 & 23 & 5.67 & 98.67 & BIS & & FALSE \\
\hline 2008 & A & 1 & 24 & 5.85 & 98.71 & EW & & FALSE \\
\hline 2008 & A & 1 & 25 & 5.97 & 98.74 & BIS & & FALSE \\
\hline 2008 & $\mathrm{~A}$ & 1 & 26 & 6.13 & 98.79 & BIS & & FALSE \\
\hline 2008 & $\mathrm{~A}$ & 1 & 27 & 6.31 & 99.02 & ТОВ & & FALSE \\
\hline 2008 & $\mathrm{~A}$ & 1 & 28 & 6.55 & 99.09 & BIS & & FALSE \\
\hline 2008 & $\mathrm{~A}$ & 1 & 29 & 6.71 & 99.16 & BIS & & FALSE \\
\hline 2008 & $\mathrm{~A}$ & 1 & 30 & 6.95 & 99.25 & BIS & & FALSE \\
\hline 2008 & $\mathrm{~A}$ & 1 & 31 & 7.16 & 99.36 & BIS & & FALSE \\
\hline 2008 & $\mathrm{~A}$ & 1 & 32 & 7.44 & 99.43 & BIS & & FALSE \\
\hline 2008 & $\mathrm{~A}$ & 1 & 33 & 7.68 & 99.46 & BIS & & FALSE \\
\hline 2008 & $\mathrm{~A}$ & 1 & 34 & 7.92 & 99.47 & BIS & & FALSE \\
\hline 2008 & $\mathrm{~A}$ & 1 & 35 & 8.23 & 99.51 & BIS & & FALSE \\
\hline 2008 & $\mathrm{~A}$ & 1 & 36 & 8.60 & 99.59 & BIS & & FALSE \\
\hline 2008 & $\mathrm{~A}$ & 1 & 37 & 8.84 & 99.67 & BIS & & FALSE \\
\hline 2008 & A & 1 & 38 & 8.99 & 99.73 & BIS & & FALSE \\
\hline 2008 & $\mathrm{~A}$ & 1 & 39 & 9.11 & 99.81 & BOT X1R & & FALSE \\
\hline 2008 & $\mathrm{~A}$ & 1 & 40 & 9.11 & 100.00 & TOP X1R & & FALSE \\
\hline 2008 & $\mathrm{~A}$ & 2 & 1 & 0.00 & 100.00 & Top X2L & & FALSE \\
\hline 2008 & $\mathrm{~A}$ & 2 & 2 & 0.00 & 99.72 & Bot X2L & & FALSE \\
\hline 2008 & $\mathrm{~A}$ & 2 & 3 & 0.70 & 99.56 & BIS & & FALSE \\
\hline 2008 & $\mathrm{~A}$ & 2 & 4 & 1.40 & 99.43 & BIS & & FALSE \\
\hline 2008 & $\mathrm{~A}$ & 2 & 5 & 2.23 & 98.95 & BIS & & FALSE \\
\hline 2008 & A & 2 & 6 & 2.96 & 98.80 & BIS & & FALSE \\
\hline 2008 & A & 2 & 7 & 3.47 & 98.62 & BIS & & FALSE \\
\hline 2008 & $\mathrm{~A}$ & 2 & 8 & 3.96 & 98.42 & TBL & & FALSE \\
\hline 2008 & $\mathrm{~A}$ & 2 & 9 & 4.48 & 98.22 & $\mathrm{BF}$ & & FALSE \\
\hline 2008 & $\mathrm{~A}$ & 2 & 10 & 4.94 & 98.11 & EWL & & FALSE \\
\hline 2008 & A & 2 & 11 & 5.12 & 98.04 & BIS & & FALSE \\
\hline 2008 & A & 2 & 12 & 5.40 & 98.06 & BIS & & FALSE \\
\hline 2008 & $\mathrm{~A}$ & 2 & 13 & 5.70 & 98.06 & BIS & & FALSE \\
\hline 2008 & $\mathrm{~A}$ & 2 & 14 & 5.88 & 98.14 & BIS & top of rock & FALSE \\
\hline 2008 & $\mathrm{~A}$ & 2 & 15 & 6.07 & 98.03 & BIS & & FALSE \\
\hline 2008 & $\mathrm{~A}$ & 2 & 16 & 6.28 & 98.08 & BIS & & FALSE \\
\hline 2008 & A & 2 & 17 & 6.71 & 97.98 & $\mathrm{TW}$ & & FALSE \\
\hline 2008 & A & 2 & 18 & 6.86 & 98.02 & EWR & EWR continues under log & FALSE \\
\hline 2008 & A & 2 & 19 & 6.98 & 98.63 & BIS & on $\log$ - over water & TRUE \\
\hline 2008 & A & 2 & 20 & 7.56 & 98.60 & TBR & on $\log$ & TRUE \\
\hline 2008 & A & 2 & 21 & 8.23 & 98.75 & BIS & & FALSE \\
\hline 2008 & $\mathrm{~A}$ & 2 & 22 & 8.53 & 99.13 & BIS & & FALSE \\
\hline 2008 & $\mathrm{~A}$ & 2 & 23 & 9.24 & 98.96 & BIS & & FALSE \\
\hline 2008 & A & 2 & 24 & 10.00 & 99.46 & BIS & & FALSE \\
\hline 2008 & A & 2 & 25 & 10.36 & 99.58 & BIS & & FALSE \\
\hline
\end{tabular}

Continued on Next Page... 
Table D.1 - Continued

\begin{tabular}{|c|c|c|c|c|c|c|c|c|}
\hline Year & Reach & Section & Point & Dist. (m) & Elev. $(\mathrm{m})$ & Desc. & Notes & Exclude \\
\hline 2008 & $\mathrm{~A}$ & 2 & 26 & 10.76 & 99.70 & BOT X2R & & FALSE \\
\hline 2008 & A & 2 & 27 & 10.76 & 100.00 & TOP X2R & & FALSE \\
\hline 2008 & A & 3 & 1 & 0.00 & 100.00 & TOP X3L & & FALSE \\
\hline 2008 & A & 3 & 2 & 0.00 & 99.54 & BOT X3L & & FALSE \\
\hline 2008 & $\mathrm{~A}$ & 3 & 3 & 0.12 & 99.59 & BIS & & FALSE \\
\hline 2008 & $\mathrm{~A}$ & 3 & 4 & 0.61 & 99.83 & BIS & On downed alder & TRUE \\
\hline 2008 & $\mathrm{~A}$ & 3 & 5 & 0.98 & 98.85 & BIS & & FALSE \\
\hline 2008 & $\mathrm{~A}$ & 3 & 6 & 1.16 & 98.81 & BIS & & FALSE \\
\hline 2008 & $\mathrm{~A}$ & 3 & 7 & 1.37 & 98.77 & ТОВ & & FALSE \\
\hline 2008 & $\mathrm{~A}$ & 3 & 8 & 1.52 & 98.16 & BIS & & FALSE \\
\hline 2008 & $\mathrm{~A}$ & 3 & 9 & 1.92 & 98.23 & BIS & & FALSE \\
\hline 2008 & $\mathrm{~A}$ & 3 & 10 & 2.07 & 98.18 & BIS & & FALSE \\
\hline 2008 & A & 3 & 11 & 2.23 & 97.94 & BIS & & FALSE \\
\hline 2008 & $\mathrm{~A}$ & 3 & 12 & 2.44 & 97.82 & EW & & FALSE \\
\hline 2008 & A & 3 & 13 & 2.96 & 97.85 & BIS & & FALSE \\
\hline 2008 & A & 3 & 14 & 3.26 & 97.83 & BIS & & FALSE \\
\hline 2008 & $\mathrm{~A}$ & 3 & 15 & 3.63 & 97.76 & $\mathrm{TW}$ & & FALSE \\
\hline 2008 & A & 3 & 16 & 4.15 & 97.78 & BIS & & FALSE \\
\hline 2008 & $\mathrm{~A}$ & 3 & 17 & 4.42 & 97.81 & BIS & & FALSE \\
\hline 2008 & A & 3 & 18 & 4.63 & 97.84 & EW & & FALSE \\
\hline 2008 & $\mathrm{~A}$ & 3 & 19 & 5.03 & 98.03 & BIS & & FALSE \\
\hline 2008 & $\mathrm{~A}$ & 3 & 20 & 5.40 & 98.19 & BIS & & FALSE \\
\hline 2008 & A & 3 & 21 & 5.52 & 98.37 & ТОВ & & FALSE \\
\hline 2008 & $\mathrm{~A}$ & 3 & 22 & 6.00 & 98.49 & BIS & & FALSE \\
\hline 2008 & A & 3 & 23 & 6.58 & 98.54 & BIS & & FALSE \\
\hline 2008 & A & 3 & 24 & 6.98 & 98.58 & BIS & & FALSE \\
\hline 2008 & $\mathrm{~A}$ & 3 & 25 & 7.50 & 98.53 & BIS & & FALSE \\
\hline 2008 & A & 3 & 26 & 7.65 & 98.45 & BIS & & FALSE \\
\hline 2008 & $\mathrm{~A}$ & 3 & 27 & 7.96 & 98.56 & BIS & & FALSE \\
\hline 2008 & $\mathrm{~A}$ & 3 & 28 & 8.44 & 98.66 & BIS & & FALSE \\
\hline 2008 & A & 3 & 29 & 8.90 & 98.74 & BIS & & FALSE \\
\hline 2008 & A & 3 & 30 & 9.72 & 99.26 & BIS & & FALSE \\
\hline 2008 & A & 3 & 31 & 9.97 & 99.38 & BIS & & FALSE \\
\hline 2008 & A & 3 & 32 & 10.33 & 99.45 & BIS & & FALSE \\
\hline 2008 & A & 3 & 33 & 10.61 & 99.68 & BIS & & FALSE \\
\hline 2008 & $\mathrm{~A}$ & 3 & 34 & 10.79 & 99.68 & BOT X3R & & FALSE \\
\hline 2008 & $\mathrm{~A}$ & 3 & 35 & 10.79 & 100.08 & TOP X3R & & FALSE \\
\hline 2008 & A & 4 & 1 & 0.00 & 100.00 & TOP X4L & & FALSE \\
\hline 2008 & A & 4 & 2 & 0.00 & 99.70 & BOT X4L & & FALSE \\
\hline 2008 & $\mathrm{~A}$ & 4 & 3 & 0.58 & 99.66 & BIS & & FALSE \\
\hline 2008 & A & 4 & 4 & 1.07 & 99.57 & BIS & & FALSE \\
\hline 2008 & $\mathrm{~A}$ & 4 & 5 & 1.43 & 99.48 & BIS & & FALSE \\
\hline 2008 & $\mathrm{~A}$ & 4 & 6 & 1.77 & 99.40 & BIS & & FALSE \\
\hline 2008 & $\mathrm{~A}$ & 4 & 7 & 2.01 & 99.37 & BIS & & FALSE \\
\hline 2008 & A & 4 & 8 & 2.26 & 99.33 & BIS & & FALSE \\
\hline 2008 & A & 4 & 9 & 2.53 & 99.19 & тОВ & & FALSE \\
\hline 2008 & A & 4 & 10 & 2.74 & 98.87 & BIS & & FALSE \\
\hline 2008 & A & 4 & 11 & 2.90 & 98.70 & BIS & & FALSE \\
\hline 2008 & $\mathrm{~A}$ & 4 & 12 & 3.11 & 98.62 & BIS & & FALSE \\
\hline 2008 & $\mathrm{~A}$ & 4 & 13 & 3.41 & 98.59 & BIS & & FALSE \\
\hline 2008 & A & 4 & 14 & 3.75 & 98.58 & BIS & & FALSE \\
\hline 2008 & A & 4 & 15 & 4.05 & 98.53 & EW & & FALSE \\
\hline
\end{tabular}

Continued on Next Page... 
Table D.1 - Continued

\begin{tabular}{|c|c|c|c|c|c|c|c|c|}
\hline Year & Reach & Section & Point & Dist. (m) & Elev. $(\mathrm{m})$ & Desc. & Notes & Exclude \\
\hline 2008 & A & 4 & 16 & 4.42 & 98.45 & BIS & & FALSE \\
\hline 2008 & A & 4 & 17 & 4.91 & 98.41 & TW & & FALSE \\
\hline 2008 & A & 4 & 18 & 5.33 & 98.51 & BIS & & FALSE \\
\hline 2008 & A & 4 & 19 & 5.58 & 98.53 & EW & & FALSE \\
\hline 2008 & $\mathrm{~A}$ & 4 & 20 & 5.88 & 98.58 & BIS & & FALSE \\
\hline 2008 & A & 4 & 21 & 6.22 & 98.70 & BIS & & FALSE \\
\hline 2008 & $\mathrm{~A}$ & 4 & 22 & 6.28 & 98.81 & $\mathrm{BF}$ & & FALSE \\
\hline 2008 & $\mathrm{~A}$ & 4 & 23 & 6.77 & 98.98 & BIS & & FALSE \\
\hline 2008 & $\mathrm{~A}$ & 4 & 24 & 7.16 & 99.04 & BIS & & FALSE \\
\hline 2008 & $\mathrm{~A}$ & 4 & 25 & 7.47 & 99.09 & BIS & & FALSE \\
\hline 2008 & A & 4 & 26 & 7.89 & 99.23 & BIS & & FALSE \\
\hline 2008 & A & 4 & 27 & 8.23 & 99.41 & BIS & Rock & FALSE \\
\hline 2008 & $\mathrm{~A}$ & 4 & 28 & 8.53 & 99.42 & BIS & & FALSE \\
\hline 2008 & $\mathrm{~A}$ & 4 & 29 & 8.78 & 99.45 & BOT X4R & & FALSE \\
\hline 2008 & $\mathrm{~A}$ & 4 & 30 & 8.78 & 99.83 & TOP X4R & & FALSE \\
\hline 2008 & $\mathrm{~A}$ & 5 & 1 & 0.00 & 100.00 & TOP X5L & & FALSE \\
\hline 2008 & $\mathrm{~A}$ & 5 & 2 & 0.00 & 99.75 & BOT X5L & & FALSE \\
\hline 2008 & $\mathrm{~A}$ & 5 & 3 & 0.61 & 99.87 & BIS & & FALSE \\
\hline 2008 & $\mathrm{~A}$ & 5 & 4 & 0.91 & 99.81 & BIS & & FALSE \\
\hline 2008 & A & 5 & 5 & 1.58 & 99.73 & BIS & On $\log$ & FALSE \\
\hline 2008 & $\mathrm{~A}$ & 5 & 6 & 2.04 & 99.58 & ТОВ & On $\log$ & FALSE \\
\hline 2008 & $\mathrm{~A}$ & 5 & 7 & 2.32 & 99.16 & BIS & On $\log$ & FALSE \\
\hline 2008 & $\mathrm{~A}$ & 5 & 8 & 2.59 & 98.81 & BIS & & FALSE \\
\hline 2008 & $\mathrm{~A}$ & 5 & 9 & 2.96 & 98.74 & BIS & & FALSE \\
\hline 2008 & $\mathrm{~A}$ & 5 & 10 & 3.26 & 98.69 & EW & & FALSE \\
\hline 2008 & $\mathrm{~A}$ & 5 & 11 & 3.63 & 98.64 & BIS & & FALSE \\
\hline 2008 & $\mathrm{~A}$ & 5 & 12 & 4.11 & 98.57 & TW & & FALSE \\
\hline 2008 & $\mathrm{~A}$ & 5 & 13 & 4.54 & 98.59 & BIS & & FALSE \\
\hline 2008 & $\mathrm{~A}$ & 5 & 14 & 4.97 & 98.69 & EW & & FALSE \\
\hline 2008 & $\mathrm{~A}$ & 5 & 15 & 5.27 & 98.99 & BIS & On rock & FALSE \\
\hline 2008 & $\mathrm{~A}$ & 5 & 16 & 5.85 & 99.02 & $\mathrm{BF}$ & & FALSE \\
\hline 2008 & $\mathrm{~A}$ & 5 & 17 & 6.16 & 99.12 & BIS & & FALSE \\
\hline 2008 & A & 5 & 18 & 6.64 & 99.26 & BIS & & FALSE \\
\hline 2008 & $\mathrm{~A}$ & 5 & 19 & 7.19 & 99.58 & BIS & & FALSE \\
\hline 2008 & $\mathrm{~A}$ & 5 & 20 & 7.83 & 99.60 & BOT X5R & & FALSE \\
\hline 2008 & $\mathrm{~A}$ & 5 & 21 & 7.83 & 99.98 & TOP X5R & & FALSE \\
\hline 2008 & $\mathrm{~A}$ & 6 & 1 & 0.00 & 100.00 & TOP X6L & & FALSE \\
\hline 2008 & A & 6 & 2 & 0.00 & 99.79 & BOT X6L & & FALSE \\
\hline 2008 & A & 6 & 3 & 0.15 & 99.71 & ТОВ & & FALSE \\
\hline 2008 & $\mathrm{~A}$ & 6 & 4 & 1.04 & 98.59 & BIS & & FALSE \\
\hline 2008 & $\mathrm{~A}$ & 6 & 5 & 1.40 & 98.36 & $\mathrm{BF}$ & & FALSE \\
\hline 2008 & $\mathrm{~A}$ & 6 & 6 & 1.77 & 98.15 & BIS & & FALSE \\
\hline 2008 & A & 6 & 7 & 2.10 & 98.17 & BIS & & FALSE \\
\hline 2008 & A & 6 & 8 & 2.59 & 98.06 & EW & & FALSE \\
\hline 2008 & $\mathrm{~A}$ & 6 & 9 & 2.83 & 98.05 & BIS & & FALSE \\
\hline 2008 & A & 6 & 10 & 3.08 & 98.03 & TW & & FALSE \\
\hline 2008 & A & 6 & 11 & 3.51 & 98.05 & BIS & & FALSE \\
\hline 2008 & A & 6 & 12 & 4.02 & 98.12 & BIS & & FALSE \\
\hline 2008 & $\mathrm{~A}$ & 6 & 13 & 4.11 & 98.06 & EW & & FALSE \\
\hline 2008 & $\mathrm{~A}$ & 6 & 14 & 4.39 & 98.71 & ТОВ & & FALSE \\
\hline 2008 & A & 6 & 15 & 5.18 & 99.07 & BIS & & FALSE \\
\hline 2008 & A & 6 & 16 & 6.68 & 99.31 & BIS & & FALSE \\
\hline
\end{tabular}

Continued on Next Page... 
Table D.1 - Continued

\begin{tabular}{|c|c|c|c|c|c|c|c|c|}
\hline Year & Reach & Section & Point & Dist. (m) & Elev. $(\mathrm{m})$ & Desc. & Notes & Exclude \\
\hline 2008 & A & 6 & 17 & 7.89 & 99.36 & BIS & & FALSE \\
\hline 2008 & A & 6 & 18 & 9.14 & 99.34 & BIS & & FALSE \\
\hline 2008 & A & 6 & 19 & 10.36 & 99.38 & BIS & & FALSE \\
\hline 2008 & A & 6 & 20 & 11.52 & 99.34 & BIS & & FALSE \\
\hline 2008 & $\mathrm{~A}$ & 6 & 21 & 12.80 & 99.27 & BIS & & FALSE \\
\hline 2008 & A & 6 & 22 & 13.17 & 99.32 & BIS & & FALSE \\
\hline 2008 & $\mathrm{~A}$ & 6 & 23 & 14.33 & 99.28 & BIS & & FALSE \\
\hline 2008 & $\mathrm{~A}$ & 6 & 24 & 14.72 & 99.29 & BIS & & FALSE \\
\hline 2008 & $\mathrm{~A}$ & 6 & 25 & 15.03 & 99.56 & BIS & & FALSE \\
\hline 2008 & $\mathrm{~A}$ & 6 & 26 & 15.18 & 99.58 & BOT X6R & & FALSE \\
\hline 2008 & A & 6 & 27 & 15.18 & 99.97 & TOB X6R & & FALSE \\
\hline 2008 & A & 7 & 1 & 0.00 & 100.00 & TOP X7L & & FALSE \\
\hline 2008 & $\mathrm{~A}$ & 7 & 2 & 0.00 & 99.79 & BOT X7L & & FALSE \\
\hline 2008 & $\mathrm{~A}$ & 7 & 3 & 0.44 & 99.73 & BIS & & FALSE \\
\hline 2008 & $\mathrm{~A}$ & 7 & 4 & 0.58 & 99.64 & ТОВ & & FALSE \\
\hline 2008 & $\mathrm{~A}$ & 7 & 5 & 1.46 & 98.75 & BIS & & FALSE \\
\hline 2008 & $\mathrm{~A}$ & 7 & 6 & 1.77 & 98.66 & BIS & On rock & FALSE \\
\hline 2008 & A & 7 & 7 & 2.16 & 98.78 & BIS & On rock & FALSE \\
\hline 2008 & $\mathrm{~A}$ & 7 & 8 & 2.41 & 98.61 & BIS & On rock & FALSE \\
\hline 2008 & $\mathrm{~A}$ & 7 & 9 & 2.56 & 98.24 & BIS & On rock & FALSE \\
\hline 2008 & $\mathrm{~A}$ & 7 & 10 & 2.68 & 98.17 & EW & & FALSE \\
\hline 2008 & $\mathrm{~A}$ & 7 & 11 & 3.20 & 98.19 & BIS & On rock & FALSE \\
\hline 2008 & $\mathrm{~A}$ & 7 & 12 & 3.41 & 98.25 & BIS & On rock & FALSE \\
\hline 2008 & $\mathrm{~A}$ & 7 & 13 & 3.66 & 98.06 & BIS & On rock & FALSE \\
\hline 2008 & $\mathrm{~A}$ & 7 & 14 & 3.84 & 97.99 & TW & & FALSE \\
\hline 2008 & $\mathrm{~A}$ & 7 & 15 & 4.02 & 98.01 & BIS & & FALSE \\
\hline 2008 & $\mathrm{~A}$ & 7 & 16 & 4.21 & 98.03 & BIS & & FALSE \\
\hline 2008 & $\mathrm{~A}$ & 7 & 17 & 4.42 & 98.09 & EW & & FALSE \\
\hline 2008 & $\mathrm{~A}$ & 7 & 18 & 4.45 & 98.22 & BIS & & FALSE \\
\hline 2008 & $\mathrm{~A}$ & 7 & 19 & 4.66 & 98.39 & BIS & & FALSE \\
\hline 2008 & $\mathrm{~A}$ & 7 & 20 & 4.88 & 98.54 & $\mathrm{BF}$ & & FALSE \\
\hline 2008 & $\mathrm{~A}$ & 7 & 21 & 5.24 & 98.62 & BIS & & FALSE \\
\hline 2008 & A & 7 & 22 & 5.88 & 98.81 & BIS & & FALSE \\
\hline 2008 & A & 7 & 23 & 6.31 & 98.89 & BIS & & FALSE \\
\hline 2008 & $\mathrm{~A}$ & 7 & 24 & 6.80 & 98.97 & BIS & & FALSE \\
\hline 2008 & $\mathrm{~A}$ & 7 & 25 & 7.68 & 99.06 & BIS & & FALSE \\
\hline 2008 & $\mathrm{~A}$ & 7 & 26 & 8.02 & 99.16 & BIS & & FALSE \\
\hline 2008 & A & 7 & 27 & 8.38 & 99.25 & BIS & & FALSE \\
\hline 2008 & A & 7 & 28 & 8.63 & 99.35 & BIS & On rock & FALSE \\
\hline 2008 & $\mathrm{~A}$ & 7 & 29 & 8.90 & 99.44 & BIS & On rock & FALSE \\
\hline 2008 & $\mathrm{~A}$ & 7 & 30 & 9.45 & 99.46 & BIS & & FALSE \\
\hline 2008 & $\mathrm{~A}$ & 7 & 31 & 10.67 & 99.58 & BIS & & FALSE \\
\hline 2008 & $\mathrm{~A}$ & 7 & 32 & 12.01 & 99.51 & BIS & & FALSE \\
\hline 2008 & $\mathrm{~A}$ & 7 & 33 & 13.66 & 99.45 & BIS & & FALSE \\
\hline 2008 & $\mathrm{~A}$ & 7 & 34 & 14.75 & 99.34 & BIS & & FALSE \\
\hline 2008 & A & 7 & 35 & 16.61 & 99.37 & BIS & & FALSE \\
\hline 2008 & A & 7 & 36 & 17.25 & 99.38 & BIS & & FALSE \\
\hline 2008 & A & 7 & 37 & 17.98 & 99.54 & BIS & & FALSE \\
\hline 2008 & $\mathrm{~A}$ & 7 & 38 & 18.29 & 99.64 & BIS & & FALSE \\
\hline 2008 & $\mathrm{~A}$ & 7 & 39 & 18.71 & 99.96 & BOT X7R & & FALSE \\
\hline 2008 & A & 7 & 40 & 18.71 & 100.22 & TOP X7R & & FALSE \\
\hline 2008 & A & 8 & 1 & 0.00 & 100.00 & TOP X8L & & FALSE \\
\hline
\end{tabular}

Continued on Next Page... 
Table D.1 - Continued

\begin{tabular}{|c|c|c|c|c|c|c|c|c|}
\hline Year & Reach & Section & Point & Dist. (m) & Elev. $(\mathrm{m})$ & Desc. & Notes & Exclude \\
\hline 2008 & A & 8 & 2 & 0.00 & 99.65 & BOT X8L & & FALSE \\
\hline 2008 & A & 8 & 3 & 0.61 & 99.60 & BIS & & FALSE \\
\hline 2008 & A & 8 & 4 & 0.98 & 99.41 & ТОВ & & FALSE \\
\hline 2008 & A & 8 & 5 & 1.13 & 98.53 & BIS & & FALSE \\
\hline 2008 & $\mathrm{~A}$ & 8 & 6 & 1.77 & 98.39 & BIS & & FALSE \\
\hline 2008 & A & 8 & 7 & 2.23 & 98.24 & $\mathrm{BF}$ & & FALSE \\
\hline 2008 & A & 8 & 8 & 2.77 & 98.05 & BIS & & FALSE \\
\hline 2008 & $\mathrm{~A}$ & 8 & 9 & 3.17 & 97.91 & EW & & FALSE \\
\hline 2008 & $\mathrm{~A}$ & 8 & 10 & 3.63 & 97.84 & TW & & FALSE \\
\hline 2008 & $\mathrm{~A}$ & 8 & 11 & 4.24 & 97.85 & BIS & & FALSE \\
\hline 2008 & A & 8 & 12 & 4.54 & 97.92 & BIS & & FALSE \\
\hline 2008 & A & 8 & 13 & 5.18 & 97.90 & BIS & & FALSE \\
\hline 2008 & $\mathrm{~A}$ & 8 & 14 & 5.58 & 97.97 & EW & & FALSE \\
\hline 2008 & $\mathrm{~A}$ & 8 & 15 & 5.88 & 97.92 & BIS & & FALSE \\
\hline 2008 & $\mathrm{~A}$ & 8 & 16 & 6.25 & 98.39 & ТОВ & & FALSE \\
\hline 2008 & A & 8 & 17 & 6.80 & 98.51 & BIS & & FALSE \\
\hline 2008 & $\mathrm{~A}$ & 8 & 18 & 7.62 & 98.71 & BIS & Point may be 1' off. & FALSE \\
\hline 2008 & $\mathrm{~A}$ & 8 & 19 & 8.32 & 98.69 & BIS & & FALSE \\
\hline 2008 & $\mathrm{~A}$ & 8 & 20 & 9.08 & 98.77 & BIS & & FALSE \\
\hline 2008 & $\mathrm{~A}$ & 8 & 21 & 9.75 & 98.80 & BIS & & FALSE \\
\hline 2008 & $\mathrm{~A}$ & 8 & 22 & 10.36 & 98.87 & BIS & & FALSE \\
\hline 2008 & $\mathrm{~A}$ & 8 & 23 & 10.97 & 98.94 & BIS & & FALSE \\
\hline 2008 & $\mathrm{~A}$ & 8 & 24 & 11.86 & 99.13 & BIS & & FALSE \\
\hline 2008 & $\mathrm{~A}$ & 8 & 25 & 12.65 & 99.23 & BIS & & FALSE \\
\hline 2008 & $\mathrm{~A}$ & 8 & 26 & 13.11 & 99.25 & BIS & & FALSE \\
\hline 2008 & $\mathrm{~A}$ & 8 & 27 & 13.72 & 99.21 & BIS & & FALSE \\
\hline 2008 & $\mathrm{~A}$ & 8 & 28 & 14.60 & 99.18 & BIS & & FALSE \\
\hline 2008 & $\mathrm{~A}$ & 8 & 29 & 15.64 & 99.07 & BIS & & FALSE \\
\hline 2008 & $\mathrm{~A}$ & 8 & 30 & 16.40 & 98.99 & BIS & & FALSE \\
\hline 2008 & $\mathrm{~A}$ & 8 & 31 & 16.86 & 99.00 & BIS & & FALSE \\
\hline 2008 & A & 8 & 32 & 17.71 & 99.14 & BIS & & FALSE \\
\hline 2008 & $\mathrm{~A}$ & 8 & 33 & 18.47 & 99.14 & BIS & & FALSE \\
\hline 2008 & A & 8 & 34 & 19.63 & 99.65 & BOT X8R & & FALSE \\
\hline 2008 & $\mathrm{~A}$ & 8 & 35 & 19.63 & 100.00 & TOP X8R & & FALSE \\
\hline 2008 & $\mathrm{~A}$ & 9 & 1 & 0.00 & 100.00 & TOP X9L & & FALSE \\
\hline 2008 & $\mathrm{~A}$ & 9 & 2 & 0.00 & 99.67 & BOT X9L & & FALSE \\
\hline 2008 & $\mathrm{~A}$ & 9 & 3 & 0.49 & 99.70 & BIS & & FALSE \\
\hline 2008 & A & 9 & 4 & 0.79 & 99.64 & ТОВ & & FALSE \\
\hline 2008 & A & 9 & 5 & 0.88 & 98.70 & BIS & & FALSE \\
\hline 2008 & $\mathrm{~A}$ & 9 & 6 & 1.95 & 98.57 & BIS & & FALSE \\
\hline 2008 & A & 9 & 7 & 2.35 & 98.60 & BIS & & FALSE \\
\hline 2008 & $\mathrm{~A}$ & 9 & 8 & 2.71 & 98.50 & BIS & & FALSE \\
\hline 2008 & A & 9 & 9 & 3.14 & 98.52 & BIS & & FALSE \\
\hline 2008 & A & 9 & 10 & 3.51 & 98.45 & $\mathrm{BF}$ & & FALSE \\
\hline 2008 & A & 9 & 11 & 4.05 & 98.20 & BIS & & FALSE \\
\hline 2008 & A & 9 & 12 & 4.42 & 98.15 & EW & & FALSE \\
\hline 2008 & A & 9 & 13 & 5.18 & 97.99 & TW & & FALSE \\
\hline 2008 & A & 9 & 14 & 5.67 & 98.05 & BIS & & FALSE \\
\hline 2008 & $\mathrm{~A}$ & 9 & 15 & 6.43 & 98.09 & BIS & & FALSE \\
\hline 2008 & $\mathrm{~A}$ & 9 & 16 & 6.68 & 98.15 & EW & & FALSE \\
\hline 2008 & A & 9 & 17 & 6.95 & 98.20 & BIS & & FALSE \\
\hline 2008 & A & 9 & 18 & 7.38 & 98.40 & BIS & & FALSE \\
\hline
\end{tabular}

Continued on Next Page... 
Table D.1 - Continued

\begin{tabular}{|c|c|c|c|c|c|c|c|c|}
\hline Year & Reach & Section & Point & Dist. (m) & Elev. $(\mathrm{m})$ & Desc. & Notes & Exclude \\
\hline 2008 & $\mathrm{~A}$ & 9 & 19 & 8.02 & 98.54 & BIS & & FALSE \\
\hline 2008 & A & 9 & 20 & 8.47 & 98.78 & ТОВ & & FALSE \\
\hline 2008 & A & 9 & 21 & 9.66 & 98.88 & BIS & & FALSE \\
\hline 2008 & A & 9 & 22 & 10.67 & 99.03 & BIS & & FALSE \\
\hline 2008 & $\mathrm{~A}$ & 9 & 23 & 12.07 & 98.98 & BIS & & FALSE \\
\hline 2008 & A & 9 & 24 & 12.74 & 99.16 & BIS & & FALSE \\
\hline 2008 & A & 9 & 25 & 13.05 & 99.39 & BIS & & FALSE \\
\hline 2008 & $\mathrm{~A}$ & 9 & 26 & 13.90 & 99.43 & BIS & & FALSE \\
\hline 2008 & $\mathrm{~A}$ & 9 & 27 & 14.94 & 99.34 & BIS & & FALSE \\
\hline 2008 & $\mathrm{~A}$ & 9 & 28 & 16.28 & 99.31 & BIS & & FALSE \\
\hline 2008 & A & 9 & 29 & 17.16 & 99.21 & BIS & & FALSE \\
\hline 2008 & $\mathrm{~A}$ & 9 & 30 & 18.29 & 99.35 & BIS & & FALSE \\
\hline 2008 & $\mathrm{~A}$ & 9 & 31 & 19.51 & 99.40 & BIS & & FALSE \\
\hline 2008 & $\mathrm{~A}$ & 9 & 32 & 20.15 & 99.67 & BIS & & FALSE \\
\hline 2008 & $\mathrm{~A}$ & 9 & 33 & 20.67 & 99.98 & BOT X9R & Rebar bent in 0.3 ' & FALSE \\
\hline 2008 & $\mathrm{~A}$ & 9 & 34 & 20.67 & 100.24 & TOP X9R & & FALSE \\
\hline 2008 & $\mathrm{~A}$ & 10 & 1 & 0.00 & 100.00 & TOP X10L & & FALSE \\
\hline 2008 & $\mathrm{~A}$ & 10 & 2 & 0.00 & 99.59 & BOT X10L & & FALSE \\
\hline 2008 & $\mathrm{~A}$ & 10 & 3 & 0.49 & 99.57 & ТОВ & & FALSE \\
\hline 2008 & $\mathrm{~A}$ & 10 & 4 & 0.61 & 98.67 & BIS & undercut bank $0.4^{\prime}$ & FALSE \\
\hline 2008 & $\mathrm{~A}$ & 10 & 5 & 2.10 & 98.52 & BIS & & FALSE \\
\hline 2008 & $\mathrm{~A}$ & 10 & 6 & 2.32 & 98.55 & BIS & & FALSE \\
\hline 2008 & $\mathrm{~A}$ & 10 & 7 & 2.59 & 98.45 & BIS & & FALSE \\
\hline 2008 & $\mathrm{~A}$ & 10 & 8 & 2.96 & 98.35 & BIS & & FALSE \\
\hline 2008 & $\mathrm{~A}$ & 10 & 9 & 3.51 & 98.34 & BIS & & FALSE \\
\hline 2008 & $\mathrm{~A}$ & 10 & 10 & 3.84 & 98.36 & BIS & & FALSE \\
\hline 2008 & $\mathrm{~A}$ & 10 & 11 & 3.93 & 98.34 & BIS & & FALSE \\
\hline 2008 & $\mathrm{~A}$ & 10 & 12 & 4.48 & 98.22 & BIS & & FALSE \\
\hline 2008 & $\mathrm{~A}$ & 10 & 13 & 4.63 & 98.29 & BIS & & FALSE \\
\hline 2008 & $\mathrm{~A}$ & 10 & 14 & 4.91 & 98.20 & BIS & & FALSE \\
\hline 2008 & $\mathrm{~A}$ & 10 & 15 & 5.24 & 98.21 & BIS & & FALSE \\
\hline 2008 & $\mathrm{~A}$ & 10 & 16 & 5.43 & 98.31 & BIS & & FALSE \\
\hline 2008 & A & 10 & 17 & 5.73 & 98.17 & $\mathrm{BF}$ & on rock & FALSE \\
\hline 2008 & A & 10 & 18 & 5.76 & 97.88 & EW & & FALSE \\
\hline 2008 & $\mathrm{~A}$ & 10 & 19 & 5.97 & 97.53 & BIS & & FALSE \\
\hline 2008 & $\mathrm{~A}$ & 10 & 20 & 6.16 & 97.57 & BIS & & FALSE \\
\hline 2008 & $\mathrm{~A}$ & 10 & 21 & 6.37 & 97.50 & BIS & & FALSE \\
\hline 2008 & A & 10 & 22 & 7.07 & 97.53 & BIS & & FALSE \\
\hline 2008 & A & 10 & 23 & 7.41 & 97.64 & BIS & & FALSE \\
\hline 2008 & $\mathrm{~A}$ & 10 & 24 & 7.71 & 98.10 & BIS & on rock & FALSE \\
\hline 2008 & $\mathrm{~A}$ & 10 & 25 & 8.44 & 97.64 & BIS & & FALSE \\
\hline 2008 & $\mathrm{~A}$ & 10 & 26 & 8.90 & 97.47 & $\mathrm{TW}$ & & FALSE \\
\hline 2008 & $\mathrm{~A}$ & 10 & 27 & 9.17 & 98.26 & BIS & on rock & FALSE \\
\hline 2008 & $\mathrm{~A}$ & 10 & 28 & 9.45 & 97.87 & EW & & FALSE \\
\hline 2008 & $\mathrm{~A}$ & 10 & 29 & 10.09 & 98.23 & ТОВ & & FALSE \\
\hline 2008 & A & 10 & 30 & 10.58 & 98.59 & BIS & & FALSE \\
\hline 2008 & A & 10 & 31 & 11.73 & 98.82 & BIS & & FALSE \\
\hline 2008 & A & 10 & 32 & 12.62 & 98.92 & BIS & & FALSE \\
\hline 2008 & $\mathrm{~A}$ & 10 & 33 & 14.02 & 98.94 & BIS & & FALSE \\
\hline 2008 & $\mathrm{~A}$ & 10 & 34 & 15.54 & 99.06 & BIS & & FALSE \\
\hline 2008 & A & 10 & 35 & 17.07 & 99.00 & BIS & & FALSE \\
\hline 2008 & A & 10 & 36 & 17.98 & 99.12 & BIS & & FALSE \\
\hline
\end{tabular}

Continued on Next Page... 
Table D.1 - Continued

\begin{tabular}{|c|c|c|c|c|c|c|c|c|}
\hline Year & Reach & Section & Point & Dist. (m) & Elev. $(\mathrm{m})$ & Desc. & Notes & Exclude \\
\hline 2008 & $\mathrm{~A}$ & 10 & 37 & 18.50 & 99.29 & BIS & & FALSE \\
\hline 2008 & A & 10 & 38 & 20.30 & 99.80 & BOT X10R & & FALSE \\
\hline 2008 & A & 10 & 39 & 20.30 & 100.00 & BOT X10R & & FALSE \\
\hline 2008 & B & 1 & 1 & 0.00 & 100.00 & TOP X1L & & FALSE \\
\hline 2008 & B & 1 & 2 & 0.00 & 99.86 & BOT X1L & & FALSE \\
\hline 2008 & B & 1 & 3 & 0.27 & 99.75 & BIS & & FALSE \\
\hline 2008 & B & 1 & 4 & 0.40 & 99.26 & BIS & & FALSE \\
\hline 2008 & B & 1 & 5 & 0.85 & 98.58 & BIS & & FALSE \\
\hline 2008 & $\mathrm{~B}$ & 1 & 6 & 1.19 & 98.33 & BIS & on trail & FALSE \\
\hline 2008 & B & 1 & 7 & 1.62 & 98.22 & ТОВ & & FALSE \\
\hline 2008 & B & 1 & 8 & 1.83 & 98.12 & BIS & & FALSE \\
\hline 2008 & B & 1 & 9 & 2.04 & 97.94 & BIS & & FALSE \\
\hline 2008 & B & 1 & 10 & 2.44 & 97.96 & BIS & & FALSE \\
\hline 2008 & B & 1 & 11 & 2.83 & 97.62 & EW & true EW under rock & FALSE \\
\hline 2008 & B & 1 & 12 & 3.14 & 97.56 & BIS & & FALSE \\
\hline 2008 & $\mathrm{~B}$ & 1 & 13 & 3.47 & 97.62 & BIS & & FALSE \\
\hline 2008 & B & 1 & 14 & 3.75 & 97.63 & BIS & & FALSE \\
\hline 2008 & B & 1 & 15 & 4.39 & 97.60 & BIS & & FALSE \\
\hline 2008 & B & 1 & 16 & 5.49 & 97.54 & BIS & & FALSE \\
\hline 2008 & B & 1 & 17 & 5.76 & 97.52 & TW & & FALSE \\
\hline 2008 & B & 1 & 18 & 5.94 & 97.61 & EW & & FALSE \\
\hline 2008 & B & 1 & 19 & 6.19 & 97.63 & BIS & & FALSE \\
\hline 2008 & B & 1 & 20 & 6.58 & 97.70 & BIS & & FALSE \\
\hline 2008 & B & 1 & 21 & 6.95 & 97.81 & $\mathrm{BF}$ & & FALSE \\
\hline 2008 & B & 1 & 22 & 7.22 & 98.04 & BIS & & FALSE \\
\hline 2008 & B & 1 & 23 & 7.96 & 98.51 & BIS & & FALSE \\
\hline 2008 & B & 1 & 24 & 8.44 & 98.72 & BIS & & FALSE \\
\hline 2008 & B & 1 & 25 & 8.90 & 99.05 & BIS & & FALSE \\
\hline 2008 & B & 1 & 26 & 9.14 & 99.24 & BIS & & FALSE \\
\hline 2008 & B & 1 & 27 & 9.45 & 99.65 & BIS & & FALSE \\
\hline 2008 & $\mathrm{~B}$ & 1 & 28 & 9.88 & 99.76 & BIS & & FALSE \\
\hline 2008 & B & 1 & 29 & 10.06 & 99.81 & BOT X1R & & FALSE \\
\hline 2008 & B & 1 & 30 & 10.06 & 99.99 & TOP X1R & & FALSE \\
\hline 2008 & B & 2 & 1 & 0.00 & 100.00 & TOP X2L & $\begin{array}{l}\text { right rebar bent inwards; rebent } \\
\text { into upward }\end{array}$ & FALSE \\
\hline 2008 & $\mathrm{~B}$ & 2 & 2 & 0.00 & 99.59 & BOT X2L & & FALSE \\
\hline 2008 & B & 2 & 3 & 0.18 & 99.51 & BIS & On horse trail & FALSE \\
\hline 2008 & B & 2 & 4 & 0.34 & 99.09 & BIS & On horse trail & FALSE \\
\hline 2008 & B & 2 & 5 & 0.61 & 98.98 & BIS & On horse trail & FALSE \\
\hline 2008 & B & 2 & 6 & 0.82 & 99.17 & BIS & On horse trail & FALSE \\
\hline 2008 & B & 2 & 7 & 1.55 & 98.62 & ТОВ & edge of horsetrail & FALSE \\
\hline 2008 & B & 2 & 8 & 1.95 & 98.28 & BIS & & FALSE \\
\hline 2008 & B & 2 & 9 & 2.16 & 98.16 & BIS & & FALSE \\
\hline 2008 & B & 2 & 10 & 2.41 & 98.01 & BIS & & FALSE \\
\hline 2008 & B & 2 & 11 & 2.74 & 97.85 & BIS & & FALSE \\
\hline 2008 & B & 2 & 12 & 3.08 & 97.51 & BIS & & FALSE \\
\hline 2008 & B & 2 & 13 & 3.35 & 97.46 & BIS & & FALSE \\
\hline 2008 & B & 2 & 14 & 3.84 & 97.43 & EW & & FALSE \\
\hline 2008 & $\mathrm{~B}$ & 2 & 15 & 4.48 & 97.39 & BIS & & FALSE \\
\hline 2008 & $\mathrm{~B}$ & 2 & 16 & 4.66 & 97.40 & BIS & & FALSE \\
\hline 2008 & $\mathrm{~B}$ & 2 & 17 & 5.03 & 97.35 & BIS & & FALSE \\
\hline 2008 & B & 2 & 18 & 5.24 & 97.32 & TW & & FALSE \\
\hline
\end{tabular}

Continued on Next Page... 
Table D.1 - Continued

\begin{tabular}{|c|c|c|c|c|c|c|c|c|}
\hline Year & Reach & Section & Point & Dist. (m) & Elev. $(\mathrm{m})$ & Desc. & Notes & Exclude \\
\hline 2008 & B & 2 & 19 & 5.52 & 97.33 & BIS & & FALSE \\
\hline 2008 & B & 2 & 20 & 5.79 & 97.35 & BIS & & FALSE \\
\hline 2008 & $\mathrm{~B}$ & 2 & 21 & 6.07 & 97.35 & BIS & & FALSE \\
\hline 2008 & B & 2 & 22 & 6.46 & 97.40 & EW & & FALSE \\
\hline 2008 & B & 2 & 23 & 6.74 & 97.45 & BIS & & FALSE \\
\hline 2008 & B & 2 & 24 & 7.04 & 97.47 & $\mathrm{BF}$ & & FALSE \\
\hline 2008 & B & 2 & 25 & 7.44 & 97.57 & BIS & & FALSE \\
\hline 2008 & B & 2 & 26 & 7.56 & 97.86 & BIS & on rock & TRUE \\
\hline 2008 & $\mathrm{~B}$ & 2 & 27 & 7.92 & 97.82 & BIS & on edge of rock & FALSE \\
\hline 2008 & B & 2 & 28 & 8.44 & 97.97 & BIS & & FALSE \\
\hline 2008 & B & 2 & 29 & 9.05 & 98.65 & ТОВ & & FALSE \\
\hline 2008 & $\mathrm{~B}$ & 2 & 30 & 9.30 & 98.68 & BIS & & FALSE \\
\hline 2008 & B & 2 & 31 & 9.75 & 99.11 & BIS & & FALSE \\
\hline 2008 & B & 2 & 32 & 10.18 & 99.31 & BIS & & FALSE \\
\hline 2008 & B & 2 & 33 & 10.36 & 99.52 & BIS & & FALSE \\
\hline 2008 & B & 2 & 34 & 10.61 & 99.54 & $\mathrm{BOT} \times 2 \mathrm{R}$ & & FALSE \\
\hline 2008 & B & 2 & 35 & 10.61 & 99.99 & TOP X2R & & FALSE \\
\hline 2008 & B & 3 & 1 & 0.00 & 100.00 & TOP X3L & & FALSE \\
\hline 2008 & B & 3 & 2 & 0.00 & 99.48 & BOT X3L & & FALSE \\
\hline 2008 & B & 3 & 3 & 0.55 & 99.23 & ТОВ & & FALSE \\
\hline 2008 & B & 3 & 4 & 1.13 & 98.84 & BIS & & FALSE \\
\hline 2008 & B & 3 & 5 & 1.52 & 98.53 & BIS & & FALSE \\
\hline 2008 & B & 3 & 6 & 1.86 & 98.44 & BIS & & FALSE \\
\hline 2008 & B & 3 & 7 & 2.41 & 98.23 & BIS & & FALSE \\
\hline 2008 & B & 3 & 8 & 2.87 & 98.28 & BIS & & FALSE \\
\hline 2008 & B & 3 & 9 & 3.54 & 98.26 & BIS & & FALSE \\
\hline 2008 & B & 3 & 10 & 3.84 & 98.18 & $\mathrm{BF}$ & & FALSE \\
\hline 2008 & B & 3 & 11 & 4.30 & 98.03 & EW & & FALSE \\
\hline 2008 & B & 3 & 12 & 4.45 & 97.97 & BIS & & FALSE \\
\hline 2008 & B & 3 & 13 & 4.94 & 97.96 & BIS & & FALSE \\
\hline 2008 & B & 3 & 14 & 5.18 & 97.95 & TW & & FALSE \\
\hline 2008 & B & 3 & 15 & 5.49 & 97.96 & BIS & & FALSE \\
\hline 2008 & B & 3 & 16 & 5.73 & 97.96 & EW & true EW under rock & FALSE \\
\hline 2008 & B & 3 & 17 & 6.13 & 98.12 & BIS & & FALSE \\
\hline 2008 & $\mathrm{~B}$ & 3 & 18 & 6.34 & 98.16 & BIS & & FALSE \\
\hline 2008 & $\mathrm{~B}$ & 3 & 19 & 6.64 & 98.42 & BIS & & FALSE \\
\hline 2008 & B & 3 & 20 & 7.19 & 98.70 & BIS & & FALSE \\
\hline 2008 & B & 3 & 21 & 7.68 & 98.96 & BIS & & FALSE \\
\hline 2008 & B & 3 & 22 & 8.20 & 99.27 & BIS & & FALSE \\
\hline 2008 & B & 3 & 23 & 8.47 & 99.72 & BOT X3R & & FALSE \\
\hline 2008 & B & 3 & 24 & 8.47 & 99.98 & TOP X3R & & FALSE \\
\hline 2008 & B & 4 & 1 & 0.00 & 100.00 & TOP X4L & & FALSE \\
\hline 2008 & B & 4 & 2 & 0.00 & 99.88 & BOT X4L & & FALSE \\
\hline 2008 & $\mathrm{~B}$ & 4 & 3 & 0.24 & 99.81 & BIS & & FALSE \\
\hline 2008 & B & 4 & 4 & 1.16 & 99.66 & BIS & & FALSE \\
\hline 2008 & B & 4 & 5 & 1.34 & 99.48 & TBL & & FALSE \\
\hline 2008 & B & 4 & 6 & 1.71 & 99.00 & BIS & & FALSE \\
\hline 2008 & B & 4 & 7 & 2.90 & 98.95 & BIS & & FALSE \\
\hline 2008 & B & 4 & 8 & 3.66 & 98.97 & BIS & & FALSE \\
\hline 2008 & B & 4 & 9 & 3.84 & 98.88 & $\mathrm{BF}$ & & FALSE \\
\hline 2008 & B & 4 & 10 & 4.21 & 98.71 & EWL & & FALSE \\
\hline 2008 & B & 4 & 11 & 4.85 & 98.68 & BIS & & FALSE \\
\hline
\end{tabular}

Continued on Next Page... 
Table D.1 - Continued

\begin{tabular}{cccccclcl}
\hline Year & Reach & Section & Point & Dist. $(\mathrm{m})$ & Elev. $(\mathrm{m})$ & Desc. & Notes & Exclude \\
\hline 2008 & B & 4 & 12 & 5.70 & 98.62 & TW & FALSE \\
2008 & B & 4 & 13 & 6.31 & 98.66 & BIS & FALSE \\
2008 & B & 4 & 14 & 6.64 & 98.70 & EWR & FALSE \\
2008 & B & 4 & 15 & 7.16 & 99.22 & BIS & FALSE \\
2008 & B & 4 & 16 & 7.35 & 99.50 & BOT X4R & & FALSE \\
2008 & B & 4 & 17 & 7.35 & 100.02 & TOP X4R & & FALSE \\
2008 & B & 5 & 1 & 0.00 & 100.00 & TOP X5L & RB paralled to ground. REBAR & FALSE \\
& & & & & & & 14" ABOVE GROUND AT END
\end{tabular}

GLE

\begin{tabular}{|c|c|c|c|c|c|c|c|c|}
\hline 2008 & B & 5 & 2 & 0.00 & 99.57 & BOT X5L & & FALSE \\
\hline 2008 & B & 5 & 3 & 0.15 & 99.35 & BIS & & FALSE \\
\hline 2008 & B & 5 & 4 & 0.40 & 99.19 & BIS & & FALSE \\
\hline 2008 & B & 5 & 5 & 0.91 & 98.91 & BIS & & FALSE \\
\hline 2008 & B & 5 & 6 & 1.25 & 98.75 & BIS & & FALSE \\
\hline 2008 & B & 5 & 7 & 1.71 & 98.44 & ТОВ & & FALSE \\
\hline 2008 & B & 5 & 8 & 2.07 & 98.37 & BIS & & FALSE \\
\hline 2008 & B & 5 & 9 & 2.29 & 98.31 & BIS & & FALSE \\
\hline 2008 & B & 5 & 10 & 2.59 & 98.16 & $\mathrm{BF}$ & & FALSE \\
\hline 2008 & B & 5 & 11 & 2.80 & 98.05 & BIS & ON ROCK & FALSE \\
\hline 2008 & B & 5 & 12 & 3.11 & 97.92 & EW & & FALSE \\
\hline 2008 & B & 5 & 13 & 3.54 & 97.79 & BIS & & FALSE \\
\hline 2008 & B & 5 & 14 & 3.96 & 97.79 & BIS & on rock & FALSE \\
\hline 2008 & B & 5 & 15 & 4.02 & 97.68 & TW & & FALSE \\
\hline 2008 & B & 5 & 16 & 4.51 & 97.76 & BIS & & FALSE \\
\hline 2008 & B & 5 & 17 & 4.72 & 97.83 & BIS & & FALSE \\
\hline 2008 & B & 5 & 18 & 5.03 & 97.82 & BIS & $\begin{array}{l}\text { cross section through small log } \\
\text { jam }\end{array}$ & FALSE \\
\hline 2008 & B & 5 & 19 & 5.24 & 98.42 & BIS & on $\log$ & TRUE \\
\hline 2008 & B & 5 & 20 & 5.64 & 98.43 & BIS & on $\log$ & TRUE \\
\hline 2008 & B & 5 & 21 & 6.00 & 98.06 & BIS & & FALSE \\
\hline 2008 & B & 5 & 22 & 6.34 & 97.98 & BIS & & FALSE \\
\hline 2008 & B & 5 & 23 & 6.59 & 97.99 & EW & & FALSE \\
\hline 2008 & B & 5 & 24 & 7.01 & 98.59 & BIS & & FALSE \\
\hline 2008 & B & 5 & 25 & 7.35 & 99.44 & BIS & & FALSE \\
\hline 2008 & B & 5 & 26 & 7.83 & 99.93 & BOT X5R & & FALSE \\
\hline 2008 & B & 5 & 27 & 7.83 & 100.07 & TOP X5R & & FALSE \\
\hline 2008 & B & 6 & 1 & 0.00 & 100.00 & TOP X6L & & FALSE \\
\hline 2008 & B & 6 & 2 & 0.00 & 99.95 & BOT X6L & & FALSE \\
\hline 2008 & B & 6 & 3 & 0.49 & 99.57 & BIS & & FALSE \\
\hline 2008 & B & 6 & 4 & 0.91 & 99.42 & BIS & & FALSE \\
\hline 2008 & B & 6 & 5 & 1.10 & 99.35 & ТОВ & & FALSE \\
\hline 2008 & B & 6 & 6 & 1.52 & 99.10 & BIS & & FALSE \\
\hline 2008 & B & 6 & 7 & 1.95 & 98.94 & BIS & & FALSE \\
\hline 2008 & B & 6 & 8 & 2.32 & 98.73 & BIS & & FALSE \\
\hline 2008 & B & 6 & 9 & 2.77 & 98.54 & BIS & & FALSE \\
\hline 2008 & B & 6 & 10 & 3.17 & 98.45 & BIS & on rock & FALSE \\
\hline 2008 & B & 6 & 11 & 3.51 & 98.28 & EW & & FALSE \\
\hline 2008 & B & 6 & 12 & 3.60 & 98.28 & TW & & FALSE \\
\hline 2008 & B & 6 & 13 & 3.66 & 98.35 & BIS & & FALSE \\
\hline 2008 & B & 6 & 14 & 3.84 & 98.30 & BIS & & FALSE \\
\hline 2008 & B & 6 & 15 & 4.51 & 98.38 & BIS & & FALSE \\
\hline
\end{tabular}

Continued on Next Page... 
Table D.1 - Continued

\begin{tabular}{|c|c|c|c|c|c|c|c|c|}
\hline Year & Reach & Section & Point & Dist. (m) & Elev. $(\mathrm{m})$ & Desc. & Notes & Exclude \\
\hline 2008 & B & 6 & 16 & 5.18 & 98.36 & EW & & FALSE \\
\hline 2008 & B & 6 & 17 & 5.46 & 98.63 & BIS & on rock & TRUE \\
\hline 2008 & B & 6 & 18 & 5.61 & 98.55 & $\mathrm{BF}$ & & FALSE \\
\hline 2008 & B & 6 & 19 & 6.00 & 98.58 & BIS & & FALSE \\
\hline 2008 & B & 6 & 20 & 6.40 & 98.73 & BIS & & FALSE \\
\hline 2008 & B & 6 & 21 & 6.55 & 98.73 & BIS & & FALSE \\
\hline 2008 & B & 6 & 22 & 6.98 & 98.98 & BIS & & FALSE \\
\hline 2008 & B & 6 & 23 & 7.38 & 99.32 & BIS & & FALSE \\
\hline 2008 & B & 6 & 24 & 7.86 & 99.85 & BOT X6R & & FALSE \\
\hline 2008 & B & 6 & 25 & 7.86 & 99.99 & TOP X6R & & FALSE \\
\hline 2008 & B & 7 & 1 & 0.00 & 100.00 & TOP X7L & top of rb bent in 6 " from base & FALSE \\
\hline 2008 & B & 7 & 2 & 0.00 & 99.42 & BOT X7L & & FALSE \\
\hline 2008 & B & 7 & 3 & 0.43 & 99.18 & BIS & & FALSE \\
\hline 2008 & B & 7 & 4 & 0.88 & 99.04 & ТОВ & & FALSE \\
\hline 2008 & B & 7 & 5 & 1.28 & 98.76 & BIS & & FALSE \\
\hline 2008 & B & 7 & 6 & 1.83 & 98.44 & BIS & & FALSE \\
\hline 2008 & B & 7 & 7 & 2.26 & 98.34 & BIS & & FALSE \\
\hline 2008 & B & 7 & 8 & 2.65 & 98.25 & BIS & & FALSE \\
\hline 2008 & B & 7 & 9 & 3.11 & 98.13 & $\mathrm{BF}$ & & FALSE \\
\hline 2008 & B & 7 & 10 & 3.57 & 97.99 & EW & & FALSE \\
\hline 2008 & B & 7 & 11 & 3.81 & 97.92 & BIS & & FALSE \\
\hline 2008 & B & 7 & 12 & 3.96 & 97.89 & TW & & FALSE \\
\hline 2008 & B & 7 & 13 & 4.30 & 97.94 & BIS & & FALSE \\
\hline 2008 & B & 7 & 14 & 4.51 & 97.94 & BIS & & FALSE \\
\hline 2008 & B & 7 & 15 & 4.85 & 97.90 & BIS & & FALSE \\
\hline 2008 & B & 7 & 16 & 5.64 & 97.97 & EW & & FALSE \\
\hline 2008 & B & 7 & 17 & 6.04 & 98.06 & BIS & & FALSE \\
\hline 2008 & B & 7 & 18 & 6.64 & 98.39 & BIS & & FALSE \\
\hline 2008 & B & 7 & 19 & 7.04 & 98.74 & ТОВ & & FALSE \\
\hline 2008 & B & 7 & 20 & 7.35 & 98.93 & BIS & & FALSE \\
\hline 2008 & B & 7 & 21 & 7.86 & 99.20 & BIS & & FALSE \\
\hline 2008 & B & 7 & 22 & 8.35 & 99.27 & BIS & & FALSE \\
\hline 2008 & B & 7 & 23 & 8.93 & 99.45 & BIS & & FALSE \\
\hline 2008 & B & 7 & 24 & 9.27 & 99.55 & BIS & Dist changed to 30.4 -dp & FALSE \\
\hline 2008 & $\mathrm{~B}$ & 7 & 25 & 9.72 & 99.75 & BOT X7R & & FALSE \\
\hline 2008 & B & 7 & 26 & 9.72 & 100.02 & TOP X7R & & FALSE \\
\hline 2008 & B & 8 & 1 & 0.00 & 100.00 & TOP X8L & tape place $4 "$ above rb base & FALSE \\
\hline 2008 & B & 8 & 2 & 0.00 & 99.55 & BOT X8L & & FALSE \\
\hline 2008 & $\mathrm{~B}$ & 8 & 3 & 0.21 & 99.42 & BIS & & FALSE \\
\hline 2008 & B & 8 & 4 & 0.34 & 99.37 & BIS & on $\log$ & FALSE \\
\hline 2008 & $\mathrm{~B}$ & 8 & 5 & 0.58 & 99.31 & ТОВ & on $\log$ & FALSE \\
\hline 2008 & $\mathrm{~B}$ & 8 & 6 & 0.70 & 98.89 & $\mathrm{BF}$ & $\begin{array}{l}\text { overhanging log-bf hard to deter- } \\
\text { mine }\end{array}$ & FALSE \\
\hline 2008 & $\mathrm{~B}$ & 8 & 7 & 1.19 & 98.86 & BIS & & FALSE \\
\hline 2008 & B & 8 & 8 & 1.40 & 98.85 & BIS & & FALSE \\
\hline 2008 & $\mathrm{~B}$ & 8 & 9 & 1.65 & 98.82 & BIS & & FALSE \\
\hline 2008 & $\mathrm{~B}$ & 8 & 10 & 1.89 & 98.79 & EW & & FALSE \\
\hline 2008 & B & 8 & 11 & 2.13 & 98.74 & BIS & overhanging log blocking & FALSE \\
\hline 2008 & B & 8 & 12 & 2.90 & 98.70 & $\mathrm{TW}$ & overhanging log blocking & FALSE \\
\hline 2008 & $\mathrm{~B}$ & 8 & 13 & 3.20 & 98.74 & EWR & $\begin{array}{l}\text { overhanging log-not real waters } \\
\text { edge }\end{array}$ & FALSE \\
\hline 2008 & $\mathrm{~B}$ & 8 & 14 & 3.41 & 99.68 & BIS & on $\log$ & FALSE \\
\hline
\end{tabular}

Continued on Next Page... 
Table D.1 - Continued

\begin{tabular}{|c|c|c|c|c|c|c|c|c|}
\hline Year & Reach & Section & Point & Dist. (m) & Elev. $(\mathrm{m})$ & Desc. & Notes & Exclude \\
\hline 2008 & $\mathrm{~B}$ & 8 & 15 & 3.75 & 99.57 & BIS & on $\log$ & FALSE \\
\hline 2008 & B & 8 & 16 & 4.15 & 99.75 & BIS & on $\log$ & FALSE \\
\hline 2008 & $\mathrm{~B}$ & 8 & 17 & 4.48 & 99.69 & BIS & on $\log$ & FALSE \\
\hline 2008 & $\mathrm{~B}$ & 8 & 18 & 4.82 & 99.50 & ТОВ & & FALSE \\
\hline 2008 & $\mathrm{~B}$ & 8 & 19 & 5.03 & 99.55 & BIS & & FALSE \\
\hline 2008 & B & 8 & 20 & 5.33 & 99.63 & BOT X8R & & FALSE \\
\hline 2008 & B & 8 & 21 & 5.33 & 99.82 & TOP X8R & & FALSE \\
\hline 2008 & B & 9 & 1 & 0.00 & 100.00 & TOP X9L & rebar sideways. fixed & FALSE \\
\hline 2008 & $\mathrm{~B}$ & 9 & 2 & 0.00 & 99.62 & BOT X9L & & FALSE \\
\hline 2008 & B & 9 & 3 & 0.30 & 99.48 & ТОВ & & FALSE \\
\hline 2008 & B & 9 & 4 & 0.79 & 97.46 & EW & & FALSE \\
\hline 2008 & B & 9 & 5 & 1.22 & 97.31 & BIS & & FALSE \\
\hline 2008 & B & 9 & 6 & 1.55 & 97.28 & BIS & & FALSE \\
\hline 2008 & B & 9 & 7 & 1.95 & 97.28 & BIS & & FALSE \\
\hline 2008 & B & 9 & 8 & 2.23 & 97.26 & TW & & FALSE \\
\hline 2008 & B & 9 & 9 & 2.59 & 97.27 & EW & & FALSE \\
\hline 2008 & B & 9 & 10 & 3.29 & 97.32 & BIS & & FALSE \\
\hline 2008 & B & 9 & 11 & 3.69 & 97.41 & BIS & & FALSE \\
\hline 2008 & B & 9 & 12 & 3.84 & 97.43 & $\mathrm{BF}$ & & FALSE \\
\hline 2008 & B & 9 & 13 & 4.05 & 97.56 & BIS & & FALSE \\
\hline 2008 & B & 9 & 14 & 4.27 & 97.73 & ТОВ & & FALSE \\
\hline 2008 & B & 9 & 15 & 4.60 & 97.85 & BIS & & FALSE \\
\hline 2008 & B & 9 & 16 & 5.12 & 98.07 & BIS & & FALSE \\
\hline 2008 & B & 9 & 17 & 5.58 & 98.10 & BIS & & FALSE \\
\hline 2008 & B & 9 & 18 & 6.10 & 98.20 & BIS & & FALSE \\
\hline 2008 & B & 9 & 19 & 6.98 & 98.31 & BIS & & FALSE \\
\hline 2008 & B & 9 & 20 & 7.53 & 98.50 & BIS & & FALSE \\
\hline 2008 & B & 9 & 21 & 8.23 & 98.62 & BIS & & FALSE \\
\hline 2008 & B & 9 & 22 & 9.14 & 98.56 & BIS & & FALSE \\
\hline 2008 & B & 9 & 23 & 10.03 & 98.49 & BIS & & FALSE \\
\hline 2008 & B & 9 & 24 & 10.85 & 98.59 & BIS & & FALSE \\
\hline 2008 & B & 9 & 25 & 11.55 & 98.83 & BIS & & FALSE \\
\hline 2008 & B & 9 & 26 & 12.28 & 99.24 & BIS & & FALSE \\
\hline 2008 & B & 9 & 27 & 12.92 & 99.45 & BIS & & FALSE \\
\hline 2008 & B & 9 & 28 & 13.90 & 99.93 & BOT X9R & & FALSE \\
\hline 2008 & $\mathrm{~B}$ & 9 & 29 & 13.90 & 99.97 & TOP X9R & & FALSE \\
\hline 2008 & B & 10 & 1 & 0.00 & 100.00 & TOP X10L & & FALSE \\
\hline 2008 & B & 10 & 2 & 0.00 & 99.68 & BOT X10L & & FALSE \\
\hline 2008 & B & 10 & 3 & 0.21 & 99.54 & TBL & & FALSE \\
\hline 2008 & B & 10 & 4 & 0.49 & 97.74 & BIS & & FALSE \\
\hline 2008 & B & 10 & 5 & 0.64 & 97.67 & BIS & & FALSE \\
\hline 2008 & B & 10 & 6 & 0.91 & 97.45 & BIS & & FALSE \\
\hline 2008 & B & 10 & 7 & 1.19 & 97.39 & BIS & & FALSE \\
\hline 2008 & B & 10 & 8 & 1.40 & 97.35 & EW & & FALSE \\
\hline 2008 & B & 10 & 9 & 1.68 & 97.38 & BIS & & FALSE \\
\hline 2008 & B & 10 & 10 & 1.86 & 97.36 & BIS & & FALSE \\
\hline 2008 & B & 10 & 11 & 2.19 & 97.35 & BIS & & FALSE \\
\hline 2008 & B & 10 & 12 & 2.41 & 97.32 & BIS & & FALSE \\
\hline 2008 & B & 10 & 13 & 2.68 & 97.36 & BIS & & FALSE \\
\hline 2008 & B & 10 & 14 & 2.93 & 97.35 & BIS & & FALSE \\
\hline 2008 & B & 10 & 15 & 3.26 & 97.31 & BIS & & FALSE \\
\hline 2008 & B & 10 & 16 & 3.72 & 97.27 & TW & & FALSE \\
\hline
\end{tabular}

Continued on Next Page... 
Table D.1 - Continued

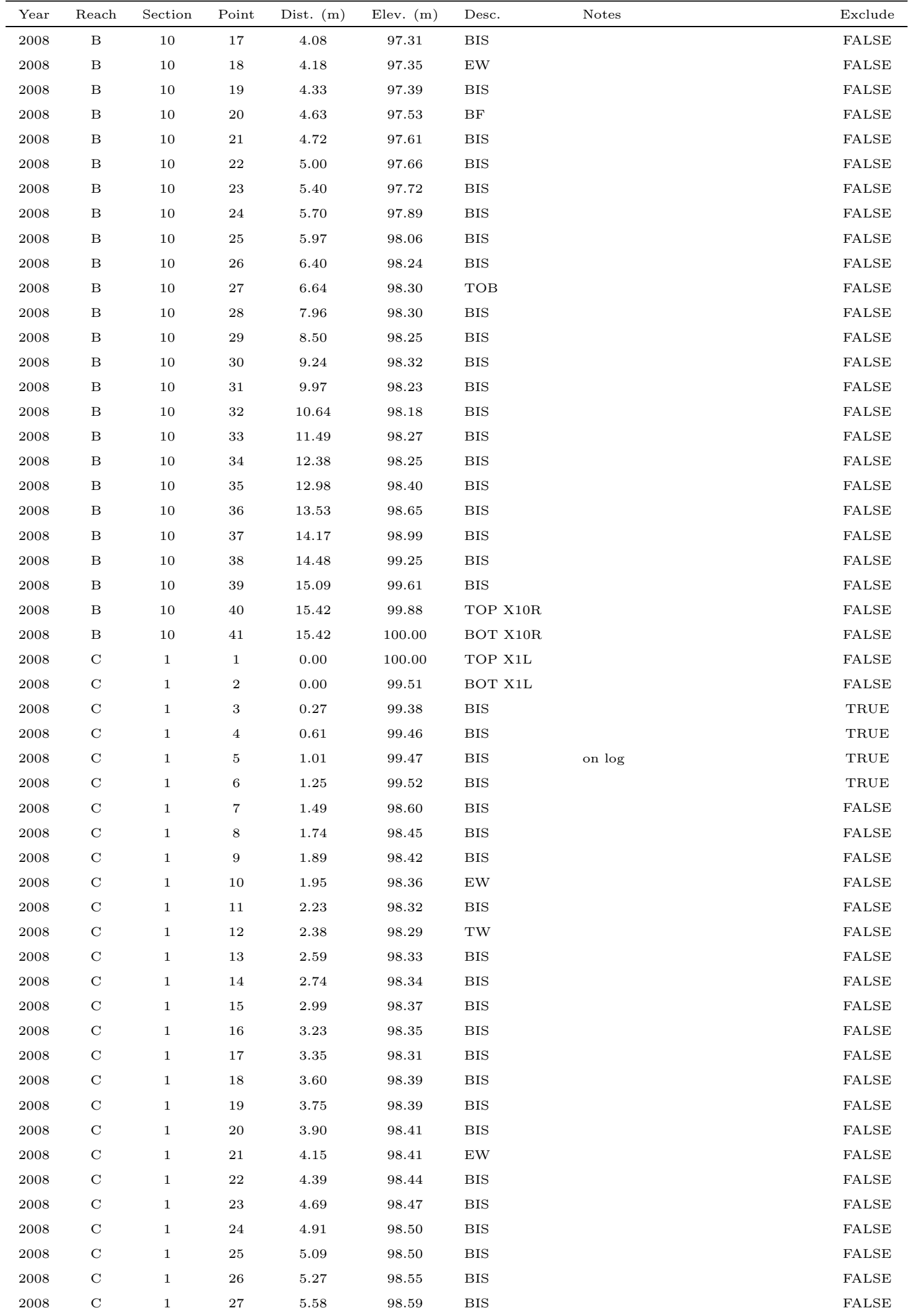

Continued on Next Page... 
APPENDIX D. SURVEY DATA

Table D.1 - Continued

\begin{tabular}{|c|c|c|c|c|c|c|c|c|}
\hline Year & Reach & Section & Point & Dist. (m) & Elev. $(\mathrm{m})$ & Desc. & Notes & Exclude \\
\hline 2008 & $\mathrm{C}$ & 1 & 28 & 5.82 & 98.62 & $\mathrm{BF}$ & & FALSE \\
\hline 2008 & $\mathrm{C}$ & 1 & 29 & 6.04 & 98.76 & BIS & on $\log$ & TRUE \\
\hline 2008 & $\mathrm{C}$ & 1 & 30 & 6.28 & 98.70 & BIS & & FALSE \\
\hline 2008 & $\mathrm{C}$ & 1 & 31 & 6.49 & 98.74 & BIS & & FALSE \\
\hline 2008 & $\mathrm{C}$ & 1 & 32 & 6.77 & 98.74 & BIS & & FALSE \\
\hline 2008 & $\mathrm{C}$ & 1 & 33 & 7.07 & 98.80 & BIS & & FALSE \\
\hline 2008 & $\mathrm{C}$ & 1 & 34 & 7.28 & 98.82 & BIS & & FALSE \\
\hline 2008 & $\mathrm{C}$ & 1 & 35 & 7.62 & 98.86 & BIS & & FALSE \\
\hline 2008 & $\mathrm{C}$ & 1 & 36 & 7.89 & 98.90 & BIS & & FALSE \\
\hline 2008 & $\mathrm{C}$ & 1 & 37 & 8.20 & 99.02 & BIS & & FALSE \\
\hline 2008 & $\mathrm{C}$ & 1 & 38 & 8.47 & 99.07 & BIS & & FALSE \\
\hline 2008 & $\mathrm{C}$ & 1 & 39 & 9.30 & 99.09 & BIS & & FALSE \\
\hline 2008 & $\mathrm{C}$ & 1 & 40 & 10.15 & 99.01 & BIS & & FALSE \\
\hline 2008 & $\mathrm{C}$ & 1 & 41 & 10.94 & 99.05 & BIS & & FALSE \\
\hline 2008 & $\mathrm{C}$ & 1 & 42 & 11.64 & 99.29 & BIS & & FALSE \\
\hline 2008 & $\mathrm{C}$ & 1 & 43 & 12.19 & 99.52 & BIS & & FALSE \\
\hline 2008 & $\mathrm{C}$ & 1 & 44 & 12.62 & 99.69 & BOT X1R & & FALSE \\
\hline 2008 & $\mathrm{C}$ & 1 & 45 & 12.62 & 100.08 & TOP X1R & & FALSE \\
\hline 2008 & $\mathrm{C}$ & 2 & 1 & 0.00 & 100.00 & TOP X2L & & FALSE \\
\hline 2008 & $\mathrm{C}$ & 2 & 2 & 0.00 & 99.61 & BOT X2L & & FALSE \\
\hline 2008 & $\mathrm{C}$ & 2 & 3 & 0.40 & 99.38 & BIS & & FALSE \\
\hline 2008 & $\mathrm{C}$ & 2 & 4 & 0.67 & 99.11 & BIS & & FALSE \\
\hline 2008 & $\mathrm{C}$ & 2 & 5 & 0.79 & 99.09 & BIS & & FALSE \\
\hline 2008 & $\mathrm{C}$ & 2 & 6 & 1.04 & 98.91 & BIS & & FALSE \\
\hline 2008 & $\mathrm{C}$ & 2 & 7 & 2.13 & 98.83 & BIS & & FALSE \\
\hline 2008 & $\mathrm{C}$ & 2 & 8 & 2.50 & 98.75 & BIS & & FALSE \\
\hline 2008 & $\mathrm{C}$ & 2 & 9 & 2.93 & 98.65 & BIS & & FALSE \\
\hline 2008 & $\mathrm{C}$ & 2 & 10 & 3.63 & 98.52 & BIS & & FALSE \\
\hline 2008 & $\mathrm{C}$ & 2 & 11 & 4.24 & 98.37 & BIS & & FALSE \\
\hline 2008 & $\mathrm{C}$ & 2 & 12 & 4.66 & 98.32 & BIS & & FALSE \\
\hline 2008 & $\mathrm{C}$ & 2 & 13 & 4.91 & 98.18 & TBL & & FALSE \\
\hline 2008 & $\mathrm{C}$ & 2 & 14 & 5.27 & 98.07 & BIS & & FALSE \\
\hline 2008 & $\mathrm{C}$ & 2 & 15 & 5.64 & 97.95 & $\mathrm{BF}$ & & FALSE \\
\hline 2008 & $\mathrm{C}$ & 2 & 16 & 5.94 & 97.85 & BIS & & FALSE \\
\hline 2008 & $\mathrm{C}$ & 2 & 17 & 6.10 & 97.74 & BIS & & FALSE \\
\hline 2008 & $\mathrm{C}$ & 2 & 18 & 6.28 & 97.70 & EW & & FALSE \\
\hline 2008 & $\mathrm{C}$ & 2 & 19 & 6.64 & 97.67 & BIS & & FALSE \\
\hline 2008 & $\mathrm{C}$ & 2 & 20 & 6.83 & 97.69 & BIS & & FALSE \\
\hline 2008 & $\mathrm{C}$ & 2 & 21 & 7.56 & 97.63 & BIS & & FALSE \\
\hline 2008 & $\mathrm{C}$ & 2 & 22 & 7.71 & 97.60 & TW & & FALSE \\
\hline 2008 & $\mathrm{C}$ & 2 & 23 & 7.92 & 97.67 & BIS & & FALSE \\
\hline 2008 & $\mathrm{C}$ & 2 & 24 & 8.35 & 97.68 & BIS & & FALSE \\
\hline 2008 & $\mathrm{C}$ & 2 & 25 & 8.53 & 97.62 & BIS & & FALSE \\
\hline 2008 & $\mathrm{C}$ & 2 & 26 & 8.84 & 97.62 & BIS & & FALSE \\
\hline 2008 & $\mathrm{C}$ & 2 & 27 & 9.02 & 97.65 & BIS & & FALSE \\
\hline 2008 & $\mathrm{C}$ & 2 & 28 & 9.08 & 97.65 & BIS & & FALSE \\
\hline 2008 & $\mathrm{C}$ & 2 & 29 & 9.39 & 97.68 & ER & & FALSE \\
\hline 2008 & $\mathrm{C}$ & 2 & 30 & 9.75 & 97.80 & BIS & & FALSE \\
\hline 2008 & $\mathrm{C}$ & 2 & 31 & 10.03 & 97.97 & BIS & & FALSE \\
\hline 2008 & $\mathrm{C}$ & 2 & 32 & 10.21 & 98.13 & TBR & & FALSE \\
\hline 2008 & $\mathrm{C}$ & 2 & 33 & 10.85 & 98.26 & BIS & & FALSE \\
\hline 2008 & C & 2 & 34 & 12.04 & 98.21 & BIS & & FALSE \\
\hline
\end{tabular}

Continued on Next Page... 
Table D.1 - Continued

\begin{tabular}{|c|c|c|c|c|c|c|c|c|}
\hline Year & Reach & Section & Point & Dist. (m) & Elev. $(\mathrm{m})$ & Desc. & Notes & Exclude \\
\hline 2008 & $\mathrm{C}$ & 2 & 35 & 12.28 & 98.24 & BIS & & FALSE \\
\hline 2008 & C & 2 & 36 & 12.41 & 98.33 & BIS & & FALSE \\
\hline 2008 & $\mathrm{C}$ & 2 & 37 & 12.47 & 99.57 & BIS & on rw stump & FALSE \\
\hline 2008 & $\mathrm{C}$ & 2 & 38 & 12.62 & 99.58 & BOT X2R & on rw stump & FALSE \\
\hline 2008 & $\mathrm{C}$ & 2 & 39 & 12.47 & 99.97 & TOP X2R & on rw stump-rebar bent inwards & FALSE \\
\hline 2008 & $\mathrm{C}$ & 3 & 1 & 0.00 & 100.00 & TOP X3L & & FALSE \\
\hline 2008 & $\mathrm{C}$ & 3 & 2 & 0.00 & 99.62 & BOT X3L & & FALSE \\
\hline 2008 & $\mathrm{C}$ & 3 & 3 & 0.55 & 99.41 & BIS & & FALSE \\
\hline 2008 & $\mathrm{C}$ & 3 & 4 & 0.82 & 99.32 & BIS & & FALSE \\
\hline 2008 & $\mathrm{C}$ & 3 & 5 & 1.22 & 99.12 & BIS & & FALSE \\
\hline 2008 & $\mathrm{C}$ & 3 & 6 & 1.77 & 98.92 & BIS & & FALSE \\
\hline 2008 & $\mathrm{C}$ & 3 & 7 & 2.23 & 98.77 & BIS & & FALSE \\
\hline 2008 & $\mathrm{C}$ & 3 & 8 & 2.53 & 98.71 & BIS & & FALSE \\
\hline 2008 & $\mathrm{C}$ & 3 & 9 & 3.02 & 98.72 & BIS & & FALSE \\
\hline 2008 & $\mathrm{C}$ & 3 & 10 & 3.54 & 98.81 & BIS & & FALSE \\
\hline 2008 & $\mathrm{C}$ & 3 & 11 & 4.18 & 98.72 & BIS & & FALSE \\
\hline 2008 & $\mathrm{C}$ & 3 & 12 & 4.66 & 98.66 & BIS & & FALSE \\
\hline 2008 & $\mathrm{C}$ & 3 & 13 & 5.18 & 98.45 & BIS & & FALSE \\
\hline 2008 & $\mathrm{C}$ & 3 & 14 & 5.73 & 98.45 & BIS & & FALSE \\
\hline 2008 & $\mathrm{C}$ & 3 & 15 & 6.46 & 98.43 & BIS & & FALSE \\
\hline 2008 & $\mathrm{C}$ & 3 & 16 & 6.71 & 98.38 & TBL & & FALSE \\
\hline 2008 & $\mathrm{C}$ & 3 & 17 & 6.98 & 98.27 & $\mathrm{BF}$ & & FALSE \\
\hline 2008 & $\mathrm{C}$ & 3 & 18 & 7.19 & 98.21 & BIS & & FALSE \\
\hline 2008 & $\mathrm{C}$ & 3 & 19 & 7.47 & 98.09 & EW & & FALSE \\
\hline 2008 & $\mathrm{C}$ & 3 & 20 & 7.92 & 98.09 & BIS & & FALSE \\
\hline 2008 & $\mathrm{C}$ & 3 & 21 & 8.26 & 98.08 & BIS & & FALSE \\
\hline 2008 & $\mathrm{C}$ & 3 & 22 & 8.47 & 98.03 & BIS & & FALSE \\
\hline 2008 & $\mathrm{C}$ & 3 & 23 & 8.75 & 98.03 & BIS & & FALSE \\
\hline 2008 & $\mathrm{C}$ & 3 & 24 & 9.14 & 97.96 & $\mathrm{TW}$ & & FALSE \\
\hline 2008 & $\mathrm{C}$ & 3 & 25 & 9.48 & 97.99 & BIS & & FALSE \\
\hline 2008 & $\mathrm{C}$ & 3 & 26 & 9.91 & 98.06 & EW & & FALSE \\
\hline 2008 & $\mathrm{C}$ & 3 & 27 & 10.15 & 98.12 & BIS & & FALSE \\
\hline 2008 & $\mathrm{C}$ & 3 & 28 & 10.36 & 98.21 & BIS & & FALSE \\
\hline 2008 & $\mathrm{C}$ & 3 & 29 & 10.55 & 98.53 & BIS & & FALSE \\
\hline 2008 & $\mathrm{C}$ & 3 & 30 & 10.82 & 98.58 & TBR & & FALSE \\
\hline 2008 & $\mathrm{C}$ & 3 & 31 & 11.13 & 98.82 & BIS & & FALSE \\
\hline 2008 & $\mathrm{C}$ & 3 & 32 & 11.89 & 98.94 & BIS & & FALSE \\
\hline 2008 & $\mathrm{C}$ & 3 & 33 & 12.50 & 99.09 & BIS & & FALSE \\
\hline 2008 & $\mathrm{C}$ & 3 & 34 & 13.32 & 99.40 & BIS & & FALSE \\
\hline 2008 & $\mathrm{C}$ & 3 & 35 & 13.69 & 99.56 & BIS & & FALSE \\
\hline 2008 & $\mathrm{C}$ & 3 & 36 & 14.14 & 99.77 & BOT X3R & & FALSE \\
\hline 2008 & $\mathrm{C}$ & 3 & 37 & 14.14 & 100.01 & TOPX3R & & FALSE \\
\hline 2008 & $\mathrm{C}$ & 4 & 1 & 0.00 & 100.00 & TOP X4L & & FALSE \\
\hline 2008 & $\mathrm{C}$ & 4 & 2 & 0.00 & 99.69 & BOT X4L & & FALSE \\
\hline 2008 & $\mathrm{C}$ & 4 & 3 & 0.30 & 99.59 & BID & & FALSE \\
\hline 2008 & $\mathrm{C}$ & 4 & 4 & 0.52 & 99.43 & BIS & & FALSE \\
\hline 2008 & $\mathrm{C}$ & 4 & 5 & 0.76 & 99.27 & BIS & & FALSE \\
\hline 2008 & $\mathrm{C}$ & 4 & 6 & 0.91 & 99.51 & BIS & on stump & TRUE \\
\hline 2008 & $\mathrm{C}$ & 4 & 7 & 1.22 & 99.21 & BIS & on stump & TRUE \\
\hline 2008 & $\mathrm{C}$ & 4 & 8 & 1.62 & 98.63 & BIS & & FALSE \\
\hline 2008 & $\mathrm{C}$ & 4 & 9 & 1.89 & 98.64 & BIS & & FALSE \\
\hline 2008 & C & 4 & 10 & 2.23 & 98.47 & BIS & & FALSE \\
\hline
\end{tabular}

Continued on Next Page... 
Table D.1 - Continued

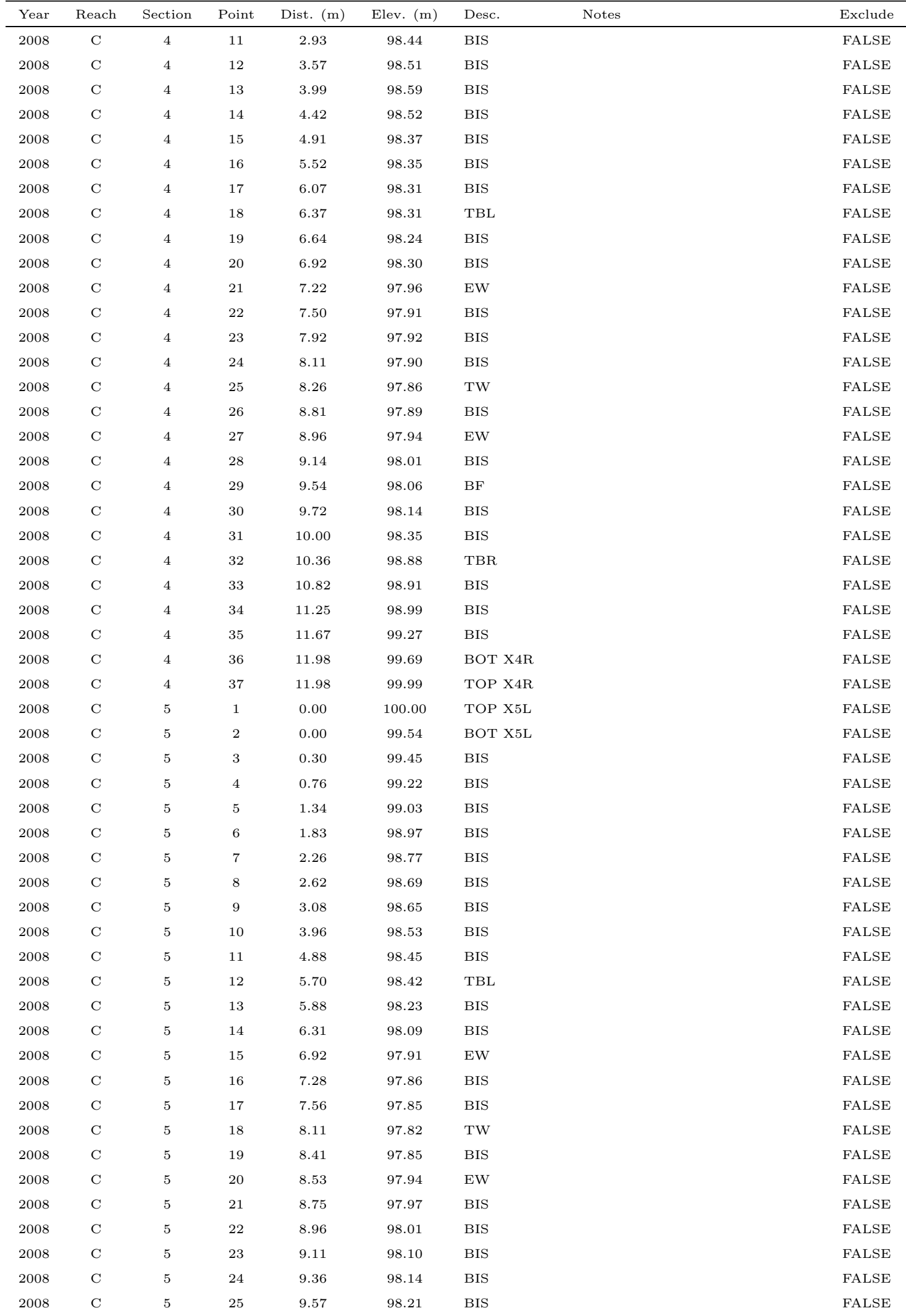

Continued on Next Page... 
Table D.1 - Continued

\begin{tabular}{|c|c|c|c|c|c|c|c|c|}
\hline Year & Reach & Section & Point & Dist. (m) & Elev. $(\mathrm{m})$ & Desc. & Notes & Exclude \\
\hline 2008 & $\mathrm{C}$ & 5 & 26 & 9.63 & 98.30 & BIS & & FALSE \\
\hline 2008 & C & 5 & 27 & 9.94 & 98.41 & BIS & & FALSE \\
\hline 2008 & $\mathrm{C}$ & 5 & 28 & 10.18 & 98.63 & BIS & & FALSE \\
\hline 2008 & $\mathrm{C}$ & 5 & 29 & 10.45 & 98.73 & TBR & & FALSE \\
\hline 2008 & $\mathrm{C}$ & 5 & 30 & 11.00 & 98.78 & BIS & & FALSE \\
\hline 2008 & $\mathrm{C}$ & 5 & 31 & 11.40 & 98.80 & BIS & & FALSE \\
\hline 2008 & $\mathrm{C}$ & 5 & 32 & 11.83 & 98.99 & BIS & & FALSE \\
\hline 2008 & $\mathrm{C}$ & 5 & 33 & 12.50 & 99.39 & BIS & & FALSE \\
\hline 2008 & $\mathrm{C}$ & 5 & 34 & 12.68 & 99.48 & BOT X5R & & FALSE \\
\hline 2008 & $\mathrm{C}$ & 5 & 35 & 12.59 & 99.99 & TOPX5R & rebar bent inward & FALSE \\
\hline 2008 & $\mathrm{C}$ & 6 & 1 & 0.00 & 100.00 & TOP X6L & & FALSE \\
\hline 2008 & $\mathrm{C}$ & 6 & 2 & 0.00 & 99.71 & BOT X6L & & FALSE \\
\hline 2008 & $\mathrm{C}$ & 6 & 3 & 0.24 & 99.48 & BIS & & FALSE \\
\hline 2008 & $\mathrm{C}$ & 6 & 4 & 0.46 & 99.24 & BIS & & FALSE \\
\hline 2008 & $\mathrm{C}$ & 6 & 5 & 0.76 & 99.13 & BIS & & FALSE \\
\hline 2008 & $\mathrm{C}$ & 6 & 6 & 1.19 & 98.91 & BIS & & FALSE \\
\hline \multirow[t]{2}{*}{2008} & $\mathrm{C}$ & 6 & 7 & 1.68 & 98.88 & BIS & TRUE TOP OF BANK L ABOVE & FALSE \\
\hline & & & & & & & REBAR & \\
\hline 2008 & $\mathrm{C}$ & 6 & 8 & 2.16 & 98.78 & BIS & & FALSE \\
\hline 2008 & $\mathrm{C}$ & 6 & 9 & 2.56 & 98.54 & BIS & & FALSE \\
\hline 2008 & $\mathrm{C}$ & 6 & 10 & 2.83 & 98.44 & BIS & & FALSE \\
\hline 2008 & $\mathrm{C}$ & 6 & 11 & 3.14 & 98.59 & BIS & ON LOG & TRUE \\
\hline 2008 & $\mathrm{C}$ & 6 & 12 & 3.35 & 98.20 & $\mathrm{BF}$ & & FALSE \\
\hline 2008 & $\mathrm{C}$ & 6 & 13 & 3.57 & 97.88 & BIS & & FALSE \\
\hline 2008 & $\mathrm{C}$ & 6 & 14 & 3.66 & 97.83 & EW & & FALSE \\
\hline 2008 & $\mathrm{C}$ & 6 & 15 & 3.93 & 97.87 & BIS & & FALSE \\
\hline 2008 & $\mathrm{C}$ & 6 & 16 & 4.42 & 97.90 & BIS & & FALSE \\
\hline 2008 & $\mathrm{C}$ & 6 & 17 & 5.03 & 97.93 & BIS & & FALSE \\
\hline 2008 & $\mathrm{C}$ & 6 & 18 & 5.55 & 97.91 & BIS & & FALSE \\
\hline 2008 & $\mathrm{C}$ & 6 & 19 & 6.07 & 97.91 & BIS & & FALSE \\
\hline 2008 & $\mathrm{C}$ & 6 & 20 & 6.55 & 97.84 & BIS & & FALSE \\
\hline 2008 & $\mathrm{C}$ & 6 & 21 & 6.98 & 97.85 & BIS & & FALSE \\
\hline 2008 & $\mathrm{C}$ & 6 & 22 & 7.44 & 97.81 & $\mathrm{TW}$ & & FALSE \\
\hline 2008 & $\mathrm{C}$ & 6 & 23 & 7.80 & 97.83 & BIS & & FALSE \\
\hline 2008 & $\mathrm{C}$ & 6 & 24 & 7.96 & 97.90 & EW & & FALSE \\
\hline 2008 & $\mathrm{C}$ & 6 & 25 & 8.26 & 97.98 & BIS & & FALSE \\
\hline 2008 & $\mathrm{C}$ & 6 & 26 & 8.50 & 98.04 & BIS & & FALSE \\
\hline 2008 & $\mathrm{C}$ & 6 & 27 & 8.81 & 98.10 & BIS & & FALSE \\
\hline 2008 & $\mathrm{C}$ & 6 & 28 & 9.42 & 98.62 & BIS & & FALSE \\
\hline 2008 & $\mathrm{C}$ & 6 & 29 & 10.09 & 98.76 & TBR & & FALSE \\
\hline 2008 & $\mathrm{C}$ & 6 & 30 & 10.49 & 98.92 & BIS & & FALSE \\
\hline 2008 & $\mathrm{C}$ & 6 & 31 & 10.91 & 99.22 & BIS & & FALSE \\
\hline 2008 & $\mathrm{C}$ & 6 & 32 & 11.25 & 99.35 & BIS & & FALSE \\
\hline 2008 & $\mathrm{C}$ & 6 & 33 & 11.70 & 99.10 & BIS & & FALSE \\
\hline 2008 & $\mathrm{C}$ & 6 & 34 & 12.25 & 99.19 & BIS & & FALSE \\
\hline 2008 & $\mathrm{C}$ & 6 & 35 & 12.65 & 99.38 & BIS & & FALSE \\
\hline 2008 & $\mathrm{C}$ & 6 & 36 & 13.02 & 99.76 & BOT X6R & & FALSE \\
\hline 2008 & $\mathrm{C}$ & 6 & 37 & 13.02 & 99.99 & TOP X6R & & FALSE \\
\hline 2008 & $\mathrm{C}$ & 7 & 1 & 0.00 & 100.00 & TOP X7L & & FALSE \\
\hline 2008 & $\mathrm{C}$ & 7 & 2 & 0.00 & 99.78 & BOTX7L & & FALSE \\
\hline 2008 & $\mathrm{C}$ & 7 & 3 & 0.21 & 99.43 & BIS & & FALSE \\
\hline 2008 & C & 7 & 4 & 0.58 & 99.13 & BIS & & FALSE \\
\hline
\end{tabular}

Continued on Next Page... 
Table D.1 - Continued

\begin{tabular}{|c|c|c|c|c|c|c|c|c|}
\hline Year & Reach & Section & Point & Dist. (m) & Elev. $(\mathrm{m})$ & Desc. & Notes & Exclude \\
\hline 2008 & $\mathrm{C}$ & 7 & 5 & 1.19 & 98.94 & BIS & & FALSE \\
\hline 2008 & C & 7 & 6 & 1.71 & 98.90 & BIS & & FALSE \\
\hline 2008 & $\mathrm{C}$ & 7 & 7 & 1.98 & 98.91 & BIS & & FALSE \\
\hline 2008 & $\mathrm{C}$ & 7 & 8 & 2.35 & 98.50 & BIS & EDGE OF SMALL BANK & FALSE \\
\hline 2008 & $\mathrm{C}$ & 7 & 9 & 2.80 & 98.32 & BIS & & FALSE \\
\hline 2008 & $\mathrm{C}$ & 7 & 10 & 3.02 & 97.93 & $\mathrm{BF}$ & & FALSE \\
\hline 2008 & $\mathrm{C}$ & 7 & 11 & 3.41 & 97.86 & EW & & FALSE \\
\hline 2008 & $\mathrm{C}$ & 7 & 12 & 3.66 & 98.03 & BIS & ON ROCK & FALSE \\
\hline 2008 & $\mathrm{C}$ & 7 & 13 & 3.90 & 97.72 & BIS & & FALSE \\
\hline 2008 & $\mathrm{C}$ & 7 & 14 & 4.27 & 97.70 & $\mathrm{TW}$ & & FALSE \\
\hline 2008 & $\mathrm{C}$ & 7 & 15 & 4.51 & 97.78 & BIS & & FALSE \\
\hline 2008 & $\mathrm{C}$ & 7 & 16 & 4.88 & 97.78 & EW & & FALSE \\
\hline 2008 & $\mathrm{C}$ & 7 & 17 & 5.18 & 97.86 & BIS & & FALSE \\
\hline 2008 & $\mathrm{C}$ & 7 & 18 & 5.43 & 97.93 & BIS & & FALSE \\
\hline 2008 & $\mathrm{C}$ & 7 & 19 & 5.64 & 97.85 & BIS & & FALSE \\
\hline 2008 & $\mathrm{C}$ & 7 & 20 & 6.10 & 98.22 & BIS & & FALSE \\
\hline 2008 & $\mathrm{C}$ & 7 & 21 & 6.52 & 98.26 & BIS & & FALSE \\
\hline 2008 & $\mathrm{C}$ & 7 & 22 & 7.01 & 98.39 & BIS & & FALSE \\
\hline 2008 & $\mathrm{C}$ & 7 & 23 & 7.38 & 98.66 & BIS & ON LOG & FALSE \\
\hline 2008 & $\mathrm{C}$ & 7 & 24 & 7.44 & 98.74 & BIS & & FALSE \\
\hline 2008 & $\mathrm{C}$ & 7 & 25 & 7.77 & 99.15 & TBR & & FALSE \\
\hline 2008 & $\mathrm{C}$ & 7 & 26 & 8.38 & 99.15 & BIS & & FALSE \\
\hline 2008 & $\mathrm{C}$ & 7 & 27 & 9.05 & 98.94 & BIS & & FALSE \\
\hline 2008 & $\mathrm{C}$ & 7 & 28 & 9.57 & 98.86 & BIS & & FALSE \\
\hline 2008 & $\mathrm{C}$ & 7 & 29 & 10.09 & 99.02 & BIS & ON LOG & TRUE \\
\hline 2008 & $\mathrm{C}$ & 7 & 30 & 10.49 & 99.26 & BIS & ON LOG & TRUE \\
\hline 2008 & $\mathrm{C}$ & 7 & 31 & 11.06 & 98.79 & BIS & & FALSE \\
\hline 2008 & $\mathrm{C}$ & 7 & 32 & 11.58 & 98.86 & BIS & & FALSE \\
\hline 2008 & $\mathrm{C}$ & 7 & 33 & 12.25 & 99.08 & BIS & & FALSE \\
\hline 2008 & $\mathrm{C}$ & 7 & 34 & 12.89 & 99.35 & BIS & & FALSE \\
\hline 2008 & $\mathrm{C}$ & 7 & 35 & 13.20 & 99.65 & BIS & & FALSE \\
\hline 2008 & $\mathrm{C}$ & 7 & 36 & 13.44 & 99.83 & BOT X7R & & FALSE \\
\hline 2008 & $\mathrm{C}$ & 7 & 37 & 13.44 & 100.03 & TOP X7R & & FALSE \\
\hline 2008 & $\mathrm{C}$ & 8 & 1 & 0.00 & 100.00 & TOP X8L & & FALSE \\
\hline 2008 & $\mathrm{C}$ & 8 & 2 & 0.00 & 99.56 & BOT X8L & & FALSE \\
\hline 2008 & $\mathrm{C}$ & 8 & 3 & 0.27 & 99.21 & BIS & & FALSE \\
\hline 2008 & $\mathrm{C}$ & 8 & 4 & 0.46 & 98.40 & BIS & & FALSE \\
\hline 2008 & $\mathrm{C}$ & 8 & 5 & 0.67 & 97.69 & EW & & FALSE \\
\hline 2008 & $\mathrm{C}$ & 8 & 6 & 1.04 & 97.64 & TW & & FALSE \\
\hline 2008 & $\mathrm{C}$ & 8 & 7 & 1.31 & 97.67 & BIS & & FALSE \\
\hline 2008 & $\mathrm{C}$ & 8 & 8 & 1.52 & 97.73 & BIS & & FALSE \\
\hline 2008 & $\mathrm{C}$ & 8 & 9 & 1.89 & 97.72 & BIS & & FALSE \\
\hline 2008 & $\mathrm{C}$ & 8 & 10 & 2.13 & 97.66 & BIS & & FALSE \\
\hline 2008 & $\mathrm{C}$ & 8 & 11 & 2.68 & 97.77 & EW & & FALSE \\
\hline 2008 & $\mathrm{C}$ & 8 & 12 & 2.93 & 97.79 & BIS & & FALSE \\
\hline 2008 & $\mathrm{C}$ & 8 & 13 & 3.35 & 97.84 & BIS & & FALSE \\
\hline 2008 & $\mathrm{C}$ & 8 & 14 & 3.81 & 97.94 & BIS & & FALSE \\
\hline 2008 & $\mathrm{C}$ & 8 & 15 & 4.15 & 98.16 & $\mathrm{BF}$ & & FALSE \\
\hline 2008 & $\mathrm{C}$ & 8 & 16 & 4.33 & 98.17 & BIS & ON LOG-UNDER CUT & FALSE \\
\hline 2008 & $\mathrm{C}$ & 8 & 17 & 4.57 & 98.20 & BIS & ON LOG & FALSE \\
\hline 2008 & $\mathrm{C}$ & 8 & 18 & 4.82 & 98.33 & BIS & & FALSE \\
\hline 2008 & C & 8 & 19 & 5.18 & 98.44 & BIS & & FALSE \\
\hline
\end{tabular}

Continued on Next Page... 
Table D.1 - Continued

\begin{tabular}{|c|c|c|c|c|c|c|c|c|}
\hline Year & Reach & Section & Point & Dist. (m) & Elev. $(\mathrm{m})$ & Desc. & Notes & Exclude \\
\hline 2008 & $\mathrm{C}$ & 8 & 20 & 5.46 & 98.55 & BIS & & FALSE \\
\hline 2008 & C & 8 & 21 & 5.73 & 98.67 & BIS & & FALSE \\
\hline 2008 & $\mathrm{C}$ & 8 & 22 & 5.94 & 98.95 & TBR & & FALSE \\
\hline 2008 & $\mathrm{C}$ & 8 & 23 & 6.25 & 99.04 & BIS & & FALSE \\
\hline 2008 & $\mathrm{C}$ & 8 & 24 & 6.74 & 99.03 & BIS & & FALSE \\
\hline 2008 & $\mathrm{C}$ & 8 & 25 & 7.53 & 98.98 & BIS & & FALSE \\
\hline 2008 & $\mathrm{C}$ & 8 & 26 & 8.20 & 98.91 & BIS & & FALSE \\
\hline 2008 & $\mathrm{C}$ & 8 & 27 & 9.17 & 98.84 & BIS & & FALSE \\
\hline 2008 & $\mathrm{C}$ & 8 & 28 & 10.00 & 98.74 & BIS & & FALSE \\
\hline 2008 & $\mathrm{C}$ & 8 & 29 & 11.03 & 98.63 & BIS & & FALSE \\
\hline 2008 & $\mathrm{C}$ & 8 & 30 & 11.92 & 98.59 & BIS & & FALSE \\
\hline 2008 & $\mathrm{C}$ & 8 & 31 & 12.89 & 98.84 & BIS & & FALSE \\
\hline 2008 & $\mathrm{C}$ & 8 & 32 & 13.62 & 99.28 & BIS & & FALSE \\
\hline 2008 & $\mathrm{C}$ & 8 & 33 & 13.93 & 99.47 & BIS & & FALSE \\
\hline 2008 & $\mathrm{C}$ & 8 & 34 & 14.26 & 99.63 & BOT X8R & & FALSE \\
\hline 2008 & $\mathrm{C}$ & 8 & 35 & 14.26 & 99.99 & TOP X8R & & FALSE \\
\hline 2008 & $\mathrm{C}$ & 9 & 1 & 0.00 & 100.00 & TOP X9L & $\begin{array}{l}\text { REBAR BENT INWARD AT AP- } \\
\text { PROX } 45 \text { DEG; } .7 \mathrm{FT}\end{array}$ & FALSE \\
\hline 2008 & $\mathrm{C}$ & 9 & 2 & 0.21 & 99.72 & BOTX9L & & FALSE \\
\hline 2008 & $\mathrm{C}$ & 9 & 3 & 0.21 & 99.44 & BIS & & FALSE \\
\hline 2008 & $\mathrm{C}$ & 9 & 4 & 0.24 & 98.67 & BIS & & FALSE \\
\hline 2008 & $\mathrm{C}$ & 9 & 5 & 0.61 & 98.32 & BIS & & FALSE \\
\hline 2008 & $\mathrm{C}$ & 9 & 6 & 1.19 & 98.02 & BIS & & FALSE \\
\hline 2008 & $\mathrm{C}$ & 9 & 7 & 1.95 & 97.88 & BIS & & FALSE \\
\hline 2008 & $\mathrm{C}$ & 9 & 8 & 2.47 & 97.81 & $\mathrm{BF}$ & & FALSE \\
\hline 2008 & $\mathrm{C}$ & 9 & 9 & 2.96 & 97.78 & BIS & ON ROCK & FALSE \\
\hline 2008 & $\mathrm{C}$ & 9 & 10 & 3.08 & 97.62 & EW & TRUE EW UNDER ROCK & FALSE \\
\hline 2008 & $\mathrm{C}$ & 9 & 11 & 3.32 & 97.48 & BIS & & FALSE \\
\hline 2008 & $\mathrm{C}$ & 9 & 12 & 3.81 & 97.52 & BIS & & FALSE \\
\hline 2008 & $\mathrm{C}$ & 9 & 13 & 4.18 & 97.46 & BIS & & FALSE \\
\hline 2008 & $\mathrm{C}$ & 9 & 14 & 4.91 & 97.33 & TW & & FALSE \\
\hline 2008 & $\mathrm{C}$ & 9 & 15 & 5.43 & 97.43 & EW & & FALSE \\
\hline 2008 & $\mathrm{C}$ & 9 & 16 & 5.64 & 97.49 & BIS & & FALSE \\
\hline 2008 & $\mathrm{C}$ & 9 & 17 & 5.76 & 97.68 & BIS & & FALSE \\
\hline 2008 & $\mathrm{C}$ & 9 & 18 & 5.91 & 97.82 & BIS & & FALSE \\
\hline 2008 & $\mathrm{C}$ & 9 & 19 & 6.10 & 98.17 & BIS & & FALSE \\
\hline 2008 & $\mathrm{C}$ & 9 & 20 & 6.68 & 98.57 & TBR & & FALSE \\
\hline 2008 & $\mathrm{C}$ & 9 & 21 & 7.22 & 98.71 & BIS & & FALSE \\
\hline 2008 & C & 9 & 22 & 8.23 & 98.78 & BIS & & FALSE \\
\hline 2008 & $\mathrm{C}$ & 9 & 23 & 9.30 & 98.73 & BIS & & FALSE \\
\hline 2008 & $\mathrm{C}$ & 9 & 24 & 10.36 & 98.69 & BIS & & FALSE \\
\hline 2008 & $\mathrm{C}$ & 9 & 25 & 11.28 & 98.60 & BIS & TBL ABOVE REBAR & FALSE \\
\hline 2008 & $\mathrm{C}$ & 9 & 26 & 11.89 & 98.61 & BIS & & FALSE \\
\hline 2008 & $\mathrm{C}$ & 9 & 27 & 12.95 & 98.52 & BIS & & FALSE \\
\hline 2008 & $\mathrm{C}$ & 9 & 28 & 13.78 & 98.57 & BIS & & FALSE \\
\hline 2008 & $\mathrm{C}$ & 9 & 29 & 14.87 & 98.75 & BIS & & FALSE \\
\hline 2008 & $\mathrm{C}$ & 9 & 30 & 15.24 & 98.96 & BIS & & FALSE \\
\hline 2008 & $\mathrm{C}$ & 9 & 31 & 15.58 & 99.09 & BIS & & FALSE \\
\hline 2008 & $\mathrm{C}$ & 9 & 32 & 16.00 & 99.47 & BOT X9R & REBAR HORIZONTAL & FALSE \\
\hline 2008 & $\mathrm{C}$ & 9 & 33 & 15.82 & 99.51 & TOP X9R & & FALSE \\
\hline 2008 & $\mathrm{C}$ & 10 & 1 & 0.00 & 100.00 & TOP X10L & & FALSE \\
\hline 2008 & $\mathrm{C}$ & 10 & 2 & 0.00 & 99.76 & BOT10L & & FALSE \\
\hline
\end{tabular}

Continued on Next Page... 
Table D.1 - Continued

\begin{tabular}{|c|c|c|c|c|c|c|c|c|}
\hline Year & Reach & Section & Point & Dist. (m) & Elev. $(\mathrm{m})$ & Desc. & Notes & Exclude \\
\hline 2008 & $\mathrm{C}$ & 10 & 3 & 0.12 & 99.74 & BIS & & FALSE \\
\hline 2008 & C & 10 & 4 & 0.49 & 99.42 & BIS & & FALSE \\
\hline 2008 & $\mathrm{C}$ & 10 & 5 & 0.88 & 99.19 & BIS & & FALSE \\
\hline 2008 & $\mathrm{C}$ & 10 & 6 & 1.52 & 98.90 & BIS & ON BOULDER & FALSE \\
\hline 2008 & $\mathrm{C}$ & 10 & 7 & 2.23 & 98.52 & BIS & & FALSE \\
\hline 2008 & $\mathrm{C}$ & 10 & 8 & 2.99 & 98.10 & BIS & & FALSE \\
\hline 2008 & $\mathrm{C}$ & 10 & 9 & 3.44 & 98.21 & BIS & & FALSE \\
\hline 2008 & $\mathrm{C}$ & 10 & 10 & 4.18 & 97.97 & BIS & & FALSE \\
\hline 2008 & $\mathrm{C}$ & 10 & 11 & 4.48 & 97.97 & BIS & & FALSE \\
\hline 2008 & $\mathrm{C}$ & 10 & 12 & 4.79 & 98.11 & BIS & & FALSE \\
\hline 2008 & $\mathrm{C}$ & 10 & 13 & 5.15 & 97.63 & BIS & & FALSE \\
\hline 2008 & $\mathrm{C}$ & 10 & 14 & 5.49 & 97.48 & EW & & FALSE \\
\hline 2008 & $\mathrm{C}$ & 10 & 15 & 6.10 & 97.43 & BIS & & FALSE \\
\hline 2008 & $\mathrm{C}$ & 10 & 16 & 6.52 & 97.41 & TW & & FALSE \\
\hline 2008 & $\mathrm{C}$ & 10 & 17 & 6.95 & 97.45 & BIS & & FALSE \\
\hline 2008 & $\mathrm{C}$ & 10 & 18 & 7.32 & 97.54 & BIS & & FALSE \\
\hline 2008 & $\mathrm{C}$ & 10 & 19 & 8.08 & 97.52 & EW & & FALSE \\
\hline 2008 & $\mathrm{C}$ & 10 & 20 & 8.53 & 97.80 & $\mathrm{BF}$ & & FALSE \\
\hline 2008 & $\mathrm{C}$ & 10 & 21 & 9.14 & 97.85 & BIS & & FALSE \\
\hline 2008 & $\mathrm{C}$ & 10 & 22 & 10.18 & 97.94 & BIS & & FALSE \\
\hline 2008 & $\mathrm{C}$ & 10 & 23 & 11.28 & 98.21 & BIS & & FALSE \\
\hline 2008 & $\mathrm{C}$ & 10 & 24 & 11.49 & 98.55 & BIS & & FALSE \\
\hline 2008 & $\mathrm{C}$ & 10 & 25 & 12.86 & 98.78 & BIS & & FALSE \\
\hline 2008 & $\mathrm{C}$ & 10 & 26 & 14.02 & 98.81 & BIS & & FALSE \\
\hline 2008 & $\mathrm{C}$ & 10 & 27 & 15.24 & 98.82 & BIS & & FALSE \\
\hline 2008 & $\mathrm{C}$ & 10 & 28 & 15.85 & 98.90 & BIS & & FALSE \\
\hline 2008 & $\mathrm{C}$ & 10 & 29 & 16.19 & 99.01 & BIS & & FALSE \\
\hline 2008 & $\mathrm{C}$ & 10 & 30 & 16.55 & 99.31 & BIS & & FALSE \\
\hline 2008 & $\mathrm{C}$ & 10 & 31 & 16.58 & 99.87 & BIS & & FALSE \\
\hline 2008 & $\mathrm{C}$ & 10 & 32 & 16.86 & 99.91 & BOT X10R & & FALSE \\
\hline 2008 & $\mathrm{C}$ & 10 & 33 & 16.86 & 100.00 & TOP X10R & & FALSE \\
\hline 2008 & $\mathrm{D}$ & 1 & 1 & 0.00 & 100.00 & TOP X1L & & FALSE \\
\hline 2008 & $\mathrm{D}$ & 1 & 2 & 0.00 & 99.81 & BOT X1L & & FALSE \\
\hline 2008 & $\mathrm{D}$ & 1 & 3 & 0.58 & 99.73 & TBL & & FALSE \\
\hline 2008 & $\mathrm{D}$ & 1 & 4 & 1.07 & 98.62 & BIS & & FALSE \\
\hline 2008 & $\mathrm{D}$ & 1 & 5 & 1.49 & 98.21 & BIS & & FALSE \\
\hline 2008 & $\mathrm{D}$ & 1 & 6 & 1.80 & 98.06 & $\mathrm{BFL}$ & & FALSE \\
\hline 2008 & $\mathrm{D}$ & 1 & 7 & 2.13 & 97.94 & BIS & & FALSE \\
\hline 2008 & $\mathrm{D}$ & 1 & 8 & 2.41 & 97.99 & BIS & & FALSE \\
\hline 2008 & $\mathrm{D}$ & 1 & 9 & 2.90 & 97.88 & EW & & FALSE \\
\hline 2008 & $\mathrm{D}$ & 1 & 10 & 3.32 & 97.78 & $\mathrm{TW}$ & TRUE TW UNDER ROCK & FALSE \\
\hline 2008 & $\mathrm{D}$ & 1 & 11 & 3.54 & 97.80 & BIS & ON ROCK & FALSE \\
\hline 2008 & $\mathrm{D}$ & 1 & 12 & 3.66 & 97.86 & EW & $\begin{array}{l}\text { ON ROCK; TRUE EW UNDER } \\
\text { ROCK }\end{array}$ & FALSE \\
\hline 2008 & $\mathrm{D}$ & 1 & 13 & 3.78 & 98.02 & BIS & ON ROCK & FALSE \\
\hline 2008 & $\mathrm{D}$ & 1 & 14 & 4.24 & 98.11 & BIS & ON ROCK & FALSE \\
\hline 2008 & $\mathrm{D}$ & 1 & 15 & 4.36 & 98.01 & BIS & & FALSE \\
\hline 2008 & $\mathrm{D}$ & 1 & 16 & 5.03 & 98.20 & BIS & & FALSE \\
\hline 2008 & $\mathrm{D}$ & 1 & 17 & 5.40 & 98.34 & BIS & & FALSE \\
\hline 2008 & $\mathrm{D}$ & 1 & 18 & 5.70 & 98.31 & BIS & & FALSE \\
\hline 2008 & $\mathrm{D}$ & 1 & 19 & 6.28 & 98.54 & BIS & & FALSE \\
\hline 2008 & $\mathrm{D}$ & 1 & 20 & 6.34 & 98.54 & BIS & & FALSE \\
\hline
\end{tabular}

Continued on Next Page... 
Table D.1 - Continued

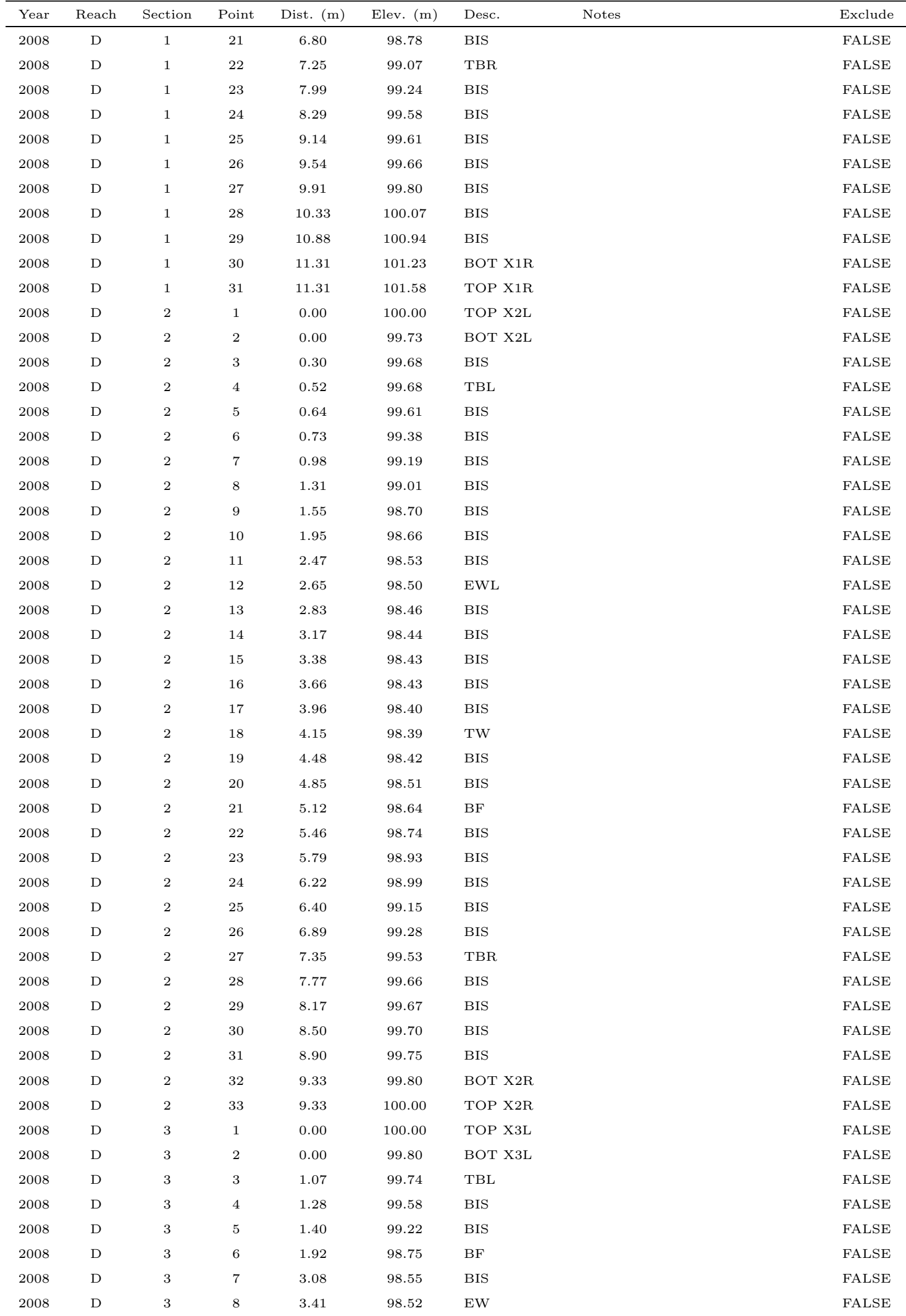

Continued on Next Page... 
Table D.1 - Continued

\begin{tabular}{|c|c|c|c|c|c|c|c|c|}
\hline Year & Reach & Section & Point & Dist. (m) & Elev. $(\mathrm{m})$ & Desc. & Notes & Exclude \\
\hline 2008 & $\mathrm{D}$ & 3 & 9 & 3.87 & 98.40 & BIS & & FALSE \\
\hline 2008 & D & 3 & 10 & 4.15 & 98.36 & BIS & & FALSE \\
\hline 2008 & $\mathrm{D}$ & 3 & 11 & 4.24 & 98.35 & TW & & FALSE \\
\hline 2008 & $\mathrm{D}$ & 3 & 12 & 4.66 & 98.52 & EW & & FALSE \\
\hline 2008 & $\mathrm{D}$ & 3 & 13 & 5.03 & 98.99 & BIS & & FALSE \\
\hline 2008 & $\mathrm{D}$ & 3 & 14 & 5.43 & 99.19 & BIS & & FALSE \\
\hline 2008 & $\mathrm{D}$ & 3 & 15 & 6.04 & 99.82 & BIS & & FALSE \\
\hline 2008 & $\mathrm{D}$ & 3 & 16 & 6.46 & 99.91 & BIS & & FALSE \\
\hline 2008 & $\mathrm{D}$ & 3 & 17 & 7.01 & 99.76 & BIS & & FALSE \\
\hline 2008 & $\mathrm{D}$ & 3 & 18 & 7.47 & 99.19 & BIS & & FALSE \\
\hline 2008 & $\mathrm{D}$ & 3 & 19 & 8.23 & 99.24 & BIS & & FALSE \\
\hline 2008 & $\mathrm{D}$ & 3 & 20 & 9.45 & 99.32 & BIS & & FALSE \\
\hline 2008 & $\mathrm{D}$ & 3 & 21 & 10.55 & 99.50 & BIS & & FALSE \\
\hline 2008 & D & 3 & 22 & 10.91 & 99.82 & BIS & & FALSE \\
\hline 2008 & $\mathrm{D}$ & 3 & 23 & 11.03 & 99.91 & BOT X3R & & FALSE \\
\hline 2008 & $\mathrm{D}$ & 3 & 24 & 11.03 & 100.00 & TOP X3R & & FALSE \\
\hline 2008 & $\mathrm{D}$ & 4 & 1 & 0.15 & 100.00 & TOP X4L & $\begin{array}{l}\text { ON STUMP; TAPE STATED AT } \\
.3\end{array}$ & FALSE \\
\hline 2008 & $\mathrm{D}$ & 4 & 2 & 0.09 & 99.31 & BOT X4L & ON STUMP & FALSE \\
\hline 2008 & $\mathrm{D}$ & 4 & 3 & 0.52 & 98.24 & BIS & BANK IS UNDERCUT & FALSE \\
\hline 2008 & $\mathrm{D}$ & 4 & 4 & 0.85 & 97.84 & BIS & & FALSE \\
\hline 2008 & $\mathrm{D}$ & 4 & 5 & 1.22 & 96.68 & BIS & ON ROCK & FALSE \\
\hline 2008 & $\mathrm{D}$ & 4 & 6 & 1.46 & 96.72 & BIS & ON ROCK & FALSE \\
\hline 2008 & $\mathrm{D}$ & 4 & 7 & 1.62 & 96.52 & EW & & FALSE \\
\hline 2008 & $\mathrm{D}$ & 4 & 8 & 1.80 & 96.53 & BIS & & FALSE \\
\hline 2008 & $\mathrm{D}$ & 4 & 9 & 1.98 & 96.45 & BIS & & FALSE \\
\hline 2008 & $\mathrm{D}$ & 4 & 10 & 2.13 & 96.42 & BIS & & FALSE \\
\hline 2008 & $\mathrm{D}$ & 4 & 11 & 2.38 & 96.55 & BIS & & FALSE \\
\hline 2008 & $\mathrm{D}$ & 4 & 12 & 2.74 & 96.48 & BIS & & FALSE \\
\hline 2008 & $\mathrm{D}$ & 4 & 13 & 2.93 & 96.41 & TW & & FALSE \\
\hline 2008 & $\mathrm{D}$ & 4 & 14 & 3.14 & 96.53 & EW & & FALSE \\
\hline 2008 & $\mathrm{D}$ & 4 & 15 & 3.35 & 96.59 & BIS & & FALSE \\
\hline 2008 & $\mathrm{D}$ & 4 & 16 & 3.66 & 96.64 & BIS & & FALSE \\
\hline 2008 & $\mathrm{D}$ & 4 & 17 & 3.99 & 96.78 & $\mathrm{BF}$ & & FALSE \\
\hline 2008 & $\mathrm{D}$ & 4 & 18 & 4.27 & 96.88 & BIS & & FALSE \\
\hline 2008 & $\mathrm{D}$ & 4 & 19 & 4.57 & 96.97 & BIS & & FALSE \\
\hline 2008 & $\mathrm{D}$ & 4 & 20 & 5.03 & 97.06 & BIS & & FALSE \\
\hline 2008 & $\mathrm{D}$ & 4 & 21 & 5.30 & 97.11 & BIS & & FALSE \\
\hline 2008 & $\mathrm{D}$ & 4 & 22 & 5.73 & 97.27 & BIS & & FALSE \\
\hline 2008 & $\mathrm{D}$ & 4 & 23 & 6.28 & 97.38 & BIS & & FALSE \\
\hline 2008 & $\mathrm{D}$ & 4 & 24 & 7.10 & 97.33 & BIS & & FALSE \\
\hline 2008 & $\mathrm{D}$ & 4 & 25 & 7.74 & 97.34 & BIS & & FALSE \\
\hline 2008 & $\mathrm{D}$ & 4 & 26 & 8.41 & 97.67 & BIS & & FALSE \\
\hline 2008 & $\mathrm{D}$ & 4 & 27 & 9.11 & 97.86 & BIS & & FALSE \\
\hline 2008 & $\mathrm{D}$ & 4 & 28 & 9.48 & 98.00 & BIS & & FALSE \\
\hline 2008 & $\mathrm{D}$ & 4 & 29 & 9.75 & 98.19 & TBR & & FALSE \\
\hline 2008 & D & 4 & 30 & 11.09 & 98.24 & BIS & & FALSE \\
\hline 2008 & $\mathrm{D}$ & 4 & 31 & 11.80 & 98.48 & BIS & & FALSE \\
\hline 2008 & $\mathrm{D}$ & 4 & 32 & 12.28 & 99.01 & BIS & & FALSE \\
\hline 2008 & D & 4 & 33 & 12.62 & 99.20 & BIS & & FALSE \\
\hline 2008 & $\mathrm{D}$ & 4 & 34 & 12.95 & 99.69 & BIS & & FALSE \\
\hline 2008 & $\mathrm{D}$ & 4 & 35 & 13.41 & 100.16 & BIS & & FALSE \\
\hline
\end{tabular}

Continued on Next Page... 
Table D.1 - Continued

\begin{tabular}{|c|c|c|c|c|c|c|c|c|}
\hline Year & Reach & Section & Point & Dist. (m) & Elev. $(\mathrm{m})$ & Desc. & Notes & Exclude \\
\hline 2008 & $\mathrm{D}$ & 4 & 36 & 13.90 & 100.58 & BOT X4R & & FALSE \\
\hline 2008 & $\mathrm{D}$ & 4 & 37 & 13.90 & 101.00 & TOP X4R & & FALSE \\
\hline 2008 & $\mathrm{D}$ & 5 & 1 & 0.00 & 100.00 & TOP X5L & & FALSE \\
\hline 2008 & $\mathrm{D}$ & 5 & 2 & 0.00 & 99.64 & BOT X5L & & FALSE \\
\hline 2008 & $\mathrm{D}$ & 5 & 3 & 0.24 & 99.57 & BIS & & FALSE \\
\hline 2008 & $\mathrm{D}$ & 5 & 4 & 0.30 & 99.51 & TBL & & FALSE \\
\hline 2008 & $\mathrm{D}$ & 5 & 5 & 0.43 & 99.36 & BIS & & FALSE \\
\hline 2008 & $\mathrm{D}$ & 5 & 6 & 0.61 & 99.18 & BIS & & FALSE \\
\hline 2008 & $\mathrm{D}$ & 5 & 7 & 0.85 & 98.45 & BIS & & FALSE \\
\hline 2008 & $\mathrm{D}$ & 5 & 8 & 1.28 & 98.37 & BIS & & FALSE \\
\hline 2008 & $\mathrm{D}$ & 5 & 9 & 2.13 & 98.28 & BIS & & FALSE \\
\hline 2008 & $\mathrm{D}$ & 5 & 10 & 2.44 & 98.25 & BIS & & FALSE \\
\hline 2008 & $\mathrm{D}$ & 5 & 11 & 2.80 & 98.15 & BIS & & FALSE \\
\hline 2008 & $\mathrm{D}$ & 5 & 12 & 3.32 & 98.09 & BIS & & FALSE \\
\hline 2008 & $\mathrm{D}$ & 5 & 13 & 3.63 & 98.03 & BIS & & FALSE \\
\hline 2008 & $\mathrm{D}$ & 5 & 14 & 3.81 & 98.05 & BIS & & FALSE \\
\hline 2008 & $\mathrm{D}$ & 5 & 15 & 4.11 & 97.98 & EW & & FALSE \\
\hline 2008 & $\mathrm{D}$ & 5 & 16 & 4.30 & 97.92 & $\mathrm{TW}$ & & FALSE \\
\hline 2008 & $\mathrm{D}$ & 5 & 17 & 4.39 & 97.93 & BIS & & FALSE \\
\hline 2008 & $\mathrm{D}$ & 5 & 18 & 4.51 & 98.02 & BIS & & FALSE \\
\hline 2008 & $\mathrm{D}$ & 5 & 19 & 4.63 & 97.99 & BIS & & FALSE \\
\hline 2008 & $\mathrm{D}$ & 5 & 20 & 4.88 & 97.97 & EW & & FALSE \\
\hline 2008 & $\mathrm{D}$ & 5 & 21 & 5.06 & 98.01 & BIS & & FALSE \\
\hline 2008 & $\mathrm{D}$ & 5 & 22 & 5.24 & 98.09 & BIS & & FALSE \\
\hline 2008 & $\mathrm{D}$ & 5 & 23 & 5.46 & 98.13 & BIS & & FALSE \\
\hline 2008 & $\mathrm{D}$ & 5 & 24 & 5.61 & 98.24 & $\mathrm{BF}$ & & FALSE \\
\hline 2008 & $\mathrm{D}$ & 5 & 25 & 5.88 & 98.35 & BIS & & FALSE \\
\hline 2008 & $\mathrm{D}$ & 5 & 26 & 6.19 & 98.46 & BIS & & FALSE \\
\hline 2008 & $\mathrm{D}$ & 5 & 27 & 6.68 & 98.63 & BIS & & FALSE \\
\hline 2008 & $\mathrm{D}$ & 5 & 28 & 7.28 & 98.87 & BIS & & FALSE \\
\hline 2008 & $\mathrm{D}$ & 5 & 29 & 7.89 & 99.03 & BIS & & FALSE \\
\hline 2008 & $\mathrm{D}$ & 5 & 30 & 8.05 & 99.45 & $\mathrm{TBR}$ & & FALSE \\
\hline 2008 & $\mathrm{D}$ & 5 & 31 & 8.26 & 99.55 & BIS & & FALSE \\
\hline 2008 & $\mathrm{D}$ & 5 & 32 & 8.53 & 99.59 & BIS & & FALSE \\
\hline 2008 & $\mathrm{D}$ & 5 & 33 & 9.14 & 99.69 & BIS & & FALSE \\
\hline 2008 & $\mathrm{D}$ & 5 & 34 & 10.06 & 99.65 & BIS & & FALSE \\
\hline 2008 & $\mathrm{D}$ & 5 & 35 & 10.91 & 99.66 & BOT X5R & & FALSE \\
\hline 2008 & $\mathrm{D}$ & 5 & 36 & 10.91 & 100.02 & TOP X5R & & FALSE \\
\hline 2008 & $\mathrm{D}$ & 6 & 1 & 0.00 & 100.00 & TOP X6L & & FALSE \\
\hline 2008 & $\mathrm{D}$ & 6 & 2 & 0.00 & 99.83 & BOT X6L & & FALSE \\
\hline 2008 & $\mathrm{D}$ & 6 & 3 & 0.24 & 99.78 & BIS & log supported bank & FALSE \\
\hline 2008 & $\mathrm{D}$ & 6 & 4 & 0.40 & 99.68 & BIS & & FALSE \\
\hline 2008 & $\mathrm{D}$ & 6 & 5 & 0.61 & 99.60 & BIS & & FALSE \\
\hline 2008 & $\mathrm{D}$ & 6 & 6 & 0.70 & 99.60 & TBL & & FALSE \\
\hline 2008 & $\mathrm{D}$ & 6 & 7 & 0.98 & 98.36 & BIS & & FALSE \\
\hline 2008 & $\mathrm{D}$ & 6 & 8 & 1.13 & 98.26 & BIS & & FALSE \\
\hline 2008 & $\mathrm{D}$ & 6 & 9 & 1.31 & 98.24 & BIS & & FALSE \\
\hline 2008 & $\mathrm{D}$ & 6 & 10 & 1.52 & 98.17 & BIS & & FALSE \\
\hline 2008 & $\mathrm{D}$ & 6 & 11 & 1.92 & 98.13 & BIS & & FALSE \\
\hline 2008 & $\mathrm{D}$ & 6 & 12 & 2.38 & 98.08 & BIS & & FALSE \\
\hline 2008 & $\mathrm{D}$ & 6 & 13 & 2.74 & 98.03 & BIS & & FALSE \\
\hline 2008 & $\mathrm{D}$ & 6 & 14 & 2.93 & 97.92 & $\mathrm{BF}$ & & FALSE \\
\hline
\end{tabular}

Continued on Next Page... 
APPENDIX D. SURVEY DATA

Table D.1 - Continued

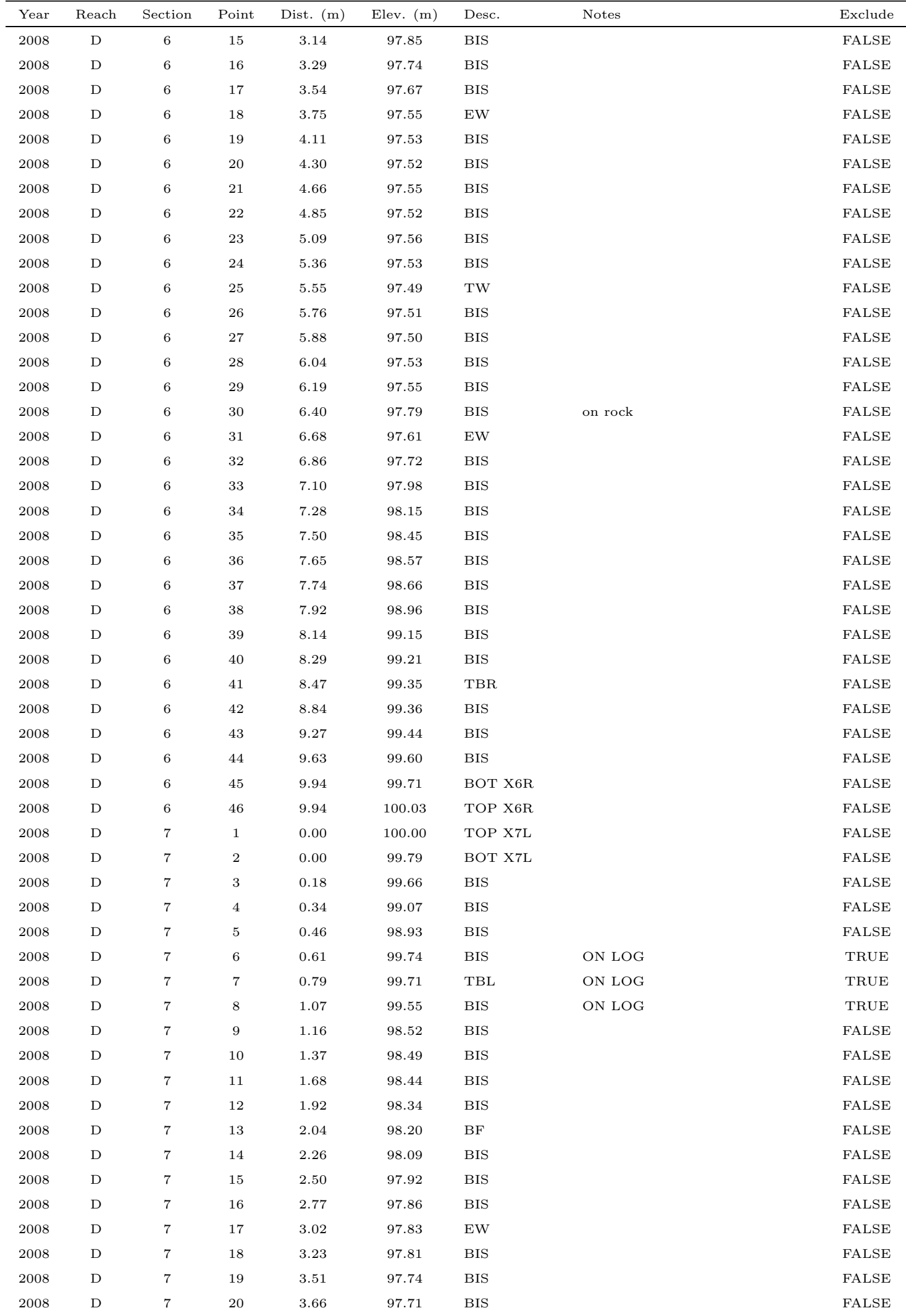

Continued on Next Page... 
Table D.1 - Continued

\begin{tabular}{|c|c|c|c|c|c|c|c|c|}
\hline Year & Reach & Section & Point & Dist. $(\mathrm{m})$ & Elev. $(\mathrm{m})$ & Desc. & Notes & Exclude \\
\hline 2008 & $\mathrm{D}$ & 7 & 21 & 3.81 & 97.69 & $\mathrm{TW}$ & & FALSE \\
\hline 2008 & D & 7 & 22 & 3.96 & 97.73 & BIS & & FALSE \\
\hline 2008 & $\mathrm{D}$ & 7 & 23 & 4.15 & 97.77 & BIS & & FALSE \\
\hline 2008 & $\mathrm{D}$ & 7 & 24 & 4.33 & 97.79 & EW & TRUE EW UNDER LOG & FALSE \\
\hline 2008 & $\mathrm{D}$ & 7 & 25 & 4.45 & 98.31 & BIS & ON LOG & TRUE \\
\hline 2008 & $\mathrm{D}$ & 7 & 26 & 4.72 & 98.29 & BIS & ON LOG & TRUE \\
\hline 2008 & $\mathrm{D}$ & 7 & 27 & 5.09 & 98.23 & BIS & ON LOG & TRUE \\
\hline 2008 & $\mathrm{D}$ & 7 & 28 & 5.30 & 98.20 & BIS & ON LOG & TRUE \\
\hline 2008 & $\mathrm{D}$ & 7 & 29 & 5.61 & 98.09 & BIS & & FALSE \\
\hline 2008 & $\mathrm{D}$ & 7 & 30 & 5.79 & 98.11 & BIS & & FALSE \\
\hline 2008 & D & 7 & 31 & 5.94 & 98.08 & BIS & & FALSE \\
\hline 2008 & $\mathrm{D}$ & 7 & 32 & 6.10 & 98.02 & BIS & & FALSE \\
\hline 2008 & $\mathrm{D}$ & 7 & 33 & 6.19 & 98.52 & BIS & & FALSE \\
\hline 2008 & $\mathrm{D}$ & 7 & 34 & 6.40 & 98.84 & BIS & & FALSE \\
\hline 2008 & $\mathrm{D}$ & 7 & 35 & 6.61 & 99.10 & BIS & & FALSE \\
\hline 2008 & $\mathrm{D}$ & 7 & 36 & 6.83 & 99.21 & BIS & & FALSE \\
\hline 2008 & $\mathrm{D}$ & 7 & 37 & 7.01 & 99.33 & BIS & & FALSE \\
\hline 2008 & $\mathrm{D}$ & 7 & 38 & 7.22 & 99.30 & BIS & & FALSE \\
\hline 2008 & $\mathrm{D}$ & 7 & 39 & 7.38 & 99.55 & TBR & & FALSE \\
\hline 2008 & $\mathrm{D}$ & 7 & 40 & 7.50 & 99.73 & BIS & & FALSE \\
\hline 2008 & $\mathrm{D}$ & 7 & 41 & 7.65 & 99.80 & BOT X7R & & FALSE \\
\hline 2008 & $\mathrm{D}$ & 7 & 42 & 7.65 & 99.97 & TOP X7R & & FALSE \\
\hline 2008 & $\mathrm{D}$ & 8 & 1 & 0.12 & 100.00 & TOP X8L & BENT IN WARD & FALSE \\
\hline 2008 & $\mathrm{D}$ & 8 & 2 & 0.00 & 99.76 & BOT X8L & & FALSE \\
\hline 2008 & $\mathrm{D}$ & 8 & 3 & 0.73 & 99.43 & BIS & & FALSE \\
\hline 2008 & $\mathrm{D}$ & 8 & 4 & 0.94 & 99.27 & BIS & & FALSE \\
\hline 2008 & $\mathrm{D}$ & 8 & 5 & 1.19 & 99.04 & BIS & & FALSE \\
\hline 2008 & $\mathrm{D}$ & 8 & 6 & 1.52 & 98.06 & BIS & & FALSE \\
\hline 2008 & $\mathrm{D}$ & 8 & 7 & 1.86 & 97.90 & BIS & & FALSE \\
\hline 2008 & $\mathrm{D}$ & 8 & 8 & 2.23 & 97.82 & EW & & FALSE \\
\hline 2008 & $\mathrm{D}$ & 8 & 9 & 2.38 & 97.79 & BIS & & FALSE \\
\hline 2008 & $\mathrm{D}$ & 8 & 10 & 2.62 & 97.78 & TW & & FALSE \\
\hline 2008 & $\mathrm{D}$ & 8 & 11 & 2.90 & 97.82 & BIS & & FALSE \\
\hline 2008 & $\mathrm{D}$ & 8 & 12 & 3.20 & 97.81 & BIS & & FALSE \\
\hline 2008 & $\mathrm{D}$ & 8 & 13 & 3.57 & 97.79 & EW & & FALSE \\
\hline 2008 & $\mathrm{D}$ & 8 & 14 & 3.78 & 97.77 & BIS & & FALSE \\
\hline 2008 & $\mathrm{D}$ & 8 & 15 & 4.05 & 97.81 & BIS & & FALSE \\
\hline 2008 & $\mathrm{D}$ & 8 & 16 & 4.30 & 97.84 & BIS & & FALSE \\
\hline 2008 & $\mathrm{D}$ & 8 & 17 & 4.63 & 97.91 & BIS & & FALSE \\
\hline 2008 & $\mathrm{D}$ & 8 & 18 & 4.94 & 97.99 & $\mathrm{BF}$ & & FALSE \\
\hline 2008 & $\mathrm{D}$ & 8 & 19 & 5.36 & 98.15 & BIS & & FALSE \\
\hline 2008 & $\mathrm{D}$ & 8 & 20 & 5.64 & 98.20 & BIS & & FALSE \\
\hline 2008 & $\mathrm{D}$ & 8 & 21 & 6.04 & 98.39 & BIS & & FALSE \\
\hline 2008 & $\mathrm{D}$ & 8 & 22 & 6.19 & 98.52 & BIS & & FALSE \\
\hline 2008 & $\mathrm{D}$ & 8 & 23 & 6.31 & 98.68 & BIS & & FALSE \\
\hline 2008 & $\mathrm{D}$ & 8 & 24 & 6.64 & 99.74 & BIS & ON STUMP & FALSE \\
\hline 2008 & $\mathrm{D}$ & 8 & 25 & 6.95 & 99.79 & BOT X8R & & FALSE \\
\hline 2008 & $\mathrm{D}$ & 8 & 26 & 6.95 & 100.03 & TOP X8R & & FALSE \\
\hline 2008 & $\mathrm{D}$ & 9 & 1 & 0.18 & 100.00 & TOP X9L & BENT INWARD & FALSE \\
\hline 2008 & $\mathrm{D}$ & 9 & 2 & 0.00 & 99.80 & BOT X9L & TOP OF BANK ABOVE REBAR & FALSE \\
\hline 2008 & $\mathrm{D}$ & 9 & 3 & 0.18 & 99.55 & BIS & & FALSE \\
\hline 2008 & $\mathrm{D}$ & 9 & 4 & 0.30 & 99.34 & BIS & & FALSE \\
\hline
\end{tabular}

Continued on Next Page... 
Table D.1 - Continued

\begin{tabular}{|c|c|c|c|c|c|c|c|c|}
\hline Year & Reach & Section & Point & Dist. (m) & Elev. $(\mathrm{m})$ & Desc. & Notes & Exclude \\
\hline 2008 & $\mathrm{D}$ & 9 & 5 & 0.49 & 99.07 & BIS & & FALSE \\
\hline 2008 & D & 9 & 6 & 0.70 & 98.92 & BIS & & FALSE \\
\hline 2008 & $\mathrm{D}$ & 9 & 7 & 0.88 & 98.82 & BIS & & FALSE \\
\hline 2008 & $\mathrm{D}$ & 9 & 8 & 1.19 & 98.69 & $\mathrm{BF}$ & & FALSE \\
\hline 2008 & $\mathrm{D}$ & 9 & 9 & 1.58 & 98.52 & EW & & FALSE \\
\hline 2008 & $\mathrm{D}$ & 9 & 10 & 1.80 & 98.58 & BIS & & FALSE \\
\hline 2008 & $\mathrm{D}$ & 9 & 11 & 2.26 & 98.63 & BIS & & FALSE \\
\hline 2008 & $\mathrm{D}$ & 9 & 12 & 2.74 & 98.59 & BIS & ON LOG & FALSE \\
\hline 2008 & $\mathrm{D}$ & 9 & 13 & 3.17 & 98.42 & TW & & FALSE \\
\hline 2008 & $\mathrm{D}$ & 9 & 14 & 3.38 & 98.45 & BIS & & FALSE \\
\hline 2008 & $\mathrm{D}$ & 9 & 15 & 3.54 & 98.44 & BIS & & FALSE \\
\hline 2008 & $\mathrm{D}$ & 9 & 16 & 3.63 & 98.43 & BIS & & FALSE \\
\hline 2008 & $\mathrm{D}$ & 9 & 17 & 3.84 & 98.46 & EW & TRUE EW UNDER ROCK & FALSE \\
\hline 2008 & $\mathrm{D}$ & 9 & 18 & 4.05 & 98.69 & BIS & ON ROCK & TRUE \\
\hline 2008 & $\mathrm{D}$ & 9 & 19 & 4.24 & 98.57 & BIS & & FALSE \\
\hline 2008 & $\mathrm{D}$ & 9 & 20 & 4.51 & 98.57 & BIS & & FALSE \\
\hline 2008 & $\mathrm{D}$ & 9 & 21 & 4.79 & 98.73 & BIS & & FALSE \\
\hline 2008 & $\mathrm{D}$ & 9 & 22 & 4.94 & 98.86 & BIS & & FALSE \\
\hline 2008 & $\mathrm{D}$ & 9 & 23 & 5.24 & 98.91 & BIS & & FALSE \\
\hline 2008 & $\mathrm{D}$ & 9 & 24 & 5.58 & 98.94 & BIS & & FALSE \\
\hline 2008 & $\mathrm{D}$ & 9 & 25 & 5.91 & 98.98 & BIS & & FALSE \\
\hline 2008 & $\mathrm{D}$ & 9 & 26 & 6.31 & 99.03 & BIS & & FALSE \\
\hline 2008 & $\mathrm{D}$ & 9 & 27 & 6.77 & 99.04 & BIS & & FALSE \\
\hline 2008 & $\mathrm{D}$ & 9 & 28 & 7.07 & 99.16 & BIS & & FALSE \\
\hline 2008 & $\mathrm{D}$ & 9 & 29 & 7.32 & 99.38 & BIS & & FALSE \\
\hline 2008 & $\mathrm{D}$ & 9 & 30 & 7.59 & 99.73 & BOT X9R & & FALSE \\
\hline 2008 & $\mathrm{D}$ & 9 & 31 & 7.41 & 100.02 & TOP X9R & BENT INWARD & FALSE \\
\hline 2008 & $\mathrm{D}$ & 10 & 1 & 0.00 & 100.00 & TOP X10L & top of bank above rebar & FALSE \\
\hline 2008 & $\mathrm{D}$ & 10 & 2 & 0.00 & 99.68 & BOT X10L & & FALSE \\
\hline 2008 & $\mathrm{D}$ & 10 & 3 & 0.24 & 99.56 & BIS & & FALSE \\
\hline 2008 & $\mathrm{D}$ & 10 & 4 & 0.37 & 99.40 & BIS & & FALSE \\
\hline 2008 & $\mathrm{D}$ & 10 & 5 & 0.49 & 99.23 & BIS & & FALSE \\
\hline 2008 & $\mathrm{D}$ & 10 & 6 & 0.61 & 99.13 & BIS & & FALSE \\
\hline 2008 & $\mathrm{D}$ & 10 & 7 & 0.88 & 98.97 & BIS & & FALSE \\
\hline 2008 & $\mathrm{D}$ & 10 & 8 & 1.13 & 98.93 & BIS & & FALSE \\
\hline 2008 & $\mathrm{D}$ & 10 & 9 & 1.49 & 98.76 & BIS & & FALSE \\
\hline 2008 & $\mathrm{D}$ & 10 & 10 & 1.74 & 98.49 & $\mathrm{BF}$ & & FALSE \\
\hline 2008 & $\mathrm{D}$ & 10 & 11 & 2.10 & 98.42 & BIS & & FALSE \\
\hline 2008 & $\mathrm{D}$ & 10 & 12 & 2.38 & 98.33 & BIS & & FALSE \\
\hline 2008 & $\mathrm{D}$ & 10 & 13 & 2.65 & 98.31 & BIS & & FALSE \\
\hline 2008 & $\mathrm{D}$ & 10 & 14 & 2.99 & 98.29 & BIS & on rock & FALSE \\
\hline 2008 & $\mathrm{D}$ & 10 & 15 & 3.05 & 98.25 & EW & & FALSE \\
\hline 2008 & $\mathrm{D}$ & 10 & 16 & 3.41 & 98.20 & BIS & & FALSE \\
\hline 2008 & $\mathrm{D}$ & 10 & 17 & 3.60 & 98.12 & BIS & & FALSE \\
\hline 2008 & $\mathrm{D}$ & 10 & 18 & 3.78 & 98.08 & $\mathrm{TW}$ & & FALSE \\
\hline 2008 & $\mathrm{D}$ & 10 & 19 & 3.96 & 98.15 & BIS & & FALSE \\
\hline 2008 & $\mathrm{D}$ & 10 & 20 & 4.27 & 98.11 & BIS & & FALSE \\
\hline 2008 & $\mathrm{D}$ & 10 & 21 & 4.48 & 98.16 & BIS & & FALSE \\
\hline 2008 & $\mathrm{D}$ & 10 & 22 & 4.69 & 98.19 & BIS & & FALSE \\
\hline 2008 & $\mathrm{D}$ & 10 & 23 & 4.97 & 98.25 & EW & & FALSE \\
\hline 2008 & $\mathrm{D}$ & 10 & 24 & 5.36 & 98.36 & BIS & & FALSE \\
\hline 2008 & $\mathrm{D}$ & 10 & 25 & 5.55 & 98.31 & BIS & & FALSE \\
\hline
\end{tabular}

Continued on Next Page... 
Table D.1 - Continued

\begin{tabular}{|c|c|c|c|c|c|c|c|c|}
\hline Year & Reach & Section & Point & Dist. (m) & Elev. $(\mathrm{m})$ & Desc. & Notes & Exclude \\
\hline 2008 & $\mathrm{D}$ & 10 & 26 & 5.76 & 98.50 & BIS & on rock & FALSE \\
\hline 2008 & D & 10 & 27 & 6.07 & 98.47 & BIS & & FALSE \\
\hline 2008 & $\mathrm{D}$ & 10 & 28 & 6.43 & 98.58 & BIS & & FALSE \\
\hline 2008 & $\mathrm{D}$ & 10 & 29 & 6.71 & 98.81 & BIS & & FALSE \\
\hline 2008 & $\mathrm{D}$ & 10 & 30 & 6.95 & 98.92 & BIS & & FALSE \\
\hline 2008 & $\mathrm{D}$ & 10 & 31 & 7.13 & 99.52 & BIS & & FALSE \\
\hline 2008 & $\mathrm{D}$ & 10 & 32 & 7.47 & 99.79 & BOT X10R & & FALSE \\
\hline 2008 & $\mathrm{D}$ & 10 & 33 & 7.47 & 99.99 & TOP X10R & top of bank above rebar & FALSE \\
\hline 2008 & $\mathrm{E}$ & 1 & 1 & 0.00 & 100.00 & TOP X1L & TB ABOUVE REBAR & FALSE \\
\hline 2008 & $\mathrm{E}$ & 1 & 2 & 0.00 & 99.62 & BOT X1L & & FALSE \\
\hline 2008 & $\mathrm{E}$ & 1 & 3 & 0.46 & 99.37 & BIS & & FALSE \\
\hline 2008 & $\mathrm{E}$ & 1 & 4 & 1.10 & 99.10 & BIS & & FALSE \\
\hline 2008 & $\mathrm{E}$ & 1 & 5 & 1.68 & 98.95 & BIS & & FALSE \\
\hline 2008 & $\mathrm{E}$ & 1 & 6 & 2.56 & 98.84 & BIS & & FALSE \\
\hline 2008 & $\mathrm{E}$ & 1 & 7 & 3.32 & 98.77 & BIS & & FALSE \\
\hline 2008 & $\mathrm{E}$ & 1 & 8 & 4.05 & 98.67 & BIS & & FALSE \\
\hline 2008 & $\mathrm{E}$ & 1 & 9 & 4.54 & 98.50 & BIS & & FALSE \\
\hline 2008 & $\mathrm{E}$ & 1 & 10 & 5.24 & 98.33 & $\mathrm{BF}$ & & FALSE \\
\hline 2008 & $\mathrm{E}$ & 1 & 11 & 5.73 & 98.21 & BIS & & FALSE \\
\hline 2008 & $\mathrm{E}$ & 1 & 12 & 5.97 & 98.24 & BIS & $\begin{array}{l}\text { ON ROCK/ TRUE EDGE OF } \\
\text { WATER }\end{array}$ & FALSE \\
\hline 2008 & $\mathrm{E}$ & 1 & 13 & 6.16 & 98.06 & $\mathrm{EW} / \mathrm{TW}$ & & FALSE \\
\hline 2008 & $\mathrm{E}$ & 1 & 14 & 6.34 & 98.11 & BIS & $\begin{array}{l}\text { ON ROCK/ TRUE EDGE OF } \\
\text { WATER }\end{array}$ & FALSE \\
\hline 2008 & $\mathrm{E}$ & 1 & 15 & 6.58 & 98.07 & BIS & & FALSE \\
\hline 2008 & $\mathrm{E}$ & 1 & 16 & 6.95 & 98.13 & $\mathrm{EW} / \mathrm{TW}$ & ROOT OVERHANG & FALSE \\
\hline 2008 & $\mathrm{E}$ & 1 & 17 & 7.19 & 99.68 & BIS & & FALSE \\
\hline 2008 & $\mathrm{E}$ & 1 & 18 & 7.47 & 99.81 & BOT X1R & & FALSE \\
\hline 2008 & $\mathrm{E}$ & 1 & 19 & 7.47 & 99.88 & TOP X1R & & FALSE \\
\hline 2008 & $\mathrm{E}$ & 2 & 1 & 0.00 & 100.00 & TOP X2L & & FALSE \\
\hline 2008 & $\mathrm{E}$ & 2 & 2 & 0.00 & 99.42 & BOT X2L & & FALSE \\
\hline 2008 & $\mathrm{E}$ & 2 & 3 & 0.43 & 99.18 & TBL & & FALSE \\
\hline 2008 & $\mathrm{E}$ & 2 & 4 & 0.79 & 98.95 & BIS & & FALSE \\
\hline 2008 & $\mathrm{E}$ & 2 & 5 & 1.46 & 98.55 & $\mathrm{BF}$ & & FALSE \\
\hline 2008 & $\mathrm{E}$ & 2 & 6 & 1.89 & 98.42 & BIS & & FALSE \\
\hline 2008 & $\mathrm{E}$ & 2 & 7 & 2.07 & 98.51 & BIS & ON ROCK/TRUE EW & FALSE \\
\hline 2008 & $\mathrm{E}$ & 2 & 8 & 2.13 & 98.30 & EW & & FALSE \\
\hline 2008 & $\mathrm{E}$ & 2 & 9 & 2.19 & 98.33 & BIS & & FALSE \\
\hline 2008 & $\mathrm{E}$ & 2 & 10 & 2.23 & 98.43 & BIS & ON ROCK & FALSE \\
\hline 2008 & $\mathrm{E}$ & 2 & 11 & 2.77 & 98.32 & BIS & ON ROCK & FALSE \\
\hline 2008 & $\mathrm{E}$ & 2 & 12 & 2.87 & 98.26 & TW & & FALSE \\
\hline 2008 & $\mathrm{E}$ & 2 & 13 & 3.29 & 98.34 & EW & & FALSE \\
\hline 2008 & $\mathrm{E}$ & 2 & 14 & 3.57 & 98.36 & BIS & & FALSE \\
\hline 2008 & $\mathrm{E}$ & 2 & 15 & 3.63 & 98.60 & BIS & & FALSE \\
\hline 2008 & $\mathrm{E}$ & 2 & 16 & 3.93 & 98.72 & BIS & ON ROCK & FALSE \\
\hline 2008 & $\mathrm{E}$ & 2 & 17 & 4.02 & 98.61 & BIS & ON ROCK & FALSE \\
\hline 2008 & $\mathrm{E}$ & 2 & 18 & 4.27 & 98.62 & BIS & & FALSE \\
\hline 2008 & $\mathrm{E}$ & 2 & 19 & 4.75 & 99.09 & BIS & & FALSE \\
\hline 2008 & $\mathrm{E}$ & 2 & 20 & 5.09 & 99.34 & TBR & & FALSE \\
\hline 2008 & $\mathrm{E}$ & 2 & 21 & 5.61 & 99.42 & BIS & & FALSE \\
\hline 2008 & $\mathrm{E}$ & 2 & 22 & 5.82 & 99.57 & BIS & & FALSE \\
\hline 2008 & $\mathrm{E}$ & 2 & 23 & 5.97 & 99.62 & BOT X2R & & FALSE \\
\hline
\end{tabular}

Continued on Next Page... 
Table D.1 - Continued

\begin{tabular}{|c|c|c|c|c|c|c|c|c|}
\hline Year & Reach & Section & Point & Dist. (m) & Elev. $(\mathrm{m})$ & Desc. & Notes & Exclude \\
\hline 2008 & $\mathrm{E}$ & 2 & 24 & 5.97 & 100.01 & TOP X2R & & FALSE \\
\hline 2008 & $\mathrm{E}$ & 3 & 1 & 0.00 & 100.00 & TOP X3L & & FALSE \\
\hline 2008 & $\mathrm{E}$ & 3 & 2 & 0.00 & 99.52 & BOT X3L & & FALSE \\
\hline 2008 & $\mathrm{E}$ & 3 & 3 & 0.40 & 99.22 & TBL & & FALSE \\
\hline 2008 & $\mathrm{E}$ & 3 & 4 & 0.79 & 98.90 & BIS & & FALSE \\
\hline 2008 & $\mathrm{E}$ & 3 & 5 & 1.13 & 98.69 & BIS & & FALSE \\
\hline 2008 & $\mathrm{E}$ & 3 & 6 & 1.83 & 98.36 & BIS & & FALSE \\
\hline 2008 & $\mathrm{E}$ & 3 & 7 & 1.95 & 98.05 & EW & & FALSE \\
\hline 2008 & $\mathrm{E}$ & 3 & 8 & 2.10 & 97.97 & BIS & ON ROCK & FALSE \\
\hline 2008 & $\mathrm{E}$ & 3 & 9 & 2.32 & 97.96 & BIS & ON ROCK & FALSE \\
\hline 2008 & $\mathrm{E}$ & 3 & 10 & 2.93 & 98.16 & BIS & ON ROCK & FALSE \\
\hline 2008 & $\mathrm{E}$ & 3 & 11 & 3.14 & 97.87 & BIS & & FALSE \\
\hline 2008 & $\mathrm{E}$ & 3 & 12 & 3.57 & 97.75 & TW & & FALSE \\
\hline 2008 & $\mathrm{E}$ & 3 & 13 & 3.78 & 97.84 & EW & TRUE EW UNDER ROCK & FALSE \\
\hline 2008 & $\mathrm{E}$ & 3 & 14 & 4.08 & 98.25 & BIS & ON ROCK & FALSE \\
\hline 2008 & $\mathrm{E}$ & 3 & 15 & 4.18 & 98.02 & $\mathrm{BF}$ & & FALSE \\
\hline 2008 & $\mathrm{E}$ & 3 & 16 & 4.57 & 98.28 & BIS & & FALSE \\
\hline 2008 & $\mathrm{E}$ & 3 & 17 & 4.79 & 98.42 & BIS & & FALSE \\
\hline 2008 & $\mathrm{E}$ & 3 & 18 & 5.03 & 98.45 & BIS & & FALSE \\
\hline 2008 & $\mathrm{E}$ & 3 & 19 & 5.30 & 98.49 & BIS & & FALSE \\
\hline 2008 & $\mathrm{E}$ & 3 & 20 & 5.49 & 98.89 & TBR & & FALSE \\
\hline 2008 & $\mathrm{E}$ & 3 & 21 & 5.97 & 99.05 & BIS & & FALSE \\
\hline 2008 & $\mathrm{E}$ & 3 & 22 & 7.16 & 99.20 & BIS & & FALSE \\
\hline 2008 & $\mathrm{E}$ & 3 & 23 & 7.32 & 99.53 & BOT X3R & & FALSE \\
\hline 2008 & $\mathrm{E}$ & 3 & 24 & 7.32 & 100.01 & TOP X3R & & FALSE \\
\hline 2008 & $\mathrm{E}$ & 4 & 1 & 0.00 & 100.00 & TOP X4L & & FALSE \\
\hline 2008 & $\mathrm{E}$ & 4 & 2 & 0.00 & 99.77 & BOT X4L & & FALSE \\
\hline 2008 & $\mathrm{E}$ & 4 & 3 & 0.37 & 99.50 & BIS & & FALSE \\
\hline 2008 & $\mathrm{E}$ & 4 & 4 & 0.61 & 99.44 & TBL & & FALSE \\
\hline 2008 & $\mathrm{E}$ & 4 & 5 & 0.70 & 99.13 & BIS & & FALSE \\
\hline 2008 & $\mathrm{E}$ & 4 & 6 & 1.01 & 99.03 & BIS & & FALSE \\
\hline 2008 & $\mathrm{E}$ & 4 & 7 & 1.10 & 98.90 & BIS & & FALSE \\
\hline 2008 & $\mathrm{E}$ & 4 & 8 & 1.62 & 98.93 & BIS & ON ROCK & FALSE \\
\hline 2008 & $\mathrm{E}$ & 4 & 9 & 1.98 & 98.70 & EW & & FALSE \\
\hline 2008 & $\mathrm{E}$ & 4 & 10 & 2.62 & 98.58 & BIS & & FALSE \\
\hline 2008 & $\mathrm{E}$ & 4 & 11 & 2.90 & 98.61 & BIS & & FALSE \\
\hline 2008 & $\mathrm{E}$ & 4 & 12 & 3.11 & 98.58 & TW & & FALSE \\
\hline 2008 & $\mathrm{E}$ & 4 & 13 & 3.60 & 98.84 & BIS & ON ROCK & FALSE \\
\hline 2008 & $\mathrm{E}$ & 4 & 14 & 3.96 & 98.63 & BIS & ON ROCK & FALSE \\
\hline 2008 & $\mathrm{E}$ & 4 & 15 & 4.39 & 98.76 & BIS & ON ROCK & FALSE \\
\hline 2008 & $\mathrm{E}$ & 4 & 16 & 4.72 & 98.60 & EW & & FALSE \\
\hline 2008 & $\mathrm{E}$ & 4 & 17 & 5.12 & 98.86 & BIS & ON ROCK & FALSE \\
\hline 2008 & $\mathrm{E}$ & 4 & 18 & 5.30 & 98.70 & $\mathrm{BF}$ & & FALSE \\
\hline 2008 & $\mathrm{E}$ & 4 & 19 & 5.70 & 98.76 & BIS & & FALSE \\
\hline 2008 & $\mathrm{E}$ & 4 & 20 & 5.94 & 99.47 & TBL & & FALSE \\
\hline 2008 & $\mathrm{E}$ & 4 & 21 & 6.40 & 99.60 & BIS & & FALSE \\
\hline 2008 & $\mathrm{E}$ & 4 & 22 & 7.16 & 99.70 & BIS & & FALSE \\
\hline 2008 & $\mathrm{E}$ & 4 & 23 & 7.68 & 99.81 & BOT X4R & & FALSE \\
\hline 2008 & $\mathrm{E}$ & 4 & 24 & 7.68 & 100.03 & TOP X4R & & FALSE \\
\hline 2008 & $\mathrm{E}$ & 5 & 1 & 0.00 & 100.00 & TOP X5L & UNABLE TO DETERMINE BF & FALSE \\
\hline 2008 & $\mathrm{E}$ & 5 & 2 & 0.00 & 99.73 & BOT X5L & & FALSE \\
\hline 2008 & $\mathrm{E}$ & 5 & 3 & 0.46 & 99.62 & BIS & & FALSE \\
\hline
\end{tabular}

Continued on Next Page... 
Table D.1 - Continued

\begin{tabular}{|c|c|c|c|c|c|c|c|c|}
\hline Year & Reach & Section & Point & Dist. (m) & Elev. $(\mathrm{m})$ & Desc. & Notes & Exclude \\
\hline 2008 & $\mathrm{E}$ & 5 & 4 & 0.88 & 99.45 & TBL & & FALSE \\
\hline 2008 & $\mathrm{E}$ & 5 & 5 & 1.19 & 99.22 & BIS & & FALSE \\
\hline 2008 & $\mathrm{E}$ & 5 & 6 & 1.55 & 98.93 & BIS & & FALSE \\
\hline 2008 & $\mathrm{E}$ & 5 & 7 & 2.19 & 98.87 & BIS & & FALSE \\
\hline 2008 & $\mathrm{E}$ & 5 & 8 & 2.38 & 98.81 & BIS & & FALSE \\
\hline 2008 & $\mathrm{E}$ & 5 & 9 & 2.62 & 98.66 & EW & & FALSE \\
\hline 2008 & $\mathrm{E}$ & 5 & 10 & 2.90 & 98.64 & TW & & FALSE \\
\hline 2008 & $\mathrm{E}$ & 5 & 11 & 3.11 & 98.64 & BIS & & FALSE \\
\hline 2008 & $\mathrm{E}$ & 5 & 12 & 3.41 & 98.69 & BIS & & FALSE \\
\hline 2008 & $\mathrm{E}$ & 5 & 13 & 3.57 & 98.66 & BIS & & FALSE \\
\hline 2008 & $\mathrm{E}$ & 5 & 14 & 3.78 & 98.66 & BIS & & FALSE \\
\hline 2008 & $\mathrm{E}$ & 5 & 15 & 4.08 & 98.73 & BIS & ON ROCK & FALSE \\
\hline 2008 & $\mathrm{E}$ & 5 & 16 & 4.24 & 98.76 & EW & TRUE EW UNDER ROCK & FALSE \\
\hline 2008 & $\mathrm{E}$ & 5 & 17 & 4.39 & 99.01 & BIS & ON ROCK & FALSE \\
\hline 2008 & $\mathrm{E}$ & 5 & 18 & 4.57 & 98.99 & BIS & ON ROCK & FALSE \\
\hline 2008 & $\mathrm{E}$ & 5 & 19 & 4.63 & 99.13 & BIS & ON ROCK & FALSE \\
\hline 2008 & $\mathrm{E}$ & 5 & 20 & 4.91 & 99.22 & BIS & ON ROCK & FALSE \\
\hline 2008 & $\mathrm{E}$ & 5 & 21 & 5.24 & 99.26 & BIS & ON ROCK & FALSE \\
\hline 2008 & $\mathrm{E}$ & 5 & 22 & 5.49 & 99.69 & TBR & & FALSE \\
\hline 2008 & $\mathrm{E}$ & 5 & 23 & 5.85 & 99.79 & BIS & & FALSE \\
\hline 2008 & $\mathrm{E}$ & 5 & 24 & 6.55 & 99.87 & BIS & & FALSE \\
\hline 2008 & $\mathrm{E}$ & 5 & 25 & 7.25 & 99.92 & BIS & & FALSE \\
\hline 2008 & $\mathrm{E}$ & 5 & 26 & 7.86 & 99.90 & BOT X5R & & FALSE \\
\hline 2008 & $\mathrm{E}$ & 5 & 27 & 7.86 & 100.31 & TOP X5R & & FALSE \\
\hline 2008 & $\mathrm{E}$ & 6 & 1 & 0.00 & 100.00 & TOP X6L & & FALSE \\
\hline 2008 & $\mathrm{E}$ & 6 & 2 & 0.00 & 99.58 & BOT X6L & & FALSE \\
\hline 2008 & $\mathrm{E}$ & 6 & 3 & 0.18 & 99.51 & TBL & & FALSE \\
\hline 2008 & $\mathrm{E}$ & 6 & 4 & 0.34 & 99.39 & BIS & & FALSE \\
\hline 2008 & $\mathrm{E}$ & 6 & 5 & 0.55 & 98.98 & BIS & & FALSE \\
\hline 2008 & $\mathrm{E}$ & 6 & 6 & 0.76 & 98.70 & BIS & & FALSE \\
\hline 2008 & $\mathrm{E}$ & 6 & 7 & 1.10 & 98.70 & BIS & ON ROCK & FALSE \\
\hline 2008 & $\mathrm{E}$ & 6 & 8 & 1.25 & 98.38 & EW & & FALSE \\
\hline 2008 & $\mathrm{E}$ & 6 & 9 & 1.46 & 98.35 & BIS & & FALSE \\
\hline 2008 & $\mathrm{E}$ & 6 & 10 & 1.89 & 98.30 & TW & & FALSE \\
\hline 2008 & $\mathrm{E}$ & 6 & 11 & 2.07 & 98.41 & EW & ON ROCK & FALSE \\
\hline 2008 & $\mathrm{E}$ & 6 & 12 & 2.23 & 98.41 & BIS & ON ROCK & FALSE \\
\hline 2008 & $\mathrm{E}$ & 6 & 13 & 2.56 & 98.41 & BIS & & FALSE \\
\hline 2008 & $\mathrm{E}$ & 6 & 14 & 3.05 & 98.48 & BIS & & FALSE \\
\hline 2008 & $\mathrm{E}$ & 6 & 15 & 3.35 & 98.53 & $\mathrm{BF}$ & & FALSE \\
\hline 2008 & $\mathrm{E}$ & 6 & 16 & 3.41 & 98.77 & BIS & ON ROCK & FALSE \\
\hline 2008 & $\mathrm{E}$ & 6 & 17 & 3.63 & 98.84 & BIS & ON ROCK & FALSE \\
\hline 2008 & $\mathrm{E}$ & 6 & 18 & 3.87 & 98.83 & BIS & ON ROCK & FALSE \\
\hline 2008 & $\mathrm{E}$ & 6 & 19 & 3.96 & 98.68 & BIS & & FALSE \\
\hline 2008 & $\mathrm{E}$ & 6 & 20 & 4.42 & 98.70 & BIS & & FALSE \\
\hline 2008 & $\mathrm{E}$ & 6 & 21 & 4.60 & 98.90 & BIS & & FALSE \\
\hline 2008 & $\mathrm{E}$ & 6 & 22 & 4.82 & 99.49 & TBR & & FALSE \\
\hline 2008 & $\mathrm{E}$ & 6 & 23 & 5.43 & 99.65 & BOT X6R & & FALSE \\
\hline 2008 & $\mathrm{E}$ & 6 & 24 & 5.43 & 99.99 & TOP X6R & & FALSE \\
\hline 2008 & $\mathrm{E}$ & 7 & 1 & 0.00 & 100.00 & TOP X7L & & FALSE \\
\hline 2008 & $\mathrm{E}$ & 7 & 2 & 0.00 & 99.78 & BOT X7L & & FALSE \\
\hline 2008 & $\mathrm{E}$ & 7 & 3 & 0.61 & 99.48 & TBL & & FALSE \\
\hline 2008 & $\mathrm{E}$ & 7 & 4 & 0.88 & 99.30 & BIS & & FALSE \\
\hline
\end{tabular}

Continued on Next Page... 
Table D.1 - Continued

\begin{tabular}{|c|c|c|c|c|c|c|c|c|}
\hline Year & Reach & Section & Point & Dist. (m) & Elev. $(\mathrm{m})$ & Desc. & Notes & Exclude \\
\hline 2008 & $\mathrm{E}$ & 7 & 5 & 1.01 & 98.95 & BIS & & FALSE \\
\hline 2008 & $\mathrm{E}$ & 7 & 6 & 1.31 & 98.91 & BIS & ON ROCK & FALSE \\
\hline 2008 & $\mathrm{E}$ & 7 & 7 & 1.46 & 98.64 & BIS & ON ROCK & FALSE \\
\hline 2008 & $\mathrm{E}$ & 7 & 8 & 1.55 & 98.42 & EW & TRUE EW UNDER ROCK & FALSE \\
\hline 2008 & $\mathrm{E}$ & 7 & 9 & 1.83 & 98.46 & BIS & & FALSE \\
\hline 2008 & $\mathrm{E}$ & 7 & 10 & 2.10 & 98.38 & TW & & FALSE \\
\hline 2008 & $\mathrm{E}$ & 7 & 11 & 2.74 & 98.46 & BIS & & FALSE \\
\hline 2008 & $\mathrm{E}$ & 7 & 12 & 2.99 & 98.44 & BIS & & FALSE \\
\hline 2008 & $\mathrm{E}$ & 7 & 13 & 3.32 & 98.47 & BIS & & FALSE \\
\hline 2008 & $\mathrm{E}$ & 7 & 14 & 3.87 & 98.53 & EW & & FALSE \\
\hline 2008 & $\mathrm{E}$ & 7 & 15 & 4.18 & 98.71 & BIS & ON ROCK & FALSE \\
\hline 2008 & $\mathrm{E}$ & 7 & 16 & 4.54 & 98.99 & BIS & & FALSE \\
\hline 2008 & $\mathrm{E}$ & 7 & 17 & 4.72 & 99.63 & $\mathrm{TBR}$ & & FALSE \\
\hline 2008 & $\mathrm{E}$ & 7 & 18 & 4.94 & 99.73 & BIS & & FALSE \\
\hline 2008 & $\mathrm{E}$ & 7 & 19 & 5.43 & 99.76 & BIS & & FALSE \\
\hline 2008 & $\mathrm{E}$ & 7 & 20 & 5.70 & 99.68 & BIS & & FALSE \\
\hline 2008 & $\mathrm{E}$ & 7 & 21 & 6.52 & 99.72 & BOT X7R & & FALSE \\
\hline 2008 & $\mathrm{E}$ & 7 & 22 & 6.52 & 100.00 & TOP X7R & & FALSE \\
\hline 2008 & $\mathrm{E}$ & 8 & 1 & 0.00 & 100.00 & TOP X8L & & FALSE \\
\hline \multirow[t]{2}{*}{2008} & $\mathrm{E}$ & 8 & 2 & 0.00 & 99.65 & BOT & & FALSE \\
\hline & & & & & & $\mathrm{X} 8 \mathrm{~L} / \mathrm{TBL}$ & & \\
\hline 2008 & $\mathrm{E}$ & 8 & 3 & 0.27 & 99.45 & BIS & & FALSE \\
\hline 2008 & $\mathrm{E}$ & 8 & 4 & 0.58 & 99.24 & BIS & & FALSE \\
\hline 2008 & $\mathrm{E}$ & 8 & 5 & 0.91 & 98.95 & BIS & & FALSE \\
\hline 2008 & $\mathrm{E}$ & 8 & 6 & 1.16 & 98.71 & BIS & & FALSE \\
\hline 2008 & $\mathrm{E}$ & 8 & 7 & 1.77 & 98.60 & EW & & FALSE \\
\hline 2008 & $\mathrm{E}$ & 8 & 8 & 1.92 & 98.56 & BIS & & FALSE \\
\hline 2008 & $\mathrm{E}$ & 8 & 9 & 2.01 & 98.54 & TW & & FALSE \\
\hline 2008 & $\mathrm{E}$ & 8 & 10 & 2.29 & 98.56 & BIS & & FALSE \\
\hline 2008 & $\mathrm{E}$ & 8 & 11 & 2.59 & 98.57 & BIS & & FALSE \\
\hline 2008 & $\mathrm{E}$ & 8 & 12 & 2.90 & 98.62 & EW & TRUE EW UNDER ROCK & FALSE \\
\hline 2008 & $\mathrm{E}$ & 8 & 13 & 3.14 & 98.77 & BIS & ON ROCK & FALSE \\
\hline 2008 & $\mathrm{E}$ & 8 & 14 & 3.20 & 98.76 & BIS & ON ROCK & FALSE \\
\hline 2008 & $\mathrm{E}$ & 8 & 15 & 3.29 & 98.68 & BIS & & FALSE \\
\hline 2008 & $\mathrm{E}$ & 8 & 16 & 3.72 & 98.75 & $\mathrm{BF}$ & & FALSE \\
\hline 2008 & $\mathrm{E}$ & 8 & 17 & 4.02 & 98.87 & BIS & & FALSE \\
\hline 2008 & $\mathrm{E}$ & 8 & 18 & 4.51 & 99.41 & TBR & & FALSE \\
\hline 2008 & $\mathrm{E}$ & 8 & 19 & 4.60 & 99.58 & BIS & & FALSE \\
\hline 2008 & $\mathrm{E}$ & 8 & 20 & 5.18 & 99.64 & BIS & & FALSE \\
\hline 2008 & $\mathrm{E}$ & 8 & 21 & 5.40 & 99.64 & BIS & & FALSE \\
\hline 2008 & $\mathrm{E}$ & 8 & 22 & 6.58 & 99.79 & BOT X8R & & FALSE \\
\hline 2008 & $\mathrm{E}$ & 8 & 23 & 6.58 & 100.00 & TOP X8R & & FALSE \\
\hline 2008 & $\mathrm{E}$ & 9 & 1 & 0.00 & 100.00 & TOP X9L & & FALSE \\
\hline 2008 & $\mathrm{E}$ & 9 & 2 & 0.00 & 99.49 & BOT X9L & & FALSE \\
\hline 2008 & $\mathrm{E}$ & 9 & 3 & 0.30 & 99.25 & BIS & & FALSE \\
\hline 2008 & $\mathrm{E}$ & 9 & 4 & 0.58 & 99.06 & BIS & & FALSE \\
\hline 2008 & $\mathrm{E}$ & 9 & 5 & 0.91 & 98.87 & TBL & & FALSE \\
\hline 2008 & $\mathrm{E}$ & 9 & 6 & 1.13 & 98.50 & BIS & & FALSE \\
\hline 2008 & $\mathrm{E}$ & 9 & 7 & 1.40 & 98.32 & BIS & & FALSE \\
\hline 2008 & $\mathrm{E}$ & 9 & 8 & 1.74 & 98.07 & BIS & & FALSE \\
\hline 2008 & $\mathrm{E}$ & 9 & 9 & 2.10 & 97.95 & BIS & & FALSE \\
\hline 2008 & $\mathrm{E}$ & 9 & 10 & 2.47 & 97.87 & BIS & & FALSE \\
\hline
\end{tabular}

Continued on Next Page... 
Table D.1 - Continued

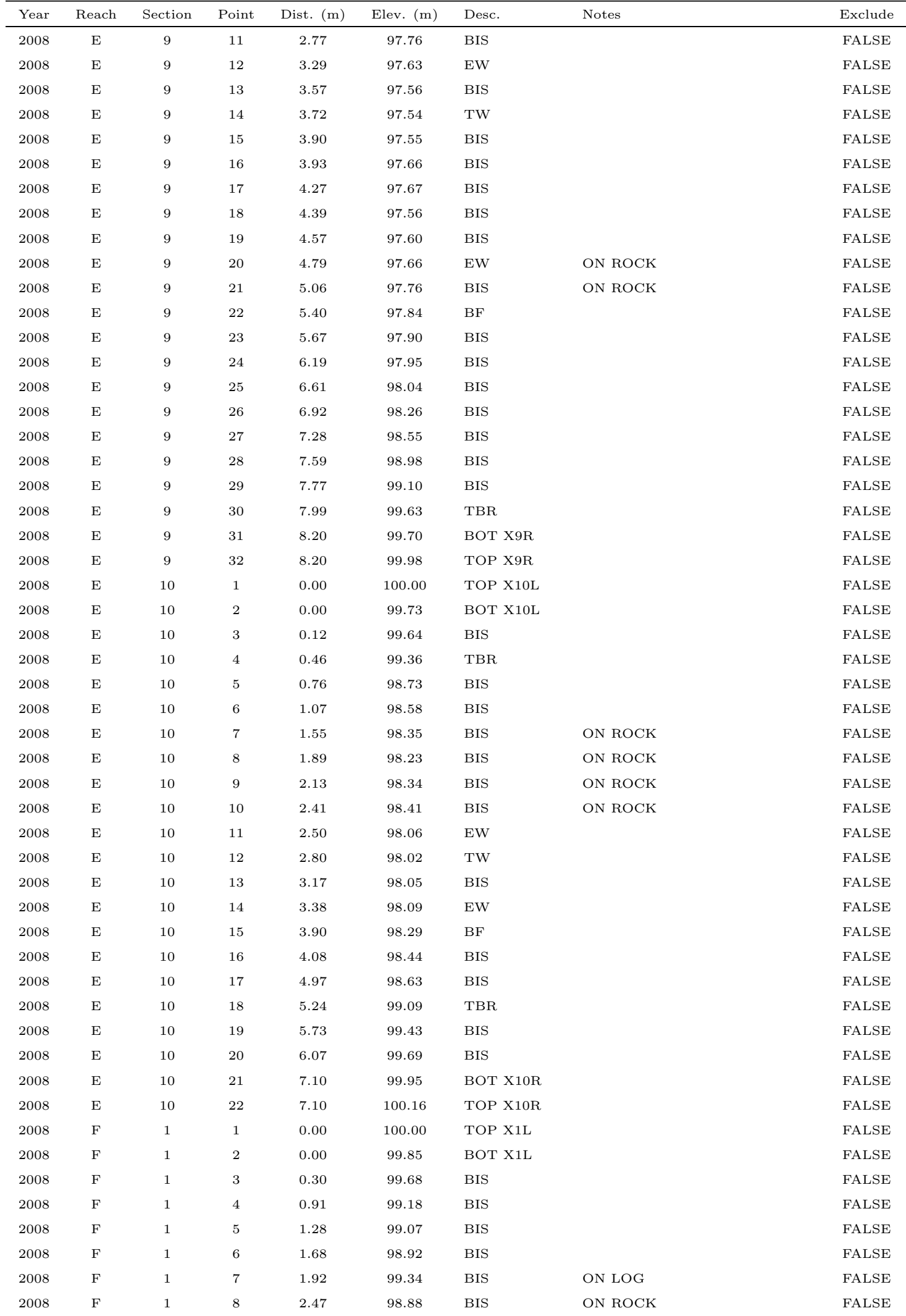

Continued on Next Page... 
Table D.1 - Continued

\begin{tabular}{|c|c|c|c|c|c|c|c|c|}
\hline Year & Reach & Section & Point & Dist. (m) & Elev. $(\mathrm{m})$ & Desc. & Notes & Exclude \\
\hline 2008 & $\mathrm{~F}$ & 1 & 9 & 2.68 & 98.58 & BIS & & FALSE \\
\hline 2008 & $\mathrm{~F}$ & 1 & 10 & 3.23 & 98.33 & BIS & & FALSE \\
\hline 2008 & $\mathrm{~F}$ & 1 & 11 & 4.42 & 98.26 & $\mathrm{BF}$ & & FALSE \\
\hline 2008 & $\mathrm{~F}$ & 1 & 12 & 5.18 & 98.11 & BIS & & FALSE \\
\hline 2008 & $\mathrm{~F}$ & 1 & 13 & 5.46 & 98.24 & BIS & ON ROCK & FALSE \\
\hline 2008 & F & 1 & 14 & 5.76 & 97.99 & EW & & FALSE \\
\hline 2008 & $\mathrm{~F}$ & 1 & 15 & 5.94 & 97.88 & BIS & & FALSE \\
\hline 2008 & $\mathrm{~F}$ & 1 & 16 & 6.04 & 97.88 & TW & & FALSE \\
\hline 2008 & $\mathrm{~F}$ & 1 & 17 & 6.07 & 98.02 & EW & & FALSE \\
\hline 2008 & $\mathrm{~F}$ & 1 & 18 & 6.46 & 98.07 & BIS & Adjusted 1 foot up & FALSE \\
\hline 2008 & F & 1 & 19 & 6.58 & 98.25 & BIS & & FALSE \\
\hline 2008 & $\mathrm{~F}$ & 1 & 20 & 7.41 & 98.28 & BIS & $\begin{array}{l}\text { ON ROCK; TRUE EW UNDER } \\
\text { ROCK }\end{array}$ & FALSE \\
\hline 2008 & $\mathrm{~F}$ & 1 & 21 & 7.68 & 98.40 & BIS & & FALSE \\
\hline 2008 & F & 1 & 22 & 8.23 & 98.69 & BIS & ON ROCK & FALSE \\
\hline 2008 & F & 1 & 23 & 8.41 & 99.04 & BIS & & FALSE \\
\hline 2008 & $\mathrm{~F}$ & 1 & 24 & 9.02 & 99.44 & BIS & & FALSE \\
\hline 2008 & $\mathrm{~F}$ & 1 & 25 & 9.21 & 99.59 & BIS & & FALSE \\
\hline 2008 & $\mathrm{~F}$ & 1 & 26 & 9.39 & 99.71 & BOT X1R & & FALSE \\
\hline 2008 & $\mathrm{~F}$ & 1 & 27 & 9.39 & 99.81 & TOP X1R & $\begin{array}{l}\text { REBAR SLIGHTLY BEND IN- } \\
\text { WARD }\end{array}$ & FALSE \\
\hline 2008 & $\mathrm{~F}$ & 2 & 1 & 0.00 & 100.00 & TOP X2L & & FALSE \\
\hline 2008 & $\mathrm{~F}$ & 2 & 2 & 0.00 & 99.82 & BOT X2L & & FALSE \\
\hline 2008 & $\mathrm{~F}$ & 2 & 3 & 0.30 & 99.67 & BIS & & FALSE \\
\hline 2008 & $\mathrm{~F}$ & 2 & 4 & 0.40 & 99.45 & BIS & & FALSE \\
\hline 2008 & F & 2 & 5 & 0.67 & 99.31 & BIS & & FALSE \\
\hline 2008 & $\mathrm{~F}$ & 2 & 6 & 0.91 & 99.13 & BIS & & FALSE \\
\hline 2008 & $\mathrm{~F}$ & 2 & 7 & 1.25 & 99.04 & BIS & & FALSE \\
\hline 2008 & $\mathrm{~F}$ & 2 & 8 & 1.43 & 98.89 & BIS & & FALSE \\
\hline 2008 & $\mathrm{~F}$ & 2 & 9 & 2.13 & 98.59 & BIS & & FALSE \\
\hline 2008 & $\mathrm{~F}$ & 2 & 10 & 2.56 & 98.42 & TBL & & FALSE \\
\hline 2008 & $\mathrm{~F}$ & 2 & 11 & 2.65 & 98.05 & BIS & & FALSE \\
\hline 2008 & $\mathrm{~F}$ & 2 & 12 & 3.08 & 97.92 & BIS & & FALSE \\
\hline 2008 & $\mathrm{~F}$ & 2 & 13 & 3.60 & 97.75 & $\mathrm{BF}$ & & FALSE \\
\hline 2008 & F & 2 & 14 & 4.15 & 97.77 & BIS & & FALSE \\
\hline 2008 & $\mathrm{~F}$ & 2 & 15 & 4.54 & 97.70 & BIS & & FALSE \\
\hline 2008 & $\mathrm{~F}$ & 2 & 16 & 4.72 & 97.61 & EW & & FALSE \\
\hline 2008 & $\mathrm{~F}$ & 2 & 17 & 4.97 & 97.56 & BIS & & FALSE \\
\hline 2008 & $\mathrm{~F}$ & 2 & 18 & 5.30 & 97.54 & TW & & FALSE \\
\hline 2008 & $\mathrm{~F}$ & 2 & 19 & 5.64 & 97.60 & BIS & & FALSE \\
\hline 2008 & F & 2 & 20 & 6.04 & 97.60 & BIS & & FALSE \\
\hline 2008 & $\mathrm{~F}$ & 2 & 21 & 6.31 & 97.57 & BIS & & FALSE \\
\hline 2008 & $\mathrm{~F}$ & 2 & 22 & 6.52 & 97.61 & EW & & FALSE \\
\hline 2008 & $\mathrm{~F}$ & 2 & 23 & 6.95 & 97.81 & BIS & & FALSE \\
\hline 2008 & $\mathrm{~F}$ & 2 & 24 & 7.13 & 97.83 & BIS & & FALSE \\
\hline 2008 & $\mathrm{~F}$ & 2 & 25 & 7.41 & 98.07 & BIS & & FALSE \\
\hline 2008 & $\mathrm{~F}$ & 2 & 26 & 7.74 & 98.22 & BIS & & FALSE \\
\hline 2008 & $\mathrm{~F}$ & 2 & 27 & 8.08 & 98.44 & BIS & & FALSE \\
\hline 2008 & $\mathrm{~F}$ & 2 & 28 & 8.17 & 98.48 & BIS & & FALSE \\
\hline 2008 & $\mathrm{~F}$ & 2 & 29 & 8.35 & 99.47 & TBR & & FALSE \\
\hline 2008 & $\mathrm{~F}$ & 2 & 30 & 8.72 & 99.81 & BOT X2R & & FALSE \\
\hline 2008 & $\mathrm{~F}$ & 2 & 31 & 8.72 & 99.90 & TOP X2R & & FALSE \\
\hline
\end{tabular}

Continued on Next Page... 
Table D.1 - Continued

\begin{tabular}{|c|c|c|c|c|c|c|c|}
\hline Year & Reach & Section & Point & Dist. (m) & Elev. $(\mathrm{m})$ & Notes & Exclude \\
\hline 2008 & $\mathrm{~F}$ & 3 & 1 & 0.00 & 100.00 & TOP X3L & FALSE \\
\hline 2008 & F & 3 & 2 & 0.00 & 99.86 & BOT X3L & FALSE \\
\hline 2008 & $\mathrm{~F}$ & 3 & 3 & 0.61 & 99.61 & BIS & FALSE \\
\hline 2008 & $\mathrm{~F}$ & 3 & 4 & 1.13 & 99.37 & TBL & FALSE \\
\hline 2008 & $\mathrm{~F}$ & 3 & 5 & 1.34 & 98.61 & BIS & FALSE \\
\hline 2008 & $\mathrm{~F}$ & 3 & 6 & 1.83 & 98.52 & BIS & FALSE \\
\hline 2008 & $\mathrm{~F}$ & 3 & 7 & 2.04 & 98.43 & $\mathrm{BF}$ & FALSE \\
\hline 2008 & $\mathrm{~F}$ & 3 & 8 & 2.59 & 98.38 & BIS & FALSE \\
\hline 2008 & $\mathrm{~F}$ & 3 & 9 & 2.90 & 98.42 & BIS & FALSE \\
\hline 2008 & $\mathrm{~F}$ & 3 & 10 & 3.05 & 98.36 & BIS & FALSE \\
\hline 2008 & F & 3 & 11 & 3.57 & 98.44 & BIS & FALSE \\
\hline 2008 & $\mathrm{~F}$ & 3 & 12 & 3.99 & 98.37 & BIS & FALSE \\
\hline 2008 & $\mathrm{~F}$ & 3 & 13 & 4.54 & 98.21 & BIS & FALSE \\
\hline 2008 & $\mathrm{~F}$ & 3 & 14 & 4.79 & 98.16 & BIS & FALSE \\
\hline 2008 & $\mathrm{~F}$ & 3 & 15 & 4.91 & 98.12 & EW & FALSE \\
\hline 2008 & $\mathrm{~F}$ & 3 & 16 & 5.09 & 98.09 & BIS & FALSE \\
\hline 2008 & $\mathrm{~F}$ & 3 & 17 & 5.27 & 98.10 & BIS & FALSE \\
\hline 2008 & $\mathrm{~F}$ & 3 & 18 & 5.43 & 98.07 & TW & FALSE \\
\hline 2008 & $\mathrm{~F}$ & 3 & 19 & 5.64 & 98.18 & EW & FALSE \\
\hline 2008 & $\mathrm{~F}$ & 3 & 20 & 6.25 & 98.63 & BIS & FALSE \\
\hline 2008 & F & 3 & 21 & 6.46 & 98.67 & BIS & FALSE \\
\hline 2008 & F & 3 & 22 & 6.95 & 98.78 & BIS & FALSE \\
\hline 2008 & $\mathrm{~F}$ & 3 & 23 & 7.41 & 99.77 & TBR & FALSE \\
\hline 2008 & $\mathrm{~F}$ & 3 & 24 & 7.80 & 100.01 & BOT X3R & FALSE \\
\hline 2008 & $\mathrm{~F}$ & 3 & 25 & 7.80 & 100.07 & TOP X3R & FALSE \\
\hline 2008 & F & 4 & 1 & 0.00 & 100.00 & TOP X4L & FALSE \\
\hline 2008 & F & 4 & 2 & 0.00 & 99.95 & BOT X4L & FALSE \\
\hline 2008 & $\mathrm{~F}$ & 4 & 3 & 1.22 & 99.06 & BIS & FALSE \\
\hline 2008 & $\mathrm{~F}$ & 4 & 4 & 1.43 & 99.03 & TBL & FALSE \\
\hline 2008 & $\mathrm{~F}$ & 4 & 5 & 1.92 & 98.43 & BIS & FALSE \\
\hline 2008 & $\mathrm{~F}$ & 4 & 6 & 2.56 & 98.03 & BIS & FALSE \\
\hline 2008 & $\mathrm{~F}$ & 4 & 7 & 2.74 & 97.87 & BIS & FALSE \\
\hline 2008 & $\mathrm{~F}$ & 4 & 8 & 3.26 & 97.85 & BIS & FALSE \\
\hline 2008 & $\mathrm{~F}$ & 4 & 9 & 3.47 & 97.88 & BIS & FALSE \\
\hline 2008 & $\mathrm{~F}$ & 4 & 10 & 3.81 & 97.87 & BIS & FALSE \\
\hline 2008 & $\mathrm{~F}$ & 4 & 11 & 4.11 & 97.76 & BIS & FALSE \\
\hline 2008 & $\mathrm{~F}$ & 4 & 12 & 4.48 & 97.74 & BIS & FALSE \\
\hline 2008 & $\mathrm{~F}$ & 4 & 13 & 4.63 & 97.74 & BIS & FALSE \\
\hline 2008 & $\mathrm{~F}$ & 4 & 14 & 4.75 & 97.74 & EWL & FALSE \\
\hline 2008 & F & 4 & 15 & 5.03 & 97.66 & TW & FALSE \\
\hline 2008 & F & 4 & 16 & 5.18 & 97.67 & BIS & FALSE \\
\hline 2008 & $\mathrm{~F}$ & 4 & 17 & 5.55 & 97.70 & BIS & FALSE \\
\hline 2008 & $\mathrm{~F}$ & 4 & 18 & 5.79 & 97.76 & EWR & FALSE \\
\hline 2008 & $\mathrm{~F}$ & 4 & 19 & 6.16 & 97.79 & BIS & FALSE \\
\hline 2008 & F & 4 & 20 & 6.40 & 97.78 & BIS & FALSE \\
\hline 2008 & $\mathrm{~F}$ & 4 & 21 & 6.74 & 98.15 & BIS & FALSE \\
\hline 2008 & $\mathrm{~F}$ & 4 & 22 & 7.19 & 98.76 & BIS & FALSE \\
\hline 2008 & F & 4 & 23 & 7.47 & 99.22 & BIS & FALSE \\
\hline 2008 & $\mathrm{~F}$ & 4 & 24 & 7.77 & 99.56 & BIS & FALSE \\
\hline 2008 & F & 4 & 25 & 8.53 & 99.89 & BIS & FALSE \\
\hline 2008 & $\mathrm{~F}$ & 4 & 26 & 8.84 & 99.92 & BOT X4R & FALSE \\
\hline 2008 & $\mathrm{~F}$ & 4 & 27 & 8.84 & 100.14 & TOP X4R & FALSE \\
\hline
\end{tabular}

Continued on Next Page... 
Table D.1 - Continued

\begin{tabular}{|c|c|c|c|c|c|c|c|c|}
\hline Year & Reach & Section & Point & Dist. (m) & Elev. $(\mathrm{m})$ & Desc. & Notes & Exclude \\
\hline 2008 & $\mathrm{~F}$ & 5 & 1 & 0.00 & 100.00 & TOP X5L & & FALSE \\
\hline 2008 & $\mathrm{~F}$ & 5 & 2 & 0.00 & 99.95 & BOT X5L & & FALSE \\
\hline 2008 & F & 5 & 3 & 0.21 & 99.74 & BIS & & FALSE \\
\hline 2008 & F & 5 & 4 & 0.58 & 99.36 & BIS & & FALSE \\
\hline 2008 & F & 5 & 5 & 1.01 & 99.19 & BIS & & FALSE \\
\hline 2008 & F & 5 & 6 & 1.28 & 99.00 & TBL & & FALSE \\
\hline 2008 & F & 5 & 7 & 1.52 & 98.91 & BIS & & FALSE \\
\hline 2008 & F & 5 & 8 & 1.77 & 98.72 & $\mathrm{BF}$ & & FALSE \\
\hline 2008 & F & 5 & 9 & 2.10 & 98.66 & BIS & & FALSE \\
\hline 2008 & $\mathrm{~F}$ & 5 & 10 & 2.47 & 98.68 & BIS & & FALSE \\
\hline 2008 & F & 5 & 11 & 2.83 & 98.56 & BIS & & FALSE \\
\hline 2008 & F & 5 & 12 & 3.05 & 98.51 & EW & & FALSE \\
\hline 2008 & F & 5 & 13 & 3.26 & 98.46 & BIS & & FALSE \\
\hline 2008 & F & 5 & 14 & 3.29 & 99.29 & BIS & ON OVERHANGING LOG & TRUE \\
\hline 2008 & F & 5 & 15 & 3.57 & 99.15 & BIS & ON OVERHANGING LOG & TRUE \\
\hline 2008 & F & 5 & 16 & 3.78 & 98.48 & BIS & & FALSE \\
\hline \multirow[t]{2}{*}{2008} & $\mathrm{~F}$ & 5 & 17 & 3.87 & 98.45 & $\mathrm{TW}$ & TRUE TW UNDER OVER- & FALSE \\
\hline & & & & & & & HANGING LOG & \\
\hline 2008 & $\mathrm{~F}$ & 5 & 18 & 4.21 & 98.49 & BIS & & FALSE \\
\hline 2008 & F & 5 & 19 & 4.79 & 98.52 & EW & & FALSE \\
\hline 2008 & F & 5 & 20 & 4.94 & 98.65 & BIS & & FALSE \\
\hline 2008 & $\mathrm{~F}$ & 5 & 21 & 5.30 & 98.62 & BIS & & FALSE \\
\hline 2008 & F & 5 & 22 & 5.40 & 98.92 & BIS & ON BOULDER & FALSE \\
\hline 2008 & $\mathrm{~F}$ & 5 & 23 & 5.76 & 99.07 & BIS & ON BOULDER & FALSE \\
\hline 2008 & F & 5 & 24 & 6.10 & 99.12 & BIS & ON BOULDER & FALSE \\
\hline 2008 & $\mathrm{~F}$ & 5 & 25 & 6.37 & 98.80 & BIS & & FALSE \\
\hline 2008 & $\mathrm{~F}$ & 5 & 26 & 6.80 & 99.03 & BIS & TBR ABOVE REBAR & FALSE \\
\hline 2008 & F & 5 & 27 & 7.22 & 99.41 & BIS & & FALSE \\
\hline 2008 & $\mathrm{~F}$ & 5 & 28 & 7.65 & 99.81 & BOT X5R & & FALSE \\
\hline 2008 & $\mathrm{~F}$ & 5 & 29 & 7.65 & 99.91 & TOP X5R & & FALSE \\
\hline 2008 & $\mathrm{~F}$ & 6 & 1 & 0.00 & 100.00 & TOP X6L & & FALSE \\
\hline 2008 & $\mathrm{~F}$ & 6 & 2 & 0.00 & 99.83 & BOT X6L & & FALSE \\
\hline 2008 & $\mathrm{~F}$ & 6 & 3 & 0.34 & 99.73 & BIS & & FALSE \\
\hline 2008 & $\mathrm{~F}$ & 6 & 4 & 0.58 & 99.51 & BIS & & FALSE \\
\hline 2008 & F & 6 & 5 & 0.91 & 99.33 & BIS & & FALSE \\
\hline 2008 & F & 6 & 6 & 1.28 & 99.03 & BIS & & FALSE \\
\hline 2008 & $\mathrm{~F}$ & 6 & 7 & 1.77 & 98.56 & BIS & $\begin{array}{l}\text { ON ROCK; POSSIBLE BF, IM- } \\
\text { POSSIBLE TO TELL DUE TO } \\
\text { DEBRIS/LOGJAM }\end{array}$ & FALSE \\
\hline 2008 & $\mathrm{~F}$ & 6 & 8 & 1.86 & 98.35 & BIS & ON DEBRIS & FALSE \\
\hline 2008 & $\mathrm{~F}$ & 6 & 9 & 2.04 & 98.41 & BIS & & FALSE \\
\hline 2008 & $\mathrm{~F}$ & 6 & 10 & 2.29 & 98.63 & BIS & ON LOG & FALSE \\
\hline 2008 & $\mathrm{~F}$ & 6 & 11 & 2.56 & 98.53 & BIS & & FALSE \\
\hline 2008 & F & 6 & 12 & 2.80 & 98.48 & BIS & ON LOG & FALSE \\
\hline 2008 & $\mathrm{~F}$ & 6 & 13 & 3.32 & 98.48 & BIS & & FALSE \\
\hline 2008 & $\mathrm{~F}$ & 6 & 14 & 3.41 & 98.27 & BIS & & FALSE \\
\hline 2008 & F & 6 & 15 & 3.60 & 98.31 & BIS & ON LOG & FALSE \\
\hline 2008 & $\mathrm{~F}$ & 6 & 16 & 3.69 & 98.26 & EW & & FALSE \\
\hline 2008 & $\mathrm{~F}$ & 6 & 17 & 3.81 & 98.17 & TW & & FALSE \\
\hline 2008 & $\mathrm{~F}$ & 6 & 18 & 4.08 & 98.19 & BIS & & FALSE \\
\hline 2008 & $\mathrm{~F}$ & 6 & 19 & 4.48 & 98.18 & BIS & & FALSE \\
\hline 2008 & $\mathrm{~F}$ & 6 & 20 & 4.88 & 98.22 & EW & TRUE EW UNDER BOULDER & FALSE \\
\hline
\end{tabular}

Continued on Next Page... 
Table D.1 - Continued

\begin{tabular}{|c|c|c|c|c|c|c|c|c|}
\hline Year & Reach & Section & Point & Dist. (m) & Elev. $(\mathrm{m})$ & Desc. & Notes & Exclude \\
\hline 2008 & $\mathrm{~F}$ & 6 & 21 & 4.94 & 98.78 & BIS & ON BOULDER & FALSE \\
\hline 2008 & $\mathrm{~F}$ & 6 & 22 & 5.06 & 98.87 & BIS & ON BOULDER & FALSE \\
\hline 2008 & $\mathrm{~F}$ & 6 & 23 & 5.40 & 98.85 & BIS & ON BOULDER & FALSE \\
\hline 2008 & $\mathrm{~F}$ & 6 & 24 & 5.67 & 98.92 & BIS & ON BOULDER & FALSE \\
\hline 2008 & $\mathrm{~F}$ & 6 & 25 & 6.07 & 98.96 & BIS & ON BOULDER & FALSE \\
\hline 2008 & F & 6 & 26 & 6.31 & 99.15 & BIS & & FALSE \\
\hline 2008 & $\mathrm{~F}$ & 6 & 27 & 6.71 & 99.21 & BIS & & FALSE \\
\hline 2008 & $\mathrm{~F}$ & 6 & 28 & 7.35 & 99.38 & BIS & & FALSE \\
\hline 2008 & $\mathrm{~F}$ & 6 & 29 & 7.89 & 99.52 & BIS & & FALSE \\
\hline 2008 & $\mathrm{~F}$ & 6 & 30 & 8.47 & 99.87 & BOT X6R & & FALSE \\
\hline 2008 & $\mathrm{~F}$ & 6 & 31 & 8.47 & 99.98 & TOP X6R & & FALSE \\
\hline 2008 & F & 7 & 1 & 0.00 & 100.00 & TOP X7L & & FALSE \\
\hline 2008 & F & 7 & 2 & 0.00 & 99.95 & BOT X7L & & FALSE \\
\hline 2008 & $\mathrm{~F}$ & 7 & 3 & 0.27 & 99.73 & TBL & & FALSE \\
\hline 2008 & F & 7 & 4 & 0.49 & 99.38 & BIS & & FALSE \\
\hline 2008 & $\mathrm{~F}$ & 7 & 5 & 0.73 & 99.15 & BIS & & FALSE \\
\hline 2008 & F & 7 & 6 & 1.01 & 98.97 & BIS & & FALSE \\
\hline 2008 & $\mathrm{~F}$ & 7 & 7 & 1.13 & 98.79 & BIS & & FALSE \\
\hline 2008 & $\mathrm{~F}$ & 7 & 8 & 1.40 & 98.69 & BIS & & FALSE \\
\hline 2008 & $\mathrm{~F}$ & 7 & 9 & 1.58 & 98.59 & BIS & & FALSE \\
\hline 2008 & F & 7 & 10 & 1.80 & 98.53 & BIS & & FALSE \\
\hline 2008 & F & 7 & 11 & 2.10 & 98.45 & BIS & & FALSE \\
\hline 2008 & $\mathrm{~F}$ & 7 & 12 & 2.59 & 98.40 & EWL & & FALSE \\
\hline 2008 & $\mathrm{~F}$ & 7 & 13 & 2.80 & 98.37 & BIS & & FALSE \\
\hline 2008 & $\mathrm{~F}$ & 7 & 14 & 2.93 & 98.36 & BIS & & FALSE \\
\hline 2008 & F & 7 & 15 & 3.23 & 98.34 & $\mathrm{TW}$ & & FALSE \\
\hline 2008 & F & 7 & 16 & 3.41 & 98.41 & EWR & & FALSE \\
\hline 2008 & $\mathrm{~F}$ & 7 & 17 & 3.66 & 98.46 & BIS & & FALSE \\
\hline 2008 & $\mathrm{~F}$ & 7 & 18 & 3.81 & 98.53 & BIS & & FALSE \\
\hline 2008 & $\mathrm{~F}$ & 7 & 19 & 3.96 & 98.56 & BIS & & FALSE \\
\hline 2008 & $\mathrm{~F}$ & 7 & 20 & 4.02 & 98.72 & BIS & ON ROCK & FALSE \\
\hline 2008 & $\mathrm{~F}$ & 7 & 21 & 4.21 & 98.82 & BIS & ON ROCK & FALSE \\
\hline 2008 & $\mathrm{~F}$ & 7 & 22 & 4.51 & 98.77 & BIS & & FALSE \\
\hline 2008 & $\mathrm{~F}$ & 7 & 23 & 5.06 & 98.87 & BIS & & FALSE \\
\hline 2008 & F & 7 & 24 & 5.61 & 99.02 & BIS & & FALSE \\
\hline 2008 & $\mathrm{~F}$ & 7 & 25 & 5.91 & 99.05 & BIS & & FALSE \\
\hline 2008 & $\mathrm{~F}$ & 7 & 26 & 6.13 & 99.16 & BIS & & FALSE \\
\hline 2008 & $\mathrm{~F}$ & 7 & 27 & 6.58 & 99.20 & BIS & & FALSE \\
\hline 2008 & $\mathrm{~F}$ & 7 & 28 & 7.01 & 99.30 & BIS & & FALSE \\
\hline 2008 & F & 7 & 29 & 7.38 & 99.29 & BIS & & FALSE \\
\hline 2008 & F & 7 & 30 & 8.14 & 99.59 & BIS & & FALSE \\
\hline 2008 & $\mathrm{~F}$ & 7 & 31 & 8.69 & 99.86 & BOT X7R & & FALSE \\
\hline 2008 & F & 7 & 32 & 8.69 & 99.95 & TOP X7R & & FALSE \\
\hline 2008 & $\mathrm{~F}$ & 8 & 1 & 0.06 & 100.00 & TOP X8L & REBAR BEND INWARDS & FALSE \\
\hline 2008 & $\mathrm{~F}$ & 8 & 2 & 0.00 & 99.91 & BOT X8L & & FALSE \\
\hline 2008 & $\mathrm{~F}$ & 8 & 3 & 0.15 & 99.82 & BIS & & FALSE \\
\hline 2008 & $\mathrm{~F}$ & 8 & 4 & 0.34 & 99.60 & BIS & & FALSE \\
\hline 2008 & $\mathrm{~F}$ & 8 & 5 & 0.70 & 99.38 & BIS & & FALSE \\
\hline 2008 & $\mathrm{~F}$ & 8 & 6 & 1.01 & 99.09 & TBL & & FALSE \\
\hline 2008 & $\mathrm{~F}$ & 8 & 7 & 1.13 & 98.62 & BIS & & FALSE \\
\hline 2008 & $\mathrm{~F}$ & 8 & 8 & 1.83 & 98.30 & BIS & & FALSE \\
\hline 2008 & $\mathrm{~F}$ & 8 & 9 & 2.29 & 98.26 & BIS & ON BOULDER & FALSE \\
\hline
\end{tabular}

Continued on Next Page... 
Table D.1 - Continued

\begin{tabular}{|c|c|c|c|c|c|c|c|c|}
\hline Year & Reach & Section & Point & Dist. (m) & Elev. $(\mathrm{m})$ & Desc. & Notes & Exclude \\
\hline 2008 & $\mathrm{~F}$ & 8 & 10 & 2.56 & 97.89 & $\mathrm{BF}$ & & FALSE \\
\hline 2008 & $\mathrm{~F}$ & 8 & 11 & 3.05 & 97.87 & BIS & & FALSE \\
\hline 2008 & F & 8 & 12 & 3.41 & 97.78 & EW & & FALSE \\
\hline 2008 & F & 8 & 13 & 3.54 & 97.74 & BIS & & FALSE \\
\hline 2008 & $\mathrm{~F}$ & 8 & 14 & 3.66 & 97.73 & BIS & & FALSE \\
\hline 2008 & $\mathrm{~F}$ & 8 & 15 & 3.84 & 97.73 & TW & & FALSE \\
\hline 2008 & F & 8 & 16 & 4.05 & 97.75 & BIS & & FALSE \\
\hline 2008 & F & 8 & 17 & 4.39 & 97.77 & BIS & & FALSE \\
\hline 2008 & F & 8 & 18 & 4.57 & 97.75 & BIS & & FALSE \\
\hline 2008 & $\mathrm{~F}$ & 8 & 19 & 4.79 & 97.78 & EW & & FALSE \\
\hline 2008 & F & 8 & 20 & 4.88 & 98.16 & BIS & & FALSE \\
\hline 2008 & F & 8 & 21 & 5.21 & 98.42 & BIS & & FALSE \\
\hline 2008 & F & 8 & 22 & 5.67 & 98.53 & BIS & & FALSE \\
\hline 2008 & F & 8 & 23 & 5.91 & 98.56 & BIS & & FALSE \\
\hline 2008 & F & 8 & 24 & 6.40 & 98.62 & BIS & & FALSE \\
\hline 2008 & F & 8 & 25 & 7.16 & 98.76 & BIS & & FALSE \\
\hline 2008 & F & 8 & 26 & 7.50 & 98.79 & BIS & & FALSE \\
\hline 2008 & F & 8 & 27 & 8.26 & 98.67 & BIS & & FALSE \\
\hline 2008 & $\mathrm{~F}$ & 8 & 28 & 8.63 & 98.68 & BIS & & FALSE \\
\hline 2008 & $\mathrm{~F}$ & 8 & 29 & 8.96 & 98.75 & BIS & & FALSE \\
\hline 2008 & F & 8 & 30 & 9.33 & 99.11 & BIS & & FALSE \\
\hline 2008 & F & 8 & 31 & 9.54 & 99.19 & BIS & & FALSE \\
\hline 2008 & $\mathrm{~F}$ & 8 & 32 & 9.75 & 99.29 & BIS & & FALSE \\
\hline 2008 & $\mathrm{~F}$ & 8 & 33 & 10.30 & 99.52 & BIS & & FALSE \\
\hline 2008 & F & 8 & 34 & 10.82 & 99.63 & BIS & & FALSE \\
\hline 2008 & F & 8 & 35 & 11.25 & 99.82 & BIS & & FALSE \\
\hline 2008 & $\mathrm{~F}$ & 8 & 36 & 11.89 & 99.82 & BIS & & FALSE \\
\hline 2008 & $\mathrm{~F}$ & 8 & 37 & 12.74 & 99.88 & BOT X8R & & FALSE \\
\hline 2008 & $\mathrm{~F}$ & 8 & 38 & 12.74 & 100.03 & TOP X8R & & FALSE \\
\hline \multirow[t]{2}{*}{2008} & $\mathrm{~F}$ & 9 & 1 & 0.00 & 100.00 & TOP & & FALSE \\
\hline & & & & & & $\mathrm{X} 9 \mathrm{~L} / \mathrm{TBL}$ & & \\
\hline 2008 & $\mathrm{~F}$ & 9 & 2 & 0.00 & 99.81 & BOT X9L & & FALSE \\
\hline 2008 & $\mathrm{~F}$ & 9 & 3 & 0.24 & 99.68 & BIS & & FALSE \\
\hline 2008 & $\mathrm{~F}$ & 9 & 4 & 0.37 & 99.28 & BIS & & FALSE \\
\hline 2008 & $\mathrm{~F}$ & 9 & 5 & 0.70 & 99.10 & BIS & & FALSE \\
\hline 2008 & $\mathrm{~F}$ & 9 & 6 & 1.10 & 98.86 & BIS & & FALSE \\
\hline 2008 & $\mathrm{~F}$ & 9 & 7 & 1.62 & 98.72 & $\mathrm{BF}$ & & FALSE \\
\hline 2008 & $\mathrm{~F}$ & 9 & 8 & 1.89 & 98.66 & BIS & & FALSE \\
\hline 2008 & $\mathrm{~F}$ & 9 & 9 & 2.16 & 98.53 & EW & & FALSE \\
\hline 2008 & F & 9 & 10 & 2.62 & 98.48 & BIS & & FALSE \\
\hline 2008 & F & 9 & 11 & 3.05 & 98.47 & BIS & & FALSE \\
\hline 2008 & F & 9 & 12 & 3.32 & 98.41 & TW & & FALSE \\
\hline 2008 & $\mathrm{~F}$ & 9 & 13 & 3.60 & 98.42 & BIS & & FALSE \\
\hline 2008 & $\mathrm{~F}$ & 9 & 14 & 3.87 & 98.45 & EW & & FALSE \\
\hline 2008 & $\mathrm{~F}$ & 9 & 15 & 4.05 & 98.89 & BIS & & FALSE \\
\hline 2008 & F & 9 & 16 & 4.45 & 99.09 & BIS & & FALSE \\
\hline 2008 & $\mathrm{~F}$ & 9 & 17 & 5.15 & 99.17 & BIS & & FALSE \\
\hline 2008 & F & 9 & 18 & 5.82 & 99.23 & BIS & & FALSE \\
\hline 2008 & $\mathrm{~F}$ & 9 & 19 & 6.37 & 99.21 & BIS & & FALSE \\
\hline 2008 & $\mathrm{~F}$ & 9 & 20 & 6.92 & 99.06 & BIS & & FALSE \\
\hline 2008 & $\mathrm{~F}$ & 9 & 21 & 7.74 & 99.11 & BIS & & FALSE \\
\hline 2008 & $\mathrm{~F}$ & 9 & 22 & 8.38 & 99.06 & BIS & & FALSE \\
\hline
\end{tabular}

Continued on Next Page... 
Table D.1 - Continued

\begin{tabular}{|c|c|c|c|c|c|c|c|c|}
\hline Year & Reach & Section & Point & Dist. (m) & Elev. $(\mathrm{m})$ & Desc. & Notes & Exclude \\
\hline 2008 & $\mathrm{~F}$ & 9 & 23 & 8.72 & 99.04 & BIS & & FALSE \\
\hline 2008 & $\mathrm{~F}$ & 9 & 24 & 9.63 & 99.05 & BIS & & FALSE \\
\hline 2008 & $\mathrm{~F}$ & 9 & 25 & 9.88 & 99.12 & BIS & & FALSE \\
\hline 2008 & $\mathrm{~F}$ & 9 & 26 & 10.15 & 99.55 & TBR & ON ROCK & FALSE \\
\hline 2008 & $\mathrm{~F}$ & 9 & 27 & 10.52 & 99.58 & BIS & & FALSE \\
\hline 2008 & $\mathrm{~F}$ & 9 & 28 & 11.25 & 99.77 & BOT X9R & & FALSE \\
\hline 2008 & $\mathrm{~F}$ & 9 & 29 & 11.25 & 99.96 & TOP X9R & & FALSE \\
\hline \multirow[t]{2}{*}{2008} & $\mathrm{~F}$ & 10 & 1 & 0.00 & 100.00 & TOP & & FALSE \\
\hline & & & & & & X10L/TBL & & \\
\hline 2008 & $\mathrm{~F}$ & 10 & 2 & 0.00 & 99.83 & BOT X10L & & FALSE \\
\hline 2008 & $\mathrm{~F}$ & 10 & 3 & 0.27 & 99.10 & BIS & & FALSE \\
\hline 2008 & $\mathrm{~F}$ & 10 & 4 & 0.52 & 98.94 & BIS & & FALSE \\
\hline 2008 & $\mathrm{~F}$ & 10 & 5 & 0.70 & 98.88 & BIS & & FALSE \\
\hline 2008 & $\mathrm{~F}$ & 10 & 6 & 0.91 & 98.69 & BIS & & FALSE \\
\hline 2008 & $\mathrm{~F}$ & 10 & 7 & 1.25 & 98.55 & EW & & FALSE \\
\hline 2008 & $\mathrm{~F}$ & 10 & 8 & 1.40 & 98.49 & BIS & & FALSE \\
\hline 2008 & $\mathrm{~F}$ & 10 & 9 & 1.80 & 98.43 & TW & & FALSE \\
\hline 2008 & $\mathrm{~F}$ & 10 & 10 & 2.07 & 98.49 & BIS & & FALSE \\
\hline 2008 & $\mathrm{~F}$ & 10 & 11 & 2.35 & 98.51 & BIS & & FALSE \\
\hline 2008 & $\mathrm{~F}$ & 10 & 12 & 2.68 & 98.49 & BIS & & FALSE \\
\hline 2008 & $\mathrm{~F}$ & 10 & 13 & 2.87 & 98.54 & EW & & FALSE \\
\hline 2008 & $\mathrm{~F}$ & 10 & 14 & 3.14 & 98.55 & BIS & & FALSE \\
\hline 2008 & $\mathrm{~F}$ & 10 & 15 & 3.26 & 98.65 & BIS & & FALSE \\
\hline 2008 & $\mathrm{~F}$ & 10 & 16 & 3.75 & 98.70 & BIS & & FALSE \\
\hline 2008 & $\mathrm{~F}$ & 10 & 17 & 4.11 & 98.86 & BIS & & FALSE \\
\hline 2008 & $\mathrm{~F}$ & 10 & 18 & 4.60 & 99.18 & BIS & & FALSE \\
\hline 2008 & $\mathrm{~F}$ & 10 & 19 & 5.12 & 99.28 & BIS & & FALSE \\
\hline 2008 & $\mathrm{~F}$ & 10 & 20 & 5.79 & 99.18 & BIS & & FALSE \\
\hline 2008 & $\mathrm{~F}$ & 10 & 21 & 6.37 & 99.18 & BIS & & FALSE \\
\hline 2008 & $\mathrm{~F}$ & 10 & 22 & 6.98 & 99.05 & BIS & & FALSE \\
\hline 2008 & $\mathrm{~F}$ & 10 & 23 & 7.83 & 99.05 & BIS & & FALSE \\
\hline 2008 & $\mathrm{~F}$ & 10 & 24 & 8.29 & 98.97 & BIS & & FALSE \\
\hline 2008 & $\mathrm{~F}$ & 10 & 25 & 8.96 & 98.95 & BIS & & FALSE \\
\hline 2008 & $\mathrm{~F}$ & 10 & 26 & 9.21 & 99.39 & BIS & & FALSE \\
\hline 2008 & $\mathrm{~F}$ & 10 & 27 & 9.45 & 99.59 & $\mathrm{TBR}$ & & FALSE \\
\hline 2008 & $\mathrm{~F}$ & 10 & 28 & 9.75 & 99.66 & BOT X10R & & FALSE \\
\hline 2008 & $\mathrm{~F}$ & 10 & 29 & 9.75 & 99.80 & TOP X10R & & FALSE \\
\hline
\end{tabular}

\section{D.2 Cross Section Offsets}

These values are added to the surveyed cross section data to correct for rebar movement. 
Table D.2: Cross section offsets

\begin{tabular}{|c|c|c|}
\hline Section Code & Distance Offset $(\mathrm{m})$ & Elevation Offset $(\mathrm{m})$ \\
\hline 2002A1 & 0.000 & 0.000 \\
\hline $2002 \mathrm{~A} 10$ & 0.000 & 0.000 \\
\hline $2002 \mathrm{~A} 2$ & 0.000 & 0.000 \\
\hline $2002 \mathrm{~A} 3$ & 0.000 & 0.000 \\
\hline $2002 \mathrm{~A} 4$ & 0.000 & 0.000 \\
\hline $2002 \mathrm{~A} 5$ & 0.000 & 0.000 \\
\hline $2002 \mathrm{~A} 6$ & 0.000 & 0.000 \\
\hline $2002 \mathrm{~A} 7$ & 0.000 & 0.000 \\
\hline $2002 \mathrm{~A} 8$ & 0.000 & 0.000 \\
\hline 2002A9 & 0.000 & 0.000 \\
\hline 2002B1 & 0.000 & 0.000 \\
\hline 2002B10 & 0.000 & 0.000 \\
\hline 2002B2 & 0.000 & 0.000 \\
\hline 2002B3 & 0.000 & 0.000 \\
\hline 2002B 4 & 0.000 & 0.000 \\
\hline 2002B5 & 0.000 & 0.000 \\
\hline 2002B 6 & 0.000 & 0.000 \\
\hline 2002B7 & 0.000 & 0.000 \\
\hline 2002B 8 & 0.000 & 0.000 \\
\hline 2002B9 & 0.000 & 0.000 \\
\hline $2002 \mathrm{C} 1$ & 0.000 & 0.000 \\
\hline $2002 \mathrm{C} 10$ & 0.000 & 0.000 \\
\hline $2002 \mathrm{C} 2$ & 0.000 & 0.000 \\
\hline $2002 \mathrm{C} 3$ & 0.000 & 0.000 \\
\hline $2002 \mathrm{C} 4$ & 0.000 & 0.000 \\
\hline $2002 \mathrm{C} 5$ & 0.000 & 0.000 \\
\hline $2002 \mathrm{C} 6$ & 0.000 & 0.000 \\
\hline $2002 \mathrm{C} 7$ & 0.000 & 0.000 \\
\hline $2002 \mathrm{C} 8$ & 0.000 & 0.000 \\
\hline $2002 \mathrm{C} 9$ & 0.000 & -0.305 \\
\hline 2002D1 & 6.889 & -1.158 \\
\hline 2002D10 & 0.000 & 0.000 \\
\hline 2002D2 & 0.000 & 0.000 \\
\hline 2002D3 & 0.000 & 0.000 \\
\hline 2002D5 & 0.000 & 0.000 \\
\hline 2002D6 & 0.000 & 0.000 \\
\hline $2002 \mathrm{D} 7$ & 0.000 & 0.000 \\
\hline 2002D 8 & -0.091 & 0.000 \\
\hline 2002D9 & -0.305 & 0.152 \\
\hline $2002 \mathrm{E} 1$ & 0.000 & 0.000 \\
\hline $2002 \mathrm{E} 10$ & 0.000 & 0.000 \\
\hline $2002 \mathrm{E} 2$ & 0.000 & 0.000 \\
\hline $2002 \mathrm{E} 3$ & 0.000 & 0.000 \\
\hline $2002 \mathrm{E} 4$ & 0.000 & 0.671 \\
\hline 2002E5 & 0.000 & 0.000 \\
\hline 2002E6 & 0.000 & 0.000 \\
\hline $2002 \mathrm{E} 7$ & 0.000 & 0.000 \\
\hline $2002 \mathrm{E} 8$ & 0.000 & 0.000 \\
\hline 2002E9 & 0.061 & 0.000 \\
\hline $2002 \mathrm{~F} 1$ & -0.061 & 0.000 \\
\hline $2002 \mathrm{~F} 10$ & 0.000 & 0.000 \\
\hline
\end{tabular}

Continued on Next Page. 
Table D.2 - Continued

\begin{tabular}{|c|c|c|}
\hline Section Code & Distance Offset (m) & Elevation Offset $(\mathrm{m})$ \\
\hline $2002 \mathrm{~F} 2$ & 0.000 & 0.000 \\
\hline $2002 \mathrm{~F} 3$ & 0.000 & 0.000 \\
\hline $2002 \mathrm{~F} 4$ & 0.000 & 0.000 \\
\hline $2002 \mathrm{~F} 5$ & 0.000 & 0.000 \\
\hline $2002 \mathrm{~F} 6$ & -0.183 & 0.000 \\
\hline $2002 \mathrm{~F} 7$ & 0.000 & 0.000 \\
\hline $2002 \mathrm{~F} 8$ & 0.000 & 0.000 \\
\hline $2002 \mathrm{~F} 9$ & 0.000 & 0.000 \\
\hline 2003A1 & 0.000 & 0.000 \\
\hline 2003A10 & 0.000 & 0.000 \\
\hline 2003A2 & 0.000 & 0.000 \\
\hline $2003 \mathrm{~A} 3$ & 0.000 & 0.000 \\
\hline 2003A4 & 0.000 & 0.000 \\
\hline 2003A5 & 0.000 & 0.000 \\
\hline $2003 \mathrm{~A} 6$ & 0.101 & -0.113 \\
\hline $2003 \mathrm{~A} 7$ & 0.000 & 0.000 \\
\hline $2003 \mathrm{~A} 8$ & 0.000 & 0.000 \\
\hline 2003A9 & 0.000 & 0.000 \\
\hline 2003B1 & 0.000 & 0.000 \\
\hline 2003B10 & 0.000 & -0.244 \\
\hline 2003B2 & 0.000 & 0.000 \\
\hline 2003B3 & 0.000 & 0.000 \\
\hline 2003B4 & 0.000 & 0.000 \\
\hline 2003B5 & -0.238 & 0.030 \\
\hline 2003B 6 & 0.000 & 0.000 \\
\hline 2003B7 & 0.000 & -0.549 \\
\hline 2003B8 & 0.000 & 0.000 \\
\hline 2003B9 & 0.000 & 0.000 \\
\hline 2004A1 & 0.000 & 0.000 \\
\hline $2004 \mathrm{~A} 10$ & 0.000 & 0.000 \\
\hline 2004A2 & 0.000 & 0.000 \\
\hline 2004A3 & 0.000 & 0.000 \\
\hline $2004 \mathrm{~A} 4$ & 0.000 & 0.000 \\
\hline 2004A5 & 0.000 & 0.000 \\
\hline 2004A6 & 0.183 & -0.076 \\
\hline $2004 \mathrm{~A} 7$ & 0.000 & 0.000 \\
\hline $2004 \mathrm{~A} 8$ & 0.000 & 0.000 \\
\hline 2004A9 & 0.000 & 0.000 \\
\hline 2004B1 & 0.000 & 0.000 \\
\hline 2004B10 & 0.000 & 0.000 \\
\hline 2004B2 & 0.000 & 0.000 \\
\hline 2004B3 & 0.030 & 0.000 \\
\hline 2004B4 & 0.000 & 0.000 \\
\hline 2004B5 & 0.030 & -0.152 \\
\hline 2004B6 & 0.000 & 0.000 \\
\hline 2004B7 & 0.000 & 0.000 \\
\hline 2004B8 & 0.000 & 0.000 \\
\hline 2004B9 & 0.122 & 0.000 \\
\hline $2004 \mathrm{C} 1$ & 0.274 & -0.140 \\
\hline $2004 \mathrm{C} 10$ & 0.000 & 0.000 \\
\hline $2004 \mathrm{C} 2$ & 0.000 & 0.000 \\
\hline $2004 \mathrm{C} 3$ & 0.000 & 0.000 \\
\hline
\end{tabular}

Continued on Next Page. . 
Table D.2 - Continued

\begin{tabular}{|c|c|c|}
\hline Section Code & Distance Offset $(\mathrm{m})$ & Elevation Offset $(\mathrm{m})$ \\
\hline $2004 \mathrm{C} 4$ & 0.000 & 0.000 \\
\hline $2004 \mathrm{C} 5$ & 0.366 & -0.091 \\
\hline $2004 \mathrm{C} 6$ & 0.000 & 0.000 \\
\hline $2004 \mathrm{C} 7$ & 0.000 & 0.000 \\
\hline $2004 \mathrm{C} 8$ & 0.000 & 0.000 \\
\hline $2004 \mathrm{C} 9$ & 0.000 & 0.000 \\
\hline 2004D1 & 0.000 & 0.000 \\
\hline $2004 \mathrm{D} 10$ & 0.000 & 0.000 \\
\hline 2004D2 & 0.000 & 0.000 \\
\hline 2004D3 & 0.000 & 0.000 \\
\hline 2004D4 & 0.000 & 0.000 \\
\hline 2004D5 & 0.000 & 0.000 \\
\hline 2004D6 & 0.030 & 0.000 \\
\hline 2004D7 & 0.000 & 0.000 \\
\hline 2004D8 & 0.000 & 0.000 \\
\hline 2004D9 & 0.000 & 0.000 \\
\hline $2004 \mathrm{E} 1$ & 0.000 & 0.000 \\
\hline $2004 \mathrm{E} 10$ & 0.000 & 0.000 \\
\hline $2004 \mathrm{E} 2$ & 0.000 & 0.000 \\
\hline $2004 \mathrm{E} 3$ & 0.000 & 0.000 \\
\hline $2004 \mathrm{E} 4$ & 0.000 & 0.000 \\
\hline 2004E5 & 0.000 & 0.000 \\
\hline $2004 \mathrm{E} 6$ & 0.000 & 0.000 \\
\hline $2004 \mathrm{E} 7$ & 0.000 & 0.000 \\
\hline $2004 \mathrm{E} 8$ & 0.000 & 0.000 \\
\hline $2004 \mathrm{E} 9$ & 0.000 & 0.000 \\
\hline $2004 \mathrm{~F} 1$ & 0.000 & 0.000 \\
\hline $2004 \mathrm{~F} 10$ & 0.000 & 0.000 \\
\hline $2004 \mathrm{~F} 2$ & 0.000 & 0.000 \\
\hline 2004F3 & 0.000 & 0.000 \\
\hline $2004 \mathrm{~F} 4$ & 0.000 & 0.000 \\
\hline $2004 \mathrm{~F} 5$ & 0.000 & 0.000 \\
\hline 2004F 6 & 0.000 & 0.000 \\
\hline $2004 \mathrm{~F} 7$ & 0.000 & 0.000 \\
\hline $2004 \mathrm{~F} 8$ & 0.000 & 0.000 \\
\hline 2004F9 & 0.000 & 0.000 \\
\hline $2005 \mathrm{~A} 1$ & 0.000 & 0.000 \\
\hline $2005 \mathrm{~A} 10$ & 0.000 & 0.000 \\
\hline $2005 \mathrm{~A} 2$ & 0.000 & 0.000 \\
\hline 2005 A 3 & 0.000 & 0.000 \\
\hline $2005 \mathrm{~A} 4$ & 0.000 & 0.000 \\
\hline 2005A5 & 0.000 & 0.000 \\
\hline 2005A 6 & 0.213 & -0.091 \\
\hline 2005A 7 & 0.000 & 0.000 \\
\hline 2005 A 8 & 0.000 & 0.000 \\
\hline 2005A9 & 0.000 & 0.000 \\
\hline 2005B1 & 0.000 & 0.000 \\
\hline 2005B10 & 0.000 & 0.000 \\
\hline 2005B2 & 0.000 & 0.000 \\
\hline 2005B3 & 0.030 & 0.000 \\
\hline 2005B4 & 0.000 & 0.000 \\
\hline 2005B5 & 0.091 & 0.000 \\
\hline
\end{tabular}

Continued on Next Page. . 
Table D.2 - Continued

\begin{tabular}{|c|c|c|}
\hline Section Code & Distance Offset $(\mathrm{m})$ & Elevation Offset $(\mathrm{m})$ \\
\hline 2005B 6 & 0.000 & 0.000 \\
\hline 2005B7 & 0.000 & 0.000 \\
\hline 2005B8 & 0.183 & -0.037 \\
\hline 2005B9 & 0.122 & -0.488 \\
\hline $2005 \mathrm{C} 1$ & 0.274 & -0.216 \\
\hline $2005 \mathrm{C} 2$ & 0.000 & 0.000 \\
\hline $2005 \mathrm{C} 3$ & 0.000 & 0.000 \\
\hline $2005 \mathrm{C} 4$ & 0.000 & 0.000 \\
\hline $2005 \mathrm{C} 5$ & 0.244 & -0.091 \\
\hline $2005 \mathrm{C} 6$ & 0.000 & 0.000 \\
\hline $2005 \mathrm{C} 7$ & 0.000 & 0.000 \\
\hline 2005D1 & 0.000 & 0.000 \\
\hline $2005 \mathrm{D} 10$ & 0.000 & 0.000 \\
\hline 2005D2 & 0.000 & 0.000 \\
\hline 2005D3 & 0.000 & 0.000 \\
\hline 2005 D4 & -0.091 & 0.000 \\
\hline 2005D5 & 0.000 & 0.000 \\
\hline 2005D6 & 0.061 & 0.000 \\
\hline 2005D7 & 0.000 & 0.000 \\
\hline 2005D8 & 0.000 & 0.000 \\
\hline 2005D9 & 0.000 & 0.000 \\
\hline $2005 \mathrm{E} 1$ & 0.000 & 0.000 \\
\hline $2005 \mathrm{E} 10$ & 0.000 & 0.000 \\
\hline $2005 \mathrm{E} 2$ & 0.000 & 0.000 \\
\hline $2005 \mathrm{E} 3$ & 0.000 & 0.000 \\
\hline $2005 \mathrm{E} 4$ & 0.091 & -0.061 \\
\hline 2005E5 & 0.000 & 0.000 \\
\hline 2005E6 & 0.000 & 0.000 \\
\hline $2005 \mathrm{E} 7$ & 0.000 & 0.000 \\
\hline $2005 \mathrm{E} 8$ & 0.000 & 0.000 \\
\hline 2005E9 & 0.000 & 0.000 \\
\hline $2005 \mathrm{~F} 1$ & 0.000 & 0.000 \\
\hline $2005 \mathrm{~F} 10$ & -0.610 & 0.000 \\
\hline $2005 \mathrm{~F} 2$ & 0.000 & 0.000 \\
\hline 2005F 3 & 0.000 & 0.000 \\
\hline $2005 \mathrm{~F} 4$ & 0.000 & 0.000 \\
\hline 2005 F 5 & 0.000 & 0.000 \\
\hline $2005 \mathrm{~F} 6$ & 0.000 & 0.000 \\
\hline $2005 \mathrm{~F} 7$ & 0.000 & 0.000 \\
\hline 2005 F 8 & 0.000 & 0.000 \\
\hline 2005F9 & 0.000 & 0.000 \\
\hline 2006A1 & 0.000 & 0.000 \\
\hline 2006A10 & 0.000 & 0.000 \\
\hline 2006A2 & 0.000 & 0.000 \\
\hline 2006A3 & 0.000 & 0.000 \\
\hline 2006A4 & 0.000 & 0.000 \\
\hline 2006A5 & 0.000 & 0.000 \\
\hline 2006A 6 & 0.274 & -0.107 \\
\hline $2006 \mathrm{~A} 7$ & 0.000 & 0.000 \\
\hline $2006 \mathrm{~A} 8$ & 0.000 & 0.000 \\
\hline 2006A9 & 0.000 & 0.000 \\
\hline 2006B1 & 0.000 & 0.000 \\
\hline
\end{tabular}

Continued on Next Page. . 
Table D.2 - Continued

\begin{tabular}{|c|c|c|}
\hline Section Code & Distance Offset $(\mathrm{m})$ & Elevation Offset $(\mathrm{m})$ \\
\hline 2006B10 & 0.000 & 0.000 \\
\hline 2006B2 & 0.000 & 0.000 \\
\hline 2006B3 & 0.061 & 0.000 \\
\hline 2006B4 & 0.000 & 0.000 \\
\hline 2006B5 & 0.061 & 0.000 \\
\hline 2006B6 & 0.000 & 0.000 \\
\hline 2006B7 & 0.000 & 0.000 \\
\hline 2006B8 & 0.244 & -0.107 \\
\hline 2006B9 & 0.122 & -0.488 \\
\hline $2006 \mathrm{C} 1$ & 0.366 & -0.442 \\
\hline $2006 \mathrm{C} 10$ & 0.000 & 0.000 \\
\hline $2006 \mathrm{C} 2$ & 0.000 & 0.000 \\
\hline $2006 \mathrm{C} 3$ & 0.000 & 0.000 \\
\hline $2006 \mathrm{C} 4$ & 0.000 & 0.000 \\
\hline 2006C5 & 0.122 & -0.091 \\
\hline $2006 \mathrm{C} 6$ & 0.000 & 0.000 \\
\hline $2006 \mathrm{C} 7$ & 0.000 & 0.000 \\
\hline $2006 \mathrm{C} 8$ & 0.000 & 0.000 \\
\hline $2006 \mathrm{C} 9$ & 0.000 & 0.000 \\
\hline 2006D1 & 0.000 & 0.000 \\
\hline $2006 \mathrm{D} 10$ & 0.000 & 0.000 \\
\hline 2006D2 & 0.000 & 0.000 \\
\hline 2006D3 & 0.000 & 0.000 \\
\hline 2006D4 & 0.000 & 0.000 \\
\hline 2006D5 & 0.000 & 0.000 \\
\hline 2006D6 & 0.091 & -0.030 \\
\hline 2006D7 & 0.000 & 0.000 \\
\hline 2006D8 & 0.000 & 0.000 \\
\hline 2006D9 & 0.000 & 0.000 \\
\hline 2006E1 & 0.000 & 0.000 \\
\hline $2006 \mathrm{E} 10$ & 0.000 & 0.091 \\
\hline 2006E2 & 0.000 & 0.000 \\
\hline $2006 \mathrm{E} 3$ & 0.000 & 0.000 \\
\hline 2006E4 & 0.091 & -0.061 \\
\hline 2006E5 & 0.000 & 0.000 \\
\hline 2006E6 & 0.000 & 0.000 \\
\hline 2006E7 & 0.000 & 0.000 \\
\hline $2006 \mathrm{E} 8$ & 0.000 & 0.000 \\
\hline 2006E9 & 0.000 & 0.000 \\
\hline $2006 \mathrm{~F} 1$ & 0.000 & 0.000 \\
\hline $2006 \mathrm{~F} 10$ & 0.000 & 0.000 \\
\hline $2006 \mathrm{~F} 2$ & 0.000 & 0.000 \\
\hline 2006F3 & 0.000 & 0.000 \\
\hline $2006 \mathrm{~F} 4$ & 0.000 & 0.000 \\
\hline 2006 F5 & 0.000 & 0.000 \\
\hline $2006 \mathrm{~F} 6$ & 0.000 & 0.000 \\
\hline $2006 \mathrm{~F} 7$ & 0.000 & 0.000 \\
\hline $2006 \mathrm{~F} 8$ & 0.000 & 0.000 \\
\hline 2006F9 & 0.000 & 0.000 \\
\hline $2007 \mathrm{~A} 1$ & 0.000 & 0.000 \\
\hline $2007 \mathrm{~A} 10$ & 0.000 & 0.000 \\
\hline 2007A2 & 0.000 & 0.000 \\
\hline
\end{tabular}

Continued on Next Page. . 
APPENDIX D. SURVEY DATA

Table D.2 - Continued

\begin{tabular}{|c|c|c|}
\hline Section Code & Distance Offset $(\mathrm{m})$ & Elevation Offset $(\mathrm{m})$ \\
\hline 2007A3 & 0.000 & 0.000 \\
\hline 2007A4 & 0.000 & 0.000 \\
\hline 2007A5 & 0.000 & 0.000 \\
\hline $2007 \mathrm{~A} 6$ & 0.366 & -0.198 \\
\hline $2007 \mathrm{~A} 7$ & 0.000 & 0.000 \\
\hline $2007 \mathrm{~A} 8$ & 0.000 & 0.000 \\
\hline 2007A9 & 0.000 & 0.000 \\
\hline 2007B1 & 0.000 & 0.000 \\
\hline 2007B10 & 0.000 & 0.000 \\
\hline 2007B2 & 0.000 & 0.000 \\
\hline 2007B3 & 0.061 & 0.000 \\
\hline 2007B4 & 0.000 & 0.000 \\
\hline 2007B5 & 0.457 & -0.094 \\
\hline 2007B6 & 0.000 & 0.000 \\
\hline 2007B7 & 0.000 & 0.000 \\
\hline 2007B8 & 0.305 & -0.168 \\
\hline 2007B9 & 0.122 & -0.488 \\
\hline $2007 \mathrm{C} 1$ & 0.335 & -0.265 \\
\hline $2007 \mathrm{C} 2$ & 0.152 & 0.000 \\
\hline $2007 \mathrm{C} 3$ & 0.000 & 0.000 \\
\hline $2007 \mathrm{C} 4$ & 0.000 & 0.000 \\
\hline $2007 \mathrm{C} 5$ & 0.122 & -0.091 \\
\hline $2007 \mathrm{C} 6$ & 0.000 & 0.000 \\
\hline $2007 \mathrm{C} 7$ & 0.000 & 0.000 \\
\hline $2007 \mathrm{C} 8$ & 0.000 & 0.000 \\
\hline 2007C9 & 0.000 & 0.000 \\
\hline 2007D1 & 0.000 & 0.000 \\
\hline 2007D10 & 0.000 & 0.000 \\
\hline 2007D2 & 0.000 & 0.000 \\
\hline 2007D3 & 0.000 & 0.000 \\
\hline 2007D4 & 0.061 & 0.000 \\
\hline 2007D5 & 0.000 & -0.128 \\
\hline 2007D6 & 0.091 & -0.030 \\
\hline 2007D7 & 0.000 & 0.000 \\
\hline 2007D8 & 0.000 & 0.000 \\
\hline 2007D9 & 0.000 & 0.000 \\
\hline $2007 \mathrm{E} 1$ & 0.000 & 0.000 \\
\hline $2007 \mathrm{E} 10$ & 0.030 & 0.000 \\
\hline 2007E2 & 0.000 & 0.000 \\
\hline 2007E3 & 0.000 & 0.000 \\
\hline $2007 \mathrm{E} 4$ & 0.091 & -0.061 \\
\hline 2007E5 & 0.000 & -0.305 \\
\hline 2007E6 & 0.000 & 0.000 \\
\hline $2007 \mathrm{E} 7$ & 0.000 & 0.000 \\
\hline $2007 \mathrm{E} 8$ & 0.000 & 0.000 \\
\hline 2007E9 & 0.000 & 0.000 \\
\hline $2007 \mathrm{~F} 1$ & -0.030 & 0.000 \\
\hline $2007 \mathrm{~F} 10$ & 0.000 & 0.000 \\
\hline 2007F2 & 0.000 & 0.000 \\
\hline 2007F3 & 0.000 & 0.000 \\
\hline $2007 \mathrm{~F} 4$ & 0.000 & 0.000 \\
\hline $2007 \mathrm{~F} 5$ & 0.000 & 0.000 \\
\hline
\end{tabular}

Continued on Next Page. . 
Table D.2 - Continued

\begin{tabular}{|c|c|c|}
\hline Section Code & Distance Offset $(\mathrm{m})$ & Elevation Offset $(\mathrm{m})$ \\
\hline $2007 \mathrm{~F} 6$ & 0.000 & 0.000 \\
\hline $2007 \mathrm{~F} 7$ & 0.000 & 0.000 \\
\hline $2007 \mathrm{~F} 8$ & 0.000 & 0.000 \\
\hline 2007F9 & 0.000 & 0.000 \\
\hline 2008A1 & 0.000 & 0.000 \\
\hline $2008 \mathrm{~A} 10$ & 0.000 & 0.000 \\
\hline 2008A2 & 0.000 & 0.000 \\
\hline 2008 A 3 & 0.000 & 0.000 \\
\hline $2008 \mathrm{~A} 4$ & 0.000 & 0.000 \\
\hline 2008A5 & 0.000 & 0.000 \\
\hline $2008 \mathrm{~A} 6$ & 0.122 & -0.241 \\
\hline $2008 \mathrm{~A} 7$ & 0.000 & 0.000 \\
\hline $2008 \mathrm{~A} 8$ & 0.000 & 0.000 \\
\hline 2008A9 & 0.000 & 0.000 \\
\hline 2008B1 & 0.000 & 0.000 \\
\hline 2008B10 & 0.000 & 0.000 \\
\hline 2008B2 & 0.000 & 0.000 \\
\hline 2008B3 & 0.000 & 0.000 \\
\hline 2008B4 & 0.000 & 0.000 \\
\hline 2008B5 & 0.427 & -0.070 \\
\hline 2008B6 & 0.000 & 0.000 \\
\hline 2008B7 & -0.152 & 0.000 \\
\hline 2008B8 & 0.091 & -0.061 \\
\hline 2008B9 & 0.000 & 0.000 \\
\hline $2008 \mathrm{C} 1$ & 0.000 & -0.073 \\
\hline $2008 \mathrm{C} 10$ & 0.000 & 0.000 \\
\hline $2008 \mathrm{C} 2$ & 0.000 & 0.000 \\
\hline $2008 \mathrm{C} 3$ & 0.000 & 0.000 \\
\hline $2008 \mathrm{C} 4$ & 0.000 & 0.000 \\
\hline $2008 \mathrm{C} 5$ & 0.000 & 0.000 \\
\hline $2008 \mathrm{C} 6$ & 0.000 & 0.000 \\
\hline $2008 \mathrm{C} 7$ & 0.000 & 0.000 \\
\hline $2008 \mathrm{C} 8$ & 0.000 & 0.000 \\
\hline $2008 \mathrm{C} 9$ & 0.000 & 0.000 \\
\hline 2008D1 & 6.706 & -1.158 \\
\hline 2008D10 & 0.000 & 0.000 \\
\hline 2008D2 & 0.000 & 0.000 \\
\hline 2008D3 & 0.000 & 0.000 \\
\hline 2008D4 & -0.030 & -0.107 \\
\hline 2008D5 & 0.000 & -0.024 \\
\hline 2008D6 & 0.091 & -0.030 \\
\hline 2008D7 & 0.000 & 0.000 \\
\hline 2008D8 & -0.122 & 0.000 \\
\hline 2008D9 & -0.183 & 0.000 \\
\hline 2008E1 & 0.000 & 0.000 \\
\hline $2008 \mathrm{E} 10$ & 0.000 & 0.000 \\
\hline 2008E2 & -0.091 & 0.000 \\
\hline 2008E3 & 0.000 & 0.000 \\
\hline $2008 \mathrm{E} 4$ & 0.030 & -0.061 \\
\hline 2008E5 & 0.000 & 0.000 \\
\hline 2008E6 & -0.091 & 0.000 \\
\hline 2008E7 & 0.000 & 0.000 \\
\hline
\end{tabular}

Continued on Next Page... 
Table D.2 - Continued

\begin{tabular}{ccc}
\hline Section Code & Distance Offset $(\mathrm{m})$ & Elevation Offset $(\mathrm{m})$ \\
\hline $2008 \mathrm{E} 8$ & 0.030 & 0.000 \\
$2008 \mathrm{E} 9$ & 0.000 & 0.000 \\
$2008 \mathrm{~F} 1$ & 0.000 & 0.000 \\
$2008 \mathrm{~F} 10$ & 0.000 & 0.000 \\
$2008 \mathrm{~F} 2$ & 0.000 & 0.000 \\
$2008 \mathrm{~F} 3$ & 0.000 & 0.000 \\
$2008 \mathrm{~F} 4$ & 0.000 & 0.000 \\
$2008 \mathrm{~F} 5$ & 0.000 & 0.000 \\
$2008 \mathrm{~F} 6$ & 0.000 & 0.000 \\
$2008 \mathrm{~F} 7$ & 0.000 & 0.000 \\
$2008 \mathrm{~F} 8$ & 0.000 & 0.000 \\
$2008 \mathrm{~F} 9$ & 0.000 & 0.000 \\
\hline \hline
\end{tabular}

\section{D.3 Longitudinal Profile Data}

Table D.3: Permanent point and rebar coordinates. Units are in feet, CA State Plane Coordinates.

\begin{tabular}{ccccc}
\hline Reach & Northing & Easting & Elevation & Description \\
\hline A & 1850675.23 & 6058274.92 & 92.42 & CONTROL A1 \\
A & 1850663.37 & 6058322.63 & 93.81 & PPA \\
A & 1850788.23 & 6058374.22 & 91.32 & CONTROL A2 \\
A & 1850728.22 & 6058268.43 & 91.37 & PPA2 \\
A & 1850770.94 & 6058340.74 & 93.96 & PPA3 \\
A & 1850783.74 & 6058300.56 & 107.53 & ST8 SPK ANGL PT \\
A & 1850743.00 & 6058265.17 & 93.80 & TOP REB XS10R \\
A & 1850750.93 & 6058280.51 & 94.37 & TOP REB XS789R \\
A & 1850756.40 & 6058297.08 & 94.12 & TOP REB XS6R \\
A & 1850677.39 & 6058275.46 & 93.79 & TOP REB XS10L \\
A & 1850684.12 & 6058290.75 & 93.60 & TOP REB XS9L \\
A & 1850691.69 & 6058305.56 & 94.40 & TOP REB XS8L \\
A & 1850703.99 & 6058319.87 & 93.65 & TOP REB XS7L \\
A & 1850717.63 & 6058327.78 & 94.25 & TOP REB XS6L \\
A & 1850769.33 & 6058350.90 & 93.29 & TOP REB XS45R \\
A & 1850791.80 & 6058375.80 & 96.76 & TOP REB XS3R \\
A & 1850793.58 & 6058386.66 & 95.78 & TOP REB XS2R \\
A & 1850799.00 & 6058399.20 & 94.02 & TOP REB XS1R \\
A & 1850772.58 & 6058413.34 & 94.20 & TOP REB XS1L \\
A & 1850763.93 & 6058405.56 & 95.79 & TOP REB XS2L \\
A & 1850758.65 & 6058388.21 & 96.48 & TOP REB XS3L \\
A & 1850751.20 & 6058373.33 & 93.81 & TOP REB XS4L \\
A & 1850746.39 & 6058363.02 & 93.32 & TOP REB XS5L \\
\hline B & 1851072.04 & 6059560.93 & 143.43 & PPB \\
B & 1851103.87 & 6059487.00 & 139.72 & PPB2 \\
B & 1851092.38 & 6059602.13 & 152.06 & PPB3 \\
B & 1851044.01 & 6059487.05 & 131.45 & TOP REB XS10L \\
B & 1851054.18 & 6059498.98 & 131.71 & TOP REB XS9L \\
B & 1851067.45 & 6059510.11 & 127.22 & TOP REB XS8L \\
\hline D Next Page. . & & \\
\hline
\end{tabular}


Table D.3 - Continued

\begin{tabular}{|c|c|c|c|c|}
\hline Reach & Northing & Easting & Elevation & Description \\
\hline B & 1851074.17 & 6059524.77 & 129.96 & TOP REB XS7L \\
\hline B & 1851081.90 & 6059534.57 & 128.96 & TOP REB XS6L \\
\hline B & 1851090.89 & 6059545.80 & 130.41 & TOP REB XS5L \\
\hline B & 1851090.11 & 6059546.32 & 129.84 & BOTT REB XS5L \\
\hline B & 1851101.28 & 6059556.62 & 129.16 & TOP REB XS4L \\
\hline B & 1851107.80 & 6059568.05 & 131.61 & TOP REB XS3L \\
\hline B & 1851111.79 & 6059580.57 & 134.22 & TOP REB XS2L \\
\hline B & 1851118.79 & 6059589.80 & 133.88 & TOP REB XS1L \\
\hline B & 1851088.31 & 6059462.29 & 131.40 & TOP REB XS10R \\
\hline B & 1851091.57 & 6059473.15 & 131.56 & TOP REB XS9R \\
\hline B & 1851081.95 & 6059500.42 & 126.58 & TOP REB XS8R \\
\hline $\mathrm{B}$ & 1851098.04 & 6059504.61 & 130.03 & TOP REB XS7R \\
\hline B & 1851102.96 & 6059519.67 & 128.92 & TOP REB XS6R \\
\hline B & 1851111.27 & 6059530.21 & 130.78 & TOP REB XS5R \\
\hline B & 1851120.53 & 6059541.88 & 129.11 & TOP REB XS4R \\
\hline B & 1851131.02 & 6059553.50 & 131.43 & TOP REB XS3R \\
\hline $\mathrm{B}$ & 1851142.87 & 6059565.40 & 134.19 & TOP REB XS2R \\
\hline B & 1851148.35 & 6059574.71 & 133.76 & TOP REB XS1R \\
\hline $\mathrm{C}$ & 1852046.61 & 6062592.30 & 318.45 & PPC \\
\hline $\mathrm{C}$ & 1852027.81 & 6062502.69 & 297.64 & $\mathrm{PPC} 2$ \\
\hline $\mathrm{C}$ & 1852064.69 & 6062656.95 & 321.92 & PPC3 \\
\hline $\mathrm{C}$ & 1852025.69 & 6062642.64 & 304.62 & CONTROL C1 \\
\hline C & 1852030.25 & 6062656.94 & 309.67 & TOP REB XS2R \\
\hline $\mathrm{C}$ & 1852034.01 & 6062642.21 & 308.14 & TOP REB XS3R \\
\hline $\mathrm{C}$ & 1852025.54 & 6062628.83 & 308.02 & TOP REB XS4R \\
\hline C & 1852021.17 & 6062613.46 & 307.58 & TOP REB XS5R \\
\hline $\mathrm{C}$ & 1851980.64 & 6062620.57 & 307.65 & TOP REB XS5L \\
\hline $\mathrm{C}$ & 1851987.42 & 6062638.08 & 308.05 & TOP REB XS4L \\
\hline $\mathrm{C}$ & 1851988.63 & 6062651.26 & 308.14 & TOP REB XS3L \\
\hline C & 1851989.71 & 6062661.85 & 309.77 & TOP REB XS2L \\
\hline $\mathrm{C}$ & 1852004.94 & 6062679.47 & 307.90 & TOP REB XS1L \\
\hline $\mathrm{C}$ & 1852058.78 & 6062489.78 & 301.13 & TOP REB XS10R \\
\hline $\mathrm{C}$ & 1852049.11 & 6062500.43 & 300.03 & TOP REB XS9R \\
\hline $\mathrm{C}$ & 1852049.62 & 6062500.61 & 299.96 & BOT REB XS9R \\
\hline $\mathrm{C}$ & 1852042.50 & 6062508.94 & 301.61 & TOP REB XS8R \\
\hline $\mathrm{C}$ & 1852037.51 & 6062519.04 & 301.74 & TOP REB XS7R \\
\hline $\mathrm{C}$ & 1852034.28 & 6062534.01 & 302.55 & TOP REB XS6R \\
\hline $\mathrm{C}$ & 1851992.13 & 6062528.45 & 302.58 & TOP REB XS6L \\
\hline $\mathrm{C}$ & 1851993.73 & 6062516.73 & 301.61 & TOP REB XS7L \\
\hline $\mathrm{C}$ & 1851997.98 & 6062497.22 & 301.51 & TOP REB XS8L \\
\hline $\mathrm{C}$ & 1852003.12 & 6062478.68 & 301.72 & TOP REB XS9L \\
\hline $\mathrm{C}$ & 1852009.98 & 6062464.05 & 301.10 & TOP REB XS10L \\
\hline $\mathrm{C}$ & 1852043.26 & 6062664.67 & 308.30 & TOP REB XS1R \\
\hline $\mathrm{D}$ & 1852517.08 & 6063094.09 & 356.55 & PPD2 \\
\hline $\mathrm{D}$ & 1852635.66 & 6063198.30 & 363.37 & PPD3 \\
\hline $\mathrm{D}$ & 1852530.48 & 6063167.67 & 362.94 & PPD \\
\hline $\mathrm{D}$ & 1852478.55 & 6063071.62 & 353.01 & TOP REB XS10R \\
\hline $\mathrm{D}$ & 1852494.53 & 6063082.69 & 352.81 & TOP REB XS9R \\
\hline $\mathrm{D}$ & 1852495.05 & 6063082.35 & 351.96 & BOT REB XS9R \\
\hline $\mathrm{D}$ & 1852503.49 & 6063097.82 & 355.52 & TOP REB XS8R \\
\hline $\mathrm{D}$ & 1852515.42 & 6063105.98 & 355.89 & TOP REB XS7R \\
\hline $\mathrm{D}$ & 1852529.86 & 6063109.67 & 357.60 & TOP REB XS6R \\
\hline
\end{tabular}

Continued on Next Page... 
Table D.3 - Continued

\begin{tabular}{|c|c|c|c|c|}
\hline Reach & Northing & Easting & Elevation & Description \\
\hline $\mathrm{D}$ & 1852505.87 & 6063131.92 & 357.48 & TOP REB XS6L \\
\hline $\mathrm{D}$ & 1852498.54 & 6063124.78 & 355.95 & TOP REB XS7L \\
\hline $\mathrm{D}$ & 1852488.30 & 6063114.37 & 355.35 & TOP REB XS8L \\
\hline $\mathrm{D}$ & 1852479.96 & 6063101.61 & 352.60 & TOP REB XS9L \\
\hline $\mathrm{D}$ & 1852479.51 & 6063102.03 & 352.04 & BOT REB XS9L \\
\hline $\mathrm{D}$ & 1852465.18 & 6063092.16 & 352.97 & TOP REB XS10L \\
\hline $\mathrm{D}$ & 1852677.68 & 6063220.75 & 372.48 & TOP REB XS1R \\
\hline $\mathrm{D}$ & 1852661.14 & 6063219.74 & 364.87 & TOP REB XS2R \\
\hline $\mathrm{D}$ & 1852653.05 & 6063211.35 & 363.89 & TOP REB XS3R \\
\hline $\mathrm{D}$ & 1852652.38 & 6063194.90 & 372.86 & TOP UP REB XS4R \\
\hline $\mathrm{D}$ & 1852609.19 & 6063218.50 & 364.78 & TOP REB XS5L \\
\hline $\mathrm{D}$ & 1852622.97 & 6063229.45 & 369.16 & TOP UP REB XS4L \\
\hline $\mathrm{D}$ & 1852635.89 & 6063243.17 & 363.90 & TOP REB XS3L \\
\hline $\mathrm{D}$ & 1852648.04 & 6063247.80 & 364.86 & TOP REB XS2L \\
\hline $\mathrm{D}$ & 1852661.76 & 6063253.66 & 367.30 & TOP REB XS1L \\
\hline $\mathrm{D}$ & 1852638.51 & 6063197.87 & 364.76 & TOP REB XS5R \\
\hline $\mathrm{E}$ & 1854582.28 & 6065303.16 & 631.69 & PPE \\
\hline $\mathrm{E}$ & 1854568.56 & 6065221.48 & 627.37 & PPE2 \\
\hline $\mathrm{E}$ & 1854635.41 & 6065364.13 & 635.21 & TOP REB XS1L \\
\hline $\mathrm{E}$ & 1854628.78 & 6065349.74 & 634.10 & TOP REB XS2L \\
\hline $\mathrm{E}$ & 1854617.12 & 6065341.15 & 634.61 & TOP REB XS3L \\
\hline $\mathrm{E}$ & 1854607.69 & 6065327.61 & 631.80 & TOP REB XS4L \\
\hline $\mathrm{E}$ & 1854598.02 & 6065316.33 & 629.85 & TOP REB XS5L \\
\hline $\mathrm{E}$ & 1854590.05 & 6065303.43 & 629.84 & TOP REB XS6L \\
\hline $\mathrm{E}$ & 1854580.32 & 6065292.22 & 628.77 & TOP REB XS7L \\
\hline $\mathrm{E}$ & 1854572.58 & 6065279.19 & 628.10 & TOP REB XS8L \\
\hline $\mathrm{E}$ & 1854561.33 & 6065269.11 & 630.99 & TOP REB XS9L \\
\hline $\mathrm{E}$ & 1854557.00 & 6065253.93 & 628.75 & TOP REB XS10L \\
\hline $\mathrm{E}$ & 1854577.47 & 6065243.01 & 629.35 & TOP REB XS10R \\
\hline $\mathrm{E}$ & 1854584.69 & 6065255.81 & 630.93 & TOP REB XS9R \\
\hline $\mathrm{E}$ & 1854590.89 & 6065267.28 & 628.08 & TOP REB XS8R \\
\hline $\mathrm{E}$ & 1854597.62 & 6065279.69 & 628.74 & TOP REB XS7R \\
\hline $\mathrm{E}$ & 1854603.59 & 6065292.09 & 629.82 & TOP REB XS6R \\
\hline $\mathrm{E}$ & 1854618.75 & 6065300.66 & 630.86 & TOP REB XS5R \\
\hline $\mathrm{E}$ & 1854628.79 & 6065314.04 & 631.89 & TOP REB XS4R \\
\hline $\mathrm{E}$ & 1854635.85 & 6065326.00 & 634.70 & TOP REB XS3R \\
\hline $\mathrm{E}$ & 1854644.06 & 6065337.93 & 634.12 & TOP REB XS2R \\
\hline $\mathrm{E}$ & 1854653.38 & 6065347.15 & 634.81 & TOP REB XS1R \\
\hline $\mathrm{F}$ & 1852073.82 & 6063156.87 & 351.78 & PPF3 \\
\hline $\mathrm{F}$ & 1852041.64 & 6063244.30 & 355.36 & PPF2 \\
\hline $\mathrm{F}$ & 1851998.21 & 6063315.19 & 362.51 & PPF \\
\hline $\mathrm{F}$ & 1851970.33 & 6063350.19 & 361.64 & TOP REB XS1R \\
\hline $\mathrm{F}$ & 1851977.17 & 6063335.34 & 362.88 & TOP REB XS2R \\
\hline $\mathrm{F}$ & 1851983.38 & 6063324.55 & 361.18 & TOP REB XS3R \\
\hline $\mathrm{F}$ & 1851992.17 & 6063311.65 & 361.30 & TOP REB XS4R \\
\hline $\mathrm{F}$ & 1851998.95 & 6063295.77 & 357.69 & TOP REB XS5R \\
\hline $\mathrm{F}$ & 1852006.60 & 6063286.44 & 357.78 & TOP REB XS6R \\
\hline F & 1852015.00 & 6063274.12 & 356.37 & TOP REB XS7R \\
\hline F & 1852031.55 & 6063267.53 & 357.91 & TOP REB XS8R \\
\hline $\mathrm{F}$ & 1852037.06 & 6063250.62 & 355.05 & TOP REB XS9R \\
\hline $\mathrm{F}$ & 1852038.56 & 6063238.99 & 354.40 & TOP REB XS10R \\
\hline $\mathrm{F}$ & 1852012.32 & 6063220.05 & 355.06 & TOP REB XS10L \\
\hline
\end{tabular}

Continued on Next Page... 
Table D.3 - Continued

\begin{tabular}{ccccc}
\hline Reach & Northing & Easting & Elevation & Description \\
\hline F & 1852004.17 & 6063233.56 & 355.16 & TOP REB XS9L \\
F & 1851996.66 & 6063245.26 & 357.82 & TOP REB XS8L \\
F & 1851989.68 & 6063261.36 & 356.54 & TOP REB XS7L \\
F & 1851982.08 & 6063273.75 & 357.84 & TOP REB XS6L \\
F & 1851976.47 & 6063284.86 & 357.99 & TOP REB XS5L \\
F & 1851966.33 & 6063298.71 & 360.77 & TOP REB XS4L \\
F & 1851960.62 & 6063313.19 & 360.93 & TOP REB XS3L \\
F & 1851951.59 & 6063322.67 & 363.19 & TOP REB XS2L \\
F & 1851942.28 & 6063337.05 & 362.23 & TOP REB XS1L \\
\hline \hline
\end{tabular}

Table D.4: Adjusted longitudinal profile data.

\begin{tabular}{|c|c|c|c|c|c|}
\hline Year & Reach & Point Number & Adjusted Dist (m) & Adjusted Elev (m) & Point Description \\
\hline 2002 & A & 1 & 0.00 & 25.50 & TAILPOOL \\
\hline 2002 & A & 2 & 1.61 & 25.10 & MIDPOOL2.16 \\
\hline 2002 & A & 3 & 2.68 & 25.19 & HEADPOOL. 15 \\
\hline 2002 & A & 4 & 6.55 & 25.74 & TOPRIFF.58 \\
\hline 2002 & A & 5 & 11.01 & 25.75 & TOPRIFF.68 \\
\hline 2002 & A & 6 & 15.17 & 25.98 & TOPRIFF.50 \\
\hline 2002 & A & 7 & 19.65 & 25.91 & TAILRIFF. 83 \\
\hline 2002 & A & 8 & 23.38 & 25.99 & HEADRIFF. 71 \\
\hline 2002 & A & 9 & 25.44 & 26.21 & TOPRIFF.56 \\
\hline 2002 & A & 10 & 26.26 & 26.22 & TAILPOOL. 62 \\
\hline 2002 & A & 11 & 29.12 & 26.05 & MIDPOOL1.25 \\
\hline 2002 & A & 11.5 & 29.39 & 26.07 & 10 \\
\hline 2002 & A & 12 & 32.32 & 26.31 & HEADPOOL.50 \\
\hline 2002 & A & 13 & 33.77 & 26.57 & TOPRIFTPOOL \\
\hline 2002 & A & 13.5 & 34.75 & 26.45 & 9 \\
\hline 2002 & A & 14 & 36.41 & 26.24 & MIDPOOL1.50 \\
\hline 2002 & A & 14.5 & 38.73 & 26.46 & 8 \\
\hline 2002 & A & 15 & 39.42 & 26.52 & TOPPOOL. 57 \\
\hline 2002 & A & 16 & 42.70 & 26.53 & TOPRIFF.20 \\
\hline 2002 & A & 17 & 43.08 & 26.68 & TAILRUN.40 \\
\hline 2002 & A & 17.4 & 43.50 & 26.69 & 7 \\
\hline 2002 & A & 17.5 & 47.04 & 26.84 & 6 \\
\hline 2002 & A & 18 & 49.15 & 26.85 & TAILRUN.40 \\
\hline 2002 & A & 19 & 49.85 & 26.63 & TAILRUN.40 \\
\hline 2002 & A & 19.5 & 63.21 & 26.87 & 5 \\
\hline 2002 & A & 20 & 64.16 & 26.87 & TAILRIFF. 83 \\
\hline 2002 & A & 20.5 & 67.19 & 27.05 & 4 \\
\hline 2002 & A & 21 & 67.52 & 27.07 & TOPRIFF.60 \\
\hline 2002 & A & 22 & 70.04 & 27.09 & TAILPOOL.35 \\
\hline 2002 & A & 23 & 71.40 & 26.81 & MIDPOOL1.33 \\
\hline 2002 & A & 23.5 & 72.40 & 27.10 & 3 \\
\hline 2002 & A & 24 & 72.73 & 27.20 & TOPRIFF.33 \\
\hline 2002 & A & 25 & 74.36 & 27.02 & TAILPOOL. 79 \\
\hline 2002 & A & 26 & 76.66 & 27.00 & MIDPOOL1.08 \\
\hline 2002 & A & 26.5 & 77.34 & 27.06 & 2 \\
\hline 2002 & A & 27 & 79.10 & 27.21 & HEADPOOL. 63 \\
\hline 2002 & A & 27.5 & 81.20 & 27.28 & 1 \\
\hline 2002 & A & 28 & 83.25 & 27.34 & BISRUN.62 \\
\hline
\end{tabular}

Continued on Next Page. . 
Table D.4 - Continued

\begin{tabular}{|c|c|c|c|c|c|}
\hline Year & Reach & Point Number & Adjusted Dist. (m) & Adjusted Elev. (m) & Point Description \\
\hline 2002 & $\mathrm{~A}$ & 29 & 86.57 & 27.41 & TOPRIFF.46 \\
\hline 2002 & A & 30 & 90.49 & 27.52 & TOPRIFF.33 \\
\hline 2002 & A & 31 & 96.68 & 27.54 & TAILRIFF.58 \\
\hline 2002 & A & 32 & 101.28 & 28.02 & TOPRIFF.79 \\
\hline 2003 & $\mathrm{~A}$ & 1 & 24.33 & 26.16 & MID RIFF \\
\hline 2003 & A & 2 & 25.28 & 26.25 & TAIL POOL \\
\hline 2003 & A & 3 & 27.07 & 26.09 & MID POOL \\
\hline 2003 & A & 3.5 & 29.39 & 26.19 & 10 \\
\hline 2003 & $\mathrm{~A}$ & 4 & 29.57 & 26.20 & MID POOL \\
\hline 2003 & A & 5 & 31.36 & 26.31 & HEAD POOL \\
\hline 2003 & A & 6 & 32.35 & 26.38 & MID RIFF \\
\hline 2003 & A & 7 & 33.40 & 26.63 & TAIL POOL \\
\hline 2003 & A & 7.5 & 34.75 & 26.53 & 9 \\
\hline 2003 & A & 8 & 36.31 & 26.39 & MID POOL \\
\hline 2003 & A & 8.5 & 38.73 & 26.52 & 8 \\
\hline 2003 & A & 9 & 39.50 & 26.56 & HEAD POOL \\
\hline 2003 & A & 10 & 43.14 & 26.61 & MID RIFF \\
\hline 2003 & A & 10.5 & 43.50 & 26.72 & 7 \\
\hline 2003 & A & 11 & 43.55 & 26.74 & MID RIFF \\
\hline 2003 & A & 12 & 44.67 & 26.73 & GLIDE \\
\hline 2003 & A & 13 & 46.76 & 26.76 & GLIDE \\
\hline 2003 & A & 13.5 & 47.04 & 26.76 & 6 \\
\hline 2003 & A & 14 & 49.41 & 26.74 & GLIDE \\
\hline 2003 & A & 15 & 52.40 & 26.82 & GLIDE \\
\hline 2003 & A & 16 & 55.75 & 26.85 & GLIDE \\
\hline 2003 & A & 17 & 58.24 & 26.84 & GLIDE \\
\hline 2003 & A & 18 & 58.74 & 26.88 & MID RIFF \\
\hline 2003 & A & 19 & 59.42 & 26.86 & MID RIFF \\
\hline 2003 & A & 20 & 60.07 & 26.89 & MID RIFF \\
\hline 2003 & $\mathrm{~A}$ & 21 & 60.81 & 26.98 & TAIL POOL \\
\hline 2003 & $\mathrm{~A}$ & 22 & 62.65 & 26.79 & MID POOL \\
\hline 2003 & A & 22.5 & 63.21 & 26.84 & 5 \\
\hline 2003 & A & 23 & 64.07 & 26.92 & HEAD POOL \\
\hline 2003 & A & 24 & 65.70 & 27.04 & MID RIFF \\
\hline 2003 & A & 24.5 & 67.19 & 27.07 & 4 \\
\hline 2003 & A & 25 & 67.69 & 27.08 & MID RIFF \\
\hline 2003 & A & 26 & 69.52 & 27.19 & TAIL POOL \\
\hline 2003 & A & 27 & 70.80 & 27.04 & MID POOL \\
\hline 2003 & A & 28 & 71.62 & 26.96 & MID POOL \\
\hline 2003 & A & 29 & 72.38 & 27.14 & HEAD POOL \\
\hline 2003 & A & 29.5 & 72.40 & 27.14 & 3 \\
\hline 2003 & A & 30 & 72.92 & 27.20 & TAIL POOL \\
\hline 2003 & A & 31 & 75.08 & 27.10 & MID POOL \\
\hline 2003 & A & 31.5 & 77.34 & 27.20 & 2 \\
\hline 2003 & A & 32 & 77.91 & 27.23 & HEAD POOL \\
\hline 2003 & $\mathrm{~A}$ & 33 & 80.26 & 27.34 & MID RIFF \\
\hline 2003 & A & 33.5 & 81.20 & 27.35 & 1 \\
\hline 2003 & A & 34 & 82.65 & 27.37 & GLIDE \\
\hline 2003 & A & 35 & 84.89 & 27.43 & GLIDE \\
\hline 2003 & A & 36 & 87.49 & 27.51 & RIFF \\
\hline 2003 & A & 37 & 90.17 & 27.54 & RIFF \\
\hline 2003 & A & 38 & 91.49 & 27.61 & TAIL POOL \\
\hline
\end{tabular}

Continued on Next Page... 
Table D.4 - Continued

\begin{tabular}{|c|c|c|c|c|c|}
\hline Year & Reach & Point Number & Adjusted Dist. (m) & Adjusted Elev. (m) & Point Description \\
\hline 2003 & $\mathrm{~A}$ & 39 & 92.29 & 27.51 & MID POOL \\
\hline 2003 & A & 40 & 94.44 & 27.57 & MID POOL \\
\hline 2003 & A & 41 & 95.97 & 27.69 & HEAD POOL \\
\hline 2003 & A & 42 & 97.63 & 27.66 & MID RIFF \\
\hline 2004 & A & 1 & 22.55 & 26.19 & MID RIFF \\
\hline 2004 & A & 2 & 24.75 & 26.21 & MID RIFF \\
\hline 2004 & A & 3 & 26.72 & 26.22 & MID RIFF \\
\hline 2004 & A & 4 & 28.24 & 26.31 & TAIL POOL \\
\hline 2004 & A & 4.5 & 29.39 & 26.10 & 10 \\
\hline 2004 & $\mathrm{~A}$ & 5 & 29.66 & 26.05 & MID POOL \\
\hline 2004 & A & 6 & 30.12 & 26.13 & HEAD POOL \\
\hline 2004 & A & 7 & 30.47 & 26.63 & TOP STEP \\
\hline 2004 & A & 8 & 32.70 & 26.61 & MID RIFF \\
\hline 2004 & A & 9 & 34.73 & 26.67 & MID RIFF \\
\hline 2004 & A & 9.5 & 34.75 & 26.67 & 9 \\
\hline 2004 & A & 10 & 36.20 & 26.53 & MID RIFF \\
\hline 2004 & A & 11 & 37.10 & 26.49 & MID RIFF \\
\hline 2004 & A & 11.5 & 38.73 & 26.67 & 8 \\
\hline 2004 & A & 12 & 39.25 & 26.72 & MID RIFF \\
\hline 2004 & A & 13 & 41.53 & 26.76 & MID RIFF \\
\hline 2004 & A & 14 & 43.00 & 26.64 & MID RIFF \\
\hline 2004 & A & 14.5 & 43.50 & 26.66 & 7 \\
\hline 2004 & A & 15 & 44.48 & 26.74 & MID RIFF \\
\hline 2004 & A & 16 & 45.87 & 26.80 & MID RIFF \\
\hline 2004 & A & 16.5 & 47.04 & 26.83 & 6 \\
\hline 2004 & A & 17 & 47.46 & 26.84 & MID RIFF \\
\hline 2004 & A & 18 & 49.10 & 26.82 & MID RIFF \\
\hline 2004 & A & 19 & 51.18 & 27.00 & MID RIFF \\
\hline 2004 & $\mathrm{~A}$ & 20 & 52.94 & 26.98 & MID RIFF \\
\hline 2004 & A & 21 & 54.48 & 26.93 & MID RIFF \\
\hline 2004 & $\mathrm{~A}$ & 22 & 55.90 & 27.03 & MID RIFF \\
\hline 2004 & A & 23 & 57.71 & 27.06 & MID RIFF \\
\hline 2004 & A & 24 & 59.07 & 26.95 & MID RIFF \\
\hline 2004 & A & 25 & 61.01 & 27.03 & MID RIFF \\
\hline 2004 & A & 26 & 62.59 & 26.93 & MID RIFF \\
\hline 2004 & A & 26.5 & 63.21 & 26.94 & 5 \\
\hline 2004 & A & 27 & 63.82 & 26.94 & MID RIFF \\
\hline 2004 & A & 28 & 65.16 & 27.09 & MID RIFF \\
\hline 2004 & A & 29 & 66.55 & 27.02 & MID RIFF \\
\hline 2004 & A & 29.5 & 67.19 & 27.10 & 4 \\
\hline 2004 & A & 30 & 67.51 & 27.15 & MID RIFF \\
\hline 2004 & A & 31 & 69.28 & 27.17 & MID RIFF \\
\hline 2004 & A & 32 & 71.38 & 27.16 & MID RIFF \\
\hline 2004 & A & 32.5 & 72.40 & 27.15 & 3 \\
\hline 2004 & A & 33 & 74.35 & 27.12 & MID RIFF \\
\hline 2004 & A & 34 & 76.33 & 27.01 & MID RIFF \\
\hline 2004 & A & 34.5 & 77.34 & 27.15 & 2 \\
\hline 2004 & A & 35 & 77.76 & 27.21 & MID RIFF \\
\hline 2004 & $\mathrm{~A}$ & 36 & 79.52 & 27.31 & MID RIFF \\
\hline 2004 & A & 37 & 81.03 & 27.29 & MID RIFF \\
\hline 2004 & A & 37.5 & 81.20 & 27.30 & 1 \\
\hline 2004 & A & 38 & 82.70 & 27.38 & MID RIFF \\
\hline
\end{tabular}

Continued on Next Page... 
Table D.4 - Continued

\begin{tabular}{|c|c|c|c|c|c|}
\hline Year & Reach & Point Number & Adjusted Dist. (m) & Adjusted Elev. (m) & Point Description \\
\hline 2004 & $\mathrm{~A}$ & 39 & 84.78 & 27.40 & MID RIFF \\
\hline 2004 & A & 40 & 86.51 & 27.43 & MID RIFF \\
\hline 2004 & $\mathrm{~A}$ & 41 & 88.82 & 27.55 & MID RIFF \\
\hline 2004 & $\mathrm{~A}$ & 42 & 90.75 & 27.49 & MID RIFF \\
\hline 2004 & $\mathrm{~A}$ & 43 & 92.34 & 27.62 & MID RIFF \\
\hline 2004 & $\mathrm{~A}$ & 44 & 94.12 & 27.61 & MID RIFF \\
\hline 2004 & A & 45 & 96.17 & 27.58 & MID RIFF \\
\hline 2005 & A & 1 & 23.04 & 26.13 & MID RIFF \\
\hline 2005 & $\mathrm{~A}$ & 2 & 25.07 & 26.23 & MID RIFF \\
\hline 2005 & $\mathrm{~A}$ & 3 & 27.53 & 26.26 & MID RIFF \\
\hline 2005 & $\mathrm{~A}$ & 4 & 28.89 & 26.25 & MID RIFF \\
\hline 2005 & $\mathrm{~A}$ & 4.5 & 29.39 & 26.19 & 10 \\
\hline 2005 & $\mathrm{~A}$ & 5 & 30.26 & 26.08 & MID RIFF \\
\hline 2005 & $\mathrm{~A}$ & 6 & 30.86 & 26.17 & BOTTOM ROCK STEP \\
\hline 2005 & $\mathrm{~A}$ & 7 & 31.17 & 26.29 & TOP ROCK STEP \\
\hline 2005 & $\mathrm{~A}$ & 8 & 31.97 & 26.37 & MID RIFF \\
\hline 2005 & $\mathrm{~A}$ & 9 & 32.47 & 26.35 & BOTTOM ROCK STEP \\
\hline 2005 & A & 10 & 32.97 & 26.54 & TOP ROCK STEP \\
\hline 2005 & $\mathrm{~A}$ & 11 & 33.96 & 26.51 & MID RIFF \\
\hline 2005 & $\mathrm{~A}$ & 11.5 & 34.75 & 26.55 & 9 \\
\hline 2005 & $\mathrm{~A}$ & 12 & 35.51 & 26.59 & MID RIFF \\
\hline 2005 & A & 13 & 36.84 & 26.58 & MID RIFF \\
\hline 2005 & A & 14 & 37.59 & 26.63 & MID RIFF \\
\hline 2005 & $\mathrm{~A}$ & 15 & 38.02 & 26.59 & MID RIFF \\
\hline 2005 & $\mathrm{~A}$ & 15.5 & 38.73 & 26.63 & 8 \\
\hline 2005 & $\mathrm{~A}$ & 16 & 39.05 & 26.65 & MID RIFF \\
\hline 2005 & A & 17 & 40.35 & 26.61 & MID RIFF \\
\hline 2005 & A & 18 & 41.97 & 26.67 & MID RIFF \\
\hline 2005 & $\mathrm{~A}$ & 19 & 42.96 & 26.65 & MID RIFF \\
\hline 2005 & A & 19.5 & 43.50 & 26.69 & 7 \\
\hline 2005 & A & 20 & 44.24 & 26.78 & MID RIFF \\
\hline 2005 & A & 21 & 45.23 & 26.73 & MID RIFF \\
\hline 2005 & A & 22 & 46.02 & 26.81 & MID RIFF \\
\hline 2005 & $\mathrm{~A}$ & 23 & 47.00 & 26.86 & MID RIFF \\
\hline 2005 & $\mathrm{~A}$ & 23.5 & 47.04 & 26.86 & 6 \\
\hline 2005 & A & 24 & 48.20 & 26.77 & MID RIFF \\
\hline 2005 & A & 25 & 49.27 & 26.77 & MID RIFF \\
\hline 2005 & A & 26 & 49.83 & 26.88 & MID RIFF \\
\hline 2005 & $\mathrm{~A}$ & 27 & 51.05 & 26.97 & MID RIFF \\
\hline 2005 & $\mathrm{~A}$ & 28 & 52.10 & 26.93 & MID RIFF \\
\hline 2005 & A & 29 & 53.16 & 26.95 & MID RIFF \\
\hline 2005 & A & 30 & 55.62 & 27.05 & MID RIFF \\
\hline 2005 & A & 31 & 56.76 & 26.94 & MID RIFF \\
\hline 2005 & A & 32 & 59.70 & 26.94 & MID RIFF \\
\hline 2005 & A & 33 & 63.16 & 26.86 & MID RIFF \\
\hline 2005 & A & 33.5 & 63.21 & 26.87 & 5 \\
\hline 2005 & $\mathrm{~A}$ & 34 & 63.77 & 26.94 & MID RIFF \\
\hline 2005 & A & 35 & 64.96 & 27.05 & MID RIFF \\
\hline 2005 & A & 36 & 65.69 & 27.06 & MID RIFF \\
\hline 2005 & $\mathrm{~A}$ & 37 & 67.01 & 27.01 & MID RIFF \\
\hline 2005 & A & 37.5 & 67.19 & 27.03 & 4 \\
\hline 2005 & A & 38 & 67.43 & 27.05 & BOTTOM ROCK STEP \\
\hline
\end{tabular}

Continued on Next Page... 
Table D.4 - Continued

\begin{tabular}{|c|c|c|c|c|c|}
\hline Year & Reach & Point Number & Adjusted Dist. (m) & Adjusted Elev. (m) & Point Description \\
\hline 2005 & A & 39 & 67.68 & 27.20 & TOP ROCK STEP \\
\hline 2005 & $\mathrm{~A}$ & 40 & 69.37 & 27.21 & MID RIFF \\
\hline 2005 & $\mathrm{~A}$ & 41 & 70.81 & 27.18 & MID RIFF \\
\hline 2005 & A & 42 & 72.32 & 27.15 & MID RIFF \\
\hline 2005 & A & 42.5 & 72.40 & 27.15 & 3 \\
\hline 2005 & A & 43 & 73.78 & 27.17 & MID RIFF \\
\hline 2005 & A & 44 & 75.33 & 27.02 & TAIL POOL \\
\hline 2005 & A & 45 & 75.91 & 26.99 & MID POOL \\
\hline 2005 & A & 46 & 76.36 & 27.02 & HEAD POOL \\
\hline 2005 & A & 47 & 77.07 & 27.11 & MID RIFF \\
\hline 2005 & A & 47.5 & 77.34 & 27.14 & 2 \\
\hline 2005 & A & 48 & 77.60 & 27.18 & MID RIFF \\
\hline 2005 & A & 49 & 77.98 & 27.29 & MID RIFF \\
\hline 2005 & A & 50 & 79.44 & 27.30 & MID RIFF \\
\hline 2005 & A & 51 & 80.35 & 27.28 & MID RIFF \\
\hline 2005 & A & 51.5 & 81.20 & 27.30 & 1 \\
\hline 2005 & A & 52 & 81.84 & 27.31 & MID RIFF \\
\hline 2005 & A & 53 & 83.77 & 27.31 & MID RIFF \\
\hline 2005 & A & 54 & 85.48 & 27.40 & MID RIFF \\
\hline 2005 & A & 55 & 87.17 & 27.43 & MID RIFF \\
\hline 2006 & A & 1 & 19.29 & 26.02 & MID RIFF \\
\hline 2006 & A & 2 & 21.33 & 26.12 & MID RIFF \\
\hline 2006 & A & 3 & 23.35 & 26.22 & MID RIFF \\
\hline 2006 & A & 4 & 25.56 & 26.31 & MID RIFF \\
\hline 2006 & A & 5 & 26.27 & 26.22 & TAIL POOL \\
\hline 2006 & A & 6 & 27.49 & 25.98 & MID POOL \\
\hline 2006 & A & 6.5 & 29.39 & 26.03 & 10 \\
\hline 2006 & A & 7 & 29.73 & 26.04 & MID POOL \\
\hline 2006 & A & 8 & 31.38 & 26.14 & MID POOL \\
\hline 2006 & A & 9 & 32.12 & 26.30 & HEAD POOL \\
\hline 2006 & A & 10 & 33.53 & 26.52 & MID RIFF \\
\hline 2006 & A & 11 & 34.45 & 26.49 & TAIL POOL \\
\hline 2006 & A & 11.5 & 34.75 & 26.48 & 9 \\
\hline 2006 & A & 12 & 36.05 & 26.40 & MID POOL \\
\hline 2006 & A & 13 & 37.36 & 26.45 & HEAD POOL \\
\hline 2006 & A & 13.5 & 38.73 & 26.50 & 8 \\
\hline 2006 & A & 14 & 39.30 & 26.51 & MID RIFF \\
\hline 2006 & A & 15 & 42.04 & 26.47 & MID RIFF \\
\hline 2006 & A & 16 & 43.15 & 26.46 & BOT ROCK STEP \\
\hline 2006 & A & 16.5 & 43.50 & 26.59 & 7 \\
\hline 2006 & A & 17 & 43.66 & 26.67 & TOP ROCK STEP \\
\hline 2006 & A & 18 & 45.16 & 26.59 & MID RIFF \\
\hline 2006 & A & 18.5 & 47.04 & 26.74 & 6 \\
\hline 2006 & $\mathrm{~A}$ & 19 & 48.36 & 26.81 & MID RIFF \\
\hline 2006 & $\mathrm{~A}$ & 20 & 53.87 & 26.88 & MID RIFF \\
\hline 2006 & A & 21 & 57.35 & 26.73 & MID RIFF \\
\hline 2006 & A & 22 & 60.86 & 26.89 & MID RIFF \\
\hline 2006 & $\mathrm{~A}$ & 22.5 & 63.21 & 26.96 & 5 \\
\hline 2006 & $\mathrm{~A}$ & 23 & 64.93 & 27.02 & MID RIFF \\
\hline 2006 & A & 23.5 & 67.19 & 27.12 & 4 \\
\hline 2006 & A & 24 & 67.36 & 27.13 & TAIL POOL \\
\hline 2006 & A & 25 & 69.03 & 26.90 & MID POOL \\
\hline
\end{tabular}

Continued on Next Page... 
Table D.4 - Continued

\begin{tabular}{|c|c|c|c|c|c|}
\hline Year & Reach & Point Number & Adjusted Dist. (m) & Adjusted Elev. (m) & Point Description \\
\hline 2006 & A & 26 & 70.53 & 27.04 & HEAD POOL \\
\hline 2006 & $\mathrm{~A}$ & 27 & 71.83 & 27.18 & MID RIFF \\
\hline 2006 & A & 27.5 & 72.40 & 27.17 & 3 \\
\hline 2006 & A & 28 & 73.66 & 27.15 & MID RIFF \\
\hline 2006 & A & 29 & 74.94 & 27.11 & TAIL POOL \\
\hline 2006 & A & 30 & 76.51 & 26.96 & MID POOL \\
\hline 2006 & A & 30.5 & 77.34 & 27.16 & 2 \\
\hline 2006 & A & 31 & 77.47 & 27.19 & HEAD POOL \\
\hline 2006 & A & 32 & 81.04 & 27.26 & MID RIFF \\
\hline 2006 & A & 32.5 & 81.20 & 27.27 & 1 \\
\hline 2006 & A & 33 & 83.31 & 27.29 & MID RIFF \\
\hline 2006 & A & 34 & 86.14 & 27.34 & MID RIFF \\
\hline 2007 & A & 1 & 22.08 & 26.19 & MID RIFF \\
\hline 2007 & A & 2 & 23.72 & 26.25 & MID RIFF \\
\hline 2007 & A & 3 & 25.38 & 26.30 & TAIL POOL \\
\hline 2007 & A & 4 & 27.35 & 26.00 & MID POOL \\
\hline 2007 & A & 4.5 & 29.39 & 26.08 & 10 \\
\hline 2007 & A & 5 & 29.57 & 26.09 & MID POOL \\
\hline 2007 & A & 6 & 31.00 & 26.13 & MID POOL \\
\hline 2007 & A & 7 & 32.41 & 26.33 & HEAD POOL \\
\hline 2007 & A & 8 & 33.33 & 26.44 & MID RIFF \\
\hline 2007 & A & 9 & 34.44 & 26.51 & TAIL POOL \\
\hline 2007 & A & 9.5 & 34.75 & 26.49 & 9 \\
\hline 2007 & A & 10 & 36.44 & 26.38 & MID POOL \\
\hline 2007 & A & 11 & 37.69 & 26.50 & HEAD POOL \\
\hline 2007 & A & 11.5 & 38.73 & 26.51 & 8 \\
\hline 2007 & A & 12 & 38.74 & 26.51 & MID RIFF \\
\hline 2007 & A & 13 & 39.55 & 26.51 & TAIL POOL \\
\hline 2007 & A & 14 & 40.46 & 26.46 & MID POOL \\
\hline 2007 & A & 15 & 41.41 & 26.52 & MID POOL \\
\hline 2007 & A & 16 & 42.31 & 26.46 & HEAD POOL \\
\hline 2007 & A & 16.5 & 43.50 & 26.60 & 7 \\
\hline 2007 & A & 17 & 43.57 & 26.61 & MID RIFF \\
\hline 2007 & A & 18 & 44.22 & 26.63 & TAIL POOL \\
\hline 2007 & A & 19 & 45.23 & 26.58 & MID POOL \\
\hline 2007 & A & 20 & 45.64 & 26.58 & HEAD POOL \\
\hline 2007 & A & 21 & 46.51 & 26.71 & MID RIFF \\
\hline 2007 & A & 21.5 & 47.04 & 26.75 & 6 \\
\hline 2007 & A & 22 & 49.14 & 26.86 & MID RIFF \\
\hline 2007 & A & 23 & 52.60 & 26.93 & TAIL POOL \\
\hline 2007 & A & 24 & 55.26 & 26.89 & MID POOL \\
\hline 2007 & A & 25 & 56.62 & 26.74 & MID POOL \\
\hline 2007 & A & 26 & 59.01 & 26.89 & HEAD POOL \\
\hline 2007 & A & 27 & 60.63 & 26.96 & MID RIFF \\
\hline 2007 & A & 28 & 62.81 & 26.89 & MID RIFF \\
\hline 2007 & A & 28.5 & 63.21 & 26.92 & 5 \\
\hline 2007 & A & 29 & 64.74 & 27.02 & MID RIFF \\
\hline 2007 & $\mathrm{~A}$ & 29.5 & 67.19 & 27.12 & 4 \\
\hline 2007 & $\mathrm{~A}$ & 30 & 67.80 & 27.14 & TAIL POOL \\
\hline 2007 & A & 31 & 69.14 & 27.00 & MID POOL \\
\hline 2007 & A & 32 & 71.13 & 27.19 & HEAD POOL \\
\hline 2007 & A & 32.5 & 72.40 & 27.17 & 3 \\
\hline
\end{tabular}

Continued on Next Page... 
Table D.4 - Continued

\begin{tabular}{|c|c|c|c|c|c|}
\hline Year & Reach & Point Number & Adjusted Dist. (m) & Adjusted Elev. (m) & Point Description \\
\hline 2007 & $\mathrm{~A}$ & 33 & 72.55 & 27.16 & MID RIFF \\
\hline 2007 & A & 34 & 73.94 & 27.15 & TAIL POOL \\
\hline 2007 & A & 35 & 76.31 & 26.98 & MID POOL \\
\hline 2007 & A & 35.5 & 77.34 & 27.11 & 2 \\
\hline 2007 & A & 36 & 77.73 & 27.16 & HEAD POOL \\
\hline 2007 & A & 37 & 79.69 & 27.29 & MID RIFF \\
\hline 2007 & A & 37.5 & 81.20 & 27.31 & 1 \\
\hline 2007 & A & 38 & 83.15 & 27.32 & MID RIFF \\
\hline 2007 & A & 39 & 85.65 & 27.33 & MID RIFF \\
\hline 2008 & A & 1 & 16.67 & 25.77 & MID POOL \\
\hline 2008 & A & 2 & 18.17 & 25.78 & HEAD POOL \\
\hline 2008 & A & 3 & 19.95 & 26.06 & MID RIFF \\
\hline 2008 & A & 4 & 21.17 & 26.02 & MID RIFF \\
\hline 2008 & A & 5 & 22.26 & 26.17 & MID RIFF \\
\hline 2008 & $\mathrm{~A}$ & 6 & 22.90 & 26.19 & MID RIFF \\
\hline 2008 & $\mathrm{~A}$ & 7 & 23.96 & 26.35 & MID RIFF \\
\hline 2008 & A & 8 & 25.88 & 26.44 & TAIL POOL \\
\hline 2008 & A & 9 & 27.45 & 26.02 & MID POOL \\
\hline 2008 & A & 9.5 & 29.39 & 26.02 & 10 \\
\hline 2008 & A & 10 & 29.43 & 26.02 & MID POOL \\
\hline 2008 & A & 11 & 30.14 & 26.21 & TAIL RIFF \\
\hline 2008 & A & 12 & 31.17 & 26.30 & MID RIFF \\
\hline 2008 & A & 13 & 31.99 & 26.35 & MID RIFF \\
\hline 2008 & A & 14 & 32.95 & 26.47 & MID RIFF \\
\hline 2008 & A & 15 & 33.52 & 26.52 & MID RIFF \\
\hline 2008 & A & 16 & 34.32 & 26.54 & TAIL POOL \\
\hline 2008 & A & 16.5 & 34.75 & 26.49 & 9 \\
\hline 2008 & A & 17 & 35.17 & 26.44 & MID POOL \\
\hline 2008 & A & 18 & 36.29 & 26.37 & MID POOL \\
\hline 2008 & A & 19 & 37.32 & 26.49 & HEAD POOL \\
\hline 2008 & $\mathrm{~A}$ & 20 & 37.96 & 26.52 & MID RIFF \\
\hline 2008 & A & 21 & 38.34 & 26.54 & MID RIFF \\
\hline 2008 & A & 21.5 & 38.73 & 26.54 & 8 \\
\hline 2008 & A & 22 & 39.08 & 26.54 & MID RIFF \\
\hline 2008 & A & 23 & 39.44 & 26.58 & MID RIFF \\
\hline 2008 & $\mathrm{~A}$ & 24 & 39.96 & 26.60 & MID RIFF \\
\hline 2008 & $\mathrm{~A}$ & 25 & 40.65 & 26.56 & MID RIFF \\
\hline 2008 & A & 26 & 41.56 & 26.56 & MID RIFF \\
\hline 2008 & A & 27 & 42.30 & 26.62 & MID RIFF \\
\hline 2008 & A & 28 & 42.59 & 26.59 & TAIL POOL \\
\hline 2008 & $\mathrm{~A}$ & 29 & 43.18 & 26.47 & MID POOL \\
\hline 2008 & $\mathrm{~A}$ & 29.5 & 43.50 & 26.52 & 7 \\
\hline 2008 & $\mathrm{~A}$ & 30 & 43.55 & 26.54 & HEAD POOL \\
\hline 2008 & A & 31 & 43.87 & 26.58 & MID RIFF \\
\hline 2008 & A & 32 & 44.61 & 26.62 & MID RIFF \\
\hline 2008 & A & 33 & 45.18 & 26.57 & MID RIFF \\
\hline 2008 & A & 34 & 45.94 & 26.61 & MID RIFF \\
\hline 2008 & $\mathrm{~A}$ & 35 & 46.31 & 26.60 & MID RIFF \\
\hline 2008 & $\mathrm{~A}$ & 36 & 46.47 & 26.64 & MID RIFF \\
\hline 2008 & A & 37 & 46.77 & 26.59 & MID RIFF \\
\hline 2008 & A & 37.5 & 47.04 & 26.71 & 6 \\
\hline 2008 & A & 38 & 47.09 & 26.73 & MID RIFF \\
\hline
\end{tabular}

Continued on Next Page... 
Table D.4 - Continued

\begin{tabular}{|c|c|c|c|c|c|}
\hline Year & Reach & Point Number & Adjusted Dist. (m) & Adjusted Elev. (m) & Point Description \\
\hline 2008 & A & 39 & 47.70 & 26.70 & TAIL POOL \\
\hline 2008 & A & 40 & 48.46 & 26.68 & MID POOL \\
\hline 2008 & $\mathrm{~A}$ & 41 & 49.03 & 26.67 & HEAD POOL \\
\hline 2008 & A & 42 & 49.39 & 26.77 & MID RIFF \\
\hline 2008 & $\mathrm{~A}$ & 43 & 50.08 & 26.78 & MID RIFF \\
\hline 2008 & A & 44 & 50.91 & 26.80 & MID RIFF \\
\hline 2008 & A & 45 & 52.03 & 26.86 & MID RIFF \\
\hline 2008 & A & 46 & 52.85 & 26.88 & MID RIFF \\
\hline 2008 & A & 47 & 53.84 & 26.87 & MID RIFF \\
\hline 2008 & A & 48 & 54.28 & 26.92 & MID RIFF \\
\hline 2008 & $\mathrm{~A}$ & 49 & 55.01 & 26.93 & MID RIFF \\
\hline 2008 & $\mathrm{~A}$ & 50 & 55.97 & 26.93 & MID RIFF \\
\hline 2008 & $\mathrm{~A}$ & 51 & 56.44 & 26.90 & MID RIFF \\
\hline 2008 & A & 52 & 56.81 & 26.95 & MID RIFF \\
\hline 2008 & $\mathrm{~A}$ & 53 & 57.32 & 26.91 & TAIL POOL \\
\hline 2008 & A & 54 & 58.20 & 26.85 & MID POOL \\
\hline 2008 & $\mathrm{~A}$ & 55 & 59.34 & 26.87 & HEAD POOL \\
\hline 2008 & $\mathrm{~A}$ & 56 & 59.83 & 26.94 & MID RIFF \\
\hline 2008 & A & 57 & 60.51 & 26.92 & MID RIFF \\
\hline 2008 & A & 58 & 61.10 & 26.99 & MID RIFF \\
\hline 2008 & A & 59 & 61.53 & 27.00 & MID RIFF \\
\hline 2008 & A & 60 & 62.02 & 27.01 & MID RIFF \\
\hline 2008 & $\mathrm{~A}$ & 61 & 62.57 & 27.01 & MID RIFF \\
\hline 2008 & $\mathrm{~A}$ & 62 & 63.11 & 26.98 & MID RIFF \\
\hline 2008 & A & 62.5 & 63.21 & 26.98 & 5 \\
\hline 2008 & A & 63 & 63.66 & 26.97 & MID RIFF \\
\hline 2008 & A & 64 & 64.26 & 26.95 & MID RIFF \\
\hline 2008 & $\mathrm{~A}$ & 65 & 64.77 & 26.99 & MID RIFF \\
\hline 2008 & A & 66 & 65.34 & 27.00 & MID RIFF \\
\hline 2008 & $\mathrm{~A}$ & 67 & 66.08 & 27.00 & MID RIFF \\
\hline 2008 & $\mathrm{~A}$ & 68 & 66.62 & 27.07 & MID RIFF \\
\hline 2008 & $\mathrm{~A}$ & 69 & 67.10 & 27.05 & MID RIFF \\
\hline 2008 & $\mathrm{~A}$ & 69.5 & 67.19 & 27.05 & 4 \\
\hline 2008 & A & 70 & 67.92 & 27.01 & MID RIFF \\
\hline 2008 & $\mathrm{~A}$ & 71 & 68.43 & 27.08 & MID RIFF \\
\hline 2008 & $\mathrm{~A}$ & 72 & 68.98 & 27.05 & MID RIFF \\
\hline 2008 & A & 73 & 69.27 & 27.08 & TAIL POOL \\
\hline 2008 & $\mathrm{~A}$ & 74 & 69.91 & 26.97 & MID POOL \\
\hline 2008 & A & 75 & 70.27 & 27.04 & HEAD POOL \\
\hline 2008 & $\mathrm{~A}$ & 76 & 70.55 & 27.11 & MID RIFF \\
\hline 2008 & $\mathrm{~A}$ & 77 & 71.09 & 27.14 & MID RIFF \\
\hline 2008 & A & 78 & 71.49 & 27.15 & MID RIFF \\
\hline 2008 & $\mathrm{~A}$ & 79 & 72.29 & 27.13 & MID RIFF \\
\hline 2008 & $\mathrm{~A}$ & 79.5 & 72.40 & 27.13 & 3 \\
\hline 2008 & $\mathrm{~A}$ & 80 & 73.44 & 27.08 & MID RIFF \\
\hline 2008 & $\mathrm{~A}$ & 81 & 74.31 & 27.12 & MID RIFF \\
\hline 2008 & A & 82 & 74.77 & 27.11 & MID RIFF \\
\hline 2008 & A & 83 & 75.16 & 27.05 & TAIL POOL \\
\hline 2008 & $\mathrm{~A}$ & 84 & 75.96 & 26.94 & MID POOL \\
\hline 2008 & $\mathrm{~A}$ & 85 & 76.78 & 27.08 & HEAD POOL \\
\hline 2008 & A & 86 & 77.24 & 27.13 & MID RIFF \\
\hline 2008 & A & 86.5 & 77.34 & 27.14 & 2 \\
\hline
\end{tabular}

Continued on Next Page... 
Table D.4 - Continued

\begin{tabular}{|c|c|c|c|c|c|}
\hline Year & Reach & Point Number & Adjusted Dist. (m) & Adjusted Elev. (m) & Point Description \\
\hline 2008 & $\mathrm{~A}$ & 87 & 78.15 & 27.28 & MID RIFF \\
\hline 2008 & $\mathrm{~A}$ & 88 & 78.61 & 27.25 & MID RIFF \\
\hline 2008 & A & 89 & 79.11 & 27.31 & MID RIFF \\
\hline 2008 & A & 90 & 79.72 & 27.25 & MID RIFF \\
\hline 2008 & $\mathrm{~A}$ & 91 & 80.40 & 27.28 & MID RIFF \\
\hline 2008 & A & 91.5 & 81.20 & 27.26 & 1 \\
\hline 2008 & A & 92 & 81.51 & 27.26 & MID RIFF \\
\hline 2008 & $\mathrm{~A}$ & 93 & 83.27 & 27.28 & MID RIFF \\
\hline 2008 & $\mathrm{~A}$ & 94 & 83.93 & 27.26 & MID RIFF \\
\hline 2008 & $\mathrm{~A}$ & 95 & 85.79 & 27.33 & MID RIFF \\
\hline 2008 & A & 96 & 87.26 & 27.36 & MID RIFF \\
\hline 2008 & A & 97 & 88.50 & 27.43 & MID RIFF \\
\hline 2008 & $\mathrm{~A}$ & 98 & 89.89 & 27.43 & MID RIFF \\
\hline 2008 & $\mathrm{~A}$ & 99 & 90.86 & 27.49 & MID RIFF \\
\hline 2008 & $\mathrm{~A}$ & 100 & 91.81 & 27.50 & MID RIFF \\
\hline 2008 & A & 101 & 92.51 & 27.42 & MID RIFF \\
\hline 2008 & A & 102 & 93.45 & 27.40 & MID RIFF \\
\hline 2002 & $\mathrm{~B}$ & 1 & -42.06 & 36.10 & TPOOL.41 \\
\hline 2002 & $\mathrm{~B}$ & 2 & -40.18 & 36.07 & MIDPOOL.60 \\
\hline 2002 & $\mathrm{~B}$ & 3 & -38.79 & 36.10 & HEADPOOL.45TG \\
\hline 2002 & $\mathrm{~B}$ & 4 & -33.91 & 36.09 & HEADGLI. 54 \\
\hline 2002 & $\mathrm{~B}$ & 5 & -33.47 & 36.24 & TAILGLI.40 \\
\hline 2002 & $\mathrm{~B}$ & 6 & -31.94 & 36.21 & MIDGLI.53 \\
\hline 2002 & B & 7 & -29.64 & 36.30 & HEADGLI.19 \\
\hline 2002 & $\mathrm{~B}$ & 8 & -29.39 & 36.36 & TAILRIFF.25 \\
\hline 2002 & B & 9 & -27.15 & 36.38 & HEADRIFF.36 \\
\hline 2002 & $\mathrm{~B}$ & 10 & -23.98 & 36.35 & MIDGLI.61 \\
\hline 2002 & $\mathrm{~B}$ & 11 & -20.18 & 36.43 & HEADGLI.45 \\
\hline 2002 & $\mathrm{~B}$ & 12 & -19.79 & 36.45 & TAILRIFF.54 \\
\hline 2002 & B & 13 & -14.03 & 36.74 & HEADRIFF..27 \\
\hline 2002 & $\mathrm{~B}$ & 14 & -13.94 & 36.75 & TAILGLI.35 \\
\hline 2002 & $\mathrm{~B}$ & 15 & -11.15 & 36.70 & MIDGLI.54 \\
\hline 2002 & $\mathrm{~B}$ & 16 & -6.61 & 36.76 & HEADGLI.43 \\
\hline 2002 & B & 17 & -6.37 & 36.72 & TAILRIFF.54 \\
\hline 2002 & B & 18 & -5.92 & 36.70 & HEADRIFF.56 \\
\hline 2002 & $\mathrm{~B}$ & 19 & -5.39 & 36.89 & TAILRIFF.31 \\
\hline 2002 & $\mathrm{~B}$ & 20 & -3.41 & 36.95 & HEADRIFF.38TGLI \\
\hline 2002 & $\mathrm{~B}$ & 21 & -2.26 & 36.88 & MIDGLI.67 \\
\hline 2002 & $\mathrm{~B}$ & 22 & -0.32 & 36.95 & HEADGLI.50TPOOL \\
\hline 2002 & $\mathrm{~B}$ & 23 & 0.13 & 36.90 & MIDPOOL.63 \\
\hline 2002 & $\mathrm{~B}$ & 24 & 1.01 & 37.04 & HEADPOOL.28TRIFF \\
\hline 2002 & $\mathrm{~B}$ & 25 & 1.93 & 37.11 & BIS.35 \\
\hline 2002 & B & 26 & 3.60 & 37.18 & HEADRIFF.43TGLI \\
\hline 2002 & $\mathrm{~B}$ & 27 & 5.25 & 37.12 & MIDGLI.65 \\
\hline 2002 & $\mathrm{~B}$ & 28 & 7.25 & 37.19 & HEADGLI.49TGLI \\
\hline 2002 & B & 29 & 8.93 & 37.20 & MIDGLI.59 \\
\hline 2002 & B & 30 & 10.81 & 37.18 & HEADGLI.46 \\
\hline 2002 & $\mathrm{~B}$ & 31 & 12.67 & 37.20 & MIDGLI.55 \\
\hline 2002 & $\mathrm{~B}$ & 32 & 13.60 & 37.31 & BIS.23 \\
\hline 2002 & $\mathrm{~B}$ & 33 & 14.28 & 37.23 & MID.54 \\
\hline 2002 & B & 33.5 & 15.21 & 37.28 & 10 \\
\hline 2002 & B & 34 & 16.39 & 37.34 & TAILRIFF.24 \\
\hline
\end{tabular}

Continued on Next Page... 
Table D.4 - Continued

\begin{tabular}{|c|c|c|c|c|c|}
\hline Year & Reach & Point Number & Adjusted Dist. (m) & Adjusted Elev. (m) & Point Description \\
\hline 2002 & B & 34.5 & 19.65 & 37.35 & 9 \\
\hline 2002 & B & 35 & 21.78 & 37.35 & HEADRIFF.28 \\
\hline 2002 & B & 36 & 22.02 & 37.36 & TAILRIFF.46 \\
\hline 2002 & B & 36.5 & 25.00 & 37.43 & 8 \\
\hline 2002 & B & 37 & 26.71 & 37.47 & HEADRIFF.24TPOOL \\
\hline 2002 & B & 38 & 28.57 & 37.36 & MIDPOOL.62 \\
\hline 2002 & B & 39 & 29.46 & 37.47 & BIS.31 \\
\hline 2002 & B & 39.5 & 29.62 & 37.45 & 7 \\
\hline 2002 & B & 40 & 30.71 & 37.32 & MIDPOOL.76 \\
\hline 2002 & B & 41 & 31.28 & 37.48 & HEADPOOL. 29 \\
\hline 2002 & B & 42 & 31.80 & 37.55 & TAILRIFF.51 \\
\hline 2002 & B & 42.5 & 34.07 & 37.55 & 6 \\
\hline 2002 & B & 43 & 34.25 & 37.55 & HEADRIFF.32 \\
\hline 2002 & B & 44 & 35.18 & 37.70 & TAILPOOL.30 \\
\hline 2002 & B & 45 & 36.60 & 37.54 & MIDPOOL.81 \\
\hline 2002 & $\mathrm{~B}$ & 46 & 38.21 & 37.59 & HEADPOOL.69 \\
\hline 2002 & B & 46.5 & 38.47 & 37.74 & 5 \\
\hline 2002 & $\mathrm{~B}$ & 47 & 38.48 & 37.74 & TAILRIFF.20 \\
\hline 2002 & $\mathrm{~B}$ & 48 & 39.37 & 37.72 & HEADRIFF. 25 \\
\hline 2002 & B & 49 & 39.64 & 37.74 & TAILPOOL.31 \\
\hline 2002 & B & 50 & 41.37 & 37.70 & MIDPOOL.56 \\
\hline 2002 & B & 51 & 43.10 & 37.78 & HEADPOOL. 29 \\
\hline 2002 & $\mathrm{~B}$ & 51.5 & 43.37 & 37.83 & 4 \\
\hline 2002 & $\mathrm{~B}$ & 52 & 43.80 & 37.93 & TOPSTEP.4 \\
\hline 2002 & B & 53 & 45.54 & 38.08 & HEADRIFF. 17 \\
\hline 2002 & B & 54 & 46.07 & 38.04 & TAILPOOL.41 \\
\hline 2002 & B & 55 & 47.45 & 38.04 & MIDPOOL.50 \\
\hline 2002 & B & 56 & 47.60 & 38.05 & BIS.36 \\
\hline 2002 & $\mathrm{~B}$ & 57 & 48.36 & 37.99 & MIDPOOL.67 \\
\hline 2002 & $\mathrm{~B}$ & 57.5 & 48.53 & 37.99 & 3 \\
\hline 2002 & $\mathrm{~B}$ & 58 & 50.60 & 38.08 & HEADPOOL.35 \\
\hline 2002 & $\mathrm{~B}$ & 59 & 53.25 & 38.31 & TOPRIFF.33 \\
\hline 2002 & B & 59.4 & 53.29 & 38.31 & 2 \\
\hline 2002 & B & 59.5 & 56.65 & 38.36 & 1 \\
\hline 2002 & B & 60 & 63.49 & 38.39 & TOPRIFF.29 \\
\hline 2002 & $\mathrm{~B}$ & 61 & 68.10 & 38.53 & TAILPOOL.12TRIFF \\
\hline 2002 & B & 62 & 70.00 & 38.42 & MIDPOOL.55 \\
\hline 2002 & B & 63 & 72.05 & 38.44 & HEADPOOL.53 \\
\hline 2002 & B & 64 & 72.28 & 38.49 & TAILGLI.37 \\
\hline 2002 & B & 65 & 73.67 & 38.39 & MIDGLI.69 \\
\hline 2002 & $\mathrm{~B}$ & 66 & 74.65 & 38.49 & BIS.24 \\
\hline 2002 & B & 67 & 79.48 & 38.48 & HEADGLI.37 \\
\hline 2002 & $\mathrm{~B}$ & 68 & 79.85 & 38.58 & TAILGLI.20 \\
\hline 2002 & $\mathrm{~B}$ & 69 & 81.70 & 38.44 & MIDGLI.86 \\
\hline 2002 & B & 70 & 82.40 & 38.46 & HEADGLI.61 \\
\hline 2002 & B & 71 & 82.62 & 38.60 & TAILRIFF.17 \\
\hline 2002 & B & 72 & 85.81 & 38.88 & HEADRIFF.23 \\
\hline 2002 & $\mathrm{~B}$ & 73 & 90.50 & 39.05 & TOPRIFF.19TPOOL \\
\hline 2002 & $\mathrm{~B}$ & 74 & 94.44 & 39.05 & BIS.30 \\
\hline 2002 & $\mathrm{~B}$ & 75 & 97.87 & 38.61 & MIDPOOL1.58 \\
\hline 2002 & B & 76 & 99.17 & 38.75 & HEADPOOL. 86 \\
\hline 2003 & B & 1 & 11.86 & 37.30 & GLIDE \\
\hline
\end{tabular}

Continued on Next Page... 
Table D.4 - Continued

\begin{tabular}{|c|c|c|c|c|c|}
\hline Year & Reach & Point Number & Adjusted Dist. (m) & Adjusted Elev. (m) & Point Description \\
\hline 2003 & $\mathrm{~B}$ & 1.5 & 15.21 & 37.30 & 10 \\
\hline 2003 & B & 2 & 16.36 & 37.30 & GLIDE \\
\hline 2003 & B & 2.5 & 19.65 & 37.35 & 9 \\
\hline 2003 & B & 3 & 21.56 & 37.39 & GLIDE \\
\hline 2003 & B & 3.5 & 25.00 & 37.41 & 8 \\
\hline 2003 & B & 4 & 25.12 & 37.41 & GLIDE \\
\hline 2003 & B & 5 & 27.14 & 37.47 & TAIL POOL \\
\hline 2003 & B & 6 & 29.16 & 37.38 & MID POOL \\
\hline 2003 & B & 6.5 & 29.62 & 37.40 & 7 \\
\hline 2003 & B & 7 & 31.33 & 37.45 & HEAD POOL \\
\hline 2003 & B & 8 & 33.19 & 37.60 & MID RIFF \\
\hline 2003 & B & 8.5 & 34.07 & 37.61 & 6 \\
\hline 2003 & B & 9 & 34.99 & 37.63 & TAIL POOL \\
\hline 2003 & B & 10 & 36.78 & 37.56 & MID POOL \\
\hline 2003 & B & 11 & 38.28 & 37.69 & HEAD POOL \\
\hline 2003 & B & 11.5 & 38.47 & 37.69 & 5 \\
\hline 2003 & B & 12 & 41.20 & 37.71 & GLIDE \\
\hline 2003 & B & 12.5 & 43.37 & 37.74 & 4 \\
\hline 2003 & B & 13 & 43.47 & 37.74 & RIFF \\
\hline 2003 & B & 14 & 45.35 & 37.86 & TAIL POOL \\
\hline 2003 & B & 15 & 47.79 & 38.02 & MID POOL \\
\hline 2003 & B & 15.5 & 48.53 & 38.03 & 3 \\
\hline 2003 & B & 16 & 50.70 & 38.07 & HEAD POOL \\
\hline 2003 & B & 16.5 & 53.29 & 38.19 & 2 \\
\hline 2003 & B & 17 & 54.21 & 38.24 & MID RIFF \\
\hline 2003 & B & 17.5 & 56.65 & 38.31 & 1 \\
\hline 2003 & B & 18 & 57.91 & 38.34 & MID RIFF \\
\hline 2003 & B & 19 & 61.21 & 38.46 & MID RIFF \\
\hline 2003 & B & 20 & 63.39 & 38.52 & TAIL POOL \\
\hline 2003 & B & 21 & 66.79 & 38.38 & MID POOL \\
\hline 2003 & B & 22 & 70.73 & 38.38 & MID POOL \\
\hline 2003 & B & 23 & 74.21 & 38.51 & HEAD POOL \\
\hline 2004 & B & 1 & 12.64 & 37.22 & MID RIFF \\
\hline 2004 & B & 2 & 13.30 & 37.31 & MID RIFF \\
\hline 2004 & B & 2.5 & 15.21 & 37.33 & 10 \\
\hline 2004 & B & 3 & 16.67 & 37.35 & MID RIFF \\
\hline 2004 & B & 3.5 & 19.65 & 37.33 & 9 \\
\hline 2004 & B & 4 & 21.12 & 37.32 & MID RIFF \\
\hline 2004 & B & 5 & 22.57 & 37.41 & MID RIFF \\
\hline 2004 & B & 6 & 24.71 & 37.46 & MID RIFF \\
\hline 2004 & B & 6.5 & 25.00 & 37.46 & 8 \\
\hline 2004 & B & 7 & 27.23 & 37.48 & MID RIFF \\
\hline 2004 & B & 7.5 & 29.62 & 37.54 & 7 \\
\hline 2004 & B & 8 & 29.64 & 37.54 & MID RIFF \\
\hline 2004 & B & 9 & 31.46 & 37.48 & MID RIFF \\
\hline 2004 & B & 10 & 33.33 & 37.54 & MID RIFF \\
\hline 2004 & B & 10.5 & 34.07 & 37.55 & 6 \\
\hline 2004 & B & 11 & 35.21 & 37.57 & MID RIFF \\
\hline 2004 & B & 12 & 37.01 & 37.61 & MID RIFF \\
\hline 2004 & B & 13 & 38.36 & 37.66 & MID RIFF \\
\hline 2004 & B & 13.5 & 38.47 & 37.66 & 5 \\
\hline 2004 & B & 14 & 39.81 & 37.73 & MID RIFF \\
\hline
\end{tabular}

Continued on Next Page... 
Table D.4 - Continued

\begin{tabular}{|c|c|c|c|c|c|}
\hline Year & Reach & Point Number & Adjusted Dist. (m) & Adjusted Elev. (m) & Point Description \\
\hline 2004 & B & 15 & 41.33 & 37.73 & MID RIFF \\
\hline 2004 & B & 16 & 43.31 & 37.77 & BOT STEP \\
\hline 2004 & B & 16.5 & 43.37 & 37.80 & 4 \\
\hline 2004 & B & 17 & 43.74 & 37.94 & TOP STEP \\
\hline 2004 & B & 18 & 45.01 & 37.89 & MID RIFF \\
\hline 2004 & B & 19 & 48.42 & 38.06 & MID RIFF \\
\hline 2004 & $\mathrm{~B}$ & 19.5 & 48.53 & 38.06 & 3 \\
\hline 2004 & B & 20 & 50.40 & 38.06 & MID RIFF \\
\hline 2004 & B & 21 & 51.70 & 38.16 & MID RIFF \\
\hline 2004 & B & 21.5 & 53.29 & 38.24 & 2 \\
\hline 2004 & B & 22 & 53.82 & 38.27 & MID RIFF \\
\hline 2004 & $\mathrm{~B}$ & 23 & 55.07 & 38.34 & MID RIFF \\
\hline 2004 & B & 23.5 & 56.65 & 38.40 & 1 \\
\hline 2004 & B & 24 & 57.00 & 38.41 & MID RIFF \\
\hline 2004 & B & 25 & 58.42 & 38.44 & MID RIFF \\
\hline 2004 & B & 26 & 60.07 & 38.41 & GLIDE \\
\hline 2004 & $\mathrm{~B}$ & 27 & 64.18 & 38.38 & MID GLIDE \\
\hline 2004 & B & 28 & 67.50 & 38.36 & TOP GLIDE \\
\hline 2004 & B & 29 & 70.12 & 38.46 & MID RIFF \\
\hline 2004 & B & 30 & 72.37 & 38.50 & MID RIFF \\
\hline 2004 & $\mathrm{~B}$ & 31 & 75.02 & 38.62 & MID RIFF \\
\hline 2005 & B & 41 & 6.69 & 37.20 & MID RIFF \\
\hline 2005 & B & 40 & 7.79 & 37.21 & MID RIFF \\
\hline 2005 & B & 39 & 8.80 & 37.15 & MID RIFF \\
\hline 2005 & B & 38 & 9.44 & 37.28 & MID RIFF \\
\hline 2005 & B & 37 & 11.81 & 37.30 & MID RIFF \\
\hline 2005 & B & 36 & 12.95 & 37.38 & MID RIFF \\
\hline 2005 & B & 35.5 & 15.21 & 37.33 & 10 \\
\hline 2005 & B & 35 & 15.27 & 37.33 & MID RIFF \\
\hline 2005 & B & 34 & 17.21 & 37.36 & MID RIFF \\
\hline 2005 & B & 33.5 & 19.65 & 37.50 & 9 \\
\hline 2005 & B & 33 & 20.42 & 37.54 & MID RIFF \\
\hline 2005 & B & 32 & 22.40 & 37.42 & MID RIFF \\
\hline 2005 & B & 31 & 24.38 & 37.51 & MID RIFF \\
\hline 2005 & B & 30.5 & 25.00 & 37.53 & 8 \\
\hline 2005 & B & 30 & 25.46 & 37.54 & MID RIFF \\
\hline 2005 & B & 29 & 26.34 & 37.57 & MID RIFF \\
\hline 2005 & B & 28 & 27.25 & 37.57 & MID RIFF \\
\hline 2005 & B & 27 & 28.43 & 37.50 & MID RIFF \\
\hline 2005 & B & 26.5 & 29.62 & 37.50 & 7 \\
\hline 2005 & B & 26 & 29.62 & 37.50 & MID RIFF \\
\hline 2005 & B & 25 & 30.56 & 37.50 & MID RIFF \\
\hline 2005 & B & 24 & 31.38 & 37.48 & MID RIFF \\
\hline 2005 & B & 23 & 32.60 & 37.51 & MID RIFF \\
\hline 2005 & B & 22 & 33.48 & 37.53 & MID RIFF \\
\hline 2005 & B & 21.5 & 34.07 & 37.54 & 6 \\
\hline 2005 & B & 21 & 34.74 & 37.55 & MID RIFF \\
\hline 2005 & B & 20 & 35.76 & 37.59 & MID RIFF \\
\hline 2005 & B & 19 & 36.73 & 37.58 & MID RIFF \\
\hline 2005 & B & 18 & 37.64 & 37.62 & MID RIFF \\
\hline 2005 & B & 17 & 38.44 & 37.64 & MID RIFF \\
\hline 2005 & B & 16.5 & 38.47 & 37.65 & 5 \\
\hline
\end{tabular}

Continued on Next Page... 
Table D.4 - Continued

\begin{tabular}{|c|c|c|c|c|c|}
\hline Year & Reach & Point Number & Adjusted Dist. (m) & Adjusted Elev. (m) & Point Description \\
\hline 2005 & B & 16 & 38.88 & 37.68 & MID RIFF \\
\hline 2005 & B & 15 & 40.25 & 37.77 & MID RIFF \\
\hline 2005 & B & 14 & 41.95 & 37.77 & MID RIFF \\
\hline 2005 & B & 13 & 43.37 & 37.77 & BOTTOM ROCK STEP \\
\hline 2005 & B & 12.5 & 43.37 & 37.77 & 4 \\
\hline 2005 & $\mathrm{~B}$ & 12 & 43.64 & 37.95 & TOP ROCK STEP \\
\hline 2005 & B & 11 & 44.59 & 37.92 & MID RIFF \\
\hline 2005 & $\mathrm{~B}$ & 10 & 45.33 & 37.98 & BOTTOM ROCK STEP \\
\hline 2005 & $\mathrm{~B}$ & 9 & 45.57 & 38.04 & TOP ROCK STEP \\
\hline 2005 & B & 8 & 46.51 & 38.03 & MID RIFF \\
\hline 2005 & B & 7 & 48.16 & 38.07 & MID RIFF \\
\hline 2005 & B & 6.5 & 48.53 & 38.07 & 3 \\
\hline 2005 & $\mathrm{~B}$ & 6 & 49.77 & 38.04 & MID RIFF \\
\hline 2005 & $\mathrm{~B}$ & 5 & 50.67 & 38.15 & MID RIFF \\
\hline 2005 & B & 4 & 51.98 & 38.23 & MID RIFF \\
\hline 2005 & B & 3.5 & 53.29 & 38.27 & 2 \\
\hline 2005 & B & 3 & 53.49 & 38.27 & MID RIFF \\
\hline 2005 & B & 2 & 55.53 & 38.31 & MID RIFF \\
\hline 2005 & B & 1.5 & 56.65 & 38.34 & 1 \\
\hline 2005 & B & 1 & 57.91 & 38.38 & MID RIFF \\
\hline 2006 & B & 49 & 7.29 & 36.89 & MID RIF \\
\hline 2006 & B & 48 & 7.53 & 36.95 & MID RIF \\
\hline 2006 & B & 47 & 8.37 & 37.03 & MID RIF \\
\hline 2006 & B & 46 & 8.77 & 36.99 & MID RIF \\
\hline 2006 & B & 45 & 9.85 & 36.98 & MID RIF \\
\hline 2006 & B & 44 & 10.73 & 37.07 & MID RIF \\
\hline 2006 & B & 43 & 11.33 & 37.20 & MID RIF \\
\hline 2006 & B & 42 & 12.83 & 37.28 & MID RIF \\
\hline 2006 & B & 41 & 13.59 & 37.24 & MID RIF \\
\hline 2006 & B & 40 & 14.29 & 37.34 & MID RIF \\
\hline 2006 & B & 39 & 14.91 & 37.37 & MID RIF \\
\hline 2006 & B & 38.5 & 15.21 & 37.36 & 10 \\
\hline 2006 & B & 38 & 16.98 & 37.26 & AFA \\
\hline 2006 & B & 37 & 17.89 & 37.22 & MID RIF \\
\hline 2006 & B & 36 & 19.14 & 37.39 & MID RIF \\
\hline 2006 & B & 35.5 & 19.65 & 37.42 & 9 \\
\hline 2006 & B & 35 & 20.09 & 37.45 & MID RIF \\
\hline 2006 & B & 34 & 23.44 & 37.50 & MID RIF \\
\hline 2006 & B & 33.5 & 25.00 & 37.51 & 8 \\
\hline 2006 & B & 33 & 26.81 & 37.52 & MID RIF \\
\hline 2006 & B & 32 & 28.78 & 37.55 & MID RIF \\
\hline 2006 & B & 31.5 & 29.62 & 37.55 & 7 \\
\hline 2006 & B & 31 & 30.18 & 37.55 & MID RIF \\
\hline 2006 & B & 30 & 31.76 & 37.52 & MID RIF \\
\hline 2006 & B & 29 & 33.84 & 37.55 & MID RIF \\
\hline 2006 & B & 28.5 & 34.07 & 37.54 & 6 \\
\hline 2006 & B & 28 & 35.27 & 37.51 & MID RIF \\
\hline 2006 & B & 27 & 36.03 & 37.57 & MID RIF \\
\hline 2006 & B & 26 & 37.12 & 37.53 & MID RIF \\
\hline 2006 & B & 25 & 37.69 & 37.62 & MID RIF \\
\hline 2006 & B & 24 & 38.40 & 37.69 & MID RIF \\
\hline 2006 & B & 23.5 & 38.47 & 37.70 & 5 \\
\hline
\end{tabular}

Continued on Next Page... 
Table D.4 - Continued

\begin{tabular}{|c|c|c|c|c|c|}
\hline Year & Reach & Point Number & Adjusted Dist. (m) & Adjusted Elev. (m) & Point Description \\
\hline 2006 & B & 23 & 39.13 & 37.73 & MID RIF \\
\hline 2006 & B & 22 & 39.55 & 37.70 & MID RIF \\
\hline 2006 & B & 21 & 40.41 & 37.73 & MID RIF \\
\hline 2006 & B & 20 & 41.36 & 37.74 & MID RIF \\
\hline 2006 & B & 19 & 42.31 & 37.73 & MID RIF \\
\hline 2006 & B & 18.5 & 43.37 & 37.77 & 4 \\
\hline 2006 & $\mathrm{~B}$ & 18 & 43.42 & 37.77 & BOTTOM LOG STEP \\
\hline 2006 & B & 17 & 43.65 & 37.89 & TOP LOG STEP \\
\hline 2006 & $\mathrm{~B}$ & 16 & 45.22 & 37.91 & MID RIF \\
\hline 2006 & B & 15 & 45.79 & 37.97 & MID RIF \\
\hline 2006 & B & 14 & 46.89 & 37.99 & MID RIF \\
\hline 2006 & $\mathrm{~B}$ & 13.5 & 48.53 & 38.03 & 3 \\
\hline 2006 & B & 13 & 48.91 & 38.04 & MID RIF \\
\hline 2006 & B & 12 & 49.52 & 38.12 & MID RIF \\
\hline 2006 & B & 11 & 50.24 & 38.08 & MID RIF \\
\hline 2006 & B & 10 & 50.88 & 38.14 & MID RIF \\
\hline 2006 & B & 9 & 51.79 & 38.12 & MID RIF \\
\hline 2006 & B & 8 & 52.32 & 38.20 & MID RIF \\
\hline 2006 & B & 7 & 53.04 & 38.22 & MID RIF \\
\hline 2006 & B & 6.5 & 53.29 & 38.24 & 2 \\
\hline 2006 & $\mathrm{~B}$ & 6 & 53.88 & 38.27 & MID RIF \\
\hline 2006 & B & 5 & 54.83 & 38.28 & MID RIF \\
\hline 2006 & B & 4 & 55.73 & 38.33 & MID RIF \\
\hline 2006 & B & 3 & 56.45 & 38.32 & MID RIF \\
\hline 2006 & B & 2.5 & 56.65 & 38.32 & 1 \\
\hline 2006 & B & 2 & 57.27 & 38.33 & MID RIF \\
\hline 2006 & B & 1 & 58.14 & 38.33 & MID RIF \\
\hline 2007 & B & 1 & 6.61 & 36.91 & TAIL POOL \\
\hline 2007 & B & 2 & 7.87 & 37.03 & MID RIFF \\
\hline 2007 & B & 3 & 9.70 & 37.06 & MID RIFF \\
\hline 2007 & B & 4 & 11.60 & 37.20 & MID RIFF \\
\hline 2007 & B & 5 & 13.17 & 37.31 & MID RIFF \\
\hline 2007 & B & 6 & 14.59 & 37.38 & TAIL POOL \\
\hline 2007 & B & 6.5 & 15.21 & 37.24 & 10 \\
\hline 2007 & B & 7 & 15.22 & 37.24 & MID POOL \\
\hline 2007 & B & 8 & 16.78 & 37.28 & MID POOL \\
\hline 2007 & B & 9 & 18.57 & 37.37 & HEAD POOL \\
\hline 2007 & B & 9.5 & 19.65 & 37.43 & 9 \\
\hline 2007 & B & 10 & 20.99 & 37.49 & MID RIFF \\
\hline 2007 & B & 11 & 24.68 & 37.48 & MID RIFF \\
\hline 2007 & B & 11.5 & 25.00 & 37.48 & 8 \\
\hline 2007 & B & 12 & 27.86 & 37.51 & MID RIFF \\
\hline 2007 & B & 12.5 & 29.62 & 37.56 & 7 \\
\hline 2007 & B & 13 & 30.26 & 37.58 & MID RIFF \\
\hline 2007 & B & 14 & 33.31 & 37.51 & MID RIFF \\
\hline 2007 & B & 14.5 & 34.07 & 37.55 & 6 \\
\hline 2007 & B & 15 & 34.83 & 37.59 & MID RIFF \\
\hline 2007 & B & 16 & 36.16 & 37.57 & MID RIFF \\
\hline 2007 & B & 17 & 37.38 & 37.70 & MID RIFF \\
\hline 2007 & B & 17.5 & 38.47 & 37.76 & 5 \\
\hline 2007 & B & 18 & 39.39 & 37.80 & MID RIFF \\
\hline 2007 & B & 19 & 41.48 & 37.78 & MID RIFF \\
\hline
\end{tabular}

Continued on Next Page... 
Table D.4 - Continued

\begin{tabular}{|c|c|c|c|c|c|}
\hline Year & Reach & Point Number & Adjusted Dist. (m) & Adjusted Elev. (m) & Point Description \\
\hline 2007 & B & 19.5 & 43.37 & 37.90 & 4 \\
\hline 2007 & B & 20 & 44.45 & 37.96 & MID RIFF \\
\hline 2007 & B & 21 & 46.13 & 38.01 & MID RIFF \\
\hline 2007 & B & 22 & 48.18 & 38.05 & MID RIFF \\
\hline 2007 & B & 22.5 & 48.53 & 38.06 & 3 \\
\hline 2007 & B & 23 & 49.31 & 38.11 & MID RIFF \\
\hline 2007 & B & 24 & 51.06 & 38.11 & MID RIFF \\
\hline 2007 & B & 25 & 52.41 & 38.24 & MID RIFF \\
\hline 2007 & B & 25.5 & 53.29 & 38.28 & 2 \\
\hline 2007 & B & 26 & 54.17 & 38.32 & MID RIFF \\
\hline 2007 & B & 27 & 56.64 & 38.32 & MID RIFF \\
\hline 2007 & B & 27.5 & 56.65 & 38.32 & 1 \\
\hline 2007 & B & 28 & 58.55 & 38.38 & MID RIFF \\
\hline 2007 & B & 29 & 59.73 & 38.39 & TAIL POOL \\
\hline 2007 & B & 30 & 61.50 & 38.25 & MID POOL \\
\hline 2007 & B & 31 & 63.77 & 38.39 & MID POOL \\
\hline 2007 & B & 32 & 66.14 & 38.23 & MID POOL \\
\hline 2007 & B & 33 & 68.89 & 38.43 & HEAD POOL \\
\hline 2008 & B & 1 & 6.04 & 36.79 & MID POOL \\
\hline 2008 & B & 2 & 7.52 & 36.83 & HEAD POOL \\
\hline 2008 & $\mathrm{~B}$ & 3 & 8.34 & 36.93 & MID RIFF \\
\hline 2008 & B & 4 & 8.87 & 36.91 & MID RIFF \\
\hline 2008 & B & 5 & 9.69 & 36.92 & MID RIFF \\
\hline 2008 & B & 6 & 11.29 & 37.14 & MID RIFF \\
\hline 2008 & B & 7 & 12.31 & 37.11 & MID RIFF \\
\hline 2008 & B & 8 & 13.01 & 37.17 & MID RIFF \\
\hline 2008 & B & 9 & 14.60 & 37.29 & MID RIFF \\
\hline 2008 & B & 9.5 & 15.21 & 37.31 & 10 \\
\hline 2008 & B & 10 & 15.63 & 37.32 & MID RIFF \\
\hline 2008 & B & 11 & 16.50 & 37.34 & MID RIFF \\
\hline 2008 & B & 12 & 18.73 & 37.33 & MID RIFF \\
\hline 2008 & B & 12.5 & 19.65 & 37.35 & 9 \\
\hline 2008 & B & 13 & 20.17 & 37.36 & MID RIFF \\
\hline 2008 & $\mathrm{~B}$ & 14 & 21.68 & 37.34 & MID RIFF \\
\hline 2008 & B & 15 & 22.66 & 37.46 & MID RIFF \\
\hline 2008 & B & 16 & 24.17 & 37.46 & MID RIFF \\
\hline 2008 & B & 16.5 & 25.00 & 37.47 & 8 \\
\hline 2008 & B & 17 & 25.76 & 37.47 & MID RIFF \\
\hline 2008 & B & 18 & 27.94 & 37.41 & MID RIFF \\
\hline 2008 & B & 19 & 29.24 & 37.47 & MID RIFF \\
\hline 2008 & B & 19.5 & 29.62 & 37.48 & 7 \\
\hline 2008 & B & 20 & 32.10 & 37.52 & MID RIFF \\
\hline 2008 & B & 21 & 33.27 & 37.51 & MID RIFF \\
\hline 2008 & B & 21.5 & 34.07 & 37.56 & 6 \\
\hline 2008 & B & 22 & 34.56 & 37.59 & MID RIFF \\
\hline 2008 & B & 23 & 35.70 & 37.61 & TAIL POOL \\
\hline 2008 & B & 24 & 37.11 & 37.50 & MID POOL \\
\hline 2008 & B & 24.5 & 38.47 & 37.59 & 5 \\
\hline 2008 & B & 25 & 38.60 & 37.60 & HEAD POOL \\
\hline 2008 & B & 26 & 39.70 & 37.86 & MID RIFF \\
\hline 2008 & B & 27 & 41.04 & 37.92 & MID RIFF \\
\hline 2008 & B & 28 & 41.89 & 37.87 & MID RIFF \\
\hline
\end{tabular}

Continued on Next Page... 
Table D.4 - Continued

\begin{tabular}{|c|c|c|c|c|c|}
\hline Year & Reach & Point Number & Adjusted Dist. (m) & Adjusted Elev. (m) & Point Description \\
\hline 2008 & B & 28.5 & 43.37 & 37.95 & 4 \\
\hline 2008 & $\mathrm{~B}$ & 29 & 44.64 & 38.00 & MID RIFF \\
\hline 2008 & B & 30 & 45.98 & 38.01 & MID RIFF \\
\hline 2008 & B & 31 & 48.35 & 38.05 & MID RIFF \\
\hline 2008 & $\mathrm{~B}$ & 31.5 & 48.53 & 38.04 & 3 \\
\hline 2008 & $\mathrm{~B}$ & 32 & 48.97 & 38.03 & MID RIFF \\
\hline 2008 & B & 33 & 49.83 & 38.09 & MID RIFF \\
\hline 2008 & $\mathrm{~B}$ & 34 & 50.68 & 38.09 & MID RIFF \\
\hline 2008 & $\mathrm{~B}$ & 35 & 51.82 & 38.14 & MID RIFF \\
\hline 2008 & B & 36 & 52.99 & 38.23 & MID RIFF \\
\hline 2008 & $\mathrm{~B}$ & 36.5 & 53.29 & 38.24 & 2 \\
\hline 2008 & $\mathrm{~B}$ & 37 & 54.48 & 38.30 & MID RIFF \\
\hline 2008 & $\mathrm{~B}$ & 38 & 55.73 & 38.34 & MID RIFF \\
\hline 2008 & $\mathrm{~B}$ & 38.5 & 56.65 & 38.33 & 1 \\
\hline 2008 & B & 39 & 56.85 & 38.33 & MID RIFF \\
\hline 2002 & $\mathrm{C}$ & 1 & 11.28 & 89.13 & BIS.52 \\
\hline 2002 & $\mathrm{C}$ & 1.5 & 14.48 & 89.22 & 10 \\
\hline 2002 & $\mathrm{C}$ & 2 & 17.05 & 89.29 & BIS.65 \\
\hline 2002 & $\mathrm{C}$ & 2.5 & 19.86 & 89.51 & 9 \\
\hline 2002 & $\mathrm{C}$ & 3 & 20.20 & 89.53 & BIS.52 \\
\hline 2002 & $\mathrm{C}$ & 4 & 23.27 & 89.55 & MIDPOOL.82 \\
\hline 2002 & $\mathrm{C}$ & 4.5 & 26.26 & 89.74 & 8 \\
\hline 2002 & $\mathrm{C}$ & 5 & 28.06 & 89.86 & BIS.38 \\
\hline 2002 & $\mathrm{C}$ & 6 & 30.54 & 89.98 & MIDRUN.51 \\
\hline 2002 & $\mathrm{C}$ & 7 & 32.54 & 89.97 & MSC4TW.64 \\
\hline 2002 & $\mathrm{C}$ & 7.5 & 32.62 & 89.98 & 7 \\
\hline 2002 & $\mathrm{C}$ & 8 & 36.71 & 90.18 & MSC5TW.62 \\
\hline 2002 & $\mathrm{C}$ & 9 & 37.48 & 90.05 & TOPRIFF.62 \\
\hline 2002 & $\mathrm{C}$ & 9.5 & 38.34 & 90.07 & 6 \\
\hline 2002 & $\mathrm{C}$ & 10 & 39.57 & 90.10 & TAILPOOL.72 \\
\hline 2002 & $\mathrm{C}$ & 11 & 42.29 & 89.42 & MIDPOOL3.51 \\
\hline 2002 & $\mathrm{C}$ & 12 & 43.17 & 89.56 & ВОТТЈАМ2.53 \\
\hline 2002 & $\mathrm{C}$ & 13 & 43.30 & 90.69 & TAIPODJ.20 \\
\hline 2002 & $\mathrm{C}$ & 14 & 44.96 & 90.48 & MIDPOOL1.14 \\
\hline 2002 & $\mathrm{C}$ & 15 & 45.92 & 90.66 & HEADPOOL.72 \\
\hline 2002 & $\mathrm{C}$ & 16 & 47.00 & 90.80 & BOTRIFF.21 \\
\hline 2002 & $\mathrm{C}$ & 17 & 48.08 & 90.96 & TOPRIFF.50 \\
\hline 2002 & $\mathrm{C}$ & 18 & 49.76 & 90.95 & TAILPOOL.42 \\
\hline 2002 & $\mathrm{C}$ & 19 & 50.56 & 90.99 & MIDPOOL.50 \\
\hline 2002 & $\mathrm{C}$ & 20 & 53.79 & 90.77 & ВОТТЈАМ1.12 \\
\hline 2002 & $\mathrm{C}$ & 21 & 54.02 & 91.68 & TOPJAM. 22 \\
\hline 2002 & $\mathrm{C}$ & 22 & 57.94 & 91.84 & TOPRIFF.50 \\
\hline 2002 & $\mathrm{C}$ & 23 & 60.83 & 91.79 & MIDRIFBND.35 \\
\hline 2002 & $\mathrm{C}$ & 24 & 64.07 & 91.90 & TAILPOOL.43 \\
\hline 2002 & $\mathrm{C}$ & 24.5 & 66.25 & 91.69 & 5 \\
\hline 2002 & $\mathrm{C}$ & 25 & 67.35 & 91.58 & MIDPOOL1.52 \\
\hline 2002 & $\mathrm{C}$ & 26 & 69.02 & 91.89 & BOTRIFTPO.40 \\
\hline 2002 & $\mathrm{C}$ & 26.5 & 71.42 & 91.99 & 4 \\
\hline 2002 & $\mathrm{C}$ & 27 & 73.53 & 92.08 & TOPRIFF.52 \\
\hline 2002 & $\mathrm{C}$ & 27.5 & 75.83 & 92.11 & 3 \\
\hline 2002 & $\mathrm{C}$ & 28 & 76.56 & 92.12 & TOPRIFF.29 \\
\hline 2002 & $\mathrm{C}$ & 28.5 & 79.71 & 92.12 & 2 \\
\hline
\end{tabular}

Continued on Next Page... 
Table D.4 - Continued

\begin{tabular}{|c|c|c|c|c|c|}
\hline Year & Reach & Point Number & Adjusted Dist. (m) & Adjusted Elev. (m) & Point Description \\
\hline 2002 & $\mathrm{C}$ & 29 & 80.54 & 92.12 & TAILPOOL. 48 \\
\hline 2002 & $\mathrm{C}$ & 30 & 83.17 & 91.80 & MIDPOOL1.32 \\
\hline 2002 & $\mathrm{C}$ & 31 & 84.78 & 92.21 & TOPPOOL.46 \\
\hline 2002 & C & 32 & 85.87 & 92.24 & TOPRIFF.47 \\
\hline 2002 & $\mathrm{C}$ & 32.5 & 86.06 & 92.25 & 1 \\
\hline 2002 & $\mathrm{C}$ & 33 & 90.20 & 92.34 & TOPRIFF.49 \\
\hline 2002 & $\mathrm{C}$ & 34 & 92.35 & 92.54 & TOPRIFF.24 \\
\hline 2002 & $\mathrm{C}$ & 35 & 93.71 & 92.52 & TOPRIFF.26 \\
\hline 2002 & $\mathrm{C}$ & 36 & 99.47 & 92.50 & TAILPOOL.59 \\
\hline 2002 & $\mathrm{C}$ & 37 & 100.91 & 92.39 & MIDPOOL1.0 \\
\hline 2002 & $\mathrm{C}$ & 38 & 102.19 & 92.56 & TOPPOOL.43 \\
\hline 2002 & $\mathrm{C}$ & 39 & 104.61 & 92.59 & BEND.50 \\
\hline 2002 & $\mathrm{C}$ & 40 & 108.10 & 92.69 & TOPRIFF.48 \\
\hline 2003 & $\mathrm{C}$ & 1 & 0.75 & 88.77 & MID RIFF \\
\hline 2003 & $\mathrm{C}$ & 2 & 3.79 & 88.92 & MID RIFF \\
\hline 2003 & $\mathrm{C}$ & 3 & 5.55 & 89.08 & TAIL POOL \\
\hline 2003 & $\mathrm{C}$ & 4 & 8.48 & 89.00 & MID POOL \\
\hline 2003 & $\mathrm{C}$ & 5 & 10.45 & 88.99 & HEAD POOL \\
\hline 2003 & $\mathrm{C}$ & 6 & 11.26 & 89.11 & MID RIFF \\
\hline 2003 & $\mathrm{C}$ & 7 & 12.75 & 89.15 & MID RIFF \\
\hline 2003 & $\mathrm{C}$ & 7.5 & 14.48 & 89.22 & 10 \\
\hline 2003 & $\mathrm{C}$ & 8 & 14.64 & 89.22 & MID RIFF \\
\hline 2003 & $\mathrm{C}$ & 9 & 16.15 & 89.32 & MID RIFF \\
\hline 2003 & $\mathrm{C}$ & 10 & 17.65 & 89.43 & TAIL POOL \\
\hline 2003 & $\mathrm{C}$ & 11 & 19.28 & 89.40 & MID POOL \\
\hline 2003 & $\mathrm{C}$ & 12 & 19.48 & 89.44 & HEAD POOL \\
\hline 2003 & $\mathrm{C}$ & 12.5 & 19.86 & 89.51 & 9 \\
\hline 2003 & $\mathrm{C}$ & 13 & 20.89 & 89.65 & MID RIFF \\
\hline 2003 & $\mathrm{C}$ & 14 & 22.91 & 89.59 & GLIDE \\
\hline 2003 & $\mathrm{C}$ & 15 & 26.11 & 89.66 & GLIDE \\
\hline 2003 & $\mathrm{C}$ & 15.5 & 26.26 & 89.66 & 8 \\
\hline 2003 & $\mathrm{C}$ & 16 & 27.80 & 89.67 & MID RIFF \\
\hline 2003 & $\mathrm{C}$ & 17 & 28.68 & 89.88 & MID RIFF \\
\hline 2003 & $\mathrm{C}$ & 18 & 30.56 & 89.97 & MID RIFF \\
\hline 2003 & $\mathrm{C}$ & 19 & 32.58 & 89.99 & MID RIFF \\
\hline 2003 & $\mathrm{C}$ & 19.5 & 32.62 & 89.99 & 7 \\
\hline 2003 & $\mathrm{C}$ & 20 & 33.71 & 90.08 & MID RIFF \\
\hline 2003 & $\mathrm{C}$ & 21 & 35.77 & 90.07 & MID RIFF \\
\hline 2003 & $\mathrm{C}$ & 22 & 37.38 & 90.08 & MID RIFF \\
\hline 2003 & $\mathrm{C}$ & 22.5 & 38.34 & 90.10 & 6 \\
\hline 2003 & $\mathrm{C}$ & 23 & 39.72 & 90.13 & TAIL POOL \\
\hline 2003 & $\mathrm{C}$ & 24 & 41.60 & 90.11 & MID POOL \\
\hline 2003 & $\mathrm{C}$ & 25 & 44.37 & 89.88 & MID POOL \\
\hline 2003 & $\mathrm{C}$ & 26 & 45.09 & 90.21 & HEAD POOL \\
\hline 2003 & $\mathrm{C}$ & 27 & 47.76 & 90.62 & MID LOGJAM \\
\hline 2003 & $\mathrm{C}$ & 28 & 50.60 & 90.81 & UPPER LOGJAM \\
\hline 2003 & $\mathrm{C}$ & 29 & 52.27 & 90.88 & MID RIFF \\
\hline 2003 & $\mathrm{C}$ & 30 & 54.26 & 91.03 & TAIL POOL \\
\hline 2003 & $\mathrm{C}$ & 31 & 56.98 & 90.61 & MID RIFF \\
\hline 2003 & $\mathrm{C}$ & 32 & 58.14 & 90.93 & HEAD POOL \\
\hline 2003 & $\mathrm{C}$ & 33 & 59.24 & 91.62 & TOP LOG STOP \\
\hline 2004 & $\mathrm{C}$ & 1 & 8.50 & 88.97 & MID RIFF \\
\hline
\end{tabular}

Continued on Next Page. . 
Table D.4 - Continued

\begin{tabular}{|c|c|c|c|c|c|}
\hline Year & Reach & Point Number & Adjusted Dist. (m) & Adjusted Elev. (m) & Point Description \\
\hline 2004 & $\mathrm{C}$ & 2 & 10.72 & 89.06 & MID RIFF \\
\hline 2004 & $\mathrm{C}$ & 3 & 12.91 & 89.23 & MID RIFF \\
\hline 2004 & $\mathrm{C}$ & 3.5 & 14.48 & 89.29 & 10 \\
\hline 2004 & $\mathrm{C}$ & 4 & 14.93 & 89.31 & MID RIFF \\
\hline 2004 & $\mathrm{C}$ & 5 & 16.30 & 89.37 & MID RIFF \\
\hline 2004 & $\mathrm{C}$ & 6 & 18.09 & 89.40 & MID RIFF \\
\hline 2004 & $\mathrm{C}$ & 6.5 & 19.86 & 89.44 & 9 \\
\hline 2004 & $\mathrm{C}$ & 7 & 21.68 & 89.47 & MID RIFF \\
\hline 2004 & $\mathrm{C}$ & 8 & 23.69 & 89.30 & MID RIFF \\
\hline 2004 & $\mathrm{C}$ & 9 & 25.30 & 89.34 & MID RIFF \\
\hline 2004 & $\mathrm{C}$ & 9.5 & 26.26 & 89.44 & 8 \\
\hline 2004 & $\mathrm{C}$ & 10 & 27.02 & 89.51 & MID RIFF \\
\hline 2004 & $\mathrm{C}$ & 11 & 29.17 & 89.75 & MID RIFF \\
\hline 2004 & $\mathrm{C}$ & 12 & 30.84 & 89.97 & MID RIFF \\
\hline 2004 & $\mathrm{C}$ & 12.5 & 32.62 & 89.97 & 7 \\
\hline 2004 & $\mathrm{C}$ & 13 & 33.56 & 89.97 & MID RIFF \\
\hline 2004 & $\mathrm{C}$ & 14 & 36.58 & 90.07 & MID RIFF \\
\hline 2004 & $\mathrm{C}$ & 14.5 & 38.34 & 90.19 & 6 \\
\hline 2004 & $\mathrm{C}$ & 15 & 38.60 & 90.21 & MID RIFF \\
\hline 2004 & $\mathrm{C}$ & 16 & 40.09 & 90.22 & MID RIFF \\
\hline 2004 & $\mathrm{C}$ & 17 & 42.03 & 90.12 & MID RIFF \\
\hline 2004 & $\mathrm{C}$ & 18 & 43.26 & 90.06 & BOT STEP \\
\hline 2004 & $\mathrm{C}$ & 19 & 43.81 & 90.51 & TOP STEP \\
\hline 2004 & $\mathrm{C}$ & 20 & 45.16 & 90.76 & MID RIFF \\
\hline 2004 & $\mathrm{C}$ & 21 & 46.34 & 90.79 & MID RIFF \\
\hline 2004 & $\mathrm{C}$ & 22 & 47.94 & 90.78 & MID RIFF \\
\hline 2004 & $\mathrm{C}$ & 23 & 49.30 & 90.90 & MID RIFF \\
\hline 2004 & $\mathrm{C}$ & 24 & 50.61 & 90.98 & MID RIFF \\
\hline 2004 & $\mathrm{C}$ & 25 & 51.61 & 91.04 & TAIL POOL \\
\hline 2004 & $\mathrm{C}$ & 26 & 53.23 & 90.41 & MID POOL \\
\hline 2004 & $\mathrm{C}$ & 27 & 54.04 & 90.68 & HEAD POOL \\
\hline 2004 & $\mathrm{C}$ & 28 & 54.74 & 91.63 & TOP STEP \\
\hline 2004 & $\mathrm{C}$ & 29 & 57.26 & 91.75 & MID RIFF \\
\hline 2004 & $\mathrm{C}$ & 30 & 59.35 & 91.74 & MID RIFF \\
\hline 2004 & $\mathrm{C}$ & 31 & 61.40 & 91.80 & MID RIFF \\
\hline 2004 & $\mathrm{C}$ & 32 & 62.80 & 91.84 & BOT GLIDE \\
\hline 2004 & $\mathrm{C}$ & 33 & 64.59 & 91.83 & MID GLIDE \\
\hline 2004 & $\mathrm{C}$ & 33.5 & 66.25 & 91.78 & 5 \\
\hline 2004 & $\mathrm{C}$ & 34 & 67.62 & 91.74 & TOP GLIDE \\
\hline 2004 & $\mathrm{C}$ & 35 & 68.84 & 91.82 & MID RIFF \\
\hline 2004 & $\mathrm{C}$ & 36 & 70.38 & 91.97 & MID RIFF \\
\hline 2004 & C & 36.5 & 71.42 & 91.96 & 4 \\
\hline 2004 & $\mathrm{C}$ & 37 & 71.87 & 91.96 & MID RIFF \\
\hline 2004 & $\mathrm{C}$ & 38 & 73.76 & 92.05 & MID RIFF \\
\hline 2004 & $\mathrm{C}$ & 38.5 & 75.83 & 92.11 & 3 \\
\hline 2004 & $\mathrm{C}$ & 39 & 76.12 & 92.11 & MID RIFF \\
\hline 2004 & $\mathrm{C}$ & 40 & 78.46 & 92.17 & MID RIFF \\
\hline 2004 & $\mathrm{C}$ & 40.5 & 79.71 & 92.07 & 2 \\
\hline 2004 & $\mathrm{C}$ & 41 & 80.74 & 92.00 & MID RIFF \\
\hline 2004 & $\mathrm{C}$ & 42 & 83.04 & 91.89 & MID RIFF \\
\hline 2004 & $\mathrm{C}$ & 43 & 84.10 & 92.01 & MID RIFF \\
\hline 2004 & $\mathrm{C}$ & 44 & 85.82 & 92.13 & MID RIFF \\
\hline
\end{tabular}

Continued on Next Page... 
Table D.4 - Continued

\begin{tabular}{|c|c|c|c|c|c|}
\hline Year & Reach & Point Number & Adjusted Dist. (m) & Adjusted Elev. (m) & Point Description \\
\hline 2004 & $\mathrm{C}$ & 44.5 & 86.06 & 92.15 & 1 \\
\hline 2004 & $\mathrm{C}$ & 45 & 87.30 & 92.24 & MID RIFF \\
\hline 2004 & $\mathrm{C}$ & 46 & 89.51 & 92.33 & MID RIFF \\
\hline 2004 & C & 47 & 91.51 & 92.44 & MID RIFF \\
\hline 2004 & $\mathrm{C}$ & 48 & 93.96 & 92.48 & MID RIFF \\
\hline 2005 & $\mathrm{C}$ & 1 & 7.09 & 89.00 & MID RIFF \\
\hline 2005 & $\mathrm{C}$ & 2 & 9.61 & 88.95 & MID RIFF \\
\hline 2005 & $\mathrm{C}$ & 3 & 10.60 & 88.91 & MID RIFF \\
\hline 2005 & $\mathrm{C}$ & 4 & 11.26 & 88.90 & MID RIFF \\
\hline 2005 & $\mathrm{C}$ & 5 & 12.08 & 89.08 & MID RIFF \\
\hline 2005 & $\mathrm{C}$ & 6 & 12.85 & 89.18 & MID RIFF \\
\hline 2005 & $\mathrm{C}$ & 7 & 13.91 & 89.24 & MID RIFF \\
\hline 2005 & $\mathrm{C}$ & 7.5 & 14.48 & 89.26 & 10 \\
\hline 2005 & $\mathrm{C}$ & 8 & 15.30 & 89.29 & MID RIFF \\
\hline 2005 & $\mathrm{C}$ & 9 & 16.19 & 89.37 & MID RIFF \\
\hline 2005 & $\mathrm{C}$ & 10 & 17.68 & 89.32 & MID RIFF \\
\hline 2005 & $\mathrm{C}$ & 11 & 19.29 & 89.40 & MID RIFF \\
\hline 2005 & $\mathrm{C}$ & 11.5 & 19.86 & 89.41 & 9 \\
\hline 2005 & $\mathrm{C}$ & 12 & 22.19 & 89.42 & TAIL POOL \\
\hline 2005 & C & 13 & 22.92 & 89.33 & MID POOL \\
\hline 2005 & $\mathrm{C}$ & 14 & 23.77 & 89.35 & HEAD POOL \\
\hline 2005 & $\mathrm{C}$ & 15 & 25.72 & 89.45 & MID RIFF \\
\hline 2005 & $\mathrm{C}$ & 15.5 & 26.26 & 89.48 & 8 \\
\hline 2005 & $\mathrm{C}$ & 16 & 26.97 & 89.53 & MID RIFF \\
\hline 2005 & $\mathrm{C}$ & 17 & 27.95 & 89.72 & MID RIFF \\
\hline 2005 & $\mathrm{C}$ & 18 & 28.52 & 89.77 & MID RIFF \\
\hline 2005 & $\mathrm{C}$ & 19 & 29.51 & 89.70 & MID RIFF \\
\hline 2005 & $\mathrm{C}$ & 20 & 30.39 & 89.93 & MID RIFF \\
\hline 2005 & $\mathrm{C}$ & 21 & 30.76 & 89.94 & MID RIFF \\
\hline 2005 & $\mathrm{C}$ & 22 & 31.73 & 89.96 & MID RIFF \\
\hline 2005 & $\mathrm{C}$ & 22.5 & 32.62 & 89.97 & 7 \\
\hline 2005 & $\mathrm{C}$ & 23 & 33.27 & 89.97 & MID RIFF \\
\hline 2005 & $\mathrm{C}$ & 24 & 34.54 & 90.03 & MID RIFF \\
\hline 2005 & $\mathrm{C}$ & 25 & 35.64 & 90.08 & MID RIFF \\
\hline 2005 & $\mathrm{C}$ & 26 & 36.91 & 90.16 & MID RIFF \\
\hline 2005 & $\mathrm{C}$ & 27 & 38.09 & 90.17 & MID RIFF \\
\hline 2005 & $\mathrm{C}$ & 27.5 & 38.34 & 90.18 & 6 \\
\hline 2005 & $\mathrm{C}$ & 28 & 39.58 & 90.24 & MID RIFF \\
\hline 2005 & $\mathrm{C}$ & 29 & 40.62 & 90.29 & TAIL POOL \\
\hline 2005 & $\mathrm{C}$ & 30 & 41.47 & 90.04 & MID POOL \\
\hline 2005 & $\mathrm{C}$ & 31 & 41.99 & 90.01 & HEAD POOL \\
\hline 2005 & $\mathrm{C}$ & 32 & 43.07 & 90.22 & BOTTOM LOG STEP \\
\hline 2005 & $\mathrm{C}$ & 33 & 43.57 & 90.71 & TOP LOG STEP \\
\hline 2005 & $\mathrm{C}$ & 34 & 45.13 & 90.67 & MID RIFF \\
\hline 2005 & $\mathrm{C}$ & 35 & 45.88 & 90.57 & MID RIFF \\
\hline 2005 & $\mathrm{C}$ & 36 & 46.88 & 90.82 & MID RIFF \\
\hline 2005 & $\mathrm{C}$ & 37 & 47.49 & 90.75 & MID RIFF \\
\hline 2005 & $\mathrm{C}$ & 38 & 48.15 & 90.83 & MID RIFF \\
\hline 2005 & $\mathrm{C}$ & 39 & 49.31 & 90.84 & MID RIFF \\
\hline 2005 & $\mathrm{C}$ & 40 & 50.48 & 90.93 & MID RIFF \\
\hline 2005 & $\mathrm{C}$ & 41 & 52.52 & 90.99 & TAIL POOL \\
\hline 2005 & $\mathrm{C}$ & 42 & 53.43 & 90.68 & MID POOL \\
\hline
\end{tabular}

Continued on Next Page... 
Table D.4 - Continued

\begin{tabular}{|c|c|c|c|c|c|}
\hline Year & Reach & Point Number & Adjusted Dist. (m) & Adjusted Elev. (m) & Point Description \\
\hline 2005 & $\mathrm{C}$ & 43 & 54.04 & 90.57 & HEAD POOL \\
\hline 2005 & $\mathrm{C}$ & 44 & 55.30 & 90.78 & BOTTOM LOG STEP \\
\hline 2005 & $\mathrm{C}$ & 45 & 55.79 & 91.62 & TOP LOG STEP \\
\hline 2005 & $\mathrm{C}$ & 46 & 56.81 & 91.70 & MID RIFF \\
\hline 2005 & $\mathrm{C}$ & 47 & 57.82 & 91.75 & MID RIFF \\
\hline 2005 & $\mathrm{C}$ & 48 & 59.04 & 91.75 & MID RIFF \\
\hline 2005 & $\mathrm{C}$ & 49 & 60.80 & 91.78 & MID RIFF \\
\hline 2005 & $\mathrm{C}$ & 50 & 61.73 & 91.76 & MID RIFF \\
\hline 2005 & $\mathrm{C}$ & 51 & 62.78 & 91.81 & MID RIFF \\
\hline 2005 & $\mathrm{C}$ & 52 & 63.22 & 91.78 & MID RIFF \\
\hline 2005 & $\mathrm{C}$ & 53 & 63.84 & 91.81 & MID RIFF \\
\hline 2005 & $\mathrm{C}$ & 54 & 64.47 & 91.75 & MID RIFF \\
\hline 2005 & $\mathrm{C}$ & 55 & 65.60 & 91.75 & MID RIFF \\
\hline 2005 & $\mathrm{C}$ & 55.5 & 66.25 & 91.74 & 5 \\
\hline 2005 & $\mathrm{C}$ & 56 & 67.20 & 91.72 & MID RIFF \\
\hline 2005 & $\mathrm{C}$ & 57 & 68.96 & 91.84 & MID RIFF \\
\hline 2005 & $\mathrm{C}$ & 57.5 & 71.42 & 91.95 & 4 \\
\hline 2005 & $\mathrm{C}$ & 58 & 71.52 & 91.95 & MID RIFF \\
\hline 2005 & $\mathrm{C}$ & 59 & 74.05 & 92.02 & MID RIFF \\
\hline 2005 & $\mathrm{C}$ & 59.5 & 75.83 & 92.04 & 3 \\
\hline 2005 & $\mathrm{C}$ & 60 & 76.54 & 92.04 & MID RIFF \\
\hline 2005 & $\mathrm{C}$ & 61 & 78.42 & 92.18 & MID RIFF \\
\hline 2005 & $\mathrm{C}$ & 61.5 & 79.71 & 92.06 & 2 \\
\hline 2005 & $\mathrm{C}$ & 62 & 80.87 & 91.96 & TAIL POOL \\
\hline 2005 & $\mathrm{C}$ & 63 & 82.24 & 91.90 & MID POOL \\
\hline 2005 & $\mathrm{C}$ & 64 & 83.64 & 91.96 & HEAD POOL \\
\hline 2005 & $\mathrm{C}$ & 65 & 85.00 & 92.16 & MID RIFF \\
\hline 2005 & $\mathrm{C}$ & 65.5 & 86.06 & 92.22 & 1 \\
\hline 2005 & $\mathrm{C}$ & 66 & 92.61 & 92.23 & MID RIFF \\
\hline 2005 & $\mathrm{C}$ & 67 & 93.99 & 92.26 & MID RIFF \\
\hline 2005 & $\mathrm{C}$ & 68 & 95.27 & 92.30 & MID RIFF \\
\hline 2005 & $\mathrm{C}$ & 69 & 97.07 & 92.40 & MID RIFF \\
\hline 2005 & $\mathrm{C}$ & 70 & 98.42 & 92.35 & MID RIFF \\
\hline 2005 & $\mathrm{C}$ & 71 & 100.43 & 92.54 & MID RIFF \\
\hline 2006 & $\mathrm{C}$ & 1 & 14.30 & 89.12 & MID RIF \\
\hline 2006 & $\mathrm{C}$ & 1.5 & 14.48 & 89.11 & 10 \\
\hline 2006 & $\mathrm{C}$ & 2 & 14.80 & 89.08 & MID RIF \\
\hline 2006 & $\mathrm{C}$ & 3 & 15.41 & 89.10 & MID RIF \\
\hline 2006 & $\mathrm{C}$ & 4 & 16.10 & 89.22 & MID RIF \\
\hline 2006 & $\mathrm{C}$ & 5 & 16.38 & 89.28 & MID RIF \\
\hline 2006 & $\mathrm{C}$ & 6 & 17.89 & 89.29 & MID RIF \\
\hline 2006 & $\mathrm{C}$ & 7 & 18.84 & 89.39 & MID RIF \\
\hline 2006 & $\mathrm{C}$ & 8 & 19.85 & 89.34 & MID RIF \\
\hline 2006 & $\mathrm{C}$ & 8.5 & 19.86 & 89.34 & 9 \\
\hline 2006 & $\mathrm{C}$ & 9 & 21.11 & 89.30 & MID RIF \\
\hline 2006 & $\mathrm{C}$ & 10 & 22.08 & 89.42 & MID RIF \\
\hline 2006 & $\mathrm{C}$ & 11 & 22.92 & 89.47 & MID RIF \\
\hline 2006 & $\mathrm{C}$ & 12 & 24.42 & 89.51 & MID RIF \\
\hline 2006 & $\mathrm{C}$ & 13 & 25.90 & 89.56 & MID RIF \\
\hline 2006 & $\mathrm{C}$ & 13.5 & 26.26 & 89.54 & 8 \\
\hline 2006 & $\mathrm{C}$ & 14 & 27.28 & 89.50 & MID RIF \\
\hline 2006 & $\mathrm{C}$ & 15 & 28.89 & 89.74 & DOWN WOODY DEBRI \\
\hline
\end{tabular}

Continued on Next Page... 
Table D.4 - Continued

\begin{tabular}{|c|c|c|c|c|c|}
\hline Year & Reach & Point Number & Adjusted Dist. (m) & Adjusted Elev. (m) & Point Description \\
\hline 2006 & $\mathrm{C}$ & 16 & 29.30 & 89.72 & UP WOODY DEBRIS \\
\hline 2006 & $\mathrm{C}$ & 17 & 29.71 & 89.70 & MID RIF \\
\hline 2006 & $\mathrm{C}$ & 18 & 30.86 & 89.62 & MID RIF \\
\hline 2006 & $\mathrm{C}$ & 19 & 31.63 & 89.59 & MID RIF \\
\hline 2006 & $\mathrm{C}$ & 20 & 32.22 & 89.72 & MID RIF \\
\hline 2006 & $\mathrm{C}$ & 20.5 & 32.62 & 89.75 & 7 \\
\hline 2006 & $\mathrm{C}$ & 21 & 33.13 & 89.79 & MID RIF \\
\hline 2006 & $\mathrm{C}$ & 22 & 34.15 & 89.84 & MID RIF \\
\hline 2006 & $\mathrm{C}$ & 23 & 35.13 & 89.83 & MID RIF \\
\hline 2006 & $\mathrm{C}$ & 24 & 35.68 & 89.92 & MID RIF \\
\hline 2006 & $\mathrm{C}$ & 25 & 36.20 & 89.90 & DOWN WOODY DEBRI \\
\hline 2006 & $\mathrm{C}$ & 26 & 36.74 & 90.01 & UP WOODY DEBRIS \\
\hline 2006 & $\mathrm{C}$ & 27 & 37.20 & 90.03 & MID RIF \\
\hline 2006 & $\mathrm{C}$ & 28 & 38.12 & 90.13 & MID RIF \\
\hline 2006 & $\mathrm{C}$ & 28.5 & 38.34 & 90.10 & 6 \\
\hline 2006 & $\mathrm{C}$ & 29 & 38.73 & 90.05 & MID RIF \\
\hline 2006 & $\mathrm{C}$ & 30 & 39.59 & 90.13 & MID RIF \\
\hline 2006 & $\mathrm{C}$ & 31 & 40.69 & 90.07 & MID RIF \\
\hline 2006 & $\mathrm{C}$ & 32 & 40.93 & 89.98 & TAIL POOL \\
\hline 2006 & $\mathrm{C}$ & 33 & 41.28 & 89.83 & MID POOL \\
\hline 2006 & $\mathrm{C}$ & 34 & 41.48 & 89.85 & HEAD POOL \\
\hline 2006 & $\mathrm{C}$ & 35 & 41.79 & 89.95 & BOTTOM ROCK STEP \\
\hline 2006 & $\mathrm{C}$ & 36 & 41.90 & 90.26 & TOP ROCK STEP \\
\hline 2006 & $\mathrm{C}$ & 37 & 42.45 & 90.05 & MID RIF \\
\hline 2006 & $\mathrm{C}$ & 38 & 43.08 & 89.95 & BOTTOM LOG STEP \\
\hline 2006 & $\mathrm{C}$ & 39 & 43.20 & 90.65 & TOP LOG STEP \\
\hline 2006 & $\mathrm{C}$ & 40 & 43.53 & 90.61 & MID RIF \\
\hline 2006 & $\mathrm{C}$ & 41 & 44.76 & 90.76 & MID RIF \\
\hline 2006 & $\mathrm{C}$ & 42 & 45.31 & 90.78 & MID RIF \\
\hline 2006 & $\mathrm{C}$ & 43 & 46.86 & 90.85 & MID RIF \\
\hline 2006 & $\mathrm{C}$ & 44 & 47.26 & 90.87 & MID RIF \\
\hline 2006 & $\mathrm{C}$ & 45 & 47.95 & 90.75 & MID RIF \\
\hline 2006 & $\mathrm{C}$ & 46 & 48.71 & 90.80 & MID RIF \\
\hline 2006 & $\mathrm{C}$ & 47 & 49.01 & 90.87 & MID RIF \\
\hline 2006 & $\mathrm{C}$ & 48 & 49.91 & 90.95 & MID RIF \\
\hline 2006 & $\mathrm{C}$ & 49 & 50.33 & 90.96 & MID RIF \\
\hline 2006 & $\mathrm{C}$ & 50 & 50.99 & 91.03 & MID RIF \\
\hline 2006 & $\mathrm{C}$ & 51 & 52.18 & 90.95 & TAIL POOL \\
\hline 2006 & $\mathrm{C}$ & 52 & 53.56 & 90.61 & MID POOL \\
\hline 2006 & $\mathrm{C}$ & 53 & 54.23 & 90.67 & MID POOL \\
\hline 2006 & $\mathrm{C}$ & 54 & 54.55 & 90.65 & HEAD POOL \\
\hline 2006 & $\mathrm{C}$ & 55 & 54.66 & 91.31 & TOP LOG STEP \\
\hline 2006 & $\mathrm{C}$ & 56 & 55.14 & 91.22 & MID RIF \\
\hline 2006 & $\mathrm{C}$ & 57 & 55.92 & 91.37 & MID RIF \\
\hline 2006 & $\mathrm{C}$ & 58 & 56.17 & 91.38 & MID RIF \\
\hline 2006 & C & 59 & 57.22 & 91.39 & MID RIF \\
\hline 2006 & $\mathrm{C}$ & 60 & 58.74 & 91.42 & MID RIF \\
\hline 2006 & $\mathrm{C}$ & 61 & 59.94 & 91.43 & MID RIF \\
\hline 2006 & $\mathrm{C}$ & 62 & 60.59 & 91.46 & MID RIF \\
\hline 2006 & $\mathrm{C}$ & 63 & 61.05 & 91.54 & MID RIF \\
\hline 2006 & $\mathrm{C}$ & 64 & 61.19 & 91.55 & MID RIF \\
\hline 2006 & $\mathrm{C}$ & 65 & 62.47 & 91.54 & MID RIF \\
\hline
\end{tabular}

Continued on Next Page... 
Table D.4 - Continued

\begin{tabular}{|c|c|c|c|c|c|}
\hline Year & Reach & Point Number & Adjusted Dist. (m) & Adjusted Elev. (m) & Point Description \\
\hline 2006 & $\mathrm{C}$ & 66 & 62.96 & 91.57 & MID RIF \\
\hline 2006 & $\mathrm{C}$ & 67 & 64.53 & 91.61 & MID RIF \\
\hline 2006 & C & 68 & 65.34 & 91.62 & MID RIF \\
\hline 2006 & C & 69 & 66.09 & 91.64 & MID RIF \\
\hline 2006 & $\mathrm{C}$ & 69.5 & 66.25 & 91.62 & 5 \\
\hline 2006 & $\mathrm{C}$ & 70 & 66.85 & 91.59 & MID RIF \\
\hline 2006 & C & 71 & 67.39 & 91.58 & TAIL POOL \\
\hline 2006 & $\mathrm{C}$ & 72 & 68.19 & 91.47 & MID POOL \\
\hline 2006 & $\mathrm{C}$ & 73 & 68.62 & 91.51 & HEAD POOL \\
\hline 2006 & $\mathrm{C}$ & 74 & 68.83 & 91.62 & MID RIF \\
\hline 2006 & C & 75 & 69.25 & 91.75 & MID RIF \\
\hline 2006 & $\mathrm{C}$ & 76 & 70.17 & 91.80 & MID RIF \\
\hline 2006 & $\mathrm{C}$ & 77 & 71.21 & 91.85 & MID RIF \\
\hline 2006 & $\mathrm{C}$ & 77.5 & 71.42 & 91.84 & 4 \\
\hline 2006 & C & 78 & 72.02 & 91.82 & MID RIF \\
\hline 2006 & C & 79 & 72.55 & 91.80 & MID RIF \\
\hline 2006 & $\mathrm{C}$ & 80 & 73.78 & 91.88 & MID RIF \\
\hline 2006 & $\mathrm{C}$ & 81 & 74.93 & 91.87 & MID RIF \\
\hline 2006 & $\mathrm{C}$ & 82 & 75.81 & 91.93 & MID RIF \\
\hline 2006 & C & 82.5 & 75.83 & 91.93 & 3 \\
\hline 2006 & $\mathrm{C}$ & 83 & 77.24 & 92.03 & MID RIF \\
\hline 2006 & $\mathrm{C}$ & 84 & 77.93 & 92.04 & MID RIF \\
\hline 2006 & $\mathrm{C}$ & 85 & 78.64 & 91.97 & MID RIF \\
\hline 2006 & $\mathrm{C}$ & 85.5 & 79.71 & 92.04 & 2 \\
\hline 2006 & $\mathrm{C}$ & 86 & 80.02 & 92.05 & MID RIF \\
\hline 2006 & $\mathrm{C}$ & 87 & 80.78 & 91.96 & TAIL POOL \\
\hline 2006 & $\mathrm{C}$ & 88 & 81.66 & 91.77 & MID POOL \\
\hline 2006 & $\mathrm{C}$ & 89 & 82.56 & 91.75 & HEAD POOL \\
\hline 2006 & $\mathrm{C}$ & 90 & 83.46 & 91.79 & MID RIF \\
\hline 2006 & $\mathrm{C}$ & 91 & 84.36 & 91.96 & MID RIF \\
\hline 2006 & $\mathrm{C}$ & 92 & 85.04 & 92.11 & MID RIF \\
\hline 2006 & $\mathrm{C}$ & 92.5 & 86.06 & 92.16 & 1 \\
\hline 2006 & $\mathrm{C}$ & 93 & 86.35 & 92.17 & MID RIF \\
\hline 2006 & $\mathrm{C}$ & 94 & 87.41 & 92.24 & MID RIF \\
\hline 2006 & $\mathrm{C}$ & 95 & 89.37 & 92.30 & MID RIF \\
\hline 2006 & $\mathrm{C}$ & 96 & 89.85 & 92.31 & MID RIF \\
\hline 2006 & $\mathrm{C}$ & 97 & 89.98 & 92.37 & MID RIF \\
\hline 2006 & $\mathrm{C}$ & 98 & 90.41 & 92.33 & MID RIF \\
\hline 2006 & $\mathrm{C}$ & 99 & 91.17 & 92.32 & MID RIF \\
\hline 2006 & $\mathrm{C}$ & 100 & 92.39 & 92.38 & MID RIF \\
\hline 2006 & $\mathrm{C}$ & 101 & 93.45 & 92.37 & MID RIF \\
\hline 2006 & C & 102 & 94.79 & 92.43 & MID RIF \\
\hline 2007 & $\mathrm{C}$ & 1 & 5.77 & 88.98 & TAIL POOL \\
\hline 2007 & $\mathrm{C}$ & 2 & 7.84 & 88.99 & MID POOL \\
\hline 2007 & $\mathrm{C}$ & 3 & 9.99 & 88.83 & MID POOL \\
\hline 2007 & $\mathrm{C}$ & 4 & 10.96 & 88.95 & HEAD POOL \\
\hline 2007 & $\mathrm{C}$ & 5 & 12.88 & 89.14 & MID RIFF \\
\hline 2007 & $\mathrm{C}$ & 6 & 13.98 & 89.16 & TAIL POOL \\
\hline 2007 & $\mathrm{C}$ & 6.5 & 14.48 & 89.10 & 10 \\
\hline 2007 & $\mathrm{C}$ & 7 & 14.57 & 89.09 & MID POOL \\
\hline 2007 & $\mathrm{C}$ & 8 & 15.28 & 89.09 & HEAD POOL \\
\hline 2007 & $\mathrm{C}$ & 9 & 15.85 & 89.16 & MID RIFF \\
\hline
\end{tabular}

Continued on Next Page... 
Table D.4 - Continued

\begin{tabular}{|c|c|c|c|c|c|}
\hline Year & Reach & Point Number & Adjusted Dist. (m) & Adjusted Elev. (m) & Point Description \\
\hline 2007 & $\mathrm{C}$ & 10 & 16.38 & 89.24 & TAIL POOL \\
\hline 2007 & $\mathrm{C}$ & 11 & 17.16 & 89.24 & MID POOL \\
\hline 2007 & C & 12 & 17.70 & 89.24 & HEAD POOL \\
\hline 2007 & C & 13 & 18.44 & 89.32 & MID RIFF \\
\hline 2007 & $\mathrm{C}$ & 14 & 19.29 & 89.45 & TAIL POOL \\
\hline 2007 & $\mathrm{C}$ & 14.5 & 19.86 & 89.39 & 9 \\
\hline 2007 & $\mathrm{C}$ & 15 & 20.28 & 89.35 & MID POOL \\
\hline 2007 & $\mathrm{C}$ & 16 & 21.85 & 89.37 & HEAD POOL \\
\hline 2007 & $\mathrm{C}$ & 17 & 22.71 & 89.41 & MID RIFF \\
\hline 2007 & $\mathrm{C}$ & 18 & 23.45 & 89.48 & TAIL POOL \\
\hline 2007 & $\mathrm{C}$ & 19 & 24.84 & 89.49 & MID POOL \\
\hline 2007 & $\mathrm{C}$ & 20 & 25.79 & 89.50 & HEAD POOL \\
\hline 2007 & $\mathrm{C}$ & 20.5 & 26.26 & 89.55 & 8 \\
\hline 2007 & $\mathrm{C}$ & 21 & 26.62 & 89.59 & MID RIFF \\
\hline 2007 & $\mathrm{C}$ & 22 & 28.20 & 89.68 & TAIL POOL \\
\hline 2007 & $\mathrm{C}$ & 23 & 30.10 & 89.70 & MID POOL \\
\hline 2007 & $\mathrm{C}$ & 24 & 31.87 & 89.75 & HEAD POOL \\
\hline 2007 & $\mathrm{C}$ & 24.5 & 32.62 & 89.78 & 7 \\
\hline 2007 & $\mathrm{C}$ & 25 & 35.17 & 89.89 & MID RIFF \\
\hline 2007 & C & 26 & 37.72 & 90.12 & MID RIFF \\
\hline 2007 & $\mathrm{C}$ & 26.5 & 38.34 & 90.11 & 6 \\
\hline 2007 & $\mathrm{C}$ & 27 & 39.12 & 90.10 & MID RIFF \\
\hline 2007 & $\mathrm{C}$ & 28 & 40.31 & 90.14 & TAIL POOL \\
\hline 2007 & $\mathrm{C}$ & 29 & 41.62 & 89.90 & MID POOL \\
\hline 2007 & $\mathrm{C}$ & 30 & 42.80 & 90.09 & BOT STEP \\
\hline 2007 & $\mathrm{C}$ & 31 & 43.16 & 90.66 & TOP STEP \\
\hline 2007 & $\mathrm{C}$ & 32 & 45.05 & 90.80 & MID RIFF \\
\hline 2007 & $\mathrm{C}$ & 33 & 46.08 & 90.81 & MID RIFF \\
\hline 2007 & $\mathrm{C}$ & 34 & 46.85 & 90.84 & TAIL POOL \\
\hline 2007 & $\mathrm{C}$ & 35 & 47.75 & 90.77 & MID POOL \\
\hline 2007 & $\mathrm{C}$ & 36 & 48.40 & 90.82 & HEAD POOL \\
\hline 2007 & $\mathrm{C}$ & 37 & 50.06 & 90.97 & MID RIFF \\
\hline 2007 & $\mathrm{C}$ & 38 & 51.01 & 91.04 & TAIL POOL \\
\hline 2007 & $\mathrm{C}$ & 39 & 52.35 & 90.97 & MID POOL \\
\hline 2007 & $\mathrm{C}$ & 40 & 53.74 & 90.61 & MID POOL \\
\hline 2007 & $\mathrm{C}$ & 41 & 54.34 & 90.72 & BOT STEP \\
\hline 2007 & $\mathrm{C}$ & 42 & 55.22 & 91.22 & TOP STEP \\
\hline 2007 & $\mathrm{C}$ & 43 & 57.30 & 91.37 & MID RIFF \\
\hline 2007 & $\mathrm{C}$ & 44 & 59.80 & 91.42 & MID RIFF \\
\hline 2007 & $\mathrm{C}$ & 45 & 62.14 & 91.56 & MID RIFF \\
\hline 2007 & $\mathrm{C}$ & 46 & 64.04 & 91.58 & TAIL POOL \\
\hline 2007 & $\mathrm{C}$ & 46.5 & 66.25 & 91.59 & 5 \\
\hline 2007 & $\mathrm{C}$ & 47 & 67.48 & 91.60 & MID POOL \\
\hline 2007 & $\mathrm{C}$ & 48 & 68.58 & 91.51 & HEAD POOL \\
\hline 2007 & $\mathrm{C}$ & 49 & 69.63 & 91.79 & MID RIFF \\
\hline 2007 & $\mathrm{C}$ & 50 & 71.08 & 91.87 & TAIL POOL \\
\hline 2007 & $\mathrm{C}$ & 50.5 & 71.42 & 91.86 & 4 \\
\hline 2007 & $\mathrm{C}$ & 51 & 72.06 & 91.84 & MID POOL \\
\hline 2007 & $\mathrm{C}$ & 52 & 73.72 & 91.88 & HEAD POOL \\
\hline 2007 & $\mathrm{C}$ & 53 & 74.59 & 91.90 & MID RIFF \\
\hline 2007 & $\mathrm{C}$ & 54 & 75.67 & 91.95 & MID RIFF \\
\hline 2007 & $\mathrm{C}$ & 54.5 & 75.83 & 91.96 & 3 \\
\hline
\end{tabular}

Continued on Next Page... 
Table D.4 - Continued

\begin{tabular}{|c|c|c|c|c|c|}
\hline Year & Reach & Point Number & Adjusted Dist. (m) & Adjusted Elev. (m) & Point Description \\
\hline 2007 & $\mathrm{C}$ & 55 & 76.70 & 92.03 & TAIL POOL \\
\hline 2007 & $\mathrm{C}$ & 56 & 77.49 & 92.00 & MID POOL \\
\hline 2007 & C & 57 & 78.76 & 92.01 & HEAD POOL \\
\hline 2007 & C & 58 & 79.19 & 92.03 & MID RIFF \\
\hline 2007 & $\mathrm{C}$ & 59 & 79.40 & 92.05 & TAIL POOL \\
\hline 2007 & $\mathrm{C}$ & 59.5 & 79.71 & 92.03 & 2 \\
\hline 2007 & C & 60 & 80.77 & 91.98 & MID POOL \\
\hline 2007 & $\mathrm{C}$ & 61 & 82.08 & 91.77 & MID POOL \\
\hline 2007 & $\mathrm{C}$ & 62 & 84.30 & 92.03 & HEAD POOL \\
\hline 2007 & $\mathrm{C}$ & 62.5 & 86.06 & 92.15 & 1 \\
\hline 2007 & C & 63 & 89.65 & 92.21 & MID RIFF \\
\hline 2008 & $\mathrm{C}$ & 1 & -3.31 & 88.59 & MID RIFF \\
\hline 2008 & $\mathrm{C}$ & 2 & -2.59 & 88.59 & MID RIFF \\
\hline 2008 & $\mathrm{C}$ & 3 & -1.24 & 88.52 & MID RIFF \\
\hline 2008 & $\mathrm{C}$ & 4 & 0.25 & 88.58 & MID RIFF \\
\hline 2008 & C & 5 & 2.51 & 88.70 & MID RIFF \\
\hline 2008 & $\mathrm{C}$ & 6 & 3.96 & 88.88 & MID RIFF \\
\hline 2008 & $\mathrm{C}$ & 7 & 5.64 & 88.97 & MID RIFF \\
\hline 2008 & $\mathrm{C}$ & 8 & 6.78 & 88.99 & MID RIFF \\
\hline 2008 & C & 9 & 7.80 & 88.93 & MID RIFF \\
\hline 2008 & $\mathrm{C}$ & 10 & 8.89 & 88.86 & MID RIFF \\
\hline 2008 & $\mathrm{C}$ & 11 & 10.08 & 89.01 & MID RIFF \\
\hline 2008 & $\mathrm{C}$ & 12 & 11.33 & 89.06 & MID RIFF \\
\hline 2008 & $\mathrm{C}$ & 13 & 12.26 & 89.07 & MID RIFF \\
\hline 2008 & $\mathrm{C}$ & 14 & 13.03 & 89.10 & MID RIFF \\
\hline 2008 & $\mathrm{C}$ & 15 & 13.78 & 89.19 & MID RIFF \\
\hline 2008 & $\mathrm{C}$ & 15.5 & 14.48 & 89.18 & 10 \\
\hline 2008 & $\mathrm{C}$ & 16 & 14.60 & 89.18 & MID RIFF \\
\hline 2008 & $\mathrm{C}$ & 17 & 15.76 & 89.18 & MID RIFF \\
\hline 2008 & $\mathrm{C}$ & 18 & 16.62 & 89.23 & MID RIFF \\
\hline 2008 & $\mathrm{C}$ & 19 & 17.42 & 89.13 & MID RIFF \\
\hline 2008 & $\mathrm{C}$ & 20 & 18.68 & 89.17 & MID RIFF \\
\hline 2008 & $\mathrm{C}$ & 21 & 19.78 & 89.26 & MID RIFF \\
\hline 2008 & $\mathrm{C}$ & 21.5 & 19.86 & 89.26 & 9 \\
\hline 2008 & $\mathrm{C}$ & 22 & 21.48 & 89.35 & MID RIFF \\
\hline 2008 & $\mathrm{C}$ & 23 & 22.20 & 89.37 & MID RIFF \\
\hline 2008 & $\mathrm{C}$ & 24 & 23.38 & 89.35 & MID RIFF \\
\hline 2008 & $\mathrm{C}$ & 25 & 24.22 & 89.43 & TAIL POOL \\
\hline 2008 & $\mathrm{C}$ & 26 & 24.90 & 89.32 & MID POOL \\
\hline 2008 & $\mathrm{C}$ & 27 & 25.65 & 89.46 & HEAD POOL \\
\hline 2008 & $\mathrm{C}$ & 27.5 & 26.26 & 89.51 & 8 \\
\hline 2008 & C & 28 & 27.06 & 89.57 & MID RIFF \\
\hline 2008 & $\mathrm{C}$ & 29 & 28.51 & 89.53 & MID RIFF \\
\hline 2008 & $\mathrm{C}$ & 30 & 30.56 & 89.59 & MID RIFF \\
\hline 2008 & $\mathrm{C}$ & 31 & 32.49 & 89.61 & MID RIFF \\
\hline 2008 & $\mathrm{C}$ & 31.5 & 32.62 & 89.62 & 7 \\
\hline 2008 & $\mathrm{C}$ & 32 & 33.88 & 89.74 & MID RIFF \\
\hline 2008 & $\mathrm{C}$ & 33 & 35.04 & 89.83 & MID RIFF \\
\hline 2008 & $\mathrm{C}$ & 34 & 37.13 & 90.01 & MID RIFF \\
\hline 2008 & $\mathrm{C}$ & 35 & 38.22 & 90.03 & MID RIFF \\
\hline 2008 & $\mathrm{C}$ & 35.5 & 38.34 & 90.02 & 6 \\
\hline 2008 & $\mathrm{C}$ & 36 & 39.12 & 89.96 & MID RIFF \\
\hline
\end{tabular}

Continued on Next Page. . 
Table D.4 - Continued

\begin{tabular}{|c|c|c|c|c|c|}
\hline Year & Reach & Point Number & Adjusted Dist. (m) & Adjusted Elev. (m) & Point Description \\
\hline 2008 & $\mathrm{C}$ & 37 & 40.64 & 90.16 & TAIL POOL \\
\hline 2008 & $\mathrm{C}$ & 38 & 41.45 & 89.81 & MID POOL \\
\hline 2008 & $\mathrm{C}$ & 39 & 42.44 & 89.63 & MID POOL \\
\hline 2008 & C & 40 & 43.12 & 89.81 & HEAD POOL \\
\hline 2008 & $\mathrm{C}$ & 41 & 43.79 & 90.53 & MID RIFF \\
\hline 2008 & $\mathrm{C}$ & 42 & 45.35 & 90.64 & MID RIFF \\
\hline 2008 & $\mathrm{C}$ & 43 & 47.76 & 90.84 & TAIL POOL \\
\hline 2008 & $\mathrm{C}$ & 44 & 48.52 & 90.61 & MID POOL \\
\hline 2008 & $\mathrm{C}$ & 45 & 49.39 & 90.87 & HEAD POOL \\
\hline 2008 & $\mathrm{C}$ & 46 & 50.08 & 90.94 & TAIL POOL \\
\hline 2008 & $\mathrm{C}$ & 47 & 51.88 & 90.74 & MID POOL \\
\hline 2008 & $\mathrm{C}$ & 48 & 53.16 & 90.81 & MID POOL \\
\hline 2008 & $\mathrm{C}$ & 49 & 54.40 & 91.03 & HEAD POOL \\
\hline 2008 & $\mathrm{C}$ & 50 & 56.13 & 91.25 & MID RIFF \\
\hline 2008 & $\mathrm{C}$ & 51 & 56.25 & 91.23 & MID RIFF \\
\hline 2008 & $\mathrm{C}$ & 52 & 58.22 & 91.38 & MID RIFF \\
\hline 2008 & $\mathrm{C}$ & 53 & 59.37 & 91.40 & MID RIFF \\
\hline 2008 & $\mathrm{C}$ & 54 & 60.45 & 91.42 & MID RIFF \\
\hline 2008 & $\mathrm{C}$ & 55 & 61.67 & 91.47 & MID RIFF \\
\hline 2008 & C & 56 & 63.39 & 91.48 & MID RIFF \\
\hline 2008 & $\mathrm{C}$ & 57 & 64.76 & 91.58 & MID RIFF \\
\hline 2008 & $\mathrm{C}$ & 57.5 & 66.25 & 91.58 & 5 \\
\hline 2008 & $\mathrm{C}$ & 58 & 66.30 & 91.58 & MID RIFF \\
\hline 2008 & $\mathrm{C}$ & 59 & 67.29 & 91.53 & MID RIFF \\
\hline 2008 & $\mathrm{C}$ & 60 & 68.29 & 91.45 & MID RIFF \\
\hline 2008 & $\mathrm{C}$ & 61 & 69.13 & 91.47 & MID RIFF \\
\hline 2008 & $\mathrm{C}$ & 62 & 70.80 & 91.63 & MID RIFF \\
\hline 2008 & $\mathrm{C}$ & 62.5 & 71.42 & 91.65 & 4 \\
\hline 2008 & $\mathrm{C}$ & 63 & 72.62 & 91.70 & MID RIFF \\
\hline 2008 & $\mathrm{C}$ & 64 & 74.09 & 91.73 & MID RIFF \\
\hline 2008 & $\mathrm{C}$ & 64.5 & 75.83 & 91.87 & 3 \\
\hline 2008 & $\mathrm{C}$ & 65 & 75.91 & 91.88 & MID RIFF \\
\hline 2008 & $\mathrm{C}$ & 66 & 77.27 & 91.96 & MID RIFF \\
\hline 2008 & $\mathrm{C}$ & 67 & 79.64 & 92.00 & MID RIFF \\
\hline 2008 & $\mathrm{C}$ & 67.5 & 79.71 & 92.00 & 2 \\
\hline 2008 & $\mathrm{C}$ & 68 & 80.55 & 91.99 & TAIL POOL \\
\hline 2008 & $\mathrm{C}$ & 69 & 81.48 & 91.74 & MID POOL \\
\hline 2008 & $\mathrm{C}$ & 70 & 83.17 & 91.73 & MID POOL \\
\hline 2008 & $\mathrm{C}$ & 71 & 84.57 & 91.97 & HEAD POOL \\
\hline 2008 & $\mathrm{C}$ & 72 & 85.84 & 92.11 & MID RIFF \\
\hline 2008 & $\mathrm{C}$ & 72.5 & 86.06 & 92.12 & 1 \\
\hline 2008 & $\mathrm{C}$ & 73 & 87.27 & 92.15 & MID RIFF \\
\hline 2008 & $\mathrm{C}$ & 74 & 88.74 & 92.23 & MID RIFF \\
\hline 2008 & $\mathrm{C}$ & 75 & 90.14 & 92.27 & MID RIFF \\
\hline 2008 & $\mathrm{C}$ & 76 & 91.42 & 92.28 & MID RIFF \\
\hline 2002 & $\mathrm{D}$ & 1 & 0.00 & 105.15 & TAILFLUME \\
\hline 2002 & $\mathrm{D}$ & 2 & 3.65 & 105.07 & HEADPOOL \\
\hline 2002 & $\mathrm{D}$ & 3 & 10.22 & 105.35 & TRIFF.57 \\
\hline 2002 & $\mathrm{D}$ & 4 & 12.36 & 105.44 & HRIFF.41 \\
\hline 2002 & $\mathrm{D}$ & 5 & 13.79 & 105.36 & OUT \\
\hline 2002 & $\mathrm{D}$ & 6 & 15.44 & 105.52 & OUT \\
\hline 2002 & $\mathrm{D}$ & 7 & 15.48 & 105.52 & STEP. 28 \\
\hline
\end{tabular}

Continued on Next Page... 
Table D.4 - Continued

\begin{tabular}{|c|c|c|c|c|c|}
\hline Year & Reach & Point Number & Adjusted Dist. (m) & Adjusted Elev. (m) & Point Description \\
\hline 2002 & $\mathrm{D}$ & 8 & 15.91 & 105.66 & TOPSTEP.44 \\
\hline 2002 & $\mathrm{D}$ & 8.5 & 17.49 & 105.67 & 10 \\
\hline 2002 & $\mathrm{D}$ & 9 & 17.87 & 105.67 & HEADRIFF.65 \\
\hline 2002 & $\mathrm{D}$ & 10 & 21.09 & 105.74 & TRIFF.53BOTTRIFF \\
\hline 2002 & $\mathrm{D}$ & 11 & 22.29 & 105.88 & TRIFF.31HEADRIFF \\
\hline 2002 & $\mathrm{D}$ & 11.5 & 23.13 & 105.91 & 9 \\
\hline 2002 & $\mathrm{D}$ & 12 & 24.46 & 105.95 & HEADRIFF.26 \\
\hline 2002 & $\mathrm{D}$ & 13 & 24.84 & 106.01 & TAILRIFF. 28 \\
\hline 2002 & $\mathrm{D}$ & 14 & 26.03 & 106.05 & HEADRIFF. 24 \\
\hline 2002 & $\mathrm{D}$ & 15 & 28.66 & 106.04 & TAILRIFF.43 \\
\hline 2002 & $\mathrm{D}$ & 15.5 & 28.67 & 106.04 & 8 \\
\hline 2002 & $\mathrm{D}$ & 16 & 31.43 & 106.10 & HEADRIFF.30TAILR \\
\hline 2002 & $\mathrm{D}$ & 17 & 32.83 & 106.22 & TOPRIFF.37TPOOL \\
\hline 2002 & $\mathrm{D}$ & 17.5 & 33.21 & 106.16 & 7 \\
\hline 2002 & $\mathrm{D}$ & 18 & 33.91 & 106.04 & MIDPOOL.97 \\
\hline 2002 & $\mathrm{D}$ & 19 & 34.62 & 106.24 & HEADPOO.36TRIFF \\
\hline 2002 & $\mathrm{D}$ & 20 & 35.53 & 106.40 & HEADRIFF.13 \\
\hline 2002 & $\mathrm{D}$ & 21 & 36.69 & 106.44 & $.18 \mathrm{HEADTAILRIFF}$ \\
\hline 2002 & $\mathrm{D}$ & 21.5 & 36.88 & 106.48 & 6 \\
\hline 2002 & $\mathrm{D}$ & 22 & 37.55 & 106.61 & TAILRIFF.27TPOOL \\
\hline 2002 & $\mathrm{D}$ & 23 & 38.77 & 106.52 & MIDPOOL.57 \\
\hline 2002 & $\mathrm{D}$ & 24 & 39.64 & 106.52 & TOPPOOL.51TAILPO \\
\hline 2002 & $\mathrm{D}$ & 25 & 40.65 & 106.33 & MIDPOOL1.12 \\
\hline 2002 & $\mathrm{D}$ & 26 & 41.37 & 106.61 & HEADPOOL.15TAILR \\
\hline 2002 & $\mathrm{D}$ & 27 & 42.38 & 106.64 & $.37 \mathrm{BOTSTEP}$ \\
\hline 2002 & $\mathrm{D}$ & 28 & 42.61 & 106.75 & .28 TOPSTEPTAILRI \\
\hline 2002 & $\mathrm{D}$ & 29 & 46.32 & 106.85 & HEAD.34TAILRIFF \\
\hline 2002 & $\mathrm{D}$ & 30 & 46.90 & 106.79 & BOTSTEP.58 \\
\hline 2002 & $\mathrm{D}$ & 31 & 47.16 & 106.87 & TOPSTEP.37 \\
\hline 2002 & $\mathrm{D}$ & 32 & 47.98 & 106.98 & BOTSTEP.25 \\
\hline 2002 & $\mathrm{D}$ & 33 & 48.48 & 107.10 & TOPSTEP.29 \\
\hline 2002 & $\mathrm{D}$ & 34 & 49.60 & 107.07 & BEND.45 \\
\hline 2002 & $\mathrm{D}$ & 35 & 50.12 & 107.06 & TAILRIF.33 \\
\hline 2002 & $\mathrm{D}$ & 36 & 50.47 & 107.12 & HEADRIFF.31 \\
\hline 2002 & $\mathrm{D}$ & 37 & 52.34 & 107.25 & TRIFF.34 \\
\hline 2002 & $\mathrm{D}$ & 38 & 52.66 & 107.28 & HRIFF.38 \\
\hline 2002 & $\mathrm{D}$ & 39 & 55.07 & 107.35 & TRIFF.27 \\
\hline 2002 & $\mathrm{D}$ & 40 & 55.58 & 107.45 & TAILPOOL. 28 \\
\hline 2002 & $\mathrm{D}$ & 41 & 56.68 & 107.39 & MIDPOOL.52 \\
\hline 2002 & $\mathrm{D}$ & 42 & 58.47 & 107.08 & BEND.96 \\
\hline 2002 & $\mathrm{D}$ & 43 & 60.37 & 107.33 & HEADPOOL.36 \\
\hline 2002 & $\mathrm{D}$ & 44 & 60.83 & 107.33 & BIS.30 \\
\hline 2002 & $\mathrm{D}$ & 45 & 62.02 & 107.38 & BIS.27 \\
\hline 2002 & $\mathrm{D}$ & 46 & 63.49 & 107.97 & TOPBIS.43TRIFF \\
\hline 2002 & $\mathrm{D}$ & 47 & 64.90 & 107.91 & BEND.65 \\
\hline 2002 & $\mathrm{D}$ & 48 & 69.23 & 108.41 & HEADRIFF. 35 \\
\hline 2002 & $\mathrm{D}$ & 49 & 69.41 & 108.49 & TAILRIFF.36 \\
\hline 2002 & $\mathrm{D}$ & 50 & 70.39 & 108.55 & HRIFF.32TRIFF \\
\hline 2002 & $\mathrm{D}$ & 51 & 71.38 & 108.58 & HRIFF.20TAILRIFF \\
\hline 2002 & $\mathrm{D}$ & 52 & 73.95 & 108.60 & HRIFF.18TRIFF \\
\hline 2002 & $\mathrm{D}$ & 53 & 74.21 & 108.72 & BIS.15 \\
\hline 2002 & $\mathrm{D}$ & 54 & 76.36 & 108.65 & TRIFF.33 \\
\hline
\end{tabular}

Continued on Next Page... 
Table D.4 - Continued

\begin{tabular}{|c|c|c|c|c|c|}
\hline Year & Reach & Point Number & Adjusted Dist. (m) & Adjusted Elev. (m) & Point Description \\
\hline 2002 & $\mathrm{D}$ & 55 & 80.25 & 108.70 & HRIFF.36 \\
\hline 2002 & $\mathrm{D}$ & 56 & 82.72 & 108.88 & TRIFF.26 \\
\hline 2002 & $\mathrm{D}$ & 57 & 84.63 & 109.02 & HRIFF.24 \\
\hline 2002 & D & 58 & 86.68 & 109.12 & TRIFF.38TPOOL \\
\hline 2002 & $\mathrm{D}$ & 58.5 & 87.13 & 109.09 & 5 \\
\hline 2002 & $\mathrm{D}$ & 59 & 87.83 & 109.04 & MIDPOOL.53 \\
\hline 2002 & $\mathrm{D}$ & 60 & 88.96 & 109.08 & HPOOL.37TPOOL \\
\hline 2002 & $\mathrm{D}$ & 61 & 90.84 & 108.94 & MIDPOOL.83 \\
\hline 2002 & $\mathrm{D}$ & 62 & 91.12 & 109.05 & HPOOL.65 \\
\hline 2002 & $\mathrm{D}$ & 63 & 91.58 & 109.15 & TRIFF.39STEP \\
\hline 2002 & $\mathrm{D}$ & 63.5 & 92.08 & 109.18 & 4 \\
\hline 2002 & $\mathrm{D}$ & 64 & 93.11 & 109.24 & HRIFF.41TRIFF \\
\hline 2002 & $\mathrm{D}$ & 65 & 95.00 & 109.37 & HRIFF.23 \\
\hline 2002 & $\mathrm{D}$ & 66 & 96.17 & 109.38 & TRIFF.33 \\
\hline 2002 & $\mathrm{D}$ & 67 & 96.35 & 109.37 & TPOOL.31 \\
\hline 2002 & $\mathrm{D}$ & 67.5 & 97.36 & 109.31 & 3 \\
\hline 2002 & $\mathrm{D}$ & 68 & 98.37 & 109.26 & MIDPOOL.70 \\
\hline 2002 & $\mathrm{D}$ & 69 & 100.49 & 109.49 & HEADPOOL. 24 \\
\hline 2002 & $\mathrm{D}$ & 70 & 101.17 & 109.68 & TRIFF.26STEP \\
\hline 2002 & $\mathrm{D}$ & 70.5 & 101.37 & 109.68 & 2 \\
\hline 2002 & $\mathrm{D}$ & 71 & 103.45 & 109.66 & HRIFF.39 \\
\hline 2002 & $\mathrm{D}$ & 72 & 104.02 & 109.73 & TRIFF.32HRIFF \\
\hline 2002 & $\mathrm{D}$ & 73 & 105.94 & 109.64 & BIS.31 \\
\hline 2002 & $\mathrm{D}$ & 73.5 & 106.03 & 109.67 & 1 \\
\hline 2002 & $\mathrm{D}$ & 74 & 106.70 & 109.92 & HRIFF. 22 \\
\hline 2002 & $\mathrm{D}$ & 75 & 110.52 & 109.92 & UNDERCUT. 37 \\
\hline 2002 & $\mathrm{D}$ & 76 & 112.21 & 110.05 & UCTRIFF.21 \\
\hline 2002 & $\mathrm{D}$ & 77 & 113.03 & 110.25 & BIS.26 \\
\hline 2002 & $\mathrm{D}$ & 78 & 115.36 & 110.36 & HRIFF.17 \\
\hline 2002 & $\mathrm{D}$ & 79 & 117.40 & 110.43 & TRIFF.32 \\
\hline 2002 & $\mathrm{D}$ & 80 & 118.44 & 110.51 & HRIFF.47 \\
\hline 2002 & $\mathrm{D}$ & 81 & 120.12 & 110.63 & TPOOL.20 \\
\hline 2002 & $\mathrm{D}$ & 82 & 121.39 & 110.35 & MIDPOOL1.07 \\
\hline 2002 & $\mathrm{D}$ & 83 & 121.96 & 110.50 & HPOOL. 41 \\
\hline 2002 & $\mathrm{D}$ & 84 & 124.31 & 110.78 & HRIFF.30 \\
\hline 2002 & $\mathrm{D}$ & 85 & 125.05 & 110.76 & BEND.23 \\
\hline 2002 & $\mathrm{D}$ & 86 & 127.07 & 110.67 & BIS.71 \\
\hline 2002 & $\mathrm{D}$ & 87 & 128.26 & 111.04 & STEP.39 \\
\hline 2002 & $\mathrm{D}$ & 88 & 129.83 & 111.05 & TRIFF.2 \\
\hline 2004 & $\mathrm{D}$ & 1 & 12.98 & 105.43 & MID RIFF \\
\hline 2004 & $\mathrm{D}$ & 2 & 15.71 & 105.53 & BOT STEP \\
\hline 2004 & $\mathrm{D}$ & 3 & 15.93 & 105.77 & TOP STEP \\
\hline 2004 & $\mathrm{D}$ & 3.5 & 17.49 & 105.77 & 10 \\
\hline 2004 & $\mathrm{D}$ & 4 & 18.39 & 105.78 & MID RIFF \\
\hline 2004 & $\mathrm{D}$ & 5 & 21.07 & 105.78 & MID RIFF \\
\hline 2004 & $\mathrm{D}$ & 6 & 22.11 & 105.85 & MID RIFF \\
\hline 2004 & $\mathrm{D}$ & 6.5 & 23.13 & 105.88 & 9 \\
\hline 2004 & $\mathrm{D}$ & 7 & 24.20 & 105.90 & MID RIFF \\
\hline 2004 & $\mathrm{D}$ & 8 & 25.31 & 105.94 & MID RIFF \\
\hline 2004 & $\mathrm{D}$ & 9 & 27.62 & 106.04 & MID RIFF \\
\hline 2004 & $\mathrm{D}$ & 9.5 & 28.67 & 106.07 & 8 \\
\hline 2004 & D & 10 & 30.04 & 106.12 & MID RIFF \\
\hline
\end{tabular}

Continued on Next Page... 
Table D.4 - Continued

\begin{tabular}{|c|c|c|c|c|c|}
\hline Year & Reach & Point Number & Adjusted Dist. (m) & Adjusted Elev. (m) & Point Description \\
\hline 2004 & $\mathrm{D}$ & 11 & 32.16 & 106.16 & MID RIFF \\
\hline 2004 & $\mathrm{D}$ & 12 & 32.88 & 106.17 & TAIL GLIDE \\
\hline 2004 & $\mathrm{D}$ & 12.5 & 33.21 & 106.16 & 7 \\
\hline 2004 & $\mathrm{D}$ & 13 & 33.95 & 106.13 & MID GLIDE \\
\hline 2004 & $\mathrm{D}$ & 14 & 34.34 & 106.20 & TOP GLIDE \\
\hline 2004 & $\mathrm{D}$ & 15 & 34.88 & 106.25 & MID RIFF \\
\hline 2004 & $\mathrm{D}$ & 16 & 35.29 & 106.39 & MID RIFF \\
\hline 2004 & $\mathrm{D}$ & 17 & 36.56 & 106.44 & MID RIFF \\
\hline 2004 & $\mathrm{D}$ & 17.5 & 36.88 & 106.49 & 6 \\
\hline 2004 & $\mathrm{D}$ & 18 & 37.60 & 106.60 & TAIL POOL \\
\hline 2004 & $\mathrm{D}$ & 19 & 38.64 & 106.51 & MID POOL \\
\hline 2004 & $\mathrm{D}$ & 20 & 39.59 & 106.47 & HEAD POOL \\
\hline 2004 & $\mathrm{D}$ & 21 & 40.33 & 106.33 & TAIL GLIDE \\
\hline 2004 & $\mathrm{D}$ & 22 & 40.92 & 106.50 & HEAD GLIDE \\
\hline 2004 & $\mathrm{D}$ & 23 & 41.53 & 106.67 & TOP STEP \\
\hline 2004 & $\mathrm{D}$ & 24 & 43.97 & 106.80 & MID RIFF \\
\hline 2004 & $\mathrm{D}$ & 25 & 46.68 & 106.82 & MID RIFF \\
\hline 2004 & $\mathrm{D}$ & 26 & 47.67 & 106.94 & MID RIFF \\
\hline 2004 & $\mathrm{D}$ & 27 & 48.27 & 107.10 & MID RIFF \\
\hline 2004 & $\mathrm{D}$ & 28 & 49.44 & 107.01 & MID RIFF \\
\hline 2004 & $\mathrm{D}$ & 29 & 50.91 & 107.11 & MID RIFF \\
\hline 2004 & $\mathrm{D}$ & 30 & 51.81 & 107.13 & MID RIFF \\
\hline 2004 & $\mathrm{D}$ & 31 & 52.48 & 107.21 & MID RIFF \\
\hline 2004 & $\mathrm{D}$ & 32 & 54.80 & 107.32 & MID RIFF \\
\hline 2004 & $\mathrm{D}$ & 33 & 54.98 & 107.40 & MID RIFF \\
\hline 2004 & $\mathrm{D}$ & 34 & 55.99 & 107.35 & TAIL POOL \\
\hline 2004 & $\mathrm{D}$ & 35 & 56.65 & 107.28 & MID POOL \\
\hline 2004 & $\mathrm{D}$ & 36 & 57.74 & 107.33 & HEAD POOL \\
\hline 2004 & $\mathrm{D}$ & 37 & 58.43 & 107.27 & MID GLIDE \\
\hline 2004 & $\mathrm{D}$ & 38 & 59.12 & 107.23 & TOP GLIDE \\
\hline 2004 & $\mathrm{D}$ & 39 & 60.87 & 107.51 & MID RIFF \\
\hline 2004 & $\mathrm{D}$ & 40 & 62.11 & 107.38 & MID GLIDE \\
\hline 2004 & $\mathrm{D}$ & 41 & 63.37 & 107.42 & TOP GLIDE \\
\hline 2004 & $\mathrm{D}$ & 42 & 64.72 & 108.01 & TOP ROOT \\
\hline 2004 & $\mathrm{D}$ & 43 & 66.47 & 108.05 & BOT STEP \\
\hline 2004 & $\mathrm{D}$ & 44 & 66.63 & 108.30 & TOP STEP \\
\hline 2004 & $\mathrm{D}$ & 45 & 67.55 & 108.16 & MID RIFF \\
\hline 2004 & $\mathrm{D}$ & 46 & 68.76 & 108.32 & MID RIFF \\
\hline 2004 & $\mathrm{D}$ & 47 & 69.51 & 108.59 & MID RIFF \\
\hline 2004 & $\mathrm{D}$ & 48 & 72.22 & 108.66 & MID RIFF \\
\hline 2004 & $\mathrm{D}$ & 49 & 73.97 & 108.75 & MID RIFF \\
\hline 2004 & $\mathrm{D}$ & 50 & 78.40 & 108.74 & MID RIFF \\
\hline 2004 & $\mathrm{D}$ & 51 & 80.82 & 108.85 & MID RIFF \\
\hline 2004 & $\mathrm{D}$ & 52 & 84.61 & 108.99 & MID RIFF \\
\hline 2004 & $\mathrm{D}$ & 53 & 86.39 & 109.03 & MID RIFF \\
\hline 2004 & $\mathrm{D}$ & 54 & 86.80 & 109.12 & MID RIFF \\
\hline 2004 & $\mathrm{D}$ & 54.5 & 87.13 & 109.08 & 5 \\
\hline 2004 & $\mathrm{D}$ & 55 & 87.23 & 109.07 & BOT GLIDE \\
\hline 2004 & $\mathrm{D}$ & 56 & 89.35 & 109.07 & MID GLIDE \\
\hline 2004 & $\mathrm{D}$ & 57 & 90.57 & 108.91 & TOP GLIDE \\
\hline 2004 & $\mathrm{D}$ & 58 & 91.54 & 109.02 & MID RIFF \\
\hline 2004 & $\mathrm{D}$ & 58.5 & 92.08 & 109.09 & 4 \\
\hline
\end{tabular}

Continued on Next Page... 
Table D.4 - Continued

\begin{tabular}{|c|c|c|c|c|c|}
\hline Year & Reach & Point Number & Adjusted Dist. (m) & Adjusted Elev. (m) & Point Description \\
\hline 2004 & $\mathrm{D}$ & 59 & 92.84 & 109.19 & MID RIFF \\
\hline 2004 & $\mathrm{D}$ & 60 & 93.31 & 109.18 & MID RIFF \\
\hline 2004 & $\mathrm{D}$ & 61 & 94.30 & 109.26 & MID RIFF \\
\hline 2004 & $\mathrm{D}$ & 62 & 95.14 & 109.34 & MID RIFF \\
\hline 2004 & $\mathrm{D}$ & 63 & 96.24 & 109.37 & TAIL GLIDE \\
\hline 2004 & $\mathrm{D}$ & 63.5 & 97.36 & 109.27 & 3 \\
\hline 2004 & $\mathrm{D}$ & 64 & 97.38 & 109.27 & MID GLIDE \\
\hline 2004 & $\mathrm{D}$ & 65 & 98.57 & 109.34 & HEAD GLIDE \\
\hline 2004 & $\mathrm{D}$ & 66 & 101.01 & 109.48 & MID RIFF \\
\hline 2004 & $\mathrm{D}$ & 66.5 & 101.37 & 109.50 & 2 \\
\hline 2004 & $\mathrm{D}$ & 67 & 102.43 & 109.56 & MID RIFF \\
\hline 2004 & $\mathrm{D}$ & 68 & 104.08 & 109.73 & MID RIFF \\
\hline 2004 & $\mathrm{D}$ & 69 & 105.82 & 109.70 & MID RIFF \\
\hline 2004 & $\mathrm{D}$ & 69.5 & 106.03 & 109.75 & 1 \\
\hline 2004 & $\mathrm{D}$ & 70 & 106.59 & 109.88 & MID RIFF \\
\hline 2004 & $\mathrm{D}$ & 71 & 108.37 & 109.82 & MID RIFF \\
\hline 2004 & $\mathrm{D}$ & 72 & 110.14 & 109.85 & MID RIFF \\
\hline 2004 & $\mathrm{D}$ & 73 & 110.70 & 110.07 & MID RIFF \\
\hline 2004 & $\mathrm{D}$ & 74 & 111.23 & 110.08 & MID RIFF \\
\hline 2004 & $\mathrm{D}$ & 75 & 111.60 & 110.17 & MID RIFF \\
\hline 2004 & $\mathrm{D}$ & 76 & 112.86 & 110.30 & MID RIFF \\
\hline 2004 & $\mathrm{D}$ & 77 & 114.26 & 110.23 & MID RIFF \\
\hline 2004 & $\mathrm{D}$ & 78 & 115.61 & 110.37 & MID RIFF \\
\hline 2004 & $\mathrm{D}$ & 79 & 117.36 & 110.51 & MID RIFF \\
\hline 2005 & $\mathrm{D}$ & 1 & 13.84 & 105.41 & MID RIFF \\
\hline 2005 & $\mathrm{D}$ & 2 & 15.28 & 105.53 & BTM LOG STEP \\
\hline 2005 & $\mathrm{D}$ & 3 & 15.79 & 105.71 & TOP LOG STEP \\
\hline 2005 & $\mathrm{D}$ & 4 & 17.02 & 105.74 & MID RIFF \\
\hline 2005 & $\mathrm{D}$ & 4.5 & 17.49 & 105.74 & 10 \\
\hline 2005 & $\mathrm{D}$ & 5 & 19.64 & 105.76 & MID RIFF \\
\hline 2005 & $\mathrm{D}$ & 6 & 22.09 & 105.79 & MID RIFF \\
\hline 2005 & $\mathrm{D}$ & 6.5 & 23.13 & 105.80 & 9 \\
\hline 2005 & $\mathrm{D}$ & 7 & 24.45 & 105.80 & MID RIFF \\
\hline 2005 & $\mathrm{D}$ & 8 & 24.94 & 105.99 & MID RIFF \\
\hline 2005 & $\mathrm{D}$ & 9 & 26.48 & 106.14 & MID RIFF \\
\hline 2005 & $\mathrm{D}$ & 10 & 27.04 & 105.99 & MID RIFF \\
\hline 2005 & $\mathrm{D}$ & 10.5 & 28.67 & 106.07 & 8 \\
\hline 2005 & $\mathrm{D}$ & 11 & 30.05 & 106.14 & MID RIFF \\
\hline 2005 & $\mathrm{D}$ & 12 & 31.88 & 106.12 & MID RIFF \\
\hline 2005 & $\mathrm{D}$ & 13 & 32.83 & 106.14 & TAIL POOL \\
\hline 2005 & $\mathrm{D}$ & 13.5 & 33.21 & 106.12 & 7 \\
\hline 2005 & $\mathrm{D}$ & 14 & 33.62 & 106.10 & MID POOL \\
\hline 2005 & $\mathrm{D}$ & 15 & 34.63 & 106.15 & HEAD POOL \\
\hline 2005 & $\mathrm{D}$ & 16 & 36.16 & 106.42 & MID RIF \\
\hline 2005 & $\mathrm{D}$ & 16.5 & 36.88 & 106.49 & 6 \\
\hline 2005 & $\mathrm{D}$ & 17 & 37.80 & 106.57 & TAIL POOL \\
\hline 2005 & $\mathrm{D}$ & 18 & 39.34 & 106.53 & MID POOL \\
\hline 2005 & $\mathrm{D}$ & 19 & 40.32 & 106.30 & MID POOL \\
\hline 2005 & $\mathrm{D}$ & 20 & 41.31 & 106.62 & HEAD POOL \\
\hline 2005 & $\mathrm{D}$ & 21 & 42.73 & 106.76 & MID RIF \\
\hline 2005 & $\mathrm{D}$ & 22 & 45.43 & 106.83 & MID RIF \\
\hline 2005 & $\mathrm{D}$ & 23 & 46.74 & 106.85 & MID RIF \\
\hline
\end{tabular}

Continued on Next Page. . 
Table D.4 - Continued

\begin{tabular}{|c|c|c|c|c|c|}
\hline Year & Reach & Point Number & Adjusted Dist. (m) & Adjusted Elev. (m) & Point Description \\
\hline 2005 & $\mathrm{D}$ & 24 & 47.71 & 106.99 & MID RIF \\
\hline 2005 & $\mathrm{D}$ & 25 & 50.59 & 107.11 & MID RIF \\
\hline 2005 & $\mathrm{D}$ & 26 & 52.17 & 107.21 & MID RIF \\
\hline 2005 & $\mathrm{D}$ & 27 & 54.68 & 107.35 & MID RIF \\
\hline 2005 & $\mathrm{D}$ & 28 & 55.97 & 107.39 & TAIL POOL \\
\hline 2005 & $\mathrm{D}$ & 29 & 56.97 & 107.28 & MID POOL \\
\hline 2005 & $\mathrm{D}$ & 30 & 58.01 & 107.32 & HEAD POOL \\
\hline 2005 & $\mathrm{D}$ & 31 & 60.26 & 107.52 & MID RIFF \\
\hline 2005 & $\mathrm{D}$ & 32 & 61.54 & 107.47 & TAIL POOL \\
\hline 2005 & $\mathrm{D}$ & 33 & 62.71 & 107.34 & MID POOL \\
\hline 2005 & $\mathrm{D}$ & 34 & 63.74 & 107.47 & HEAD POOL \\
\hline 2005 & $\mathrm{D}$ & 35 & 64.11 & 107.72 & ROCK STEP \\
\hline 2005 & $\mathrm{D}$ & 36 & 64.84 & 107.93 & ROCK STEP \\
\hline 2005 & $\mathrm{D}$ & 37 & 65.89 & 108.05 & MID RIFF \\
\hline 2005 & $\mathrm{D}$ & 38 & 66.86 & 108.21 & MID RIFF \\
\hline 2005 & $\mathrm{D}$ & 39 & 68.00 & 108.23 & MID RIFF \\
\hline 2005 & $\mathrm{D}$ & 40 & 68.67 & 108.45 & MID RIFF \\
\hline 2005 & $\mathrm{D}$ & 41 & 69.40 & 108.52 & MID RIFF \\
\hline 2005 & $\mathrm{D}$ & 42 & 70.79 & 108.54 & MID RIFF \\
\hline 2005 & $\mathrm{D}$ & 43 & 72.03 & 108.62 & MID RIFF \\
\hline 2005 & $\mathrm{D}$ & 44 & 73.24 & 108.70 & MID RIFF \\
\hline 2005 & $\mathrm{D}$ & 45 & 75.17 & 108.74 & MID RIFF \\
\hline 2005 & $\mathrm{D}$ & 46 & 76.82 & 108.79 & MID RIFF \\
\hline 2005 & $\mathrm{D}$ & 47 & 78.21 & 108.75 & MID RIFF \\
\hline 2005 & $\mathrm{D}$ & 48 & 79.96 & 108.82 & MID RIFF \\
\hline 2005 & $\mathrm{D}$ & 49 & 81.29 & 108.86 & MID RIFF \\
\hline 2005 & $\mathrm{D}$ & 50 & 82.87 & 108.91 & MID RIFF \\
\hline 2005 & $\mathrm{D}$ & 51 & 85.12 & 108.97 & MID RIFF \\
\hline 2005 & $\mathrm{D}$ & 52 & 86.80 & 109.03 & MID RIFF \\
\hline 2005 & $\mathrm{D}$ & 52.5 & 87.13 & 109.06 & 5 \\
\hline 2005 & $\mathrm{D}$ & 53 & 87.35 & 109.08 & TAIL POOL \\
\hline 2005 & $\mathrm{D}$ & 54 & 88.77 & 109.05 & MID POOL \\
\hline 2005 & $\mathrm{D}$ & 55 & 89.64 & 108.93 & MID POOL \\
\hline 2005 & $\mathrm{D}$ & 56 & 90.54 & 108.87 & MID POOL \\
\hline 2005 & $\mathrm{D}$ & 57 & 91.88 & 109.13 & HEAD POOL \\
\hline 2005 & $\mathrm{D}$ & 57.5 & 92.08 & 109.14 & 4 \\
\hline 2005 & $\mathrm{D}$ & 58 & 92.67 & 109.17 & MID RIFF \\
\hline 2005 & $\mathrm{D}$ & 59 & 93.27 & 109.21 & MID RIFF \\
\hline 2005 & $\mathrm{D}$ & 60 & 94.58 & 109.30 & MID RIFF \\
\hline 2005 & $\mathrm{D}$ & 61 & 95.85 & 109.38 & MID RIFF \\
\hline 2005 & $\mathrm{D}$ & 62 & 96.25 & 109.37 & TAIL POOL \\
\hline 2005 & $\mathrm{D}$ & 62.5 & 97.36 & 109.28 & 3 \\
\hline 2005 & $\mathrm{D}$ & 63 & 97.66 & 109.26 & MID POOL \\
\hline 2005 & $\mathrm{D}$ & 64 & 98.83 & 109.31 & HEAD POOL \\
\hline 2005 & $\mathrm{D}$ & 65 & 100.39 & 109.45 & MID RIFF \\
\hline 2005 & $\mathrm{D}$ & 65.5 & 101.37 & 109.65 & 2 \\
\hline 2005 & $\mathrm{D}$ & 66 & 101.50 & 109.68 & MID RIFF \\
\hline 2005 & $\mathrm{D}$ & 67 & 102.33 & 109.57 & MID RIFF \\
\hline 2005 & $\mathrm{D}$ & 68 & 103.38 & 109.64 & MID RIFF \\
\hline 2005 & $\mathrm{D}$ & 69 & 104.90 & 109.70 & MID RIFF \\
\hline 2005 & $\mathrm{D}$ & 70 & 105.88 & 109.71 & MID RIFF \\
\hline 2005 & $\mathrm{D}$ & 70.5 & 106.03 & 109.76 & 1 \\
\hline
\end{tabular}

Continued on Next Page... 
Table D.4 - Continued

\begin{tabular}{|c|c|c|c|c|c|}
\hline Year & Reach & Point Number & Adjusted Dist. (m) & Adjusted Elev. (m) & Point Description \\
\hline 2005 & $\mathrm{D}$ & 71 & 106.50 & 109.90 & MID RIFF \\
\hline 2005 & D & 72 & 108.22 & 109.88 & MID RIFF \\
\hline 2005 & $\mathrm{D}$ & 73 & 109.09 & 109.74 & MID RIFF \\
\hline 2005 & $\mathrm{D}$ & 74 & 110.92 & 109.91 & MID RIFF \\
\hline 2005 & $\mathrm{D}$ & 75 & 111.41 & 110.01 & MID RIFF \\
\hline 2005 & $\mathrm{D}$ & 76 & 111.88 & 110.16 & MID RIFF \\
\hline 2005 & $\mathrm{D}$ & 77 & 112.49 & 110.25 & MID RIFF \\
\hline 2005 & $\mathrm{D}$ & 78 & 113.64 & 110.30 & MID RIFF \\
\hline 2005 & $\mathrm{D}$ & 79 & 116.16 & 110.39 & MID RIFF \\
\hline 2006 & $\mathrm{D}$ & 1 & 13.20 & 105.39 & MID RIF \\
\hline 2006 & $\mathrm{D}$ & 2 & 14.54 & 105.56 & MID RIF \\
\hline 2006 & $\mathrm{D}$ & 3 & 15.55 & 105.49 & BOT LOG STEP \\
\hline 2006 & $\mathrm{D}$ & 4 & 15.76 & 105.77 & TOP LOG STEP \\
\hline 2006 & $\mathrm{D}$ & 5 & 16.24 & 105.65 & MID RIFF \\
\hline 2006 & $\mathrm{D}$ & 6 & 17.38 & 105.71 & MID RIFF \\
\hline 2006 & $\mathrm{D}$ & 6.5 & 17.49 & 105.72 & 10 \\
\hline 2006 & $\mathrm{D}$ & 7 & 18.22 & 105.80 & MID RIFF \\
\hline 2006 & $\mathrm{D}$ & 8 & 19.18 & 105.75 & TAIL POOL \\
\hline 2006 & $\mathrm{D}$ & 9 & 20.61 & 105.57 & MID POOL \\
\hline 2006 & $\mathrm{D}$ & 10 & 21.57 & 105.78 & HEAD POOL \\
\hline 2006 & $\mathrm{D}$ & 11 & 21.99 & 105.83 & MID RIFF \\
\hline 2006 & $\mathrm{D}$ & 11.5 & 23.13 & 105.83 & 9 \\
\hline 2006 & $\mathrm{D}$ & 12 & 23.34 & 105.83 & MID RIFF \\
\hline 2006 & $\mathrm{D}$ & 13 & 25.10 & 105.96 & MID RIFF \\
\hline 2006 & $\mathrm{D}$ & 14 & 26.23 & 106.00 & MID RIFF \\
\hline 2006 & $\mathrm{D}$ & 15 & 27.05 & 105.89 & MID RIFF \\
\hline 2006 & $\mathrm{D}$ & 16 & 28.10 & 105.95 & MID RIFF \\
\hline 2006 & $\mathrm{D}$ & 16.5 & 28.67 & 106.01 & 8 \\
\hline 2006 & $\mathrm{D}$ & 17 & 29.51 & 106.12 & MID RIFF \\
\hline 2006 & $\mathrm{D}$ & 18 & 31.81 & 106.19 & MID RIFF \\
\hline 2006 & $\mathrm{D}$ & 18.5 & 33.21 & 106.17 & 7 \\
\hline 2006 & $\mathrm{D}$ & 19 & 33.45 & 106.17 & MID RIFF \\
\hline 2006 & $\mathrm{D}$ & 20 & 34.69 & 106.19 & MID RIFF \\
\hline 2006 & $\mathrm{D}$ & 20.5 & 36.88 & 106.42 & 6 \\
\hline 2006 & $\mathrm{D}$ & 21 & 36.89 & 106.42 & MID RIFF \\
\hline 2006 & $\mathrm{D}$ & 22 & 37.50 & 106.51 & MID RIFF \\
\hline 2006 & $\mathrm{D}$ & 23 & 37.90 & 106.57 & MID RIFF \\
\hline 2006 & $\mathrm{D}$ & 24 & 38.55 & 106.49 & MID RIFF \\
\hline 2006 & $\mathrm{D}$ & 25 & 39.61 & 106.54 & TAIL POOL \\
\hline 2006 & $\mathrm{D}$ & 26 & 40.58 & 106.33 & MID POOL \\
\hline 2006 & $\mathrm{D}$ & 27 & 41.29 & 106.70 & HEAD POOL \\
\hline 2006 & $\mathrm{D}$ & 28 & 42.53 & 106.76 & MID RIFF \\
\hline 2006 & $\mathrm{D}$ & 29 & 43.50 & 106.79 & MID RIFF \\
\hline 2006 & $\mathrm{D}$ & 30 & 44.41 & 106.74 & MID RIFF \\
\hline 2006 & $\mathrm{D}$ & 31 & 45.09 & 106.81 & MID RIFF \\
\hline 2006 & $\mathrm{D}$ & 32 & 46.01 & 106.87 & MID RIFF \\
\hline 2006 & $\mathrm{D}$ & 33 & 46.46 & 106.82 & MID RIFF \\
\hline 2006 & $\mathrm{D}$ & 34 & 46.75 & 106.80 & MID RIFF \\
\hline 2006 & $\mathrm{D}$ & 35 & 47.40 & 106.90 & MID RIFF \\
\hline 2006 & $\mathrm{D}$ & 36 & 48.29 & 107.07 & MID RIFF \\
\hline 2006 & $\mathrm{D}$ & 37 & 49.36 & 106.97 & MID RIFF \\
\hline 2006 & D & 38 & 50.32 & 107.10 & MID RIFF \\
\hline
\end{tabular}

Continued on Next Page... 
Table D.4 - Continued

\begin{tabular}{|c|c|c|c|c|c|}
\hline Year & Reach & Point Number & Adjusted Dist. (m) & Adjusted Elev. (m) & Point Description \\
\hline 2006 & $\mathrm{D}$ & 39 & 51.05 & 107.11 & MID RIFF \\
\hline 2006 & $\mathrm{D}$ & 40 & 52.21 & 107.16 & MID RIFF \\
\hline 2006 & $\mathrm{D}$ & 41 & 52.95 & 107.19 & MID RIFF \\
\hline 2006 & $\mathrm{D}$ & 42 & 53.91 & 107.21 & MID RIFF \\
\hline 2006 & $\mathrm{D}$ & 43 & 55.47 & 107.20 & MID RIFF \\
\hline 2006 & $\mathrm{D}$ & 44 & 57.38 & 107.22 & MID RIFF \\
\hline 2006 & $\mathrm{D}$ & 45 & 59.04 & 107.25 & MID RIFF \\
\hline 2006 & $\mathrm{D}$ & 46 & 60.84 & 107.52 & MID RIFF \\
\hline 2006 & $\mathrm{D}$ & 47 & 61.89 & 107.38 & MID RIFF \\
\hline 2006 & $\mathrm{D}$ & 48 & 63.25 & 107.41 & MID RIFF \\
\hline 2006 & $\mathrm{D}$ & 49 & 65.09 & 108.06 & MID RIFF \\
\hline 2006 & $\mathrm{D}$ & 50 & 66.35 & 108.08 & MID RIFF \\
\hline 2006 & $\mathrm{D}$ & 51 & 66.82 & 108.17 & MID RIFF \\
\hline 2006 & $\mathrm{D}$ & 52 & 67.34 & 108.14 & MID RIFF \\
\hline 2006 & $\mathrm{D}$ & 53 & 68.07 & 108.13 & MID RIFF \\
\hline 2006 & $\mathrm{D}$ & 54 & 69.37 & 108.50 & MID RIFF \\
\hline 2006 & $\mathrm{D}$ & 55 & 70.51 & 108.59 & MID RIFF \\
\hline 2006 & $\mathrm{D}$ & 56 & 71.42 & 108.51 & MID RIFF \\
\hline 2006 & $\mathrm{D}$ & 57 & 71.98 & 108.49 & MID RIFF \\
\hline 2006 & $\mathrm{D}$ & 58 & 73.14 & 108.66 & MID RIFF \\
\hline 2006 & $\mathrm{D}$ & 59 & 74.52 & 108.72 & MID RIFF \\
\hline 2006 & $\mathrm{D}$ & 60 & 75.42 & 108.70 & MID RIFF \\
\hline 2006 & $\mathrm{D}$ & 61 & 79.06 & 108.82 & MID RIFF \\
\hline 2006 & $\mathrm{D}$ & 62 & 80.59 & 108.83 & MID RIFF \\
\hline 2006 & $\mathrm{D}$ & 63 & 81.97 & 108.83 & MID RIFF \\
\hline 2006 & $\mathrm{D}$ & 64 & 83.37 & 108.92 & MID RIFF \\
\hline 2006 & $\mathrm{D}$ & 65 & 84.06 & 108.92 & MID RIFF \\
\hline 2006 & $\mathrm{D}$ & 66 & 85.09 & 108.92 & MID RIFF \\
\hline 2006 & $\mathrm{D}$ & 67 & 86.43 & 108.98 & MID RIFF \\
\hline 2006 & $\mathrm{D}$ & 67.5 & 87.13 & 109.03 & 5 \\
\hline 2006 & $\mathrm{D}$ & 68 & 87.65 & 109.06 & TAIL POOL \\
\hline 2006 & $\mathrm{D}$ & 69 & 89.20 & 109.02 & MID POOL \\
\hline 2006 & $\mathrm{D}$ & 70 & 90.60 & 108.93 & MID POOL \\
\hline 2006 & $\mathrm{D}$ & 71 & 91.71 & 109.06 & HEAD POOL \\
\hline 2006 & $\mathrm{D}$ & 71.5 & 92.08 & 109.09 & 4 \\
\hline 2006 & $\mathrm{D}$ & 72 & 92.41 & 109.13 & MID RIFF \\
\hline 2006 & $\mathrm{D}$ & 73 & 93.28 & 109.23 & MID RIFF \\
\hline 2006 & $\mathrm{D}$ & 74 & 94.52 & 109.27 & MID RIFF \\
\hline 2006 & $\mathrm{D}$ & 75 & 96.05 & 109.35 & MID RIFF \\
\hline 2006 & $\mathrm{D}$ & 76 & 96.90 & 109.27 & MID RIFF \\
\hline 2006 & $\mathrm{D}$ & 76.5 & 97.36 & 109.26 & 3 \\
\hline 2006 & $\mathrm{D}$ & 77 & 98.33 & 109.23 & MID RIFF \\
\hline 2006 & $\mathrm{D}$ & 78 & 100.38 & 109.44 & MID RIFF \\
\hline 2006 & $\mathrm{D}$ & 79 & 101.21 & 109.57 & MID RIFF \\
\hline 2006 & $\mathrm{D}$ & 79.5 & 101.37 & 109.56 & 2 \\
\hline 2006 & $\mathrm{D}$ & 80 & 102.35 & 109.50 & MID RIFF \\
\hline 2006 & $\mathrm{D}$ & 81 & 103.30 & 109.62 & MID RIFF \\
\hline 2006 & $\mathrm{D}$ & 82 & 104.16 & 109.74 & MID RIFF \\
\hline 2006 & $\mathrm{D}$ & 83 & 104.63 & 109.69 & MID RIFF \\
\hline 2006 & $\mathrm{D}$ & 84 & 105.70 & 109.68 & MID RIFF \\
\hline 2006 & $\mathrm{D}$ & 84.5 & 106.03 & 109.74 & 1 \\
\hline 2006 & $\mathrm{D}$ & 85 & 106.76 & 109.88 & MID RIFF \\
\hline
\end{tabular}

Continued on Next Page... 
Table D.4 - Continued

\begin{tabular}{|c|c|c|c|c|c|}
\hline Year & Reach & Point Number & Adjusted Dist. (m) & Adjusted Elev. (m) & Point Description \\
\hline 2006 & $\mathrm{D}$ & 86 & 107.53 & 109.88 & MID RIFF \\
\hline 2006 & $\mathrm{D}$ & 87 & 108.50 & 109.80 & TAIL POOL \\
\hline 2006 & $\mathrm{D}$ & 88 & 109.50 & 109.66 & MID POOL \\
\hline 2006 & $\mathrm{D}$ & 89 & 110.45 & 109.90 & HEAD POOL \\
\hline 2006 & $\mathrm{D}$ & 90 & 111.02 & 109.98 & MID RIFF \\
\hline 2006 & $\mathrm{D}$ & 91 & 112.04 & 110.04 & BOT LOG STEP \\
\hline 2006 & $\mathrm{D}$ & 92 & 113.02 & 110.62 & TOP LOG STEP \\
\hline 2006 & $\mathrm{D}$ & 93 & 115.22 & 110.60 & MID RIFF \\
\hline 2006 & $\mathrm{D}$ & 94 & 116.78 & 110.53 & MID RIFF \\
\hline 2007 & $\mathrm{D}$ & 1 & 10.12 & 105.41 & MID RIFF \\
\hline 2007 & $\mathrm{D}$ & 2 & 11.65 & 105.45 & TAIL POOL \\
\hline 2007 & $\mathrm{D}$ & 3 & 12.48 & 105.31 & MID POOL \\
\hline 2007 & $\mathrm{D}$ & 4 & 13.55 & 105.46 & HEAD POOL \\
\hline 2007 & $\mathrm{D}$ & 5 & 14.46 & 105.52 & MID RIFF \\
\hline 2007 & $\mathrm{D}$ & 6 & 15.32 & 105.56 & BOT STEP \\
\hline 2007 & $\mathrm{D}$ & 7 & 15.48 & 105.74 & TOP STEP \\
\hline 2007 & $\mathrm{D}$ & 8 & 16.61 & 105.75 & MID RIFF \\
\hline 2007 & $\mathrm{D}$ & 8.5 & 17.49 & 105.77 & 10 \\
\hline 2007 & $\mathrm{D}$ & 9 & 18.25 & 105.78 & MID RIFF \\
\hline 2007 & $\mathrm{D}$ & 10 & 19.11 & 105.79 & TAIL POOL \\
\hline 2007 & $\mathrm{D}$ & 11 & 20.61 & 105.58 & MID POOL \\
\hline 2007 & $\mathrm{D}$ & 12 & 21.48 & 105.92 & HEAD POOL \\
\hline 2007 & $\mathrm{D}$ & 13 & 22.98 & 105.87 & MID RIFF \\
\hline 2007 & $\mathrm{D}$ & 13.5 & 23.13 & 105.88 & 9 \\
\hline 2007 & $\mathrm{D}$ & 14 & 24.41 & 105.94 & MID RIFF \\
\hline 2007 & $\mathrm{D}$ & 15 & 25.72 & 106.01 & TAIL POOL \\
\hline 2007 & $\mathrm{D}$ & 16 & 26.65 & 105.84 & MID POOL \\
\hline 2007 & $\mathrm{D}$ & 17 & 27.80 & 106.07 & HEAD POOL \\
\hline 2007 & $\mathrm{D}$ & 18 & 28.57 & 106.07 & MID RIFF \\
\hline 2007 & $\mathrm{D}$ & 18.5 & 28.67 & 106.08 & 8 \\
\hline 2007 & $\mathrm{D}$ & 19 & 29.33 & 106.14 & MID RIFF \\
\hline 2007 & $\mathrm{D}$ & 20 & 30.62 & 106.27 & MID RIFF \\
\hline 2007 & $\mathrm{D}$ & 21 & 32.58 & 106.20 & TAIL POOL \\
\hline 2007 & $\mathrm{D}$ & 21.5 & 33.21 & 106.15 & 7 \\
\hline 2007 & $\mathrm{D}$ & 22 & 34.08 & 106.08 & MID POOL \\
\hline 2007 & $\mathrm{D}$ & 23 & 34.82 & 106.32 & HEAD POOL \\
\hline 2007 & $\mathrm{D}$ & 24 & 36.31 & 106.45 & MID RIFF \\
\hline 2007 & $\mathrm{D}$ & 24.5 & 36.88 & 106.50 & 6 \\
\hline 2007 & $\mathrm{D}$ & 25 & 37.82 & 106.59 & TAIL POOL \\
\hline 2007 & $\mathrm{D}$ & 26 & 38.88 & 106.52 & MID POOL \\
\hline 2007 & $\mathrm{D}$ & 27 & 40.38 & 106.33 & MID POOL \\
\hline 2007 & $\mathrm{D}$ & 28 & 41.19 & 106.60 & HEAD POOL \\
\hline 2007 & $\mathrm{D}$ & 29 & 42.89 & 106.78 & HEAD POOL \\
\hline 2007 & $\mathrm{D}$ & 30 & 45.29 & 106.83 & MID RIFF \\
\hline 2007 & $\mathrm{D}$ & 31 & 47.11 & 106.98 & MID RIFF \\
\hline 2007 & $\mathrm{D}$ & 32 & 47.96 & 107.08 & MID RIFF \\
\hline 2007 & $\mathrm{D}$ & 33 & 49.83 & 107.10 & MID RIFF \\
\hline 2007 & $\mathrm{D}$ & 34 & 52.11 & 107.21 & MID RIFF \\
\hline 2007 & $\mathrm{D}$ & 35 & 54.99 & 107.41 & TAIL POOL \\
\hline 2007 & $\mathrm{D}$ & 36 & 56.59 & 107.28 & MID POOL \\
\hline 2007 & $\mathrm{D}$ & 37 & 57.62 & 107.23 & MID POOL \\
\hline 2007 & $\mathrm{D}$ & 38 & 59.12 & 107.46 & HEAD POOL \\
\hline
\end{tabular}

Continued on Next Page... 
APPENDIX D. SURVEY DATA

Table D.4 - Continued

\begin{tabular}{|c|c|c|c|c|c|}
\hline Year & Reach & Point Number & Adjusted Dist. (m) & Adjusted Elev. (m) & Point Description \\
\hline 2007 & $\mathrm{D}$ & 39 & 60.15 & 107.56 & TAIL POOL \\
\hline 2007 & $\mathrm{D}$ & 40 & 61.86 & 107.29 & MID POOL \\
\hline 2007 & $\mathrm{D}$ & 41 & 64.37 & 108.09 & MID RIFF \\
\hline 2007 & $\mathrm{D}$ & 42 & 66.20 & 108.20 & MID RIFF \\
\hline 2007 & $\mathrm{D}$ & 43 & 68.03 & 108.25 & MID RIFF \\
\hline 2007 & $\mathrm{D}$ & 44 & 68.99 & 108.50 & MID RIFF \\
\hline 2007 & $\mathrm{D}$ & 45 & 71.40 & 108.60 & MID RIFF \\
\hline 2007 & $\mathrm{D}$ & 46 & 72.71 & 108.66 & MID RIFF \\
\hline 2007 & $\mathrm{D}$ & 47 & 76.75 & 108.79 & MID RIFF \\
\hline 2007 & $\mathrm{D}$ & 48 & 78.94 & 108.81 & MID RIFF \\
\hline 2007 & $\mathrm{D}$ & 49 & 81.93 & 108.88 & MID RIFF \\
\hline 2007 & $\mathrm{D}$ & 50 & 83.17 & 108.95 & MID RIFF \\
\hline 2007 & $\mathrm{D}$ & 51 & 84.54 & 108.95 & MID RIFF \\
\hline 2007 & $\mathrm{D}$ & 51.5 & 87.13 & 109.06 & 5 \\
\hline 2007 & $\mathrm{D}$ & 52 & 87.26 & 109.06 & TAIL POOL \\
\hline 2007 & $\mathrm{D}$ & 53 & 89.12 & 109.00 & MID POOL \\
\hline 2007 & $\mathrm{D}$ & 54 & 90.70 & 108.92 & MID POOL \\
\hline 2007 & $\mathrm{D}$ & 55 & 91.72 & 109.08 & HEAD POOL \\
\hline 2007 & $\mathrm{D}$ & 55.5 & 92.08 & 109.12 & 4 \\
\hline 2007 & $\mathrm{D}$ & 56 & 93.10 & 109.23 & MID RIFF \\
\hline 2007 & $\mathrm{D}$ & 57 & 94.45 & 109.27 & MID RIFF \\
\hline 2007 & $\mathrm{D}$ & 58 & 95.95 & 109.37 & TAIL POOL \\
\hline 2007 & $\mathrm{D}$ & 58.5 & 97.36 & 109.25 & 3 \\
\hline 2007 & $\mathrm{D}$ & 59 & 97.59 & 109.23 & MID POOL \\
\hline 2007 & $\mathrm{D}$ & 60 & 100.00 & 109.43 & HEAD POOL \\
\hline 2007 & $\mathrm{D}$ & 61 & 100.91 & 109.60 & MID RIFF \\
\hline 2007 & $\mathrm{D}$ & 61.5 & 101.37 & 109.61 & 2 \\
\hline 2007 & $\mathrm{D}$ & 62 & 103.01 & 109.66 & MID RIFF \\
\hline 2007 & $\mathrm{D}$ & 63 & 104.17 & 109.76 & TAIL POOL \\
\hline 2007 & $\mathrm{D}$ & 64 & 105.49 & 109.68 & MID POOL \\
\hline 2007 & $\mathrm{D}$ & 65 & 105.95 & 109.76 & HEAD POOL \\
\hline 2007 & $\mathrm{D}$ & 65.5 & 106.03 & 109.78 & 1 \\
\hline 2007 & $\mathrm{D}$ & 66 & 106.56 & 109.90 & MID RIFF \\
\hline 2007 & $\mathrm{D}$ & 67 & 108.18 & 109.93 & TAIL POOL \\
\hline 2008 & $\mathrm{D}$ & 1 & 11.85 & 105.38 & MID RIFF \\
\hline 2008 & $\mathrm{D}$ & 2 & 12.48 & 105.39 & TAIL POOL \\
\hline 2008 & $\mathrm{D}$ & 3 & 13.50 & 105.26 & MID POOL \\
\hline 2008 & $\mathrm{D}$ & 4 & 13.96 & 105.36 & HEAD POOL \\
\hline 2008 & $\mathrm{D}$ & 5 & 14.42 & 105.53 & MID RIFF \\
\hline 2008 & $\mathrm{D}$ & 6 & 15.07 & 105.55 & MID RIFF \\
\hline 2008 & $\mathrm{D}$ & 7 & 15.43 & 105.67 & MID RIFF \\
\hline 2008 & $\mathrm{D}$ & 8 & 16.00 & 105.58 & MID RIFF \\
\hline 2008 & $\mathrm{D}$ & 9 & 16.96 & 105.69 & MID RIFF \\
\hline 2008 & $\mathrm{D}$ & 9.5 & 17.49 & 105.66 & 10 \\
\hline 2008 & $\mathrm{D}$ & 10 & 17.76 & 105.65 & MID RIFF \\
\hline 2008 & $\mathrm{D}$ & 11 & 19.11 & 105.73 & TAIL POOL \\
\hline 2008 & $\mathrm{D}$ & 12 & 19.80 & 105.71 & MID POOL \\
\hline 2008 & $\mathrm{D}$ & 13 & 21.35 & 105.73 & HEAD POOL \\
\hline 2008 & $\mathrm{D}$ & 14 & 22.28 & 105.83 & MID RIFF \\
\hline 2008 & $\mathrm{D}$ & 14.5 & 23.13 & 105.86 & 9 \\
\hline 2008 & $\mathrm{D}$ & 15 & 23.46 & 105.87 & MID RIFF \\
\hline 2008 & $\mathrm{D}$ & 16 & 24.66 & 105.86 & MID RIFF \\
\hline
\end{tabular}

Continued on Next Page... 
Table D.4 - Continued

\begin{tabular}{|c|c|c|c|c|c|}
\hline Year & Reach & Point Number & Adjusted Dist. (m) & Adjusted Elev. (m) & Point Description \\
\hline 2008 & $\mathrm{D}$ & 17 & 25.49 & 105.95 & MID RIFF \\
\hline 2008 & $\mathrm{D}$ & 18 & 27.32 & 105.91 & MID RIFF \\
\hline 2008 & $\mathrm{D}$ & 19 & 28.11 & 105.99 & MID RIFF \\
\hline 2008 & D & 19.5 & 28.67 & 106.06 & 8 \\
\hline 2008 & $\mathrm{D}$ & 20 & 28.99 & 106.11 & MID RIFF \\
\hline 2008 & $\mathrm{D}$ & 21 & 29.84 & 106.14 & MID RIFF \\
\hline 2008 & $\mathrm{D}$ & 22 & 31.11 & 106.22 & MID RIFF \\
\hline 2008 & $\mathrm{D}$ & 23 & 32.02 & 106.23 & MID RIFF \\
\hline 2008 & $\mathrm{D}$ & 24 & 33.08 & 106.19 & MID RIFF \\
\hline 2008 & $\mathrm{D}$ & 24.5 & 33.21 & 106.19 & 7 \\
\hline 2008 & $\mathrm{D}$ & 25 & 34.76 & 106.25 & MID RIFF \\
\hline 2008 & $\mathrm{D}$ & 26 & 35.97 & 106.46 & MID RIFF \\
\hline 2008 & $\mathrm{D}$ & 26.5 & 36.88 & 106.44 & 6 \\
\hline 2008 & $\mathrm{D}$ & 27 & 36.96 & 106.43 & MID RIFF \\
\hline 2008 & $\mathrm{D}$ & 28 & 37.94 & 106.52 & MID RIFF \\
\hline 2008 & $\mathrm{D}$ & 29 & 38.40 & 106.49 & TAIL POOL \\
\hline 2008 & $\mathrm{D}$ & 30 & 39.11 & 106.48 & MID POOL \\
\hline 2008 & $\mathrm{D}$ & 31 & 40.36 & 106.33 & MID POOL \\
\hline 2008 & $\mathrm{D}$ & 32 & 40.88 & 106.46 & HEAD POOL \\
\hline 2008 & $\mathrm{D}$ & 33 & 41.79 & 106.62 & MID RIFF \\
\hline 2008 & $\mathrm{D}$ & 34 & 42.71 & 106.76 & MID RIFF \\
\hline 2008 & $\mathrm{D}$ & 35 & 44.41 & 106.75 & MID RIFF \\
\hline 2008 & $\mathrm{D}$ & 36 & 45.68 & 106.84 & MID RIFF \\
\hline 2008 & $\mathrm{D}$ & 37 & 46.49 & 106.81 & MID RIFF \\
\hline 2008 & $\mathrm{D}$ & 38 & 47.39 & 106.90 & MID RIFF \\
\hline 2008 & $\mathrm{D}$ & 39 & 48.31 & 106.97 & MID RIFF \\
\hline 2008 & $\mathrm{D}$ & 40 & 49.49 & 107.03 & MID RIFF \\
\hline 2008 & $\mathrm{D}$ & 41 & 50.37 & 107.07 & MID RIFF \\
\hline 2008 & $\mathrm{D}$ & 42 & 51.27 & 107.10 & MID RIFF \\
\hline 2008 & $\mathrm{D}$ & 43 & 52.19 & 107.19 & MID RIFF \\
\hline 2008 & $\mathrm{D}$ & 44 & 53.17 & 107.24 & MID RIFF \\
\hline 2008 & $\mathrm{D}$ & 45 & 54.13 & 107.15 & MID RIFF \\
\hline 2008 & $\mathrm{D}$ & 46 & 54.93 & 107.38 & MID RIFF \\
\hline 2008 & $\mathrm{D}$ & 47 & 55.51 & 107.35 & TAIL POOL \\
\hline 2008 & $\mathrm{D}$ & 48 & 56.23 & 107.23 & MID POOL \\
\hline 2008 & $\mathrm{D}$ & 49 & 56.72 & 107.01 & MID POOL \\
\hline 2008 & $\mathrm{D}$ & 50 & 57.54 & 107.10 & HEAD POOL \\
\hline 2008 & $\mathrm{D}$ & 51 & 58.04 & 107.35 & MID RIFF \\
\hline 2008 & $\mathrm{D}$ & 52 & 59.29 & 107.55 & MID RIFF \\
\hline 2008 & $\mathrm{D}$ & 53 & 59.96 & 107.50 & TAIL POOL \\
\hline 2008 & $\mathrm{D}$ & 54 & 60.49 & 107.41 & MID POOL \\
\hline 2008 & $\mathrm{D}$ & 55 & 61.02 & 107.41 & HEAD POOL \\
\hline 2008 & $\mathrm{D}$ & 56 & 62.46 & 107.57 & MID RIFF \\
\hline 2008 & $\mathrm{D}$ & 57 & 63.99 & 107.32 & MID RIFF \\
\hline 2008 & $\mathrm{D}$ & 58 & 65.51 & 107.95 & MID RIFF \\
\hline 2008 & $\mathrm{D}$ & 59 & 66.43 & 108.21 & TAIL POOL \\
\hline 2008 & $\mathrm{D}$ & 60 & 68.06 & 108.05 & MID POOL \\
\hline 2008 & $\mathrm{D}$ & 61 & 69.58 & 108.30 & HEAD POOL \\
\hline 2008 & $\mathrm{D}$ & 62 & 70.25 & 108.52 & MID RIFF \\
\hline 2008 & $\mathrm{D}$ & 63 & 72.03 & 108.50 & MID RIFF \\
\hline 2008 & $\mathrm{D}$ & 64 & 73.37 & 108.60 & MID RIFF \\
\hline 2008 & D & 65 & 74.82 & 108.72 & MID RIFF \\
\hline
\end{tabular}

Continued on Next Page... 
Table D.4 - Continued

\begin{tabular}{|c|c|c|c|c|c|}
\hline Year & Reach & Point Number & Adjusted Dist. (m) & Adjusted Elev. (m) & Point Description \\
\hline 2008 & $\mathrm{D}$ & 66 & 76.29 & 108.70 & MID RIFF \\
\hline 2008 & $\mathrm{D}$ & 67 & 77.93 & 108.77 & MID RIFF \\
\hline 2008 & $\mathrm{D}$ & 68 & 80.01 & 108.83 & MID RIFF \\
\hline 2008 & $\mathrm{D}$ & 69 & 81.56 & 108.81 & MID RIFF \\
\hline 2008 & $\mathrm{D}$ & 70 & 83.29 & 108.92 & MID RIFF \\
\hline 2008 & $\mathrm{D}$ & 71 & 84.41 & 108.87 & MID RIFF \\
\hline 2008 & $\mathrm{D}$ & 72 & 85.54 & 108.89 & MID RIFF \\
\hline 2008 & $\mathrm{D}$ & 73 & 87.04 & 109.05 & TAIL POOL \\
\hline 2008 & $\mathrm{D}$ & 73.5 & 87.13 & 109.04 & 5 \\
\hline 2008 & $\mathrm{D}$ & 74 & 88.85 & 108.97 & MID POOL \\
\hline 2008 & $\mathrm{D}$ & 75 & 90.51 & 108.84 & MID POOL \\
\hline 2008 & $\mathrm{D}$ & 75.5 & 92.08 & 109.12 & 4 \\
\hline 2008 & $\mathrm{D}$ & 76 & 92.19 & 109.14 & HEAD POOL \\
\hline 2008 & $\mathrm{D}$ & 77 & 93.53 & 109.22 & MID RIFF \\
\hline 2008 & $\mathrm{D}$ & 78 & 94.43 & 109.26 & MID RIFF \\
\hline 2008 & $\mathrm{D}$ & 79 & 95.75 & 109.31 & MID RIFF \\
\hline 2008 & $\mathrm{D}$ & 80 & 96.29 & 109.35 & TAIL POOL \\
\hline 2008 & $\mathrm{D}$ & 81 & 97.36 & 109.24 & MID POOL \\
\hline 2008 & $\mathrm{D}$ & 81.5 & 97.36 & 109.24 & 3 \\
\hline 2008 & $\mathrm{D}$ & 82 & 98.50 & 109.22 & MID POOL \\
\hline 2008 & $\mathrm{D}$ & 83 & 99.52 & 109.43 & HEAD POOL \\
\hline 2008 & $\mathrm{D}$ & 84 & 101.29 & 109.62 & MID RIFF \\
\hline 2008 & $\mathrm{D}$ & 84.5 & 101.37 & 109.62 & 2 \\
\hline 2008 & $\mathrm{D}$ & 85 & 102.66 & 109.56 & MID RIFF \\
\hline 2008 & $\mathrm{D}$ & 86 & 104.44 & 109.65 & MID RIFF \\
\hline 2008 & $\mathrm{D}$ & 87 & 105.68 & 109.65 & MID RIFF \\
\hline 2008 & $\mathrm{D}$ & 87.5 & 106.03 & 109.71 & 1 \\
\hline 2008 & $\mathrm{D}$ & 88 & 107.09 & 109.90 & TAIL POOL \\
\hline 2008 & $\mathrm{D}$ & 89 & 107.72 & 109.85 & MID POOL \\
\hline 2008 & $\mathrm{D}$ & 90 & 109.48 & 109.71 & MID POOL \\
\hline 2008 & $\mathrm{D}$ & 91 & 110.57 & 110.06 & HEAD POOL \\
\hline 2008 & $\mathrm{D}$ & 92 & 112.15 & 110.06 & MID RIFF \\
\hline 2008 & $\mathrm{D}$ & 93 & 113.57 & 110.29 & MID RIFF \\
\hline 2008 & $\mathrm{D}$ & 94 & 114.60 & 110.36 & MID RIFF \\
\hline 2008 & $\mathrm{D}$ & 95 & 116.40 & 110.44 & MID RIFF \\
\hline 2008 & $\mathrm{D}$ & 96 & 117.39 & 110.48 & TAIL POOL \\
\hline 2008 & $\mathrm{D}$ & 97 & 118.58 & 110.47 & MID POOL \\
\hline 2008 & $\mathrm{D}$ & 98 & 120.05 & 110.30 & MID POOL \\
\hline 2008 & $\mathrm{D}$ & 99 & 120.65 & 110.71 & HEAD POOL \\
\hline 2008 & $\mathrm{D}$ & 100 & 121.86 & 110.69 & MID RIFF \\
\hline 2008 & $\mathrm{D}$ & 101 & 123.32 & 110.71 & MID RIFF \\
\hline 2008 & $\mathrm{D}$ & 102 & 124.83 & 110.67 & MID RIFF \\
\hline 2008 & $\mathrm{D}$ & 103 & 126.02 & 110.88 & MID RIFF \\
\hline 2008 & $\mathrm{D}$ & 104 & 127.26 & 110.97 & MID RIFF \\
\hline 2008 & $\mathrm{D}$ & 105 & 128.96 & 111.11 & MID RIFF \\
\hline 2008 & $\mathrm{D}$ & 106 & 133.01 & 110.66 & MID RIFF \\
\hline 2008 & $\mathrm{D}$ & 107 & 133.66 & 110.84 & MID RIFF \\
\hline 2008 & $\mathrm{D}$ & 108 & 134.61 & 110.86 & MID RIFF \\
\hline 2008 & $\mathrm{D}$ & 109 & 135.66 & 110.98 & MID RIFF \\
\hline 2008 & $\mathrm{D}$ & 110 & 136.81 & 111.14 & MID RIFF \\
\hline 2008 & $\mathrm{D}$ & 111 & 137.63 & 111.10 & MID RIFF \\
\hline 2008 & $\mathrm{D}$ & 112 & 138.56 & 111.15 & MID RIFF \\
\hline
\end{tabular}

Continued on Next Page... 
Table D.4 - Continued

\begin{tabular}{|c|c|c|c|c|c|}
\hline Year & Reach & Point Number & Adjusted Dist. (m) & Adjusted Elev. (m) & Point Description \\
\hline 2008 & $\mathrm{D}$ & 113 & 140.13 & 111.09 & MID RIFF \\
\hline 2002 & $\mathrm{E}$ & 1 & 7.62 & 189.17 & BOTTRIFF.30 \\
\hline 2002 & $\mathrm{E}$ & 2 & 8.98 & 189.29 & TOPRIFF.58 \\
\hline 2002 & $\mathrm{E}$ & 3 & 11.41 & 189.31 & BOTTRIFF.47 \\
\hline 2002 & $\mathrm{E}$ & 4 & 13.97 & 188.58 & TOPRIFF.17 \\
\hline 2002 & $\mathrm{E}$ & 5 & 16.11 & 188.55 & STEP.48 \\
\hline 2002 & $\mathrm{E}$ & 6 & 16.29 & 189.10 & TAILPOOL.59 \\
\hline 2002 & $\mathrm{E}$ & 7 & 17.41 & 189.01 & MIDPOOL.98 \\
\hline 2002 & $\mathrm{E}$ & 8 & 18.61 & 189.22 & HEADPOOL. 25 \\
\hline 2002 & $\mathrm{E}$ & 9 & 20.49 & 189.27 & BOTTRIFF.49 \\
\hline 2002 & $\mathrm{E}$ & 10 & 22.19 & 189.33 & BEND.50 \\
\hline 2002 & $\mathrm{E}$ & 11 & 23.02 & 189.43 & STEP.495 \\
\hline 2002 & $\mathrm{E}$ & 12 & 24.36 & 189.48 & TAILPOOL. 15 \\
\hline 2002 & $\mathrm{E}$ & 13 & 25.46 & 189.15 & MIDPOOL1.67 \\
\hline 2002 & $\mathrm{E}$ & 14 & 26.16 & 189.47 & BOTTSTEP 1.5 \\
\hline 2002 & $\mathrm{E}$ & 15 & 26.21 & 189.65 & STEP.52 \\
\hline 2002 & $\mathrm{E}$ & 15.5 & 26.77 & 189.66 & 10.0 \\
\hline 2002 & $\mathrm{E}$ & 16 & 28.82 & 189.68 & TAILRIFF.50 \\
\hline 2002 & $\mathrm{E}$ & 17 & 30.98 & 189.92 & TAILPOOL. 26 \\
\hline 2002 & $\mathrm{E}$ & 17.5 & 31.46 & 189.88 & 9.0 \\
\hline 2002 & $\mathrm{E}$ & 18 & 32.59 & 189.78 & MIDPOOL.60 \\
\hline 2002 & $\mathrm{E}$ & 19 & 33.02 & 189.90 & HEADPOOL. 48 \\
\hline 2002 & $\mathrm{E}$ & 20 & 34.68 & 189.85 & MIDRIFF.51 \\
\hline 2002 & $\mathrm{E}$ & 20.5 & 35.84 & 189.89 & 8 \\
\hline 2002 & $\mathrm{E}$ & 21 & 40.44 & 190.06 & BEND.47 \\
\hline 2002 & $\mathrm{E}$ & 21.5 & 40.46 & 190.06 & 7.0 \\
\hline 2002 & $\mathrm{E}$ & 22 & 41.41 & 190.06 & STEP.71 \\
\hline 2002 & $\mathrm{E}$ & 23 & 41.90 & 190.28 & TAILPOOL. 21 \\
\hline 2002 & $\mathrm{E}$ & 24 & 43.09 & 190.21 & MIDPOOL.68 \\
\hline 2002 & $\mathrm{E}$ & 24.5 & 45.21 & 190.23 & 6.0 \\
\hline 2002 & $\mathrm{E}$ & 25 & 45.55 & 190.23 & BRIFFTOPPO.6 \\
\hline 2002 & $\mathrm{E}$ & 26 & 48.73 & 190.48 & TOPRIFF.44 \\
\hline 2002 & $\mathrm{E}$ & 26.5 & 50.32 & 190.56 & 5 \\
\hline 2002 & $\mathrm{E}$ & 27 & 50.67 & 190.58 & MIDRIFF.47 \\
\hline 2002 & $\mathrm{E}$ & 28 & 52.47 & 190.79 & BEND.465 \\
\hline 2002 & $\mathrm{E}$ & 29 & 54.86 & 191.02 & BEND. 25 \\
\hline 2002 & $\mathrm{E}$ & 30 & 55.77 & 191.14 & TOPRIFF.28 \\
\hline 2002 & $\mathrm{E}$ & 30.5 & 56.44 & 191.15 & 4.0 \\
\hline 2002 & $\mathrm{E}$ & 31 & 57.81 & 191.17 & TAILPOOL. 25 \\
\hline 2002 & $\mathrm{E}$ & 32 & 59.23 & 190.91 & MIDPOOL1.18 \\
\hline 2002 & $\mathrm{E}$ & 33 & 60.96 & 191.12 & HEADPOOL.59 \\
\hline 2002 & $\mathrm{E}$ & 33.5 & 61.48 & 191.18 & 3 \\
\hline 2002 & $\mathrm{E}$ & 34 & 62.55 & 191.32 & MIDRIFF.47 \\
\hline 2002 & $\mathrm{E}$ & 34.5 & 66.03 & 191.46 & 2.0 \\
\hline 2002 & $\mathrm{E}$ & 35 & 67.16 & 191.51 & BEND.58 \\
\hline 2002 & $\mathrm{E}$ & 36 & 68.52 & 191.61 & BEND.49 \\
\hline 2002 & $\mathrm{E}$ & 37 & 70.83 & 191.61 & TAILRIFF.61 \\
\hline 2002 & $\mathrm{E}$ & 37.5 & 71.17 & 191.64 & 1.0 \\
\hline 2002 & $\mathrm{E}$ & 38 & 75.17 & 192.03 & TOPRIFF.36 \\
\hline 2002 & $\mathrm{E}$ & 39 & 77.67 & 192.15 & BEND.5 \\
\hline 2002 & $\mathrm{E}$ & 40 & 79.20 & 192.21 & BEND.25 \\
\hline 2002 & $\mathrm{E}$ & 41 & 80.66 & 192.20 & BEND.5 \\
\hline
\end{tabular}

Continued on Next Page... 
Table D.4 - Continued

\begin{tabular}{|c|c|c|c|c|c|}
\hline Year & Reach & Point Number & Adjusted Dist. (m) & Adjusted Elev. (m) & Point Description \\
\hline 2002 & $\mathrm{E}$ & 42 & 87.22 & 193.01 & TOPRIFF.20 \\
\hline 2004 & $\mathrm{E}$ & 1 & 20.96 & 189.26 & MID RIF \\
\hline 2004 & $\mathrm{E}$ & 2 & 22.52 & 189.27 & MID RIF \\
\hline 2004 & $\mathrm{E}$ & 3 & 23.94 & 189.40 & TAIL POOL \\
\hline 2004 & $\mathrm{E}$ & 4 & 25.10 & 189.05 & MID POOL \\
\hline 2004 & $\mathrm{E}$ & 5 & 25.87 & 189.31 & HEAD POOL \\
\hline 2004 & $\mathrm{E}$ & 6 & 26.37 & 189.62 & TOP STEP \\
\hline 2004 & $\mathrm{E}$ & 6.5 & 26.77 & 189.63 & 10.0 \\
\hline 2004 & $\mathrm{E}$ & 7 & 27.98 & 189.67 & MID RIFF \\
\hline 2004 & $\mathrm{E}$ & 8 & 28.61 & 189.68 & MID RIFF \\
\hline 2004 & $\mathrm{E}$ & 9 & 29.26 & 189.70 & MID RIFF \\
\hline 2004 & $\mathrm{E}$ & 10 & 30.17 & 189.71 & MID RIFF \\
\hline 2004 & $\mathrm{E}$ & 11 & 30.46 & 189.86 & MID RIFF \\
\hline 2004 & $\mathrm{E}$ & 11.5 & 31.46 & 189.84 & 9.0 \\
\hline 2004 & $\mathrm{E}$ & 12 & 32.71 & 189.81 & MID RIFF \\
\hline 2004 & $\mathrm{E}$ & 13 & 34.86 & 189.93 & MID RIFF \\
\hline 2004 & $\mathrm{E}$ & 13.5 & 35.84 & 189.95 & 8.0 \\
\hline 2004 & $\mathrm{E}$ & 14 & 37.22 & 189.99 & MID RIFF \\
\hline 2004 & $\mathrm{E}$ & 15 & 39.75 & 190.08 & MID RIFF \\
\hline 2004 & $\mathrm{E}$ & 15.5 & 40.46 & 190.11 & 7 \\
\hline 2004 & $\mathrm{E}$ & 16 & 41.17 & 190.13 & MID RIFF \\
\hline 2004 & $\mathrm{E}$ & 17 & 43.53 & 190.24 & MID RIFF \\
\hline 2004 & $\mathrm{E}$ & 17.5 & 45.21 & 190.30 & 6 \\
\hline 2004 & $\mathrm{E}$ & 18 & 45.94 & 190.32 & MID RIFF \\
\hline 2004 & $\mathrm{E}$ & 19 & 47.47 & 190.33 & MID RIFF \\
\hline 2004 & $\mathrm{E}$ & 20 & 49.01 & 190.49 & MID RIFF \\
\hline 2004 & $\mathrm{E}$ & 20.5 & 50.32 & 190.58 & 5.0 \\
\hline 2004 & $\mathrm{E}$ & 21 & 50.63 & 190.60 & MID RIFF \\
\hline 2004 & $\mathrm{E}$ & 22 & 53.60 & 190.76 & MID RIFF \\
\hline 2004 & $\mathrm{E}$ & 23 & 55.18 & 190.98 & MID RIFF \\
\hline 2004 & $\mathrm{E}$ & 24 & 55.91 & 191.13 & MID RIFF \\
\hline 2004 & $\mathrm{E}$ & 24.5 & 56.44 & 191.14 & 4 \\
\hline 2004 & $\mathrm{E}$ & 25 & 56.78 & 191.14 & MID RIFF \\
\hline 2004 & $\mathrm{E}$ & 26 & 58.34 & 190.90 & POOL \\
\hline 2004 & $\mathrm{E}$ & 27 & 59.21 & 191.06 & TAIL POOL \\
\hline 2004 & $\mathrm{E}$ & 28 & 60.40 & 191.00 & HEAD POOL \\
\hline 2004 & $\mathrm{E}$ & 28.5 & 61.48 & 191.16 & 3 \\
\hline 2004 & $\mathrm{E}$ & 29 & 61.73 & 191.19 & MID RIFF \\
\hline 2004 & $\mathrm{E}$ & 30 & 62.92 & 191.33 & MID RIFF \\
\hline 2004 & $\mathrm{E}$ & 31 & 64.84 & 191.50 & MID RIFF \\
\hline 2004 & $\mathrm{E}$ & 31.5 & 66.03 & 191.49 & 2.0 \\
\hline 2004 & $\mathrm{E}$ & 32 & 67.02 & 191.49 & MID RIFF \\
\hline 2004 & $\mathrm{E}$ & 33 & 67.77 & 191.52 & MID RIFF \\
\hline 2004 & $\mathrm{E}$ & 34 & 68.99 & 191.60 & MID RIFF \\
\hline 2004 & $\mathrm{E}$ & 35 & 70.40 & 191.55 & MID RIFF \\
\hline 2004 & $\mathrm{E}$ & 35.5 & 71.17 & 191.63 & 1.0 \\
\hline 2004 & $\mathrm{E}$ & 36 & 71.47 & 191.67 & MID RIFF \\
\hline 2004 & $\mathrm{E}$ & 37 & 72.27 & 191.74 & MID RIFF \\
\hline 2004 & $\mathrm{E}$ & 38 & 73.19 & 191.68 & BOT STEP \\
\hline 2004 & $\mathrm{E}$ & 39 & 73.46 & 191.90 & TOP STEP \\
\hline 2005 & $\mathrm{E}$ & 1 & 20.07 & 189.25 & MID RIFF \\
\hline 2005 & $\mathrm{E}$ & 2 & 21.29 & 189.25 & MID RIFF \\
\hline
\end{tabular}

Continued on Next Page... 
Table D.4 - Continued

\begin{tabular}{|c|c|c|c|c|c|}
\hline Year & Reach & Point Number & Adjusted Dist. (m) & Adjusted Elev. (m) & Point Description \\
\hline 2005 & $\mathrm{E}$ & 3 & 22.08 & 189.30 & MID RIFF \\
\hline 2005 & $\mathrm{E}$ & 4 & 23.32 & 189.46 & MID RIFF \\
\hline 2005 & $\mathrm{E}$ & 5 & 24.17 & 189.45 & MID RIFF \\
\hline 2005 & $\mathrm{E}$ & 6 & 24.56 & 189.41 & TAIL POOL \\
\hline 2005 & $\mathrm{E}$ & 7 & 24.92 & 189.10 & MID POOL \\
\hline 2005 & $\mathrm{E}$ & 8 & 25.47 & 189.13 & HEAD POOL \\
\hline 2005 & $\mathrm{E}$ & 9 & 25.89 & 189.41 & BOTTOM ROCK STEP \\
\hline 2005 & $\mathrm{E}$ & 10 & 26.34 & 189.65 & TOP ROCK STEP \\
\hline 2005 & $\mathrm{E}$ & 10.5 & 26.77 & 189.66 & 10.0 \\
\hline 2005 & $\mathrm{E}$ & 11 & 27.98 & 189.70 & MID RIFF \\
\hline 2005 & $\mathrm{E}$ & 12 & 28.72 & 189.75 & MID RIFF \\
\hline 2005 & $\mathrm{E}$ & 13 & 29.21 & 189.69 & BOTTOM ROCK STEP \\
\hline 2005 & $\mathrm{E}$ & 14 & 29.59 & 189.74 & TOP ROCK STEP \\
\hline 2005 & $\mathrm{E}$ & 15 & 30.04 & 189.72 & MID RIFF \\
\hline 2005 & $\mathrm{E}$ & 16 & 30.23 & 189.74 & BOTTOM ROCK STEP \\
\hline 2005 & $\mathrm{E}$ & 17 & 30.45 & 189.84 & TOP ROCK STEP \\
\hline 2005 & $\mathrm{E}$ & 17.5 & 31.46 & 189.87 & 9 \\
\hline 2005 & $\mathrm{E}$ & 18 & 31.73 & 189.87 & MID RIFF \\
\hline 2005 & $\mathrm{E}$ & 19 & 32.86 & 189.86 & MID RIFF \\
\hline 2005 & $\mathrm{E}$ & 20 & 34.50 & 189.92 & MID RIFF \\
\hline 2005 & $\mathrm{E}$ & 21 & 35.73 & 189.98 & MID RIFF \\
\hline 2005 & $\mathrm{E}$ & 21.5 & 35.84 & 189.99 & 8.0 \\
\hline 2005 & $\mathrm{E}$ & 22 & 36.61 & 190.04 & MID RIFF \\
\hline 2005 & $\mathrm{E}$ & 23 & 38.03 & 190.06 & MID RIFF \\
\hline 2005 & $\mathrm{E}$ & 24 & 38.85 & 190.06 & MID RIFF \\
\hline 2005 & $\mathrm{E}$ & 25 & 39.70 & 190.08 & MID RIFF \\
\hline 2005 & $\mathrm{E}$ & 25.5 & 40.46 & 190.09 & 7.0 \\
\hline 2005 & $\mathrm{E}$ & 26 & 41.09 & 190.11 & MID RIFF \\
\hline 2005 & $\mathrm{E}$ & 27 & 41.74 & 190.20 & MID RIFF \\
\hline 2005 & $\mathrm{E}$ & 28 & 43.09 & 190.19 & MID RIFF \\
\hline 2005 & $\mathrm{E}$ & 29 & 44.09 & 190.21 & MID RIFF \\
\hline 2005 & $\mathrm{E}$ & 30 & 44.85 & 190.19 & MID RIFF \\
\hline 2005 & $\mathrm{E}$ & 30.5 & 45.21 & 190.22 & 6.0 \\
\hline 2005 & $\mathrm{E}$ & 31 & 45.49 & 190.25 & MID RIFF \\
\hline 2005 & $\mathrm{E}$ & 32 & 46.12 & 190.34 & MID RIFF \\
\hline 2005 & $\mathrm{E}$ & 33 & 47.15 & 190.33 & MID RIFF \\
\hline 2005 & $\mathrm{E}$ & 34 & 48.19 & 190.40 & MID RIFF \\
\hline 2005 & $\mathrm{E}$ & 35 & 48.87 & 190.49 & MID RIFF \\
\hline 2005 & $\mathrm{E}$ & 36 & 50.20 & 190.60 & MID RIFF \\
\hline 2005 & $\mathrm{E}$ & 36.5 & 50.32 & 190.60 & 5 \\
\hline 2005 & $\mathrm{E}$ & 37 & 51.67 & 190.61 & MID RIFF \\
\hline 2005 & $\mathrm{E}$ & 38 & 52.39 & 190.79 & MID RIFF \\
\hline 2005 & $\mathrm{E}$ & 39 & 53.22 & 190.78 & MID RIFF \\
\hline 2005 & $\mathrm{E}$ & 40 & 54.08 & 190.89 & MID RIFF \\
\hline 2005 & $\mathrm{E}$ & 41 & 54.98 & 190.97 & MID RIFF \\
\hline 2005 & $\mathrm{E}$ & 42 & 55.64 & 191.08 & MID RIFF \\
\hline 2005 & $\mathrm{E}$ & 42.5 & 56.44 & 191.14 & 4 \\
\hline 2005 & $\mathrm{E}$ & 43 & 57.33 & 191.20 & MID RIFF \\
\hline 2005 & $\mathrm{E}$ & 44 & 57.96 & 191.11 & TAIL POOL \\
\hline 2005 & $\mathrm{E}$ & 45 & 58.74 & 190.99 & MID POOL \\
\hline 2005 & $\mathrm{E}$ & 46 & 59.58 & 191.03 & HEAD POOL \\
\hline 2005 & $\mathrm{E}$ & 47 & 60.86 & 191.13 & MID RIFF \\
\hline
\end{tabular}

Continued on Next Page... 
Table D.4 - Continued

\begin{tabular}{|c|c|c|c|c|c|}
\hline Year & Reach & Point Number & Adjusted Dist. (m) & Adjusted Elev. (m) & Point Description \\
\hline 2005 & $\mathrm{E}$ & 47.5 & 61.48 & 191.21 & 3 \\
\hline 2005 & $\mathrm{E}$ & 48 & 62.15 & 191.31 & MID RIFF \\
\hline 2005 & $\mathrm{E}$ & 49 & 62.94 & 191.38 & MID RIFF \\
\hline 2005 & $\mathrm{E}$ & 50 & 63.67 & 191.38 & MID RIFF \\
\hline 2005 & $\mathrm{E}$ & 51 & 64.33 & 191.42 & MID RIFF \\
\hline 2005 & $\mathrm{E}$ & 52 & 65.13 & 191.43 & MID RIFF \\
\hline 2005 & $\mathrm{E}$ & 52.5 & 66.03 & 191.51 & 2.0 \\
\hline 2005 & $\mathrm{E}$ & 53 & 66.18 & 191.52 & MID RIFF \\
\hline 2005 & $\mathrm{E}$ & 54 & 66.60 & 191.48 & MID RIFF \\
\hline 2005 & $\mathrm{E}$ & 55 & 67.96 & 191.56 & MID RIFF \\
\hline 2005 & $\mathrm{E}$ & 56 & 69.52 & 191.57 & MID RIFF \\
\hline 2005 & $\mathrm{E}$ & 57 & 71.04 & 191.62 & MID RIFF \\
\hline 2005 & $\mathrm{E}$ & 57.5 & 71.17 & 191.63 & 1.0 \\
\hline 2005 & $\mathrm{E}$ & 58 & 72.11 & 191.70 & MID RIFF \\
\hline 2005 & $\mathrm{E}$ & 59 & 73.04 & 191.83 & MID RIFF \\
\hline 2005 & $\mathrm{E}$ & 60 & 74.12 & 191.84 & MID RIFF \\
\hline 2005 & $\mathrm{E}$ & 61 & 75.00 & 192.02 & MID RIFF \\
\hline 2005 & $\mathrm{E}$ & 62 & 76.06 & 192.07 & MID RIFF \\
\hline 2005 & $\mathrm{E}$ & 63 & 77.26 & 191.95 & MID RIFF \\
\hline 2005 & $\mathrm{E}$ & 64 & 77.86 & 192.02 & MID RIFF \\
\hline 2005 & $\mathrm{E}$ & 65 & 78.79 & 192.13 & MID RIFF \\
\hline 2006 & $\mathrm{E}$ & 1 & 19.67 & 189.17 & MID POOL \\
\hline 2006 & $\mathrm{E}$ & 2 & 20.16 & 189.28 & HEAD POOL \\
\hline 2006 & $\mathrm{E}$ & 3 & 20.85 & 189.29 & MID RIFF \\
\hline 2006 & $\mathrm{E}$ & 4 & 21.38 & 189.30 & TAIL POOL \\
\hline 2006 & $\mathrm{E}$ & 5 & 21.95 & 189.19 & MID POOL \\
\hline 2006 & $\mathrm{E}$ & 6 & 22.78 & 189.32 & HEAD POOL \\
\hline 2006 & $\mathrm{E}$ & 7 & 23.41 & 189.39 & MID RIFF \\
\hline 2006 & $\mathrm{E}$ & 8 & 24.02 & 189.34 & TAIL POOL \\
\hline 2006 & $\mathrm{E}$ & 9 & 24.92 & 189.19 & MID POOL \\
\hline 2006 & $\mathrm{E}$ & 10 & 25.49 & 189.15 & MID POOL \\
\hline 2006 & $\mathrm{E}$ & 11 & 26.21 & 189.48 & HEAD POOL \\
\hline 2006 & $\mathrm{E}$ & 11.5 & 26.77 & 189.56 & 10.0 \\
\hline 2006 & $\mathrm{E}$ & 12 & 27.36 & 189.65 & MID RIFF \\
\hline 2006 & $\mathrm{E}$ & 13 & 28.87 & 189.71 & MID RIFF \\
\hline 2006 & $\mathrm{E}$ & 14 & 30.06 & 189.74 & MID RIFF \\
\hline 2006 & $\mathrm{E}$ & 15 & 30.94 & 189.89 & MID RIFF \\
\hline 2006 & $\mathrm{E}$ & 15.5 & 31.46 & 189.85 & 9.0 \\
\hline 2006 & $\mathrm{E}$ & 16 & 32.07 & 189.81 & MID RIFF \\
\hline 2006 & $\mathrm{E}$ & 17 & 33.21 & 189.84 & MID RIFF \\
\hline 2006 & $\mathrm{E}$ & 18 & 34.38 & 189.93 & MID RIFF \\
\hline 2006 & $\mathrm{E}$ & 18.5 & 35.84 & 189.96 & 8.0 \\
\hline 2006 & $\mathrm{E}$ & 19 & 36.05 & 189.96 & MID RIFF \\
\hline 2006 & $\mathrm{E}$ & 20 & 37.55 & 190.04 & MID RIFF \\
\hline 2006 & $\mathrm{E}$ & 21 & 38.61 & 190.04 & MID RIFF \\
\hline 2006 & $\mathrm{E}$ & 22 & 39.27 & 190.10 & TAIL POOL \\
\hline 2006 & $\mathrm{E}$ & 23 & 40.01 & 190.02 & MID POOL \\
\hline 2006 & $\mathrm{E}$ & 23.5 & 40.46 & 190.02 & 7 \\
\hline 2006 & $\mathrm{E}$ & 24 & 40.53 & 190.02 & MID POOL \\
\hline 2006 & $\mathrm{E}$ & 25 & 40.98 & 190.18 & HEAD POOL \\
\hline 2006 & $\mathrm{E}$ & 26 & 42.04 & 190.18 & MID RIFF \\
\hline 2006 & $\mathrm{E}$ & 27 & 43.35 & 190.20 & MID RIFF \\
\hline
\end{tabular}

Continued on Next Page... 
Table D.4 - Continued

\begin{tabular}{|c|c|c|c|c|c|}
\hline Year & Reach & Point Number & Adjusted Dist. (m) & Adjusted Elev. (m) & Point Description \\
\hline 2006 & $\mathrm{E}$ & 28 & 44.43 & 190.20 & MID RIFF \\
\hline 2006 & $\mathrm{E}$ & 28.5 & 45.21 & 190.24 & 6.0 \\
\hline 2006 & $\mathrm{E}$ & 29 & 45.39 & 190.25 & MID RIFF \\
\hline 2006 & $\mathrm{E}$ & 30 & 46.39 & 190.34 & MID RIFF \\
\hline 2006 & $\mathrm{E}$ & 31 & 48.82 & 190.51 & MID RIFF \\
\hline 2006 & $\mathrm{E}$ & 32 & 49.65 & 190.41 & MID RIFF \\
\hline 2006 & $\mathrm{E}$ & 33 & 49.97 & 190.53 & MID RIFF \\
\hline 2006 & $\mathrm{E}$ & 33.5 & 50.32 & 190.55 & 5 \\
\hline 2006 & $\mathrm{E}$ & 34 & 51.57 & 190.65 & MID RIFF \\
\hline 2006 & $\mathrm{E}$ & 35 & 52.46 & 190.80 & MID RIFF \\
\hline 2006 & $\mathrm{E}$ & 36 & 53.39 & 190.79 & MID RIFF \\
\hline 2006 & $\mathrm{E}$ & 37 & 54.82 & 190.98 & MID RIFF \\
\hline 2006 & $\mathrm{E}$ & 38 & 55.83 & 191.09 & MID RIFF \\
\hline 2006 & $\mathrm{E}$ & 38.5 & 56.44 & 191.08 & 4.0 \\
\hline 2006 & $\mathrm{E}$ & 39 & 56.66 & 191.08 & MID RIFF \\
\hline 2006 & $\mathrm{E}$ & 40 & 57.88 & 191.14 & TAIL POOL \\
\hline 2006 & $\mathrm{E}$ & 41 & 58.74 & 191.01 & MID POOL \\
\hline 2006 & $\mathrm{E}$ & 42 & 59.67 & 190.97 & MID POOL \\
\hline 2006 & $\mathrm{E}$ & 43 & 61.01 & 191.14 & HEAD POOL \\
\hline 2006 & $\mathrm{E}$ & 43.5 & 61.48 & 191.20 & 3.0 \\
\hline 2006 & $\mathrm{E}$ & 44 & 61.95 & 191.26 & MID RIFF \\
\hline 2006 & $\mathrm{E}$ & 45 & 62.95 & 191.34 & MID RIFF \\
\hline 2006 & $\mathrm{E}$ & 46 & 63.45 & 191.41 & MID RIFF \\
\hline 2006 & $\mathrm{E}$ & 47 & 64.16 & 191.39 & MID RIFF \\
\hline 2006 & $\mathrm{E}$ & 48 & 64.83 & 191.48 & MID RIFF \\
\hline 2006 & $\mathrm{E}$ & 49 & 65.62 & 191.50 & MID RIFF \\
\hline 2006 & $\mathrm{E}$ & 49.5 & 66.03 & 191.48 & 2 \\
\hline 2006 & $\mathrm{E}$ & 50 & 66.55 & 191.45 & MID RIFF \\
\hline 2006 & $\mathrm{E}$ & 51 & 67.39 & 191.54 & MID RIFF \\
\hline 2006 & $\mathrm{E}$ & 52 & 67.95 & 191.54 & MID RIFF \\
\hline 2006 & $\mathrm{E}$ & 53 & 69.12 & 191.60 & MID RIFF \\
\hline 2006 & $\mathrm{E}$ & 53.5 & 71.17 & 191.69 & 1.0 \\
\hline 2006 & $\mathrm{E}$ & 54 & 71.71 & 191.72 & MID RIFF \\
\hline 2006 & $\mathrm{E}$ & 55 & 72.34 & 191.72 & TAIL POOL \\
\hline 2006 & $\mathrm{E}$ & 56 & 73.26 & 191.70 & MID POOL \\
\hline 2006 & $\mathrm{E}$ & 57 & 73.65 & 191.88 & HEAD POOL \\
\hline 2006 & $\mathrm{E}$ & 58 & 74.35 & 191.82 & MID RIFF \\
\hline 2006 & $\mathrm{E}$ & 59 & 74.99 & 192.00 & MID RIFF \\
\hline 2006 & $\mathrm{E}$ & 60 & 76.78 & 192.10 & MID RIFF \\
\hline 2006 & $\mathrm{E}$ & 61 & 78.20 & 192.12 & MID RIFF \\
\hline 2006 & $\mathrm{E}$ & 62 & 79.45 & 192.18 & MID RIFF \\
\hline 2007 & $\mathrm{E}$ & 1 & 23.48 & 189.40 & TAIL POOL \\
\hline 2007 & $\mathrm{E}$ & 2 & 25.10 & 189.08 & MID POOL \\
\hline 2007 & $\mathrm{E}$ & 3 & 25.87 & 189.25 & BOT STEP \\
\hline 2007 & $\mathrm{E}$ & 4 & 26.30 & 189.68 & TOP STEP \\
\hline 2007 & $\mathrm{E}$ & 4.5 & 26.77 & 189.69 & 10.0 \\
\hline 2007 & $\mathrm{E}$ & 5 & 28.63 & 189.73 & MID RIFF \\
\hline 2007 & $\mathrm{E}$ & 6 & 30.88 & 189.90 & MID RIFF \\
\hline 2007 & $\mathrm{E}$ & 6.5 & 31.46 & 189.90 & 9 \\
\hline 2007 & $\mathrm{E}$ & 7 & 34.59 & 189.87 & MID RIFF \\
\hline 2007 & $\mathrm{E}$ & 7.5 & 35.84 & 189.94 & 8.0 \\
\hline 2007 & $\mathrm{E}$ & 8 & 37.75 & 190.06 & MID RIFF \\
\hline
\end{tabular}

Continued on Next Page... 
Table D.4 - Continued

\begin{tabular}{|c|c|c|c|c|c|}
\hline Year & Reach & Point Number & Adjusted Dist. (m) & Adjusted Elev. (m) & Point Description \\
\hline 2007 & $\mathrm{E}$ & 8.5 & 40.46 & 190.09 & 7.0 \\
\hline 2007 & $\mathrm{E}$ & 9 & 41.15 & 190.10 & MID RIFF \\
\hline 2007 & $\mathrm{E}$ & 10 & 44.45 & 190.18 & MID RIFF \\
\hline 2007 & $\mathrm{E}$ & 10.5 & 45.21 & 190.24 & 6 \\
\hline 2007 & $\mathrm{E}$ & 11 & 46.46 & 190.34 & MID RIFF \\
\hline 2007 & $\mathrm{E}$ & 12 & 48.19 & 190.35 & MID RIFF \\
\hline 2007 & $\mathrm{E}$ & 12.5 & 50.32 & 190.56 & 5 \\
\hline 2007 & $\mathrm{E}$ & 13 & 50.77 & 190.61 & MID RIFF \\
\hline 2007 & $\mathrm{E}$ & 14 & 53.00 & 190.72 & MID RIFF \\
\hline 2007 & $\mathrm{E}$ & 15 & 55.22 & 190.97 & MID RIFF \\
\hline 2007 & $\mathrm{E}$ & 16 & 56.29 & 191.12 & MID RIFF \\
\hline 2007 & $\mathrm{E}$ & 16.5 & 56.44 & 191.12 & 4 \\
\hline 2007 & $\mathrm{E}$ & 17 & 57.88 & 191.15 & TAIL POOL \\
\hline 2007 & $\mathrm{E}$ & 18 & 59.40 & 190.98 & MID POOL \\
\hline 2007 & $\mathrm{E}$ & 19 & 60.69 & 191.09 & HEAD POOL \\
\hline 2007 & $\mathrm{E}$ & 19.5 & 61.48 & 191.17 & 3.0 \\
\hline 2007 & $\mathrm{E}$ & 20 & 62.88 & 191.31 & MID RIFF \\
\hline 2007 & $\mathrm{E}$ & 20.5 & 66.03 & 191.45 & 2 \\
\hline 2007 & $\mathrm{E}$ & 21 & 66.56 & 191.48 & MID RIFF \\
\hline 2007 & $\mathrm{E}$ & 22 & 69.58 & 191.52 & MID RIFF \\
\hline 2007 & $\mathrm{E}$ & 22.5 & 71.17 & 191.61 & 1 \\
\hline 2007 & $\mathrm{E}$ & 23 & 72.98 & 191.71 & MID RIFF \\
\hline 2007 & $\mathrm{E}$ & 24 & 76.49 & 192.09 & MID RIFF \\
\hline 2008 & $\mathrm{E}$ & 1 & 21.02 & 189.29 & MID RIFF \\
\hline 2008 & $\mathrm{E}$ & 2 & 22.06 & 189.19 & MID RIFF \\
\hline 2008 & $\mathrm{E}$ & 3 & 23.18 & 189.40 & MID RIFF \\
\hline 2008 & $\mathrm{E}$ & 4 & 24.14 & 189.30 & TAIL POOL \\
\hline 2008 & $\mathrm{E}$ & 5 & 25.18 & 189.04 & MID POOL \\
\hline 2008 & $\mathrm{E}$ & 6 & 25.88 & 189.26 & HEAD POOL \\
\hline 2008 & $\mathrm{E}$ & 7 & 26.51 & 189.62 & MID RIFF \\
\hline 2008 & $\mathrm{E}$ & 7.5 & 26.77 & 189.61 & 10 \\
\hline 2008 & $\mathrm{E}$ & 8 & 28.08 & 189.59 & MID RIFF \\
\hline 2008 & $\mathrm{E}$ & 9 & 29.24 & 189.71 & MID RIFF \\
\hline 2008 & $\mathrm{E}$ & 10 & 31.14 & 189.86 & MID RIFF \\
\hline 2008 & $\mathrm{E}$ & 10.5 & 31.46 & 189.85 & 9 \\
\hline 2008 & $\mathrm{E}$ & 11 & 32.71 & 189.81 & MID RIFF \\
\hline 2008 & $\mathrm{E}$ & 12 & 34.65 & 189.91 & MID RIFF \\
\hline 2008 & $\mathrm{E}$ & 12.5 & 35.84 & 189.91 & 8 \\
\hline 2008 & $\mathrm{E}$ & 13 & 36.28 & 189.92 & MID RIFF \\
\hline 2008 & $\mathrm{E}$ & 14 & 37.94 & 190.01 & MID RIFF \\
\hline 2008 & $\mathrm{E}$ & 15 & 39.79 & 189.97 & MID RIFF \\
\hline 2008 & $\mathrm{E}$ & 15.5 & 40.46 & 190.04 & 7 \\
\hline 2008 & $\mathrm{E}$ & 16 & 40.93 & 190.08 & MID RIFF \\
\hline 2008 & $\mathrm{E}$ & 17 & 41.82 & 190.20 & MID RIFF \\
\hline 2008 & $\mathrm{E}$ & 18 & 43.79 & 190.19 & MID RIFF \\
\hline 2008 & $\mathrm{E}$ & 19 & 44.92 & 190.20 & MID RIFF \\
\hline 2008 & $\mathrm{E}$ & 19.5 & 45.21 & 190.23 & 6 \\
\hline 2008 & $\mathrm{E}$ & 20 & 46.19 & 190.35 & MID RIFF \\
\hline 2008 & $\mathrm{E}$ & 21 & 47.59 & 190.35 & MID RIFF \\
\hline 2008 & $\mathrm{E}$ & 22 & 48.65 & 190.46 & MID RIFF \\
\hline 2008 & $\mathrm{E}$ & 23 & 49.63 & 190.39 & MID RIFF \\
\hline 2008 & $\mathrm{E}$ & 23.5 & 50.32 & 190.57 & 5 \\
\hline
\end{tabular}

Continued on Next Page... 
Table D.4 - Continued

\begin{tabular}{|c|c|c|c|c|c|}
\hline Year & Reach & Point Number & Adjusted Dist. (m) & Adjusted Elev. (m) & Point Description \\
\hline 2008 & $\mathrm{E}$ & 24 & 50.47 & 190.61 & MID RIFF \\
\hline 2008 & $\mathrm{E}$ & 25 & 51.27 & 190.69 & MID RIFF \\
\hline 2008 & $\mathrm{E}$ & 26 & 52.59 & 190.69 & MID RIFF \\
\hline 2008 & $\mathrm{E}$ & 27 & 53.02 & 190.69 & MID RIFF \\
\hline 2008 & $\mathrm{E}$ & 28 & 53.95 & 190.80 & MID RIFF \\
\hline 2008 & $\mathrm{E}$ & 29 & 55.71 & 190.95 & MID RIFF \\
\hline 2008 & $\mathrm{E}$ & 29.5 & 56.44 & 191.10 & 4 \\
\hline 2008 & $\mathrm{E}$ & 30 & 56.72 & 191.17 & MID RIFF \\
\hline 2008 & $\mathrm{E}$ & 31 & 57.99 & 191.18 & TAIL POOL \\
\hline 2008 & $\mathrm{E}$ & 32 & 59.45 & 190.99 & MID POOL \\
\hline 2008 & $\mathrm{E}$ & 33 & 60.62 & 191.06 & MID POOL \\
\hline 2008 & $\mathrm{E}$ & 33.5 & 61.48 & 191.16 & 3 \\
\hline 2008 & $\mathrm{E}$ & 34 & 61.53 & 191.17 & HEAD POOL \\
\hline 2008 & $\mathrm{E}$ & 35 & 62.98 & 191.30 & MID RIFF \\
\hline 2008 & $\mathrm{E}$ & 36 & 64.13 & 191.40 & MID RIFF \\
\hline 2008 & $\mathrm{E}$ & 37 & 65.08 & 191.41 & MID RIFF \\
\hline 2008 & $\mathrm{E}$ & 37.5 & 66.03 & 191.46 & 2 \\
\hline 2008 & $\mathrm{E}$ & 38 & 66.58 & 191.49 & MID RIFF \\
\hline 2008 & $\mathrm{E}$ & 39 & 68.09 & 191.54 & MID RIFF \\
\hline 2008 & $\mathrm{E}$ & 40 & 70.10 & 191.60 & MID RIFF \\
\hline 2008 & $\mathrm{E}$ & 40.5 & 71.17 & 191.65 & 1 \\
\hline 2008 & $\mathrm{E}$ & 41 & 71.98 & 191.70 & MID RIFF \\
\hline 2008 & $\mathrm{E}$ & 42 & 73.58 & 191.71 & MID RIFF \\
\hline 2008 & $\mathrm{E}$ & 43 & 75.56 & 192.04 & MID RIFF \\
\hline 2008 & $\mathrm{E}$ & 44 & 77.08 & 191.94 & MID RIFF \\
\hline 2008 & $\mathrm{E}$ & 45 & 78.20 & 192.04 & MID RIFF \\
\hline 2008 & $\mathrm{E}$ & 46 & 79.64 & 192.18 & MID RIFF \\
\hline 2008 & $\mathrm{E}$ & 47 & 80.94 & 192.17 & MID RIFF \\
\hline 2008 & $\mathrm{E}$ & 48 & 82.86 & 192.43 & TAIL POOL \\
\hline 2008 & $\mathrm{E}$ & 49 & 84.20 & 192.33 & MID POOL \\
\hline 2008 & $\mathrm{E}$ & 50 & 85.17 & 192.39 & HEAD POOL \\
\hline 2002 & $\mathrm{~F}$ & 1 & 0.00 & 104.93 & TAILPOOL.30 \\
\hline 2002 & $\mathrm{~F}$ & 2 & 1.79 & 104.56 & MIDPOOL1.52 \\
\hline 2002 & $\mathrm{~F}$ & 3 & 2.82 & 104.83 & HEADPOOL. 57 \\
\hline 2002 & $\mathrm{~F}$ & 4 & 3.38 & 105.40 & TOPSTEP.08TRIFF \\
\hline 2002 & $\mathrm{~F}$ & 5 & 8.02 & 105.63 & HEADRIFF.20TPOOL \\
\hline 2002 & $\mathrm{~F}$ & 6 & 9.93 & 105.46 & MIDPOOL.77 \\
\hline 2002 & $\mathrm{~F}$ & 7 & 12.20 & 105.64 & HEADPOOL.18TRIFF \\
\hline 2002 & $\mathrm{~F}$ & 8 & 13.72 & 105.57 & BEND.54 \\
\hline 2002 & $\mathrm{~F}$ & 9 & 16.02 & 105.76 & HEADRIFF.33 \\
\hline 2002 & $\mathrm{~F}$ & 10 & 17.20 & 105.70 & MIDPOOL.50 \\
\hline 2002 & $\mathrm{~F}$ & 11 & 17.55 & 105.76 & HEADPOOL.27TRIFF \\
\hline 2002 & $\mathrm{~F}$ & 12 & 20.62 & 106.06 & HEADRIFF.21TGLI \\
\hline 2002 & $\mathrm{~F}$ & 13 & 21.35 & 106.05 & MIDGLI.49 \\
\hline 2002 & $\mathrm{~F}$ & 14 & 22.48 & 106.14 & HEADGLI.21TRIFF \\
\hline 2002 & $\mathrm{~F}$ & 15 & 26.31 & 106.31 & HEADRIFF.28TRIFF \\
\hline 2002 & $\mathrm{~F}$ & 16 & 28.04 & 106.44 & HEADRIFF.05TPOOL \\
\hline 2002 & $\mathrm{~F}$ & 17 & 28.93 & 106.18 & MIDPOOL.70 \\
\hline 2002 & $\mathrm{~F}$ & 18 & 29.42 & 106.29 & HEADPOOL.47BSTEP \\
\hline 2002 & $\mathrm{~F}$ & 19 & 29.90 & 106.49 & TOPSTEP.14TRIFF \\
\hline 2002 & F & 20 & 31.94 & 106.57 & HEADRIFF.12TGLI \\
\hline 2002 & $\mathrm{~F}$ & 20.5 & 33.09 & 106.51 & 10.0 \\
\hline
\end{tabular}


Table D.4 - Continued

\begin{tabular}{|c|c|c|c|c|c|}
\hline Year & Reach & Point Number & Adjusted Dist. (m) & Adjusted Elev. (m) & Point Description \\
\hline 2002 & F & 21 & 33.46 & 106.49 & MIDGLI.46 \\
\hline 2002 & $\mathrm{~F}$ & 22 & 36.35 & 106.60 & HEADGLI.18BSTEP \\
\hline 2002 & $\mathrm{~F}$ & 23 & 36.74 & 106.66 & TAILPOOL.31TOPST \\
\hline 2002 & $\mathrm{~F}$ & 24 & 37.43 & 106.62 & BEND.44 \\
\hline 2002 & $\mathrm{~F}$ & 25 & 37.79 & 106.54 & MIDPOOL.67 \\
\hline 2002 & $\mathrm{~F}$ & 25.5 & 38.27 & 106.60 & 9 \\
\hline 2002 & $\mathrm{~F}$ & 26 & 38.29 & 106.61 & HEADPOOL. 48 \\
\hline 2002 & $\mathrm{~F}$ & 27 & 38.47 & 106.69 & TAILRIFF.25TSTEP \\
\hline 2002 & $\mathrm{~F}$ & 28 & 39.18 & 106.72 & BEND.29 \\
\hline 2002 & $\mathrm{~F}$ & 29 & 39.76 & 106.70 & BEND.31 \\
\hline 2002 & $\mathrm{~F}$ & 30 & 40.59 & 106.72 & HEADRIFF.27TGLI \\
\hline 2002 & $\mathrm{~F}$ & 31 & 42.79 & 106.71 & MID.44LOW \\
\hline 2002 & $\mathrm{~F}$ & 32 & 43.19 & 106.73 & MID.35HIGH \\
\hline 2002 & $\mathrm{~F}$ & 32.5 & 43.28 & 106.73 & 8.0 \\
\hline 2002 & $\mathrm{~F}$ & 33 & 43.83 & 106.71 & MID.43LOW \\
\hline 2002 & $\mathrm{~F}$ & 34 & 44.60 & 106.73 & HEADGLI.29BOTSTE \\
\hline 2002 & $\mathrm{~F}$ & 35 & 44.78 & 106.80 & TAILRIFF.33TSTEP \\
\hline 2002 & $\mathrm{~F}$ & 36 & 47.24 & 106.92 & HRIFF.23TRIFF \\
\hline 2002 & $\mathrm{~F}$ & 37 & 47.76 & 107.00 & TAIL.20HEADRIFF \\
\hline 2002 & $\mathrm{~F}$ & 37.5 & 48.49 & 107.00 & 7.0 \\
\hline 2002 & F & 38 & 49.69 & 107.01 & HRIFF.17TRIFF \\
\hline 2002 & $\mathrm{~F}$ & 39 & 50.24 & 107.09 & TRIFF.28HRIFF \\
\hline 2002 & $\mathrm{~F}$ & 40 & 51.63 & 107.11 & HRIFF.13 \\
\hline 2002 & $\mathrm{~F}$ & 41 & 52.29 & 107.21 & MIDRIFF.21 \\
\hline 2002 & $\mathrm{~F}$ & 41.5 & 53.18 & 107.20 & 6 \\
\hline 2002 & $\mathrm{~F}$ & 42 & 55.17 & 107.19 & HEADGLI. 29 \\
\hline 2002 & $\mathrm{~F}$ & 43 & 56.76 & 107.29 & HRIFF.26TGLI \\
\hline 2002 & $\mathrm{~F}$ & 44 & 57.00 & 107.34 & HEADGLI. 29 \\
\hline 2002 & $\mathrm{~F}$ & 44.5 & 57.03 & 107.34 & 5.0 \\
\hline 2002 & $\mathrm{~F}$ & 45 & 58.98 & 107.30 & TRIFF.41HRIFF \\
\hline 2002 & $\mathrm{~F}$ & 46 & 59.76 & 107.43 & TRIFF.14HRIFF \\
\hline 2002 & $\mathrm{~F}$ & 47 & 61.06 & 107.45 & HRIFF.34TPOOL \\
\hline 2002 & $\mathrm{~F}$ & 47.5 & 62.45 & 107.32 & 4 \\
\hline 2002 & $\mathrm{~F}$ & 48 & 62.93 & 107.28 & MIDPOOL.90 \\
\hline 2002 & $\mathrm{~F}$ & 49 & 65.26 & 107.57 & HEADPOOL.08BSTEP \\
\hline 2002 & $\mathrm{~F}$ & 50 & 65.65 & 107.71 & TRIFF.37TSTEP \\
\hline 2002 & $\mathrm{~F}$ & 51 & 66.69 & 107.78 & HRIFF.24 \\
\hline 2002 & $\mathrm{~F}$ & 51.5 & 67.36 & 107.91 & 3.0 \\
\hline 2002 & $\mathrm{~F}$ & 52 & 67.50 & 107.94 & HTRIFF.14 \\
\hline 2002 & $\mathrm{~F}$ & 52.5 & 71.19 & 108.07 & 2 \\
\hline 2002 & $\mathrm{~F}$ & 53 & 73.02 & 108.14 & TRIFF.22TGLI \\
\hline 2002 & $\mathrm{~F}$ & 54 & 73.39 & 108.21 & TGLI.37 \\
\hline 2002 & $\mathrm{~F}$ & 55 & 75.32 & 108.21 & HGLI.51 \\
\hline 2002 & $\mathrm{~F}$ & 56 & 75.63 & 108.25 & TRIFF.23 \\
\hline 2002 & $\mathrm{~F}$ & 56.5 & 76.30 & 108.29 & 1 \\
\hline 2002 & $\mathrm{~F}$ & 57 & 79.37 & 108.45 & HRIFF.28 \\
\hline 2002 & $\mathrm{~F}$ & 58 & 80.07 & 108.57 & TPOOL.30 \\
\hline 2002 & $\mathrm{~F}$ & 59 & 80.89 & 108.47 & MIDPOOL.56 \\
\hline 2002 & $\mathrm{~F}$ & 60 & 81.92 & 108.58 & HPOOL. 28 \\
\hline 2002 & $\mathrm{~F}$ & 61 & 83.67 & 108.70 & TGLI.20 \\
\hline 2002 & $\mathrm{~F}$ & 62 & 85.10 & 108.57 & MIDGLI.61 \\
\hline 2002 & $\mathrm{~F}$ & 63 & 86.98 & 108.58 & HRIFFBSTEP.58 \\
\hline
\end{tabular}

Continued on Next Page... 
Table D.4 - Continued

\begin{tabular}{|c|c|c|c|c|c|}
\hline Year & Reach & Point Number & Adjusted Dist. (m) & Adjusted Elev. (m) & Point Description \\
\hline 2002 & $\mathrm{~F}$ & 64 & 87.11 & 108.70 & BIS.33 \\
\hline 2004 & F & 1 & 26.20 & 106.15 & MID RIFF \\
\hline 2004 & $\mathrm{~F}$ & 2 & 27.84 & 106.31 & MID RIFF \\
\hline 2004 & $\mathrm{~F}$ & 3 & 29.53 & 106.34 & MID RIFF \\
\hline 2004 & F & 4 & 31.18 & 106.47 & MID RIFF \\
\hline 2004 & $\mathrm{~F}$ & 5 & 32.22 & 106.51 & TAIL GLIDE \\
\hline 2004 & F & 5.5 & 33.09 & 106.51 & 10.0 \\
\hline 2004 & $\mathrm{~F}$ & 6 & 34.12 & 106.52 & MID GLIDE \\
\hline 2004 & F & 7 & 35.95 & 106.55 & HEAD GLIDE \\
\hline 2004 & F & 8 & 37.91 & 106.56 & MID RIFF \\
\hline 2004 & $\mathrm{~F}$ & 8.5 & 38.27 & 106.59 & 9.0 \\
\hline 2004 & F & 9 & 39.66 & 106.68 & MID RIFF \\
\hline 2004 & $\mathrm{~F}$ & 10 & 42.28 & 106.74 & MID RIFF \\
\hline 2004 & F & 10.5 & 43.28 & 106.73 & 8.0 \\
\hline 2004 & $\mathrm{~F}$ & 11 & 44.63 & 106.72 & MID RIFF \\
\hline 2004 & $\mathrm{~F}$ & 12 & 47.45 & 106.92 & MID RIFF \\
\hline 2004 & $\mathrm{~F}$ & 12.5 & 48.49 & 106.98 & 7.0 \\
\hline 2004 & $\mathrm{~F}$ & 13 & 50.75 & 107.10 & MID RIFF \\
\hline 2004 & $\mathrm{~F}$ & 14 & 52.88 & 107.16 & MID RIFF \\
\hline 2004 & $\mathrm{~F}$ & 14.5 & 53.18 & 107.17 & 6.0 \\
\hline 2004 & $\mathrm{~F}$ & 15 & 54.63 & 107.20 & MID RIFF \\
\hline 2004 & $\mathrm{~F}$ & 16 & 56.82 & 107.31 & MID RIFF \\
\hline 2004 & $\mathrm{~F}$ & 16.5 & 57.03 & 107.30 & 5 \\
\hline 2004 & $\mathrm{~F}$ & 17 & 58.91 & 107.27 & MID RIFF \\
\hline 2004 & $\mathrm{~F}$ & 18 & 61.09 & 107.45 & MID RIFF \\
\hline 2004 & F & 18.5 & 62.45 & 107.47 & 4.0 \\
\hline 2004 & $\mathrm{~F}$ & 19 & 63.13 & 107.48 & MID RIFF \\
\hline 2004 & $\mathrm{~F}$ & 20 & 65.54 & 107.57 & MID RIFF \\
\hline 2004 & $\mathrm{~F}$ & 21 & 67.18 & 107.73 & BOT STEP \\
\hline 2004 & F & 21.5 & 67.36 & 107.82 & 3.0 \\
\hline 2004 & $\mathrm{~F}$ & 22 & 67.71 & 107.98 & TOP STEP \\
\hline 2004 & $\mathrm{~F}$ & 23 & 69.81 & 108.07 & MID RIFF \\
\hline 2004 & $\mathrm{~F}$ & 23.5 & 71.19 & 108.01 & 2 \\
\hline 2004 & F & 24 & 71.70 & 107.99 & MID RIFF \\
\hline 2004 & $\mathrm{~F}$ & 25 & 73.45 & 108.22 & MID RIFF \\
\hline 2004 & $\mathrm{~F}$ & 26 & 75.00 & 108.10 & MID RIFF \\
\hline 2004 & $\mathrm{~F}$ & 26.5 & 76.30 & 108.24 & 1.0 \\
\hline 2004 & $\mathrm{~F}$ & 27 & 76.79 & 108.29 & MID RIFF \\
\hline 2004 & $\mathrm{~F}$ & 28 & 78.55 & 108.45 & MID RIFF \\
\hline 2004 & F & 29 & 80.81 & 108.48 & MID RIFF \\
\hline 2004 & $\mathrm{~F}$ & 30 & 82.61 & 108.65 & MID RIFF \\
\hline 2005 & $\mathrm{~F}$ & 1 & 23.46 & 106.16 & MID RIFF \\
\hline 2005 & $\mathrm{~F}$ & 2 & 25.41 & 106.28 & MID RIFF \\
\hline 2005 & $\mathrm{~F}$ & 3 & 26.18 & 106.26 & MID RIFF \\
\hline 2005 & $\mathrm{~F}$ & 4 & 27.68 & 106.30 & MID RIFF \\
\hline 2005 & $\mathrm{~F}$ & 5 & 28.33 & 106.36 & MID RIFF \\
\hline 2005 & F & 6 & 29.11 & 106.35 & MID RIFF \\
\hline 2005 & $\mathrm{~F}$ & 7 & 29.78 & 106.34 & MID RIFF \\
\hline 2005 & $\mathrm{~F}$ & 8 & 31.74 & 106.44 & MID RIFF \\
\hline 2005 & $\mathrm{~F}$ & 8.5 & 33.09 & 106.46 & 10.0 \\
\hline 2005 & $\mathrm{~F}$ & 9 & 33.26 & 106.46 & MID RIFF \\
\hline 2005 & F & 10 & 35.73 & 106.52 & MID RIFF \\
\hline
\end{tabular}

Continued on Next Page... 
Table D.4 - Continued

\begin{tabular}{|c|c|c|c|c|c|}
\hline Year & Reach & Point Number & Adjusted Dist. (m) & Adjusted Elev. (m) & Point Description \\
\hline 2005 & F & 11 & 37.38 & 106.65 & MID RIFF \\
\hline 2005 & F & 11.5 & 38.27 & 106.67 & 9.0 \\
\hline 2005 & $\mathrm{~F}$ & 12 & 39.17 & 106.69 & MID RIFF \\
\hline 2005 & $\mathrm{~F}$ & 13 & 41.67 & 106.78 & MID RIFF \\
\hline 2005 & F & 13.5 & 43.28 & 106.78 & 8.0 \\
\hline 2005 & $\mathrm{~F}$ & 14 & 43.29 & 106.78 & MID RIFF \\
\hline 2005 & F & 15 & 43.92 & 106.75 & MID RIFF \\
\hline 2005 & F & 16 & 44.72 & 106.72 & MID RIFF \\
\hline 2005 & F & 17 & 44.90 & 106.82 & MID RIFF \\
\hline 2005 & F & 18 & 45.28 & 106.90 & MID RIFF \\
\hline 2005 & $\mathrm{~F}$ & 19 & 45.77 & 106.88 & MID RIFF \\
\hline 2005 & $\mathrm{~F}$ & 20 & 46.90 & 106.92 & MID RIFF \\
\hline 2005 & F & 21 & 47.79 & 106.94 & MID RIFF \\
\hline 2005 & F & 22 & 48.46 & 107.02 & MID RIFF \\
\hline 2005 & F & 22.5 & 48.49 & 107.02 & 7.0 \\
\hline 2005 & F & 23 & 50.10 & 107.07 & MID RIFF \\
\hline 2005 & $\mathrm{~F}$ & 24 & 50.51 & 107.17 & MID RIFF \\
\hline 2005 & $\mathrm{~F}$ & 25 & 51.40 & 107.08 & MID RIFF \\
\hline 2005 & $\mathrm{~F}$ & 26 & 51.84 & 107.23 & MID RIFF \\
\hline 2005 & F & 26.5 & 53.18 & 107.09 & 6.0 \\
\hline 2005 & F & 27 & 53.20 & 107.08 & MID RIFF \\
\hline 2005 & F & 28 & 54.09 & 107.19 & MID RIFF \\
\hline 2005 & $\mathrm{~F}$ & 29 & 55.81 & 107.25 & MID RIFF \\
\hline 2005 & $\mathrm{~F}$ & 29.5 & 57.03 & 107.35 & 5 \\
\hline 2005 & $\mathrm{~F}$ & 30 & 57.20 & 107.36 & MID RIFF \\
\hline 2005 & $\mathrm{~F}$ & 31 & 58.99 & 107.39 & MID RIFF \\
\hline 2005 & F & 32 & 59.68 & 107.39 & MID RIFF \\
\hline 2005 & F & 33 & 60.09 & 107.40 & MID RIFF \\
\hline 2005 & F & 34 & 61.27 & 107.46 & TAIL POOL \\
\hline 2005 & F & 35 & 62.39 & 107.43 & MID POOL \\
\hline 2005 & F & 35.5 & 62.45 & 107.42 & 4.0 \\
\hline 2005 & $\mathrm{~F}$ & 36 & 62.77 & 107.37 & MID POOL \\
\hline 2005 & F & 37 & 63.15 & 107.22 & MID POOL \\
\hline 2005 & F & 38 & 63.90 & 107.57 & HEAD POOL \\
\hline 2005 & $\mathrm{~F}$ & 39 & 64.83 & 107.53 & MID RIFF \\
\hline 2005 & F & 40 & 65.70 & 107.63 & MID RIFF \\
\hline 2005 & F & 41 & 66.94 & 107.66 & MID RIFF \\
\hline 2005 & F & 41.5 & 67.36 & 107.86 & 3 \\
\hline 2005 & F & 42 & 67.69 & 108.02 & MID RIFF \\
\hline 2005 & $\mathrm{~F}$ & 43 & 69.53 & 108.09 & MID RIFF \\
\hline 2005 & F & 43.5 & 71.19 & 108.04 & 2 \\
\hline 2005 & F & 44 & 71.45 & 108.03 & MID RIFF \\
\hline 2005 & F & 45 & 72.94 & 108.14 & TAIL POOL \\
\hline 2005 & $\mathrm{~F}$ & 46 & 74.11 & 108.25 & MID POOL \\
\hline 2005 & $\mathrm{~F}$ & 47 & 75.08 & 108.17 & HEAD POOL \\
\hline 2005 & F & 48 & 76.00 & 108.27 & MID RIFF \\
\hline 2005 & $\mathrm{~F}$ & 48.5 & 76.30 & 108.29 & 1.0 \\
\hline 2005 & $\mathrm{~F}$ & 49 & 78.23 & 108.44 & MID RIFF \\
\hline 2005 & F & 50 & 79.45 & 108.51 & MID RIFF \\
\hline 2005 & $\mathrm{~F}$ & 51 & 81.34 & 108.59 & MID RIFF \\
\hline 2005 & F & 52 & 83.40 & 108.65 & MID RIFF \\
\hline 2006 & $\mathrm{~F}$ & 1 & 28.56 & 106.32 & TAIL POOL \\
\hline
\end{tabular}

Continued on Next Page. . 
Table D.4 - Continued

\begin{tabular}{|c|c|c|c|c|c|}
\hline Year & Reach & Point Number & Adjusted Dist. (m) & Adjusted Elev. (m) & Point Description \\
\hline 2006 & $\mathrm{~F}$ & 2 & 28.95 & 106.30 & MID POOL \\
\hline 2006 & F & 3 & 29.61 & 106.29 & HEAD POOL \\
\hline 2006 & $\mathrm{~F}$ & 4 & 30.29 & 106.58 & MID RIF \\
\hline 2006 & F & 5 & 32.47 & 106.60 & MID RIF \\
\hline 2006 & F & 5.5 & 33.09 & 106.59 & 10 \\
\hline 2006 & F & 6 & 35.44 & 106.57 & MID RIF \\
\hline 2006 & $\mathrm{~F}$ & 7 & 36.62 & 106.61 & MID RIF \\
\hline 2006 & $\mathrm{~F}$ & 8 & 36.93 & 106.69 & MID RIF \\
\hline 2006 & F & 9 & 37.43 & 106.62 & MID RIF \\
\hline 2006 & F & 9.5 & 38.27 & 106.60 & 9 \\
\hline 2006 & F & 10 & 38.35 & 106.60 & MID RIF \\
\hline 2006 & F & 11 & 38.60 & 106.69 & MID RIF \\
\hline 2006 & F & 12 & 40.73 & 106.76 & MID RIF \\
\hline 2006 & $\mathrm{~F}$ & 13 & 41.51 & 106.75 & MID RIF \\
\hline 2006 & F & 14 & 43.14 & 106.79 & TAIL POOL \\
\hline 2006 & F & 14.5 & 43.28 & 106.77 & 8 \\
\hline 2006 & $\mathrm{~F}$ & 15 & 43.84 & 106.71 & MID POOL \\
\hline 2006 & F & 16 & 44.57 & 106.72 & HEAD POOL \\
\hline 2006 & F & 17 & 44.97 & 106.80 & MID RIF \\
\hline 2006 & F & 18 & 45.66 & 106.88 & MID RIF \\
\hline 2006 & $\mathrm{~F}$ & 19 & 47.59 & 106.93 & MID RIF \\
\hline 2006 & F & 20 & 47.91 & 106.99 & MID RIF \\
\hline 2006 & $\mathrm{~F}$ & 21 & 48.16 & 107.02 & MID RIF \\
\hline 2006 & F & 21.5 & 48.49 & 107.02 & 7.0 \\
\hline 2006 & F & 22 & 50.05 & 107.04 & MID RIF \\
\hline 2006 & $\mathrm{~F}$ & 23 & 51.36 & 107.07 & BOTTOM ROCK STEP \\
\hline 2006 & $\mathrm{~F}$ & 24 & 51.57 & 107.27 & TOP ROCK STEP \\
\hline 2006 & $\mathrm{~F}$ & 25 & 52.98 & 107.30 & MID RIF \\
\hline 2006 & F & 25.5 & 53.18 & 107.29 & 6.0 \\
\hline 2006 & $\mathrm{~F}$ & 26 & 54.02 & 107.24 & WOOD DEBRIS DWN \\
\hline 2006 & $\mathrm{~F}$ & 27 & 54.86 & 107.28 & WOODY DEBRIS UP \\
\hline 2006 & $\mathrm{~F}$ & 28 & 56.54 & 107.41 & MID RIF \\
\hline 2006 & $\mathrm{~F}$ & 28.5 & 57.03 & 107.39 & 5.0 \\
\hline 2006 & $\mathrm{~F}$ & 29 & 57.16 & 107.38 & TAIL POOL \\
\hline 2006 & $\mathrm{~F}$ & 30 & 58.00 & 107.32 & MID POOL \\
\hline 2006 & $\mathrm{~F}$ & 31 & 58.42 & 107.36 & HEAD POOL \\
\hline 2006 & $\mathrm{~F}$ & 32 & 59.08 & 107.46 & MID POOL \\
\hline 2006 & $\mathrm{~F}$ & 33 & 59.75 & 107.49 & MID POOL \\
\hline 2006 & $\mathrm{~F}$ & 34 & 61.04 & 107.55 & MID POOL \\
\hline 2006 & $\mathrm{~F}$ & 35 & 62.31 & 107.48 & TAIL POOL \\
\hline 2006 & $\mathrm{~F}$ & 35.5 & 62.45 & 107.45 & 4.0 \\
\hline 2006 & $\mathrm{~F}$ & 36 & 63.00 & 107.32 & MID POOL \\
\hline 2006 & $\mathrm{~F}$ & 37 & 63.87 & 107.36 & HEAD POOL \\
\hline 2006 & $\mathrm{~F}$ & 38 & 64.31 & 107.57 & MID RIF \\
\hline 2006 & $\mathrm{~F}$ & 39 & 65.24 & 107.46 & WOODY DEBRIS DWN \\
\hline 2006 & $\mathrm{~F}$ & 40 & 65.77 & 107.55 & WOODY DEBRIS UP \\
\hline 2006 & $\mathrm{~F}$ & 41 & 66.40 & 107.60 & MID RIF \\
\hline 2006 & $\mathrm{~F}$ & 42 & 67.07 & 107.75 & MID RIF \\
\hline 2006 & $\mathrm{~F}$ & 42.5 & 67.36 & 107.83 & 3 \\
\hline 2006 & $\mathrm{~F}$ & 43 & 67.57 & 107.88 & MID RIF \\
\hline 2006 & $\mathrm{~F}$ & 44 & 69.44 & 108.02 & MID RIF \\
\hline 2006 & $\mathrm{~F}$ & 45 & 70.74 & 108.09 & MID RIF \\
\hline
\end{tabular}

Continued on Next Page. . 
Table D.4 - Continued

\begin{tabular}{|c|c|c|c|c|c|}
\hline Year & Reach & Point Number & Adjusted Dist. (m) & Adjusted Elev. (m) & Point Description \\
\hline 2006 & $\mathrm{~F}$ & 45.5 & 71.19 & 108.08 & 2 \\
\hline 2006 & $\mathrm{~F}$ & 46 & 71.81 & 108.08 & MID RIF \\
\hline 2006 & $\mathrm{~F}$ & 47 & 72.97 & 108.08 & MID RIF \\
\hline 2006 & $\mathrm{~F}$ & 48 & 75.04 & 108.10 & MID RIF \\
\hline 2006 & $\mathrm{~F}$ & 49 & 75.69 & 108.28 & MID RIF \\
\hline 2006 & $\mathrm{~F}$ & 49.5 & 76.30 & 108.31 & 1.0 \\
\hline 2006 & $\mathrm{~F}$ & 50 & 77.10 & 108.34 & MID RIF \\
\hline 2006 & $\mathrm{~F}$ & 51 & 77.95 & 108.46 & MID RIF \\
\hline 2006 & $\mathrm{~F}$ & 52 & 78.17 & 108.56 & MID RIF \\
\hline 2006 & $\mathrm{~F}$ & 53 & 80.02 & 108.56 & MID RIF \\
\hline 2006 & $\mathrm{~F}$ & 54 & 80.82 & 108.48 & MID RIF \\
\hline 2006 & $\mathrm{~F}$ & 55 & 81.46 & 108.63 & MID RIF \\
\hline 2007 & $\mathrm{~F}$ & 1 & 24.03 & 106.20 & MID RIFF \\
\hline 2007 & $\mathrm{~F}$ & 2 & 26.27 & 106.28 & MID RIFF \\
\hline 2007 & $\mathrm{~F}$ & 3 & 28.36 & 106.32 & TAIL POOL \\
\hline 2007 & $\mathrm{~F}$ & 4 & 29.09 & 106.12 & MID POOL \\
\hline 2007 & $\mathrm{~F}$ & 5 & 29.73 & 106.32 & HEAD POOL \\
\hline 2007 & $\mathrm{~F}$ & 6 & 30.16 & 106.56 & MID RIFF \\
\hline 2007 & $\mathrm{~F}$ & 7 & 31.66 & 106.59 & TAIL POOL \\
\hline 2007 & $\mathrm{~F}$ & 7.5 & 33.09 & 106.57 & 10.0 \\
\hline 2007 & $\mathrm{~F}$ & 8 & 34.49 & 106.56 & MID POOL \\
\hline 2007 & $\mathrm{~F}$ & 9 & 36.63 & 106.63 & HEAD POOL \\
\hline 2007 & $\mathrm{~F}$ & 9.5 & 38.27 & 106.69 & 9 \\
\hline 2007 & $\mathrm{~F}$ & 10 & 38.51 & 106.70 & MID RIFF \\
\hline 2007 & $\mathrm{~F}$ & 11 & 40.14 & 106.77 & MID RIFF \\
\hline 2007 & $\mathrm{~F}$ & 12 & 42.76 & 106.80 & TAIL POOL \\
\hline 2007 & $\mathrm{~F}$ & 12.5 & 43.28 & 106.77 & 8.0 \\
\hline 2007 & $\mathrm{~F}$ & 13 & 44.14 & 106.72 & MID POOL \\
\hline 2007 & $\mathrm{~F}$ & 14 & 44.73 & 106.82 & HEAD POOL \\
\hline 2007 & $\mathrm{~F}$ & 15 & 46.47 & 106.93 & MID RIFF \\
\hline 2007 & $\mathrm{~F}$ & 16 & 47.82 & 106.93 & MID RIFF \\
\hline 2007 & $\mathrm{~F}$ & 17 & 48.34 & 107.07 & MID RIFF \\
\hline 2007 & $\mathrm{~F}$ & 17.5 & 48.49 & 107.07 & 7.0 \\
\hline 2007 & $\mathrm{~F}$ & 18 & 49.97 & 107.13 & MID RIFF \\
\hline 2007 & $\mathrm{~F}$ & 19 & 51.33 & 107.23 & MID RIFF \\
\hline 2007 & $\mathrm{~F}$ & 19.5 & 53.18 & 107.30 & 6.0 \\
\hline 2007 & $\mathrm{~F}$ & 20 & 53.21 & 107.30 & MID RIFF \\
\hline 2007 & $\mathrm{~F}$ & 21 & 54.48 & 107.31 & MID RIFF \\
\hline 2007 & $\mathrm{~F}$ & 22 & 55.54 & 107.33 & MID RIFF \\
\hline 2007 & $\mathrm{~F}$ & 23 & 56.07 & 107.38 & MID RIFF \\
\hline 2007 & $\mathrm{~F}$ & 23.5 & 57.03 & 107.40 & 5.0 \\
\hline 2007 & $\mathrm{~F}$ & 24 & 58.73 & 107.42 & MID RIFF \\
\hline 2007 & $\mathrm{~F}$ & 25 & 60.62 & 107.60 & TAIL POOL \\
\hline 2007 & $\mathrm{~F}$ & 25.5 & 62.45 & 107.41 & 4 \\
\hline 2007 & $\mathrm{~F}$ & 26 & 62.77 & 107.37 & MID POOL \\
\hline 2007 & $\mathrm{~F}$ & 27 & 63.91 & 107.58 & MID POOL \\
\hline 2007 & $\mathrm{~F}$ & 28 & 64.67 & 107.28 & MID POOL \\
\hline 2007 & $\mathrm{~F}$ & 29 & 65.76 & 107.65 & HEAD POOL \\
\hline 2007 & $\mathrm{~F}$ & 30 & 67.10 & 107.78 & MID RIFF \\
\hline 2007 & $\mathrm{~F}$ & 30.5 & 67.36 & 107.84 & 3.0 \\
\hline 2007 & $\mathrm{~F}$ & 31 & 67.94 & 107.97 & MID RIFF \\
\hline 2007 & $\mathrm{~F}$ & 32 & 69.12 & 108.06 & MID RIFF \\
\hline
\end{tabular}

Continued on Next Page. . 
Table D.4 - Continued

\begin{tabular}{|c|c|c|c|c|c|}
\hline Year & Reach & Point Number & Adjusted Dist. (m) & Adjusted Elev. (m) & Point Description \\
\hline 2007 & F & 33 & 70.99 & 108.10 & MID RIFF \\
\hline 2007 & F & 33.5 & 71.19 & 108.11 & 2 \\
\hline 2007 & F & 34 & 72.19 & 108.19 & MID RIFF \\
\hline 2007 & $\mathrm{~F}$ & 35 & 73.60 & 108.29 & TAIL POOL \\
\hline 2007 & F & 36 & 73.64 & 108.28 & TAIL POOL \\
\hline 2007 & $\mathrm{~F}$ & 37 & 75.02 & 108.10 & MID POOL \\
\hline 2007 & F & 38 & 75.48 & 108.30 & HEAD POOL \\
\hline 2007 & F & 38.5 & 76.30 & 108.33 & 1 \\
\hline 2007 & F & 39 & 77.25 & 108.37 & MID RIFF \\
\hline 2007 & F & 40 & 78.05 & 108.52 & MID RIFF \\
\hline 2007 & $\mathrm{~F}$ & 41 & 80.41 & 108.66 & MID RIFF \\
\hline 2007 & $\mathrm{~F}$ & 42 & 82.37 & 108.67 & MID RIFF \\
\hline 2007 & F & 43 & 84.97 & 108.73 & MID RIFF \\
\hline 2008 & F & 1 & 18.80 & 106.17 & MID RIFF \\
\hline 2008 & F & 2 & 21.01 & 106.26 & MID RIFF \\
\hline 2008 & F & 3 & 22.60 & 106.26 & MID RIFF \\
\hline 2008 & $\mathrm{~F}$ & 4 & 24.11 & 106.18 & MID RIFF \\
\hline 2008 & $\mathrm{~F}$ & 5 & 24.92 & 106.38 & MID RIFF \\
\hline 2008 & $\mathrm{~F}$ & 6 & 27.21 & 106.33 & MID RIFF \\
\hline 2008 & F & 7 & 29.45 & 106.70 & MID RIFF \\
\hline 2008 & F & 8 & 32.02 & 106.69 & MID RIFF \\
\hline 2008 & F & 8.5 & 33.09 & 106.67 & 10 \\
\hline 2008 & $\mathrm{~F}$ & 9 & 34.25 & 106.66 & MID RIFF \\
\hline 2008 & $\mathrm{~F}$ & 9.5 & 38.27 & 106.72 & 9 \\
\hline 2008 & $\mathrm{~F}$ & 10 & 39.07 & 106.73 & MID RIFF \\
\hline 2008 & $\mathrm{~F}$ & 11 & 42.30 & 106.76 & MID RIFF \\
\hline 2008 & F & 11.5 & 43.28 & 106.78 & 8 \\
\hline 2008 & F & 12 & 44.88 & 106.80 & MID RIFF \\
\hline 2008 & F & 13 & 47.55 & 106.90 & MID RIFF \\
\hline 2008 & F & 13.5 & 48.49 & 106.95 & 7 \\
\hline 2008 & F & 14 & 50.52 & 107.07 & MID RIFF \\
\hline 2008 & $\mathrm{~F}$ & 15 & 52.72 & 107.14 & MID RIFF \\
\hline 2008 & F & 15.5 & 53.18 & 107.20 & 6 \\
\hline 2008 & F & 16 & 55.64 & 107.51 & MID RIFF \\
\hline 2008 & $\mathrm{~F}$ & 16.5 & 57.03 & 107.48 & 5 \\
\hline 2008 & F & 17 & 58.61 & 107.44 & MID RIFF \\
\hline 2008 & F & 17.5 & 62.45 & 107.59 & 4 \\
\hline 2008 & F & 18 & 62.52 & 107.59 & TAIL POOL \\
\hline 2008 & F & 19 & 64.64 & 107.24 & MID POOL \\
\hline 2008 & $\mathrm{~F}$ & 20 & 65.48 & 107.58 & HEAD POOL \\
\hline 2008 & F & 21 & 65.89 & 107.94 & MID RIFF \\
\hline 2008 & $\mathrm{~F}$ & 21.5 & 67.36 & 108.04 & 3 \\
\hline 2008 & F & 22 & 68.68 & 108.14 & MID RIFF \\
\hline 2008 & $\mathrm{~F}$ & 22.5 & 71.19 & 108.18 & 2 \\
\hline 2008 & $\mathrm{~F}$ & 23 & 72.46 & 108.20 & MID RIFF \\
\hline 2008 & F & 24 & 75.17 & 108.13 & MID RIFF \\
\hline 2008 & $\mathrm{~F}$ & 24.5 & 76.30 & 108.26 & 1 \\
\hline 2008 & $\mathrm{~F}$ & 25 & 78.87 & 108.54 & MID RIFF \\
\hline 2008 & F & 26 & 80.95 & 108.47 & MID RIFF \\
\hline 2008 & $\mathrm{~F}$ & 27 & 83.79 & 108.61 & MID RIFF \\
\hline 2008 & F & 28 & 86.79 & 108.66 & MID RIFF \\
\hline 2008 & $\mathrm{~F}$ & 29 & 88.14 & 109.33 & TAIL POOL \\
\hline
\end{tabular}

Continued on Next Page... 
Table D.4 - Continued

\begin{tabular}{cccccc}
\hline Year & Reach & Point Number & Adjusted Dist. $(\mathrm{m})$ & Adjusted Elev. $(\mathrm{m})$ & Point Description \\
\hline 2008 & F & 30 & 89.77 & 109.15 & MID POOL \\
2008 & F & 31 & 90.50 & 109.53 & HEAD POOL \\
2008 & F & 32 & 92.99 & 109.62 & MID RIFF \\
2008 & F & 33 & 94.46 & 109.70 & MID RIFF \\
2008 & F & 34 & 95.54 & 109.65 & MID RIFF \\
2008 & F & 35 & 96.99 & 109.94 & TAIL POOL \\
2008 & F & 36 & 97.99 & 109.82 & MID POOL \\
2008 & F & 37 & 99.28 & 109.73 & MID POOL \\
2008 & F & 38 & 100.52 & 109.93 & HEAD POOL \\
2008 & F & 39 & 102.28 & 110.05 & MID RIFF \\
2008 & F & 40 & 104.08 & 109.98 & MID RIFF \\
2008 & F & 41 & 105.07 & 110.27 & MID RIFF \\
2008 & F & 42 & 106.95 & 110.32 & MID RIFF \\
2008 & F & 43 & 108.03 & 110.55 & TAIL POOL \\
2008 & F & 44 & 109.67 & 110.25 & MID POOL \\
2008 & F & 45 & 110.65 & 110.58 & HEAD POOL \\
2008 & F & 46 & 111.47 & 110.52 & MID RIFF \\
2008 & F & 47 & 113.03 & 110.67 & TAIL POOL \\
2008 & F & 48 & 113.70 & 110.71 & MID POOL \\
2008 & F & 49 & & & HEAD POOL \\
\hline \hline
\end{tabular}




\section{Appendix E}

\section{VBA code}

This code was written in VBA for Excel ${ }^{\mathrm{TM}} 2003$ and was used in the cross-section viewer spreadsheet along with parameter queries to access the database and graph the data.

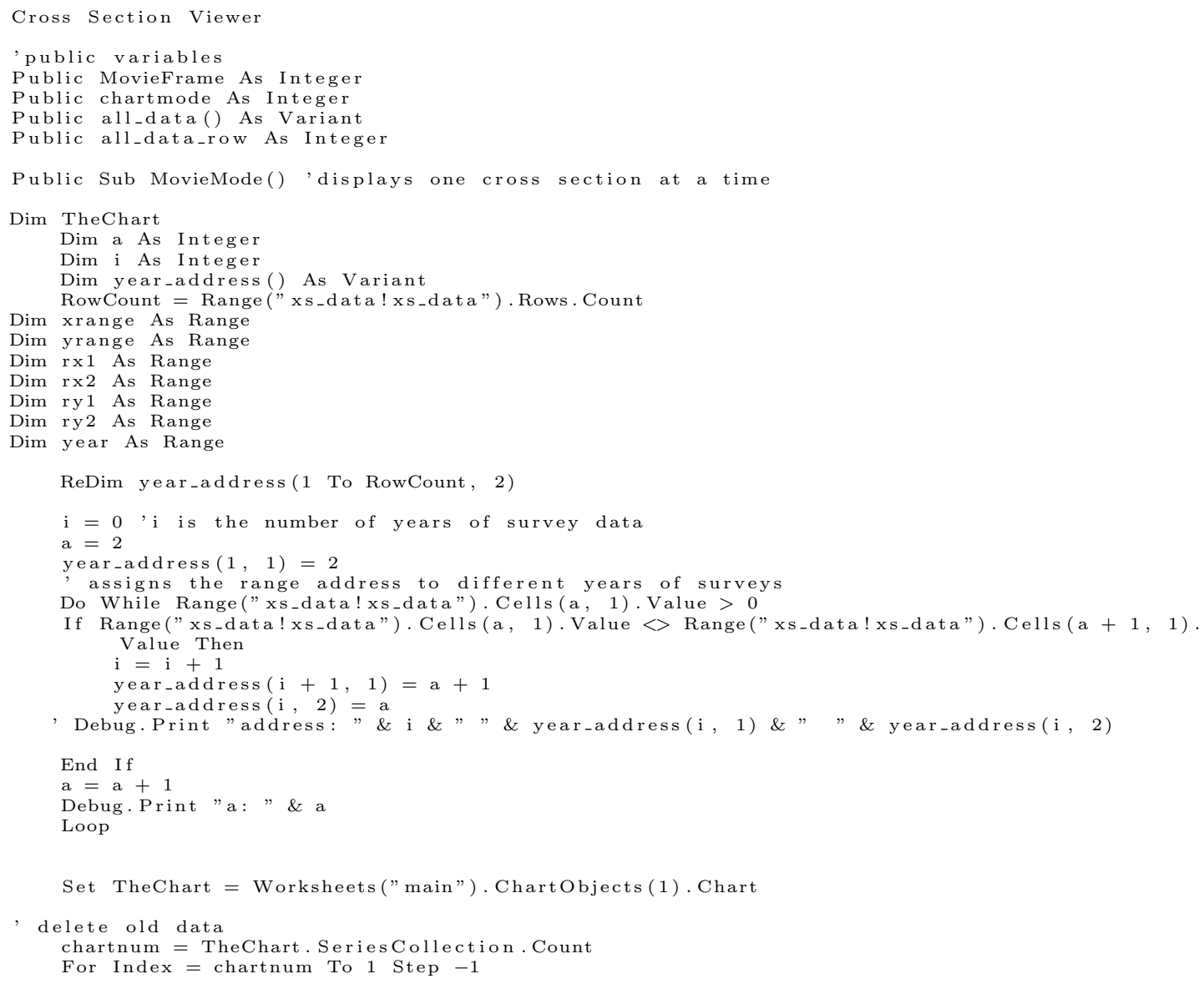




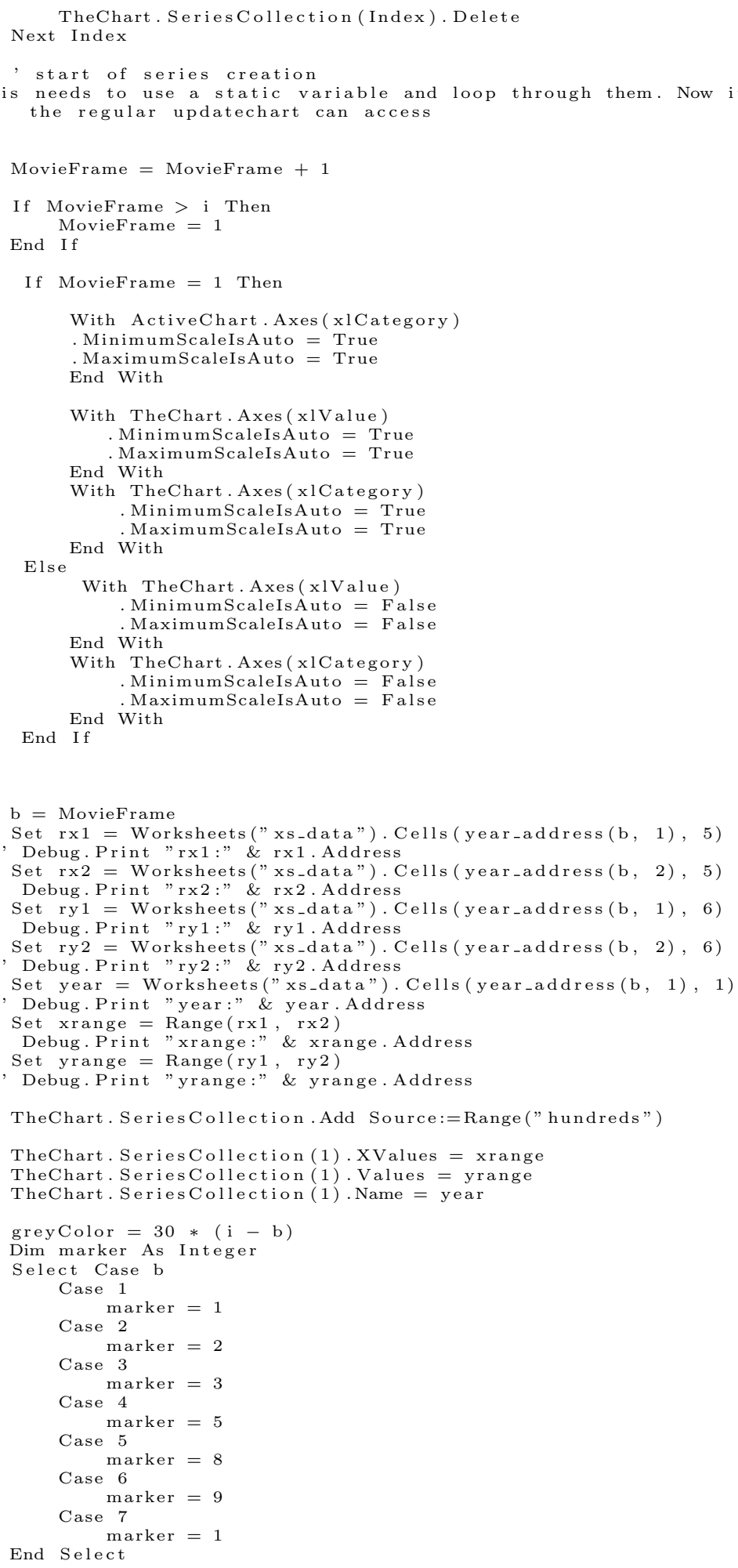

With TheChart.SeriesCollection (1)

Border.ColorIndex $=(17+\mathrm{i}-\mathrm{b})$ 'RGB(greyColor, greyColor, greyColor) Border. Weight $=$ xlMedium

MarkerStyle = marker

. MarkerBackgroundColorIndex $=x l$ None

MarkerForegroundColorIndex $=(17+\mathrm{i}-\mathrm{b})$ 'RGB(greyColor, greyColor, greyColor)

. MarkerSize $=5$ 


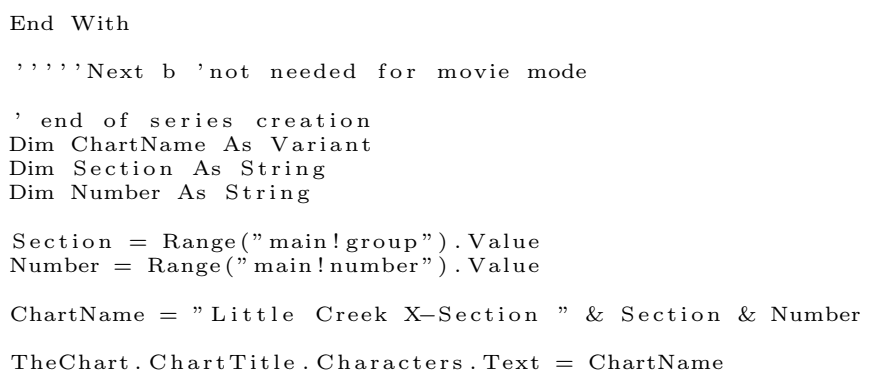

End Sub

Public Sub UpdateChart()

'update data ranges

Range ("Main! offset-query 1"). QueryTable. Refresh BackgroundQuery:=False

Range("xs_data! xs_data"). QueryTable. Refresh BackgroundQuery:=Fals

Range ("Main! Query_from_MS_Access_Database_1"). QueryTable.Refresh BackgroundQuery:=False

chartmode $=1$

Dim TheChart

Dim a As Integer

Dim i As Integer

Dim year-address () As Variant

RowCount $=$ Range("xs_data! xs_data"). Rows. Count

Dim xrange As Range

Dim yrange As Range

Dim $\mathrm{rx} 1$ As Range

Dim $r x 2$ As Range

Dim ry 1 As Range

Dim ry 2 As Range

Dim year As Range

ReDim year_address (1 To RowCount, 2)

$\mathrm{i}=0$

year-address $(1,1)=2$

assigns the range address to different years of surveys

Do While Range("xs_data! xs_data"). Cells (a, 1). Value $>0$

If Range("xs_data! xs_data"). Cells(a, 1). Value $<$ Range("xs_data!xs_data"). Cells (a + 1, 1).

Value Then

year-address $(\mathrm{i}+1,1)=\mathrm{a}+1$

Debug. Print "address: "\& i \& " \& year-address (i, 1) \& " " \& year_address (i, 2)

End If

$\mathrm{a}=\mathrm{a}+1$

'Debug.Print "a: "\& a

Loop

Set TheChart $=$ Worksheets ("main"). ChartObjects (1). Chart

chartnum $=$ TheChart. SeriesCollection. Count

For Index $=$ chartnum To 1 Step -1

TheChart. SeriesCollection (Index). Delete

Next Index

For $b=1$ To $\mathrm{i}$

Set $\mathrm{rx} 1=$ Worksheets ("xs_data"). Cells (year_address $(\mathrm{b}, 1), 5)$

Debug.Print "rxil:" \& $\mathrm{rx} 1$. Address

Set $\mathrm{rx} 2=$ Worksheets ("xs_data"). Cells (year_address (b, 2), 5)

Debug.Print "rx2:" \& rx2. Address

Set ry 1 = Worksheets ("xs_data"). Cells (year_address (b, 1), 6)

Debug. Print "ry1:" \& ry 1 . Address

Set ry $2=$ Worksheets ("xs_data"). Cells (year-address $(b, 2), 6)$

'Debug.Print "ry2:" \& ry2.Address

Set year = Worksheets ("xs_data"). Cells (year_address (b, 1), 1)

Debug.Print "year:" \& year.Address

Set $\mathrm{xrange}=$ Range $(\mathrm{rx} 1, \mathrm{rx} 2)$

Debug.Print "xrange:" \& xrange.Address

Set yrange $=$ Range( $\mathrm{ry} 1$, ry 2$)$

Debug.Print "yrange:" \& yrange. Address

TheChart. SeriesCollection. Add Source:=Range("hundreds") 


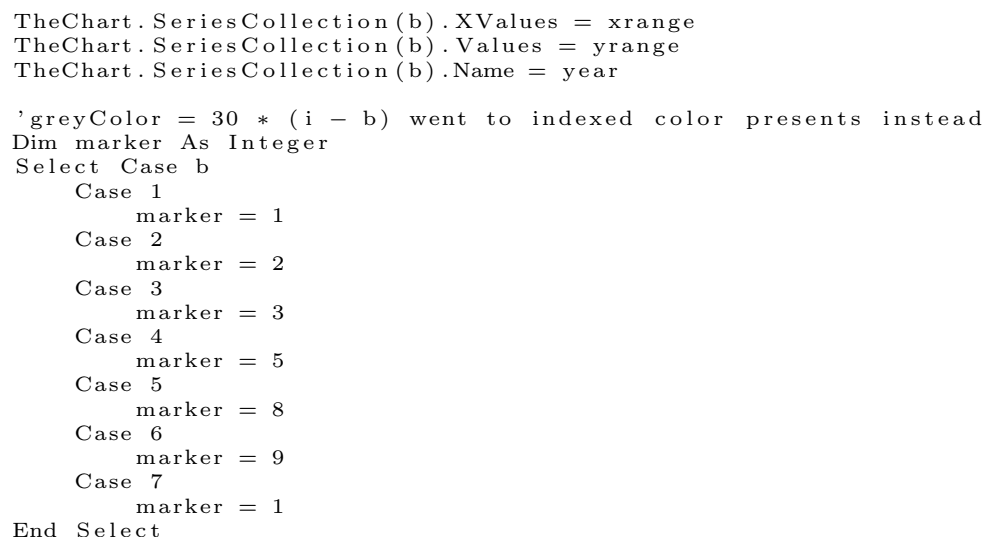

With TheChart. Seriescollection (b)

Border. ColorIndex $=(17+\mathrm{i}-\mathrm{b})$ 'RGB(greyColor, greyColor, greyColor)

Border. Weight $=$ xlMedium

MarkerStyle $=$ marker

MarkerBackgroundColorIndex $=x$ lNone

MarkerForegroundColorIndex $=(17+\mathrm{i}-\mathrm{b})$ 'RGB(greyColor, greyColor, greyColor) End With

Next b

Dim ChartName As Variant

Dim Section As String

Dim Number As String.

Section = Range("main! group"). Value

Number = Range ("main! number"). Value

ChartName = "Little Creek X-Section " \& Section \& Number

TheChart. ChartTitle. Characters. Text $=$ ChartName

TheChart. Legend. Left $=325$

MovieFrame $=0$

'Worksheets ("main"). Activate

Call thalweg_calc

Call area_calc

Call meanelev_calc

End Sub

Sub deletecheck()

Set TheChart $=$ Worksheets ("main"). ChartObjects (1). Chart

For chartnum $=$ TheChart. SeriesCollection. Count To 1 Step TheChart. Seriescollection (chartnum). Delete

Next chartnum

End Sub

Sub selectxs ()
Range("xs_data! xs_data"). BorderAround Weight:=xlThin

End Sub

Sub Create_Excel_Palette_By_RGB (

Purpose: Assigns the RGB value to each of 56 colors available to Excel

'Special Notes: The Excel index values are not in logical order in Excel 97 and 2000 and 2002 .

'The index values as follows work row by row from the top and left to right within each row.

'The sixth row The first default row for charts

ActiveWorkbook. Colors $(17)=\operatorname{RGB}(255,140,0)$

ActiveWorkbook. Colors $(18)=\operatorname{RGB}(0,0,0)$

ActiveWorkbook. Colors $(19)=\operatorname{RGB}(50,50,50)$

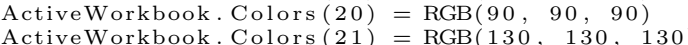

'swatch 1 from left

'swatch 2 from left

'swatch 3 from left 


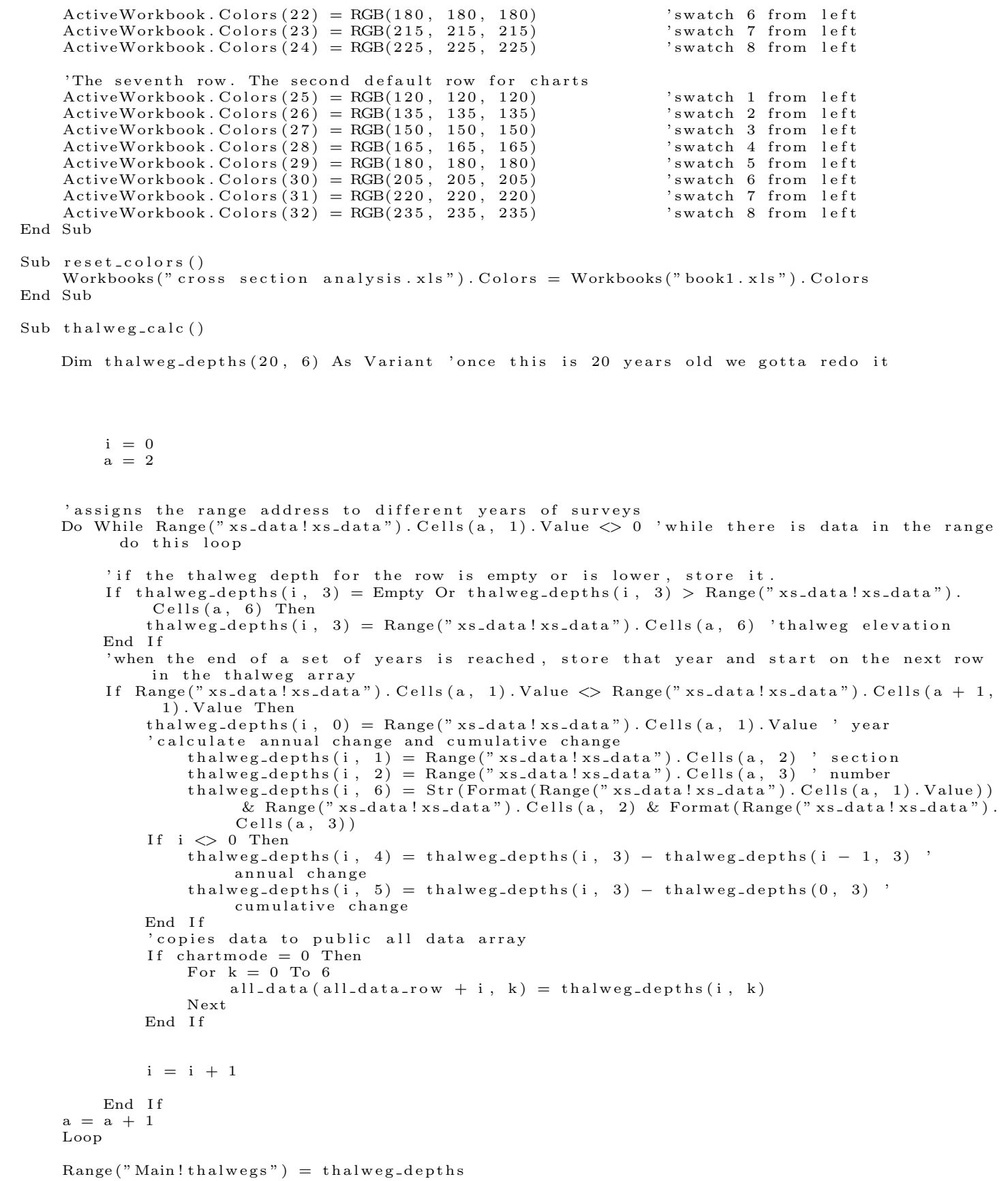

End Sub

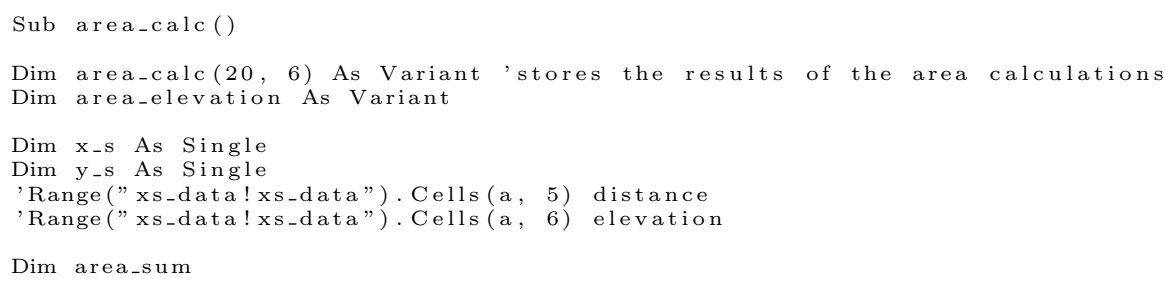




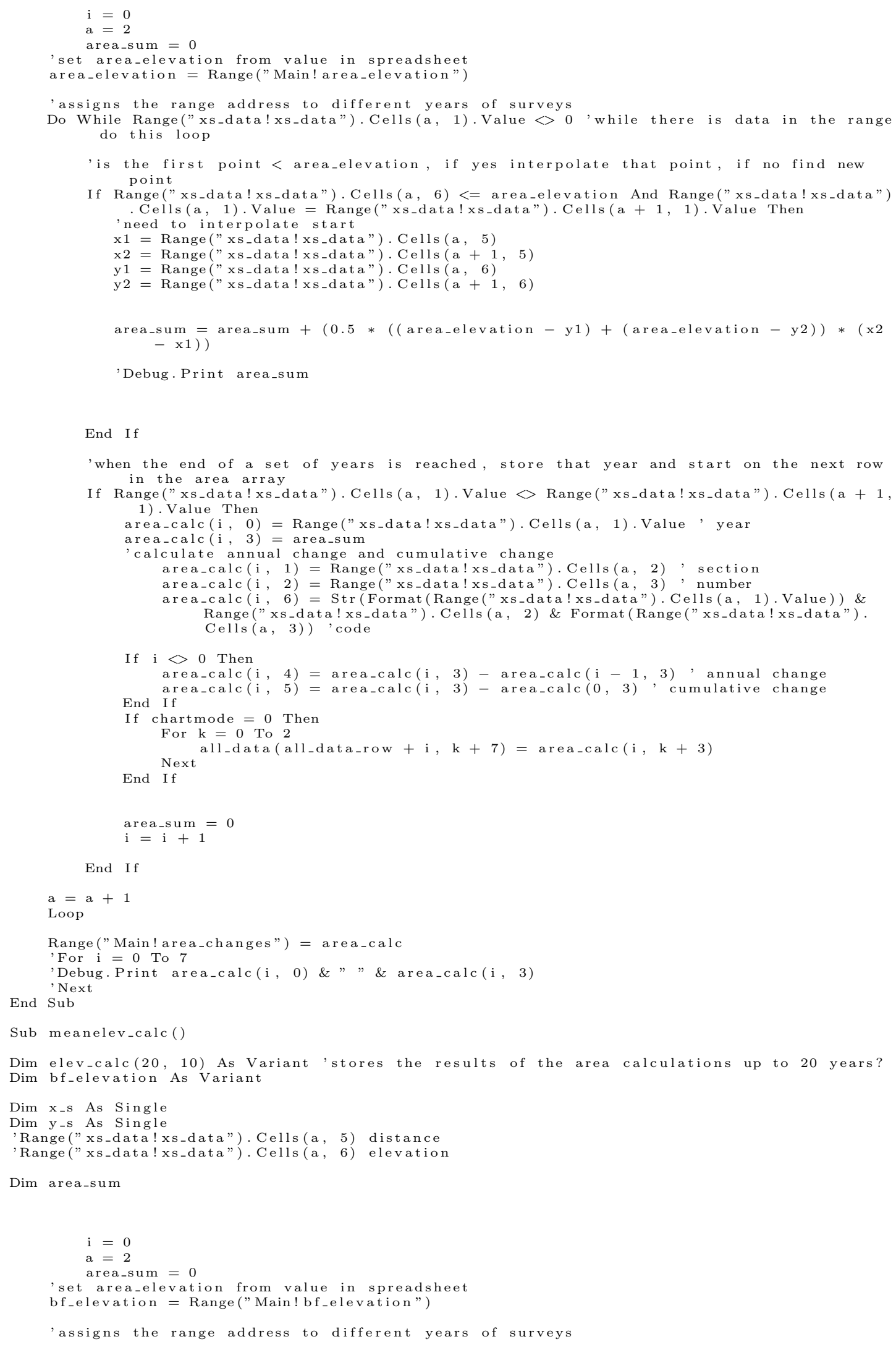


Do While Range("xs_data!xs_data"). Cells(a, 1). Value $<>0$ 'while there is data in the range do this loop

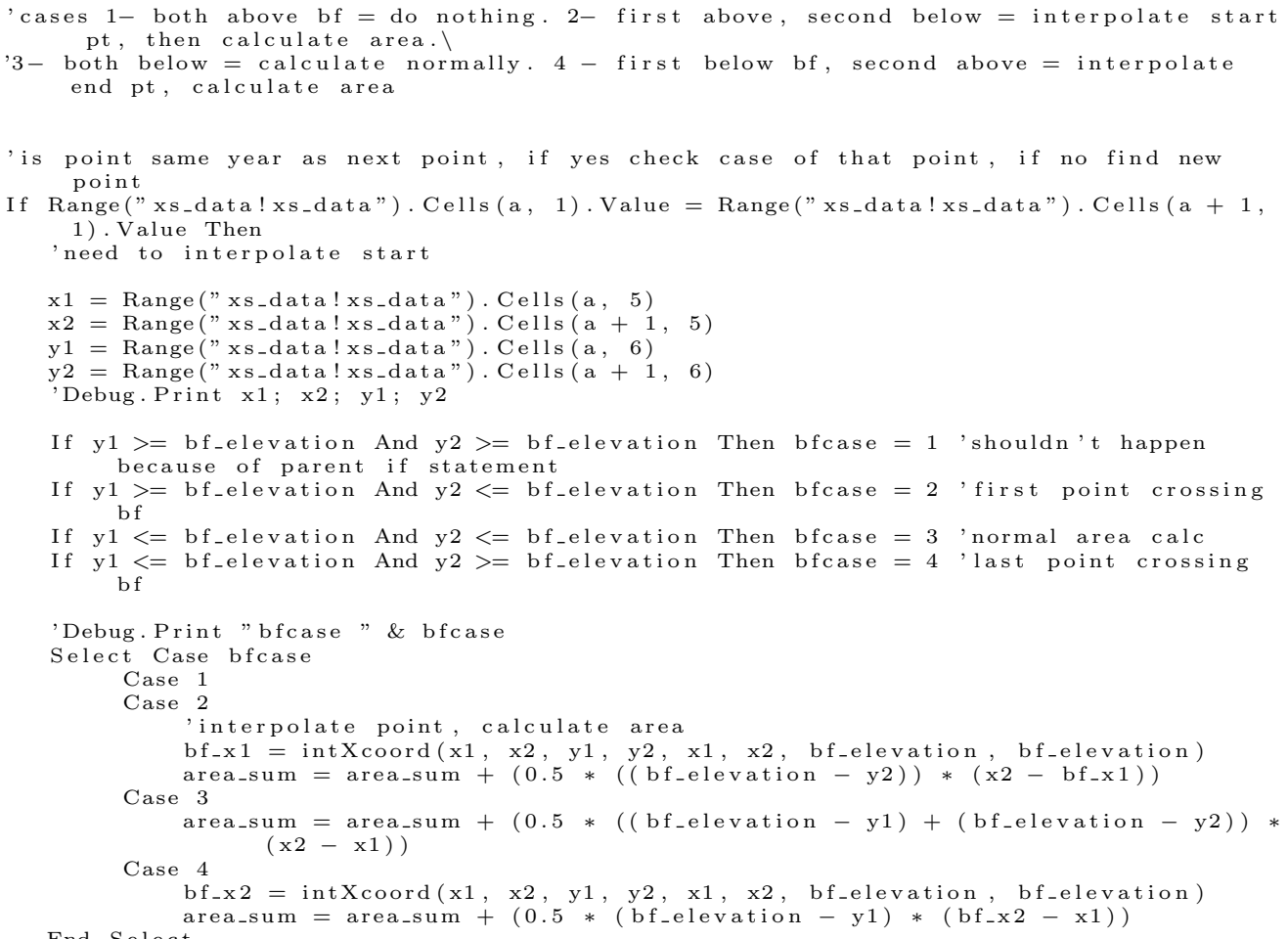
End Select

'Debug. Print "bf $f_{-} 1=" \& f_{-x} \& " \quad b f_{-x} 2 " \& f_{-} 2$

End If

'when the end of a set of years is reached, store that year and start on the next row in the area array

If Range("xs_data! xs_data"). Cells (a, 1). Value $<>$ Range("xs_data!xs_data"). Cells (a+ 1

1). Value Then

elev_calc $(i, 0)=$ Range("xs_data! xs_data"). Cells(a, 1) Value , year

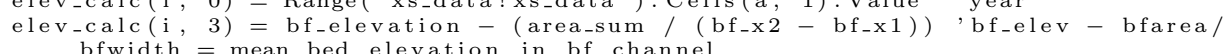

elev_calc $(i, 7)=$ area_sum, area under bf elevation

elev_calc $(\mathrm{i}, 10)=\mathrm{bf}_{-} 2_{2}-\mathrm{bf}_{-\mathrm{x} 1}$, bf width

calculate annual change and cumulative change

elev_calc $(\mathrm{i}, 1)=$ Range ("xs_data! xs_data"). Cells (a, 2), section

elev_calc $(\mathrm{i}, 2)=$ Range ("xs_data! xs_data"). Cells (a, 3) ' number

elev_calc $(\mathrm{i}, 6)$ Format (Range("xs_data! xs_data"). Cells (a, 1). Value) \& Range(" xs_data!xs_data"). Cells (a, 2) \& Format (Range("xs_data!xs_data"). Cells (a,

If $i<0$ ) ' $\operatorname{cod}$

elev_calc $(\mathrm{i}, 4)=$ elev_calc $(\mathrm{i}, 3)-$ elev_calc $(\mathrm{i}-1,3)$ ' annual change

elev_calc $(i, 5)=$ elev_calc $(i, 3)$ - elev_calc $(0,3)$, cumulative change

elev-calc $(\mathrm{i}, 8)$ elev-calc $(\mathrm{i}, 7)-$ elev-calc $(\mathrm{i}-1,7)$, annual change bf

elev_calc $(i, 9)=$ elev_calc $(i, 7)-$ elev_calc $(0,7)$, cumulative change bf

End If

If chartmode $=0$ Then

For $\mathrm{k}=0$ To

all_data( all_data_row $+\mathrm{i}, \mathrm{k}+10)=$ elev_calc $(\mathrm{i}, \mathrm{k}+3)$

all-data (all-data-row $+\mathrm{i}, \mathrm{k}+13)=\mathrm{elev-calc}(\mathrm{i}, \mathrm{k}+7)$ 'skips the code Next column

End If

all_data( all_data_row $+\mathrm{i}, 16)=\operatorname{elev-calc(i,10)}$ 'bf width

area_sum $=0$

$\mathrm{i}=\mathrm{i}+1$ 
End If

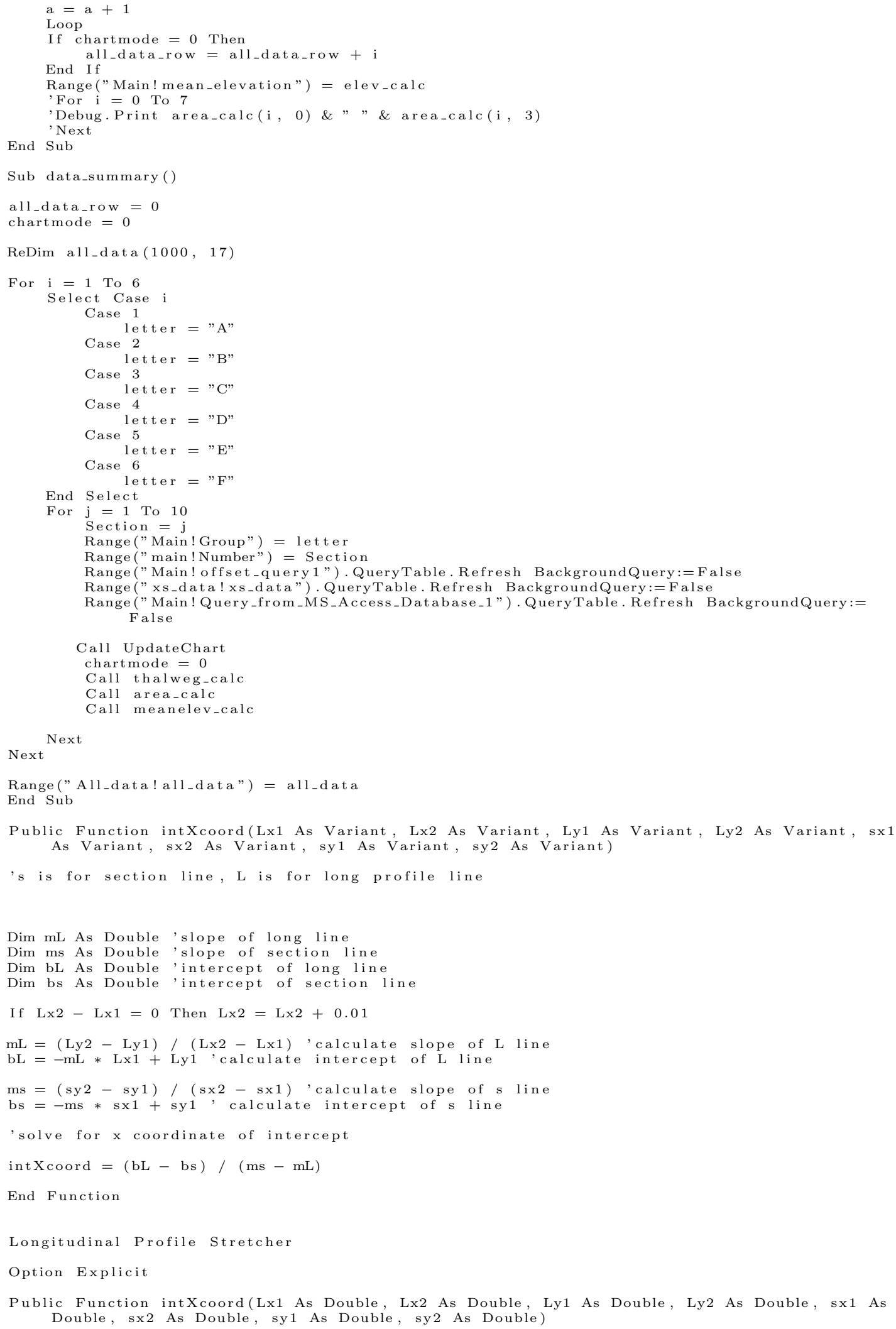


's is for section line, $L$ is for long profile line

Dim $\mathrm{mL}$ As Double 'slope of long line

Dim ms As Double, slope of section line

Dim bL As Double, intercept of long line

Dim bs As Double, intercept of section line

$\mathrm{mL}=(\operatorname{Ly} 2-\mathrm{Ly} 1) /(\mathrm{Lx} 2-\mathrm{Lx} 1)$ 'calculate slope of L line

$\mathrm{bL}=-\mathrm{mL} * \mathrm{Lx} 1+\mathrm{Ly} 1$ ' calculate intercept of L line

$\mathrm{ms}=(\mathrm{sy} 2-\mathrm{sy} 1) /(\mathrm{sx} 2-\mathrm{sx} 1)$ 'calculate slope of s line

$\mathrm{bs}=-\mathrm{ms} * \mathrm{sx} 1+\mathrm{sy} 1$, calculate intercept of s line

'solve for $x$ coordinate of intercept

intXcoord $=(\mathrm{bL}-\mathrm{bs}) /(\mathrm{ms}-\mathrm{mL})$

End Function 